NISTIR 5024

COMPOSITE STRUTS FOR SMES PLANTS

Edited by

R.P. Reed

J.D. McColskey

QC 



\section{COMPOSITE STRUTS FOR SMES PLANTS}

Edited by:

R.P. Reed*

J.D. McColskey

Materials Reliability Division

Materials Science and Engineering Laboratory

National Institute of Standards and Technology

325 Broadway

Boulder, Colorado 80303-3328

${ }^{*}$ Cryogenic Materials, Inc.

2625 Iliff

Boulder, Colorado 80303-7017

Sponsored by:

Defense Nuclear Agency

under DNA IACRO No. 91-854

and Work Unit 00034

October 1994

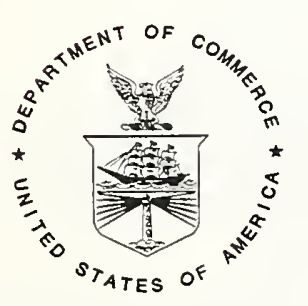

U.S. DEPARTMENT OF COMMERCE, Ronald H. Brown, Secretary TECHNOLOGY ADMINISTRATION, Mary L. Good, Under Secretary for Technology NATIONAL INSTITUTE OF STANDARDS AND TECHNOLOGY, Arati Prabhakar, Director 
Certain commercial equipment, instruments, or materials are identified in this report in order to adequately specify the experimental procedure. Such identification does not imply recommendation or endorsement by the National Institute of Standards and Technology, nor does it imply that the materials or equipment identified are necessarily the best available for the purpose. 


\section{Contents}

I. Executive Summary . . . . . . . . . . . . . . . . . . . . . I-1

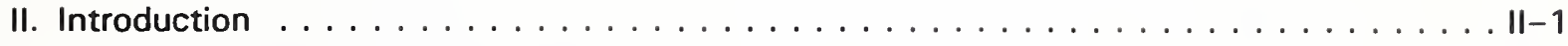

III. Properties of Neat-Resin Systems $\ldots \ldots \ldots \ldots \ldots \ldots \ldots \ldots \ldots \ldots \ldots$. . . . . . . . . . . .

1. Summary . . . . . . . . . . . . . . . . . . . . . . . III-1

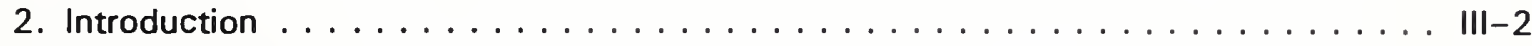

3. Production Characteristics $\ldots \ldots \ldots \ldots \ldots \ldots \ldots \ldots \ldots \ldots \ldots \ldots \ldots$. . . . . . . . . . .

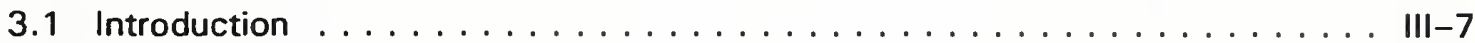

3.2 Resin Types . . . . . . . . . . . . . . . . . . . . . . III-7

3.3 Procedures ............................ III-7

3.3.1 Production Techniques $\ldots \ldots \ldots \ldots \ldots \ldots \ldots \ldots \ldots \ldots$ III-7

3.3.1.1 Flat Plates . . . . . . . . . . . . . . . . . III-7

3.3.1.2 Rods .......................... III-8

3.3.2 Resin Characteristics . . . . . . . . . . . . . . . . . III-9

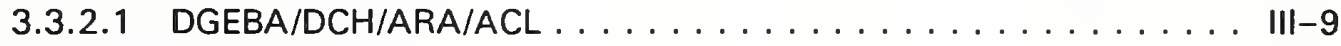

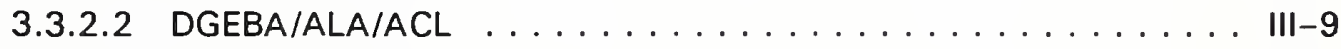

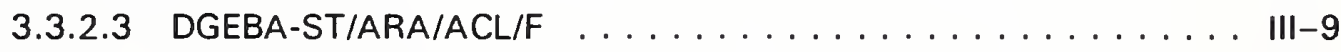

3.3.2.4 DGEBF/CTBN/AN .................. III-10

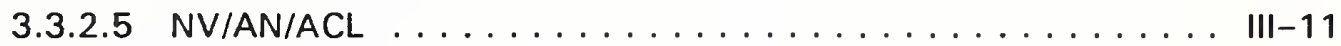

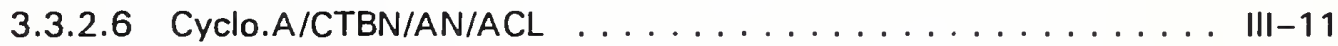

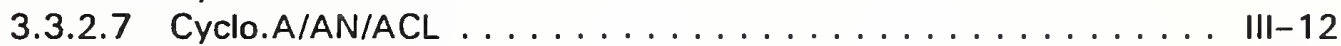

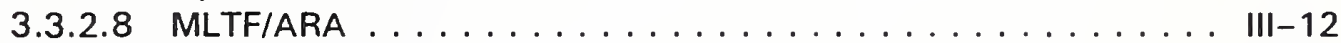

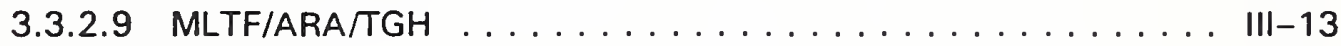

3.3.2.10 CE/VT/Co.AA ................... III-13

3.3.2.11 CE ........................ . . . $\ldots \ldots$

3.3.2.12 CE(PT) ....................... III-15

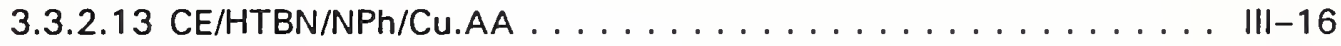

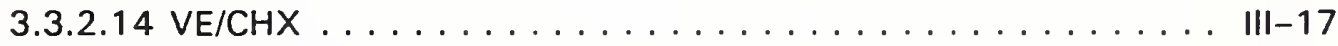

3.3.2.15 VE-EL/MEKP . . . . . . . . . . . . . . . III-18

3.3.2.16 VE/CHP/F . . . . . . . . . . . . . . . . III-18

3.3.2.17 VE/CHP/CoNap/2,4-P . . . . . . . . . . . . . . . III-19

3.3.2.18 PE/MEKP ..................... III-19

3.4 Discussion . . . . . . . . . . . . . . . . . . . . III-20

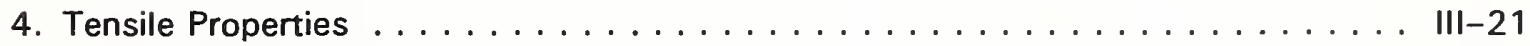

4.1 Introduction ......................... III-22

4.2 Literature Review . . . . . . . . . . . . . . . . . . III-22

4.3 Resins . . . . . . . . . . . . . . . . . . . . III-26

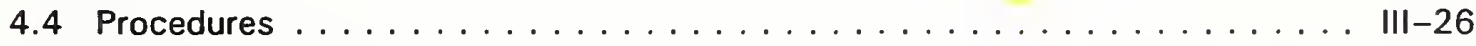

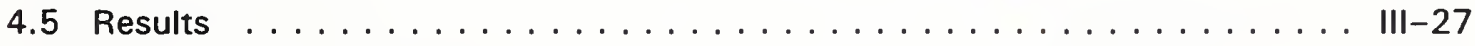

4.5 .1 Young's Modulus . . . . . . . . . . . . . . . . III-27

4.5 .2 Poisson's Ratio . . . . . . . . . . . . . . . . . . III-36

4.5 .3 Ultimate Tensile Strength . . . . . . . . . . . . . . . III-37

4.5 .4 Fractography . . . . . . . . . . . . . . . . . . . . III-54

4.6 Discussion .......................... III-66 
5. Shear Properties . . . . . . . . . . . . . . . . . . . . . III-72

5.1 Introduction .......................... III-72

5.2 Review of Literature $\ldots \ldots \ldots \ldots \ldots \ldots \ldots \ldots \ldots \ldots \ldots \ldots$ III-72

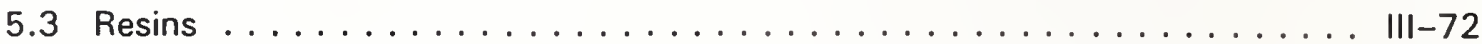

5.4 Procedures .............................. . . . .73

5.4.1 Specimen Preparation ................... III-73

5.4 .2 Test Apparatus and Method . . . . . . . . . . . . . . . . III-73

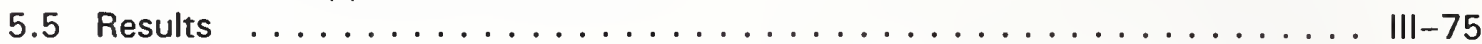

5.6 Discussion . . . . . . . . . . . . . . . . . . . III-82

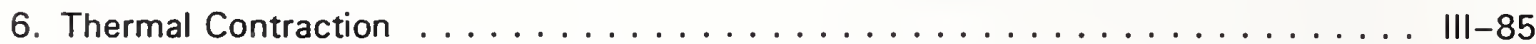

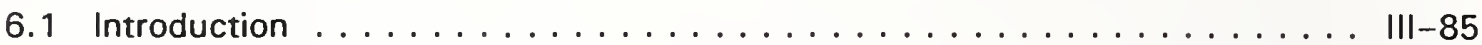

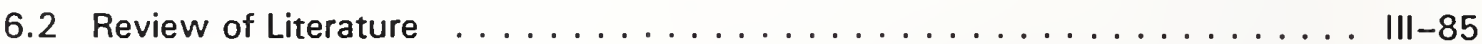

6.3 Resins . . . . . . . . . . . . . . . . . . . . . . III-87

6.4 Procedures . . . . . . . . . . . . . . . . . . . . . . III-87

6.4.1 Description of Equipment . . . . . . . . . . . . . . . III-88

6.4 .2 Methods . . . . . . . . . . . . . . . . . . . . . . III-88

6.4 .3 Calibration . . . . . . . . . . . . . . . . . . . III-89

6.5 Results .............................. III-92

6.6 Discussion ............................ . $111-100$

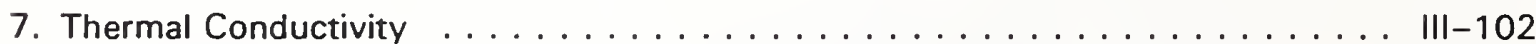

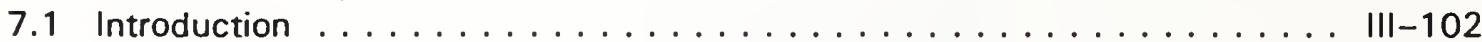

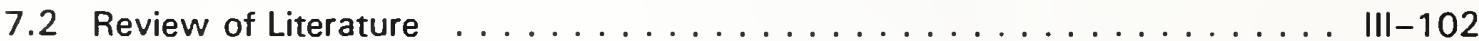

7.3 Description of Specimens . . . . . . . . . . . . . . . . . III-103

7.4 Procedure ................................... . III-104

7.4.1 Description of Apparatus .................... III-104

7.4.2 Experimental Procedure ..................... . III-105

7.4.3 Computation of Thermal Conductivity ................ . III-106

7.4.4 Accuracy and Reproducibility of Measurements . . . . . . . . . . . III-106

7.5 Results ............................. III-106

7.6 Discussion ............................ III-117

8. Thermal Cycling . . . . . . . . . . . . . . . . . . . . . . III-122

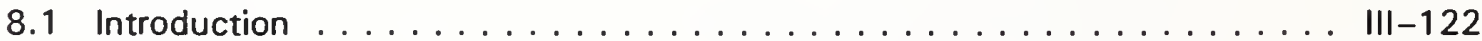

8.2 Materials .............................. . . . . 122

8.3 Procedures ............................. $\ldots \ldots 122$

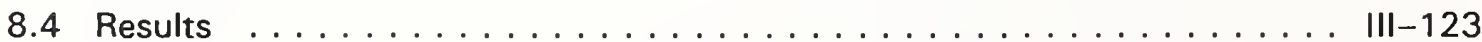

8.5 Discussion . . . . . . . . . . . . . . . . . . . . . III-124

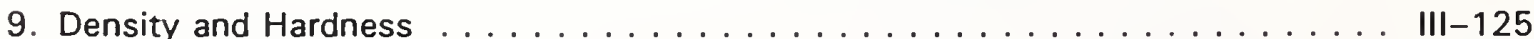

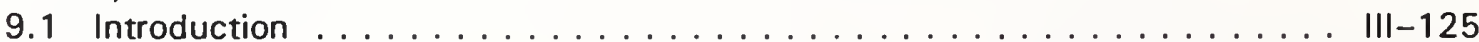

9.2 Hardness . . . . . . . . . . . . . . . . . . . . . . . III-125

9.3 Density . . . . . . . . . . . . . . . . . . . . . . . . III-125

9.4 Comparison of Hardness and Density . . . . . . . . . . . . . . . . III-128

10. Dynamic Elastic Properties . . . . . . . . . . . . . . . . . . . . . III-130

10.1 Introduction .......................... III-130

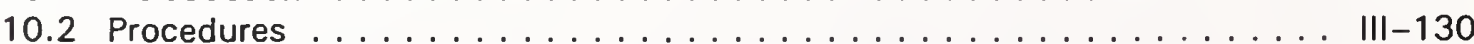

10.2.1 Materials ......................... III-130

10.2.2 Measurement Methods . . . . . . . . . . . . . . . . III-131

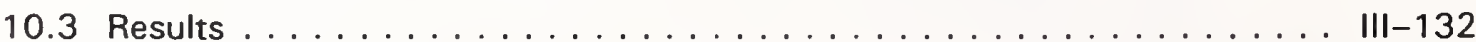

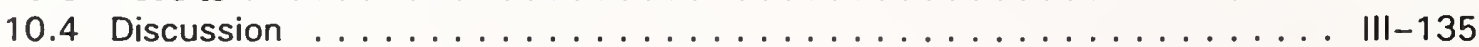


III. Properties of Neat-Resin Systems, continued

11. Discussion $\ldots \ldots \ldots \ldots \ldots \ldots \ldots \ldots \ldots \ldots \ldots \ldots \ldots \ldots \ldots \ldots$ III-137

11.1 Mechanical Properties . . . . . . . . . . . . . . . . . III-137

11.1 .1 Elastic Moduli . . . . . . . . . . . . . . . . . . . III-137

11.1.2 Tensile and Shear Strength . . . . . . . . . . . . . . . . III-144

11.1.3 Relationships between Elastic and Strength Properties . . . . . . III-147

11.1.4 Effects of Flaws on Mechanical Properties . . . . . . . . . . III-151

11.2 Thermal Properties . . . . . . . . . . . . . . . . . . . III-151

11.3 Effects of Chemical Composition . . . . . . . . . . . . . . . III-154

11.4 Resin Selection . . . . . . . . . . . . . . . . . . . III-154

12. References .......................... III-156

13. Appendixes .............................. . III-160

Appendix A. Tensile Strength, Elastic Modulus, and Poisson's Ratio . . . . . . . III-160

Appendix B. Shear Properties . . . . . . . . . . . . . . . . . . III-169

IV. Review of the Low-Temperature Properties of E-Glass $\ldots \ldots \ldots \ldots \ldots \ldots \ldots$. . . . IV-1

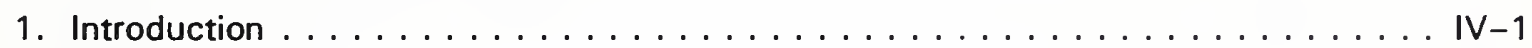

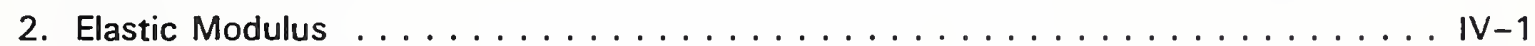

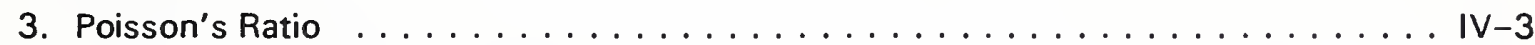

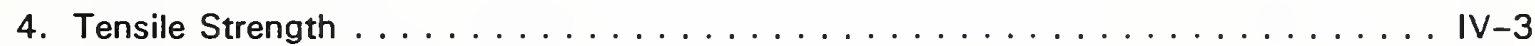

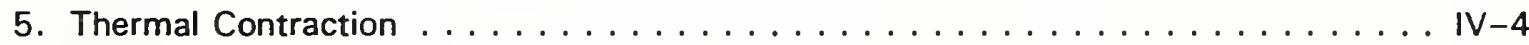

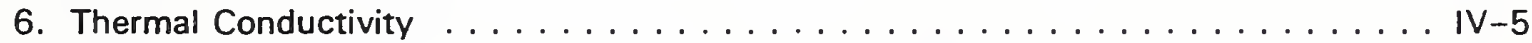

7. References .......................... IV-6

V. Properties of Directionally Reinforced Composites $\ldots \ldots \ldots \ldots \ldots \ldots \ldots \ldots \ldots$. . . . . .

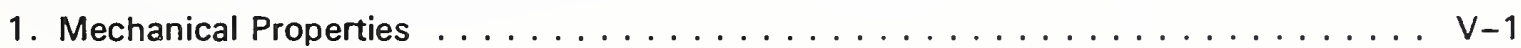

1.1 Introduction ...........................

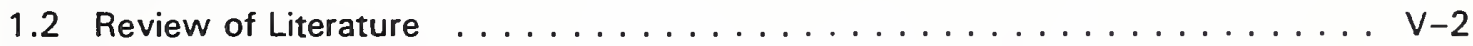

1.3 Materials .............................

1.4 Procedures ..............................

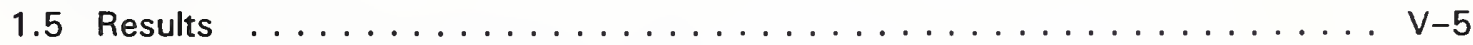

1.5.1 Epoxy-Resin-Matrix Composites . . . . . . . . . . . . . V-5

1.5 .2 Cyanate-Ester-Resin-Matrix Composites .............. $v-12$

1.6. Discussion .......................... . . . . . . . .

1.6.1 Epoxy-Resin-Matrix Composites . . . . . . . . . . . . . . V V-13

1.6.2 Cyanate-Ester-Resin-Matrix Composites .............. $v-13$

2. Thermal Contraction ........................ . . . .

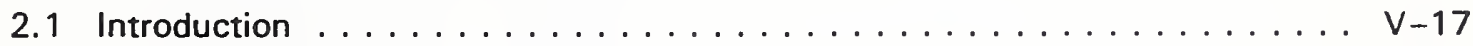

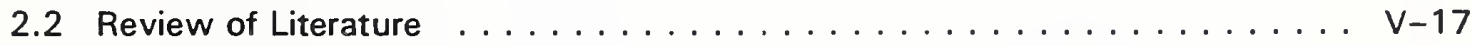

2.3 Materials ............................ $v-18$

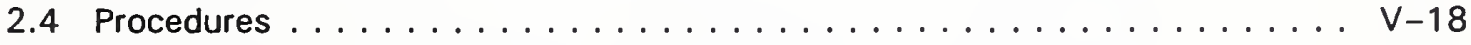

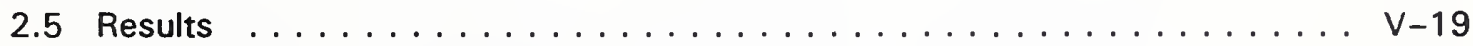

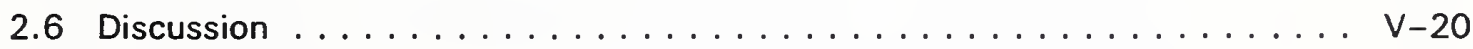

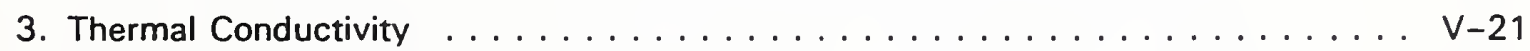

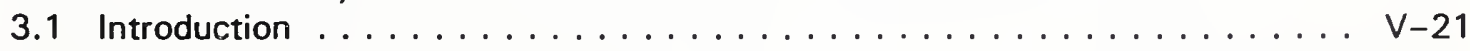

3.2 Review of Literature . . . . . . . . . . . . . . . . . . . . . V

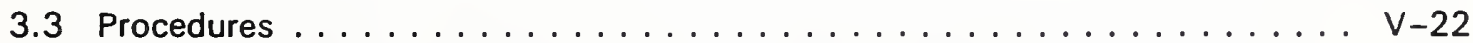

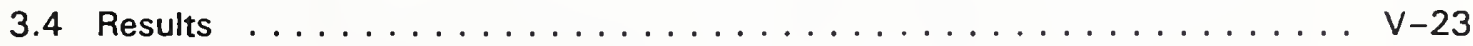

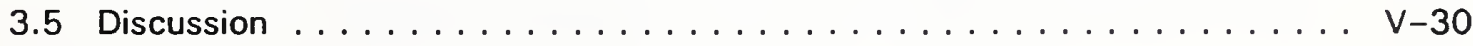


V. Properties of Directionally Reinforced Composites, continued

4. Interfacial Residual Thermal Stress . . . . . . . . . . . . . . . . . V V-31

4.1 Introduction ............................

4.2 Specimen and Test-Method Development ................. . $V_{-32}$

4.3 Experimental Materials . . . . . . . . . . . . . . . . . . . . . V

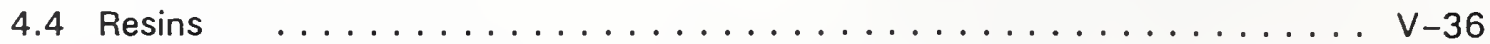

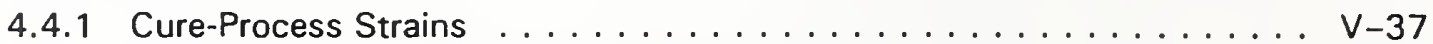

4.4 .2 Cryogenic Cooling Strains . . . . . . . . . . . . . . . V V-38

4.4 .3 Total Strain . . . . . . . . . . . . . . . . . . . . . . . V-39

4.5 Analysis of Cure-Cycle Strain Curves . . . . . . . . . . . . . . . . . . V-40

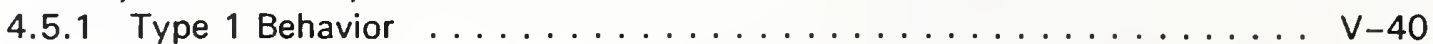

4.5 .2 Type 2 Behavior ....................... $V-40$

4.5 .3 Type 3 Behavior ....................... $v-43$

4.6 Analysis of the Cryogenic Cooling Curve ................ .

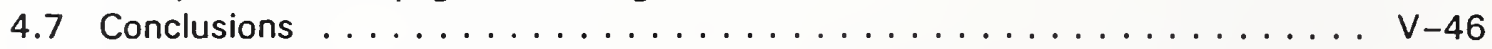

4.8 Recommendations for Future Work . . . . . . . . . . . . . . . V V-47

5. References .............................. V-49

VI. Properties of Struts $\ldots \ldots \ldots \ldots \ldots \ldots \ldots \ldots \ldots \ldots \ldots \ldots \ldots \ldots \ldots$

1. Introduction ............................ VI-2

2. Review of Literature . . . . . . . . . . . . . . . . . . VI-2

3. Materials .............................. . . . .

4. Procedures .............................. . . . . .

4.1 Tube Fabrication Methods . . . . . . . . . . . . . . . . . VI-4

4.1 .1 Filament Winding . . . . . . . . . . . . . . . . VI-4

4.1 .2 Pultrusion ......................... VI-5

4.1.3 Rubber Expansion ....................... VI-6

4.2 Specimens ........................... . . 6

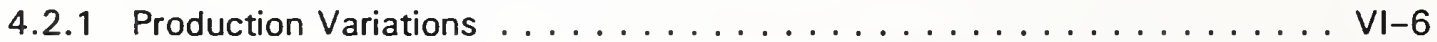

4.2 .2 Lay-ups ............................ . . . .

4.2 .3 Specimen Description . . . . . . . . . . . . . . . . . . . VI-8

4.2 .4 Specimen Instrumentation . . . . . . . . . . . . . . . . . VI-9

4.2 Tests . . . . . . . . . . . . . . . . . . . . . . . VI-11

4.2 .1 Compression Tests ....................... . . . 11

4.2 .2 Fatigue Tests ........................ VI-11

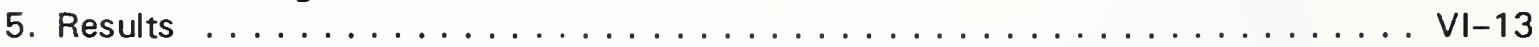

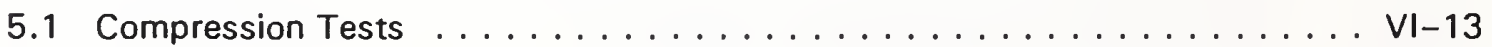

5.2 Fatigue Tests .......................... . . . . . . . . .

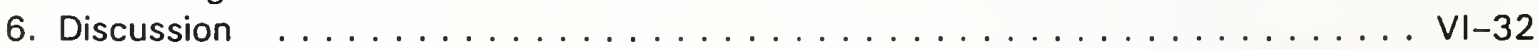

6.1 Fiber Lay-up and Temperature . . . . . . . . . . . . . . . VI-32

6.2 Type of $\operatorname{Resin} \ldots \ldots \ldots \ldots \ldots \ldots \ldots \ldots \ldots \ldots \ldots \ldots$

6.3 Tube Geometry . . . . . . . . . . . . . . . . . . . . . . VI-37

6.4 Filament Winding versus Pultrusion . . . . . . . . . . . . . . . VI-40

6.5 Strain to Failure . . . . . . . . . . . . . . . . . . . . . VI-41

6.6 Optimization of Strength and Thermal Conductivity . . . . . . . . . . VI-42

6.7 Comparison of Strengths . . . . . . . . . . . . . . . . VI-42

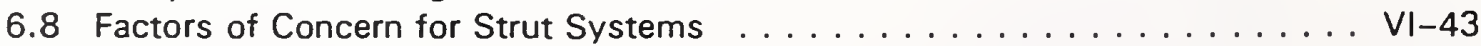

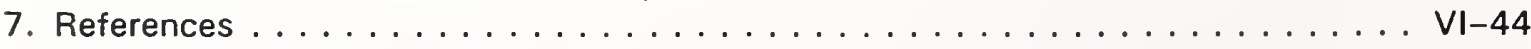

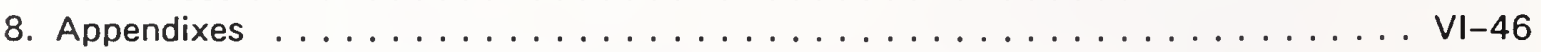

Appendix A. Tube Construction . . . . . . . . . . . . . . . . VI-46

Appendix B. Photographs of Test Equipment and Specimens . . . . . . . . . VI-71 
VII. Analyses of the Response of Fiber-Reinforced Composite Cylinders to Loads . . . . . . VII-1

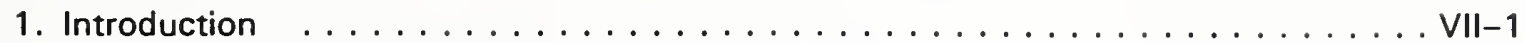

2. Problem Statement . . . . . . . . . . . . . . . . . . . . VII-2

3. Governing Equations . . . . . . . . . . . . . . . . . . . . . VII-5

4. Boundary Conditions . . . . . . . . . . . . . . . . . . . . . VII-6

5. General Solution of the Governing Equations . . . . . . . . . . . . . . VII-8

5.1 Displacements, $0^{\text {th }}$ Terms . . . . . . . . . . . . . . . . . VII-8

5.2 Displacements, $t^{\text {th }}$ Term of the Unstarred Series . . . . . . . . . . . VII-11

5.3 Displacements, $i^{\text {th }}$ Term of the Starred Series . . . . . . . . . . . . VII-13

6. Solutions for the Displacements, Stresses, and Strains . . . . . . . . . . . . . . VII-14

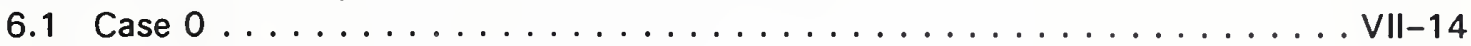

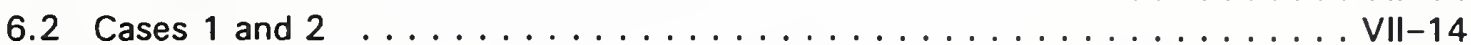

6.3 Cases 3 through $6 \ldots \ldots \ldots \ldots \ldots \ldots \ldots \ldots \ldots \ldots \ldots$. . . . . . . . . . . . . . . . . . .

6.4 General Solutions . . . . . . . . . . . . . . . . . . . . VII-21

6.5 Method of Solution . . . . . . . . . . . . . . . . . . VII-21

7. Sample Problems . . . . . . . . . . . . . . . . . . . . . . . VII-25

7.1 Composite Cylinder Subjected to Axial Displacement . . . . . . . . . . . . V VII-26

7.2 Composite Cylinder Subjected to Temperature Load . . . . . . . . . . . . . . VII-26

8. Failure of Axially Loaded Composite Cylinders . . . . . . . . . . . . . . . . VII-31

9. References . . . . . . . . . . . . . . . . . . . . . . . . . . VII-37

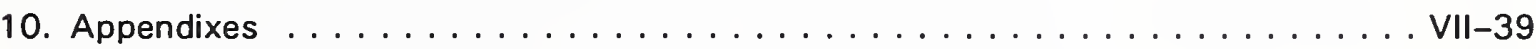

Appendix A. Axial Temperature Distribution in the Cylinder . . . . . . . . . VII-39

Appendix B. The Series of the Temperature and Surface Loads . . . . . . . . . . VII-42

Appendix C. Solution of the Differential Equation $5.6 \ldots \ldots \ldots \ldots \ldots \ldots$. . . . . . . . . .

Appendix D. Sample Problems for the CYLINDER 2 Code . . . . . . . . . VII-48

VIII. Analysis of End-Fitting-Induced Strains

in Axially Loaded Glass-Epoxy Cylinders . . . . . . . . . . . . . . . . . . . . VIII-1

1. Introduction ............................. VIII-1

2. Procedures $\ldots \ldots \ldots \ldots \ldots \ldots \ldots \ldots \ldots \ldots \ldots \ldots \ldots \ldots \ldots \ldots \ldots$ VIII-2

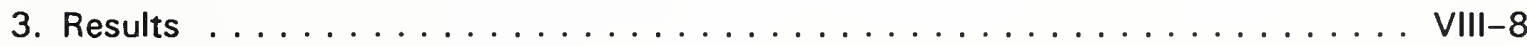

3.1 [10] Cylinders . . . . . . . . . . . . . . . . . . . . . . VIII-12

3.1.1 Thermally Induced Strain - Baseline-Design End Fitting . . . . . . . . . VIII-12

3.1.2 Thermally Induced Strain - Alternative-Design End Fitting . . . . . . . . VIII-16

3.1.3 Strains due to Load and Low-Temperature Material Properties -

Baseline-Design End Fitting . . . . . . . . . . . . . . . . . . VIII-16

3.1.4 Strains due to Load and Low-Temperature Material Properties -

Alternative-Design End Fitting . . . . . . . . . . . . . . . VIII-21

3.1.5 Strains due to Load and Room-Temperature Material Properties -

Baseline-Design End Fitting . . . . . . . . . . . . . . . VIII-23

3.1.6 Strains due to Load and Room-Temperature Material Properties -

Alternative-Design End Fitting . . . . . . . . . . . . . VIII-25

$3.2[82 / 45 / 10]$ Cylinders . . . . . . . . . . . . . . . . . . . . . VIII-27

3.2.1 Thermally Induced Strains - Baseline-Design End Fitting . . . . . . . . VIII-27

3.2.2 Thermally Induced Strain - Alternative-Design End Fitting . . . . . . . . VIII-31

3.2.3 Strains due to Load and Low-Temperature Material Properties -

Baseline-Design End Fitting . . . . . . . . . . . . . . . . . VIII-35

3.2.4 Strains due to Load and Low-Temperature Material Properties -

Alternative-Design End Fitting .................... VIII-40

3.2.5 Strains due to Load and Room-Temperature Material Properties -

Baseline-Design End Fitting . . . . . . . . . . . . . . . . . . VIII-43

3.2.6 Strains due to Load and Room-Temperature Material Properties -

Alternative-Design End Fitting . . . . . . . . . . . . VIII-48

3.3 Thinner Cylinders . . . . . . . . . . . . . . . . . . . VIII-51 
VIII. Analysis of End-Fitting-Induced Strains in Axially Loaded Glass-Epoxy Cylinders, continued

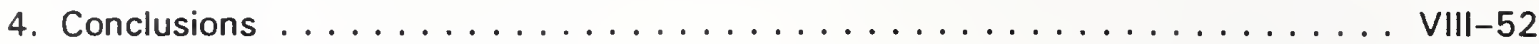

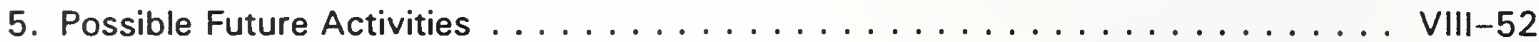

6. References ............................... VIII-52

7. Appendixes ........................... VIII-53

Appendix A. Determination of Ply Properties from Micromechanics . . . . . . VIII-53

Appendix B. Equivalent Laminate Properties . . . . . . . . . . . . . . . VIII-62

Appendix C. Strain Response for Thinner Laminates . . . . . . . . . . . VIII-76

IX. Failure and Design Analyses for SMES Fiberglass Tubes $\ldots \ldots \ldots \ldots \ldots \ldots \ldots$

1. Introduction ............................. IX-1

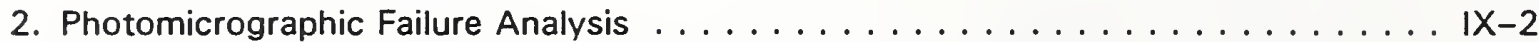

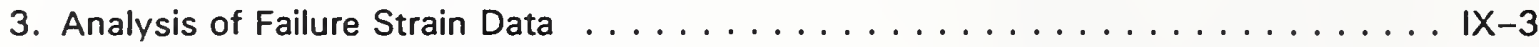

4. Material Elastic Properties Estimation . . . . . . . . . . . . . . . . . . . . IX-20

5. Tube Design Studies . . . . . . . . . . . . . . . . . . . . . IX-22

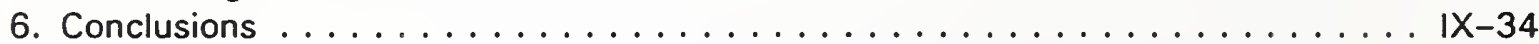

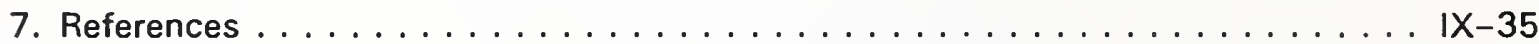

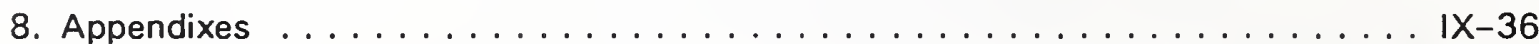

Appendix A. Micrographic Examination of Failed SMES Tube Samples . . . . . . IX-36

Appendix B. FORTRAN Program for Estimating Layer Elastic Properties

of Fiberglass Tubes . . . . . . . . . . . . . . . . . . . IX-77 


\title{
Composite Struts for SMES Plants
}

\author{
R.P. Reed* \\ J.D. McColskey \\ Materials Reliability Division \\ National Institute of Standards and Technology \\ Boulder, Colorado \\ ${ }^{*}$ Cryogenic Materials, Inc. \\ Boulder, Colorado
}

This report summarizes NIST research efforts in the development of composite struts for Superconducting Magnetic Energy Storage (SMES) plants. Large SMES plants require radial structural support for the superconducting coils to the outer walls to assist in restraining the coil cool-down and Lorentz forces. The struts proposed for this structural support must have good axial compressive strength and low axial thermal conductivity, as well as low cost.

A variety of E-glass fibers, resins, lay-up angles and manufacturing methods were evaluated in the course of this program, at temperatures ranging from 295 to $76 \mathrm{~K}$. Analysis methods are presented that describe the response of fiber-reinforced composite cylinders and end-fitting designs to axially varying mechanical and thermal loads. Finally, failure-mode and tube-design studies were conducted to optimize support-tube design based on minimum cross-sectional area with allowable strain values used as design constraints.

Key words: composite; compression; cryogenic; fibers; resin; struts; superconductivity; supports; tubes

\section{Executive Summary}

A comprehensive program was undertaken to improve the compressive strength and technology base of glass/resin tubes for use in future Superconducting Magnetic Energy Storage (SMES) plants. Because these tubes must restrain the cool-down and Lorentz forces of the superconducting magnet coils, the program emphasized the low-temperature mechani$\mathrm{cal}$ and thermal properties of neat resins, unidirectional composites, and tubes of various fiber lay-ups. The thermal and mechanical properties of E-glass fibers, which were used as the tube reinforcement, were critically reviewed. Eighteen different epoxy, vinyl-ester, polyester, and cyanate-ester resin systems were tested. Five different epoxy, vinyl-ester, and cyanate-ester resin systems were used for tube construction. Two methods of tube production, filament-winding and pultrusion, were also evaluated. 
In general, the compressive strengths of E-glass/resin-matrix composite tubes are about $50 \%$ higher at low temperatures than at room temperature. Lay-ups that produced the strongest tubes contained fiber layers with near $-0^{\circ}$ (axial) orientation plus a few layers with near $-90^{\circ}$ (hoop) orientation. The strongest tubes were those that consisted of filament-wound layers of epoxy resin (DGEBA) reinforced with E-glass. Nevertheless, vinyl-ester and polyester resins should be considered on the basis of resin and production costs.

Tubes were analyzed, on the basis of strain and stress criteria, to predict the thermal and mechanical performance of struts, to assess the possibility of predicting compressive failure based on strain readings at lower stress levels, and to study end fixtures.

A relatively large amount of scatter was observed in the mechanical-property data of both neat resins and tubes. The scatter of compressive-strength data for the tubes was judged to be related to material inconsistencies, such as porosity, resin-rich areas, and fiber waviness. The scatter of neat-resin tensile data corresponded to the square root of the size of the defects, such as pores and second-phase particles, that had been identified as crack initiation sites. For neat resins, extrapolation of the $295-\mathrm{K}$ and $76-$ to-4-K data sets to zero defect sizes provided inherent tensile-strength values of about $100 \mathrm{MPa}(14.5 \mathrm{ksi})$ at $295 \mathrm{~K}$ and $170 \mathrm{MPa}$ $(24.5 \mathrm{ksi})$ at 76 to $4 \mathrm{~K}$. The initiation of virtually all neat-resin failures at defect sites suggests that processing is a significant factor in the initiation of matrix microcracking and subsequent failure of composite tubes.

Mechanical failures in the strut system, which will operate in the temperature range 4 to $295 \mathrm{~K}$, are most probable at room temperature. It was determined that tubes can be produced in sizes up to $250 \mathrm{~mm}$ (10 in) in diameter with 480 to $500 \mathrm{MPa}$ (70 to $80 \mathrm{ksi}$ ) compressive strength at room temperature. At $10^{4}$ cycles, the compressive fatigue strength at room temperature is expected to be about $25 \%$ lower. To maintain this strength level, production techniques must be improved to reduce tube defects.

We recommend future research to establish acceptance criteria for tubes and tube systems (including end caps and thermal intercepts). This research should entail

1. development of a screening test to eliminate tubes with low strength

2. establishment of maximum operating stress levels

3. establishment of appropriate safety factors that consider structural redundancy, fatigue, and in-service inspection, repair, and replacement of SMES plant strut systems 


\title{
II. Introduction
}

\author{
R.P. Reed* \\ J.D. McColskey \\ Materials Reliability Division \\ National Institute of Standards and Technology \\ Boulder, Colorado \\ "Cryogenic Materials, Inc. \\ Boulder, Colorado
}





\title{
II. Introduction
}

\author{
R.P. Reed ${ }^{*}$ \\ J.D. McColskey \\ Materials Reliability Division \\ National Institute of Standards and Technology \\ Boulder, Colorado \\ ${ }^{*}$ Cryogenic Materials, Inc. \\ Boulder, Colorado
}

In 1987 a program was initiated to demonstrate the feasibility of superconducting magnetic energy storage (SMES) and to consider the construction of an engineering test model (ETM). It was primarily sponsored by the Strategic Defense Initiative Office (SDIO), partially supported by the Electric Power Research Institute (EPRI), and managed by the Defense Nuclear Agency (DNA). The program objectives were to assess the feasibility of using SMES technology to provide electrical power for defense purposes (SDIO) and to provide grid stability and control and load leveling to benefit the electric-power utilities (EPRI). During Phase I of this program, a $72 \mathrm{GJ}(20 \mathrm{MW} \cdot \mathrm{h})$ ETM was designed by two teams. These industrial teams were led by EBASCO Services and Bechtel National. Their final report for Phase I activities was submitted to DNA in 1991.

Large SMES plants require radial structural support from the superconducting coils to the outer walls to assist in restraining the coil cool-down and Lorentz forces. The inner columns of struts transmit most of the forces from coil contraction during cool-down to the inner trench wall, and the outer struts transmit the Lorentz forces produced during coil operation to the outer trench wall. Between 1,000 and 2,000 struts are estimated to be needed for the engineering test model (ETM), and as many as 30,000 have been estimated for full-scale plants. Typical sizes of struts range from about 0.15 to $0.30 \mathrm{~m}$ in diameter and from 1.5 to $2.5 \mathrm{~m}$ in length.

For operational efficiency, the struts must have good axial compressive strength and low axial thermal conductivity. Both teams selected E-glass-fiber-reinforced, epoxy-matrix composites as the structural material for the struts. Technical variables for property optimization include fiber lay-up, resin type, manufacturing method, and optimization of the manufacturing process. Problems were encountered by both teams in achieving strength levels in ETM-size struts comparable to those based on smaller coupon or tube tests. These results had not been anticipated, and neither team allocated adequate program resources to address this shortcoming.

This program continued the research on struts that was initiated by both industrial teams during Phase I. It was needed for the following reasons: Support struts are an essential component of SMES plants; they contribute substantially to capital and operational costs and to plant safety and reliability. Neither industrial team developed a technical basis for analyses, production of full-scale support struts, or improvement of compressive strengths of larger struts. Since many struts are required, cost savings are achievable through optimization of strut design, materials, production technique, and performance. 
Our program was divided into four principal tasks:

1. Various generic classes of resins that are suitable for either filament-winding or pultrusion manufacturing processes were tested at room and low temperatures. In all, eighteen different resins-including epoxy, cyanate-ester, vinyl-ester, and polyester systems-were studied.

2. Properties of composite plates with unidirectionally oriented E-glass fibers in both epoxy and cyanate-ester-resin matrices were measured at 295, 76, and $4 \mathrm{~K}$.

3. Compressive properties of composite struts, ranging from $100 \mathrm{~mm}$ (4 in) to $250 \mathrm{~mm}$ (10 in) in diameter, were obtained at 295,76 , and $4 \mathrm{~K}$. Fiber lay-up, tube thickness and length, and resin system were varied in these experiments. Limited fatigue data were also obtained.

4. Analytic and failure analyses studies were conducted. These included the development of stress- and strain-controlled thermal-mechanical modeling for property prediction and quality control, the modeling of end fixtures, and analyses of failed tubes.

This comprehensive program on composite strut properties with composite component (reinforcement/matrix) characterization and on analytic modeling should provide a firm technical foundation on which to base future strut design and manufacturing. The specific objectives of the program were (1) to provide a technical basis for the future production of struts by developing thermal-mechanical analyses and supplying process-selection information and thermal and mechanical design data and (2) to improve the ultimate compressive strength of full-scale struts at both room and low temperatures. Achievement of these objectives should result in a significant reduction in the cost of the strut systems as a result of improved thermal efficiency and the reduction in the size of the struts and, thus, in the amount of composite material required.

The program, "Development of Composite Supports for SMES," has been sponsored by the Defense Nuclear Agency, under Cost Reimbursable Order Number 91-854, with Lt. Col. William Dungan, followed by Col. Kevin Yelmgren and Capt. David Jacobs, project monitors. We also appreciate the support of Michael Superczynski of the David Taylor Research Center and leader of the SMES Technical Advisory Group and the contributions of

- R. Lyon and S. DeTeresa (Lawrence Livermore National Laboratories), J. Keester and S. Hansen (Addax), and D. Longburg (Ershigs) for their recommendations of resin systems for evaluation

- Brant Goldsworthy (consultant) for advice on pultrusion

- Bob Baucom (NASA-Langley) for advice and assistance in tube production

- Renee Deal (University of Colorado) for countless hours of data reduction and graphs

- Gerald Velarde and David Mason (University of Colorado) for conducting mechanical property tests 


\title{
III. Properties of Neat-Resin Systems
}

\author{
R.P. Reed \\ J.D. McColskey \\ Materials Reliability Division \\ National Institute of Standards and Technology \\ Boulder, Colorado \\ *Cryogenic Materials, Inc. \\ Boulder, Colorado
}





\title{
III. Properties of Neat-Resin Systems
}

\author{
R.P. Reed* \\ J.D. McColskey \\ Materials Reliability Division \\ National Institute of Standards and Technology \\ Boulder, Colorado \\ ${ }^{*}$ Cryogenic Materials, Inc. \\ Boulder, Colorado
}

\section{Summary}

Eighteen different neat-resin systems with potential for use as the matrix resin in filament-wound or pultruded support struts for SMES plants were studied at low temperatures. They included four resin classes-epoxies, vinyl esters, cyanate esters, and polyesters-and three types of resins systems-filled, multifunctional, and toughened. The properties measured were shear, tensile, thermal conductivity, and thermal contraction from room to liquid-helium temperatures. A new type of residual-stress measurement technique was developed to assess the effects of resin shrinkage during curing and cooling.

Correlations were found between elastic moduli and resin density, thermal contraction and type of resin system, thermal conductivity and resin density, tensile and shear strength and type of resin system, shear and tensile moduli and resin hardness and density. Tensile failures were identified as originating from flaws in the neat-resin specimens, and a good correlation was obtained between flaw size and tensile strength. Extrapolation to zero flaw size enabled estimation of inherent strength at each test temperature, and use of the dependency of strength on flaw size enabled estimation of the inherent fracture toughness.

Neither a resin class nor an individual resin was found to be superior in low-temperature performance. However, the effects of polymer chemistry and variations, such as the addition of fillers, tougheners, or multifunctional components, were identified: they affected not only the absolute values of the mechanical and thermal properties, but also the temperature dependence of these properties.

Effective utilization of the information in this study requires knowledge of composite structural failure mechanisms and analytic modeling. The influence of matrix properties on the failure mechanisms must be understood, and resin selection should be based on the matrix properties that minimize failure. 


\section{Introduction}

For future superconducting magnetic energy storage (SMES) plants, composite struts are proposed to be used for radial support of the superconducting coil against the trench wall. Cool-down (coil-contraction) forces are transmitted to the inner trench wall and Lorentz (coilhoop-expansion) forces are transmitted to the outer wall of the trench. The requirements of high compressive strength, low thermal conductivity, and low capital costs suggest the use of fiberreinforced, resin-matrix composite tubes.

Composite properties are dependent on (1) the properties of the matrix and reinforcement, (2) the interactions of these matrix and reinforcement properties and their influence on other properties, and (3) the bond strength between matrix and reinforcement. Both the mechanical and physical properties of a composite are derived from some combination of the properties of the matrix and reinforcement; usually a rule-of-mixtures approach gives the best approximation. The properties of the composite are also influenced by the disparity between the properties of the matrix and the reinforcement. For instance, on cooling from a resin cure temperature, the thermal contraction of the resin is considerably larger than that of a glass-fiber reinforcement, causing a large residual stress in the vicinity of the fiber-matrix interface. Under certain loading conditions in the composite, this residual stress results in reduced strength or toughness. The adhesive strength between the reinforcement and matrix also contributes to the composite strength. Premature failures at the interfaces may provide a series of microcracks that affect the elastic, thermal, and strength properties of the composite.

Selection of the resin is also important from a cost standpoint. The bisphenol A and $F$ and cycloalphiatic epoxy resins included in this report cost between $\$ 3$ and $\$ 4$ per pound. Multifunctional epoxies and cyanate esters are much more expensive ( $\$ 20$ to $\$ 50$ per pound). On the other hand, polyester resins are inexpensive, costing between $\$ 1.00$ and $\$ 1.20$ per pound, and vinyl esters are about $\$ 2$ per pound. We estimate that a full-scale SMES plant will use about $4,000,000$ pounds of resin for the radial support struts. Thus, each dollar saved per pound in the cost of the resin results in an overall saving of $\$ 4,000,000$ ! For this reason, unless their price falls considerably, it is very unlikely that multifunctional epoxy or cyanate-ester resin systems will be used.

The objectives of this program were (1) to conduct sufficient property measurements and testing to enable evaluation of the candidate neat-resin systems on the basis of their lowtemperature characteristics and (2) to provide accurate property data of neat-resin systems for input into thermomechanical models to predict composite strut behavior. Correlation between neat-resin properties and composite performance may provide insight for the screening of neat resins in the future.

Since the overall purpose of the program was to improve the performance and lower the cost of composite struts, the resin systems included in this program were those that are candidates for filament winding or pultrusion of large glass-fiber-reinforced struts. Four generic classes of resin systems were studied: epoxy, vinyl ester, cyanate ester, and polyester. Since typical pultrusion fabrication includes the use of filler material in the resin system, two different resins were evaluated that contained fillers. Newer epoxy resins developed for aerospace or 
defense applications include multifunctional, toughened, and multifunctional toughened systems; typical examples of these systems were included. In some cases, filament-winding or pultrusion companies had previously worked extensively with specific resin systems, and these systems were included in the program. Altogether, 18 different resin systems were studied. They are identified in Table 2.1; the abbreviations used throughout this report to designate the resin systems are defined in Table 2.2 .

Knowledge of resin properties is essential for accurate design and modeling of composite structural components. The struts used for radial support of superconducting coils in future SMES plants are tubes. The failure stress for localized and column (Euler) buckling of cylinders is linearly related to the compressive elastic modulus. The fibers aligned in the near$0^{\circ}$ direction (parallel to the tube axis) contribute very substantially to the magnitude of the elastic moduli [2.1] $]^{*}$ so that matrix contributions to the compressive elastic modulus are relatively small and may be insignificant. However, the buckling or kinking of individual fibers within an elastic medium, also called microbuckling, is strongly affected by the stiffness of the elastic resin system [2.1-2.3]. As the tensile and shear moduli of the resin system increase, the stress level required to initiate microbuckling increases. This dependence on matrix stiffness has been reported to be much stronger in unidirectional ply (graphite-fiber) composites [2.1] than in quasi-isotropic (graphite-fiber) lay-ups [2.2] or in unidirectional ply (stainless-steel-reinforced) composites [2.3].

Other resin selection criteria have been developed from a mechanics approach that relate the properties of composites to the properties of unidirectional ply and component properties [2.4]. For improvement of laminate strength, ply shear energy, and composite toughness, the ratio of resin strength to modulus must be increased. Although this ratio may be referred to as an apparent strain, it is clearly not a strain to failure unless the stress-strain curve is linear elastic.

Under compressive loads, composite failures that are dominated by delamination (i.e., interlaminar strength) or by micromechanics (i.e., compressive strength) of the fiber have been empirically related to the resin tensile strength and Young's modulus, respectively [2.5].

Other major programs that use composites at low temperatures have also conducted resin-evaluation programs. The Gravity Probe-B program studied resin alternatives to be used in the gamma-alumina fiber-reinforced neck tubes and support struts [2.6]. The selection criteria included microcracking and helium permeability tendencies, shear modulus at room temperature, and processibility with gamma-alumina fiber. Four candidates, including three DGEBA epoxies and one cyanate-ester resin were included in the final evaluation. An anhydride-cured epoxy was selected for use.

The National Aerospace Plane program also has an ongoing resin-evaluation program for cryogenic applications. In this program, flexure testing at $77 \mathrm{~K}$ was used as the key mechanical property to screen candidate neat resins.

${ }^{*}$ Numbers within square brackets denote references at the end of the section.

III-3 
Table 2.1. Resin identification.

\begin{tabular}{|c|c|c|c|}
\hline Type \& Designation & Description & Supplier & $\begin{array}{l}\text { Trade Name } \\
\text { or Number }\end{array}$ \\
\hline \multicolumn{4}{|l|}{ Epoxy } \\
\hline DGEBA/DCH/ARA/ACL & Addax proprietary & Dow Chemical & Tactix 123 \\
\hline DGEBA/ALA/ACL & CTD proprietary & $\begin{array}{l}\text { Composite } \\
\text { Technology } \\
\text { Development }\end{array}$ & 501 \\
\hline DGEBA-ST/ARA/ACL/F & used for pultrusion & Shell & 9310 \\
\hline DGEBF/CTBN/AN & Addax proprietary & Shell & DPL 862 \\
\hline NV/AN/ACL & CTD proprietary & $\begin{array}{l}\text { Composite } \\
\text { Technology } \\
\text { Development }\end{array}$ & 102 \\
\hline Cyclo.A/CTBN/AN/ACL & LLNL developed & Ciba-Geigy & 179 \\
\hline Cyclo.A/AN/ACL & LLNL developed & Ciba-Geigy & 179 \\
\hline MLTF/ARA & Hercules proprietary & Hercules & 3502 \\
\hline MLTF/ARA/TGH & Hercules proprietary & Hercules & $8553-50$ \\
\hline \multicolumn{4}{|l|}{ Cyanate Ester } \\
\hline $\mathrm{CE} / \mathrm{VT} / \mathrm{Co} . \mathrm{AA}$ & LLNL developed & Dow Chemical & XU71781.02 \\
\hline $\mathrm{CE}$ & ICI proprietary & ICI & $954-3$ \\
\hline $\mathrm{CE}(\mathrm{PT})$ & Allied-Signal proprietary & Allied-Signal & Cryorad \\
\hline $\mathrm{CE} / \mathrm{HTBN} / \mathrm{NPh} / \mathrm{Cu} . \mathrm{AA}$ & LLNL developed & Rhone-Poulenc & REX-378 \\
\hline \multicolumn{4}{|l|}{ Vinyl Ester } \\
\hline $\mathrm{VE} / \mathrm{CHX}$ & $\begin{array}{l}\text { available from foreign } \\
\text { source }\end{array}$ & $\begin{array}{l}\text { Dainippon Ink } \\
\text { and Chemicals }\end{array}$ & Diclite UE-3505 \\
\hline VE-EL/MEKP & $\begin{array}{l}\text { commonly used for } \\
\text { pultrusion }\end{array}$ & Dow Chemical & Derakane 8084 \\
\hline $\mathrm{VE} / \mathrm{CHP} / \mathrm{F}$ & CTD proprietary & $\begin{array}{c}\text { Composite } \\
\text { Technology } \\
\text { Development }\end{array}$ & $\mathrm{X} 700$ \\
\hline VE/CHP/CoNap/2,4-P & CTD proprietary & $\begin{array}{c}\text { Composite } \\
\text { Technology } \\
\text { Development }\end{array}$ & $\mathrm{X} 800$ \\
\hline \multicolumn{4}{|l|}{ Polyester } \\
\hline PE/MEKP & commonly used & Owens Corning & 701 \\
\hline
\end{tabular}


Table 2.2. Abbreviations.

Resins:

CE cyanate ester

$\mathrm{CE}(\mathrm{PT}) \quad$ cyanate ester (phenolic triazine)

Cyclo.A cycloaliphatic epoxy

DGEBA diglycidylether of bisphenol-A epoxy

DGEBA-ST diglycidylether of bisphenol-A epoxy, styrene modified

DGEBF diglycidylether of bisphenol-F epoxy

MLTF multifunctional epoxy

NV novolac epoxy

PE polyester

VE vinyl ester

VE-EL vinyl ester, elastomer modified

Curing Agents:

ALA aliphatic amine

AN anhydride

ARA aromatic amine

Tougheners and Modifiers:

CTBN carbonyl terminated butadiene acrylonitrile copolymer (rubber)

DCH diaminocyclohexane (epoxy modifier)

F filler

HTBN hydroxyl terminated butadiene acrylonitrile copolymer (rubber)

TGH toughened

Accelerators, Catalysts, Promotors, and Retarders:

2,4-P

2,4-pentanedione

ACL accelerator for epoxy resins

CHP cumene hydroperoxide

CHX cyclohexanone peroxide

Co.AA cobalt (III) acetyl acetonate

CoNap cobalt naphthenate

Cu.AA cupric acetyl acetonate

DMA dimethyl aniline

MEKP methyl ethyl ketone peroxide

$\mathrm{NPh}$ nonyl phenol

VT vinyl toluene 
This study of neat resins addressed the need for matrix property data and some of the concerns about property interactions. Bond strengths were not studied in this program. Mechanical properties included tensile and shear; physical properties included thermal conductivity and thermal expansion. The effects of residual stress and thermal cycling were also studied. Hardness and density data were obtained at room temperature. Property data were measured at room $(295 \mathrm{~K})$, boiling liquid-nitrogen $(76 \mathrm{~K})$, and boiling liquid-helium $(4 \mathrm{~K})$ temperatures.

For our program, it was considered essential to test at temperatures that spanned the operational range of struts (4 to $295 \mathrm{~K}$ ). Shear and tensile tests were selected for neat-resin mechanical tests since clear definition of strain-stress characteristics could be obtained from these measurements. These comparisons enable simultaneous characterization of ultimate strength, strain to failure, and modulus. Furthermore, for modeling and data-comparison purposes, test data with only one type of applied stress are preferred. (In flexure, specimens are exposed to combinations of tensile, compression, and shear forces.) Knowledge of neat-resin mechanical and thermal property data should lead to better modeling and correlations with composite strut performance. 


\title{
3. Production Characteristics
}

\author{
J. B. Schutz and N. A. Munshi \\ Composite Technology Development, Inc. \\ Boulder, Colorado
}

\subsection{Introduction}

Eighteen neat-resin systems were produced and evaluated for their potential to be optimized for support strut fabrication for the SMES program. In the Introduction to Properties of Neat-Resin Systems (§III.2), the resin systems are identified in Table 2.1, and their abbreviations, in Table 2.2. The choice of resins was based on their suitability for filament winding or pultrusion production of glass-fiber-reinforced tubes.

Rods and flat plates of each resin were produced, except for two resins (see §3.3.2.9 and $\$ 3.3 .2 .12$ below), whose characteristics precluded flat-plate fabrication.

\subsection{Resin Types}

Eighteen resin systems, suitable for filament winding or pultrusion, were selected for evaluation. They included nine epoxy, five vinyl-ester, one polyester, and three cyanate-ester resin systems. Rods and flat plates were fabricated from all but two systems, as described in $\S 3.3 .1$. The chemical composition, formulation, handling characteristics, and processing information are described in $\$ 3.3 .2$.

\subsection{Procedures}

\subsubsection{Production Techniques}

\subsubsection{Flat Plates}

Flat plates $(300 \times 300 \times 3.2 \mathrm{~mm})$ of each material were produced by casting the liquid resin between glass-plate molds. The glass plates were treated with at least three coats of mold release prior to use. Rubber or silicone tubing was used to form a gasket between the glass plates, on the bottom and sides of the mold. One-eighth-inch $(3.2-\mathrm{mm})$ thick steel stock was used as a spacer to ensure consistent plate thickness. The mold assembly was held together by large binder clips.

Three different methods were used to cast the liquid resins into the glass molds, depending on the resin characteristics:

- For low-to-medium viscosity materials that are mixed within a container and can be processed at relatively low temperatures (typically epoxy materials): Resin was transferred into the mold by pressurizing the container with bottled nitrogen gas. Resin was transferred through tubing into the top center of the mold. 
- For low-viscosity materials that can be processed at ambient temperature or mixed by hand lowviscosity epoxies and most of the vinyl esters): A funnel was attached to the glass mold for the liquid resin.

- For high-viscosity resins that require high processing temperatures (prepreg-type resins, some of the cyanate esters, and the phenolic triazine): Glass molds were filled by pouring resin into an aluminum trough that was attached to the molds. To maintain the temperature of the resin and molds, the assembly was replaced in the oven after filling the trough. Since the trough did not hold enough resin to completely fill the mold, the trough was refilled and the molds placed in the oven as many times as needed to fill the mold.

\subsubsection{Rods}

From each resin material, high-quality, 300-mm-long rods were produced in the system shown in Figure 3.1. The resin was preheated in the lower chambers and then drawn into 3.2-mmdiameter molds in the upper chambers. After an oven cure, the rods were removed from the molds and postcured.

Both neat and fiber-reinforced specimens can be produced by the proprietary system illustrated in Figure 3.1. Each production line consists of two chambers-a lower chamber (B) for resin impregation and an upper chamber (D) containing a specimen mold. Each chamber can be isolated by a system of clamps and valves (A). The temperature of each chamber is controlled by a microprocessor. The heated resin is simultaneously mixed and degassed in a mixing pot $(\mathrm{C})$. The prepared resin is vacuum-transferred into the lower chamber, and atmospheric pressure is introduced to achieve impregnation. The specimens (in molds) are transferred to an autoclave where they are cured under the proper temperature and pressure.

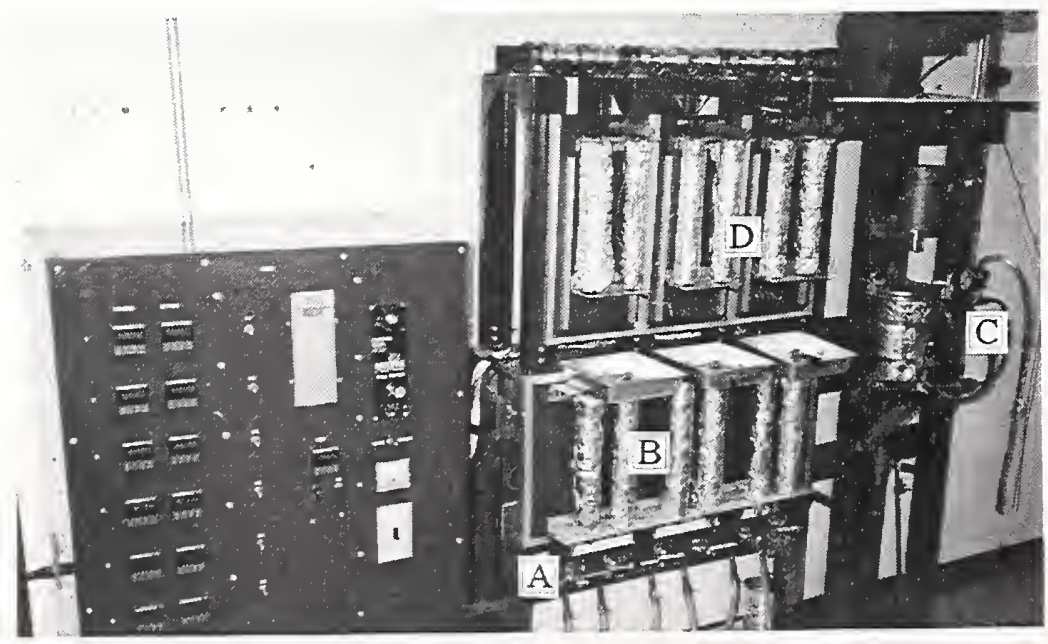

Figure 3.1. The rod-production system with six production lines for neat and fiber-reinforced specimens.

A - System of clamps and valves to isolate each chamber

B - Lower chamber

C - Mixing pot

D - Upper chamber 


\subsubsection{Resin Characteristics}

\subsubsection{DGEBA/DCH/ARA/ACL}

Chemical composition: a proprietary system

Formulation: proprietary

Production and handling:

- Resin ingredients were weighed into the mixing container, which was maintained at $305 \mathrm{~K}\left(32^{\circ} \mathrm{C}\right)$ during mixing and degassing operations. The resin was mixed for $15 \mathrm{~min}$ before it was degassed to $3.3 \mathrm{kPa}(25 \mathrm{~mm} \mathrm{Hg})$ in about $30 \mathrm{~min}$.

- The glass flat-plate mold was warmed to $316 \mathrm{~K}\left(43^{\circ} \mathrm{C}\right)$ and maintained at this temperature until just before resin transfer. From the pressurized mix container, the resin was transferred through tubing into the mold.

- The flat-plate and rod temperatures were ramped to $394 \mathrm{~K}\left(121^{\circ} \mathrm{C}\right)$ over a period of $2 \mathrm{~h}$ and held at this cure temperature for $4 \mathrm{~h}$.

\section{Comments:}

The resin system is a relatively low-viscosity liquid at the processing temperature of $305 \mathrm{~K}$ $\left(32^{\circ} \mathrm{C}\right)$; it processed well at that temperature.

\subsubsection{DGEBA/ALA/ACL}

Chemical composition: diglycidylether of bisphenol-A epoxy resin with an aliphatic amine curing agent and an accelerator

Formulation: 100 parts resin

45 parts curing agent

Production and handling:

- This system was handled at ambient temperature for all processes. The ingredients were weighed into the mixing container and mixed for 30 min before the resin blend was degassed to $3.3 \mathrm{kPa}$ $(25 \mathrm{~mm} \mathrm{Hg}$ ) in about $30 \mathrm{~min}$.

- Flat plates were cast both by transferring resin through a tube and by pouring it into an open mold.

- Flat plates and rods were cured at ambient temperature for 24 to $72 \mathrm{~h}$, followed by $16 \mathrm{~h}$ at $358 \mathrm{~K}$ $\left(85^{\circ} \mathrm{C}\right)$. The minimum cure for this resin is $24 \mathrm{~h}$ at $293 \mathrm{~K}\left(20^{\circ} \mathrm{C}\right)$.

\section{Comments:}

The resin system is a low-viscosity liquid that handled well at ambient temperature.

\subsubsection{DGEBA-ST/ARA/ACL/F}

Chemical composition:

diglycidylether of bisphenol-A epoxy resin, styrene modified, with an aromatic amine curing agent, an accelerator, and a filler 
Formulation: 60.7 parts resin

$\begin{array}{rll}0.42 & \text { part } & \text { surfactant } \\ 17.0 & \text { parts } & \text { filler } \\ 0.61 & \text { part } & \text { zinc stearate internal release agent } \\ 0.85 & \text { part } & \text { internal release agent } \\ 0.39 & \text { part } & \text { accelerator } \\ 20.03 & \text { parts } & \text { curing agent }\end{array}$

The formulation for this resin system was provided by a company that uses it for pultruded-parts fabrication.

\section{Production and handling:}

- All processing was done with the material at ambient temperature. The components were weighed into the mix container in the order given and mixed for approximately $20 \mathrm{~min}$. Since the styrene contained in this resin would have rapidly boiled away under vacuum and changed the resin formulation, the resin was not degassed. However, a partial vacuum was applied to the resin momentarily to help break filler agglomerates and to aid in mixing.

- Flat-plate and rod molds were warmed to $353 \mathrm{~K}\left(80^{\circ} \mathrm{C}\right)$ before casting. Two methods were used to cast plates of this material: (1) The resin was vacuum-transferred into a hot metal mold. The mold was then closed off and placed back in the oven, and 69-kPa-gauge (10-psig) nitrogen pressure was applied. (2) Another flat plate was cast by pouring the resin through a funnel into a prewarmed flat glass mold. Rods were cast normally.

- Flat plates and rods were cured at $353 \mathrm{~K}\left(80^{\circ} \mathrm{C}\right)$ for $1.5 \mathrm{~h}$ followed by $1.5 \mathrm{~h}$ at $450 \mathrm{~K}\left(177^{\circ} \mathrm{C}\right)$ and a free-standing postcure of $2 \mathrm{~h}$ at $450 \mathrm{~K}$.

\section{Comments:}

The resin system is a low-viscosity liquid, even with the addition of inorganic filler, and it handled easily at ambient temperature. The plate that was cast in the metal mold had a darker color when cured, which may have been due to exotherm of the material.

\subsubsection{DGEBF/CTBN/AN}

\section{Chemical composition: a proprietary system}

Formulation: proprietary

\section{Production and handling:}

- All mixing and processing was done at ambient temperature. The base resin and an additive were weighed into the mixing container and then mixed and degassed for about $15 \mathrm{~min}$. The curing agent was then weighed into a new mixing can, and the correct proportion of the resin blend was added. The curing agent and resin blend were mixed for $15 \mathrm{~min}$ and then degassed for $15 \mathrm{~min}$.

- The flat-plate and rod molds were warmed to $305 \mathrm{~K}\left(32^{\circ} \mathrm{C}\right)$ before the resin was transferred into them. Resin was transferred through tubing from the pressurized mix container into the mold.

- The flat-plate and rod temperatures were ramped to $394 \mathrm{~K}\left(121^{\circ} \mathrm{C}\right)$ over a period of $5 \mathrm{~h}$ and held at this cure temperature for $10 \mathrm{~h}$.

\section{Comments:}

The resin system is a relatively low-viscosity liquid that handled well at ambient temperature with no particular problems. 


\subsubsection{NV/AN/ACL}

Chemical composition: novolac epoxy-resin blend with an anhydride curing agent and an accelerator

Formulation: 100 parts resin

100 parts hardener

0.25 part accelerator

Production and handling:

- The ingredients were weighed into the mixing container, which was maintained at $293 \mathrm{~K}\left(70^{\circ} \mathrm{C}\right)$ during mixing and degassing operations. The resin was mixed for $10 \mathrm{~min}$ and then degassed to $3.3 \mathrm{kPa}(25 \mathrm{~mm} \mathrm{Hg})$ in about $25 \mathrm{~min}$ at $293 \mathrm{~K}$. The resin container temperature was raised to $353 \mathrm{~K}\left(80^{\circ} \mathrm{C}\right)$ prior to transfer of resin into the glass flat-plate mold.

- The glass mold was warmed to the resin cure temperature, $393 \mathrm{~K}\left(120^{\circ} \mathrm{C}\right)$, and maintained at this temperature until just before the resin was transferred from the pressurized mix container into the glass-plate mold.

- Flat plates and rods were cured at $393 \mathrm{~K}$ for $24 \mathrm{~h}$. This system does not require a postcure.

\section{Comments:}

The resin system, a low-viscosity liquid, handled well at 343 to $353 \mathrm{~K}\left(70^{\circ} \mathrm{C}\right.$ to $\left.80^{\circ} \mathrm{C}\right)$ with no particular problems.

\subsubsection{Cyclo.A/CTBN/AN/ACL}

Chemical composition: cycloaliphatic epoxy resin with a carbonyl terminated butadiene acrylonitrile copolymer (rubber) toughener, an anhydride curing agent, and an accelerator

Formulation: 100 parts epoxy resin

100 parts curing agent

10 parts toughener

1 part 1-methyl imidazole catalyst

Production and handling:

- All processing was done at ambient temperature because the material has a low viscosity. The base resin, curing agent, reactive rubber modifier, and catalyst were weighed into the mixing container. Degassing was begun immediately after mixing was started. The resin was very foamy during degassing; it required as long as $90 \mathrm{~min}$ to degas.

- Plate and rod molds were warmed to $368 \mathrm{~K}\left(95^{\circ} \mathrm{C}\right)$ before transfer of the resin.

- The first flat plate produced bonded to one of the glass plates, probably owing to inadequate mold release. This resulted in a broken resin sheet and glass plate. A replacement resin plate was made with the same processing conditions and cure times.

- Flat plates and rods were cured $6 \mathrm{~h}$ at $368 \mathrm{~K}\left(95^{\circ} \mathrm{C}\right)$ with an oven cool-down.

\section{Comments:}

The resin system, a low-viscosity liquid, processed easily at ambient temperature, except for the long degassing time required. 


\subsubsection{Cyclo.A/AN/ACL}

Chemical composition: cycloaliphatic epoxy resin with an anhydride curing agent and an accelerator

Formulation: 100 parts epoxy resin

100 parts curing agent

1 part 1-methyl imidazole catalyst

Production and handling:

- All processing was done at ambient temperature because the material has a low viscosity. The base resin, curing agent, and catalyst were weighed into the mixing container. Degassing was begun immediately after mixing was started.

- Plate and rod molds were warmed to $368 \mathrm{~K}\left(95^{\circ} \mathrm{C}\right)$ before transfer of the resin.

- Flat plates and rods were cured $6 \mathrm{~h}$ at $368 \mathrm{~K}$ with an oven cool-down.

Comments:

Processing and curing of this resin system was identical to that of the CTBN-toughened resin described in $\$ 3.3 .2 .6$, except that the toughener was omitted.

The resin system, a low-viscosity liquid, processed easily at ambient temperature, except for the long degassing time required.

\subsubsection{MLTF/ARA}

Chemical composition: Proprietary, multifunctional epoxy resin with an aromatic-amine curing agent

Formulation: One-part resin system

Production and handling:

- Chunks of frozen resin were placed in the mix can, which was then put into the mix chamber, and full vacuum was applied. The chamber temperature was maintained at $353 \mathrm{~K}\left(80^{\circ} \mathrm{C}\right)$. During heating, vacuum was occasionally broken to help release entrapped bubbles. To heat and degas the resin, 3 to $4 \mathrm{~h}$ were required.

- Rod and plate molds were warmed to $363 \mathrm{~K}\left(90^{\circ} \mathrm{C}\right)$ prior to casting of the resin. Owing to the extremely high viscosity of this resin, the glass-plate molds were fitted with an aluminum trough along the top opening. Liquid resin was poured into this trough, and then the entire mold assembly was placed in the oven, which was kept at 363 to $373 \mathrm{~K}\left(90^{\circ}\right.$ to $\left.100^{\circ} \mathrm{C}\right)$. This procedure enabled the mold and resin temperature to be maintained while the liquid flowed into the mold. At this temperature, the resin flow was quite slow. The process of filling the trough and waiting for the flow was repeated as many times as necessary to fill the mold.

\section{Comments:}

The material must be shipped and stored at or below $255 \mathrm{~K}\left(-18^{\circ} \mathrm{C}\right)$ and should be kept in a sealed container to minimize moisture absorption.

Because it has a very high viscosity at the processing temperature, the resin system was difficult to process. 


\subsubsection{MLTF/ARA/TGH}

Chemical composition: a proprietary, multifunctional epoxy resin with an aromatic amine curing agent and a toughener

Formulation: premixed blend

Production and handling:

- The frozen resin was fractured inside the sealed container. Chunks of the resin were placed into the mix container and evacuated immediately to avoid moisture absorption. Unused resin was resealed and returned to cold storage immediately. Several attempts were made to fabricate flat plates from this material. In the first attempt, the resin container was maintained under vacuum at $377 \mathrm{~K}$ $\left(104^{\circ} \mathrm{C}\right)$ while the material was melted. After about $30 \mathrm{~min}$ in the resin container, mixing was begun. Initially, only the top layer of the resin could be mixed owing to its slow melting rate and extremely high viscosity. A thermocouple was inserted into the hollow paddle wheel to monitor the resin temperature. It took more than $2 \mathrm{~h}$ for the resin to reach the processing temperature of $377 \mathrm{~K}$. Although the mix-container temperature was controlled to $377 \mathrm{~K}$, the resin temperature continued to rise beyond this set point, indicating a possible exothermic reaction.

- Molds were prewarmed to $377 \mathrm{~K}$ prior to transfer of the resin through tubing from the pressurized mix container. When the temperature of this resin drops, its viscosity increases significantly. During casting of the flat plate, the resin in the tubing cooled sufficiently to stop the flow. With other resins processed in this manner, flow could be resumed by warming the tubing with a heat gun. In this case, the temperature required to reinitiate flow was so high that the tubing began to soften and bulge, and flow could not be maintained. A flat plate could not be made with this material using the pressurized-mix-container transfer method. Approximately $1 \mathrm{~h}$ after reaching the desired processing temperature of $377 \mathrm{~K}$, the material had gelled to a solid and begun to smoke, indicating an exothermic chemical reaction.

- There was another attempt to make a resin plate, after consultation with the manufacturer's laboratory personnel. They recommended processing the resin at $357 \mathrm{~K}\left(84^{\circ} \mathrm{C}\right)$ without stirring. Resin was poured into a trough attached to the prewarmed mold, and the mold assembly was placed back in the oven to keep warm while the resin flowed. The resin viscosity was so high that only onequarter of a plate could be cast before resin flow stopped.

- Rods were cast as before, but the viscosity of this material was the highest of any in this program.

- The rod temperature was ramped from $377 \mathrm{~K}\left(104^{\circ} \mathrm{C}\right)$ to the cure temperature of $450 \mathrm{~K}\left(177^{\circ} \mathrm{C}\right)$ at a rate of 2 to $3 \mathrm{~K}$ per min and held at that temperature for $2 \mathrm{~h}$. The rods were allowed to ovencool slowly to ambient temperature after cure.

\section{Comments:}

This resin system is primarily a prepreg system. It must be shipped and stored at or below $255 \mathrm{~K}$ $\left(-18^{\circ} \mathrm{C}\right)$, and it must be kept in a sealed container to minimize moisture absorption. It must be processed at $377 \mathrm{~K}\left(104^{\circ} \mathrm{C}\right)$ owing to its extremely high viscosity. The viscosity of the resin system, the highest of the materials evaluated, precluded flat-plate fabrication.

\subsubsection{CE/VT/Co.AA}

Chemical composition: cyanate-ester resin with vinyl toluene and a cobalt (III) acetyl acetonate catalyst

Formulation: $\begin{array}{rll}950 & \text { parts } & \text { resin } \\ 50 & \text { parts } & \text { vinyl toluene } \\ & 1.875 \text { parts } & \text { catalyst }\end{array}$ 


\section{Production and handling:}

- This resin, which is semisolid at ambient temperature, was warmed to $366 \mathrm{~K}\left(93^{\circ} \mathrm{C}\right)$ prior to mixing. The catalyst Co.AA is a powder that must be dissolved in a solvent (VT) before it can be mixed properly with the resin. A solution was made by weighing $1.875 \mathrm{~g}$ of catalyst into $50 \mathrm{~g}$ of VT. The solution was added to the prewarmed resin in the proportion of 50 parts by weight VT/ Co.AA solution to 950 parts by weight resin. This catalyst system is thermally latent, meaning that cure will not initiate until some activation temperature is reached, in this case, at about $373 \mathrm{~K}$ $\left(100^{\circ} \mathrm{C}\right)$. Thus, the material has a long pot life once mixed, as long as the activation temperature is not reached.

- The prewarmed resin and catalyst were placed in the resin-mixing container, which was maintained at $355 \mathrm{~K}\left(82^{\circ} \mathrm{C}\right)$ during mixing, degassing, and resin-transfer operations. The blend was mixed for $15 \mathrm{~min}$, before application of $3.3-\mathrm{kPa}(25-\mathrm{mm}-\mathrm{Hg})$ vacuum to degas.

- The plate and rod molds were warmed to $366 \mathrm{~K}\left(93^{\circ} \mathrm{C}\right)$ before the resin was transferred.

- The plates and rods were cured for $2 \mathrm{~h}$ at $394 \mathrm{~K}\left(121^{\circ} \mathrm{C}\right)$, followed by $2 \mathrm{~h}$ at $449 \mathrm{~K}\left(176^{\circ} \mathrm{C}\right)$. The parts were removed from their respective molds prior to postcuring at $519 \mathrm{~K}\left(246^{\circ} \mathrm{C}\right)$ for $2 \mathrm{~h}$.

\section{Comments:}

This resin system processed relatively well when maintained at a high temperature; however, its viscosity increased significantly when its temperature dropped. During casting of the flat plates, the resin cooled in the tubing, completely blocking the flow. It was necessary to warm the resin with a heat gun to reinitiate flow. Once a good flow rate was achieved, the resin retained enough heat to maintain a viscosity suitable for processing.

The material appears to expand upon cure. Release of the rods from the molds was difficult, and the plates apparently expanded within the flat mold. The finished rod diameters were approximately 0.05 to $0.08 \mathrm{~mm}(0.002$ to $0.003 \mathrm{in})$ larger than those of the other materials.

Two versions of the cyanate-ester resin are available commercially: one was tested in this program; the other contains $10 \%$ elastomeric rubber modifier. The use of the latter, or a blend of the two versions of resin, may be desirable to improve the toughness of the material, but at the cost of a reduced modulus. Since the primary application for this material is compression, it was decided to test the stiffer formulation.

\subsubsection{CE}

Chemical composition: a cyanate-ester resin

Formulation: one-part resin system

\section{Production and handling:}

- Chunks of the frozen resin were placed in the mix container and evacuated immediately to minimize moisture absorption. The resin container was maintained at $373 \mathrm{~K}\left(100^{\circ} \mathrm{C}\right)$. After approximately $1 \mathrm{~h}$, more frozen resin was added to the container. The resin was stirred occasionally with a paint stick during the melting and degassing process. Two hours after the start, the resin was fully liquefied and degassed.

- Rod and plate molds were warmed to $373 \mathrm{~K}$ before casting. Flat plates were made by pouring the liquid resin into an open, vertical glass mold equipped with a funnel. The resin was warmed locally with a heat gun to ensure good flow and to release any bubbles that formed. This liquid-pouring process avoided the problem of the resin cooling in the tubing and stopping the flow into the mold. Rods were cast as usual.

- Both flat plates and rods were cured for $1 \mathrm{~h}$ at $394 \mathrm{~K}\left(121^{\circ} \mathrm{C}\right)$, followed by $2 \mathrm{~h}$ at $450 \mathrm{~K}\left(177^{\circ} \mathrm{C}\right)$. 


\section{Comments:}

This material must be shipped and stored at or below $255 \mathrm{~K}\left(-18^{\circ} \mathrm{C}\right)$, and it must be kept in a sealed container to avoid moisture absorption.

The resin system has a relatively high viscosity at $373 \mathrm{~K}$, but it processed relatively easily as long as this temperature was maintained.

\subsubsection{CE(PT)}

Chemical composition: cyanate ester (phenolic triazine); no catalyst was used, although it is optional

Formulation: one-part resin system

\section{Production and handling:}

- The resin was allowed to warm to ambient temperature, at which point its viscosity was extremely high. The resin was removed from the container and weighed into a mix can. Chilled tools were used to handle the resin to prevent it from sticking to the tools. The mix can was placed into the mix chamber, and full vacuum was applied. The chamber temperature was slowly raised from ambient to the processing temperature of $343 \mathrm{~K}\left(70^{\circ} \mathrm{C}\right)$. During heating, the vacuum was occasionally broken to help release entrapped bubbles. To heat and degas the resin required 2 to $3 \mathrm{~h}$.

- Rod and plate molds were warmed to $353 \mathrm{~K}\left(80^{\circ} \mathrm{C}\right)$ prior to casting of the resin. Owing to the extremely high viscosity of this resin, the alternate flat-plate process was used. The glass-plate molds were assembled and fitted with an aluminum trough along the top opening. Liquid resin was poured into this trough, and then the entire mold assembly was placed in the oven, which was kept at $353 \mathrm{~K}$. This procedure enabled the mold and resin temperature to be maintained while the liquid flowed down into the mold. Even at $343 \mathrm{~K}$, the resin viscosity was very high, and resin flow was quite slow. The process of filling the trough and waiting for the flow was repeated as many times as necessary to fill the molds. The oven temperature was then slowly raised to the cure temperature of $383 \mathrm{~K}\left(110^{\circ} \mathrm{C}\right)$ and held for $12 \mathrm{~h}$. This cure schedule, which was suggested by consultants, differs from the one recommended in the manufacturer's literature (see last paragraph under Comments on the following page).

- There were two attempts to make flat plates from this material. In the first attempt, everything went smoothly until the mold was disassembled. When the mold plates were pried apart, the resin fractured through its thickness, leaving a rough half plate stuck to each mold plate. The mold plates had been thoroughly treated with mold release prior to use, but apparently the resin adhesion to the plates was greater than the resin cohesive strength. In the second attempt, the resin also fractured in the mold, in the plane perpendicular to the plate. The fracture is probably attributable to cure shrinkage or thermal shrinkage upon cooling from the cure to ambient temperature.

- Rod casting was similar to that for the other materials. Evidence of exotherm on the first rods produced dictated, for subsequent rods, the use of a very slow ramp time of $6 \mathrm{~h}$ to raise the temperature from $333 \mathrm{~K}\left(60^{\circ} \mathrm{C}\right)$ to the cure temperature of $383 \mathrm{~K}\left(110^{\circ} \mathrm{C}\right)$. Rods were cured at $383 \mathrm{~K}$ for $12 \mathrm{~h}$.

\section{Comments:}

The material specifications indicate that it must be shipped and stored at or below $277 \mathrm{~K}\left(4^{\circ} \mathrm{C}\right)$. When received, the material had apparently been stored at ambient temperature for approximately two days. The resin supplier said that the time at ambient temperature probably would not affect the material performance, and the resin could be used. 
The cured resin system exhibits some rather curious properties. During trial casting of rods, when cured material was removed from the mold while it was still warm, it could easily be bent and deformed by hand. Upon cooling, it regained its stiffness. These characteristics may be indicative of a relatively low glass-transition temperature. Cured rods were extremely brittle; they could be easily broken by hand, and multiple fractures often occurred. That the cure schedule used for the CE(PT) yielded a $40 \%$ cure of the resin was discovered only after the tests were completed. Therefore, all test results for the $\mathrm{CE}(\mathrm{PT})$ were affected by this undercure.

\subsubsection{CE/HTBN/NPh/Cu.AA}

Chemical composition: cyanate-ester resin with a hydroxyl terminated butadiene acrylonitrile copolymer (rubber) toughener, nonyl phenol, and a cupric acetyl acetonate catalyst

Formulation: 100 parts resin

6 parts toughener

6 parts nonyl phenol

0.1 part cupric acetyl acetonate

Production and handling:

- The resin and toughener were warmed to about $363 \mathrm{~K}\left(90^{\circ} \mathrm{C}\right)$ before processing, because their viscosity is extremely high at ambient temperature. These two components were measured into the mix container and degassed at $353 \mathrm{~K}\left(80^{\circ} \mathrm{C}\right)$; no curing agents were present in the blend.

- The resin was catalyzed with $\mathrm{Cu}$.AA, a powder which must be dissolved in a solvent before it can be mixed properly with the resin. A solution was made by weighing 0.1 parts of catalyst into 6 parts of nonyl phenol. This mixture was warmed to about $353 \mathrm{~K}\left(80^{\circ} \mathrm{C}\right)$ and stirred to assist in the dissolution of the catalyst.

- The viscosity of the resin blend at $353 \mathrm{~K}$ was too high for processing purposes, so the mix container temperature was raised to $358 \mathrm{~K}$. The catalyst package was added to the resin blend and mixed. The entire blend was degassed for $10 \mathrm{~min}$.

- The glass-plate and rod molds were warmed to nearly the cure temperature of $394 \mathrm{~K}\left(121^{\circ} \mathrm{C}\right)$ before resin transfer. Resin was transferred from the pressurized mix container through tubing into the mold.

- The flat plates and rods were cured at $394 \mathrm{~K}$ for $4 \mathrm{~h}$. After removal from the molds, the flat plates and rods were postcured at $394 \mathrm{~K}$ for $4.5 \mathrm{~h}$.

\section{Comments:}

The resin system processed relatively easily when maintained at an adequate temperature; however, its viscosity increased significantly when its temperature dropped. During casting of the flat plates, the resin cooled in the tubing sufficiently to slow the flow. It was necessary to warm the resin with a heat gun to get it flowing again. Once a good flow rate was achieved, the resin retained enough heat to keep its viscosity low enough for processing.

The catalyst system is thermally latent, meaning that it will not initiate cure until some activation temperature is reached. The material should have a long pot life once mixed, as long as it is maintained below the activation temperature. 


\subsubsection{VE/CHX}

Chemical composition: vinyl-ester resin with cyclohexanone peroxide catalyst

Formulation: 100 parts resin

1.00 part cyclohexane peroxide catalyst

This resin is manufactured in Japan. A limited quantity, approximately $1 \ell$, was obtained for the purposes of this program.

Two catalysts, also made in Japan, were recommended by the manufacturer; their chemical names are bis-tertiary-butyl-cyclohexyl-peroxydicarbonate and cyclohexanone peroxide. Sample quantities of chemical equivalents of these curing agents were obtained domestically. These materials are hazardous and extremely flammable peroxides which must be shipped under refrigeration. One of these materials could be shipped only via a refrigerated freight truck.

The desired mix ratios of these curing agents were not contained in the report of the previous study, and only sketchy cure data were included. The United States representative of the Japanese manufacturer recommended methyl cyclohexanone peroxide at a level of one part per hundred by weight. A cure schedule of $428 \mathrm{~K}\left(155^{\circ} \mathrm{C}\right)$ for $1 \mathrm{~h}$ was obtained from the report of the previous study.

\section{Production and handling:}

- This resin was handled at ambient temperature for all processes. The base resin and catalyst were measured into the mix can and stirred by hand for $2 \mathrm{~min}$. The resin was not vacuum-degassed during mixing owing to the volatility of the components of the base resin.

- Flat plates were produced by pouring the liquid resin into an open glass mold. The plates were cured by ramping the temperature from ambient to the cure temperature in $1 \mathrm{~h}$ and holding for $1 \mathrm{~h}$ at $428 \mathrm{~K}\left(155^{\circ} \mathrm{C}\right)$. Rods were produced by casting the material into rod molds, and the same cure schedule was followed.

- The material exhibited a high degree of shrinkage upon cure. The flat plates exhibited a shrinkage of approximately $6 \mathrm{~mm}$ over the $350-\mathrm{mm}$ plate length. A portion of the resin plate broke during the cure cycle, probably as a result of cure shrinkage and thermally induced stresses.

- The rods and plates exhibited surface waviness and roughness generally associated with an exotherm during the resin cure. This was the only material in the program that exhibited an exotherm during the manufacturing of flat plates.

- The quality of the rods initially produced was too poor for testing. Subsequently, rods of reasonable quality were produced by increasing the ramping time from ambient to the cure temperature to $3 \mathrm{~h}$. These rods still exhibited some surface waviness, but the best parts of the rods were selected for testing.

- Only one plate could be produced because only a small quantity of liquid resin was available.

\section{Comments:}

The material exhibited a high degree of shrinkage upon cure. The rods and plates exhibited surface waviness and roughness generally associated with an exotherm during the resin cure. This was the only material in the program that exhibited an exotherm during the manufacturing of plates.

The resin system has a low viscosity at ambient temperature, and it can be easily processed. The resin has a strong styrene odor. 


\subsubsection{VE-EL/MEKP}

Chemical composition: vinyl-ester resin, elastomer modified, with a methyl ethyl ketone peroxide catalyst

Formulation: 100 parts resin

1 part methyl ethyl ketone peroxide catalyst

The resin received was prepromoted with cobalt naphthenate and dimethyl aniline.

Production and handling:

- All mixing and handling was done with the resin at ambient temperature. Since this material has a very short pot life, several mixes were made. In the first batch, the resin and catalyst were weighed into the mix container and mixed for $5 \mathrm{~min}$. Transfer of the resin into the glass-plate mold was begun immediately after mixing. After mixing was started, the resin gelled into a solid mass in $10 \mathrm{~min}$, which corresponds to a working pot life of about $5 \mathrm{~min}$ after completion of mixing. In this time, only one-half of a flat plate could be cast before the resin gelled, and no rods could be made. The minimum cure for this material is $72 \mathrm{~h}$ at ambient temperature, followed by a postcure of 16 to $20 \mathrm{~h}$ at $344 \mathrm{~K}\left(71^{\circ} \mathrm{C}\right)$ or 2 to $4 \mathrm{~h}$ at $366 \mathrm{~K}\left(93^{\circ} \mathrm{C}\right)$. The resin plate was allowed to cure for 5 days at ambient temperature, followed by a free-standing postcure of $3 \mathrm{~h}$ at $366 \mathrm{~K}$.

- Upon consultation, the manufacturer suggested that mixing be done by hand and only for a period of about $1 \mathrm{~min}$. In the second batch, components were weighed into the mix container and mixed by hand with a paint stick for 1 min. Rods were then produced. The resin gelled 9 min after completion of mixing, which corresponds to a 10 min pot life after mixing has begun, regardless of whether the resin is mixed mechanically or by hand.

- Flat plates were made by pouring the material into an open mold. The resin was mixed by hand for $1 \mathrm{~min}$; then it was poured into a vertical open mold. Bubbles tended to form during mixing, although extra care was taken to ensure that no bubbles were entrapped. Presumably these bubbles were due to a reaction product. Void-free castings could not be made by pouring into an open mold because the material gelled before the bubbles could rise to the surface. A better casting was made by mixing for $1 \mathrm{~min}$ by hand and then using the resin container to transfer material into the vertical mold. By drawing resin from the bottom of the mix container, most of the bubbles on top of the liquid could be avoided.

- Rods were cast normally, but as a result of the energetic chemical reaction, a slight exotherm occurred, resulting in a slightly rough surface on the rods. To reduce the reaction rate, and hence the exotherm, a set of rods were cast into cold molds, which were immediately placed in a freezer at $273 \mathrm{~K}\left(0^{\circ} \mathrm{C}\right)$. This extended the gel time from $10 \mathrm{~min}$ to several hours and reduced the exotherm such that a good surface finish on the rods was obtained. The rods were subjected to the normal ambient cure and elevated postcure schedule after gelling.

Comments:

The resin system is a very low viscosity material that could be easily processed at ambient temperature as long as all processes were completed within the gel time.

\subsubsection{VE/CHP/F}

Chemical composition: vinyl-ester resin with a cumene hydroperoxide catalyst and a filler

Formulation: 100 parts filled vinyl ester

0.85 part catalyst 


\section{Production and handling:}

- This resin was handled at ambient temperature for all processes. Prior to use, the filled base-resin component had to be stirred carefully to prevent the filler from setting. Resin components were weighed into a mixing can and stirred by hand until the filler was thoroughly dispersed. The mixture was allowed to sit for several minutes between mixings to enable the filler to disperse and any entrapped bubbles to release. Typical of vinyl esters, the resin was not vacuum-degassed during mixing owing to the volatility of the components of the base resin.

- Flat plates were produced by pouring the liquid resin through a funnel into the open glass mold. The plates were cured for $1 \mathrm{~h}$ at $394 \mathrm{~K}\left(121^{\circ} \mathrm{C}\right)$.

- Rods were produced by casting the material into rod molds at ambient temperature and placing the molds in an oven maintained at $394 \mathrm{~K}$ for $1 \mathrm{~h}$.

\section{Comments:}

The resin system, with a low viscosity at ambient temperature, handles easily at that temperature, and it can be easily processed. It exhibits no foaming due to reaction of the catalyst, and it has a very long pot life, in excess of several days, although the filler has a tendency to settle out. The resin has a strong styrene odor.

\subsubsection{VE/CHIP/CoNap/2,4-P}

Chemical composition: vinyl-ester resin with a cumene hydroperoxide catalyst, a cobalt napthenate promotor and 2,4-pentanedione catalyst

Formulation: $\begin{aligned} 100 & \text { parts resin } \\ 1 & \text { part catalyst }\end{aligned}$

Production and handling:

- This resin was handled at ambient temperature for all processes. Resin components were weighed into a mixing can and stirred by hand for $2 \mathrm{~min}$. Typical of vinyl esters, the resin was not vacuumdegassed during mixing owing to the volatility of the components of the base resin.

- Flat plates were produced by pouring the liquid resin into an open glass mold and allowing the material to gel at ambient temperature for at least $12 \mathrm{~h}$. A postcure of $3 \mathrm{~h}$ at $366 \mathrm{~K}\left(93^{\circ} \mathrm{C}\right)$ followed.

- Rods were produced by casting the liquid resin into the rod molds and allowing the material to gel at ambient temperature. A 3-h postcure at $366 \mathrm{~K}$ followed.

\section{Comments:}

This low-viscosity resin system handles easily at ambient temperature; it can be easily processed. It exhibits no foaming due to reaction of the catalyst, and it has a relatively long pot life, in excess of $4 \mathrm{~h}$, which can be easily tailored for specific applications. The resin has a strong styrene odor.

\subsubsection{PE/MEKP}

Chemical composition: polyester resin with a methyl ethyl ketone peroxide catalyst

Formulation: 100 parts polyester

0.02 part cobalt naphthenate

0.01 part dimethyl aniline (DMA)

1 part MEKP 


\section{Production and handling:}

- This resin was handled at ambient temperature for all processes. The base resin, the promoter (CoNap), and accelerator (DMA) were weighed into a mixing can and stirred by hand until thoroughly blended. The mixture was allowed to sit for $30 \mathrm{~min}$ to release any entrained air. The catalyst (MEKP) was then measured into the mix can, and the mixture was stirred by hand for $2 \mathrm{~min}$. A specific formulation was not given by the supplier, and several trial mixes were necessary to produce a material that had a low enough exotherm so that high-quality rods could be fabricated. The polyester resin is similar to the vinyl esters in that the resin was not vacuum-degassed during mixing owing to the volatility of the components of the base resin.

- Flat plates were produced by pouring the liquid resin into an open glass mold and allowing the material to gel at ambient temperature for at least 3 days. A postcure of $1 \mathrm{~h}$ at $394 \mathrm{~K}\left(121^{\circ} \mathrm{C}\right)$ followed. Rods were produced by casting the material into rod molds and following the same gel and cure schedule.

\section{Comments:}

The material has a low viscosity at ambient temperature, and it can be processed easily. The resin has a strong styrene odor.

\subsection{Discussion}

Optimizing a resin system for cryogenic structural applications is a challenge because very few relevant data exist to guide the choice of matrix material for the specified mechanical and physical environment. Generating a comprehensive database on these materials is very costly and timeconsuming. Thus, certain assumptions have been made in order to select a material from the very limited database; they lead to the following observations:

- Tougheners. When a resin is toughened with an elastomer, its high-temperature impact strength may improve, but its low-temperature performance may deteriorate; e.g., Cyclo.A/AN/ACL and Cyclo.A/CTBN/AN/ACL. However, when the "flexibilizer" is a material that fully reacts with the system, adding small amounts may improve low-temperature performance: The performance of $\mathrm{CE} / \mathrm{HTBN} / \mathrm{NPh} / \mathrm{Cu} . \mathrm{AA}$ is better than the other cyanate-ester resin systems and that of VEEL/MEKP is better than the other vinyl-ester resin systems.

- Fillers. Fillers are often added to pultrusion systems to regulate viscosity and flow. Owing to the very high shear forces generated in the pultrusion process, selection of the filler is crucial. Hard fillers tend to cut through the fibers and thus lower the performance of the composite at cryogenic temperatures. Addition of fillers also lowers the strain to failure and increases the modulus; usually strength is not affected, except at very high filler concentrations. At cryogenic temperatures, fillers can also act as stress concentrators owing to the thermal-contraction mismatch between the filler and resin matrix. Thus, the filled vinyl-ester resin system VE/CHP/F has a lower strain to failure but a higher modulus than the unfilled vinyl ester VE/CHP/CoNap/2,4-P.

- Matrix material. Selection of a matrix material cannot be based solely on neat-resin properties. Data indicate that interfacial strength and residual stress at the fiber-matrix interface play key roles in the cryogenic performance of a composite material. Therefore, a composite material can possess a lower shear strength than the matrix material if its fiber-matrix interface is very poor. 


\title{
4. Tensile Properties
}

\author{
R.P. Reed* \\ R.P. Walsh, E.S. Drexler, C.N. McCowan, \\ R.L. Tobler, and J.D. McColskey \\ Materials Reliability Division \\ National Institute of Standards and Technology \\ Boulder, Colorado \\ ${ }^{*}$ Cryogenic Materials, Inc. \\ Boulder, Colorado
}

The tensile properties of eight epoxy, four cyanate-ester, four vinyl-ester, and one polyester resin systems have been measured at 295,76 , and $4 \mathrm{~K}$. Several filled resin systems and multifunctional epoxy systems were included. The resin systems were selected on the basis of their potential for use in filament winding or pultrusion production of fiberglass-reinforced struts. Most of the resin systems are currently available from commercial vendors.

Tensile failures occurred at single, embedded or surface flaws at all temperatures. The tensile strength was correlated with the flaw size, and two distinct trend lines were constructed for $295-\mathrm{K}$ data and for combined $76-$ and $4-\mathrm{K}$ data. By extrapolating the trend lines to zero flaw size, an inherent resin strength was obtained; approximate values of $100 \mathrm{MPa}$ at $295 \mathrm{~K}$ and of $170 \mathrm{MPa}$ at 77 to $4 \mathrm{~K}$ were predicted. This analysis included specimens from all but the two filled resin systems. When the stress-intensity-factor approach was used to estimate fracture toughness, values as high as about $2.4 \mathrm{MPa} \sqrt{\mathrm{m}}$ were calculated from the $77-$ to-4- $\mathrm{K}$ data and as high as $1.75 \mathrm{MPa} \sqrt{\mathrm{m}}$, from the $295-\mathrm{K}$ data, depending on the tensile strength.

The dependence of the tensile strength of neat-resin systems on flaw size suggests a strong dependence of cracking during loading on resin processability. Fewer and smaller flaws within the resin lead to higher strengths.

Resins that exhibited high tensile strengths were not the same as those that had a high Young's modulus or a high strain to failure. For the purpose of resin selection for composites produced by filament winding or pultrusion, these property distinctions among resin systems will be very important in strut failure analyses and modeling. For an optimum resin to be selected from this set of data, the matrix property that has the greatest impact on strut mechanical performance must be identified. Furthermore, the strong dependence of resin strength on flaw size suggests that the ability to produce a resin with low flaw concentrations and size may be the most significant selection parameter.

At room temperature, all classes of resin systems in this study had similar strengths, but filled systems and multifunctional epoxy systems had higher Young's moduli. At low temperatures, some vinyl esters had higher tensile strengths, and the cyanate esters had lower tensile strengths. The filled systems and multifunctional epoxies also had relatively low tensile strengths. 


\section{I Introduction}

The strength of fiber-reinforced composites is affected by the strength of the matrix resin. Similarly, the stiffness of a composite is influenced by the elastic properties of the resin; this is particularly true for through-thickness and transverse (to the fiber) orientations. Therefore, to obtain Young's modulus $(E)$, ultimate tensile strength $\left(\sigma_{u}\right)$, and strain to failure (by using $\sigma_{u} / E$ ) of candidate neat-resin systems, tensile tests were carried out at 295, 76, and $4 \mathrm{~K}$. All resin systems (epoxies, vinyl esters, cyanate esters, and polyesters) were included in this test program. Neat resins that were toughened and filled were also included.

Very few tensile tests have been conducted on epoxies at $4 \mathrm{~K}$, and no tensile tests have been reported on vinyl esters, polyesters, and cyanate esters at 76 or $4 \mathrm{~K}$. Therefore, these data add very significantly to our understanding of neat-resin mechanical behavior at low temperatures.

\subsection{Literature Review}

The tensile properties of neat-resin systems at low temperatures have been measured by only a few-Hartwig [4.1, 4.2], Lee and Neville [4.3], Chiao et al. [4.4], Hacker et al. [4.5], and Pink and Campbell [4.6]. These measurements were restricted to the study of epoxy systems; they are summarized in Table 4.1.

Table 4.1 Low-temperature tensile properties of neat resins.

\begin{tabular}{|c|c|c|c|}
\hline Type & $\begin{array}{c}\text { Temperature } \\
(\mathrm{K})\end{array}$ & $\begin{array}{l}\text { Property Value* } \\
\text { (MPa) }\end{array}$ & Investigator-Year [Ref.] \\
\hline \multirow{3}{*}{$\begin{array}{l}\text { Epoxy: } \\
\text { My } 740 \text {, } \\
\text { D } 230 \text { hardener }\end{array}$} & 4 & $\begin{array}{l}E=8.11 \times 10^{3} \\
\sigma_{u}=20, \nu=0.37\end{array}$ & Hartwig-1978 [4.1] \\
\hline & 77 & $E=7.85 \times 10^{3}$ & \\
\hline & 295 & $E=3.9 \times 10^{3}$ & \\
\hline $\begin{array}{l}\text { Flexible epoxy: } \\
\text { Cy } 221 \text {, Hy } 956 \\
\text { hardener }\end{array}$ & 4 & $\begin{array}{l}E=7.35 \times 10^{3} \\
\nu=0.36\end{array}$ & Hartwig-1978 [4.1] \\
\hline $\begin{array}{l}\text { Semiflexible epoxy: } \\
\text { Cy } 221 \text {, Hy } 979 \\
\text { hardener }\end{array}$ & 4 & $\begin{array}{l}E=7.3 \times 10^{3} \\
\sigma_{u}=179, \nu=0.37\end{array}$ & Hartwig-1978 [4.1] \\
\hline $\begin{array}{l}\text { Rigid epoxy: } \\
\text { X186/2476/Hy } 905 \\
\text { hardener }\end{array}$ & 4 & $\begin{array}{l}E=7.65 \times 10^{3} \\
\sigma_{u}=142, \nu=0.37\end{array}$ & Hartwig-1978 [4.1] \\
\hline
\end{tabular}


Table 4.1. Low-temperature tensile properties of neat resins (continued).

\begin{tabular}{|c|c|c|c|}
\hline Type & $\begin{array}{c}\text { Temperature } \\
(\mathrm{K}) \\
\end{array}$ & $\begin{array}{c}\text { Property Value* } \\
(\mathrm{MPa})\end{array}$ & Investigator-Year [Ref.] \\
\hline $\begin{array}{l}\text { Semiflexible epoxy: } \\
\text { CY } 221 \text { (aliphatic), } \\
\text { HY } 979 \text { (aromatic amine) } \\
\text { hardener: }\end{array}$ & & & Hartwig-1976 [4.2] \\
\hline no quartz filler & 4 & $E=7.3 \times 10^{3}$ & \\
\hline $20 \%$ quartz filler & 4 & $E=11 \times 10^{3}$ & \\
\hline $40 \%$ quartz filler & 4 & $E=15.5 \times 10^{3}$ & \\
\hline no quartz filler & 200 & $E=4.9 \times 10^{3}$ & \\
\hline $20 \%$ quartz filler & 200 & $E=6.9 \times 10^{3}$ & \\
\hline $40 \%$ quartz filler & 200 & $E=12 \times 10^{3}$ & \\
\hline $\begin{array}{l}\text { Epoxy: DGEBA } \\
8 \text { phr DETA hardener }\end{array}$ & 295 & $\begin{array}{l}E=2.8 \times 10^{3} \\
\sigma_{u}=80\end{array}$ & $\begin{array}{l}\text { Lee and Neville-1972 } \\
\text { [4.3] }\end{array}$ \\
\hline \multirow[t]{3}{*}{14 phr DETA } & 295 & $\begin{array}{l}E=3.8 \times 10^{3} \\
\sigma_{u}=81\end{array}$ & \\
\hline & 295 & $\begin{array}{l}E=2.6 \times 10^{3} \\
\sigma_{u}=68\end{array}$ & \\
\hline & 295 & $\begin{array}{l}E=3.0 \times 10^{3} \\
\sigma_{u}=97\end{array}$ & \\
\hline $\begin{array}{l}\text { Epoxy: XD7818, } \\
\text { XD7114, Tonox 60-40 } \\
(4 \mathrm{~h}-333 \mathrm{~K}, 3 \mathrm{~h}-393 \mathrm{~K})\end{array}$ & 295 & $\sigma_{u}=95$ & Chiao et al. -1975 [4.4] \\
\hline $\begin{array}{l}\text { Epoxy: XD7818 } \\
\text { ERL } 4026 \\
\text { Tonox } 60-40\end{array}$ & 295 & $\sigma_{u}=117$ & Chiao et al. -1975 [4.4] \\
\hline \multirow[t]{2}{*}{$\begin{array}{l}\text { Epoxy: DGEBA, } \\
\text { nadicanhydride }\end{array}$} & 300 & $\begin{array}{l}E=3.0 \times 10^{3} \\
\sigma_{u}=60\end{array}$ & Hacker et al. -1984 [4.5] \\
\hline & 77 & $E=5.2 \times 10^{3}$ & \\
\hline \multirow[t]{2}{*}{ Epoxy: new system } & 300 & $\begin{array}{l}E=2 \times 10^{3} \\
\sigma_{u}=65\end{array}$ & Hacker et al. -1984 [4.5] \\
\hline & 77 & $\begin{array}{l}E=5.2 \times 10^{3} \\
\sigma_{u}=65\end{array}$ & \\
\hline
\end{tabular}


Table 4.1. Low-temperature tensile properties of neat resins (continued).

\begin{tabular}{|c|c|c|c|}
\hline Type & $\begin{array}{c}\text { Temperature } \\
\text { (K) }\end{array}$ & $\begin{array}{l}\text { Property Value } \\
\text { (MPa) }\end{array}$ & Investigator-Year [Ref.] \\
\hline \multirow{3}{*}{$\begin{array}{l}\text { Epoxy: MY } 753 \\
\text { HY 951 hardener } \\
\left(T_{g} \sim 423 \mathrm{~K}\right)\end{array}$} & 300 & $\begin{array}{l}E=2 \times 10^{3} \\
\sigma_{u}=80\end{array}$ & \multirow[t]{3}{*}{$\begin{array}{l}\text { Pink and Campbell- } \\
1974 \text { [4.6] }\end{array}$} \\
\hline & 200 & $\begin{array}{l}E=3 \times 10^{3} \\
\sigma_{u}=100\end{array}$ & \\
\hline & 77 & $\begin{array}{l}E=5 \times 10^{3} \\
\sigma_{u}=118\end{array}$ & \\
\hline
\end{tabular}

${ }^{*} \nu$ is Poisson's ratio

Hartwig studied the effects of quartz filler on the Young's modulus of a semiflexible DGEBA epoxy system [4.2]. His tests were conducted at 4,50,100, and $200 \mathrm{~K}$. The dependence on volume percent of quartz filler ( 0 to $35 \mathrm{vol} \%$ ) remained constant throughout this temperature range. The approximate dependence is $E \sim 1 /\left(1-V_{f}\right)$ where $V_{f}$ is the volume percent of the filler. At a higher volume percent ( $52 \mathrm{vol} \%)$, there was less increase in $E$ at the higher test temperatures. Poisson's ratios of 0.35 and 0.37 were measured for the semiflexible neat resin at $\leq 200 \mathrm{~K}$ and room temperature, respectively. For a highly filled system, with $66 \mathrm{wt} \% \mathrm{Al}_{2} \mathrm{O}_{3}$, Poisson's ratio was measured at 0.31 at room temperature.

Later, Hartwig extended his study to include the effects of cross-link distance of epoxy neat resins on $E$ at low temperatures [4.1]. Cross-link distances were varied from 1.5 to $10 \mathrm{~nm}$ by varying the epoxy system. Resin systems with large cross-link distances are flexible and their density, $\rho$, is lower $\left(7.5 \mathrm{~nm}\right.$ with $\left.\rho=1.1 \mathrm{~g} / \mathrm{cm}^{3}\right)$; systems with low cross-link distances are rigid and have a higher density $\left(1.5 \mathrm{~nm}\right.$ with $\left.\rho=1.26 \mathrm{~g} / \mathrm{cm}^{3}\right)$. Hartwig reports a small dependence of $E$ on cross-link distance; the rigid epoxy system (X186/2476 with anhydride hardener Hy 905 with a cross-link distance of $1.5 \mathrm{~nm}$ ) has an $E$ of about $7.65 \mathrm{GPa}$ at $4 \mathrm{~K}$, whereas the flexible system (CY 221 with HY 956 hardener and a cross-link distance of $7.5 \mathrm{~nm}$ ) has an $E$ of about $7.35 \mathrm{GPa}$ at $4 \mathrm{~K}$. Poisson's ratios of 0.36 to 0.37 are reported for all epoxy resin systems at $4 \mathrm{~K}$. Ultimate tensile strengths (fracture strengths) were also measured at $4 \mathrm{~K}$ by Hartwig [4.1]. The rigid epoxy system failed at $142 \mathrm{MPa}$, and the semiflexible systems failed at 179 to $200 \mathrm{MPa}$.

Fracture energy (area under the stress-strain curve) was found to decrease by Colyer et al. with decreasing cross-link distance (increasing resin rigidity) at $4 \mathrm{~K}$ [4.7]. Table 4.2, which reproduces the results of their study, shows the strong dependence of increasing fracture energy on decreasing resin rigidity. 
Table 4.2. Fracture energy of epoxy neat resins.

\begin{tabular}{|c|c|c|c|c|c|}
\hline Resin Type & Contents & $\begin{array}{r}\text { Parts by } \\
\text { Weight }\end{array}$ & Cure & $\begin{array}{c}\text { Test } \\
\text { Temp. (K) } \\
\end{array}$ & $\begin{array}{c}\text { Fracture } \\
\text { Energy }\left(\mathrm{J} / \mathrm{m}^{2}\right)\end{array}$ \\
\hline \multirow[t]{3}{*}{ Rigid } & MY 740 & 100 & \multirow{3}{*}{$\begin{array}{c}\text { Gel at } 80^{\circ} \mathrm{C} \text {, } \\
10 \mathrm{~h} \\
\text { at } \\
120^{\circ} \mathrm{C}\end{array}$} & 293 & 100 \\
\hline & MNA & 80 & & 4 & 72 \\
\hline & DY 062 & 0.5 & & & \\
\hline \multirow[t]{3}{*}{ Semiflexible } & MY 740 & 100 & \multirow{3}{*}{$\begin{array}{l}16 \mathrm{~h} \\
\text { at } \\
85^{\circ} \mathrm{C}\end{array}$} & \multirow[t]{3}{*}{4} & \multirow[t]{3}{*}{364} \\
\hline & POPDA 400 & 57 & & & \\
\hline & Cyclo hexyldiamine & 10 & & & \\
\hline \multirow[t]{2}{*}{ Semiflexible } & MY 740 & 100 & \multirow{2}{*}{$\begin{array}{c}16 \mathrm{~h} \\
\text { at } \\
85^{\circ} \mathrm{C} \\
\end{array}$} & 77 & 430 \\
\hline & D 230 & 44 & & 4 & 302 \\
\hline \multirow[t]{4}{*}{ Flexible } & Adiprene 400 & 50 & \multirow{4}{*}{$\begin{array}{c}5 \mathrm{~h} \\
\text { at } \\
140^{\circ} \mathrm{C}\end{array}$} & \multirow[t]{4}{*}{4} & \multirow[t]{4}{*}{905} \\
\hline & 828 & 35 & & & \\
\hline & 871 & 15 & & & \\
\hline & $\mathrm{MOCA}$ & 27.5 & & & \\
\hline
\end{tabular}

Pritchard and Rhoades reviewed the fracture of resins and concluded that cross-linking reduces the fracture energy [4.8]. Since the fracture energy includes both the energy to reach ultimate strength and the energy for crack growth (unloading), examination of these components enables assessment of their relative contributions. Cross-linking reduces or eliminates stable crack growth. Fracture surfaces of highly cross-linked resins are relatively smooth and featureless.

The deformation characteristics of a DGEBA epoxy system were studied by Pink and Campbell in the temperature range 77 to $380 \mathrm{~K}$ [4.6]. Tensile properties and activation parameters, as measured from strain-rate change experiments, were reported. Tensile modulus and strength data at three temperatures $(77,200$, and $300 \mathrm{~K}$ ) are listed in Table 4.1. From activation-energy analyses, they report three temperature ranges of district deformation mechanisms. At temperatures below $180 \mathrm{~K}$, the activation enthalpy of deformation was about $1 \mathrm{eV}$; between 180 and $320 \mathrm{~K}$, the activation enthalpy range was between 2 and $3 \mathrm{eV}$; at higher temperatures, this enthalpy was estimated between 4 and $6 \mathrm{eV}$. Other conclusions are presented regarding time, temperature, and stress dependencies for the intermediate (180 to $320 \mathrm{~K}$ ) deformation stage.

Limited tensile data were obtained by Hacker et al. on the epoxy system that was used to impregnate the Euratom LCT coil [4.5]. The elastic modulus value at $77 \mathrm{~K}$, given in Table 4.1, agrees quite well with other data at $77 \mathrm{~K}$, but a lower modulus $(2 \mathrm{GPa})$ at room temperature was reported. This resin system had a low viscosity and low cure temperature $(<400 \mathrm{~K})$. 
Ambient temperature data are presented and reviewed by Lee and Neville [4.3]. Data on effects of cure agent, temperature, and concentration are presented. Some of the results are listed in Table 4.1. Tensile data from Chiao et al. from DGEBF resins developed for filament winding [4.4] are also included in Table 4.1. Epoxy resins based on DGEBF have slightly lower viscosities $(3.4 \mathrm{~Pa} \cdot \mathrm{s}$ at $300 \mathrm{~K})$ and slightly better tensile properties.

Poisson's ratio of epoxy resins has been studied at room temperature by Warfield et al. by measuring the stress-strain characteristics with compressibility tests on single specimens [4.9]. Bulk and Young's modulus were measured simultaneously. They obtained values of Poisson's ratio ranging from 0.38 to 0.41 . Individual measurements included polyepoxide $-0.41,0.40$; polyepoxide plus DDSA - 0.38; polyepoxide plus MPDA - 0.40 .

\subsection{Resins}

All the resin systems included in the overall program were measured. They included nine epoxies, four vinyl esters, one polyester, and four cyanate esters. These resin systems are discussed in detail in §III.3; their trade names and key to their abbreviations are given in Tables 2.1 and 2.2 of the Introduction (\$III.2).

\subsection{Procedures}

Tensile tests were conducted using screw-driven, constant-deflection-rate machines at room temperature, $76 \mathrm{~K}$ (boiling liquid nitrogen), and $4 \mathrm{~K}$ ( boiling liquid helium).

Specimens were machined from the 3-mm-thick plate produced by pressure molding (§III.3.3.1). The configurations of the specimens are shown in Figure 4.1. Originally, all specimens were machined according to Figure 4.1a specifications, but at low temperatures most of these specimens failed in the grips or along the radius. Therefore, the reduced sections of these specimens were further decreased in area, following Figure $4.1 \mathrm{~b}$ specifications. Subsequently, most of the lowtemperature failures occurred within the gage length.

The specimens were held with bolted friction grips at both wide ends. The amount of compression force on the specimen, which was applied with a torque wrench on the bolts of the end grips, proved to be a sensitive variable. Too much pressure induced microcracking on cooling due to thermal-contraction mismatch of the metal grip and neat resin. Too little pressure led to specimen slippage under tensile load. The effective crosshead rate was held at $0.02 \mathrm{~cm} / \mathrm{min}$ in all tests. Specimens were cooled slowly in all low-temperature tests to reduce the stresses due to thermalcontraction mismatch.

Young's modulus and Poisson's ratio were measured by using longitudinal and transverse oriented low-temperature strain gages.

Failures always occurred at the strain gages, independent of test temperature. Thus, separate tests were conducted to obtain ultimate strength data. In practice, two specimens were used to obtain data for Young's modulus and Poisson's ratio at each test temperature, and a minimum of two specimens were used (without strain gages) to obtain ultimate strength data. Usually, four or more specimens were tested without strain gages at low temperatures. 
(a)

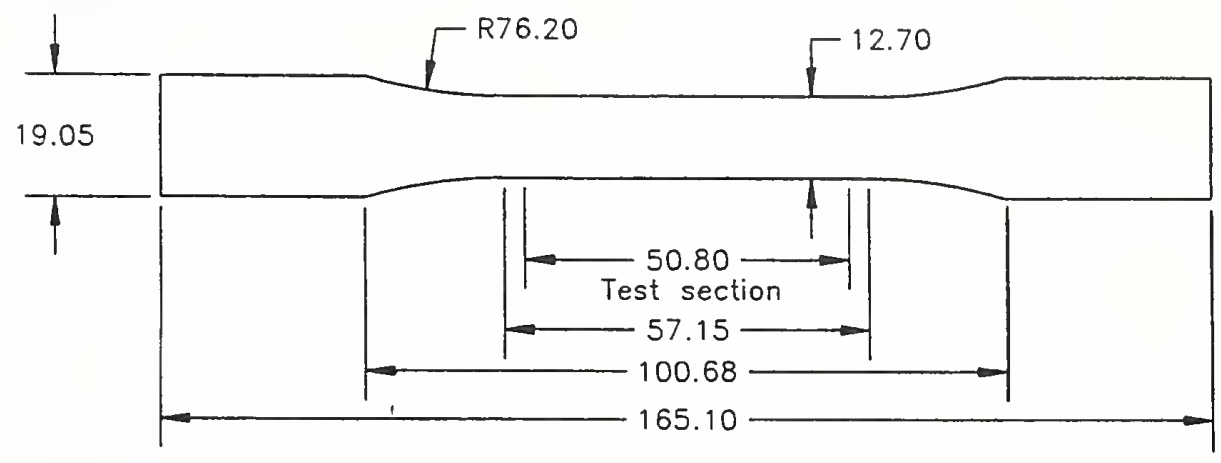

(b)

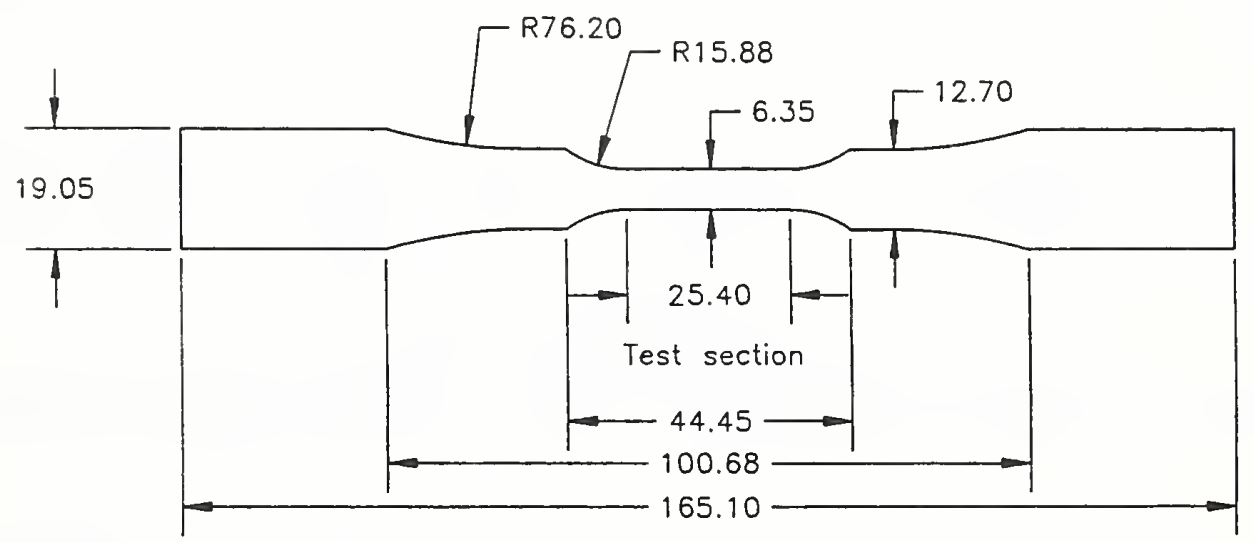

Figure 4.1 Specimen dimensions (in millimeters).

\subsection{Results}

Three sets of data were obtained for each neat resin at 295, 76, and $4 \mathrm{~K}$ : Young's modulus, Poisson's ratio, and ultimate tensile strength. These are discussed separately.

\subsubsection{Young's Modulus}

Young's moduli of the epoxy-base resin systems at 295, 76, and $4 \mathrm{~K}$ are plotted in Figures 4.2 through 4.9. Moduli data at low temperatures for the cyanate-ester systems are plotted in Figures 4.10 through 4.13; for the vinyl ester systems, in Figures 4.14 through 4.17; and for the polyester, in Figure 4.18. 


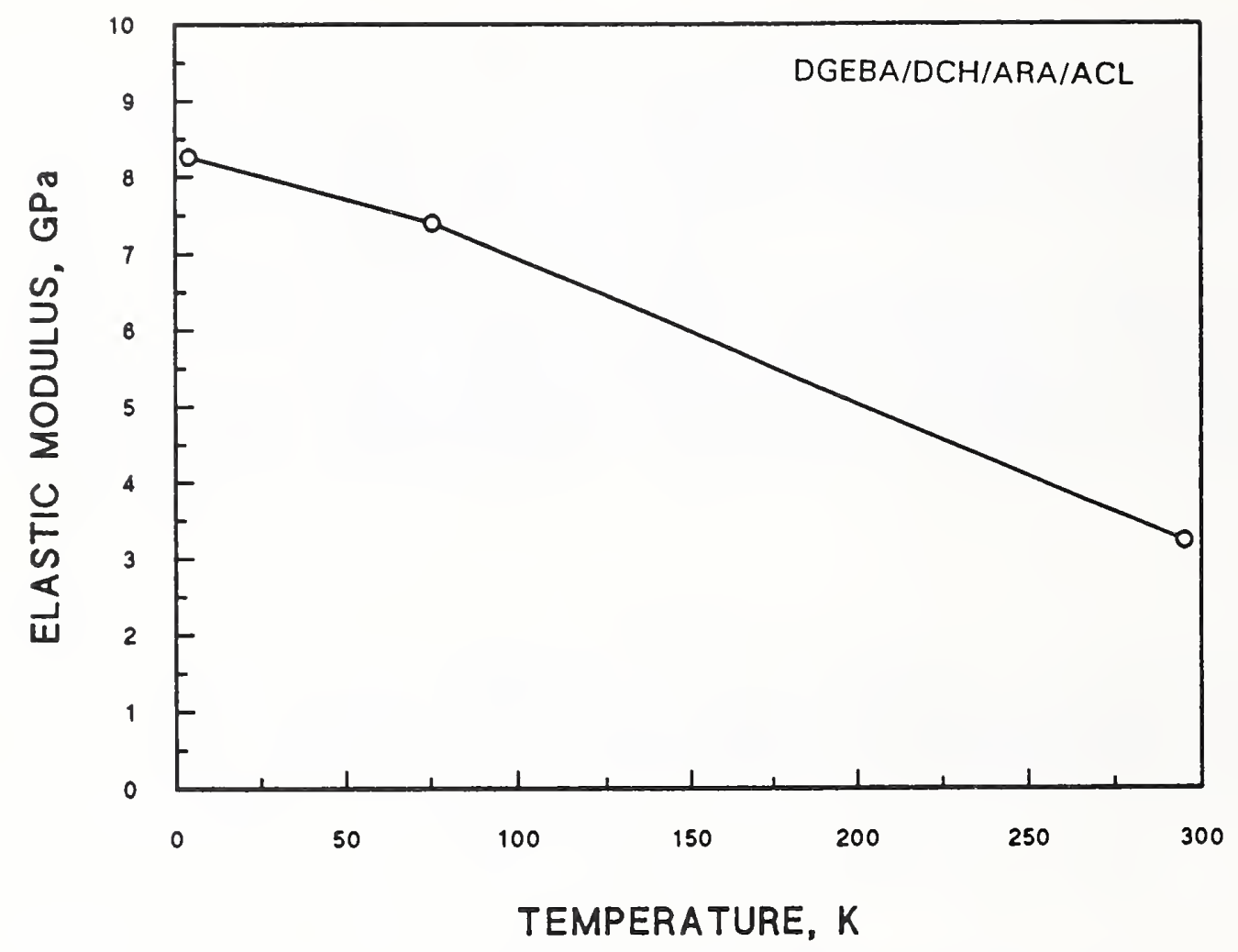

Figure 4.2. Young's modulus versus temperature-DGEBA/DCH/ARA/ACL epoxy.

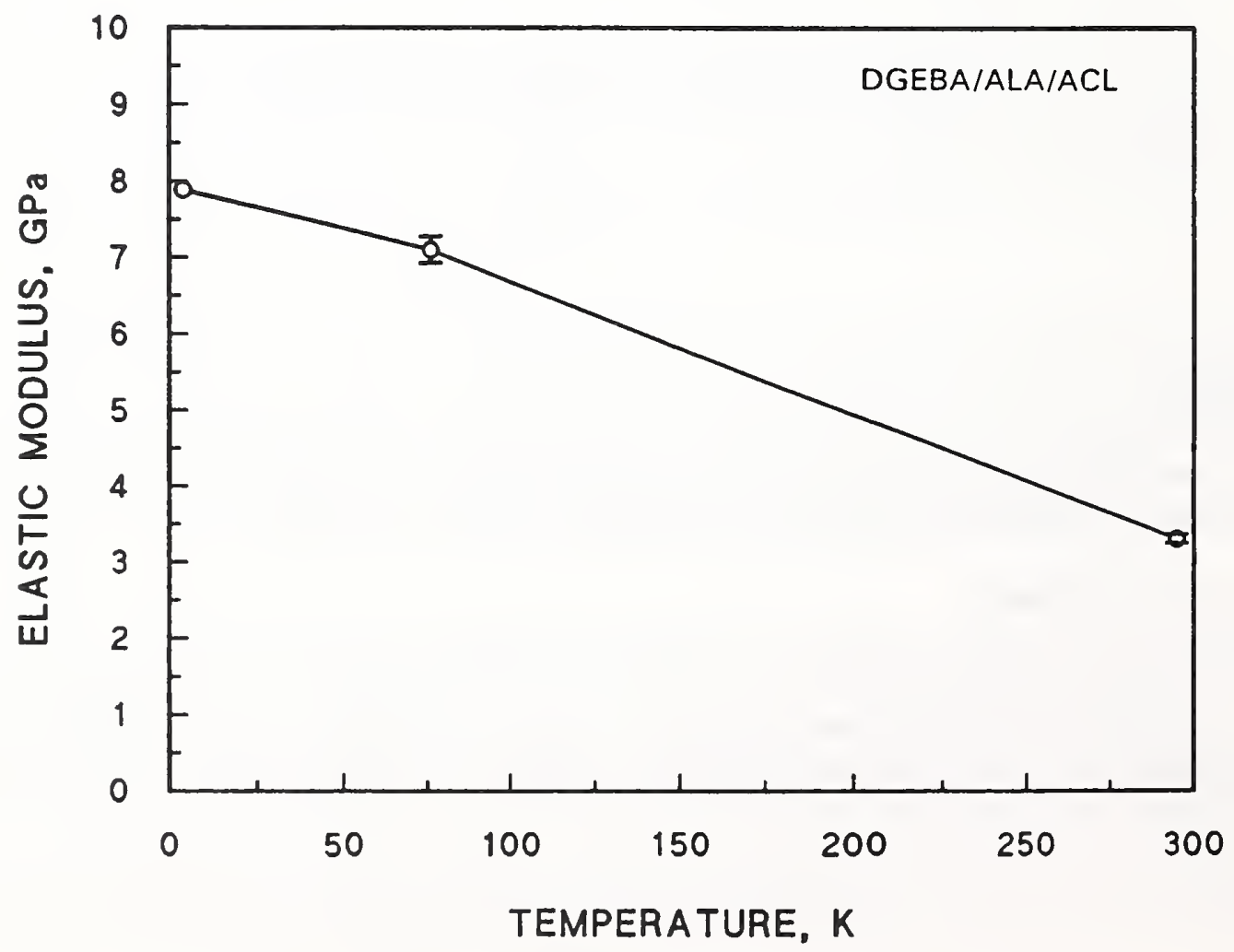

Figure 4.3. Young's modulus versus temperature-DGEBA/ALA/ACL epoxy. 


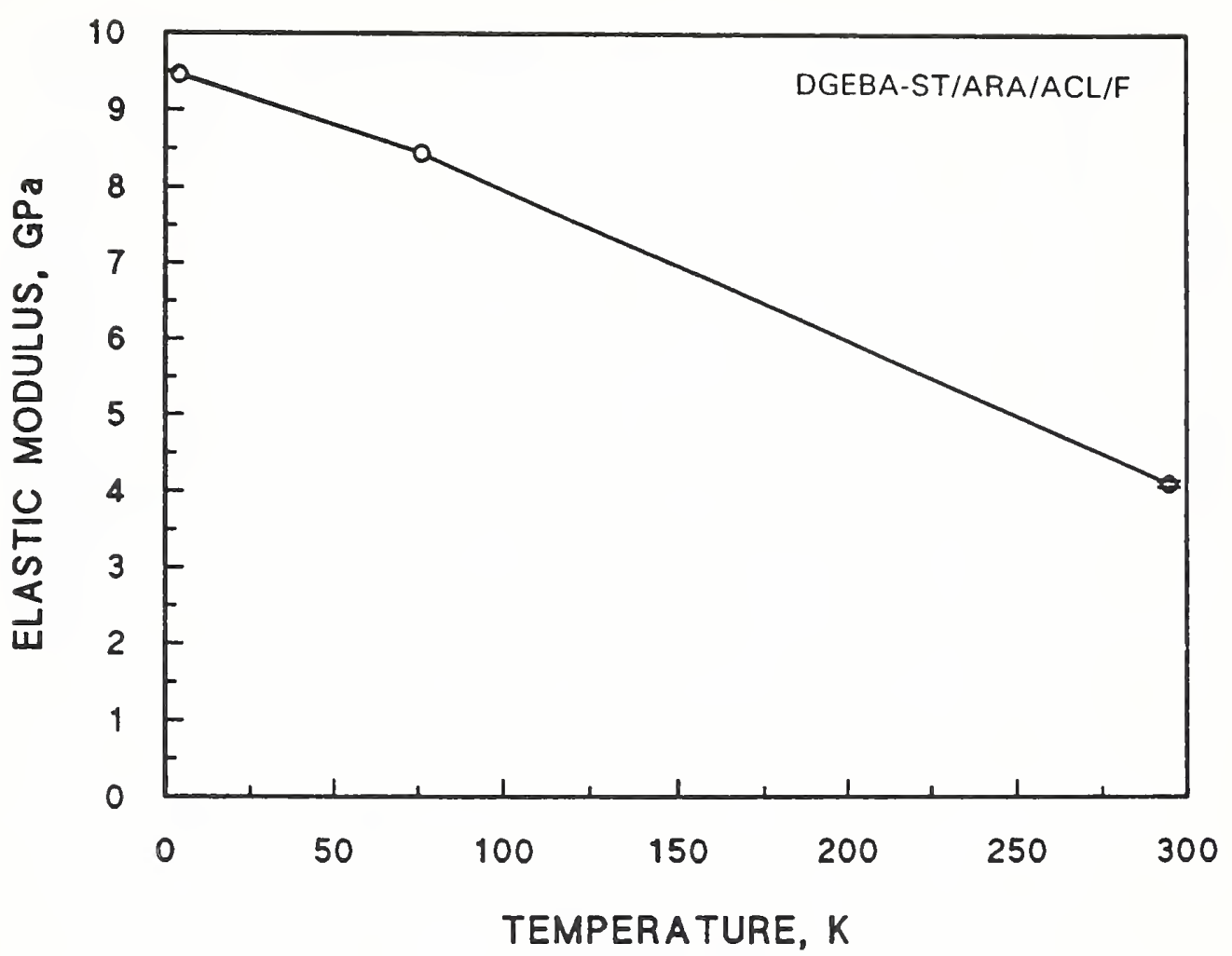

Figure 4.4. Young's modulus versus temperature-DGEBA-ST/ARA/ACL/F epoxy.

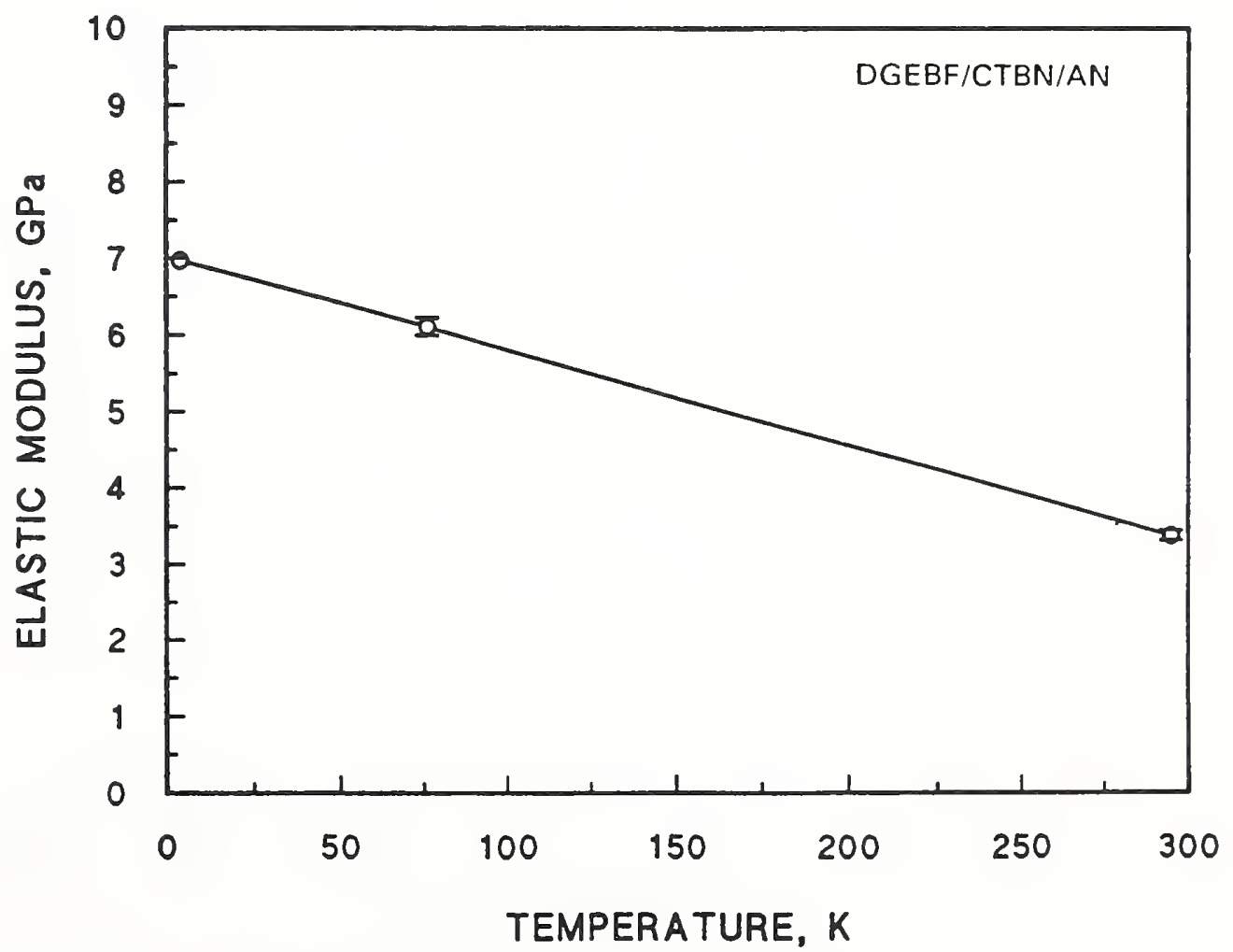

Figure 4.5. Young's modulus versus temperature-DGEBF/CTBN/AN epoxy. 


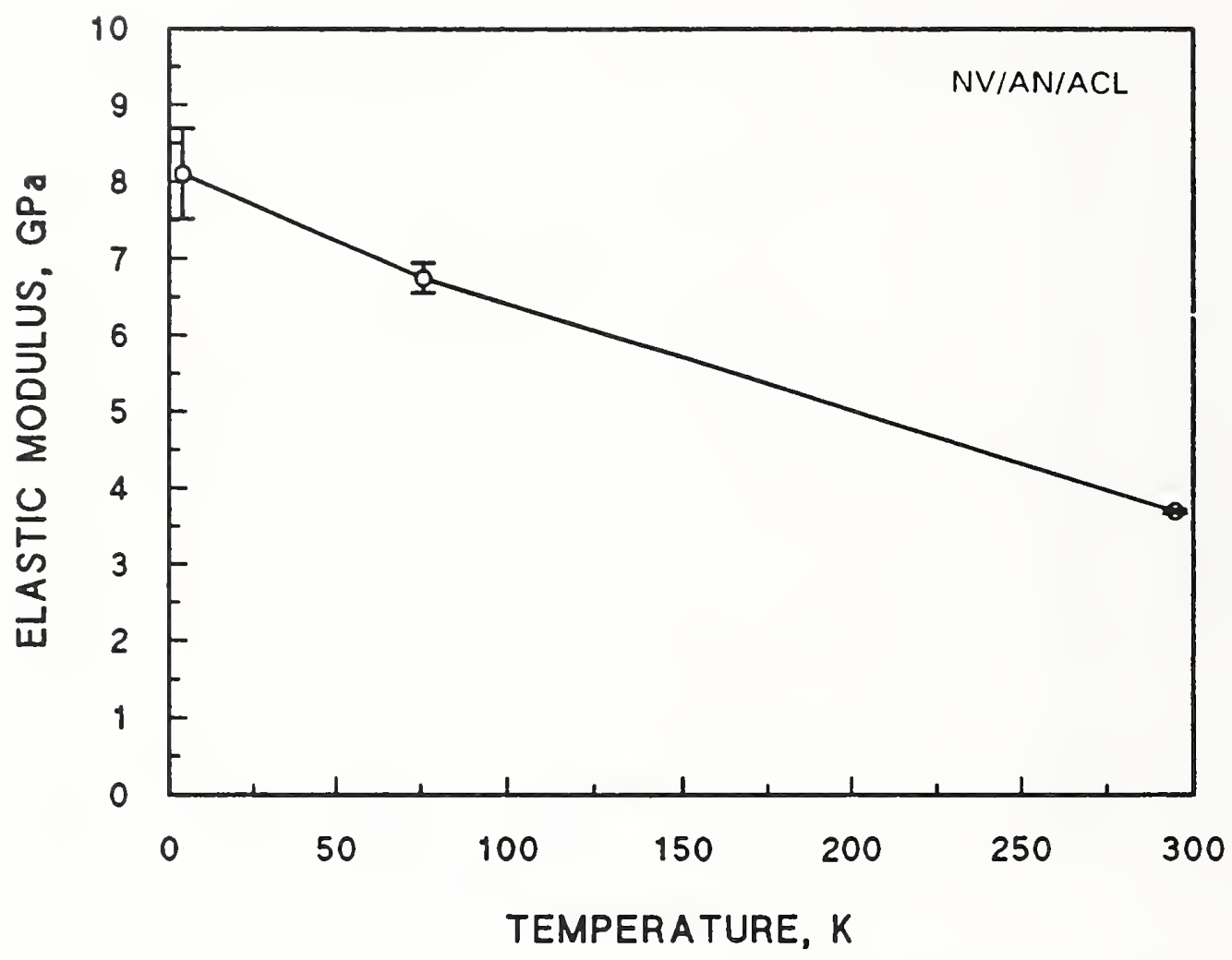

Figure 4.6. Young's modulus versus temperature-NV/AN/ACL epoxy.

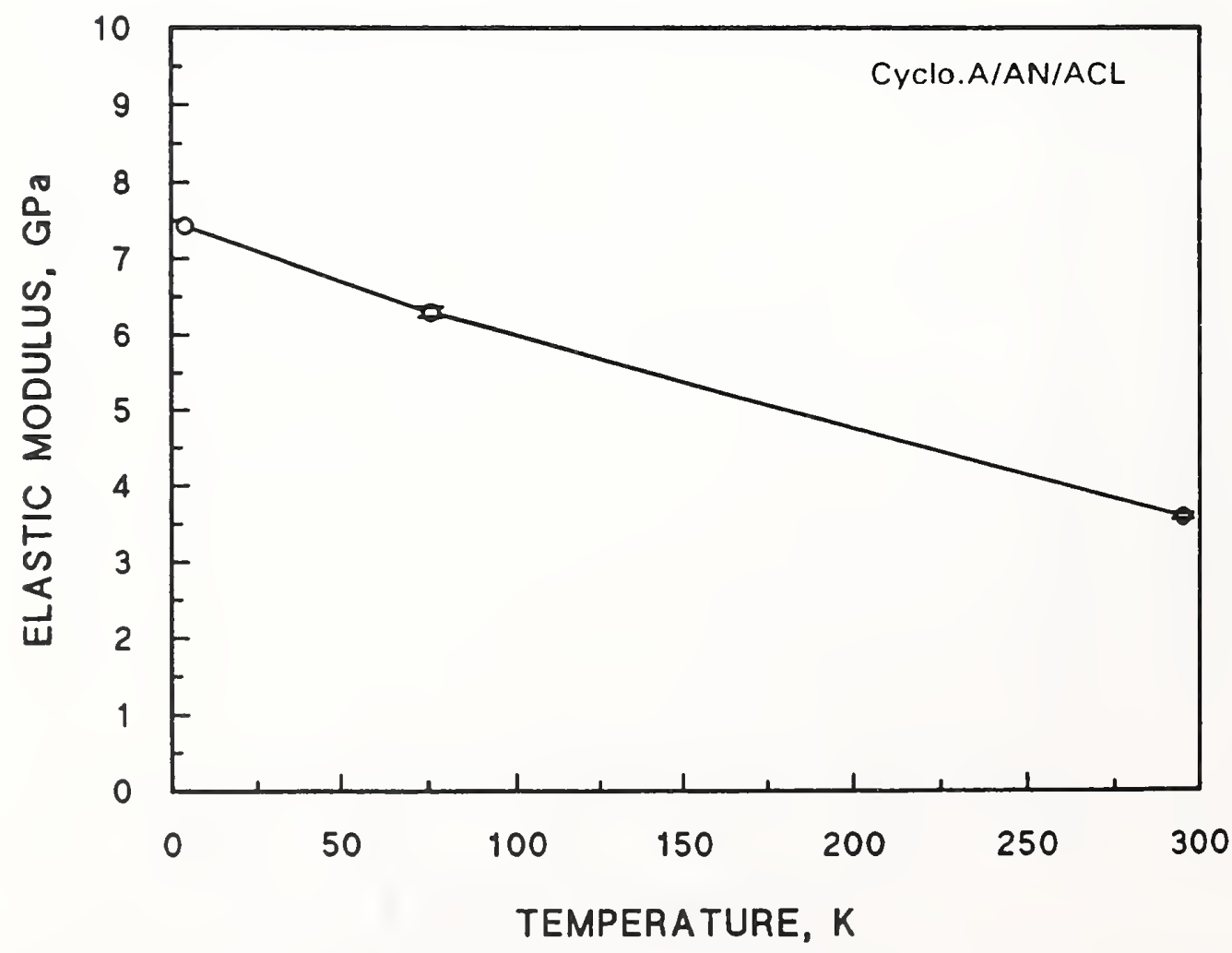

Figure 4.7. Young's modulus versus temperature-Cyclo.A/AN/ACL epoxy. 


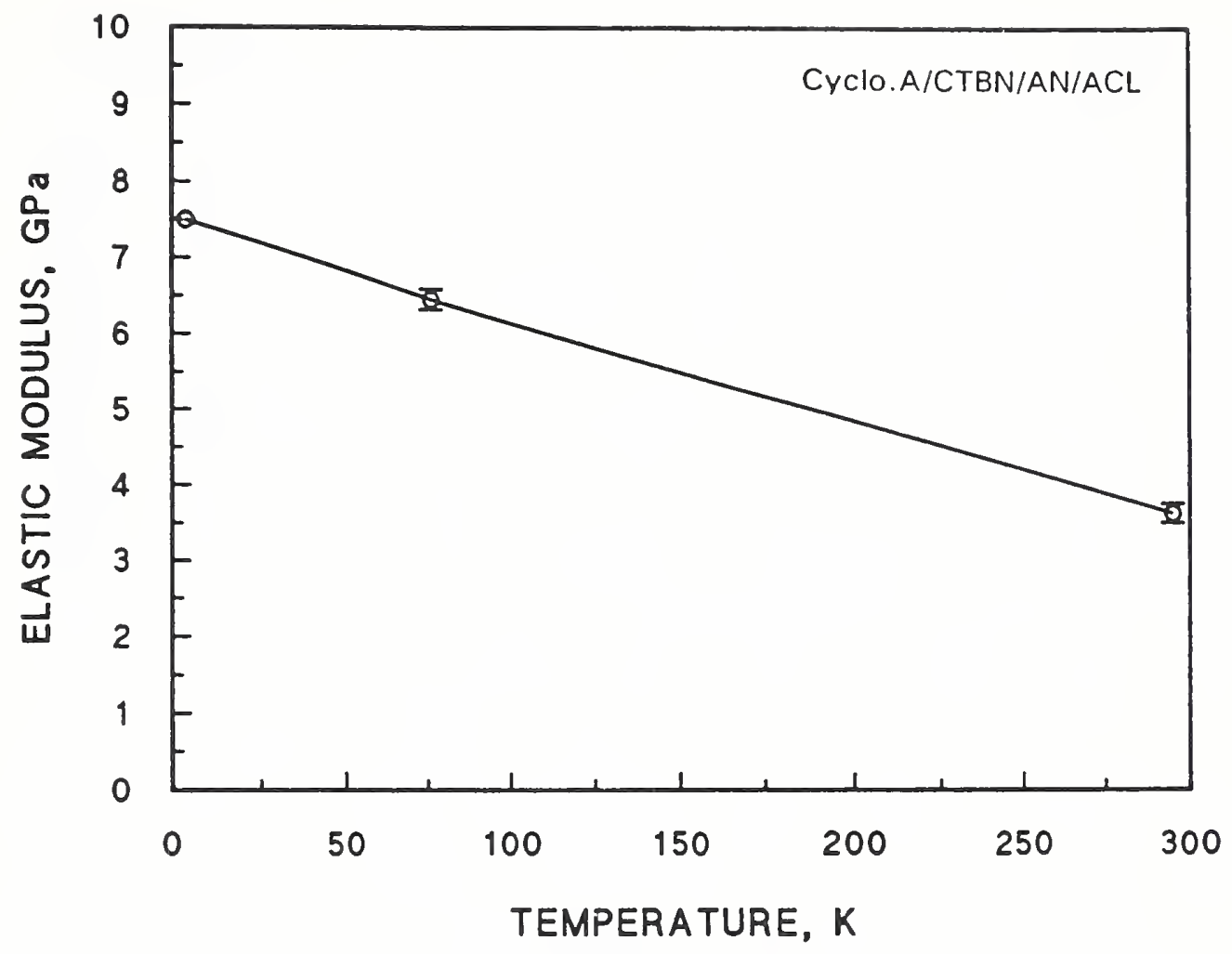

Figure 4.8. Young's modulus versus temperature-Cyclo.A/CTBN/AN/ACL epoxy.

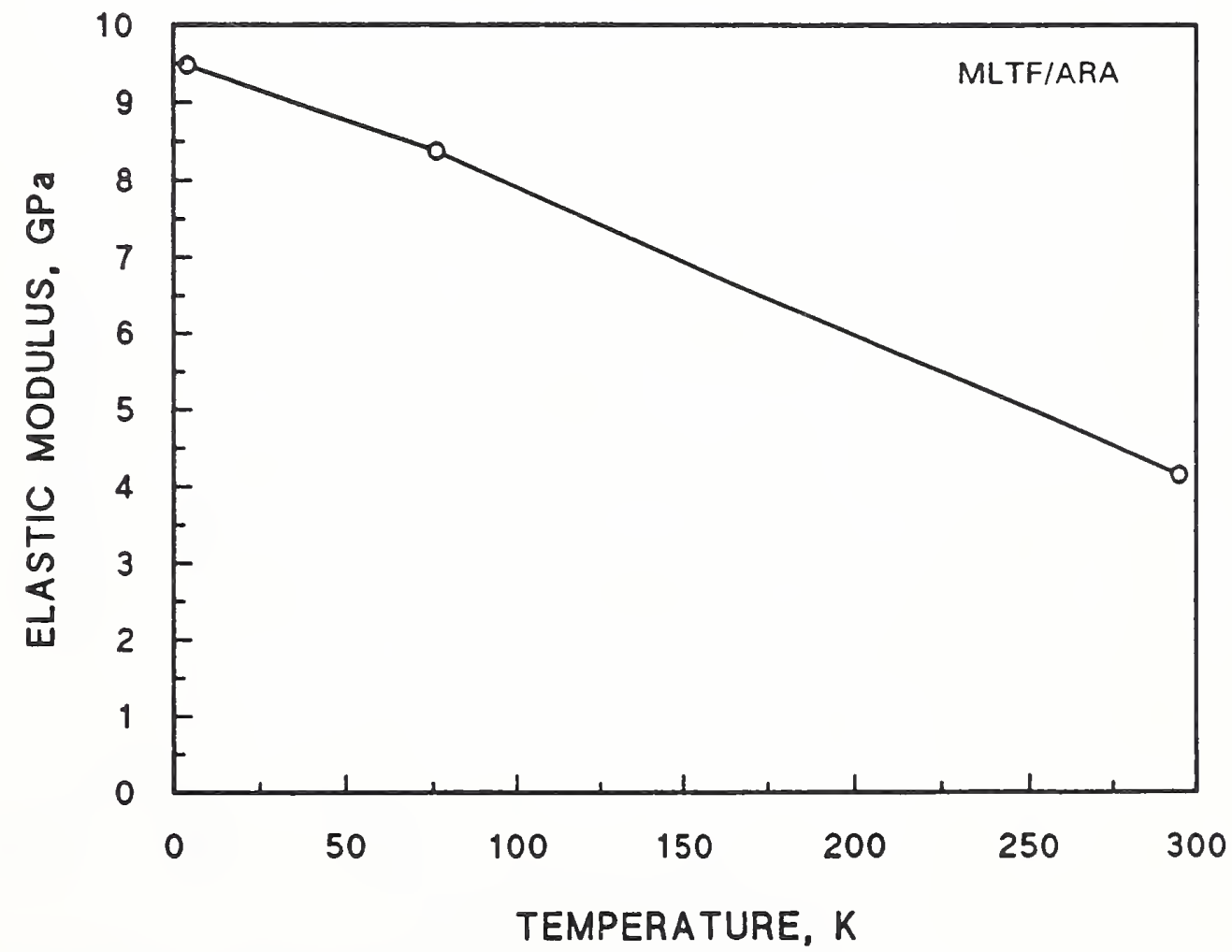

Figure 4.9. Young's modulus versus temperature-MLTF/ARA epoxy. 


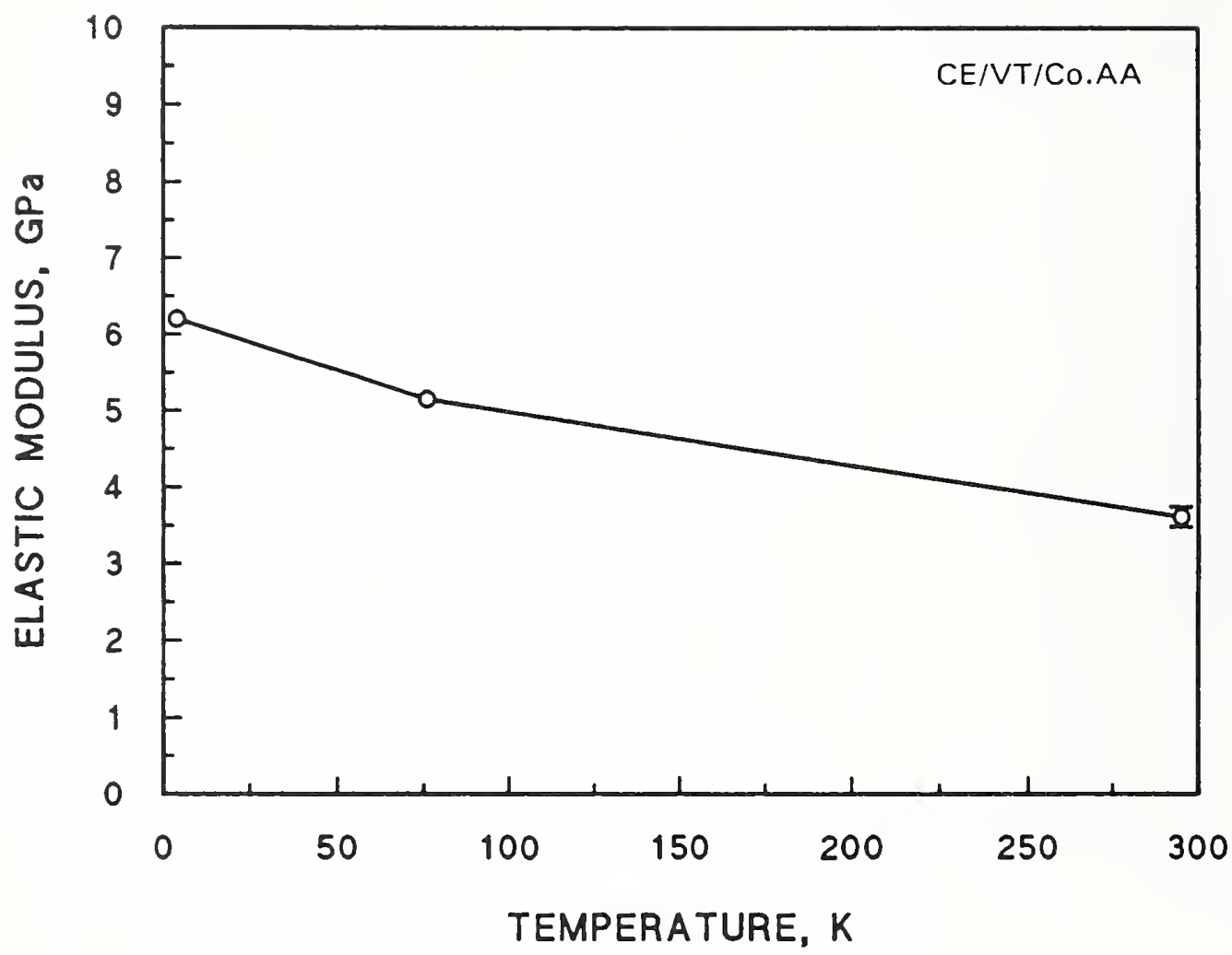

Figure 4.10. Young's modulus versus temperature-CE/VT/Co.AA cyanate ester.

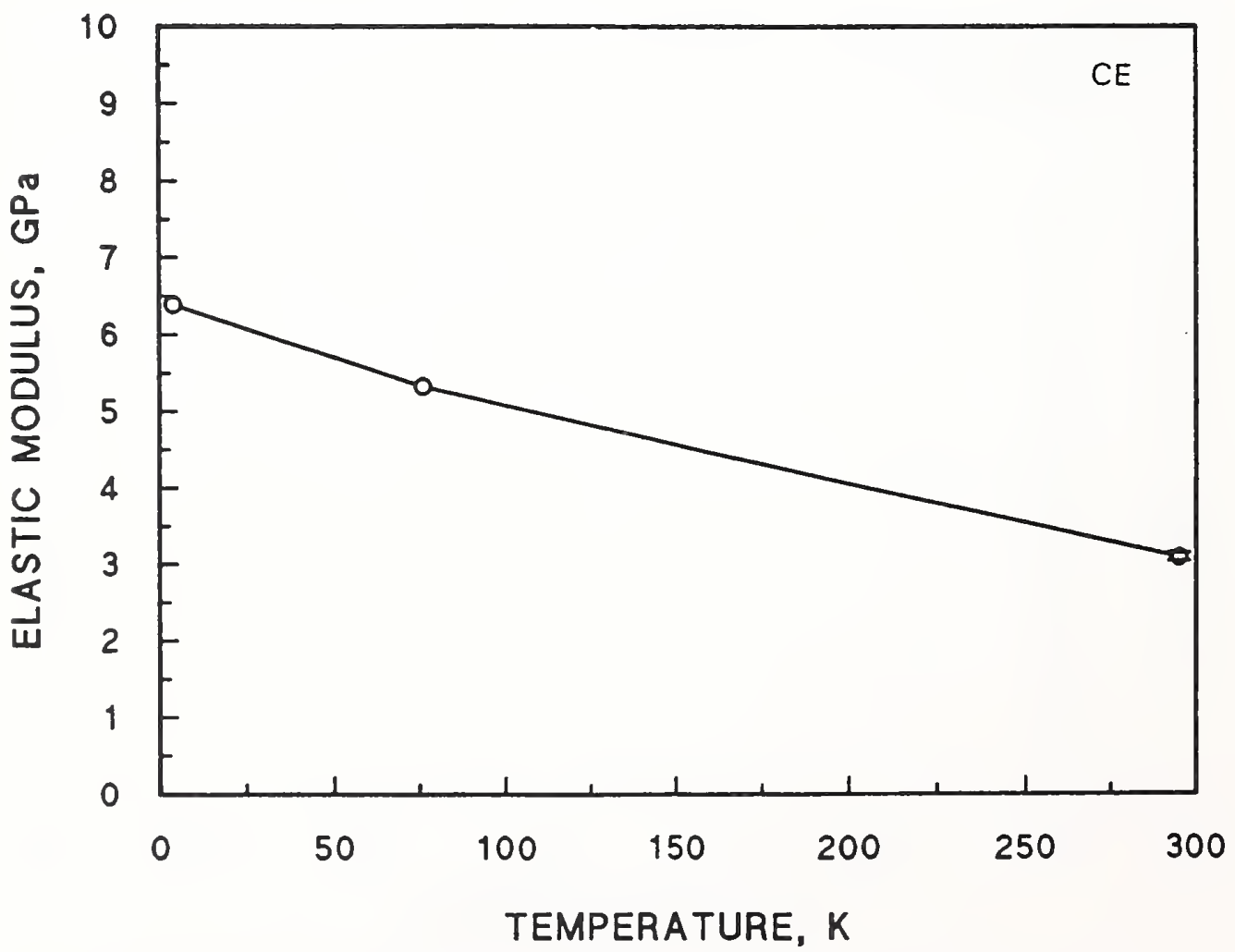

Figure 4.11. Young's modulus versus temperature-CE cyanate ester. 


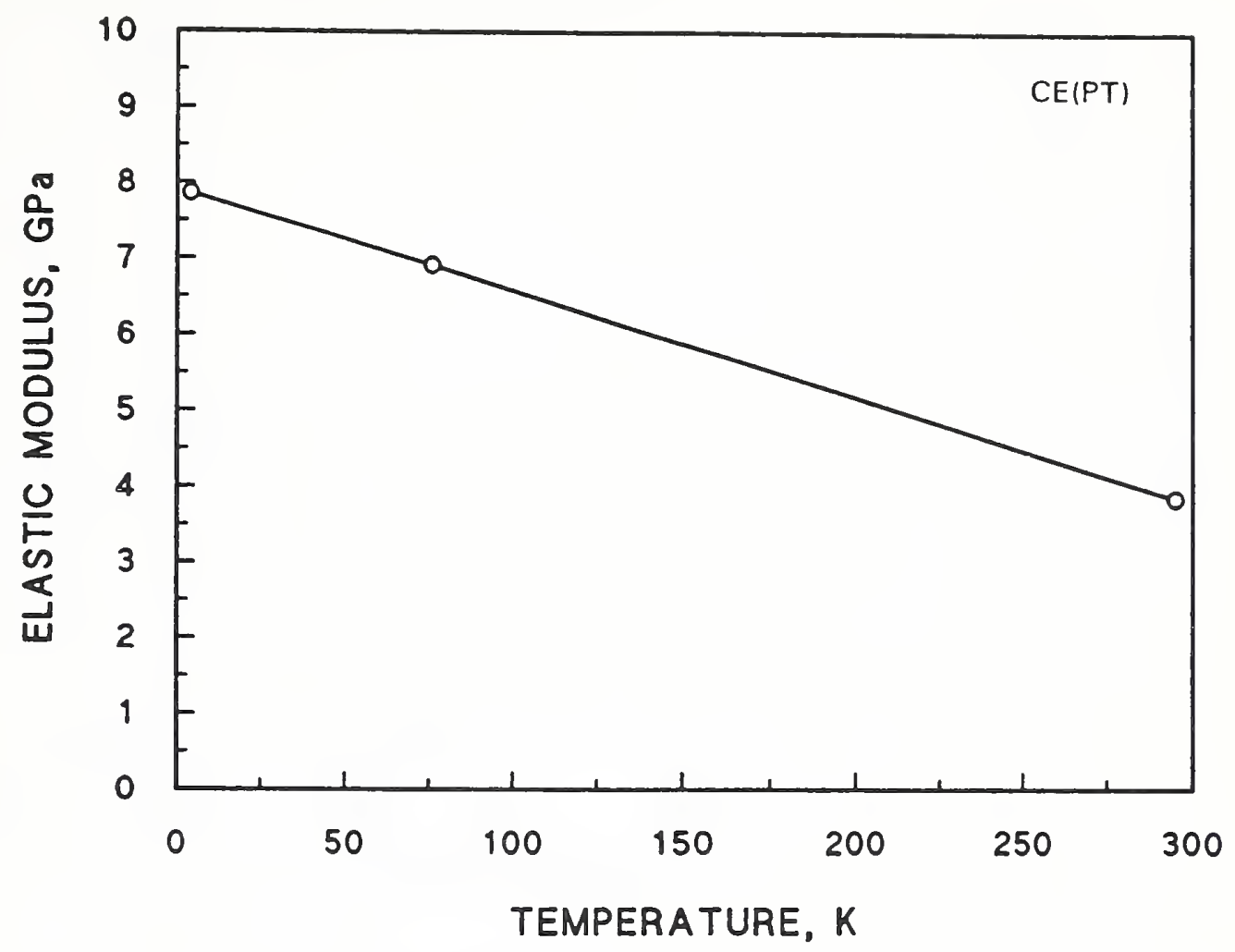

Figure 4.12. Young's modulus versus temperature-CE(PT) cyanate ester.

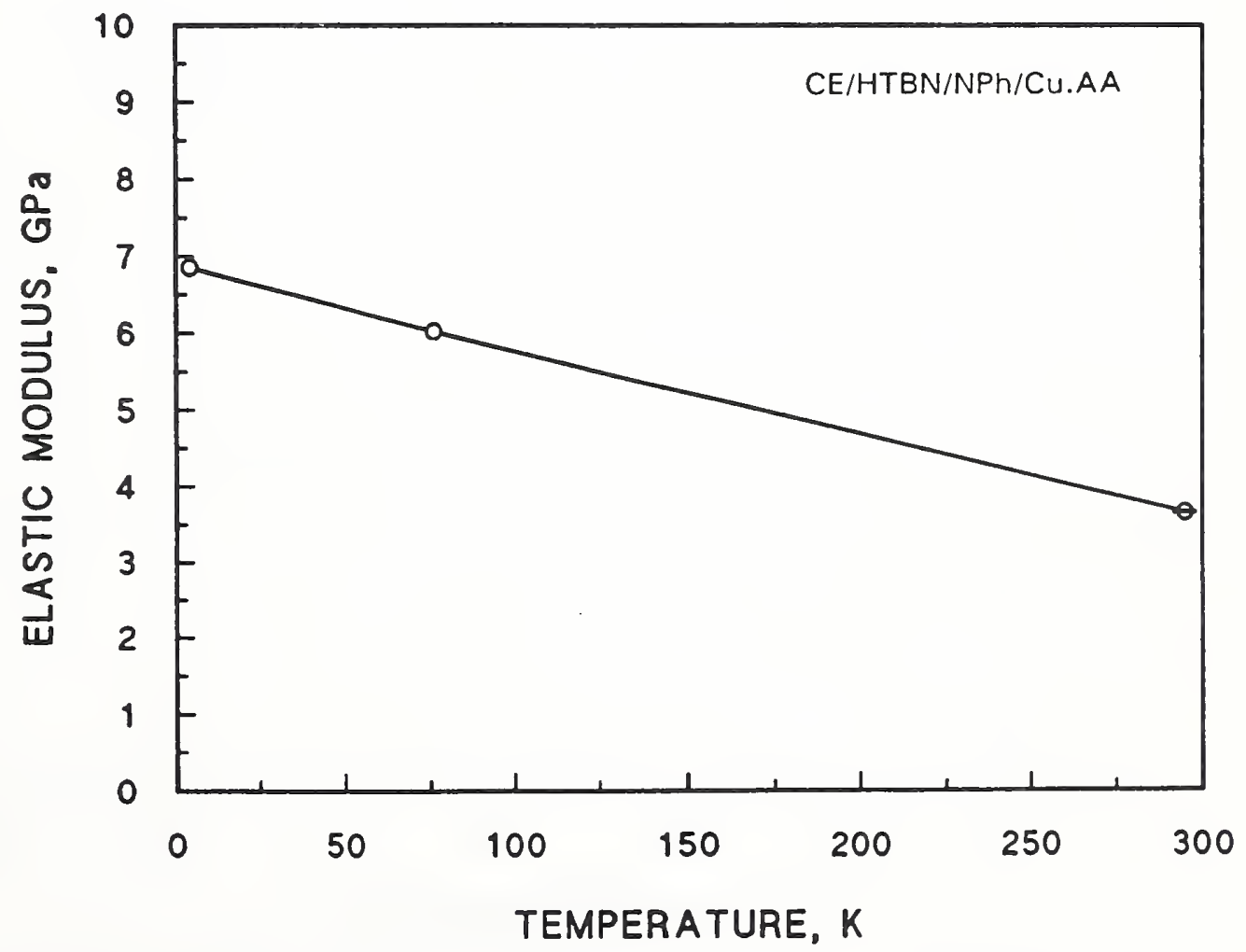

Figure 4.13. Young's modulus versus temperature-CE/HTBN/NPh/Cu.AA cyanate ester. 


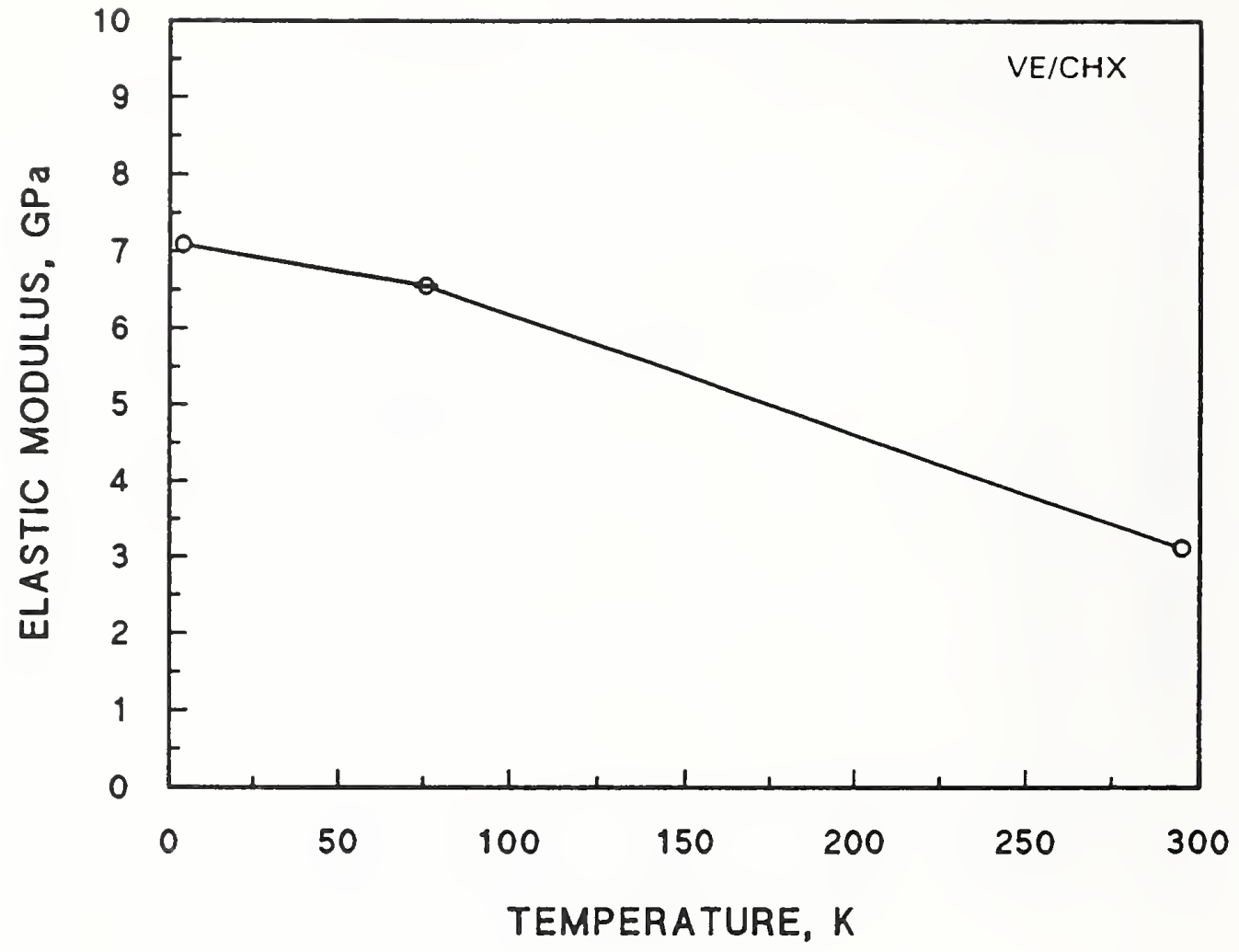

Figure 4.14. Young's modulus versus temperature-VE/CHX vinyl ester.

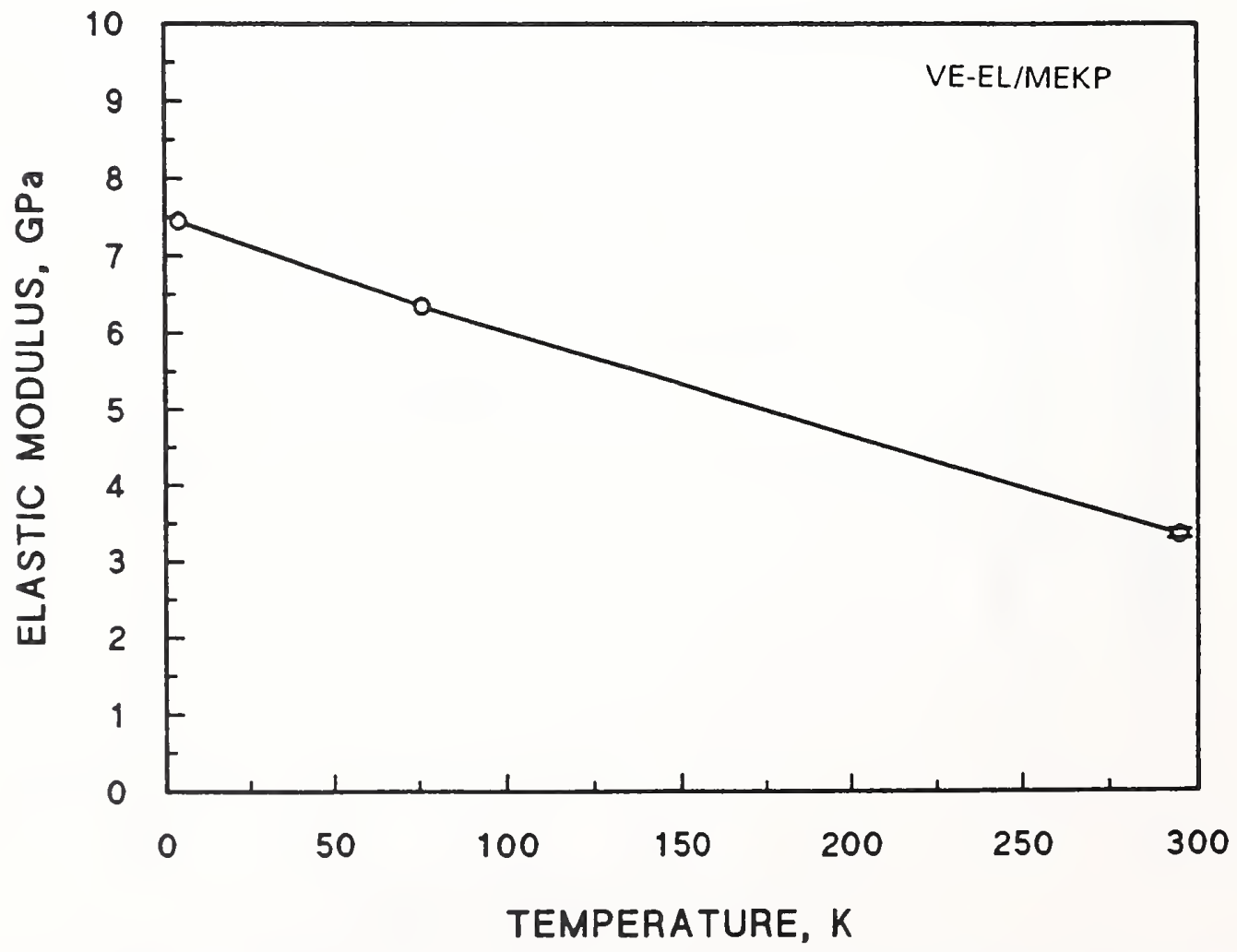

Figure 4.15. Young's modulus versus temperature-VE-EL/MEKP vinyl ester. 


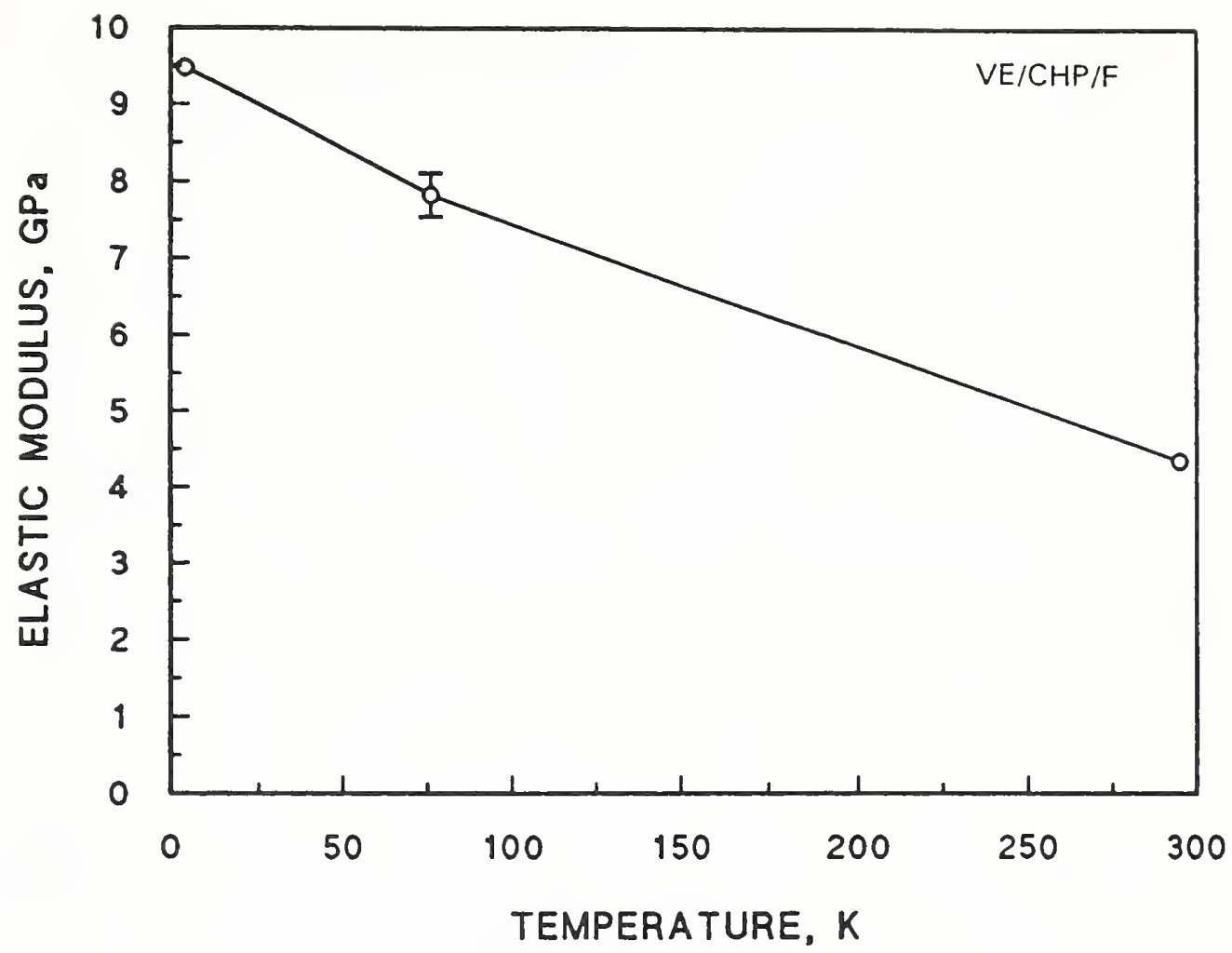

Figure 4.16. Young's modulus versus temperature-VE/CHP/F vinyl ester.

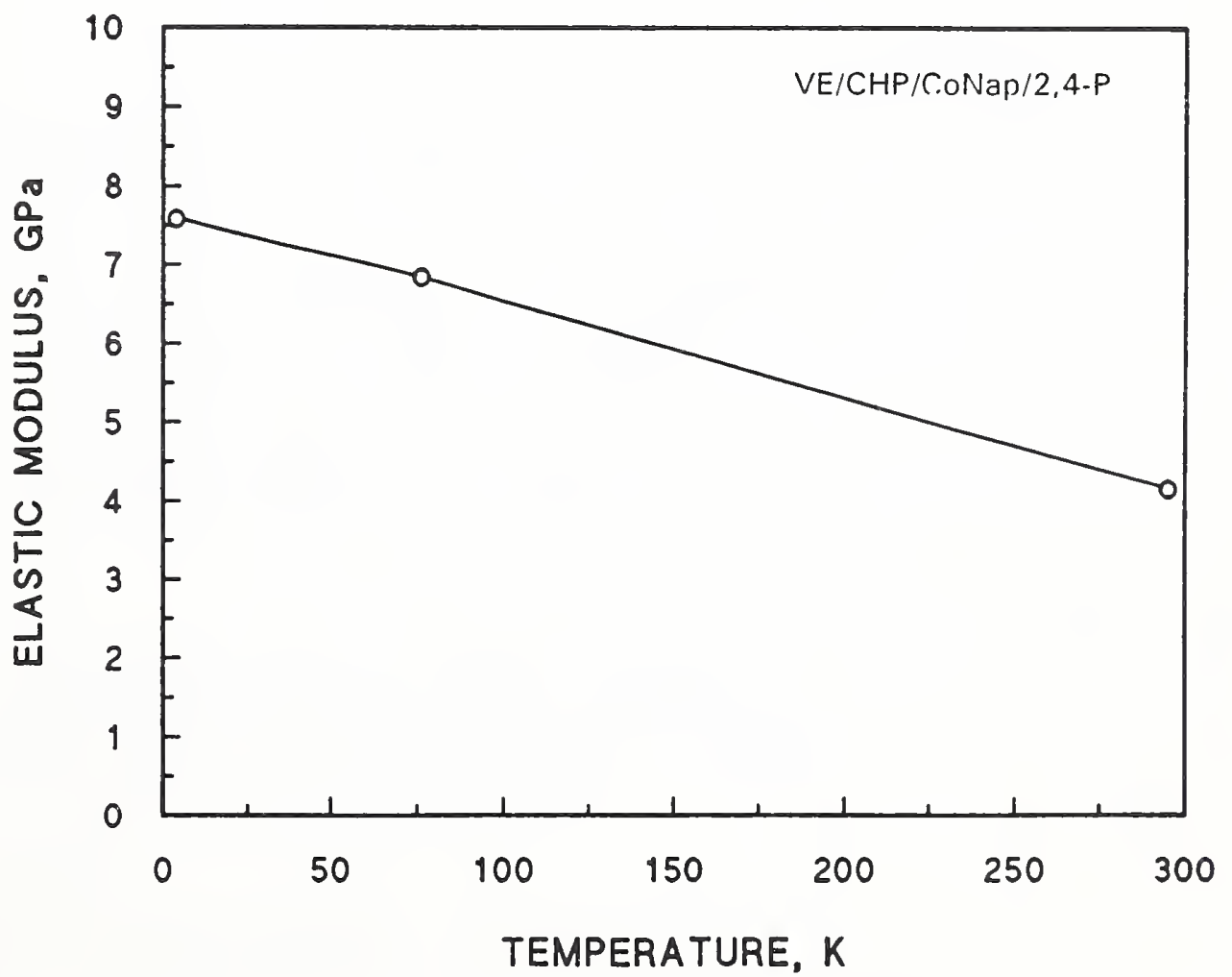

Figure 4.17. Young's modulus versus temperature-VE/CHP/CoNap/2,4-P vinyl ester. 


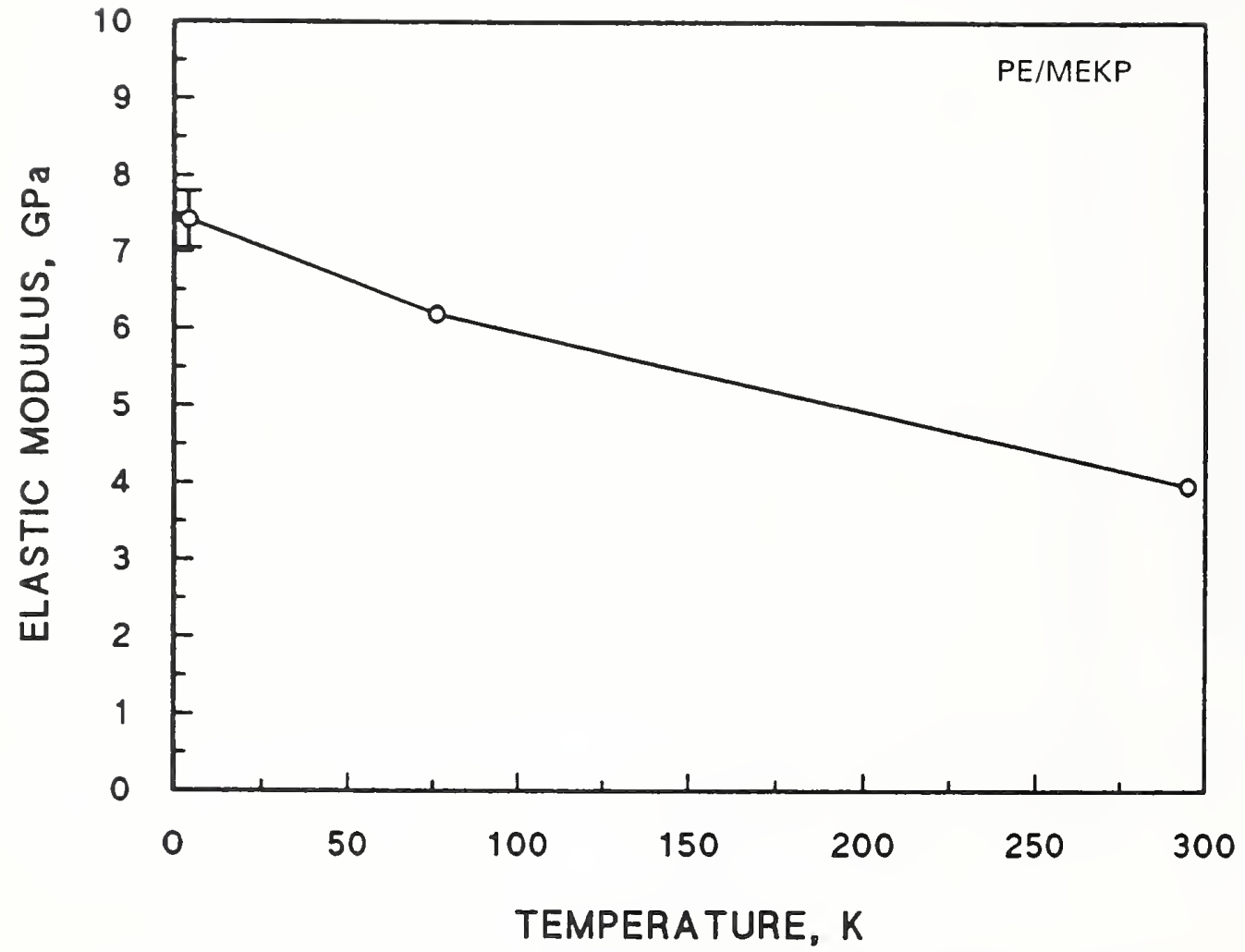

Figure 4.18. Young's modulus versus temperature-PE/MEKP polyester.

Several traits distinguish these resin systems:

1. The highest Young's moduli at room temperature (average values $\geq 4.06 \mathrm{GPa}$ ) were obtained from filled resin systems (Figs. 4.4 and 4.16), a multifunctional epoxy (Fig. 4.9), and a cyanate-ester, phenolic-triazine system (Fig. 4.12). ${ }^{*}$ The filled resin systems and the multifunctional epoxy system also had the highest average values $(9.5 \mathrm{GPa})$ at $4 \mathrm{~K}$.

2. The cyanate-ester resin systems, which exhibited a lower dependence of Young's Modulus on temperature, had the lowest Young's moduli at $4 \mathrm{~K} .^{*}$

3. The epoxy, vinyl-ester, and polyester resin systems, in general, all had comparable Young's moduli at room and low temperatures.

The data for each resin system and test temperature are presented in Table A in Appendix A (§III.13).

\subsubsection{Poisson's Ratio}

Average values of Poisson's ratio for the four resin classes are summarized in Table 4.3. In these averages the data for the filled resin systems were excluded. All data from individual tests are listed in Appendix A, Table A (\$III.13).

\footnotetext{
* See comments regarding cure schedule on page III-16.
} 
Table 4.3. Average Poisson's ratio data.

\begin{tabular}{lccc}
\hline \hline \multirow{2}{*}{$\begin{array}{c}\text { Resin Base } \\
\text { (no. of resins) }\end{array}$} & \multicolumn{3}{c}{$\begin{array}{c}\text { Average Values at Each Temperature } \\
\text { (range of values) }\end{array}$} \\
\cline { 2 - 4 } & $295 \mathrm{~K}$ & $76 \mathrm{~K}$ & $4 \mathrm{~K}$ \\
\hline \hline Epoxy (7) & 0.37 & 0.42 & 0.40 \\
& $(0.34-0.38)$ & $(0.38-0.47)$ & $(0.38-0.43)$ \\
Vinyl ester (3) & 0.36 & 0.43 & 0.42 \\
& $(0.34-0.39)$ & $(0.40-0.46)$ & $(0.40-0.43)$ \\
Cyanate ester (4) ${ }^{*}$ & 0.35 & 0.43 & 0.43 \\
& $(0.33-0.37)$ & $(0.41-0.46)$ & $(0.42-0.43)$ \\
Polyester (1) & 0.33 & 0.46 & 0.40 \\
& - & - & - \\
\hline \hline
\end{tabular}

Poisson's ratios at low temperatures ( 76 and $4 \mathrm{~K}$ ) are about $20 \%$ larger than those at room temperature. A surprising result is that the Poisson's ratios of those resins with high values at $76 \mathrm{~K}$ $(\sim 0.44$ to 0.47$)$ are apparently less at $4 \mathrm{~K}$ than at $76 \mathrm{~K}$. This behavior must be studied further. The effect of small changes of strain-gage sensitivity between 76 and $4 \mathrm{~K}$ must be considered and more than two specimens per resin system per temperature must be included. The average value of Poisson's ratios for epoxies is slightly larger at room temperature and slightly less at low temperatures than values for the other resin classes.

\subsubsection{Ultimate Tensile Strength}

The ultimate tensile strengths of 18 neat resins were measured at 4, 76, and $295 \mathrm{~K}$. In Figures 4.19 through 4.26 the results for epoxy resins are presented. Data for the cyanate-ester systems are shown in Figures 4.27 through 4.29; for the vinyl-ester systems, in Figures 4.30 through 4.33; and for the polyester system, in Figure 4.34. Averages of the highest three ultimate strengths at each temperature are used to portray the inherent strength of each resin system. The lower values reflect the effects of processing or chemical defects (e.g., porosity or surface scratches on secondary crystallites) on the fracture strength, which is discussed later. As the test temperature is lowered, there is more data scatter, which is caused by the higher sensitivity to defects at lower temperatures.

On perusal of Figures 4.19 through 4.34 , several trends are apparent:

1. The low-temperature ultimate tensile strength is generally higher than the room-temperature strength.

2. Considerably greater scatter of data occurs at low temperatures.

3. The unfilled vinyl esters are the strongest at $4 \mathrm{~K}$.

4. At room temperature, the average strengths for all resin systems fall with in a narrow band of 55 to $89 \mathrm{MPa}$.

5. The neat-resin systems with high Young's modulus have low tensile strengths at $4 \mathrm{~K}$ (see Figs. 4.21, 4.26, and 4.32). 


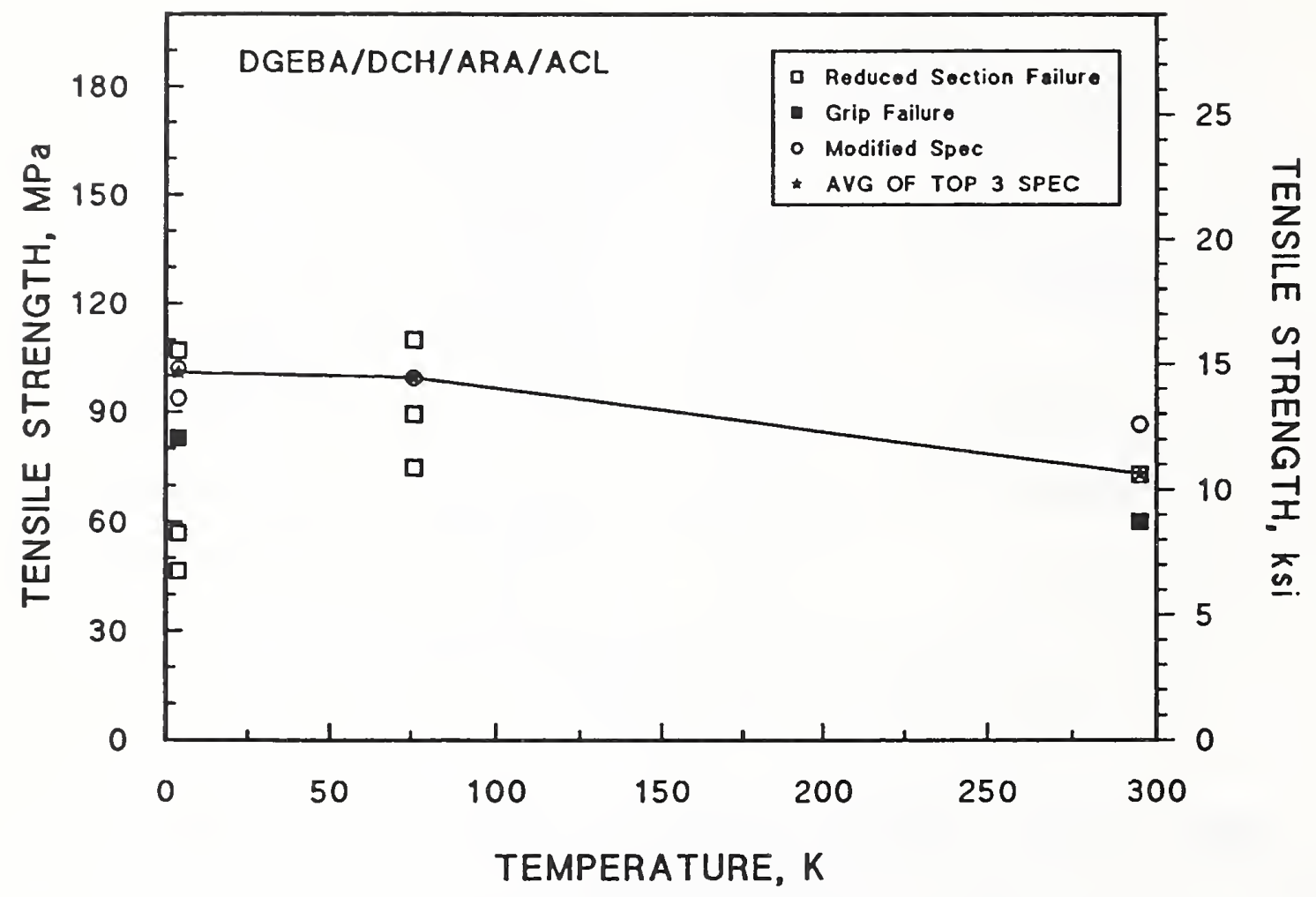

Figure 4.19. Ultimate tensile strength versus temperature-DGEBA/DCH/ARA/ACL epoxy.

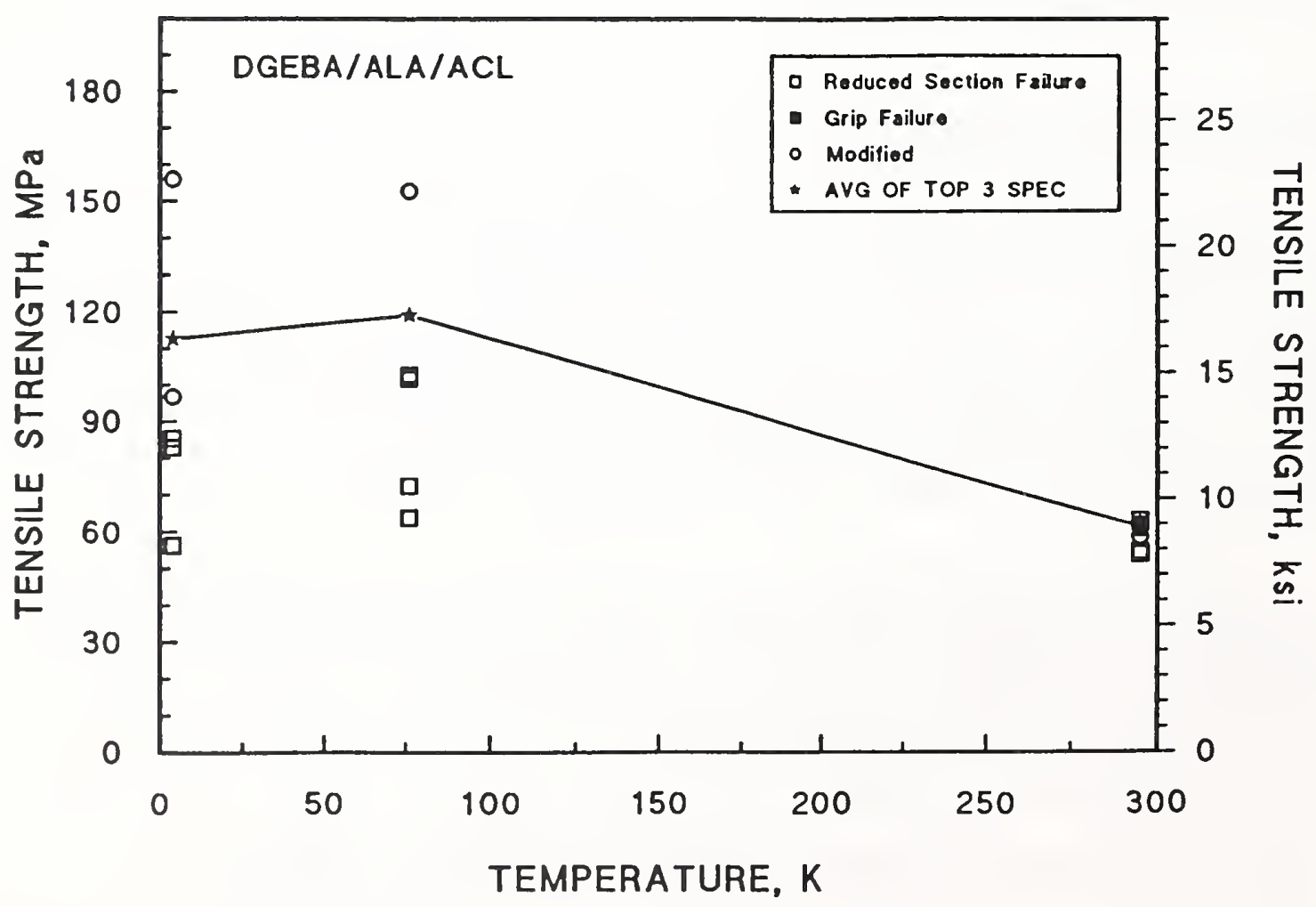

Figure 4.20. Ultimate tensile strength versus temperature-DGEBA/ALA/ACL epoxy. 


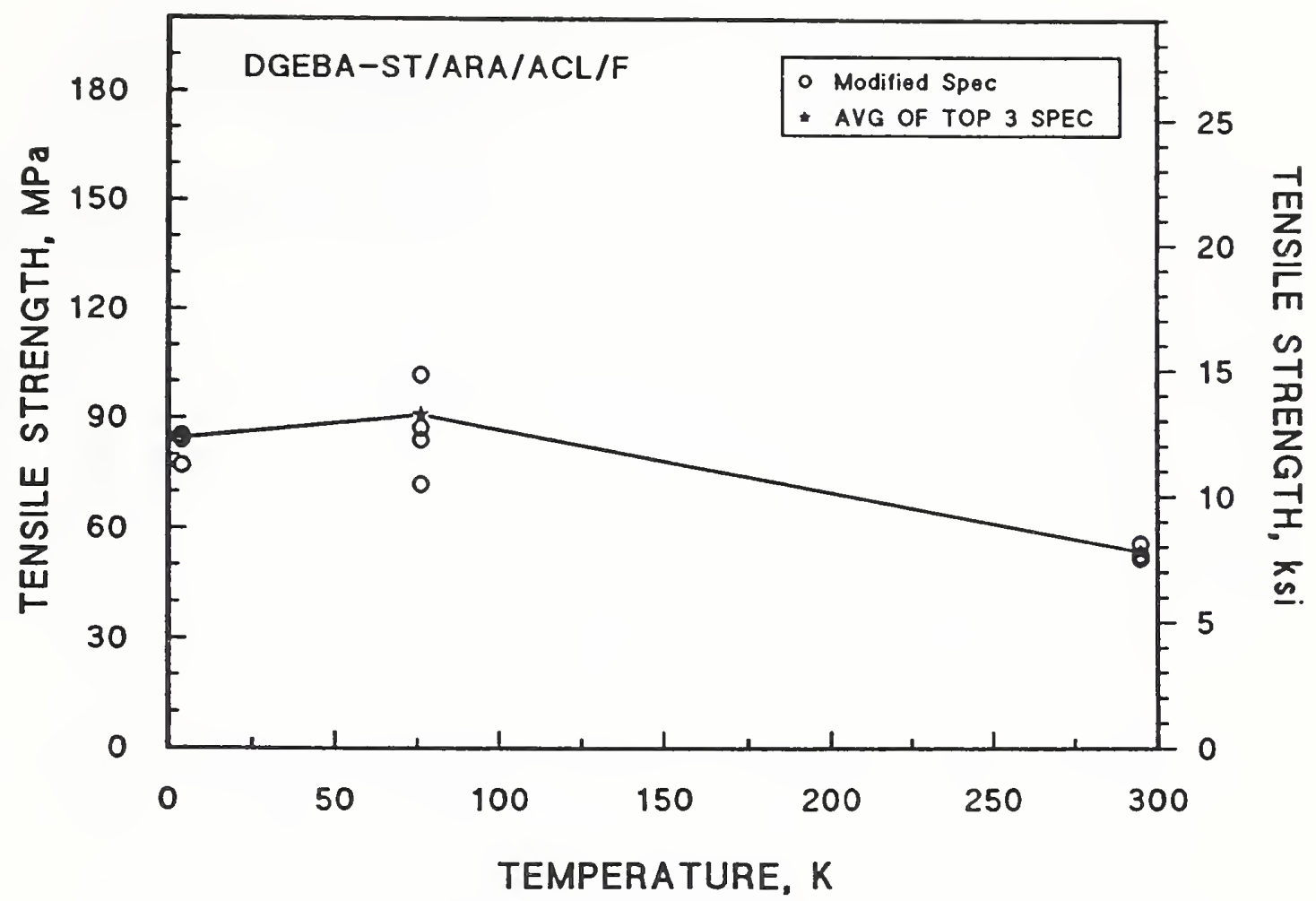

Figure 4.21. Ultimate tensile strength versus temperature-DGEBA-ST/ARA/ACL/F epoxy.

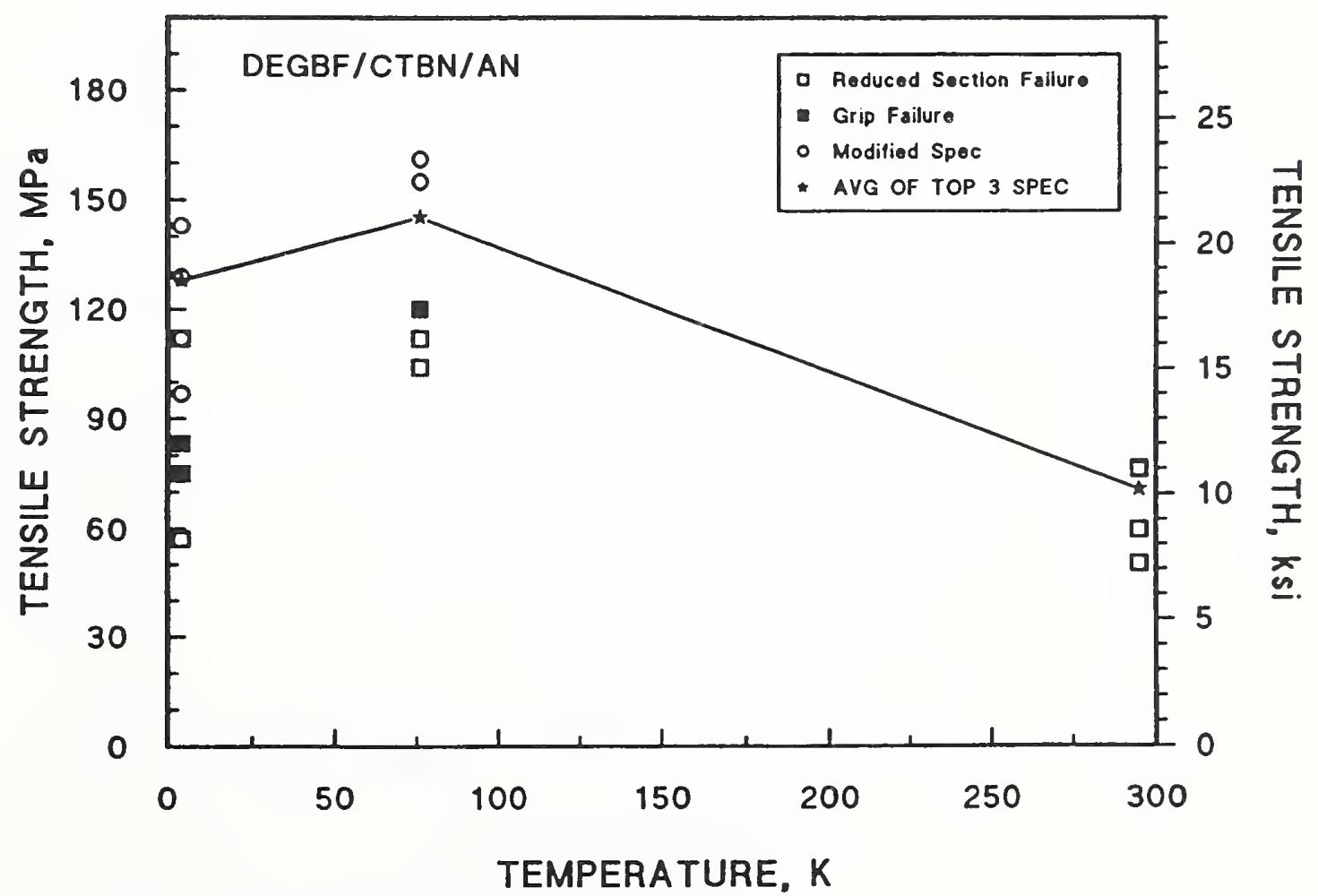

Figure 4.22. Ultimate tensile strength versus temperature-DGEBF/CTBN/AN epoxy. 


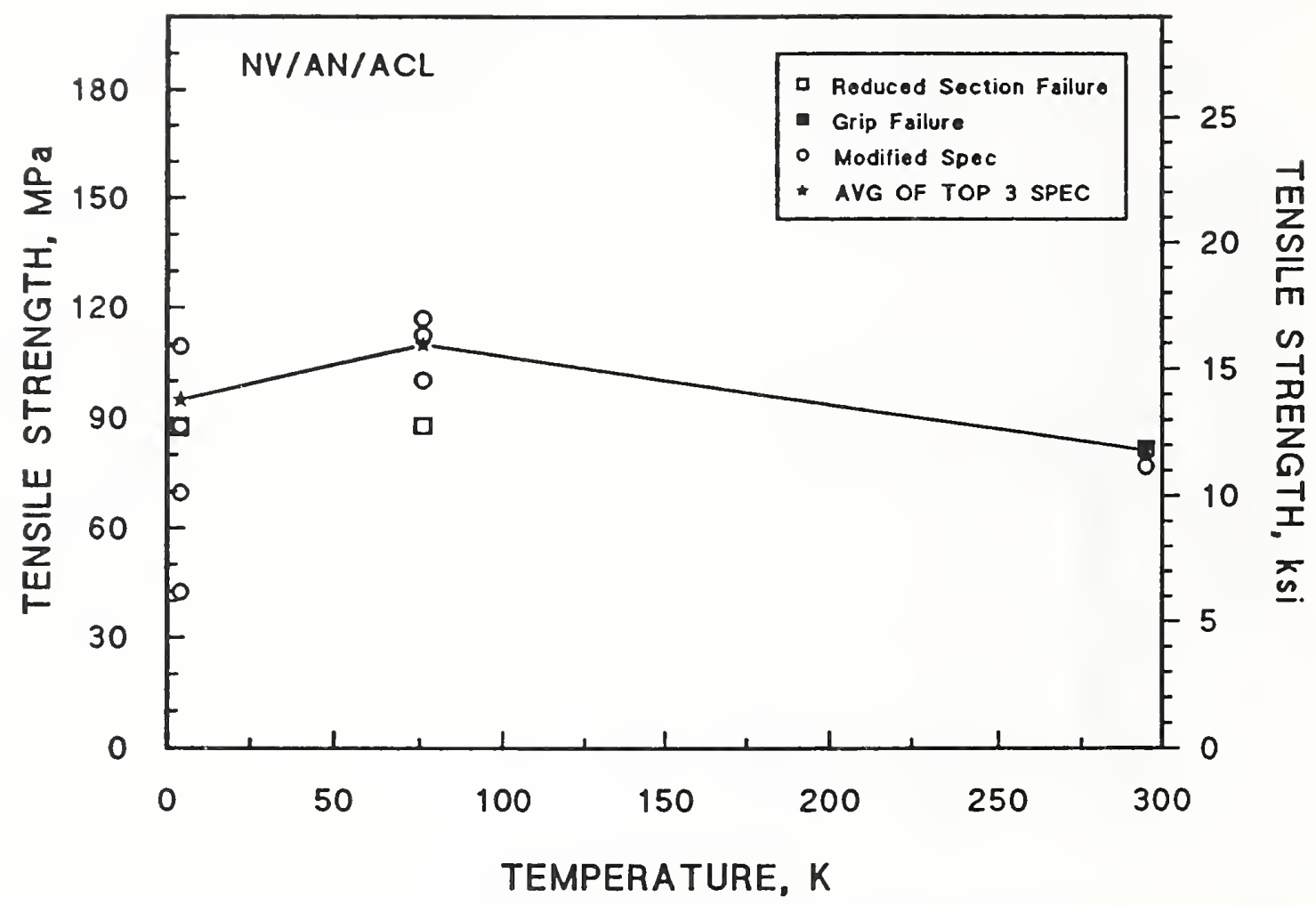

Figure 4.23. Ultimate tensile strength versus temperature-NV/AN/ACL epoxy.

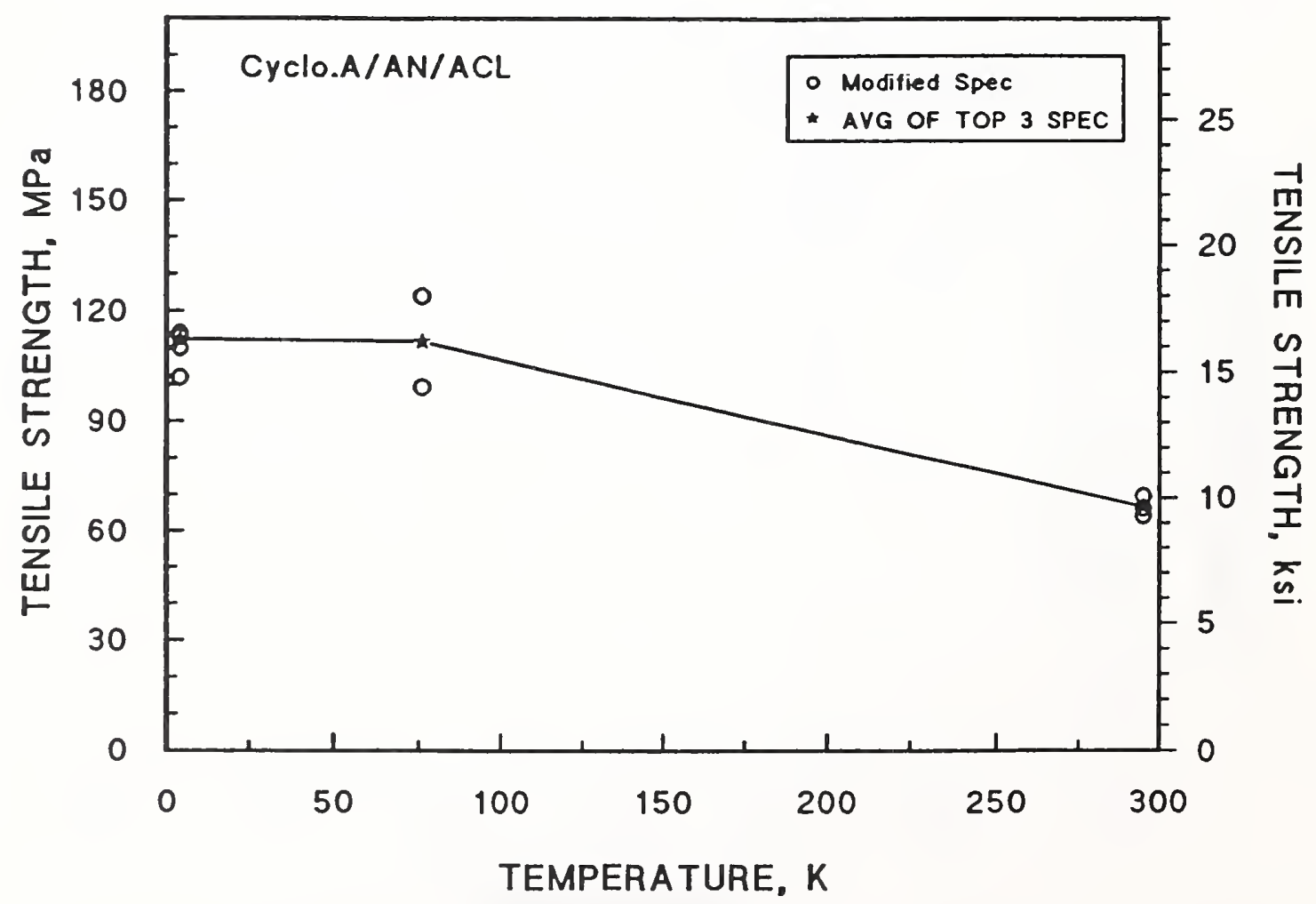

Figure 4.24. Ultimate tensile strength versus temperature-Cyclo.A/AN/ACL epoxy. 


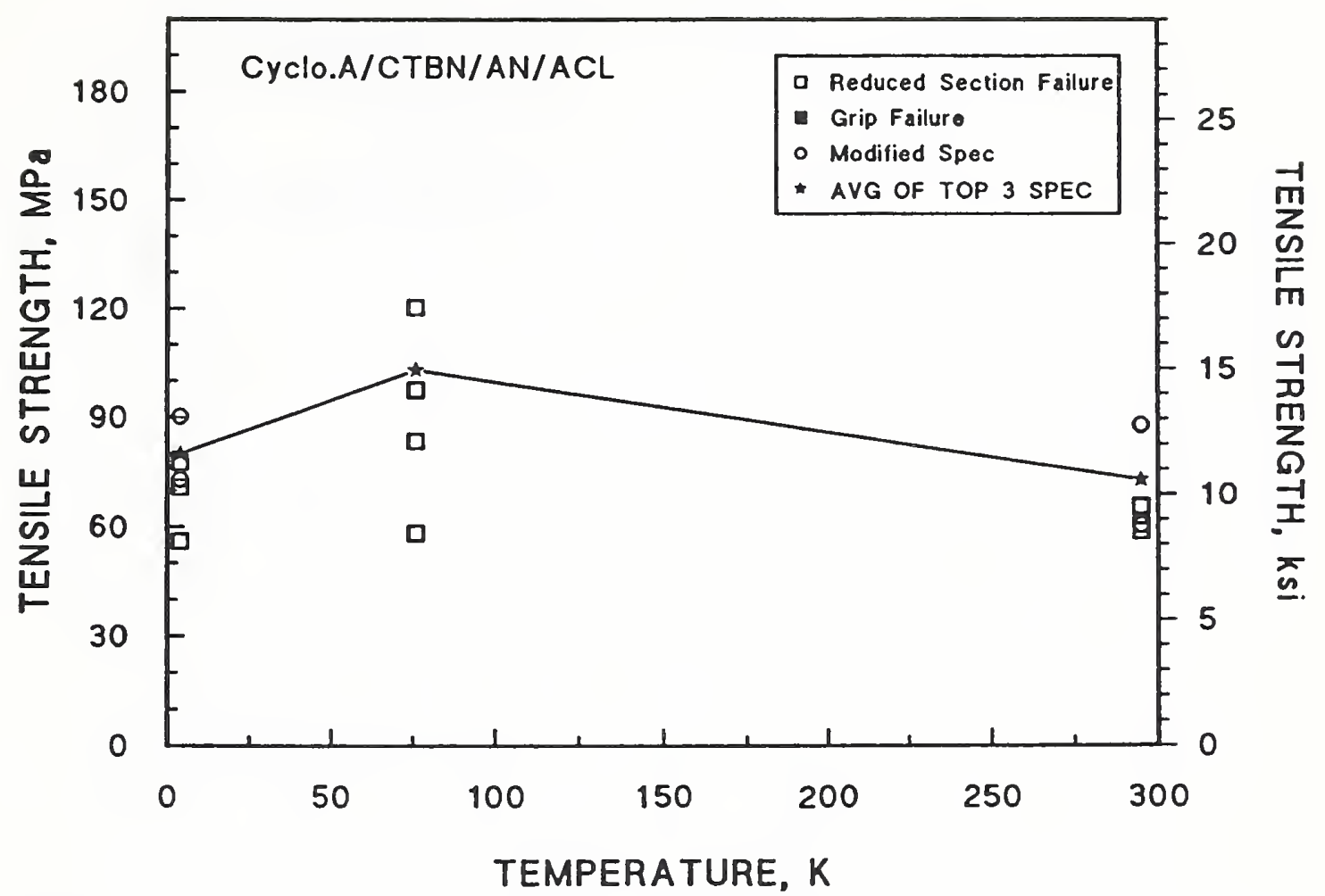

Figure 4.25. Ultimate tensile strength versus temperature-Cyclo.A/CTBN/AN/ACL epoxy.

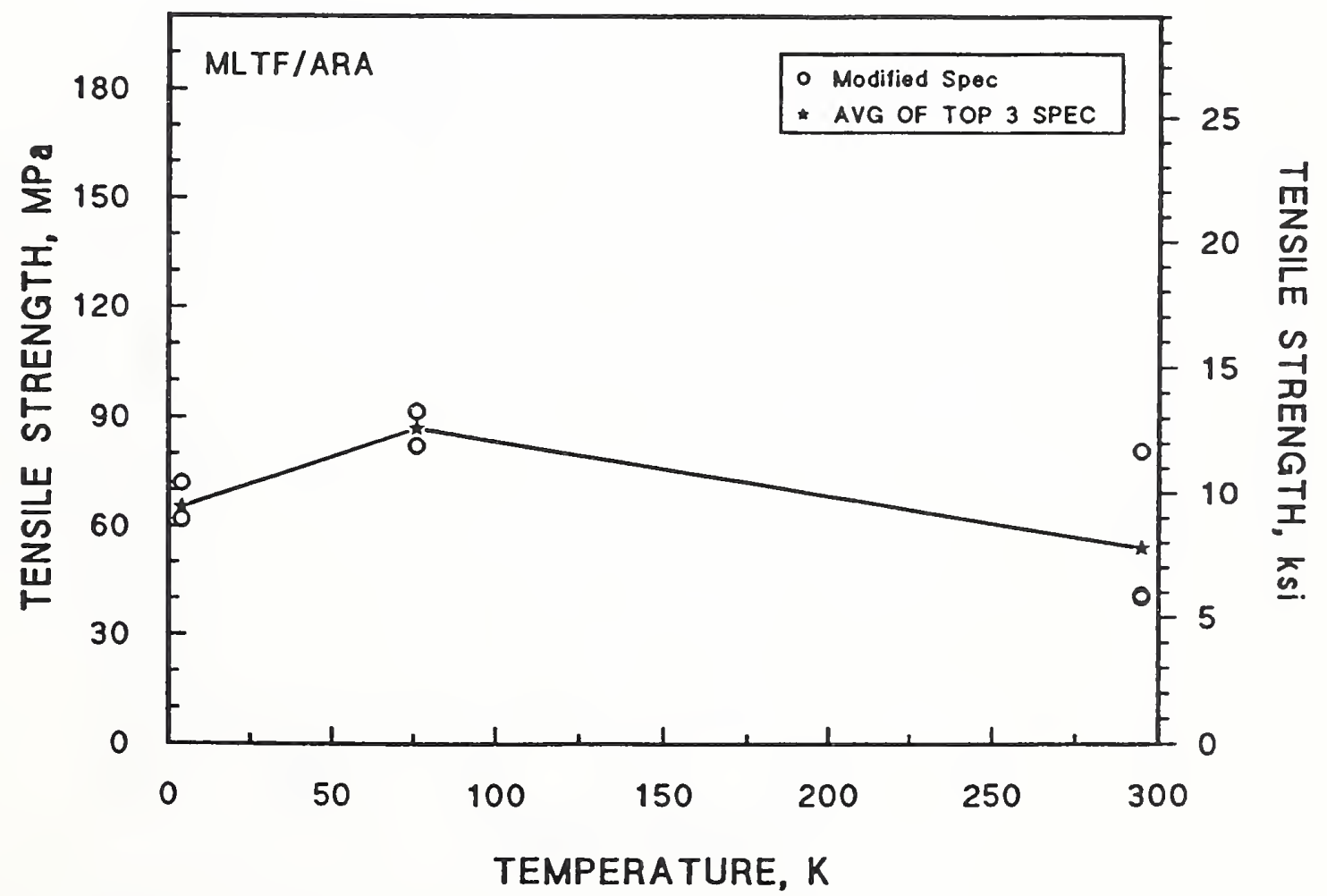

Figure 4.26. Ultimate tensile strength versus temperature-MLTF/ARA epoxy. 


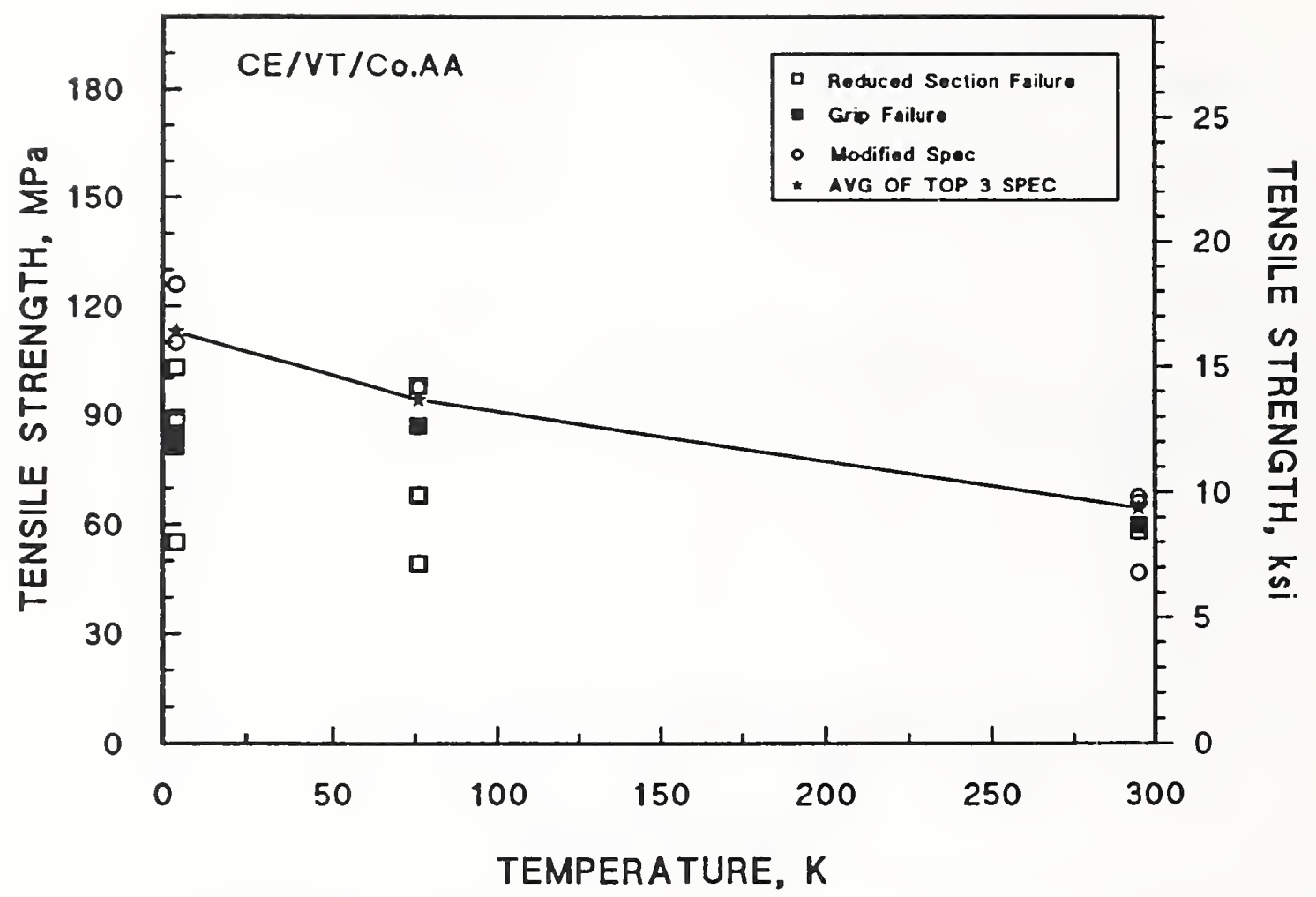

Figure 4.27. Ultimate tensile strength versus temperature-CE/VT/Co.AA cyanate ester.

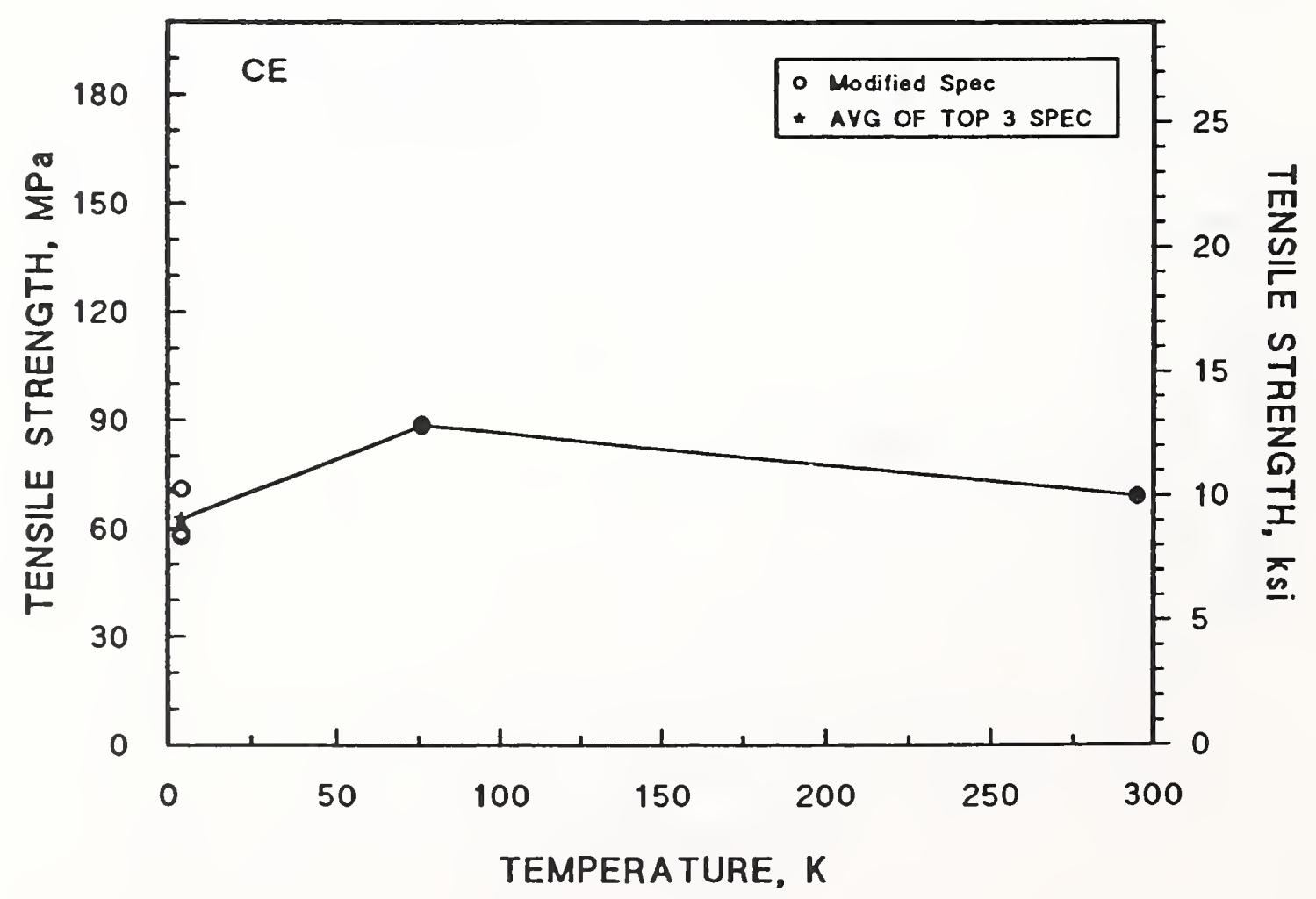

Figure 4.28. Ultimate tensile strength versus temperature-CE cyanate ester. 


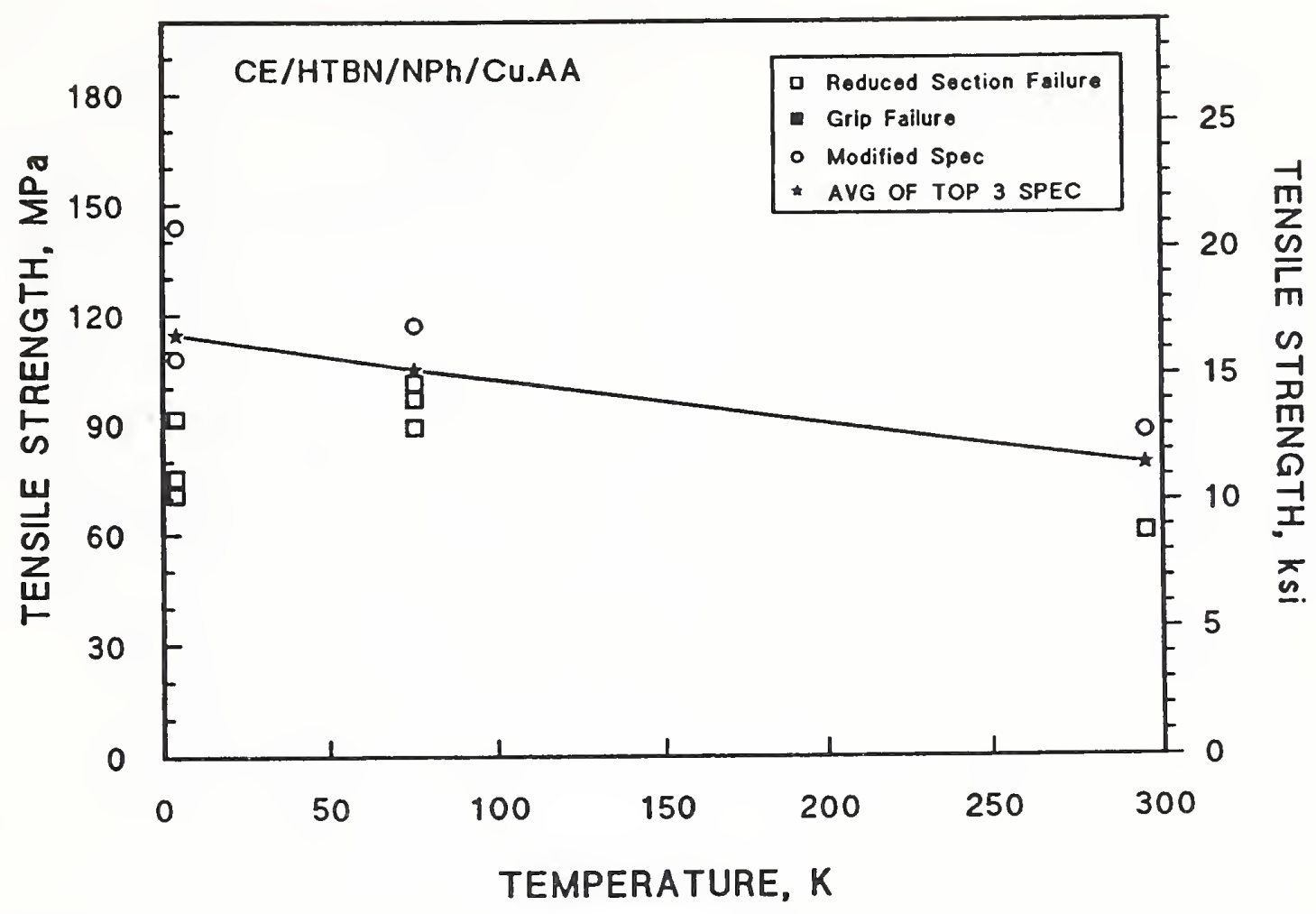

Figure 4.29. Ultimate tensile strength versus temperature-CE/HTBN/NPh/Cu.AA cyanate ester.

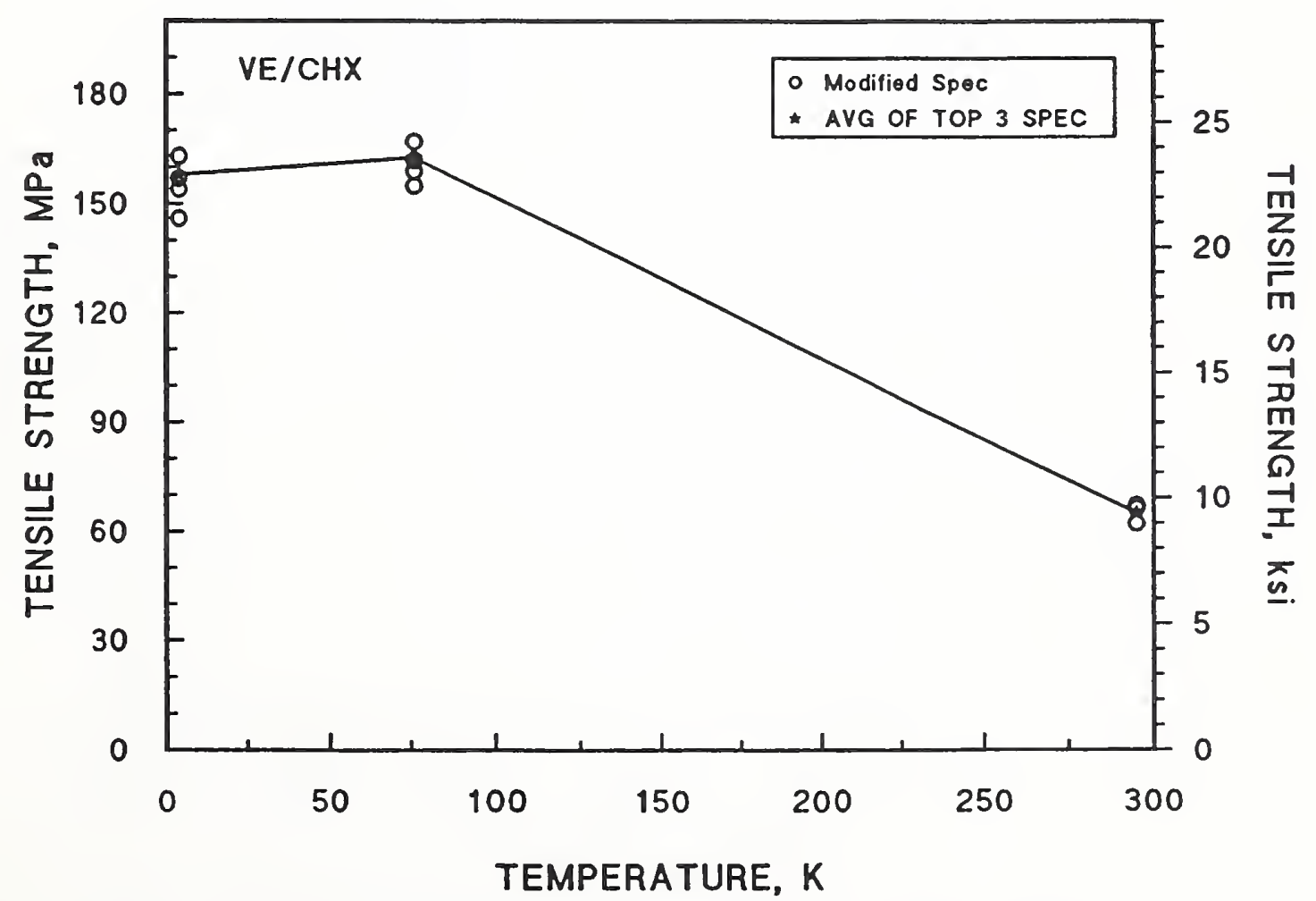

Figure 4.30. Ultimate tensile strength versus temperature-VE/CHX vinyl ester. 


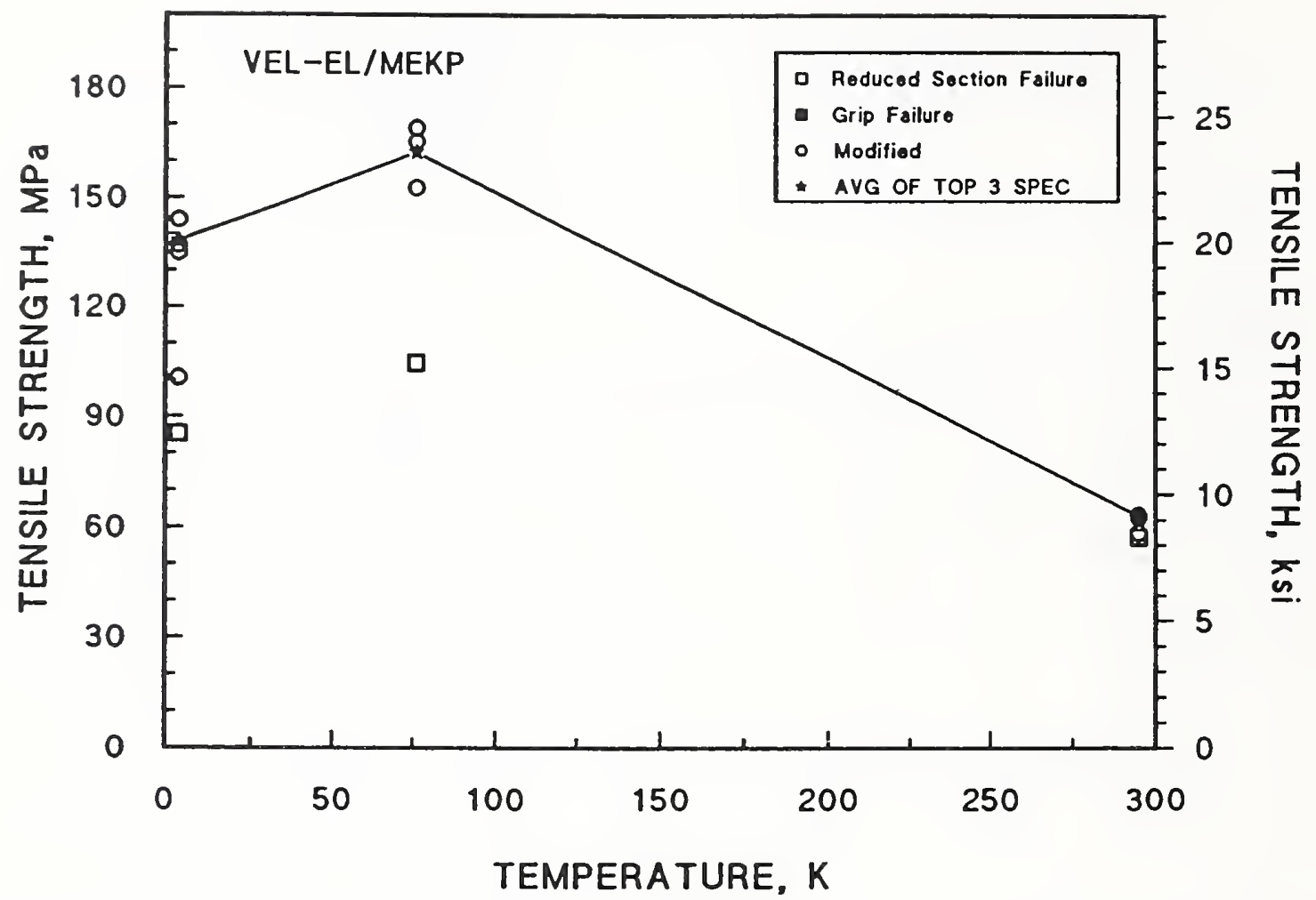

Figure 4.31. Ultimate tensile strength versus temperature-VE-EL/MEKP vinyl ester.

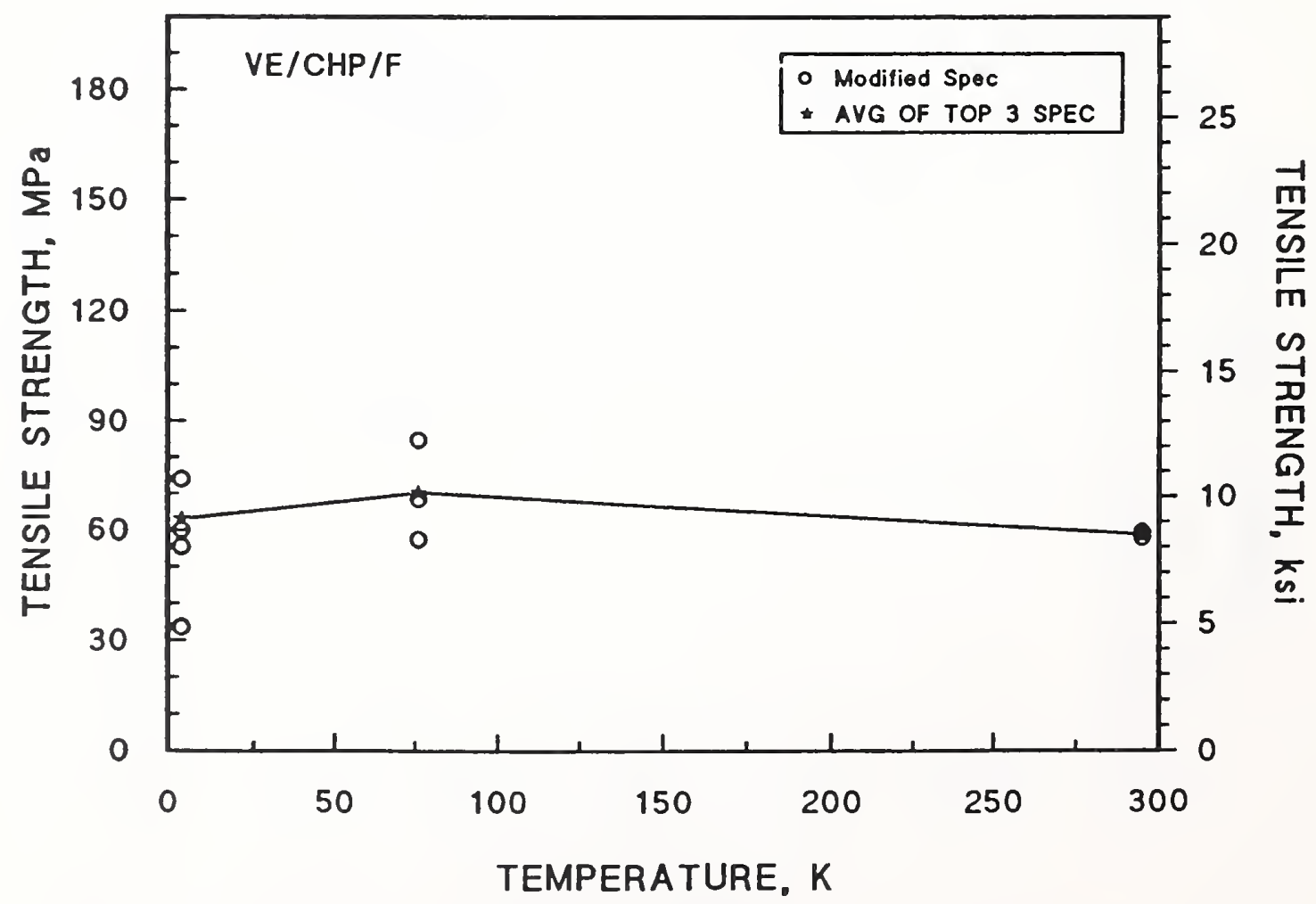

Figure 4.32. Ultimate tensile strength versus temperature-VE/CHP/F vinyl ester. 


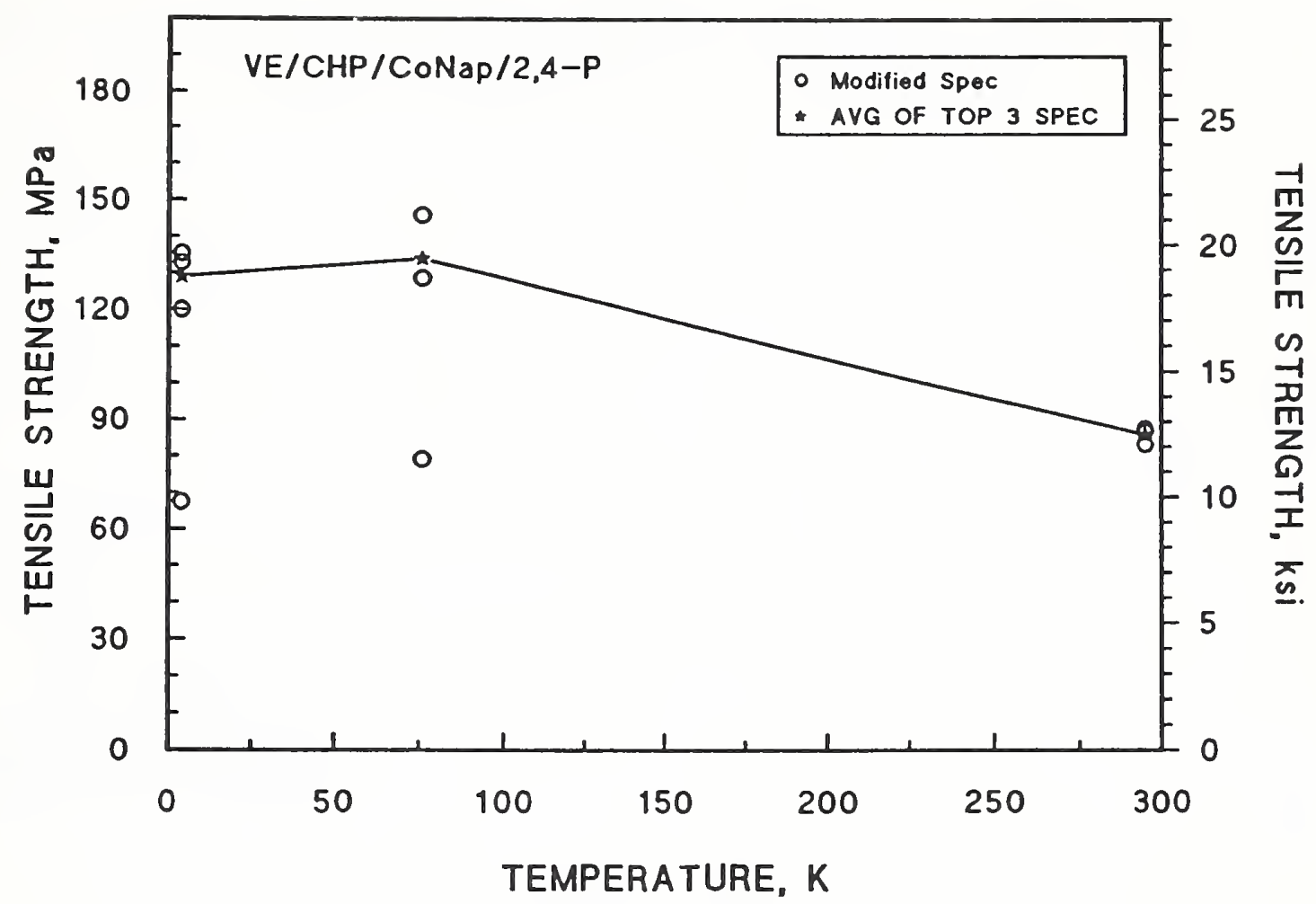

Figure 4.33. Ultimate tensile strength versus temperature-VE/CHP/CoNap/2,4-P vinyl ester.

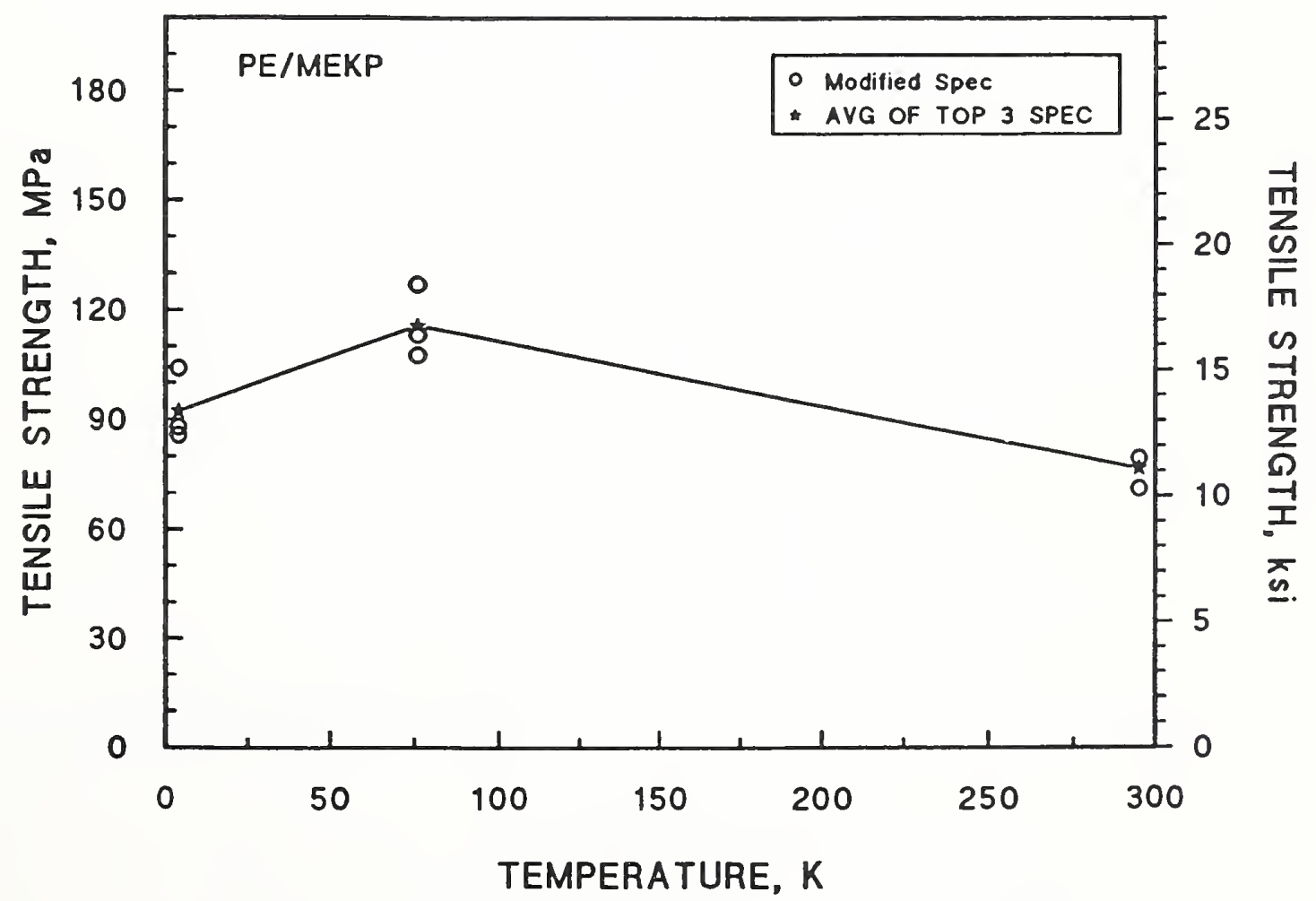

Figure 4.34. Ultimate tensile strength versus temperature-PE/MEKP polyester. 

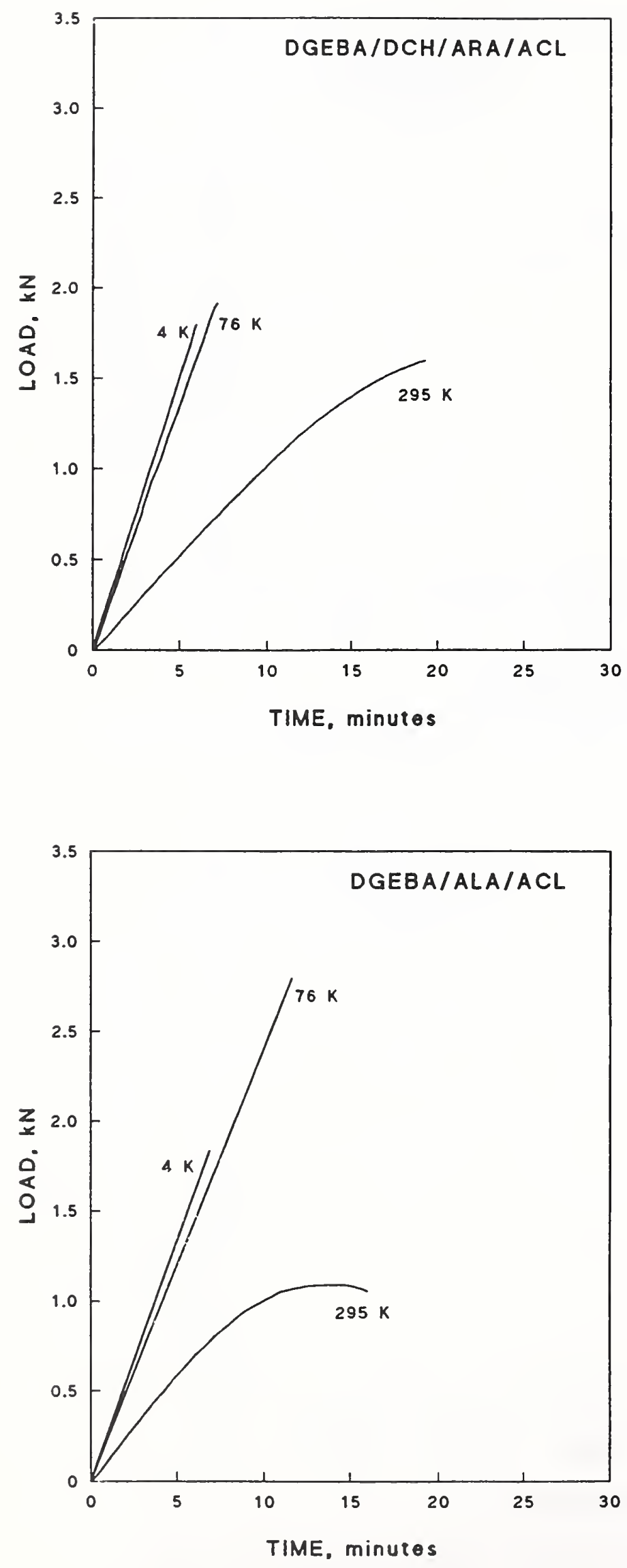

Figure 4.35. Load versus time-DGEBA/DCH/ARA/ACL epoxy.
Figure 4.36. Load versus timeDGEBA/ALA/ACL epoxy. 


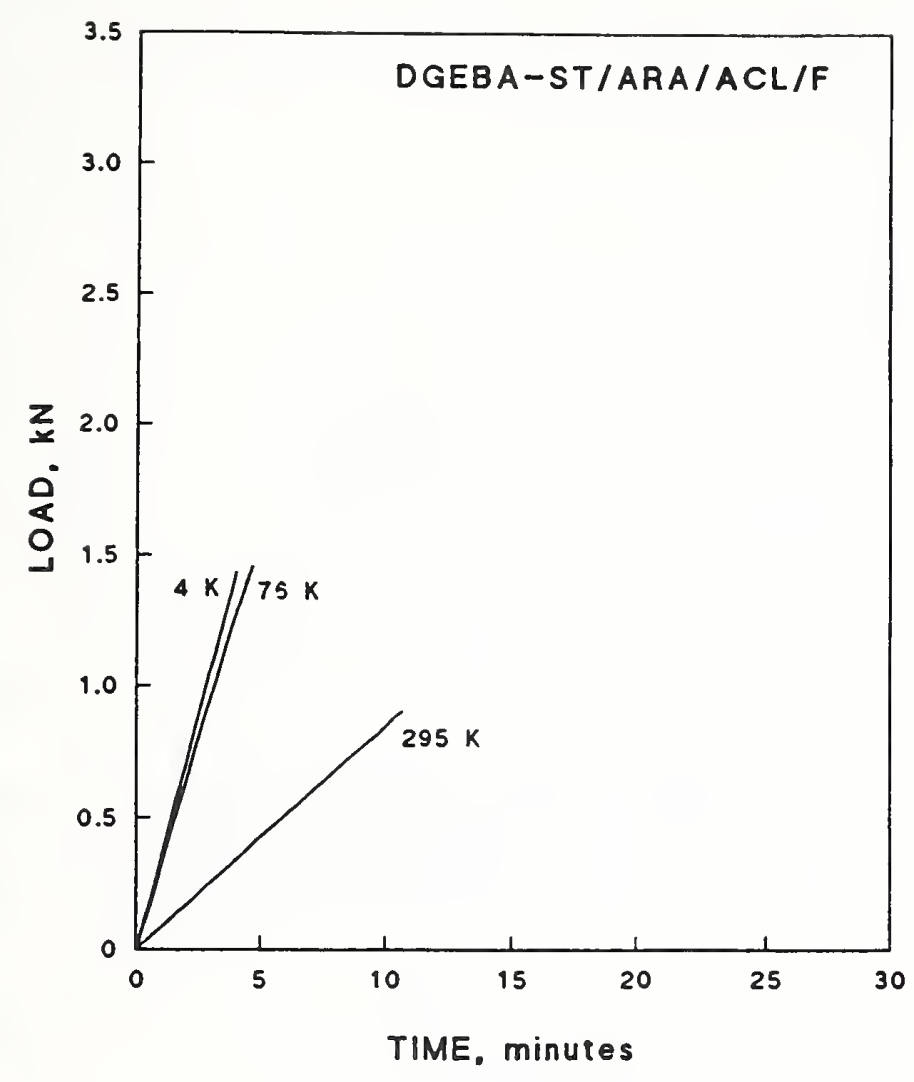

Figure 4.37. Load versus timeDGEBA-ST/ARA/ACL/F epoxy.

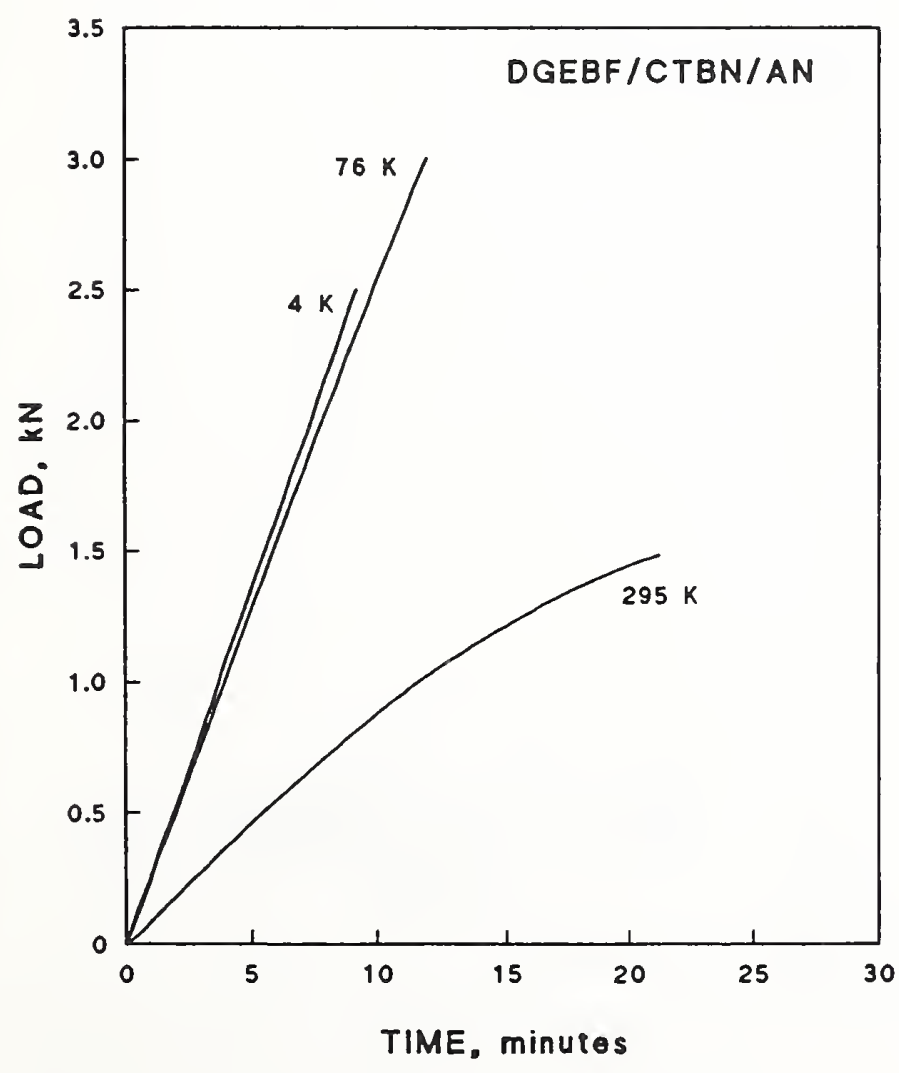

Figure 4.38. Load versus timeDGEBF/CTBN/AN epoxy. 


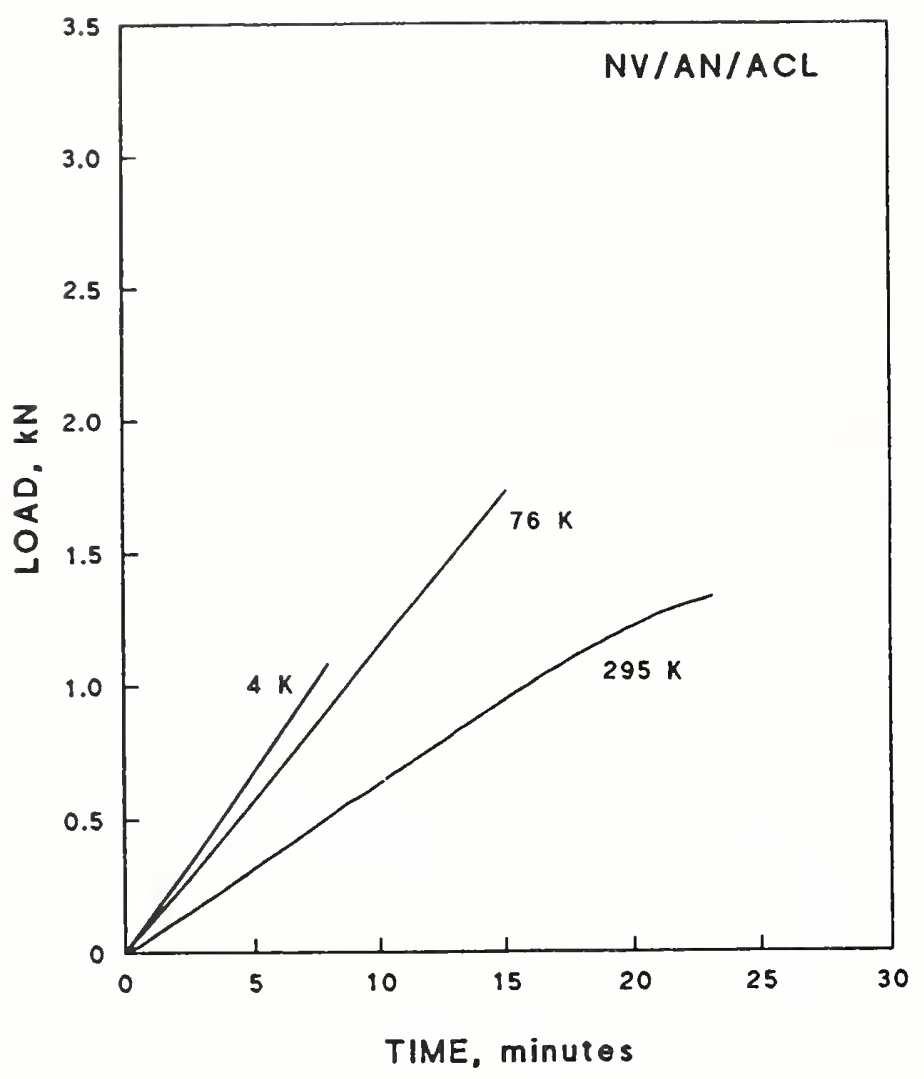

Figure 4.39. Load versus timeNV/AN/ACL epoxy.

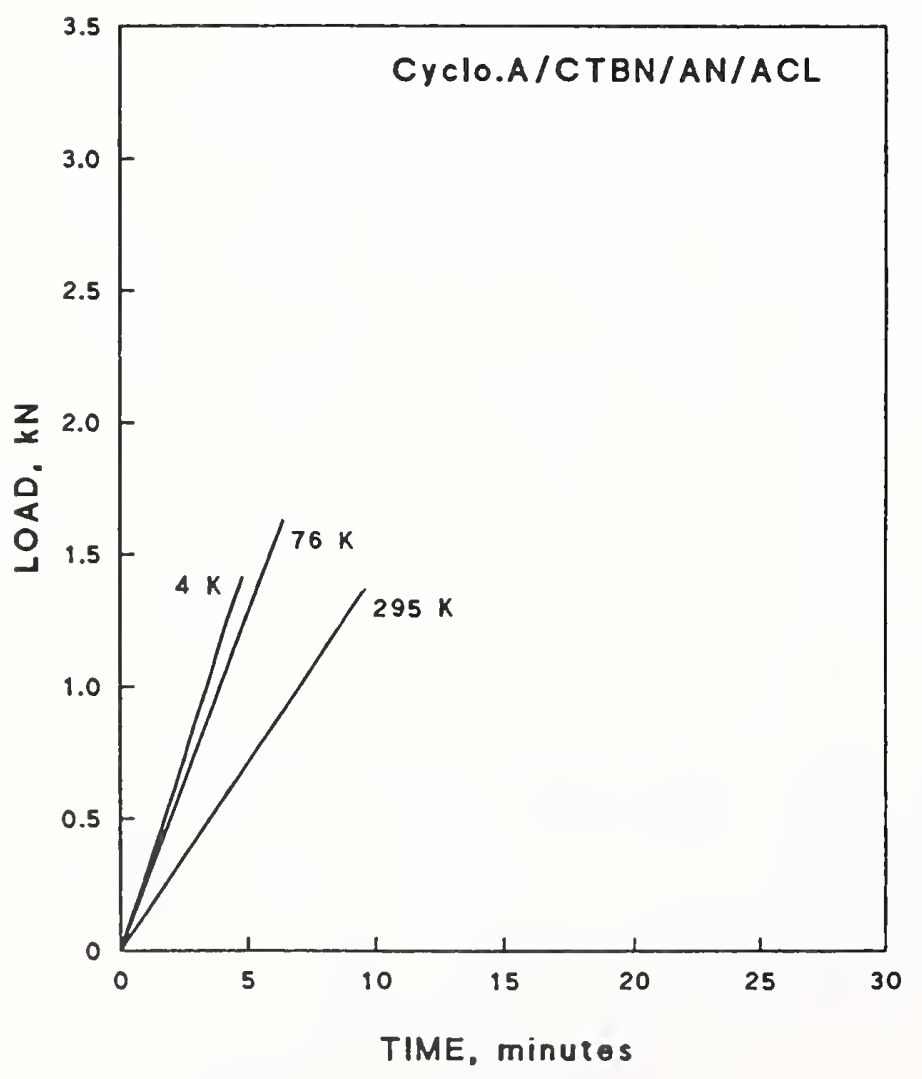

Figure 4.40. Load versus timeCyclo.A/CTBN/AN/ACL epoxy. 


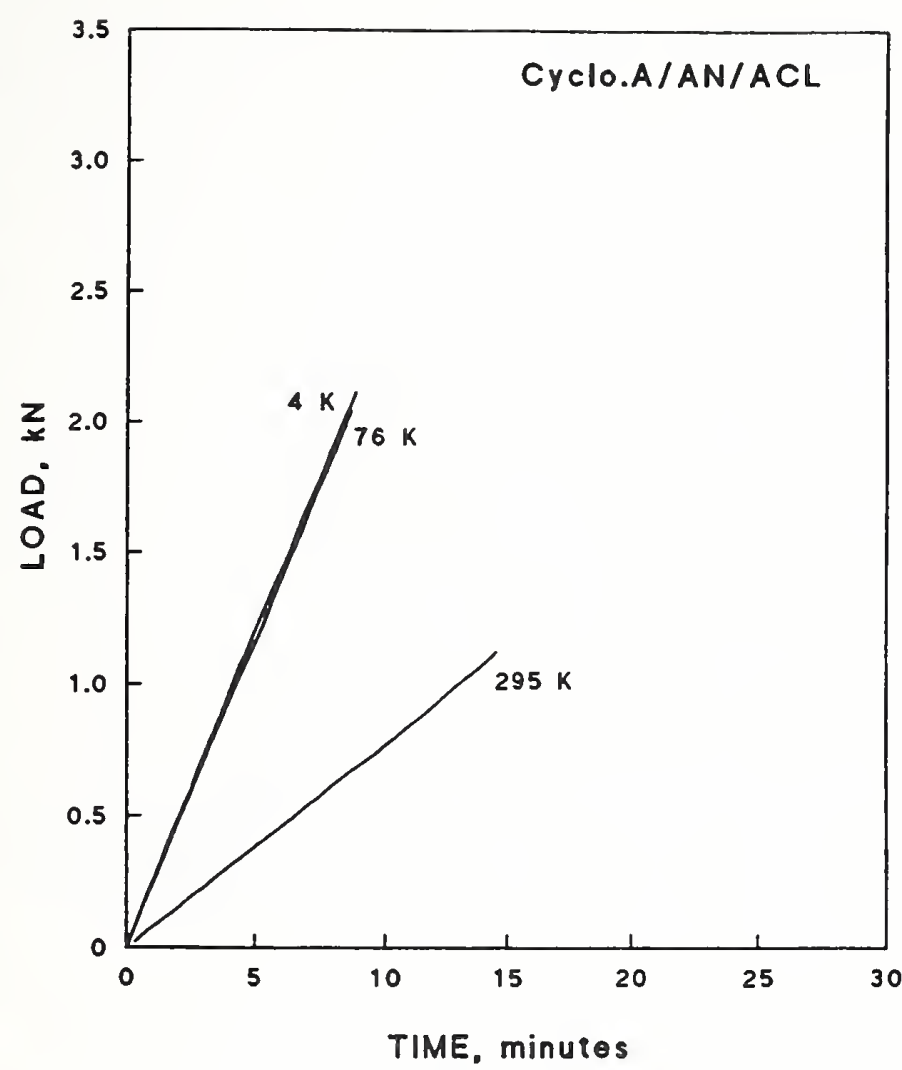

Figure 4.41. Load versus time-

Cyclo.A/AN/ACL epoxy.

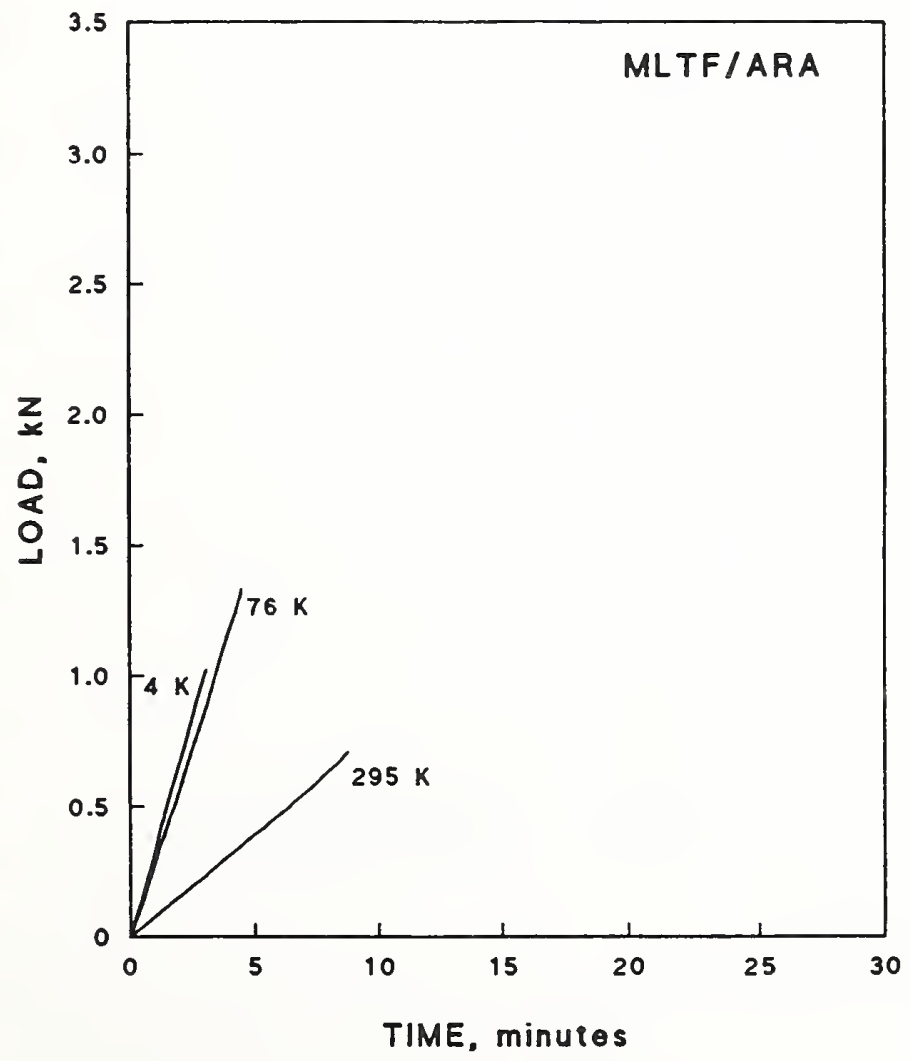

Figure 4.42. Load versus time-MLTF/ARA epoxy. 


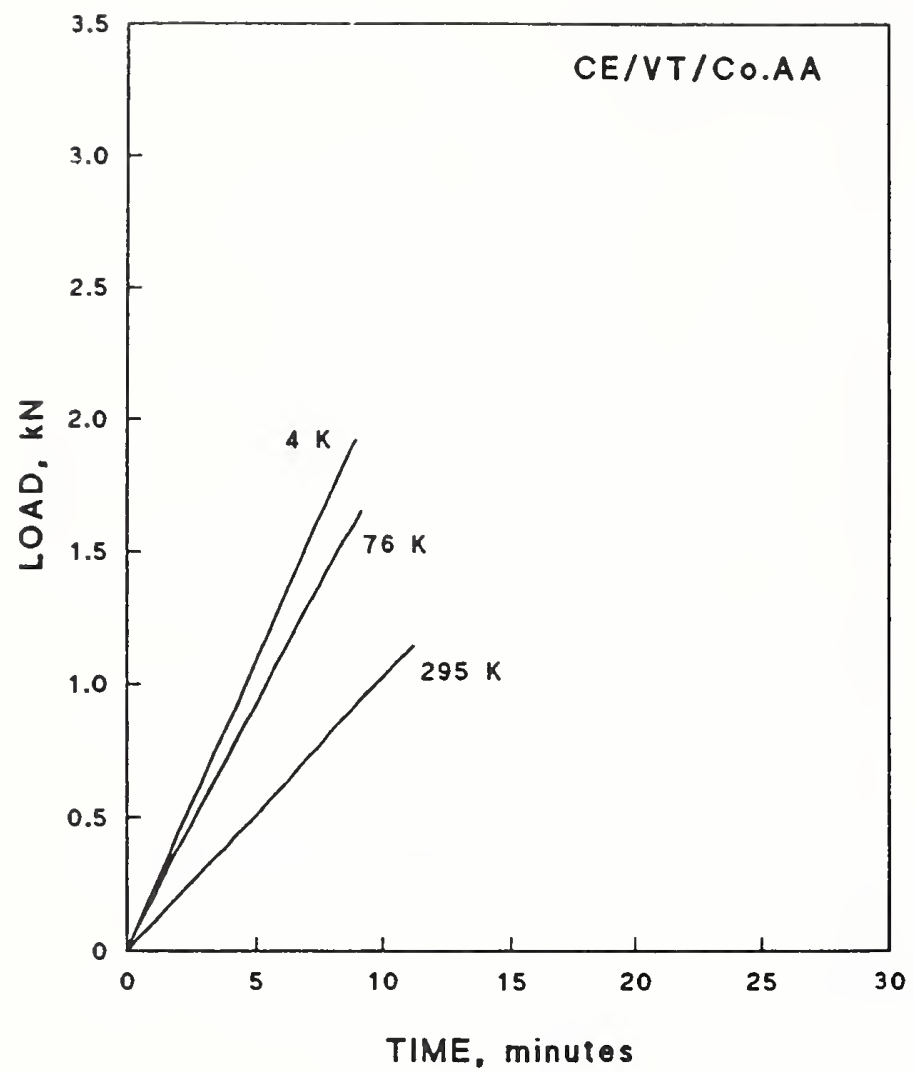

Figure 4.43. Load versus timeCE/VT/Co.AA cyanate ester.

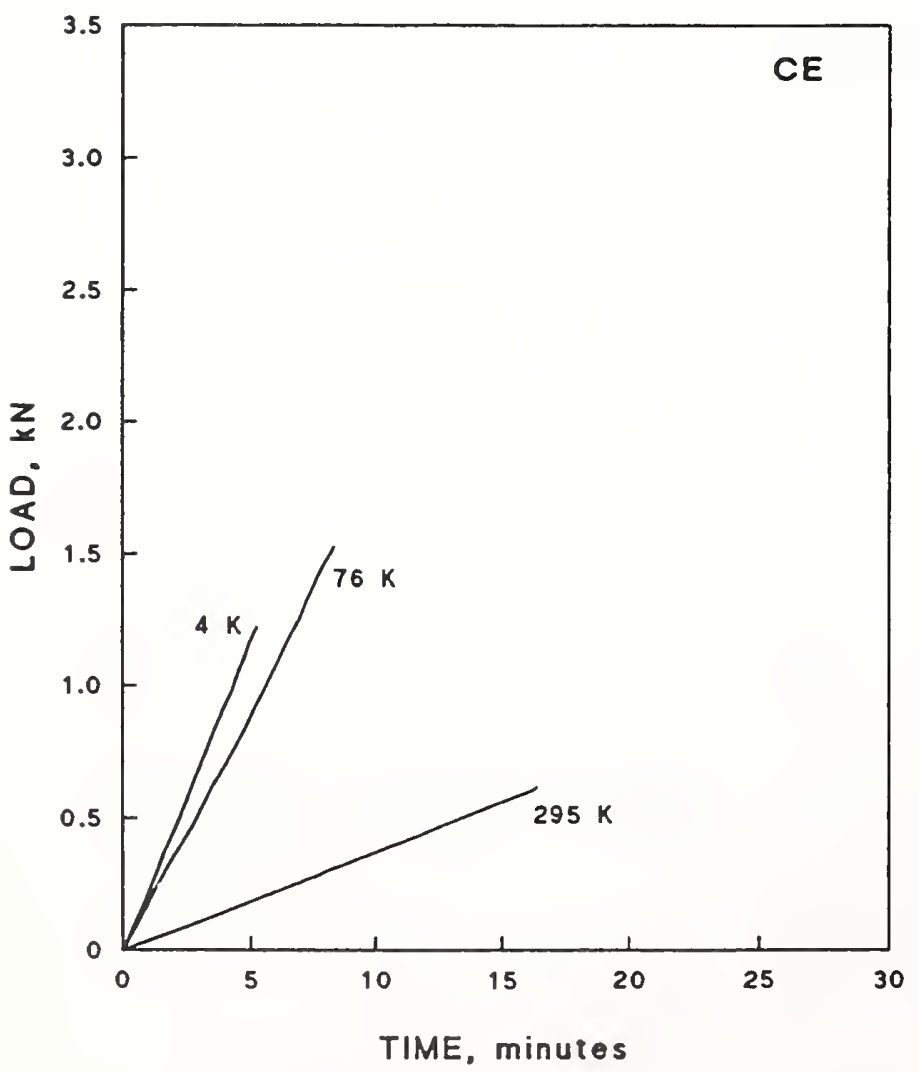

Figure 4.44. Load versus timeCE cyanate ester. 

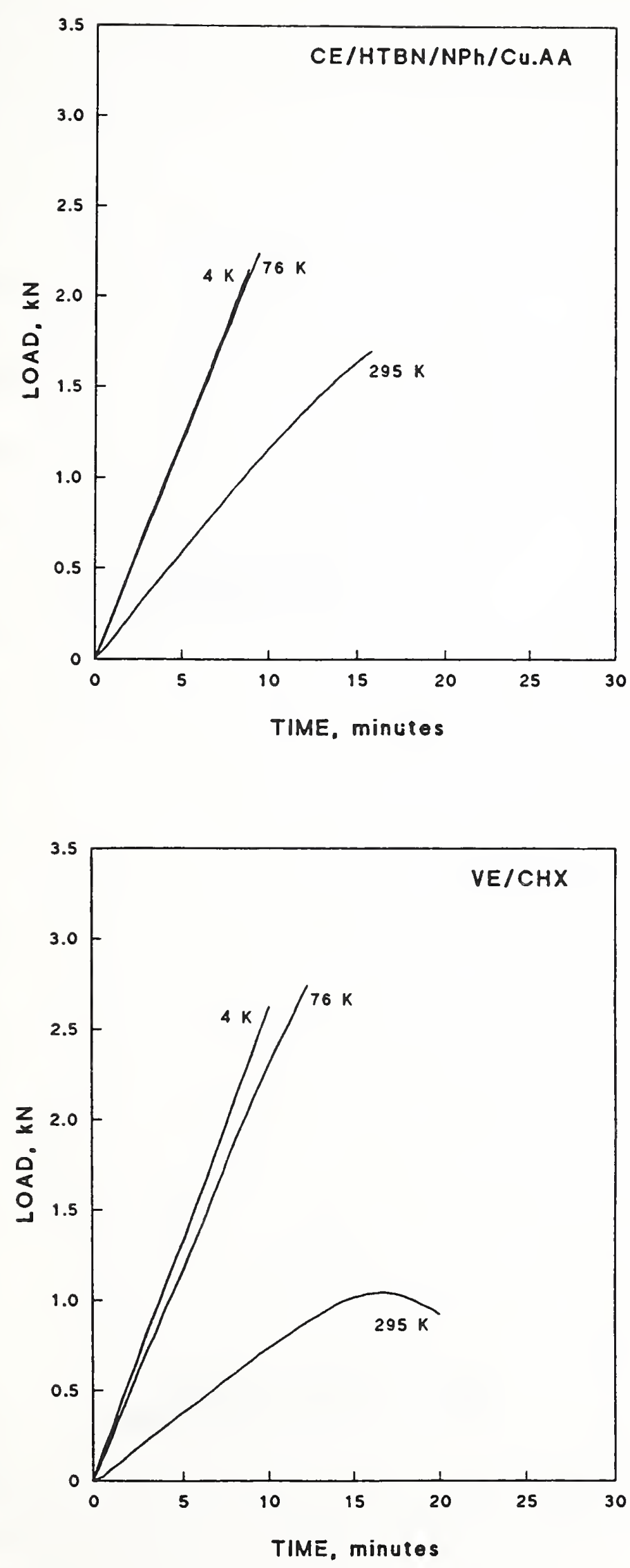

Figure 4.45. Load versus time$\mathrm{CE} / \mathrm{HTBN} / \mathrm{NPh} / \mathrm{Cu} . \mathrm{AA}$ cyanate ester.
Figure 4.46. Load versus timeVE/CHX vinyl ester. 


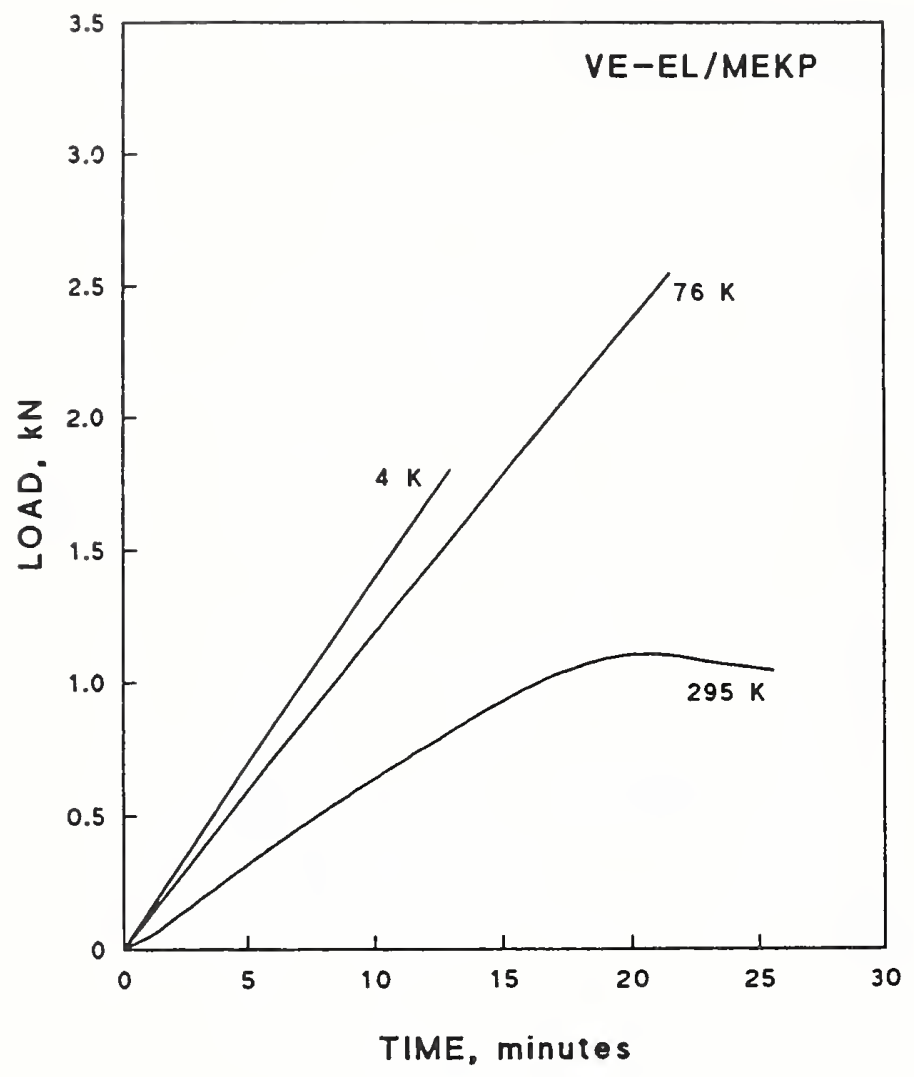

Figure 4.47. Load versus time-

VE-EL/MEKP vinyl ester.

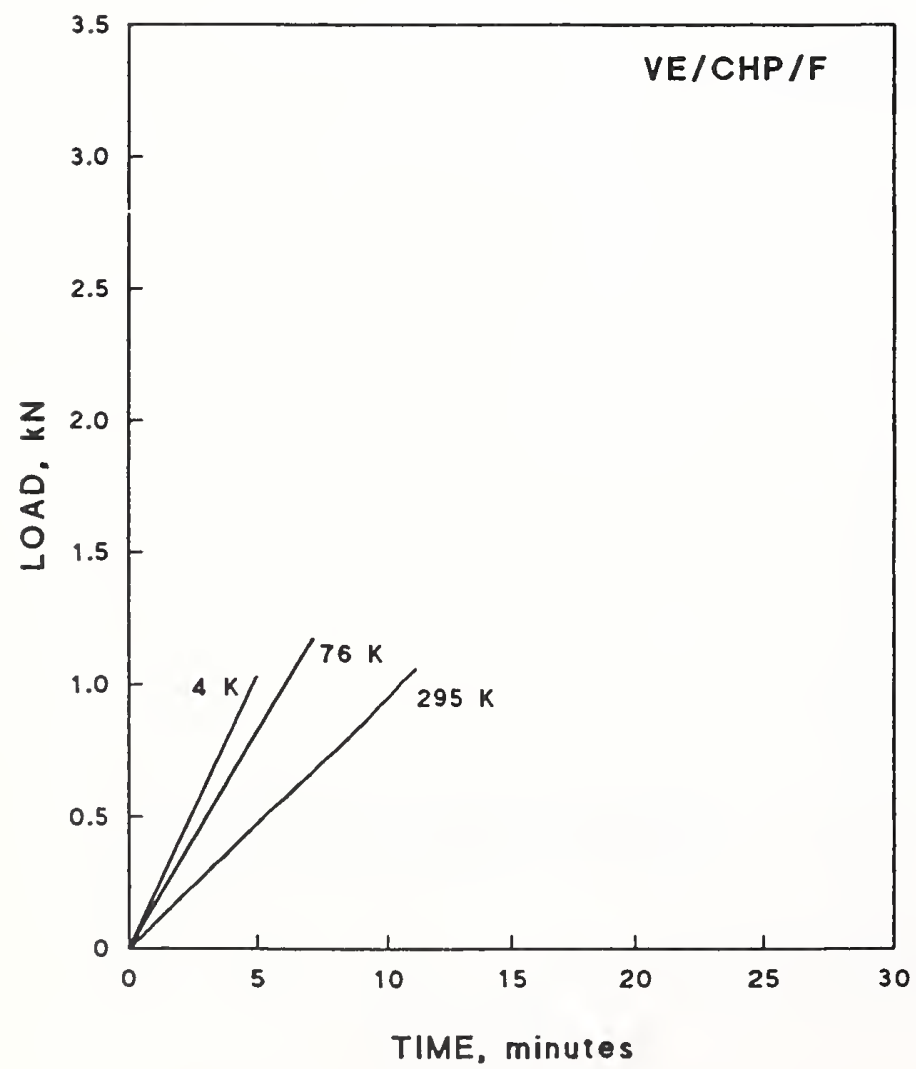

Figure 4.48. Load versus timeVE/CHP/F vinyl ester. 

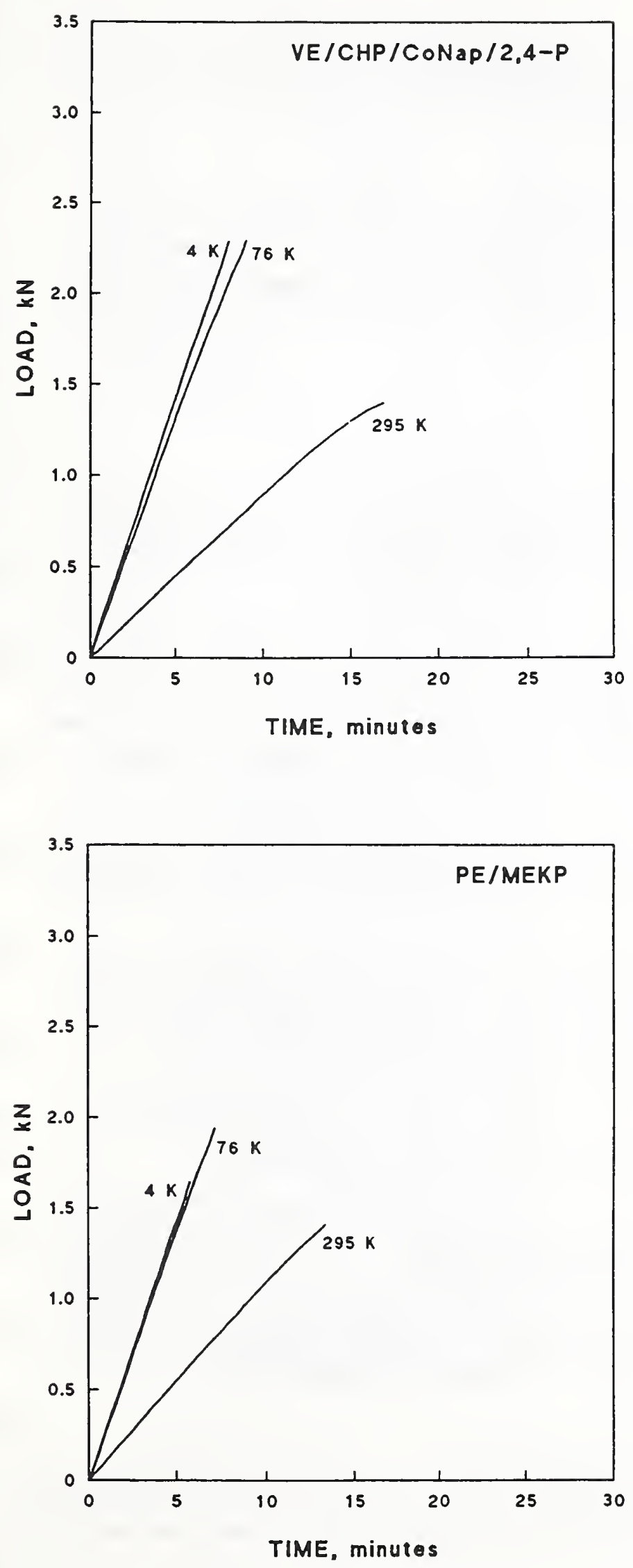

Figure 4.49. Load versus timeVE/CHP/CoNap/2,4-P vinyl ester.
Figure 4.50. Load versus time$\mathrm{PE} / \mathrm{MEKP}$ polyester. 
The $\mathrm{CE}(\mathrm{PT})$ resin system was very brittle at low temperatures, and no failures were achieved within the reduced section of the tensile specimens. Premature failures were also observed during testing at room temperature (average failure strengths were $28 \mathrm{MPa}$ at $295 \mathrm{~K}$ ). This resin system did not develop full crosslinking as a result of improper cure.

Typical load-versus-time curves for each test temperature for most neat-resin systems are shown in Figures 4.35 through 4.50. All neat resins exhibited nonlinear deformation at room temperature, but only a few (DGEBA/DCA/ARA/ACL, DGEBA-ST/ARA/ACL/F, DGEBF/CTBN/AN epoxies; CE/VT/Co.AA cyanate ester; VE/CHX, VE-EL/MEKP, VE/CHP/CoNap/2,4-P vinyl esters) showed very limited nonlinear deformation at $76 \mathrm{~K}$, and only the cyanate ester (CE/VT/Co.AA) had very limited nonlinear deformation at $4 \mathrm{~K}$.

\subsubsection{Fractography}

Optical microscopy photographs were taken of the tensile fracture surface of selected specimens that had been tested at 295, 76, and $4 \mathrm{~K}$. They are shown in Figures 4.51 through 4.55 for epoxies, Figures 4.56 through 4.57 for cyanate esters, Figures 4.58 through 4.60 for vinyl esters, and Figure 4.61 for the polyester.

The origin of all fractures was easily determined in all specimens since the flaw lines from the crack path were always observed to originate at a given location. Sometimes the fracture source was on or very near the surface of specimen; sometimes it was embedded in the bulk resin. In some cases, the origin was a pore; in other cases, its nature was not clear; but in all cases, the crack appeared to begin at a material or surface defect.

On many fracture surfaces, granular-like contours were visible. In some cases (e.g., Fig. 4.58 for the VE/CHX vinyl ester), they appeared more commonly on specimens tested at low temperatures that those tested at room temperature.

Surprisingly, in some cases, the contours of the fracture surfaces on specimens tested at low temperatures had a more fibrous appearance than the specimens tested at room temperature. For example, in Figures 4.53, 4.57, and 4.59, the fracture surface is more planar at $295 \mathrm{~K}$ than at $4 \mathrm{~K}$ for these epoxy, cyanate-ester, and vinyl-ester resins, respectively.

The white, globular-like objects on the fracture surfaces of the epoxy DGEBA-ST/ARA/ ACL/F specimens (Fig. 4.51) are caused by the styrene modification to this neat resin.

On the fracture surfaces of a few resins (e.g., Fig. 4.56 for the CE/VT/Co.AA cyanate ester at $76 \mathrm{~K}$ ), pores appeared to be present on the fracture surface. Opposite sides of these specimens also showed matching pores. The fact that these pores appeared on the surface of specimens fractured at $4 \mathrm{~K}$ (Fig. 4.56) argues against the formation of pores via diffusion of vacancies during testing. The artifacts are not present at other locations on the specimen, indicating that they appeared only during the fracture process. This interesting observation deserves careful, fundamental study.

Circumferential flaw lines were present on the fracture surface of the toughened epoxy Cyclo.A/CTBN/AN/ACL specimens that were tested at $4 \mathrm{~K}$ (Fig. 4.54). They were similar to striations found in materials that have undergone high-cycle fatigue, and in these specimens, each striation represents an increment of crack growth. However, the load-deflection curve of these specimens at 


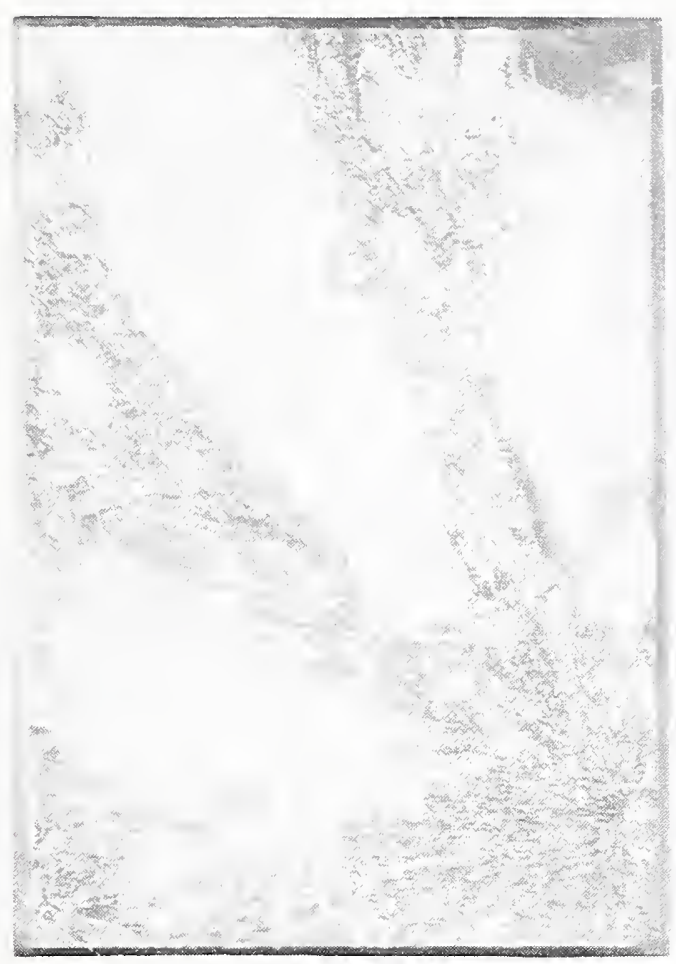

(a)

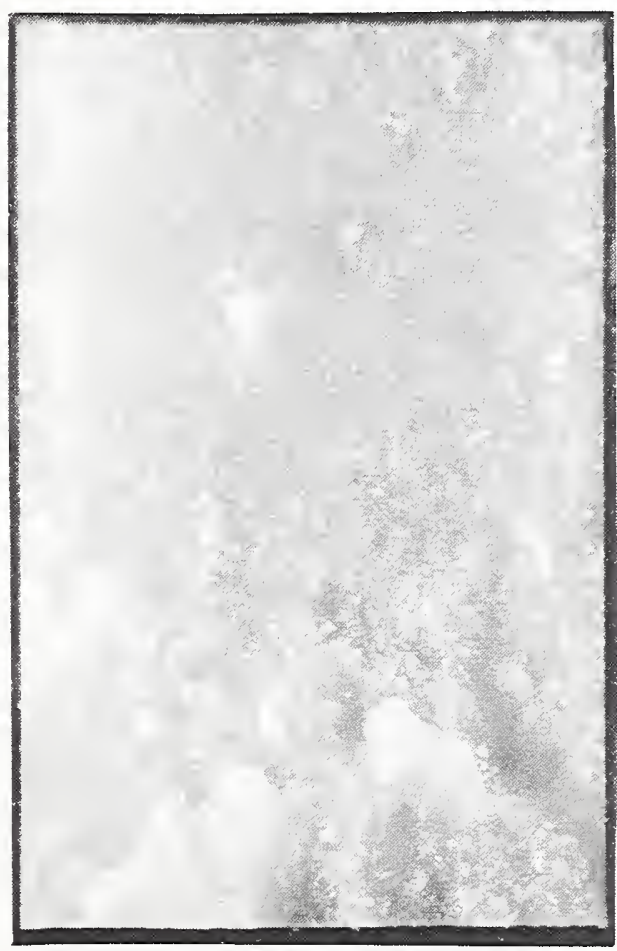

(c)

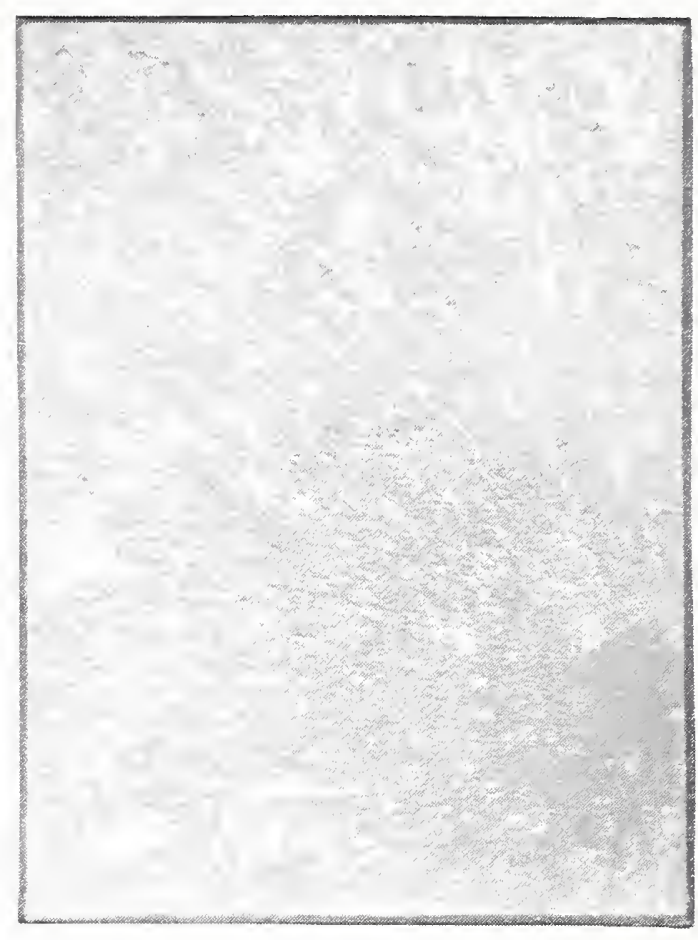

(b)

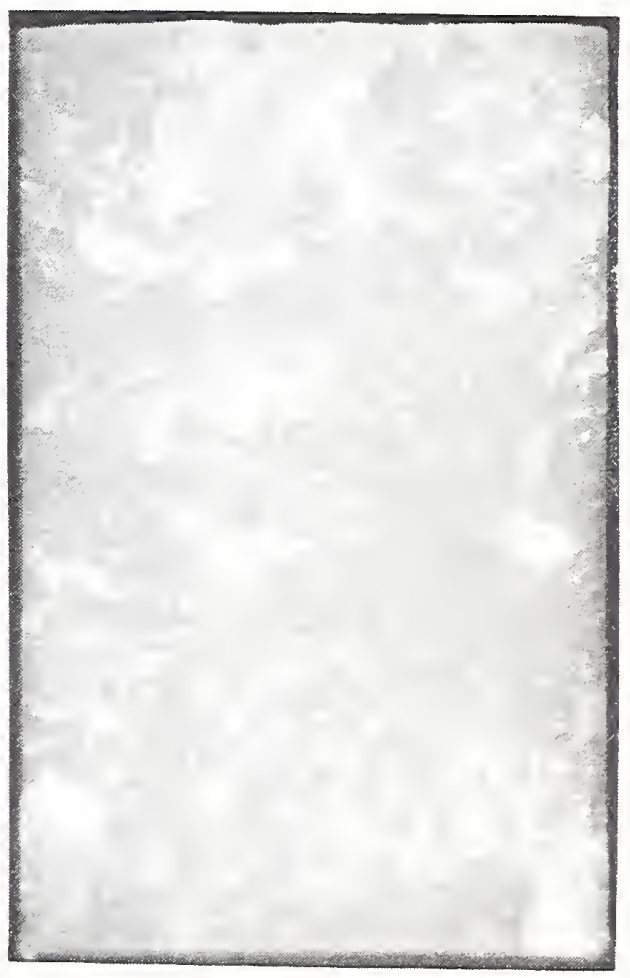

(d)

Figure 4.51. Tensile fracture surfaces of DGEBA/DCH/ARA/ACL at $295 \mathrm{~K}$ (a) and $76 \mathrm{~K}$ (b) and of DGEBA-ST/ARA/ACL/F at $295 \mathrm{~K}$ (c) and $4 \mathrm{~K}$ (d). 


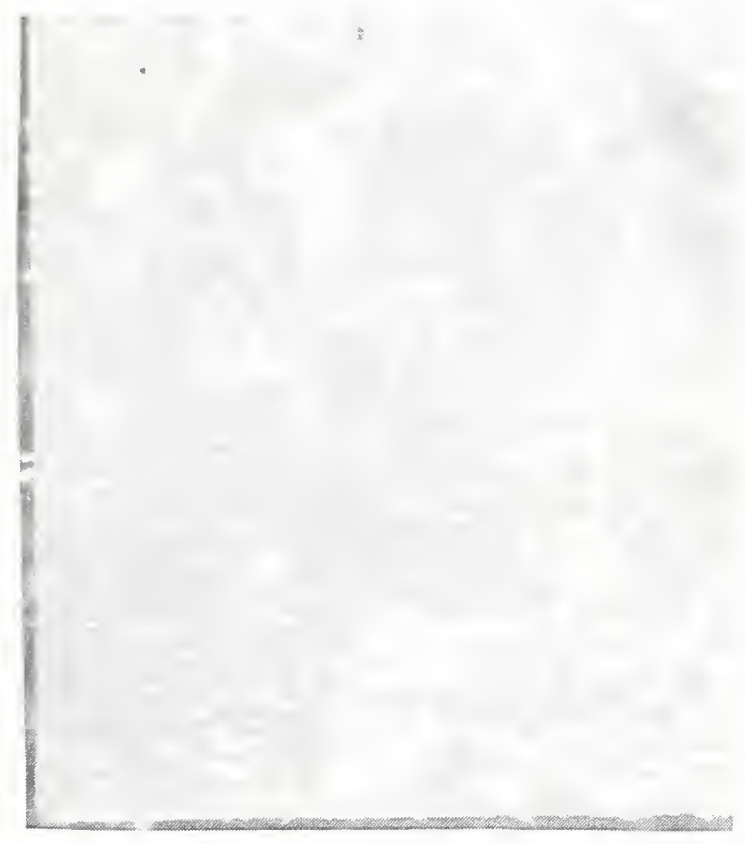

(a)

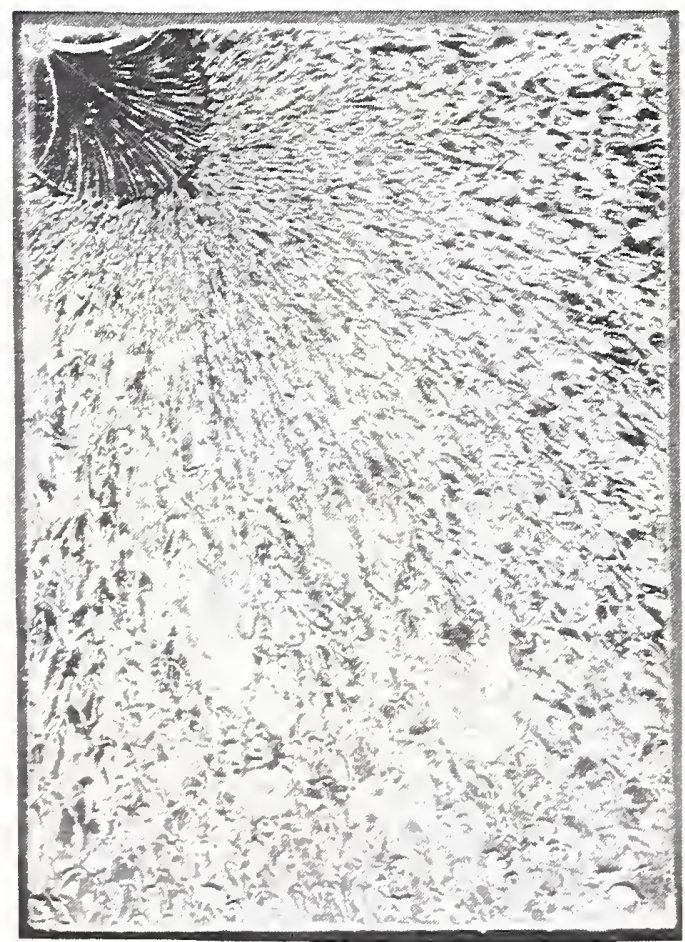

(c)

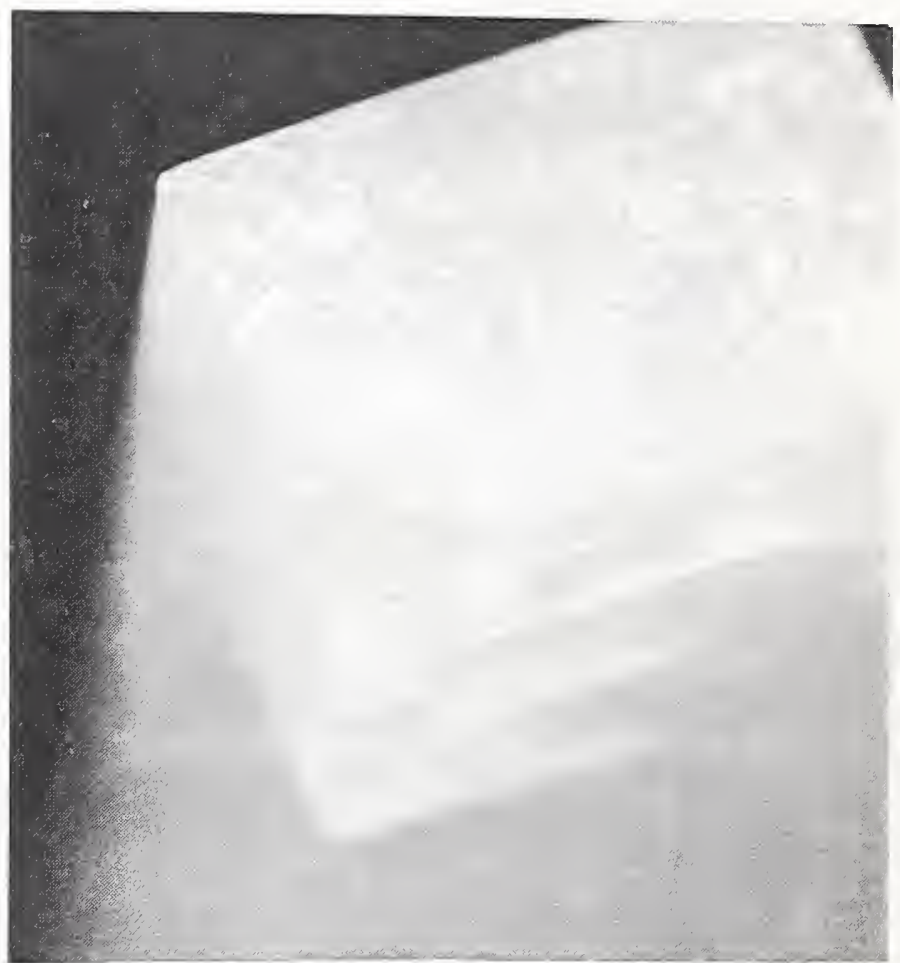

(b)

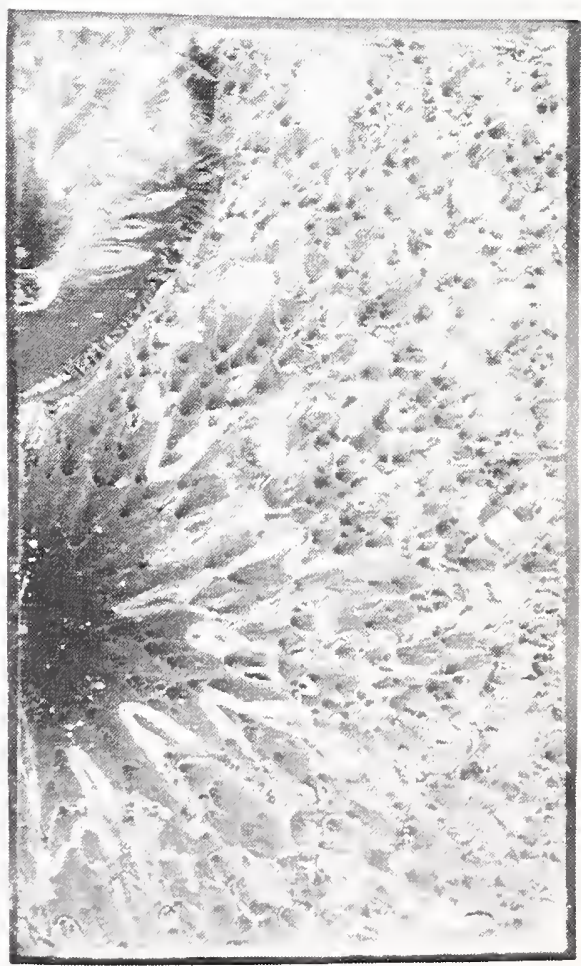

(d)

Figure 4.52. Tensile fracture surfaces of DGEBF/CTBN/AN at $76 \mathrm{~K}$ (a) and $4 \mathrm{~K}$ (b) and of DGEBA/ALA/ACL at $295 \mathrm{~K}$ (c) and NV/AN/ACL at $76 \mathrm{~K}$ (d). 


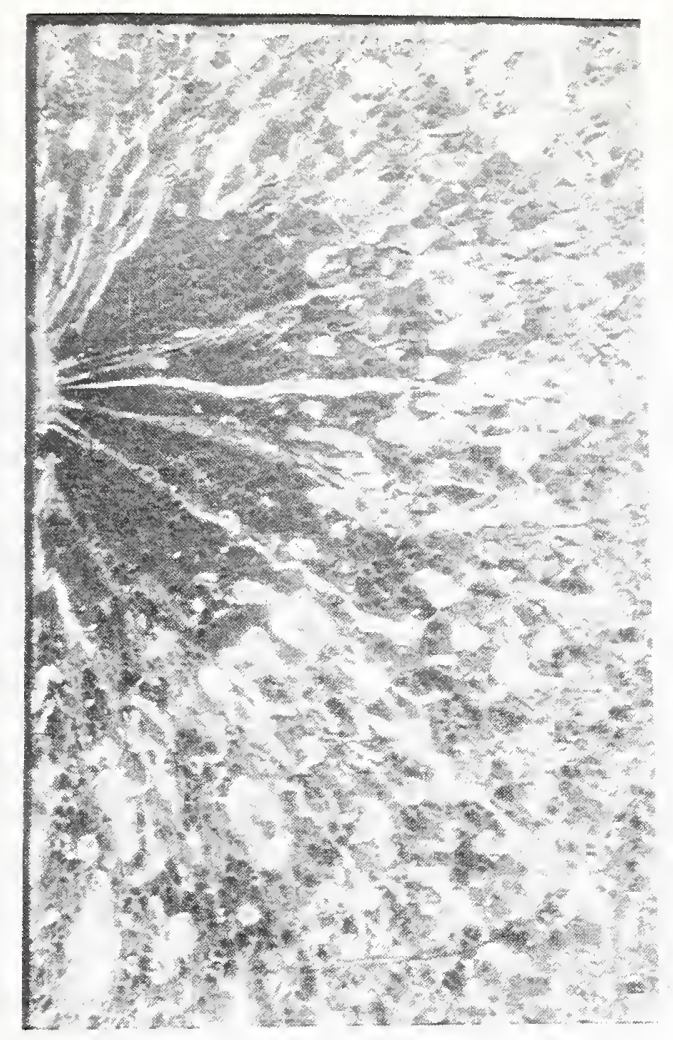

(a)

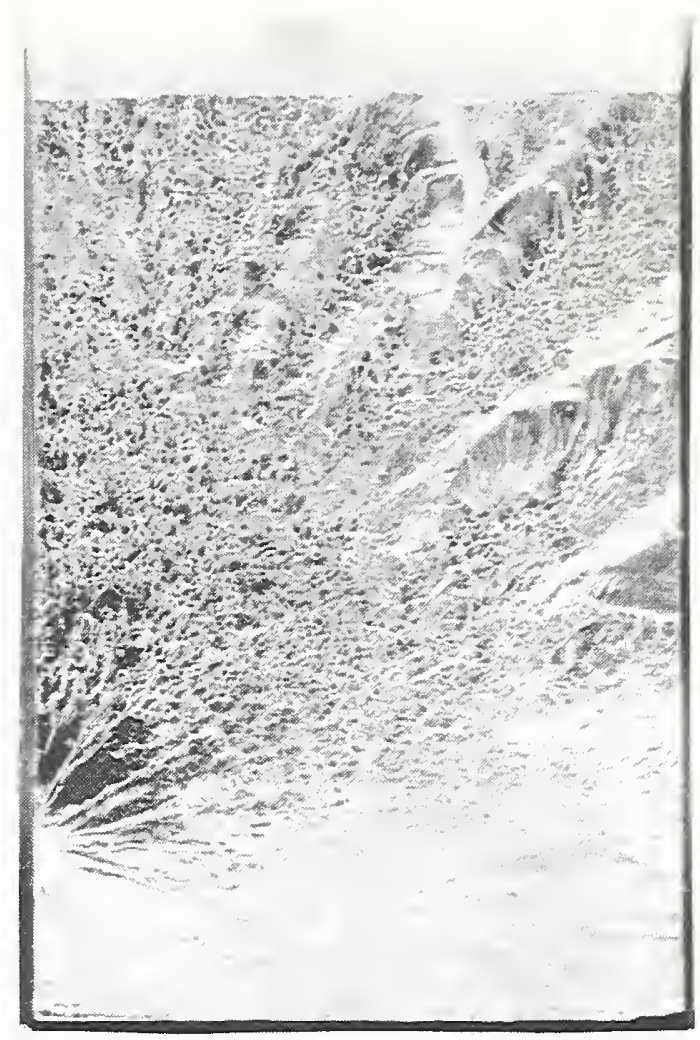

(b)

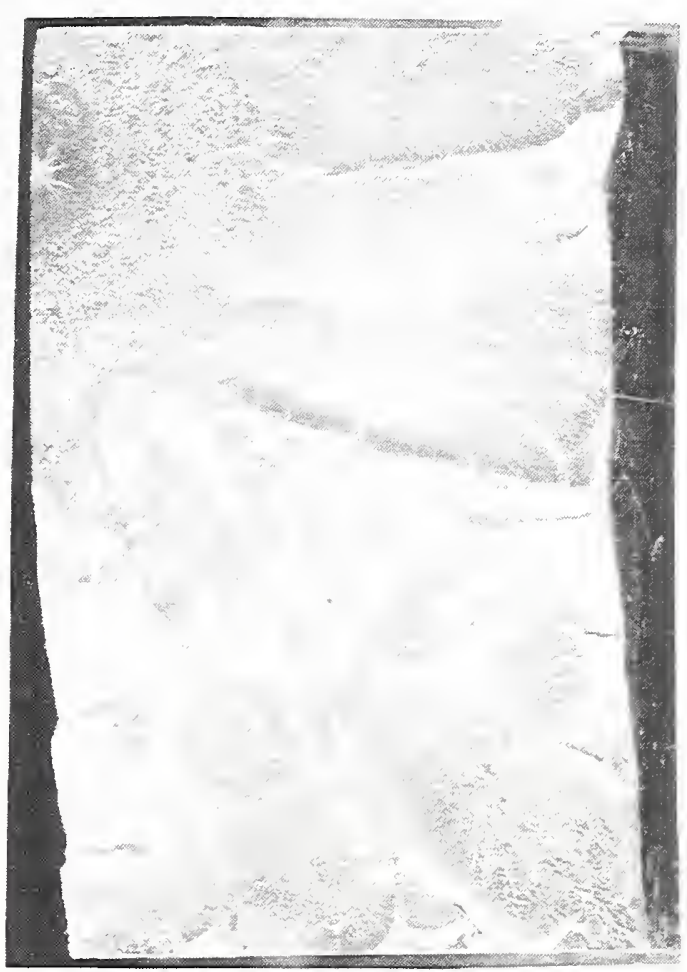

(c)

Figure 4.53. Tensile fracture surfaces of Cyclo.A/AN/ACL at $295 \mathrm{~K}$ (a) and (b) and at $4 \mathrm{~K}(\mathrm{c})$. 


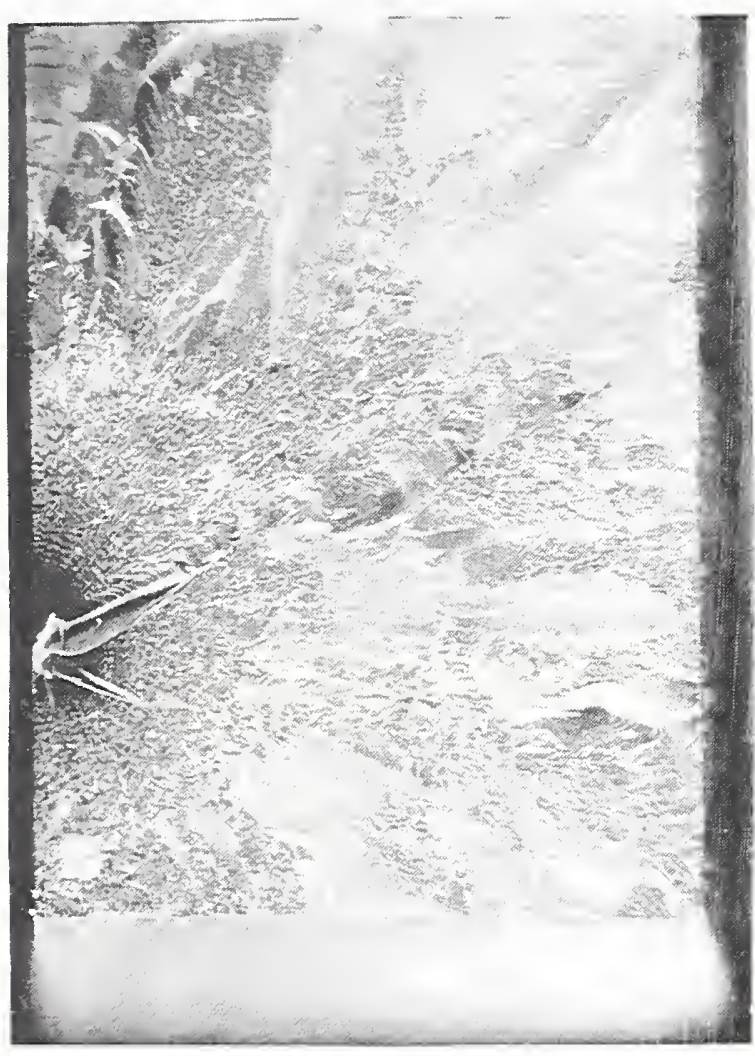

(a)

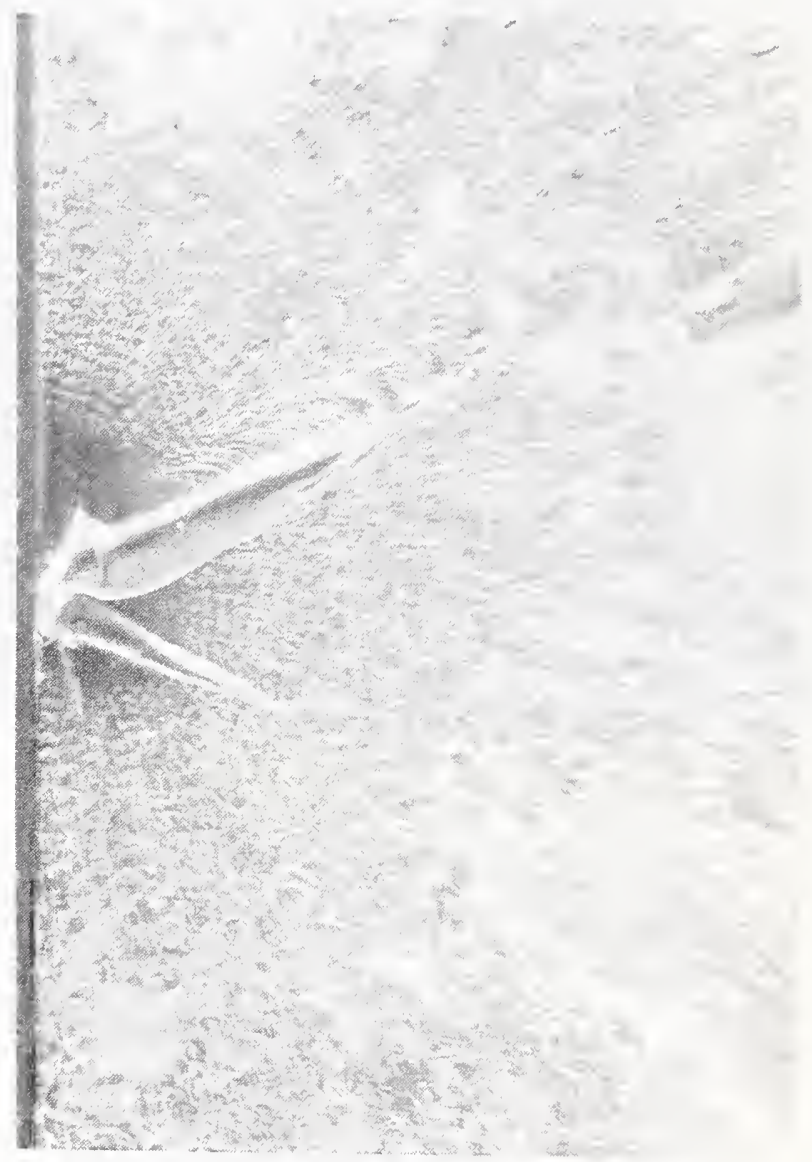

(b)

Figure 4.54. Tensile fracture surfaces of Cyclo.A/CTBN/AN/ACL at $4 \mathrm{~K}$ (a) and (b). 


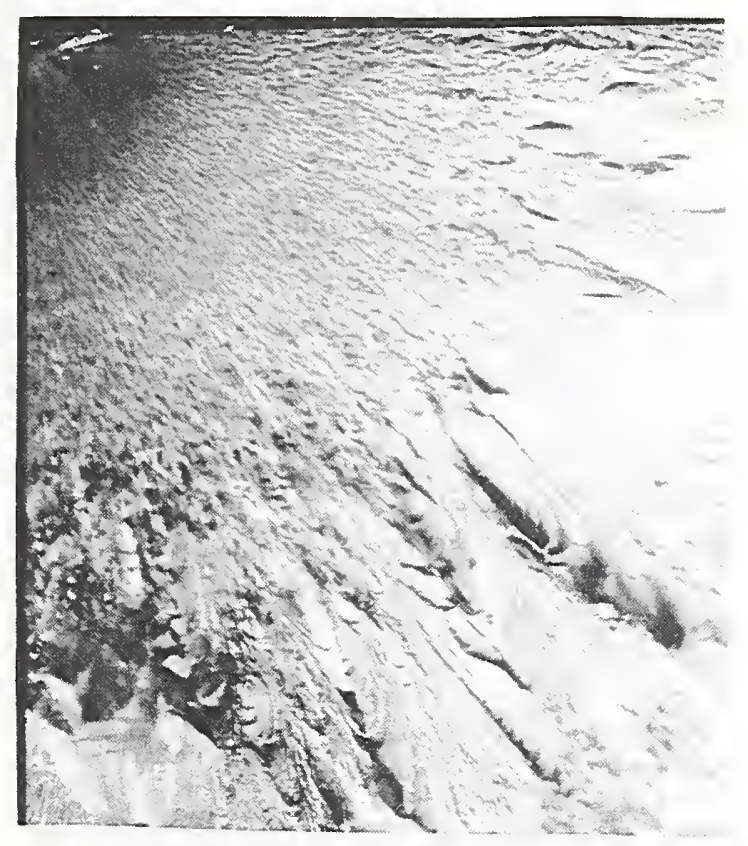

(a)

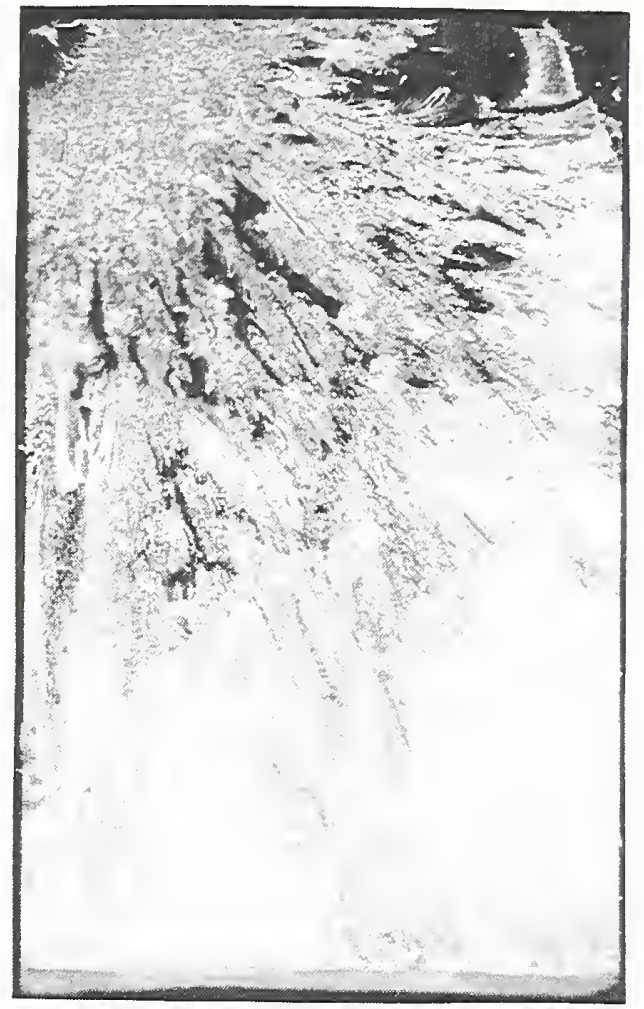

(b)

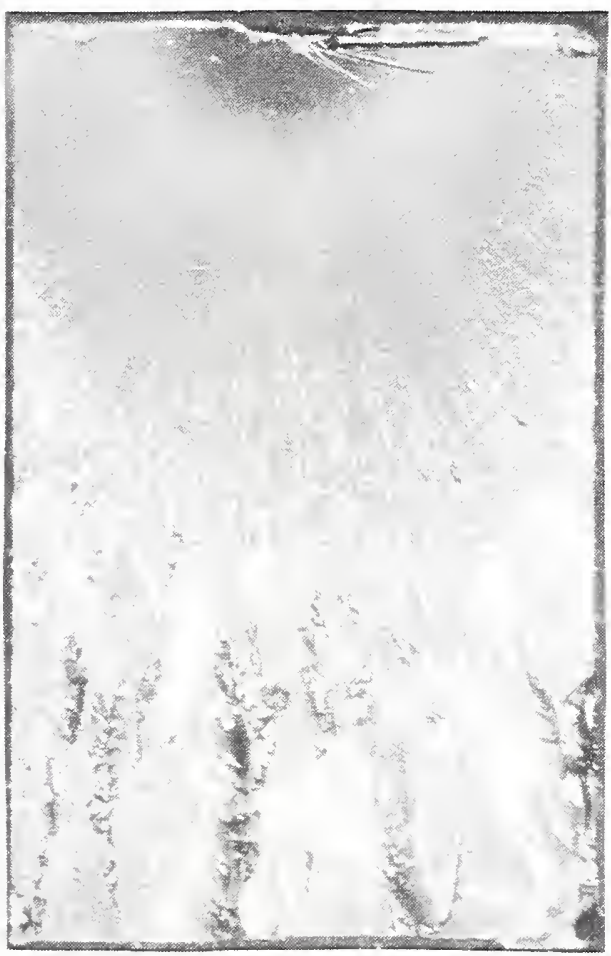

(c)

Figure 4.55. Tensile fracture surfaces of MLTF/ARA at $295 \mathrm{~K}$ (a) and (b) and at $4 \mathrm{~K}$ (c). 


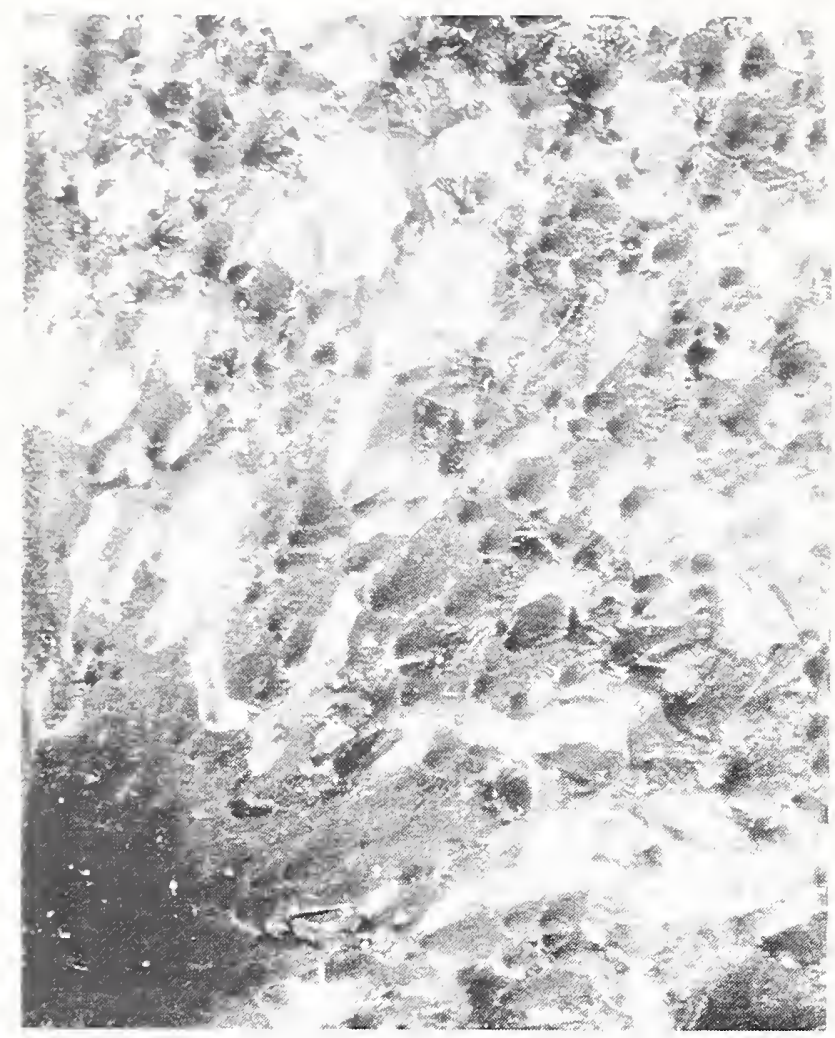

(a)

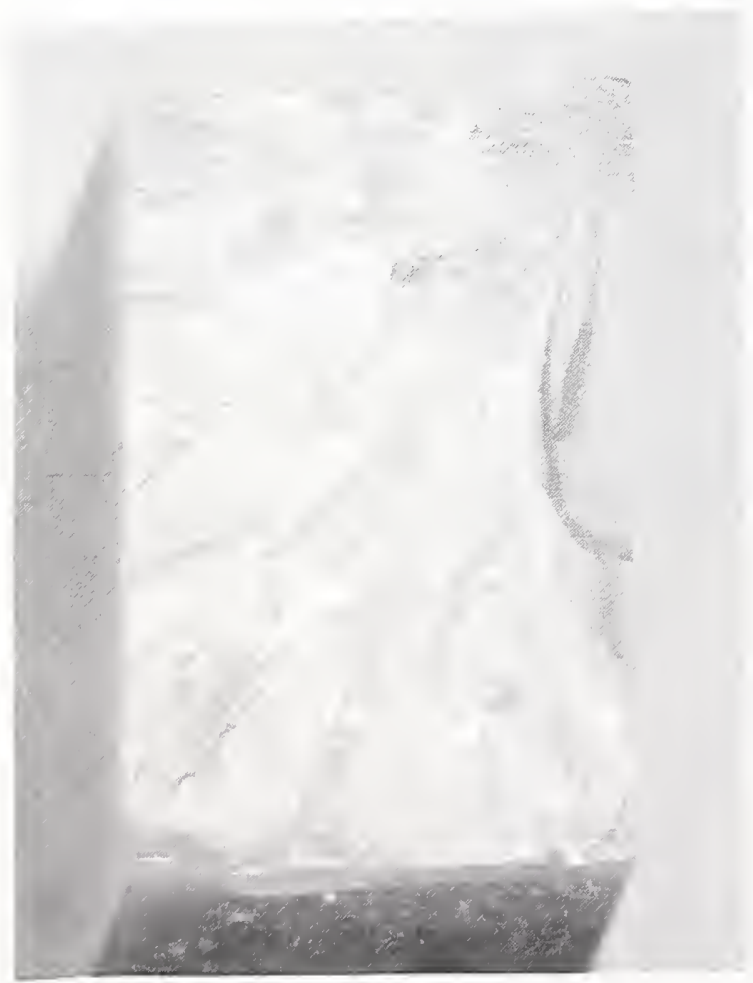

(c)

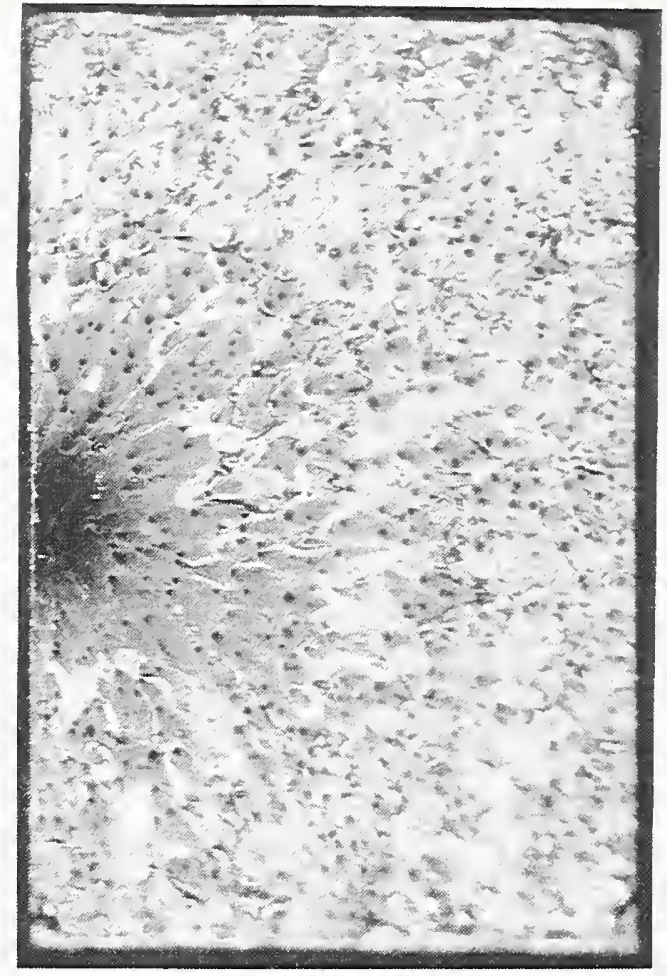

(b)

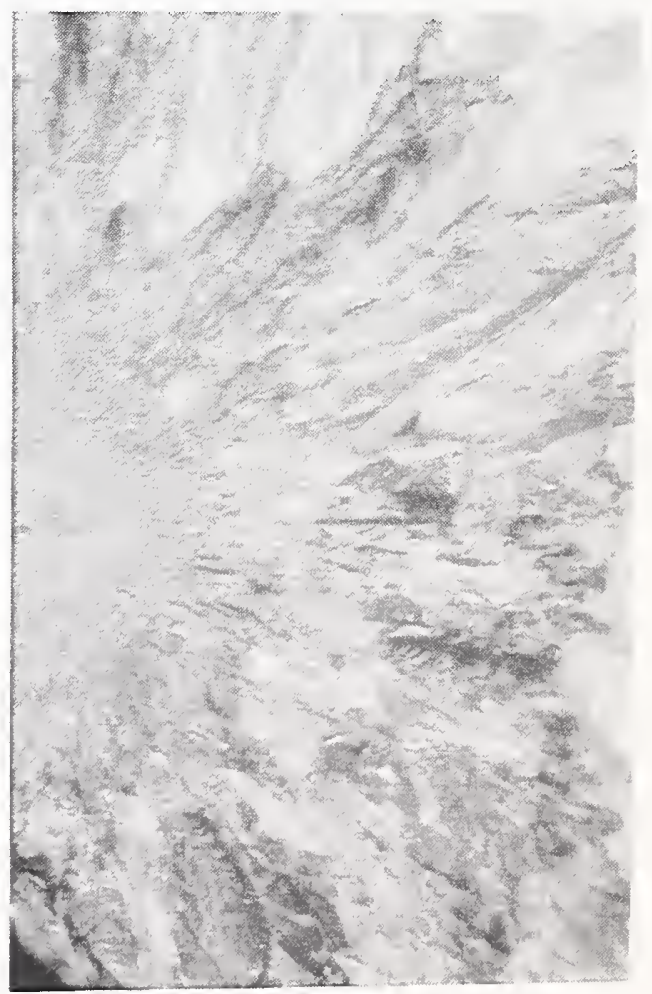

(d)

Figure 4.56. Tensile fracture surfaces of CE/VT/Co.AA at $76 \mathrm{~K}$ (a) and $4 \mathrm{~K}$ (b) and of $\mathrm{CE}$ at $76 \mathrm{~K}(\mathrm{c})$ and $4 \mathrm{~K}(\mathrm{~d})$. 


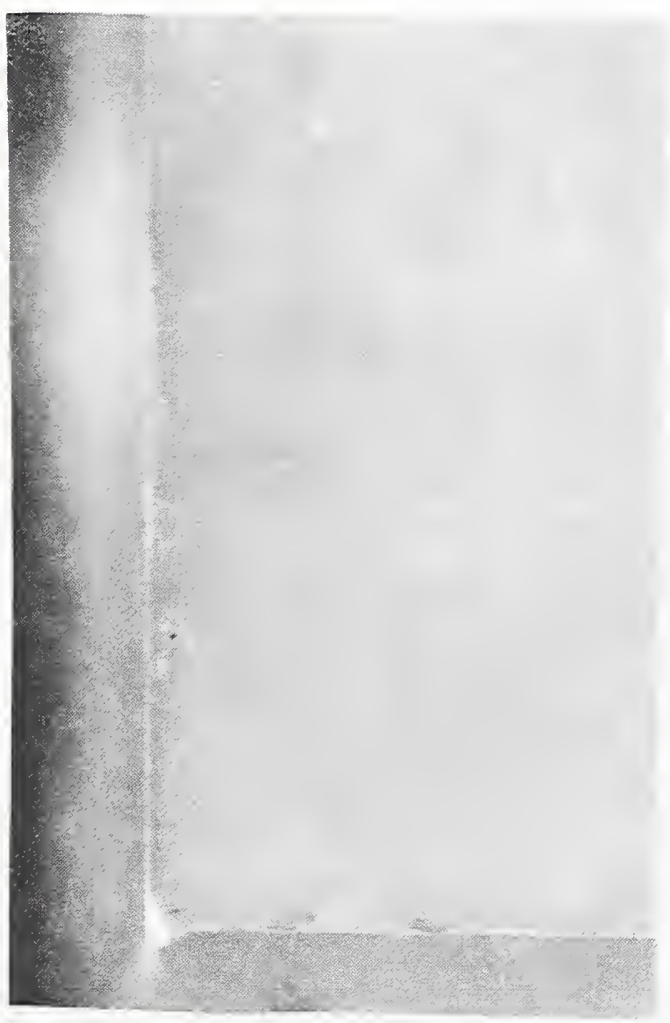

(a)

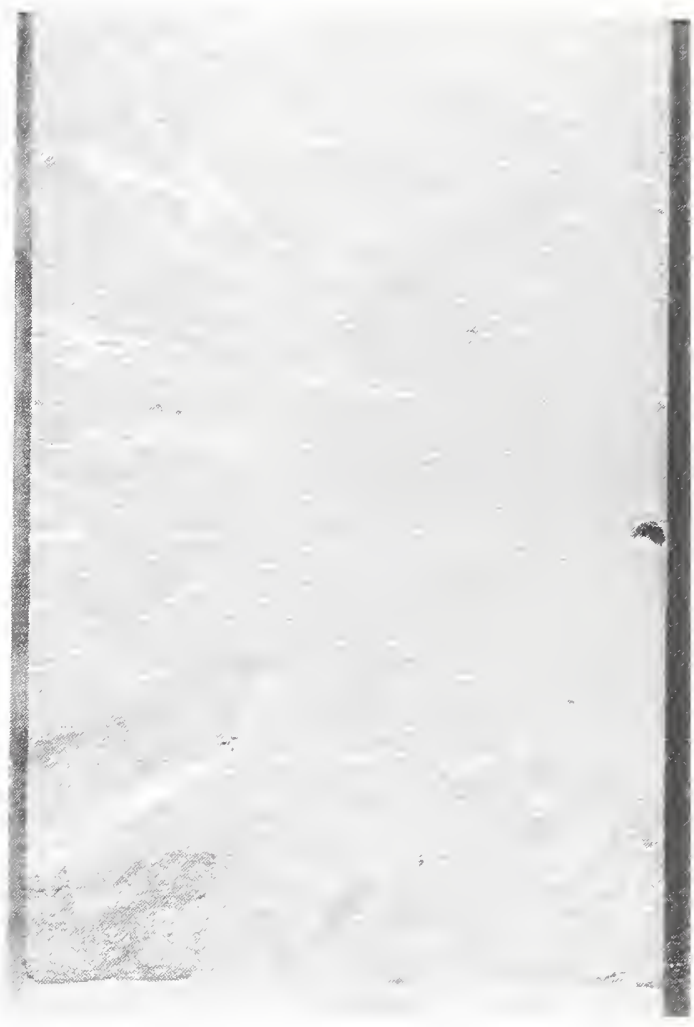

(b)

Figure 4.57. Tensile fracture surfaces of $\mathrm{CE} / \mathrm{HTBN} / \mathrm{NPh} / \mathrm{Cu} . \mathrm{AA}$ at $295 \mathrm{~K}$ (a) and $4 \mathrm{~K}$ (b). 


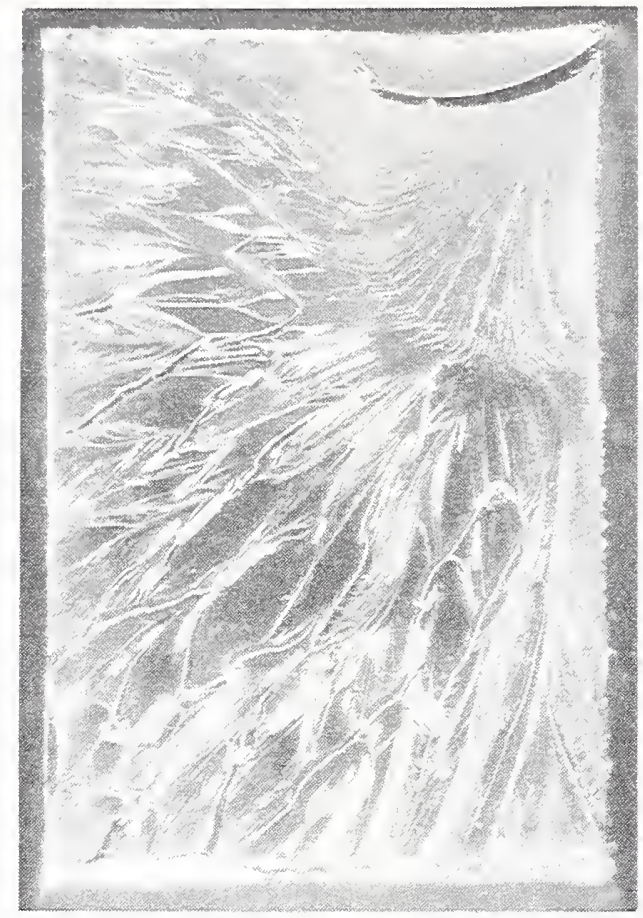

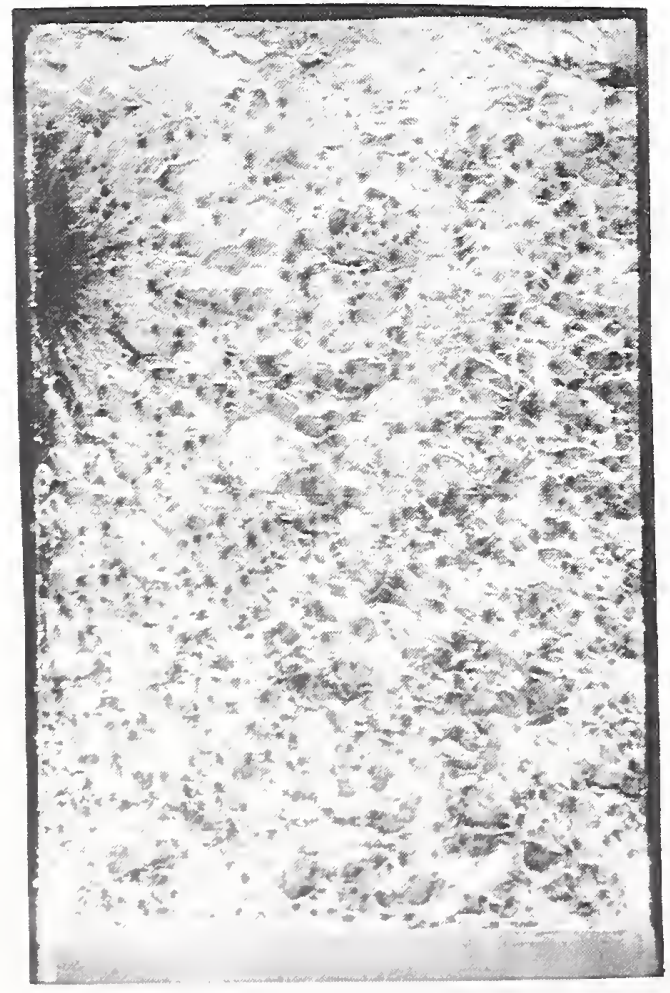

(b)

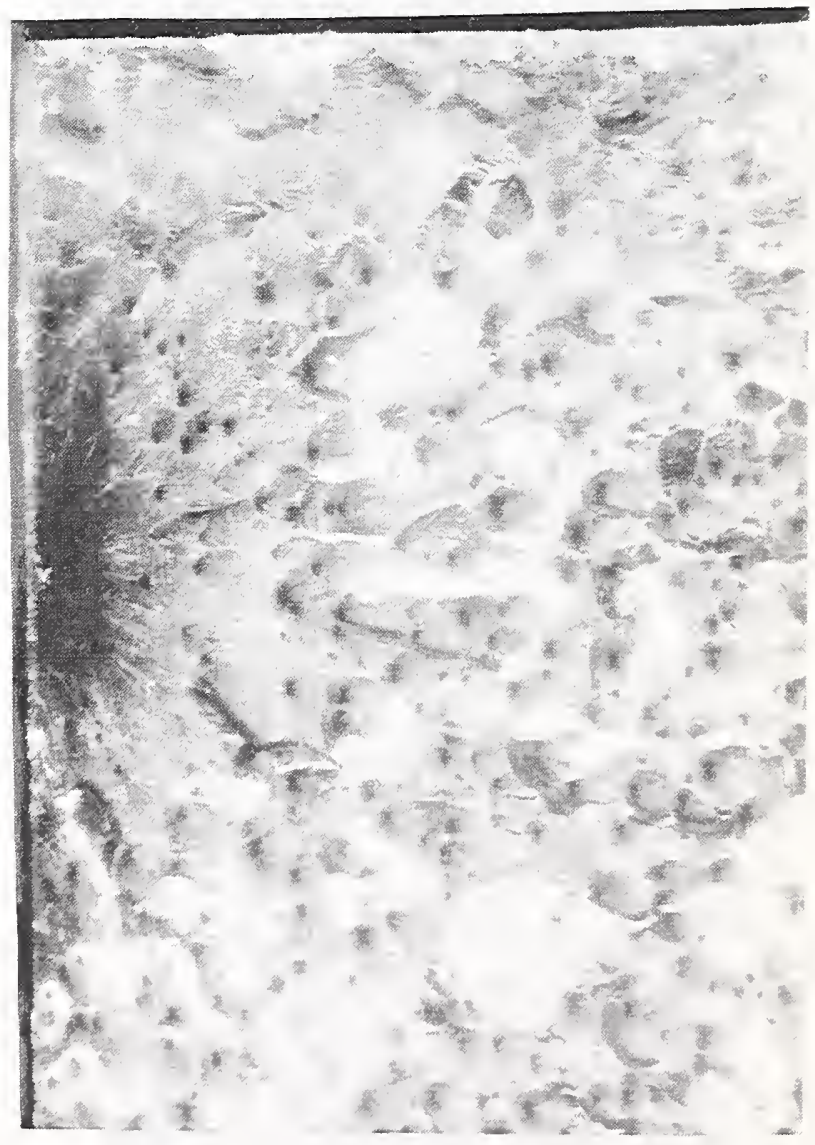

(c)

Figure 4.58. Tensile fracture surfaces of VE/CHX at $295 \mathrm{~K}$ (a) and $4 \mathrm{~K}$ (b) and (c). 


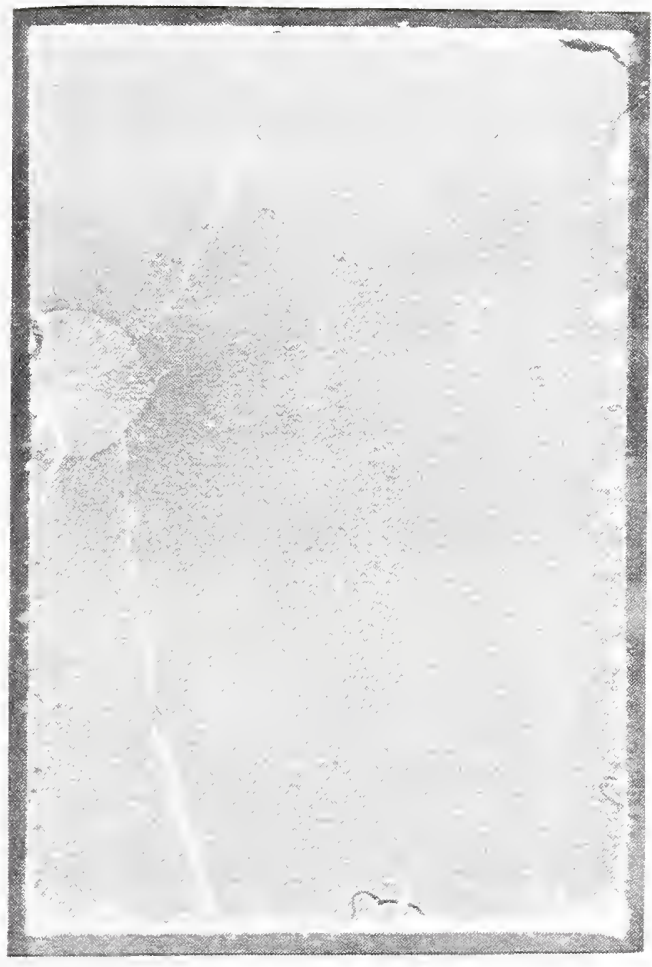

(a)

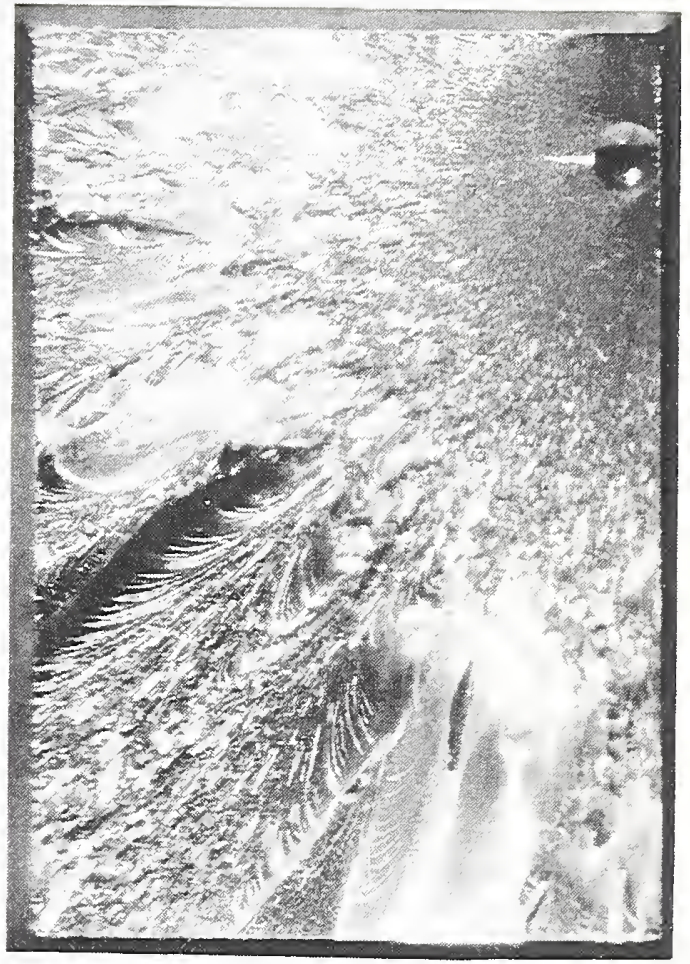

(b)

Figure 4.59. Tensile fracture surfaces of VE-EL/MEKP at $295 \mathrm{~K}$ (a) and $4 \mathrm{~K}$ (b). 


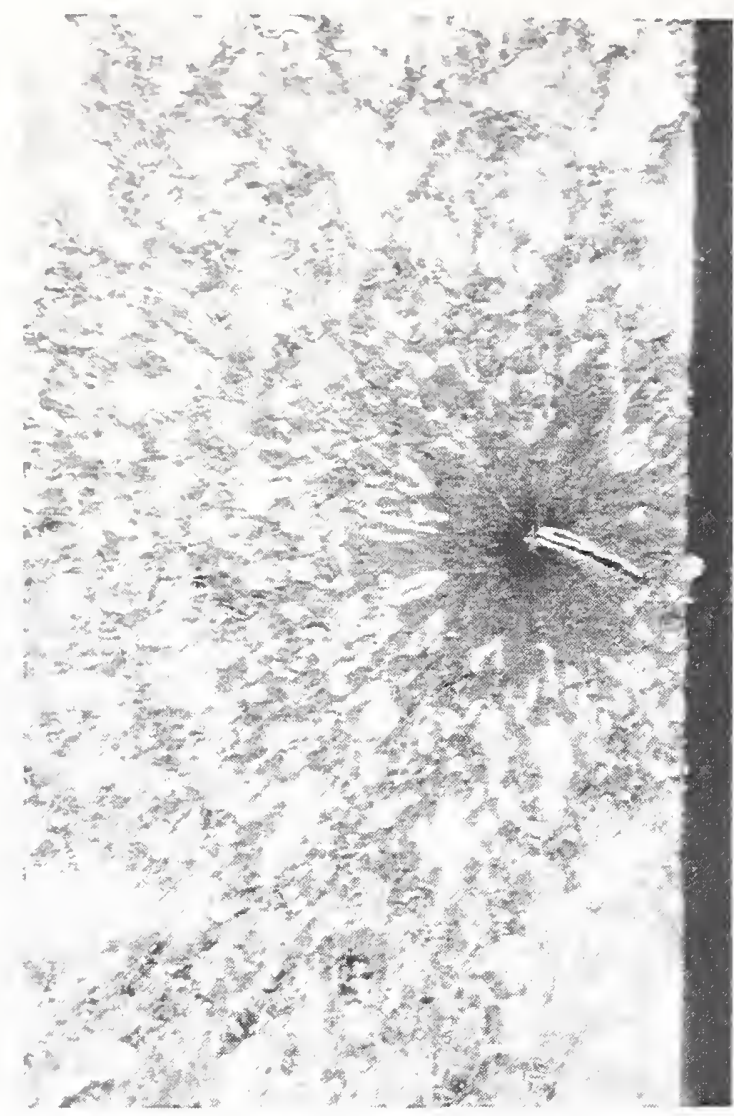

(a)

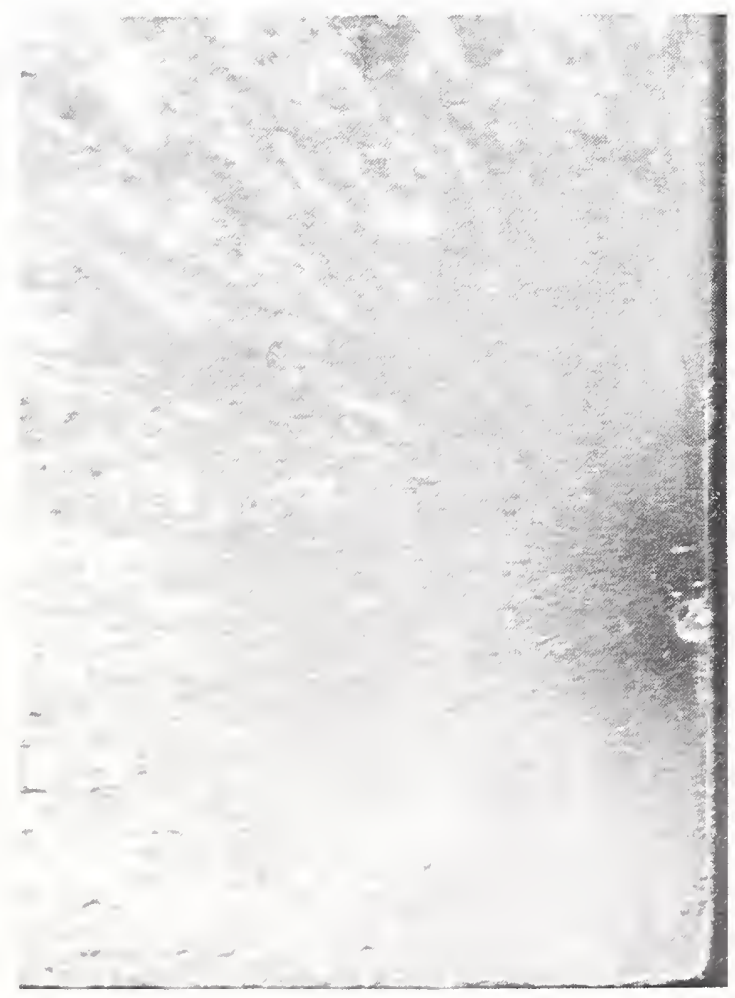

(c)

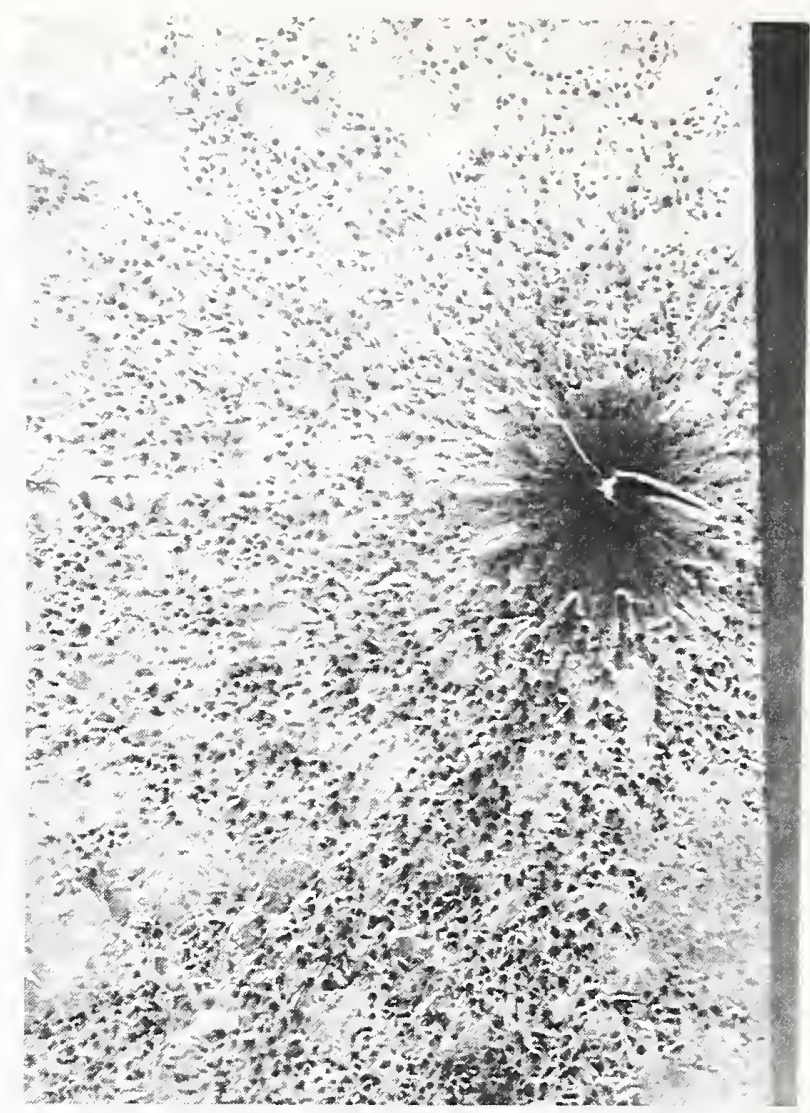

(b)

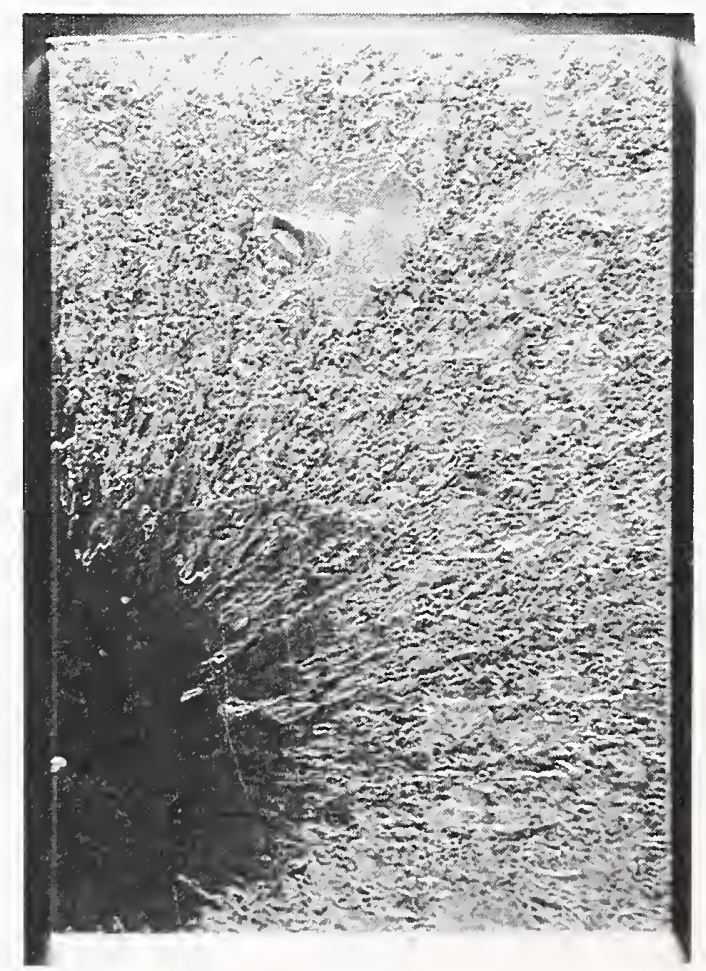

(d)

Figure 4.60. Tensile fracture surfaces of VE/CHP/CoNap/2,4-P at $76 \mathrm{~K}$ (a) and (b) and at $4 \mathrm{~K}$ (c) and (d) 


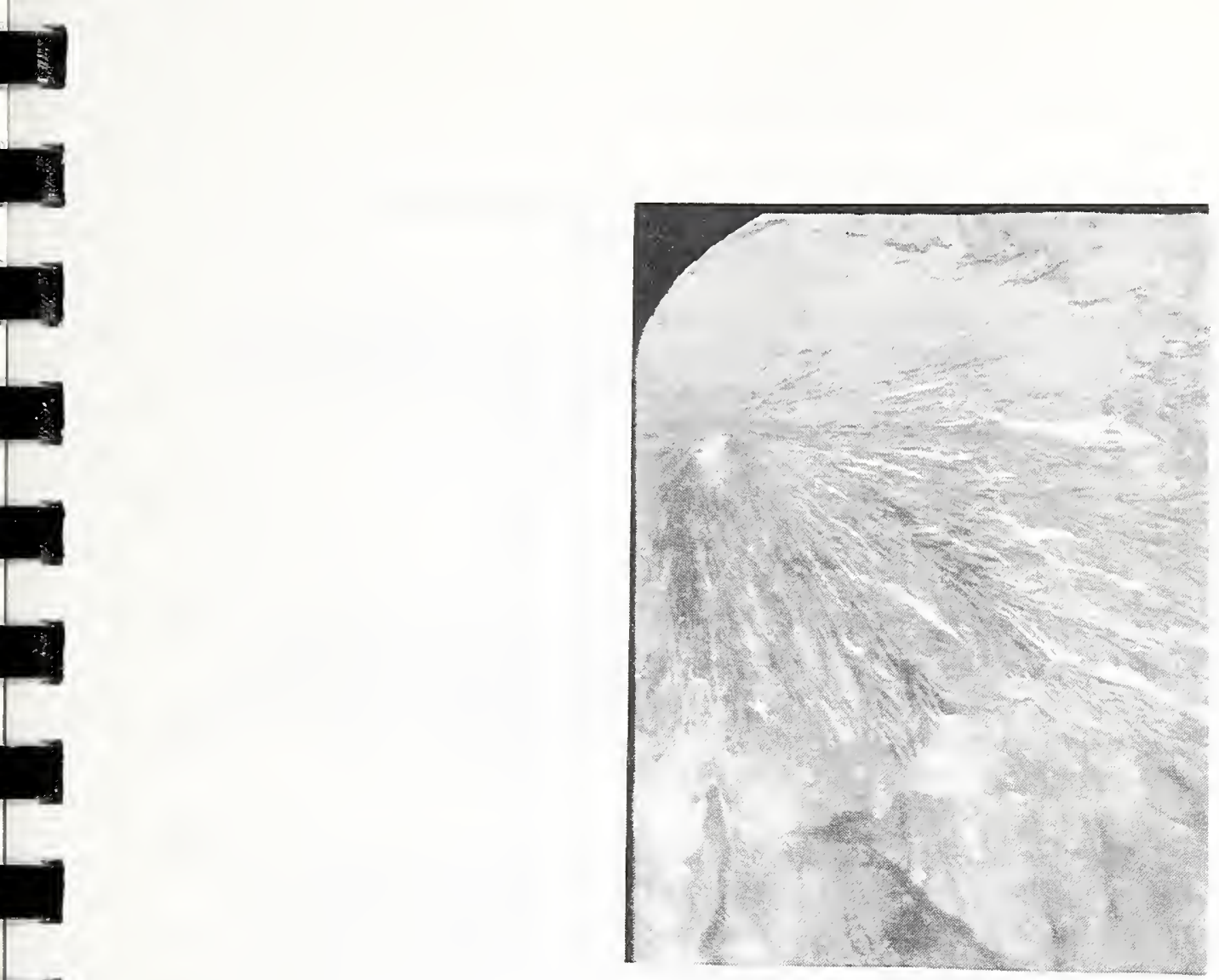

(a)

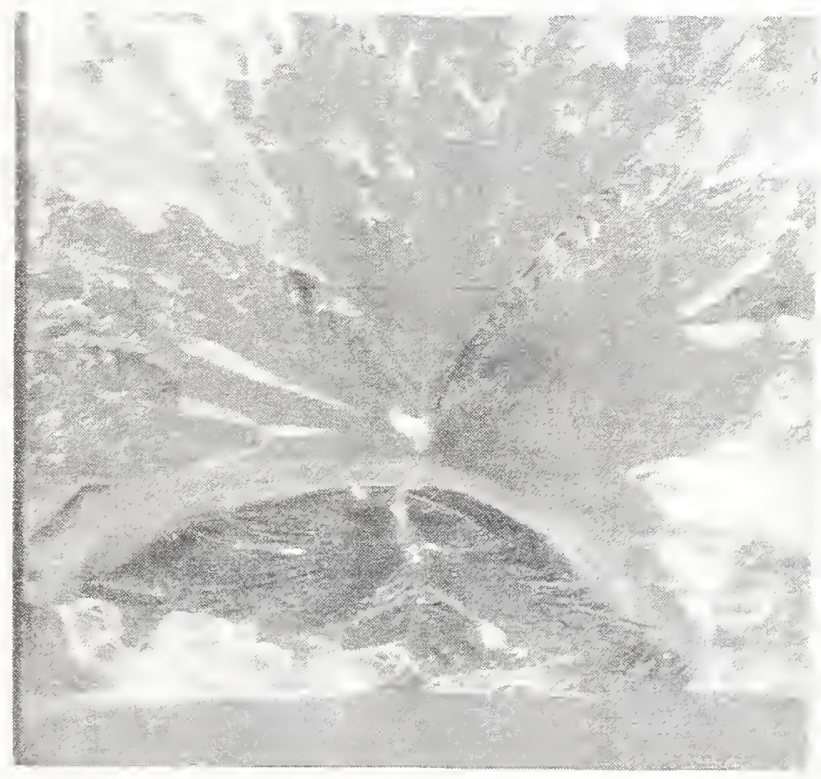

(b)

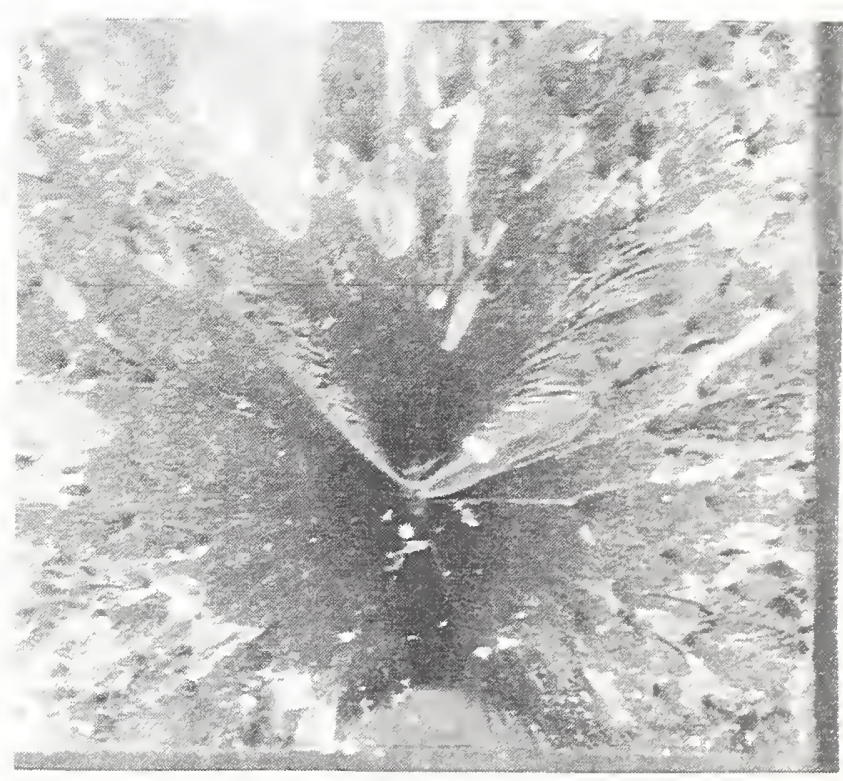

(c)

Figure 4.61. Tensile fracture surfaces of PE/MEKP at $295 \mathrm{~K}$ (a) and $76 \mathrm{~K}$ (b) and (c). 
$4 \mathrm{~K}$ was completely linear elastic. Thus, the striations probably represent sudden-arrest sites of a rapidly moving crack front. This phenomenon has not been previously reported.

In general, one cannot correlate the appearance of the fracture surfaces to the base-resin system. All the base resins (epoxy and vinyl, cyanate and polyester) that were included in this study may have similar fracture-surface topography. The distinctions among the fracture surfaces can be attributed to the use of fillers, tougheners, and other additives and to the test temperature.

\subsection{Discussion}

Analyses of the fracture surfaces of all types of resins, some of which are shown in Figures 4.51 through 4.61 , lead to the conclusion that all failures initiated from a point source and that th source represented a flaw in the neat resin. These flaws may have been either on the specimen surface or embedded in the resin. Therefore, it is natural to attempt to relate the flaw size (maximum diameter) to the failure strength.

In Figure 4.62, the tensile strength at 295,77 , and $4 \mathrm{~K}$ is plotted versus the square root of the average flaw size $(a)$ for most of the specimens whose fracture surfaces are shown in Figures 4.51 through 4.61. At both low and room temperatures, there is a linear relationship between the ultimate tensile strength $\left(\sigma_{u}\right)$ and the square root of the flaw size. In this figure, the power dependence of $\sigma_{u}$ on $a$ was chosen as $1 / 2$, in deference to that found in metals. However, it must be pointed out that the scatter of the data was large enough so that other exponents would have yielded similar trends. No regression analyses were performed on these data sets to optimize the power dependence.

Data from 76- and 4-K tests all fall within one trend line, indicating that fracture mechanisms are similar; the data from $295-\mathrm{K}$ tests are distinct and lower. Significantly, the data of all neat-resin systems fall within the trend lines except for the filled-resin systems (DGEBA-ST/ARA/ACL/F epoxy and VE/CHP/F vinyl ester); data for these two resin systems are not included.

Analysis of Figure 4.62 leads to some interesting conclusions. Equations from Figure 4.62 are for 76 and $4 \mathrm{~K}$

and for $295 \mathrm{~K}$

$$
\sigma_{u} \simeq 170-172 \sqrt{ } a \simeq 170(1-\sqrt{ } a)
$$

$$
\sigma_{u} \simeq 98-80 \sqrt{ } a \simeq 100(1-0.8 \sqrt{ } a)
$$

where the units of $\sigma_{u}$ are $\mathrm{MPa}$ and of $a, \mathrm{~mm}$.

Equations 4.1 and 4.2 suggest that the inherent low-temperature strength of neat resins is about 1.7 larger than the room-temperature strength, independent of coexisting flaws in the neat resin or on the specimen surface. Also, at low temperatures ( 76 to $4 \mathrm{~K}$ ), the sensitivity to flaws $(\sim-172 \mathrm{MPa} / \sqrt{ } a)$ is about $100 \%$ more than the room-temperature sensitivity $(\sim-80 \mathrm{MPa} / \sqrt{ } a)$. Thus, at low temperatures, the inherent resin strength increases about $75 \%$, and the sensitivity to flaws increases by about $100 \%$.

From these trend lines of Figure 4.62, the fracture toughness $\left(K_{I c}\right)$ of the resin systems can be estimated if one assumes a constant stress-intensity factor $(\beta)$ for all flaw types. Here 


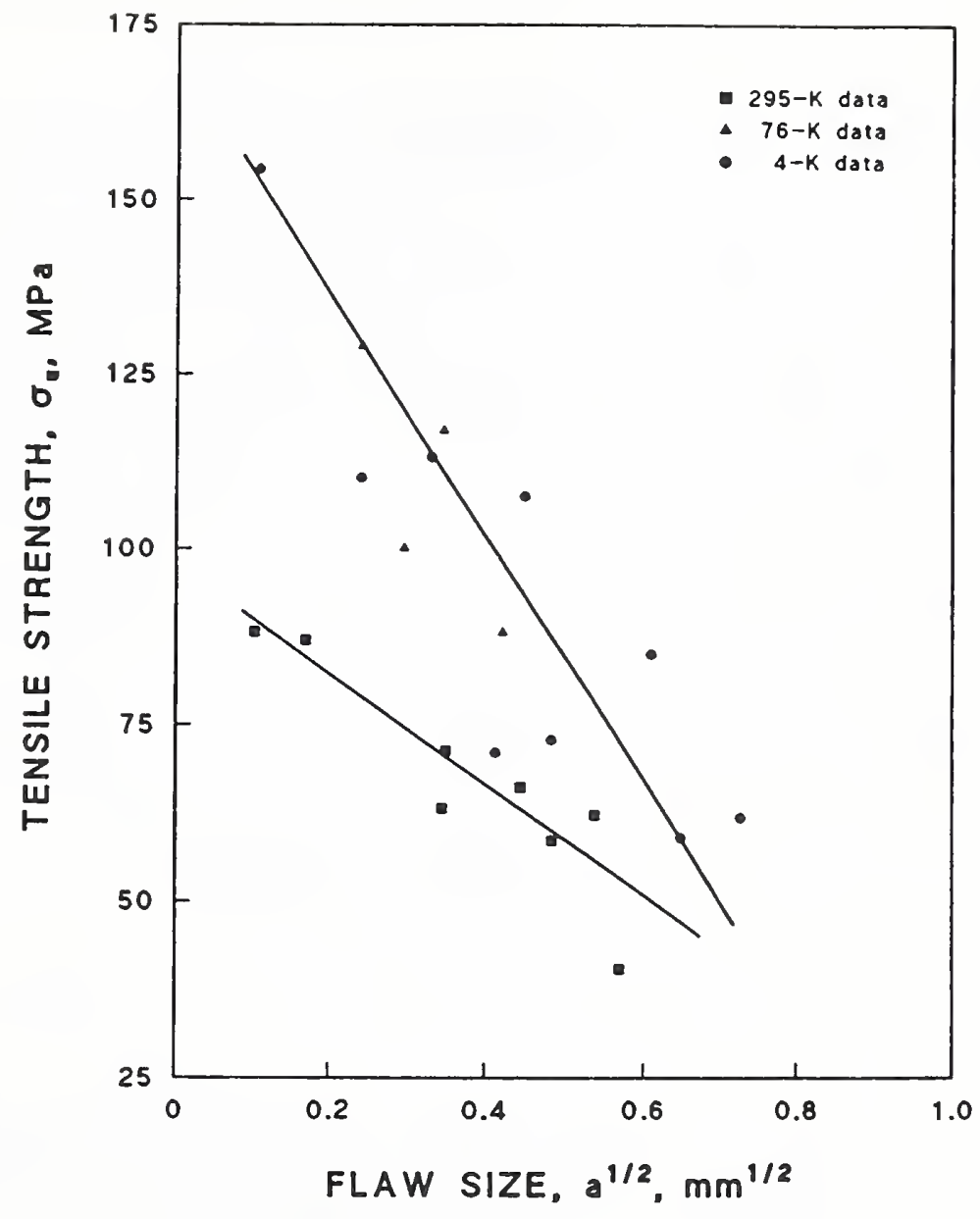

Figure 4.62. Tensile strength versus the square root of flaw size.

$$
K_{l c}=\beta \sigma_{u} \sqrt{\pi a}
$$

where $\beta$ is related to crack size, geometry, and location; it was arbitrarily chosen to be a constant and equal to 1 for these calculations. For a more detailed discussion of the stress-intensity-factor approach to describing fracture in resins, the reader is referred to Pritchard and Rhoades [4.8].

Combining Equations 4.1 and 4.2 with 4.3 and converting the units of $a$ from millimeters to meters, we obtain $K_{l c}$ in $\mathrm{MPa} \sqrt{\mathrm{m}}$,

for 76 and $4 \mathrm{~K}$

and for $295 \mathrm{~K}$

$$
K_{l c} \simeq\left(3.3 \times 10^{-4}\right) \sigma_{u}\left(170-\sigma_{u}\right)
$$

$$
K_{l c} \simeq\left(7 \times 10^{-4}\right) \sigma_{u}\left(100-\sigma_{u}\right)
$$




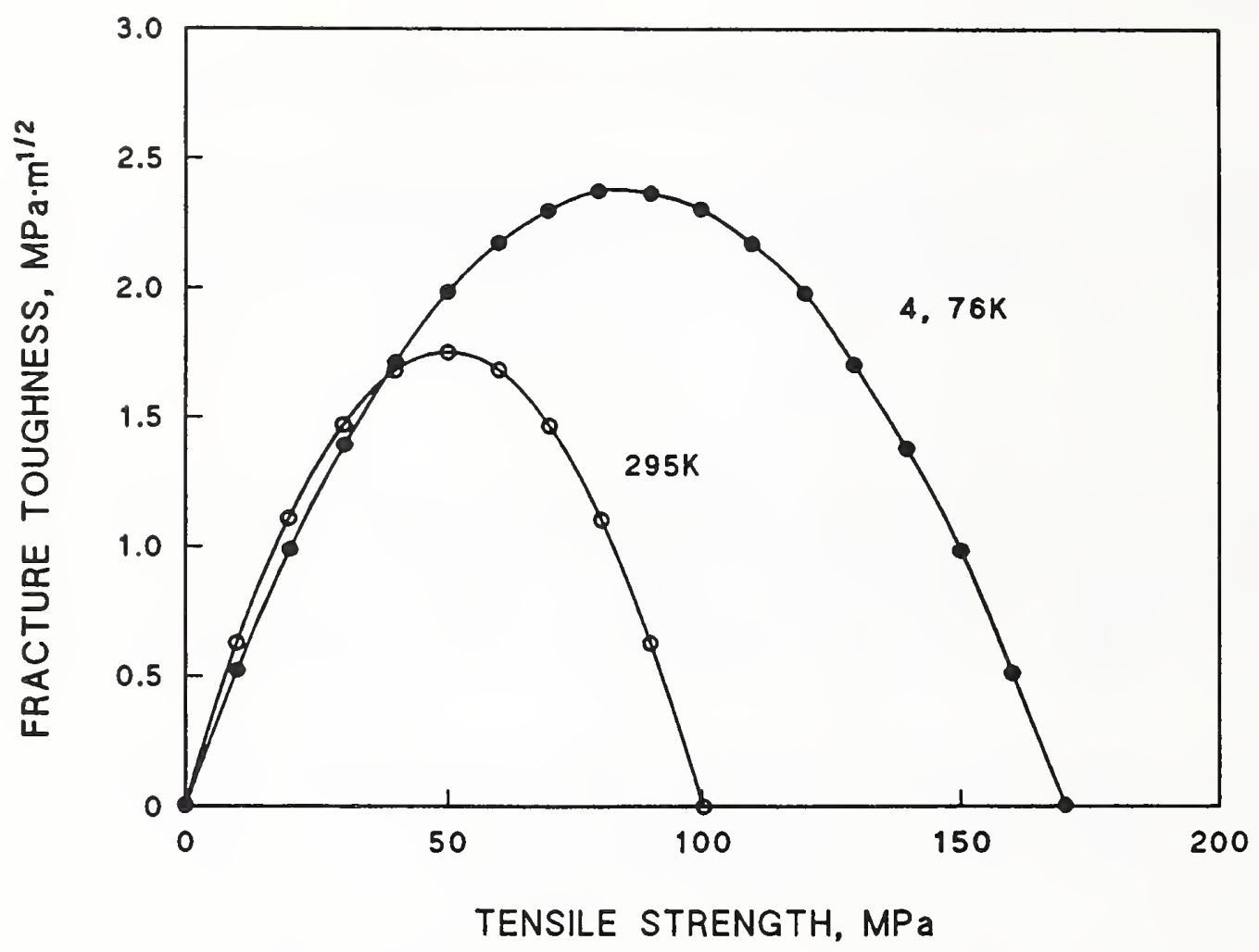

Figure 4.63. Equations 4.4 and 4.5 showing the dependence of fracture toughness on tensile strength.

These two relationships are plotted in Figure 4.63; they represent parabolic dependences with maximum $\mathrm{K}_{\mathrm{Ic}}$ values of $1.76 \mathrm{MPa} \sqrt{\mathrm{m}}$ at $50 \mathrm{MPa}, 295 \mathrm{~K}$ and of $2.38 \mathrm{MPa} \sqrt{\mathrm{m}}$ at $85 \mathrm{MPa}, 76$ to $4 \mathrm{~K}$. At low values of tensile strength, the fracture toughness at room temperature is slightly higher than at low temperatures. At tensile-strength values above about $40 \mathrm{MPa}$, the toughness at low temperatures is higher. Optimum-toughness resins have a tensile strength of about $50 \mathrm{MPa}$ at room temperature and $85 \mathrm{MPa}$ at low temperatures.

There is a reassuring aspect to the $\sigma_{u}$-versus- $\sqrt{ } a$ low-temperature trend line of Figure 4.62. The four data points that lie nearly on the trend line are from embedded flaws. It is much easier to estimate the size of flaws that lie entirely within the matrix than the size of surface flaws. The nature of surface flaws and, sometimes, the complex lighting conditions make it much more difficult to estimate their size.

Thus, considering the sensitivity of ultimate strength to flaw size, it is easy to understand the data scatter at low temperatures. Also, the apparent relative ultimate tensile strength of each resin system may, in fact, reflect processing difficulties or deficiencies rather than inherent strength distinctions. To properly sort out the inherent strength difference of the generic classes of resins demands increased emphasis on careful, reliable processing techniques. This increased processing care should go well beyond that which is currently practiced by industry in the production of components that are used primarily at room temperature.

It could be that the processing schedules provided by the various sources listed in Table 2.1 of $\S I I I .2$ result in flaw concentrations and sizes that would be generated during strut manufacture. If this 
is assumed, then the results of this study can be used for initial selection of resin systems for strut manufacture.

Apparent total strain to failure, with the assumption of linear elastic conditions, may be estimated by comparing the ratios of ultimate tensile strength to Young's modulus for different resins. Of course, since all resins exhibited some plastic deformation at room temperature, this ratio underestimates strain-to-failure at room temperature. In Figures 4.64 through 4.66 , the tensile strength is plotted versus Young's modulus for the epoxies, the vinyl esters, and the cyanate esters plus the polyester, respectively. Data from all test temperatures are included in these figures. Resin systems with relatively high strength and low modulus at each specific temperature have large apparent strains to failure. Thus, for epoxy resins, the DGEBF/CTBN/AN has the largest strain-to-failure at low temperatures. Other resin systems that have high strain to failure at low temperatures include the vinyl esters VE/CHX and VE-EL/MEKP.

From these tensile results, the following resin systems have the best properties in their class: epoxy
at low temperatures - strength:
DGEBF/CTBN/AN
modulus:
DGEBA-ST/ARA/ACL/F \& MLTF/ARA
elongation:
DGEBF/CTBN/AN
at room temperature - $\begin{aligned} & \text { strength: } \\ & \text { modulus: }\end{aligned}$
Cyclo.A/AN/ACL \& NV/AN/ACL
DGEBA-ST/ARA/ACL/F \& MLTF/ARA

cyanate ester:

$\mathrm{CE} / \mathrm{HTBN} / \mathrm{NPh} / \mathrm{CnAA}$

vinyl ester:

$\begin{array}{lll}\text { at low temperatures - } & \text { strength: } & \text { VE/CHX \& VE-EL/MEKP } \\ & \text { modulus: } & \text { VE/CHP/F } \\ & \text { elongation: } & \text { VE/CHX \& VE-EL/MEKP } \\ \text { at room temperature - } & \text { strength: } & \text { VE/CHP/CoNap/2,4-P } \\ & \text { modulus: } & \text { VE/CHP/F }\end{array}$

polyester:

PE/MEKP

The strength of PE/MEKP is better than those of cyanate esters and its modulus is better than most cyanate esters and some vinyl esters and epoxies.

Higher Young's moduli are obtained by adding filler material or by using multifunctional epoxies. However, both of these types of resins have unusually low tensile strengths at low temperatures. Higher strengths at low temperatures are found in some vinyl esters, and the lowest strengths are found in the cyanate esters. The low strength in the $\mathrm{CE}(\mathrm{PT})$ resin system is probably due to undercuring; therefore, this ranking is not absolute. The epoxy resin systems have a large range of low-temperature strengths, and from a chemical standpoint, it is not evident what influences the strength. 


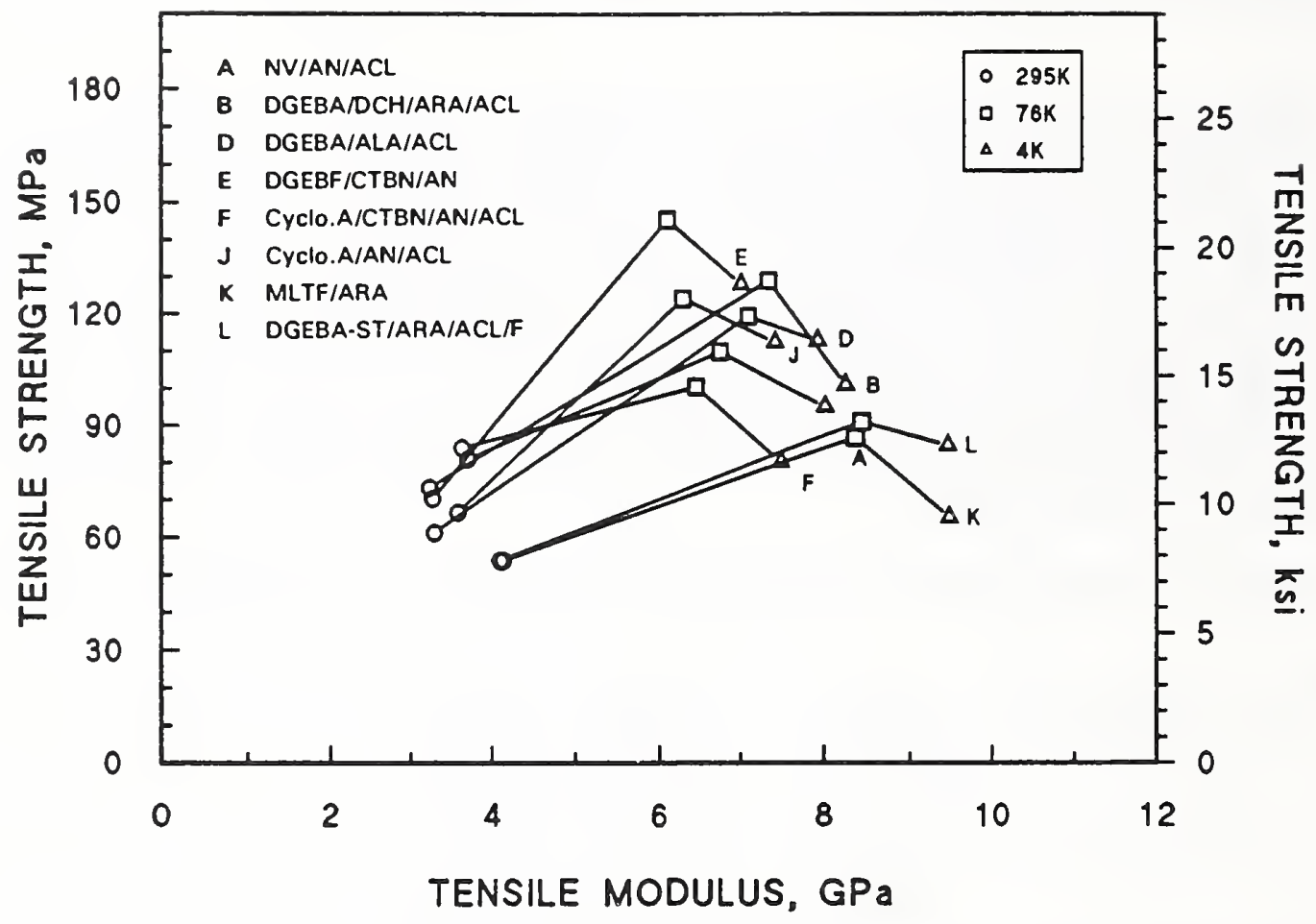

Figure 4.64. Tensile strength versus Young's modulus for epoxies.

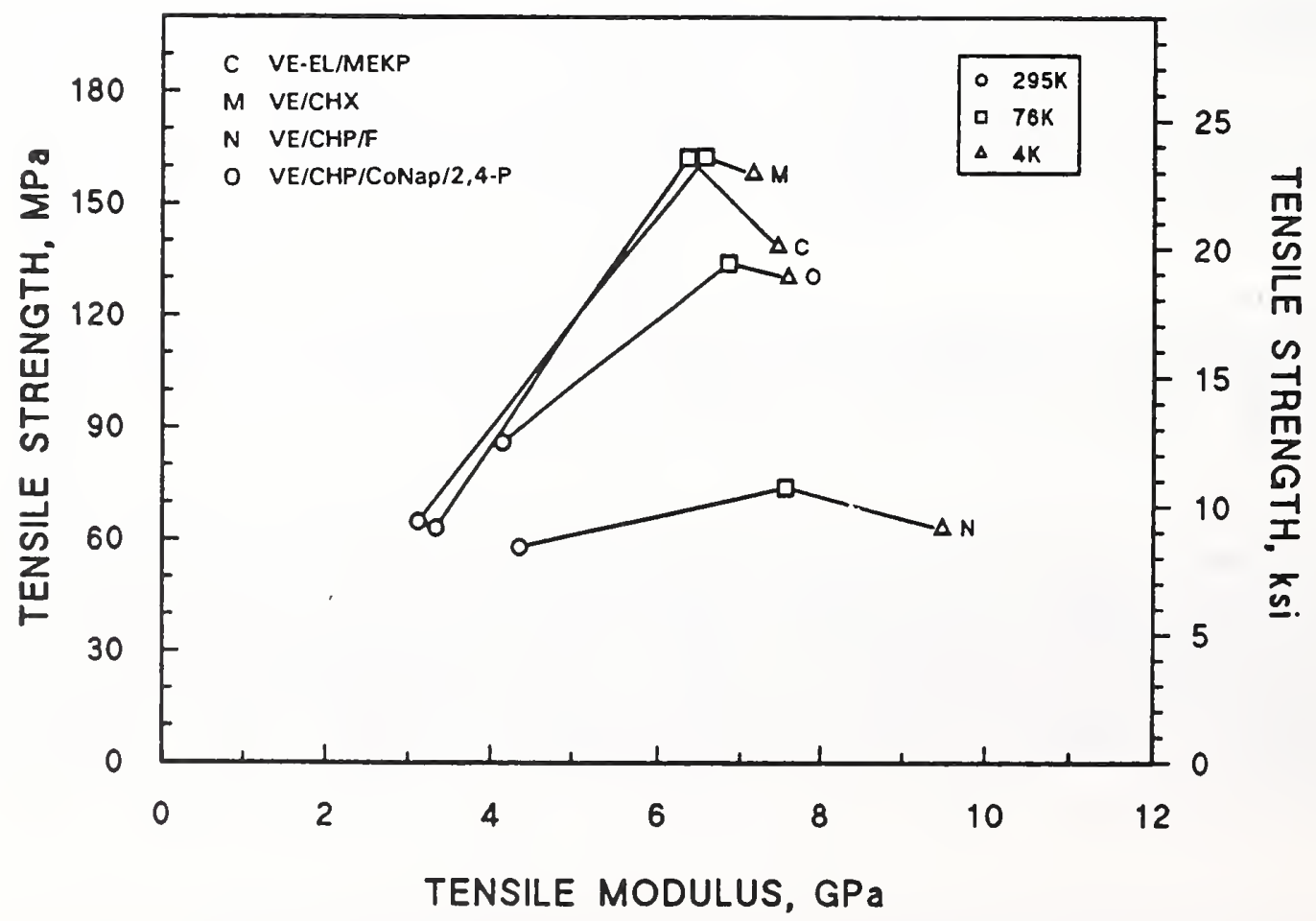

Figure 4.65. Tensile strength versus Young's modulus for vinyl esters. 


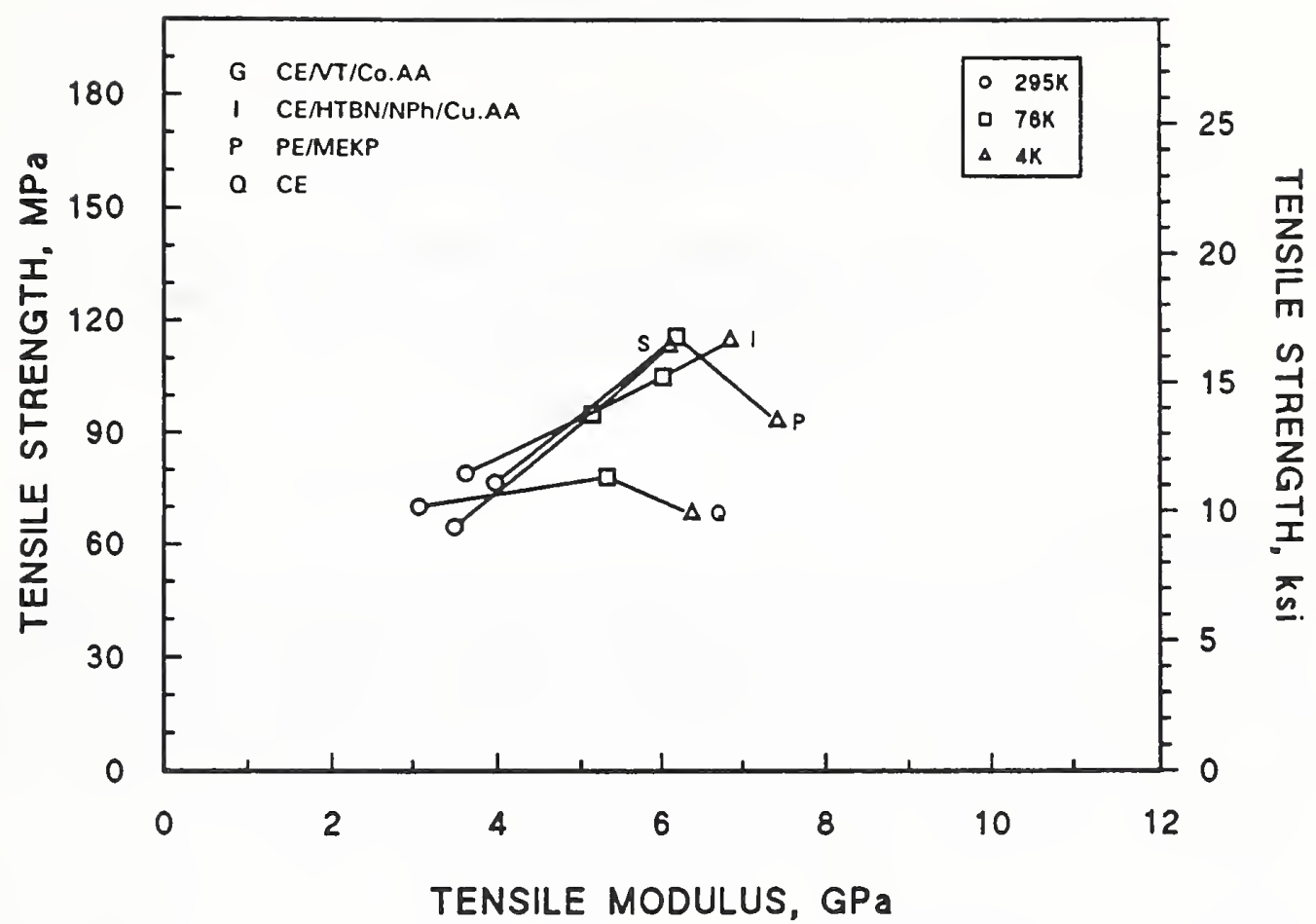

Figure 4.66. Tensile strength versus Young's modulus for cyanate esters and polyester.

Finally, the selection of the best resin systems for strut manufacture, based on the tensile data, depends on two parameters:

1. The dependence of tensile strengths on flaw size and flaw concentration places a premium on the effective processability of the resin system.

2. The distinctions between resin systems that have high Young's modulus and those that have high strength place emphasis on effective failure analyses and modeling of strut failure mechanisms. The predicted dependence of the failure mechanism on strut (and matrix) rigidity, or on matrix strength or strain to failure, will dictate resin selection criteria. 


\title{
5. Shear Properties
}

\author{
J.B. Darr and P.E. Fabian \\ R.P. Reed ${ }^{*}$ \\ Composite Technology Development, Inc. \\ Boulder, Colorado \\ ${ }^{*}$ Cryogenic Materials, Inc. \\ Boulder, Colorado
}

\subsection{Introduction}

Cylindrical, neat specimens, produced with the pultrusion process, contain very few flaws, have a very smooth surface contour and finish, and are ideal for testing in torsion. Torsion tests are an excellent method for evaluating shear properties. Shear strength, strain to failure, and modulus were measured at 295,76 , and $4 \mathrm{~K}$ for selected epoxy, vinyl-ester, cyanate-ester, and polyester resins. Toughened-epoxy and filled-epoxy resins were also tested.

\subsection{Review of Literature}

The torsion test method has been repeatedly confirmed as a very reliable, accurate test for evaluating shear properties in materials. Wilson recognized the torsion test as the test method that produces the purest state of shear stress [5.1], and Adams found that the torsion test has the ability to introduce a well-defined state of stress into the test specimen [5.2], which enables both the shear strength and shear modulus to be determined from a single specimen. Adams also showed that the easily performed, inexpensive, solid-rod torsion test consistently gives reliable values for shear properties.

Kasen introduced torsion testing at cryogenic temperatures. He found this test method to be an efficient way of screening the influence of components in unreinforced polymers, as well as composites, metals, and alloys, in a cryogenic environment $[5.3,5.4]$ and advocated its use for irradiation testing. To study composites, he developed a pultrusion method to produce unidirectional glass-reinforced resin-based materials that contain about 55 vol\% of reinforcing fibers. He showed that at low temperatures the fracture path for these unidirectional composites tended to follow the fiber-matrix interface. Thus, one should expect strong correlation between the shear strength obtained with the torsion method and the interlaminar shear strength.

Composite Technology Development has used this test procedure and method in numerous programs to screen and differentiate between different materials, including neat-resin and composite materials, at cryogenic and ambient temperatures $[5.5,5.6]$.

\subsection{Resins}

Each of the resin systems is described in detail in $\$$ III.3.3.2. Their trade names and key to their abbreviations are given in Tables 2.1 and 2.2 of $\$ I I I .2$. 


\subsection{Procedures}

\subsubsection{Specimen Preparation}

Rods of neat resin were produced by the pultrusion process described in $\S$ III.3.3.1.

Torsion specimens were cut from 3.2-mm-diameter rods to a length of $50 \mathrm{~mm}$. The diameter and length of each specimen were measured, and each specimen was visually inspected for flaws. Flawed specimens were eliminated from the test matrix.

\subsubsection{Test Apparatus and Method}

Torsion testing was done at 295,76 , and $4 \mathrm{~K}$ with the special test apparatus shown in Figures 5.1 and 5.2. This strain-controlled torsion-shear test facility and method are described further in Reference 5.3. The system was operated at an angular displacement rate of about $0.3^{\circ} \cdot \mathrm{s}^{-1}$, which resulted in a torsion strain rate of about $5.2 \times 10^{-3}$ radian $\cdot \mathrm{s}^{-1}$ for $3.2-\mathrm{mm}$ diameter specimens. The gage length of a specimen was $25 \mathrm{~mm}$.

Rod specimens were inserted into collet grips. The grips were tightened to a torque adequate to hold the specimens without undue slippage but not so tight as to cause premature failure. The specimen was inserted into the reaction tube assembly, which was then loaded into the test apparatus. When the test was to be performed at cryogenic temperature, the reaction tube assembly bearing the specimen was lowered into a Dewar containing the appropriate cryogen, either liquid nitrogen (at $76 \mathrm{~K}$ ) or liquid helium (at $4 \mathrm{~K}$ ).

A torsion load was applied via a gear assembly, and the torque was measured by a torsion load cell located between the loading point and the specimen. Rotation of the specimen was measured by the rotary variable-displacement transducer (RVDT), also in the load line. Signals from the load cell and RVDT were fed into a chart recorder, which produced a continuous record of torque versus angular displacement.

The shear moduli were calculated by converting the initial slopes of the torque-angulardisplacement curves into the appropriate shear stress and shear strain components. For each specimen, continuous measurements at low loads were taken repeatedly, at least four times, by raising and lowering the applied torque. The final three measurement trials were averaged to obtain the reported shear modulus.

After measuring the moduli at low loadings, the specimen was loaded to failure. The shear strengths and shear strains reported were calculated from the maximum loads, and angular displacements were recorded at specimen failures. When the angular displacement exceeded $90^{\circ}$, the test was terminated because this is the limit of linear response for the RVDT.

In shear tests performed at ambient temperature $(295 \mathrm{~K})$, specimens usually do not fail before $90^{\circ}$ of rotation is reached. Therefore, an effective yield shear strength and strain were calculated by taking the slope of the initial linear portion of the load-angular-rotation curve, offsetting this slope by $10^{\circ}$ of angular rotation, and finding the intersection of this line with the load-displacement curve. The calculated yield strength and strain represent the departure of the material from linear behavior and are analogous to yield in metallic materials. For the two tests 
at $76 \mathrm{~K}$ that did not result in material failure at $90^{\circ}$ of rotation, the maximum values were calculated, and the shear strengths and strains are reported as "greater than" values.

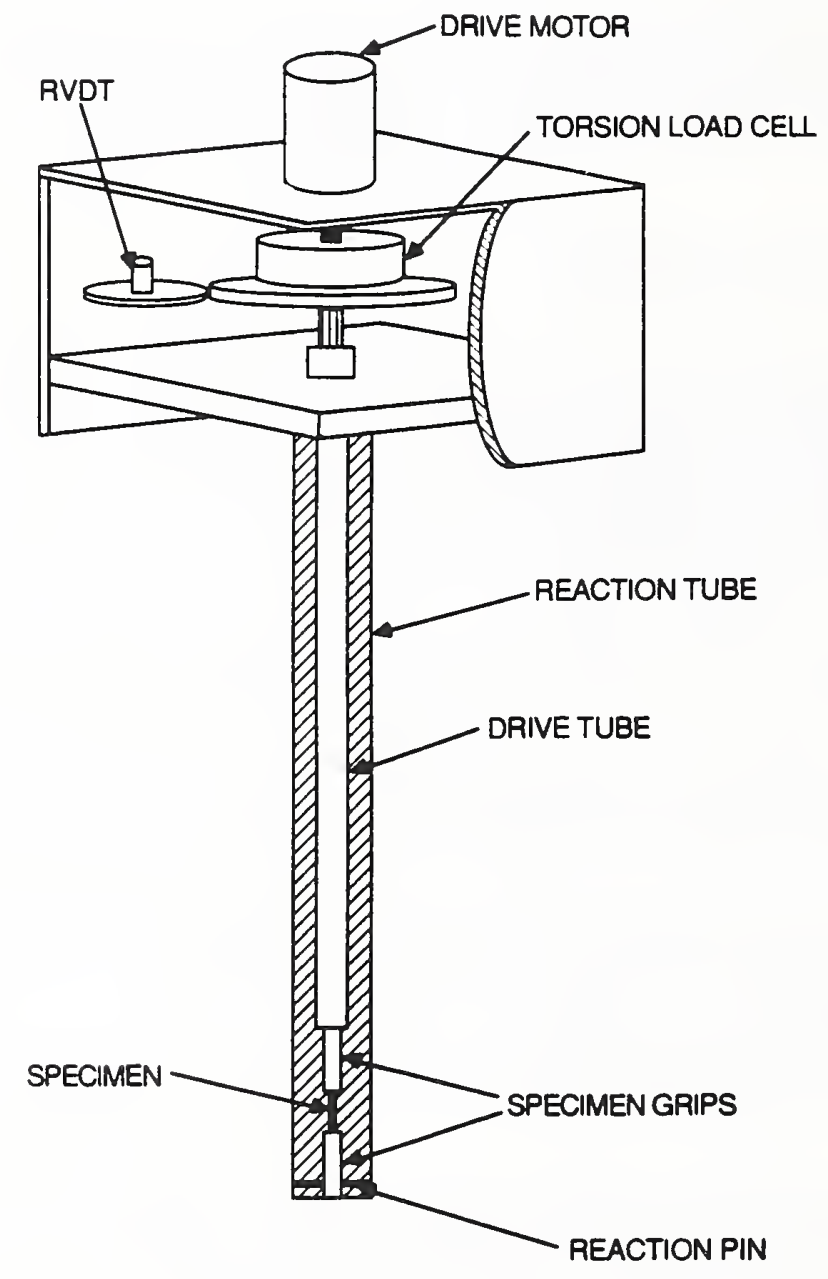

Figure 5.1. Strain-controlled torsion-shear test facility (cutaway view) optimized for rod-shaped specimens.

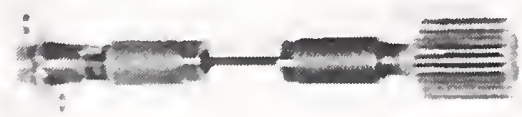

Figure 5.2. Torsion test specimens are held by collet grips in the reaction-tube assembly. 
The shear strength $(\tau)$ is calculated from

$$
\tau=\frac{T r}{l_{p}},
$$

where $T$ is the torque, $r$ is the specimen radius, and $I_{p}$ is the polar moment of inertia, which is equal to $\pi d^{4} / 32$ (where $d$ is the specimen diameter).

The shear modulus $(G)$ is found from

$$
G=\frac{T L}{\dot{\theta} I_{p}},
$$

where $L$ is the specimen gage length and $\dot{\theta}$ is the angular displacement in radians.

The shear strain $(\gamma)$ is related to $\dot{\theta}$ by

$$
\gamma=\frac{\dot{\theta} r}{L}
$$

and since $r=1.6 \mathrm{~mm}$ and $L=25.4 \mathrm{~mm}, \gamma=\dot{\theta} / 16$.

\subsection{Results}

The shear modulus $(G)$, shear strength $(\tau)$, and shear strain to failure $(\gamma)$ are presented in tabular form for each specimen of each resin system in Appendix B (§III.13). In tests conducted at room temperature, the specimens did not fracture. The data presented for room-temperature tests that are labeled "yield strength" and "strain" represent the shear strength and strain for the intersection point on the $\tau-\gamma$ curves of a $10^{\circ}$ rotational offset (from the linear elastic portion of the $\tau-\gamma$ curves) on the strain axis. Room-temperature values labeled "ultimate strength" and "strain" represent data taken at a full $90^{\circ}$ rotation and do not represent specimen failure data. Property data for epoxy, toughened-epoxy, cyanate-ester, ${ }^{*}$ and vinyl-ester plus polyester resin systems are plotted versus temperature in Figures 5.3 through 5.6, respectively.

The shear modulus has a strong dependence on temperature. For all epoxy, vinyl-ester, and polyester resin systems, $G$ is about a factor of 3 larger at $4 \mathrm{~K}$ than at $295 \mathrm{~K}$. For cyanateester systems, $G$ increases slightly less as the temperature is lowered and the ratio of 4-K to $295-\mathrm{K}$ data is about 2 . The filled and multifunctional epoxies and the filled vinyl-ester systems have higher values of $G$ at all temperatures.

At $76 \mathrm{~K}$, the shear-strength averages tend to be slightly higher than at $4 \mathrm{~K}$ for all resin systems except the cyanate esters. The cyanate esters have steadily increasing shear strengths at lower temperatures, but the magnitude of their strength is lower, in general, than that of the other resin systems. Notice, again, that the room-temperature strength data do not represent fracture strengths, but offset, yield-like strengths.

Shear strain to failure also decreases from 76 to $4 \mathrm{~K}$; this reflects either a slightly higher shear modulus, a lower shear strength, or both.

\footnotetext{
* See comments regarding cure schedule on page III-16.
} 


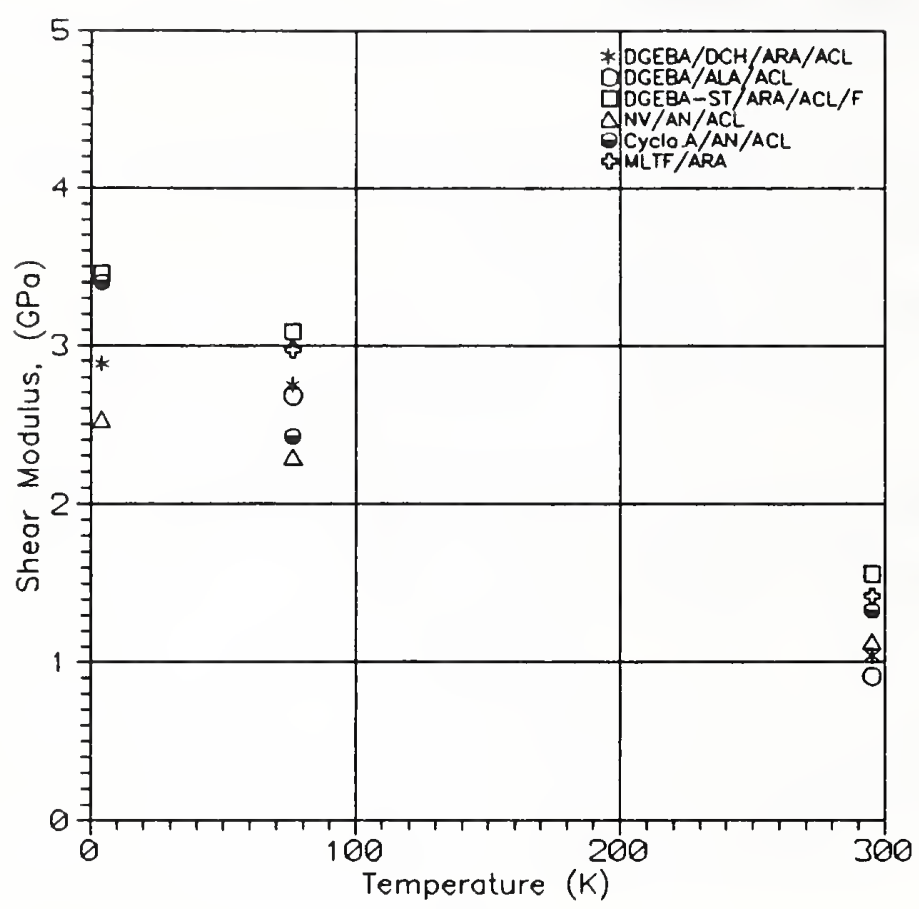

(a)

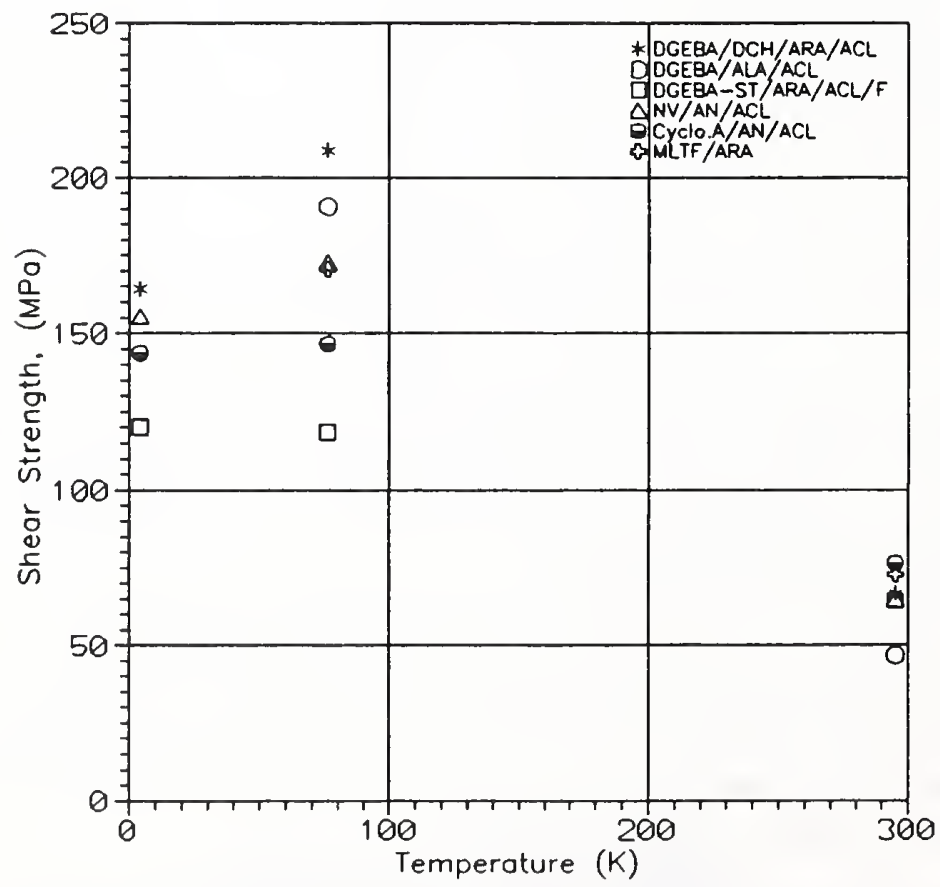

(b)

Figure 5.3. Epoxy-system properties: (a) shear modulus; (b) shear strength (continued on next page). 


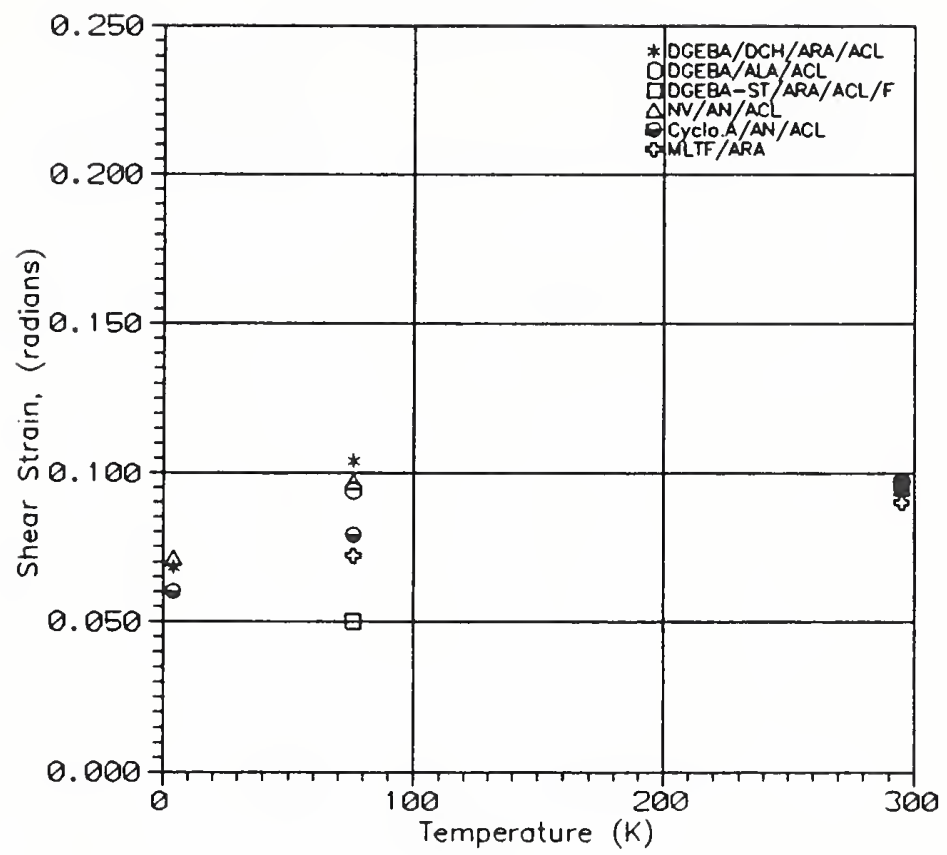

(c)

Figure 5.3. (continued). Epoxy-system properties: (c) shear strain versus temperature.

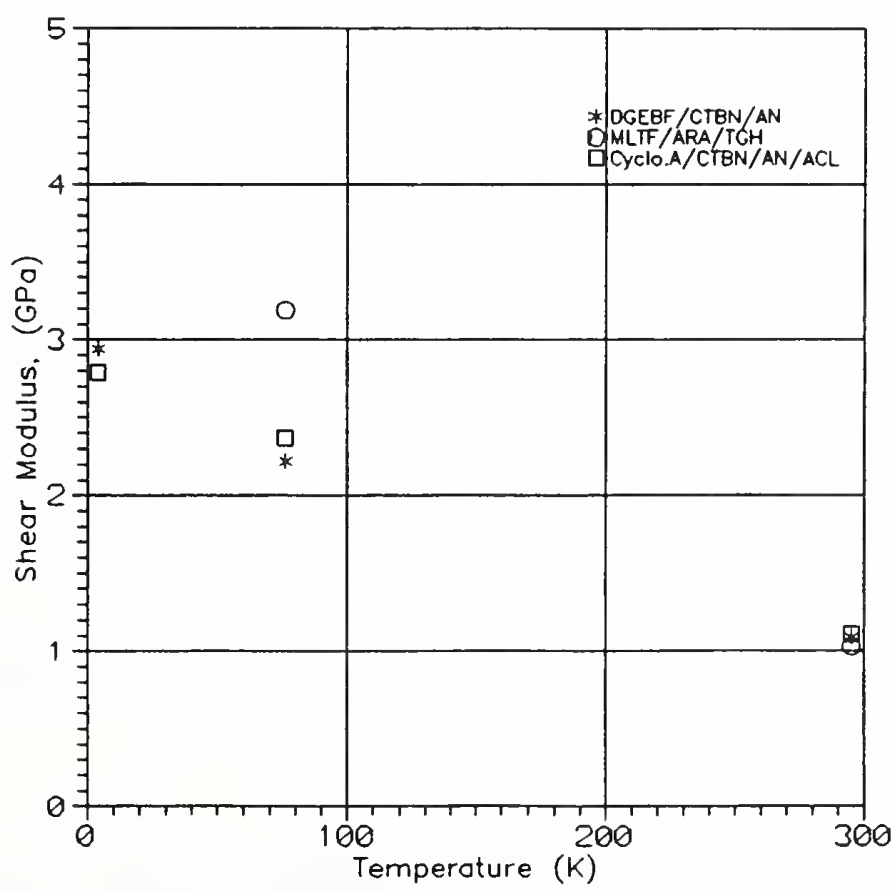

(a)

Figure 5.4. Toughened-epoxy-system properties: (a) shear modulus (continued on next page). 


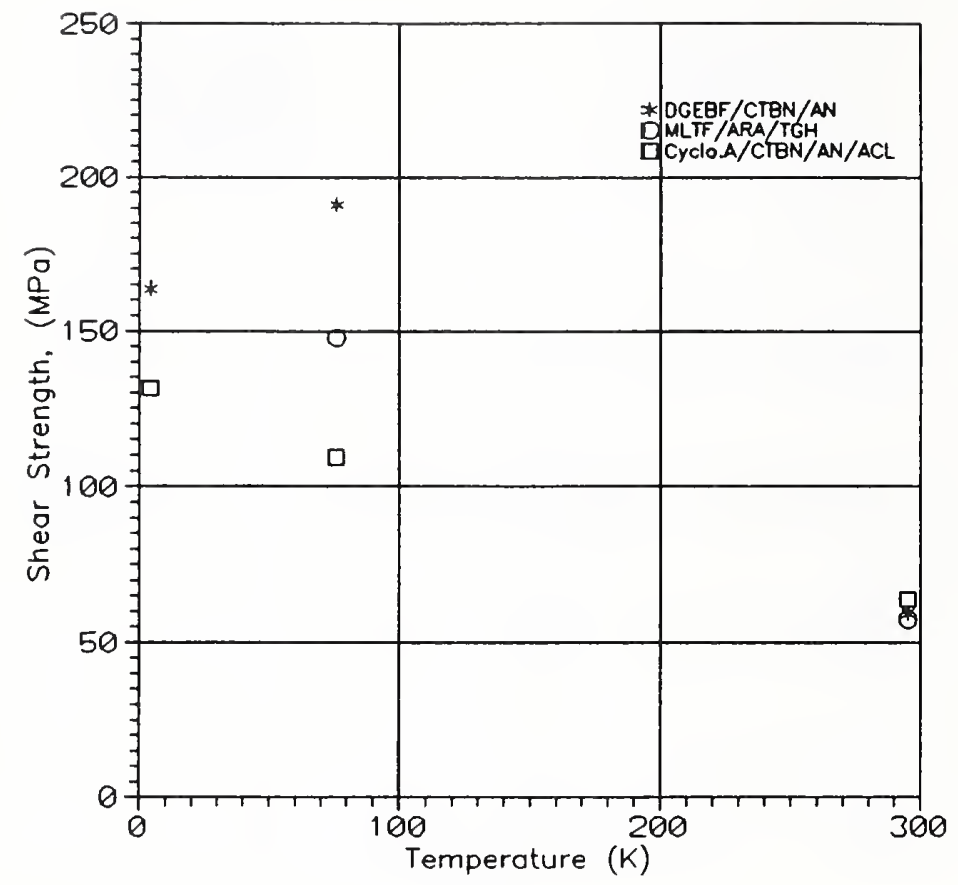

(b)

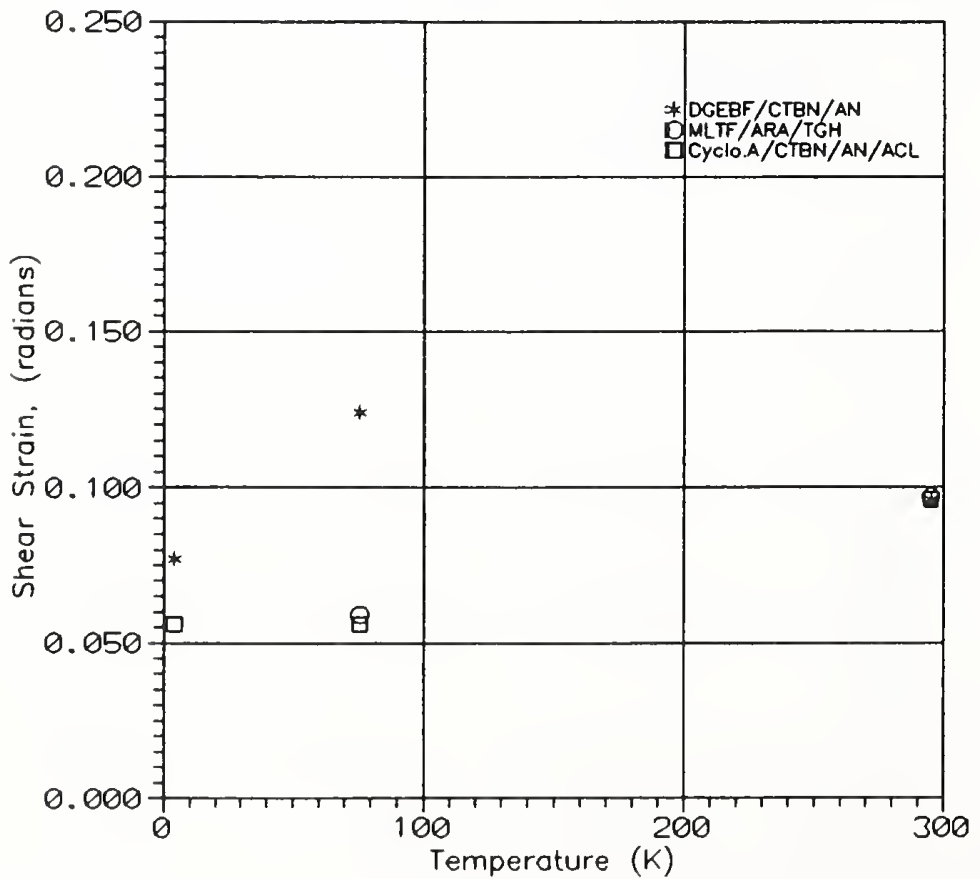

(c)

Figure 5.4. (continued). Toughened-epoxy-system properties: (b) shear strength; (c) shear strain versus temperature. 


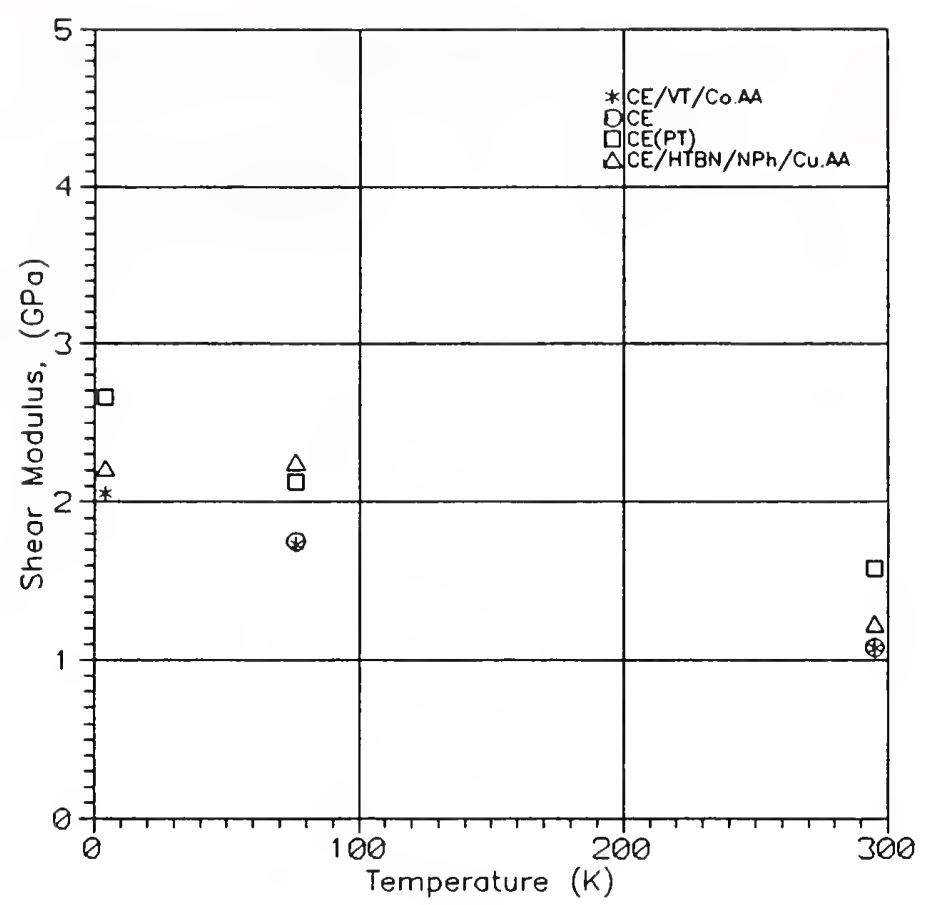

(a)

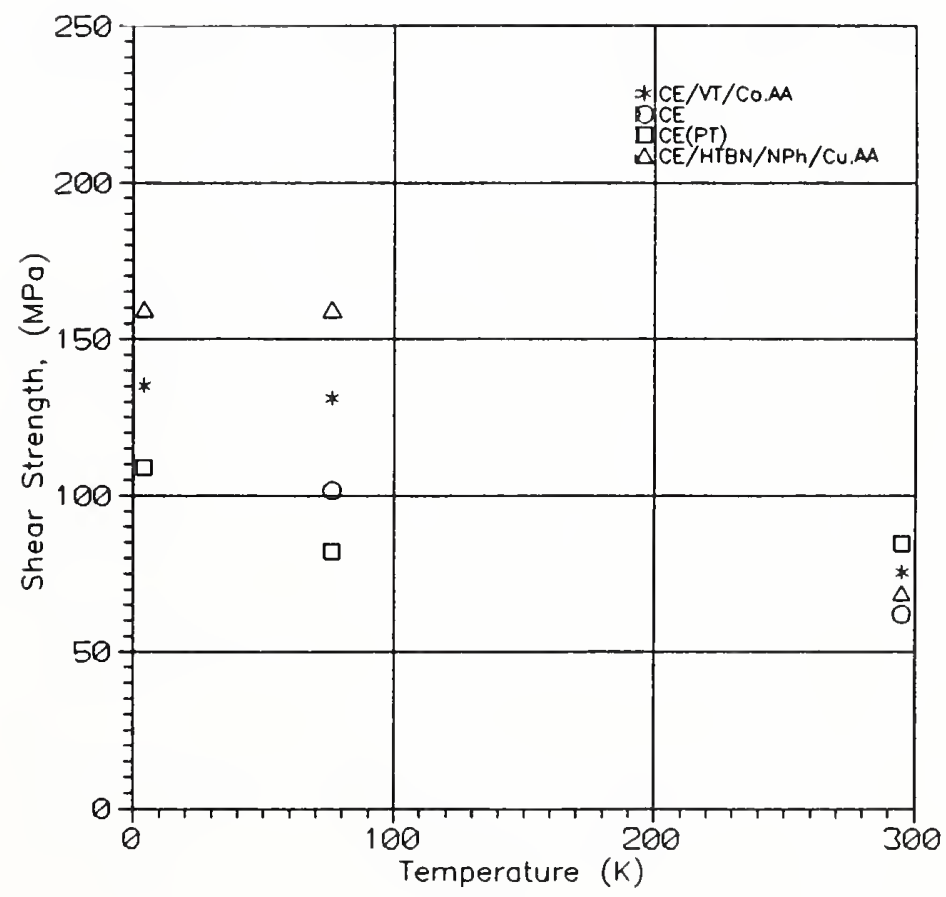

(b)

Figure 5.5. Cyanate-ester-system properties: (a) shear modulus; (b) shear strength (continued on next page). 


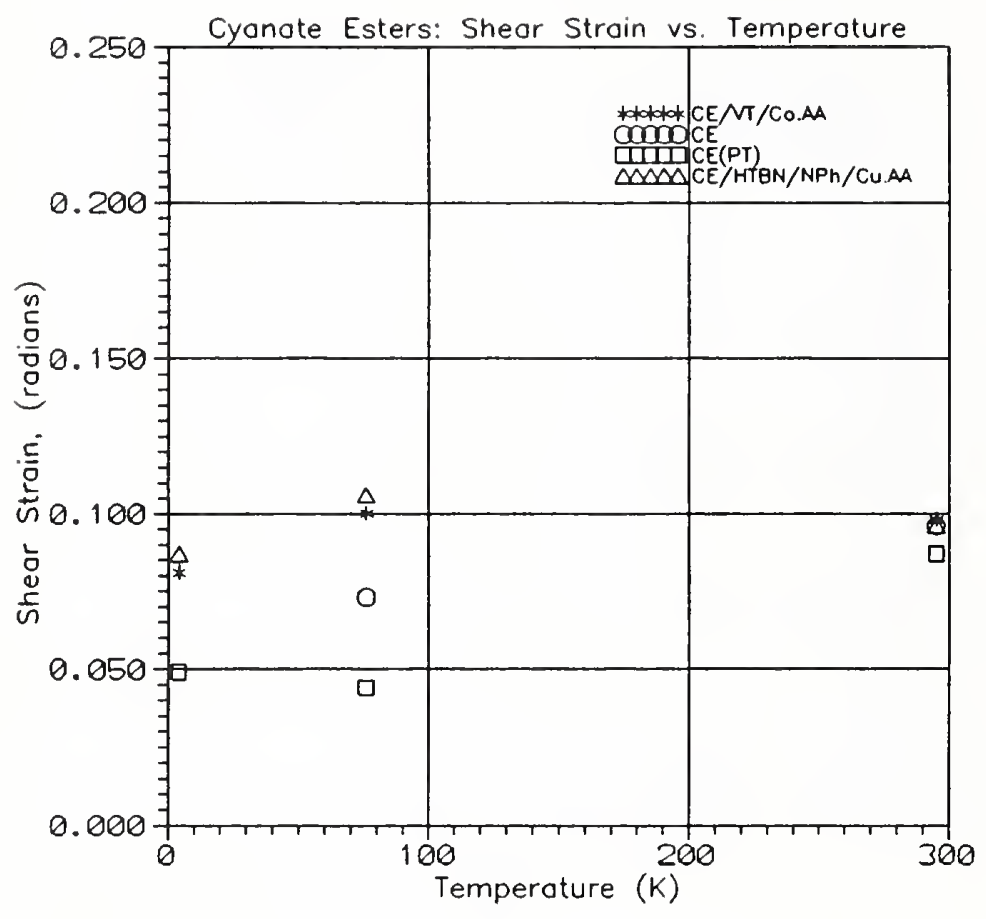

(c)

Figure 5.5 (continued). Cyanate-ester-system properties:

(c) shear strain versus temperature. 


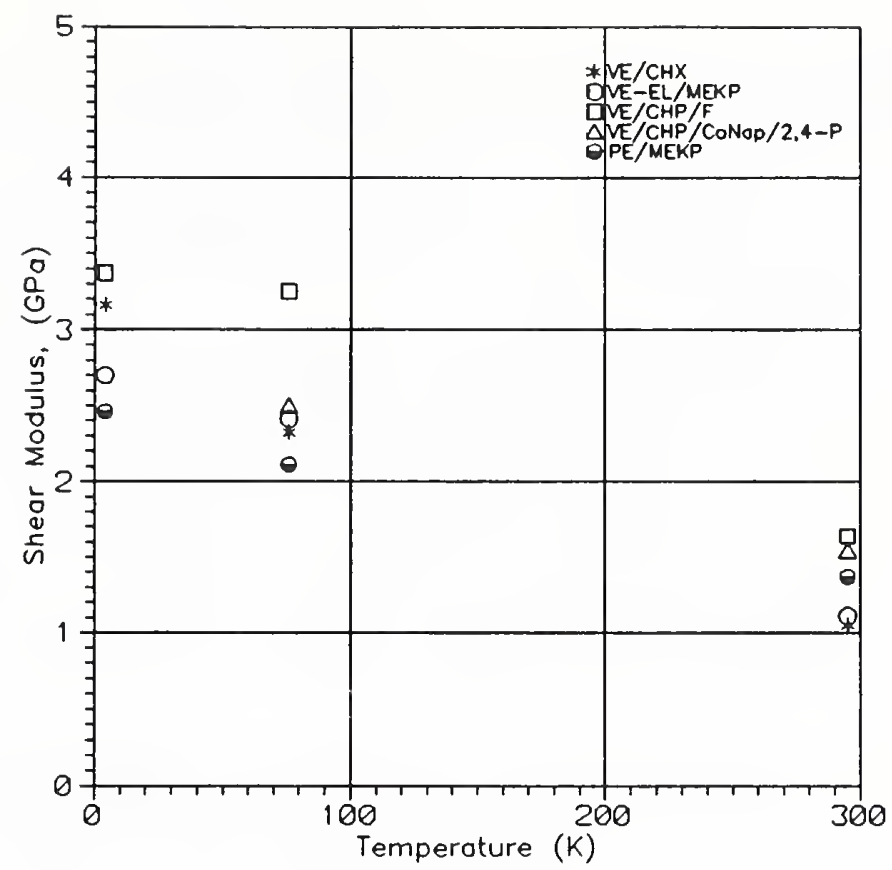

(a)

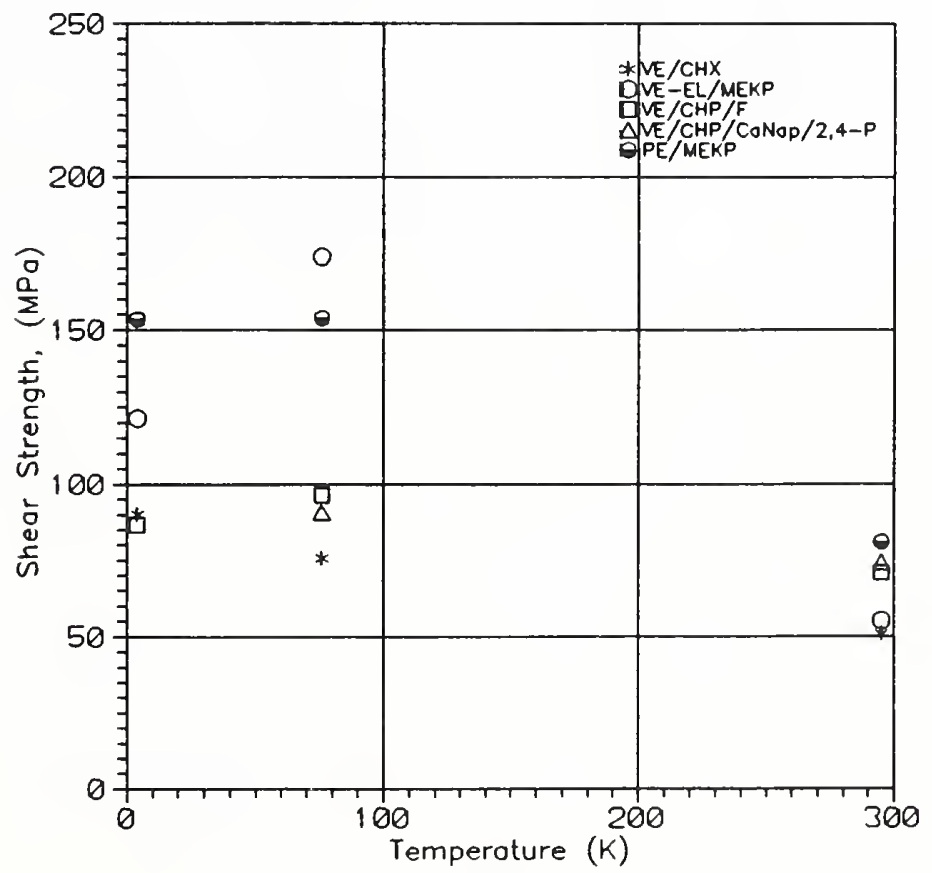

(b)

Figure 5.6. Vinyl-ester and polyester system properties: (a) shear modulus; (b) shear strength versus temperature. 


\subsection{Discussion}

From the results presented in Figures 5.3 through 5.6, several conclusions can be drawn regarding the relative performance of the resin systems ${ }^{*}$ in this program:

1. At low temperatures, the epoxies and vinyl esters had higher shear moduli than the cyanate esters and polyesters. Of the epoxies and vinyl esters, the filled and multifunctional resin systems had higher shear moduli.

2. At low temperatures, those resin systems with the highest shear strength at $76 \mathrm{~K}$ had reduced shear strength at $4 \mathrm{~K}$; conversely, those with the lowest strengths at $77 \mathrm{~K}$ had higher strengths at $4 \mathrm{~K}$. These temperature dependences reflect the sensitivity of the resin systems to surface or embedded flaws. Several epoxy systems had the highest shear strengths at low temperatures: the DGEBA/DCH/ARA/ACL, NV/AN/ACL, and DGEBA/ALA/ACL epoxy systems and the DGEBF/CTBN/AN toughened epoxy system.

3. Since most stress-strain curves at low temperatures are linear elastic, the shear-strain data usually reflect the relative strengths and moduli. Therefore, those systems that had the highest shear strengths usually had better shear strain to failure.

Plots of shear strength versus shear modulus provide a visual guide for relative strain to failure since the ratio of strength to modulus is the effective strain, assuming linear elastic loading conditions. In Figures 5.7 through 5.9, these plots are shown for epoxies, cyanate esters, and the polyester and vinyl esters, respectively. Typically, one sees a probable linear dependence between $\tau$ and $\mathrm{G}$ from 295 to $76 \mathrm{~K}$ and a random falling off of $\tau$ in those resin systems that are more notch (flaw) sensitive at low temperatures. All resin systems that have higher shear moduli at low temperatures (including filled and multifunctional epoxies) have relatively lower values of shear strength at low temperature. To construct these plots, the three highest strength data points were used; this is similar to the procedure used for some presentations of tensile data. Thus, the averages presented in Figures 5.7 through 5.9 are slightly higher than those presented in Figures 5.3 through 5.6. Clearly, the epoxy systems have better low-temperature properties. Of the other resin systems, the PE/MEKP polyester, VE-EL/MEKP vinyl-ester, and CE/HTBN/NPh/CuAA cyanate-ester systems have promise for low-temperature use.

\footnotetext{
* See comments regarding cure schedule on page III-16.
} 


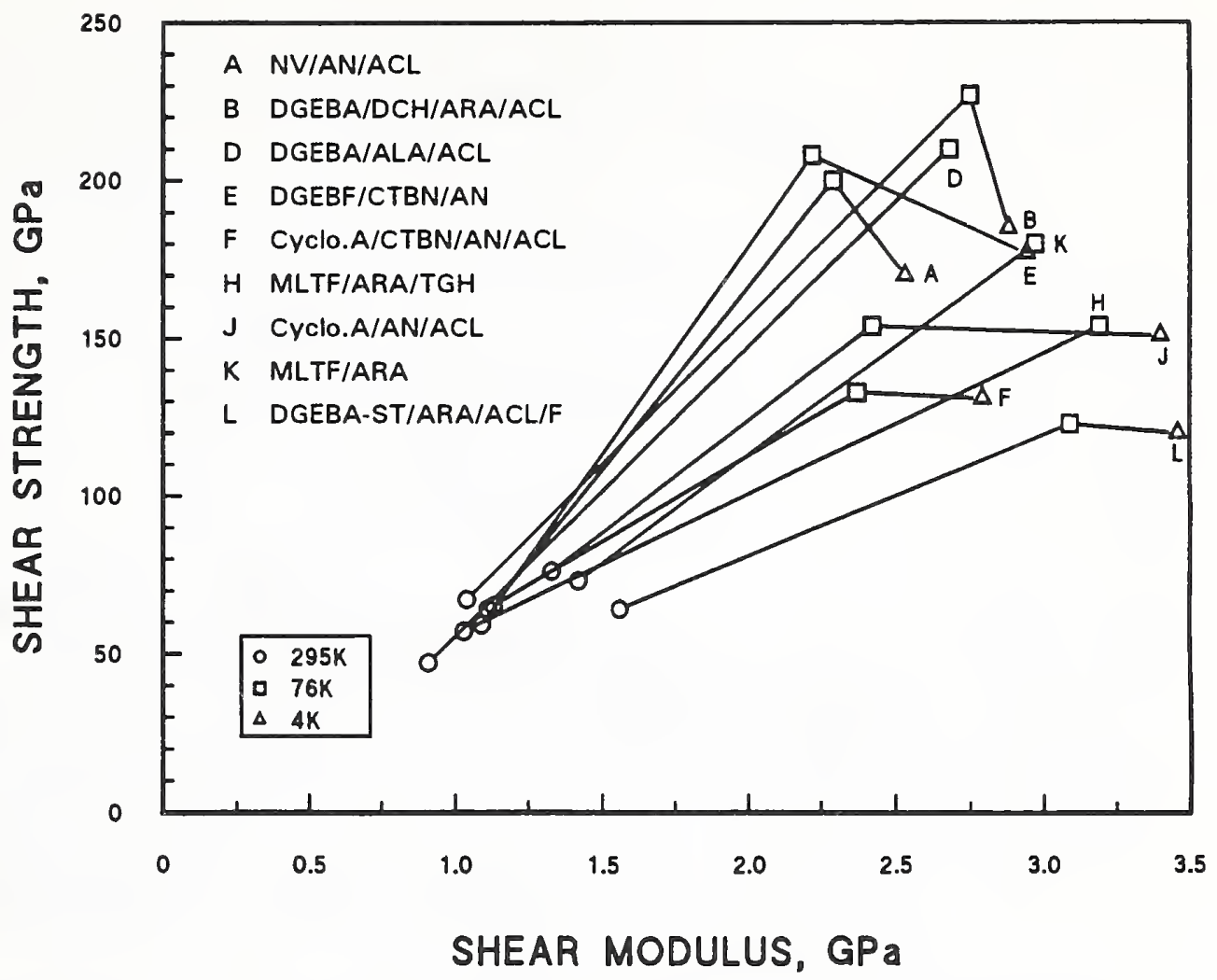

Figure 5.7. For epoxy-resin systems, shear strength versus shear modulus.

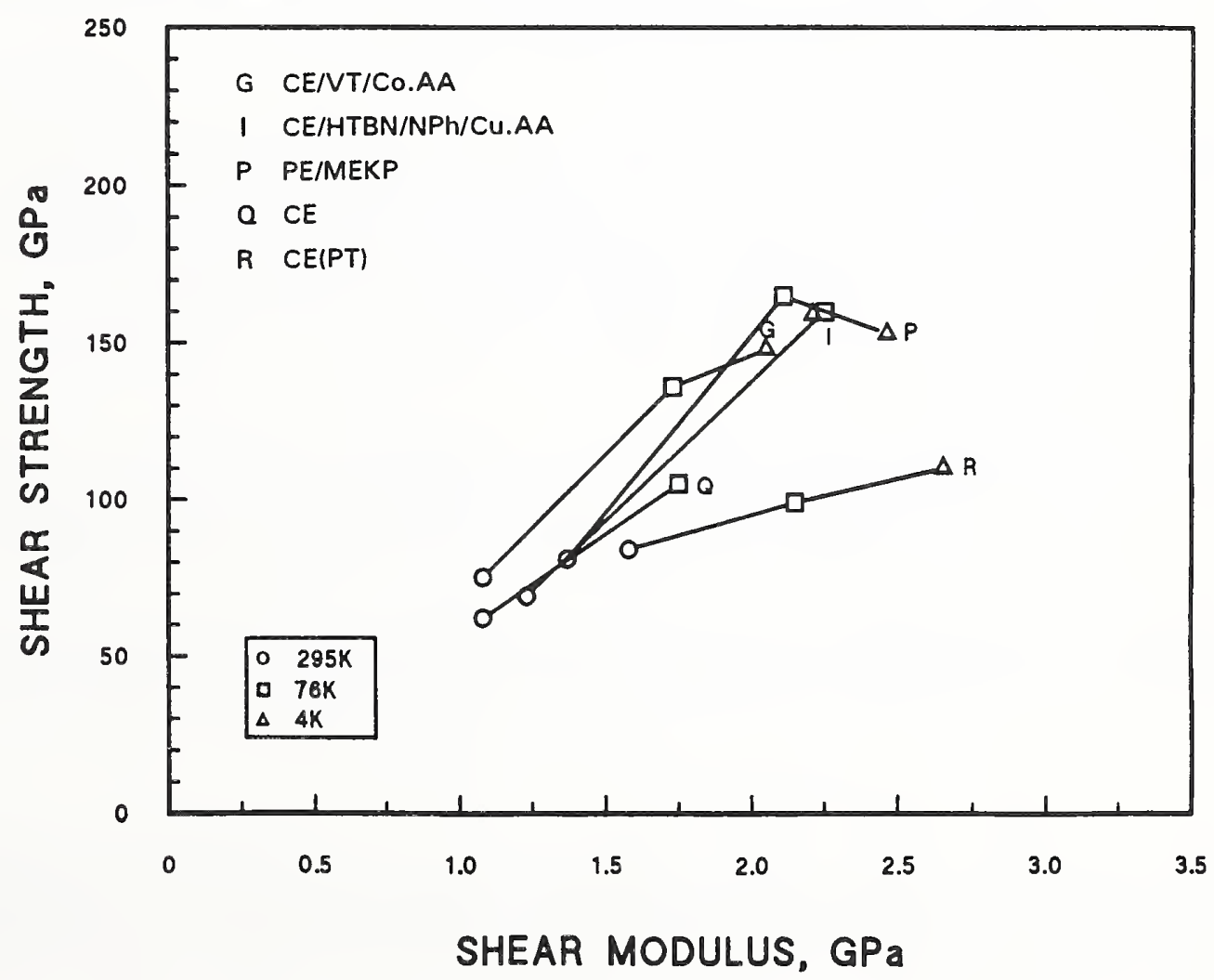

Figure 5.8. For cyanate-ester and polyester systems, shear strength versus shear modulus. 


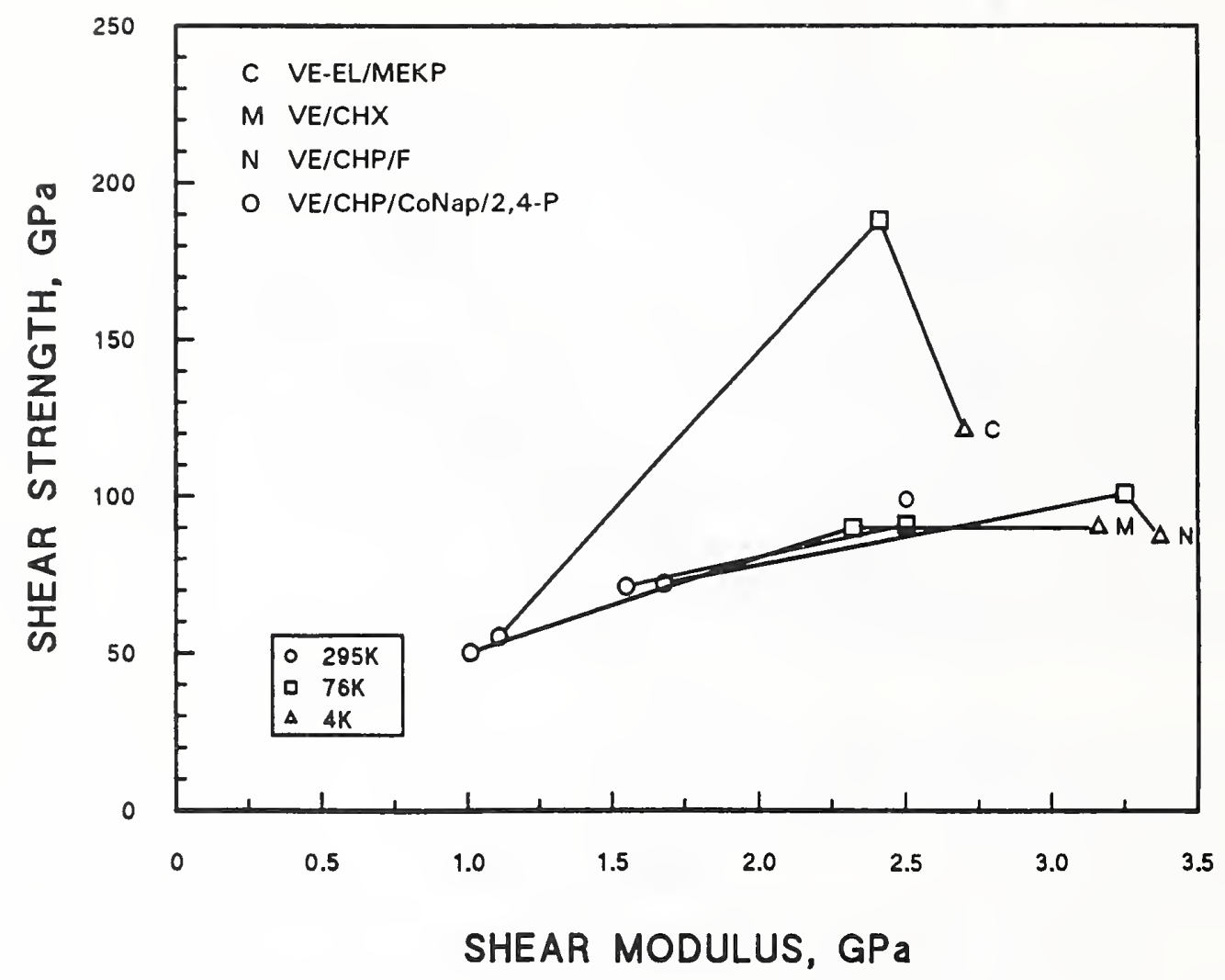

Figure 5.9. For vinyl-ester systems, shear strength versus shear modulus. 


\title{
6. Thermal Contraction
}

\author{
R.P. Reed ${ }^{*}$ \\ R.P. Walsh \\ National Institute of Standards and Technology \\ Boulder, Colorado \\ ${ }^{*}$ Cryogenic Materials, Inc. \\ Boulder, Colorado
}

\begin{abstract}
The thermal contraction of 15 different neat-resin systems, on cooling from room temperature to $4 \mathrm{~K}$, was measured with strain gages. Epoxy-, vinyl-ester-, polyester-, and cyanate-ester-based systems were studied. In general, the vinyl esters and polyesters contracted more than the epoxies during cool-down. Filled resin systems and tetrafunctional epoxy systems contracted less on cooling. A very general correlation for neat-resin systems was found between (increasing) elastic moduli and (decreasing) thermal contraction during cooling.
\end{abstract}

To the best of our knowledge, this study represents the first time that the thermal contractions of these resin systems have been measured at low temperatures. The use of strain gages to measure thermal contraction at low temperatures has been shown to produce reliable data.

\subsection{Introduction}

The amount of contraction of the resin on cooling to low temperatures affects the residual stress of the composite and contributes to the overall length change of a strut system. Thermal contraction is an essential parameter for analytic models. Surprisingly, as discussed in $\$ 6.2$, the thermal contraction of only a few epoxy resins has been measured to $4 \mathrm{~K}$, and there is no information on the contraction characteristics at low temperatures of vinyl-ester-, polyester-, and cyanate-ester-based resin systems.

Measurements from room temperature to boiling liquid-helium temperature $(4 \mathrm{~K})$ were conducted on selected epoxy, vinyl-ester, polyester, and cyanate-ester neat resins. Neat resins that are toughened and filled were also included to assess the influence of the additions.

We measured the thermal contraction of 15 different neat resins with a unique, relatively inexpensive technique that uses strain gages to measure the change in specimen length. The technique is also very versatile; it can be used to measure specimens of different shapes.

\subsection{Review of Literature}

The thermal contraction of a limited number of epoxies has been measured from room temperature to $4 \mathrm{~K}[6.1-6.5]$. The data from these studies are given in Table 6.1 in percent thermal 
contraction, $\left\{\left(L_{\mathrm{RT}}-L_{4}\right) / L_{\mathrm{RT}}\right\} \times 100$, where $L_{\mathrm{RT}}$ is the original specimen length at room temperature, and $L_{4}$ is the specimen length at $4 \mathrm{~K}$. For unfilled epoxies, the reported values range from about 1.1 to $1.5 \%$. Filled resins have less contraction on cooling; for example, with $40 \%$ filler, the contraction is about one-half that of the unfilled epoxy system [6.1-6.3]. The disparity among the data in Table 6.1 is rather large, and none of the reports specify the components of the epoxy systems; thus, it is not possible to differentiate between measurement errors and material differences. A large difference, $1.5 \%$ compared to $1.1 \%$, was observed $[6.4,6.5]$ for data on flexible epoxies. Another feature of the data found in the literature is the large variability, typically \pm 0.2 to $\pm 0.4 \%$, of values for similar resin systems.

Table 6.1. Percent thermal contraction of resins at low temperatures

\begin{tabular}{lcl}
\hline \multicolumn{1}{c}{ Resin } & $\frac{L_{\mathrm{RT}}-L_{4}}{L_{\mathrm{RT}}} \times 100$ & Source \\
\hline \hline Various unfilled epoxies & $1.4 \pm 0.4$ & M. Van de Voorde [6.1] \\
Filled epoxies & $0.5 \pm 0.2$ & M. Van de Voorde [6.1] \\
Shell linear epoxy R & 1.25 & M. Van de Voorde [6.1] \\
with 20 vol\% quartz powder & 0.75 & \\
with 40 vol\% quartz powder & 0.62 & \\
Epoxy resins & $1.2 \pm 0.2$ & G. Hartwig [6.2, 6.3] \\
with 20 vol\% filler & \\
with 40 vol\% filler & & \\
Flexibilized epoxy & $0.9 \pm 0.1$ & \\
Rigid epoxy & $0.65 \pm 0.05$ & H. Hacker et al. [6.4] \\
Semiflexible epoxy & 1.09 & D. Evans and J. T. Morgan [6.5] \\
\hline \hline
\end{tabular}

*includes quartz, zirconium silicate, or aluminum oxide

The effects of fillers in epoxy on the low-temperature thermal contraction were studied extensively by Hartwig [6.3]. Quartz, zirconium silicate, aluminum oxide, and glass spheres with particle diameters ranging from 5 to $1000 \mu \mathrm{m}$ were used in the study. Data $(\Delta L / L)$ from 300 to $4 \mathrm{~K}$ were presented as a function of volume percent of filler. Data for all filler types and particle sizes fell between a linear mixing dependence and a nonlinear dependence that also accounts for the thermal stress distribution between the spherical glass particles and resin. In these mixtures, the following linear dependence holds: $(\Delta L / L)_{\text {filled }} /(\Delta L / L)_{\text {unfilled }} \approx 1-V_{f}$, where $\Delta L$ is the change in length between 295 and $4 \mathrm{~K}$ and $V_{f}$ is the volume fraction of filler. Average values of the data taken from this study for 20 and 40 vol\% fillers are listed in Table 6.1 .

Hartwig pointed out that most low-temperature polymer properties are determined by intrachain interactions, in contrast with the high-temperature properties that are affected by interchain interactions [6.2]. He studied the effects of polymer-chain cross-link distance on low-temperature thermal expansion. For those studies, special resins (MY990, Araldite B, A6084, and A6097) with a 
hexyhydrophthalic acid, anhydride hardener supplied by Ciba-Gigy were chosen. Thermal contraction (from 100 to $15 \mathrm{~K}$ ) increased as the cross-link distance increased from about 1.5 to $10 \mathrm{~nm}$. At $15 \mathrm{~K}$, the thermal-expansion coefficient increased by about a factor of 3 when the cross-link distance changed from 10 to $1.5 \mathrm{~nm}$.

\subsection{Resins}

Epoxy, vinyl-ester, polyester, and cyanate-ester resin systems were studied, including the DGEBA, DGEBF, novolac, and cycloaliphatic type of epoxies. Resin systems were also chosen to assess the effects of toughness and fillers. Both filament winding and pultrusion systems were included. Table 2.1 of $\$ I I I .2$ summarizes the resin systems that were included in this program.

\subsection{Procedures}

Strain gages were used to sense the change of length of neat-resin specimens during cooldown to boiling liquid-helium temperature $(4 \mathrm{~K})$ and warm-up to room temperature $(293 \mathrm{~K})$. This technique has been previously described [6.6-6.8] and used at low temperatures [6.9].

In our studies, strain gages were bonded to each side of neat-resin plate specimens, $3 \mathrm{~mm}$ (0.125 in) thick. A silicon-diode temperature sensor was also bonded to one side of each specimen. Temperature was controlled by positioning the specimen at various heights above boiling liquid helium, which was in a long stainless steel Dewar. Data were recorded during cool-down and warmup. A schematic of this technique is shown in Figure 6.1.

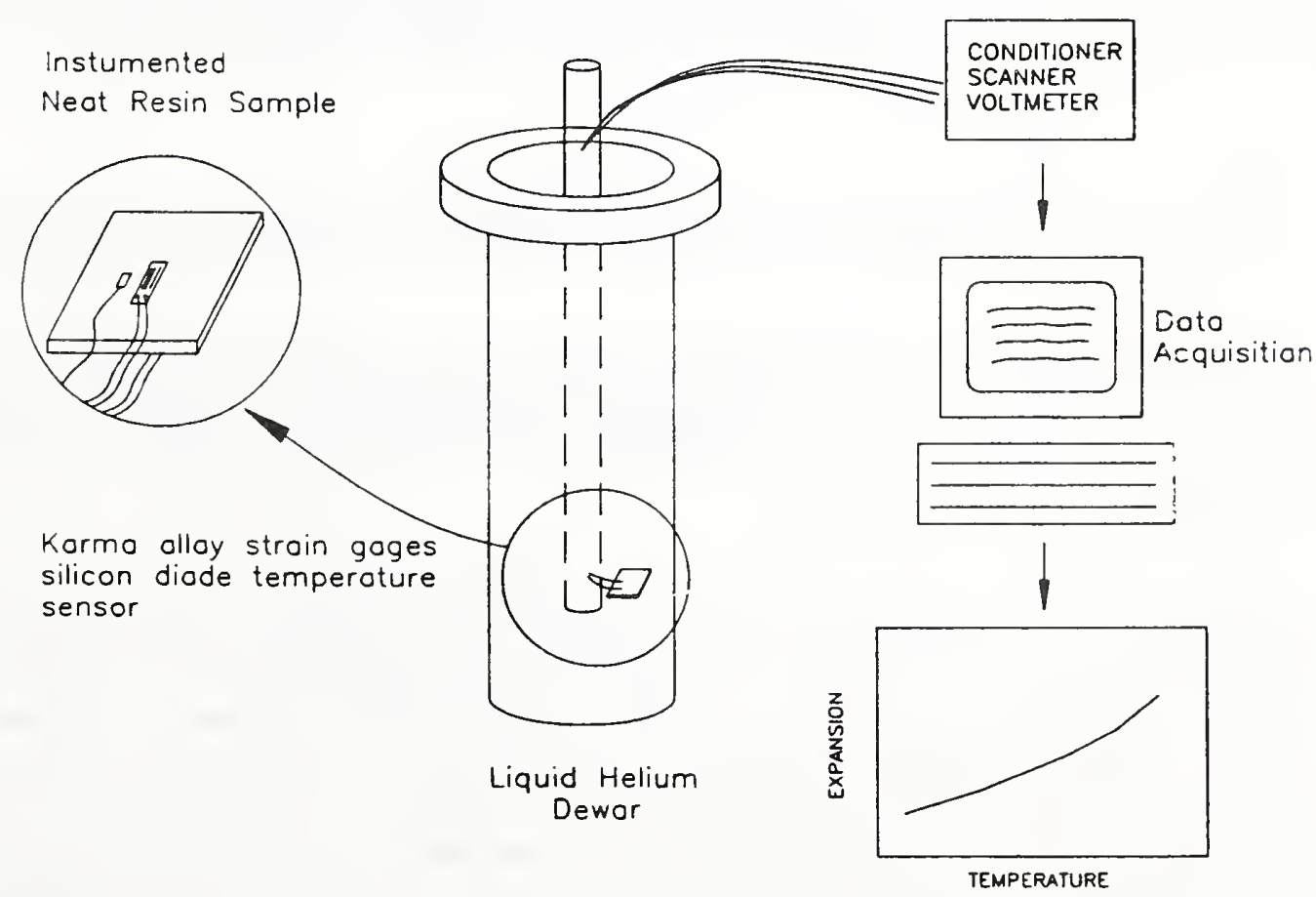

Figure 6.1. Thermal-contraction measurement technique. 


\subsubsection{Description of Equipment}

The gages used for these measurements were commercially available nickel-chromium-foil resistance gages. All the gages were from the same production lot and had $350-\Omega$ resistance and a 3.2-mm active gage length.

The adhesive was a two-component epoxy resin that is commercially available. When properly cured, the adhesive is useful at temperatures ranging from 4 to $300 \mathrm{~K}$. The adhesive was cured at $328 \mathrm{~K}\left(65^{\circ} \mathrm{C}\right)$ for $2 \mathrm{~h}$ under clamping pressure.

The lead wires were 0.255 -mm-diameter, stranded, silver-plated copper with Teflon insulation. Each gage was wired with a 2.2-m-long, braided, shielded cable containing three wires. The wires were connected to the strain-gage lead wires with a $63 \% \mathrm{Sn}-37 \% \mathrm{~Pb}$ resin-core solder.

The electronic equipment used to monitor the strain gages was a commercially available multichannel system with constant bridge excitation voltage and internal bridge completion circuitry. This system has a resolution specification of $1 \mu \epsilon$.

Temperature was measured with a silicon diode sensor and a commercial digital temperature controller. A layer of thermally conductive grease was used to ensure thermal contact between the sensor and the specimen. A thin layer of foam was used to insulate the sensor from the surrounding Dewar environment. The sensor was placed on the specimen as close to the strain gages as possible.

An automated computer system was used for data acquisition. The system consisted of a desktop computer equipped with an IEEE 488 interface card, a digital voltmeter, and 20-channel scanner in combination with an IEEE 488 bus. A computer code was written to scan two channels and also to read the temperature measured by the digital temperature controller. The program was capable of recording the data at regular time intervals or at intermittent times selected by the user.

\subsubsection{Methods}

The strain gages were applied to the specimens in the manner prescribed by the manufacturer for the epoxy adhesive.

The gages were electrically connected to the indicator as a fourth bridge element. A standard three-lead-wire configuration was used to desensitize the effect of lead-wire resistance. The excitation voltage used for all measurements was a constant $1.00 \mathrm{Vdc}$. For these measurements, the roomtemperature gage factor of 2.05 was used.

Single-specimen tests were conducted by hanging the test plate from a wooden dowel that could be used to lower the specimen into the test Dewar. The strain indicators were balanced at room temperature $(295 \mathrm{~K})$ to give a zero microstrain read-out and zero analog output. The test specimen was thermally cycled from room temperature $(295 \mathrm{~K})$ to liquid-helium temperature $(4 \mathrm{~K})$ three times before the start of the measurements. After ensuring that all gages were performing properly and that the strain-gage signal returned to zero at $295 \mathrm{~K}$, the test was started. The specimen was slowly cooled $(\sim 1 \mathrm{~h})$ from 295 to $76 \mathrm{~K}$ by lowering it into a Dewar containing liquid nitrogen. Data were taken during the cool-down with the automated computer setup described previously. The specimen was cooled close to liquid-nitrogen temperature $(76 \mathrm{~K})$ and then immersed in the liquid to obtain the 
apparent strain data at $76 \mathrm{~K}$. It was cooled from 76 to $4 \mathrm{~K}$ by slowly lowering the test plate into a Dewar containing a small quantity of liquid helium. The specimen was immersed in liquid helium at atmospheric pressure to obtain the lowest temperature (4-K) datum point. The specimen was then raised out of the liquid and suspended above it. The system was allowed to warm naturally while data were recorded at regular time intervals of $15 \mathrm{~min}$. Thus, the thermal output of the strain gages was monitored during cool-down and warm-up of the test plates.

\subsubsection{Calibration}

When low-temperature strain gages are mounted on a stress-free specimen and the temperature is changed, the output (defined as change of resistance per unit strain) of the gages is complex and not linear with temperature. This temperature-dependent gage effect must be accounted for when using strain gages in a variable-temperature environment. Measured strains that have not been corrected for this gage effect are commonly referred to as apparent strains.

The gage effect used in the analysis of the following data for thermal expansion of neat resins was computed by comparing observed values to reference values of strain for OFHC copper [6.10]. Observed values (apparent strain) are given in Figure 6.2(a); reference values are given in Figure 2.6(b). The computed gage effect is shown in Figure 6.2(c). Three independent tests, using three different OFHC specimens and three different strain gages, were used to determine the gage effect.

The contraction of a DGEBA/DCH/ARA/ACL epoxy resin was also measured [6.11] by using the quartz-tube dilatometer technique [6.12]; the data from both techniques are presented in Figure 6.3(a) (strain gage) and 6.3(b) (quartz-tube dilatometer). Comparisons of these data suggest that the strain-gage method is valid for determination of the thermal contraction of neat resins in the cryogenic temperature range.

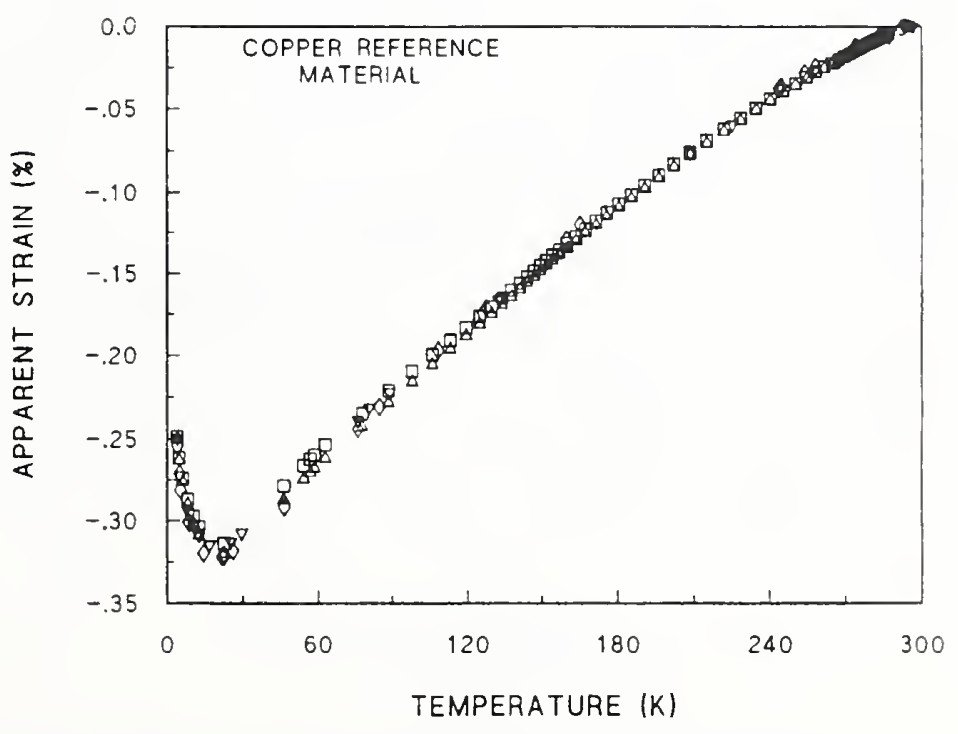

(a)

Figure 6.2. (a) Output of strain gages on OFHC copper specimens (continued on next page). 


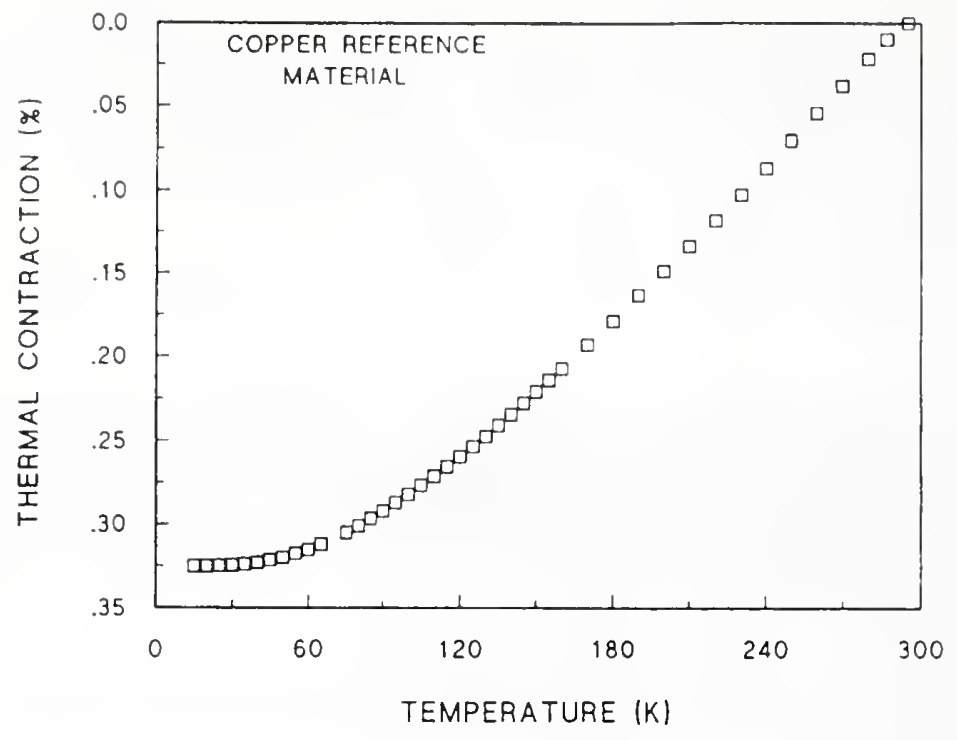

(b)

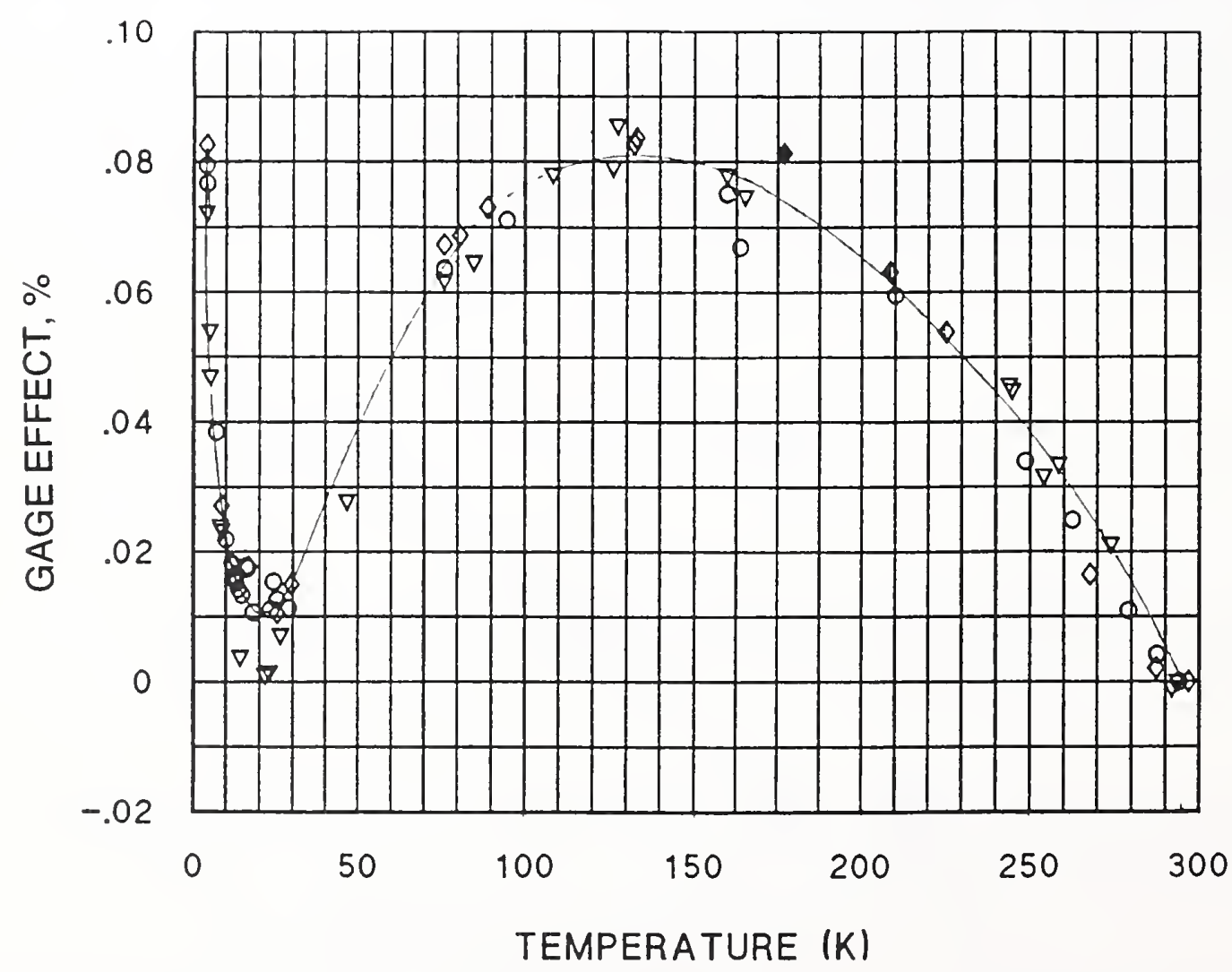

(c)

Figure 6.2. (continued). (b) Reference data for thermal contraction of OFHC copper; (c) computed gage effect. 


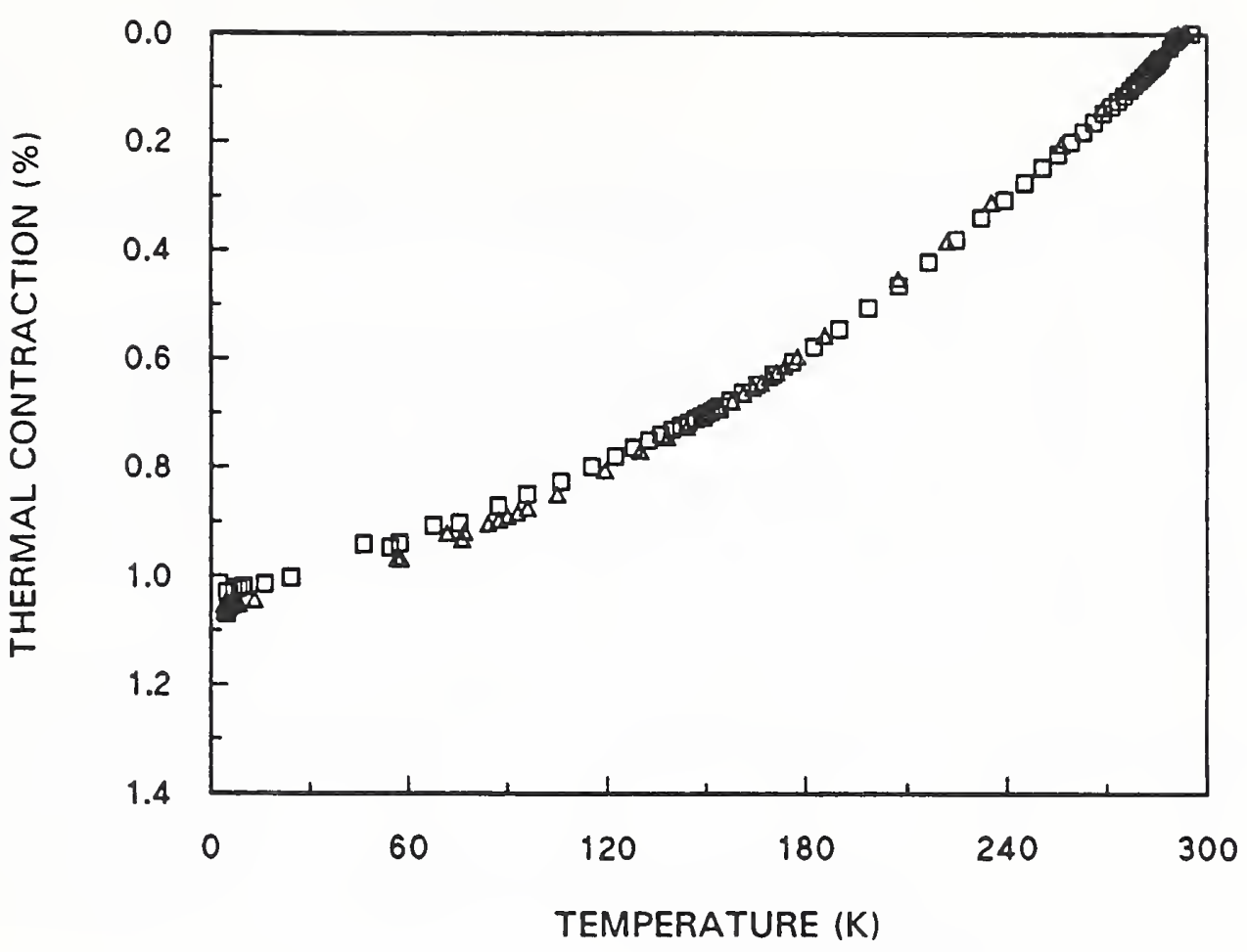

(b)

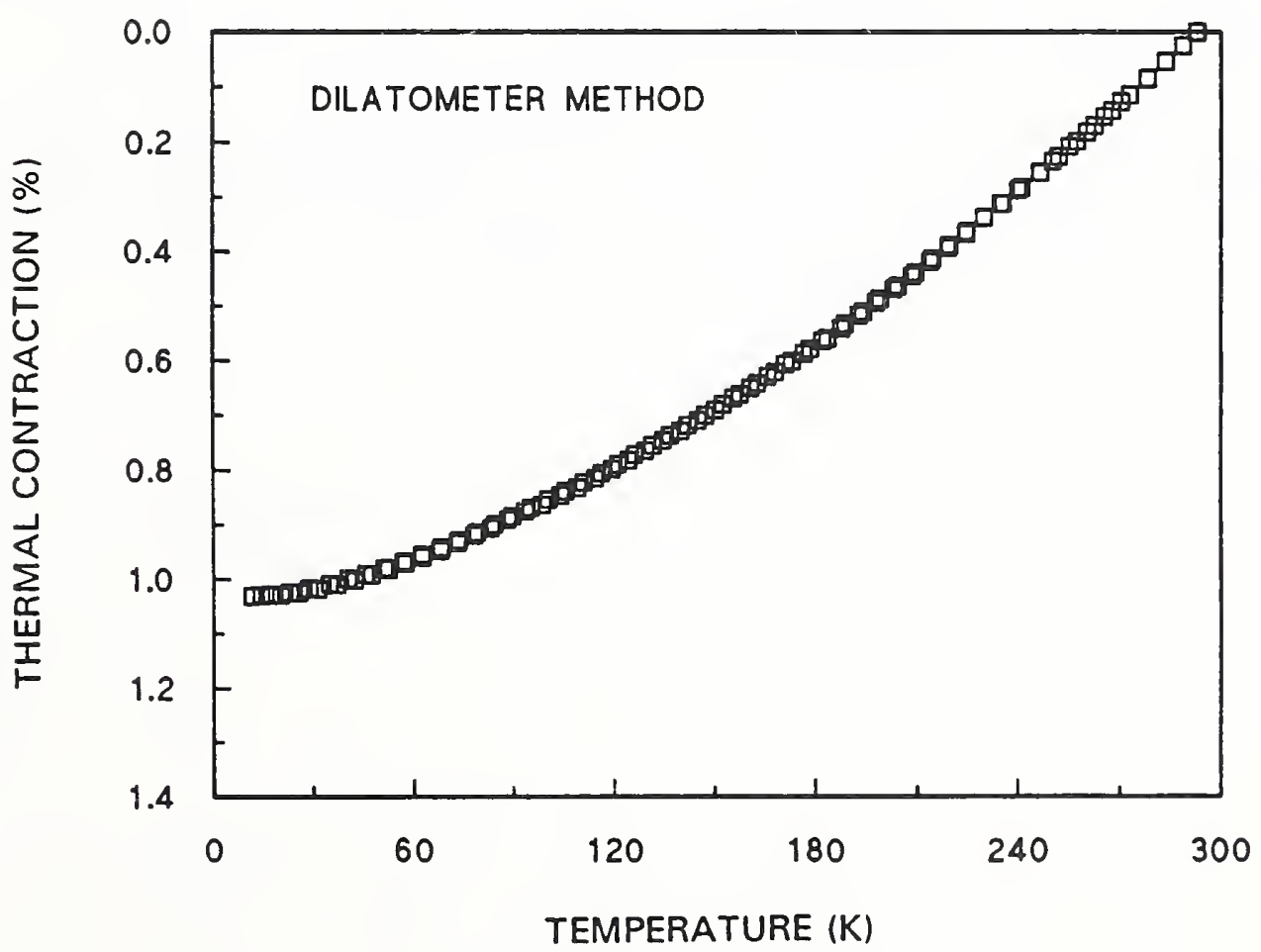

Figure 6.3. Thermal contraction of DGEBA/DCH/ARA/ACL epoxy resin measured with a strain gage (a) and with a quartz-tube dilatometer (b). 


\subsection{Results}

The thermal contraction data are presented as the percent contraction on cooling: $\left\{\left(L_{293 \mathrm{~K}}-\right.\right.$ $\left.\left.L_{T}\right) / L_{293 \mathrm{~K}}\right\} \times 100$. Here $L_{293 \mathrm{~K}}$ is the length at $293 \mathrm{~K}$ and $L_{T}$ is the length at temperature $T$; length refers to the effective gage length of the strain gages.

The epoxy-base resin data are presented in Figures 6.4 through 6.10. The percent contraction is dependent on the type of epoxy system. The DGEBA-based systems contract between 0.86 and $1.15 \%$ on cooling to $4 \mathrm{~K}$ (Figs. 6.4 and 6.5 ). Toughened-epoxy systems contract more (1.2 and $1.35 \%$, Figs. 6.6 and 6.9). The tetrafunctional epoxy system (Fig. 6.10) contracts much less $(0.80 \%)$. The contraction of the novolak epoxy system (Fig. 6.7) is similar to the DGEBA epoxy systems. Adding filler to an epoxy-base system decreases the contraction, as is shown in Figure 6.5 for a DGEBA-base system.

The thermal contractions of cyanate-ester-based systems ${ }^{*}$ are presented in Figures 6.11 through 6.14. They contract approximately the same as DGEBA and novolac epoxy systems $(0.97$ to $1.15 \%)$.

The vinyl-ester data are shown in Figures 6.15 through 6.17. The unfilled systems contract more than the epoxy and cyanate-ester systems (1.25 to $1.30 \%$, Figs. $6.15,6.16)$. The filled system, shown in Figure 6.18, follows the same trend shown by the filled epoxy system (Fig. 6.5), contracting much less $(1.00 \%)$ than the unfilled systems.

The contraction of the polyester-base system is shown in Figure 6.18. The thermal contraction of this system is very similar to that of the vinyl esters.

Several of the data sets shown in Figures 6.4 through 6.18 contain deviations from smooth and continuous behavior, e.g., Figures 6.14 and 6.15 in the temperature range 60 to $90 \mathrm{~K}$. These deviations are not of engineering significance, and their cause was not determined. Either phase changes or experimental procedures could produce similar results.

* See comments regarding cure schedule on page III-16. 


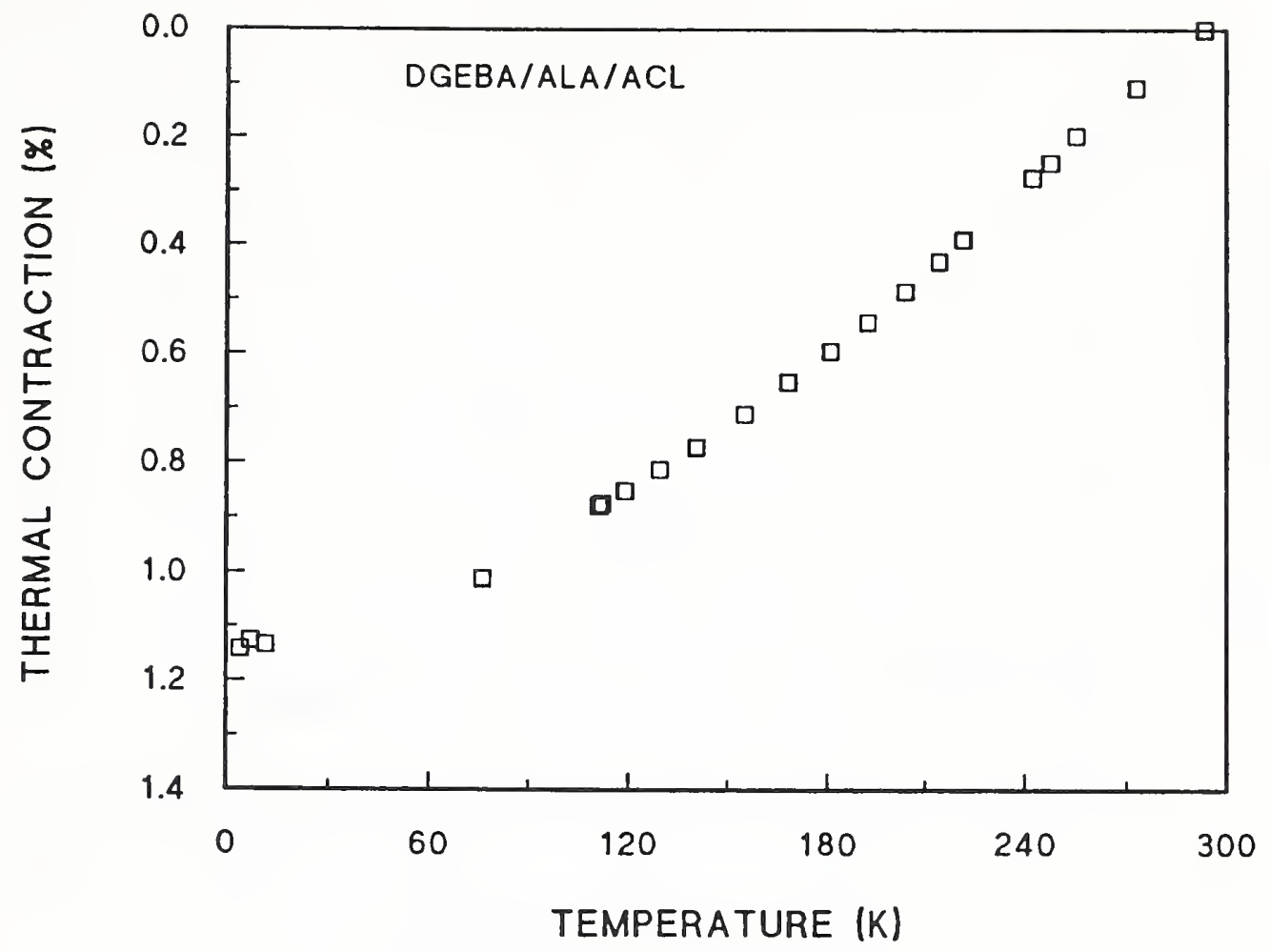

Figure 6.4. Thermal contraction of DGEBA/ARA/ACL epoxy resin.

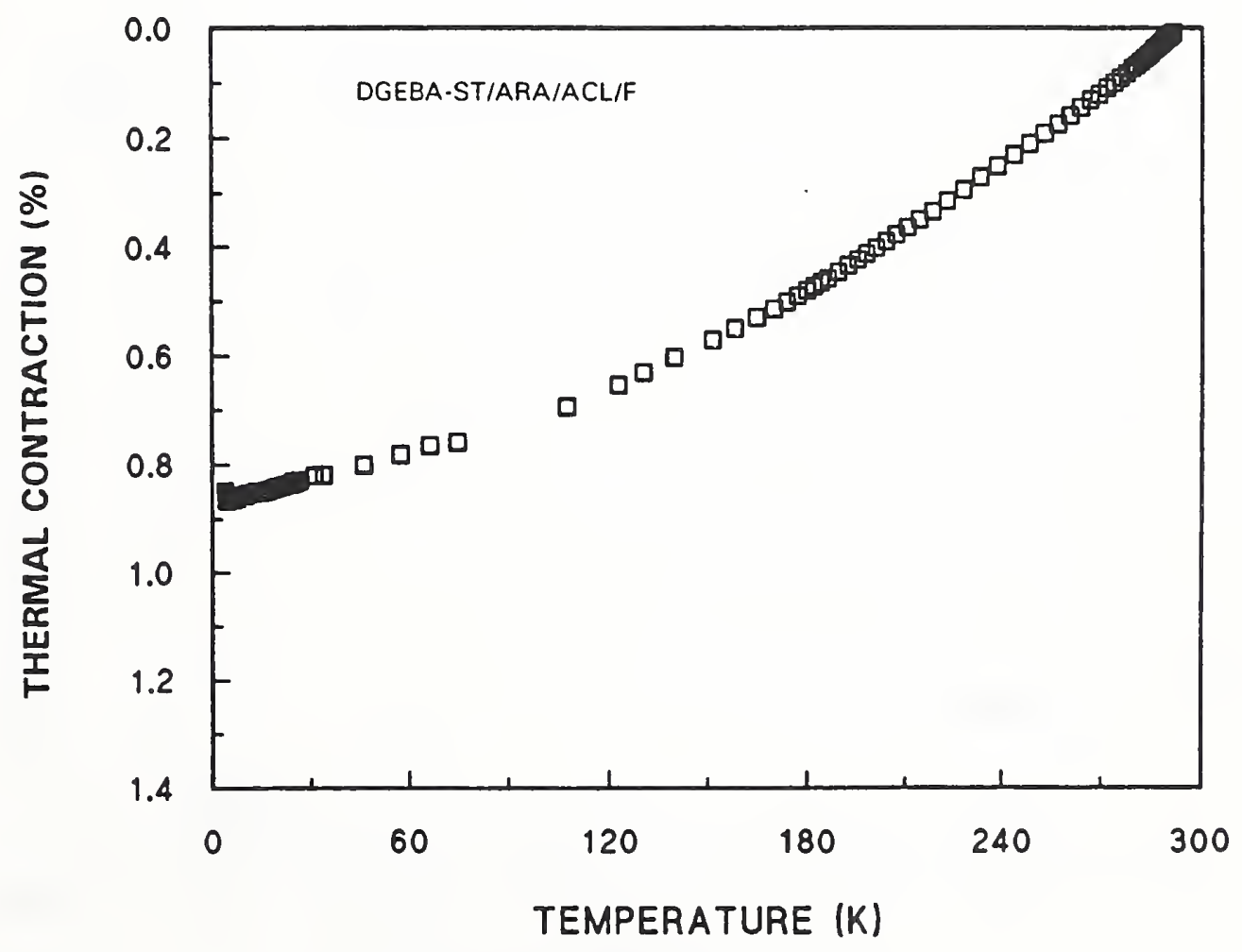

Figure 6.5. Thermal contraction of DGEBA-ST/ARA/ACL/F epoxy resin. 


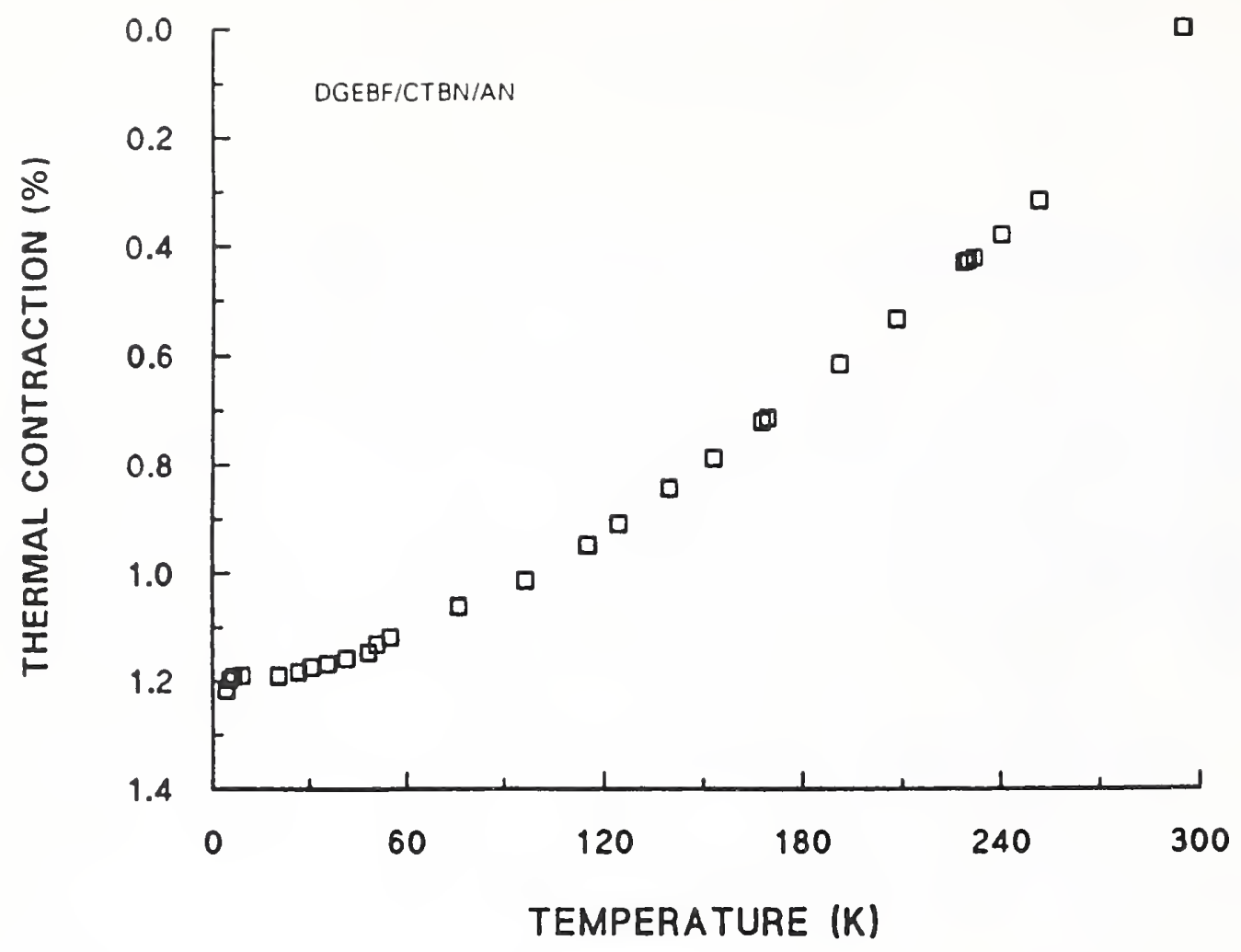

Figure 6.6. Thermal contraction of DGEBF/CTBN/AN epoxy resin.

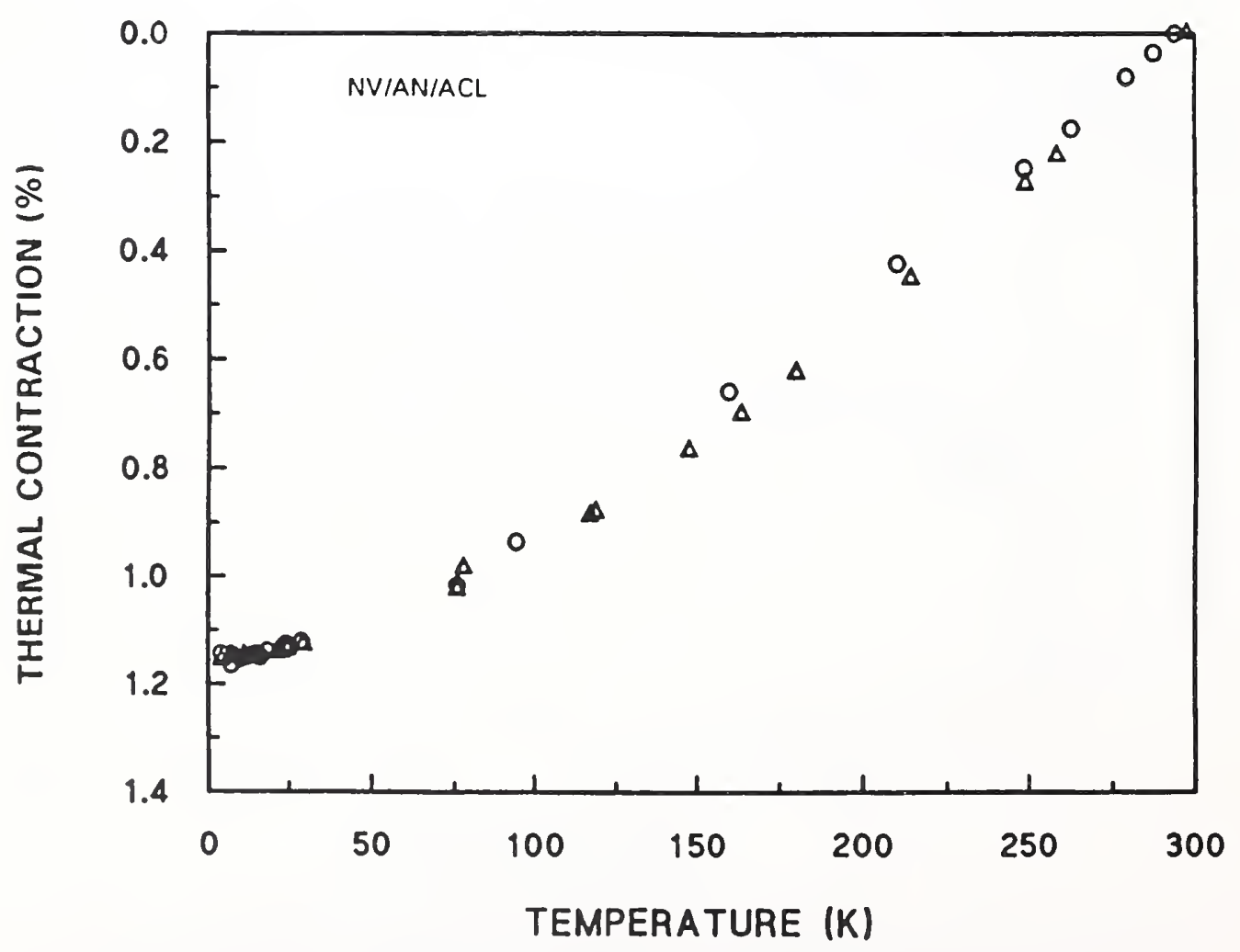

Figure 6.7. Thermal contraction of NV/AN/ACL epoxy resin. 


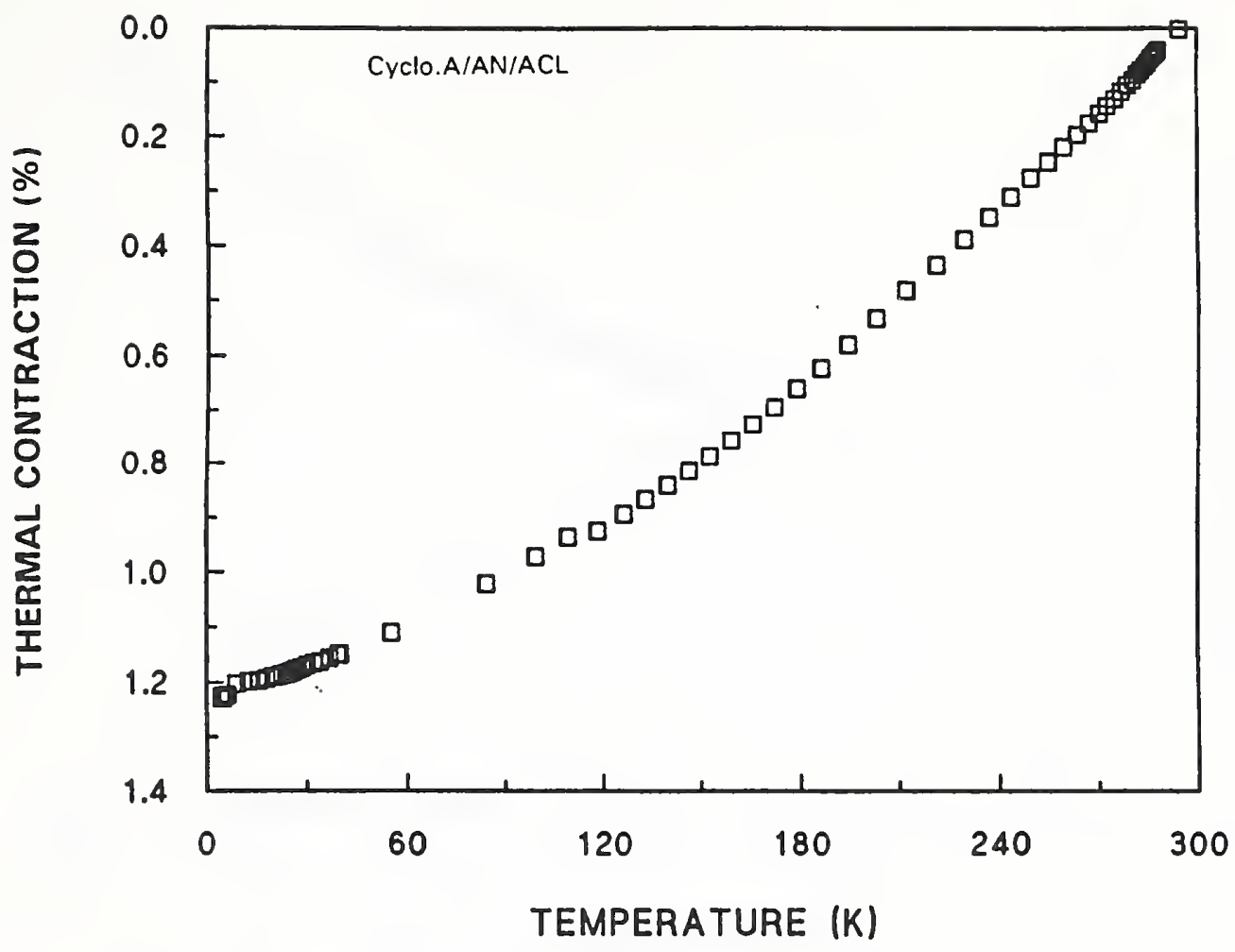

Figure 6.8. Thermal contraction of Cyclo.A/AN/ACL epoxy resin.

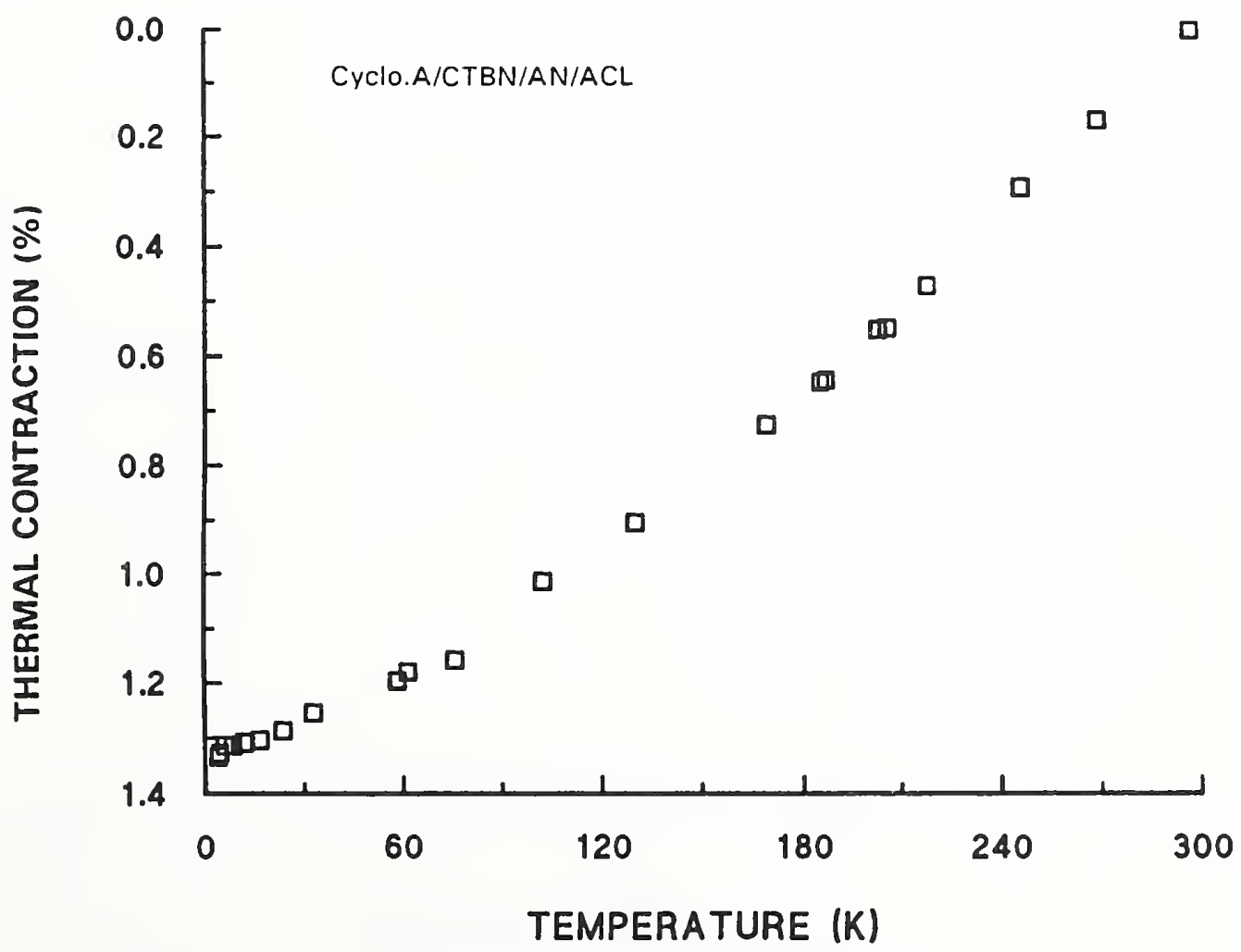

Figure 6.9. Thermal contraction of Cyclo.A/CTBN/AN/ACL epoxy resin. 


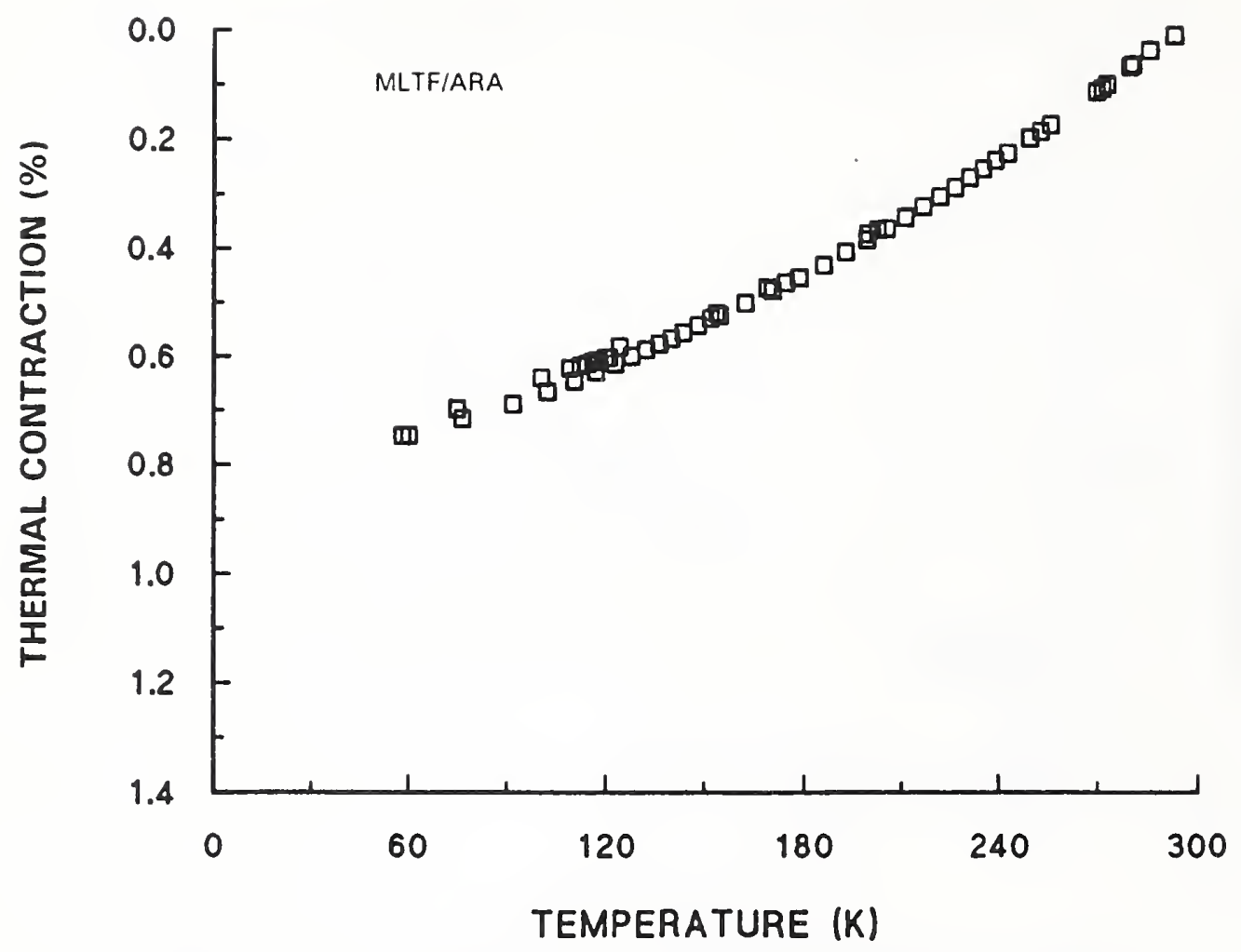

Figure 6.10. Thermal contraction of MLTF/ARA epoxy resin.

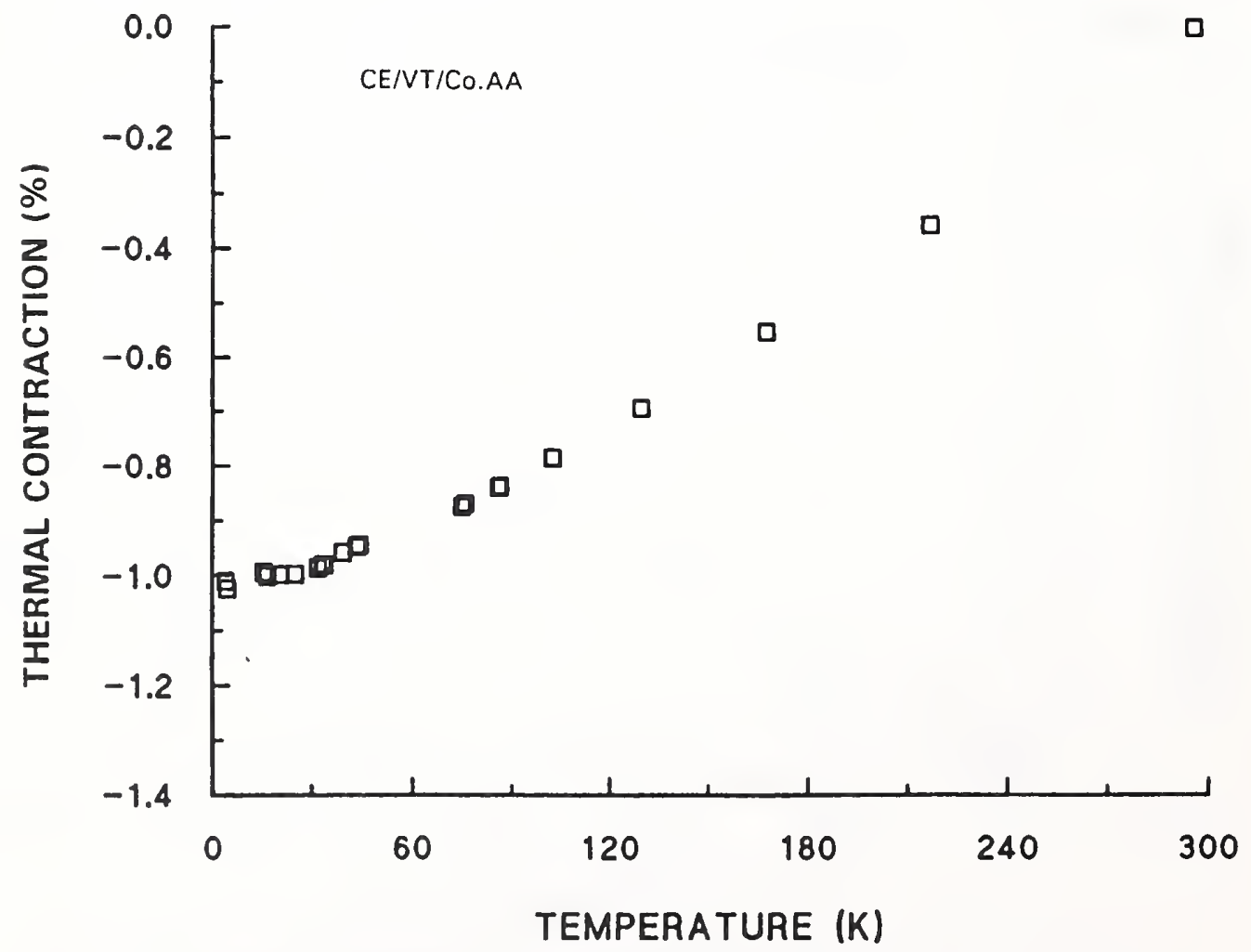

Figure 6.11. Thermal contraction of CE/VT/Co.AA cyanate-ester resin. 


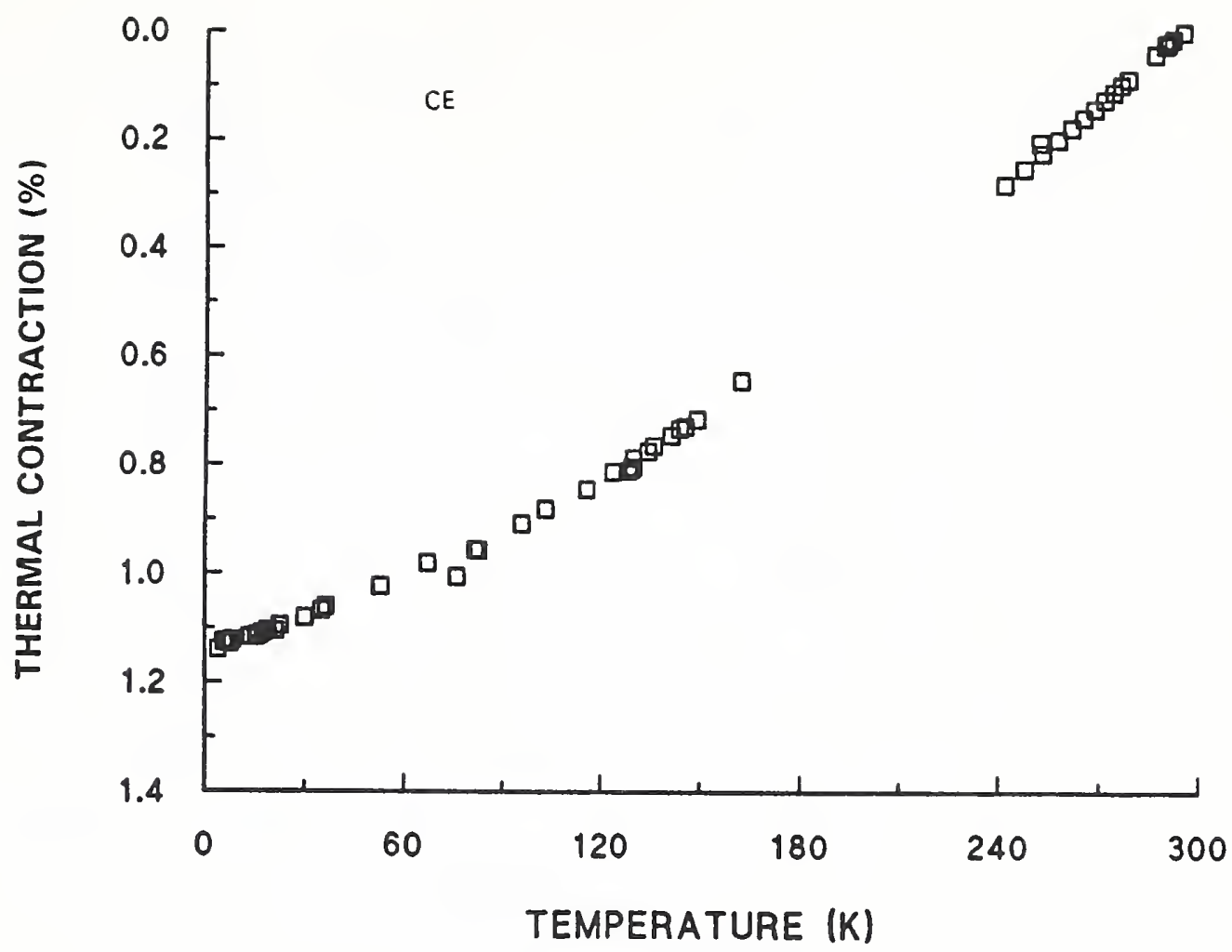

Figure 6.12. Thermal contraction of CE cyanate-ester resin.

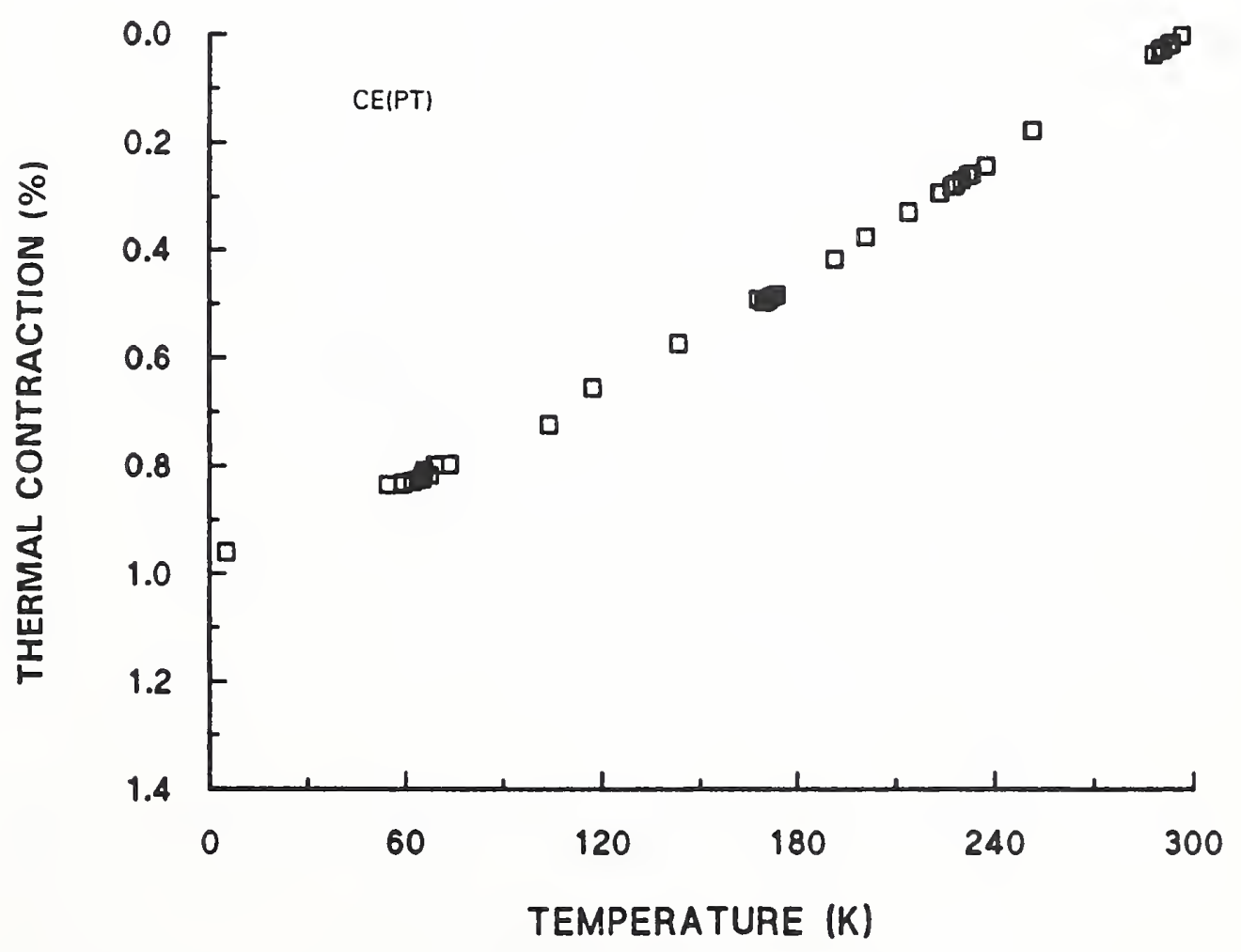

Figure 6.13. Thermal contraction of $\mathrm{CE}(\mathrm{PT})$ cyanate-ester resin. 


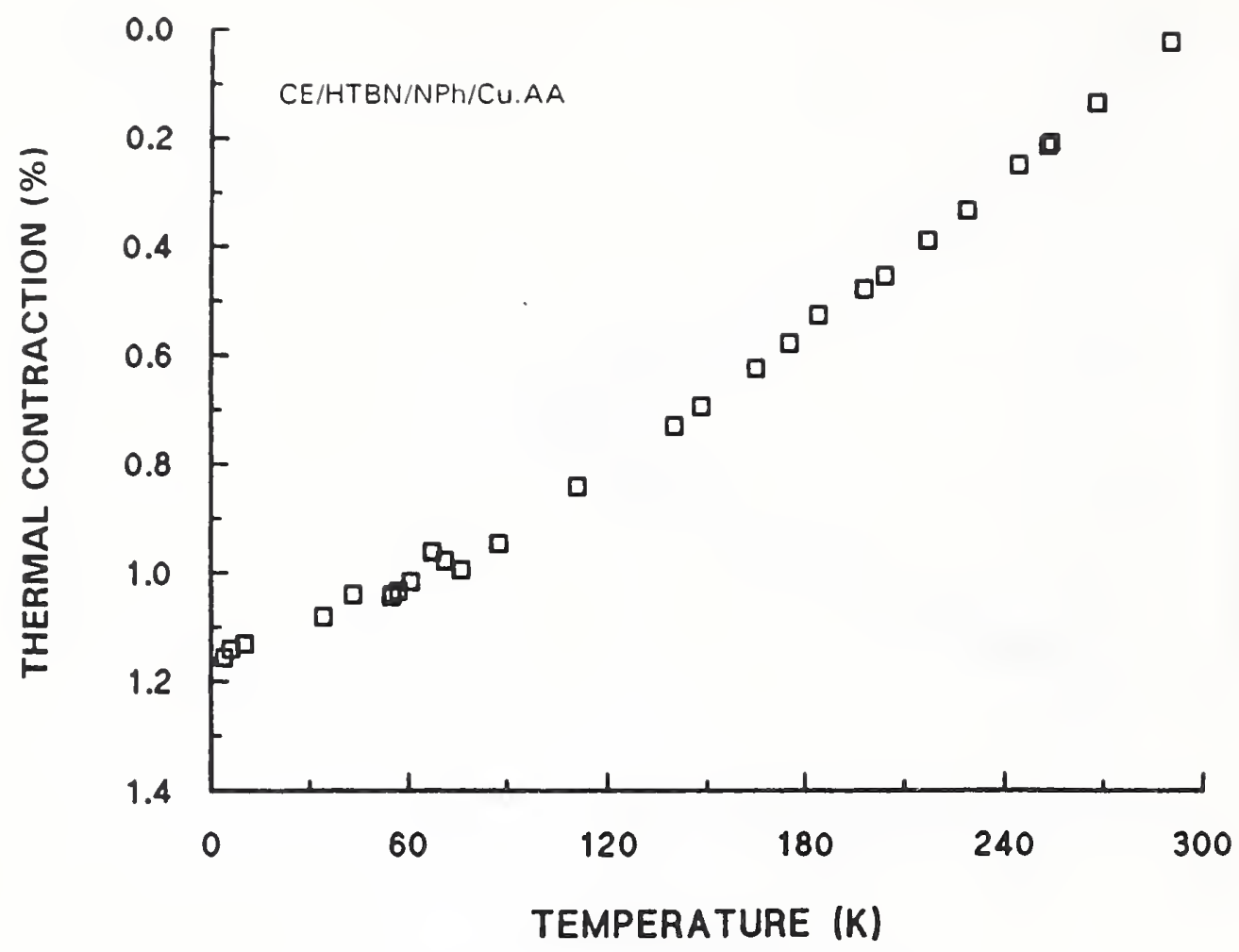

Figure 6.14. Thermal contraction of CE/HTBN/NPh/Cu.AA cyanate-ester resin.

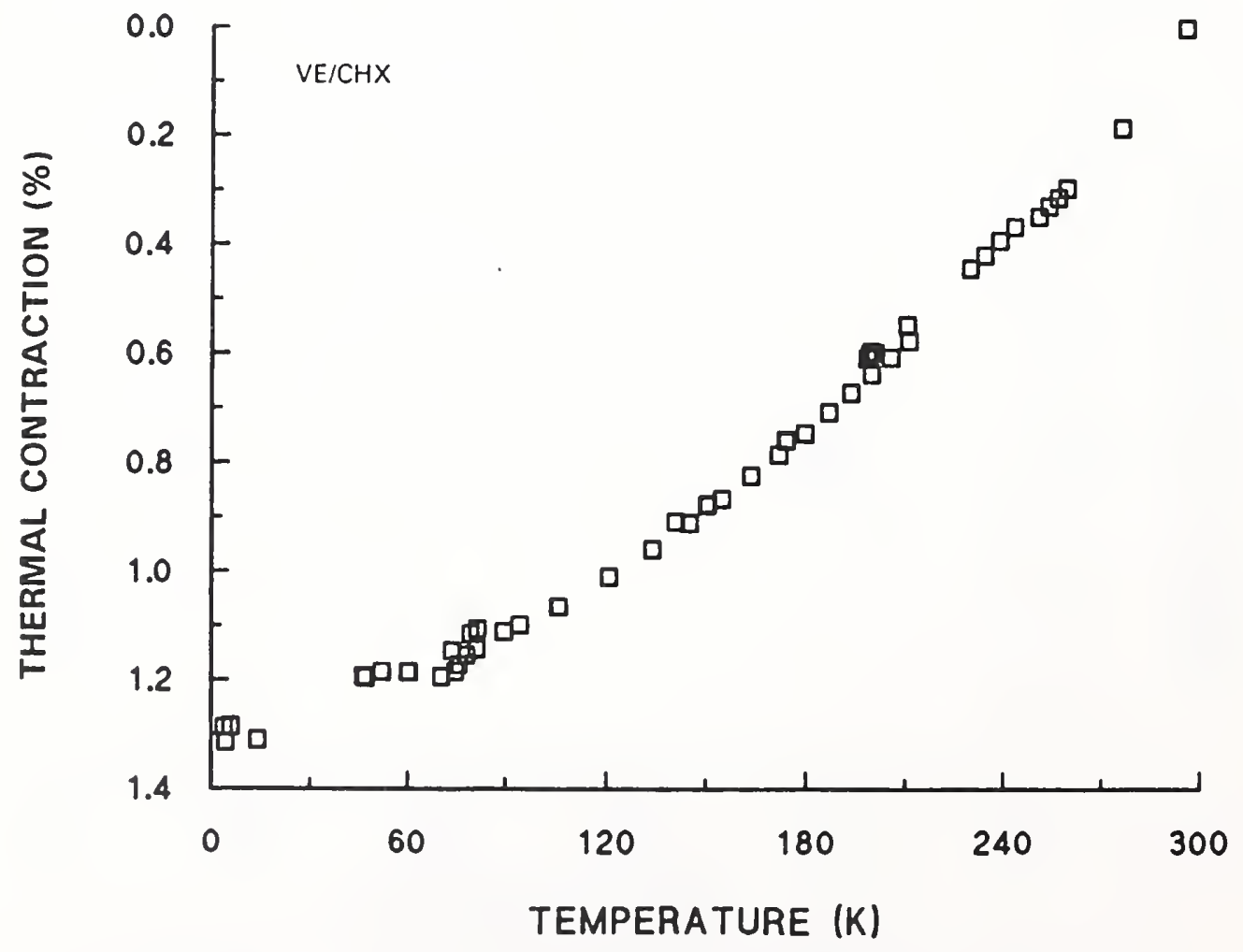

Figure 6.15. Thermal contraction of VE/CHX vinyl-ester resin. 


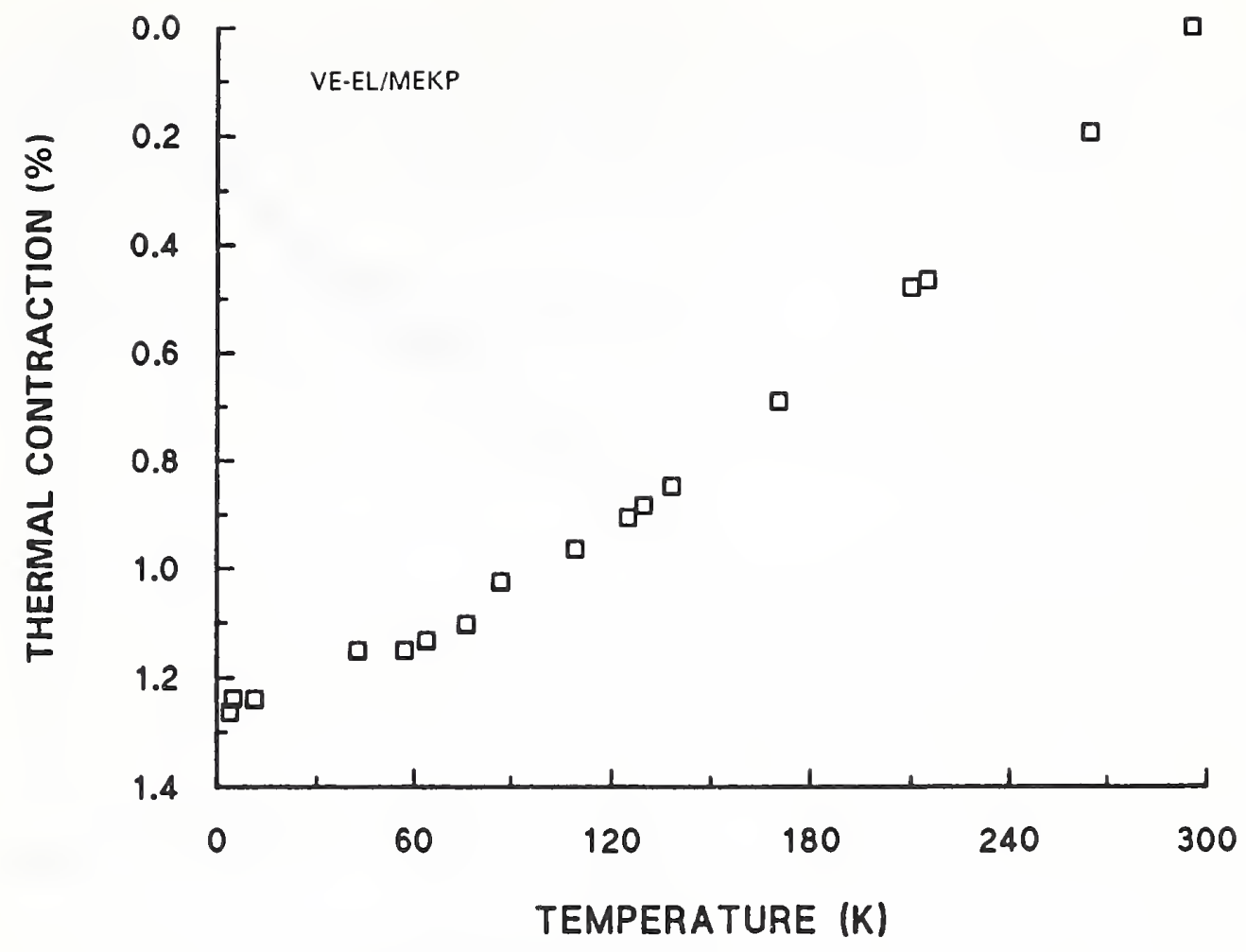

Figure 6.16. Thermal contraction of VE-EL/MEKP vinyl-ester resin.

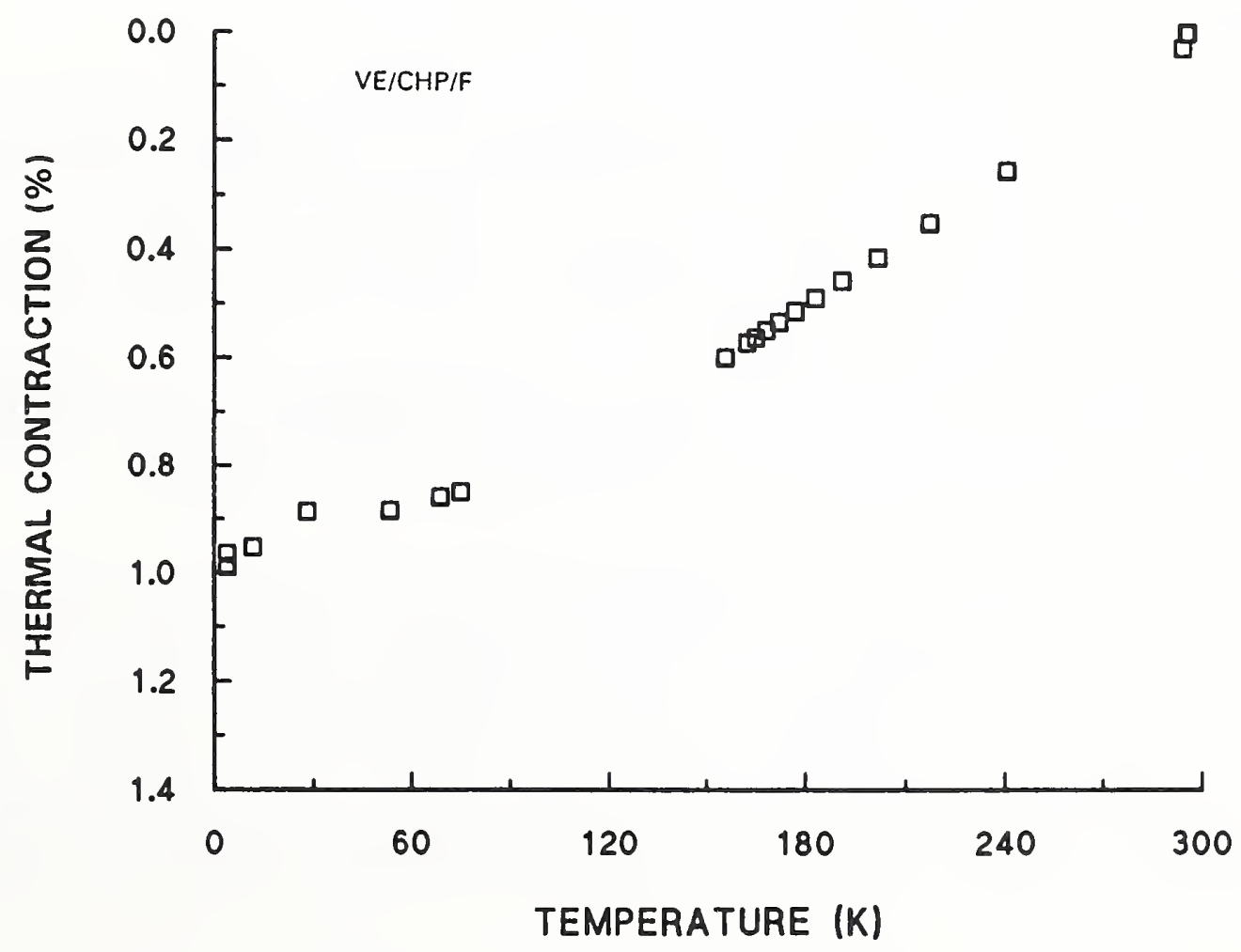

Figure 6.17. Thermal contraction of VE/CHP/F vinyl-ester resin. 


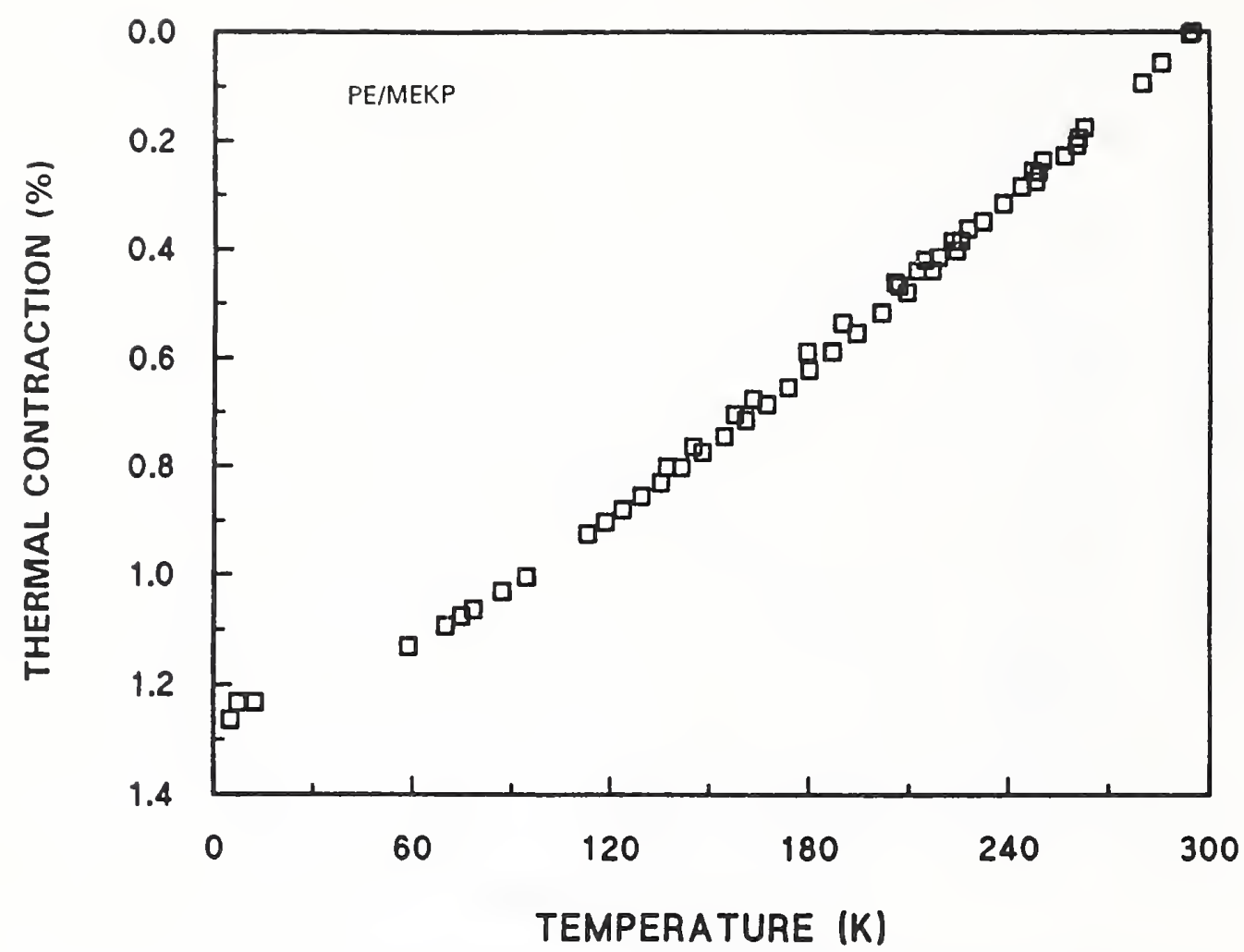

Figure 6.18. Thermal contraction of PE/MEKP polyester resin.

\subsection{Discussion}

This study demonstrates that the thermal contractions of different classes of resin systems are distinct and that, even within the epoxy class, wide differences may result from variations in chemical composition, processing, or both. The lowest contraction on cooling to $4 \mathrm{~K}$ was obtained in tetrafunctional epoxies and filled-resin systems. The highest thermal contraction was found in vinyl-ester and polyester systems. Toughened-resin systems contract more than equivalent untoughened systems.

No previous studies were conducted to measure the thermal contraction of these particular resin systems at low temperatures. However, our data for epoxies range from 1.1 to $1.2 \%$ contraction, in agreement with the data from Table 6.I of Hacker [6.4] and Evans and Morgan [6.5]. The other reported contraction data in Table 6.1 for unfilled epoxies may be too large.

Since low-temperature thermal contraction is dependent on cross-link distance [6.3], a correlation should exist between thermal contraction and the elastic or shear modulus. The data for the resins in this study were assessed for a moduli-thermal contraction correlation. We found very general correlation between an increase of elastic moduli with a decrease in contraction on cooling. Yet, attempts to use $E, G, E+2 G$, and $B$ did not yield a strong correlation with all resin systems or individual groups of resin systems. (Here $E$ is the Young's modulus, $G$ is the shear modulus, and $B$ is the bulk modulus.) However, filled systems have higher $E$ and $G$ values, in general, and lower thermal contraction. Tetrafunctional epoxy-resin systems also have higher moduli and lower thermal contraction. 
To assess the residual stress in the vicinity of the fiber-matrix interface, it is not sufficient to compare the thermal contraction of the resin system with that of the E-glass reinforcement. Shrinkage due to resin cure while in solid form must also be included. The studies reported in the section on residual stress $(\$ \mathrm{~V} .4)$ address the relative cure shrinkage of the resins in this study.

The strain-gage measurement technique has proven to yield reproducible data. It should be considered for other studies to obtain thermal-contraction data for use in engineering applications. 


\title{
7. Thermal Conductivity
}

\author{
D.L. Rule \\ Materials Reliability Division \\ National Institute of Standards and Technology \\ Boulder, Colorado
}

The thermal conductivities of five neat resins were determined from 4.6 to $290 \mathrm{~K}$. The conductivities of three different types of epoxies, one vinyl-ester resin, and one polyester resin were measured. All the neat resins had similar thermal conductivities at $4 \mathrm{~K}$; however, one epoxy had a much higher conductivity at room temperature than all the other materials.

\subsection{Introduction}

The Superconducting Magnetic Energy Storage (SMES) system will employ polymer composite tubes that must provide thermal isolation as well as mechanical support. Many polymeric resins are capable of being utilized as the matrix in a composite, but very few have been studied for possible applications at cryogenic temperatures.

\subsection{Review of Literature}

For many of the resins in this program, little or no experimental data were available at cryogenic temperatures. An examination of some previous thermal conductivity studies on neat resins relevant to this study revealed some interesting information. Unfortunately, very little material characterization accompanied the available data. The effects of possible material variations cause the comparison of neat-resin data to be rather qualitative.

Hust measured the thermal conductivities of the neat epoxies that are used in G-10CR and G-11CR. He used the same measurment apparatus (described in §III.7.4.1) before automation and reported that the results were virtually the same for the two epoxies [7.1]. The epoxies used in G-10CR and G-11CR generally were a diglycidyl ether of bisphenol-A epoxy (DGEBA), but the chemical composition and hardener used for these specimens were not noted.

Nicholls and Rosenberg measured the thermal conductivity (from 4 to $80 \mathrm{~K}$ ) of the neat epoxy Araldite MY $750^{*}$ [7.2]. Various diamine hardeners were used to cure the epoxy, and the specimens were heat-treated. These data appear to be about $40 \%$ higher at $4 \mathrm{~K}$ than data from an earlier report on the same neat epoxy: Rosenberg had previously measured the conductivity of the same base

\footnotetext{
* The epoxy Araldite MY 750 is a registered trademark for a proprietary epoxy. This product, as well as other products named in this report are identified only for better material characterization; endorsement of these products or their manufacturers by NIST or by the U.S government is neither intended nor implied.
} 
report on the same neat epoxy: Rosenberg had previously measured the conductivity of the same base resin, Araldite MY 750, with various types of particle fillers over the temperature range 4 to $295 \mathrm{~K}$ [7.3]. Possible reasons for these variations between the two studies were not discussed.

Evans and Morgan reported the thermal conductivity of an unspecified neat epoxy at low temperatures [7.4]. In this article, they discussed a variety of materials involved in the fabrication of a cryogenic Dewar and specifics about the neat epoxy material, but the sources of the measurements were not discussed.

In another study, the thermal conductivity of polyimide material poly pyro mellitimide (PPMI) was measured with the same apparatus as that used in this study [7.5]. The PPMI material was a thin film, commonly known as Kapton. However, the results compared favorably to the known data on the similar (bulk) castable material $[7.6,7.7]$.

\subsection{Description of Specimens}

Tests were performed on five different neat polymers. This study included three different types of epoxies: two of the epoxies were a diglycidyl ether of bisphenol-A epoxy (DGEBA) mixture, and one was a diglycidyl ether of bisphenol-F epoxy (DGEBF) mixture. The study also included a commonly used polyester and a vinyl-ester resin. The polymers were made according to the manufacturers' specifications. The specimens and nomenclature used in this section are identified in Table 7.1.

The five specimens in this study were fabricated by cutting several small sections from the manufactured plate, then cementing the sections together with a very thin layer of cyanoacrylate adhesive, and finally, cutting the specimen with a diamond saw to the sizes listed in Table 7.2. All dimensions were obtained as an average of eight measurements; they are accurate to $\pm 12 \mu \mathrm{m}$ $( \pm 0.0005$ in).

Table 7.1. Specimen identification.

\begin{tabular}{|c|c|c|c|}
\hline \multicolumn{2}{|c|}{$\begin{array}{l}\text { Type and Specimen } \\
\text { Identification }\end{array}$} & \multirow[b]{2}{*}{ DGEBA/DCH/ARA/ACL } & \multirow{2}{*}{$\begin{array}{l}\text { Components } \\
\text { DGEBA epoxy, armomatic amine cure, } \\
\text { H41 + other, catalyst }\end{array}$} \\
\hline Epoxies: & Resin B & & \\
\hline & Resin E & DGEBF/CTBN/AN & $\begin{array}{l}\text { DGEBF epoxy, anhydride cure, } \\
\text { catalyst, CTBN toughener }\end{array}$ \\
\hline & Resin L & DGEBA-ST/ARA/ACL/F & $\begin{array}{l}\text { DGEBA epoxy, styrene modified, } \\
\text { aromatic amine cure, accelerator, filler }\end{array}$ \\
\hline Polyester: & Resin $\mathrm{P}$ & PE/MEKP & polyester, MEKP (peroxide) \\
\hline Vinyl ester: & Resin C & VE-EL/MEKP & vinyl ester, MEKP (peroxide) \\
\hline
\end{tabular}


Table 7.2. Physical characteristics of the specimens.

\begin{tabular}{ccc}
\hline $\begin{array}{c}\text { Specimen } \\
\text { Identification }\end{array}$ & $\begin{array}{c}\text { Length } \\
(\mathrm{cm})\end{array}$ & $\begin{array}{c}\text { Area } \\
\left(\mathrm{cm}^{2}\right)\end{array}$ \\
\hline Resin B & 6.19 & 171.0 \\
Resin E & 6.19 & 182.0 \\
Resin L & 6.13 & 177.0 \\
Resin P & 6.50 & 174.4 \\
Resin C & 6.43 & 172.9 \\
\hline \hline
\end{tabular}

\subsection{Procedure}

\subsubsection{Description of the Apparatus}

To measure thermal conductivity, we used an unguarded, "fixed-point" apparatus [7.8] that is based on a method of axial one-dimensional heat flow. Some modifications have been made since the original description was published. The specimen chamber is shown schematically in Figure 7.1. A specimen of known length and cross-sectional area is compressed between two isothermal copper blocks. Three stainless-steel bolts clamp the specimen between the two copper blocks; the flow of heat bypassing the specimen through the bolts is known and well-characterized from previous calibration runs.

The upper, cooled, isothermal block is anchored to the top of the specimen chamber, which is usually immersed in one of four constant-temperature (fixed-point) baths. This upper block determines the cold-side temperature of the specimen. The nominal temperatures of these fixed-point baths are: $4 \mathrm{~K}$ (liquid helium), $76 \mathrm{~K}$ (liquid nitrogen), $194 \mathrm{~K}$ (a mixture of dry ice and alcohol), or $273 \mathrm{~K}$ (a mixture of pure ice and water).

The temperature of the lower, heated block is fixed by controlling the electric power supplied to its attached heater. This determines the hot-side temperature of the specimen and establishes a fixed temperature difference across the specimen. Total power supplied to the hot block is determined from measurements of the voltage and current to its resistive heating element. Heat flowing through the specimen is computed by subtracting the calibrated heat flow through the compression bolts from the total power.

Type AuFe-NiCr thermocouples are used to determine the cold-block temperature and the difference in temperature between the hot and cold blocks. The temperature differences established between the hot and cold blocks can be as much as $64 \mathrm{~K}$. These adjustable temperature differences enable different mean temperatures to be established within the specimen.

Each specimen was clamped between the copper blocks by tightening the stainless steel bolts to $1.4 \mathrm{~N} \cdot \mathrm{m}(12 \mathrm{in} \cdot \mathrm{lb})$ of torque, applied at room temperature. This resulted in a compressive pressure on the specimen of approximately $27 \mathrm{MPa}(3.9 \mathrm{kpsi})$. The change in this load resulting from the 


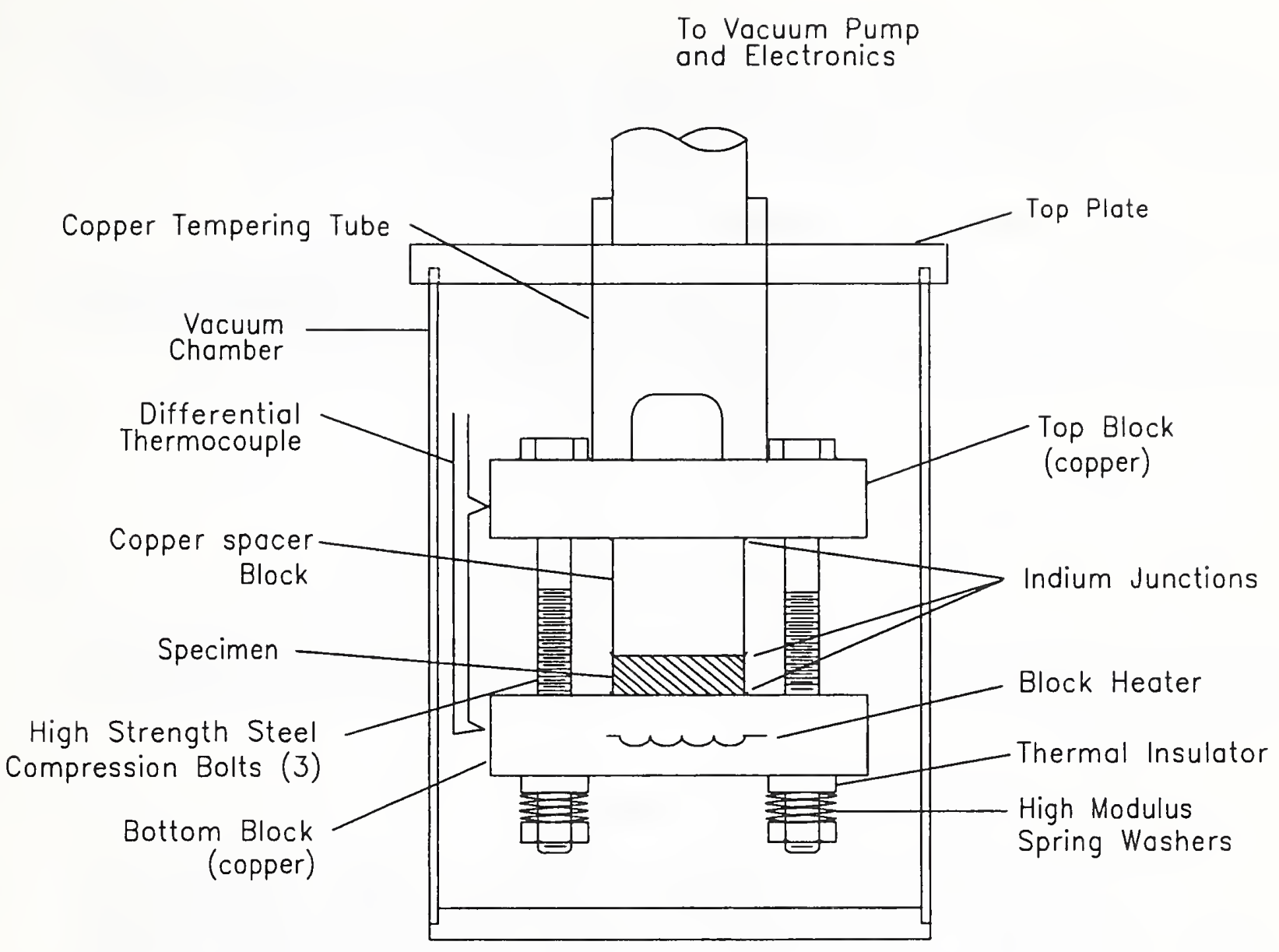

Figure 7.1. Specimen chamber for fixed-point compression probe.

cooling of a specimen to cryogenic temperatures, while not accurately known, is believed to be relatively small and did not affect the measurements of conductivity reported here.

This compressive pressure of $27 \mathrm{MPa}(3.9 \mathrm{kpsi}$ ) approached the compressive strength of the neat-resin specimens. Two specimens deformed permanently when compressed in the apparatus under a slightly larger load. The thermal conductivities of these damaged specimens were not measured.

\subsubsection{Experimental Procedure}

Steady-state measurements of conductivity were made with the specimen in a vacuum environment of $8 \times 10^{-3} \mathrm{~Pa}\left(6 \times 10^{-5}\right.$ torr $)$ and with the specimen chamber in one of the fixed-point baths described earlier. A $76-\mu \mathrm{m}(0.003$-in) indium foil and thermally conductive grease were applied to the two contact surfaces between the specimen and the apparatus. The grease was used as a lubricant to prevent the indium foil from "cold welding" to the specimen and apparatus. 
Experience with this apparatus indicates that the use of indium-grease contacts coupled with enough contact pressure is sufficient to eliminate significant thermal-contact resistance. After the specimen was compressed in the apparatus, excess grease and indium were removed.

\subsubsection{Computation of Thermal Conductivity}

From the power $\left(Q_{\text {spec }}\right)$ through the specimen, specimen length $(\ell)$, cross-sectional area $(A)$, and temperature difference $(\Delta T)$ across the specimen, the mean thermal conductivity $(\lambda)$ can be calculated from the one-dimensional approximation of Fourier's law:

$$
Q_{\text {spec }}=-\lambda \cdot A \cdot(d T / d x)
$$

recast into the approximate form

$$
\lambda=\left(Q_{\mathrm{spec}} / \Delta T\right) \cdot(\ell / A)
$$

Here, the ratio $(\Delta T / \ell)$ approximates the derivative $d T / d x$ in Equation (7.1), and the conductivity obtained is the value averaged over the range of temperature defined by the temperature difference $\Delta T$ across the specimen.

\subsubsection{Accuracy and Reproducibility of Measurements}

The uncertainty associated with the original apparatus was $\pm 10 \%$ [7.1]. After some modifications to the apparatus and automating the system, tests on a stainless steel standard reference material (SRM 735) indicated an overall system uncertainty of $\pm 5 \%$ (when measuring specimens having a conductivity similar to that of stainless steel). The conductivities of the stainless steel SRM are $0.352 \mathrm{~W} /(\mathrm{m} \cdot \mathrm{K})$ at $4 \mathrm{~K}, 8.16 \mathrm{~W} /(\mathrm{m} \cdot \mathrm{K})$ at $77 \mathrm{~K}, 12.01 \mathrm{~W} /(\mathrm{m} \cdot \mathrm{K})$ at $192 \mathrm{~K}$, and $13.79 \mathrm{~W} /(\mathrm{m} \cdot \mathrm{K})$ at $273 \mathrm{~K}$. The low conductivities of the polymer specimens cause a significant fraction of the total heat flow to go through the compression bolts, increasing the uncertainty of the results presented here to an estimated $\pm 10 \%$.

\subsection{Results}

The thermal conductivities of the neat resins were measured over the temperature range 4.6 to $290 \mathrm{~K}$. The experimental conductivity data obtained for the Resin B specimen is given in Table 7.3 and Figure 7.2; the relative (percent) deviations of the data points from the appropriate fitted curve are shown in Figure 7.3. Likewise, the thermal conductivity data for the other four neat-resin systems are presented in Tables 7.4 through 7.7 and Figures 7.4 through 7.11. As is conventional, all conductivity data in the figures are plotted as a function of the (arithmetic) mean temperature of the specimen. 
Table 7.3. Experimental conductivity as a function of temperature for the neat-resin specimen Resin B.

\begin{tabular}{|c|c|c|c|}
\hline $\begin{array}{l}\text { Temperature } \\
\text { Difference } \\
(\Delta \mathrm{K})\end{array}$ & $\begin{array}{c}\text { Average } \\
\text { Temperature } \\
(\mathrm{K}) \\
\end{array}$ & $\begin{array}{c}\text { Thermal } \\
\text { Conductivity } \\
{[\mathrm{W} /(\mathrm{m} \cdot \mathrm{K})]}\end{array}$ & $\begin{array}{l}\text { Cold } \\
\text { Bath }\end{array}$ \\
\hline 1 & 4.649 & 0.065 & $\mathrm{LHe}$ \\
\hline 2 & 5.170 & 0.065 & \\
\hline 4 & 6.234 & 0.066 & \\
\hline 8 & 8.352 & 0.069 & \\
\hline 16 & 12.640 & 0.075 & \\
\hline 32 & 21.365 & 0.089 & \\
\hline 64 & 39.636 & 0.116 & \\
\hline 1 & 76.534 & 0.151 & $\mathrm{LN}_{2}$ \\
\hline 1.9 & 76.974 & 0.147 & \\
\hline 2.2 & 77.118 & 0.153 & \\
\hline 3.9 & 78.007 & 0.148 & \\
\hline 8 & 80.153 & 0.151 & \\
\hline 15.7 & 84.147 & 0.152 & \\
\hline 32 & 92.650 & 0.157 & \\
\hline 64 & 109.48 & 0.165 & \\
\hline 2 & 193.11 & 0.231 & $\mathrm{CO}_{2}$-alcohol \\
\hline 4.1 & 194.22 & 0.217 & \\
\hline 8.2 & 196.47 & 0.209 & \\
\hline 16 & 200.65 & 0.195 & \\
\hline 32 & 209.37 & 0.191 & \\
\hline 47.7 & 218.03 & 0.191 & \\
\hline 48 & 218.16 & 0.188 & \\
\hline 61.7 & 225.72 & 0.181 & \\
\hline 64 & 227.02 & 0.186 & \\
\hline 64 & 227.04 & 0.188 & \\
\hline 1 & 273.70 & 0.182 & Ice-water \\
\hline 2 & 274.23 & 0.183 & \\
\hline 4 & 275.36 & 0.183 & \\
\hline 8 & 277.54 & 0.181 & \\
\hline 16 & 281.92 & 0.176 & \\
\hline 32 & 290.76 & 0.173 & \\
\hline 64 & 308.72 & 0.169 & \\
\hline
\end{tabular}




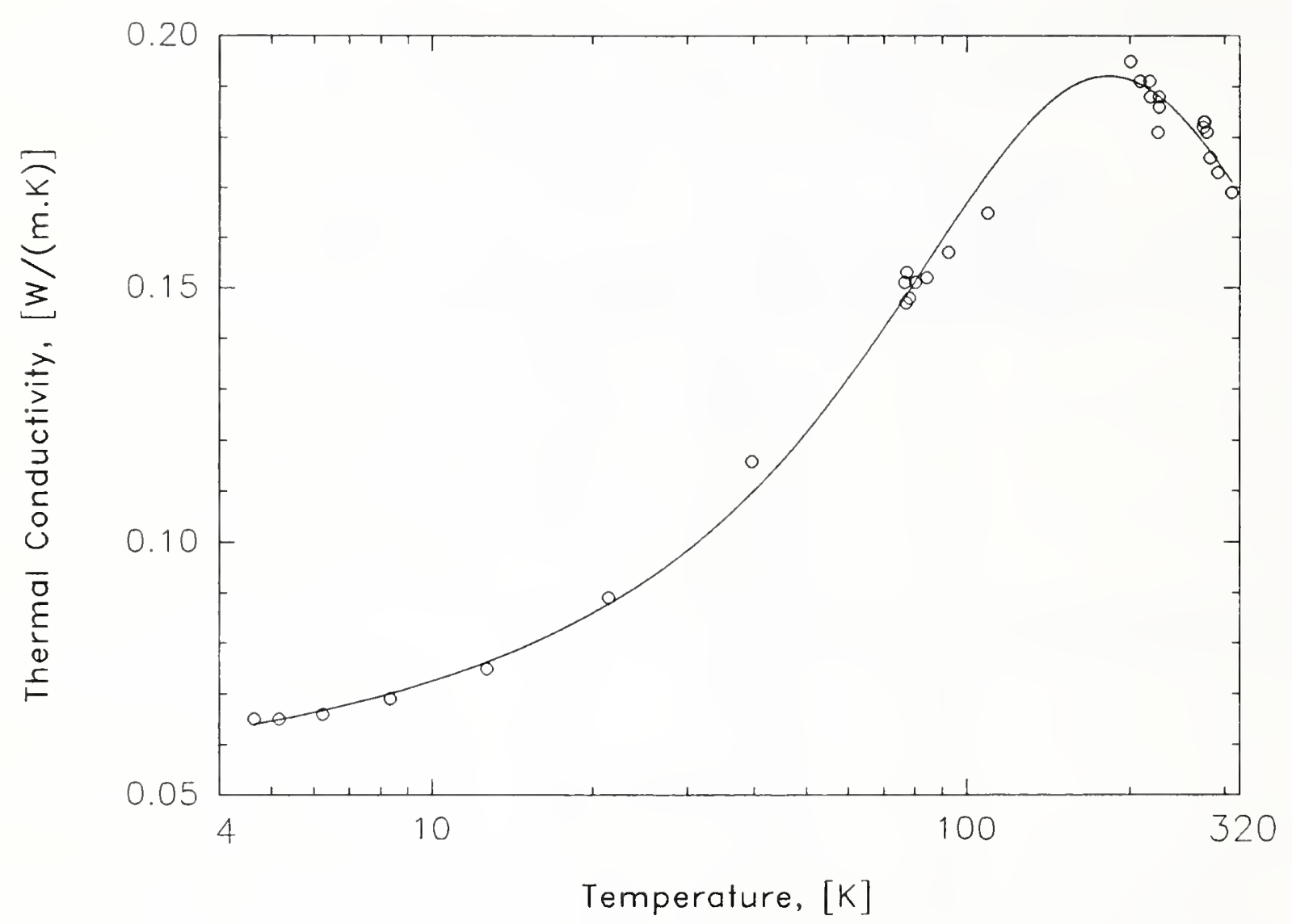

Figure 7.2. Thermal conductivity of the neat-resin specimen Resin B. Experimental data are presented as discrete points.

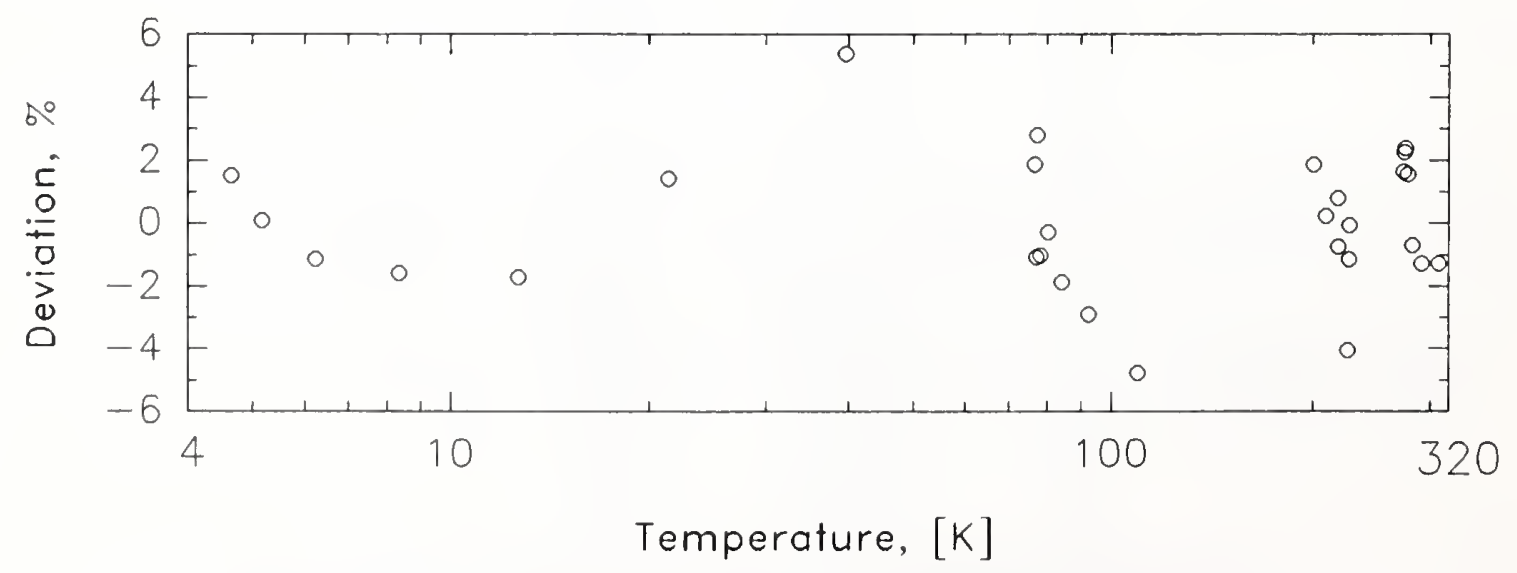

Figure 7.3. Relative deviations of the experimental and calculated thermal conductivity for the neat-resin specimen Resin B. 
Table 7.4. Experimental conductivity as a function of temperature for the neat-resin specimen Resin E.

\begin{tabular}{|c|c|c|c|}
\hline $\begin{array}{c}\text { Temperature } \\
\text { Difference } \\
(\Delta K)\end{array}$ & $\begin{array}{c}\text { Average } \\
\text { Temperature } \\
(\mathrm{K})\end{array}$ & $\begin{array}{l}\text { Thermal } \\
\text { Conductivity } \\
{[\mathrm{W} /(\mathrm{m} \cdot \mathrm{K})]}\end{array}$ & $\begin{array}{l}\text { Cold } \\
\text { Bath }\end{array}$ \\
\hline 1 & 4.638 & 0.043 & \multirow[t]{7}{*}{ LHe } \\
\hline 2 & 5.156 & 0.042 & \\
\hline 4 & 6.204 & 0.043 & \\
\hline 8 & 8.299 & 0.046 & \\
\hline 16 & 12.561 & 0.055 & \\
\hline 32 & 21.291 & 0.073 & \\
\hline 63.3 & 38.541 & 0.098 & \\
\hline 0.9 & 76.485 & 0.132 & \multirow[t]{11}{*}{$\mathrm{LN}_{2}$} \\
\hline 2 & 77.032 & 0.135 & \\
\hline 4 & 78.091 & 0.136 & \\
\hline 7.9 & 80.082 & 0.135 & \\
\hline 15.9 & 84.274 & 0.142 & \\
\hline 16 & 84.316 & 0.138 & \\
\hline 24 & 88.463 & 0.139 & \\
\hline 32 & 92.637 & 0.142 & \\
\hline 32 & 92.650 & 0.147 & \\
\hline 48 & 101.00 & 0.145 & \\
\hline 64 & 109.39 & 0.150 & \\
\hline 4 & 194.14 & 0.192 & \multirow[t]{7}{*}{$\mathrm{CO}_{2}$-alcohol } \\
\hline 7.9 & 196.29 & 0.190 & \\
\hline 16 & 200.67 & 0.189 & \\
\hline 24 & 205.02 & 0.188 & \\
\hline 32 & 209.39 & 0.187 & \\
\hline 48 & 218.19 & 0.185 & \\
\hline 64 & 227.06 & 0.181 & \\
\hline 1 & 273.69 & 0.189 & \multirow[t]{10}{*}{ Ice-water } \\
\hline 1 & 273.71 & 0.194 & \\
\hline 2 & 274.26 & 0.190 & \\
\hline 4 & 275.34 & 0.188 & \\
\hline 8 & 277.51 & 0.186 & \\
\hline 8 & 277.54 & 0.190 & \\
\hline 16 & 281.92 & 0.187 & \\
\hline 16 & 281.94 & 0.188 & \\
\hline 24 & 286.37 & 0.187 & \\
\hline 24 & 286.52 & 0.187 & \\
\hline
\end{tabular}




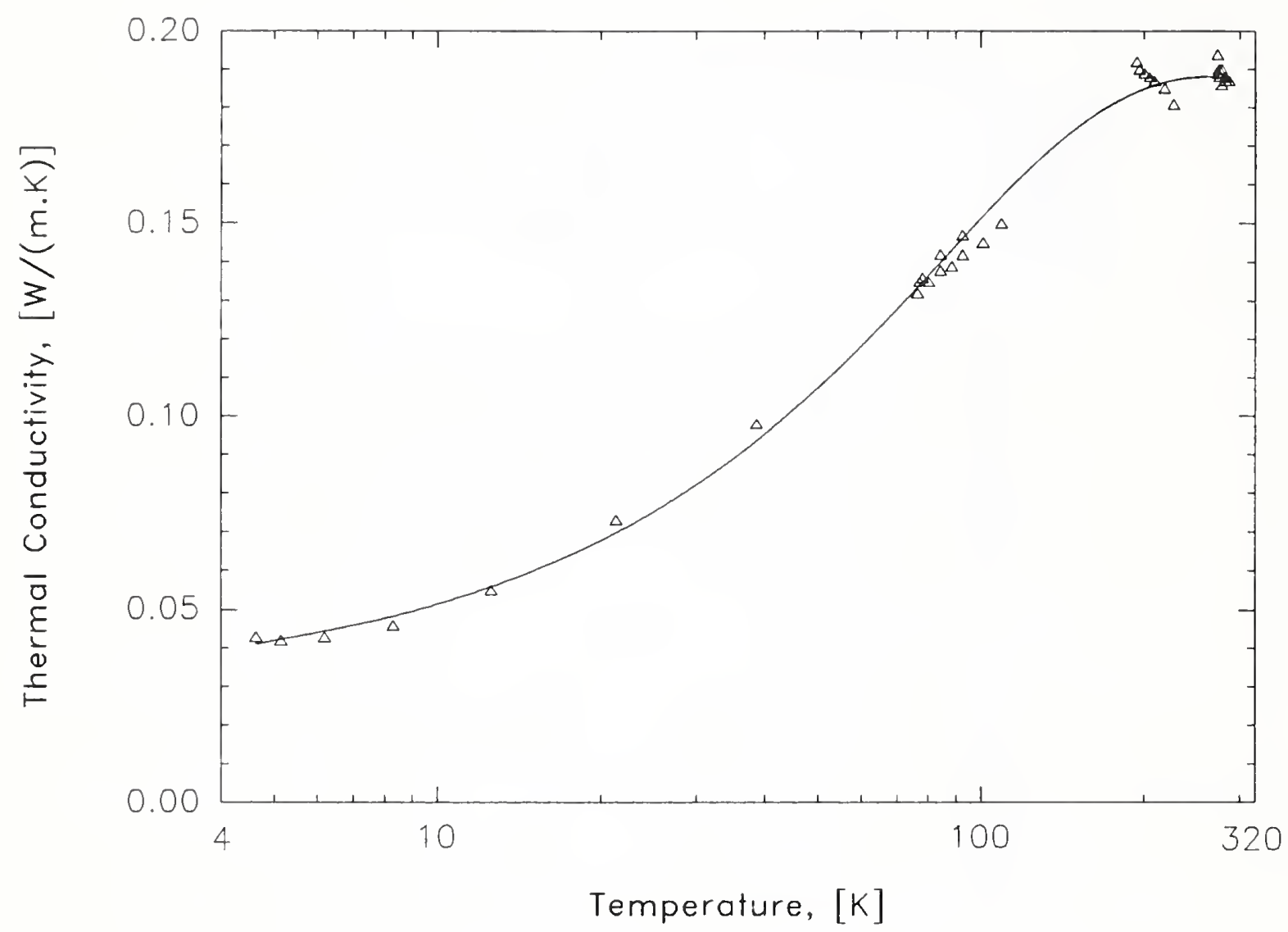

Figure 7.4. Thermal conductivity of the neat-resin specimen Resin E. Experimental data are presented as discrete points.

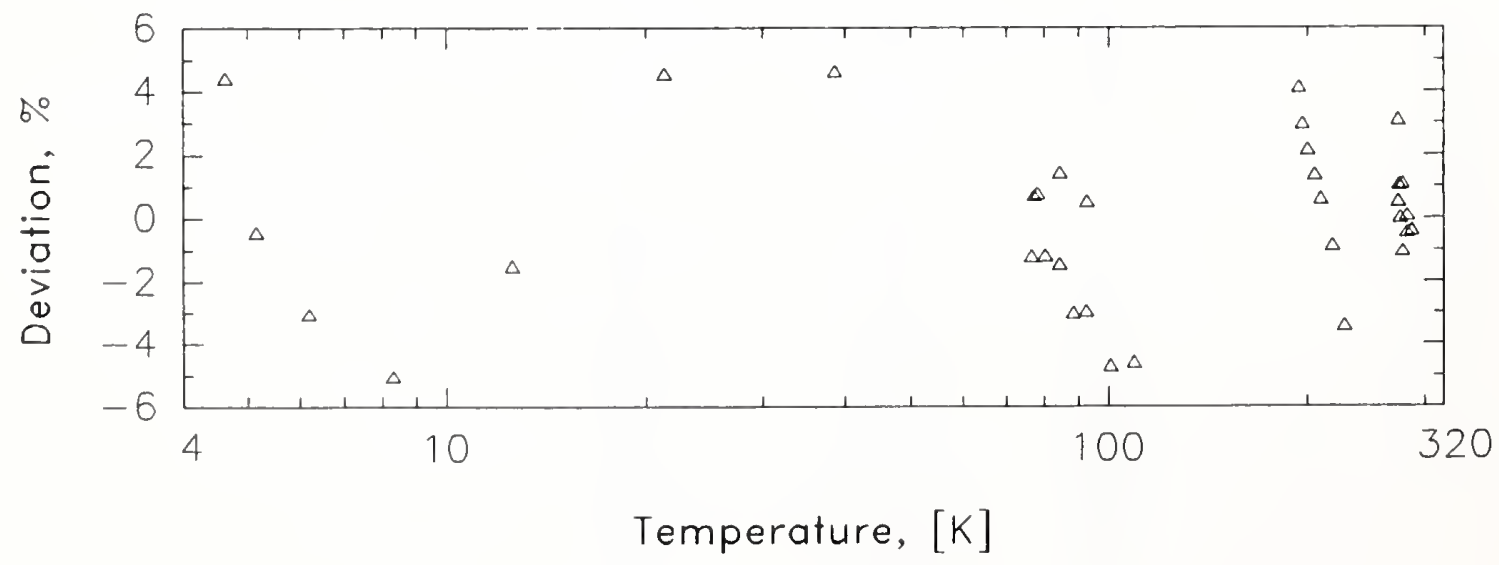

Figure 7.5. Relative deviations of the experimental and calculated thermal conductivity for the neat-resin specimen Resin E. 
Table 7.5. Experimental conductivity as a function of temperature for the neat-resin specimen Resin L.

\begin{tabular}{|c|c|c|c|}
\hline $\begin{array}{c}\text { Temperature } \\
\text { Difference } \\
(\Delta \mathrm{K})\end{array}$ & $\begin{array}{c}\text { Average } \\
\text { Temperature } \\
(\mathrm{K})\end{array}$ & $\begin{array}{c}\text { Thermal } \\
\text { Conductivity } \\
{[\mathrm{W} /(\mathrm{m} \cdot \mathrm{K})]}\end{array}$ & $\begin{array}{l}\text { Cold } \\
\text { Bath }\end{array}$ \\
\hline 1 & 4.638 & 0.045 & LHe \\
\hline 2 & 5.159 & 0.047 & \\
\hline 4 & 6.211 & 0.052 & \\
\hline 8 & 8.331 & 0.061 & \\
\hline 16 & 12.701 & 0.079 & \\
\hline 32.1 & 21.701 & 0.112 & \\
\hline 48.6 & 31.040 & 0.142 & \\
\hline 62.5 & 38.916 & 0.161 & \\
\hline 1 & 76.517 & 0.233 & $\mathrm{LN}_{2}$ \\
\hline 1 & 76.525 & 0.229 & \\
\hline 2 & 77.016 & 0.225 & \\
\hline 2 & 77.037 & 0.231 & \\
\hline 3.9 & 78.073 & 0.233 & \\
\hline 4 & 78.130 & 0.229 & \\
\hline 7.9 & 80.162 & 0.23 & \\
\hline 8 & 80.215 & 0.236 & \\
\hline 8 & 80.226 & 0.233 & \\
\hline 16 & 84.408 & 0.233 & \\
\hline 16 & 84.414 & 0.234 & \\
\hline 24 & 88.646 & 0.249 & \\
\hline 24 & 88.646 & 0.243 & \\
\hline 31.9 & 92.866 & 0.241 & \\
\hline 32 & 92.866 & 0.245 & \\
\hline 32 & 92.898 & 0.254 & \\
\hline 48 & 101.35 & 0.247 & \\
\hline 48 & 101.37 & 0.254 & \\
\hline 48 & 101.41 & 0.262 & \\
\hline 64 & 109.91 & 0.261 & \\
\hline 64 & 109.93 & 0.266 & \\
\hline 64 & 109.95 & 0.27 & \\
\hline 4.1 & 194.26 & 0.32 & $\mathrm{CO}_{2}$-alcohol \\
\hline 16 & 200.84 & 0.318 & \\
\hline 24 & 205.29 & 0.318 & \\
\hline 32 & 209.76 & 0.32 & \\
\hline 48 & 218.75 & 0.318 & \\
\hline 64 & 227.79 & 0.313 & \\
\hline 64 & 227.81 & 0.314 & \\
\hline 2 & 274.27 & 0.333 & Ice-water \\
\hline 4 & 275.39 & 0.333 & \\
\hline 8 & 277.61 & 0.329 & \\
\hline 16 & 282.09 & 0.331 & \\
\hline 24 & 286.60 & 0.334 & \\
\hline
\end{tabular}




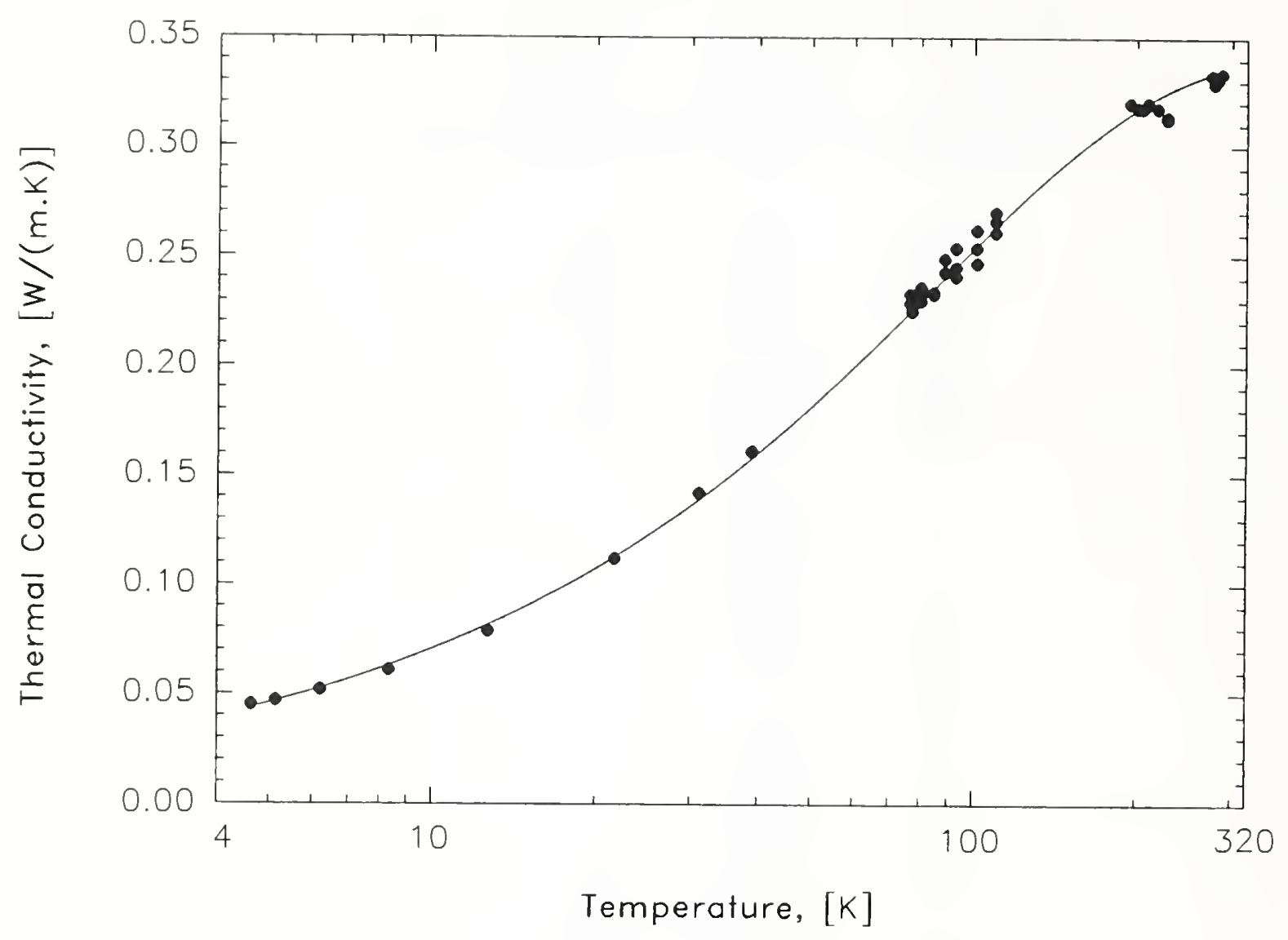

Figure 7.6. Thermal conductivity of the neat-resin specimen Resin L. Experimental data are presented as discrete points.

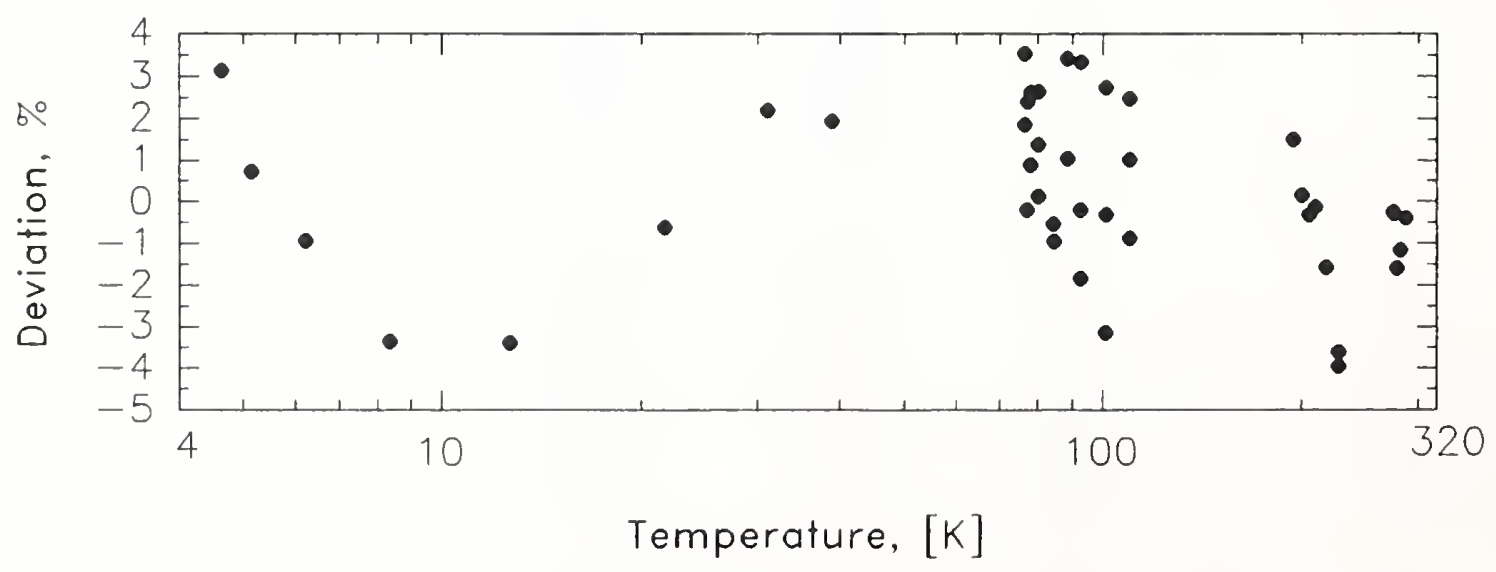

Figure 7.7. Relative deviations of the experimental and calculated thermal conductivity for the neat-resin specimen Resin L. 
Table 7.6. Experimental conductivity as a function of temperature for the neat-resin specimen Resin P.

\begin{tabular}{|c|c|c|c|}
\hline $\begin{array}{c}\text { Temperature } \\
\text { Difference } \\
(\Delta \mathrm{K})\end{array}$ & $\begin{array}{c}\text { Average } \\
\text { Temperature } \\
\text { (K) }\end{array}$ & $\begin{array}{c}\text { Thermal } \\
\text { Conductivity } \\
{[\mathrm{W} /(\mathrm{m} \cdot \mathrm{K})]}\end{array}$ & $\begin{array}{l}\text { Cold } \\
\text { Bath }\end{array}$ \\
\hline 1 & 4.632 & 0.030 & LHe \\
\hline 2 & 5.146 & 0.030 & \\
\hline 4 & 6.174 & 0.031 & \\
\hline 8 & 8.254 & 0.035 & \\
\hline 16 & 12.477 & 0.045 & \\
\hline 24 & 16.786 & 0.055 & \\
\hline 32 & 21.145 & 0.064 & \\
\hline 32 & 21.143 & 0.064 & \\
\hline 64.1 & 38.852 & 0.094 & \\
\hline 2 & 77.072 & 0.138 & $\mathrm{LN}_{2}$ \\
\hline 4 & 78.060 & 0.137 & \\
\hline 7.9 & 80.082 & 0.136 & \\
\hline 16 & 84.265 & 0.138 & \\
\hline 32 & 92.604 & 0.142 & \\
\hline 48 & 100.95 & 0.145 & \\
\hline 64 & 109.32 & 0.148 & \\
\hline 3.9 & 194.13 & 0.199 & $\mathrm{CO}_{2}$-alcohol \\
\hline 7.9 & 196.27 & 0.193 & \\
\hline 16 & 200.63 & 0.193 & \\
\hline 32 & 209.35 & 0.195 & \\
\hline 48 & 218.12 & 0.190 & \\
\hline 64 & 226.96 & 0.185 & \\
\hline 1 & 273.68 & 0.204 & Ice-water \\
\hline 2 & 274.23 & 0.200 & \\
\hline 4 & 275.36 & 0.199 & \\
\hline 8 & 277.53 & 0.198 & \\
\hline 8 & 277.54 & 0.196 & \\
\hline 16 & 281.93 & 0.196 & \\
\hline 24 & 286.34 & 0.195 & \\
\hline
\end{tabular}




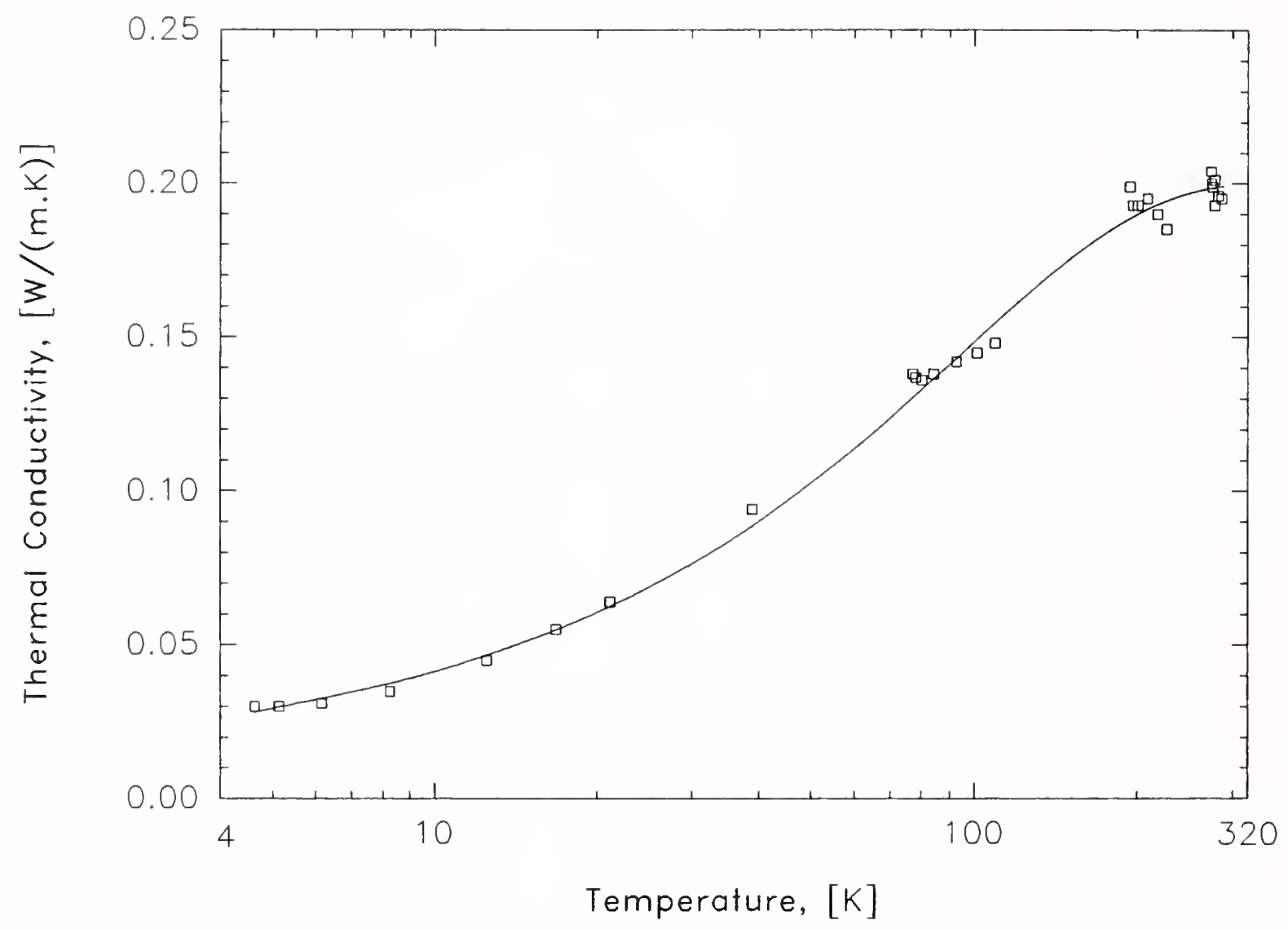

Figure 7.8. Thermal conductivity of the neat-resin specimen Resin P. Experimental data are presented as discrete points.

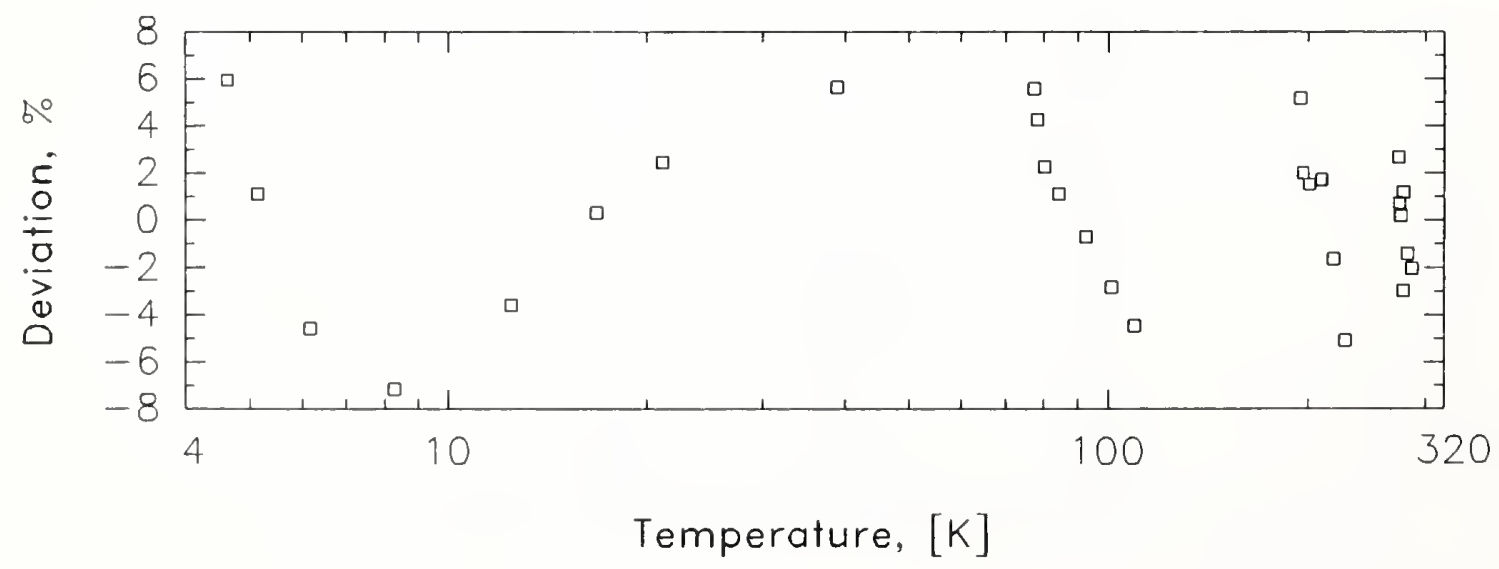

Figure 7.9. Relative deviations of the experimental and calculated thermal conductivity for the neat-resin specimen Resin P. 
Table 7.7. Experimental conductivity as a function of temperature for the neat-resin specimen Resin C.

\begin{tabular}{|c|c|c|c|}
\hline $\begin{array}{c}\text { Temperature } \\
\text { Difference } \\
(\Delta K)\end{array}$ & $\begin{array}{c}\text { Average } \\
\text { Temperature } \\
(\mathrm{K})\end{array}$ & $\begin{array}{l}\text { Thermal } \\
\text { Conductivity } \\
{[\mathrm{W} /(\mathrm{m} \cdot \mathrm{K})]}\end{array}$ & $\begin{array}{l}\text { Cold } \\
\text { Bath }\end{array}$ \\
\hline 1 & 4.637 & 0.042 & \multirow[t]{7}{*}{ LHe } \\
\hline 2 & 5.154 & 0.042 & \\
\hline 4 & 6.190 & 0.043 & \\
\hline 8 & 8.288 & 0.047 & \\
\hline 16 & 12.550 & 0.056 & \\
\hline 31.6 & 21.039 & 0.074 & \\
\hline 64.6 & 39.232 & 0.103 & \\
\hline 2 & 77.041 & 0.139 & \multirow[t]{7}{*}{$\mathrm{LN}_{2}$} \\
\hline 4 & 78.114 & 0.140 & \\
\hline 8 & 80.143 & 0.141 & \\
\hline 16 & 84.287 & 0.143 & \\
\hline 32 & 92.618 & 0.147 & \\
\hline 48 & 100.98 & 0.152 & \\
\hline 64 & 109.36 & 0.155 & \\
\hline 1 & 192.55 & 0.193 & \multirow{8}{*}{$\mathrm{CO}_{2}$-alcohol } \\
\hline 2 & 193.12 & 0.199 & \\
\hline 4 & 194.15 & 0.195 & \\
\hline 7.9 & 196.28 & 0.193 & \\
\hline 16 & 200.64 & 0.192 & \\
\hline 16 & 200.63 & 0.192 & \\
\hline 32 & 209.35 & 0.190 & \\
\hline 48 & 218.15 & 0.191 & \\
\hline 2 & 274.27 & 0.197 & \multirow[t]{4}{*}{ Ice-water } \\
\hline 4 & 275.33 & 0.195 & \\
\hline 16 & 281.93 & 0.194 & \\
\hline 24 & 286.35 & 0.195 & \\
\hline
\end{tabular}




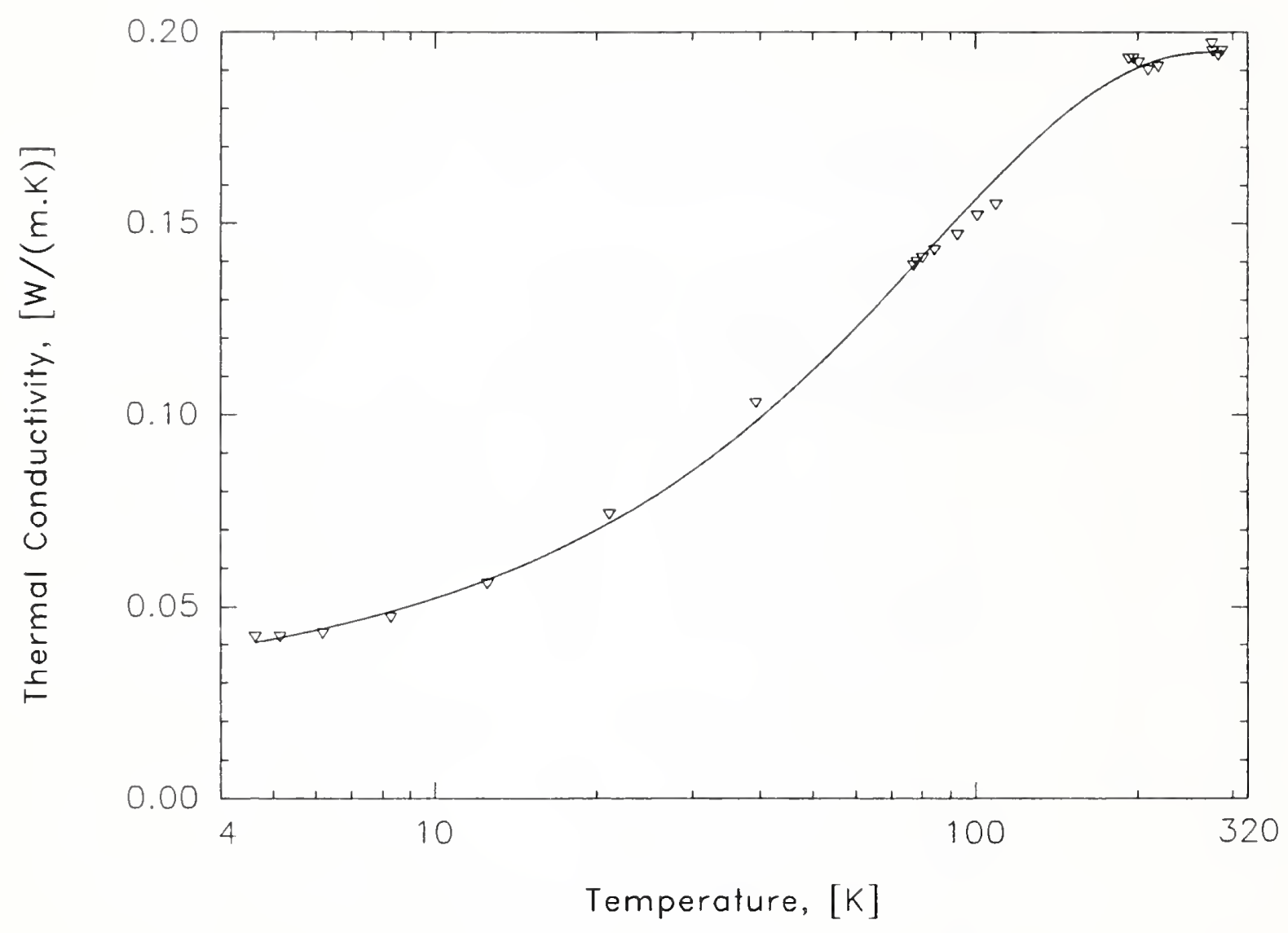

Figure 7.10. Thermal conductivity of the neat-resin specimen Resin C. Experimental data are presented as discrete points.

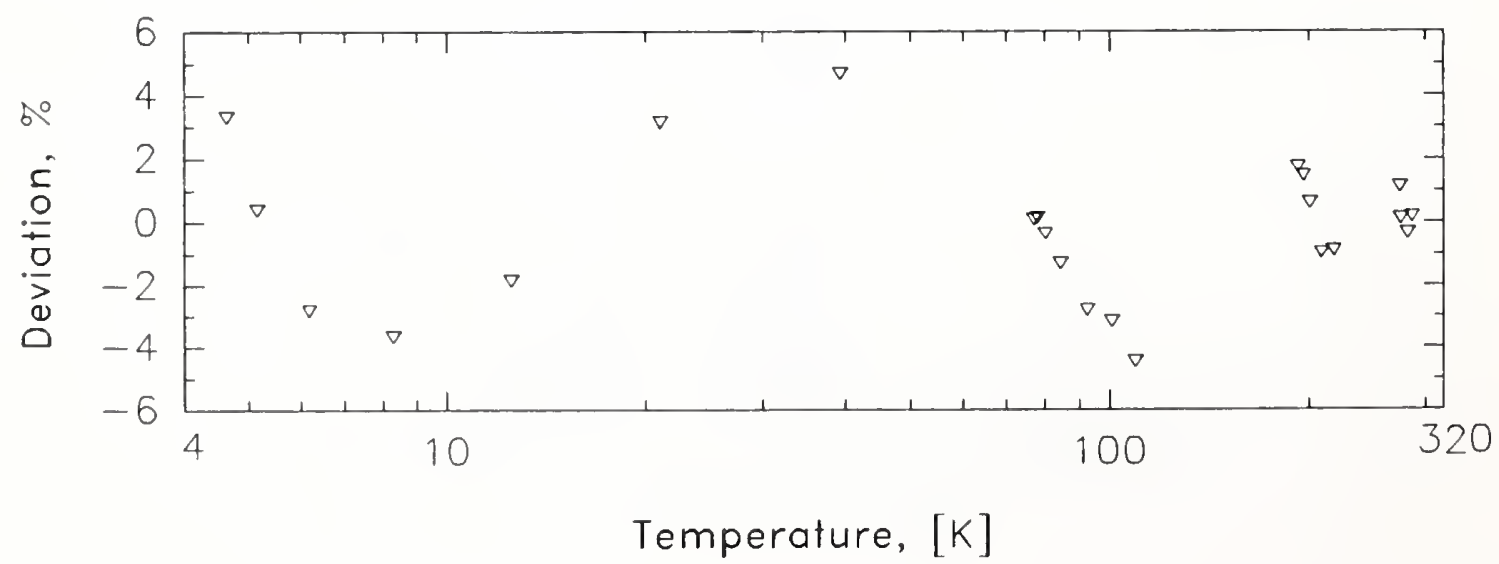

Figure 7.11. Relative deviations of the experimental and calculated thermal conductivity for the neat-resin specimen Resin C. 
The function chosen to fit the thermal conductivity data for each of the five neat-resin specimens is of the form

$$
\lambda(T)=\frac{a_{1}+a_{3} \ln (T)+a_{5}\{\ln (T)\}^{2}}{1+a_{2} \ln (T)+a_{4}\{\ln (T)\}^{2}} .
$$

The coefficients in this equation for the conductivity of the five neat-resin specimens are listed in Table 7.8 .

Table 7.8. Coefficients of $\lambda(T)$ in Equation 7.3 for the five neat-resin specimens.

\begin{tabular}{rrrrrr}
\hline \hline$a_{i}$ & Resin B & Resin E & Resin L & Resin P & \multicolumn{1}{c}{ Resin C } \\
\hline \hline 1 & 0.05557 & 0.03190 & 0.01446 & 0.01470 & 0.02992 \\
2 & -0.35843 & -0.33762 & -0.30000 & -0.31759 & -0.33241 \\
3 & -0.01643 & -0.00739 & 0.00800 & 0.00093 & -0.00592 \\
4 & 0.03410 & 0.03212 & 0.02676 & 0.02857 & 0.03143 \\
5 & 0.00152 & 0.00100 & -0.00020 & 0.00011 & 0.00087 \\
\hline \hline
\end{tabular}

\subsection{Discussion}

The conductivities of the five neat-resin specimens are compared in Figures 7.12 and 7.13. The polyester material had the lowest conductivity at $4 \mathrm{~K}$; it was $53 \%$ lower than the thermal conductivity of the DGEBA neat-resin specimen at that temperature. At room temperature, the thermal conductivity of Resin $\mathrm{L}$ was 40 to $50 \%$ higher than the conductivities of the other four neatresin specimens. As is noted in Table 7.1, Resin L contained an unknown filler. Inclusion of a filler affects the observed conductivity; the extent depends on the type and amount of filler. This unknown filler had little effect on the conductivity at $4 \mathrm{~K}$, but it is likely to be the cause for the higher conductivity at room temperature.

Figures 7.14 and 7.15 include the thermal-conductivity results from other studies. Previous measurements on DGEBA resin by Hust [7.1] compare very well to measurements of the same type of resin in this study (Resin $\mathrm{B}$ ) at $4 \mathrm{~K}$, but they diverge above $15 \mathrm{~K}$. This divergence may be due to added fillers, but the exact chemical composition of the resin tested was not known. Rosenberg's results on another DGEBA resin [7.3] also compare fairly well to Resin B. The results of Resin B also closely match the undefined epoxy that was noted by Evans and Morgan [7.4]. Also shown in the figures are the two specimens that had the maximum and minimum conductivity in a study to determine the effects of using various diamine hardeners in the DGEBA epoxy Araldite MY 750 [7.2]. Both of these curves show a higher conductivity than that of Resin B in this study.

The polyester resin (Resin P) measured in this study had the lowest conductivity at $4 \mathrm{~K}$. This compares closely to one neat-polyimide specimen (Type $\mathrm{H}$ ) previously reported but quite different from another neat specimen (Type HN) [7.5]. The two neat-polyimide specimens were manufactured differently, but the details of their manufacture are proprietary. 


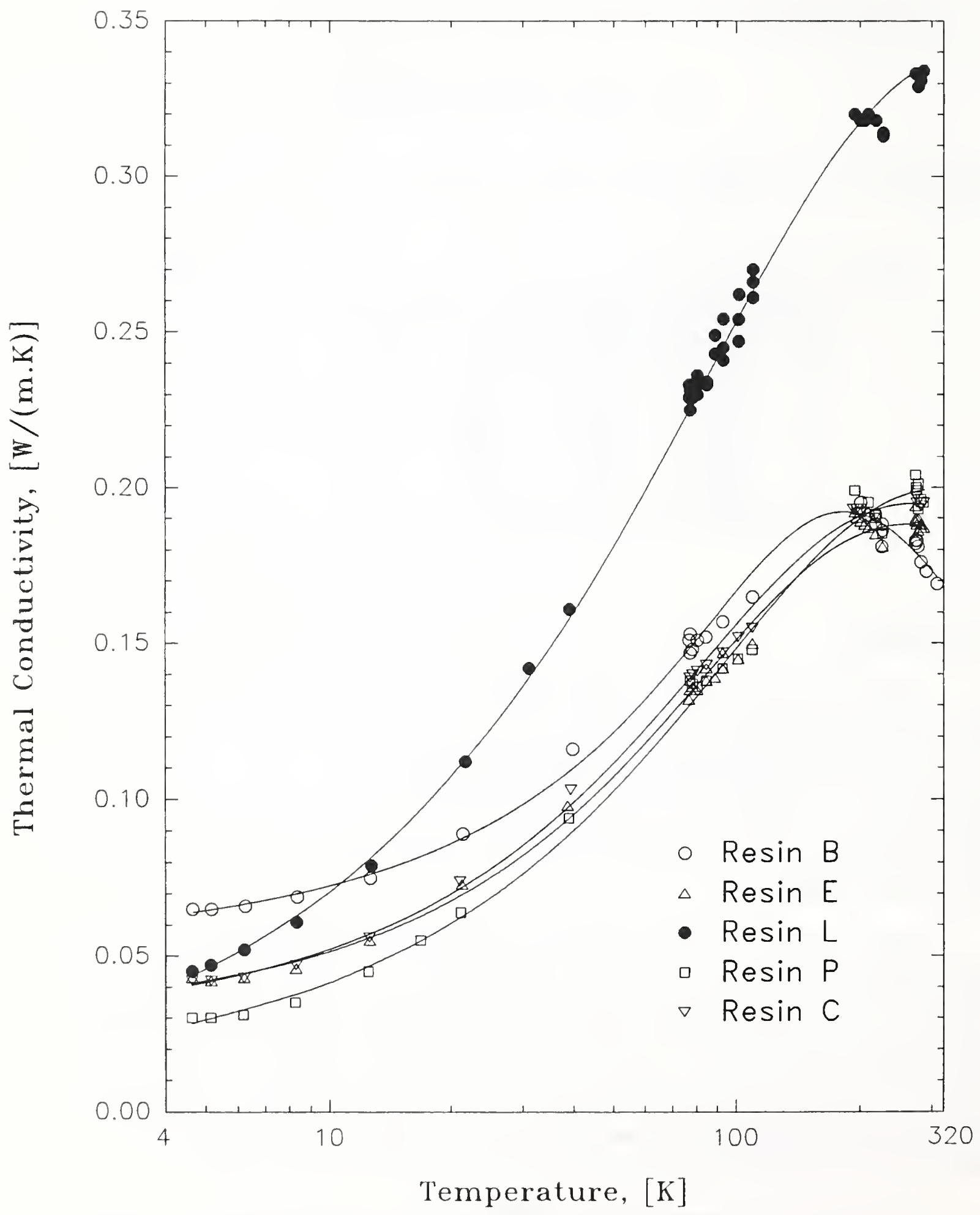

Figure 7.12. Thermal conductivity of the five neat-resin specimens measured in this study. Experimental data are presented as discrete points. The temperature scale is logarithmic, which clarifies the behavior at low temperatures. 


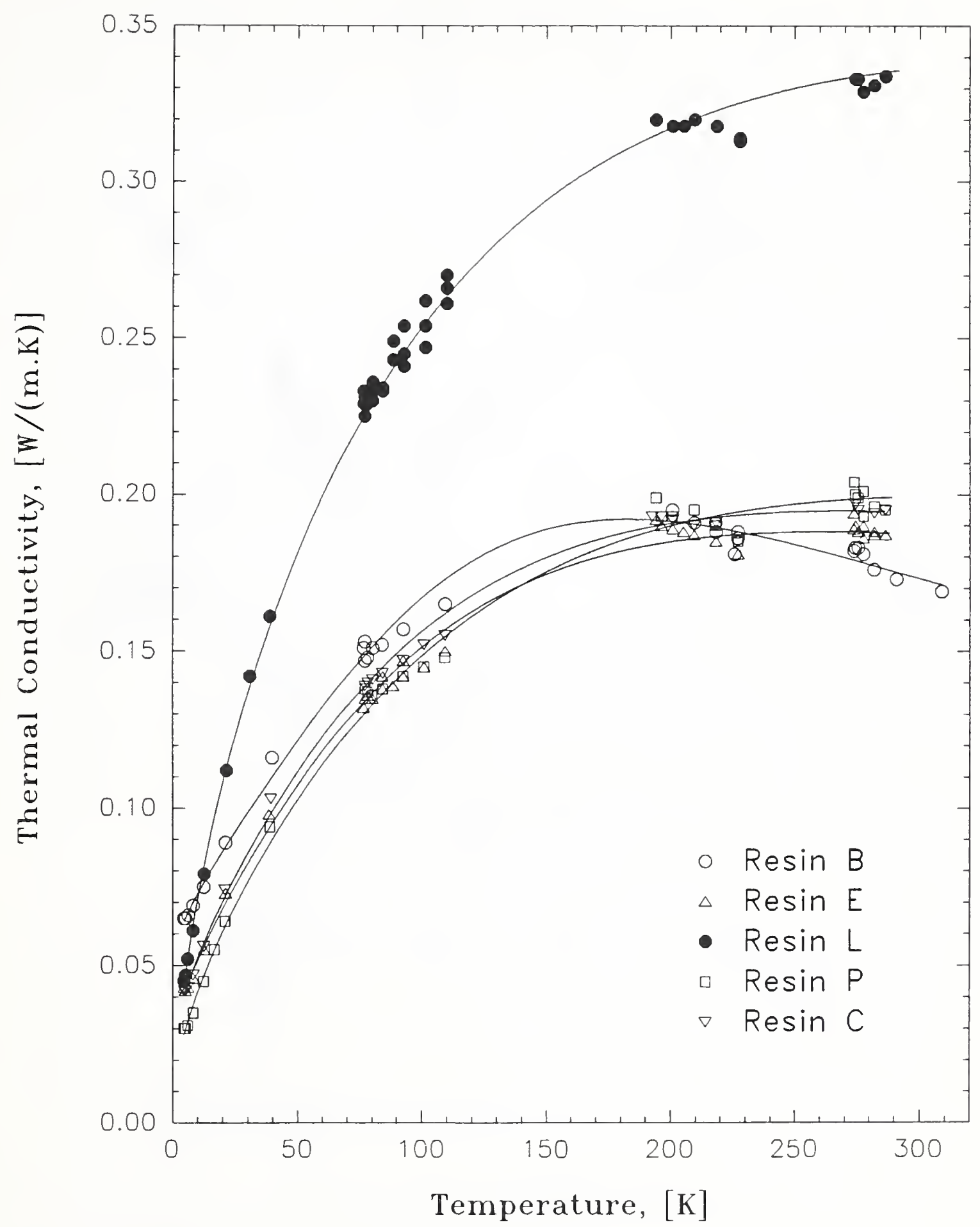

Figure 7.13 Thermal conductivity of the five neat-resin specimens measured in this study. Experimental data are presented as discrete points. The temperature scale is linear, which clarifies the behavior at high temperatures. 


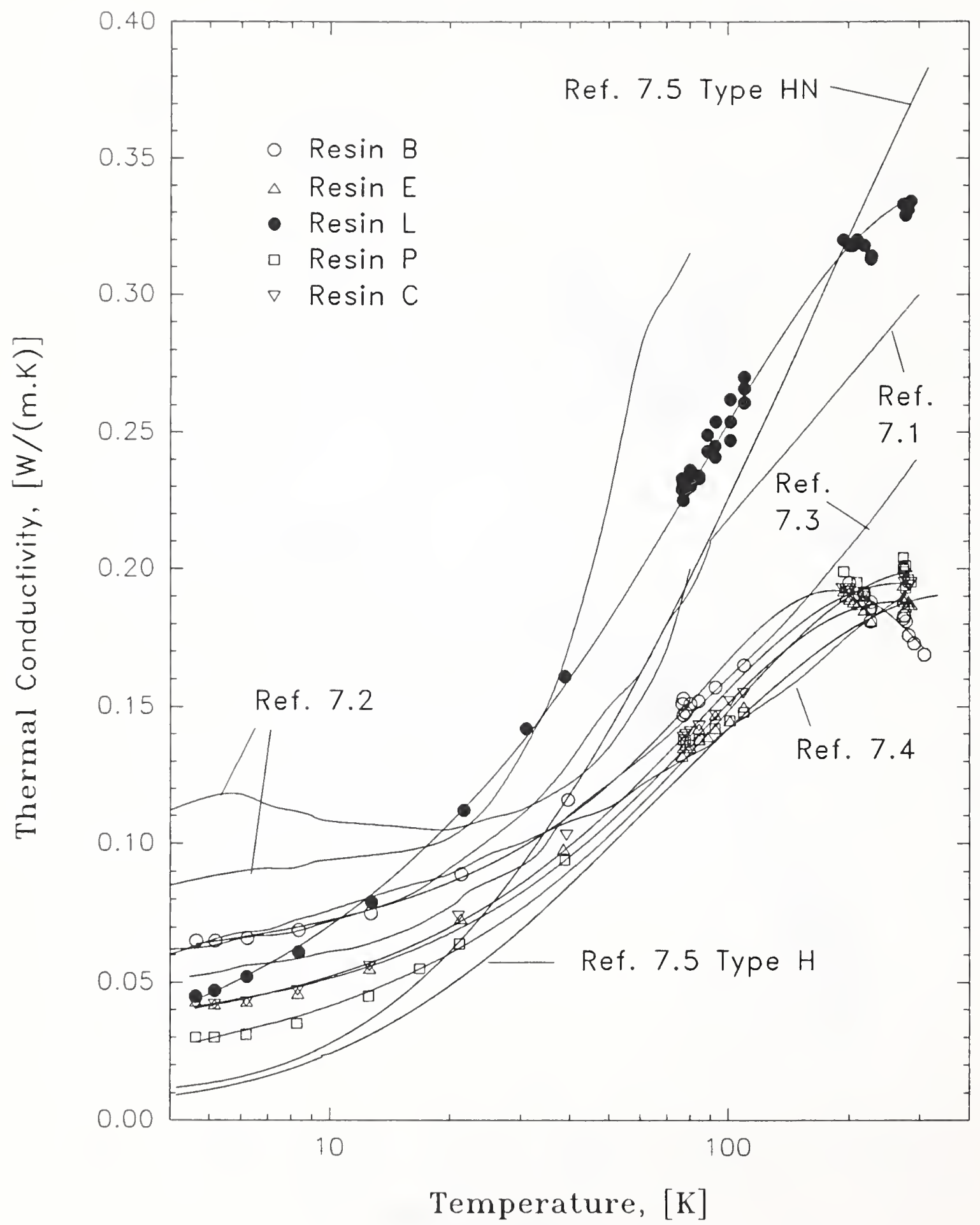

Figure 7.14 Thermal conductivity of the neat-resins measured in this study. Conductivity data for four other epoxies [7.1-7.4] and one polyimide [7.5] are also given for comparison. The temperature scale is logarithmic, which clarifies the behavior at low temperatures. 


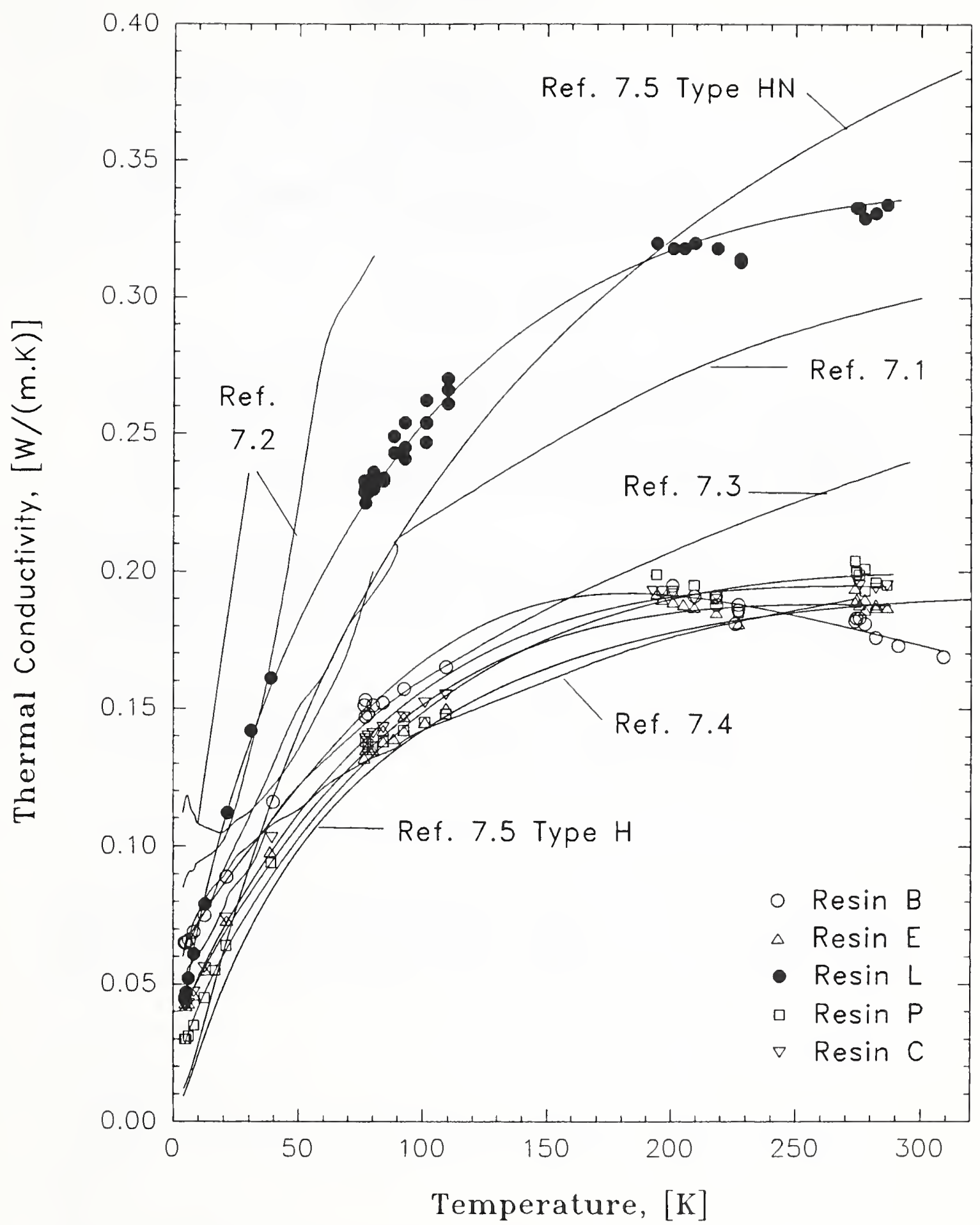

Figure 7.15 Thermal conductivity of the neat-resins measured in this study. Conductivity data for four other epoxies [7.1-7.4] and one polyimide [7.5] are also given for comparison. The temperature scale is linear, which clarifies the behavior at high temperatures. 


\title{
8. Thermal Cycling
}

\author{
R.P. Walsh \\ R.P. Reed ${ }^{*}$ \\ National Institute of Standards and Technology \\ Boulder, Colorado \\ ${ }^{*}$ Cryogenics Materials, Inc. \\ Boulder, Colorado
}

All neat resins were cycled within the temperature range 295 to $76 \mathrm{~K}$. The cooling was accomplished by rapid immersion in liquid nitrogen; the warming, by holding in air at ambient temperature. The thermal cycles were repeated 10 times. Specimens with rough edges and specimens with smooth edges were used.

None of the specimens with smooth edges cracked from thermal cycling. When specimens with rough edges were used, ten different types of neat resins cracked: four epoxies, three cyanate esters, two vinyl esters, and the polyester.

These tests were not considered to be sufficiently definitive to provide guidance for selection of resins for glass-fiber-reinforced composites that are to be used in cryogenic structures.

\subsection{Introduction}

Many resin-selection programs for cryogenic applications have included thermalcycling tests. Simple thermal-cycling procedures and specimen configurations were used in this program to assess the effect of cycling neat resins between room and liquid-nitrogen temperature.

\subsection{Materials}

The materials included in this study have been discussed in $§ I I I .3$ of this report.

\subsection{Procedures}

Tensile specimens were used for the thermal-cycling tests. The cool-down to $76 \mathrm{~K}$ was accomplished by using a cage that contained three specimens. The cage was quickly lowered into liquid nitrogen boiling at atmospheric pressure. The warm-up from $76 \mathrm{~K}$ to room temperature took about $10 \mathrm{~min}$, since the specimens were simply allowed to warm in still, ambient-temperature air. 


\subsection{Results}

The response of neat resins to 10 thermal cycles between 295 and $76 \mathrm{~K}$ is summarized in Table 8:1. Specimens that had only smooth (machined) edges did not crack during cycling. Specimens that had a rough edge (previously tested tensile specimens that contained a fracture surface) sometimes cracked during cycling. All cracking occurred during cool-down. As Table 8.1 indicates, the specimens that cracked included four epoxies, three cyanate esters, two vinyl esters, and the polyester. A few specimens exhibited extensive cracking: one epoxy, one cyanate ester, and one vinyl ester.

Table 8.1. Results of thermal-cycling tests ( 295 to $76 \mathrm{~K} ; 10$ cycles).

\begin{tabular}{|c|c|c|c|c|}
\hline \multirow[t]{2}{*}{ Resin Type } & \multicolumn{2}{|c|}{$\begin{array}{l}\text { Specimens with } \\
\text { Rough Edges }\end{array}$} & \multicolumn{2}{|c|}{$\begin{array}{l}\text { Specimens with } \\
\text { Smooth Edges }\end{array}$} \\
\hline & No Cracks & Cracking & No Cracks & Cracking \\
\hline NV/AN/ACL & $\mathrm{O}$ & & $\mathrm{O}$ & \\
\hline DGEBA/DCH/ARA/ACL & $\mathrm{O}$ & & $\mathrm{O}$ & \\
\hline VE-EL/MEKP & $\mathrm{O}$ & & $\mathrm{O}$ & \\
\hline DGEBA/ALA/ACL & & $\mathrm{X}$ & $\mathrm{O}$ & \\
\hline DGEBA/CTBN/AN & & $\mathrm{X}$ & $\mathrm{O}$ & \\
\hline Cyclo.A/CTBN/AN/ACL & & $\mathrm{XX}$ & $\mathrm{O}$ & \\
\hline CE/VT/Co.AA & & $\mathrm{X}$ & $\mathrm{O}$ & \\
\hline CT/HTBN/NPh/Cu.AA & $\mathrm{O}$ & & $\mathrm{O}$ & \\
\hline Cyclo.A/AN/ACL & & $\mathrm{X}$ & $\mathrm{O}$ & \\
\hline MLTF/ARA & & $\mathrm{X}$ & $\mathrm{O}$ & \\
\hline DGEBA-ST/ARA/ACL/F & $\mathrm{O}$ & & $\mathrm{O}$ & \\
\hline $\mathrm{VE} / \mathrm{CHX}$ & $\mathrm{O}$ & & $\mathrm{O}$ & \\
\hline $\mathrm{VE} / \mathrm{CHP} / \mathrm{F}$ & & $\mathrm{X}$ & $\mathrm{O}$ & \\
\hline VE/CHP/CoNap/2,4-P & & $\mathrm{XX}$ & $\mathrm{O}$ & \\
\hline PE/MEKP & & $\mathrm{X}$ & $\mathrm{O}$ & \\
\hline $\mathrm{CE}$ & $\mathrm{O}$ & & $\mathrm{O}$ & \\
\hline CE/HTBN/NPh/Cu.AA & & $\mathrm{XX}$ & $\mathrm{O}$ & \\
\hline
\end{tabular}

$\mathrm{X}$ - Small cracks

$\mathrm{XX}$ - Extensive cracking 


\subsection{Discussion}

There appears to be no correlation of the tendency to crack during thermal cycles and resin composition ( $\$ I I I .3)$, thermal contraction ( $\$ I I I .6)$, or thermal conductivity ( $\$ I I I .7)$. Therefore, perhaps the local intensity of residual stresses from the tensile failure led to cracking during the thermal cycling of individual specimens. For this reason, we chose not to use these data to assist in screening resin candidates. However, in defense of these results, the neat resins that were the most difficult to grip in the low-temperature tensile tests (CE/HTBN/NPh/Cu.AA and MLTF/ARA) were the ones that cracked during these thermal-cycling tests. 


\title{
9. Density and Hardness
}

\author{
R.P. Reed ${ }^{*}$ \\ J.D. McColskey \\ J.B. $\operatorname{Darr}^{\dagger}$ \\ National Institute of Standards and Technology \\ Boulder, Colorado \\ ${ }^{*}$ Cryogenics Materials, Inc. \\ Boulder, Colorado \\ ${ }^{\dagger}$ Composite Technology Development, Inc. \\ Boulder, Colorado
}

\subsection{Introduction}

The characterization of the resin systems, which are described in Table 2.1 of $\$$ III.2, included measurement of their density and hardness. The materials are referred to by the nomenclature defined in Table 2.2 of $\S I I I .2$.

\subsection{Hardness}

The materials tested were manufactured approximately nine months before these measurements were made. For two weeks prior to testing, the materials were conditioned at $22^{\circ} \mathrm{C}$ and approximately $50 \%$ relative humidity. The test temperature at the time of test was $22^{\circ} \mathrm{C}$.

The indentation hardness of the neat resins was determined according to ASTM Standard Test Method D2583-81 [9.1]. Nine readings were made on each sample; the averaged results are reported in Table 9.1.

The largest hardness values were found in those resin systems that had fillers, were multifunctional, or, in the case of CE(PT), contained phenolic triazine. Surprisingly, the DGEBF/CTBN/AN epoxy-resin system was very soft. Correlations between hardness, density, and other properties are presented and discussed in §III. 10.

\subsection{Density}

The density of the neat resins was measured by using a Messler balance and the Archimedes method. In the method, a neat-resin specimen of known weight in air ( $\left.W_{\mathrm{air}}\right)$ was immersed in an ethanol solution and weighed ( $\left.W_{\mathrm{ETOH}}\right)$. From knowledge of the ethanol density $\left(\rho_{\mathrm{ETOH}}\right)$ at the measured 
Table 9.1 Resin hardness.

\begin{tabular}{lc}
\hline \multicolumn{1}{c}{ Type of Resin } & $\begin{array}{c}\text { Average } \\
\text { Barcol Hardness }\end{array}$ \\
\hline \hline Epoxies: & \\
DGEBA/DCH/ARA/ACL & 34 \\
DGEBA/ALA/ACL & 30 \\
DGEBA-ST/ARA/ACL/F & 49 \\
DGEBF/CTBN/AN & 15 \\
NV/AN/ACL & 40 \\
Cyclo.A/CTBN/AN/ACL & 43 \\
Cyclo.A/AN/ACL & 45 \\
MLTF/ARA & 52 \\
Cyanate Esters: & \\
CE/VT/Co.AA & \\
CE & 47 \\
CE(PT) & \\
CE/HTBN/NPh/Cu.AA & 40 \\
Vinyl Esters: & 57 \\
VE/CHX & 42 \\
VE-EL/MEKP & \\
VE/CHP/F & \\
VE/CHP/CoNap/2,4-P & 31 \\
Polyesters: & 35 \\
PE/MEKP & $49-55$ \\
\hline \hline
\end{tabular}

*See comments regarding cure schedule on page III-16.

laboratory temperature and knowledge of the volume of the container, the density of the neat resin $\left(\rho_{\text {sample }}\right)$ can be calculated from the equations

and

$$
V_{\text {sample }}=\rho_{\mathrm{ETOH}} /\left(W_{\text {air }}-W_{\mathrm{ETOH}}\right)
$$

$$
\rho_{\text {sample }}=W_{\text {air }} / V_{\text {sample }} \text {. }
$$

This procedure is specified in ASTM Standard D3800-81 [9.2]. In Table 9.2, individual specimen measurements and calculated average densities for each resin system are summarized. Standard deviations (S.D.) and coefficients of variation (C.V.) are also included in this table.

From this study of the resin systems, there were no clear distinctions among their densities at room temperature. Filled and multifunctional systems had higher densities than the other systems. 
The average densities of the other epoxy-based resins ranged from 1.143 to $1.229 \mathrm{~g} / \mathrm{cm}^{3}$; of cyanate esters, from 1.143 to $1.186 \mathrm{~g} / \mathrm{cm}^{3}$; and of vinyl esters and polyester, from 1.116 to $1.246 \mathrm{~g} / \mathrm{cm}^{3}$.

Table 9.2. Density of neat-resin systems.

\begin{tabular}{|c|c|c|c|c|c|c|c|c|c|}
\hline Material & & $\begin{array}{l}\text { Ethanol } \\
\text { Temp. } \\
\left({ }^{\circ} \mathrm{C}\right)\end{array}$ & $\begin{array}{l}\text { Ethanol } \\
\text { Density } \\
\left(\mathrm{g} / \mathrm{cm}^{3}\right)\end{array}$ & $\begin{array}{c}\text { Specimen } \\
\text { Weight } \\
\text { (in air) } \\
\text { (g) }\end{array}$ & $\begin{array}{c}\text { Specimen } \\
\text { Weight } \\
\text { (immersed) } \\
(\mathrm{g})\end{array}$ & $\begin{array}{l}\text { Density } \\
\left(\mathrm{g} / \mathrm{cm}^{3}\right)\end{array}$ & $\begin{array}{l}\text { Average } \\
\text { Density } \\
\left(\mathrm{g} / \mathrm{cm}^{3}\right)\end{array}$ & $\begin{array}{l}\text { S.D. } \\
\left(\mathrm{g} / \mathrm{cm}^{3}\right)\end{array}$ & $\begin{array}{l}\text { C.V. } \\
(\%)\end{array}$ \\
\hline \multirow{3}{*}{ DGEBF/CTBN/AN } & $\# 1$ & 18.8 & 0.79046 & 0.2676 & 0.0935 & 1.215 & 1.215 & 0.002 & 0.18 \\
\hline & \#2 & 18.9 & 0.79037 & 0.2992 & 0.1050 & 1.218 & & & \\
\hline & $\# 3$ & 18.9 & 0.79037 & 0.3285 & 0.1145 & 1.213 & & & \\
\hline \multirow[t]{3}{*}{ NV/AN/ACL } & $\# 1$ & 19.0 & 0.79029 & 0.2666 & 0.0944 & 1.224 & 1.229 & 0.006 & 0.46 \\
\hline & $\# 2$ & 18.9 & 0.79037 & 0.3027 & 0.1080 & 1.229 & & & \\
\hline & $\# 3$ & 19.0 & 0.79029 & 0.2856 & 0.1028 & 1.235 & & & \\
\hline \multirow[t]{3}{*}{ MLTF/ARA/TGH } & $\# 1$ & 19.1 & 0.79021 & 0.3398 & 0.1353 & 1.313 & 1.318 & 0.006 & 0.46 \\
\hline & $\# 2$ & 19.1 & 0.79021 & 0.3226 & 0.1302 & 1.325 & & & \\
\hline & $\# 3$ & 19.1 & 0.79021 & 0.3401 & 0.1360 & 1.317 & & & \\
\hline \multirow[t]{3}{*}{$\mathrm{VE} / \mathrm{CHX}$} & $\# 1$ & 19.2 & 0.79012 & 0.2911 & 0.0891 & 1.139 & 1.134 & 0.005 & 0.40 \\
\hline & $\# 2$ & 19.2 & 0.79012 & 0.2017 & 0.0612 & 1.134 & & & \\
\hline & $\# 3$ & 19.2 & 0.79012 & 0.2819 & 0.0847 & 1.129 & & & \\
\hline \multirow[t]{3}{*}{ PE/MEKP } & $\# 1$ & 19.2 & 0.79012 & 0.3020 & 0.0985 & 1.173 & 1.171 & 0.002 & 0.20 \\
\hline & $\# 2$ & 18.2 & 0.79096 & 0.3027 & 0.0985 & 1.172 & & & \\
\hline & $\# 3$ & 18.2 & 0.79096 & 0.2535 & 0.0819 & 1.168 & & & \\
\hline \multirow[t]{3}{*}{ VE/CHP/F } & $\# 1$ & 18.4 & 0.79079 & 0.2623 & 0.1016 & 1.291 & 1.309 & 0.019 & 0.42 \\
\hline & $\# 2$ & 18.4 & 0.79079 & 0.3748 & 0.1516 & 1.328 & & & \\
\hline & $\# 3$ & 18.6 & 0.79063 & 0.3274 & 0.1297 & 1.309 & & & \\
\hline \multirow[t]{3}{*}{ VE-EL/MEKP } & $\# 1$ & 18.7 & 0.79054 & 0.2725 & 0.0791 & 1.114 & 1.116 & 0.002 & 0.15 \\
\hline & $\# 2$ & 18.6 & 0.79063 & 0.2689 & 0.0786 & 1.117 & & & \\
\hline & $\# 3$ & 18.6 & 0.79063 & 0.2689 & 0.0786 & 1.116 & & & \\
\hline \multirow[t]{3}{*}{$\mathrm{CE}$} & $\# 1$ & 18.7 & 0.79054 & 0.2501 & 0.0825 & 1.180 & 1.178 & 0.002 & 0.17 \\
\hline & $\# 2$ & 18.7 & 0.79054 & 0.3313 & 0.1092 & 1.179 & & & \\
\hline & $\# 3$ & 18.7 & 0.79054 & 0.2682 & 0.0879 & 1.176 & & & \\
\hline \multirow{3}{*}{$\begin{array}{l}\text { Cyclo.A/CTBN/ } \\
\text { AN/ACL }\end{array}$} & $\# 1$ & 18.8 & 0.79046 & 0.2673 & 0.0920 & 1.205 & 1.208 & 0.002 & 0.18 \\
\hline & $\# 2$ & 18.8 & 0.79046 & 0.2831 & 0.0978 & 1.208 & & & \\
\hline & $\# 3$ & 18.8 & 0.79046 & 0.3359 & 0.1164 & 1.210 & & & \\
\hline \multirow{3}{*}{$\begin{array}{l}\mathrm{CE} / \mathrm{HTBN} / \mathrm{NPh} / \\
\mathrm{Cu} . \mathrm{AA}\end{array}$} & $\# 1$ & 18.9 & 0.79037 & 0.2925 & 0.0899 & 1.141 & 1.143 & 0.002 & 0.14 \\
\hline & $\# 2$ & 18.9 & 0.79037 & 0.3115 & 0.0963 & 1.144 & & & \\
\hline & $\# 3$ & 18.9 & 0.79037 & 0.2950 & 0.9011 & 1.144 & & & \\
\hline
\end{tabular}


Table 9.2. Density of neat-resin systems (continued).

\begin{tabular}{|c|c|c|c|c|c|c|c|c|c|}
\hline Material & & $\begin{array}{l}\text { Ethanol } \\
\text { Temp. } \\
\left({ }^{\circ} \mathrm{C}\right)\end{array}$ & $\begin{array}{l}\text { Ethanol } \\
\text { Density } \\
\left(\mathrm{g} / \mathrm{cm}^{3}\right)\end{array}$ & $\begin{array}{l}\text { Specimen } \\
\text { Weight } \\
\text { (in air) } \\
\text { (g) }\end{array}$ & $\begin{array}{c}\text { Specimen } \\
\text { Weight } \\
\text { (immersed) } \\
\text { (g) }\end{array}$ & $\begin{array}{l}\text { Density } \\
\left(\mathrm{g} / \mathrm{cm}^{3}\right)\end{array}$ & $\begin{array}{l}\text { Average } \\
\text { Density } \\
\left(\mathrm{g} / \mathrm{cm}^{3}\right)\end{array}$ & $\begin{array}{l}\text { S.D. } \\
\left(\mathrm{g} / \mathrm{cm}^{3}\right)\end{array}$ & $\begin{array}{l}\text { C.V. } \\
(\%)\end{array}$ \\
\hline \multirow{3}{*}{$\begin{array}{l}\text { DGEBA-ST/ARA/ } \\
\mathrm{ACL} / \mathrm{F}\end{array}$} & $\# 1$ & 19.0 & 0.79029 & 0.2750 & 0.1048 & 1.277 & \multirow[t]{3}{*}{1.280} & \multirow[t]{3}{*}{0.003} & \multirow[t]{3}{*}{0.22} \\
\hline & $\# 2$ & 19.0 & 0.79029 & 0.2882 & 0.1106 & 1.282 & & & \\
\hline & \#3 & 19.0 & 0.79029 & 0.2661 & 0.1018 & 1.280 & & & \\
\hline \multirow{3}{*}{$\begin{array}{l}\text { DGEBA/DCH/ } \\
\text { ARA/ACL }\end{array}$} & $\# 1$ & 19.0 & 0.79029 & 0.2775 & 0.0885 & 1.160 & \multirow[t]{3}{*}{1.162} & \multirow[t]{3}{*}{0.002} & \multirow[t]{3}{*}{0.15} \\
\hline & $\# 2$ & 19.0 & 0.79029 & 0.2837 & 0.0910 & 1.163 & & & \\
\hline & $\# 3$ & 19.0 & 0.79029 & 0.2488 & 0.0794 & 1.161 & & & \\
\hline \multirow[t]{3}{*}{ Cyclo.A/AN/ACL } & $\# 1$ & 19.1 & 0.79021 & 0.3355 & 0.1192 & 1.226 & \multirow[t]{3}{*}{1.223} & \multirow[t]{3}{*}{0.003} & \multirow[t]{3}{*}{0.23} \\
\hline & $\# 2$ & 19.0 & 0.79029 & 0.2713 & 0.0958 & 1.222 & & & \\
\hline & $\# 3$ & 19.0 & 0.79029 & 0.2880 & 0.1015 & 1.220 & & & \\
\hline \multirow[t]{3}{*}{$\mathrm{CE}(\mathrm{PT})^{*}$} & $\# 1$ & 19.1 & 0.79021 & 0.2401 & 0.0931 & 1.291 & \multirow[t]{3}{*}{1.289} & \multirow[t]{3}{*}{0.001} & \multirow[t]{3}{*}{0.10} \\
\hline & $\# 2$ & 19.1 & 0.79021 & 0.3250 & 0.1258 & 1.289 & & & \\
\hline & $\# 3$ & 19.0 & 0.79029 & 0.3159 & 0.1221 & 1.288 & & & \\
\hline \multirow{3}{*}{$\begin{array}{l}\text { VE/CHP/CoNap/ } \\
2,4-\mathrm{P}\end{array}$} & $\# 1$ & 19.1 & 0.79021 & 0.2478 & 0.0907 & 1.246 & \multirow[t]{3}{*}{1.246} & \multirow[t]{3}{*}{0.001} & \multirow[t]{3}{*}{0.06} \\
\hline & $\# 2$ & 19.1 & 0.79021 & 0.2938 & 0.1075 & 1.246 & & & \\
\hline & $\# 3$ & 19.1 & 0.79021 & 0.2677 & 0.0978 & 1.246 & & & \\
\hline \multirow[t]{3}{*}{ CE/VT/Co.AA } & $\# 1$ & 19.1 & 0.79021 & 0.2423 & 0.0806 & 1.184 & \multirow[t]{3}{*}{1.186} & \multirow[t]{3}{*}{0.003} & \multirow[t]{3}{*}{0.24} \\
\hline & $\# 2$ & 19.1 & 0.79021 & 0.2465 & 0.0827 & 1.189 & & & \\
\hline & $\# 3$ & 19.1 & 0.79021 & 0.2912 & 0.0969 & 1.184 & & & \\
\hline \multirow[t]{3}{*}{ MLTF/ARA } & $\# 1$ & 19.1 & 0.79021 & 0.2692 & 0.1011 & 1.265 & \multirow[t]{3}{*}{1.264} & \multirow[t]{3}{*}{0.002} & \multirow[t]{3}{*}{0.12} \\
\hline & $\# 2$ & 19.0 & 0.79029 & 0.3392 & 0.1270 & 1.263 & & & \\
\hline & $\# 3$ & 19.1 & 0.79021 & 0.2791 & 0.1044 & 1.262 & & & \\
\hline \multirow[t]{3}{*}{ DGEBA/ALA/ACL } & $\# 1$ & 19.1 & 0.79021 & 0.2514 & 0.0778 & 1.144 & \multirow[t]{3}{*}{1.143} & \multirow[t]{3}{*}{0.001} & \multirow[t]{3}{*}{0.11} \\
\hline & $\# 2$ & 19.1 & 0.79021 & 0.3043 & 0.0938 & 1.142 & & & \\
\hline & $\# 3$ & 19.1 & 0.79021 & 0.2418 & 0.0745 & 1.142 & & & \\
\hline
\end{tabular}

S.D. = standard deviation; C.V. = coefficient of variation

See comments regarding cure schedule on page III-16.

\subsection{Comparison of Hardness and Density}

The relationship between neat-resin density and hardness at room temperature is shown in Figure 9.1. A line depicting the linear relationship between the two properties for the epoxies of this program is also drawn in this figure. The four resin systems that appear to have higher hardness than the other systems (CE/VT/Co.AA, PE/MEKP, CE/HTBN/NPh/Cu.AA, VE-EL/MEKP) have little in common since they represent cyanate-ester-, polyester-, and vinyl-ester-base resins. 
The datum point for the DGEBF/CTBN/AN epoxy resin is very curious. For this resin, the hardness was checked repeatedly on fifteen tensile specimens and found to range from 10 to 17 . The density and elastic properties of epoxy resins (see \$III.4 and §III.5) are normal for epoxy resins; only the hardness is anomalous. In this system, there is a very large amount of flexibilizer, and a twophase structure is likely to be produced. We measured the hardness of a DGEBA epoxy system without a large amount of flexibilizer, as produced in plate form by the resin supplier, and found an average hardness of about 35. A possible explanation for the low hardness data is that the softer phase dominates the microproperties, as typified by hardness measured with a pin-type loading system, and the stronger phase dominates the macroproperties, such as shear and tensile strength. Additional work on this interesting resin system may prove rewarding in increasing our understanding of the mechanical properties of multiphase neat-resin systems.

In summary, as expected, there was good correlation between the density and hardness of neat-resin systems measured at room temperature. Their linear dependency appears to be independent of the type of resin system, although there is less data scatter for the epoxy systems than for the other resin systems.

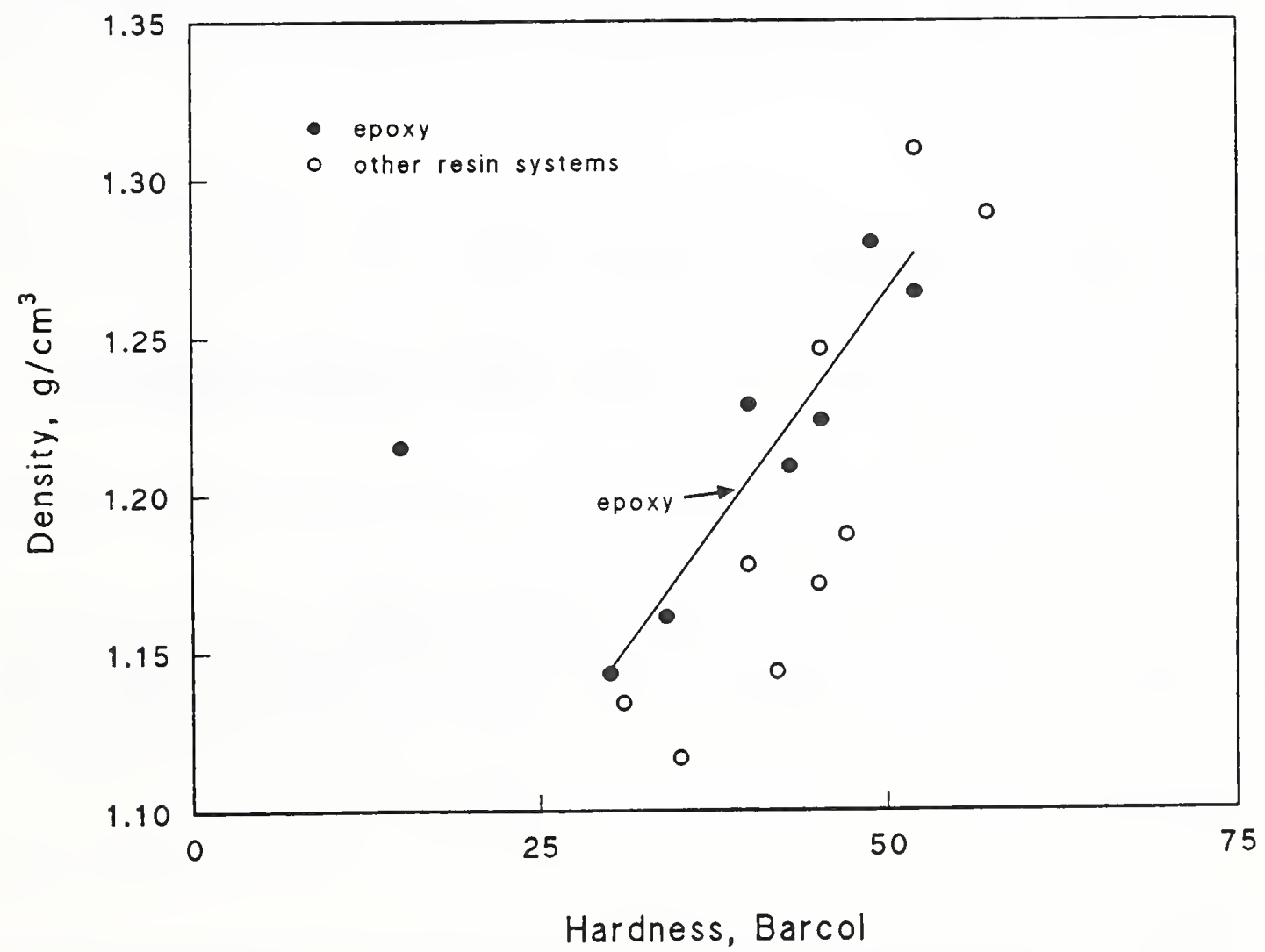

Figure 9.1. Density versus hardness at room temperature. 


\title{
10. Dynamic Elastic Properties
}

\author{
Sudook Kim and Hassel Ledbetter \\ Materials Reliability Division \\ National Institute of Standards and Technology \\ Boulder, Colorado
}

We measured the dynamic elastic constants of resins from five polymer classes: acrylate, cyanate ester, epoxy, polyester, and vinyl ester. We used two methods: Marx composite oscillator $(30 \mathrm{kHz})$ and pulse-echo superposition (1 and $5 \mathrm{MHz}$ ). The first method gives the Young's (extensional) modulus and the associated internal friction. The second method gives the complete set of isotropic elastic constants. Here, we report Young's, shear, bulk, and longitudinal moduli together with Poisson's ratio. Among the materials studied, the Poisson's ratio was the most consistent property, with a standard deviation of only $4 \%$. Young's moduli varied by a factor of 1.6. For comparison, we measured the same properties of a wellcharacterized polymer, isotropic polymethylmethacrylate (PMMA).

\subsection{Introduction}

Elastic properties play at least three important roles: engineering-design parameters, materialcharacterization parameters, and indicators of interatomic forces.

In engineering design, elastic coefficients enter considerations of stress-strain (loaddeflection), residual stress, thermoelastic stress, fracture toughness, and elastic instabilities, such as buckling and jamming.

For material characterization, elastic coefficients can provide information about cracks and voids, nonhomogeneity, and anisotropy (texture).

The macroscopic elastic coefficients follow directly from fundamental interatomic forces. In most solids, the coefficients relate simply to interatomic bond-stretching and bond-bending forces. In polymers, many more structural features affect the elastic coefficients. These features include chain length, chain shape, average molecular weight, nature and density of cross-links, and so on.

\subsection{Procedures}

\subsubsection{Materials}

The eighteen materials measured are described in \$III.3.3.2. Their designations and sources are given in Table 2.1 of $\S$ III.2. For comparison, we measured the same properties of polymethylmethacrylate (PMMA). 


\subsubsection{Measurement Methods}

In the Marx-oscillator method, we used 4.7-mm-diameter rods, typically 3-cm long. By establishing standing waves and measuring resonance frequencies $\left(f_{s}\right.$, near $\left.30 \mathrm{kHz}\right)$, one obtains Young's modulus from

$$
\mathrm{E}=4 \rho f_{s}^{2} p_{s}^{2}
$$

Here $\rho$ denotes mass density and $\ell_{s}$, specimen length.

from

From the width of the Lorentzian-shape resonance peak, we calculated the internal friction

$$
Q^{-1}=\left(f_{2}-f_{1}\right) / f_{0}
$$

Here, $f_{0}$ denotes the center frequency of the resonance peak, and $f_{2}$ and $f_{1}$, the frequencies corresponding to half-maximum in power.

In the pulse-echo-superposition method, we used plate-shape specimens a few millimeters thick. Using piezoelectric transducers, we excited an ultrasonic pulse that traveled through the specimen, reflected, and returned for detection at the original transducer. Using longitudinal-wave polarization, we measured the longitudinal velocity $\left(v_{\mathfrak{\ell}}\right)$ and obtained the modulus $\left(C_{\mathfrak{\ell}}\right)$ from

$$
C_{\ell}=\rho v_{\ell}^{2} \text {. }
$$

Similarly, transverse-wave polarization leads to the transverse modulus:

$$
C_{t}=\rho v_{t}
$$

In isotropic materials, the shear modulus $(G)$ equals the transverse modulus. Other elastic properties follow from simple, well-known relationships:

$$
\begin{aligned}
\text { bulk modulus } & =B=C_{\ell}-(4 / 3) C_{t}, \\
\text { Young's modulus } & =E=9 B G /(3 B+G), \\
\text { Poisson's ratio } & =\nu=E /(2 G)-1 .
\end{aligned}
$$

Except for the dimensionless Poisson's ratio, all these elastic-stiffness parameters have units of stress (herein GPa). standard.

To determine mass density $(\rho)$ we used Archimedes's method with distilled water as a

From our experience in measuring standard materials, we estimate the uncertainty in principal elastic constants $C_{\ell}$ and $C_{t}$ as $\pm 0.5 \%$ and the error in mass density as $0.1 \%$. 


\subsection{Results}

Tables 10.1 and 10.2 show the principal measurement results: sound velocities and complete sets of elastic coefficients at two frequencies, 1 and $5 \mathrm{MHz}$. For 30 or $35 \mathrm{kHz}$ (determined by specimen length), the Young's modulus and associated internal friction are given in Table 10.3.

Table 10.1. At $1 \mathrm{MHz}$, elastic constants of 17 resins compared with those of PMMA.

\begin{tabular}{|c|c|c|c|c|c|c|c|c|c|}
\hline Material & $\begin{array}{c}T \\
\left({ }^{\circ} \mathrm{C}\right)\end{array}$ & $\begin{array}{c}\rho \\
\left(\mathrm{g} / \mathrm{cm}^{3}\right)\end{array}$ & $\begin{array}{c}v_{\ell} \\
(\mathrm{cm} / \mu \mathrm{s})\end{array}$ & $\begin{array}{c}v_{t} \\
(\mathrm{~cm} / \mu \mathrm{s})\end{array}$ & $\begin{array}{c}C_{\ell} \\
(\mathrm{GPa})\end{array}$ & $\begin{array}{c}G \\
(\mathrm{GPa})\end{array}$ & $\begin{array}{c}B \\
(\mathrm{GPa})\end{array}$ & $\begin{array}{c}E \\
(\mathrm{GPa})\end{array}$ & $\nu$ \\
\hline \multicolumn{10}{|l|}{ Epoxies- } \\
\hline DGEBA/DCH/ARA/ACL & 22.2 & 1.166 & 0.3101 & 0.1277 & 11.21 & 1.901 & 8.679 & 5.315 & 0.3979 \\
\hline DGEBA/ALA/ACL & 23.6 & 1.149 & 0.2978 & 0.1288 & 10.19 & 1.907 & 7.651 & 5.281 & 0.3849 \\
\hline DGEBA-ST/ARA/ACL/F & 23.9 & 1.292 & 0.2930 & 0.1276 & 11.09 & 2.103 & 8.287 & 5.818 & 0.3830 \\
\hline DGEBF/CTBN/AN & 24.0 & 1.220 & 0.2722 & 0.1163 & 9.036 & 1.651 & 6.835 & 4.584 & 0.3882 \\
\hline NV/AN/ACL & 22.1 & 1.232 & 0.2808 & 0.1255 & 9.714 & 1.942 & 7.125 & 5.341 & 0.3751 \\
\hline Cyclo.A/AN/ACL & 22.9 & 1.212 & 0.2756 & 0.1216 & 9.208 & 1.793 & 6.818 & 4.944 & 0.3791 \\
\hline Cyclo.A/CTBN/AN/ACL & 23.2 & 1.214 & 0.2729 & 0.1191 & 9.043 & 1.721 & 6.748 & 4.759 & 0.3825 \\
\hline MLTF/ARA & 23.2 & 1.270 & 0.3413 & 0.1370 & 14.80 & 2.383 & 11.618 & 6.692 & 0.4040 \\
\hline \multicolumn{10}{|l|}{ Cyanate Esters- } \\
\hline CE/VT/Co.AA & 22.4 & 1.188 & 0.2715 & 0.1169 & 8.760 & 1.622 & 6.597 & 4.498 & 0.3864 \\
\hline $\mathrm{CE}$ & 22.5 & 1.184 & 0.2681 & 0.1116 & 8.511 & 1.474 & 6.545 & 4.114 & 0.3952 \\
\hline $\mathrm{CE}(\mathrm{PT})$ & 22.3 & 1.278 & 0.2995 & 0.1301 & 11.460 & 2.162 & 8.577 & 5.984 & 0.3837 \\
\hline $\mathrm{CE} / \mathrm{HTBN} / \mathrm{NPh} / \mathrm{Cu} . \mathrm{AA}$ & 22.9 & 1.147 & 0.2830 & 0.1240 & 9.185 & 1.763 & 6.834 & 4.870 & 0.3812 \\
\hline \multicolumn{10}{|l|}{ Vinyl Esters- } \\
\hline $\mathrm{VE} / \mathrm{CHX}$ & 24.8 & 1.142 & 0.2849 & 0.1222 & 9.271 & 1.706 & 6.996 & 4.734 & 0.3872 \\
\hline VE-EL/MEKP & 22.3 & 1.123 & 0.2963 & 0.1273 & 9.861 & 1.819 & 7.436 & 5.046 & 0.3869 \\
\hline $\mathrm{VE} / \mathrm{CHP} / \mathrm{F}$ & 23.3 & 1.262 & 0.2863 & 0.1281 & 10.34 & 2.069 & 7.585 & 5.690 & 0.3750 \\
\hline VE/CHP/CoNap/2,4-P & 24.1 & 1.249 & 0.2868 & 0.1253 & 10.27 & 1.962 & 7.655 & 5.423 & 0.3819 \\
\hline \multicolumn{10}{|l|}{ Polyester- } \\
\hline PE/MEKP & 23.4 & 1.179 & 0.2870 & 0.1285 & 9.710 & 1.947 & 7.114 & 5.353 & 0.3746 \\
\hline \multicolumn{10}{|l|}{ Acrylate- } \\
\hline PMMA & 22.2 & 1.187 & 0.3081 & 0.1478 & 11.27 & 2.592 & 7.812 & 7.001 & 0.3506 \\
\hline
\end{tabular}

"See comments regarding cure schedule on page III-16. 
Table 10.2. At $5 \mathrm{MHz}$, elastic constants of 17 resins compared with those of PMMA.

\begin{tabular}{|c|c|c|c|c|c|c|c|c|}
\hline Material & $\begin{array}{c}T \\
\left({ }^{\circ} \mathrm{C}\right)\end{array}$ & $\begin{array}{c}v_{\ell} \\
(\mathrm{cm} / \mu \mathrm{s})\end{array}$ & $\begin{array}{c}v_{t} \\
(\mathrm{~cm} / \mu \mathrm{s})\end{array}$ & $\begin{array}{c}C_{\ell} \\
(\mathrm{GPa})\end{array}$ & $\begin{array}{c}G \\
(\mathrm{GPa})\end{array}$ & $\begin{array}{c}B \\
(\mathrm{GPa})\end{array}$ & $\begin{array}{c}E \\
(\mathrm{GPa})\end{array}$ & $\nu$ \\
\hline \multicolumn{9}{|l|}{ Epoxies- } \\
\hline DGEBA/DCH/ARA/ACL & 22.8 & 0.3131 & 0.1278 & 11.43 & 1.904 & 8.888 & 5.332 & 0.4000 \\
\hline DGEBA/ALA/ACL & 22.9 & 0.2990 & 0.1300 & 10.27 & 1.942 & 7.685 & 5.373 & 0.3835 \\
\hline DGEBA-ST/ARA/ACL/F & 21.6 & 0.2850 & 0.1292 & 10.50 & 2.156 & 7.620 & 5.911 & 0.3707 \\
\hline DGEBF/CTBN/AN & 22.5 & 0.2749 & 0.1170 & 9.219 & 1.671 & 6.991 & 4.642 & 0.3893 \\
\hline NV/AN/ACL & 22.8 & 0.2800 & 0.1233 & 9.659 & 1.874 & 7.160 & 5.171 & 0.3796 \\
\hline Cyclo.A/AN/ACL & 22.4 & 0.2736 & 0.1226 & 9.070 & 1.821 & 6.641 & 5.006 & 0.3744 \\
\hline Cyclo.A/CTBN/AN/ACL & 22.5 & 0.2648 & 0.1194 & 8.515 & 1.731 & 6.207 & 4.752 & 0.3724 \\
\hline MLTF/ARA & 21.4 & 0.3246 & 0.1388 & 13.39 & 2.446 & 10.12 & 6.792 & 0.3882 \\
\hline \multicolumn{9}{|l|}{ Cyanate Esters- } \\
\hline CE/VT/Co.AA & 22.7 & 0.2644 & 0.1143 & 8.308 & 1.552 & 6.238 & 4.301 & 0.3851 \\
\hline $\mathrm{CE}$ & 22.8 & 0.2595 & 0.1091 & 7.971 & 1.410 & 6.091 & 3.926 & 0.3926 \\
\hline $\mathrm{CE}(\mathrm{PT})^{*}$ & 22.8 & 0.2924 & 0.1289 & 10.93 & 2.124 & 8.097 & 5.861 & 0.3794 \\
\hline CE/HTBN/NPh/Cu.AA & 22.2 & 0.2756 & 0.1221 & 8.715 & 1.711 & 6.434 & 4.714 & 0.3779 \\
\hline \multicolumn{9}{|l|}{ Vinyl Esters- } \\
\hline VE/CHX & 23.3 & 0.2788 & 0.1232 & 8.880 & 1.732 & 6.570 & 4.776 & 0.3788 \\
\hline VE-EL/MEKP & 22.6 & 0.2854 & 0.1252 & 9.147 & 1.759 & 6.802 & 4.858 & 0.3810 \\
\hline VE/CHP/F & 22.2 & 0.2794 & 0.1290 & 9.849 & 2.101 & 7.047 & 5.734 & 0.3644 \\
\hline VE/CHP/CoNap/2,4-P & 22.6 & 0.2833 & 0.1247 & 10.03 & 1.941 & 7.440 & 5.357 & 0.3800 \\
\hline \multicolumn{9}{|l|}{ Polyester- } \\
\hline PE/MEKP & 22.6 & 0.2798 & 0.1269 & 9.229 & 1.899 & 6.697 & 5.205 & 0.3705 \\
\hline \multicolumn{9}{|l|}{ Acrylate- } \\
\hline PMMA & 23.0 & 0.5052 & 0.1458 & 11.06 & 2.525 & 7.690 & 6.828 & 0.3520 \\
\hline
\end{tabular}

"See comments regarding cure schedule on page III-16. 
Table 10.3. At kilohertz frequencies, Young's modulus and internal friction of 18 resins compared with those of PMMA.

\begin{tabular}{|c|c|c|c|c|}
\hline Material & $\underset{(\mathrm{kHz})}{f}$ & $\begin{array}{c}T \\
\left({ }^{\circ} \mathrm{C}\right)\end{array}$ & $\begin{array}{c}E \\
(\mathrm{GPa})\end{array}$ & $Q^{-1}$ \\
\hline \multicolumn{5}{|l|}{ Epoxies- } \\
\hline DGEBA/DCH/ARA/ACL & 30 & 25.3 & 3.630 & 0.060 \\
\hline DGEBA/ALA/ACL & 30 & 24.4 & 3.858 & 0.067 \\
\hline DGEBA-ST/ARA/ACL/F & 35 & 24.0 & 5.038 & 0.062 \\
\hline DGEBF/CTBN/AN & 30 & 23.9 & 3.570 & 0.026 \\
\hline NV/AN/ACL & 30 & 25.2 & 4.200 & 0.041 \\
\hline Cyclo.A/AN/ACL & 30 & 24.2 & 3.931 & 0.021 \\
\hline Cyclo.A/CTBN/AN/ACL & 30 & 23.0 & 4.120 & 0.016 \\
\hline MLTF/ARA & 35 & 23.2 & 4.885 & 0.058 \\
\hline MLTF/ARA/TGH & 30 & 23.5 & 4.372 & 0.048 \\
\hline \multicolumn{5}{|l|}{ Cyanate Esters- } \\
\hline CE/VT/Co.AA & 30 & 25.6 & 3.876 & 0.014 \\
\hline $\mathrm{CE}$ & 30 & 23.0 & 3.495 & 0.0086 \\
\hline $\mathrm{CE}(\mathrm{PT})^{*}$ & 35 & 23.1 & 4.797 & 0.0055 \\
\hline $\mathrm{CE} / \mathrm{HTBN} / \mathrm{NPh} / \mathrm{Cu} . \mathrm{AA}$ & 30 & 24.5 & 4.198 & 0.017 \\
\hline \multicolumn{5}{|l|}{ Vinyl Esters- } \\
\hline $\mathrm{VE} / \mathrm{CHX}$ & 30 & 23.8 & 3.886 & 0.013 \\
\hline VE-EL/MEKP & 30 & 25.6 & 3.762 & 0.023 \\
\hline VE/CHP/F & 35 & 22.4 & 5.723 & 0.028 \\
\hline VE/CHP/CoNap/2,4-P & 30 & 23.2 & 4.347 & 0.013 \\
\hline \multicolumn{5}{|l|}{ Polyester- } \\
\hline PE/MEKP & 30 & 24.5 & 4.441 & 0.016 \\
\hline \multicolumn{5}{|l|}{ Acrylate- } \\
\hline PMMA & 30 & 23.3 & 5.890 & 0.015 \\
\hline
\end{tabular}

"See comments regarding cure schedule on page III-16. 


\subsection{Discussion}

The most surprising result is the near-constancy of the Poisson's ratio among the materials studied. If $\nu$ is nearly constant, then, from Equation 10.7, a plot of $G$ versus $E$ should be linear with little scatter. Figure 10.1 confirms such behavior and gives, by a least-squares fit to 38 points, an effective $\nu=0.380$. This near-constancy of $\nu$ reflects the similarity in type of bonding in these materials. In principle, for isotropic solids, the Poisson's ratio is bounded by $0.5 \geq \nu \geq-1$. In practice, for nearly all materials, we observe $0.4 \geq \nu \geq 0.2$, with no known negative values except for network structures. Because, in most of their properties, polymers resemble liquids rather than solids, we would expect the value of $\nu$ to be about 0.5 , a liquid's value, reflecting zero resistance to shear force. A mean value near 0.4 shows a partial transition from a liquid state to a crystalline state. The relatively high Poisson's ratio reflects the higher strength of extension-compression bends compared with that of bending-twisting bends, consistent with a polymer structure whose Young's modulus along the chain may exceed $300 \mathrm{GPa}$. This value is much higher than that for iron, whereas the Young's modulus perpendicular to the chain is a few gigapascals.

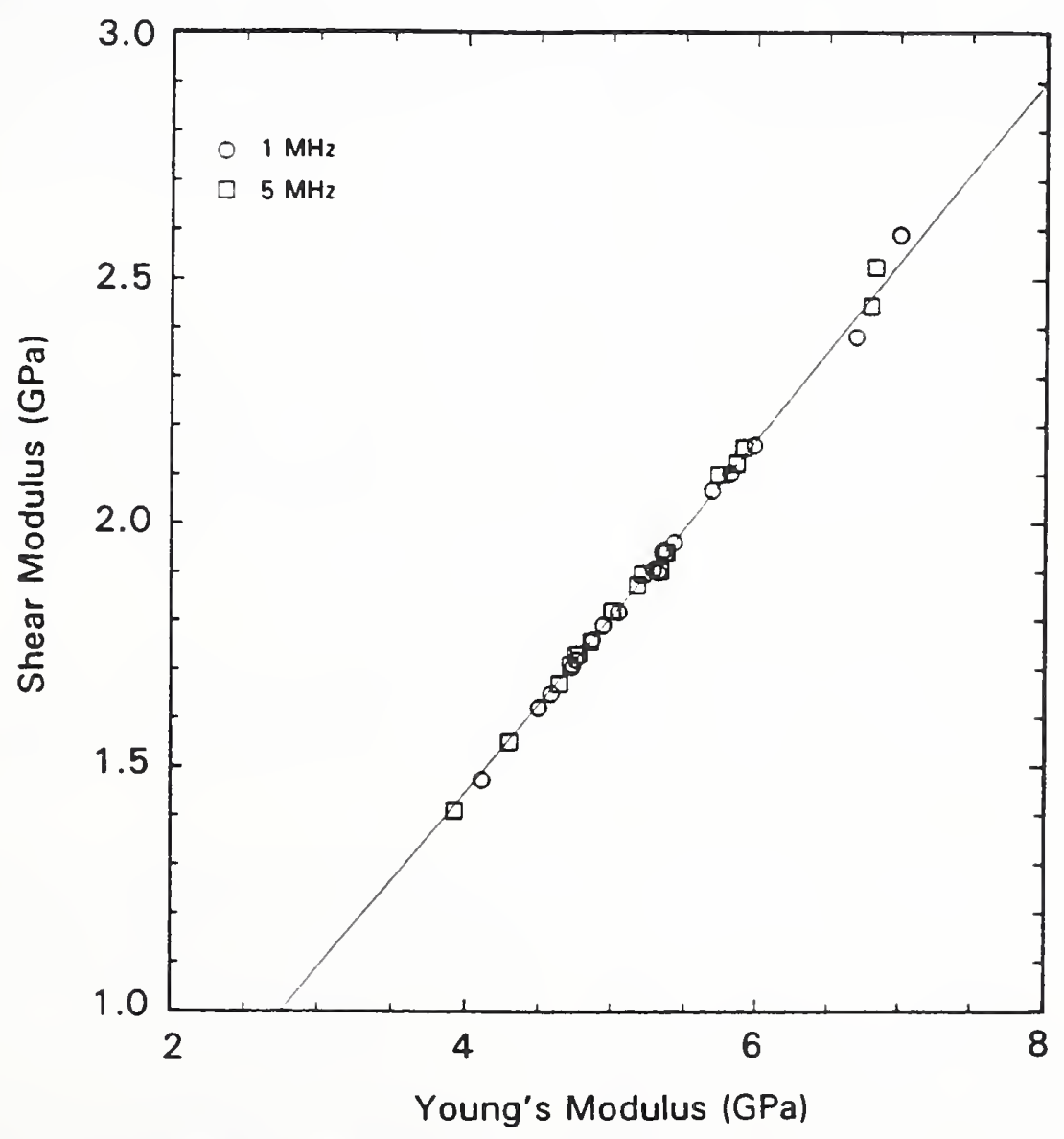

Figure 10.1. Shear-modulus-Young's-modulus diagram showing a nearly constant Poisson's ratio. A least-squares line gives $\nu=0.380$. 
Among the resins, the range of elastic stiffnesses, say the Young's moduli where $E_{\max } / E_{\min }$ $=1.6$, is normal compared with those of other groups of materials. The limited range of stiffness suggests also that the resins vary little in crystallinity. In polyethylene, for example, elastic stiffness increases by a factor of about 4 in going from amorphous to crystalline states. Thus, we conjecture that the resins vary little in crystallinity and that their stiffness differences arise from differences in other characteristics, such as molecular weight, mass density, and cross-linking.

Using Table 10.3, we can also compare the internal friction, $Q^{-1}$, of the resins with that of PMMA, a glassy polymer with a glass-transition temperature near $150^{\circ} \mathrm{C}$. Transition to the glass state increases $Q^{-1}$ substantially. We note that the various esters show $Q^{-1}$ values similar to or lower than that of PMMA, whereas the epoxies show much higher values.

Finally, a few comments on frequency dependence: In principle, one can measure dynamic elastic constants over a wide frequency range, say $10^{-3}$ to $10^{9} \mathrm{~Hz}$. In the present study, we focused on only three frequencies $-30 \mathrm{kHz}$ and 1 and $5 \mathrm{MHz}$. With regard to the Young's moduli, $E$, our results show that between zero frequency and megahertz frequencies, $E$ sometimes increases and sometimes decreases. For usual materials, we expect $E$ to increase with frequency because various relaxation mechanisms become "frozen," that is, unable to follow the rapidly varying applied stress. Compared with $E$ and other elastic stiffnesses, we found that the Poisson's ratio usually shows a much lower frequency dependence. Unless the polymer is near its glass-transition temperature, we expect $\mathrm{Q}^{-1}$ to change only slightly with frequency.

\footnotetext{
*Note also that the usual, so-called static test conditions are not really static, but low-frequency dynamic, say $10^{-3}$ to $10^{-1} \mathrm{~Hz}$. In this frequency range, we expect $E$ to vary with frequency, just as it does in the kilohertz and megahertz regions. For materials with low-frequency relaxation mechanisms, the following question arises: Can one measure the static Young's modulus? If not, then from an operationalist viewpoint, it lacks meaning.
} 


\title{
11. Discussion
}

\author{
R.P. Reed \\ Cryogenic Materials, Inc. \\ Boulder, Colorado
}

\begin{abstract}
Most of the results reported in §III represent the first measurements of these properties of these resin systems. Therefore, comparison of our data with other data for identical resin systems is not possible. Instead, this discussion compares the properties of the different resin systems that we studied. It also includes the role of defects in affecting mechanical properties, the use of data in selecting resins for cryogenic structures, and discussion of the selection of the best resin systems on the basis of neat-resin properties.
\end{abstract}

\subsection{Mechanical Properties}

Tensile and shear properties of the neat resin systems were measured at 295,76 , and $4 \mathrm{~K}$. This section includes (1) a discussion of the elastic moduli and their relationships and (2) a discussion of the tensile and shear strengths.

\subsubsection{Elastic Moduli}

The shear $(G)$ and Young's $(E)$ moduli for all resin systems are compared at 295, 76, $4 \mathrm{~K}$, and combined temperatures in Figures 11.1(a), (b), (c), and (d), respectively. ${ }^{*}$ When one constructs a best-fit linear relationship through the origin for Figure 11.1(d) and uses the isotropic expression to relate the moduli to Poisson's ratio $(\nu)$,

$$
\nu=\frac{E}{2 G}-1
$$

a value of $\nu=0.38$ is obtained. This average value (over the temperature range 4 to $295 \mathrm{~K}$ ) corresponds very well to the literature values of 0.38 to 0.41 at room temperature and 0.36 to 0.37 at $4 \mathrm{~K}$ reported in §III.4.2.

The tensile strengths and shear moduli at room temperature, with considerable scatter, increase with the increase in resin density. These dependences are shown in Figure 11.2(a) and (b) for tensile strength and shear moduli, respectively. ${ }^{*}$ Surprisingly, at low temperatures, the dependences on the room-temperature density are much less clear. This result suggests that (1) the temperature dependences of the elastic properties of neat-resin systems are much more dependent on resin chemical composition than on resin density; (2) the temperature dependence of the resin density is, in turn, dependent on the chemical composition; and (3) the room-temperature densities cannot be easily correlated with low-temperature densities.

\footnotetext{
${ }^{*}$ See comments regarding cure scheudle on page III-16.
} 
(a)

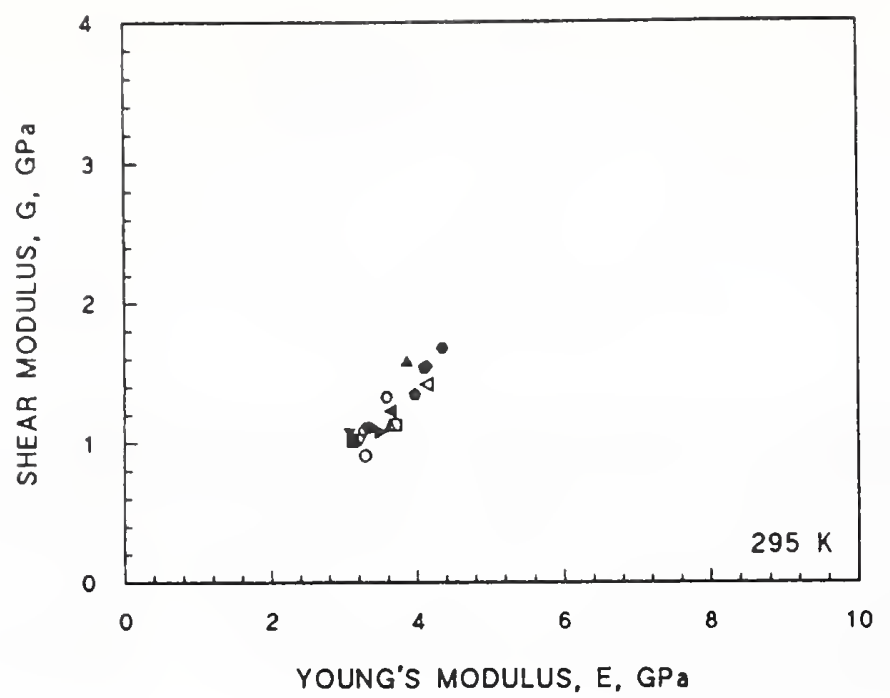

(b)
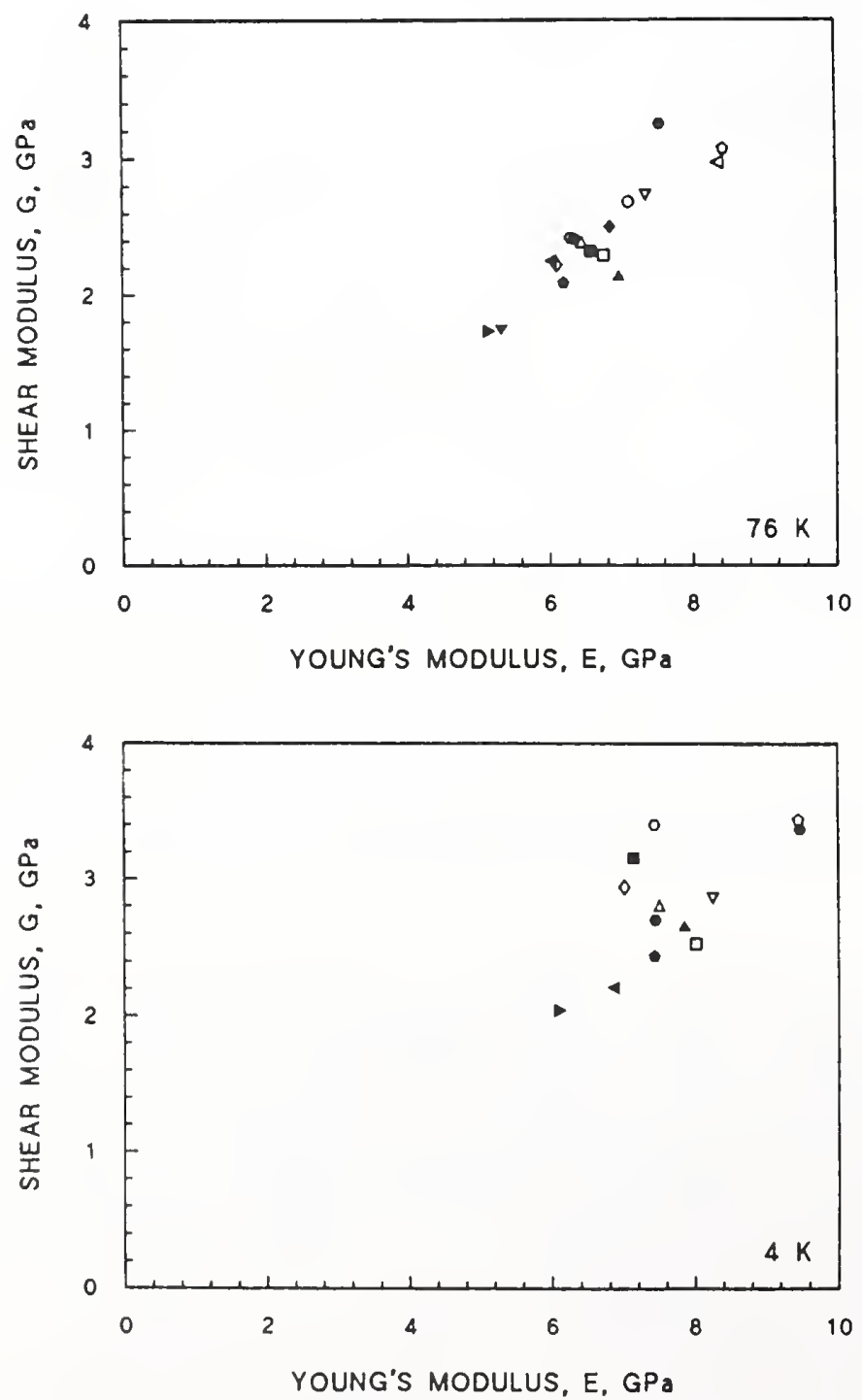

Figure 11.1. Shear and Young's moduli for all resin systems at $295 \mathrm{~K}(\mathrm{a}), 76 \mathrm{~K}$ (b), and $4 \mathrm{~K}$ (c). The material legend used in this figure is given in part (d). 
(d)

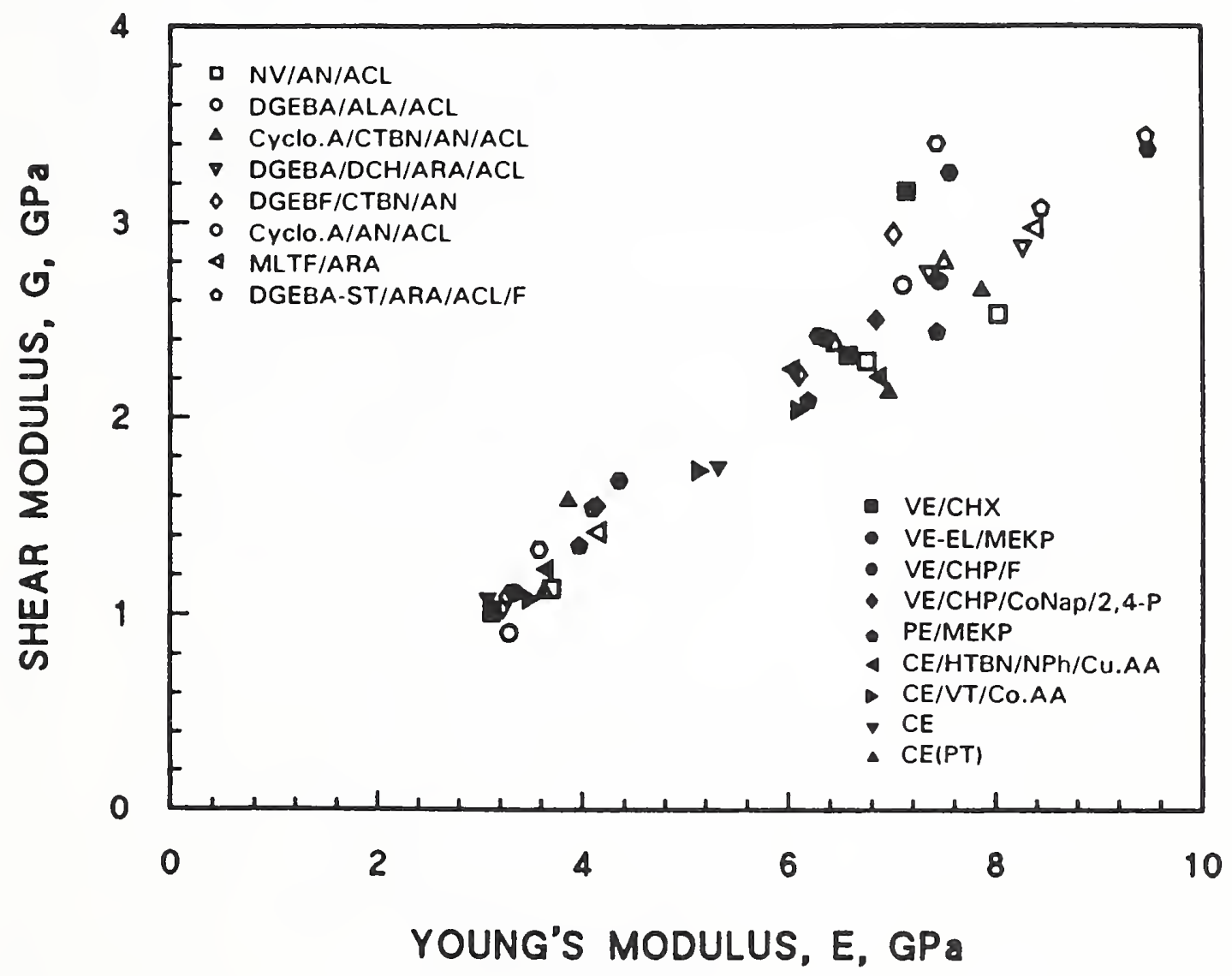

Figure 11.1. (continued). Shear and Young's moduli for all resin systems at the combined temperatures (d). 
(a)

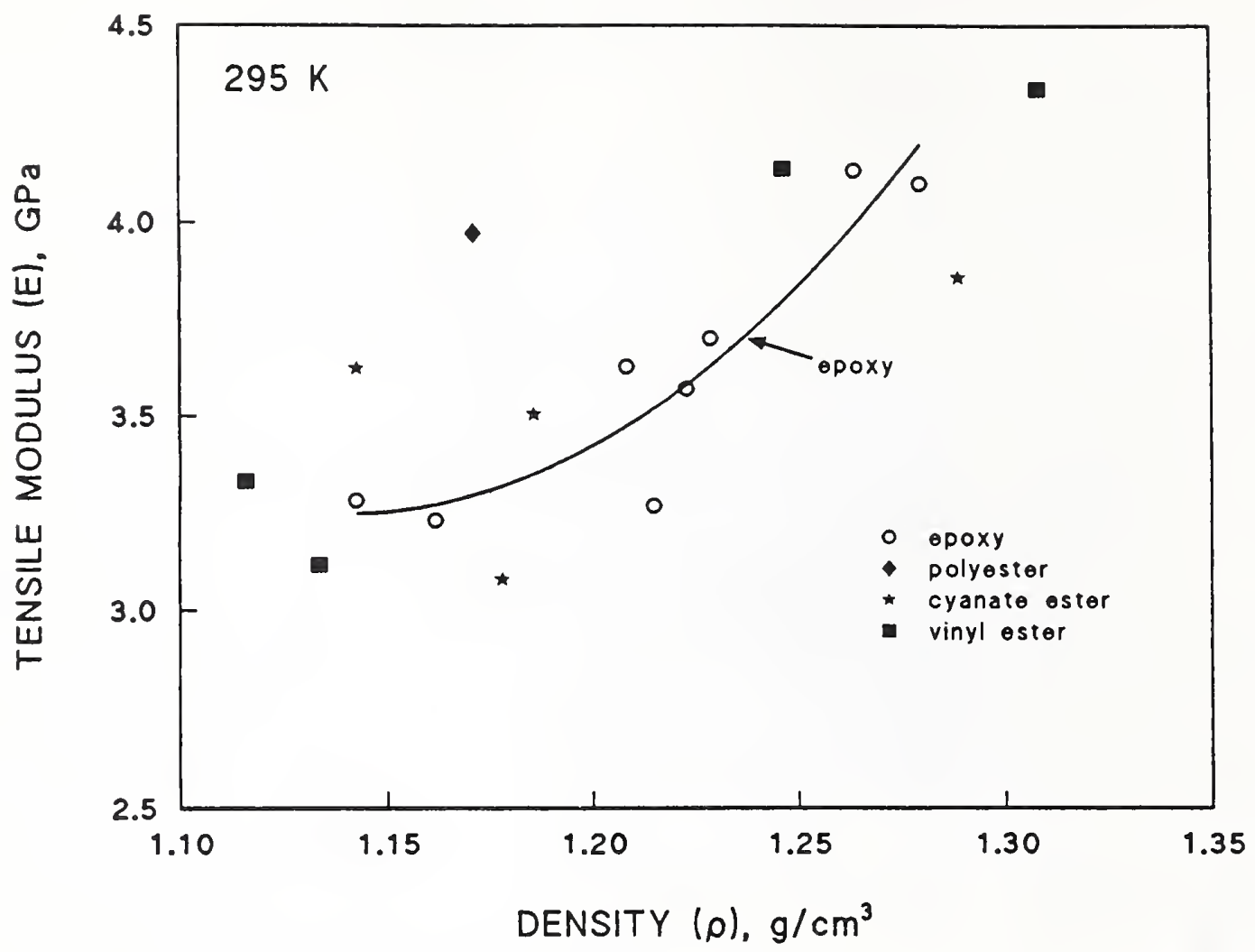

(b)

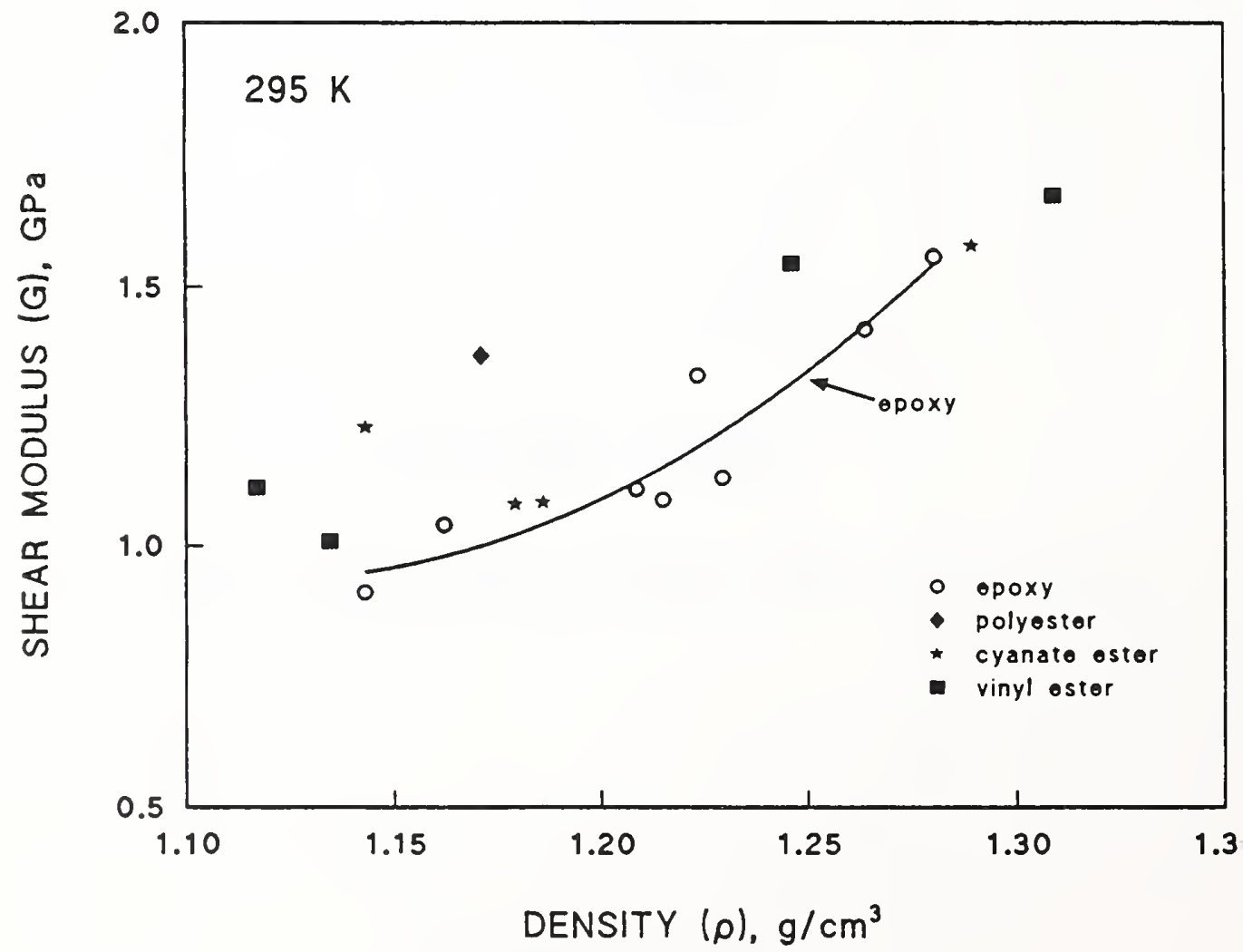

Figure 11.2. At room temperature, the dependences of tensile strengths (a) and shear moduli (b) on resin density. The trends for the epoxy resins are more defined than trends for the other classes of resins; thus, trend lines were constructed only for the epoxy resins. 
In $\$ I I I .9$, we pointed out the good relationship between the hardness and density of neat-resin systems. Therefore, since there is good correspondence between their density and elastic moduli at room temperature, one would expect good correlation between their hardness and elastic moduli at room temperature. This correlation is shown in Figure 11.3, where the elastic moduli are combined as $E+2 G$ and plotted versus the hardness. Unlike the correlation with density, the elastic-modulihardness dependence appears to be independent of the type of resin system. However, like the elasticmoduli-hardness data, there is no strong correlation between density and elastic moduli at low temperatures. Perhaps contributing to the lower scatter of elastic-moduli-hardness data is the use of the summation of the tensile and shear moduli. To assist in summarizing the trends of the elastic moduli, this same combination $(E+2 G)$ was used to analyze the temperature dependence and relative elastic strengths.

Elastic moduli $(E+2 G)$ are plotted versus temperature for epoxies (Fig. 11.4), cyanate esters (Fig. 11.5), ${ }^{*}$ and polyesters and vinyl esters (Fig. 11.6). Data variability is independent of temperature and, typically, about $\pm 3 \%$. The epoxies with the highest elastic moduli are the multifunctional and filled systems. Except for the multifunctional and filled systems, all epoxy systems have similar

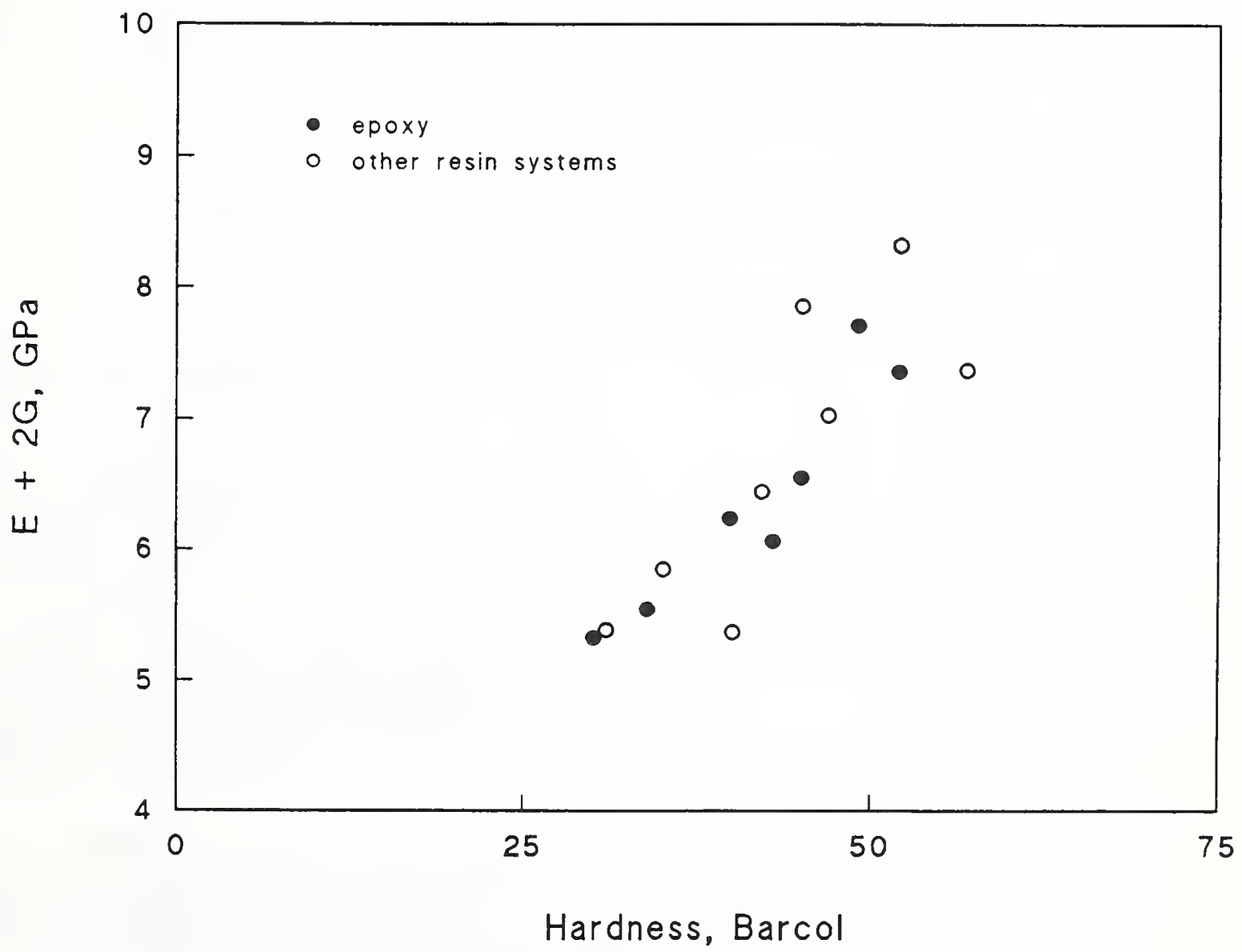

Figure 11.3. Elastic moduli $E+2 G$ at room temperature versus hardness.

\footnotetext{
*See comments regarding cure schedule on page III-16.
} 


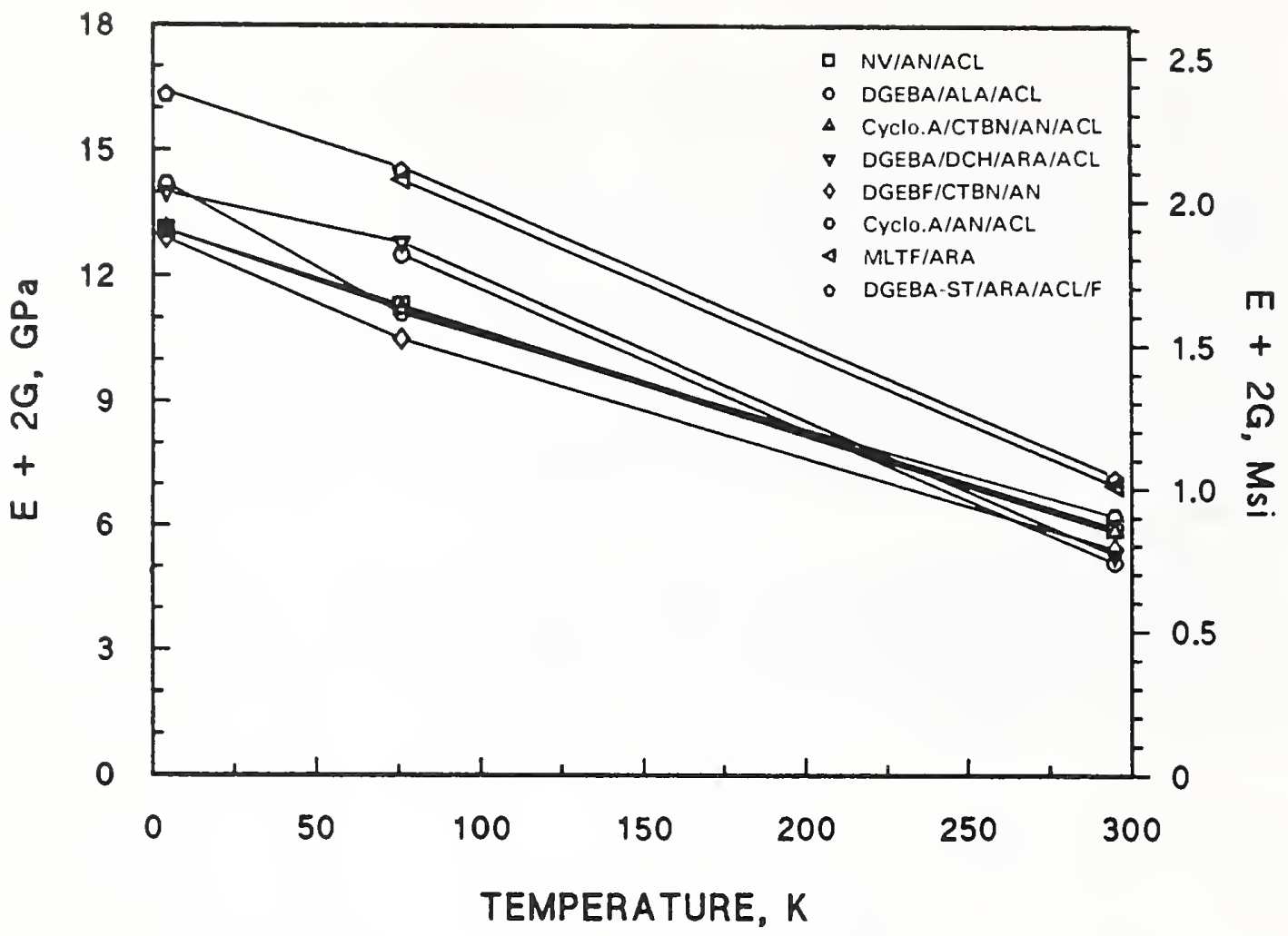

Figure 11.4. Elastic moduli $E+2 G$ versus temperature for epoxies.

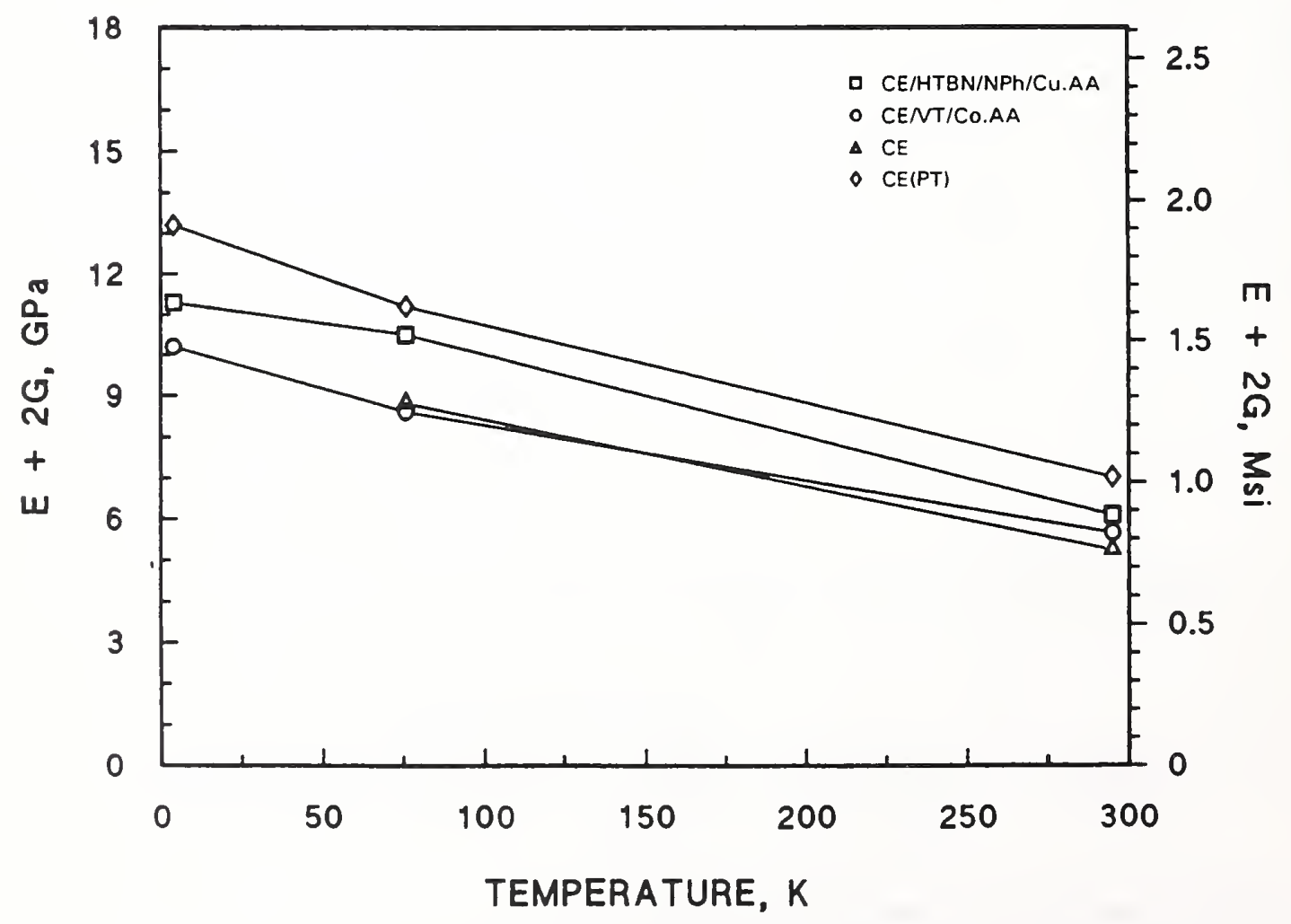

Figure 11.5. Elastic moduli $E+2 G$ versus temperature for cyanate esters. 


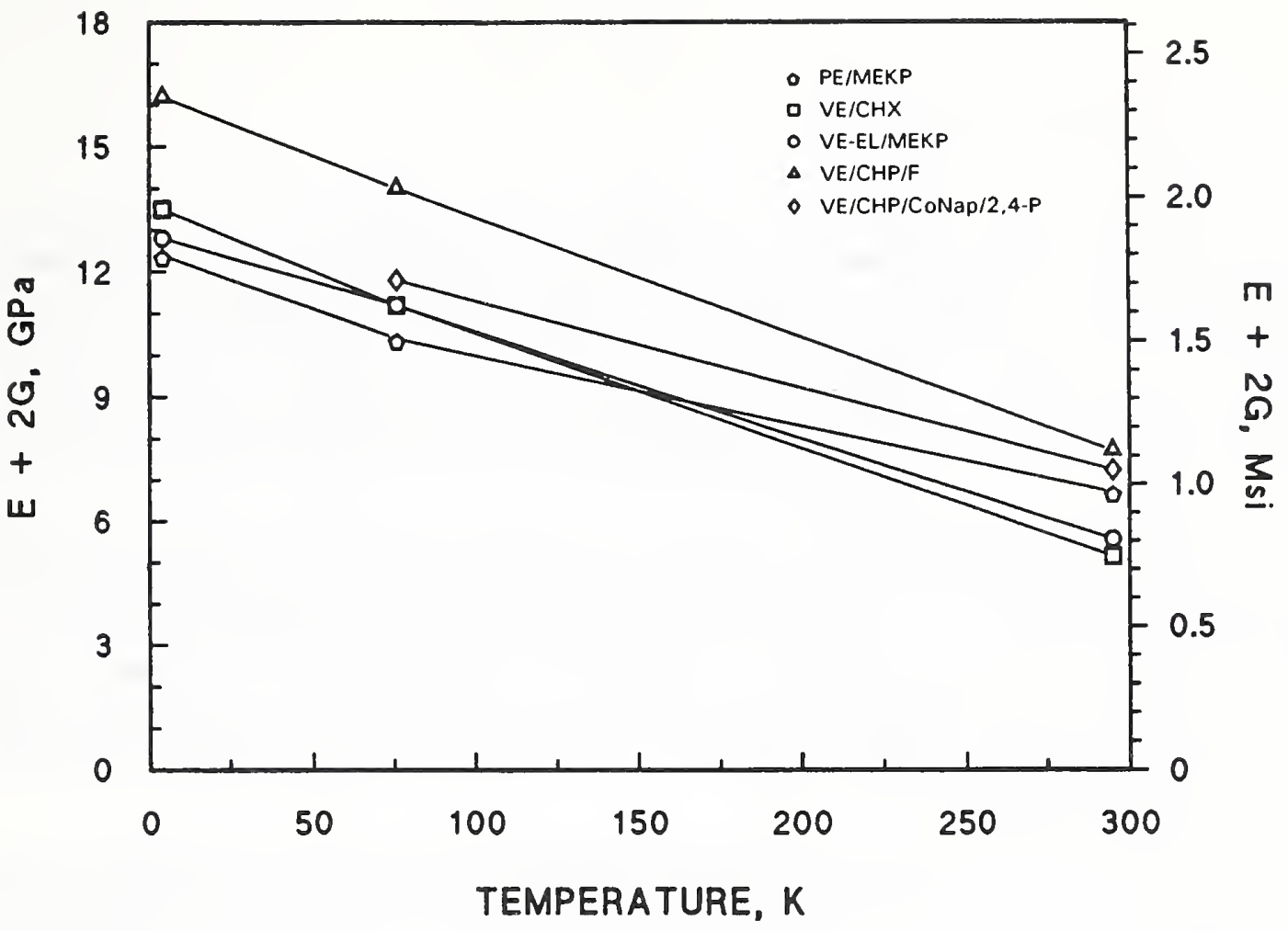

Figure 11.6. Elastic moduli $E+2 G$ versus temperature for polyesters and vinyl esters.

moduli at room temperature. The toughened epoxy systems have the lowest moduli at low temperatures. Thus, the dependence on temperature is less for the toughened epoxy systems.

The cyanate-ester resins show a relatively wide diversity of elastic moduli, and the CE(PT)* system exhibits the highest moduli. However, the cyanate-ester resins have a lower temperature dependence than the epoxy systems and, as a result, have lower elastic moduli at low temperatures. At room temperature, both resin systems have equivalent elastic moduli.

The VE/CHP/F (filled) vinyl-ester resin has the highest combined elastic moduli for the vinyl-ester and polyester resin systems. The temperature dependence of the vinyl esters is equivalent to that of the untoughened epoxy systems. Thus, the vinyl esters have good low-temperature elastic properties. The polyester has lower temperature dependence and lower elastic properties at low temperatures.

In summary, filled resin systems and multifunctional epoxy resins have the highest elastic properties at all temperatures. At room temperature, the elastic properties are dependent on resin density (and hardness). At low temperatures, untoughened epoxies and vinyl esters have higher elastic properties than the cyanate esters and polyesters.

\footnotetext{
${ }^{*}$ See comments regarding cure schedule on page $111-16$.
} 


\subsubsection{Tensile and Shear Strength}

To summarize the strengths of the neat resins at low temperatures, considering the large data scatter in general, the sum of the shear $(\tau)$ and tensile $(\sigma)$ strengths for each resin system at each temperature was used. Again, following the procedures defined in \$III.4 and §III.5, the shear and tensile strengths that were summed represent average values of the three highest data points. Typical data uncertainties are $\pm 4 \%$ at $295 \mathrm{~K}$ and $\pm 10 \%$ at 76 and $4 \mathrm{~K}$. The sum of these data is presented in Figure 11.7 for epoxies (a), cyanate esters (b), ${ }^{*}$ and polyester and vinyl esters (c). In Figure 11.7(d), the strongest resin systems for each resin class (including two epoxies) are presented.

Following a trend similar to that for elastic properties, the strongest resin systems at low temperatures were the epoxies and cyanate esters. At room temperature, the trend was reversed: the strongest resin systems were the polyester and vinyl esters, although a few epoxies that are not included in Figure 11.7(d) had equivalent room-temperature strengths. The cyanate esters were the only resins that had better strengths at $4 \mathrm{~K}$ than at $76 \mathrm{~K}$. In all other systems, apparently the sensitivity to flaws was sufficiently higher at $4 \mathrm{~K}$ than at $76 \mathrm{~K}$ to cause premature fracture. This is discussed in more detail in \$III.11.1.4.

The disparity between the relative strength of the resin systems at room temperature and that at low temperatures results in difficulties for resin screening. If resin strength is a significant factor in strut performance and if the struts are designed for a temperature gradient from 4 to $295 \mathrm{~K}$, then resin selection should be more strongly influenced by resin performance at the temperature of lowest strut strength or fatigue limit-which is room temperature. On the other hand, the greater strengths of epoxies and vinyl esters at low temperatures suggest that, perhaps, a two-strut approach is best, with the strut intercept also serving as the 76-K thermal intercept.

With regard to resin classes, it is interesting to note that the filled or multifunctional (epoxy) resin systems, with exceptionally good elastic properties, had the lowest strengths at cryogenic temperatures. These strengths, in the case of the epoxy and vinyl-ester classes, were significantly lower, by about 30 to $50 \%$, than the stronger resin systems.

To summarize-at low temperatures, several epoxy and vinyl-ester systems had the highest combined shear and tensile strength. At room temperature, several cyanate-ester and polyester systems had equivalent or higher combined strengths. Those filled systems or multifunctional epoxy systems that had the highest elastic properties had the lowest strengths at cryogenic temperatures.

\footnotetext{
*See comments regarding cure schedule on page III-16.
} 
(a)

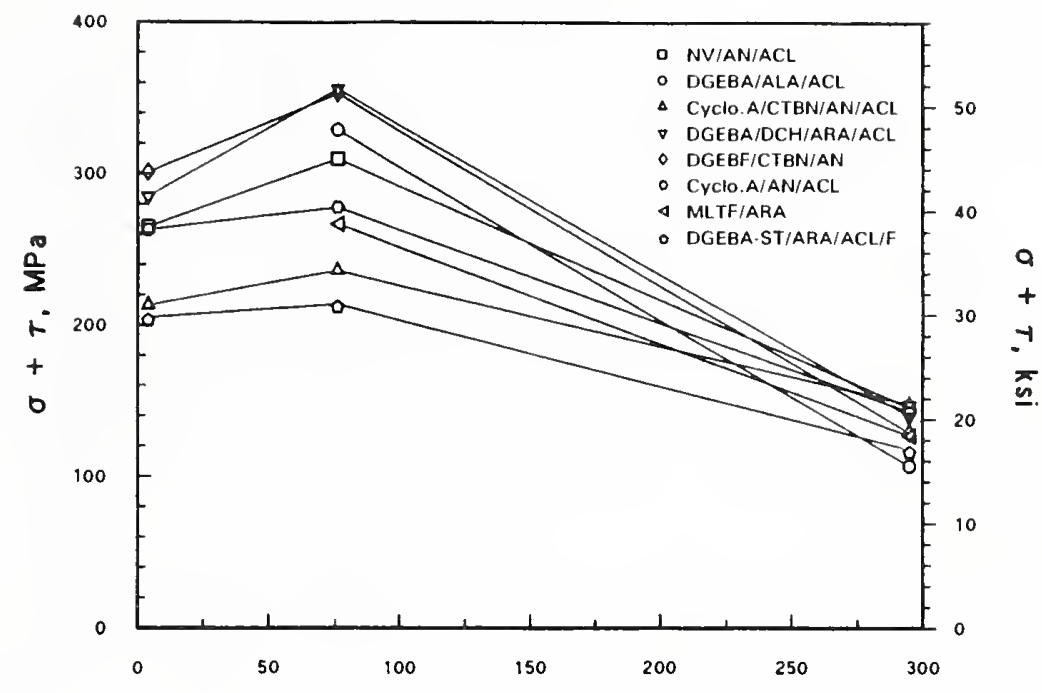

TEMPERATURE, $K$

(b)

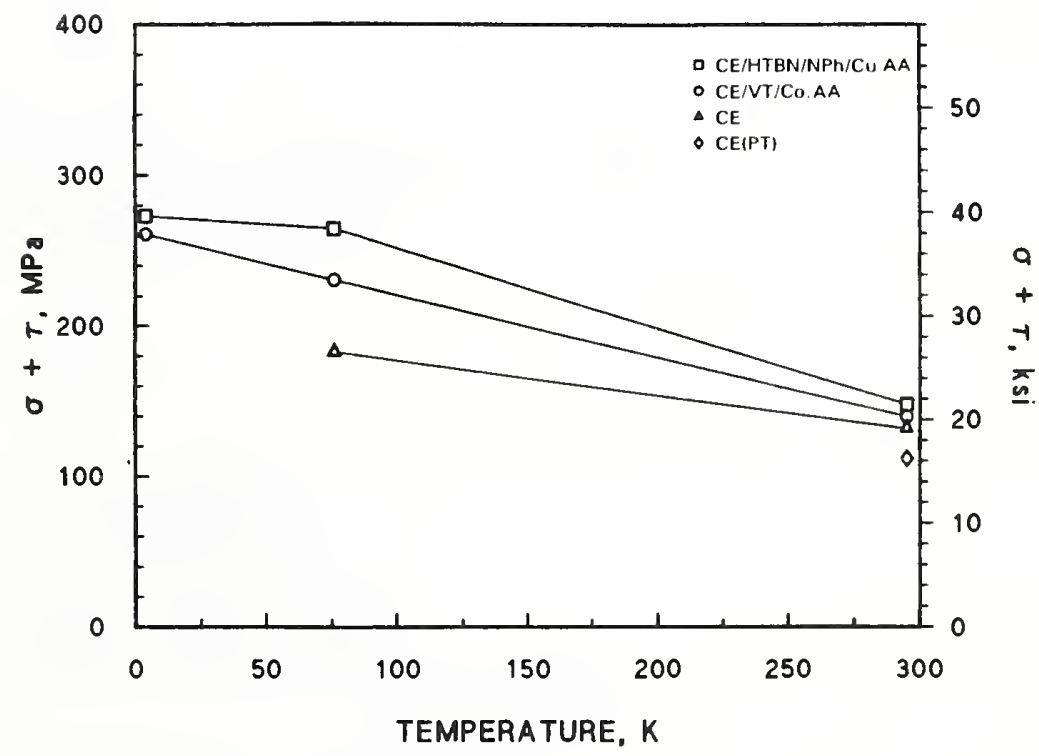

Figure 11.7. Sum of shear and tensile strength averages (three highest data points) for epoxies (a) and cyanate esters (b) (continued on next page). 
(c)

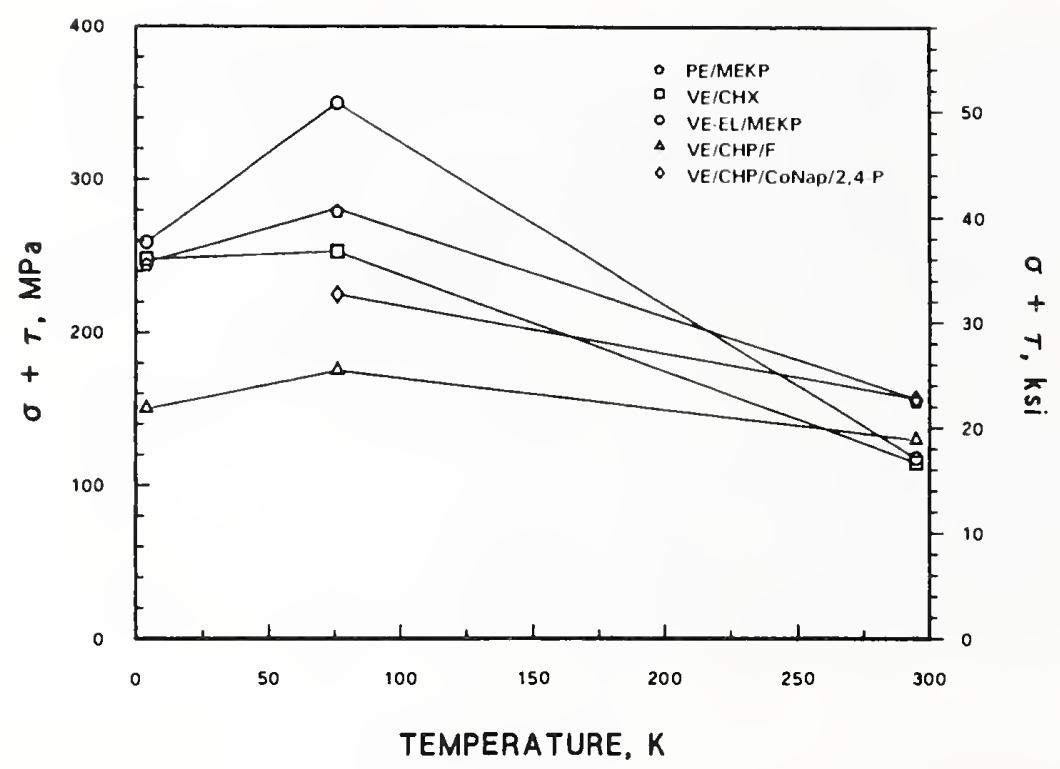

(d)

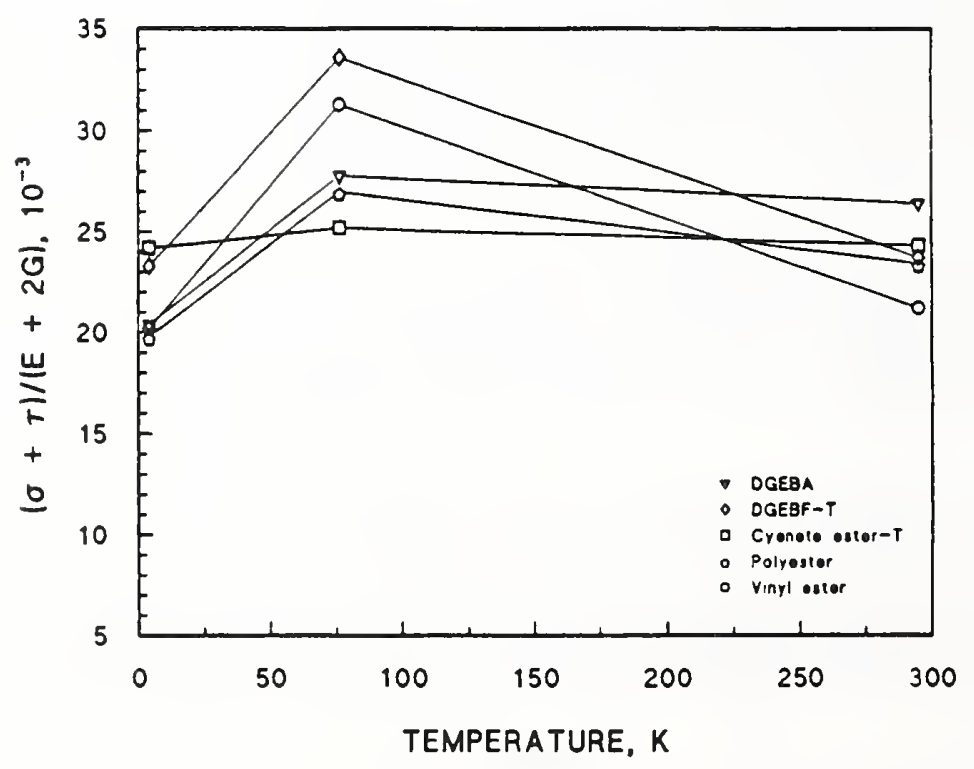

Figure 11.7. (continued). Sum of shear and tensile strength averages (three highest data points) for the polyester and vinyl esters (c) and the strongest resin systems for each resin class $(d)$. 


\subsubsection{Relationships between Elastic and Strength Properties}

The "apparent" strain in a tensile or shear specimen can be approximated by using the ratio of strength to elastic modulus. This approximation is especially valid at low temperatures, where there is little or no nonlinearity in the stress-strain curves of neat resins. For these comparisons, the strength was approximated by the term $\sigma+\tau$ and the elastic properties, by the term $E+2 G$. In Figure 11.8 , the ratio $(\sigma+\tau) /(E+2 G)$ for each resin system is plotted versus temperature. Epoxies [Fig. 11.8(a)], cyanate esters [Fig. 11.8(b)], ${ }^{*}$ and polyester and vinyl esters [Fig. 11.8(c)] are included. For all resin systems, except two cyanate esters (CE/VT/Co.AA and CE/HTBN/NPh/Cu.AA), the lowest apparent strains to failure (strength-to-modulus ratios) were at $4 \mathrm{~K}$. On the basis of apparent strain to failure, comparative data for the best resin systems of each class are replotted in Figure 11.8(d). Notice that, in addition to being less sensitive to temperature, the cyanate ester had the highest strength-to-

modulus ratio at $4 \mathrm{~K}$. In summary, on the basis of apparent strain to failure, data were lower at $4 \mathrm{~K}$ than at other temperatures, except for two cyanate ester-resin systems.

The plots of $E+2 G$ versus $\sigma+\tau$ in Figure 11.9 provide an excellent summary of the tensile and shear data of all resin systems. The data are shown for each temperature in Figures $11.7(\mathrm{a}-\mathrm{c})$ and combined in Figure 11.9(d). Several items are apparent:

1. The properties of resin systems varied less at room temperature than at low temperatures. This trend suggests that careful resin selection is more important for cryogenic applications.

2. Those resins that had comparatively better properties at room temperature did not have better properties at low temperatures. Thus, for structural components that span the temperature gradient from 4 to $295 \mathrm{~K}$, there is no clearly superior resin system based on their tensile and shear properties.

3. Those resins that had better elastic properties were not the same as those that had better strength properties. Similarly, resins more tolerant in strain-to-failure criteria were not those that had better elastic properties. These distinctions between resin systems place a premium on resin-selection criteria and on reliable static and dynamic failure-prediction models. Accurate predictive models that contain either elastic, strength, or strain parameters for the resin matrix are needed for resin screening.

4. At low temperatures, the elastic and strength properties of resin systems increased as the strain-tofailure ratios decreased.

Some contend that the energy-absorption capability of the resin may be related to the resin's matrix performance. An estimate of the energy absorbed during tensile and shear tests of neat resins is obtained by considering the area under the stress-strain curve, that is $\sigma \epsilon / 2$, or for this report, by combining the shear and tensile properties: $(1 / 2)(\tau+\sigma)^{2}(E+2 G)^{-1}$. From this quantity, which was calculated for all resin systems, we conclude:

- At low temperatures, on the basis of energy absorbed, three general resin systems are better: epoxies DGEBA/DCH/ARA/ACL and DGEBF/CTBN/AN and vinyl ester VE-EL/MEKP.

- At room temperature, although differences among resin types are not as distinct, several resin systems appear to have slightly better energy-absorption capacity: epoxies DGEBA/DCH/ARA/ ACL, Cyclo.A/CTBN/AN/ACL, and NV/AN/ACL and cyanate esters CE/VT/Co.AA, CE, and $\mathrm{CE} / \mathrm{HTBN} / \mathrm{NPh} / \mathrm{Cu} . \mathrm{AA}$.

\footnotetext{
*See comments regarding cure schedule on page III-16.
} 
(a)
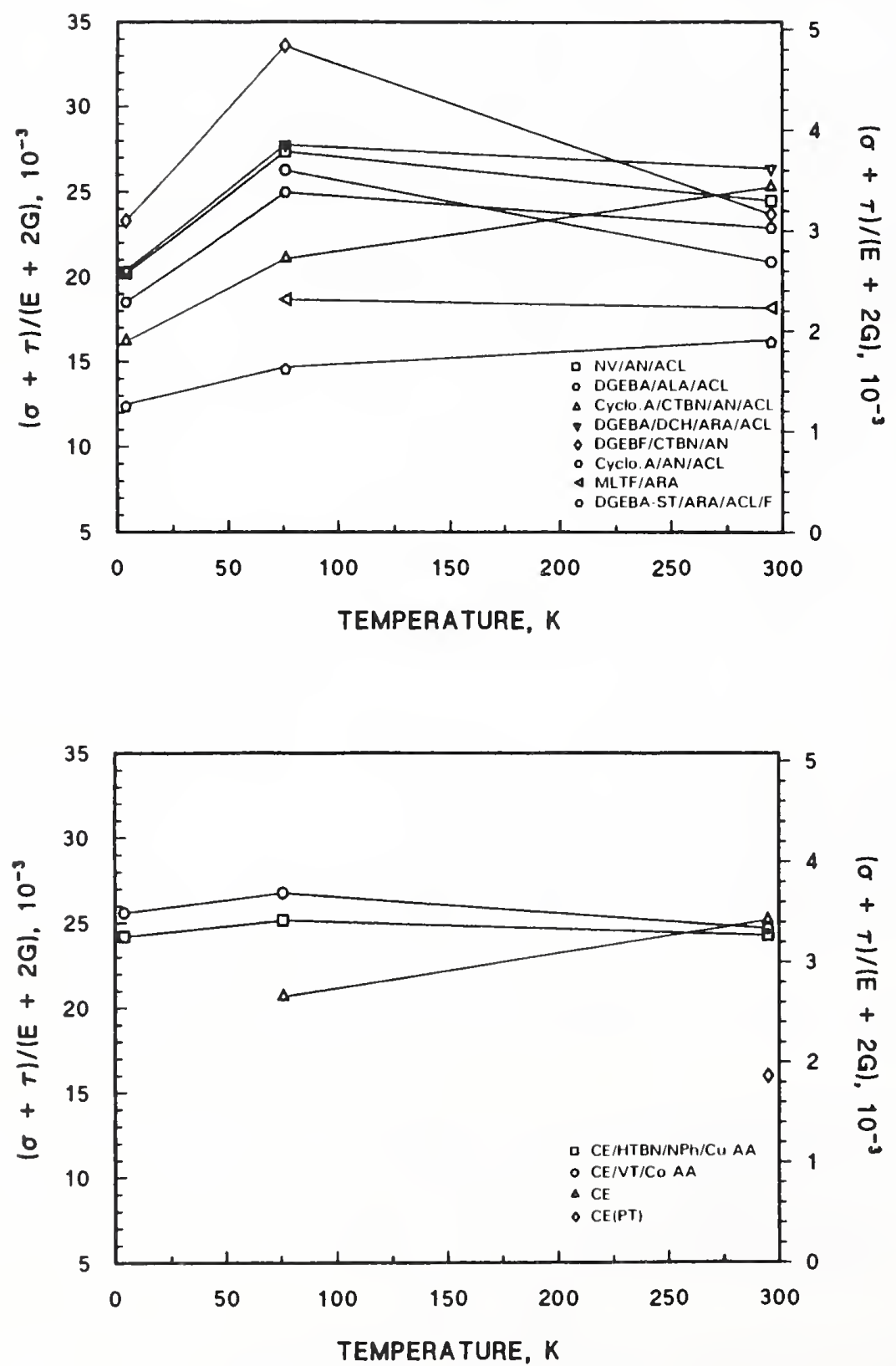

Figure 11.8. The ratio $(\sigma+\tau) /(E+2 G)$ versus temperature for epoxies (a) and cyanate esters (b) (continued on next page). 
(c)
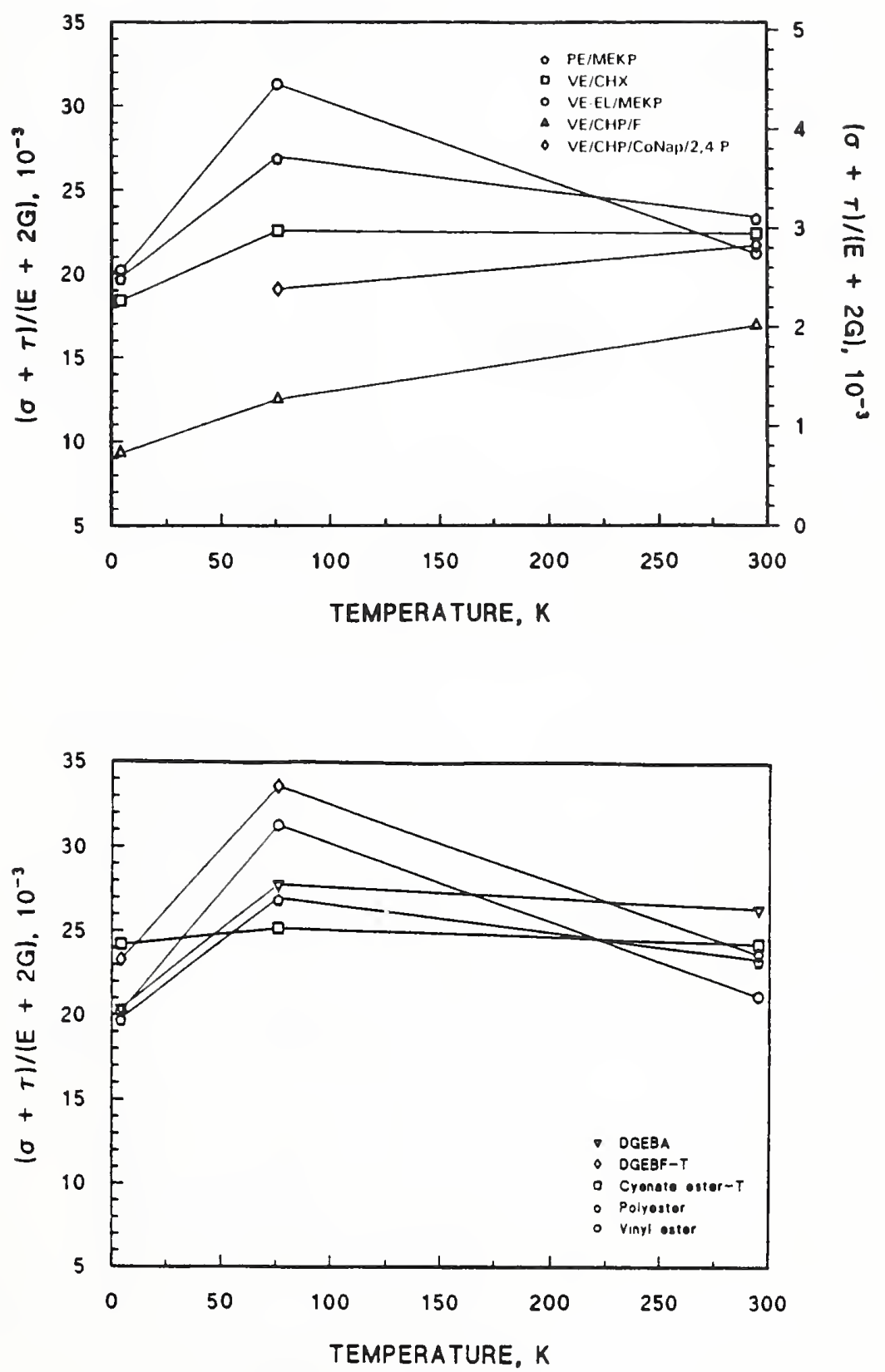

Figure 11.8. (continued). The ratio $(\sigma+\tau) /(E+2 G)$ versus temperature for the polyester and vinyl esters (c) and the best resin systems of each class (d). 
(a)

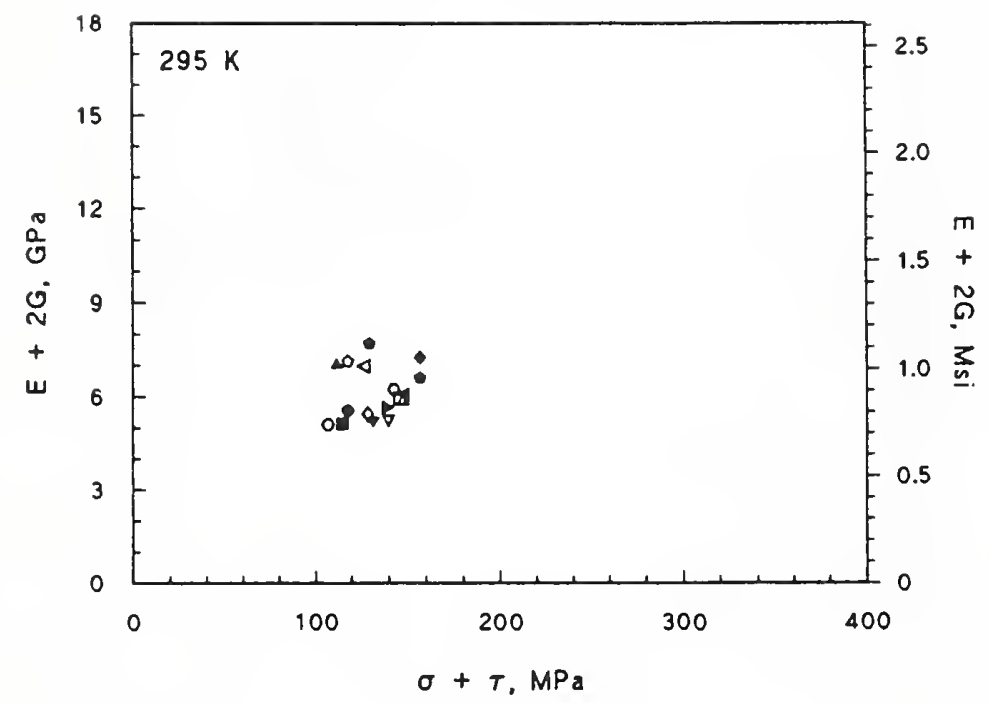

(b)

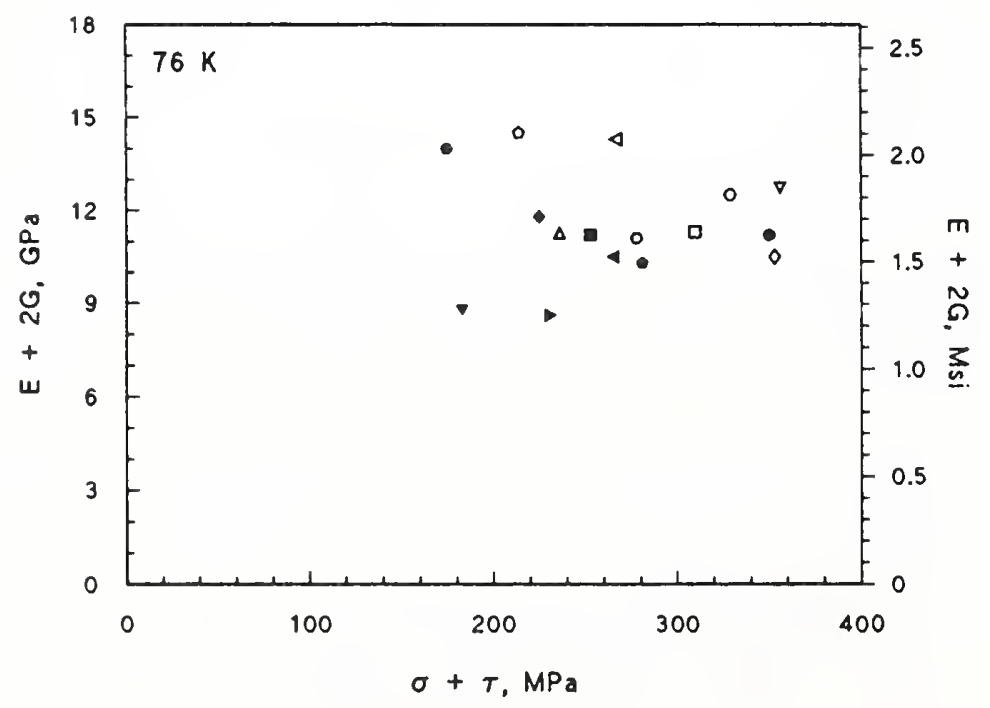

Figure 11.9. For all resin systems, $E+2 G$ versus $\sigma+\tau$ at $295 \mathrm{~K}$ (a) and $76 \mathrm{~K}$ (b) (continued on next page). The material legend used in this figure is given in part (d). 
(c)

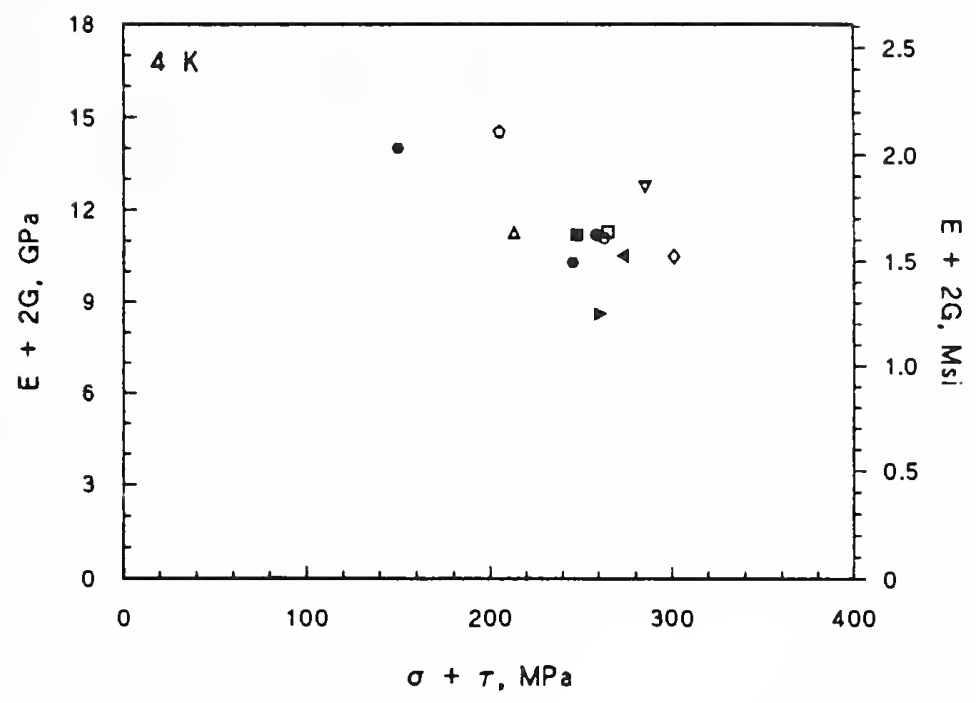

(d)

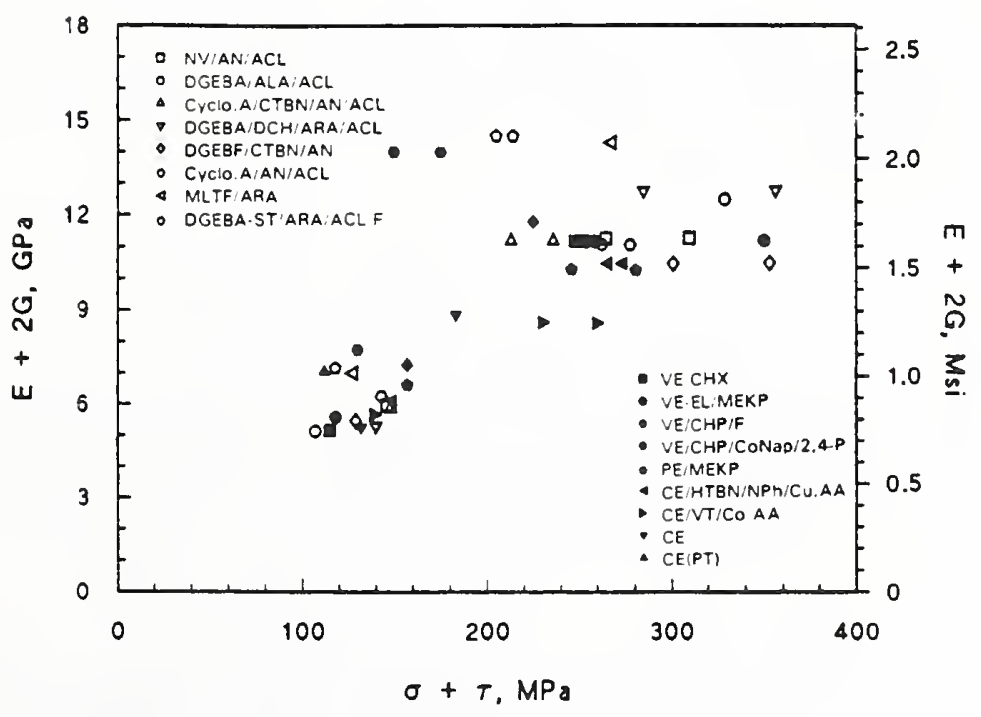

Figure 11.9. (continued). For all resin systems, $E+2 G$ versus $\sigma+\tau$ at $4 \mathrm{~K}$ (c) and all three temperatures (d). 


\subsubsection{Effects of Flaws on Mechanical Properties}

In $\$ I I I .4$ we demonstrated that the tensile strength of neat-resin systems was critically dependent on the size of surface or internal flaws. This dependence on notch sensitivity increased at low temperatures. Plots of tensile strength versus flaw size (Fig. 4.62), extrapolated to zero flaw size, indicated an inherent resin strength of about $100 \mathrm{MPa}$ at room temperature and $170 \mathrm{MPa}$ at 76 and $4 \mathrm{~K}$. These plots contained data from all classes of resin systems. It is likely, however, that more extensive analyses of individual resin systems or of each class of resin systems would reveal variations from these approximate values. From these plots we estimated the fracture toughness of neatresin systems to be as high as about $1.8 \mathrm{MPa} \sqrt{\mathrm{m}}$ at $295 \mathrm{~K}$ and $2.4 \mathrm{MPa} \sqrt{\mathrm{m}}$ at 76 and $4 \mathrm{~K}$.

The clear dependence of neat-resin strength on flaw size, as discussed in \$III.4.6, places a premium on good fabrication procedures to achieve high resin strength. Clearly, in view of the strong influence of flaws in affecting fracture, the concentration of flaws will also be a concern. In the tensile specimens that we analyzed, the volume percent of flaws was less than $1 \%$ and there were no multiple flaws within the specimen cross section that were able to affect fracture. But obviously, in composites where the porosity is typically between 3 and $10 \%$, the strength level at which microcracking will initiate will be strongly influenced by multiple flaws.

\subsection{Thermal Properties}

Jackel et al. [11.1], using DGEBA-base epoxy systems, reported increases of thermal conductivity $(\lambda)$ at low temperatures for decreased cross-link density and small increases of $E$ (calculated from their reported density and sound-velocity data) at room temperature for decreased cross-link density. Thus, from their data, $\lambda$ increases with increasing $E$, if one assumes that $E$ also decreases with cross-link density at low temperatures.

Earlier, Hartwig had reported that $\lambda$ at $4 \mathrm{~K}$ decreased as the cross-link distance between atom chains decreased [11.2]. He also reported a small increase in the Young's modulus $(E)$ as the crosslink distance decreased. Thus, his data supported an inverse dependence of $\lambda$ on $E$. Our thermal conductivity data at 295 and $4 \mathrm{~K}$ are plotted versus the combined values of the elastic moduli $(E+2 G)$ measured at 295 and $4 \mathrm{~K}$, respectively, in Figure 11.10. Notice that $\lambda$ increases as $E+2 G$ increases. Notice also, that because of the differing temperature dependences of $E$ for the resin systems in Figure 11.10, the positions of the resin systems within the trend lines change at 295 and $4 \mathrm{~K}$.

The filled epoxy (DGEBA-ST/ARA/ACL/F) produced an anomalous dependence of $\lambda$ on $E$ at $4 \mathrm{~K}$ : the $E+2 G$ value at $4 \mathrm{~K}$ was $16.4 \mathrm{GPa}$ and $\lambda$ was $0.045 \mathrm{~W} / \mathrm{m} \cdot \mathrm{K}$; these values fall well off of the trend line for the unfilled epoxies. Again, in contrast, Hartwig reported that the value of $\lambda$ for filled-epoxy systems at $4 \mathrm{~K}$ was higher than that of the unfilled epoxy systems that he studied [11.2]. A reasonable explanation for the disparity between the two sets of data is that his filled-resin system contained $60 \mathrm{wt} \%$ quartz powder, whereas our system contained only about 17 wt \% of filler $\left(\mathrm{Al}_{2} \mathrm{O}_{3}\right.$ powder).

The correlation of low-temperature thermal conductivity with room-temperature density is not as good as its correlation with elastic moduli. This suggests that chemical composition does play an important role in affecting thermal conductivity. 


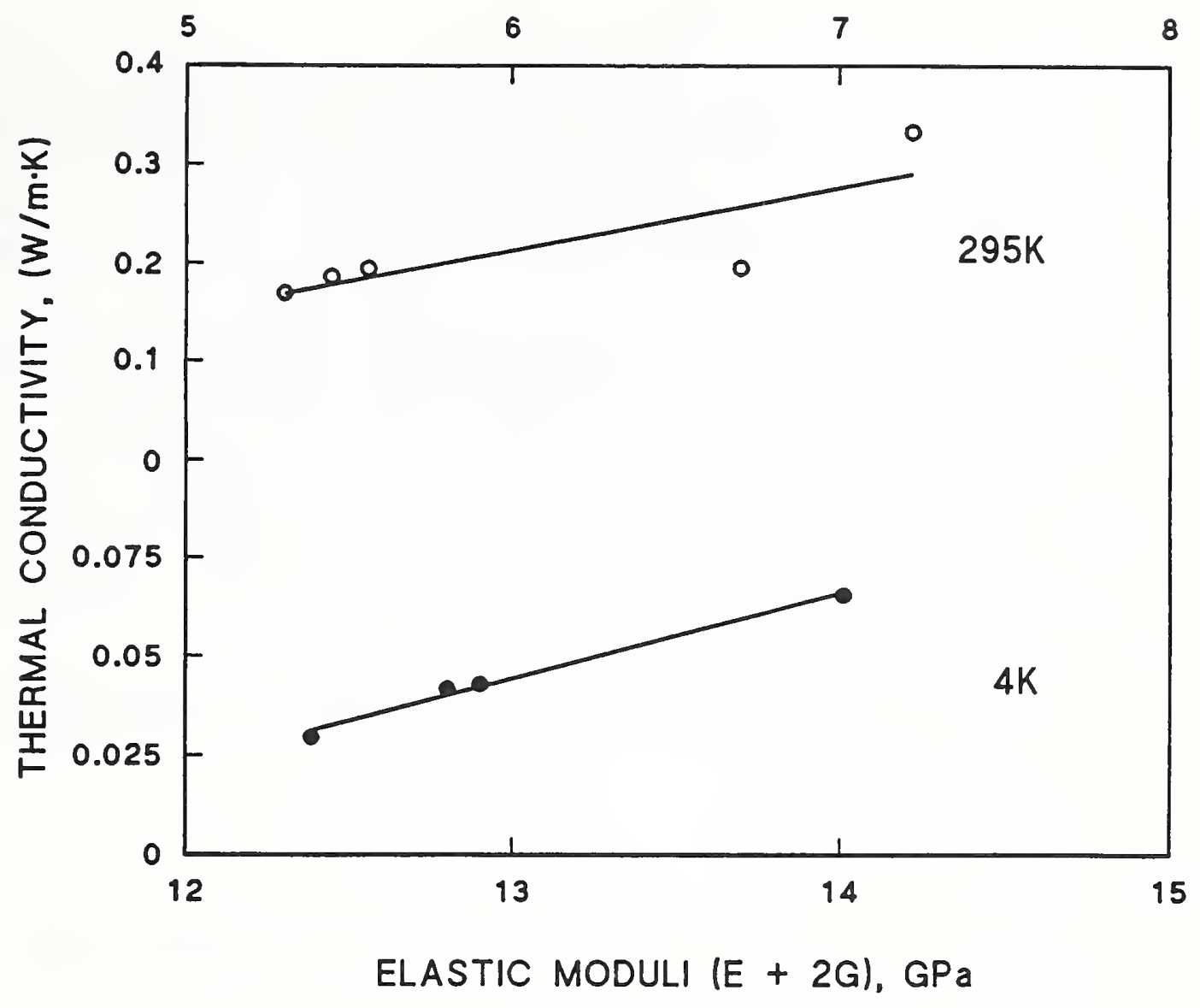

Figure 11.10. Thermal conductivity versus $E+2 G$ at 295 and $4 \mathrm{~K}$.

Similarly, the amount of thermal contraction on cooling from room temperature to $4 \mathrm{~K}$ has been shown to depend on chemical composition. Epoxies contracted between 1.0 and $1.2 \%$; cyanate esters, between 1.0 and $1.1 \%$, and polyesters and vinyl esters, about $1.25 \%$. The addition of tougheners increased the total contraction from 0.1 to $0.2 \%$. Multifunctional epoxies or filled resin systems had lower thermal contractions-between 0.80 and $0.97 \%$.

In general, the amount of thermal contraction on cooling from 295 to $4 \mathrm{~K}$ increases as the density of the resin system decreases. All systems that contracted more than $1 \%$ had densities less than $1.23 \mathrm{~g} / \mathrm{cm}^{3}$; all systems that contracted less than $1 \%$ had densities greater than $1.26 \mathrm{~g} / \mathrm{cm}^{3}$. 


\subsection{Effects of Chemical Composition}

This program has demonstrated that the polymer chemistry can be used to change the lowtemperature properties of neat resins. The addition of fillers increased the elastic moduli and thermal conductivity but lowered the strength and thermal contraction. Similarly, at low temperatures, multifunctional epoxies had higher elastic moduli but lower strength and thermal contraction than other epoxies. Differences between the epoxies and esters were not as distinct, yet some general comments can be made:

1. The temperature dependence of the elastic properties of cyanate esters was not as strong as that of the other classes of resin systems. Since all resins had similar elastic moduli at room temperature, the elastic moduli of cyanate ester systems were, therefore, lower at low temperatures.

2. The cyanate esters (and perhaps the polyesters) had distinctive strength properties. At room temperature their tensile and shear strengths were slightly higher than most epoxies and vinyl esters; at $76 \mathrm{~K}$, their strengths were decidedly lower. At $4 \mathrm{~K}$, however, all resin strengths were approximately equivalent since the strengths of all epoxies and vinyl esters decreased on cooling from 76 to $4 \mathrm{~K}$, and those of the cyanate esters did not.

3. The amount of thermal contraction was dependent on the type of resin system. Both cyanate esters and epoxy systems contracted less than the vinyl and polyester systems on cooling from 295 to $4 \mathrm{~K}$.

\subsection{Resin Selection}

These measurements on neat-resin systems at low temperatures have identified several significant factors:

1. No one resin system is clearly superior. The selection of "the best" resin system depends on the matrix properties that are required in the composite. Improved properties can be obtained by changing the chemical composition or density of the resin system. The selection of the most important properties depends on the weak link or failure mode of the composite structure. This discussion will be pursued following identification of the failure modes in the composite struts that have been tested in this program $(\S \mathrm{VI})$.

2. Defects in the resin systems strongly affect their failure under tensile or shear loads. This factor, then, suggests that the processibility of the resin system is as significant as the properties reported in this study. For, if the strength of the matrix is a significant parameter in affecting the failure of a composite structure, then the void size and content will strongly influence the performance of the composite.

3. Both the strength and thermal conductivity of neat resins tend to increase with increasing elastic moduli. Thus, in selecting resin systems for structural applications, such as support struts for SMES that span the temperature gradient from 4 to $295 \mathrm{~K}$, there may be a trade-off between optimization of strength and optimization of thermal conductance.

Finally, a word about the use of the information in this study. From the data acquired, it is not possible to identify a "best" resin for use in composite structures for three basic reasons:

1. No one resin system has clearly demonstrated the highest moduli and strength and the lowest thermal conductivity, thermal contraction, and residual stress. 
2. Selection of a resin must be based on its performance as the matrix for a specific composite, a factor more significant than data for the matrix material only. Thus, comparable unidirectional and $\pm 45^{\circ}$ (in-plane shear) composite data are also necessary to optimize neat-resin selection.

3. Resin selection depends on the composite failure mode that restricts operational usage. For example, composite struts for SMES plants are likely to experience a temperature gradient from either 4 or $76 \mathrm{~K}$ to $295 \mathrm{~K}$. Our strut data indicate that the failure stress at $295 \mathrm{~K}$ is less than the failure stress at lower temperatures. Thus, to improve operational performance, the roomtemperature strengths may have to be considered. This would require careful consideration of the resin system that has the best room-temperature data for the elastic or strength properties that address the failure mode of the strut (at room temperature). 


\section{References}

[2.1] Hahn, H.T.; Williams, J.G. Compression failure mechanisms in unidirectional composites. ASTM STP 893. Philadelphia, PA: American Society for Testing Materials; 1986. 115-139. Also NASA Tech. Memo 85834. Hampton, VA: Langley Research Center; 1984.

[2.2] Sohi, M.M.; Hahn, H.T.; Williams, J.G. The effect of resin toughness and modulus on compressive failure modes of quasi-isotropic graphite/epoxy laminates. ASTM STP 937. Philadelphia, PA: American Society of Testing and Materials; 1987.

[2.3] L.B. Greszczuk. On failure modes of unidirectional composites under compressive loading, in Fracture of composite materials. Sih, G.C. and Tamuzs, V.J.P., eds. Martinus Wijhoff Publishing Co.; 1982. 231-244.

[2.4] Chamis, C.C. and Smith, G.T. Resin selection criteria for 'tough' composite structures. NASA Tech. Memo 83449. Cleveland, OH: Lewis Research Center; 1983.

[2.5] Sinclair, J.H.; Chamisc, C.C. Compression behavior of unidirectional fibrous composites. NASA Tech. Memo. 82833. Cleveland, OH: Lewis Research Center; 1982.

[2.6] Will, E.T.. A screening program to select a resin for Gravity Probe-B composites. Cryogenics 32: $179-184 ; 1992$.

[4.1] Hartwig, G. Low-temperature properties of epoxy resins and composites, in Advances in cryogenic engineering-materials, vol. 24. New York, NY: Plenum Press; 1978. 17-36.

[4.2] Hartwig, G. Low-temperature properties of resins and their correlations, in Advances in cryogenic engineering, vol. 22. New York, NY: Plenum Press; 1976. 283-295.

[4.3] Lee, H.; Neville, K. Handbook of epoxy resins. New York, NY: McGraw-Hill Book Co.; 1972. 7-8.

[4.4] Chiao, T.T.; Jessop, E.S.; Penn, L. Screening of epoxy systems for high performance filament winding applications, in Proceedings of the 7th national SAMPE technical conference, vol. 7.; 1975. 167-176.

[4.5] Hacker, H.; Albrecht, C.; Laupenmühlen, H.; Satzburger, H., Ihlein, W. Epoxies for low temperature application impregnating technology, in Advances in cryogenic engineeringmaterials, vol. 30. New York, NY: Plenum Press; 1984. 51-60.

[4.6] Pink, E.; Campbell, J.D. The low-temperature macro deformation of an epoxide resin. Mater. Sci. Eng. 15: 187-194; 1974.

[4.7] Colyer, B.; Ball, M.T.; Campbell, M.P. Work of fracture of epoxy resins. Cryogenics 14: 281-283; 1974. 
[4.8] Pritchard, G.; Rhoades, G.V. The fracture of highly crosslinked resins. An invited review, Mater. Sci. Eng. 26: 1-11; 1976.

[4.9] Warfield, R.W.; Cuevas, J.E.; Barret, F.R. Single specimen determination of Young's and bulk moduli of polymers. Rheol. Acta 9: 439-446; 1970.

[5.1] Wilson, D.W. An overview of test methods used for shear characterization of advanced composite materials, in Advances in cryogenic engineering-materials, vol. 36. New York, NY: Plenum Press; 1989. 793-810.

[5.2] Adams, D.F.; Thomas, R.L., The solid-rod torsion test for the determination of unidirectional composite shear properties. Text. Res. J. 39(4): 339-345; 1969.

[5.3] Kasen, M.B. A strain-controlled torsional test method for screening the performance of composite materials at cryogenic temperatures. J. Mater. Sci. 23: 813-834; 1988.

[5.4] Kasen, M.B.; Stoddard, R.B. Screening the performance of organic insulators under cryogenic neutron irradiation, in Advances in cryogenic engineering-materials, vol. 32. New York, NY: Plenum Press; 1986. 153-159.

[5.5] Kasen, M.B.; Munshi, N.A. Cost-effective techniques for development of radiation-resistant organic insulators for superconducting magnets. Phase II, SBIR Contract No. DE-AC0287ER80487. U.S. Department of Energy; 1989. 2-7, 11-12, 15.

[5.6] Munshi, N.A. Effect of polymer additives and residual elements on the cryogenic performance and radiation resistance of insulators for high-field magnets. Phase II, SBIR Contract No. DEAC02-89ER80708. U.S. Department of Energy, 1990. 5, 7-9, 14.

[6.1] Van de Voorde, M. Results of physical tests on polymer materials at cryogenic temperatures. Geneva, Switzerland: CERN; 1972.

[6.2] Hartwig, G. Low-temperature properties of epoxy resins and composites, in Advances in cryogenic engineering-materials, vol. 24. New York, NY: Plenum Press; 1978. 17-36.

[6.3] Hartwig, G. Low-temperature properties of resins and their correlations, in Advances in cryogenic engineering, vol. 22. New York, NY: Plenum Press; 1976. 283-295.

[6.4] Hacker, H.; Albrecht, C.; Laupenmühlen, H.; Salzburger, H.; Ihlein, W. Epoxies for low temperature application impregnating technology, in Advances in cryogenic engineeringmaterials, vol. 30. New York, NY: Plenum Press; 1984. 51-60.

[6.5] Evans, D.; Morgan, J.T. A review of the thermal properties of epoxide resins and composites at low temperatures, in Proceedings of the international cryogenic materials conference, Kobe, Japan. Guildford, Surrey, England: Butterworths; 1983. 446-454.

[6.6] Measurement of thermal expansion coefficient using strain gages. Raleigh, NC: Measurements Group, Inc., P.O. Box 27777; 1986. 
[6.7] Finke, T.E.; Heberling, T.G. Determination of thermal expansion characteristics of metals using strain gages. Proc. SESA (now SEM) 25(1): 155-158; 1978.

[6.8] Poore, M.W.; Kesterson, K.F. Measuring the thermal expansion of solids with strain gages. J. Test. Eval. ASTM 6(2): 90-102; 1978.

[6.9] Valentich, J. Thermal expansion of solids from $-261^{\circ}$ to $173^{\circ} \mathrm{C}$ using strain gages. Cryogenics 25: 63-67; 1985.

[6.10] Hahn, T.A. Thermal expansion of copper from 20 to $800 \mathrm{~K}$-Standard Reference Material 736, J. Appl. Phys. 41: 5096; 1970.

[6.11] Austin, M.W. unpublished data. Natl. Inst. Stand. Technol.; 1992.

[6.12] Linear thermal expansion of solid materials with a vitreous silica dilatometer. ASTM Standard E228-85. Philadelphia, PA: American Society for Testing and Materials; 1991.

[7.1] Hust, J.G. Thermal conductivity of G-10CR and G-11CR industrial laminates and their component parts at low temperatures, in Materials studies for magnetic fusion energy applications at low temperatures-V, NBSIR 82-1667. Nat. Bur. Stand. (U.S.); 1982.

[7.2] Nicholls, C.I.; Rosenberg, H.M. The excitation spectrum of epoxy resins; neutron diffraction, specific heat and thermal conductivity at low temperatures. J. Phys. C: Solid State Phys. 17: $1165-78 ; 1984$.

[7.3] Rosenberg, H.M. The thermal conductivity and thermal expansion of non-metallic composite materials at low temperatures, in Nonmetallic materials and composites at low temperatures-2. New York, NY: Plenum Press; 1982. 181-195,

[7.4] Evans, D.; Morgan, J.T. Cryogen containment in composite vessels, in Advances in cryogenic engineering-materials, vol. 32. New York, NY: Plenum Press; 1986. 127-136,

[7.5] Rule, D.L.; Smith, D.R.; Sparks, L.L. Thermal conductivity of a polyimide film between 4.2 and $300 \mathrm{~K}$, with and without alumina particles as filler. NISTIR 3948. Natl. Inst. Stand. Technol.; 1990.

[7.6] Locatelli, M.; Arnaud, D.; Routin, M. Thermal conductivity of some insulating materials below 1 K. Cryogenics 16(61): 374-375; 1976.

[7.7] Van de Voorde, M. Temperature irradiation effects on materials and components for superconducting magnets for high energy physics applications. CERN 77-03. Geneva, Switzerland: CERN, ISR Division; July 1977.

[7.8] Hust, J.G.; Arvidson, J. Thermal conductivity of glass fiber/epoxy composite support bands for cryogenic Dewars. Report to sponsor. Nat. Bur. Stand. (U.S); 1978.

[9.1] Indentation hardness of rigid plastics by means of a Barcol impressor. ASTM standard test method D2583-81. Philadelphia, PA: American Society for Testing and Materials; 1987. 
[9.2] Density of high modulus fibers by archimedes and sink-float method. ASTM standard D380079. Philadelphia, PA: American Society for Testing and Materials; 1988.

[11.1] Jackel, M.; Müller, M.; Licea Claverie, A.; Arndt, K.F. Thermal conductivity of modified epoxy resins with different cross-link densities at low temperatures. Cryogenics 31: 228-230; 1991.

[11.2] Hartwig, G. Low-temperature properties of epoxy resins and composites, in Advances in cryogenic engineering-materials, vol. 24. New York, NY: Plenum Press;1978. 17-36. 


\section{Appendixes}

\section{Appendix A. Tensile Strength, Elastic Modulus, and Poisson's Ratio}

Table A.1. Tensile strength, elastic modulus, and Poisson's ratio.

\begin{tabular}{|c|c|c|c|c|c|c|}
\hline \multirow[t]{2}{*}{ Resin } & \multirow{2}{*}{$\begin{array}{c}\text { Tempera- } \\
\text { ture } \\
(\mathrm{K}) \\
\end{array}$} & \multicolumn{2}{|c|}{$\begin{array}{c}\text { Tensile } \\
\text { Strength }\end{array}$} & \multicolumn{2}{|c|}{$\begin{array}{l}\text { Elastic } \\
\text { Modulus }\end{array}$} & \multirow[t]{2}{*}{$\begin{array}{c}\text { Poisson's } \\
\text { Ratio }\end{array}$} \\
\hline & & (MPa) & (ksi) & $(\mathrm{GPa})$ & (Msi) & \\
\hline \multirow[t]{14}{*}{ NV/AN/ACL } & 295 & 76.9 & 11.15 & & & \\
\hline & 295 & 80.8 & 11.72 & & & \\
\hline & 295 & 81.4 & 11.81 & & & \\
\hline & 295 & 81.9 & 11.87 & & & \\
\hline & 295 & & & 3.67 & 0.533 & 0.335 \\
\hline & 295 & & & 3.72 & 0.540 & 0.378 \\
\hline & 295 & & & 3.73 & 0.541 & 0.376 \\
\hline & & 80.3 & 11.60 & 3.71 & 0.538 & 0.363 \\
\hline & 76 & 116.9 & 16.9 & & & \\
\hline & 76 & 100.2 & 14.5 & & & \\
\hline & 76 & 112.4 & 16.3 & & & \\
\hline & 76 & 87.9 & 12.8 & & & \\
\hline & 76 & & & 6.94 & 1.01 & 0.389 \\
\hline & 76 & & & 6.55 & 0.950 & 0.382 \\
\hline \multirow[t]{11}{*}{ Average } & & 104.4 & 15.1 & 6.75 & 0.98 & 0.385 \\
\hline & 4 & 69.7 & 10.1 & & & \\
\hline & 4 & 109.4 & 15.9 & & & \\
\hline & 4 & 47.1 & 6.83 & & & \\
\hline & 4 & 51.8 & 7.51 & & & \\
\hline & 4 & 87.9 & 12.7 & & & \\
\hline & 4 & 49.6 & 7.2 & & & \\
\hline & 4 & 87.4 & 12.7 & & & \\
\hline & 4 & 76.7 & 11.12 & & & \\
\hline & 4 & & & 8.69 & 1.26 & 0.402 \\
\hline & 4 & & & 7.52 & 1.09 & 0.371 \\
\hline Average & & 72.5 & 10.5 & 8.10 & 1.17 & 0.386 \\
\hline
\end{tabular}


Table A.l. Tensile strength, elastic modulus, and Poisson's ratio (continued).

\begin{tabular}{|c|c|c|c|c|c|c|}
\hline \multirow[t]{2}{*}{ Resin } & \multirow{2}{*}{$\begin{array}{c}\text { Tempera- } \\
\text { ture } \\
(\mathrm{K})\end{array}$} & \multicolumn{2}{|c|}{$\begin{array}{l}\text { Tensile } \\
\text { Strength }\end{array}$} & \multicolumn{2}{|c|}{$\begin{array}{c}\text { Elastic } \\
\text { Modulus }\end{array}$} & \multirow[t]{2}{*}{$\begin{array}{c}\text { Poisson's } \\
\text { Ratio }\end{array}$} \\
\hline & & (MPa) & (ksi) & $(\mathrm{GPa})$ & (Msi) & \\
\hline \multirow[t]{12}{*}{ DGEBA/DCH/ARA/ACL } & 295 & 86.8 & 12.6 & & & \\
\hline & 295 & 60.1 & 8.72 & & & \\
\hline & 295 & 73.1 & 10.6 & & & \\
\hline & 295 & & & 3.23 & 0.469 & 0.397 \\
\hline & 295 & & & 3.22 & 0.467 & 0.364 \\
\hline & & 73.3 & 10.6 & 3.23 & 0.468 & 0.381 \\
\hline & 76 & 99.5 & 14.4 & & & \\
\hline & 76 & 110 & 15.9 & & & \\
\hline & 76 & 89.4 & 13.0 & & & \\
\hline & 76 & 74.9 & 10.9 & & & \\
\hline & 76 & & & 7.40 & 1.08 & 0.418 \\
\hline & 76 & & & 7.30 & 1.06 & 0.423 \\
\hline Average & & 93.5 & 13.6 & 7.35 & 1.07 & 0.421 \\
\hline \multirow[t]{8}{*}{ DGEBA/DCH/ARA/ACL } & 4 & 102 & 14.8 & & & \\
\hline & 4 & 93.8 & 13.6 & & & \\
\hline & 4 & 56.9 & 8.25 & & & \\
\hline & 4 & 82.9 & 12.0 & & & \\
\hline & 4 & 46.5 & 6.75 & & & \\
\hline & 4 & 107 & 15.6 & & & \\
\hline & 4 & & & 8.26 & 1.20 & 0.358 \\
\hline & 4 & & & 8.26 & 1.20 & 0.426 \\
\hline Average & & 81.5 & 11.8 & 8.26 & 1.20 & 0.392 \\
\hline \multirow[t]{13}{*}{ VE-EL/MEKP } & 295 & 62.7 & 9.09 & & & \\
\hline & 295 & 63.4 & 9.20 & & & \\
\hline & 295 & 63.4 & 9.20 & & & \\
\hline & 295 & 57.4 & 8.33 & & & \\
\hline & 295 & & & 3.40 & 0.493 & 0.365 \\
\hline & 295 & & & 3.29 & 0.477 & 0.361 \\
\hline & & 61.7 & 8.96 & 3.34 & 0.485 & 0.363 \\
\hline & 76 & 153 & 22.2 & & & \\
\hline & 76 & 169 & 24.6 & & & \\
\hline & 76 & 165 & 23.9 & & & \\
\hline & 76 & 105 & 15.2 & & & \\
\hline & 76 & & & 6.01 & 0.871 & 0.429 \\
\hline & 76 & & & 6.68 & 0.968 & 0.426 \\
\hline Average & & 148. & 21.5 & 6.35 & 0.920 & 0.426 \\
\hline
\end{tabular}


Table A.l. Tensile strength, elastic modulus, and Poisson's ratio (continued).

\begin{tabular}{|c|c|c|c|c|c|c|}
\hline Resin & $\begin{array}{c}\text { Tempera- } \\
\text { ture } \\
(\mathrm{K})\end{array}$ & \multicolumn{2}{|c|}{$\begin{array}{c}\text { Tensile } \\
\text { Strength }\end{array}$} & \multicolumn{2}{|c|}{$\begin{array}{c}\text { Elastic } \\
\text { Modulus }\end{array}$} & $\begin{array}{c}\text { Poisson's } \\
\text { Ratio }\end{array}$ \\
\hline \multirow[t]{7}{*}{ VE-EL/MEKP } & 4 & 135 & 19.6 & & & \\
\hline & 4 & 144 & 20.9 & & & \\
\hline & 4 & 101 & 14.6 & & & \\
\hline & 4 & 137 & 19.9 & & & \\
\hline & 4 & 85.3 & 12.4 & & & \\
\hline & 4 & & & 7.87 & 1.14 & 0.464 \\
\hline & 4 & & & 7.02 & 1.02 & 0.378 \\
\hline Average & & 120. & 17.5 & 7.45 & 1.08 & 0.421 \\
\hline \multirow[t]{9}{*}{ DGEBA/ALA/ACL } & 295 & 58.6 & 8.50 & & & \\
\hline & 295 & 61.9 & 8.97 & & & \\
\hline & 295 & 60.7 & 8.80 & & & \\
\hline & 295 & 61.1 & 8.86 & & & \\
\hline & 295 & 54.6 & 7.92 & & & \\
\hline & 295 & 63.0 & 9.13 & & & \\
\hline & 295 & 53.9 & 7.82 & & & \\
\hline & 295 & & & 3.24 & 0.47 & 0.378 \\
\hline & 295 & & & 3.35 & 0.49 & 0.378 \\
\hline Average & & 59.1 & 8.57 & 3.29 & 0.48 & 0.378 \\
\hline \multirow[t]{15}{*}{ DGEBA/ALA/ACL } & 76 & 153 & 22.2 & & & \\
\hline & 76 & 64.0 & 9.29 & & & \\
\hline & 76 & 103 & 14.9 & & & \\
\hline & 76 & 101 & 14.6 & & & \\
\hline & 76 & 72.7 & 10.6 & & & \\
\hline & 76 & & & 6.73 & 1.00 & 0.388 \\
\hline & 76 & & & 7.28 & 1.06 & 0.449 \\
\hline & & 98.7 & 14.3 & 7.10 & 1.03 & 0.418 \\
\hline & 4 & 156 & 22.6 & & & \\
\hline & 4 & 96.8 & 14.0 & & & \\
\hline & 4 & 82.9 & 12.0 & & & \\
\hline & 4 & 56.3 & 8.16 & & & \\
\hline & 4 & 85.4 & 12.4 & & & \\
\hline & 4 & & & 7.89 & 1.14 & 0.382 \\
\hline & 4 & & & 7.99 & 1.16 & 0.435 \\
\hline Average & & 95.5 & 13.8 & 7.94 & 1.15 & 0.409 \\
\hline \multirow[t]{6}{*}{ DGEBF/CTBN/AN } & 295 & 59.3 & 8.60 & & & \\
\hline & 295 & 76.0 & 11.0 & & & \\
\hline & 295 & 49.8 & 7.22 & & & \\
\hline & 295 & 75.6 & 10.9 & & & \\
\hline & 295 & & & 3.30 & 0.48 & 0.366 \\
\hline & 295 & & & 3.43 & 0.50 & 0.373 \\
\hline Average & & 65.2 & 9.43 & 3.36 & 0.49 & 0.366 \\
\hline
\end{tabular}


Table A.I. Tensile strength, elastic modulus, and Poisson's ratio (continued).

\begin{tabular}{|c|c|c|c|c|c|c|}
\hline \multirow[t]{2}{*}{ Resin } & \multirow{2}{*}{$\begin{array}{c}\text { Tempera- } \\
\text { ture } \\
(\mathrm{K})\end{array}$} & \multicolumn{2}{|c|}{$\begin{array}{l}\text { Tensile } \\
\text { Strength }\end{array}$} & \multicolumn{2}{|c|}{$\begin{array}{l}\text { Elastic } \\
\text { Modulus }\end{array}$} & \multirow[t]{2}{*}{$\begin{array}{c}\text { Poisson's } \\
\text { Ratio }\end{array}$} \\
\hline & & $(\mathrm{MPa})$ & (ksi) & $(\mathrm{GPa})$ & (Msi) & \\
\hline \multirow[t]{7}{*}{ DGEBF/CTBN/AN } & 76 & 161 & 23.4 & & & \\
\hline & 76 & 155 & 22.5 & & & \\
\hline & 76 & 104 & 15.1 & & & \\
\hline & 76 & 112 & 16.2 & & & \\
\hline & 76 & 120 & 17.5 & & & \\
\hline & 76 & & & 5.99 & 0.87 & 0.487 \\
\hline & 76 & & & 6.22 & 0.903 & 0.447 \\
\hline \multirow[t]{10}{*}{ Average } & & 130. & 18.9 & 6.10 & 0.88 & 0.467 \\
\hline & 4 & 96.9 & 14.1 & & & \\
\hline & 4 & 129 & 18.7 & & & \\
\hline & 4 & 112 & 16.2 & & & \\
\hline & 4 & 143 & 20.7 & & & \\
\hline & 4 & 83.2 & 12.1 & & & \\
\hline & 4 & 75 & 10.9 & & & \\
\hline & 4 & 57.1 & 8.28 & & & \\
\hline & 4 & & & 6.97 & 1.01 & 0.423 \\
\hline & 4 & & & 7.04 & 1.02 & 0.435 \\
\hline \multicolumn{2}{|l|}{ Average } & 99.5 & 14.4 & 7.01 & 1.02 & 0.429 \\
\hline \multirow[t]{21}{*}{ Cyclo.A/CTBN/AN/ACL } & 295 & 60.5 & 8.78 & & & \\
\hline & 295 & 87.8 & 12.7 & & & \\
\hline & 295 & 65.6 & 9.51 & & & \\
\hline & 295 & 58.8 & 8.52 & & & \\
\hline & 295 & 65.4 & 9.49 & & & \\
\hline & 295 & & & 3.51 & 0.546 & 0.363 \\
\hline & 295 & & & 3.76 & 0.510 & 0.374 \\
\hline & & 67.6 & 9.80 & 3.63 & 0.527 & 0.368 \\
\hline & 76 & 120 & 17.4 & & & \\
\hline & 76 & 83.6 & 12.1 & & & \\
\hline & 76 & 97.5 & 14.2 & & & \\
\hline & 76 & 58.3 & 8.45 & & & \\
\hline & 76 & & & 6.59 & 0.956 & 0.396 \\
\hline & 76 & & & 6.32 & 0.917 & 0.434 \\
\hline & & 89.9 & 13.0 & 6.45 & 0.936 & 0.415 \\
\hline & 4 & 90.3 & 13.1 & & & \\
\hline & 4 & 73.1 & 10.6 & & & \\
\hline & 4 & 77.7 & 11.3 & & & \\
\hline & 4 & 56.0 & 8.12 & & & \\
\hline & 4 & & & 7.50 & 1.09 & 0.365 \\
\hline & 4 & & & 8.16 & 1.18 & 0.433 \\
\hline Average & & 74.3 & 10.8 & 7.83 & 1.14 & 0.399 \\
\hline
\end{tabular}


Table A.l. Tensile strength, elastic modulus, and Poisson's ratio (continued).

\begin{tabular}{|c|c|c|c|c|c|c|}
\hline \multirow[t]{2}{*}{ Resin } & \multirow{2}{*}{$\begin{array}{c}\text { Tempera- } \\
\text { ture } \\
(\mathrm{K})\end{array}$} & \multicolumn{2}{|c|}{$\begin{array}{l}\text { Tensile } \\
\text { Strength }\end{array}$} & \multicolumn{2}{|c|}{$\begin{array}{c}\text { Elastic } \\
\text { Modulus }\end{array}$} & \multirow[t]{2}{*}{$\begin{array}{c}\text { Poisson's } \\
\text { Ratio }\end{array}$} \\
\hline & & (MPa) & (ksi) & $(\mathrm{GPa})$ & (Msi) & \\
\hline \multirow[t]{16}{*}{ CE/VT/Co.AA } & 295 & 66.0 & 9.57 & & & \\
\hline & 295 & 46.9 & 6.80 & & & \\
\hline & 295 & 67.6 & 9.80 & & & \\
\hline & 295 & 58.2 & 8.50 & & & \\
\hline & 295 & 60.5 & 8.77 & & & \\
\hline & 295 & 66.0 & 9.57 & & & \\
\hline & 295 & & & 3.74 & 0.54 & 0.38 \\
\hline & 295 & & & 3.48 & 0.50 & 0.36 \\
\hline & & 60.8 & 8.82 & 3.61 & 0.52 & 0.37 \\
\hline & 76 & 97.6 & 14.2 & & & \\
\hline & 76 & 98.3 & 14.3 & & & \\
\hline & 76 & 49.4 & 7.17 & & & \\
\hline & 76 & 69.0 & 10.0 & & & \\
\hline & 76 & 86.9 & 12.6 & & & \\
\hline & 76 & & & 5.15 & 0.748 & 0.443 \\
\hline & 76 & & & 5.12 & 0.742 & 0.392 \\
\hline \multirow[t]{10}{*}{ Average } & & 80.2 & 11.7 & 5.14 & 0.745 & 0.418 \\
\hline & 4 & 126 & 18.3 & & & \\
\hline & 4 & 110 & 16.0 & & & \\
\hline & 4 & 81.4 & 11.8 & & & \\
\hline & 4 & 103 & 15.0 & & & \\
\hline & 4 & 55.2 & 8.06 & & & \\
\hline & 4 & 89.0 & 12.9 & & & \\
\hline & 4 & 85.0 & 12.3 & & & \\
\hline & 4 & & & 6.20 & 0.899 & 0.405 \\
\hline & 4 & & & 6.01 & 0.871 & 0.446 \\
\hline Average & & 92.8 & 13.5 & 6.11 & 0.885 & 0.426 \\
\hline \multirow[t]{12}{*}{ CE/HTBN/NPh/Cu.AA } & 295 & 88.3 & 12.8 & & & \\
\hline & 295 & 60.4 & 8.76 & & & \\
\hline & 295 & 58.8 & 8.53 & & & \\
\hline & 295 & 88.0 & 12.8 & & & \\
\hline & 295 & & & 3.62 & 0.525 & 0.334 \\
\hline & 295 & & & 3.64 & 0.528 & $\longrightarrow$ \\
\hline & & 73.9 & 10.7 & 3.63 & 0.527 & 0.334 \\
\hline & 76 & 117 & 16.9 & & & \\
\hline & 76 & 96.9 & 14.1 & & & \\
\hline & 76 & 89.4 & 13.0 & & & \\
\hline & 76 & 101 & 14.7 & & & \\
\hline & 76 & & & 6.02 & 0.873 & 0.416 \\
\hline Average & & 101. & 14.7 & 6.02 & 0.873 & 0.416 \\
\hline
\end{tabular}


Table A.I. Tensile strength, elastic modulus, and Poisson's ratio (continued).

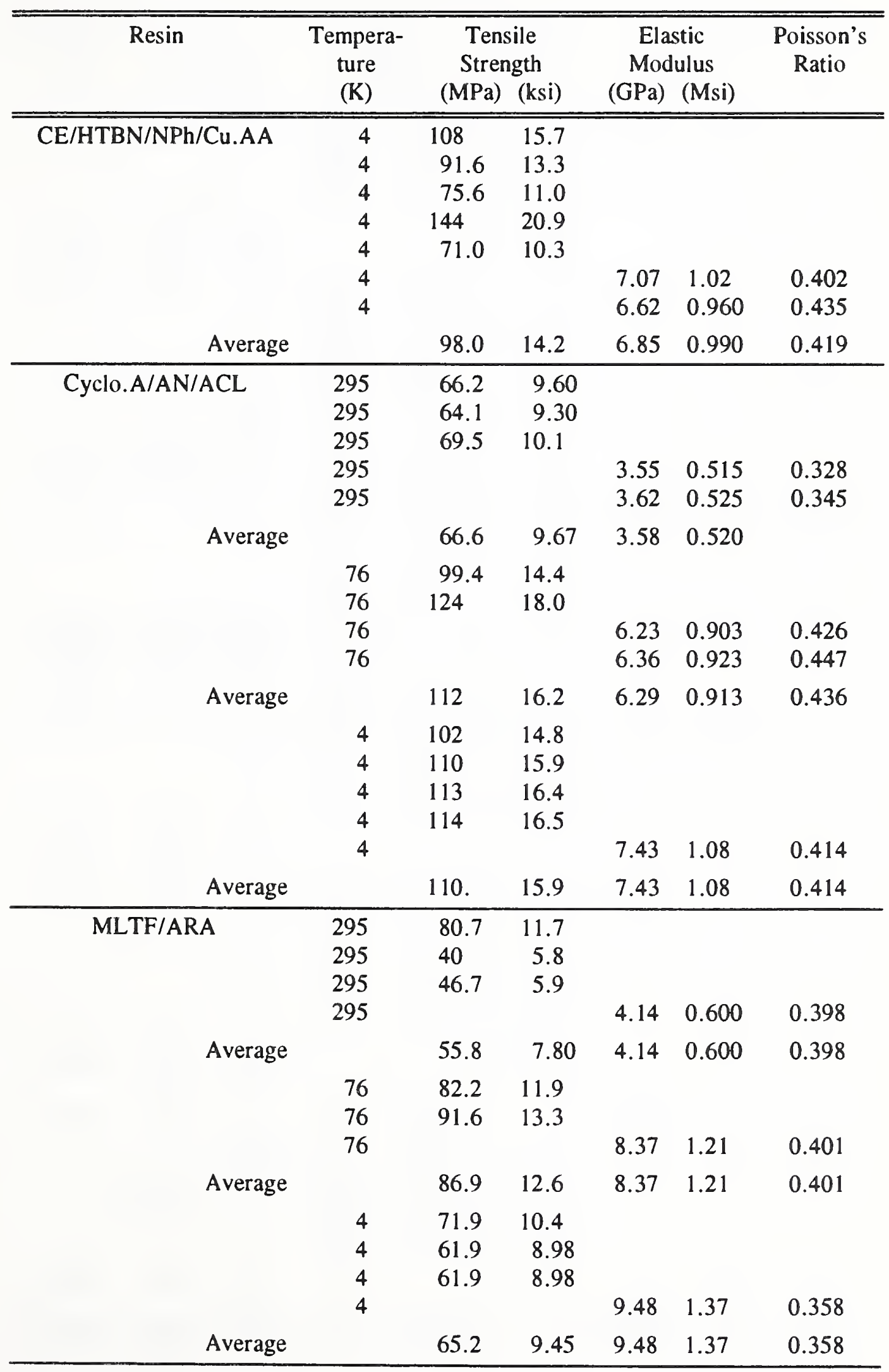


Table A.I. Tensile strength, elastic modulus, and Poisson's ratio (continued).

\begin{tabular}{|c|c|c|c|c|c|c|}
\hline \multirow[t]{2}{*}{ Resin } & \multirow{2}{*}{$\begin{array}{c}\text { Tempera- } \\
\text { ture } \\
(\mathrm{K})\end{array}$} & \multicolumn{2}{|c|}{$\begin{array}{l}\text { Tensile } \\
\text { Strength }\end{array}$} & \multicolumn{2}{|c|}{$\begin{array}{c}\text { Elastic } \\
\text { Modulus }\end{array}$} & \multirow[t]{2}{*}{$\begin{array}{c}\text { Poisson's } \\
\text { Ratio }\end{array}$} \\
\hline & & $(\mathrm{MPa})$ & (ksi) & $(\mathrm{GPa})$ & (Msi) & \\
\hline \multirow[t]{5}{*}{ DGEBA-ST/ARA/ACL/F } & 295 & 53.1 & 7.70 & & & \\
\hline & 295 & 55.8 & 8.09 & & & \\
\hline & 295 & 52.4 & 7.60 & & & \\
\hline & 295 & & & 4.14 & 0.600 & 0.353 \\
\hline & 295 & & & 4.06 & 0.589 & 0.328 \\
\hline \multirow[t]{6}{*}{ Average } & & 53.8 & 7.80 & 4.10 & 0.595 & 0.340 \\
\hline & 76 & 72.1 & 10.5 & & & \\
\hline & 76 & 84.1 & 12.2 & & & \\
\hline & 76 & 87.5 & 12.7 & & & \\
\hline & 76 & 102 & 14.8 & & & \\
\hline & 76 & & & 8.44 & 1.22 & 0.367 \\
\hline Average & & 86.4 & 12.6 & 8.44 & 1.22 & 0.367 \\
\hline \multirow[t]{6}{*}{ DGEBA-ST/ARA/ACL/F } & 4 & 84.6 & 12.3 & & & \\
\hline & 4 & 77.1 & 11.2 & & & \\
\hline & 4 & 85.3 & 12.4 & & & \\
\hline & 4 & 84.0 & 12.2 & & & \\
\hline & 4 & & & 9.57 & 1.39 & 0.350 \\
\hline & 4 & & & 9.35 & 1.36 & 0.403 \\
\hline \multicolumn{2}{|l|}{ Average } & 82.8 & 12.0 & 9.46 & 1.38 & 0.377 \\
\hline \multirow{5}{*}{$\mathrm{VE} / \mathrm{CHX}$} & 295 & 62 & 9.0 & & & \\
\hline & 295 & 67 & 9.7 & & & \\
\hline & 295 & 66 & 9.5 & & & \\
\hline & 295 & & & 3.12 & 0.453 & 0.350 \\
\hline & 295 & & & 3.11 & 0.451 & 0.313 \\
\hline \multirow[t]{7}{*}{ Average } & & 65.0 & 9.40 & 3.12 & 0.452 & 0.336 \\
\hline & 76 & 155 & 22.5 & & & \\
\hline & 76 & 159 & 23.1 & & & \\
\hline & 76 & 167 & 24.3 & & & \\
\hline & 76 & 162 & 23.5 & & & \\
\hline & 76 & & & 6.57 & 0.953 & 0.461 \\
\hline & 76 & & & 6.56 & 0.951 & 0.458 \\
\hline \multirow[t]{7}{*}{ Average } & & 161. & 23.4 & 6.57 & 0.952 & 0.460 \\
\hline & 4 & 154 & 22.3 & & & \\
\hline & 4 & 163 & 23.6 & & & \\
\hline & 4 & 157 & 22.7 & & & \\
\hline & 4 & 146 & 21.2 & & & \\
\hline & 4 & & & 7.09 & 1.03 & 0.455 \\
\hline & 4 & & & 7.19 & 1.04 & 0.400 \\
\hline Average & & 155. & 22.5 & 7.14 & 0.104 & 0.427 \\
\hline
\end{tabular}


Table A.1. Tensile strength, elastic modulus, and Poisson's ratio (continued).

\begin{tabular}{|c|c|c|c|c|c|c|}
\hline \multirow[t]{2}{*}{ Resin } & \multirow{2}{*}{$\begin{array}{c}\text { Tempera- } \\
\text { ture } \\
(\mathrm{K})\end{array}$} & \multicolumn{2}{|c|}{$\begin{array}{c}\text { Tensile } \\
\text { Strength }\end{array}$} & \multicolumn{2}{|c|}{$\begin{array}{c}\text { Elastic } \\
\text { Modulus }\end{array}$} & \multirow[t]{2}{*}{$\begin{array}{c}\text { Poisson's } \\
\text { Ratio }\end{array}$} \\
\hline & & $(\mathrm{MPa})$ & (ksi) & $(\mathrm{GPa})$ & (Msi) & \\
\hline \multirow[t]{3}{*}{$\mathrm{VE} / \mathrm{CHP} / \mathrm{F}$} & 295 & 57.8 & 8.39 & & & \\
\hline & 295 & 59.2 & 8.59 & & & \\
\hline & 295 & & & 4.35 & 0.631 & 0.329 \\
\hline \multirow[t]{6}{*}{ Average } & & 58.5 & 8.49 & 4.35 & 0.631 & 0.329 \\
\hline & 76 & 57.4 & 8.32 & & & \\
\hline & 76 & 84.7 & 12.2 & & & \\
\hline & 76 & 68.4 & 9.92 & & & \\
\hline & 76 & & & 8.11 & 1.17 & 0.406 \\
\hline & 76 & & & 7.55 & 1.10 & 0.338 \\
\hline \multicolumn{2}{|l|}{ Average } & 70.2 & 10.1 & 7.83 & 1.14 & 0.372 \\
\hline \multirow[t]{5}{*}{$\mathrm{VE} / \mathrm{CHP} / \mathrm{F}$} & 4 & 55.5 & 8.05 & & & \\
\hline & 4 & 33.6 & 4.87 & & & \\
\hline & 4 & 60.0 & 8.70 & & & \\
\hline & 4 & 73.6 & 10.7 & & & \\
\hline & 4 & & & 9.21 & 1.34 & 0.367 \\
\hline \multicolumn{2}{|l|}{ Average } & 55.6 & 8.07 & 9.21 & 1.34 & 0.367 \\
\hline \multirow[t]{4}{*}{$\mathrm{VE} / \mathrm{CHP} / \mathrm{CoNap} / 2,4-\mathrm{P}$} & 295 & 86.9 & 12.6 & & & \\
\hline & 295 & 93.4 & 12.1 & & & \\
\hline & 295 & 87.6 & 12.7 & & & \\
\hline & 295 & & & 4.14 & 0.600 & 0.391 \\
\hline \multicolumn{2}{|l|}{ Average } & 89.3 & 12.5 & 4.14 & 0.600 & 0.391 \\
\hline & 76 & 79.3 & 11.5 & & & \\
\hline & 76 & 146 & 21.2 & & & \\
\hline & 76 & 129 & 18.7 & & & \\
\hline & 76 & 129 & 18.7 & & & \\
\hline & 76 & & & 6.84 & 0.992 & 0.395 \\
\hline \multicolumn{2}{|l|}{ Average } & 121. & 17.5 & 6.84 & 0.992 & 0.395 \\
\hline & 4 & 120 & 17.4 & & & \\
\hline & 4 & 135 & 19.6 & & & \\
\hline & 4 & 67.5 & 9.76 & & & \\
\hline & 4 & 133 & 19.2 & & & \\
\hline & 4 & & & 7.58 & 1.10 & 0.400 \\
\hline \multicolumn{2}{|l|}{ Average } & 114. & 16.5 & 7.58 & 1.10 & 0.400 \\
\hline \multirow[t]{4}{*}{ PE/MEKP } & 295 & 79.3 & 11.5 & & & \\
\hline & 295 & 79.3 & 11.5 & & & \\
\hline & 295 & 71.0 & 10.3 & & & \\
\hline & 295 & & & 3.97 & 0.576 & 0.332 \\
\hline Average & & 76.5 & 11.1 & 3.97 & 0.576 & 0.332 \\
\hline
\end{tabular}


Table A.I. Tensile strength, elastic modulus, and Poisson's ratio (continued).

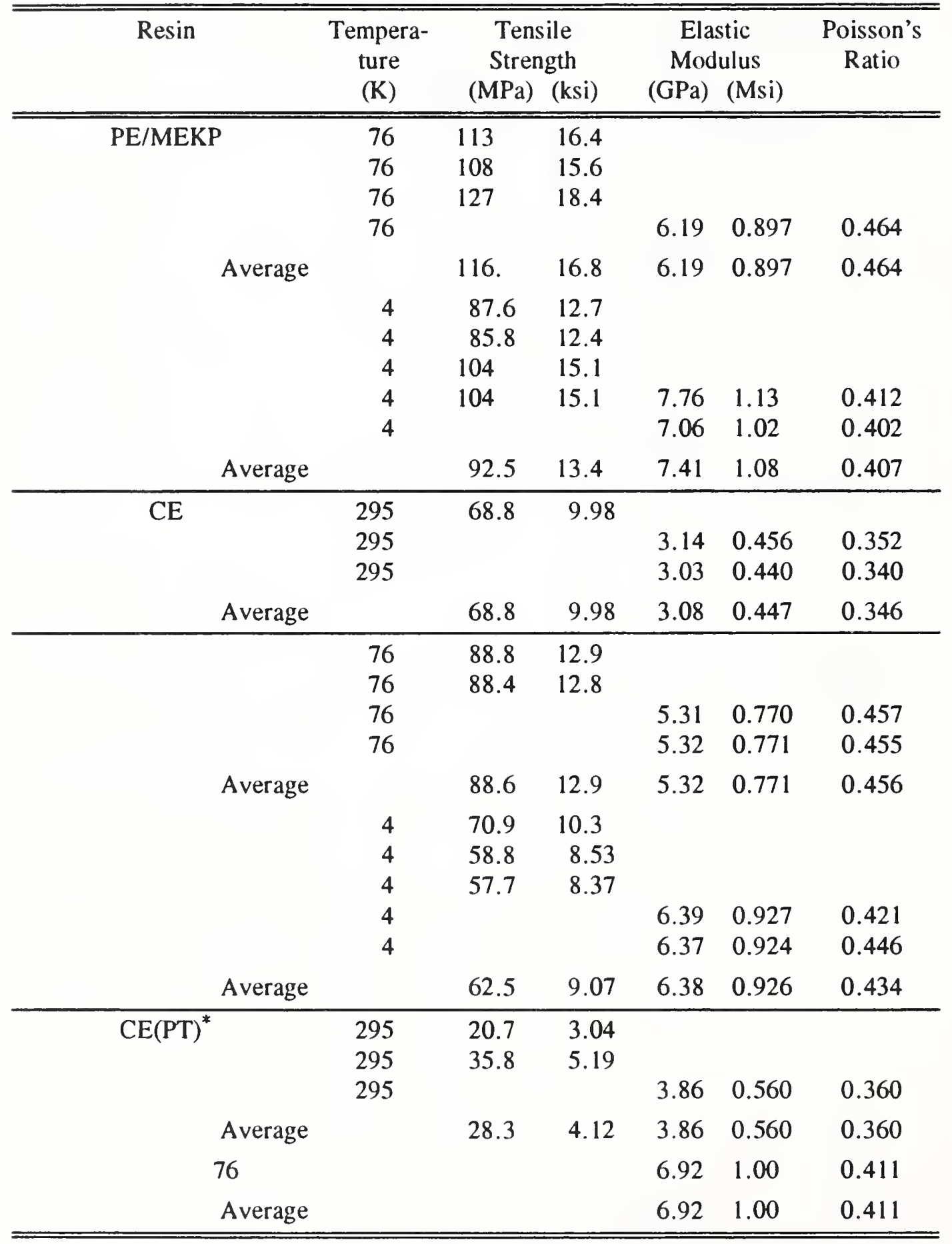

${ }^{*}$ See comments regarding cure schedule on page III-16. 


\section{Appendix B. Shear Properties}

NOTE: In the tables of this appendix, standard deviation is abbreviated S.D. and coefficient of variation, C.V.

Table B.1. Ultimate shear strength and shear modulus of epoxies at $76 \mathrm{~K}$.

\begin{tabular}{|c|c|c|c|c|c|c|c|c|}
\hline \multirow{2}{*}{$\begin{array}{c}\text { Resin } \\
\text { DGEBA/DCH/ARA/ACL }\end{array}$} & \multicolumn{2}{|c|}{$\begin{array}{l}\text { Diameter } \\
(\mathrm{mm})\end{array}$} & \multicolumn{3}{|c|}{$\begin{array}{l}\text { Ultimate Shear Strength } \\
(\mathrm{MPa})\end{array}$} & \multicolumn{3}{|c|}{$\begin{array}{c}\text { Shear Modulus } \\
(\mathrm{GPa}) \\
\end{array}$} \\
\hline & $\begin{array}{l}3.216 \\
3.208 \\
3.216 \\
3.233 \\
3.198\end{array}$ & & $\begin{array}{l}241.8 \\
232.7 \\
207.4 \\
185.0 \\
177.0\end{array}$ & $\begin{array}{l}\text { Ave. } \\
\text { S.D. } \\
\text { C.V. }\end{array}$ & $\begin{array}{r}208.8 \\
28.5 \\
13.6\end{array}$ & $\begin{array}{l}2.87 \\
2.73 \\
2.59 \\
2.68 \\
2.89\end{array}$ & $\begin{array}{l}\text { Ave. } \\
\text { S.D. } \\
\text { C.V. }\end{array}$ & $\begin{array}{l}2.75 \\
0.13 \\
4.7\end{array}$ \\
\hline DGEBA/ALA/ACL & $\begin{array}{l}3.239 \\
3.228 \\
3.231 \\
3.231 \\
3.239\end{array}$ & & $\begin{array}{l}137.8 \\
185.8 \\
194.9 \\
206.6 \\
228.3\end{array}$ & $\begin{array}{l}\text { Ave. } \\
\text { S.D. } \\
\text { C.V. }\end{array}$ & $\begin{array}{r}190.7 \\
33.6 \\
17.6\end{array}$ & $\begin{array}{l}2.85 \\
2.56 \\
2.66 \\
2.67 \\
2.67\end{array}$ & $\begin{array}{l}\text { Ave. } \\
\text { S.D. } \\
\text { C.V. }\end{array}$ & $\begin{array}{l}2.68 \\
0.105 \\
3.9\end{array}$ \\
\hline DGEBA-ST/ARA/ACL/F & $\begin{array}{l}3.193 \\
3.190 \\
3.188 \\
3.167 \\
3.165\end{array}$ & & $\begin{array}{l}131.2 \\
121.6 \\
110.3 \\
114.7 \\
115.0\end{array}$ & $\begin{array}{l}\text { Ave. } \\
\text { S.D. } \\
\text { C.V. }\end{array}$ & $\begin{array}{r}118.6 \\
8.1 \\
6.8\end{array}$ & $\begin{array}{l}3.14 \\
3.13 \\
3.16 \\
3.02 \\
2.98\end{array}$ & $\begin{array}{l}\text { Ave. } \\
\text { S.D. } \\
\text { C.V. }\end{array}$ & $\begin{array}{l}3.09 \\
0.08 \\
2.6\end{array}$ \\
\hline DGEBF/CTBN/AN & $\begin{array}{l}3.195 \\
3.223 \\
3.223 \\
3.206 \\
3.203\end{array}$ & $*$ & $\begin{array}{l}196.0 \\
211.3 \\
180.3 \\
151.9 \\
215.3\end{array}$ & $\begin{array}{l}\text { Ave. } \\
\text { S.D. } \\
\text { C.V. }\end{array}$ & $\begin{array}{r}191.0 \\
25.8 \\
13.5\end{array}$ & $\begin{array}{l}2.08 \\
2.18 \\
2.24 \\
2.31 \\
2.28\end{array}$ & $\begin{array}{l}\text { Ave. } \\
\text { S.D. } \\
\text { C.V. }\end{array}$ & $\begin{array}{l}2.22 \\
0.09 \\
4.1\end{array}$ \\
\hline NV/AN/ACL & $\begin{array}{l}3.145 \\
3.137 \\
3.150 \\
3.129 \\
3.137 \\
3.124 \\
3.150 \\
3.155 \\
3.145\end{array}$ & * & $\begin{array}{l}168.9 \\
136.6 \\
176.1 \\
157.4 \\
194.5 \\
144.1 \\
204.7 \\
170.7 \\
202.3\end{array}$ & $\begin{array}{l}\text { Ave. } \\
\text { S.D. } \\
\text { C.V. }\end{array}$ & $\begin{array}{r}172.8 \\
24.4 \\
14.1\end{array}$ & $\begin{array}{l}2.16 \\
2.15 \\
2.43 \\
2.21 \\
2.32 \\
2.40 \\
2.24 \\
2.45 \\
2.22\end{array}$ & $\begin{array}{l}\text { Ave. } \\
\text { S.D. } \\
\text { C.V. }\end{array}$ & $\begin{array}{l}2.29 \\
0.116 \\
5.1\end{array}$ \\
\hline
\end{tabular}

${ }^{*}$ Specimen did not fail. 
Table B.1. Ultimate shear strength and shear modulus of epoxies at $76 \mathrm{~K}$ (continued).

\begin{tabular}{|c|c|c|c|c|c|c|c|}
\hline \multirow{2}{*}{$\frac{\text { Resin }}{\text { Cyclo.A/CTBN/AN/ACL }}$} & \multirow{2}{*}{$\begin{array}{c}\begin{array}{c}\text { Diameter } \\
(\mathrm{mm})\end{array} \\
3.195 \\
3.216 \\
3.211 \\
3.221 \\
3.223\end{array}$} & \multicolumn{3}{|c|}{$\begin{array}{l}\text { Ultimate Shear Strength } \\
\qquad(\mathrm{MPa})\end{array}$} & \multicolumn{3}{|c|}{$\begin{array}{l}\text { Shear Modulus } \\
(\mathrm{GPa})\end{array}$} \\
\hline & & $\begin{array}{r}150.0 \\
59.1 \\
112.3 \\
138.0 \\
87.5\end{array}$ & $\begin{array}{l}\text { Ave. } \\
\text { S.D. } \\
\text { C.V. }\end{array}$ & $\begin{array}{r}109.4 \\
37.0 \\
33.8\end{array}$ & $\begin{array}{l}2.41 \\
2.29 \\
2.45 \\
2.44 \\
2.26\end{array}$ & $\begin{array}{l}\text { Ave. } \\
\text { S.D. } \\
\text { C.V. }\end{array}$ & $\begin{array}{l}2.37 \\
0.089 \\
3.7\end{array}$ \\
\hline Cyclo.A/AN/ACL & $\begin{array}{l}3.233 \\
3.223 \\
3.233 \\
3.228 \\
3.226\end{array}$ & $\begin{array}{l}143.8 \\
135.5 \\
157.5 \\
134.9 \\
160.7\end{array}$ & $\begin{array}{l}\text { Ave. } \\
\text { S.D. } \\
\text { C.V. }\end{array}$ & $\begin{array}{r}146.5 \\
12.1 \\
8.3\end{array}$ & $\begin{array}{l}2.47 \\
2.55 \\
2.31 \\
2.49 \\
2.30\end{array}$ & $\begin{array}{l}\text { Ave. } \\
\text { S.D. } \\
\text { C.V. }\end{array}$ & $\begin{array}{l}2.42 \\
0.113 \\
4.7\end{array}$ \\
\hline MLTF/ARA & $\begin{array}{l}3.155 \\
3.175 \\
3.073 \\
3.165 \\
3.172\end{array}$ & $\begin{array}{l}168.4 \\
193.7 \\
153.9 \\
178.1 \\
158.9\end{array}$ & $\begin{array}{l}\text { Ave. } \\
\text { S.D. } \\
\text { C.V. }\end{array}$ & $\begin{array}{r}170.6 \\
15.9 \\
9.3\end{array}$ & $\begin{array}{l}2.89 \\
3.01 \\
2.98 \\
2.95 \\
3.01\end{array}$ & $\begin{array}{l}\text { Ave. } \\
\text { S.D. } \\
\text { C.V. }\end{array}$ & $\begin{array}{l}2.97 \\
0.05 \\
1.7\end{array}$ \\
\hline MLTF/ARA/TGH & $\begin{array}{l}3.226 \\
3.239 \\
3.226 \\
3.231 \\
3.226\end{array}$ & $\begin{array}{l}132.0 \\
155.7 \\
144.8 \\
161.0 \\
144.8\end{array}$ & $\begin{array}{l}\text { Ave. } \\
\text { S.D. } \\
\text { C.V. }\end{array}$ & $\begin{array}{r}147.7 \\
11.2 \\
7.6\end{array}$ & $\begin{array}{l}3.07 \\
3.45 \\
3.06 \\
3.25 \\
3.12\end{array}$ & $\begin{array}{l}\text { Ave. } \\
\text { S.D. } \\
\text { C.V. }\end{array}$ & $\begin{array}{l}3.19 \\
0.164 \\
5.1\end{array}$ \\
\hline
\end{tabular}


Table B.2. Ultimate shear strain of epoxies at $76 \mathrm{~K}$.

\begin{tabular}{|c|c|c|c|c|}
\hline \multirow{2}{*}{$\frac{\text { Resin }}{\text { DGEBA/DCH/ARA/ACL }}$} & \multirow{2}{*}{$\begin{array}{c}\begin{array}{c}\text { Diameter } \\
(\mathrm{mm})\end{array} \\
3.216 \\
3.208 \\
3.216 \\
3.233 \\
3.198\end{array}$} & \multicolumn{3}{|c|}{$\begin{array}{c}\text { Ultimate Shear Strain } \\
\text { (radians) }\end{array}$} \\
\hline & & $\begin{array}{l}0.127 \\
0.119 \\
0.105 \\
0.086 \\
0.083\end{array}$ & $\begin{array}{l}\text { Ave. } \\
\text { S.D. } \\
\text { C.V. }\end{array}$ & $\begin{array}{l}0.104 \\
0.020 \\
19.2\end{array}$ \\
\hline DGEBA/ALA/ACL & $\begin{array}{l}3.239 \\
3.228 \\
3.231 \\
3.231 \\
3.239\end{array}$ & $\begin{array}{l}0.065 \\
0.091 \\
0.095 \\
0.095 \\
0.122\end{array}$ & $\begin{array}{l}\text { Ave. } \\
\text { S.D. } \\
\text { C.V. }\end{array}$ & $\begin{array}{l}0.094 \\
0.020 \\
21.3\end{array}$ \\
\hline DGEBA-ST/ARA/ACL/F & $\begin{array}{l}3.193 \\
3.190 \\
3.188 \\
3.167 \\
3.165\end{array}$ & $\begin{array}{l}0.054 \\
0.050 \\
0.043 \\
0.049 \\
0.052\end{array}$ & $\begin{array}{l}\text { Ave. } \\
\text { S.D. } \\
\text { C.V. }\end{array}$ & $\begin{array}{l}0.050 \\
0.004 \\
8.0\end{array}$ \\
\hline DGEBF/CTBN/AN & $\begin{array}{l}3.195 \\
3.223 \\
3.223 \\
3.206 \\
3.203\end{array}$ & $\begin{array}{l}0.132 \\
0.136 \\
0.114 \\
0.089 \\
0.150\end{array}$ & $\begin{array}{l}\text { Ave. } \\
\text { S.D. } \\
\text { C.V. }\end{array}$ & $\begin{array}{l}0.124 \\
0.024 \\
19.4\end{array}$ \\
\hline NV/AN/ACL & $\begin{array}{l}3.145 \\
3.137 \\
3.150 \\
3.129 \\
3.137 \\
3.124 \\
3.150 \\
3.155 \\
3.145\end{array}$ & $\begin{array}{l}0.100 \\
0.079 \\
0.100 \\
0.091 \\
0.100 \\
0.073 \\
0.120 \\
0.090 \\
0.116\end{array}$ & $\begin{array}{l}\text { Ave. } \\
\text { S.D. } \\
\text { C.V. }\end{array}$ & $\begin{array}{l}0.097 \\
0.015 \\
15.5\end{array}$ \\
\hline Cyclo.A/CTBN/AN/ACL & $\begin{array}{l}3.195 \\
3.216 \\
3.211 \\
3.221 \\
3.223\end{array}$ & $\begin{array}{l}0.077 \\
0.028 \\
0.056 \\
0.074 \\
0.044\end{array}$ & $\begin{array}{l}\text { Ave. } \\
\text { S.D. } \\
\text { C.V. }\end{array}$ & $\begin{array}{l}0.056 \\
0.021 \\
37.5\end{array}$ \\
\hline Cyclo.A/AN/ACL & $\begin{array}{l}3.233 \\
3.223 \\
3.233 \\
3.228 \\
3.226\end{array}$ & $\begin{array}{l}0.075 \\
0.073 \\
0.085 \\
0.070 \\
0.089\end{array}$ & $\begin{array}{l}\text { Ave. } \\
\text { S.D. } \\
\text { C.V. }\end{array}$ & $\begin{array}{l}0.079 \\
0.008 \\
10.1\end{array}$ \\
\hline
\end{tabular}

III-171 
Table B.2. Ultimate shear strain of epoxies at $76 \mathrm{~K}$ (continued).

\begin{tabular}{lcccc}
\hline \multicolumn{1}{c}{ Resin } & $\begin{array}{c}\text { Diameter } \\
(\mathrm{mm})\end{array}$ & \multicolumn{2}{c}{$\begin{array}{c}\text { Ultimate Shear Strain } \\
\text { (radians) }\end{array}$} \\
\hline \hline MLTF/ARA & 3.155 & 0.075 & Ave. & 0.072 \\
& 3.175 & 0.084 & S.D. & 0.009 \\
& 3.073 & 0.061 & C.V. & 12.5 \\
& 3.165 & 0.075 & & \\
MLTF/ARA/TGH & 3.172 & 0.065 & & \\
& 3.226 & 0.053 & Ave. & 0.059 \\
& 3.239 & 0.059 & S.D. & 0.004 \\
& 3.226 & 0.058 & C.V. & 6.8 \\
& 3.231 & 0.065 & & \\
\hline \hline
\end{tabular}


Table B.3. Ultimate shear strength and shear modulus of cyanate esters at $76 \mathrm{~K}$.

\begin{tabular}{|c|c|c|c|c|c|c|c|}
\hline Resin & $\begin{array}{l}\text { Diameter } \\
(\mathrm{mm})\end{array}$ & \multicolumn{3}{|c|}{$\begin{array}{l}\text { Ultimate Shear } \\
\text { Strength } \\
(\mathrm{MPa})\end{array}$} & \multicolumn{3}{|c|}{$\begin{array}{l}\text { Shear Modulus } \\
\qquad(\mathrm{GPa})\end{array}$} \\
\hline CE/VT/Co.AA & $\begin{array}{l}3.259 \\
3.259 \\
3.256 \\
3.261 \\
3.256\end{array}$ & $\begin{array}{l}114.6 \\
134.7 \\
138.7 \\
133.9 \\
133.5\end{array}$ & $\begin{array}{l}\text { Ave. } \\
\text { S.D. } \\
\text { C.V. }\end{array}$ & $\begin{array}{r}131.1 \\
9.4 \\
7.2\end{array}$ & $\begin{array}{l}1.67 \\
1.79 \\
1.72 \\
1.74 \\
1.73\end{array}$ & $\begin{array}{l}\text { Ave. } \\
\text { S.D. } \\
\text { C.V. }\end{array}$ & $\begin{array}{l}1.73 \\
0.043 \\
2.5\end{array}$ \\
\hline $\mathrm{CE}$ & $\begin{array}{l}3.193 \\
3.172 \\
3.170 \\
3.185 \\
3.185\end{array}$ & $\begin{array}{r}97.7 \\
94.0 \\
111.6 \\
102.8 \\
101.7\end{array}$ & $\begin{array}{l}\text { Ave. } \\
\text { S.D. } \\
\text { C.V. }\end{array}$ & $\begin{array}{r}101.6 \\
6.6 \\
6.5\end{array}$ & $\begin{array}{l}1.81 \\
1.64 \\
1.75 \\
1.92 \\
1.61\end{array}$ & $\begin{array}{l}\text { Ave. } \\
\text { S.D. } \\
\text { C.V. }\end{array}$ & $\begin{array}{l}1.75 \\
0.127 \\
7.2\end{array}$ \\
\hline $\mathrm{CE}(\mathrm{PT})^{*}$ & $\begin{array}{l}3.152 \\
3.157 \\
3.190 \\
3.195 \\
3.193\end{array}$ & $\begin{array}{r}102.7 \\
102.2 \\
91.3 \\
61.3 \\
52.7\end{array}$ & $\begin{array}{l}\text { Ave. } \\
\text { S.D. } \\
\text { C.V. }\end{array}$ & $\begin{array}{l}82.1 \\
23.5 \\
28.6\end{array}$ & $\begin{array}{l}2.30 \\
2.13 \\
2.03 \\
2.07 \\
2.14\end{array}$ & $\begin{array}{l}\text { Ave. } \\
\text { S.D. } \\
\text { C.V. }\end{array}$ & $\begin{array}{l}2.13 \\
0.10 \\
4.7\end{array}$ \\
\hline CE/HTBN/NPh/Cu.AA & $\begin{array}{l}3.203 \\
3.198 \\
3.200 \\
3.200 \\
3.185\end{array}$ & $\begin{array}{l}157.7 \\
157.3 \\
160.2 \\
161.3 \\
159.2\end{array}$ & $\begin{array}{l}\text { Ave. } \\
\text { S.D. } \\
\text { C.V. }\end{array}$ & $\begin{array}{r}159.2 \\
1.7 \\
1.1\end{array}$ & $\begin{array}{l}2.22 \\
2.25 \\
2.29 \\
2.28 \\
2.20\end{array}$ & $\begin{array}{l}\text { Ave. } \\
\text { S.D. } \\
\text { C.V. }\end{array}$ & $\begin{array}{l}2.25 \\
0.038 \\
1.7\end{array}$ \\
\hline
\end{tabular}

* See comments regarding cure schedule on page III-16. 
Table B.4. Ultimate shear strain of cyanate esters at $76 \mathrm{~K}$.

\begin{tabular}{|c|c|c|c|c|}
\hline \multirow{2}{*}{$\begin{array}{r}\text { Resin } \\
\text { CE/VT/Co.AA }\end{array}$} & \multirow{2}{*}{$\begin{array}{c}\begin{array}{c}\text { Diameter } \\
(\mathrm{mm})\end{array} \\
3.259 \\
3.259 \\
3.256 \\
2.261 \\
3.256\end{array}$} & \multicolumn{3}{|c|}{$\begin{array}{c}\text { Ultimate Shear Strain } \\
\text { (radians) }\end{array}$} \\
\hline & & $\begin{array}{l}0.086 \\
0.103 \\
0.108 \\
0.102 \\
0.102\end{array}$ & $\begin{array}{l}\text { Ave. } \\
\text { S.D. } \\
\text { C.V. }\end{array}$ & $\begin{array}{l}0.100 \\
0.008 \\
8.0\end{array}$ \\
\hline $\mathrm{CE}$ & $\begin{array}{l}3.193 \\
3.172 \\
3.170 \\
3.185 \\
3.185\end{array}$ & $\begin{array}{l}0.068 \\
0.067 \\
0.082 \\
0.073 \\
0.077\end{array}$ & $\begin{array}{l}\text { Ave. } \\
\text { S.D. } \\
\text { C.V. }\end{array}$ & $\begin{array}{l}0.073 \\
0.006 \\
8.2\end{array}$ \\
\hline $\mathrm{CE}(\mathrm{PT})^{*}$ & $\begin{array}{l}3.152 \\
3.157 \\
3.190 \\
3.195 \\
3.193\end{array}$ & $\begin{array}{l}0.056 \\
0.056 \\
0.050 \\
0.032 \\
0.026\end{array}$ & $\begin{array}{l}\text { Ave. } \\
\text { S.D. } \\
\text { C.V. }\end{array}$ & $\begin{array}{l}0.044 \\
0.014 \\
31.8\end{array}$ \\
\hline $\mathrm{CE} / \mathrm{HTBN} / \mathrm{NPh} / \mathrm{Cu} . \mathrm{AA}$ & $\begin{array}{l}3.203 \\
3.198 \\
3.200 \\
3.200 \\
3.185\end{array}$ & $\begin{array}{l}0.102 \\
0.105 \\
0.112 \\
0.105 \\
0.106\end{array}$ & $\begin{array}{l}\text { Ave. } \\
\text { S.D. } \\
\text { C.V. }\end{array}$ & $\begin{array}{l}0.106 \\
0.004 \\
3.8\end{array}$ \\
\hline
\end{tabular}

* See comments regarding cure schedule on page III-16. 
Table B.5. Ultimate shear strength and shear modulus of vinyl esters and polyester at $76 \mathrm{~K}$.

\begin{tabular}{|c|c|c|c|c|c|c|c|}
\hline Vinyl Esters: & $\begin{array}{l}\text { Diameter } \\
(\mathrm{mm})\end{array}$ & \multicolumn{3}{|c|}{$\begin{array}{l}\text { Ultimate Shear Strength } \\
(\mathrm{MPa})\end{array}$} & \multicolumn{3}{|c|}{$\begin{array}{l}\text { Shear Modulus } \\
(\mathrm{GPa})\end{array}$} \\
\hline $\mathrm{VE} / \mathrm{CHX}$ & $\begin{array}{l}3.068 \\
3.094 \\
3.068 \\
3.005 \\
3.073\end{array}$ & $\begin{array}{r}80.4 \\
54.9 \\
53.2 \\
126.4 \\
62.8\end{array}$ & $\begin{array}{l}\text { Ave. } \\
\text { S.D. } \\
\text { C.V. }\end{array}$ & $\begin{array}{l}75.5 \\
30.4 \\
40.3\end{array}$ & $\begin{array}{l}2.48 \\
2.38 \\
1.98 \\
2.45 \\
2.29\end{array}$ & $\begin{array}{l}\text { Ave. } \\
\text { S.D. } \\
\text { C.V. }\end{array}$ & $\begin{array}{l}2.32 \\
0.20 \\
8.6\end{array}$ \\
\hline VE-EL/MEKP & $\begin{array}{l}3.086 \\
3.119 \\
3.122 \\
3.106 \\
3.127\end{array}$ & $\begin{array}{l}203.0 \\
156.6 \\
148.0 \\
163.3 \\
198.7\end{array}$ & $\begin{array}{l}\text { Ave. } \\
\text { S.D. } \\
\text { C.V. }\end{array}$ & $\begin{array}{r}173.9 \\
25.2 \\
14.5\end{array}$ & $\begin{array}{l}2.64 \\
2.35 \\
2.63 \\
2.38 \\
2.07\end{array}$ & $\begin{array}{l}\text { Ave. } \\
\text { S.D. } \\
\text { C.V. }\end{array}$ & $\begin{array}{l}2.41 \\
0.235 \\
9.8\end{array}$ \\
\hline $\mathrm{VE} / \mathrm{CHP} / \mathrm{F}$ & $\begin{array}{l}3.162 \\
3.099 \\
3.216 \\
3.206 \\
3.221\end{array}$ & $\begin{array}{r}91.0 \\
87.7 \\
102.1 \\
100.9 \\
100.5\end{array}$ & $\begin{array}{l}\text { Ave. } \\
\text { S.D. } \\
\text { C.V. }\end{array}$ & $\begin{array}{r}96.4 \\
6.6 \\
6.8\end{array}$ & $\begin{array}{l}3.44 \\
3.39 \\
3.16 \\
3.14 \\
3.11\end{array}$ & $\begin{array}{l}\text { Ave. } \\
\text { S.D. } \\
\text { C.V. }\end{array}$ & $\begin{array}{l}3.25 \\
0.15 \\
4.6\end{array}$ \\
\hline VE/CHP/Co.Nap/2,4-P & $\begin{array}{l}3.167 \\
3.056 \\
3.180\end{array}$ & $\begin{array}{r}116.9 \\
90.2 \\
64.4\end{array}$ & $\begin{array}{l}\text { Ave. } \\
\text { S.D. } \\
\text { C.V. }\end{array}$ & $\begin{array}{l}90.5 \\
26.3 \\
29.1\end{array}$ & $\begin{array}{l}2.42 \\
2.57 \\
2.51\end{array}$ & $\begin{array}{l}\text { Ave. } \\
\text { S.D. } \\
\text { C.V. }\end{array}$ & $\begin{array}{l}2.5 \\
0.08 \\
3.2\end{array}$ \\
\hline Polyester: & & & & & & & \\
\hline $\mathrm{PE} / \mathrm{MEKP}$ & $\begin{array}{l}3.137 \\
3.117 \\
3.124 \\
3.119 \\
3.134\end{array}$ & $\begin{array}{l}155.1 \\
162.9 \\
162.9 \\
169.6 \\
118.4\end{array}$ & $\begin{array}{l}\text { Ave. } \\
\text { S.D. } \\
\text { C.V. }\end{array}$ & $\begin{array}{r}153.8 \\
20.4 \\
13.3\end{array}$ & $\begin{array}{l}2.09 \\
2.19 \\
2.05 \\
2.08 \\
2.13\end{array}$ & $\begin{array}{l}\text { Ave. } \\
\text { S.D. } \\
\text { C.V. }\end{array}$ & $\begin{array}{l}2.11 \\
0.054 \\
2.6\end{array}$ \\
\hline
\end{tabular}


Table B.6. Ultimate shear stain of vinyl esters and polyester at $76 \mathrm{~K}$.

\begin{tabular}{|c|c|c|c|c|}
\hline Resin & $\begin{array}{c}\text { Diameter } \\
(\mathrm{mm})\end{array}$ & \multicolumn{3}{|c|}{$\begin{array}{c}\text { Ultimate Shear Strain } \\
\text { (radians) }\end{array}$} \\
\hline \multicolumn{5}{|l|}{ Vinyl Esters: } \\
\hline $\mathrm{VE} / \mathrm{CHX}$ & $\begin{array}{l}3.068 \\
3.094 \\
3.068 \\
3.005 \\
3.073\end{array}$ & $\begin{array}{l}0.035 \\
0.026 \\
0.029 \\
0.060 \\
0.033\end{array}$ & $\begin{array}{l}\text { Ave. } \\
\text { S.D. } \\
\text { C.V. }\end{array}$ & $\begin{array}{c}0.037 \\
0.013 \\
35.1\end{array}$ \\
\hline VE-EL/MEKP & $\begin{array}{l}3.086 \\
3.119 \\
3.122 \\
3.106 \\
3.127\end{array}$ & $\begin{array}{l}0.115 \\
0.085 \\
0.078 \\
0.085 \\
0.111\end{array}$ & $\begin{array}{l}\text { Ave. } \\
\text { S.D. } \\
\text { C.V. }\end{array}$ & $\begin{array}{l}0.095 \\
0.017 \\
17.9\end{array}$ \\
\hline $\mathrm{VE} / \mathrm{CHP} / \mathrm{F}$ & $\begin{array}{l}3.162 \\
3.099 \\
3.216 \\
3.206 \\
3.221\end{array}$ & $\begin{array}{l}0.032 \\
0.031 \\
0.040 \\
0.040 \\
0.039\end{array}$ & $\begin{array}{l}\text { Ave. } \\
\text { S.D. } \\
\text { C.V. }\end{array}$ & $\begin{array}{l}0.036 \\
0.004 \\
11.1\end{array}$ \\
\hline VE/CHP/Co.Nap/2,4-P & $\begin{array}{l}3.167 \\
3.056 \\
3.180\end{array}$ & $\begin{array}{l}0.060 \\
0.041 \\
0.028\end{array}$ & $\begin{array}{l}\text { Ave. } \\
\text { S.D. } \\
\text { C.V. }\end{array}$ & $\begin{array}{c}0.043 \\
0.016 \\
37.2\end{array}$ \\
\hline \multicolumn{5}{|l|}{ Polyester: } \\
\hline PE/MEKP & $\begin{array}{l}3.137 \\
3.117 \\
3.124 \\
3.119 \\
3.134\end{array}$ & $\begin{array}{l}0.093 \\
0.097 \\
0.097 \\
0.099 \\
0.066\end{array}$ & $\begin{array}{l}\text { Ave. } \\
\text { S.D. } \\
\text { C.V. }\end{array}$ & $\begin{array}{l}0.090 \\
0.014 \\
15.6\end{array}$ \\
\hline
\end{tabular}


Table B.7. Yield strength and ultimate shear strength of epoxies at $295 \mathrm{~K}$.

\begin{tabular}{|c|c|c|c|c|c|c|c|}
\hline \multirow{2}{*}{$\frac{\text { Resin }}{\text { DGEBA/DCH/ARA/ACL }}$} & \multirow{2}{*}{$\begin{array}{c}\begin{array}{c}\text { Diameter } \\
(\mathrm{mm})\end{array} \\
3.233 \\
3.236 \\
3.231\end{array}$} & \multicolumn{3}{|c|}{$\begin{array}{l}\text { Yield Strength at } 10^{\circ} \\
(\mathrm{MPa})\end{array}$} & \multicolumn{3}{|c|}{$\begin{array}{l}\text { Ultimate Shear Strength } \\
\text { (MPa) }\end{array}$} \\
\hline & & $\begin{array}{l}45.45 \\
43.76 \\
49.79\end{array}$ & $\begin{array}{l}\text { Ave. } \\
\text { S.D. } \\
\text { C.V. }\end{array}$ & $\begin{array}{r}46.33 \\
3.11 \\
6.71\end{array}$ & $\begin{array}{l}* 65.53 \\
* 66.96 \\
* 67.27\end{array}$ & $\begin{array}{l}\text { Ave. } \\
\text { S.D. } \\
\text { C.V. }\end{array}$ & $\begin{array}{r}66.59 \\
0.93 \\
1.40\end{array}$ \\
\hline DGEBA/ALA/ACL & $\begin{array}{l}3.233 \\
3.216 \\
3.233\end{array}$ & $\begin{array}{l}32.50 \\
31.97 \\
32.77\end{array}$ & $\begin{array}{l}\text { Ave. } \\
\text { S.D. } \\
\text { C.V. }\end{array}$ & $\begin{array}{r}32.24 \\
0.37 \\
1.15\end{array}$ & $\begin{array}{l}* 47.03 \\
* 44.60 \\
* 48.51\end{array}$ & $\begin{array}{l}\text { Ave. } \\
\text { S.D. } \\
\text { C.V. }\end{array}$ & $\begin{array}{r}46.71 \\
1.97 \\
4.22\end{array}$ \\
\hline DGEBA-ST/ARA/ACL/F & $\begin{array}{l}3.180 \\
3.185 \\
3.178 \\
3.167 \\
3.180\end{array}$ & $\begin{array}{l}42.22 \\
43.12 \\
43.43 \\
44.98 \\
45.55\end{array}$ & $\begin{array}{l}\text { Ave. } \\
\text { S.D. } \\
\text { C.V. }\end{array}$ & $\begin{array}{r}43.86 \\
1.37 \\
3.12\end{array}$ & $\begin{array}{l}* 65.55 \\
* 56.39 \\
* 57.91 \\
* 65.78 \\
* 75.55\end{array}$ & $\begin{array}{l}\text { Ave. } \\
\text { S.D. } \\
\text { C.V. }\end{array}$ & $\begin{array}{r}64.24 \\
7.64 \\
11.89\end{array}$ \\
\hline NV/AN/ACL & $\begin{array}{l}3.200 \\
3.129 \\
3.134\end{array}$ & $\begin{array}{l}55.05 \\
48.97 \\
48.16\end{array}$ & $\begin{array}{l}\text { Ave. } \\
\text { S.D. } \\
\text { C.V. }\end{array}$ & $\begin{array}{r}50.73 \\
3.77 \\
7.43\end{array}$ & $\begin{array}{l}* 65.40 \\
* 64.13 \\
* 64.11\end{array}$ & $\begin{array}{l}\text { Ave. } \\
\text { S.D. } \\
\text { C.V. }\end{array}$ & $\begin{array}{r}64.55 \\
0.74 \\
1.15\end{array}$ \\
\hline Cyclo.A/AN/ACL & $\begin{array}{l}3.221 \\
3.221 \\
3.226 \\
3.221 \\
3.231\end{array}$ & $\begin{array}{l}53.48 \\
55.61 \\
54.29 \\
53.48 \\
55.09\end{array}$ & $\begin{array}{l}\text { Ave. } \\
\text { S.D. } \\
\text { C.V. }\end{array}$ & $\begin{array}{r}54.39 \\
0.95 \\
1.75\end{array}$ & $\begin{array}{l}* 75.93 \\
* 74.87 \\
* 76.64 \\
* 77.00 \\
* 77.34\end{array}$ & $\begin{array}{l}\text { Ave. } \\
\text { S.D. } \\
\text { C.V. }\end{array}$ & $\begin{array}{r}76.36 \\
0.98 \\
1.28\end{array}$ \\
\hline MLTF/ARA & $\begin{array}{l}3.165 \\
3.170 \\
3.167 \\
3.167 \\
3.165\end{array}$ & $\begin{array}{l}52.98 \\
51.60 \\
51.72 \\
55.10 \\
51.85\end{array}$ & $\begin{array}{l}\text { Ave. } \\
\text { S.D. } \\
\text { C.V. }\end{array}$ & $\begin{array}{r}52.65 \\
1.48 \\
2.81\end{array}$ & $\begin{array}{r}* 77.77 \\
* 74.04 \\
67.47 \\
* 74.21 \\
* 69.88\end{array}$ & $\begin{array}{l}\text { Ave. } \\
\text { S.D. } \\
\text { C.V. }\end{array}$ & $\begin{array}{r}72.67 \\
4.03 \\
5.55\end{array}$ \\
\hline \multicolumn{8}{|l|}{ Toughened Epoxies: } \\
\hline DGEBF/CTBN/AN & $\begin{array}{l}3.195 \\
3.193 \\
3.193\end{array}$ & $\begin{array}{l}43.81 \\
45.56 \\
43.36\end{array}$ & $\begin{array}{l}\text { Ave. } \\
\text { S.D. } \\
\text { C.V. }\end{array}$ & $\begin{array}{r}44.24 \\
1.16 \\
2.62\end{array}$ & $\begin{array}{l}* 58.87 \\
* 59.83 \\
* 59.56\end{array}$ & $\begin{array}{l}\text { Ave. } \\
\text { S.D. } \\
\text { C.V. }\end{array}$ & $\begin{array}{r}59.42 \\
0.50 \\
0.84\end{array}$ \\
\hline Cyclo.A/CTBN/AN/ACL & $\begin{array}{l}3.193 \\
3.195 \\
3.167\end{array}$ & $\begin{array}{l}47.21 \\
44.63 \\
47.23\end{array}$ & $\begin{array}{l}\text { Ave. } \\
\text { S.D. } \\
\text { C.V. }\end{array}$ & $\begin{array}{r}46.36 \\
1.50 \\
3.24\end{array}$ & $\begin{array}{l}* 63.95 \\
* 62.70 \\
* 64.94\end{array}$ & $\begin{array}{l}\text { Ave. } \\
\text { S.D. } \\
\text { C.V. }\end{array}$ & $\begin{array}{r}63.86 \\
1.12 \\
1.75\end{array}$ \\
\hline MLTF/ARA/TGH & $\begin{array}{l}3.226 \\
3.218 \\
3.223\end{array}$ & $\begin{array}{l}35.66 \\
40.20 \\
39.48\end{array}$ & $\begin{array}{l}\text { Ave. } \\
\text { S.D. } \\
\text { C.V. }\end{array}$ & $\begin{array}{r}38.45 \\
2.44 \\
6.35\end{array}$ & $\begin{array}{l}* 54.29 \\
* 57.89 \\
* 60.02\end{array}$ & $\begin{array}{l}\text { Ave. } \\
\text { S.D. } \\
\text { C.V. }\end{array}$ & $\begin{array}{r}57.40 \\
2.90 \\
5.05\end{array}$ \\
\hline
\end{tabular}

* Specimen did not fail prior to $90^{\circ}$. 
Table B.8. Shear modulus, shear strain, and ultimate strain of epoxies at $295 \mathrm{~K}$.

\begin{tabular}{|c|c|c|c|c|c|c|c|c|c|}
\hline \multirow{2}{*}{$\frac{\text { Resin }}{\text { DGEBA/DCH/ARA/ACL }}$} & \multicolumn{3}{|c|}{$\begin{array}{l}\text { Shear Modulus } \\
(\mathrm{GPa})\end{array}$} & \multicolumn{3}{|c|}{$\begin{array}{c}\text { Shear Strain at } 10^{\circ} \\
\text { (radians) }\end{array}$} & \multicolumn{3}{|c|}{$\begin{array}{c}\text { Ultimate Shear Strain } \\
\text { (radians) }\end{array}$} \\
\hline & $\begin{array}{l}1.05 \\
1.10 \\
0.97\end{array}$ & $\begin{array}{l}\text { Ave. } \\
\text { S.D. } \\
\text { C.V. }\end{array}$ & $\begin{array}{l}1.04 \\
0.07 \\
6.73\end{array}$ & $\begin{array}{l}0.0551 \\
0.0527 \\
0.0631\end{array}$ & $\begin{array}{l}\text { Ave. } \\
\text { S.D. } \\
\text { C.V. }\end{array}$ & $\begin{array}{l}0.0570 \\
0.0050 \\
8.77\end{array}$ & $\begin{array}{l}0.097 \\
0.097 \\
0.097\end{array}$ & $\begin{array}{l}\text { Ave. } \\
\text { S.D. } \\
\text { C.V. }\end{array}$ & $\begin{array}{l}0.097 \\
0.000 \\
0.00\end{array}$ \\
\hline DGEBA/ALA/ACL & $\begin{array}{l}0.90 \\
0.86 \\
0.97\end{array}$ & $\begin{array}{l}\text { Ave. } \\
\text { S.D. } \\
\text { C.V. }\end{array}$ & $\begin{array}{l}0.91 \\
0.06 \\
6.59\end{array}$ & $\begin{array}{l}0.0458 \\
0.0468 \\
0.0458\end{array}$ & $\begin{array}{l}\text { Ave. } \\
\text { S.D. } \\
\text { C.V. }\end{array}$ & $\begin{array}{l}0.0462 \\
0.0010 \\
2.16\end{array}$ & $\begin{array}{l}0.097 \\
0.097 \\
0.097\end{array}$ & $\begin{array}{l}\text { Ave. } \\
\text { S.D. } \\
\text { C.V. }\end{array}$ & $\begin{array}{l}0.097 \\
0.000 \\
0.00\end{array}$ \\
\hline DGEBA-ST/ARA/ACL/F & $\begin{array}{l}1.33 \\
1.69 \\
1.42 \\
1.65 \\
1.73\end{array}$ & $\begin{array}{l}\text { Ave. } \\
\text { S.D. } \\
\text { C.V. }\end{array}$ & $\begin{array}{l}1.56 \\
0.18 \\
11.54\end{array}$ & $\begin{array}{l}0.0414 \\
0.0372 \\
0.0420 \\
0.0388 \\
0.0377\end{array}$ & $\begin{array}{l}\text { Ave. } \\
\text { S.D. } \\
\text { C.V. }\end{array}$ & $\begin{array}{l}0.0394 \\
0.0020 \\
5.08\end{array}$ & $\begin{array}{l}0.096 \\
0.096 \\
0.096 \\
0.096\end{array}$ & $\begin{array}{l}\text { Ave. } \\
\text { S.D. } \\
\text { C.V. }\end{array}$ & $\begin{array}{l}0.096 \\
0.000 \\
0.00\end{array}$ \\
\hline NV/AN/ACL & $\begin{array}{l}1.11 \\
1.21 \\
1.07\end{array}$ & $\begin{array}{l}\text { Ave. } \\
\text { S.D. } \\
\text { C.V. }\end{array}$ & $\begin{array}{l}1.13 \\
0.07 \\
6.19\end{array}$ & $\begin{array}{l}0.0601 \\
0.0521 \\
0.0546\end{array}$ & $\begin{array}{l}\text { Ave. } \\
\text { S.D. } \\
\text { C.V. }\end{array}$ & $\begin{array}{l}0.0556 \\
0.0040 \\
7.19\end{array}$ & $\begin{array}{l}0.096 \\
0.094 \\
0.094\end{array}$ & $\begin{array}{l}\text { Ave. } \\
\text { S.D. } \\
\text { C.V. }\end{array}$ & $\begin{array}{l}0.095 \\
0.001 \\
1.05\end{array}$ \\
\hline Cyclo.A/AN/ACL & $\begin{array}{l}1.36 \\
1.23 \\
1.38 \\
1.33 \\
1.35\end{array}$ & $\begin{array}{l}\text { Ave. } \\
\text { S.D. } \\
\text { C.V. }\end{array}$ & $\begin{array}{l}1.33 \\
0.06 \\
4.51\end{array}$ & $\begin{array}{l}0.0506 \\
0.0561 \\
0.0506 \\
0.0518 \\
0.0526\end{array}$ & $\begin{array}{l}\text { Ave. } \\
\text { S.D. } \\
\text { C.V. }\end{array}$ & $\begin{array}{l}0.0523 \\
0.0020 \\
3.82\end{array}$ & $\begin{array}{l}0.097 \\
0.097 \\
0.097 \\
0.097 \\
0.097\end{array}$ & $\begin{array}{l}\text { Ave. } \\
\text { S.D. } \\
\text { C.V. }\end{array}$ & $\begin{array}{l}0.097 \\
0.000 \\
0.00\end{array}$ \\
\hline MLTF/ARA & $\begin{array}{l}1.43 \\
1.42 \\
1.54 \\
1.33 \\
1.39\end{array}$ & $\begin{array}{l}\text { Ave. } \\
\text { S.D. } \\
\text { C.V. }\end{array}$ & $\begin{array}{l}1.42 \\
0.08 \\
5.63\end{array}$ & $\begin{array}{l}0.0491 \\
0.0486 \\
0.0473 \\
0.0522 \\
0.0497\end{array}$ & $\begin{array}{l}\text { Ave. } \\
\text { S.D. } \\
\text { C.V. }\end{array}$ & $\begin{array}{l}0.0494 \\
0.0020 \\
4.05\end{array}$ & $\begin{array}{l}0.095 \\
0.095 \\
0.072 \\
0.095 \\
0.095\end{array}$ & $\begin{array}{l}\text { Ave. } \\
\text { S.D. } \\
\text { C.V. }\end{array}$ & $\begin{array}{c}0.090 \\
0.013 \\
14.44\end{array}$ \\
\hline \multicolumn{10}{|l|}{ Toughened Epoxies: } \\
\hline DGEBF/CTBN/AN & $\begin{array}{l}1.04 \\
1.15 \\
1.08\end{array}$ & $\begin{array}{l}\text { Ave. } \\
\text { S.D. } \\
\text { C.V. }\end{array}$ & $\begin{array}{l}1.09 \\
0.06 \\
5.50\end{array}$ & $\begin{array}{l}0.0526 \\
0.0526 \\
0.0526\end{array}$ & $\begin{array}{l}\text { Ave. } \\
\text { S.D. } \\
\text { C.V. }\end{array}$ & $\begin{array}{l}0.0526 \\
0.0000 \\
0.00\end{array}$ & $\begin{array}{l}0.096 \\
0.096 \\
0.096\end{array}$ & $\begin{array}{l}\text { Ave. } \\
\text { S.D. } \\
\text { C.V. }\end{array}$ & $\begin{array}{l}0.096 \\
0.000 \\
0.00\end{array}$ \\
\hline Cyclo.A/CTBN/AN/ACL & $\begin{array}{l}1.13 \\
1.08 \\
1.12\end{array}$ & $\begin{array}{l}\text { Ave. } \\
\text { S.D. } \\
\text { C.V. }\end{array}$ & $\begin{array}{l}1.11 \\
0.03 \\
2.70\end{array}$ & $\begin{array}{l}0.0544 \\
0.0526 \\
0.0546\end{array}$ & $\begin{array}{l}\text { Ave. } \\
\text { S.D. } \\
\text { C.V. }\end{array}$ & $\begin{array}{l}0.0539 \\
0.0010 \\
1.86\end{array}$ & $\begin{array}{l}0.096 \\
0.096 \\
0.095\end{array}$ & $\begin{array}{l}\text { Ave. } \\
\text { S.D. } \\
\text { C.V. }\end{array}$ & $\begin{array}{l}0.096 \\
0.001 \\
1.04\end{array}$ \\
\hline MLTF/ARA/TGH & $\begin{array}{l}1.03 \\
1.03 \\
1.04\end{array}$ & $\begin{array}{l}\text { Ave. } \\
\text { S.D. } \\
\text { C.V. }\end{array}$ & $\begin{array}{l}1.03 \\
0.01 \\
0.97\end{array}$ & $\begin{array}{l}0.0451 \\
0.0512 \\
0.0494\end{array}$ & $\begin{array}{l}\text { Ave. } \\
\text { S.D. } \\
\text { C.V. }\end{array}$ & $\begin{array}{l}0.0486 \\
0.0030 \\
6.17\end{array}$ & $\begin{array}{l}0.097 \\
0.097 \\
0.097\end{array}$ & $\begin{array}{l}\text { Ave. } \\
\text { S.D. } \\
\text { C.V. }\end{array}$ & $\begin{array}{l}0.097 \\
0.000 \\
0.00\end{array}$ \\
\hline
\end{tabular}

* Specimen did not fail prior to $90^{\circ}$. 
Table B.9. Yield strength and ultimate shear strength of cyanate esters at $295 \mathrm{~K}$.

\begin{tabular}{lrrrrrrr}
\hline \hline \multirow{2}{*}{ Resin } & $\begin{array}{c}\text { Diameter } \\
(\mathrm{mm})\end{array}$ & \multicolumn{2}{c}{$\begin{array}{c}\text { Yield Strength at } 10^{\circ} \\
(\mathrm{MPa})\end{array}$} & \multicolumn{3}{c}{$\begin{array}{c}\text { Ultimate Shear Strength } \\
(\mathrm{MPa})\end{array}$} \\
\hline \hline CE/VT/Co.AA & 3.264 & 51.38 & Ave. & 53.87 & $* 76.30$ & Ave. & 75.29 \\
& 3.297 & 54.34 & S.D. & 2.29 & $* 74.28$ & S.D. & 1.01 \\
& 3.256 & 55.88 & C.V. & 4.25 & $* 75.29$ & C.V. & 1.34 \\
CE & 3.193 & 46.11 & Ave. & 44.94 & $* 64.77$ & Ave. & 61.90 \\
& 3.188 & 48.54 & S.D. & 3.97 & $* 58.46$ & S.D. & 2.32 \\
& 3.178 & 40.09 & C.V. & 8.83 & $* 61.25$ & C.V. & 3.75 \\
& 3.200 & 41.42 & & & 62.13 & & \\
CE(PT) ${ }^{\dagger}$ & 3.188 & 48.54 & & & $* 62.88$ & & \\
& 3.190 & 61.63 & Ave. & 58.72 & 83.63 & Ave. & 84.43 \\
& 3.160 & 57.76 & S.D. & 2.66 & 84.95 & S.D. & 3.36 \\
& 3.155 & 54.63 & C.V. & 4.53 & 83.08 & C.V. & 3.98 \\
& 3.185 & 59.71 & & & 80.72 & & \\
& 3.183 & 59.85 & & & $* 89.78$ & & \\
CE/HTBN/NPh/Cu.AA & 3.205 & 49.90 & Ave. & 52.79 & $* 69.43$ & Ave. & 68.62 \\
& 3.205 & 53.70 & S.D. & 2.56 & $* 65.09$ & S.D. & 3.20 \\
& 3.205 & 54.78 & C.V. & 4.85 & $* 71.33$ & C.V. & 4.66 \\
\hline \hline
\end{tabular}

* Specimen did not fail prior to $90^{\circ}$.

${ }^{\dagger}$ See comments regarding cure schedule on page III-16. 
Table B.10. Shear modulus, shear strain, and ultimate strain of vinyl esters and polyester at $295 \mathrm{~K}$.

\begin{tabular}{|c|c|c|c|c|c|c|c|c|c|}
\hline Resin & \multicolumn{3}{|c|}{$\begin{array}{c}\text { Shear Modulus } \\
(\mathrm{GPa})\end{array}$} & \multicolumn{3}{|c|}{$\begin{array}{c}\text { Shear Strain at } 10^{\circ} \\
\text { (radians) }\end{array}$} & \multicolumn{3}{|c|}{$\begin{array}{c}\text { Ultimate Shear Strain } \\
\text { (radians) }\end{array}$} \\
\hline $\mathrm{VE} / \mathrm{CHX}$ & $\begin{array}{l}0.97 \\
1.02 \\
0.98 \\
1.07 \\
1.23\end{array}$ & $\begin{array}{l}\text { Ave. } \\
\text { S.D. } \\
\text { C.V. }\end{array}$ & $\begin{array}{r}1.05 \\
0.11 \\
10.48\end{array}$ & $\begin{array}{l}0.0508 \\
0.0500 \\
0.0536 \\
0.0530 \\
0.0415\end{array}$ & $\begin{array}{l}\text { Ave. } \\
\text { S.D. } \\
\text { C.V. }\end{array}$ & $\begin{array}{l}0.0498 \\
0.0050 \\
10.04\end{array}$ & $\begin{array}{l}0.094 \\
0.094 \\
0.091 \\
0.091 \\
0.084\end{array}$ & $\begin{array}{l}\text { Ave. } \\
\text { S.D. } \\
\text { C.V. }\end{array}$ & $\begin{array}{l}0.091 \\
0.002 \\
2.20\end{array}$ \\
\hline VE-EL/MEKP & $\begin{array}{l}1.14 \\
1.04 \\
1.14\end{array}$ & $\begin{array}{l}\text { Ave. } \\
\text { S.D. } \\
\text { C.V. }\end{array}$ & $\begin{array}{l}1.11 \\
0.06 \\
5.41\end{array}$ & $\begin{array}{l}0.0489 \\
0.0519 \\
0.0490\end{array}$ & $\begin{array}{l}\text { Ave. } \\
\text { S.D. } \\
\text { C.V. }\end{array}$ & $\begin{array}{l}0.0499 \\
0.0020 \\
4.01\end{array}$ & $\begin{array}{l}0.094 \\
0.094 \\
0.094\end{array}$ & $\begin{array}{l}\text { Ave. } \\
\text { S.D. } \\
\text { C.V. }\end{array}$ & $\begin{array}{l}0.094 \\
0.000 \\
0.00\end{array}$ \\
\hline $\mathrm{VE} / \mathrm{CHP} / \mathrm{F}$ & $\begin{array}{l}1.58 \\
1.65 \\
1.81 \\
1.57 \\
1.61\end{array}$ & $\begin{array}{l}\text { Ave. } \\
\text { S.D. } \\
\text { C.V. }\end{array}$ & $\begin{array}{l}1.64 \\
0.10 \\
6.10\end{array}$ & $\begin{array}{l}0.0451 \\
0.0433 \\
0.0433 \\
0.0446 \\
0.0469\end{array}$ & $\begin{array}{l}\text { Ave. } \\
\text { S.D. } \\
\text { C.V. }\end{array}$ & $\begin{array}{l}0.0446 \\
0.0010 \\
2.24\end{array}$ & $\begin{array}{l}0.082 \\
0.074 \\
0.080 \\
0.060 \\
0.079\end{array}$ & $\begin{array}{l}\text { Ave. } \\
\text { S.D. } \\
\text { C.V. }\end{array}$ & $\begin{array}{l}0.075 \\
0.004 \\
5.33\end{array}$ \\
\hline VE/CHP/CoNap/2,4-P & $\begin{array}{l}1.33 \\
1.53 \\
1.80 \\
1.48\end{array}$ & $\begin{array}{l}\text { Ave. } \\
\text { S.D. } \\
\text { C.V. }\end{array}$ & $\begin{array}{r}1.54 \\
0.20 \\
12.99\end{array}$ & $\begin{array}{l}0.0482 \\
0.0460 \\
0.0437 \\
0.0511\end{array}$ & $\begin{array}{l}\text { Ave. } \\
\text { S.D. } \\
\text { C.V. }\end{array}$ & $\begin{array}{l}0.0472 \\
0.0030 \\
6.36\end{array}$ & $\begin{array}{l}0.095 \\
0.096 \\
0.095 \\
0.093\end{array}$ & $\begin{array}{l}\text { Ave. } \\
\text { S.D. } \\
\text { C.V. }\end{array}$ & $\begin{array}{l}0.095 \\
0.001 \\
1.05\end{array}$ \\
\hline \multicolumn{10}{|l|}{ Polyester: } \\
\hline PE/MEKP & $\begin{array}{l}1.47 \\
1.42 \\
1.32 \\
1.35 \\
1.30\end{array}$ & $\begin{array}{l}\text { Ave. } \\
\text { S.D. } \\
\text { C.V. }\end{array}$ & $\begin{array}{l}1.37 \\
0.07 \\
5.11\end{array}$ & $\begin{array}{l}0.0527 \\
0.0495 \\
0.0515 \\
0.0551 \\
0.0571\end{array}$ & $\begin{array}{l}\text { Ave. } \\
\text { S.D. } \\
\text { C.V. }\end{array}$ & $\begin{array}{l}0.0532 \\
0.0030 \\
5.64\end{array}$ & $\begin{array}{l}0.093 \\
0.094 \\
0.094 \\
0.094 \\
0.094\end{array}$ & $\begin{array}{l}\text { Ave. } \\
\text { S.D. } \\
\text { C.V. }\end{array}$ & $\begin{array}{l}0.094 \\
0.001 \\
1.06\end{array}$ \\
\hline
\end{tabular}

* Specimen did not fail prior to $90^{\circ}$. 
Table B.11. Yield strength and ultimate shear strength of vinyl esters and polyester at $295 \mathrm{~K}$.

\begin{tabular}{|c|c|c|c|c|c|c|c|}
\hline Vinyl Esters: & $\begin{array}{l}\text { Diameter } \\
(\mathrm{mm})\end{array}$ & \multicolumn{3}{|c|}{$\begin{array}{l}\text { Yield Strength at } 10^{\circ} \\
(\mathrm{MPa})\end{array}$} & \multicolumn{3}{|c|}{$\begin{array}{l}\text { Ultimate Shear Strength } \\
(\mathrm{MPa})\end{array}$} \\
\hline VE/CHX & $\begin{array}{l}3.117 \\
3.106 \\
3.007 \\
3.038 \\
3.051\end{array}$ & $\begin{array}{l}38.95 \\
39.33 \\
42.04 \\
44.61 \\
40.28\end{array}$ & $\begin{array}{l}\text { Ave. } \\
\text { S.D. } \\
\text { C.V. }\end{array}$ & $\begin{array}{r}41.04 \\
2.32 \\
5.65\end{array}$ & $\begin{array}{r}* 48.39 \\
* 52.45 \\
* 47.29 \\
* 52.26 \\
52.86\end{array}$ & $\begin{array}{l}\text { Ave. } \\
\text { S.D. } \\
\text { C.V. }\end{array}$ & $\begin{array}{r}50.65 \\
2.60 \\
5.13\end{array}$ \\
\hline VE-EL/MEKP & $\begin{array}{l}3.112 \\
3.112 \\
3.122\end{array}$ & $\begin{array}{l}42.40 \\
42.11 \\
42.29\end{array}$ & $\begin{array}{l}\text { Ave. } \\
\text { S.D. } \\
\text { C.V. }\end{array}$ & $\begin{array}{r}42.27 \\
0.15 \\
0.35\end{array}$ & $\begin{array}{l}* 54.56 \\
* 53.97 \\
* 56.67\end{array}$ & $\begin{array}{l}\text { Ave. } \\
\text { S.D. } \\
\text { C.V. }\end{array}$ & $\begin{array}{r}55.07 \\
1.42 \\
2.58\end{array}$ \\
\hline $\mathrm{VE} / \mathrm{CHP} / \mathrm{F}$ & $\begin{array}{l}3.231 \\
3.233 \\
3.233 \\
3.239 \\
3.223\end{array}$ & $\begin{array}{l}54.03 \\
52.85 \\
54.96 \\
53.65 \\
55.48\end{array}$ & $\begin{array}{l}\text { Ave. } \\
\text { S.D. } \\
\text { C.V. }\end{array}$ & $\begin{array}{r}54.19 \\
1.05 \\
1.94\end{array}$ & $\begin{array}{l}73.10 \\
68.70 \\
73.99 \\
64.17 \\
73.62\end{array}$ & $\begin{array}{l}\text { Ave. } \\
\text { S.D. } \\
\text { C.V. }\end{array}$ & $\begin{array}{r}70.72 \\
4.24 \\
6.00\end{array}$ \\
\hline VE/CHP/CoNap/2,4-P & $\begin{array}{l}3.145 \\
3.203 \\
3.170 \\
3.101\end{array}$ & $\begin{array}{l}49.41 \\
52.20 \\
52.72 \\
58.69\end{array}$ & $\begin{array}{l}\text { Ave. } \\
\text { S.D. } \\
\text { C.V. }\end{array}$ & $\begin{array}{r}53.26 \\
3.90 \\
7.32\end{array}$ & $\begin{array}{l}* 68.95 \\
* 72.31 \\
* 72.91 \\
* 82.05\end{array}$ & $\begin{array}{l}\text { Ave. } \\
\text { S.D. } \\
\text { C.V. }\end{array}$ & $\begin{array}{r}74.06 \\
5.61 \\
7.57\end{array}$ \\
\hline \multicolumn{8}{|l|}{ Polyester: } \\
\hline PE/MEKP & $\begin{array}{l}3.094 \\
3.117 \\
3.127 \\
3.127 \\
31.37\end{array}$ & $\begin{array}{l}57.92 \\
55.48 \\
57.27 \\
61.95 \\
61.35\end{array}$ & $\begin{array}{l}\text { Ave. } \\
\text { S.D. } \\
\text { C.V. }\end{array}$ & $\begin{array}{r}58.79 \\
2.76 \\
4.69\end{array}$ & $\begin{array}{l}* 80.85 \\
* 80.26 \\
* 81.82 \\
* 81.24 \\
* 79.87\end{array}$ & $\begin{array}{l}\text { Ave. } \\
\text { S.D. } \\
\text { C.V. }\end{array}$ & $\begin{array}{r}80.81 \\
0.77 \\
0.95\end{array}$ \\
\hline
\end{tabular}

* Specimen did not fail prior to $90^{\circ}$. 
Table B.12. Shear modulus, shear strain, and ultimate strain of cyanate esters at $295 \mathrm{~K}$.

\begin{tabular}{|c|c|c|c|c|c|c|c|c|c|}
\hline $\begin{array}{r}\text { Resin } \\
\mathrm{CE} / \mathrm{VT} / \mathrm{Co} . \mathrm{AA}\end{array}$ & \multicolumn{3}{|c|}{$\begin{array}{l}\text { Shear Modulus } \\
\text { (GPa) }\end{array}$} & \multicolumn{3}{|c|}{$\begin{array}{c}\text { Shear Strain at } 10^{\circ} \\
\text { (radians) }\end{array}$} & \multicolumn{3}{|c|}{$\begin{array}{l}\text { Ultimate Shear Strain } \\
\text { (radians) }\end{array}$} \\
\hline CE/VT/Co.AA & $\begin{array}{l}1.08 \\
1.07 \\
1.09\end{array}$ & $\begin{array}{l}\text { Ave. } \\
\text { S.D. } \\
\text { C.V. }\end{array}$ & $\begin{array}{l}1.08 \\
0.01 \\
0.93\end{array}$ & $\begin{array}{l}0.0562 \\
0.0650 \\
0.0642\end{array}$ & $\begin{array}{l}\text { Ave. } \\
\text { S.D. } \\
\text { C.V. }\end{array}$ & $\begin{array}{l}0.0618 \\
0.0050 \\
8.09\end{array}$ & $\begin{array}{l}0.098 \\
0.099 \\
0.098\end{array}$ & $\begin{array}{l}\text { Ave. } \\
\text { S.D. } \\
\text { C.V. }\end{array}$ & $\begin{array}{l}0.098 \\
0.001 \\
1.02\end{array}$ \\
\hline $\mathrm{CE}$ & $\begin{array}{l}1.21 \\
1.03 \\
1.08 \\
1.07 \\
1.01\end{array}$ & $\begin{array}{l}\text { Ave. } \\
\text { S.D. } \\
\text { C.V. }\end{array}$ & $\begin{array}{l}1.08 \\
0.08 \\
7.41\end{array}$ & $\begin{array}{l}0.0489 \\
0.0580 \\
0.0499 \\
0.0497 \\
0.0611\end{array}$ & $\begin{array}{l}\text { Ave. } \\
\text { S.D. } \\
\text { C.V. }\end{array}$ & $\begin{array}{l}0.0535 \\
0.0060 \\
11.21\end{array}$ & $\begin{array}{l}0.096 \\
0.096 \\
0.096 \\
0.096 \\
0.096\end{array}$ & $\begin{array}{l}\text { Ave. } \\
\text { S.D. } \\
\text { C.V. }\end{array}$ & $\begin{array}{l}0.096 \\
0.000 \\
0.00\end{array}$ \\
\hline $\mathrm{CE}(\mathrm{PT})^{*}$ & $\begin{array}{l}1.52 \\
1.48 \\
1.67 \\
1.54 \\
1.67\end{array}$ & $\begin{array}{l}\text { Ave. } \\
\text { S.D. } \\
\text { C.V. }\end{array}$ & $\begin{array}{l}1.58 \\
0.09 \\
5.70\end{array}$ & $\begin{array}{l}0.0513 \\
0.0484 \\
0.0441 \\
0.0506 \\
0.0487\end{array}$ & $\begin{array}{l}\text { Ave. } \\
\text { S.D. } \\
\text { C.V. }\end{array}$ & $\begin{array}{l}0.0486 \\
0.0030 \\
6.17\end{array}$ & $\begin{array}{l}0.081 \\
0.090 \\
0.089 \\
0.077 \\
0.096\end{array}$ & $\begin{array}{l}\text { Ave. } \\
\text { S.D. } \\
\text { C.V. }\end{array}$ & $\begin{array}{l}0.087 \\
0.005 \\
5.75\end{array}$ \\
\hline CE/HTBN/NPh/Cu.AA & $\begin{array}{l}1.23 \\
1.27 \\
1.18\end{array}$ & $\begin{array}{l}\text { Ave. } \\
\text { S.D. } \\
\text { C.V. }\end{array}$ & $\begin{array}{l}1.23 \\
0.05 \\
4.07\end{array}$ & $\begin{array}{l}0.0528 \\
0.0577 \\
0.0589\end{array}$ & $\begin{array}{l}\text { Ave. } \\
\text { S.D. } \\
\text { C.V. }\end{array}$ & $\begin{array}{l}0.0565 \\
0.0030 \\
5.31\end{array}$ & $\begin{array}{l}0.096 \\
0.096 \\
0.096\end{array}$ & $\begin{array}{l}\text { Ave. } \\
\text { S.D. } \\
\text { C.V. }\end{array}$ & $\begin{array}{l}0.096 \\
0.000 \\
0.00\end{array}$ \\
\hline
\end{tabular}

* See comments regarding cure schedule on page III-16. 


\title{
IV. Review of the
}

\section{Low-Temperature Properties of E-Glass}

\author{
R.P. Reed \\ Cryogenic Materials, Inc. \\ Boulder, Colorado
}




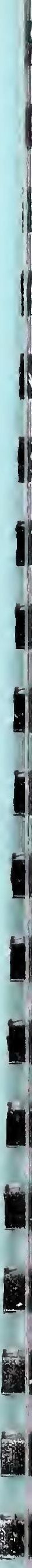




\title{
IV. Review of the Low-Temperature Properties of E-Glass
}

\author{
R.P. Reed \\ Cryogenic Materials, Inc. \\ Boulder, Colorado
}

\section{Introduction}

Selected mechanical and thermal properties of E-glass have been compiled from previous reports. These properties include the elastic modulus, Poisson's ratio, tensile strength, thermal expansion, and thermal conductivity. Elastic properties have been measured only at room temperature; the low-temperature properties were estimated from the temperature dependence of similar glasses or extrapolated from higher temperature data. Each of these properties is discussed separately.

Other physical properties of E-glass that have been measured are summarized: density: $2.54 \mathrm{~g} / \mathrm{cm}^{3}$ average Mohs hardness: 6.5 dielectric strength: $1960 \mathrm{MV} / \mathrm{m}$ dielectric constant at $295 \mathrm{~K}$

$$
\begin{aligned}
& \text { at } 60 \mathrm{~Hz}: \quad 5.9-6.4 \\
& \text { at } 10^{6} \mathrm{~Hz}: 6.3 \\
& \text { volume resistivity at } 295 \mathrm{~K} \text { and } 500 \mathrm{~V}: 10^{15} \Omega \cdot \mathrm{cm}
\end{aligned}
$$$$
\text { surface resistivity: } 10^{13} \Omega \cdot \mathrm{cm}
$$$$
\text { optical index of refraction: } 1.547
$$$$
\text { velocity of sound: } 5330 \mathrm{~m} / \mathrm{s}
$$$$
\text { specific heat of E-glass }
$$$$
\text { at } 296 \mathrm{~K}: 0.81 \mathrm{~kJ} /(\mathrm{kg} \cdot \mathrm{K})
$$$$
\text { at } 473 \mathrm{~K}: 1.03 \mathrm{~kJ} /(\mathrm{kg} \cdot \mathrm{K})
$$

E-glass was developed as an electrical grade of glass fiber, and its composition in weight percent is typically $54 \% \mathrm{SiO}_{2}, 15 \% \mathrm{Al}_{2} \mathrm{O}_{3}, 17 \% \mathrm{CaO}, 4 \% \mathrm{MgO}, 1 \% \mathrm{Na}_{2} \mathrm{O}, 8 \% \mathrm{~B}_{2} \mathrm{O}_{3}$, and $1 \% \mathrm{TiO}_{2}$ with traces of $\mathrm{F}_{2}$ and $\mathrm{Fe}_{2} \mathrm{O}_{3}$. Its softening point on heating is about $824^{\circ} \mathrm{C}$, and it anneals at $657^{\circ} \mathrm{C}$.

\section{Elastic Modulus}

Measurements or estimates of the elastic modulus $(E)$ of E-glass fiber at room temperature are compiled in Table 2.1 [2.1-2.6]. The recommended value is based mainly on the assessment by Broutman and Krock [2.3] and an estimate by McCreight [2.2]. Measurements at higher temperatures on glass plate by Spinner [2.7] and Franke [2.8] are included in Figure 2.1. Since we could find no low-temperature data on the elastic modulus, two approximations are presented in Figure 2.1. The temperature dependence of the data at higher temperatures is rather weak, and extrapolation of the trend to low temperatures, using the value of $72.4 \mathrm{GPa}$ at room temperature, gives a value of about $73.0 \mathrm{GPa}$ at $4 \mathrm{~K}$. However, the temperature dependence of $E$ in compression tests of unidirectional E-glass-reinforced composites (described in $\S \mathrm{V}$ ) is higher. If we assume that the fibers dominate this Thus, a value of about $78 \mathrm{GPa}$ is obtained for $E$ at $4 \mathrm{~K}$ for E-glass fibers. As can be seen from Figure 2.1, this latter trend is clearly steeper than those at higher temperatures. 
Table 2.1. Elastic modulus of E-glass.

\begin{tabular}{lclcc}
\hline \hline \multirow{2}{*}{ Source [ref] } & $\begin{array}{c}\text { Temperature } \\
(\mathrm{K})\end{array}$ & Comments & \multicolumn{2}{c}{$E$} \\
\cline { 4 - 5 } Murgatroyd [2.1] & room & deflection measurements & $57-61$ & $8.3-8.8$ \\
Scala from McCreight [2.2] & room & original data & 75.9 & 11.0 \\
Broutman and Krock [2.3] & 296 & critical review & 72.4 & 10.5 \\
Schmidt [2.4] & room & review & $71.5-77.4$ & $10.2-11.2$ \\
Owens-Corning [2.5] & room & recommended & $69.0-72.4$ & $10.0-10.5$ \\
Miller from Owens-Corning [2.5] & room & review & $69-85$ & $10.0-12.4$ \\
Otto [2.6] & room & as drawn & 73.1 & 10.7 \\
Recommended value & room & heat compacted (treated) & 84.8 & 12.3 \\
\hline \hline
\end{tabular}

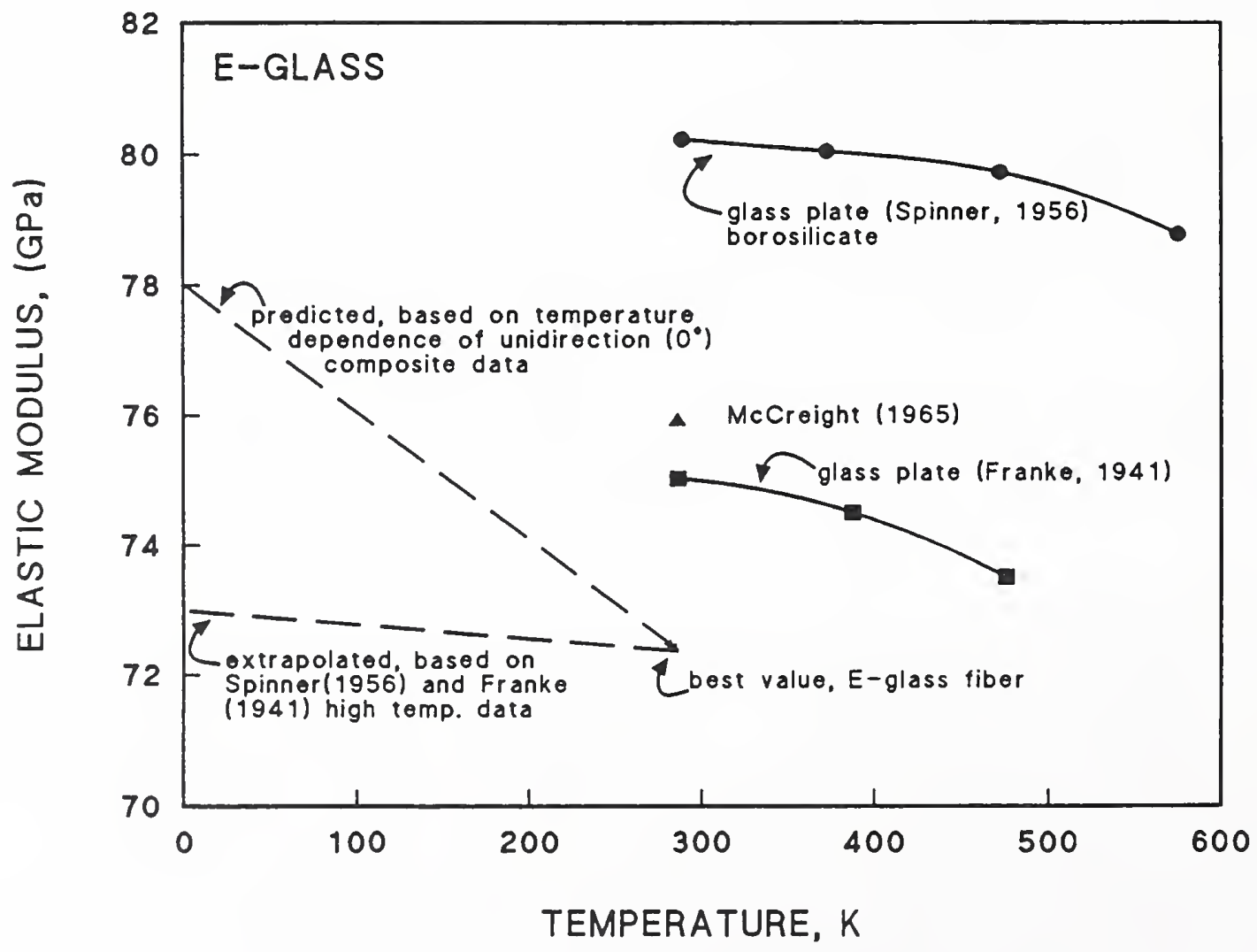

Figure. 2.1. Elastic modulus of E-glass versus temperature.

$$
\text { IV-2 }
$$




\section{Poisson's Ratio}

Brannan measured the Poisson's ratios of 50- $\mu \mathrm{m}$-diameter glass fibers at room temperature with a torsion-pendulum test assembly [3.1]. His measurements confirmed earlier glass data: a Poisson's ratio of $0.18 \pm 0.02$. The same value was obtained for as-formed glass fibers after heat treatment at $510^{\circ} \mathrm{C}$ for $2.5 \mathrm{~h}$. Earlier, using mechanical measurements of deflection under load, Murgatroyd had measured Poisson's ratios from 0.13 to 0.15 for 40 - to 110 - $\mu$ m-diameter fibers [2.1].

\section{Tensile Strength}

The strength of E-glass fibers at room temperature is strongly dependent on fiber diameter and humidity. The tensile strength typically has been shown to decrease from about $3.8 \mathrm{GPa}$ for fiber diameters less than about $5 \mu \mathrm{m}$ to $1 \mathrm{GPa}$ for fiber diameters greater than $15 \mu \mathrm{m}$. Exposure to $100 \%$ relative humidity for several days or longer results in a 20 to $50 \%$ reduction in its tensile strength. Heating the as-formed E-glass fibers to temperatures higher than about $500^{\circ} \mathrm{C}$ also results in significantly lower (10 to $75 \%$ ) tensile strengths at room temperature.

The tensile strength of glass fibers was measured at high temperatures by Thomas [4.1], Otto [4.2], Cameron [4.3], and Duflos [4.4] and below room temperature by Cameron [4.3] and Broutman and Krock [4.5]. Their results are presented in Figure 4.1. The puzzling aspect of the data in Figure 4.1 is the apparent discontinuity of the temperature dependence at room temperature, which is not likely to be a real effect. Probably the measurement technique, the type of filament used in the tests, or the peculiarities of filament processing account for the obvious disparity. The average value of the strength at room temperature is about $3.8 \mathrm{GPa}$ and, at $4 \mathrm{~K}$, about $5.8 \mathrm{GPa}$ (extrapolated).

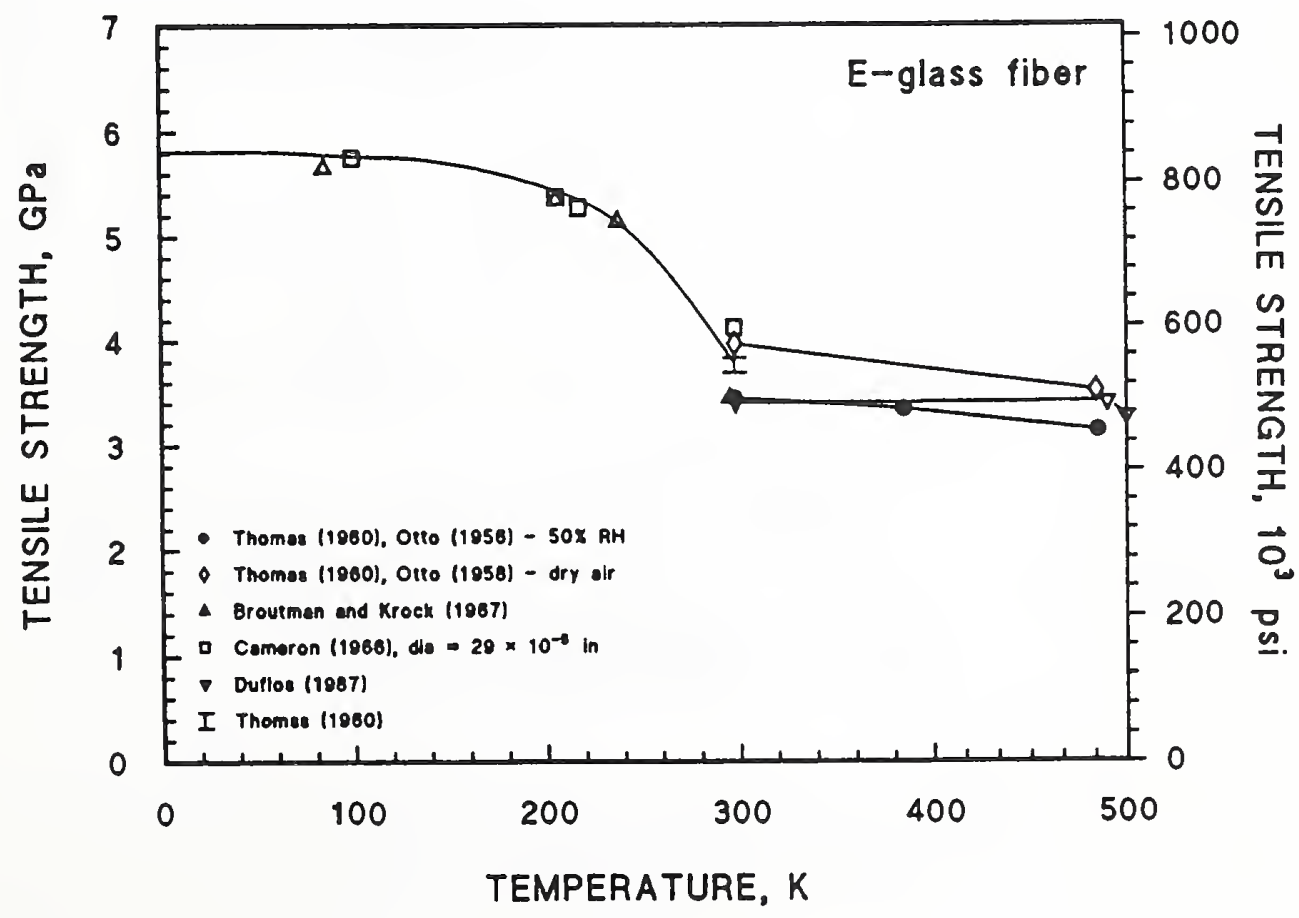

Figure 4.1. Tensile strength of E-glass fiber versus temperature. 


\section{Thermal Contraction}

The thermal contraction of glass fibers has not been measured at low temperatures. In Figure 5.1, the contraction from room temperature to $4 \mathrm{~K}$ of four types of glass from four different reports is presented. These data were taken from the critical review by Corruccini and Gniewek [5.1] of a number of earlier studies. Clearly, on cooling to $4 \mathrm{~K}$, the amount of contraction of glass is at least a factor of 10 less than that of epoxy. The contraction of the unidirectional composite of about $0.1 \%$ on cooling from 295 to $4 \mathrm{~K}$ (detailed in $\S \mathrm{V}$ ) suggests that the actual contraction of E-glass is of the same order, and it is much more than that of silica glass. The thermal contraction of E-glass is much less than that of resins-approximately $10 \%$ on cooling from 295 to 4 K. See \$III.6 for the thermal contraction data of resin systems.

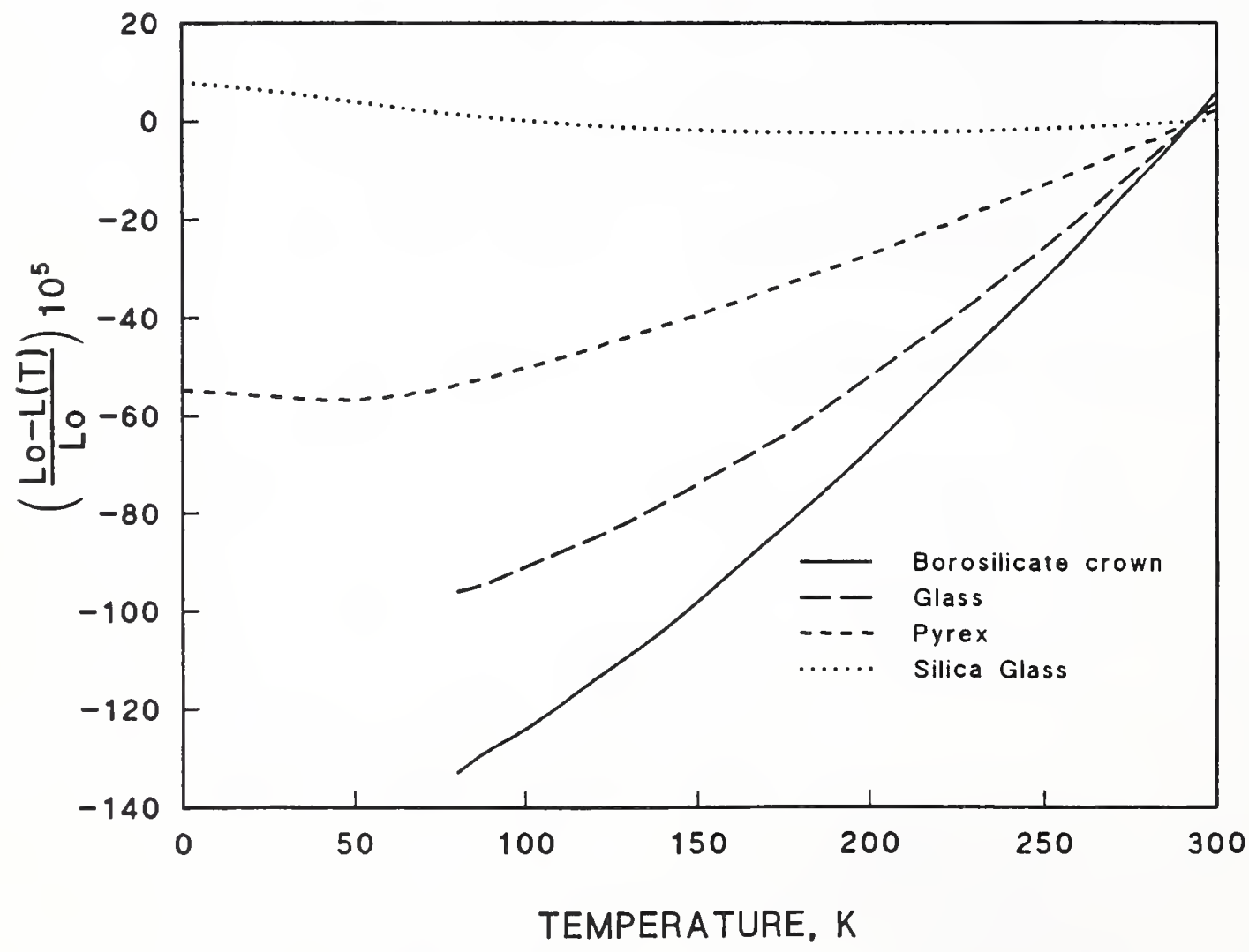

Figure 5.1. Thermal contraction of four types of glass. 


\section{Thermal Conductivity}

Low-temperature thermal-conductivity data compiled by Childs et al. [6.1] are presented in Figure 6.1. There is good agreement, despite the variety of glasses that were measured. Notice that the thermal conductivity at low temperatures is higher than that of epoxy resin by about a factor of 2 (see §III.7), but at room temperature, the E-glass thermal conductivity is about a factor of 6 higher than that of epoxy resins.

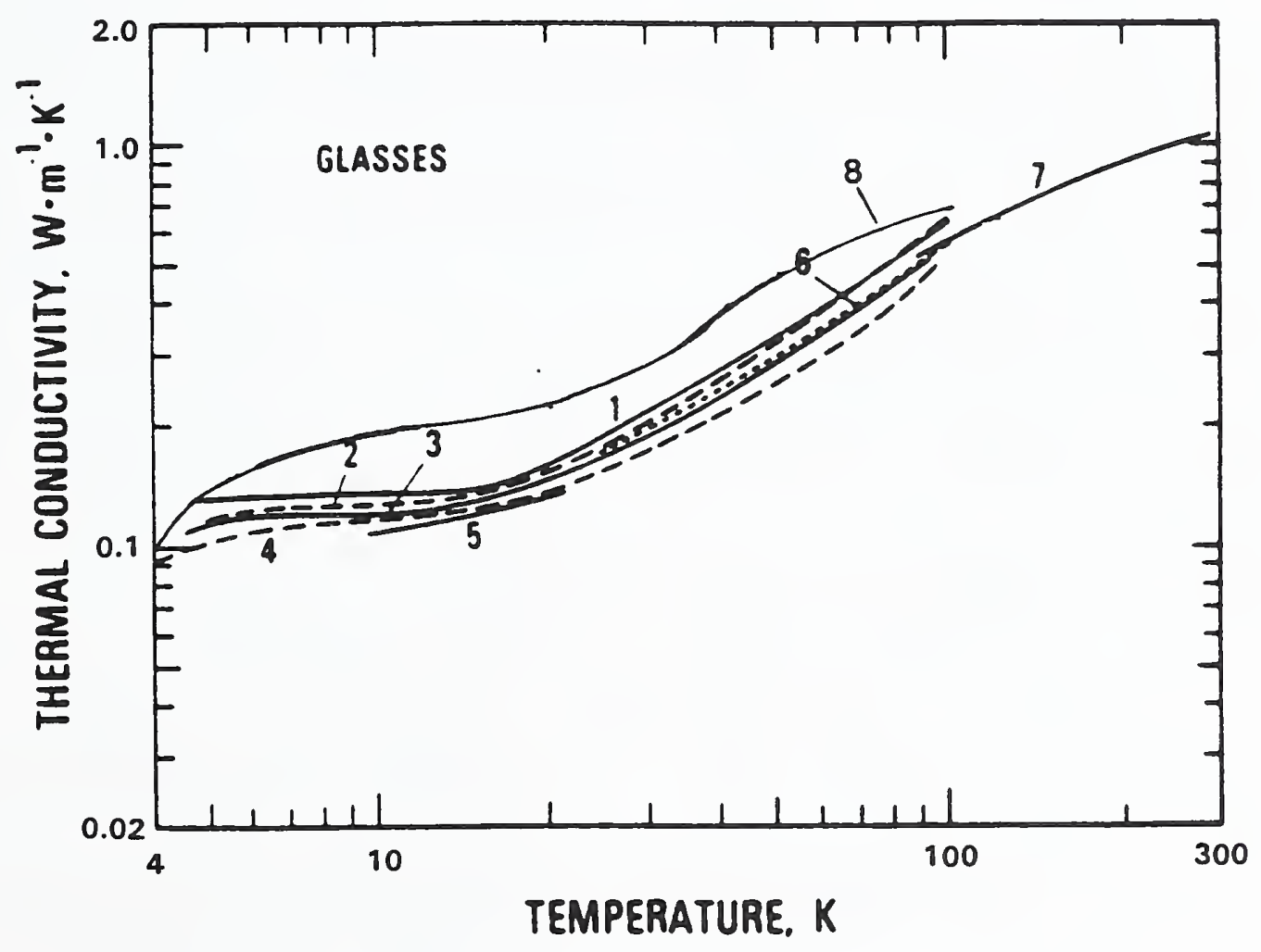

Figure 6.1. Thermal conductivity of glasses: 1 - quartz-1; 2 - quartz-2;

3 - quartz-3; 4 - Phoenix-1; 5 - Phoenix-2; 6 - glass;

7 - Pyrex; 8 - Equerore XRE fiberglass tow. 


\section{References}

[2.1] Murgatroyd, B.A. The strength of glass fibers. Part I. Elastic properties. J. Soc. Glass Technol. 28: 368-388; 1944.

[2.2] McCreight, L.R. et al. Ceramic and graphite fibers and whiskers, in Refractory materials, vol. 1. New York, NY: Academic Press; 1965.

[2.3] Broutman, L.J.; Krock, R.H. Modern composite materials. Reading, MA: Addison-Wesley; 1967. 280-301.

[2.4] Schmidt, K.A.F. Verstärkungsfasern, in Glasfaser verstärkte Kunststoffe. Berlin, Germany: Springer-Verlag; 1967. 159-293.

[2.5] Owens-Corning Fiberglas Corporation. S-2 glass fiber. Toledo, OH: Owens-Corning Fiberglas Corporation; 1990.

[2.6] Otto, W.H. Properties of glass fibers at elevated temperatures. AD228851, Contract No. 58841e. Toledo, OH: Owens-Corning Fiberglas Corporation; 1961.

Also, J. Amer. Ceram. Soc. 44: 68-72; 1961.

[2.7] Spinner, S. Elastic moduli of glasses at elevated temperatures by a dynamic method. J. Amer. Ceram. Soc. 39(3): 113-118; 1956.

[2.8] Franke, K. Modulus of elasticity of some technical glasses in relation to temperature. Glastech. Ber. 19(4): 113-119; 1941.

[3.1] Brannan, R.T. Further evidence against the orientation of structure in glass fibers. J. Amer. Ceram. Soc. 36(7): 230-231; 1953.

[4.1] Thomas, W.F. An investigation of the factors likely to affect the strength and properties of glass fibers. Prop. Chem. Glasses 1(1): 4-18; 1960.

[4.2] Otto, W.H. Properties of glass fibers at elevated temperatures. AD228851, Contract No. 58841e. Toledo, OH: Owens-Corning Fiberglas Corporation; 1958.

[4.3] Cameron, N.M. The effect of environment and temperature on the strength of E-glass fibers, Part 1, High vacuum and low temperatures. Glass Technol. 9(1): 14-21; 1968.

[4.4] Duflos, J. French developments in the field of thermal-resistant and mechanical composites in Mechanics of composite materials. New York, NY: Pergamon Press; 1967. 181-192.

[4.5] Broutman L.J.; Krock, R.H. Modern composite materials. Reading, MA: Addison-Wesley; 1967. 280-301.

[5.1] Corruccini, R.J.; Gniewek, J.T. Thermal expansion of technical solids at low temperatures, Nat. Bur. Stand. (U.S.) Monograph 29; May 19, 1961.

[6.1] Childs, G.E.; Ericks, L.J.; Powell, R.L. Thermal conductivity of solids at room temperature and below. Nat. Bur. Stand. (U.S.) Monograph 131. NBS Monograph 131; 1973. 


\title{
V. Properties of Directionally Reinforced Composites
}

\author{
R.P. Reed \\ Cryogenic Materials, Inc. \\ Boulder, Colorado \\ J.D. McColskey \\ Materials Reliability Division \\ National Institute of Standards and Technology \\ Boulder, Colorado
}




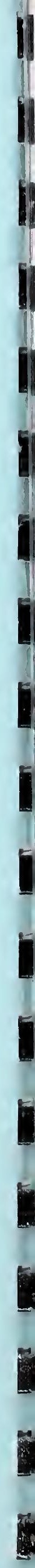




\title{
1. Mechanical Properties
}

\author{
R. P. Reed* \\ J. D. McColskey, R. P. Walsh, and D. L. Rule \\ J. B. Darr ${ }^{\dagger}$ \\ Materials Reliability Division \\ National Institute of Standards and Technology \\ Boulder, Colorado \\ ${ }^{*}$ Cryogenic Materials, Inc. \\ Boulder, Colorado \\ †Composite Technology Development, Inc. \\ Boulder, Colorado
}

The tensile, compression, and in-plane shear properties were measured at 295, 76 , and $4 \mathrm{~K}$ for $0^{\circ}$ and $90^{\circ}$ unidirectional fiber orientations and $\pm 45^{\circ}$ fiber orientations. E-glass was the fiber reinforcement for both a DGEBA-base epoxy and a cyanate ester.

In general, the elastic moduli and strengths of the cyanate-ester composite were larger than those of the epoxy composite. Yet, in resin-dominated properties (e.g., in-plane shear strength, $90^{\circ}$ tensile and compression properties), the epoxy composite data were more temperature dependent. This trend follows that found for neat-resin properties (see §III).

Strengths and elastic moduli were always larger at low temperatures. Strengths increased from 50 to $250 \%$, with the exception of the tensile strength of specimens with $0^{\circ}$ fiber orientation. For the $0^{\circ}$ fiber orientation, elastic moduli increased from 10 to $15 \%$, and for the $90^{\circ}$ and $\pm 45^{\circ}$ fiber orientations, from 25 to about $150 \%$.

The results demonstrated that resin selection plays a major role in determining all compression properties, tensile properties for specimens with $90^{\circ}$ fiber orientation, and the in-plane shear properties of specimens with $\pm 45^{\circ}$ fiber orientation.

\subsection{Introduction}

Many predictive models of fiber-reinforced-composite mechanical behavior rely on the primary data of directionally reinforced fiber composites. Data on plates made with directionally oriented fibers are relatively easy to obtain and, therefore, can be used to evaluate of the effectiveness of various resins in enhancing the low-temperature performance of composites.

In this study, only two resin systems with E-glass fiber reinforcement were evaluated: the DGEBA/DCH/ARA/ACL epoxy and the cyanate-ester resin systems. Owing to time constraints and other priorities, the vinyl-ester and polyester resin systems were not included. However, in the future, these two systems should also be studied. 
Tensile and compression tests were conducted at 295,76 , and $4 \mathrm{~K}$ on specimens with $0^{\circ}$ and $90^{\circ}$ fiber orientations. The longitudinal direction of the specimens was machined at angles of $0^{\circ}$ and $90^{\circ}$ to unidirectional fiber lay-ups and $\pm 45^{\circ}$ to a bidirectional fiber lay-up. Tensile tests were performed on specimens with $\pm 45^{\circ}$ fiber orientations to obtain in-plane shear properties. Moduli and ultimate strength data were obtained for each type of test.

Relatively few test programs have been previously conducted with E-glass-reinforced, directionally oriented fiber composites. This program generated the first mechanical-property data at low temperatures for this type of composite.

\subsection{Review of Literature}

Measurements of mechanical properties of unidirectional E-glass-reinforced epoxy composites are scarce. A summary of the tensile, compressive, and shear properties at room temperature for E-glass epoxy composites, taken from Reuton [1.1], Chamis [1.2], and Scala [1.3], is presented in Table 1.1. Great disparity exists among the reported values from these sources for the transverse $\left(90^{\circ}\right)$ tensile modulus, the transverse $\left(90^{\circ}\right)$ tensile strength, the axial $\left(G_{12}\right)$ shear modulus, and the short-beam interlaminar shear strength. Thus, for these properties, typical values could not be presented, but the range of values given in these three sources is provided in the footnotes of the table.

No low-temperature mechanical property data for either E-glass/epoxy or E-glass/ cyanate-ester unidirectional composites were found.

Table 1.1. Typical room-temperature properties of unidirectional composites (60 vol\% E-glass-reinforced epoxy).

\begin{tabular}{llc}
\hline \hline Tensile & Longitudinal $\left(E_{11}\right)$ modulus $(\mathrm{GPa})$ & 44 \\
& Transverse $\left(E_{22}\right)$ modulus $(\mathrm{GPa})$ & $(\mathrm{a})$ \\
& Poisson's ratio & 0.29 \\
& Longitudinal strength $(\mathrm{MPa})$ & 1100 \\
& Transverse strength $(\mathrm{MPa})$ & (b) \\
& Longitudinal modulus $(\mathrm{GPa})$ & 32 \\
Compression & Transverse modulus $(\mathrm{GPa})$ & - \\
& Poisson's ratio & - \\
& Longitudinal strength $(\mathrm{MPa})$ & 620 \\
& Transverse strength $(\mathrm{MPa})$ & 140 \\
& Axial $\left(G_{12}\right)$ modulus $(\mathrm{GPa})$ & $(\mathrm{c})$ \\
& Normal $\left(G_{23}\right)$ modulus $(\mathrm{GPa})$ & 3.4 \\
& In-plane (interlaminar) modulus $(\mathrm{GPa})$ & 4.8 \\
& In-plane (interlaminar) Poisson's ratio & 0.3 \\
& In-plane (intralaminar) strength (MPa) & 83 \\
& Short-beam (interlaminar) strength & (d) \\
\hline \hline
\end{tabular}

(a) Data vary from 4.8 to $12.4 \mathrm{GPa}$; no clear average.

(b) Data vary from 28 to $97 \mathrm{MPa}$; no clear average. Since the tensile strengths of neat epoxy resins vary from 60 to $90 \mathrm{MPa}$ at $295 \mathrm{~K}$, the low values of transverse tensile strengths probably reflect material or test inconsistencies.

(c) Data vary from 5.5 to $8.6 \mathrm{GPa}$; no clear average.

(d) Data vary from 69 to $97 \mathrm{MPa}$; no clear average. 


\subsection{Materials}

The unidirectional and $\pm 45^{\circ}$ fiber-reinforced plates were prepared from E-glass fibers and DGEBA/DCH/ARA/ACL epoxy and CE cyanate-ester resins.

The resins are discussed more thoroughly in $\S I I I .3 .3$ of this report. Each fiber was Type $\mathrm{M}$ with a 15.2 - to $16.5-\mu \mathrm{m}$ diameter. The tow of these fibers had a width of $25.4 \mathrm{~mm}$.

The epoxy-resin plates were prepared by wet-filament winding each ply around a mandrel, then cutting the ply, and laying each upon the others with the same filament orientation. To produce 3-mm-thick plate, eight plies were used. The effective orientation of the $0^{\circ}$ and $90^{\circ}$ fibers is estimated at $\pm 2{ }^{\circ}$ and $\pm 88^{\circ}$. Hereafter, they are labeled $0^{\circ}$ and $90^{\circ}$. Similar filamentwinding and lay-up procedures were used for the $\pm 45^{\circ}$ plates. Both 3-mm- and 6-mm-thick plates were prepared with the $0^{\circ}$ and $90^{\circ}$ fiber orientations and 3-mm-thick plates with the $\pm 45^{\circ}$ fiber orientation.

The epoxy-matrix specimens were analyzed for fiber and void content by the manufacturer. One 3-mm- and one 6-mm-thick plate were cut into three specimens each. They were initially weighed at room temperature, then warmed to $590^{\circ} \mathrm{C}$ in $1 \mathrm{~h}$ and held at that temperature for $2 \mathrm{~h}$. After cooling to room temperature, they were reweighed. Table 1.2 lists experimental measurements and calculations. Fiber volume ranged from 71 to $75 \mathrm{vol} \%$ and resin void contents ranged from 8 to 9 vol\%.

Table 1.2. Measurements of unidirectional panel characteristics.

\begin{tabular}{ccccccc}
\hline \hline $\begin{array}{c}\text { Sample Thickness } \\
(\mathrm{mm})\end{array}$ & $\begin{array}{c}\text { Air Wt. } \\
(\mathrm{g})\end{array}$ & $\begin{array}{c}\text { Water Wt. } \\
(\mathrm{g})\end{array}$ & $\begin{array}{c}\text { Density } \\
\left(\mathrm{g} / \mathrm{cm}^{3}\right)\end{array}$ & $\begin{array}{c}\text { Fiber } \\
(\text { vol\% })\end{array}$ & $\begin{array}{c}\text { Resin } \\
(\text { vol\% })\end{array}$ & $\begin{array}{c}\text { Voids } \\
(\text { vol\% })\end{array}$ \\
\hline \hline 3 & 1.0143 & 0.5441 & 1.8588 & 73.38 & 18.52 & 8.10 \\
3 & 0.8353 & 0.4996 & 1.8667 & 74.00 & 17.11 & 8.89 \\
3 & 1.2036 & 0.6616 & 1.8140 & 71.25 & 19.68 & 9.01 \\
Average & & & & 72.87 & 18.43 & 8.69 \\
\hline 6 & 0.9295 & 0.4971 & 1.8644 & 73.89 & 17.18 & 8.93 \\
6 & 0.8906 & 0.4693 & 1.8922 & 75.24 & 16.16 & 8.60 \\
6 & 0.7866 & 0.4334 & 1.8096 & 71.03 & 19.84 & 9.12 \\
Average & & & & 73.39 & 17.73 & 8.88 \\
\hline \hline
\end{tabular}

\subsection{Procedures}

Tensile and compression tests were conducted by using standard ASTM test procedures: ASTM D3039 for tensile tests [1.4], ASTM D3410 for compression tests [1.5], and ASTM D3518 for in-plane shear tests [1.6]. Specimen dimensions for each type of test are shown in Figures 1.1 and 1.2. 
For tensile tests of specimens with $0^{\circ}$ fiber orientation, tabbed ends with a bolted friction grip were used at first, but at low temperatures the tabbed ends of these specimens sheared off in the grips. To increase the tensile stress in the reduced section of the specimens relative to the shear stress in the tabbed-end area, the reduced section was machined as indicated in Figure 1.1(a). Subsequently, all failures occurred within the reduced section.
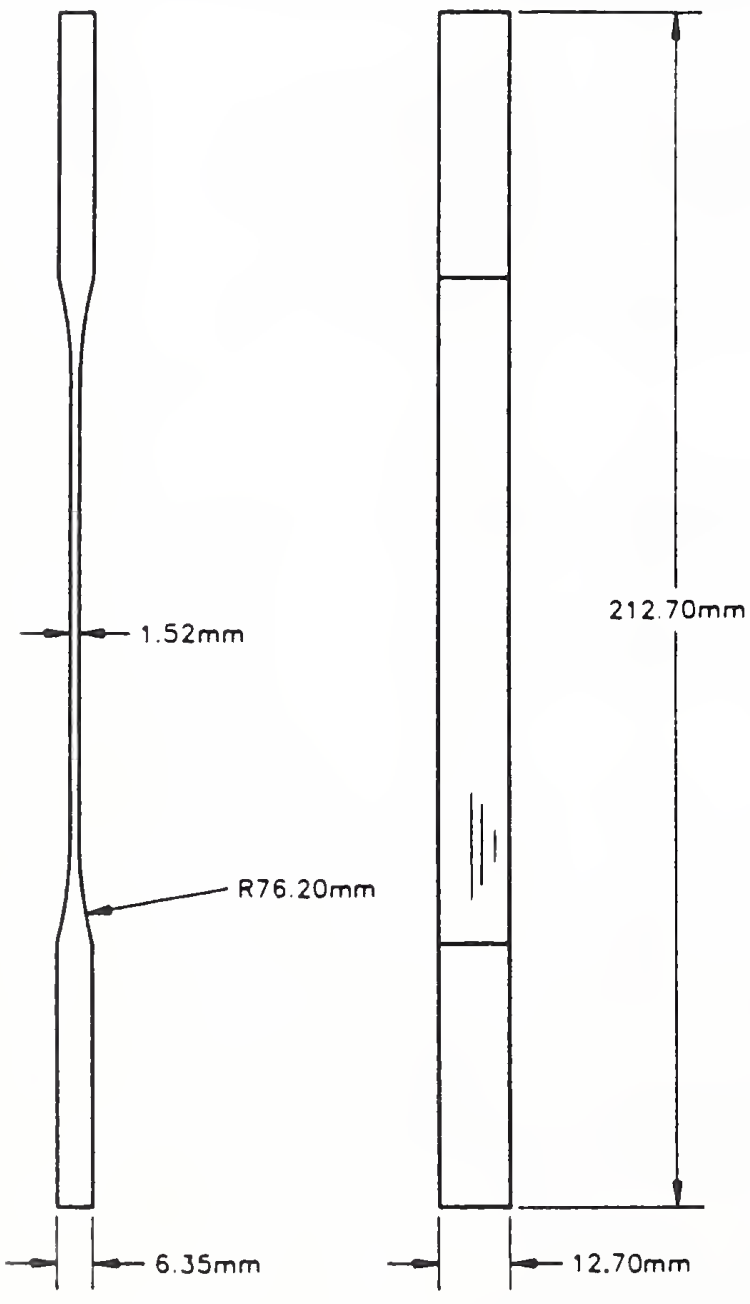

(a)

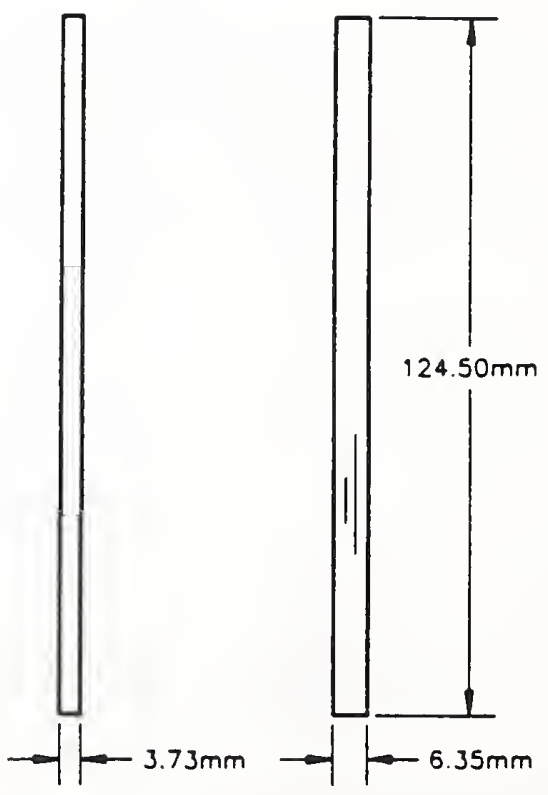

(b)

Figure 1.1. (a) Uniaxial tensile and (b) uniaxial compression specimen dimensions. 
Compression tests were conducted with a Celanese fixture, which produced satisfactory compression failures at all test temperatures. All tests were conducted with servohydraulic loading machines at a load rate of $0.2 \mathrm{~mm} / \mathrm{s}$. Cryostat systems were used that have been previously described [1.7]. Boiling liquid nitrogen and helium were used to obtain temperatures of 76 and $4 \mathrm{~K}$. Two strain gages oriented parallel to the loading direction were used to acquire the elastic moduli; for Poisson's ratios, a transverse-oriented strain gage was added.

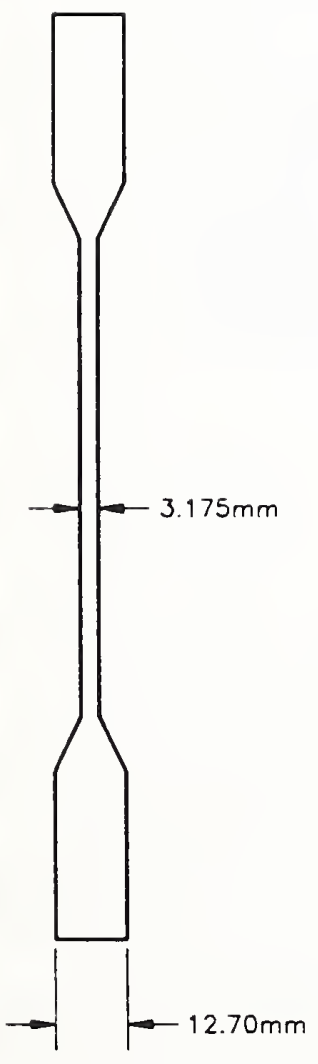

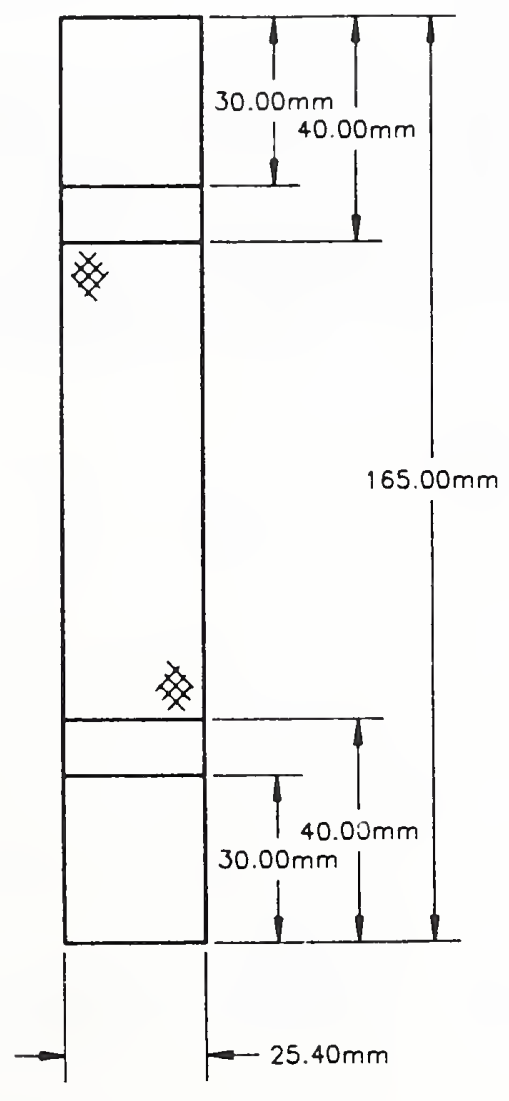

(a)
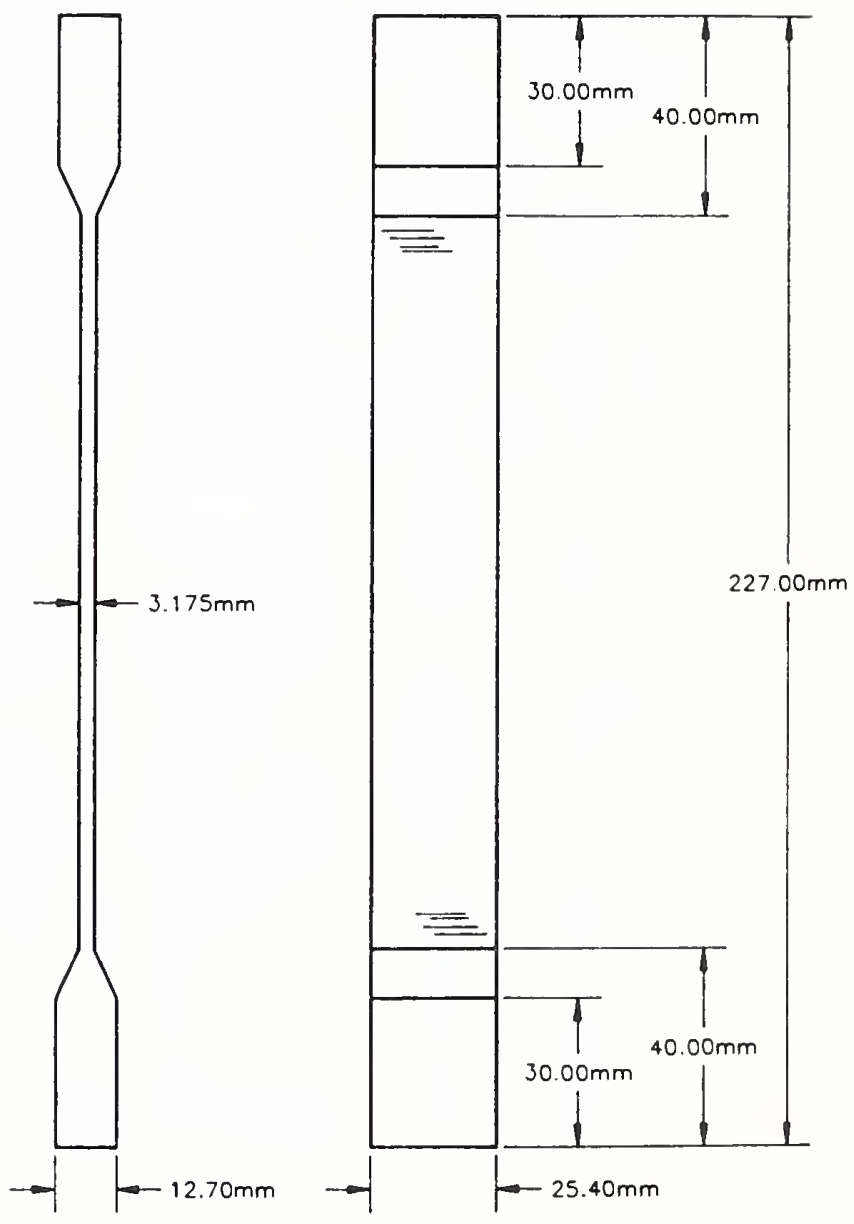

(b)

Figure 1.2. (a) $\pm 45^{\circ}$ in-plane-shear and (b) $90^{\circ}$ tensile specimen dimensions. 


\subsection{Results}

The results of the tests on unidirectional fiber-reinforced composites with epoxy and cyanate-ester resin matrices are presented below.

\subsubsection{Epoxy-Resin-Matrix Composites}

Individual test data and averages for the DGEBA/DCH/ARA/ACL epoxy-resin-matrix, Eglass fiber-reinforced, unidirectional composite are summarized in Tables 1.3, 1.4, and 1.5 for fiber orientations of $0^{\circ}, 90^{\circ}$, and $\pm 45^{\circ}$, respectively. The data are plotted as a function of test temperature in Figures 1.3 and 1.4. The compression strength and modulus were always higher than similar tensile parameters, except for the $0^{\circ}$ fiber orientation at $295 \mathrm{~K}$.

The $0^{\circ}$ tensile and compressive moduli increased about $10 \%$ at low temperatures; in strong contrast, at 76 and $4 \mathrm{~K}$ the $\pm 45^{\circ}$ shear modulus was more than $200 \%$ larger than the shear modulus at room temperature. Another strong contrast in temperature-dependent behavior was the distinction between the $90^{\circ}$ tensile strength, which increased about 20 to $30 \%$ at low temperatures, and the $90^{\circ}$ compressive strength, which showed a very strong increase at low temperatures-over $300 \%$.

Typical stress-strain curves at 295 and $4 \mathrm{~K}$ are shown in Figures $1.5,1.6$, and 1.7 for tensile tests of $0^{\circ}$-fiber-orientation specimens, compression tests of $0^{\circ}$-fiber-orientation specimens, and compression tests of $90^{\circ}$-fiber-orientation specimens, respectively. Plastic deformation for the tensile tests of $0^{\circ}$-fiber-orientation specimens at both test temperatures is clearly evident, and very limited inelastic deformation occurred at $295 \mathrm{~K}$ in the compression tests of $0^{\circ}$-fiber-oriented specimens. Tensile tests of $90^{\circ}$-fiber-oriented specimens resulted in elastic deformation only. 
Table 1.3. Properties of unidirectional E-glass-epoxy composite ( $0^{\circ}$ fiber orientation).

\begin{tabular}{|c|c|c|c|c|c|c|}
\hline $\begin{array}{l}\text { Type of Test, } \\
\text { Fiber Orientation }\end{array}$ & $\begin{array}{c}\text { Test } \\
\text { Temperature } \\
(\mathrm{K}) \\
\end{array}$ & $\begin{array}{l}\text { Elastic } \\
(\mathrm{GPa})\end{array}$ & $\begin{array}{r}\text { Modulus } \\
\text { (Msi) }\end{array}$ & $\begin{array}{c}\text { Poisson's } \\
\text { Ratio }\end{array}$ & $\begin{array}{l}\text { Stren } \\
(\mathrm{MPa})\end{array}$ & $\begin{array}{l}\text { gth } \\
\text { (ksi) }\end{array}$ \\
\hline \multirow[t]{13}{*}{ Tensile, $0^{\circ}$} & \multirow[t]{4}{*}{295} & 39.6 & 5.74 & - & 1020 & 148 \\
\hline & & 39.5 & 5.73 & - & 1010 & 146 \\
\hline & & 37.8 & 5.49 & - & 1000 & 145 \\
\hline & & 42.2 & 6.12 & 0.26 & - & - \\
\hline & Average & 39.8 & 5.77 & 0.26 & 1010 & 146 \\
\hline & \multirow[t]{3}{*}{76} & 44.7 & 6.49 & & 1370 & 198 \\
\hline & & 42.5 & 6.17 & & 1260 & 183 \\
\hline & & 44.8 & 6.50 & & 1330 & 193 \\
\hline & Average & 44.0 & 6.39 & & 1320 & 191 \\
\hline & \multirow[t]{3}{*}{4} & 50.0 & 7.25 & & 1200 & 173 \\
\hline & & 42.3 & 6.13 & & 1060 & 154 \\
\hline & & 43.2 & 6.27 & & 1190 & 193 \\
\hline & Average & 45.2 & 6.63 & & 1150 & 166 \\
\hline \multirow[t]{14}{*}{ Compression, $0^{\circ}$} & \multirow[t]{4}{*}{295} & - & - & & 807 & 117 \\
\hline & & 53.1 & 7.70 & & 845 & 122 \\
\hline & & 51.1 & 7.41 & & 749 & 109 \\
\hline & & 47.9 & 6.94 & & 675 & 98 \\
\hline & Average & 50.7 & 7.35 & & 769 & 112 \\
\hline & \multirow[t]{4}{*}{76} & - & - & & 1510 & 219 \\
\hline & & 54.1 & 7.85 & & 1350 & 196 \\
\hline & & 54.8 & 7.95 & & 1350 & 196 \\
\hline & & 54.1 & 7.85 & & - & - \\
\hline & Average & 54.3 & 7.88 & & 1400 & 204 \\
\hline & \multirow[t]{3}{*}{4} & 56.5 & 8.19 & & 1580 & 228 \\
\hline & & - & - & & 1550 & 225 \\
\hline & & 53.7 & 7.79 & & 1200 & 173 \\
\hline & Average & 55.1 & 7.99 & & 1440 & 209 \\
\hline
\end{tabular}


Table 1.4. Properties of unidirectional E-glass-epoxy composite $\left(90^{\circ}\right.$ fiber orientation).

\begin{tabular}{|c|c|c|c|c|c|}
\hline $\begin{array}{l}\text { Type of Test, } \\
\text { Fiber Orientation }\end{array}$ & $\begin{array}{c}\text { Test } \\
\text { Temperature } \\
(\mathrm{K})\end{array}$ & $\begin{array}{c}\text { Elastic } \\
(\mathrm{GPa})\end{array}$ & $\begin{array}{l}\text { Modulus } \\
\text { (Msi) }\end{array}$ & $\begin{array}{l}\text { Stre } \\
(\mathrm{MPa})\end{array}$ & $\begin{array}{l}\text { gth } \\
\text { (ksi) }\end{array}$ \\
\hline \multirow[t]{12}{*}{ Tensile, $90^{\circ}$} & \multirow[t]{3}{*}{295} & 12.3 & 1.78 & 15.1 & 2.19 \\
\hline & & 10.1 & 1.46 & 23.1 & 3.34 \\
\hline & & 10.4 & 1.51 & 26.8 & 3.88 \\
\hline & Average & 10.9 & 1.58 & 21.7 & 3.14 \\
\hline & \multirow[t]{3}{*}{76} & 16.9 & 2.45 & 15.8 & 2.29 \\
\hline & & 17.4 & 2.52 & 40.7 & 5.91 \\
\hline & & 14.9 & 2.16 & 39.0 & 5.66 \\
\hline & Average & 16.4 & 2.38 & 31.8 & 4.62 \\
\hline & \multirow[t]{3}{*}{4} & 22.3 & 3.23 & 40.5 & 5.87 \\
\hline & & 22.6 & 3.27 & 15.8 & 2.28 \\
\hline & & 17.1 & 2.48 & 39.0 & 5.65 \\
\hline & Average & 20.7 & 2.99 & 31.8 & 4.60 \\
\hline \multirow[t]{15}{*}{ Compression, $90^{\circ}$} & \multirow[t]{4}{*}{295} & 一 & - & 88.3 & 12.8 \\
\hline & & - & - & 84.8 & 12.3 \\
\hline & & - & - & 86.9 & 12.6 \\
\hline & & - & - & 86.9 & 12.6 \\
\hline & Average & & & 86.7 & 12.6 \\
\hline & \multirow[t]{4}{*}{76} & - & - & 252 & 36.6 \\
\hline & & 21.0 & 3.05 & 290 & 41.7 \\
\hline & & 22.8 & 3.31 & 315 & 45.7 \\
\hline & & 20.9 & 3.03 & 264 & 38.3 \\
\hline & Average & 21.6 & 3.13 & 280 & 39.8 \\
\hline & \multirow[t]{4}{*}{4} & 23.8 & 3.45 & 297 & 43.1 \\
\hline & & - & - & 258 & 37.4 \\
\hline & & 23.8 & 3.45 & 329 & 47.7 \\
\hline & & 20.6 & 2.99 & 328 & 47.6 \\
\hline & Average & 22.7 & 3.30 & 303 & 44.0 \\
\hline
\end{tabular}


Table 1.5. In-plane shear properties for E-glass-epoxy composite $\left( \pm 45^{\circ}\right.$ fiber orientation).

\begin{tabular}{|c|c|c|c|c|}
\hline $\begin{array}{c}\text { Test } \\
\text { Temperature } \\
\text { (K) }\end{array}$ & $\begin{array}{l}\text { Shear } \\
\text { (GPa) }\end{array}$ & $\begin{array}{l}\text { Modulus } \\
\text { (Msi) }\end{array}$ & $\begin{array}{l}\text { Shear } \\
(\mathrm{MPa})\end{array}$ & $\begin{array}{l}\text { Strength } \\
\text { (ksi) }\end{array}$ \\
\hline \multirow[t]{4}{*}{295} & 3.87 & 0.561 & 40.6 & 5.89 \\
\hline & 3.34 & 0.484 & 55.1 & 7.99 \\
\hline & 4.26 & 0.618 & 42.8 & 6.21 \\
\hline & 5.18 & 0.752 & 34.4 & 4.98 \\
\hline Average & 4.16 & 0.604 & 43.2 & 6.27 \\
\hline \multirow[t]{3}{*}{76} & 8.97 & 1.30 & 85.7 & 12.4 \\
\hline & 8.60 & 1.25 & 72.3 & 10.5 \\
\hline & 10.24 & 1.48 & 98.9 & 14.3 \\
\hline Average & 9.27 & 1.34 & 85.6 & 12.4 \\
\hline \multirow[t]{4}{*}{4} & 10.21 & 1.48 & 84.1 & 12.2 \\
\hline & 12.10 & 1.76 & 66.5 & 9.6 \\
\hline & 9.47 & 1.37 & 80.0 & 11.6 \\
\hline & 11.49 & 1.67 & 85.6 & 12.1 \\
\hline Average & 10.82 & 1.57 & 79.0 & 11.4 \\
\hline
\end{tabular}

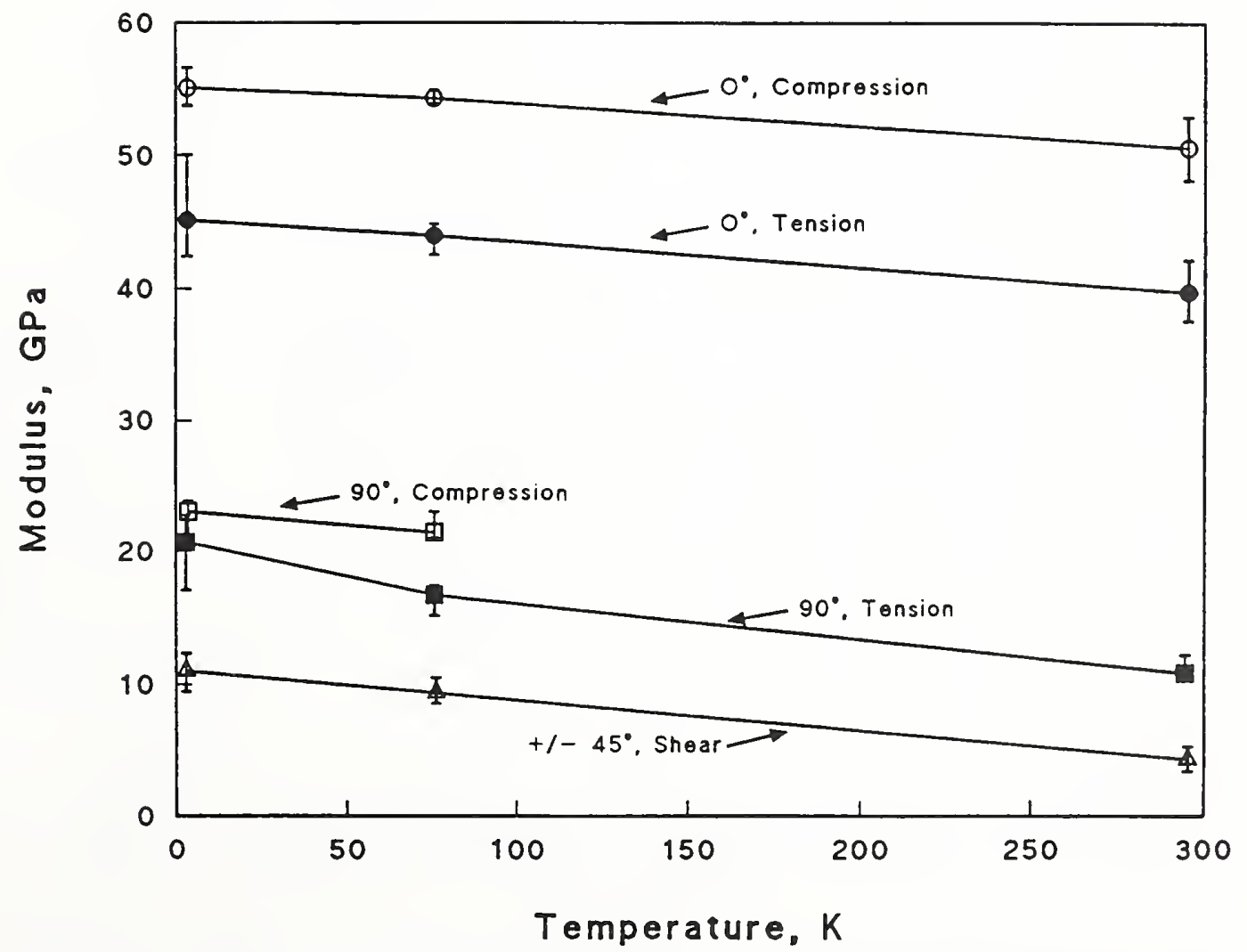

Figure 1.3. Elastic modulus versus temperature for E-glass-epoxy composite. 


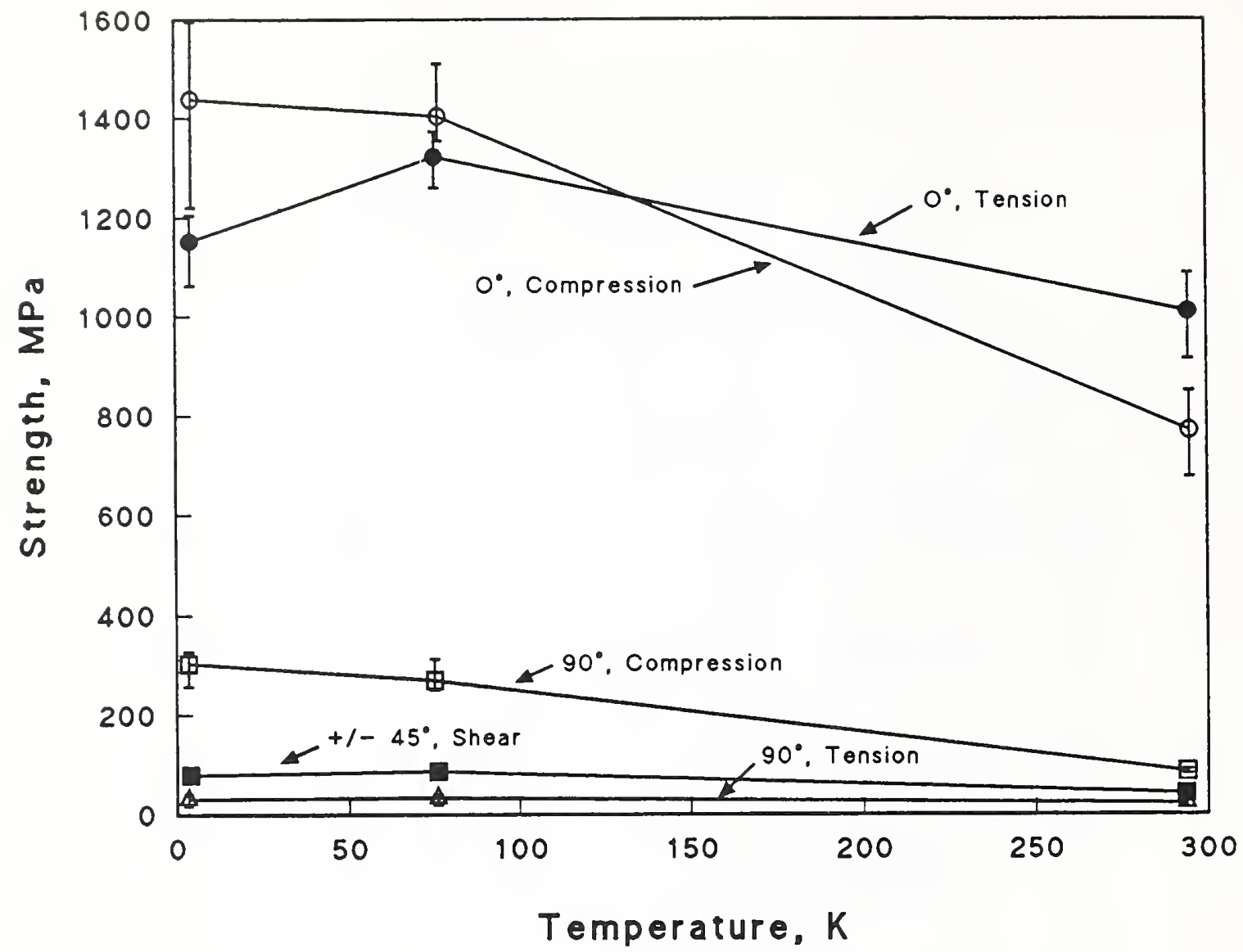

Figure 1.4. Strength versus temperature for E-glass-epoxy composite.

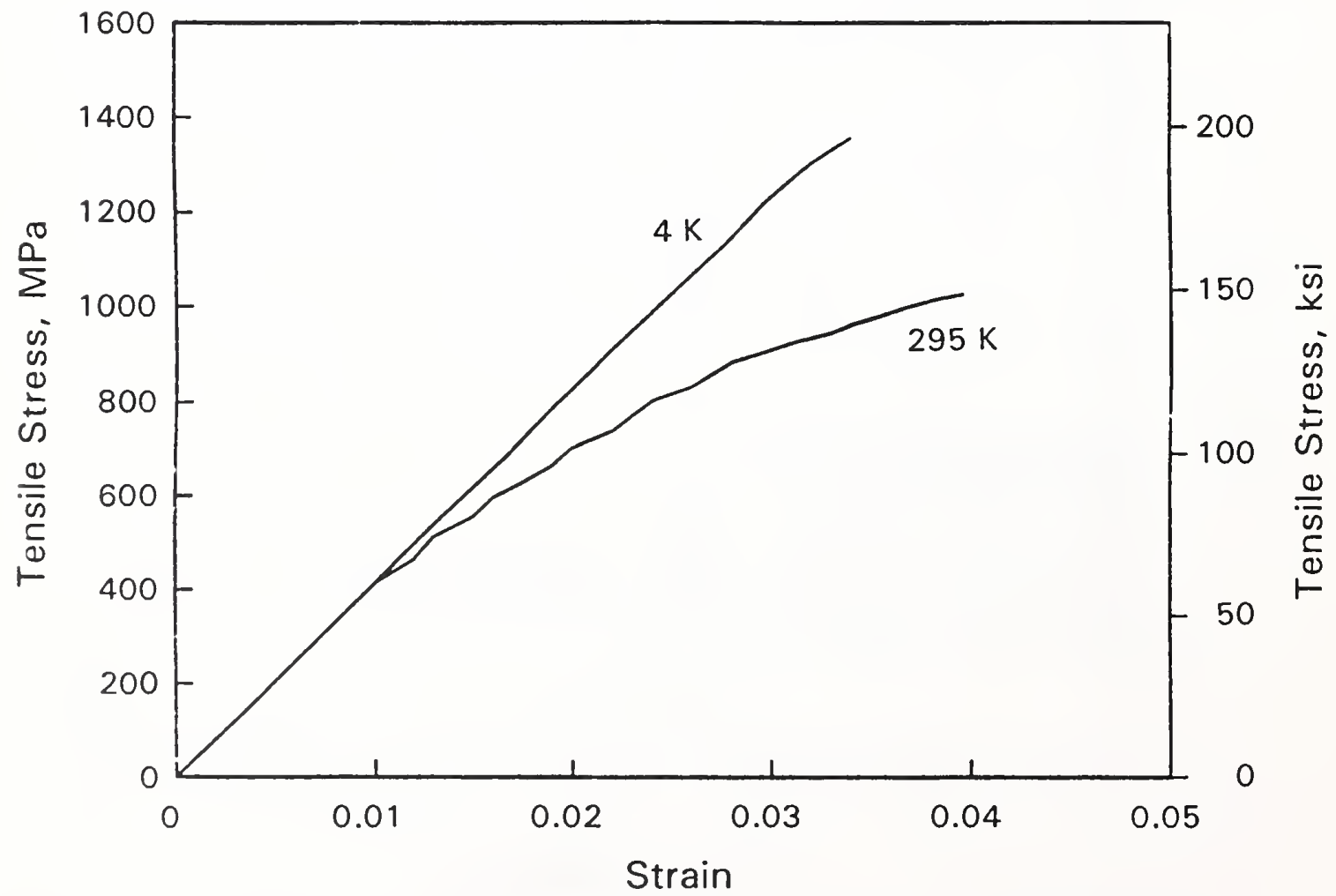

Figure 1.5. Tensile data for unidirectionally reinforced $\left(0^{\circ}\right)$ E-glass-epoxy composite. 


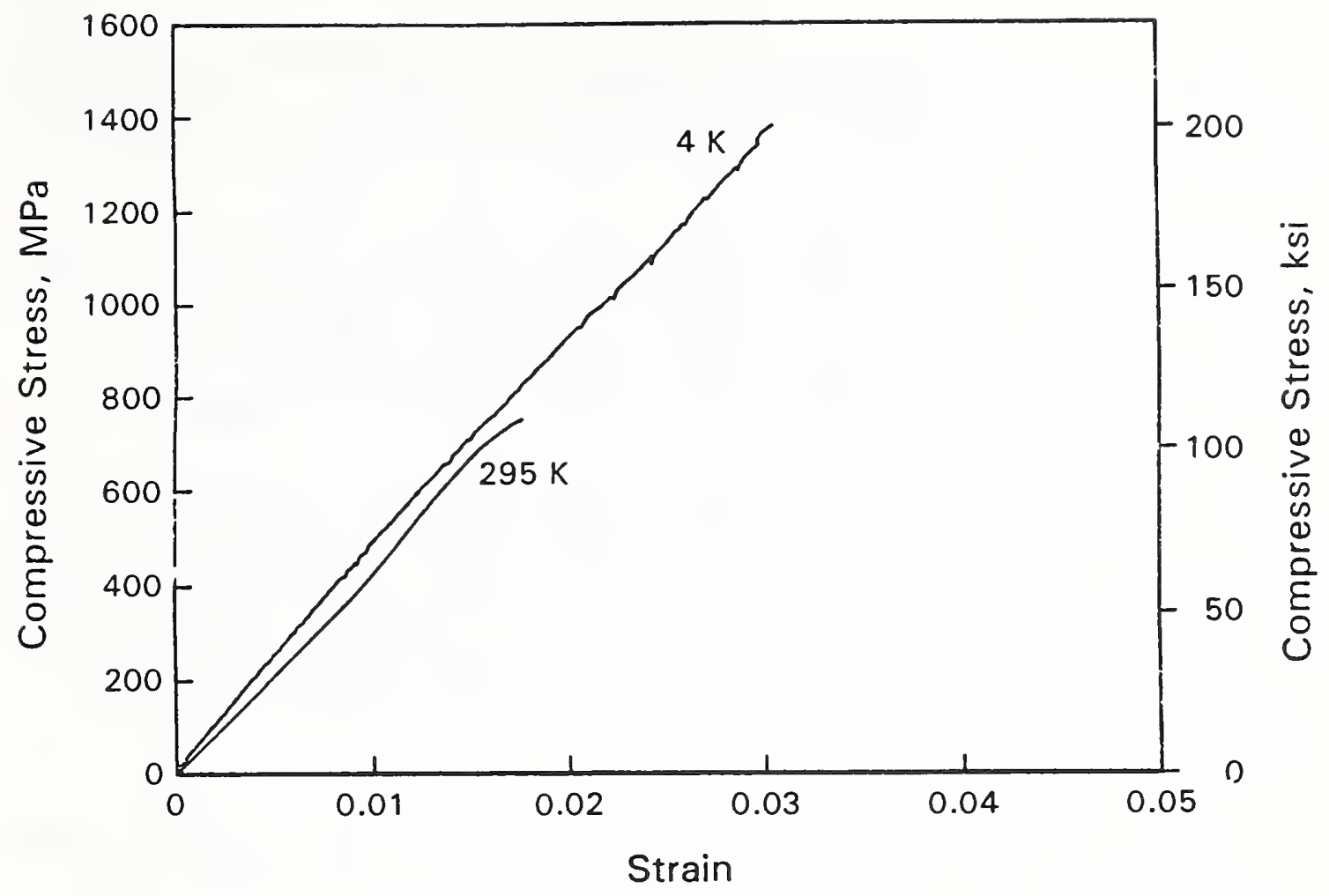

Figure 1.6. Compressive data for unidirectionally reinforced $\left(0^{\circ}\right)$ E-glass-epoxy composite.

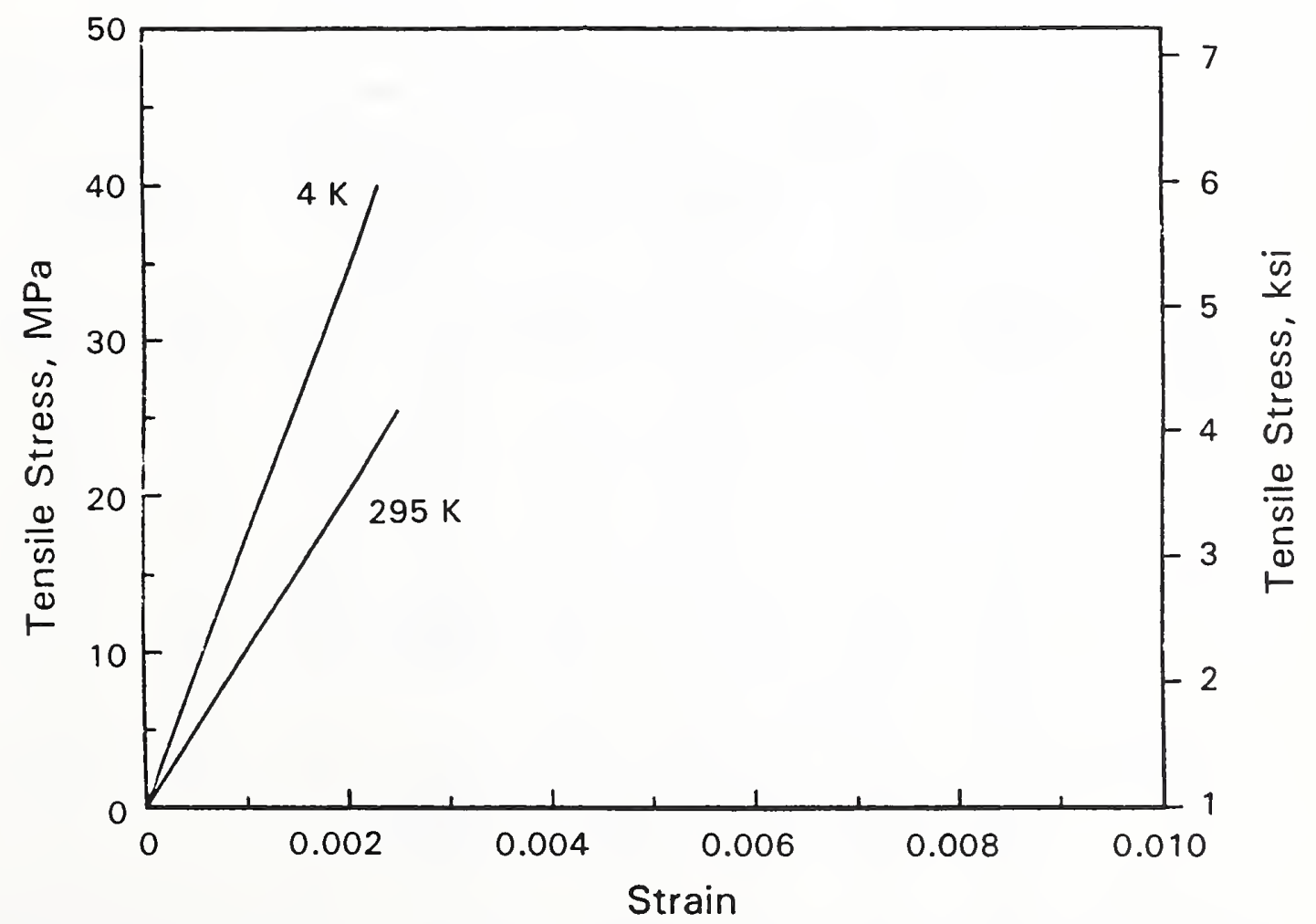

Figure 1.7. Tensile data for unidirectionally reinforced $\left(90^{\circ}\right)$ E-glass-epoxy composite. 


\subsubsection{Cyanate-Ester-Resin-Matrix Composites}

Summaries of individual test data and averages for the CE cyanate-ester-resin matrix, E-glass-reinforced, unidirectional composite are contained in Tables 1.6, 1.7, and 1.8 for fiber orientations of $0^{\circ}, 90^{\circ}$, and $\pm 45^{\circ}$, respectively. In Figures 1.8 and 1.9 the data averages are plotted as a function of test temperature.

For the $0^{\circ}$-fiber-orientation composite, the compressive strength was lower than the tensile strength at 295 but higher at $4 \mathrm{~K}$ and $76 \mathrm{~K}$. Thus, the dependence on temperature was much stronger for the compressive strength ( $107 \%$ increase from 295 to $4 \mathrm{~K}$ ) than for the tensile strength (15\% increase) for this fiber orientation. Both the tensile and compression elastic moduli showed little dependence on temperature, and they were about equivalent.

For the $90^{\circ}$-fiber-orientation composite, the compressive strengths were much higher than the tensile strengths. At $295 \mathrm{~K}$, the compressive strength was roughly 5 times higher than the tensile strength; at $4 \mathrm{~K}$, the advantage of the compressive strength increased to about a factor of 8. The compressive strength increased from 295 to $4 \mathrm{~K}$ by about a factor of $2(100 \%)$, whereas the tensile strength was less temperature dependent, increasing by only $25 \%$. The increase of both elastic moduli from 295 to $4 \mathrm{~K}$ was about $50 \%$, much larger than that of the $0^{\circ}$-fiberorientation data. We have no explanation for the wide divergence between the tensile and compressive moduli at all temperatures.

The in-plane shear strength ( $\pm 45^{\circ}$ fiber orientation) showed little dependence on temperature, increasing only about $20 \%$ at low temperatures. However, the in-plane shear modulus increased about $75 \%$ on cooling from 295 to $4 \mathrm{~K}$.

Table 1.6. Properties of unidirectional E-glass-cyanate-ester composite ( $0^{\circ}$ fiber orientation).

\begin{tabular}{ccccccccc}
\hline \hline \multirow{2}{*}{$\begin{array}{c}\text { Temperature } \\
(\mathrm{K})\end{array}$} & \multicolumn{2}{c}{$\begin{array}{c}\text { Sensile } \\
\text { Strength }\end{array}$} & \multicolumn{2}{c}{ Tensile Elastic } & \multicolumn{2}{c}{$\begin{array}{c}\text { Compressive } \\
\text { Modulus }\end{array}$} & \multicolumn{2}{c}{$\begin{array}{c}\text { Compressive } \\
\text { Elastic Modulus }\end{array}$} \\
& $(\mathrm{MPa})$ & $(\mathrm{ksi})$ & $(\mathrm{GPa})$ & $(\mathrm{Msi})$ & $(\mathrm{MPa})$ & $(\mathrm{ksi})$ & $(\mathrm{GPa})$ & $(\mathrm{Msi})$ \\
\hline \hline 295 & 1413 & 205 & 53.2 & 7.72 & 882 & 128 & 54.5 & 7.91 \\
& 1572 & 228 & 53.4 & 7.75 & 917 & 133 & 54.3 & 7.87 \\
& 1348 & 195 & 53.3 & 7.73 & 884 & 129 & 54.7 & 7.93 \\
Average & 1444 & 209 & 53.3 & 7.73 & 894 & 130 & 54.5 & 7.90 \\
\hline \multirow{2}{*}{76} & 1503 & 218 & 53.6 & 7.78 & 1589 & 230 & 56.3 & 8.16 \\
& 1710 & 248 & 54.8 & 7.95 & 1853 & 269 & 54.7 & 7.93 \\
& 1717 & 249 & 56.1 & 8.14 & 1724 & 250 & 53.4 & 7.75 \\
Average & 1643 & 238 & 54.8 & 7.96 & 1722 & 250 & 54.8 & 7.95 \\
\hline \multirow{2}{*}{4} & 1779 & 258 & 55.9 & 8.11 & 1869 & 271 & - & - \\
& 1544 & 224 & 55.8 & 8.10 & 1841 & 267 & - & - \\
Average & 1662 & 241 & 55.9 & 8.11 & 1855 & 269 & - & - \\
\hline \hline
\end{tabular}


Table 1.7. Properties of unidirectional E-glass-cyanate-ester composite ( $90^{\circ}$ fiber orientation).

\begin{tabular}{ccccccccc}
\hline \hline \multirow{2}{*}{$\begin{array}{c}\text { Temperature } \\
(\mathrm{K})\end{array}$} & \multicolumn{2}{c}{$\begin{array}{c}\text { Tensile } \\
\text { Strength }\end{array}$} & \multicolumn{2}{c}{$\begin{array}{c}\text { Tensile Elastic } \\
\text { Modulus }\end{array}$} & \multicolumn{2}{c}{$\begin{array}{c}\text { Compressive } \\
\text { Strength }\end{array}$} & \multicolumn{2}{c}{$\begin{array}{c}\text { Compressive } \\
\text { Elastic Modulus }\end{array}$} \\
\cline { 2 - 9 } & $(\mathrm{MPa})$ & $(\mathrm{ksi})$ & $(\mathrm{GPa})$ & $(\mathrm{Msi})$ & $(\mathrm{MPa})$ & $(\mathrm{ksi})$ & $(\mathrm{GPa})$ & $(\mathrm{Msi})$ \\
\hline \hline 295 & 32.1 & 4.65 & 20.0 & 2.90 & 163 & 23.6 & 18.1 & 2.62 \\
& 33.7 & 4.88 & 16.1 & 2.33 & 134 & 19.5 & 14.8 & 2.14 \\
& 29.9 & 4.34 & 15.6 & 2.27 & 177 & 25.3 & 17.7 & 2.56 \\
Average & 31.9 & 4.60 & 17.2 & 2.50 & 158 & 22.9 & 16.8 & 2.44 \\
\hline 76 & 34.7 & 5.03 & - & - & 306 & 44.4 & 16.5 & 2.39 \\
& 44.7 & 6.48 & 25.0 & 3.63 & 315 & 45.7 & 20.0 & 2.90 \\
& 34.7 & 5.04 & 27.2 & 3.95 & 316 & 45.9 & 20.9 & 3.03 \\
Average & 38.0 & 5.52 & 26.1 & 3.79 & 312 & 45.3 & 19.1 & 2.77 \\
\hline \multirow{2}{*}{4} & 42.4 & 6.15 & 25.9 & 3.75 & 302 & 43.8 & - & - \\
& 37.9 & 5.50 & - & - & 342 & 49.6 & 25.9 & 3.76 \\
& & & & & 328 & 47.6 & 23.9 & 3.47 \\
Average & 40.2 & 5.83 & 25.9 & 3.75 & 324 & 47.0 & 24.9 & 3.62 \\
\hline \hline
\end{tabular}

Table 1.8. In-plane shear properties for E-glass-cyanate-ester composite $\left( \pm 45^{\circ}\right.$ fiber orientation).

\begin{tabular}{ccccc}
\hline \hline \multirow{2}{*}{$\begin{array}{c}\text { Temperature } \\
(\mathrm{K})\end{array}$} & \multicolumn{2}{c}{ Shear Strength } & \multicolumn{2}{c}{ Shear Modulus } \\
\cline { 2 - 5 } & $(\mathrm{MPa})$ & $(\mathrm{ksi})$ & $(\mathrm{GPa})$ & $(\mathrm{Msi})$ \\
\hline \hline 295 & 105 & 15.3 & 5.11 & 0.74 \\
& 102 & 14.7 & 6.07 & 0.88 \\
& 107 & 15.5 & 5.42 & 0.79 \\
Average & 105 & 15.2 & 5.53 & 0.80 \\
\hline 76 & 129 & 18.8 & 8.96 & 1.30 \\
& 112 & 16.3 & 8.96 & 1.30 \\
& 135 & 19.5 & - & - \\
Average & 125 & 18.2 & 8.96 & 1.30 \\
\hline \multirow{2}{*}{4} & 112 & 16.2 & 10.07 & 1.46 \\
& 130 & 18.8 & 9.17 & 1.33 \\
& 121 & 17.5 & 9.62 & 1.40 \\
\hline \hline
\end{tabular}




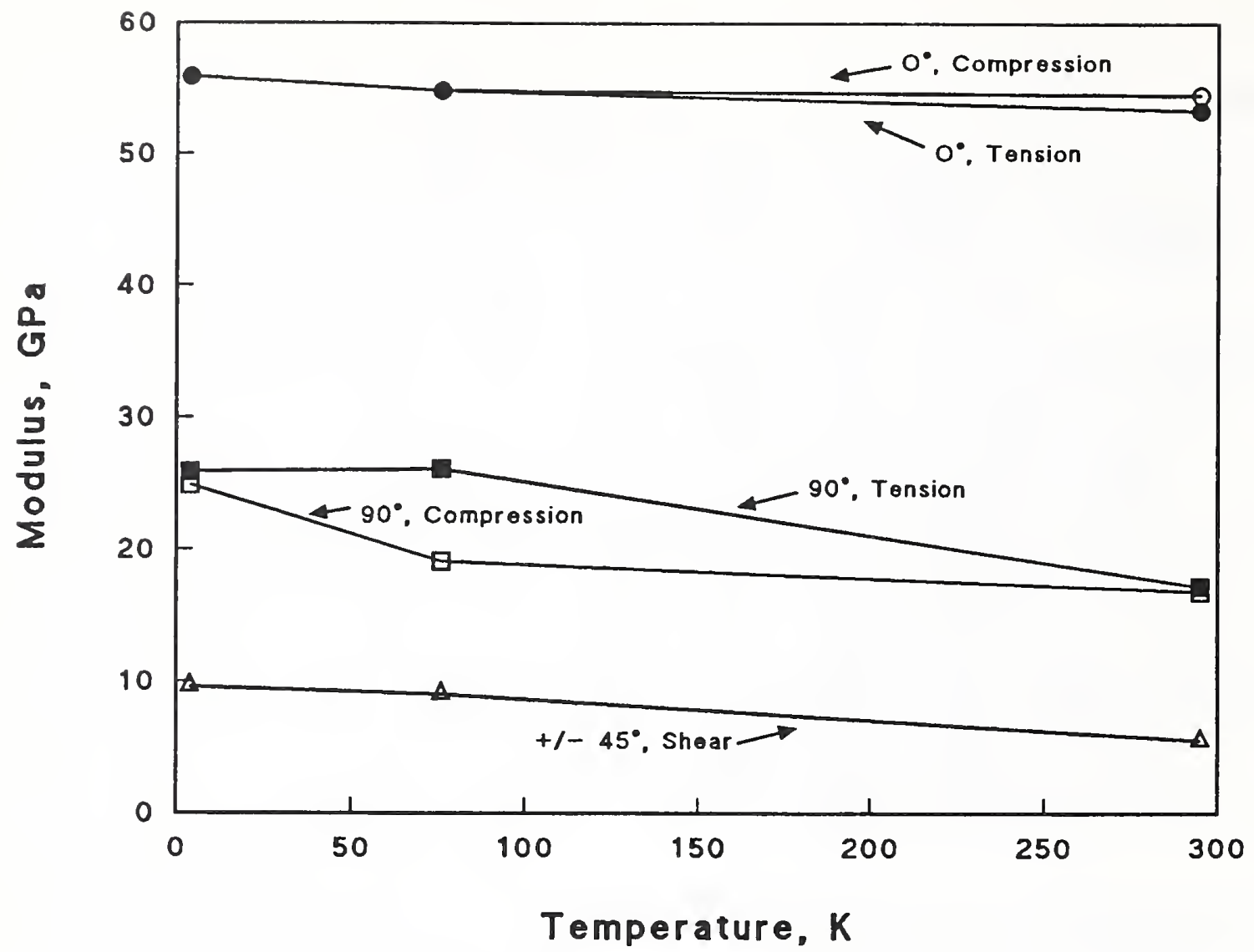

Figure 1.8. Elastic modulus versus temperature for E-glass-cyanate-ester composite.

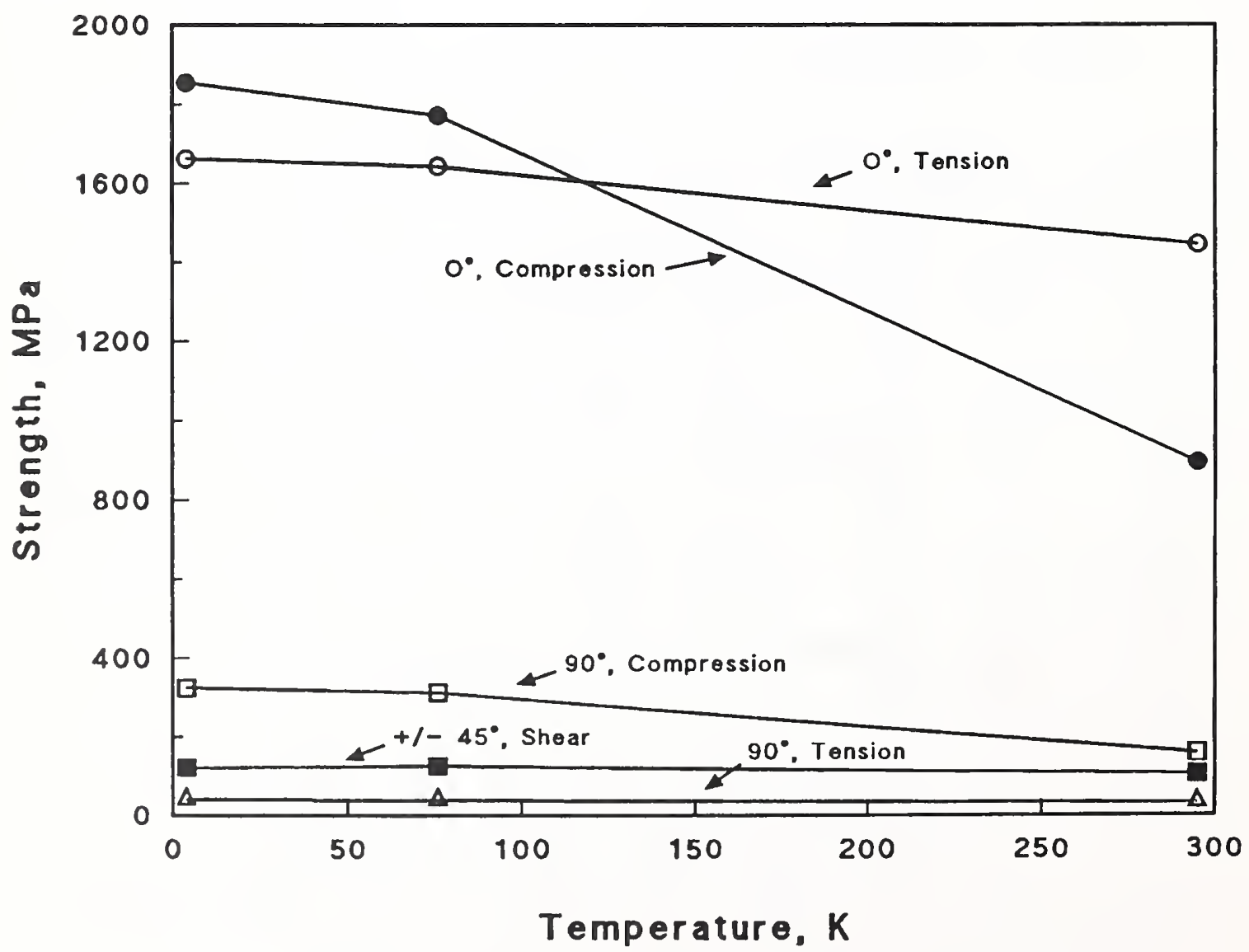

Figure 1.9. Strength versus temperature for E-glass-cyanate-ester composite. $\mathrm{V}-14$ 


\subsection{Discussion}

\subsubsection{Epoxy-Resin-Matrix Composites}

The room-temperature properties of the E-glass-epoxy specimens with $0^{\circ}$ fiber orientation compare well with the published data for epoxy-based composites with the exception of the longitudinal compression modulus. (Compare data of previous test results given in Table 1.1 with our data given in Table 1.3 and Figure 1.3.) The disparity of the compression modulus at room temperature is more than $50 \%$.

There is reasonable agreement, also, between the transverse $\left(90^{\circ}\right)$ tensile modulus and inplane shear modulus $\left( \pm 45^{\circ}\right)$ data from past test programs and the current one for epoxy-based composites. However, the strength data in these orientations $\left(90^{\circ}, \pm 45^{\circ}\right)$ do not agree well with previous data. The transverse $\left(90^{\circ}\right)$ tensile and compression strength, as well as the inplane $\left( \pm 45^{\circ}\right)$ shear strength, which were determined in this test program, are all considerably lower ( $40 \%$ or more) than those reported in Table 1.1. Indeed, the strength of our epoxy-based specimens in these orientations is lower than that of the neat resins. There are three possible reasons for these large disparities: (1) the high porosity ( 8 to $9 \%$ ) of our composite specimens led to premature failures at the fiber-matrix interface or within the resin system; (2) the high fiber volume fraction led to regions of lack of adhesion at fiber-matrix interfaces; (3) the effective specimen cross-sectional areas were too small and served to emphasize the composite inconsistencies noted in items (1) and (2).

\subsubsection{Cyanate-Ester-Resin-Matrix Composites}

We are not aware of previous tests of E-glass-cyanate-ester unidirectional fibers at low temperatures. The cyanate-ester data compare very favorably with the epoxy results. The elastic moduli for the cyanate-ester composite in tension and compression are about $25 \%$ higher than the moduli of the epoxy-based composite. This suggests the possibility of either better aligned, higher density fibers or much lower porosity for the cyanate-ester composites. Of the three possibilities, only the latter was substantiated by our characterization program.

The tensile strengths of the cyanate-ester composite are about $40 \%$ higher than those of the epoxy composite at all test temperatures. However, at room temperature, the compressive strengths of the two resin systems are much closer (that of the cyanate-ester composite is about $20 \%$ higher). At lower temperatures, the compressive strength of the cyanate-ester composite increases more than that of the epoxy system.

The in-plane shear modulus of the cyanate-ester composite is larger at room temperature than that of the epoxy composite, but at lower temperatures, the epoxy shear modulus increases much more, and at $4 \mathrm{~K}$, is about $15 \%$ higher. This temperature dependence reflects the relative temperature dependence of the elastic modulus of the neat resins. Similarly, the temperature dependence of the epoxy composites $\left(90^{\circ}\right.$ fiber orientation) in tension is greater than that of the cyanate-ester composites for the same conditions.

At all temperatures, the in-plane shear strengths of the cyanate-ester composites are larger than the shear strengths of the epoxy composites. Nevertheless, the dependence of the in-plane shear strength on temperature is much less for the cyanate-ester composite; this system has an increase of only about $15 \%$ from 295 to $4 \mathrm{~K}$, whereas the shear strength of the epoxy system nearly doubles from 295 to $4 \mathrm{~K}$. 
The percent increases in average strength and elastic moduli from 295 to $4 \mathrm{~K}$ are summarized in Table 1.9. The two data sets are very similar. Those properties that are not very dependent on temperature, and thus not strongly influenced by the base resin, are (1) tensile strength in the $0^{\circ}$ fiber orientation and (2) tensile and compressive elastic moduli in the $0^{\circ}$ fiber orientation.

All the properties of specimens with $90^{\circ}$ and $\pm 45^{\circ}$ orientation plus the compressive strength of specimens with $0^{\circ}$ fiber orientation are strongly dependent on test temperature and, concurrently, on the base resin. One anomalous result is the temperature dependence of the shear strength for the specimens with $\pm 45^{\circ}$ fiber orientation; the $15 \%$ increase appears too low in comparison to the other data of Table 1.9

In general, the properties of epoxy-base composites are more strongly dependent on temperature than are the properties of cyanate-ester-base composites.

Thus, in general, the absolute strengths and elastic moduli of the unidirectional composites with the same fiber reinforcement (E-glass) and volume percent $(\sim 75)$ do not correlate with either the shear or tensile strengths of the base-resin system. However, the relative temperature dependence of the moduli and strengths have the same trends as the neat resins. The disparity of the elastic moduli and strengths between the two resin systems should provide excellent information for analyses of the properties of filament-wound struts with similar fiber orientations but different resin systems.

Table 1.9. Percent increase in average property data from 295 to $4 \mathrm{~K}$.

\begin{tabular}{llrrr}
\hline \hline \multirow{2}{*}{ Resin System } & Property & \multicolumn{3}{c}{ Fiber Orientation } \\
& & $0^{\circ}$ & $90^{\circ}$ & $\pm 45^{\circ}$ \\
\hline \hline \multirow{2}{*}{ Epoxy } & tensile strength & 14 & 47 & - \\
& compressive strength & 87 & 248 & - \\
& shear strength & - & - & 84 \\
& tensile elastic modulus & 12 & 90 & - \\
& compressive elastic modulus & 8 & $($ a) & - \\
& shear modulus & - & - & 74 \\
& & & & \\
Cyanate Ester & tensile strength & 15 & 51 & - \\
& compressive strength & 107 & 105 & - \\
& shear strength & - & - & 15 \\
& tensile elastic modulus & 5 & 26 & - \\
& compressive elastic modulus & $(\mathrm{a})$ & 48 & - \\
& shear modulus & - & - & 74 \\
\hline \hline
\end{tabular}

(a) Data not available for comparison 


\title{
2. Thermal Contraction
}

\author{
R.P. Reed* \\ R.P. Walsh and M.W. Austin \\ Materials Reliability Division \\ National Institute of Standards and Technology \\ Boulder, Colorado \\ ${ }^{*}$ Cryogenic Materials, Inc. \\ Boulder, Colorado
}

The thermal contraction of unidirectional E-glass filaments (71 to

$75 \mathrm{vol} \%$ ) in an epoxy matrix was measured in two ways: with strain gages and a quartz-tube dilatometer. From 295 to $4 \mathrm{~K}$, the measured contraction on cooling was $0.13 \%$ in the longitudinal $\left(0^{\circ}\right)$ fiber orientation and about $0.4 \%$ in the transverse $\left(90^{\circ}\right)$ fiber orientation.

\subsection{Introduction}

For E-glass uniaxial plies with the DGEBA/DCH/ARA/ACL epoxy-resin system, thermal contraction was measured from 295 to $4 \mathrm{~K}$ with both strain gages and a quartz-tube dilatometer. Specimens were oriented parallel to the fiber direction $\left(0^{\circ}\right)$ and transverse to the fiber direction $\left(90^{\circ}\right)$.

\subsection{Review of Literature}

The thermal contractions of unidirectional fiber-reinforced epoxy-base composites have been measured as low as $4 \mathrm{~K}$ only by Hartwig and Knaak [2.1], and they have been measured to $77 \mathrm{~K}$ by Takeno et al. [2.2]. Their data are summarized in Table 2.1. For comparison, contraction measurements on cloth-reinforced laminates by Hamelin [2.3] and Kasen et al. [2.4] are also included in the table. As expected, the laminate contractions from 295 to $77 \mathrm{~K}$ are less in the warp or $0^{\circ}$ fiber orientation than in the fill or $90^{\circ}$ fiber orientation. In each case, these differences are due to the higher fiber volume fractions of specimens in the direction parallel to the fibers. To the same degree, for specimens of both fiber orientations, the differences between the contraction data of Takeno et al. and those of Hartwig and Knaak are related to the differences in fiber volume fraction (Takeno et al. - 50 vol\%; Hartwig and Knaak - 70 vol\%). Hamelin's results were similar: His measurements of the contractions for specimens with $0^{\circ}$ and $90^{\circ}$ fiber orientations were larger for specimens with lower densities; the larger contractions can be attributed to lower volume fraction, since the density of epoxies is less than that of the glass fibers. 


\subsection{Materials}

The composite measured was an E-glass-reinforced, DGEBA/DCH/ARA/ACL-epoxy matrix composite, which has been described in $\S \mathrm{V} .1 .3$. Each ply of the composite was wetfilament-wound with unidirectional E-glass tows, taken off the mandrel and cut, and then carefully laid, keeping all filaments parallel, upon the previous ply to form a plate.

\subsection{Procedures}

For each specimen, two strain gages were used on a composite plate with unidirectional fibers. One was oriented to measure strain in the direction parallel to the fibers $\left(0^{\circ}\right)$; the other was oriented to measure strain in the direction transverse to the fibers $\left(90^{\circ}\right)$. The procedures for strain-gage measurement have been extensively discussed in \$III.6. The data reported were obtained during warm-up from 4 to $295 \mathrm{~K}$.

Table 2.1. Thermal contraction of unidirectional fiber- and cloth-reinforced epoxy-base composites.

\begin{tabular}{|c|c|c|c|c|}
\hline \multirow{2}{*}{ Material } & \multirow{2}{*}{$\begin{array}{l}\text { Specimen } \\
\text { Orientation } \\
\text { (density) }\end{array}$} & \multicolumn{2}{|c|}{$\frac{\mathrm{L}_{295 \mathrm{~K}}-\mathrm{L}_{T}}{\mathrm{~L}_{295 \mathrm{~K}}} \times 100$} & \multirow[t]{2}{*}{ Source } \\
\hline & & $T=4 \mathrm{~K}$ & $T=77 \mathrm{~K}$ & \\
\hline \multirow{2}{*}{$\begin{array}{l}\text { Unidirectional fibers, } \\
\text { pultruded; } \\
\text { E-glass-Araldite } \\
\text { epoxy; } \\
-50 \text { vol\% fiber }\end{array}$} & $0^{\circ}$ & - & 0.14 & \multirow{2}{*}{$\begin{array}{l}\text { Takeno et al } \\
{[2.2]}\end{array}$} \\
\hline & $90^{\circ}$ & - & 0.57 & \\
\hline \multirow{2}{*}{$\begin{array}{l}\text { Unidirectional fibers, } \\
\text { E-glass; } \\
\text { DGEBA-base epoxy; } \\
70 \text { vol\% fiber }\end{array}$} & $0^{\circ}$ & 0.162 & 0.117 & \multirow{2}{*}{$\begin{array}{l}\text { Hartwig and } \\
\text { Knaak } \\
{[2.1]}\end{array}$} \\
\hline & $90^{\circ}$ & 0.283 & 0.333 & \\
\hline \multirow{4}{*}{$\begin{array}{l}\text { E-glass cloth, } \\
\text { laminated } \\
\text { epoxy matrix; } \\
\text { pressure varied }\end{array}$} & $0^{\circ}\left(\rho=2.15 \mathrm{~g} / \mathrm{cm}^{3}\right)$ & - & 0.15 & \multirow{4}{*}{$\begin{array}{l}\text { Hamelin } \\
{[2.3]}\end{array}$} \\
\hline & $0^{\circ}\left(\rho=1.80 \mathrm{~g} / \mathrm{cm}^{3}\right)$ & - & 0.25 & \\
\hline & $90^{\circ}\left(\rho=2.15 \mathrm{~g} / \mathrm{cm}^{3}\right)$ & - & 0.28 & \\
\hline & $90^{\circ}\left(\rho=1.80 \mathrm{~g} / \mathrm{cm}^{3}\right)$ & - & 0.71 & \\
\hline \multirow[t]{2}{*}{ G-10CR } & $0^{\circ}$ & 0.24 & 0.22 & \multirow{2}{*}{$\begin{array}{l}\text { Kasen et al. } \\
{[2.4]}\end{array}$} \\
\hline & $90^{\circ}$ & 0.71 & 0.64 & \\
\hline \multirow[t]{2}{*}{ G-11CR } & $0^{\circ}$ & 0.21 & 0.18 & \multirow{2}{*}{$\begin{array}{l}\text { Kasen et al. } \\
{[2.4]}\end{array}$} \\
\hline & $90^{\circ}$ & 0.61 & 0.54 & \\
\hline
\end{tabular}




\subsection{Results}

Using strain-gage sensors, we obtained the results shown in Figure 2.1. The axial $\left(0^{\circ}\right)$ contraction was about $0.13 \%$ on cooling from 295 to $4 \mathrm{~K}$. The contraction in the direction transverse $\left(90^{\circ}\right)$ to the unidirectional fibers over this temperature range was about $0.5 \%$.

The data from the quartz-tube dilatometer are shown in Figure 2.2. The axial $\left(0^{\circ}\right)$ specimen data, $0.13 \%$ contraction from 295 to $4 \mathrm{~K}$, agree almost exactly with the strain-gage data. There was a small difference between the transverse $\left(90^{\circ}\right)$ data of the two techniques. The dilatometer measurements indicated a contraction of about $0.41 \%$-lower than that indicated by the strain gages. The reason for the difference is not clear. Essentially no bias was detected between the strain-gage and dilatometer measurements done on a neat-epoxy resin (see Figs. 6.3 and 6.4 in $\$ 3)$.

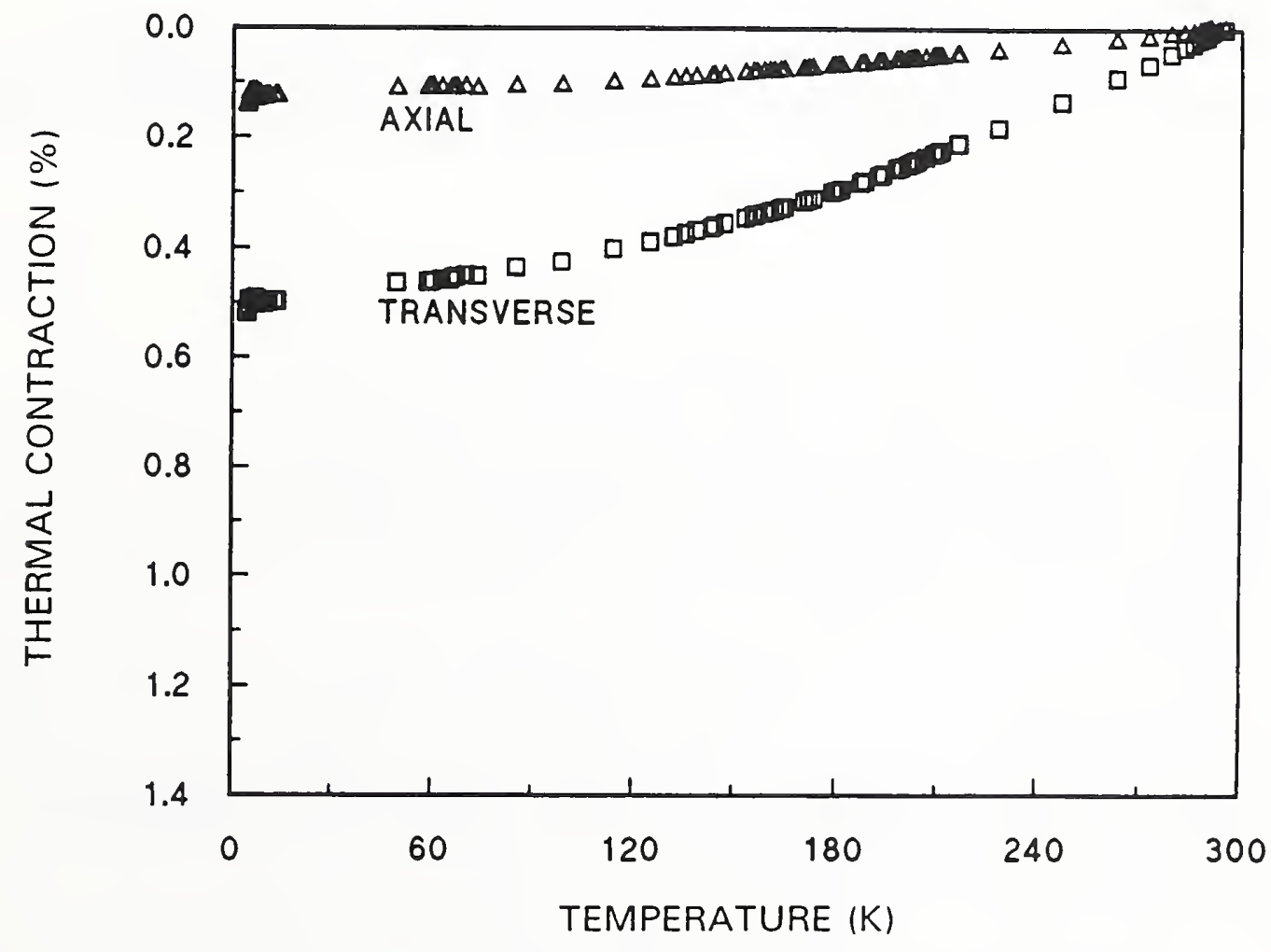

Figure 2.1. Thermal contraction of a uniaxial composite (E-GlassDGEBA/DCH/ARA/ACL) measured with strain gages. 


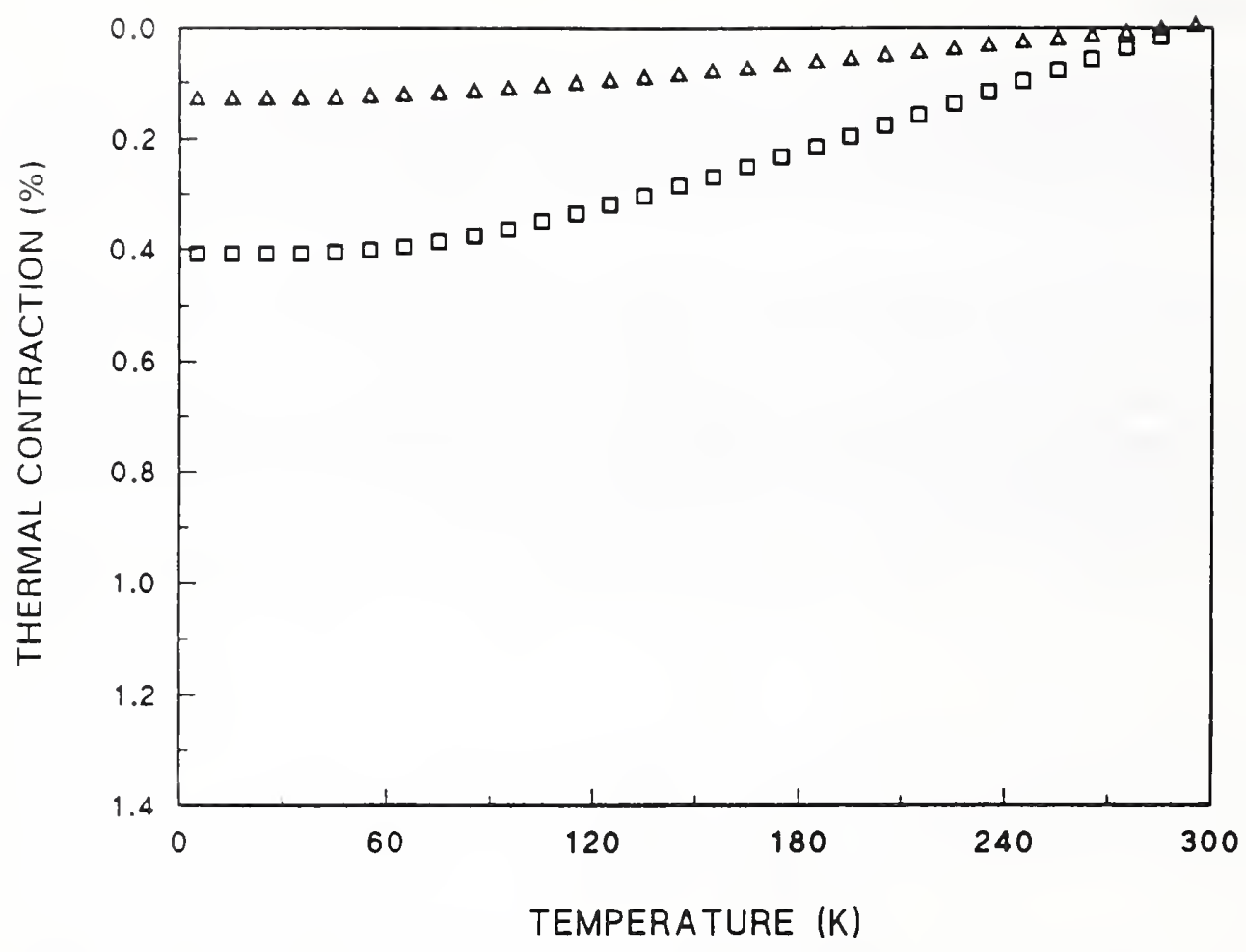

Figure 2.2. Thermal contraction of a uniaxial composite (E-Glass-DGEBA/ $\mathrm{DCH} / \mathrm{ARA} / \mathrm{ACL}$ ) measured with a quartz-tube dilatometer.

\subsection{Discussion}

The axial $\left(0^{\circ}\right)$ thermal contraction of $0.12 \%$ on cooling from 295 to $77 \mathrm{~K}$ agrees very well with the results reported by Hartwig and Knaak $(0.17 \%$ for 70 vol\% of fiber). We measured between 71 and 75 vol\% (see Table 2.1 of $\S \mathrm{V}$ ). However, our data are also comparable to the $0.14 \%$ contraction measured by Takeno et al. on a composite with a fiber volume fraction of only $50 \%$.

However, our measurements of $0.385 \%$ (dilatometer) to $0.45 \%$ (strain-gage) contraction in the transverse $\left(90^{\circ}\right)$ direction (see Figures 2.1 and 2.2 ) are higher than the $0.33 \%$ contraction measured by Hartwig and Knaak for a composite with similar fiber volume fraction on cooling from 295 to $77 \mathrm{~K}$. As one would expect from the differences in fiber volume fraction, the results of Takeno et al. ( $0.57 \%$ contraction) are higher. 


\title{
3. Thermal Conductivity
}

\author{
D.L. Rule \\ R.P. Reed ${ }^{*}$ \\ Materials Reliability \\ National Institute of Standards and Technology \\ Boulder, Colorado \\ ${ }^{*}$ Cryogenic Materials, Inc. \\ Boulder, Colorado
}

The thermal conductivity of one polymer composite was measured from 4.6 to $310 \mathrm{~K}$. The composite contained E-glass fibers in a DGEBA/DCH/ARA/ACL epoxy matrix. The conductivity of the composite material was determined with the heat flow was parallel and transverse to the fiber direction.

\subsection{Introduction}

The Superconducting Magnetic Energy Storage (SMES) system employs polymer composite tubes that must provide thermal isolation as well as mechanical support. The thermal conductivity of one E-glass polymer composite was determined parallel and transverse to the fibers over the temperature range 4.6 to $310 \mathrm{~K}$.

\subsection{Review of Literature}

The thermal conductivity of uniaxially oriented glass-fiber-epoxy-matrix composites has been measured at low temperatures [3.1-3.4]. The data between 4 and $295 \mathrm{~K}$ from these studies are summarized in Table 3.1. At $4 \mathrm{~K}$, the higher the glass volume fraction of the composite, the higher the thermal conductivity. Below $4 \mathrm{~K}$, however, Radcliffe and Rosenberg [3.2] reported a reversal of the trend for heat flow transverse to the fibers, and at $2 \mathrm{~K}$, the respective thermal conductivities were $0.020,0.025$, and 0.031 for 77,46 , and 33 vol\%. At all temperatures, specimens parallel to the fiber direction had higher thermal conductivities than those transverse to the fiber direction. In general, the data were quite similar and showed little scatter.

Radcliffe and Rosenberg [3.2] measured the thermal conductivity of both a neat-resin epoxy and a bundle of 20,000 glass fibers. For the glass, they found a dependence on temperature of about $T^{1.8}$ at very low temperatures, a plateau region from 8 to $20 \mathrm{~K}$, and thereafter a steadily increasing thermal conductivity with increasing temperature. At $100 \mathrm{~K}$, they found that the thermal conductivity of the glass fiber was approximately 4 times higher than that of the epoxy. At $2 \mathrm{~K}$, the thermal conductivities of both the glass fiber and epoxy were the same-about $0.037 \mathrm{~W} /(\mathrm{m} \cdot \mathrm{K})$. 
Treatment of the thermal conductivity of fiber-reinforced composites at very low temperatures on the basis of component properties has been discussed by Klemens [3.5] and Hartwig and Knaak [3.6].

The differences in thermal conductivity between the $0^{\circ}$ - and $90^{\circ}$ - or $\mathrm{N}$-oriented specimens at all temperatures were not very large. Considering the large differences between glassfiber and epoxy thermal conductivities at temperatures above $4 \mathrm{~K}$ found by Radcliffe and Rosenberg, the similarity of the uniaxial thermal conductivity for various specimens and fiber orientations is surprising.

Table 3.1. Thermal conductivity of directionally reinforced composites.

\begin{tabular}{|c|c|c|c|c|c|c|c|c|}
\hline \multirow{2}{*}{$\begin{array}{l}\text { Composite } \\
\text { (vol\% fiber) }\end{array}$} & \multirow{2}{*}{$\begin{array}{l}\text { Orienta- } \\
\text { tion }^{*}\end{array}$} & \multicolumn{6}{|c|}{ Thermal Conductivity, $\lambda[\mathrm{W} /(\mathrm{m} \cdot \mathrm{K})]$} & \multirow{2}{*}{$\begin{array}{c}\text { Source } \\
\text { [ref.] }\end{array}$} \\
\hline & & $4 \mathrm{~K}$ & $10 \mathrm{~K}$ & $50 \mathrm{~K}$ & $100 \mathrm{~K}$ & $200 \mathrm{~K}$ & $295 \mathrm{~K}$ & \\
\hline $\begin{array}{l}\text { E-glass-epoxy } \\
(50)\end{array}$ & $\begin{array}{r}0^{\circ} \\
90^{\circ} \\
\mathrm{N}\end{array}$ & $\begin{array}{l}0.054 \\
0.050 \\
0.045\end{array}$ & $\begin{array}{l}0.092 \\
0.085 \\
0.08\end{array}$ & $\begin{array}{l}0.22 \\
0.20 \\
0.18\end{array}$ & $\begin{array}{l}- \\
- \\
-\end{array}$ & $\begin{array}{l}- \\
- \\
-\end{array}$ & $\begin{array}{l}- \\
- \\
-\end{array}$ & $\begin{array}{l}\text { Dmitrevsky et } \\
\text { al. [3.1] }\end{array}$ \\
\hline $\begin{array}{l}\text { E-glass-epoxy } \\
(70) \\
(45) \\
(35)\end{array}$ & $\begin{array}{l}0^{\circ} \\
0^{\circ} \\
0^{\circ}\end{array}$ & $\begin{array}{l}0.087 \\
0.070 \\
0.063\end{array}$ & $\begin{array}{l}0.16 \\
0.12 \\
0.10\end{array}$ & $\begin{array}{l}0.36 \\
0.28 \\
0.24\end{array}$ & $\begin{array}{l}- \\
- \\
-\end{array}$ & $\begin{array}{l}- \\
- \\
-\end{array}$ & $\begin{array}{l}- \\
-\end{array}$ & $\begin{array}{l}\text { Radcliffe, } \\
\text { Rosenberg } \\
{[3.2]}\end{array}$ \\
\hline $\begin{array}{l}(77) \\
(46) \\
(33)\end{array}$ & $\begin{array}{l}90^{\circ} \\
90^{\circ} \\
90^{\circ}\end{array}$ & $\begin{array}{l}0.056 \\
0.051 \\
0.050\end{array}$ & $\begin{array}{l}0.12 \\
0.09 \\
0.08\end{array}$ & $\begin{array}{l}0.33 \\
0.22 \\
0.20\end{array}$ & $\begin{array}{l}- \\
-\end{array}$ & $\begin{array}{l}- \\
-\end{array}$ & $\begin{array}{l}- \\
-\end{array}$ & \\
\hline $\begin{array}{l}\text { E-glass-epoxy } \\
(-)\end{array}$ & $\begin{array}{l}0^{\circ} \\
\mathrm{N}\end{array}$ & $\begin{array}{l}0.10 \\
0.09\end{array}$ & $\begin{array}{l}0.11 \\
0.11\end{array}$ & $\begin{array}{l}0.22 \\
0.20\end{array}$ & $\begin{array}{l}0.37 \\
0.35\end{array}$ & $\begin{array}{l}0.54 \\
0.50\end{array}$ & $\begin{array}{l}0.65 \\
0.60\end{array}$ & $\begin{array}{l}\text { Takeno et al. } \\
{[3.3]}\end{array}$ \\
\hline $\begin{array}{l}\text { E-glass-epoxy } \\
(-)\end{array}$ & $0^{\circ}$ & 0.08 & 0.10 & 0.2 & 0.3 & - & 0.6 & $\begin{array}{l}\text { Evans, Morgan } \\
{[3.4]}\end{array}$ \\
\hline
\end{tabular}

\subsection{Procedures}

Two composite specimens were sectioned from a 3.17-mm (0.125-in) thick filamentwound plate of E-glass fibers in an epoxy matrix. The DGEBA/DCH/ARA/ACL resin system was described in §III.3. In this section, the resin system is labeled "B" for brevity. Porosity ranged from 8 to $9 \%$ in these plates (see $§ I V .1$ ). The manufacturer of the plate reported that the fiber volume fraction was $64 \%$.

The specimens described above were fabricated by cutting several small sections from the manufactured plate, cementing the sections together with a very thin layer of cyanoacrylate 
adhesive, and then cutting the specimens with a diamond saw to the sizes listed in Table 3.2. All dimensions, an average of eight measurements, are accurate within $\pm 12 \mu \mathrm{m}( \pm 0.0005$ in). The heat flow was directed parallel to the E-glass fibers in one specimen and transverse in the other specimen.

The procedure used to measure thermal conductivity of these composite specimens is identical to the procedure used to measure the thermal conductivity of neat resins (described in §III.7.4).

Table 3.2. Description of E-glass-Resin B composite specimens.

\begin{tabular}{lccl}
\hline Orientation & $\begin{array}{c}\text { Length } \\
(\mathrm{cm})\end{array}$ & $\begin{array}{c}\text { Area } \\
\left(\mathrm{cm}^{2}\right)\end{array}$ & Heat Flow \\
\hline Parallel to fibers $\left(0^{\circ}\right)$ & 6.34 & 160.0 & in direction of fibers \\
Transverse to fibers $\left(90^{\circ}\right)$ & 6.34 & 156.3 & across direction of fibers \\
\hline
\end{tabular}

\subsection{Results}

The thermal conductivities of a E-glass-Resin B composite were measured over the temperature range 4.6 to $310 \mathrm{~K}$ and in two directions: parallel $\left(0^{\circ}\right)$ and transverse $\left(90^{\circ}\right)$ to the filaments. The experimental data obtained for both directions are listed in Tables 3.3 and 3.4. The function that best fit the thermal conductivity data for the two directions is

$$
\lambda(T)=\sum_{i=1}^{6} a_{i}[\ln (T)]^{i-1} .
$$

The resulting values of $a_{i}$ for the two specimen orientations are listed in Table 3.3.

Table 3.3. Coefficients of $\lambda(T)$ in Equation 3.1.

\begin{tabular}{ccc}
\hline \hline \multirow{2}{*}{$a_{i}$} & \multicolumn{2}{c}{$\begin{array}{c}\text { Specimen Orientation with } \\
\text { Respect to Fibers }\end{array}$} \\
\cline { 2 - 3 } & Parallel $\left(0^{\circ}\right)$ & Transverse $\left(90^{\circ}\right)$ \\
\hline \hline 1 & 0.05846 & -0.03702 \\
2 & -0.20281 & -0.01492 \\
3 & 0.31556 & 0.13388 \\
4 & -0.15137 & -0.07778 \\
5 & 0.03088 & 0.01760 \\
6 & 0.00217 & -0.00132 \\
\hline \hline
\end{tabular}


The thermal conductivity data (Tables 3.4 and 3.5) and the relative (percent) deviation of the data points from the appropriate fitted curve are shown in Figures 3.1 and 3.2 for specimens with heat flow parallel to the fibers and in Figures 3.3 and 3.4 for specimens with heat flow transverse to the fibers. The thermal conductivities for both types of specimens and for the neat resin B are shown in Figures 3.5 and 3.6.

Table 3.4. Experimental thermal-conductivity data as a function of temperature for heat flow parallel to the fiber direction.

\begin{tabular}{|c|c|c|c|}
\hline $\begin{array}{c}\text { Temperature } \\
\text { Difference } \\
(\mathrm{K}) \\
\end{array}$ & $\begin{array}{c}\text { Average } \\
\text { Temperature } \\
(\mathrm{K}) \\
\end{array}$ & $\begin{array}{c}\text { Thermal } \\
\text { Conductivity } \\
{[\mathrm{W} /(\mathrm{m} \cdot \mathrm{K})]}\end{array}$ & Cold Bath \\
\hline 1 & 4.652 & 0.097 & LH \\
\hline 2 & 5.190 & 0.104 & \\
\hline 4 & 6.288 & 0.119 & \\
\hline 8 & 8.511 & 0.138 & \\
\hline 16 & 12.995 & 0.156 & \\
\hline 24 & 17.510 & 0.169 & \\
\hline 32 & 22.069 & 0.182 & \\
\hline 62 & 39.970 & 0.248 & \\
\hline 1 & 76.556 & 0.372 & $\mathrm{LN}_{2}$ \\
\hline 2 & 77.051 & 0.367 & \\
\hline 2 & 77.057 & 0.369 & \\
\hline 4 & 78.133 & 0.372 & \\
\hline 8 & 80.258 & 0.377 & \\
\hline 16 & 84.605 & 0.391 & \\
\hline 24 & 88.836 & 0.402 & \\
\hline 32 & 93.150 & 0.413 & \\
\hline 48 & 101.83 & 0.435 & \\
\hline 64 & 110.57 & 0.455 & \\
\hline 72 & 115.08 & 0.466 & \\
\hline 1 & 192.55 & 0.656 & $\mathrm{CO}_{2}$-alcohol \\
\hline 2 & 193.17 & 0.630 & \\
\hline 4 & 194.34 & 0.622 & \\
\hline 8 & 196.59 & 0.621 & \\
\hline 16 & 201.16 & 0.625 & \\
\hline 32 & 210.40 & 0.623 & \\
\hline 48 & 219.75 & 0.626 & \\
\hline 64 & 229.16 & 0.630 & \\
\hline 1 & 273.72 & 0.665 & Ice-water \\
\hline 2 & 274.31 & 0.669 & \\
\hline 4 & 275.46 & 0.668 & \\
\hline 32 & 291.67 & 0.671 & \\
\hline 64 & 310.60 & 0.682 & \\
\hline
\end{tabular}




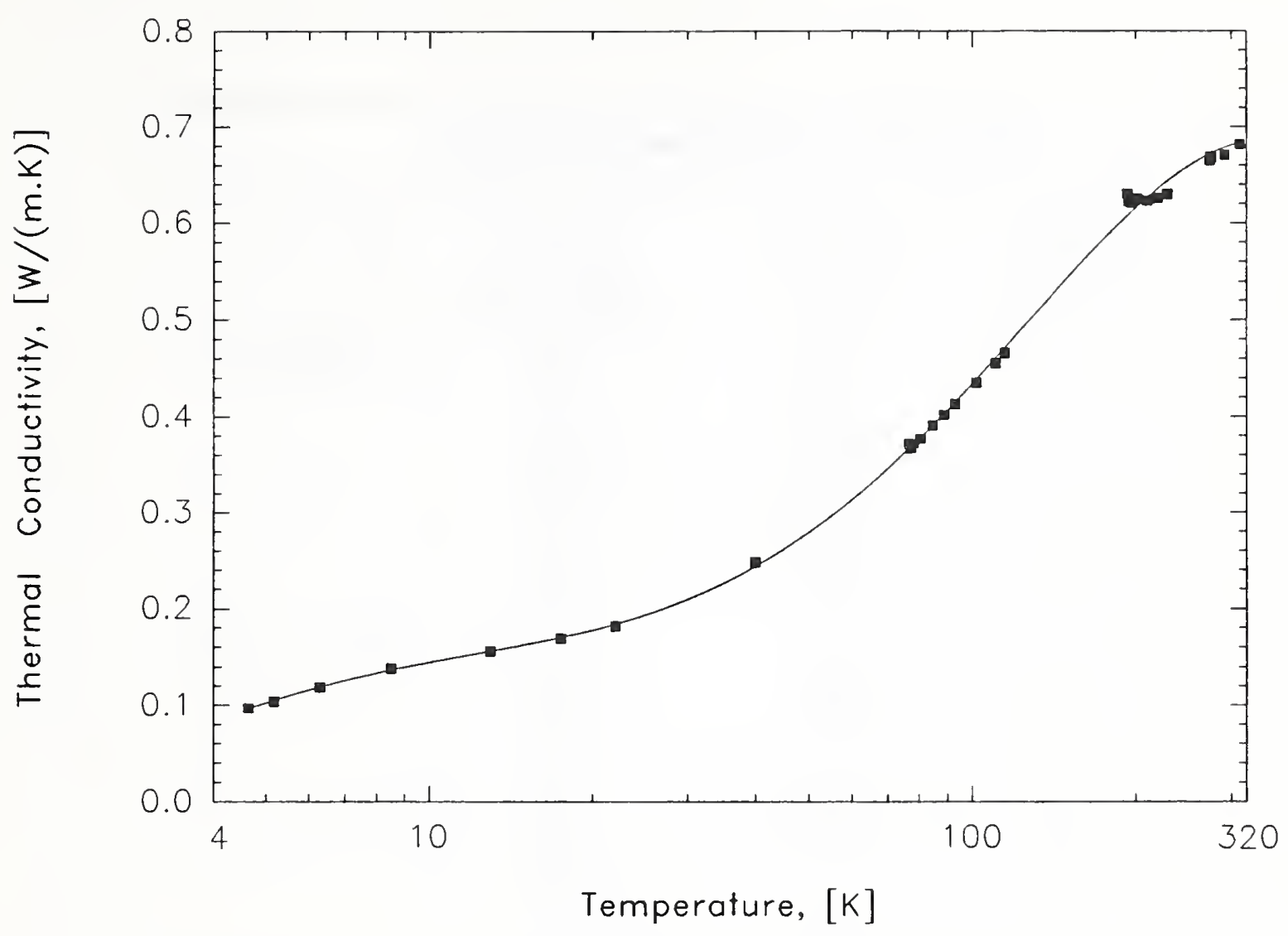

Figure 3.1. Thermal conductivity for heat tlow parallel to the fiber direction. Experimental data are presented as discrete points.

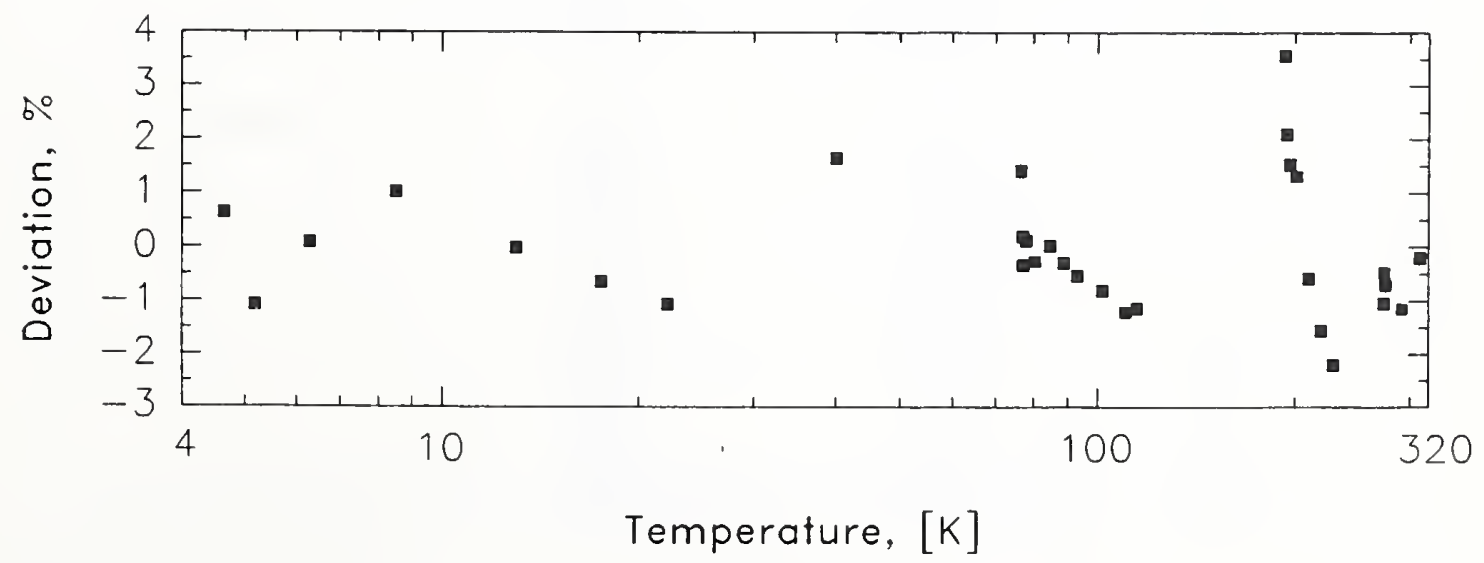

Figure 3.2. Relative deviations of the experimental and calculated thermal conductivity for an E-glass-Resin B specimen with a parallel orientation. 
Table 3.5. Experimental thermal-conductivity data as a function of temperature for heat flow transverse to the fiber direction.

\begin{tabular}{|c|c|c|c|}
\hline $\begin{array}{c}\text { Temperature } \\
\text { Difference } \\
\text { (K) }\end{array}$ & $\begin{array}{c}\text { Average } \\
\text { Temperature } \\
(\mathrm{K})\end{array}$ & $\begin{array}{c}\text { Thermal } \\
\text { Conductivity } \\
{[\mathrm{W} /(\mathrm{m} \cdot \mathrm{K})]}\end{array}$ & Cold Bath \\
\hline 1 & 4.634 & 0.062 & \multirow[t]{7}{*}{ liquid helium } \\
\hline 2 & 5.167 & 0.067 & \\
\hline 4 & 6.236 & 0.076 & \\
\hline 8 & 8.384 & 0.090 & \\
\hline 16 & 12.762 & 0.108 & \\
\hline 32 & 21.678 & 0.134 & \\
\hline 64 & 39.792 & 0.185 & \\
\hline 1 & 76.522 & 0.278 & \multirow[t]{8}{*}{ liquid nitrogen } \\
\hline 2 & 77.031 & 0.269 & \\
\hline 4 & 78.111 & 0.270 & \\
\hline 8 & 80.203 & 0.274 & \\
\hline 16 & 84.460 & 0.281 & \\
\hline 32 & 92.890 & 0.295 & \\
\hline 48 & 101.41 & 0.308 & \\
\hline 64 & 109.99 & 0.326 & \\
\hline 1 & 192.55 & 0.428 & \multirow[t]{7}{*}{$\mathrm{CO}_{2}$-alcohol } \\
\hline 2 & 193.10 & 0.425 & \\
\hline 4 & 194.24 & 0.429 & \\
\hline 8 & 196.48 & 0.431 & \\
\hline 16 & 200.91 & 0.429 & \\
\hline 32 & 209.88 & 0.425 & \\
\hline 64 & 228.06 & 0.421 & \\
\hline 1 & 273.71 & 0.438 & \multirow[t]{9}{*}{ ice-water } \\
\hline 2 & 274.27 & 0.436 & \\
\hline 4 & 275.38 & 0.435 & \\
\hline 8 & 277.63 & 0.432 & \\
\hline 8 & 277.63 & 0.433 & \\
\hline 8 & 277.64 & 0.433 & \\
\hline 16 & 282.13 & 0.430 & \\
\hline 32 & 291.20 & 0.431 & \\
\hline 64 & 309.63 & 0.434 & \\
\hline
\end{tabular}




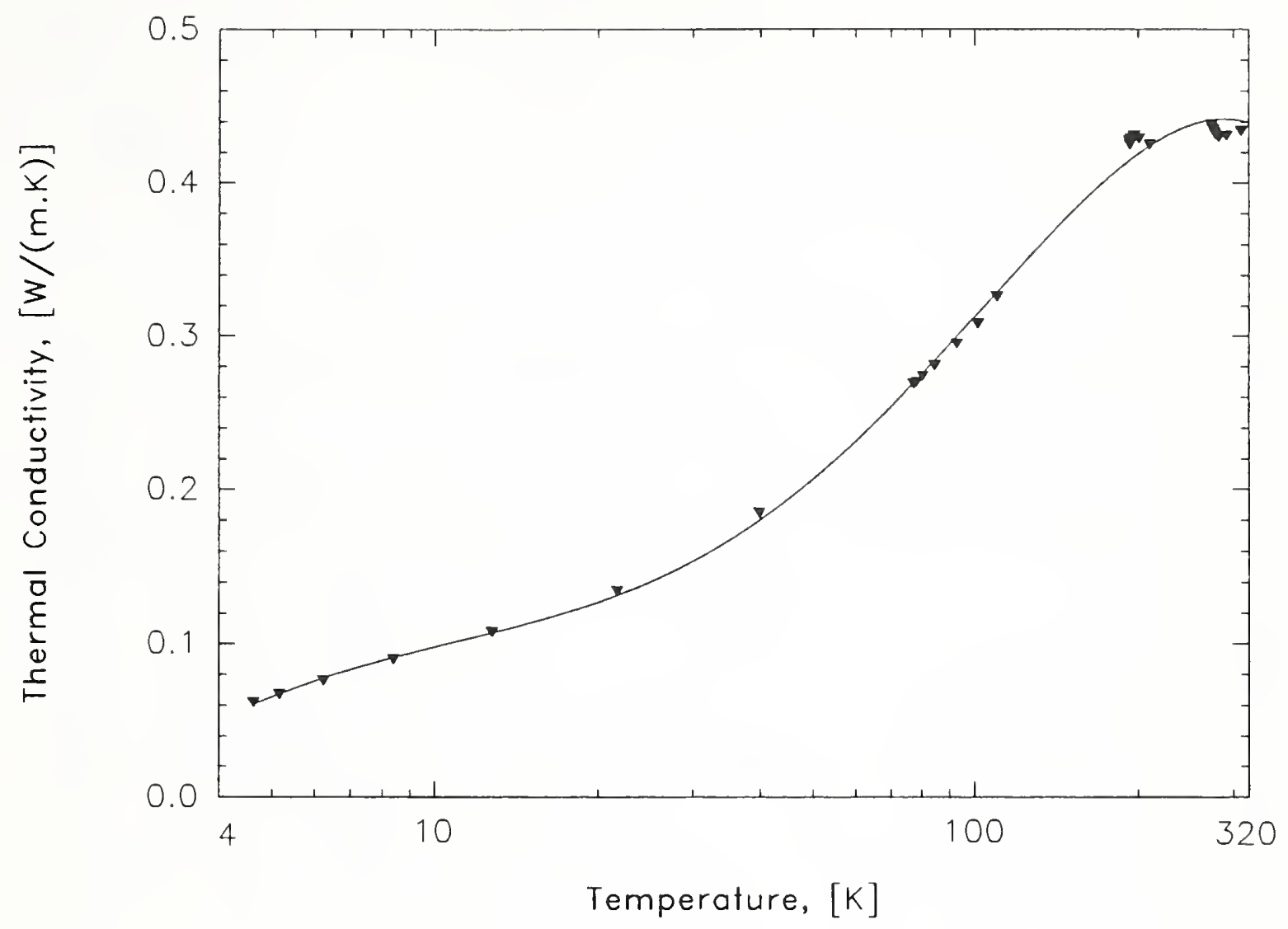

Figure 3.3. Thermal conductivity for heat flow transverse to the fiber direction. Experimental data are presented as discrete points.

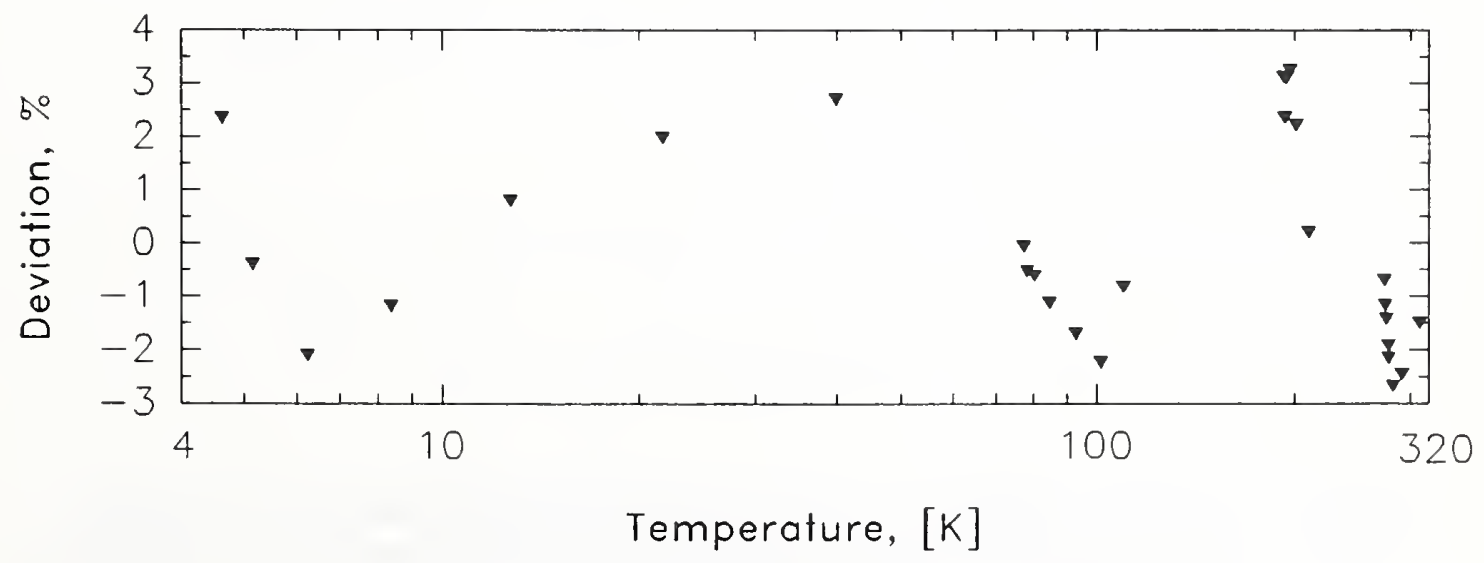

Figure 3.4. Relative deviations of the experimental and calculated thermal conductivity for the E-glass-Resin B specimen with a transverse orientation. 


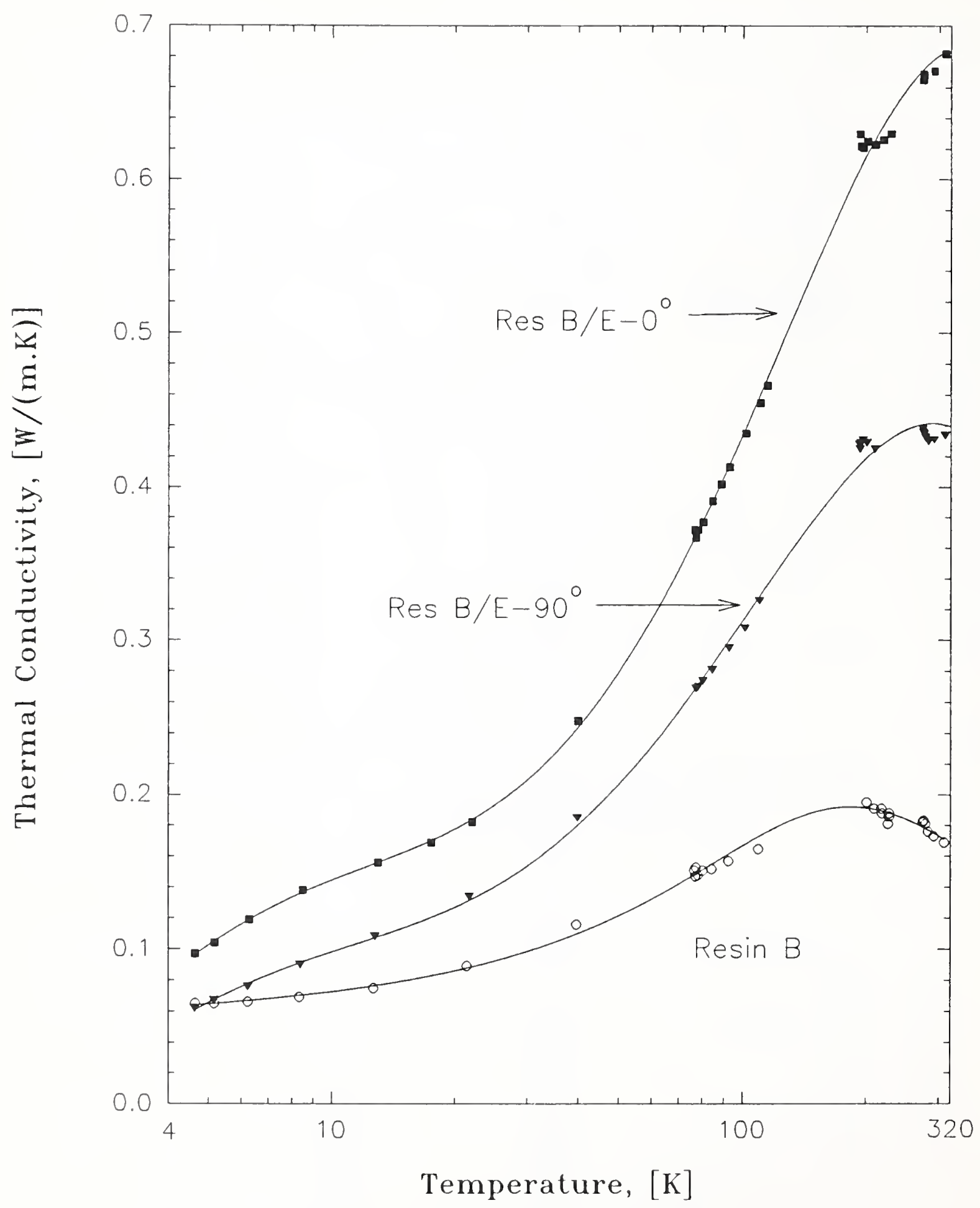

Figure 3.5. Thermal conductivity of the composite specimens measured in this study. The data of the neat resin B are also included as a reference. Experimental data are presented as discrete points. The temperature scale is logarithmic, which clarifies the behavior at low temperatures. 


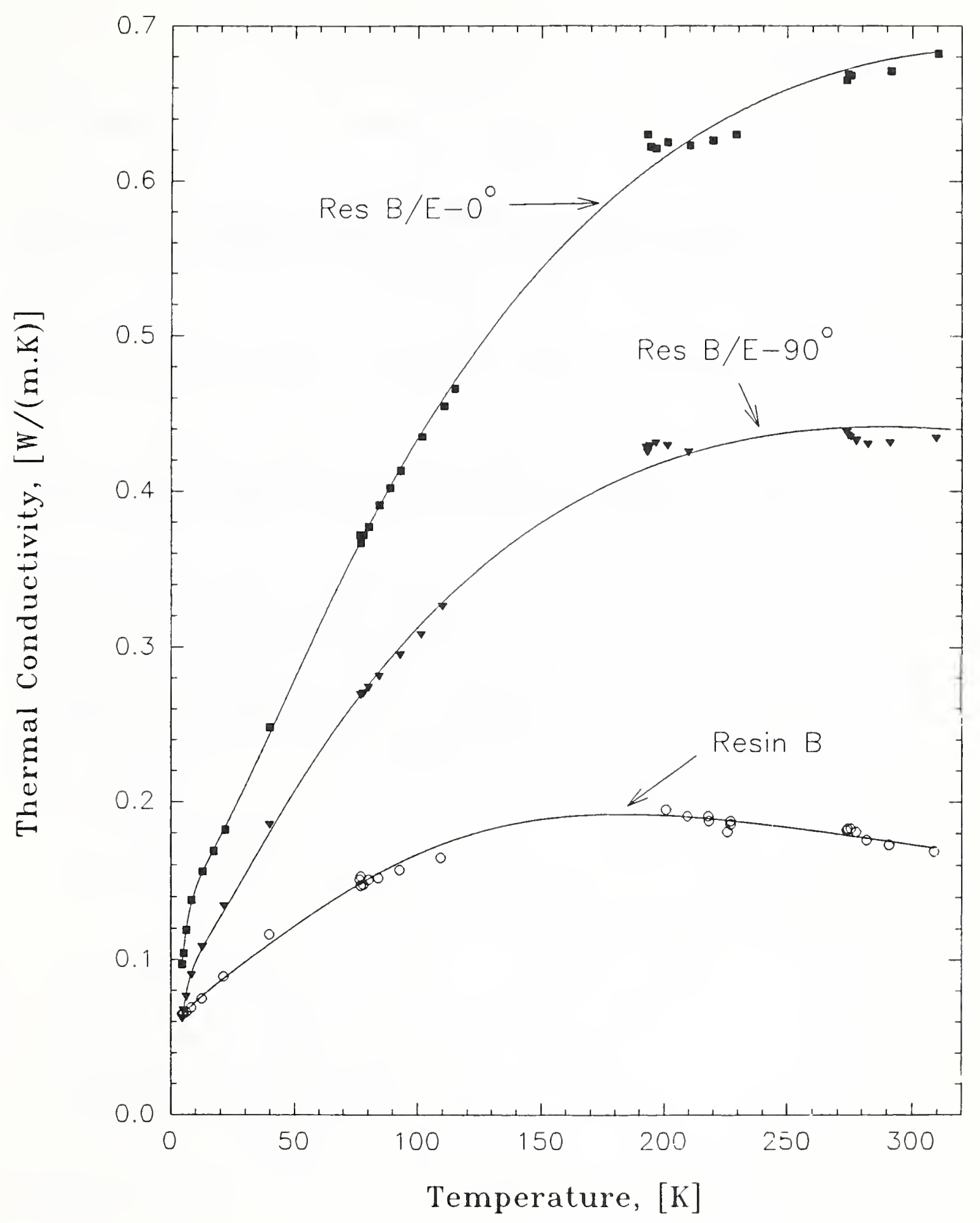

Figure 3.6. Thermal conductivity of the composite specimens measured in this study. The data of the neat resin B are also included as a reference. Experimental data are presented as discrete points. The temperature scale is linear, which clarifies the behavior at high temperatures. 


\subsection{Discussion}

The following discussion compares previous measurements with the results presented in Figures 3.5 and 3.6.

1. The thermal conductivity at $4 \mathrm{~K}$ for the specimens with heat flow transverse to the fibers was about $65 \%$ that of the specimens with heat flow parallel to the fibers. Other measurements indicated little difference in the thermal conductivities of the two orientations at $4 \mathrm{~K}$ [3.1-3.4].

2. At temperatures above $50 \mathrm{~K}$, the thermal conductivity of the transverse-oriented specimens was considerably less than that reported by Takeno et al. [3.3]. Our value of thermal conductivity at $295 \mathrm{~K}$ was about $0.44 \mathrm{~W} /(\mathrm{m} \cdot \mathrm{K})$, and the value found by Takeno et al. was about $0.60 \mathrm{~W} /(\mathrm{m} \cdot \mathrm{K})$.

3. Thus, our data indicate a much larger disparity between orientations than those found by Takeno et al. [3.3] or by Dmitrevsky et al. [3.1] at higher temperatures and Radcliffe and Rosenberg [3.2] at temperatures from 4 to $80 \mathrm{~K}$.

4. The thermal conductivity of our parallel-oriented specimens, $\sim 0.10 \mathrm{~W} /(\mathrm{m} \cdot \mathrm{K})$, agrees quite well with that of fiberglass bundles as measured by Radcliffe and Rosenberg. At temperatures from 4 to $100 \mathrm{~K}$, the thermal conductivity of the fiberglass bundles increased more rapidly than that of our parallel-oriented specimens, and at $100 \mathrm{~K}$, it was about $0.65 \mathrm{~W} /(\mathrm{m} \cdot \mathrm{K})$, whereas the thermal conductivity of our specimens was about $0.43 \mathrm{~W} /$ $(\mathrm{m} \cdot \mathrm{K})$. This difference is expected, since the thermal conductivity of the resin increases more gradually and is much less dependent on temperature. 


\title{
4. Interfacial Residual Thermal Stress
}

\author{
M. B . Kasen ${ }^{*}$ \\ R. L. Santoyo \\ Materials Reliability Division \\ National Institute of Standards and Technology \\ Boulder, Colorado \\ ${ }^{*}$ Consultant \\ Boulder, Colorado
}

A method was developed for assessing the influence of polymer chemical composition and of processing parameters on the magnitude of residual stress developed in glass-fiber-reinforced composites subjected to various cure cycles and subsequently cooled to cryogenic temperatures. The basic approach was to measure the strain induced on a glass substrate by a resin cured in contact with the substrate and during subsequent cooling to $77 \mathrm{~K}$.

Initial studies showed that curing a simple resin-glass combination produced strain levels high enough to fracture either the cured resin, the glass substrate, or both, thus negating the value of the tests. This problem was rectified by reinforcing the resin with woven fiberglass cloth. The final configuration consisted of an axially symmetric tubular specimen in which a strain-gaged glass rod was held at the centerline by an overwrap of glass cloth which was, in turn, confined within an external aluminum tube. The polymer resin was introduced by vacuum impregnation. The output of the strain gages on several specimens run simultaneously were monitored during the resin-cure cycle and upon cooling to $77 \mathrm{~K}$.

The test method was applied to a study of nine resin types including, epoxy, vinyl-ester, polyester, cyanate-ester, and phenolic formulations. Results showed that the method was capable of differentiating between strains induced by each resin type. However, the data suggest the possibility that the results obtained with certain resin types may be somewhat influenced by interaction with the mold used to contain the resin. The data also suggest that the polyester resin develops substantially less overall residual strain than do the other resin systems.

\subsection{Introduction}

A major factor controlling the ultimate strength of a fiber-reinforced composite at cryogenic temperatures is the magnitude of the residual stress existing in the composite at that temperature. High residual stress can preload the structure to the extent that failure occurs at a substantial reduction in externally applied stress. Residual stresses also reduce the fatigue life of the composite. 
Residual stresses develop due to differences in thermally induced contraction between the reinforcing fiber and the polymer matrix. For a given type of reinforcing fiber, the magnitude of the residual stress at cryogenic temperatures is determined by strain created by (a) contraction of the resin during the cure process and (b) thermal contraction of the cured resin during cooling to cryogenic temperatures. The latter of these can be determined by conventional techniques; however, little effort has been made to develop methods for assessing residual stress arising from strains induced during the cure process or to determine the influence of resin chemical composition or processing parameters on the level of residual stress.

The objective of this study was to develop a method for assessing the magnitude of residual strain arising from each of these sources and to apply the method in a comparative evaluation of a series of candidate polymer resin systems for cryogenic composite applications.

\subsection{Specimen and Test-Method Development}

Studies of laminate residual strains induced during the cure process have been conducted by means of embedded strain gages [4.1] and by development of warpage in cross-ply laminates [4.2]. The primary objective of these studies was to determine the influence of ply-stackingsequence variations on residual stress and strain. These techniques were judged inappropriate for the present work because they assess the global strain in the interior of a laminated composite, whereas we were interested in assessing the magnitude of strain developed at a glass-resin interface as a function of resin type. Our interest was confined to resins adaptable for vacuumimpregnation fabrication methods.

Our basic approach was to monitor the strain developed at a bulk glass-resin interface by means of a strain gage attached to the glass surface. We elected to use bulk glass so as to provide a substrate that applied the strain uniformly over the gage. This approach enables a quantitative ranking of interfacial strains developed by various resin systems. It does not necessarily provide data on the magnitude of strains developed in a real composite system, but it does produce data proportional to the magnitude of such strains.

We initially examined the possibility of working with an instrumented glass microscope slide immersed in pure resin. However, we found that the magnitude of strain developed during the cure cycle was sufficiently high to cause fracture of the resin, the glass substrate, or both, thus negating the value of the test. Variations in geometry of the test specimens failed to alleviate this problem.

We therefore investigated several specimen configurations in which a reinforcement was added to the resin. The most satisfactory configuration appeared to be the that shown in Figure 4.1. Here, the glass substrate was a hollow tube, $3.82-\mathrm{mm}$ i.d. and $6.36-\mathrm{mm}$ o.d. that was slightly bead-blasted and cleaned prior to bonding a single Karma-alloy strain gage to its surface about $12.7 \mathrm{~mm}(0.05 \mathrm{in})$ from the tube bottom. A type-E thermocouple was placed inside the tube directly behind the gage and held in place by sealing both ends of the tube with fiveminute epoxy. The tube was wrapped with a coarse-weave fiberglass cloth and inserted into an outer mold made from standard aluminum tubing, 63.5-mm long, 12.70-mm i.d., and 15.87mm o.d. A coating of sealer plus mold release baked at $120^{\circ} \mathrm{C}$ was applied to the aluminum mold to minimize adhesion of the resin. The fiberglass cloth was an uncharacterized material sold locally for general use. Wrapping with a 43-cm (17-in) long, 5.35-cm (2.5-in) wide section of this cloth yielded a fiber volume fraction of about $42 \%$. 


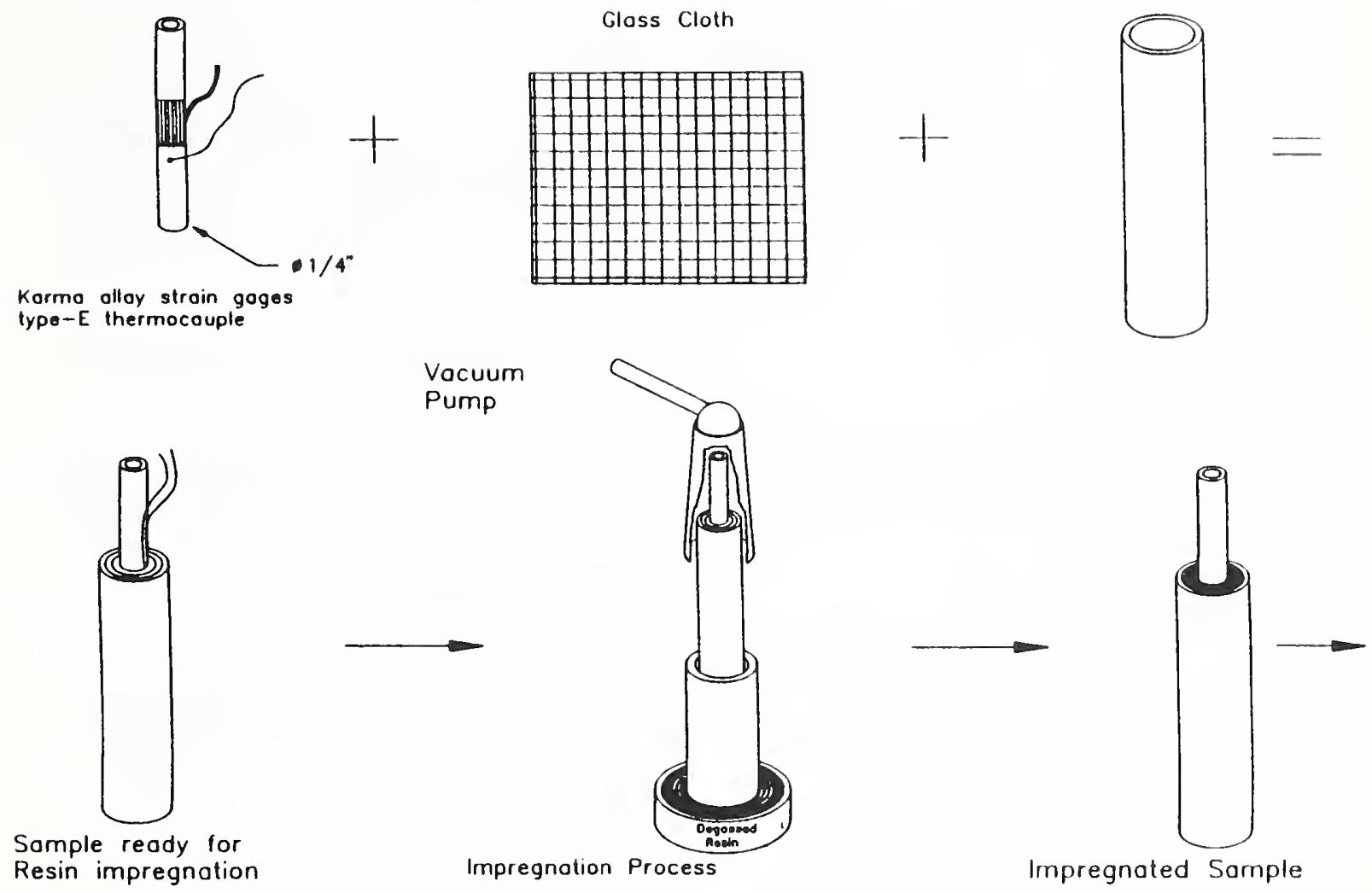

Figure 4.1. Schematic of the method for constructing and vacuum-impregnating the test specimens. See text for details.

The annular space between the glass tube and the aluminum mold was vacuum-impregnated with the desired resin system. This was accomplished by connecting the top of the mold to a vacuum system by a short length of plastic tubing and connecting the bottom of the mold to a reservoir of degassed resin with another short length of tubing. The bottom tube was pinched off, the mold was evacuated, and the lower tube was opened for specimen impregnation. The vacuum was broken and a cap, sealed with silicone rubber sealant, was placed on the bottom of the aluminum tube to prevent drainage of the resin. The two or three specimens used in each run were impregnated simultaneously; the process required from 10 to $45 \mathrm{~min}$, depending on the viscosity of the resin.

The instrumentation system schematically illustrated in Figure 4.2 enabled monitoring of the strain and temperature of several specimens run simultaneously. During the cure cycles, data points were taken every $20 \mathrm{~s}$ for resin systems requiring more than a 15 -h cure time, and every $10 \mathrm{~s}$ for systems requiring shorter cure times. Data points were taken every $3 \mathrm{~s}$ during the cryogenic cooling cycles. 


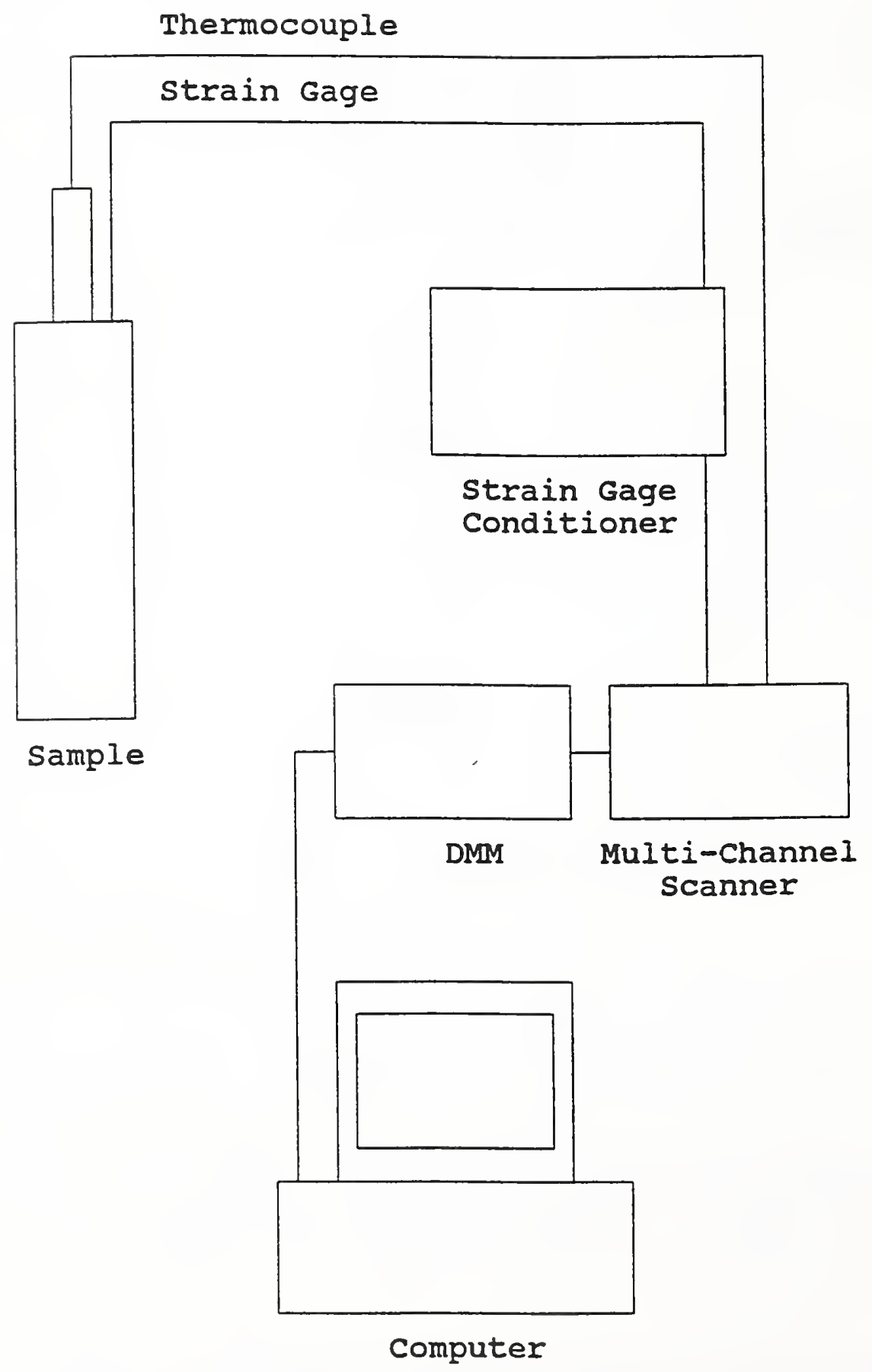

Figure 4.2. Schematic of the instrumentation system used to monitor changes in strain during the cure cycle and during cooling to $77 \mathrm{~K}$. 
The specimens were heated to the cure temperature in an air-circulating oven and were cooled by natural convection with the oven door open. Heating time to $100^{\circ} \mathrm{C}$ was approximately $50 \mathrm{~min}$, and cooling time from $100^{\circ} \mathrm{C}$ to room temperature was approximately $150 \mathrm{~min}$.

The effect of cryogenic cooling on the residual strain was determined by slowly introducing the specimens into a dewar of liquid nitrogen, using the sensible heat of the nitrogen vapor for precooling, while monitoring strain and temperature with the same apparatus as that used for monitoring the cure. Strain was also monitored during specimen warm-up, during which the specimens were removed from the dewar at the same rate as for insertion. A complete cycle required about $3 \mathrm{~h}$.

\subsection{Experimental Materials}

As summarized on Table 4.1, the program evaluated four epoxy resins, one polyester resin, one vinyl-ester resin, and three cyanate-ester resins. These systems will be referred to by the identification code given in Table 4.1. The chemical abbreviations are defined Table 2.2 of §III.2.

Table 4.1. Experimental resins.

\begin{tabular}{|c|c|c|}
\hline I.D. Letter & Abbreviation $^{*}$ & Cure Cycle \\
\hline \multicolumn{3}{|l|}{ Epoxies: } \\
\hline A & $\begin{array}{l}\text { NV/AN/ACL } \\
\text { (CTD proprietary) }\end{array}$ & $24 \mathrm{~h} @ 125^{\circ} \mathrm{C}$ \\
\hline $\mathrm{D}$ & $\begin{array}{l}\text { DGEBA/ALA/ACL } \\
\text { (CTD proprietary) }\end{array}$ & $\begin{array}{l}72 \mathrm{~h} @ 25^{\circ} \mathrm{C} \\
16 \mathrm{~h} @ 85^{\circ} \mathrm{C}\end{array}$ \\
\hline B & $\begin{array}{l}\text { DGEBA/DCH/ARA/ACL } \\
\text { (Addax proprietary) }\end{array}$ & $4 \mathrm{~h} @ 170^{\circ} \mathrm{C}$ \\
\hline $\mathrm{E}$ & $\begin{array}{l}\text { DGEBF/CTBN/AN } \\
\text { (Addax proprietary) }\end{array}$ & $\begin{array}{l}\text { ramp to } 121^{\circ} \mathrm{C} \text { over } 5 \mathrm{~h} \text {; } \\
\text { hold } 10 \mathrm{~h} @ 121^{\circ} \mathrm{C}\end{array}$ \\
\hline \multicolumn{3}{|l|}{ Polyester: } \\
\hline $\mathbf{P}$ & $\begin{array}{c}\text { PE/MEKP } \\
\text { (Owens-Corning) }\end{array}$ & $\begin{array}{l}75 \mathrm{~h} @ 25^{\circ} \mathrm{C}(\mathrm{RT}) \\
1 \mathrm{~h} @ 120^{\circ} \mathrm{C} \text { (postcure) }\end{array}$ \\
\hline \multicolumn{3}{|l|}{ Vinyl Ester: } \\
\hline $\mathrm{M}$ & $\begin{array}{c}\text { VE/CHX } \\
\text { (Dainippon Ink \& Chemicals) }\end{array}$ & $1 \mathrm{~h} @ 155^{\circ} \mathrm{C}$ \\
\hline \multicolumn{3}{|c|}{ Cyanate Esters: } \\
\hline I & $\begin{array}{l}\text { CE/HTBN/NPh/Cu.AA } \\
\text { (Rhone-Poulenc) }\end{array}$ & $\begin{array}{l}4 \mathrm{~h} @ 121^{\circ} \mathrm{C} \\
4.5 \mathrm{~h} @ 121^{\circ} \mathrm{C} \text { (postcure) }\end{array}$ \\
\hline G & $\begin{array}{l}\text { CE/VT/Co.AA } \\
\text { (Dow Chemical) }\end{array}$ & $\begin{array}{l}2 \mathrm{~h} @ 121^{\circ} \mathrm{C}+2 \mathrm{~h} @ 176^{\circ} \mathrm{C} ; \\
2 \mathrm{~h} @ 246^{\circ} \mathrm{C} \text { (postcure) }\end{array}$ \\
\hline $\mathbf{R}$ & $\begin{array}{c}\mathrm{CE}(\mathrm{PT}) \\
\text { (Allied Signal proprietary) }\end{array}$ & $12 \mathrm{~h} @ 110^{\circ} \mathrm{C}$ \\
\hline
\end{tabular}

${ }^{*}$ See Table 2.2 of $\S$ III. 2 for description. 


\subsection{Resins}

The strains measured during cure and during cryogenic cooling, plus the average total strain from both sources are reported for each polymer system in Table 4.2 and appear as bar graphs in Figures 4.3 through 4.5. The curing strains were taken as the strain differential between the heating curve and cooling curve at $30^{\circ} \mathrm{C}$. The cryogenic cooling strains were taken as the strain induced by cooling from room temperature to $77 \mathrm{~K}$.

Table 4.2. Experimental results.

\begin{tabular}{|c|c|c|c|c|c|c|c|c|c|}
\hline \multirow{2}{*}{ Resin } & \multicolumn{4}{|c|}{ Cure Strain, $\times 10^{-6}$} & \multicolumn{4}{|c|}{ Cooling Strain, $\times 10^{-6}$} & \multirow{2}{*}{$\begin{array}{c}\text { Total Strain, } \\
\times 10^{-6}\end{array}$} \\
\hline & $\# 1$ & $\# 2$ & $\# 3$ & Ave. ${ }^{*}$ & $\# 1$ & $\# 2$ & \#3 & Ave. & \\
\hline \multicolumn{10}{|c|}{ Epoxies: } \\
\hline A & -225 & -250 & - & -237 & -1090 & -1400 & - & -1245 & -1898 \\
\hline $\mathrm{D}$ & $\begin{array}{r}+8 \\
-370\end{array}$ & $\begin{array}{r}-25 \\
-730\end{array}$ & $\begin{array}{r}-70 \\
-780\end{array}$ & $\begin{array}{r}\sim 0(\mathrm{RT}) \\
-626(\mathrm{PC})\end{array}$ & -700 & -400 & -800 & -967 & -1593 \\
\hline B & -800 & -800 & - & -800 & -1500 & -1800 & - & -1650 & -2450 \\
\hline $\mathrm{E}$ & -525 & -675 & - & -600 & -1700 & -1900 & - & -1800 & -2400 \\
\hline \multicolumn{10}{|c|}{ Polyester: } \\
\hline$P$ & $\begin{array}{l}+42 \\
-70\end{array}$ & $\begin{array}{r}+25 \\
-412\end{array}$ & $\begin{array}{r}-70 \\
-177\end{array}$ & $\begin{array}{r}\sim 0(\mathrm{RT}) \\
-219(\mathrm{PC})\end{array}$ & -750 & -650 & -650 & -683 & -902 \\
\hline \multicolumn{10}{|c|}{ Vinyl Ester: } \\
\hline M & -400 & -370 & -322 & -364 & -1200 & -1380 & -1350 & -1310 & -1632 \\
\hline \multicolumn{10}{|c|}{ Cyanate Esters: } \\
\hline I & -700 & -790 & -975 & -821 & -1450 & -1500 & -1720 & -1556 & -2377 \\
\hline G & -1400 & -1550 & -1750 & -1567 & -675 & -1750 & - & -1212 & -2779 \\
\hline $\mathrm{R}$ & -818 & -586 & -737 & -714 & -950 & -1100 & -620 & -820 & -1604 \\
\hline
\end{tabular}

${ }^{*} \mathrm{RT}=$ room-temperature cure; $\mathrm{PC}=$ postcure 


\subsubsection{Cure-Process Strains}

The processing strains appeared to fall within three general groups (Fig. 4.3). The lowest strains were developed by the epoxy $A$, the polyester $\mathrm{P}$, and the vinyl-ester $\mathrm{M}$ materials. Epoxies E, B, and D and the cyanate esters I and R formed a somewhat higher strain group. The strain developed by the cyanate ester $G$ was by far the highest of this group, about 7 times higher than that developed by the epoxy $\mathrm{A}$ and polyester $\mathrm{P}$ materials.

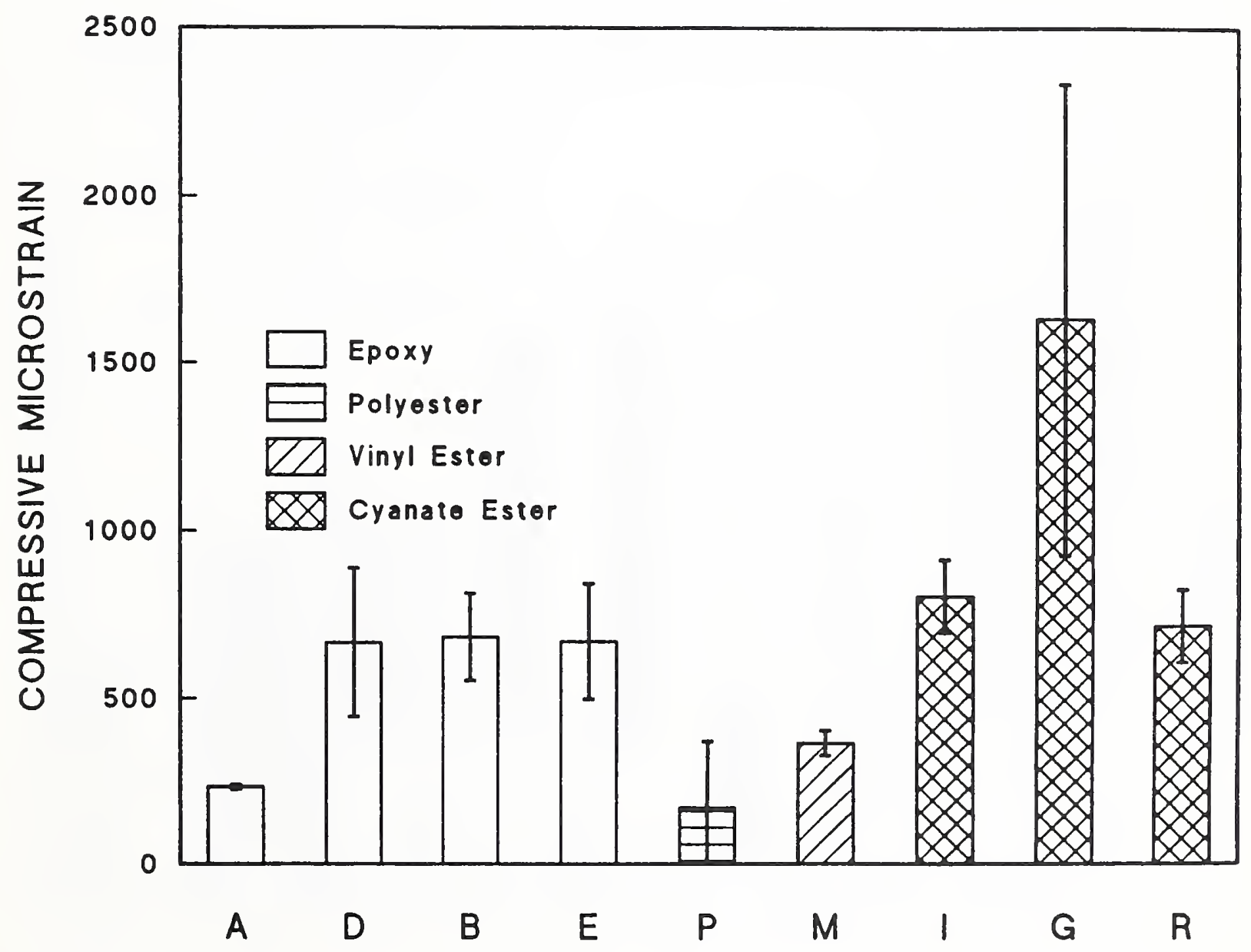

Figure 4.3. Average overall strains were determined for the studied resin systems during the cure cycle from room temperature to the cure temperature and return to room temperature. Error bars denote the spread of data for the several specimens run simultaneously. 


\subsubsection{Cryogenic Cooling Strains}

Somewhat more data scatter occurred in this test sequence (see Fig. 4.4) than in the cure cycle. However, the data indicate that the polyester $\mathbf{P}$ material develops the lowest strain, with the epoxy $D$ and the cyanate ester $R$ materials only slightly higher. The vinyl ester $M$, the epoxy $\mathrm{A}$, and the cyanate ester $\mathrm{G}$ materials form a group developing strains about 1.8 times higher than that of the polyester $\mathbf{P}$ material. Epoxies B and E, along with the cyanate ester I material, developed the highest cooling strains, about 2.5 times that of the polyester $\mathrm{P}$ material.

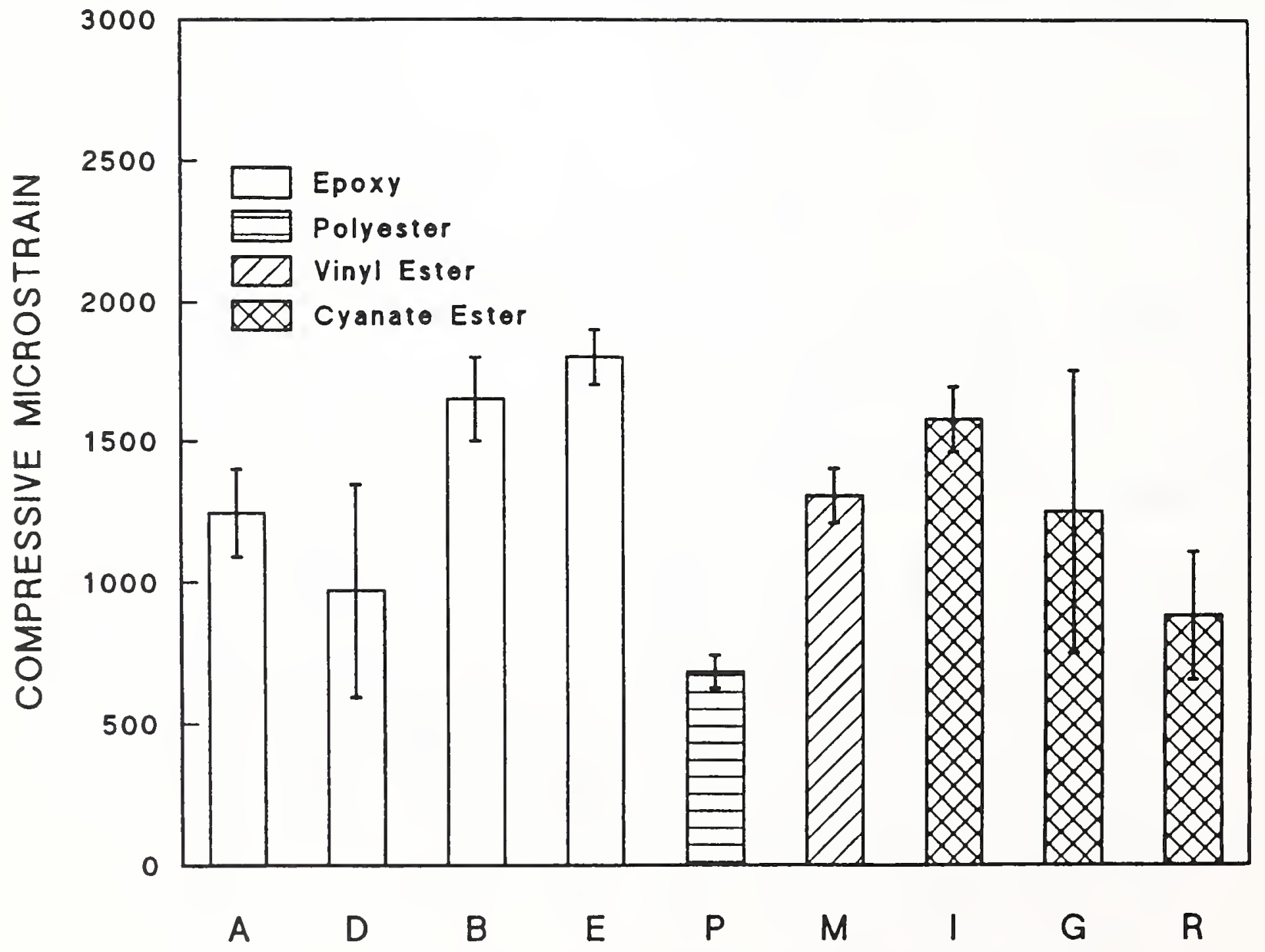

Figure 4.4. Average overall strains determined for the cured resin systems during subsequent cooling to $77 \mathrm{~K}$. Error bars reflect the spread of data on several specimens run simultaneously. 


\subsubsection{Total Strain}

The data in Figure 4.5 indicate that the least overall residual strain due to processing plus cooling to cryogenic temperatures occurs in the polyester $P$ material. The epoxies $A$ and $D$, the vinyl ester $M$, and the cyanate ester $R$ appear to form a group developing strains about 1.8 times higher than the polyester. Epoxies B and E plus the cyanate ester I developed yet higher strains, about 2.7 times that of the polyester. The highest overall strain was developed by the cyanate-ester $\mathrm{G}$ formulation.

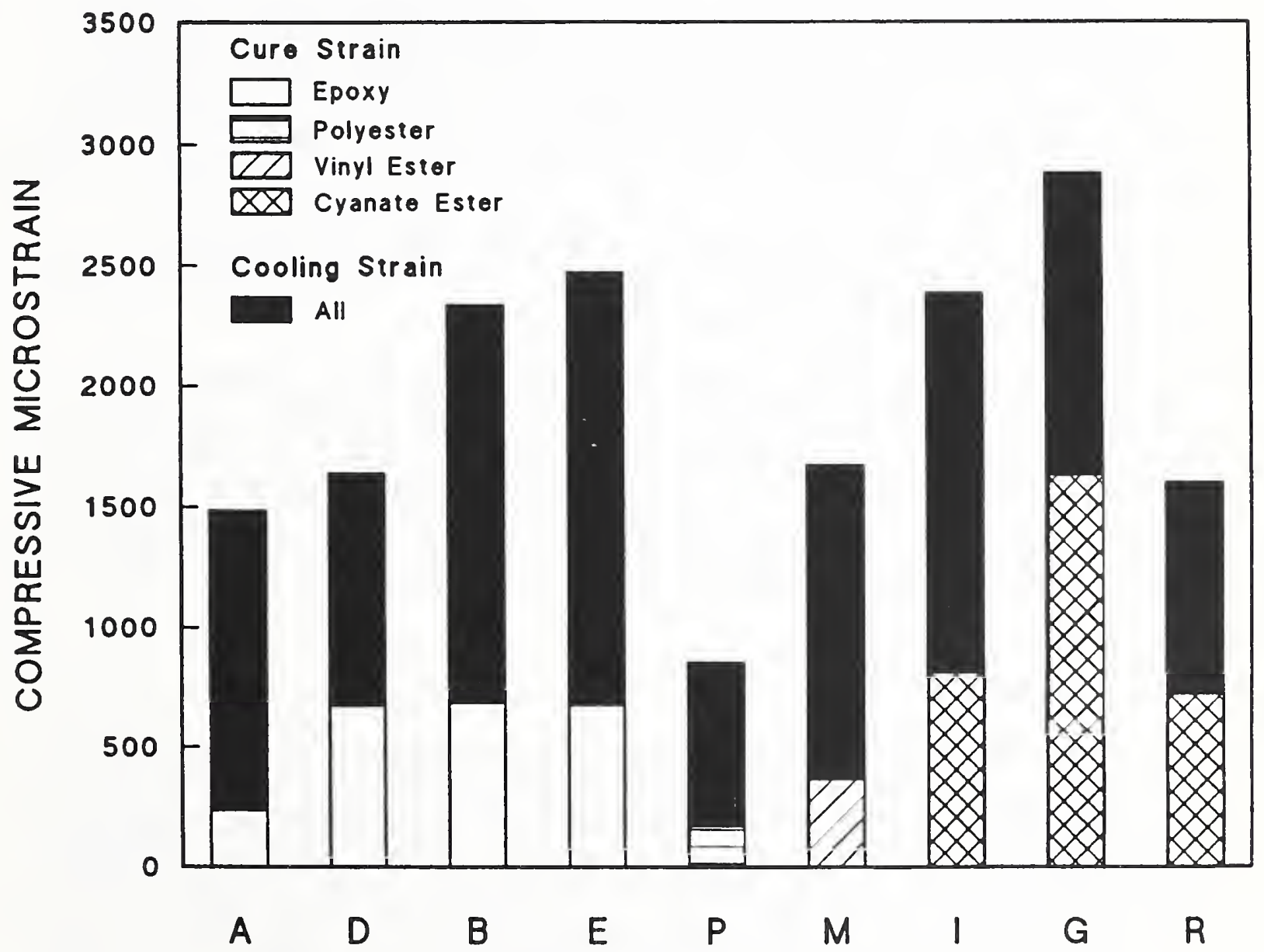

Figure 4.5. Average overall strains determined for the studied resin systems subjected to the cure cycle plus cooling to $77 \mathrm{~K}$. 


\subsection{Analysis of Cure-Cycle Strain Curves}

Three distinctly differing strain behaviors were observed during the cure cycles of the tested resin systems. They are referred to as Types 1, 2, and 3.

\subsubsection{Type 1 Behavior}

The Type 1 behavior had the appearance shown in Figure 4.6 and was characterized by a smoothly increasing compressive strain from room temperature to cure temperature, little change in strain during holding at the cure temperature, and a relatively smooth, monotonic decrease in strain from the cure temperature to room temperature. This type of behavior was observed in all the curves developed from epoxy $A$ and the vinyl ester $M$, and, for the postcure process, from the polyester P resin system. (The initial room-temperature cure of the polyester $P$ resin system produced a negligible strain change.)

The strain induced by heating the specimens from room temperature to the cure temperature primarily reflects dimensional changes in the glass substrate. The data, therefore, indicate that the glass used in this experiment has a negative coefficient of thermal expansion in the temperature range studied, inducing thermal contraction on heating and placing the gage in compression. Equivalent strain-temperature behavior was observed for all resin systems during this heating phase.

No significant strain changes were observed while holding at the cure temperature. This is attributed to the cure temperature being above the glass transition $\left(T_{g}\right)$ temperature, where relaxation processes operate to relieve the strain.

Upon cooling from the cure temperature to the $T_{g}$, a tendency toward retracing the heating curve is expected, since the strain level would continue to be dominated by the expansion characteristics of the glass substrate. However, when the $T_{g}$ point is reached, the transformation of the resin into the glassy state would introduce strains due to thermal contraction of the resin. These strains would counterbalance those arising from expansion of the glass substrate, causing a deviation of the cooling curve to a lower slope. This is observed in Figure 4.6 and suggests that the $T_{g}$ for the epoxy $\mathrm{A}$ resin is in the vicinity of $112^{\circ} \mathrm{C}$.

\subsubsection{Type 2 Behavior}

The Type 2 behavior had the appearance shown in Figure 4.7. The heating portion of the curve resembles that of the Type I behavior; however, the cooling portion of the curves are essentially horizontal and contain a series of random, sharp strain changes, in which a perturbation of the strain in one direction is usually followed by a countering perturbation in the other direction. This behavior was observed in all the curves generated with the B and E epoxy systems and with the cyanate ester I, G, and R systems. The sharpness of the perturbations suggest that they were not created by normal, thermally activated processes but rather by some repetitive mechanical phenomena.

The underlying cause of these sharp perturbations is not fully understood at this time. However, the appearance of the perturbations is consistent with the operation of a "stick-slip" 


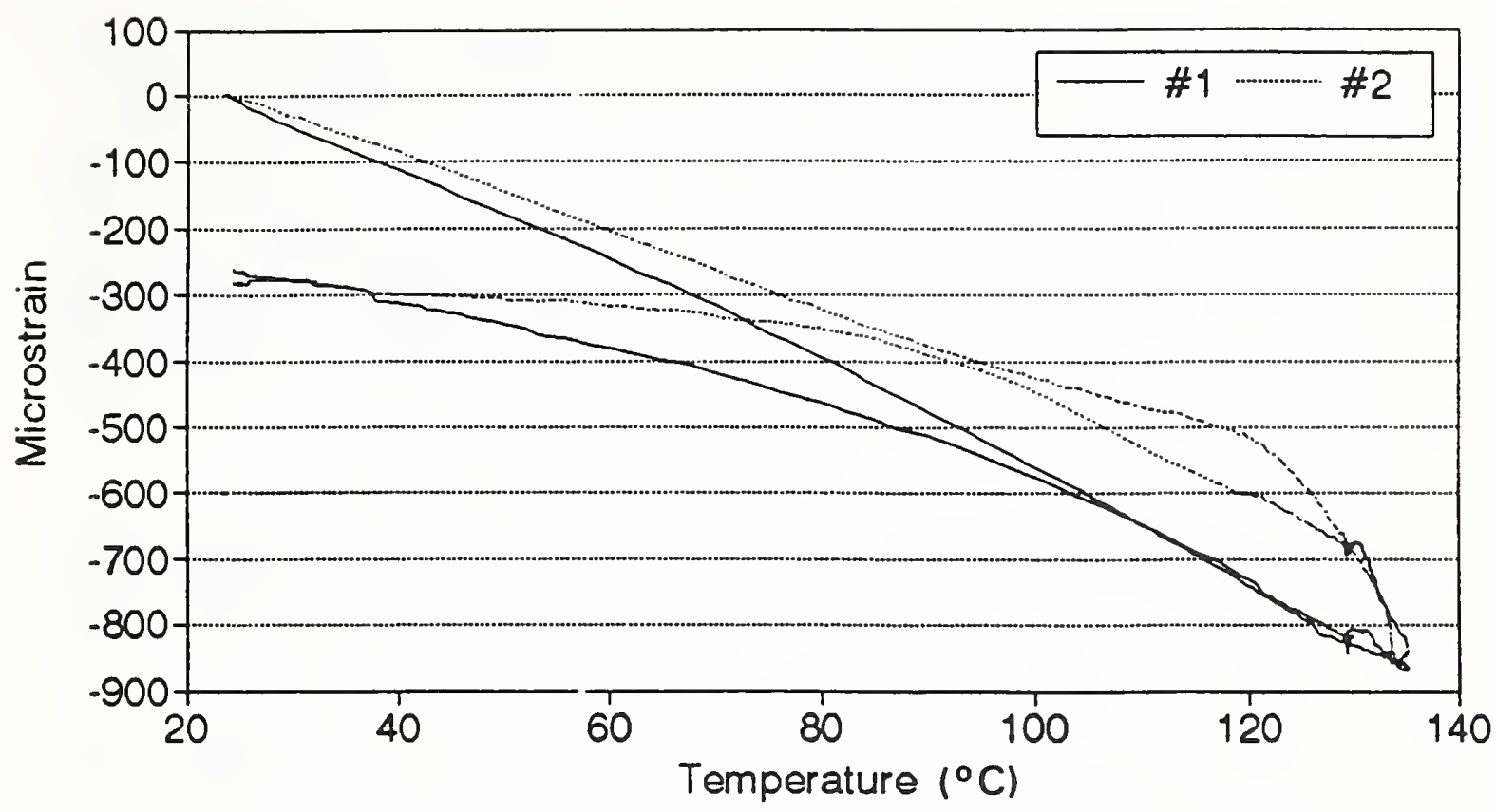

Figure 4.6. Strain curves produced by the epoxy A resin system during heating to the cure temperature and cooling to room temperature. Note the relative smoothness of the cooling curves compared with those in Figure 4.7.

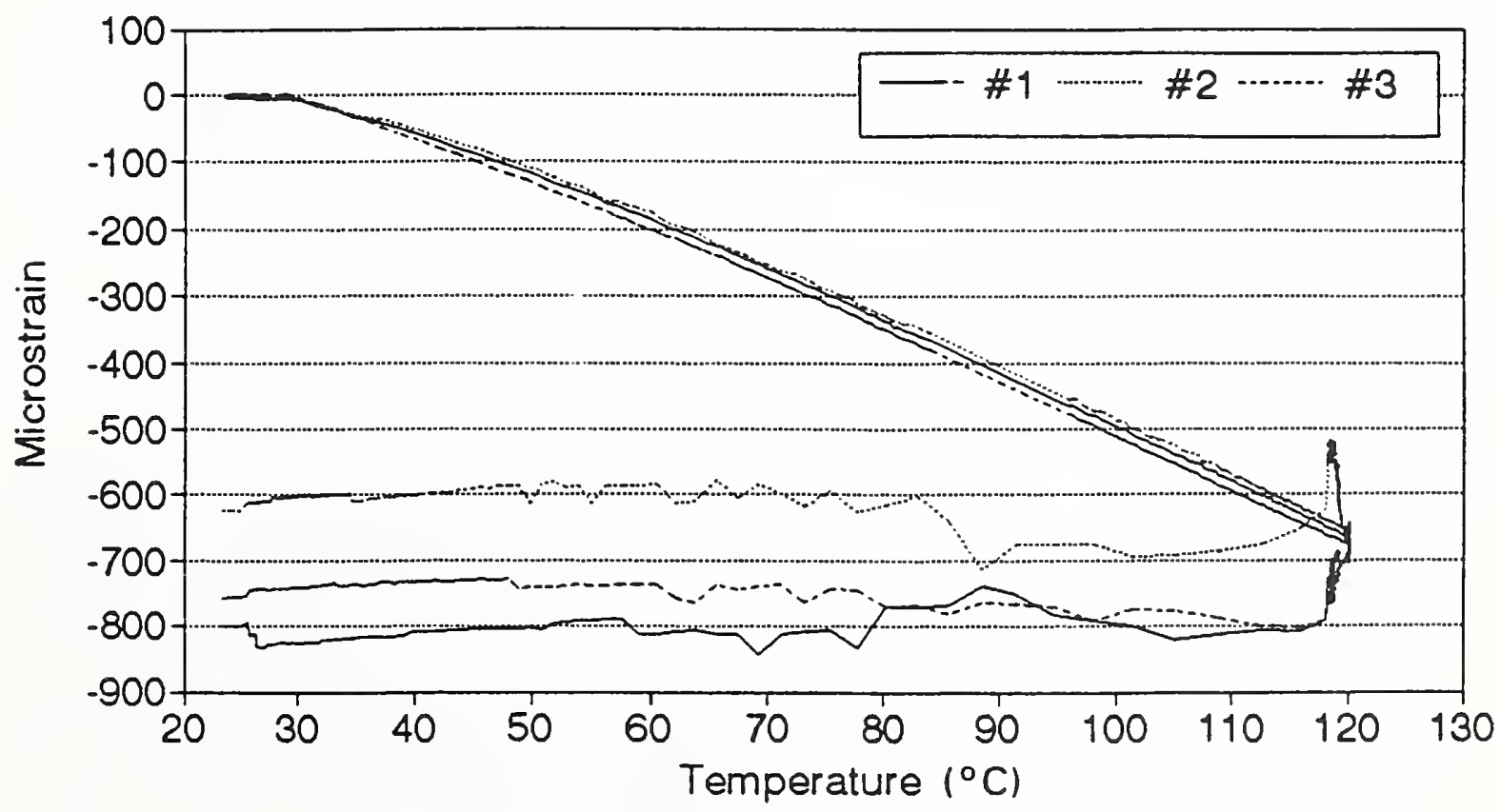

Figure 4.7. Strain curves produced by the cyanate-ester $\mathrm{R}$ resin system during heating to the cure temperature and cooling to room temperature. The curves show significant strain perturbations on the cooling cycle. 
type of phenomenon that could be taking place between the cured specimen and the inner surface of the aluminum mold. Localized sticking of the cured resin to the walls of the mold would restrict longitudinal contraction of the specimen during cooling and would cause the strain gage to sense a less than normal strain, thus driving the curve upward. Subsequent localized unsticking of the specimen from the aluminum tube wall in response to the contraction stress would cause the gage to sense a sudden increase in strain, driving the curve downward. As the driving force for contraction increases with lowering of temperature, such a mechanism could become repetitive, causing the observed strain perturbations. This theory assumes that the resin systems in which the perturbations are observed have characteristics enabling them to adhere to the aluminum mold despite the use of a mold release, while the resin systems in which the phenomenon is not observed do not. This has not been verified.

The extent to which the strain perturbations might be artificially biasing the final residual strain values obtained during the cure cycle was of some concern. We evaluated this by subjecting an epoxy E specimen that had shown substantial perturbations on the initial cooling curve to a reheating cycle from room temperature to the cure temperature and a return to room temperature. Figure 4.8 shows that the reheating yielded a strain profile very similar to that of the original curve, but without the perturbations, suggesting that the presence of the perturbations is not significantly biasing the overall strain results. The absence of perturbations on the reheating curve gives added support to the slip-stick theory of their formation.

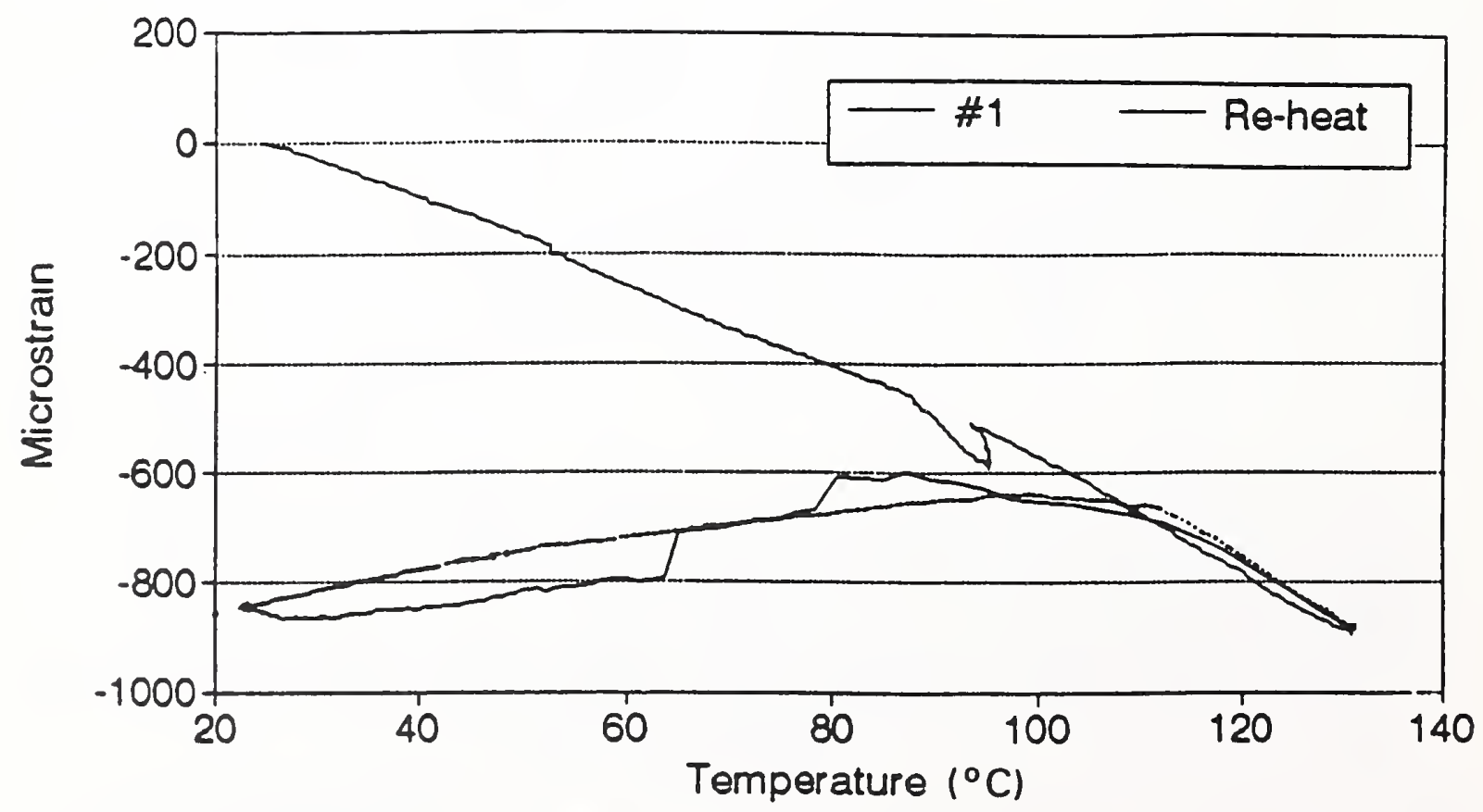

Figure 4.8. The erratic curve was developed during the initial cooling cycle of an epoxy E specimen from cure temperature to room temperature. The smooth curve was developed during recycling this specimen to and from the cure temperature. 


\subsubsection{Type 3 Behavior}

The Type 3 behavior was observed during the room-temperature cure (Fig. 4.9) and during the subsequent elevated temperature postcure (Fig. 4.10) of the epoxy D resin system.

The room-temperature cure was characterized by an increase in strain between 1500 and 2500 min of holding time, following which the strain sharply recovered and subsequently underwent a more gradual recovery such that relatively little residual strain existed at the end of the 4800-min cure cycle. From the heating curves of the previous figures (cf., Fig. 4.6), we observe that an increase in microstrain of -90 to -160 is consistent with contraction of the glass substrate in response to a temperature rise on the order of $40^{\circ} \mathrm{C}$. Such a temperature rise could be caused by isothermal heating concomitant with activation of a cross-linking reaction in the resin. Completion of the reaction would remove the heat source, allowing the glass substrate to cool, thereby decreasing the measured strain; however, it is difficult to account for the observed rapid decrease of strain on this basis.

From Figure 4.10 it is seen that, aside from a few small perturbations early in the cycle, heating to the postcure temperature produced relatively little net strain change, indicating that the strain due to contraction of the glass substrate is being counteracted by strain due to expansion of the cured resin. Subsequent holding at the postcure temperature produced a substantial increase in compressive strain. This result is consistent with the holding temperature being above the $T_{g}$ temperature where the strain in the resin could relax, allowing contraction of the glass substrate to dominate the measured strain. Note that the approximately -450 microstrain increase during this period is about that developed by heating the glass substrate to $95^{\circ} \mathrm{C}$ (cf., Fig. 4.6). Note also that, although the postcure temperature was set at $85^{\circ} \mathrm{C}$, the actual temperature developed in the specimen was 10 to $15^{\circ} \mathrm{C}$ higher, suggesting the onset of isothermal heating due to additional cross-linking reactions during the postcure. Cooling from the postcure produced little net change in strain. Again, it can be assumed that the tensile strains developed in the glass substrate and compressive strains developed in the resin neutralized each other.

\subsection{Analysis of the Cryogenic Cooling Curve}

The cryogenic cooling curves for some of the resin systems (epoxy $\mathrm{E}$, vinyl ester $\mathrm{M}$, polyester $\mathrm{P}$, and cyanate ester I) were relatively well behaved, showing a gradual increase in strain on cooling, with a few perturbations in the vicinity of $-150^{\circ} \mathrm{C}$. Figure 4.11 shows this behavior in the epoxy $\mathrm{E}$ resin. Conversely, the cooling curves for other systems (epoxies $\mathrm{A}, \mathrm{D}$, and $B$, and cyanate esters $G$ and $R$ ) showed very erratic strain excursions. Figure 4.12 illustrates this behavior in the epoxy $\mathrm{A}$ resin. As shown in Figure 4.13, repeating the cooling cycle on the latter material produced curves without the perturbations, indicating that the erratic behavior was an artifact due to adhesion of the resin to the mold or to some other factor interfering with uniform contraction of the resin. This procedure was followed for all materials showing erratic behavior on initial cooling. 


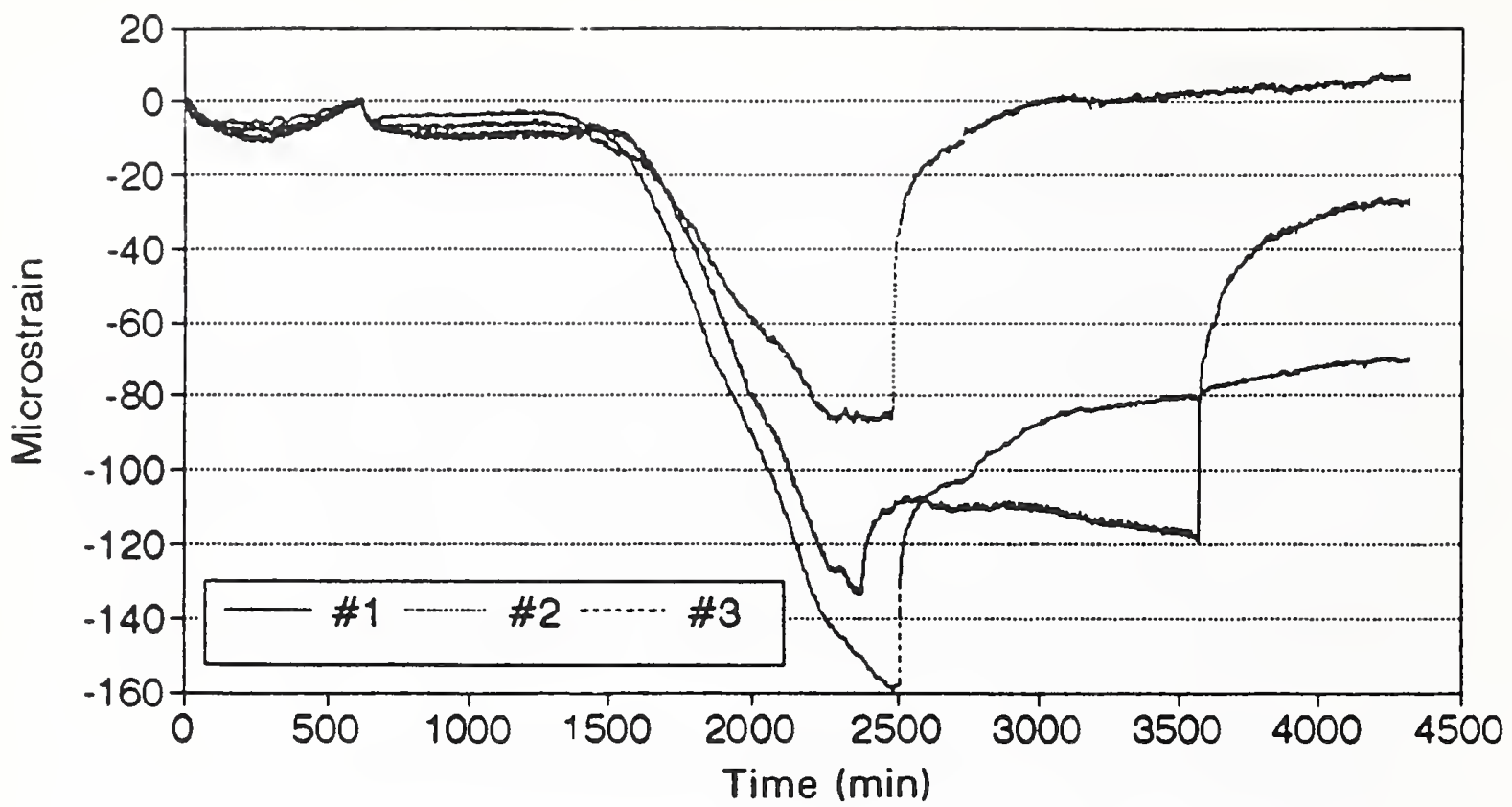

Figure 4.9. Strain curves produced during room-temperature cure of epoxy D. Note the similar behavior of the three specimens.

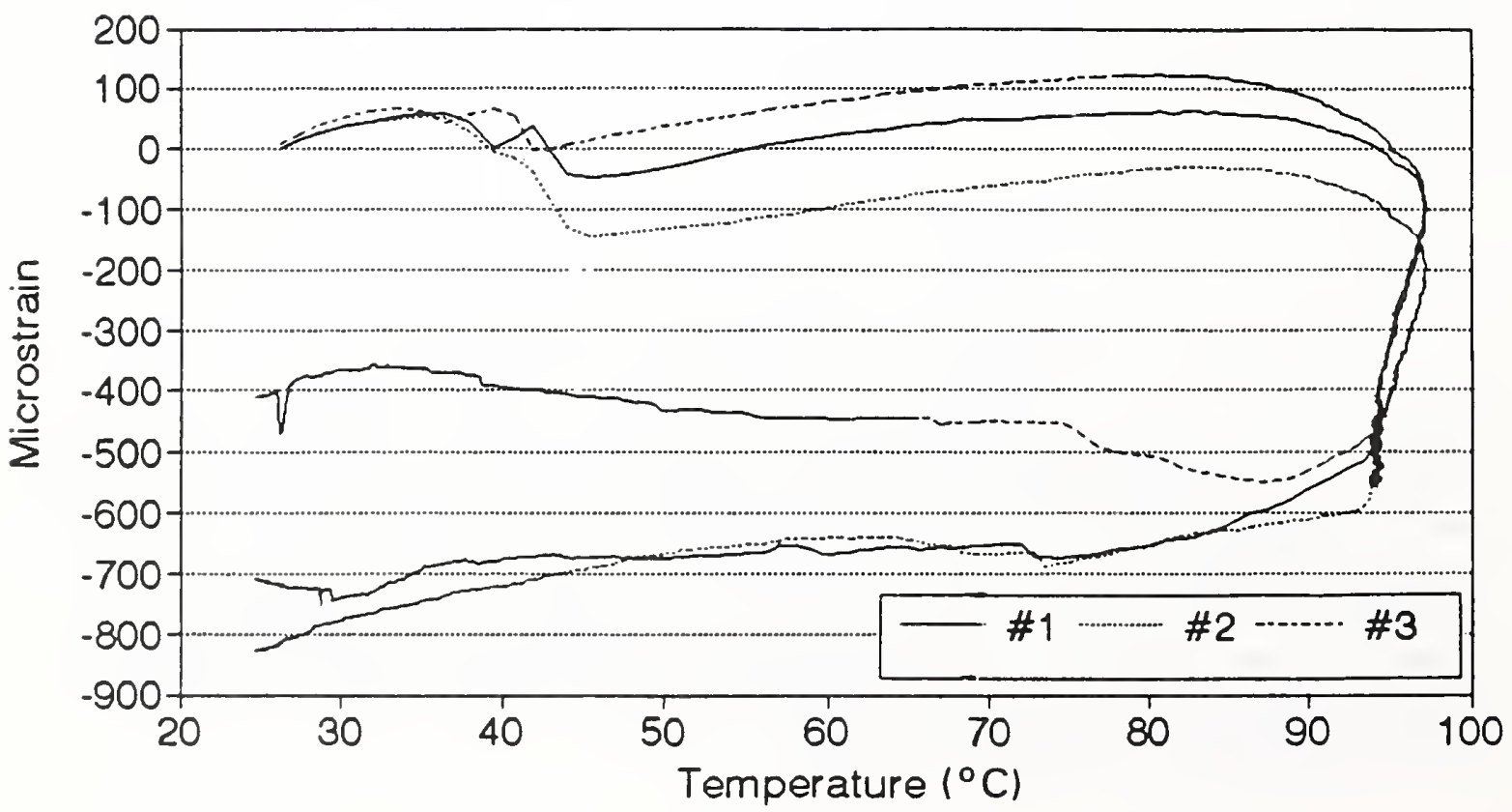

Figure 4.10. Strain curves produced during subsequent postcure treatment of epoxy D. 


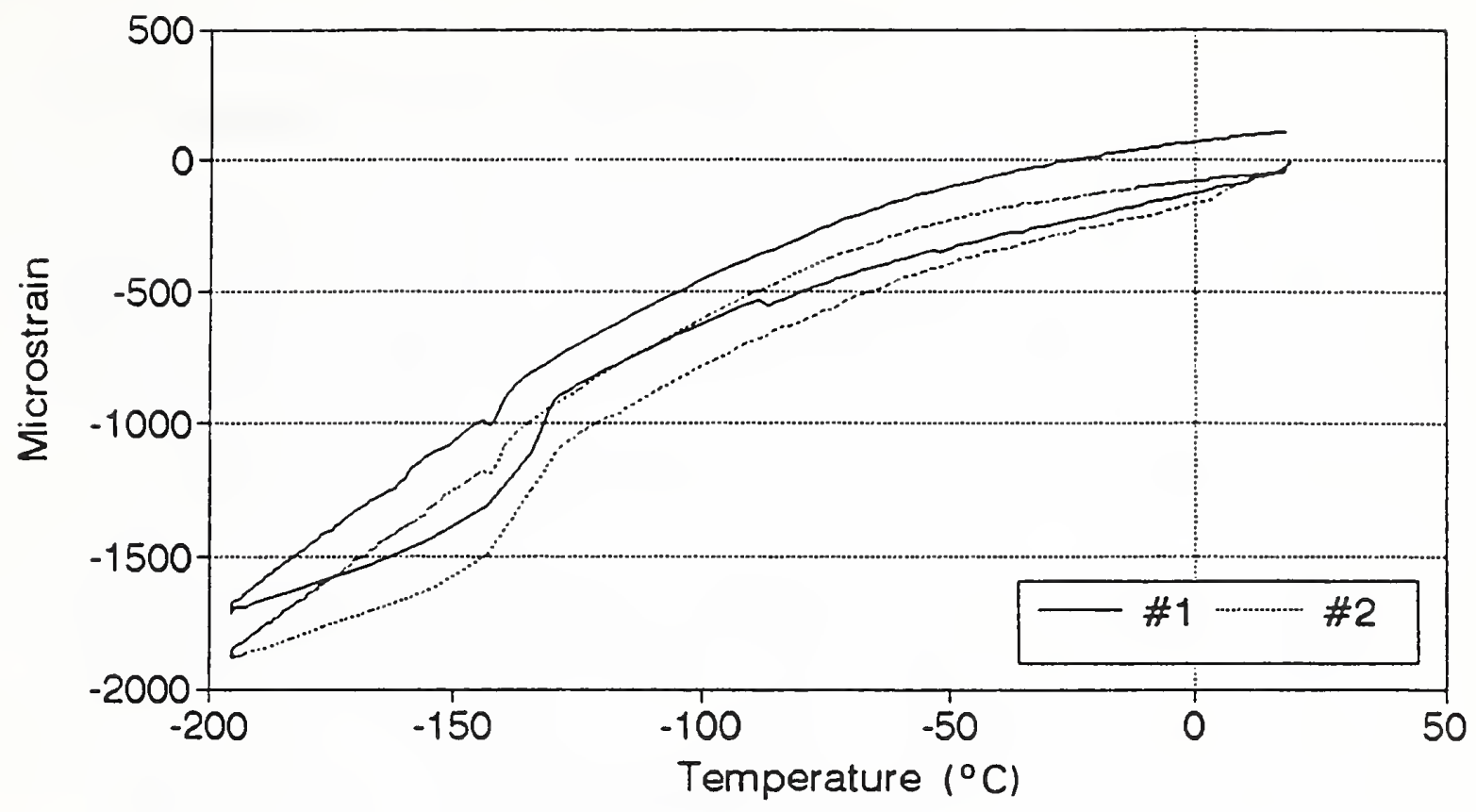

Figure 4.11. Strain curves produced by cycling epoxy E specimens to $77 \mathrm{~K}$. Note the smoothness of these curves compared to those in Figure 4.12.

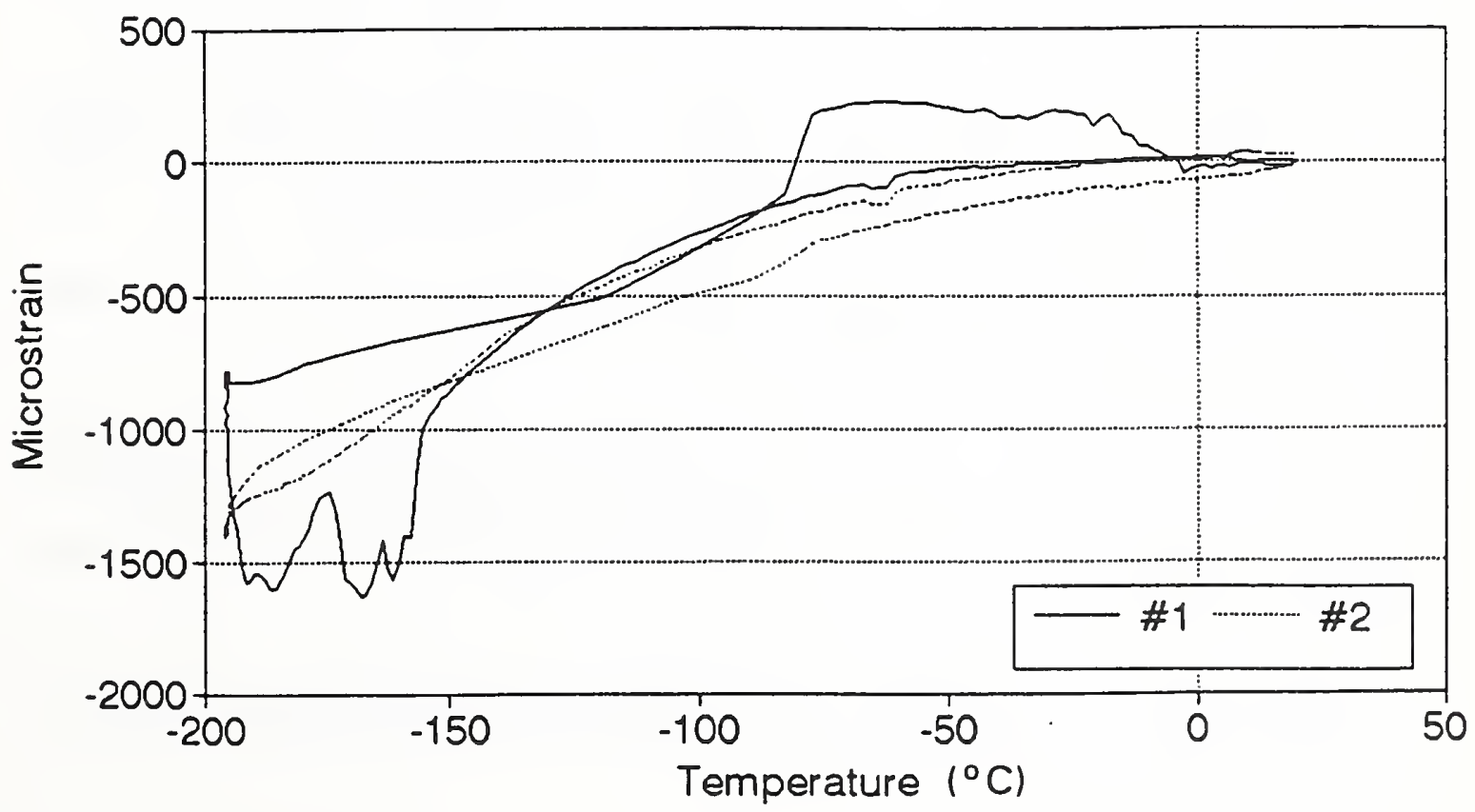

Figure 4.12. Strain curves generated by cycling epoxy A specimens to $77 \mathrm{~K}$. One of the specimens shows very erratic strain behavior. 


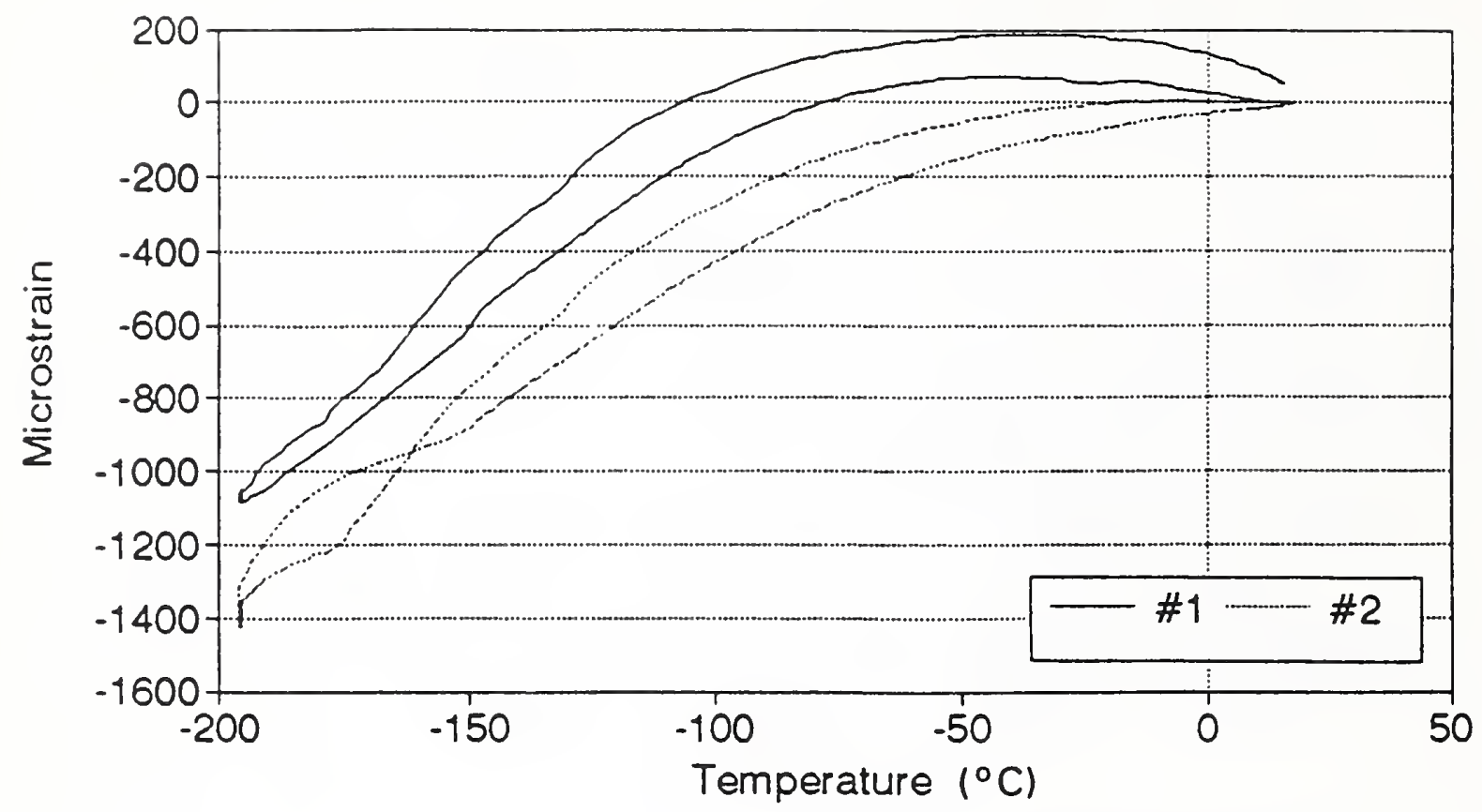

Figure 4.13. Strain curves produced by recycling the epoxy A specimens to $77 \mathrm{~K}$, showing that recycling eliminates the erratic strain excursions.

\subsection{Conclusions}

1. The experimental methodology developed in this program appears to be capable of quantitatively measuring the magnitude of residual strains developed at the glass-polymer interface of a composite material during the cure cycle and during cooling to $77 \mathrm{~K}$.

2. The experimental methodology appears to be capable of distinguishing between the residual strains developed by differing polymer systems.

3. The data indicate that, among the materials tested, the least residual stress was in the polyester $\mathrm{P}$ system. Next lowest residual stresses were found in the vinyl-ester $\mathbf{M}$, the epoxies $\mathrm{A}$ and $\mathrm{D}$, and the cyanate-ester $\mathrm{R}$ products. Higher residual stresses were observed in the epoxies $\mathrm{B}$ and $\mathrm{E}$ and in the cyanate-ester I products. The highest residual stress was developed by the cyanate-ester $\mathrm{G}$ system.

4. The presence of anomalous, sharp, strain perturbations in some systems on cooling from the cure temperature or during cooling to cryogenic temperatures indicates that the specimen design is not yet perfected. 


\subsection{Recommendations for Future Work}

Efforts should be made to improve the specimen construction so as to eliminate observed spurious strain excursions. It appears most likely that this problem is caused by failure of the contracting resin to freely release from the inner surface of the aluminum tube used in the present design. Replacement of this mold with a PTFE mold might be a workable alternative. This problem should be addressed before additional studies are undertaken.

Further studies should be undertaken to provide the basis for a systematic selection of resin systems for use in composites at cryogenic temperatures. Among these are:

1. Studies of the effect of varying processing parameters on the residual strain induced during the cure cycle. It would be of particular interest to determine if reducing the cooling rate from the cure temperature reduces residual strain, as has been suggested in the literature.

2. Studies of the effect of variations in resin chemical composition on residual strain. For example, there is some evidence in the literature that additions of small amounts of accelerator can drastically reduce fatigue life at cryogenic temperatures, suggesting that such additions substantially increase residual stress. There is also evidence that addition of certain amounts and types of flexibilizing agents can substantially increase residual stress at low temperatures. This experimental method is well suited for study of these questions.

3. A logical extension of this work would be to correlate the residual-strain data obtained by the described method with the mechanical performance of glass-fiber-reinforced composites fabricated from each of the studied resins. This would be best done by evaluating off-axis strength properties, perhaps using the $\pm 45^{\circ}$ in-plane shear test method. 


\section{References}

[1.1] Reuton, W.J. Hybrid and select metal-matrix composites. New York, NY: American Institute of Aeronautics and Astronautics; 1977. 15, 16.

[1.2] Chamis, C.C. Simplified composite micromechanics equations for mechanical, thermal, and moisture-related properties, in Engineers' guide to composite materials. Weeton, J.W.; Peters, D.M.; Thomas, K.L., eds. Metals Park, OH: ASM International; 1987. 3-8-3-24.

[1.3] Scala, E. Composite materials for combined functions. Rochelle Park, NJ: Hayden Book Co.; 1972. 200-227.

[1.4] Tensile properties of fiber-resin composites, ASTM D3039-76 (82), in ASTM standards and literature references for composite materials. Philadelphia, PA: American Society for Testing and Materials; 1987. 36-40.

[1.5] Compressive properties of unidirectional or crossply fiber-resin composites, ASTM D3410-75 (82), in ASTM standards and literature references for composite materials. Philadelphia, PA: American Society for Testing and Materials; 1987. 58-66.

[1.6] Inplane shear stress-strain response of unidirectional reinforced plastics, ASTM D351876 (82), in ASTM standards and literature references for composite materials. Philadelphia, PA: American Society for Testing and Materials; 1987. 71-60.

[1.7] Reed R.P.; Walsh, R.P. Tensile strain-rate effects in liquid helium, in Advances in cryogenic engineering-materials, vol. 34. New York, NY: Plenum Press; 1988. 199-208.

[2.1] Hartwig G.; Knaak, S. Fibre-epoxy composite at low temperatures. Cryogenics 24: $639-647 ; 1984$.

[2.2] Takeno, M.; Nishijima, S.; Okada, T.; Fujioka, K.; Tsuchida, Y.; Kuraoka, Y. Thermal and mechanical properties of advanced composite materials at low temperatures, in Advances in cryogenic engineering-materials, vol. 32. New York, NY: Plenum Press; 1986. 217-224.

[2.3] Hamelin, J. Three-dimensional contraction and mechanical properties of glass-clothreinforced epoxy materials at cryogenic temperatures, in Advances in cryogenic engineering-materials, vol. 26. New York, NY: Plenum Press; 1980. 295-299.

[2.4] Kasen, M.B.; MacDonald, G.R.; Beekman, D.H.; Schramm, R.E. Mechanical, electrical, and thermal characterization of G-10CR and G-11CR glass-cloth/epoxy laminates between room temperature and $4 \mathrm{~K}$, in Advances in cryogenic engineering-materials, vol. 26. New York, NY: Plenum Press; 1980. 235-244. 
[3.1] Dmitrevsky, Yu.P.; Kozub, S.S.; Escher, U. Thermal conductivity of various glassreinforced plastics at temperatures below $80 \mathrm{~K}$. Cryogenics 27: 429-432; 1987.

[3.2] Radcliffe, D.J.; Rosenberg, H.M. The thermal conductivity of glass-fibre and carbonfibre/epoxy composites from 2 to $80 \mathrm{~K}$. Cryogenics 22: 245-249; 1982.

[3.3] Takeno, M.; Nishijima, S.; Okada, T.; Fujioka, K.; Tsuchida, Y.; Kuroda, Y. Thermal and mechanical properties of advanced composite materials at low temperatures, in Advances in cryogenic engineering-materials, vol. 32. New York, NY: Plenum Press; 1986. 217-224.

[3.4] Evans, D. Morgan, J.T. Cryogen environment in composite vessels, in Advances in cryogenic engineering-materials, vol. 32. New York, NY: Plenum Press; 1988. 127-136.

[3.5] Klemens, P.G. Thermal conductivity of fibre composites at low temperatures. Cryogenics 31: 238-240; 1991.

[3.6] Hartwig, G.; Knaak, S. Fibre-epoxy composites at low temperatures. Cryogenics 24: $639-647 ; 1984$.

[4.1] Daniel, I.M.; Liber, T. Lamination residual strains and stresses in hybrid laminates, in Composite materials, materials testing and design. ASTM STP 617. Philadelphia, PA: American Society for Testing and Materials; 1977. 331-343,

[4.2] White, S.R.; Hahn, H.T. Mechanical property and residual stress development during cure of a graphite/BMI composite. Polym. Eng. Sci. 30(22): 1465-1473; 1990. 


\title{
VI. Properties of Struts
}

\author{
J.D. McColskey \\ Materials Reliability Division \\ National Institute of Standards and Technology \\ Boulder, Colorado \\ R.P. Reed \\ Cryogenic Materials, Inc. \\ Boulder, Colorado
}





\title{
VI. Properties of Struts
}

\author{
J.D. McColskey \\ R.P. Reed* \\ Materials Reliability Division \\ National Institute of Standards and Technology \\ Boulder, Colorado \\ ${ }^{*}$ Cryogenic Materials, Inc. \\ Boulder, Colorado
}

Tubes with different resin systems and lay-ups were tested in compression and fatigue at 295, 76, and $4 \mathrm{~K}$. Compressive strength and axial modulus, combined with axial and hoop strain, were measured for more than 180 tubes with diameters ranging from 100 to $250 \mathrm{~mm}$ (4 to $10 \mathrm{in}$ ). The principal results are

1. Tube compressive strength is linearly related (to the first order) to the axial compressive modulus.

2. Tube compressive strengths were about $50 \%$ higher at low temperatures than at room temperature. The axial compressive moduli were about $15 \%$ higher at low temperatures than at room temperature.

3. Significant, temperature-independent data scatter $(\leq \pm 25 \%)$ was attributed to material variability resulting from tube production.

4. Optimum fiber lay-up, based on both strength and thermal conductivity, consisted of predominantly $0^{\circ}$ to $10^{\circ}$ fiber-oriented layers with a few $80^{\circ}$ to $90^{\circ}$ fiber-oriented layers.

5. Strengths of vinyl-ester-resin tubes were equivalent to, or lower than, those of epoxy-resin tubes. Vinyl-ester resins should be considered only to lower resin or production costs.

6. Decreasing the diameter-to-thickness ratio from 20 to 10 resulted in improved axial compressive modulus $(8 \%)$ and strength (10\%) for tubes with predominantly $10^{\circ}$ fiber orientations.

7. Shorter tube lengths (with length-to-diameter ratios of $\leq 7$ ) resulted in higher average compressive strengths but did not influence maximum compressive strengths.

8. Compressive strengths of pultruded tubes were less than those of filamentwound tubes of equivalent lay-up.

9. Strain-to-failure values were about $30 \%$ higher at low temperatures than at room temperature.

10. The ratios of hoop to axial strain were rather constant at all temperatures, except for the room-temperature ratios of tubes with no $80^{\circ}$ to $90^{\circ}$ fiberoriented layers. The ratios for these tubes were much larger.

Very little compression data exist on E-glass-reinforced composite tubes. This study has generated an extensive amount of data on the compression response of E-glass-composite tubes at room and cryogenic temperatures. 


\section{Introduction}

The currently proposed designs for the Superconducting Magnetic Energy Storage (SMES) system require the use of support struts that can withstand high compressive loads and 10,000 load cycles; they must thermally isolate the 4-K coil from the room-temperature outer structure. The struts must be fabricated of relatively low-cost materials that provide the highest possible ratio of strength to thermal conductivity.

This study concentrated on E-glass fiber-reinforced composites. We studied six different resins with E-glass fiber reinforcement and seven different lay-ups. The struts were in the shape of tubes, with diameter-to-thickness ratios of 10 and 20 and length-to-diameter ratios of 2.25 , $3.5,4.75$, and 7.0 . For this program, two manufacturers produced tubes by filament-winding, one by pultrusion, and one by rubber expansion.

Compression tests were conducted at 295,76 , and $4 \mathrm{~K}$ on filament-wound and pultruded struts that ranged from $102 \mathrm{~mm}$ (4.0 in) in diameter and $230 \mathrm{~mm}$ (9.0 in) long to 250 $\mathrm{mm}$ (10.0 in) in diameter and $1800 \mathrm{~mm}$ (70 in) long. Ultimate compressive strengths, moduli, and strain-to-failure data were obtained from the tests.

Fatigue tests were conducted on some lay-ups at 295 and $76 \mathrm{~K}$ at 80,70 , and $60 \%$ average ultimate compressive strengths. The struts were fatigued for 40,000 cycles (four times the approximate number of cycles expected during SMES lifetime) and then loaded to failure at $295 \mathrm{~K}$ to determine residual strength. Strain to failure versus load was recorded for each test. The small number of tubes available after static testing precluded a thorough investigation of fatigue response. However, the limited amount of data obtained indicate that fatigue life increases at lower temperatures.

\section{Review of Literature}

A great deal of research has been conducted on the compression behavior of composite materials. Unfortunately, very little effort has been focused on the compression response of E-glass-epoxy tubes.

Davis reported axial compressive strengths of $1427 \mathrm{MPa}$ (207 ksi) in S-glass-epoxy tubes that were $13 \mathrm{~mm}(0.5 \mathrm{in})$ in diameter and $38 \mathrm{~mm}(1.5 \mathrm{in})$ to $76 \mathrm{~mm}$ (3.0 in) long [2.1]. The high strengths obtained in these hand-manufactured tubes were due to their small diameters and the carefully aligned axial fibers.

Using a new compression-test technique to eliminate edge effects, Swanson et al. measured axial compressive strengths as high as $1230 \mathrm{MPa}(178 \mathrm{ksi})$ in larger diameter $(38-\mathrm{mm}$, 1.5-in) graphite-epoxy tubes (AS4-3501-6) [2.2]; using the same test procedure with specimens of the same materials, lay-up, and diameter, Colvin and Swanson obtained an ultimate compressive stress of $723 \mathrm{MPa}$ (113 ksi) [2.3]. Colvin and Swanson demonstrated that hoop plies adjacent to the critical axial load-bearing plies help to stabilize the axial plies with respect to microbuckling, with the degree of stabilization depending upon the in-plane shear stiffness of the adjacent plies. 
Khitrov and Kutarzhnov demonstrated that adding a circular (hoop) wind to low-angle helical winds improved the axial compressive strength of filament-wound tubes [2.4].

The dependence of composite compressive strength on resin tensile modulus was shown by a number of researchers: Hahn and Williams [2.5] and Greszczuk [2.6] noted that the composite compressive-failure mode is governed by material properties and, along with Sohi et al. [2.7], concluded that composites containing resins with high tensile moduli have higher compressive strengths than composites containing other resins.

Shuart [2.8], Sinclair and Chamis [2.9], and Colvin and Swanson [2.3] investigated the failure modes of composites loaded in compression. Of these investigators, only Colvin and Swanson investigated the failure modes of axially loaded tubes. Their research concluded that microbuckling governs the failure in the $0^{\circ}$-fiber-dominated graphite/epoxy tubes in their program. Shuart and Sinclair and Chamis concluded that interlaminar shear is the dominant mode of failure in low-angle $\left(\leq 15^{\circ}\right)$, compressively loaded composites.

Although few data are available on the compressive strengths of axially loaded tubes, no data are currently available on the cryogenic compressive properties of E-glass/epoxy tubes. As early as 1964, Brink demonstrated that the tensile strength of E-glass/epoxy increased with a change from room temperature to $20 \mathrm{~K}\left(-424^{\circ} \mathrm{F}\right)[2.10]$. Hanson et al. investigated the strength of filament-wound glass-reinforced plastics (uniaxial, NDL rings, cylinders) at cryogenic temperatures [2.11]. His results agree with those of Brink: tensile strength increases with decreasing temperature. Neither researcher conducted compression tests at low temperatures.

\section{Materials}

For economic reasons, E-glass was chosen as the reinforcement fiber for the struts. An S2-glass fiber was used in one of the filament-wound lay-ups for comparison. Typically, the Eglass fiber was a continuous-strand, silane-treated, $1100 \mathrm{TEX}$ (450 $\mathrm{yd} / \mathrm{lb}$ ) product having the following reported nominal values:

$$
\begin{aligned}
\text { tensile strength: } & 2210 \mathrm{MPa}(320 \mathrm{ksi}) \\
\text { tensile modulus: } & 72.4 \mathrm{GPa}(10.5 \mathrm{Msi}) \\
\text { density: } & 2.59 \mathrm{~g} / \mathrm{cm}^{3}\left(0.0935 \mathrm{lb} / \mathrm{in}^{3}\right) \\
\text { coefficient of linear } & \\
\text { thermal expansion: } & 5.4 \times 10^{-6} \mathrm{~mm} / \mathrm{mm} /{ }^{\circ} \mathrm{C}\left(3 \times 10^{-6} \mathrm{in} / \mathrm{in} /{ }^{\circ} \mathrm{F}\right)
\end{aligned}
$$

The resins chosen for the fiber-reinforced composites in this study and the corresponding composite-production methods are given in Table 3.1. The characteristics of each resin are described in §III.3.3.2 and summarized in §III.2. 
Table 3.1 Resin systems and production methods.

\begin{tabular}{lll}
\hline \multicolumn{1}{c}{ Resin* } & Type & \multicolumn{1}{c}{$\begin{array}{c}\text { Production } \\
\text { Method }\end{array}$} \\
\hline \hline DGEBA/DCH/ARA/ACL & epoxy & filament winding \\
DGEBA/ARA/DTA & epoxy & filament winding \\
VE-EL/MEKP & vinyl ester & $\begin{array}{l}\text { filament winding } \\
\text { \& pultrusion }\end{array}$ \\
DGEBF/CTBN/AN & epoxy & $\begin{array}{l}\text { filament winding } \\
\text { pultrusion }\end{array}$ \\
DGEBA-ST/ARA/ACL/F & epoxy & $\begin{array}{l}\text { rubber expansion } \\
\text { (hand lay-up) }\end{array}$ \\
\hline \hline
\end{tabular}

*See Tables 2.1 and 2.2 of $\$ I I I .2$.

\section{Procedures}

\subsection{Tube Fabrication Methods}

The support tubes were fabricated by filament-winding, pultrusion, and rubber-expansion (hand lay-up) methods. Owing to the relatively high initial cost of pultrusion, most of the tubes produced for this study were filament-wound. Seven different lay-ups were fabricated by five different suppliers. Later in the study, one lay-up was chosen for the pultrusion process. Ten small-scale tubes were fabricated with a vinyl-ester resin and an epoxy resin, and ten largescale tubes were fabricated with an epoxy resin. Four tubes were fabricated with the rubberexpansion method. Filament-wound tubes were made by manufacturers referred to as A, B, and $\mathrm{C}$; pultruded tubes, by $\mathrm{P}$; and rubber-expansion tubes, by $\mathrm{R}$. The details of tube construction are given in Appendix A.

One of the objectives of this study was to compare the mechanical properties of tubes produced by pultrusion to those produced by filament winding. Although the initial tooling costs of pultrusion are relatively high, the overall costs of the automatic, continuous process may be competitive when producing a large number of tubes.

\subsubsection{Filament Winding}

Manufacturing a small number of tubes containing an off-axis lay-up is easily and inexpensively accomplished with the filament-winding process. Higher fiber volume fractions can be obtained from filament winding than from other manufacturing processes [4.1]. Other advantages of filament winding are: low initial costs and easy adaptability of fiber off-axis orientations for optimization of tube fiber lay-ups. 
The wet filament-winding process was the predominant method of fabricating struts for this study. Dry, continuous-strand, E-glass fibers (rovings) are pulled off supply creels while maintaining a given fiber tension. The rovings are gathered and formed into a band and then drawn through the resin bath, which usually has a low viscosity, enabling easy and thorough fiber saturation. The resin-saturated band is passed through a wiping device that removes excess resin. Then a numerical or computer-controlled winding apparatus is used to wind the impregnated band onto a mandrel.

The angle of the roving band with respect to the mandrel axis is called the wind angle. Wind angles between about $10^{\circ}$ (longitudinal or axial winding) and $82^{\circ}$ (transverse or hoop winding) are obtainable; for simplicity, in this section and those following, the most nearly normal and transverse angles are often referred to as $0^{\circ}$ and $90^{\circ}$. For the tubes in this study, fiber orientation was predominantly axial. To provide lateral support for the axial fibers, hoop windings (from $90^{\circ}$ to $45^{\circ}$ ) were used in six of the seven lay-ups.

The lay-ups, described and pictured in Appendix A, ranged from axial $[ \pm 10]$ (e.g., tube type 1) to near quasi-isotropic (e.g., tube type 3). Company $C$ manufactured struts that had $0^{\circ}$ oriented fibers and off-axis fibers by filament-winding the off-axis fibers $\left( \pm 23^{\circ}\right.$ to $\pm 65^{\circ}$ ) and placing layers of unidirectional weft roving at $0^{\circ}$ along the mandrel axis. The tube with this particular lay-up is designated type 11 .

\subsubsection{Pultrusion}

High compressive strengths should be attainable in tubes produced by the pultrusion process because high fiber concentrations and fiber orientations parallel to the tube (and loading) axis are possible. The high fiber tension required to pull the fiber through the fixture reduces fiber waviness.

The pultrusion process used for the fabrication of large-scale tubes required approximately 1600 E-glass supply creels. Located in bookshelf-type holders, the creels fed continuous-strand rovings into a vertical-style pultrusion machine. The small-scale tubes required fewer creels, but were also produced in the vertical machine.

Basically, continuous-strand fibers are pulled off the supply creels, passed through a guide into a resin bath and then into a heated steel forming die. As the wetted fibers enter the die, the composite starts to gel, and as it exits, the composite is cured. A continuous strand of cured composite enters the cutting station, where parts are cut to length. Small-scale tubes were constructed in the same manner as large-scale tubes, except small-scale tubes require fewer rovings. Large-scale tubes were constructed as follows:

1. At the top of the machine, nexus cloth was placed against the vertical mandrel, followed by a 0.3 -mm-thick layer of $0^{\circ}, 250$-yield E-glass. These dry fibers passed through a resin bath and formed around the mandrel; then they passed down through a bushing, which formed the fibers to the mandrel.

2. A 0.5 -mm-thick, 250 -yield, $90^{\circ}$ hoop wrap was applied onto the thin layer of $0^{\circ}$ fibers; this layer then passed through a resin bath and down through the next bushing.

3. Three wet layers of $0^{\circ}, 57$-yield rovings were applied; each 1.8 -mm-thick layer was passed through a bushing. 
4. A 0.5 -mm-thick, 250-yield, $90^{\circ}$ hoop wrap was wetted and applied onto the previous layer and passed through the next bushing.

5. Another three layers, each $1.8 \mathrm{~mm}$ thick, of $0^{\circ}, 250$-yield rovings were wetted and passed through the next three bushings.

6. Another $90^{\circ}, 250$-yield, 0.5 -mm-thick hoop was applied and passed through the bath and bushing.

7. Another nexus layer was applied to the outside of the hoop wrap.

8. The wet fibers encompassing the mandrel entered the heated die, where the resin began to harden.

9. A caterpillar-type mechanism pulled the cured tube from the die to the area where tubes were cut to the desired length.

\subsubsection{Rubber Expansion}

Manufacturer $\mathrm{R}$ fabricated four tubes from E-glass/cyanate-ester prepreg materials in two different lay-ups: $[ \pm 10]_{n}$ and $\left[90 / \pm 45 / 0_{3} / \pm 45 / 90\right]_{45}$ tube types 26 and 27 , respectively.

A 76.2-mm-diameter mandrel was covered with a 12.7-mm-thick sheet of calendered silicone rubber. A single ply of Teflon-coated, style-104 fiberglass cloth was applied to the outside of the rubber sheet. The appropriate build-up of E-glass prepreg was placed over the Teflon-coated cloth. A cover ply of Teflon-coated cloth was then added, and the assembly was placed inside a $112-\mathrm{mm}$ i.d. aluminum mold. The mold halves were bolted together and placed in an oven at $177^{\circ} \mathrm{C}$. As the rubber inside the mold expanded during heating, the composite material was compressed against the inside surface of the aluminum mold. When the composite reached $177^{\circ} \mathrm{C}$, the expanded rubber produced a constant pressure of about $690 \mathrm{kPa}(100 \mathrm{psi})$ as the tube cured. After curing, the assembly was allowed to cool; the resulting decrease in pressure caused the rubber to contract. The mold was disassembled and the fully cured composite tube was removed and prepared for testing.

\subsection{Specimens}

\subsubsection{Production Variations}

Five different manufacturers produced the 181 tubes that were tested in compression and in fatigue. Four of the manufacturers were commercial companies; the fifth was a government research laboratory. Production variations proved to be a significant problem.

Company A supplied tube types 5 and 6 and company B, tube types 7 and 8 from identical tube-production specifications. The tube lay-up construction, number of plies, and the percentage of axial and hoop fibers are similar, but void volume was significantly different (see Appendix A). The void volume fractions of tubes manufactured by A were from 0.02 to 0.04 ; those of tubes manufactured by $B$ were from 0.08 to 0.09 . This difference can be attributed to the filament-winding process. Company A used a circular (circ) winding method for fiber compaction: Several layers of $\pm 10^{\circ}$ fibers were applied, followed by a $82^{\circ}$ circ wind. Then a dry circ (for fiber compaction) was applied and removed. This procedure was repeated until the layup was complete. Company B helically wound the axial and hoop fibers with no fiber 
compaction. This method resulted in higher void volume fractions and lower ultimate compressive and fatigue strengths.

Different nomenclature was used by the various manufacturers. Some manufacturers stated that there were two plies per layer; others, two layers per ply. A request for a $\left[0_{3} / 90\right]_{T}$ filament-wound lay-up resulted in $\left[ \pm 10_{3} / 82\right]_{T},\left[10_{3} / \pm 82\right]_{T}$, or $\left[ \pm 10_{3} / \pm 82\right]_{T}$, depending on the manufacturer.

The outside surface finish was not specified on the tubes we ordered. The surface of tubes that had been wrapped with a bleeder cloth had a rougher texture. Some tubes were supplied with a smooth, resin-rich surface. When immersed in a cryogenic fluid, the roughtextured surface and the strain gages attached to it cracked less than the surface and strain gages attached to the smooth-surfaced tubes.

Pultruded tubes were made in a vertical pultrusion machine that had a floating mandrel. The small-scale (100-mm-i.d.) pultruded tubes had an eccentric outer wall and, thus, a nonuniform wall thickness caused by nonconcentricity of the mandrel and die. About $10 \%$ of the wall thickness was composed of surface veil to lubricate the mandrel and die; this material increased the calculated area of the specimen but added nothing to the load-carrying capacity of the tube. The hoop plies of pultruded tube types 22 and 23 show considerable waviness (see Appendix A). The amount of hoop fibers in the cross-sectional area of the tube remained constant throughout the wall thickness; the amount of axial fibers varied with the location on the tube diameter. The thickest section of the wall contained about $77 \%$ axial fibers; the thinnest section, as low as $54 \%$. This variation probably reduced the strengths of the small-scale pultruded tubes.

The large-scale pultruded tubes, although more difficult to manufacture, were more uniform in thickness. A floating mandrel and surfacing veil (6\% of the wall thickness) were used in their production also. The hoop plies were smooth and uniform, as seen in the photo of tube type 24 in Appendix A.

Thus, specifying a lay-up with "standard" nomenclature does not always result in a tube with the desired construction. To obtain a specific type of tube, one must specify

1. lay-up in detailed drawings (ply by ply or layer by layer)

2. the percentage of axial, off-axis, and hoop fibers

3. fiber volume fraction

4. maximum acceptable void volume fraction

5. fiber lay-up method for layers or plies (helical or circ, or a combination)

6. fiber compaction, if desired

7. exterior surface finish

8. internal diameter tolerances (external diameter tolerance may need to be flexible)

9. inner- and outer-diameter concentricity tolerance

10. maximum acceptable fiber waviness

11. fiber type, diameter, and finish

12. end-to-end tolerance (parallelism)

13. resin and cure schedule 
The first tube order achieved one of the goals of the program: to determine whether significant differences exist in tubes produced by different manufacturers. The manufacturers were very willing to work through problems in production methods, often at their own expense. Given more time and effort, the differences may have been eliminated, but production variations that affected tube strength were confirmed and the minimum specifications for tube construction became evident.

\subsubsection{Lay-ups}

Currently no data exist on composite tube design for maximizing axial compressive strength while minimizing thermal conductivity. Theoretically, a tube constructed entirely of axial $\left(0^{\circ}\right)$ fibers should have the highest compressive strength as well as the highest thermal conductivity, whereas a tube constructed entirely of hoop-wound $\left(90^{\circ}\right)$ fibers should have the lowest strength and the lowest thermal conductivity.

The lay-ups used in this study are detailed in Appendix A. To maximize strength, the lay-ups were predominately axially oriented. Off-axis plies and hoop plies were added to improve tube radial strength. The nature of helical filament-winding machines makes it difficult to filament-wind $0^{\circ}$ axial plies. It can be accomplished by filament-winding onto pins or spokes at the ends of the mandrel or by combining filament winding and hand lay-up, as shown in the lay-up of tube type 11. However, the pinned ends and the hand lay-up increase fabrication difficulty and costs and thus are not practical for SMES tube construction.

In filament-wound tubes, the so-called axial plies are approximately $9^{\circ}$ to $10^{\circ}$ off the mandrel longitudinal axis, whereas the axial plies in tube type 11 are $0^{\circ}$ to $\pm 2^{\circ}$ off the axis. Tube type 26 contained $\pm 10^{\circ}$ fibers, and tube type 27 contained true $0^{\circ}$ axial fibers. The hoop, or transverse fiber, orientation in filament winding is typically $82^{\circ}$, normal to the mandrel longitudinal axis. Off-axis plies $\left(10^{\circ}\right.$ to $\left.82^{\circ}\right)$ are easily obtained in filament-winding operations.

In the pultrusion process, true $0^{\circ}$ fiber orientation is easily obtained. Hoop winds, or $90^{\circ}$ plies, are more difficult to obtain, particularly if there are multiple hoop layers. The lay-up and percentages of the layers of the large-scale filament-wound tube (type 18) are similar to those of the large-scale pultruded tube (type 24). The ratio of axial to hoop fibers in the smaller tubes was less because relatively thick nexus and cloth were included in the 5.1 -mm-thick wall.

\subsubsection{Specimen Description}

The end-cap shown in Figure 4.1 was designed to prevent brooming of the compositetube ends. No attempt was made to minimize end-cap effects owing to the large number of tubes and the various lay-ups and wall thicknesses among the tubes studied. All tubes tested had end caps of this particular design. The annular grooves in the end caps were filled with a filled epoxy that has a room-temperature cure, and the tubes were pressed into the filled groove, squeezing out the excess resin. The capped tubes were then placed in a test machine and compressively loaded to ensure that the tube ends were in close contact with the bottom of the annular groove. Testing did not begin until the epoxy had cured. 
The small-scale tubes had an inner diameter $(d)$ of $102 \mathrm{~mm}(4.0 \mathrm{in})$, diameter-to-wallthickness ratios $(d / t)$ of 10 and 20 , and length-to-diameter ratios $(\ell / d)$ of $7.0,4.75,3.5$, or 2.25. One set of large-scale tubes had a $d$ of $177.8 \mathrm{~mm}(7 \mathrm{in}), d / t$ of 20 , and $\ell / d$ of 7.0 or 3.5 . The other large-scale tubes had a $d$ of $250 \mathrm{~mm}(10 \mathrm{in}), d / t$ of 20 , and $\ell / d$ of 7.0 or 3.5 .

The ends of the tubes were machined flat and parallel to each other within $0.05 \mathrm{~mm}$ (0.002 in) and square to the inside diameter within $0.03 \mathrm{~mm}(0.001 \mathrm{in})$.

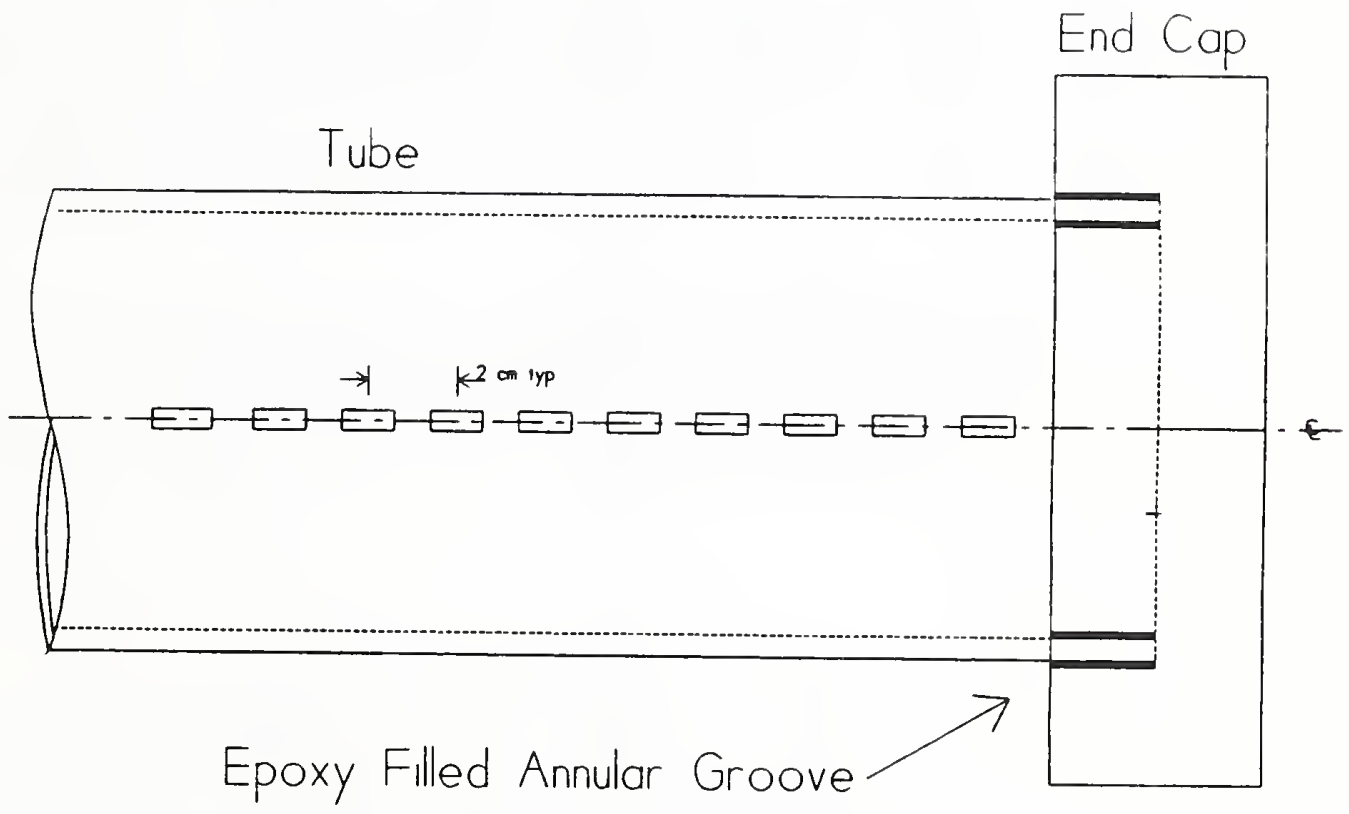

Figure 4.1. Tube with end cap and extra axial gages.

\subsubsection{Specimen Instrumentation}

All the tubes tested were instrumented with strain gages, ${ }^{*}$ which were selected on the basis of test temperature. Room-temperature (295-K) tests were instrumented with constantan alloy, self-temperature-compensating (STC), 350- $\Omega$ strain gages that were attached with a cyanoacrylate adhesive that cures at room temperature. The low-temperature tests were instrumented with a modified nickel-chromium-alloy (similar to karma), STC, 350- $\Omega$ strain gages that were attached with a two-part adhesive that has a slightly elevated temperature cure $\left(66^{\circ} \mathrm{C}\right)$.

Typically, the tubes had 18 strain gages, as shown in Figure 4.2. Note that there were strain gages inside the tube, which were used to minimize error in determining Poisson's ratio.

\footnotetext{
${ }^{*}$ Exception: Extensometers were attached to tube type 16 (between the end caps) during 76-K testing. The extensometer data are very limited because the extensometers were ruined during catastrophic failure of the tubes.
} 
A few of the tubes had extra axial gages, spaced about $2 \mathrm{~cm}$ apart, as shown in Figure 4.1. Photographs of the loading fixture and strain-gage extensometers are shown in Appendix B.

During fatigue tests, the changes in modulus were monitored on the small-scale tubes by means of an extensometer mounted between the end caps.

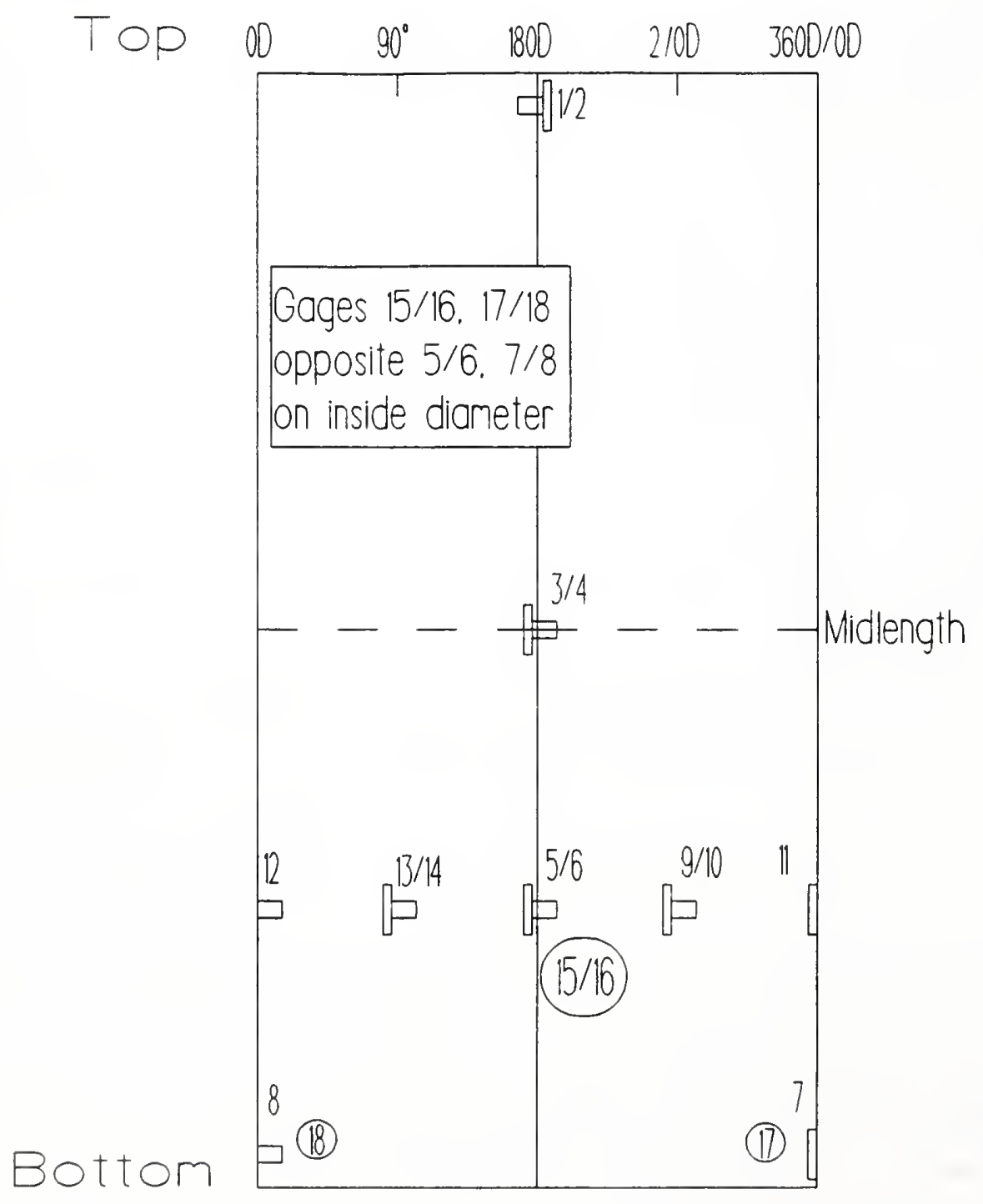

Figure 4.2. Typical strain-gage location. 


\subsection{Tests}

\subsubsection{Compression Tests}

For small-scale tube tests at room temperature, the instrumented tubes were placed in a 4.5-MN $\left(1 \times 10^{6}-\mathrm{lbf}\right)$ servohydraulic fatigue testing machine. The specimens were compressively loaded at a stroke-controlled rate of $0.01 \mathrm{\ell} / \mathrm{min}$, where $\ell$ is tube length. Specimens were loaded to failure; load and strain data were recorded at 1 -s intervals by a computer-controlled data-acquisition system.

Low-temperature tests were conducted in a specially constructed cryostat installed in the 4.5-MN test machine. The cryostat was designed so that the tests could be conducted in either liquid nitrogen $(76 \mathrm{~K})$ or liquid helium $(4 \mathrm{~K})$. The specimens were totally immersed in the cryogen, and their temperature was allowed to stabilize before testing. Strain gages were rebalanced after reaching the test temperature, and the tubes were loaded to failure while recording load and strain.

Tests of large-scale tubes were conducted at room temperature and at $76 \mathrm{~K}$ in a 52.8-MN $\left(12 \times 10^{6}-\mathrm{lbf}\right)$ test machine. The test procedures were identical to those of smallscale tubes, except that the cryogenic tests were conducted in a specially constructed, bottomloading Dewar. Again, load and strain-to-failure data were recorded with a computer-controlled data-acquisition system.

\subsubsection{Fatigue Tests}

Fatigue tests were performed on small-scale specimens in a $1-\mathrm{MN}(220,000 \mathrm{lbf})$ servohydraulic fatigue machine. The tubes were cycled in load control at $\leq 1 \mathrm{~Hz}$ (sinusoidal waveform); the frequency was adjusted to minimize heating in the tubes. A ratio of 0.1 was used for the compression/compression fatigue tests. Overall compliance was measured by using an extensometer mounted between the specimen end caps. Tests at $76 \mathrm{~K}$ used the specially constructed Dewar described in $\$ 4.2 .1$.

Initially, tests were to be conducted at 60,70 , and $80 \%$ average ultimate compressive strength (UCS) of the tubes. However, since some of the $80 \%$ UCS tests exceeded the load capacity of our test machine, most of the lay-ups were tested only at 60 and $70 \%$.

Two large-scale specimens were tested in the 4.5-MN servohydraulic fatigue machine at a frequency of $0.25 \mathrm{~Hz}$ and a load ratio of 0.1 . The test setup, shown in Fig. 4.3, enabled two fatigue specimens to be tested simultaneously. The filament-wound tubes were type 18 (see Appendix A) with a $\left[82 / \pm 10_{6} / 82 / \pm 10_{6} / 82\right]$ fiber lay-up. The lower specimen was totally immersed in liquid nitrogen. The upper specimen had a temperature gradient of $219 \mathrm{~K}(295 \mathrm{~K}$ at the upper end cap; $76 \mathrm{~K}$ at the lower end cap); its temperature was monitored throughout the test. The temperature gradient along the specimen ax is is shown in Figure 4.4. Load versus displacement was recorded for each of the two tubes during the test. The maximum fatigue stress was $345 \mathrm{MPa}, 63 \%$ of the average $295-\mathrm{K}$ UCS of the large-scale filament-wound tubes. The $76-\mathrm{K}$ tube was fatigued at $55 \%$ of the average $76-\mathrm{K}$ UCS of the large-scale filament-wound tubes. 


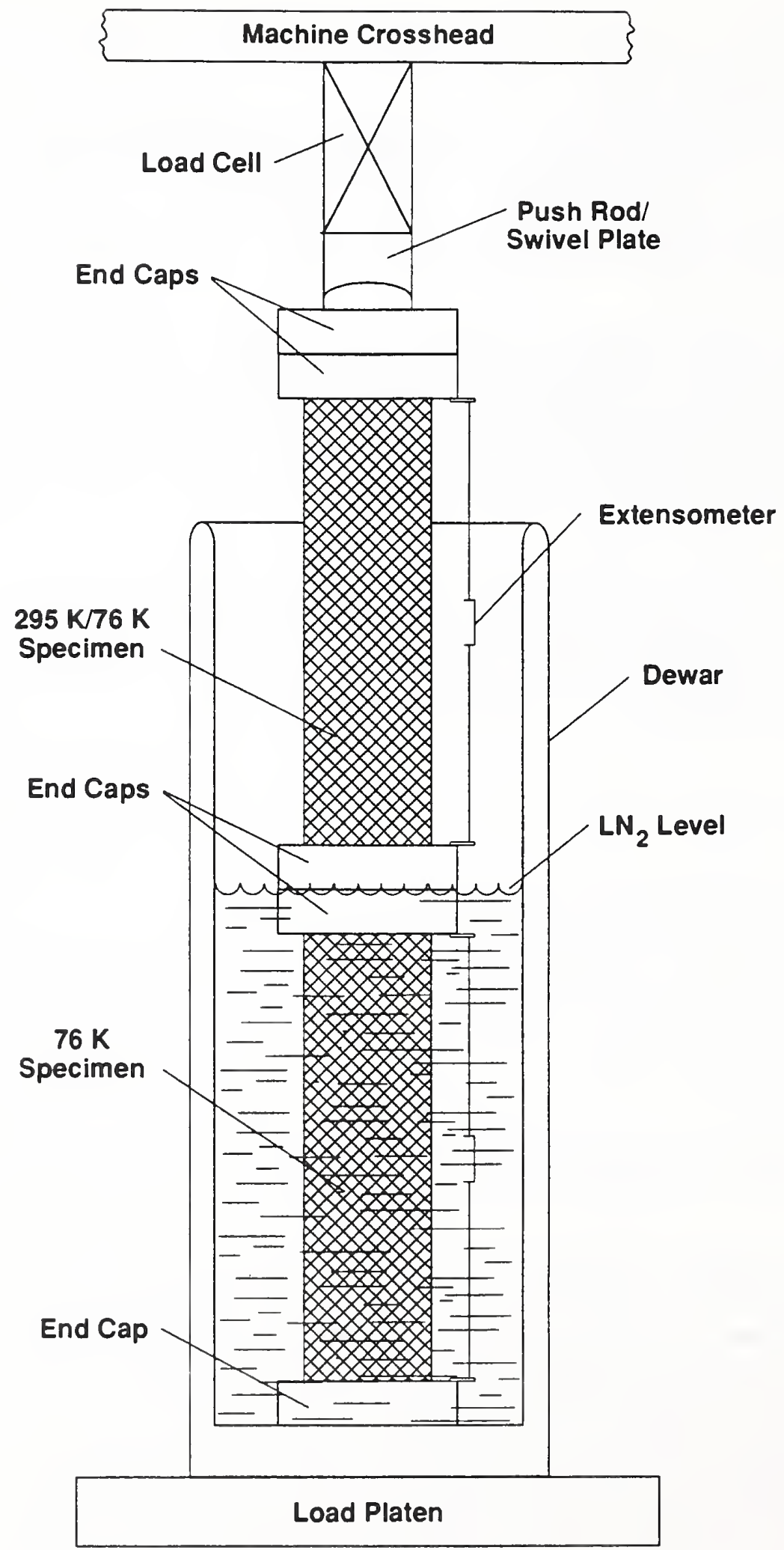

Figure 4.3. Fatigue test setup for two specimens. 


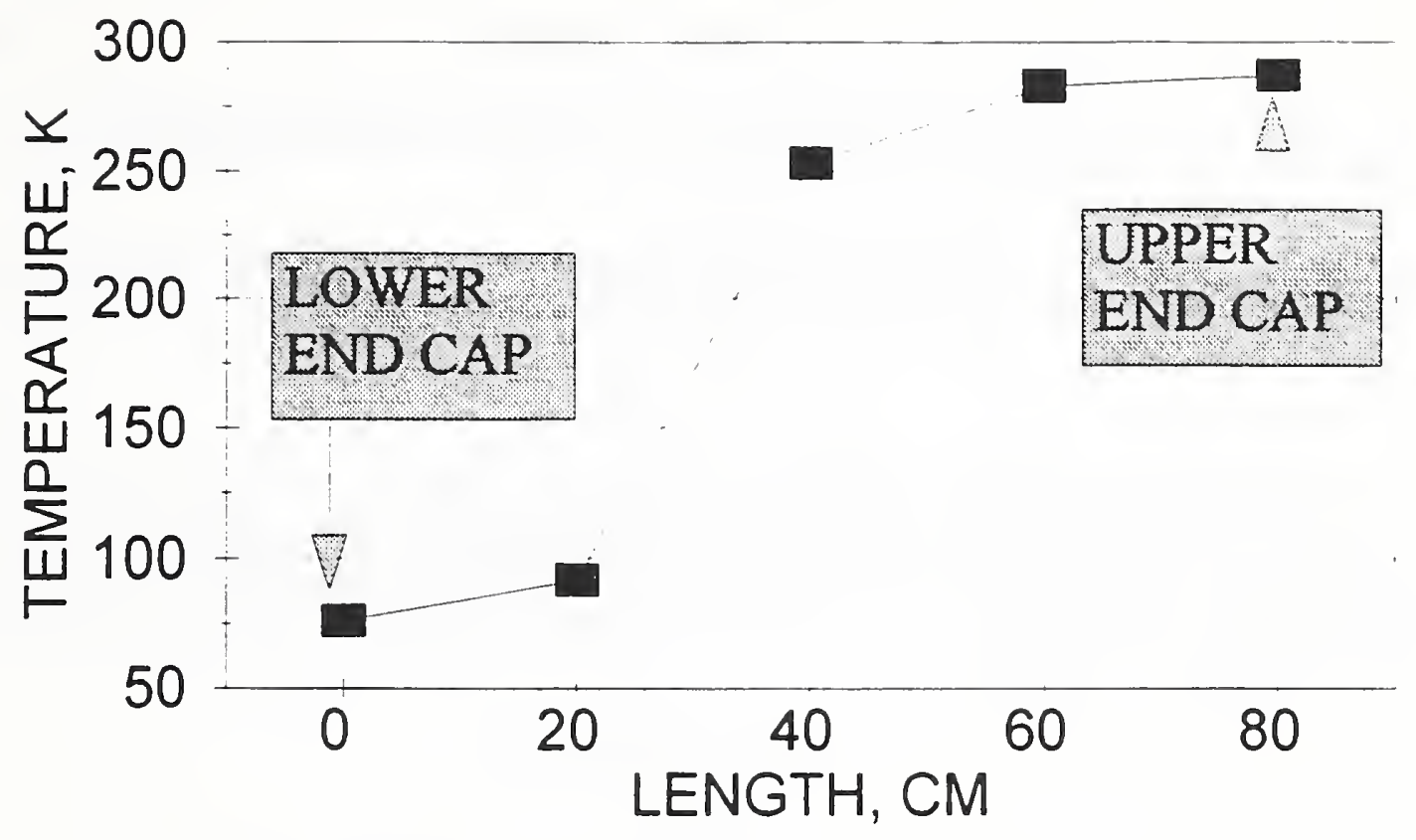

Figure 4.4. Temperature gradient along the axis of the upper specimen in the fatigue test setup shown in Figure 4.3.

\section{Results}

\subsection{Compression Tests}

Tube strength versus temperature for different materials and lay-ups are plotted in Figures 5.1 through 5.13. Compression data for the various tube types are summarized in Table 5.1.

The inside and outside diameters were measured at eight locations, and the cross-sectional area was calculated from the average of those dimensions:

$$
A=\frac{\pi}{4}\left\{(\text { o.d.avg })^{2}-(\text { i.d.avg })^{2}\right\} \text {. }
$$

The maximum load was determined by a calibrated load cell and recorded by the dataacquisition system, and the ultimate compressive stress was calculated by dividing the ultimate compressive load by the cross-sectional area of the tube. Maximum strain to failure was calculated by averaging the readings of the four axial strain gages located on the outside of the midsection of the tube. 
To minimize the error in determining Poisson's ratio $(\nu)$, the inside and outside circumferential strains were averaged and divided by the axial strain:

$$
\nu=1 / 2\left(\epsilon_{\theta o}+\epsilon_{\theta i}\right)\left(\epsilon_{z o}\right)^{-1},
$$

where $\epsilon_{\theta o}$ is the outside circumferential strain, $\epsilon_{\theta i}$ is the inside circumferential strain, and $\epsilon_{z o}$ is measured by the strain gages on the outside wall.

The moduli were calculated on the initial slope of the stress-strain $(\sigma-\epsilon)$ curve by using the average strain determined by the four circumferential axial strain gages:

$$
E=\Delta \sigma / \Delta \epsilon_{\mathrm{avg}} .
$$

The approximate failure locations are identified in Table 5.1, and photographs of typical tube failures are included in Appendix B. Also in Appendix B, a sequence of photographs at 0.001 -s intervals demonstrates that it is not possible to distinguish transverse from axial fiber crack initiation within these short time intervals. Other descriptions, analyses, and photographs of tube failures are given in $\S I X$.

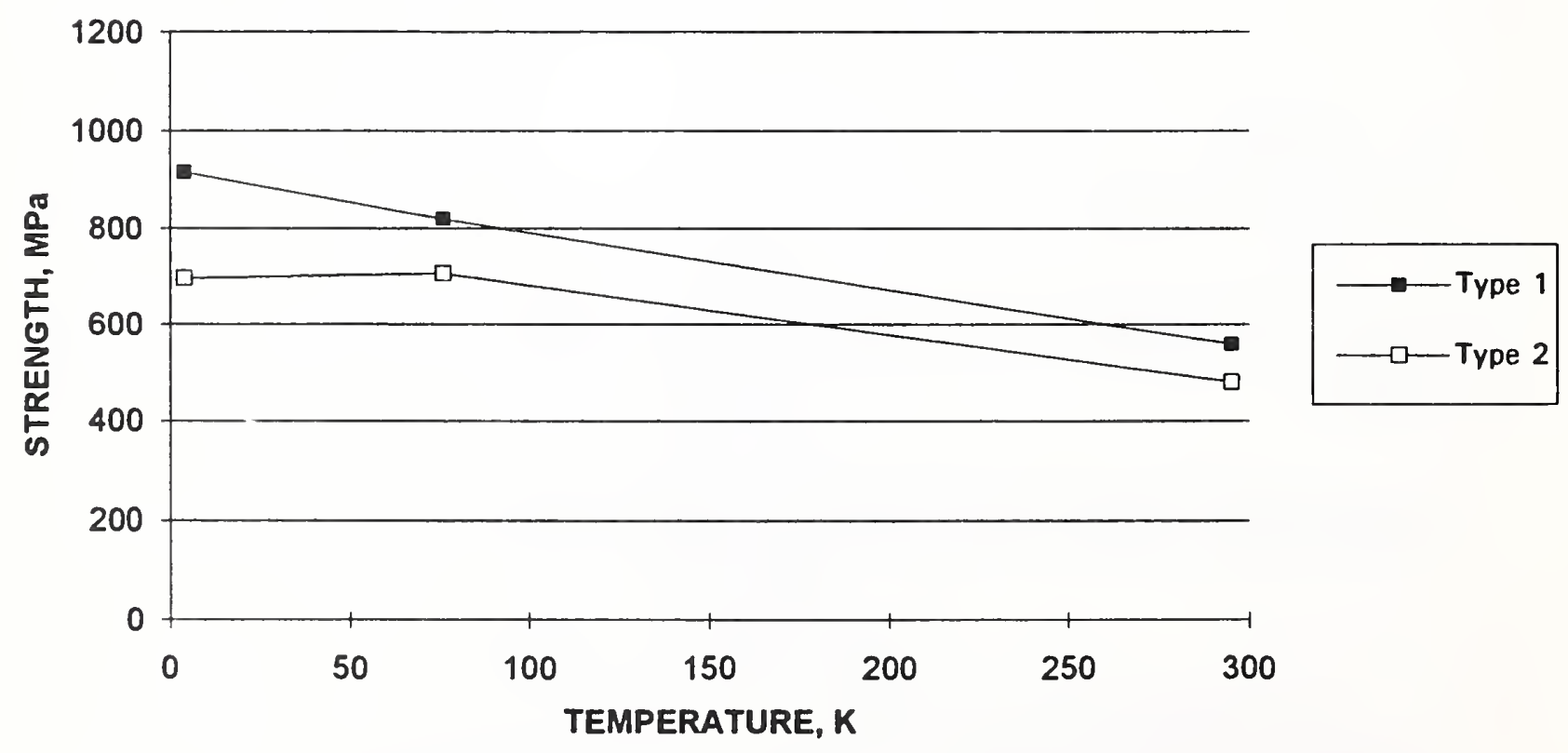

$$
\text { DGEBA/DCH/ARA/ACL epoxy; }[ \pm 10]_{n}
$$

Figure 5.1. Strength of tube types $1(t=10.2 \mathrm{~mm})$ and $2(t=5.1 \mathrm{~mm})$ versus temperature. 


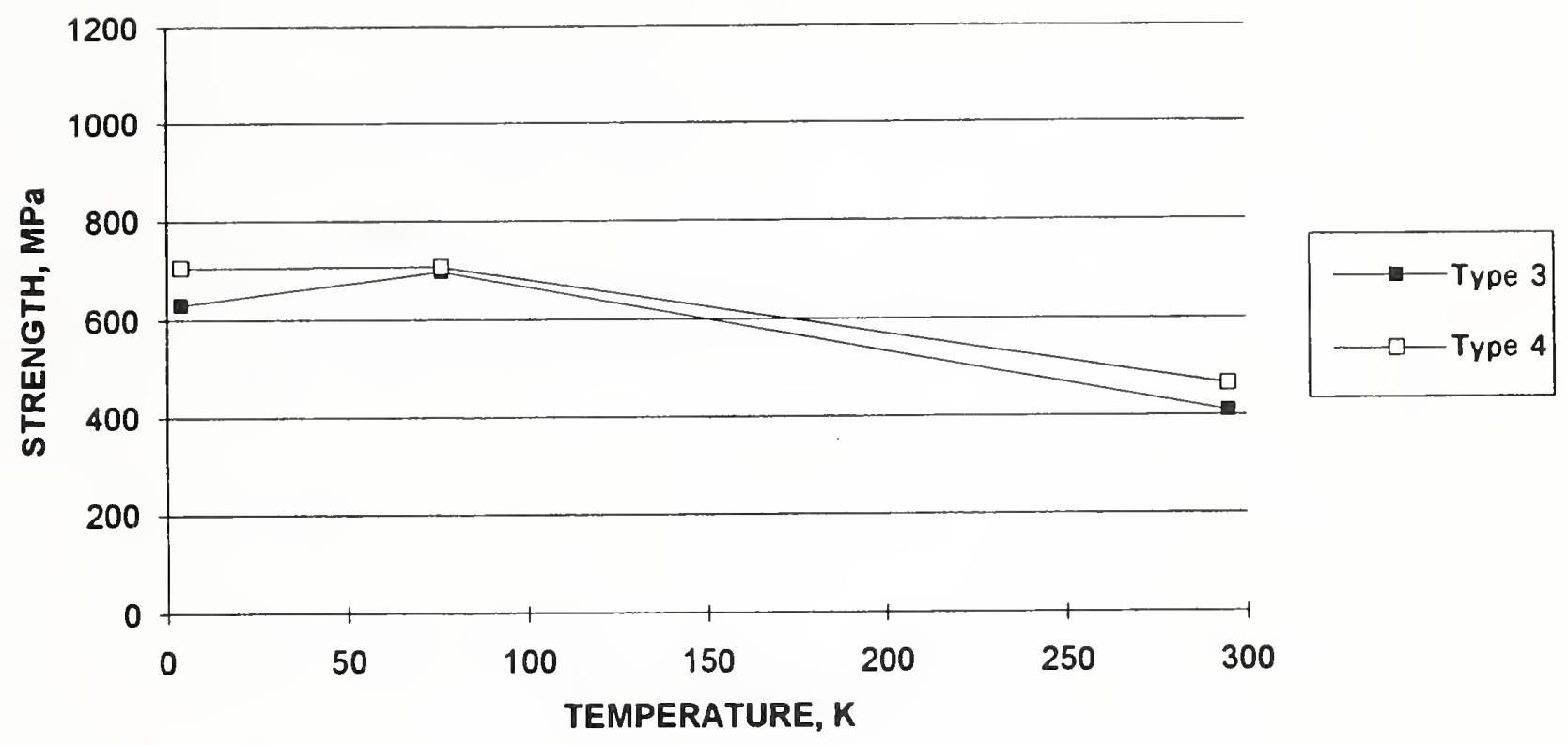

DGEBA/DCH/ARA/ACL epoxy; 50\% [ \pm 10$], 33 \%[ \pm 45], 17 \%[ \pm 82]$

Figure 5.2. Strength of tube types $3(t=10.2 \mathrm{~mm})$ and $4(t=5.1 \mathrm{~mm})$ versus temperature.

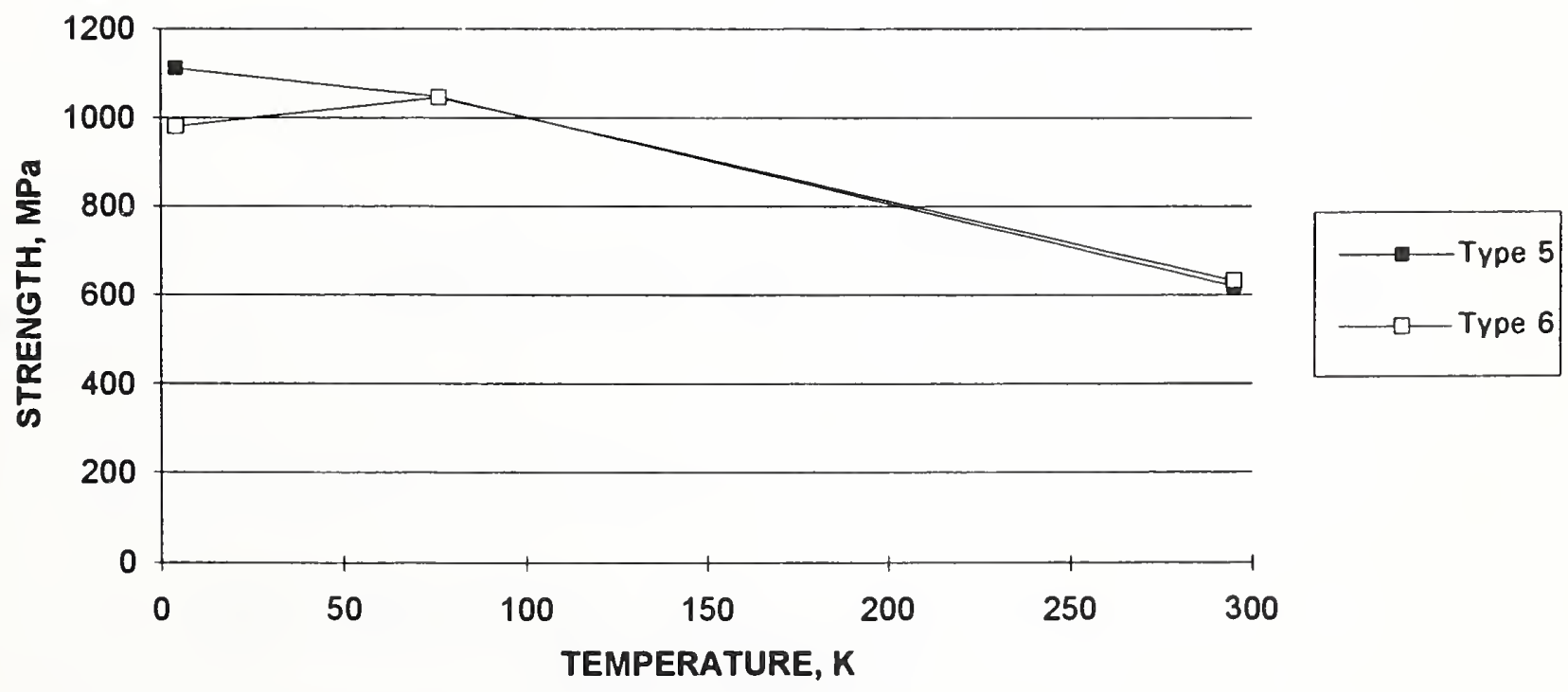

DGEBA/DCH/ARA/ACL epoxy;

tube type 5: 90\% $[ \pm 10], 10 \%[82]$; tube type 6: 85\% [ \pm 10$], 15 \%[82]$

Figure 5.3. Strength of tube types $5(t=10.2 \mathrm{~mm})$ and $6(t=5.1 \mathrm{~mm})$ versus temperature. 


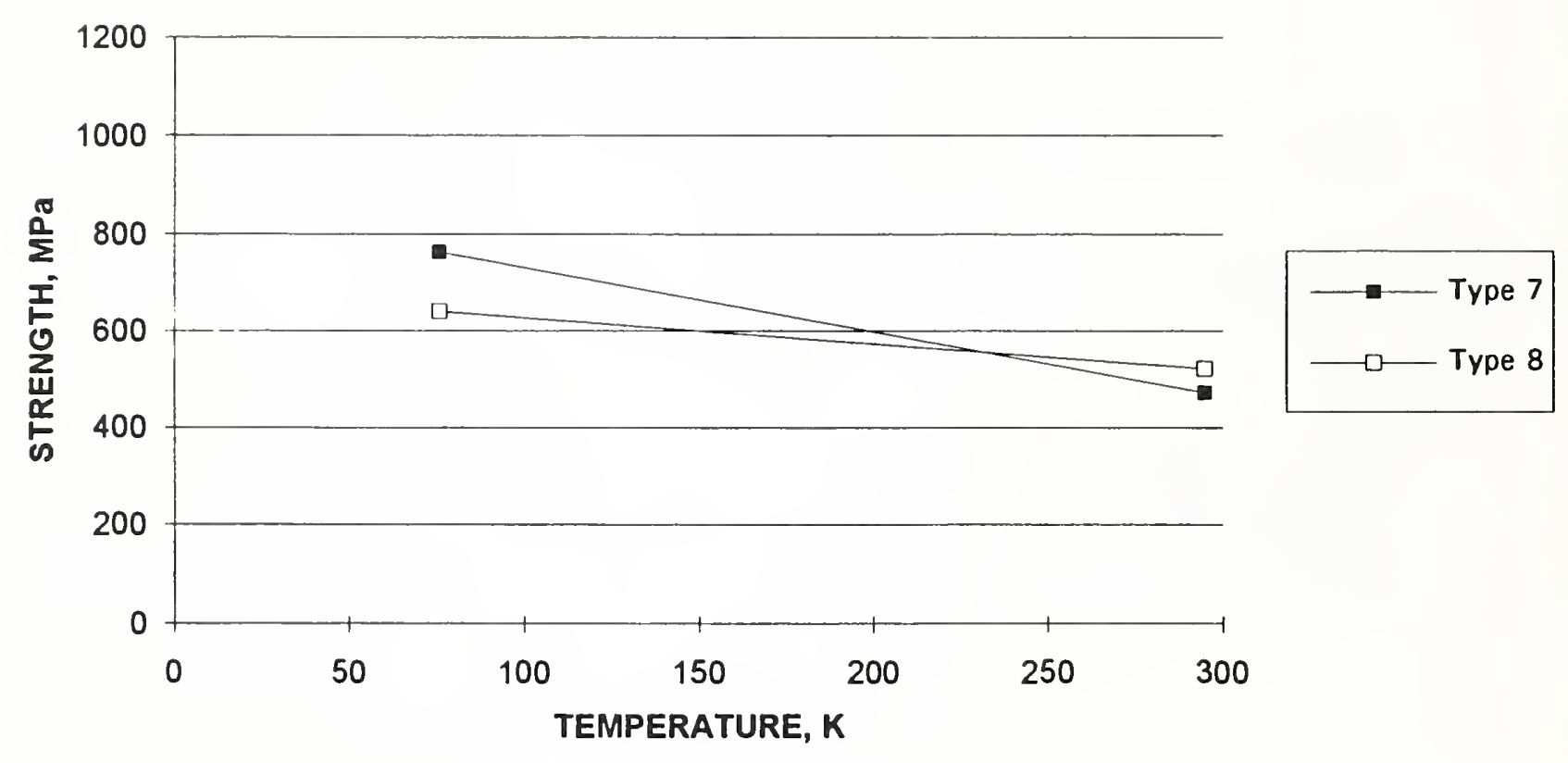

DGEBA/ARA/DTA epoxy; tube type $7: 85 \%[ \pm 9], 15 \%$ [82]; tube type $8: 84 \%[ \pm 9], 16 \%[82]$

Figure 5.4. Strength of tube types $7(t=10.2 \mathrm{~mm})$ and $8(t=5.1 \mathrm{~mm})$ versus temperature.

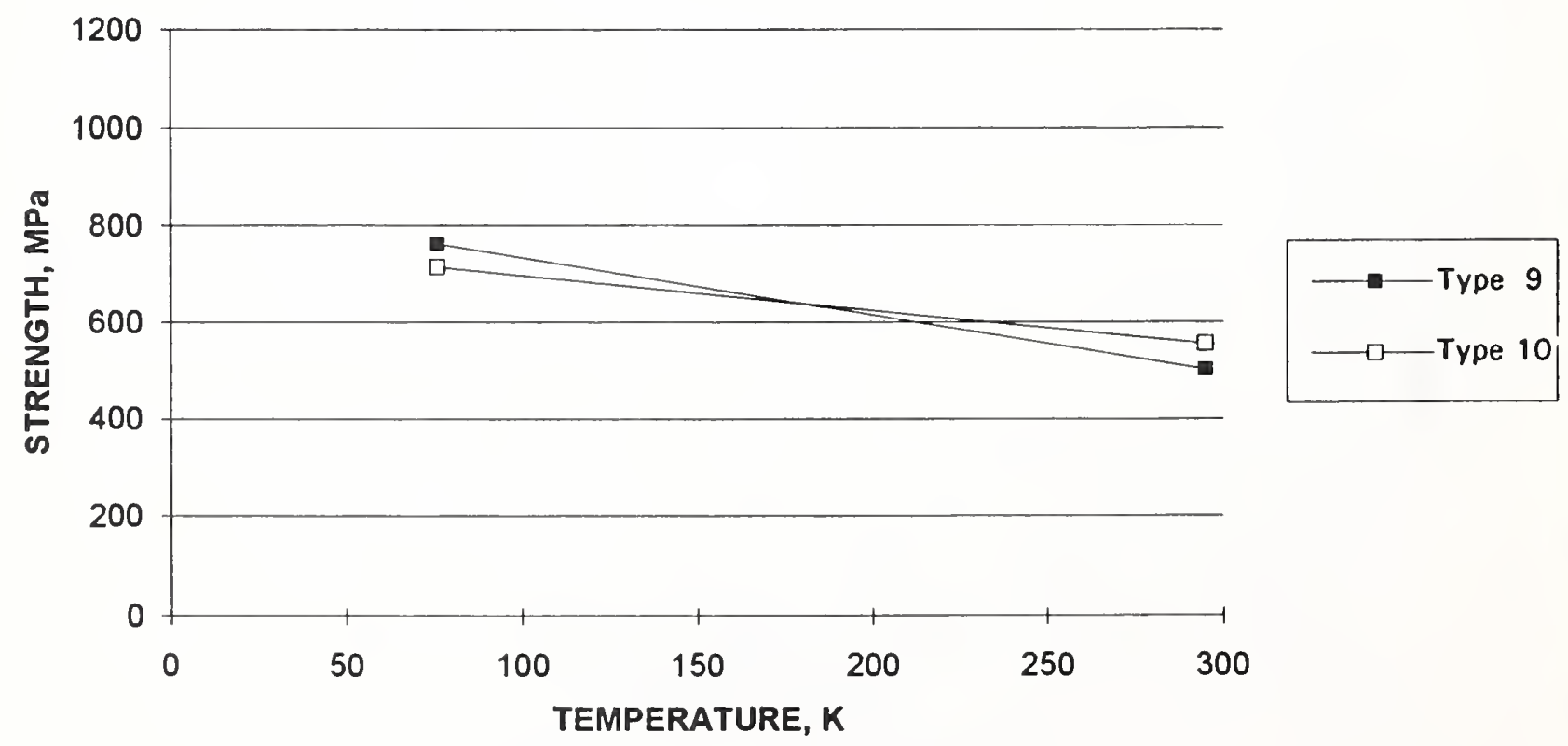

VE-EL/MEKP vinyl ester; tube type 9: $86 \%[ \pm 9], 14 \%$ [82];

tube type 10: $83 \%[ \pm 9], 17 \%[82]$

Figure 5.5. Strength of tube types $9(t=10.2 \mathrm{~mm})$ and $10(t=5.1 \mathrm{~mm})$ versus temperature. 


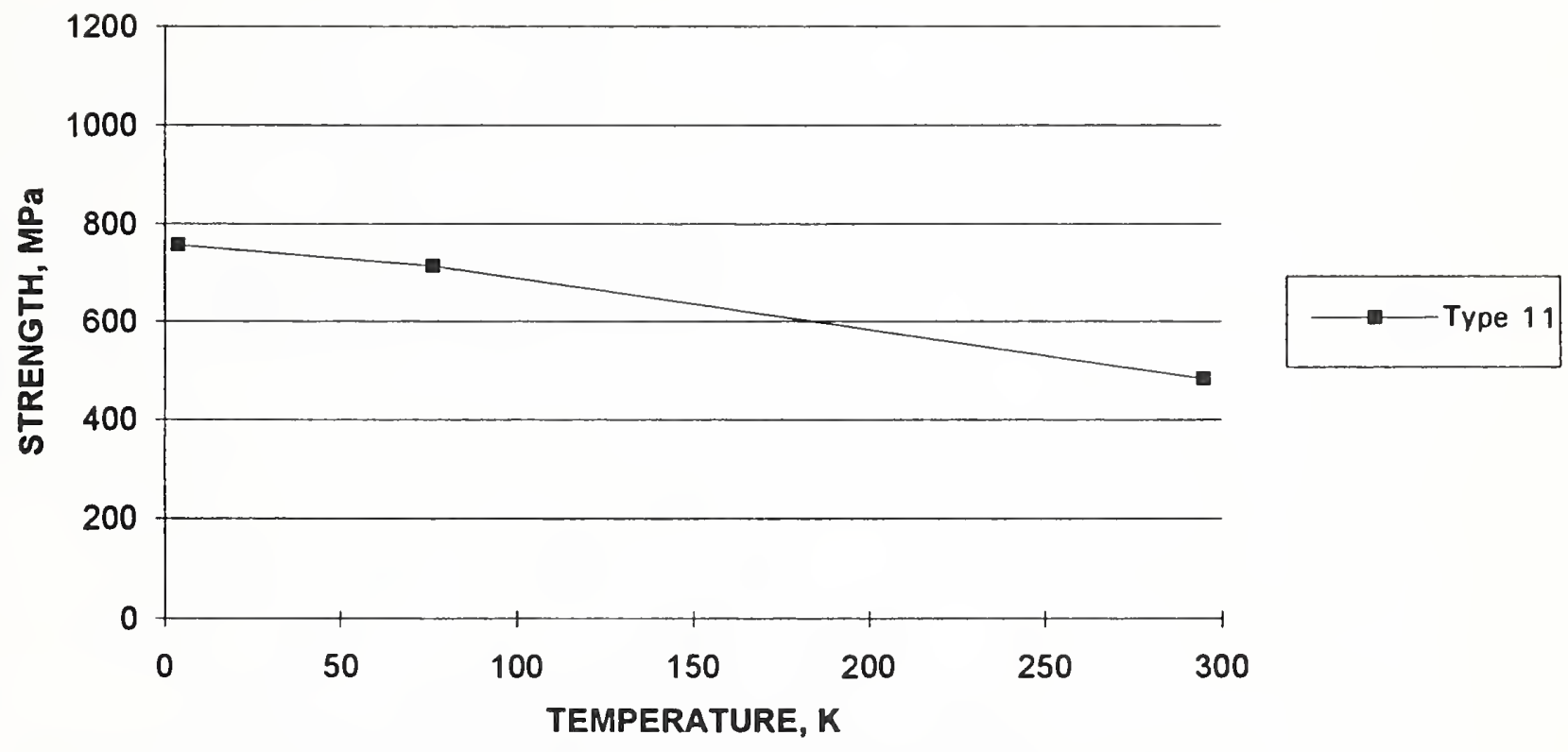

VE-EL/MEKP vinyl ester; 52\% [0], 19\% [ \pm 65$], 29 \%[ \pm 23]$

Figure 5.6. Strength of tube type $11(t=10.2 \mathrm{~mm})$ versus temperature.

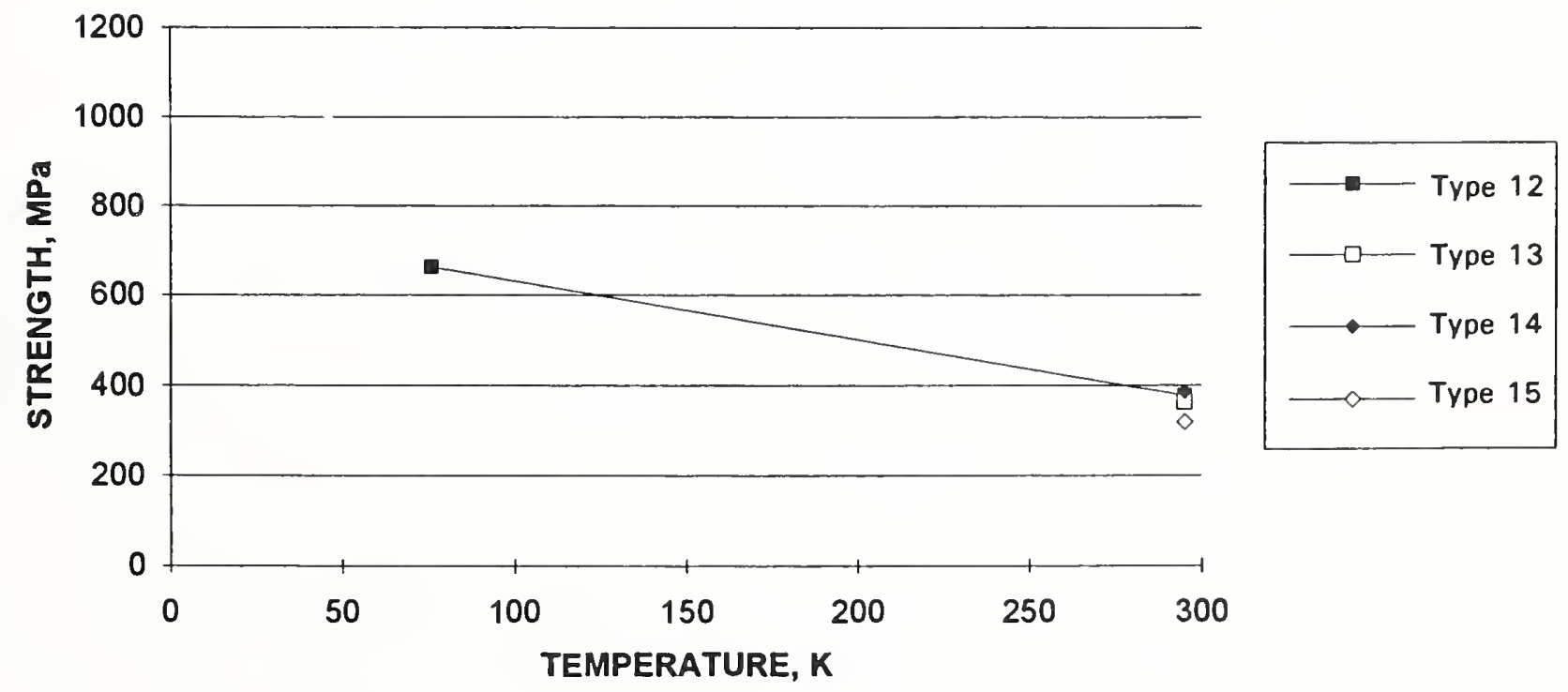

tube types 12 \& 13: DGEBA/ARA/DTA epoxy; tube types 14 \& 15:

DGEBF/CTBN/AN epoxy; 60\% [ \pm 10$], 40 \%$ [ \pm 45$]$

Figure 5.7. Strength of tube types 12 through $15(t=5.1 \mathrm{~mm})$ versus temperature. 


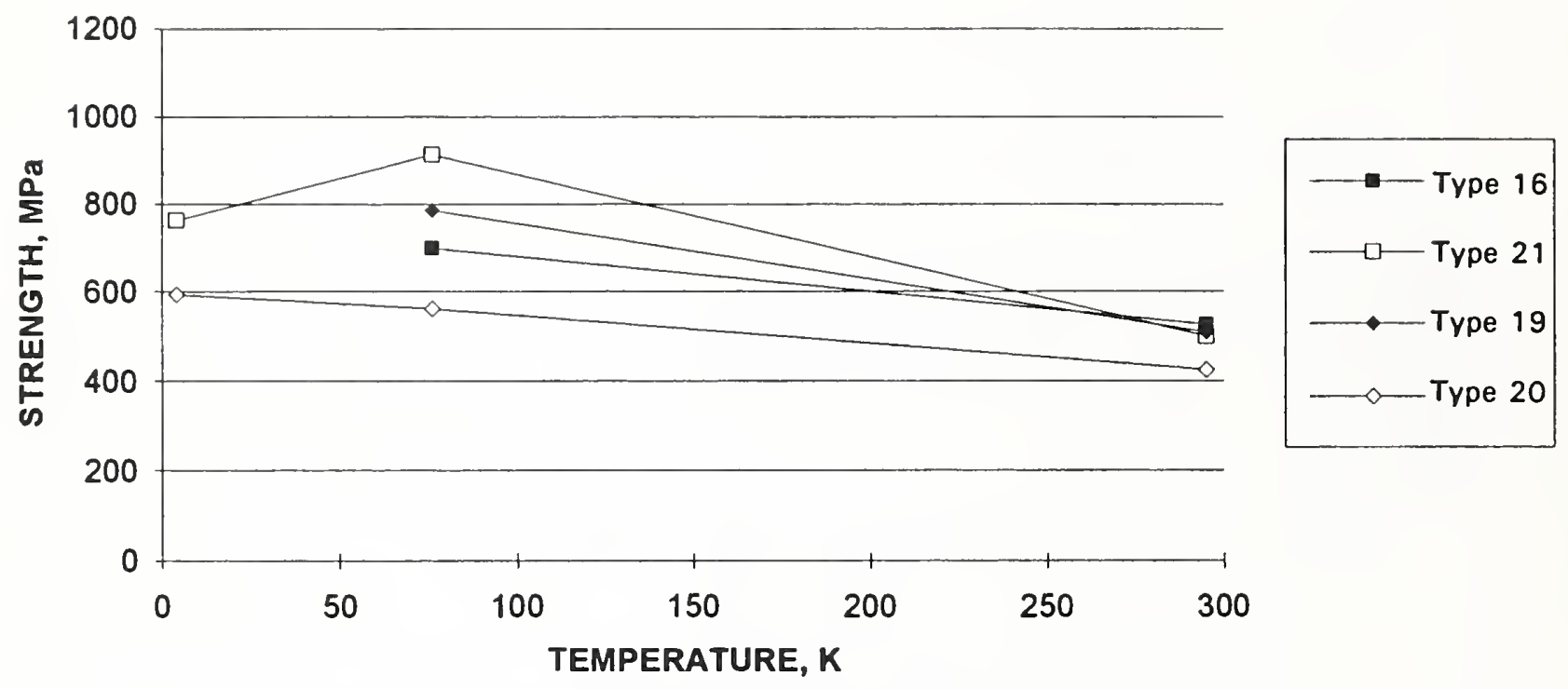

$90 \%[ \pm 10], 10 \%[82] ;$ tube type 16: DGEBA/DCH/ARA/ACL epoxy; tube types 19 \& 20: DGEBF/CTBN/AN epoxy; tube type 21: VE-EL-MEKP vinyl ester; tubes types 16, 19, 21: E-glass fibers; tube type 20: S2-glass fiber

Figure 5.8. Strength of tube types $16,19,20$, and $21(t=5.1 \mathrm{~mm})$ versus temperature.

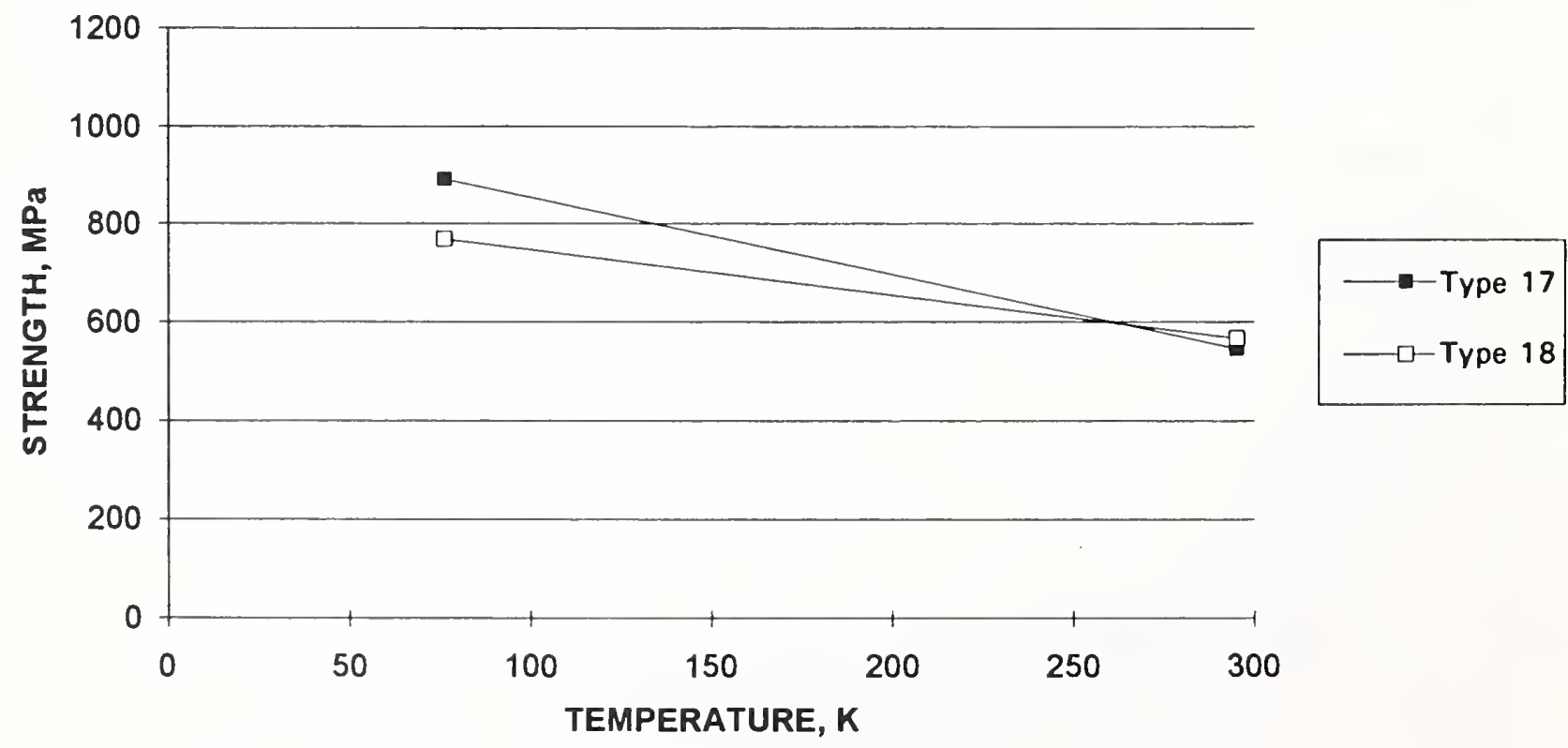

tube type 17: $92 \%[ \pm 10], 8 \%[82]$; tube type $18: 94 \%[ \pm 10], 6 \%[82]$ DGEBA/DCH/ARA/ACL epoxy

Figure 5.9. Strength of tube types $17(t=8.9 \mathrm{~mm})$ and $18(t=12.7 \mathrm{~mm})$ versus temperature. 


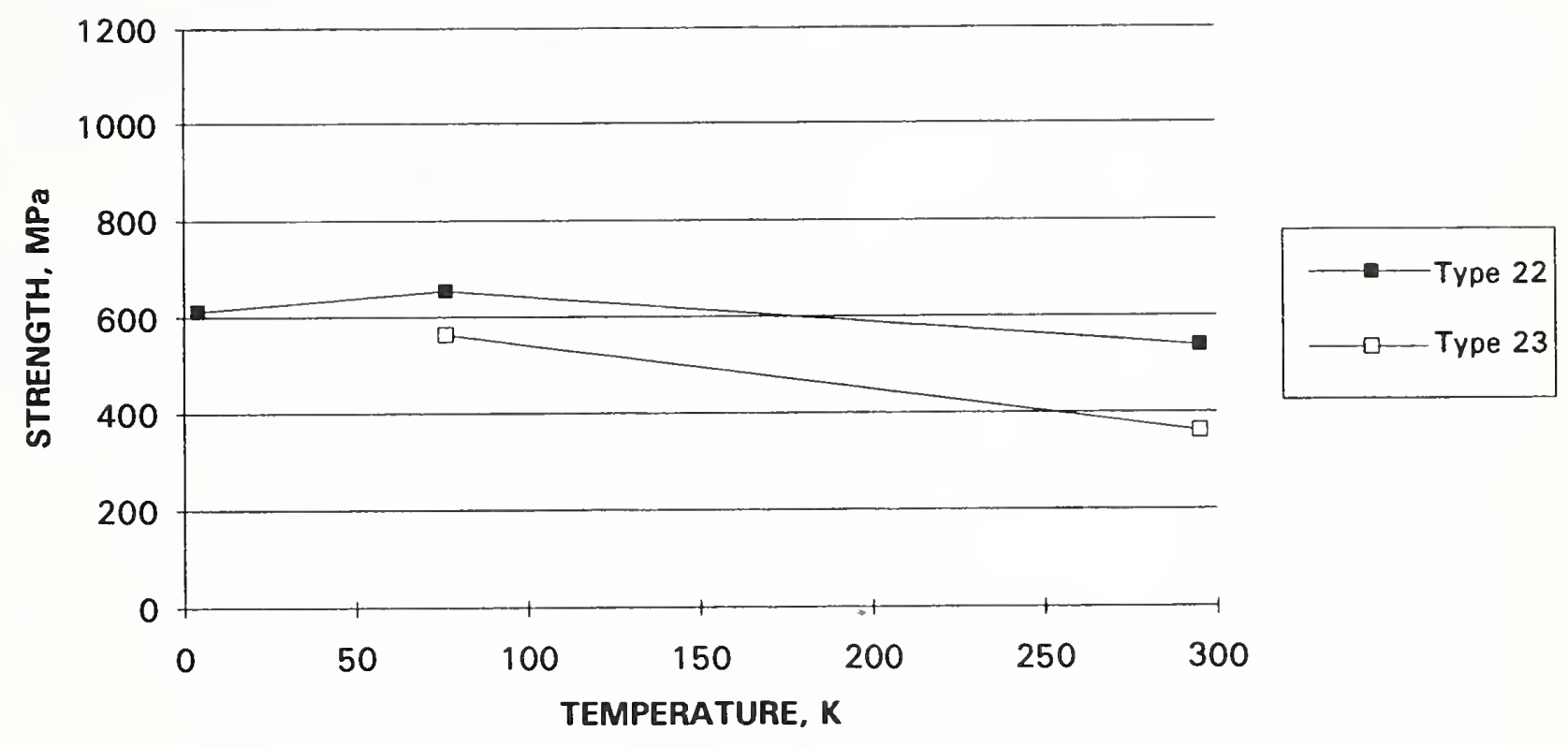

tube type 22: DGEBA-ST/ARA/ACL/F epoxy;

tube type 23: VE-EL/MEKP vinyl ester; $60 \%$ [ \pm 10$], 30 \%$ [90], 10\% Nexus and cloth; pultruded

Figure 5.10. Strength of tube types 22 and $23(t=5.1 \mathrm{~mm})$ versus temperature.

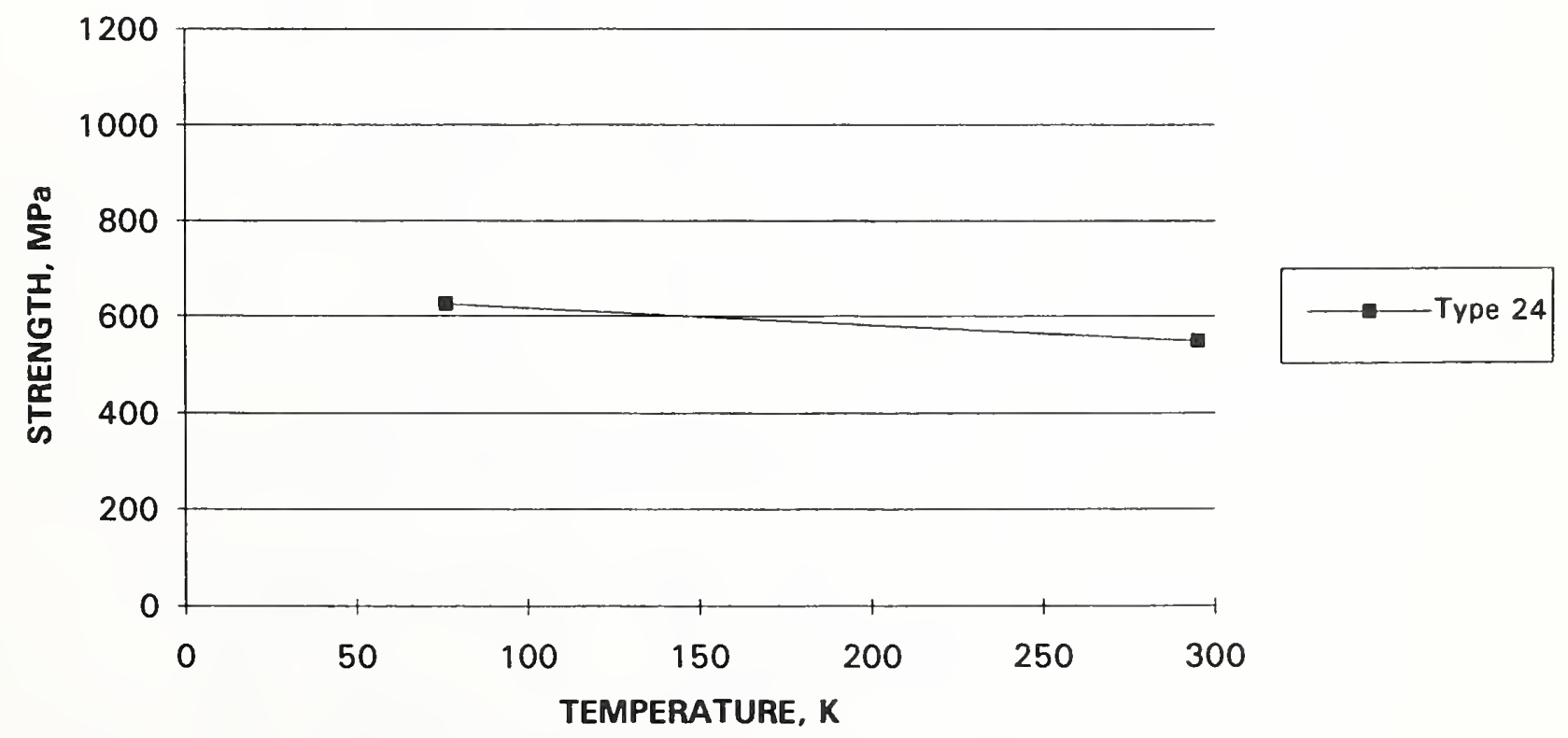

VE-EL/MEKP vinyl ester; $82 \%[ \pm 10], 12 \%$ [82], $6 \%$ Nexus and cloth; pultruded

Figure 5.11. Strength of tube type $24(t=12.7 \mathrm{~mm})$ versus temperature. 


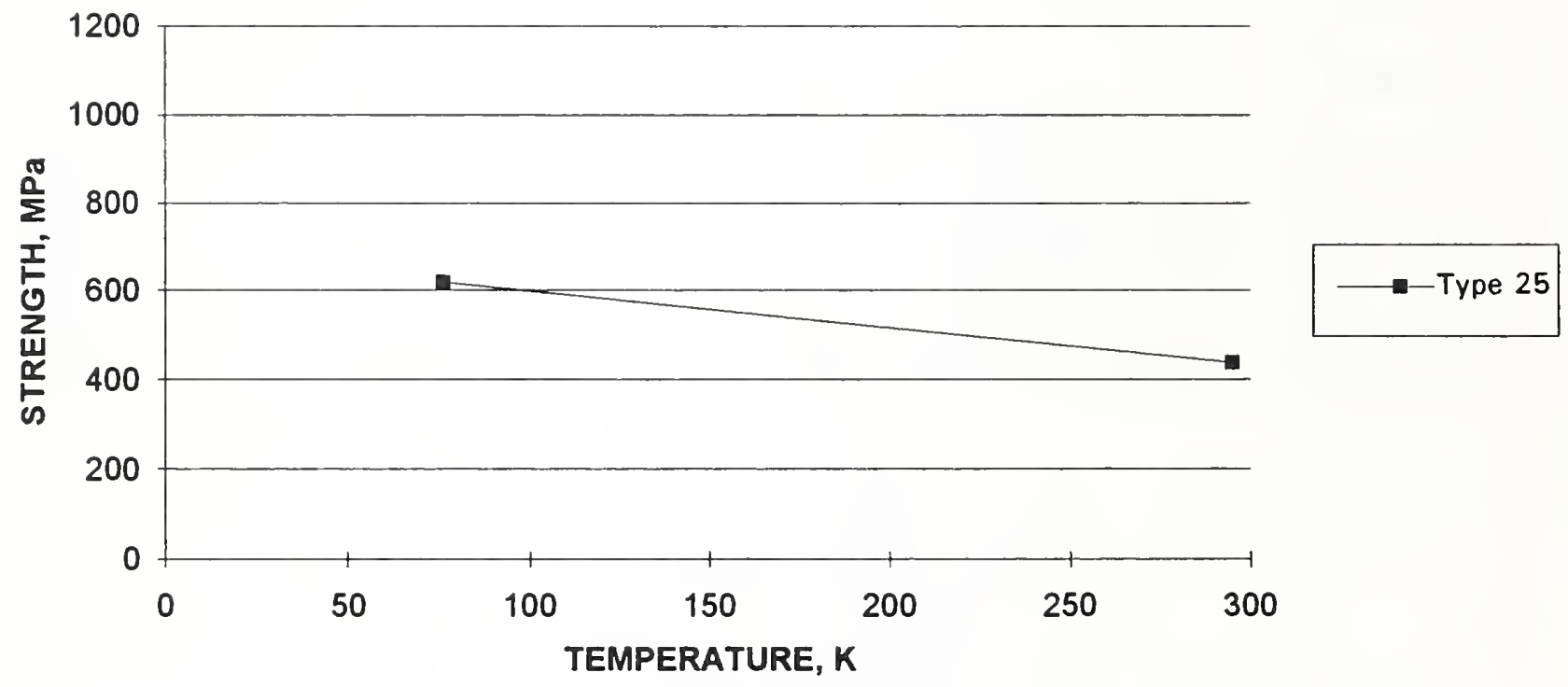

DGEBA/DCH/ARA/ACL epoxy; 77\% [ \pm 10$], 23 \%[ \pm 82]$

Figure 5.12. Strength of tube type $25(t=8.9 \mathrm{~mm})$ versus temperature.

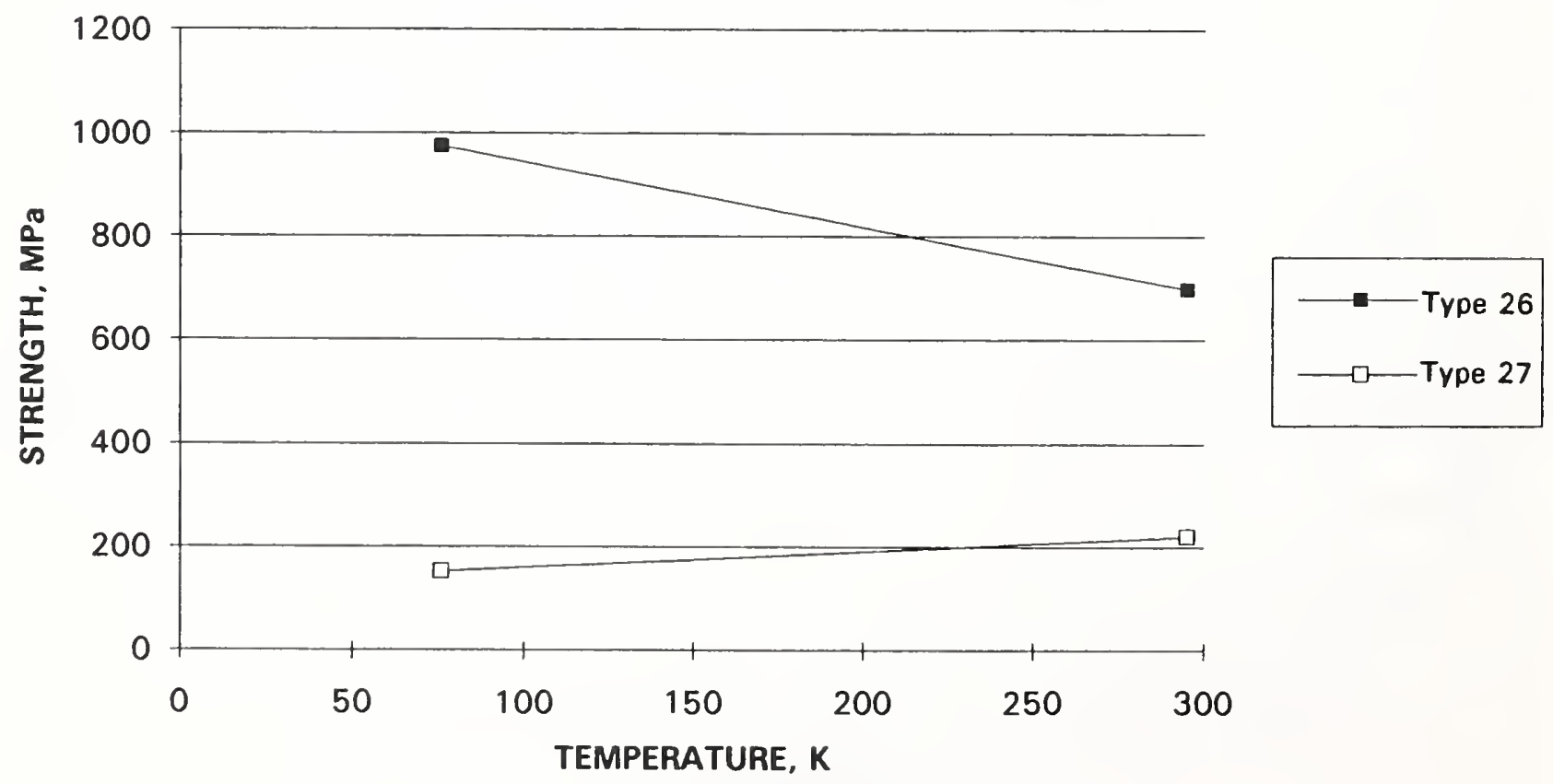

tube type $26:[ \pm 10]_{n}$; tube type $27:\left[90 / \pm 45 / 0_{3} / \pm 45 / 90\right]_{45}$;

$\mathrm{CE}$ cyanate ester; manufactured by rubber expansion + hand lay-up

Figure 5.13. Strength of tube types 26 and $27(t=5.1 \mathrm{~mm})$ versus temperature. 
Table 5.1. Results of compression tests.

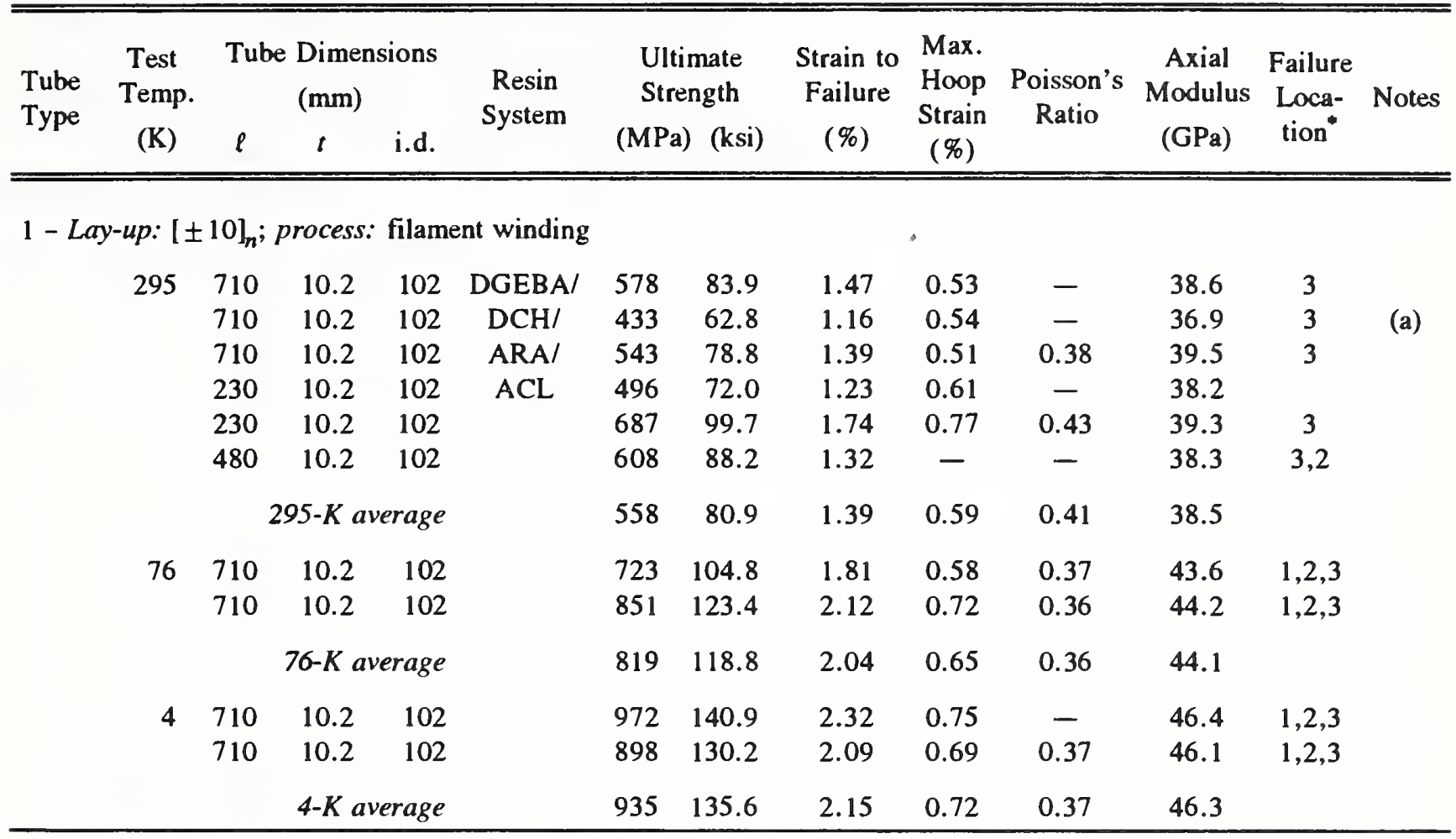

2 - Lay-up: $[ \pm 10]_{n}$; process: filament winding

\begin{tabular}{|c|c|c|c|c|c|c|c|c|c|c|c|}
\hline \multirow[t]{3}{*}{295} & 710 & 5.1 & 102 & DGEBA/ & 491 & 71.2 & 1.26 & 0.51 & - & 35.8 & 3 \\
\hline & 710 & 5.1 & 102 & $\mathrm{DCH} /$ & 466 & 67.7 & 1.41 & - & 0.34 & 35.4 & 3 \\
\hline & \multicolumn{3}{|c|}{$295-K$ average } & $\begin{array}{l}\text { ARA/ } \\
\text { ACL }\end{array}$ & 479 & 69.4 & 1.34 & 0.51 & 0.34 & 35.6 & \\
\hline \multirow[t]{3}{*}{76} & 710 & 5.1 & 102 & & 691 & 100.2 & 1.83 & 0.64 & 0.34 & 40.7 & 2,3 \\
\hline & 710 & 5.1 & 102 & & 719 & 104.3 & 1.90 & 0.62 & 0.34 & 40.8 & 1,2 \\
\hline & \multicolumn{4}{|c|}{$76-K$ average } & 705 & 102.3 & 1.87 & 0.63 & 0.34 & 40.8 & \\
\hline \multirow[t]{3}{*}{4} & 710 & 5.1 & 102 & & 803 & 116.5 & 2.05 & 0.67 & 0.35 & 42.2 & 2,3 \\
\hline & 710 & 5.1 & 102 & & 589 & 85.5 & 1.46 & 0.48 & 0.34 & 42.3 & 1,2 \\
\hline & \multicolumn{4}{|c|}{ 4- $K$ average } & 696 & 101.0 & 1.76 & 0.58 & 0.35 & 42.3 & \\
\hline
\end{tabular}

3 - Lay-up: $\left[82 / \pm 45 / \pm 10_{3} / \pm 45 / 82_{2} / \pm 45 / \pm 10_{3} / \pm 45 / 82\right]$;

process: filament winding

\begin{tabular}{ccccccccccccc}
295 & 710 & 10.2 & 102 & DGEBA/ & 329 & 47.7 & 1.17 & 0.43 & - & 26.6 & 3 & (b) \\
710 & 10.2 & 102 & DCH/ & 470 & 68.2 & 2.22 & 0.76 & 0.39 & 24.5 & 3 & \\
710 & 10.2 & 102 & ARA/ & 384 & 55.7 & 1.67 & 0.52 & 0.38 & 24.9 & 3 & (b) \\
480 & 10.2 & 102 & ACL & 429 & 62.2 & 2.05 & - & - & 21.5 & 3 & \\
230 & 10.2 & 102 & & 424 & 61.5 & 2.10 & - & - & 21.4 & 3 & \\
230 & 10.2 & 102 & & 419 & 60.7 & 2.02 & 0.82 & 0.41 & 21.8 & 3 & \\
7 \\
$295-K$ average & & 409 & 59.3 & 1.87 & 0.63 & 0.39 & 23.5 & & \\
\hline
\end{tabular}


Table 5.1. Results of compression tests (continued).

\begin{tabular}{|c|c|c|c|c|c|c|c|c|c|c|c|c|c|}
\hline \multirow{2}{*}{$\begin{array}{l}\text { Tube } \\
\text { Type }\end{array}$} & \multirow{2}{*}{$\begin{array}{l}\text { Test } \\
\text { Temp. } \\
(\mathbf{K})\end{array}$} & \multicolumn{3}{|c|}{$\begin{array}{l}\text { Tube Dimensions } \\
\qquad(\mathrm{mm})\end{array}$} & \multirow{2}{*}{$\begin{array}{l}\text { Resin } \\
\text { System }\end{array}$} & \multicolumn{2}{|c|}{$\begin{array}{l}\text { Ultimate } \\
\text { Strength }\end{array}$} & \multirow{2}{*}{$\begin{array}{c}\text { Strain to } \\
\text { Failure } \\
(\%)\end{array}$} & \multirow{2}{*}{$\begin{array}{c}\text { Max. } \\
\text { Hoop } \\
\text { Strain } \\
(\%)\end{array}$} & \multirow{2}{*}{$\begin{array}{l}\text { Poisson's } \\
\text { Ratio }\end{array}$} & \multirow{2}{*}{$\begin{array}{c}\text { Axial } \\
\text { Modulus } \\
(\mathrm{GPa})\end{array}$} & \multirow{2}{*}{$\begin{array}{l}\text { Failure } \\
\text { Loca- } \\
\text { tion" }\end{array}$} & \multirow[t]{2}{*}{ Notes } \\
\hline & & $\ell$ & $t$ & i.d. & & (MPa) & (ksi) & & & & & & \\
\hline & 76 & 710 & 10.2 & 102 & & 674 & 97.7 & 2.50 & 0.91 & 0.41 & 34.9 & 2,1 & \\
\hline & & 710 & 10.2 & 102 & & 721 & 104.6 & 2.70 & 0.85 & 0.33 & 30.4 & 2,1 & \\
\hline & \multicolumn{5}{|c|}{$76-K$ average } & 697 & 101.1 & 2.60 & 0.88 & 0.37 & 32.7 & & \\
\hline & 4 & 710 & 10.2 & 102 & & 630 & 91.4 & 2.06 & - & 0.32 & & 2 & \\
\hline
\end{tabular}

4 - Lay-up: $\left[82 / \pm 45 / \pm 10_{3} / \pm 45 / 82\right]$

process: filament winding

\begin{tabular}{|c|c|c|c|c|c|c|c|c|c|c|c|c|}
\hline \multirow[t]{4}{*}{295} & 710 & 5.1 & 102 & DGEBA/ & 474 & 68.8 & 2.03 & 0.82 & - & 23.1 & 1,2 & \\
\hline & 710 & 5.1 & 102 & $\mathrm{DCH} /$ & 463 & 67.1 & 2.50 & 0.82 & 0.43 & 26.1 & 2,1 & \\
\hline & 710 & 5.1 & 102 & ARA/ & 495 & 71.8 & 2.05 & 0.92 & 0.42 & 25.5 & 1 & \\
\hline & 710 & 5.1 & 102 & $\mathrm{ACL}$ & 429 & 62.2 & 2.23 & 0.82 & 0.37 & 22.9 & 1 & (c) \\
\hline \multicolumn{5}{|c|}{ 295-K average } & 465 & 67.5 & 2.20 & 0.85 & 0.41 & 24.4 & & \\
\hline \multirow[t]{3}{*}{76} & 710 & 5.1 & 102 & & 728 & 105.5 & 2.67 & 0.90 & 0.36 & 31.3 & 1,2 & \\
\hline & 710 & 5.1 & 102 & & 686 & 99.4 & 2.50 & 0.85 & 0.36 & 33.6 & 1,2 & \\
\hline & \multicolumn{4}{|c|}{$76-K$ average } & 707 & 102.5 & 2.59 & 0.88 & 0.36 & 32.5 & & \\
\hline 4 & 710 & 5.1 & 102 & & 706 & 102.4 & 2.27 & 0.77 & 0.33 & 33.1 & 1 & \\
\hline
\end{tabular}

5 - Lay-up: $\left[82 / \pm 10_{3} / 82 / \pm 10_{3} / 82 / \pm 10_{3} / 82\right]$;

process: filament winding

\begin{tabular}{|c|c|c|c|c|c|c|c|c|c|c|c|}
\hline \multirow{4}{*}{295} & 710 & 10.2 & 102 & DGEBA/ & 681 & 98.8 & 1.59 & 0.52 & 0.36 & 42.0 & 3 \\
\hline & 710 & 10.2 & 102 & $\mathrm{DCH} /$ & 594 & 86.2 & 1.41 & 0.43 & 0.31 & 43.0 & 3 \\
\hline & 480 & 10.2 & 102 & ARA/ & 518 & 75.2 & 1.19 & - & - & 43.0 & 3 \\
\hline & 230 & 10.2 & 102 & $\mathrm{ACL}$ & 687 & 99.6 & 1.66 & - & - & 41.5 & 3,2 \\
\hline \multicolumn{5}{|c|}{$295-K$ average } & 620 & 89.9 & 1.46 & 0.48 & 0.34 & 42.4 & \\
\hline \multirow[t]{3}{*}{76} & 710 & 10.2 & 102 & & 907 & 131.5 & 2.08 & 0.64 & 0.31 & 46.8 & 1,2 \\
\hline & 710 & 10.2 & 102 & & 1190 & 172.5 & 3.30 & 0.90 & 0.28 & 42.8 & 1,2 \\
\hline & \multicolumn{4}{|c|}{$76-K$ average } & 1048 & 152.0 & 2.69 & 0.77 & 0.30 & 44.8 & \\
\hline \multirow[t]{3}{*}{4} & 710 & 10.2 & 102 & & 1154 & 167.4 & 2.63 & 0.75 & 0.30 & 48.2 & 3 \\
\hline & 710 & 10.2 & 102 & & 1069 & 155.0 & 2.37 & 0.74 & - & 45.5 & 2 \\
\hline & \multicolumn{4}{|c|}{ 4-K average } & 1112 & 161.2 & 2.50 & 0.75 & 0.30 & 46.9 & \\
\hline
\end{tabular}


Table 5.1. Results of compression tests (continued).

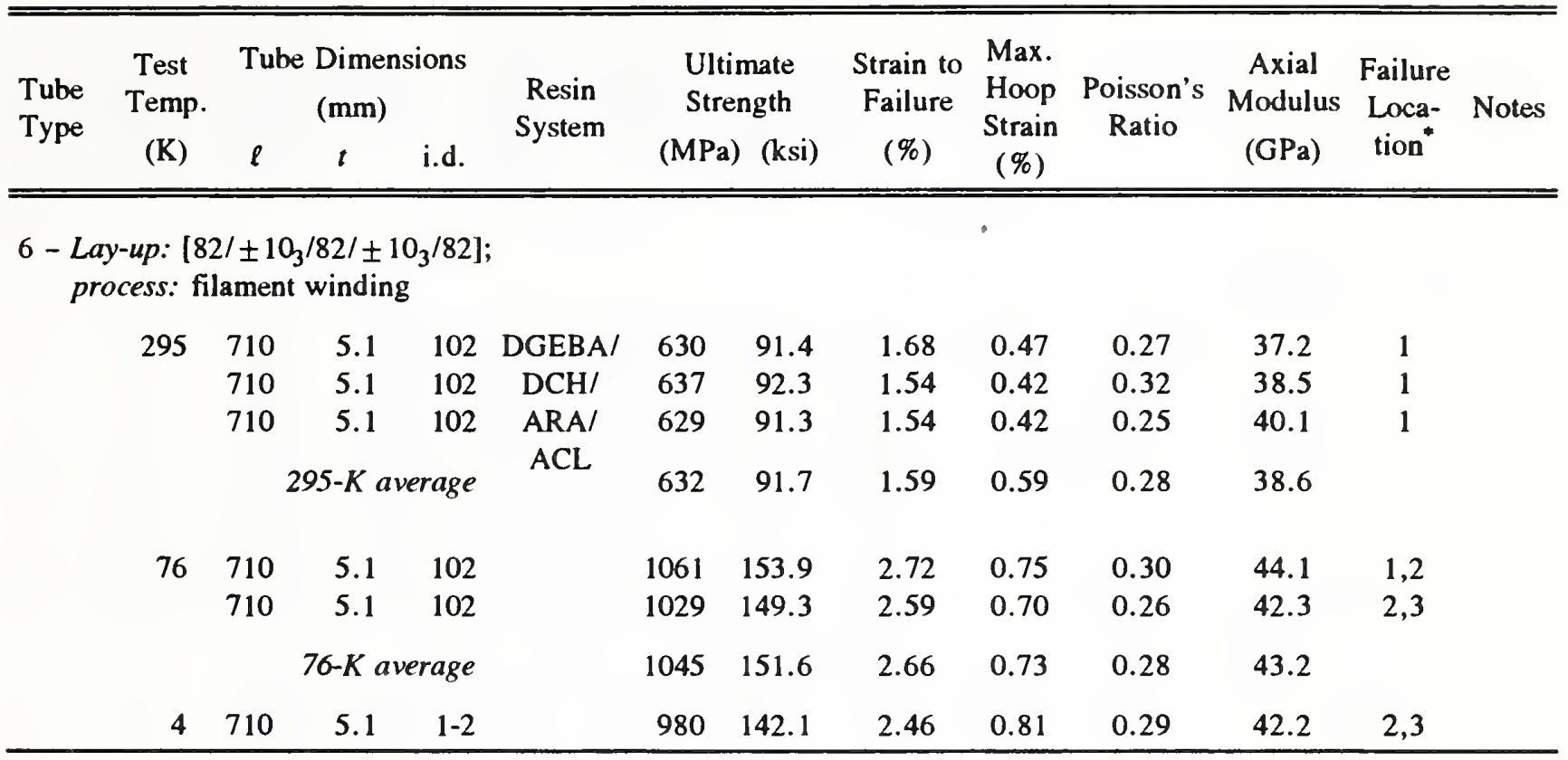

7 - Lay-up: $\left[82 / \pm 9_{2} / 82 / \pm 9_{2} / 82 / \pm 9_{2} / 82 / \pm 9_{2} / 82\right]$

process: filament winding

\begin{tabular}{|c|c|c|c|c|c|c|c|c|c|c|c|}
\hline 295 & 710 & 10.2 & 102 & DGEBA/ & 468 & 67.8 & 1.60 & 0.48 & 0.24 & 29.7 & $1,2,3$ \\
\hline & 710 & 10.2 & 102 & ARA/ & 474 & 68.7 & 1.50 & 0.43 & 0.28 & 28.5 & \\
\hline & 710 & 10.2 & 102 & DTA & 415 & 60.2 & 1.42 & 0.34 & 0.25 & 29.2 & 1 \\
\hline & 710 & 10.2 & 102 & & 526 & 76.4 & 1.69 & 0.47 & 0.27 & 31.3 & 1 \\
\hline \multicolumn{5}{|c|}{$295-K$ average } & 471 & 68.3 & 1.55 & 0.43 & 0.26 & 29.7 & \\
\hline 76 & 710 & 10.2 & 102 & & 763 & 110.6 & 2.38 & 0.64 & 0.25 & 35.5 & 3 \\
\hline
\end{tabular}

8 - Lay-up: $\left[82 / \pm 9_{2} / 82 / \pm 9_{2} / 82\right]$; process: filament winding

\begin{tabular}{rrrrrrrrrrrr}
295 & 710 & 5.1 & 102 & DGEBA/ & 517 & 74.9 & 1.57 & 0.30 & 0.20 & 33.0 & 3 \\
& 710 & 5.1 & 102 & ARA/ & 526 & 76.3 & 1.54 & 0.30 & 0.22 & 33.8 & 3 \\
& & $295-K$ average & DTA & 521 & 75.6 & 1.56 & 0.30 & 0.21 & 33.4 & \\
\multirow{2}{*}{76} & & & & & & & & & & \\
& 710 & 5.1 & 102 & & 669 & 97.0 & 2.13 & - & 0.31 & 35.0 & 3 \\
& 710 & 5.1 & 102 & & 611 & 88.7 & 1.83 & - & 0.21 & 30.8 & 3 \\
& $76-K$ average & & 640 & 92.8 & 1.98 & - & 0.26 & 32.9 &
\end{tabular}


Table 5.1. Results of compression tests (continued).

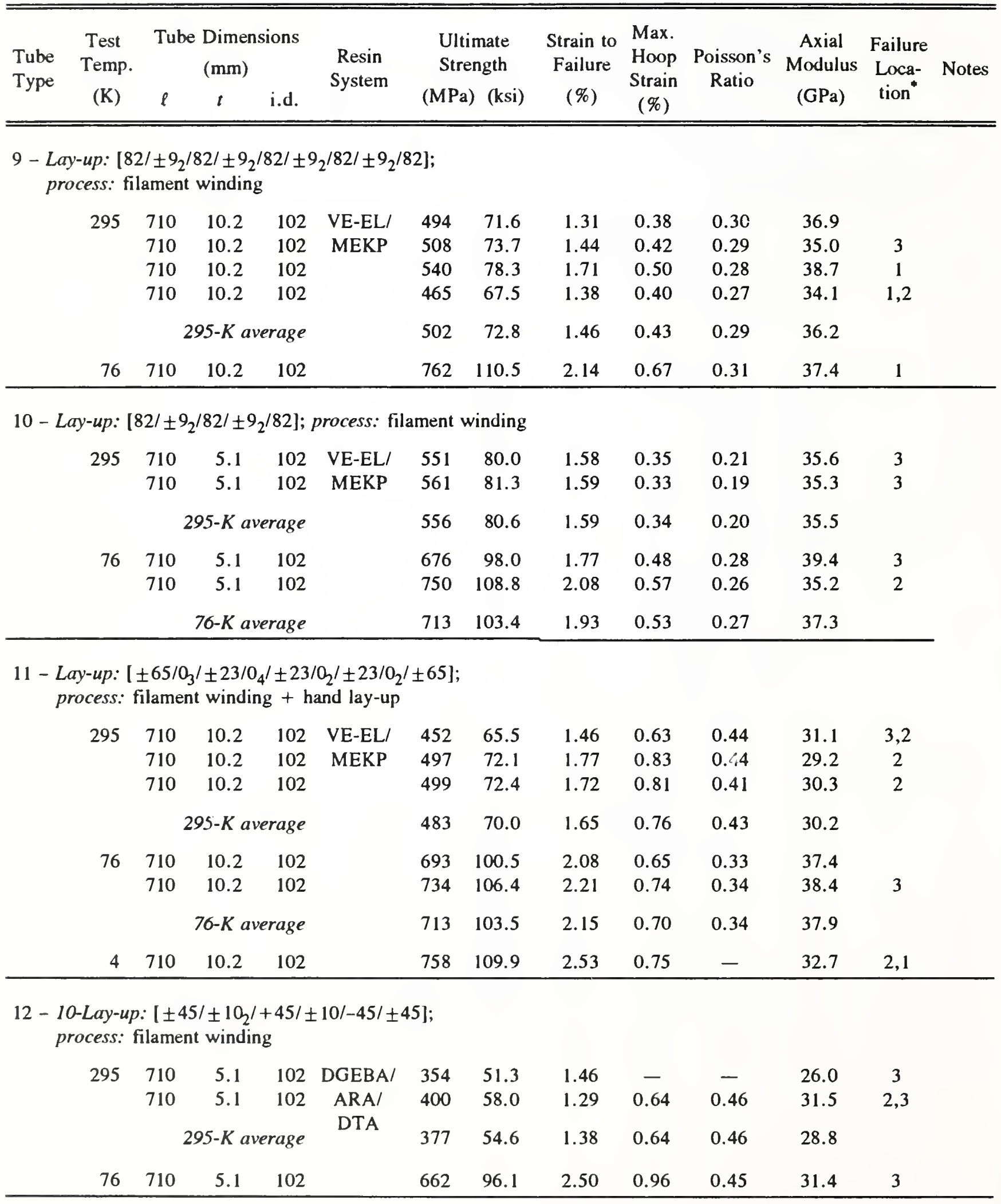


Table 5.1. Results of compression tests (continued).

\begin{tabular}{|c|c|c|c|c|c|c|c|c|c|c|c|c|}
\hline \multirow{2}{*}{$\begin{array}{l}\text { Tube } \\
\text { Type }\end{array}$} & \multirow{2}{*}{$\begin{array}{c}\text { Test } \\
\text { Temp. } \\
(\mathrm{K})\end{array}$} & \multicolumn{3}{|c|}{$\begin{array}{l}\text { Tube Dimensions } \\
\qquad(\mathrm{mm})\end{array}$} & \multirow{2}{*}{$\begin{array}{l}\text { Resin } \\
\text { System }\end{array}$} & \multirow{2}{*}{$\begin{array}{c}\text { Ultimate } \\
\text { Strength } \\
\text { (MPa) (ksi) }\end{array}$} & \multirow{2}{*}{$\begin{array}{c}\text { Strain to } \\
\text { Failure } \\
(\%)\end{array}$} & \multirow{2}{*}{$\begin{array}{c}\text { Max. } \\
\text { Hoop } \\
\text { Strain } \\
(\%)\end{array}$} & \multirow{2}{*}{$\begin{array}{l}\text { Poisson's } \\
\text { Ratio }\end{array}$} & \multirow{2}{*}{$\begin{array}{c}\text { Axial } \\
\text { Modulus } \\
(\mathrm{GPa})\end{array}$} & \multirow{2}{*}{$\begin{array}{c}\text { Failure } \\
\text { Loca- } \\
\text { tion" }\end{array}$} & \multirow[t]{2}{*}{ Notes } \\
\hline & & $\ell$ & $t$ & i.d. & & & & & & & & \\
\hline
\end{tabular}

13 - Lay-up: $\left[ \pm 45 / \pm 10_{2} /+45 / \pm 10 /-45 / \pm 45\right]$;

process: filament winding

\begin{tabular}{cccccccccccc}
295 & 710 & 5.1 & 102 & DGEBA/ & 350 & 50.8 & 1.61 & - & - & 23.8 & 3 \\
& 710 & 5.1 & 102 & ARA/ & 377 & 54.6 & 1.57 & 1.08 & 0.49 & 25.4 & 1,2 \\
\multicolumn{7}{c}{$295-K$ average } & DTA & & & &
\end{tabular}

14 - Lay-up: $\left[ \pm 45 / \pm 10_{2} /+45 / \pm 10 /-45 / \pm 45\right]$

process: filament winding

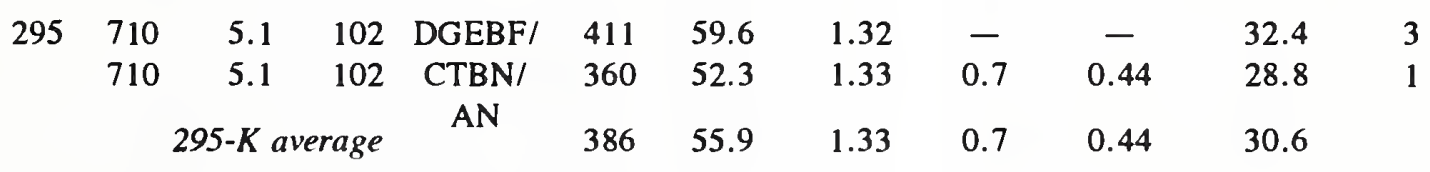

15 - Lay-up: $\left[ \pm 45 / \pm 10_{2} /+45 / \pm 10 /-45 / \pm 45\right]$;

process: filament winding

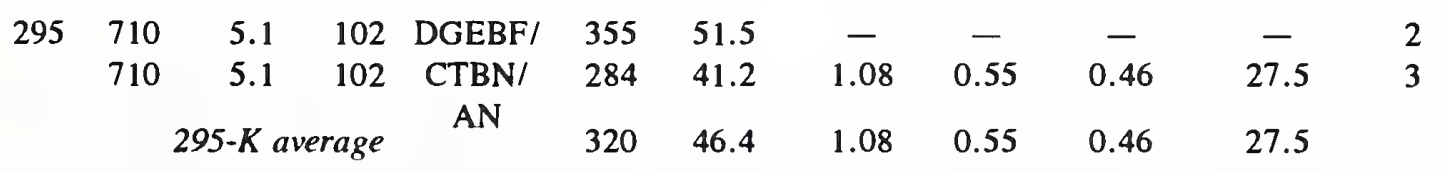

16 - Lay-up: [82/10 $/ 82]$; process: filament winding

\begin{tabular}{|c|c|c|c|c|c|c|c|c|c|c|c|c|}
\hline \multirow[t]{5}{*}{295} & 710 & 5.1 & 102 & DGEBA/ & 645 & 93.6 & 1.66 & 0.54 & 0.29 & 37.9 & 3 & \\
\hline & 710 & 5.1 & 102 & DCH/ & 659 & 95.5 & 1.73 & 0.54 & 0.29 & 38.2 & 3 & \\
\hline & 710 & 5.1 & 102 & ARA/ & 484 & 70.2 & 1.25 & 0.38 & 0.30 & 37.5 & 3 & \\
\hline & 710 & 5.1 & 102 & $\mathrm{ACL}$ & 261 & 37.8 & 0.70 & 0.25 & 0.24 & 37.6 & 3 & (c) \\
\hline & 710 & 5.1 & 102 & & 585 & 84.8 & 1.83 & 0.41 & - & 31.7 & 1 & (d) \\
\hline \multicolumn{5}{|c|}{ 295-K average } & 527 & 76.4 & 1.43 & 0.42 & 0.28 & 36.6 & & \\
\hline \multirow[t]{4}{*}{76} & 710 & 5.1 & 102 & & 896 & 130.0 & - & - & - & - & 1,2 & (e) \\
\hline & 710 & 5.1 & 102 & & 680 & 98.6 & - & - & - & - & 3 & (e) \\
\hline & 710 & 5.1 & 102 & & 519 & 75.25 & $\begin{array}{c}\text { (ext.) } \\
1.34\end{array}$ & - & - & 38.7 & 2,1 & (e) \\
\hline & \multicolumn{4}{|c|}{ 76- $K$ average } & 698 & 101.3 & 1.34 & & & 38.7 & & \\
\hline
\end{tabular}


Table 5.1. Results of compression tests (continued).

\begin{tabular}{|c|c|c|c|c|c|c|c|c|c|c|c|c|c|}
\hline \multirow{2}{*}{$\begin{array}{l}\text { Tube } \\
\text { Type }\end{array}$} & \multirow{2}{*}{$\begin{array}{l}\text { Test } \\
\text { Temp. } \\
(\mathrm{K})\end{array}$} & \multicolumn{3}{|c|}{$\begin{array}{l}\text { Tube Dimensions } \\
\qquad(\mathrm{mm})\end{array}$} & \multirow{2}{*}{$\begin{array}{l}\text { Resin } \\
\text { System }\end{array}$} & \multicolumn{2}{|c|}{$\begin{array}{l}\text { Ultimate } \\
\text { Strength }\end{array}$} & \multirow{2}{*}{$\begin{array}{l}\text { Strain to } \\
\text { Failure } \\
(\%)\end{array}$} & \multirow{2}{*}{$\begin{array}{c}\text { Max. } \\
\text { Hoop } \\
\text { Strain } \\
(\%)\end{array}$} & \multirow{2}{*}{$\begin{array}{l}\text { Poisson's } \\
\text { Ratio }\end{array}$} & \multirow{2}{*}{$\begin{array}{c}\text { Axial } \\
\text { Modulus } \\
(\mathrm{GPa})\end{array}$} & \multirow{2}{*}{$\begin{array}{c}\text { Failure } \\
\text { Loca- } \\
\text { tion }\end{array}$} & \multirow[t]{2}{*}{ Notes } \\
\hline & & $\ell$ & $t$ & i.d. & & $(\mathrm{MPa})$ & (ksi) & & & & & & \\
\hline
\end{tabular}

17 - Lay-up: $\left[82 / \pm 10_{6} / 82 / \pm 10_{6} / 82\right]$;

process: filament winding

$\begin{array}{cccccccccccc}295 & 1200 & 8.9 & 180 & \text { DGEBA/ } & 512 & 74.2 & 1.59 & 0.55 & 0.33 & 32.1 & 1 \\ 1200 & 8.9 & 180 & \text { DCH/ } & 574 & 83.3 & 1.61 & 0.51 & 0.34 & 35.2 & 1 \\ 1200 & 8.9 & 180 & \text { ARA/ } & 546 & 79.2 & 1.43 & 0.45 & 0.32 & 38.5 & 1,2 \\ 1200 & 8.9 & 180 & \text { ACL } & 546 & 79.3 & 1.64 & 0.48 & 0.29 & 33.6 & \\ 1200 & 8.9 & 180 & & 547 & 79.4 & 1.44 & 0.49 & - & 36.3 & 1 \\ 610 & 8.9 & 180 & & 546 & 79.2 & 1.29 & 0.42 & 0.33 & 39.9 & 1 \\ & 295-K \text { average } & 545 & 79.1 & 1.50 & 0.48 & 0.32 & 35.9 & \\ 76 & 1200 & 8.9 & 180 & & 897 & 130.1 & 2.29 & 0.74 & 0.32 & 42.7 & 2,1 \\ 1200 & 8.9 & 180 & & 853 & 123.8 & 2.35 & 0.74 & - & 39.2 & \\ 1200 & 8.9 & 180 & & 926 & 134.3 & 2.49 & 0.77 & 0.31 & 42.1 & 1,2,3 \\ & 76-K \text { average } & & 892 & 129.4 & 2.38 & 0.75 & 0.32 & 41.3 & \end{array}$

18 - Lay-up: $\left[82 / \pm 10_{6} / 82 / \pm 10_{6} / 82\right]$;

process: filament winding

\begin{tabular}{|c|c|c|c|c|c|c|c|c|c|c|}
\hline \multirow[t]{4}{*}{295} & 1800 & 12.7 & 250 & DGEBA/ & 488 & 70.8 & 1.31 & 0.39 & 0.32 & 38.0 \\
\hline & 1800 & 12.7 & 250 & $\mathrm{DCH} /$ & 589 & 85.4 & 1.69 & 0.53 & 0.30 & 35.0 \\
\hline & 1800 & 12.7 & 250 & ARA/ & 615 & 89.2 & 1.79 & 0.54 & 0.26 & 33.3 \\
\hline & 910 & 12.7 & 250 & $\mathrm{ACL}$ & 570 & 82.7 & 1.52 & 0.50 & - & 38.2 \\
\hline \multicolumn{5}{|c|}{$295-K$ average } & 566 & 82.0 & 1.58 & 0.49 & 0.29 & 36.1 \\
\hline \multirow[t]{4}{*}{76} & 1800 & 12.7 & 250 & & 623 & 90.4 & 1.44 & 0.49 & - & 44.6 \\
\hline & 1800 & 12.7 & 250 & & 878 & 127.4 & 2.13 & 0.70 & 0.33 & 44.4 \\
\hline & 1800 & 12.7 & 250 & & 806 & 116.9 & 2.01 & 0.62 & 0.35 & 43.4 \\
\hline & \multicolumn{3}{|c|}{$76-K$ average } & & 769 & 111.6 & 1.86 & 0.60 & 0.34 & 44.1 \\
\hline
\end{tabular}

19 - Lay-up: [82/106 $/ 82]$; process: filament winding

\begin{tabular}{|c|c|c|c|c|c|c|c|c|c|c|c|c|}
\hline \multirow[t]{4}{*}{295} & 710 & 5.1 & 102 & DGEBF/ & 535 & 77.6 & 1.50 & 0.43 & 0.28 & 35.2 & 1,2 & \\
\hline & 710 & 5.1 & 102 & CTBN/ & 389 & 56.4 & 0.98 & 0.29 & 0.29 & 39.7 & 3 & \\
\hline & 710 & 5.1 & 102 & AN & 520 & 75.4 & 1.44 & 0.42 & 0.26 & 35.7 & 3 & \\
\hline & 710 & 5.1 & 102 & & 530 & 76.9 & 1.56 & 0.47 & - & 36.3 & 3 & (c) \\
\hline \multicolumn{5}{|c|}{ 295-K average } & 494 & 71.6 & 1.37 & 0.40 & 0.28 & 36.7 & & \\
\hline 76 & 710 & 5.1 & 102 & & 933 & 135.3 & 2.57 & 0.79 & 0.30 & 39.1 & 1,2 & \\
\hline & 710 & 5.1 & 102 & & 700 & 101.6 & 1.87 & 0.57 & 0.32 & 39.5 & 2 & \\
\hline & 710 & 5.1 & 102 & & 721 & 104.6 & 1.95 & 0.66 & - & 38.7 & 3 & \\
\hline \multicolumn{5}{|c|}{ 76- $K$ average } & 785 & 113.8 & 2.13 & 0.67 & 0.31 & 39.1 & & \\
\hline
\end{tabular}


Table 5.1. Results of compression tests (continued).

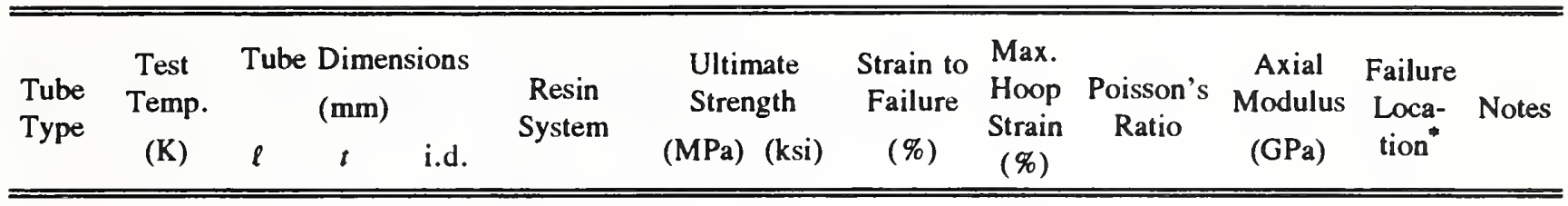

20 - Lay-up: [82/10 $6 / 82]$; process: filament winding; reinforcement: S2-glass fiber

\begin{tabular}{|c|c|c|c|c|c|c|c|c|c|c|}
\hline \multirow[t]{3}{*}{295} & 710 & 5.1 & 102 & DGEBF/ & 434 & 62.9 & 1.15 & 0.32 & - & 37.3 \\
\hline & 710 & 5.1 & 102 & CTBN/ & 430 & 62.3 & 1.18 & 0.31 & - & 35.8 \\
\hline & 710 & 5.1 & 102 & AN & 415 & 60.2 & 1.13 & 0.33 & 0.27 & 34.6 \\
\hline \multicolumn{5}{|c|}{$295-K$ average } & 426 & 61.8 & 1.15 & 0.32 & 0.27 & 35.9 \\
\hline \multirow[t]{5}{*}{76} & 710 & 5.1 & 102 & & 608 & 88.3 & 1.47 & 0.43 & 0.31 & 43.0 \\
\hline & 710 & 5.1 & 102 & & 607 & 88.1 & 1.52 & 0.47 & - & 41.5 \\
\hline & 710 & 5.1 & 102 & & 562 & 81.6 & 1.36 & 0.44 & - & 42.8 \\
\hline & 710 & 5.1 & 102 & & 470 & 68.2 & - & - & - & - \\
\hline & \multicolumn{4}{|c|}{ 76- $K$ average } & 562 & 81.5 & 1.45 & 0.45 & 0.31 & 42.4 \\
\hline & 710 & 5.1 & 102 & & 510 & 74.0 & 1.20 & 0.37 & 0.30 & 43.0 \\
\hline & 710 & 5.1 & 102 & & 678 & 98.3 & 1.55 & 0.49 & - & 45.5 \\
\hline & \multicolumn{4}{|c|}{$4-K$ average } & 594 & 86.1 & 1.38 & 0.43 & 0.30 & 44.3 \\
\hline
\end{tabular}

21 - Lay-up: [82/10 $/ 82]$; process: filament winding

\begin{tabular}{|c|c|c|c|c|c|c|c|c|c|c|c|}
\hline \multirow[t]{4}{*}{295} & 710 & 5.1 & 102 & VE-EL/ & 441 & 64.0 & 1.44 & 0.46 & - & 30.6 & 1 \\
\hline & 710 & 5.1 & 102 & MEKP & 417 & 60.5 & 1.39 & 0.42 & - & 29.1 & 1 \\
\hline & 710 & 5.1 & 102 & & 580 & 84.1 & 2.00 & 0.64 & - & 28.8 & 1 \\
\hline & 710 & 5.1 & 102 & & 559 & 81.1 & 2.01 & 0.62 & - & 27.9 & 2,1 \\
\hline \multicolumn{5}{|c|}{$295-K$ average } & 499 & 72.4 & 1.71 & 0.53 & - & 29.1 & \\
\hline \multirow[t]{4}{*}{76} & 710 & 5.1 & 102 & & 896 & 129.9 & 2.94 & 0.94 & 0.29 & 32.8 & 2,1 \\
\hline & 710 & 5.1 & 102 & & 927 & 134.4 & 3.05 & 0.94 & - & 31.7 & 2,1 \\
\hline & 710 & 5.1 & 102 & & 916 & 132.8 & 2.32 & 0.79 & - & 31.1 & 2,1 \\
\hline & \multicolumn{4}{|c|}{$76-K$ average } & 913 & 132.4 & 2.77 & 0.89 & 0.29 & 31.9 & \\
\hline \multirow[t]{3}{*}{4} & 710 & 5.1 & 102 & & 727 & 105.5 & 2.41 & 0.71 & - & 30.2 & 2,1 \\
\hline & 710 & 5.1 & 102 & & 798 & 115.7 & 2.54 & - & - & 31.2 & 2,1 \\
\hline & \multicolumn{4}{|c|}{ 4-K average } & 763 & 110.6 & 2.48 & 0.71 & - & 30.7 & \\
\hline
\end{tabular}

22 - Lay-up: [90/0 $\left./ 90 / 0_{3} / 90\right]$; process: pultrusion

\begin{tabular}{|c|c|c|c|c|c|c|c|c|c|c|}
\hline \multirow[t]{3}{*}{295} & 710 & 5.1 & 102 & DGEBA- & 549 & 79.7 & 1.58 & 0.32 & - & 34.5 \\
\hline & 710 & 5.1 & 102 & ST/ARA/ & 555 & 80.5 & 1.51 & 0.21 & - & 36.8 \\
\hline & 710 & 5.1 & 102 & $\mathrm{ACL} / \mathrm{F}$ & 514 & 74.5 & 1.48 & 0.32 & - & 34.5 \\
\hline & & $-K a$ & age & & 539 & 78.2 & 1.52 & 0.28 & - & 35.5 \\
\hline
\end{tabular}


Table 5.1. Results of compression tests (continued).

\begin{tabular}{|c|c|c|c|c|c|c|c|c|c|c|c|c|c|}
\hline \multirow{2}{*}{$\begin{array}{l}\text { Tube } \\
\text { Type }\end{array}$} & \multirow{2}{*}{$\begin{array}{l}\text { Test } \\
\text { Temp. } \\
(\mathrm{K})\end{array}$} & \multirow{2}{*}{$\begin{array}{l}\text { Tube } \\
\ell\end{array}$} & \multicolumn{2}{|c|}{$\begin{array}{l}\text { Dimensions } \\
(\mathrm{mm})\end{array}$} & \multirow{2}{*}{$\begin{array}{l}\text { Resin } \\
\text { System }\end{array}$} & \multicolumn{2}{|c|}{$\begin{array}{l}\text { Ultimate } \\
\text { Strength }\end{array}$} & \multirow{2}{*}{$\begin{array}{c}\text { Strain to } \\
\text { Failure } \\
(\%)\end{array}$} & \multirow{2}{*}{$\begin{array}{c}\text { Max. } \\
\text { Hoop } \\
\text { Strain } \\
(\%)\end{array}$} & \multirow{2}{*}{$\begin{array}{c}\text { Poisson's } \\
\text { Ratio }\end{array}$} & \multirow{2}{*}{$\begin{array}{c}\text { Axial } \\
\text { Modulus } \\
(\mathrm{GPa})\end{array}$} & \multirow{2}{*}{$\begin{array}{l}\text { Failure } \\
\text { Loca- } \\
\text { tion" }\end{array}$} & \multirow[t]{2}{*}{ Notes } \\
\hline & & & $t$ & i.d. & & $(\mathrm{MPa})$ & (ksi) & & & & & & \\
\hline & \multirow[t]{3}{*}{76} & 710 & 5.1 & 102 & & 648 & 94.0 & 1.68 & 0.48 & 0.26 & 39.8 & 2,1 & \\
\hline & & 710 & 5.1 & 102 & & 653 & 94.8 & 1.76 & 0.51 & - & 39.0 & 1,2 & \\
\hline & & 710 & 5.1 & 102 & & 660 & 95.7 & 1.64 & 0.53 & - & 39.7 & 2,1 & \\
\hline & \multicolumn{5}{|c|}{ 76- $K$ average } & 654 & 94.8 & 1.69 & 0.51 & 0.26 & 39.7 & & \\
\hline & 4 & 710 & 5.1 & 102 & & 612 & 88.7 & 1.19 & 0.44 & 0.22 & 39.5 & 2 & \\
\hline
\end{tabular}

23 - Lay-up: [90/0 $\left./ 90 / 0_{3} / 90\right]$; process: pultrusion

\begin{tabular}{|c|c|c|c|c|c|c|c|c|c|c|c|}
\hline \multirow{4}{*}{295} & 710 & 5.1 & 102 & VE-EL/ & 347 & 50.3 & 1.09 & 0.21 & 0.21 & 32.5 & 3 \\
\hline & 710 & 5.1 & 102 & MEKP & 380 & 55.2 & 1.16 & 0.34 & - & 33.3 & 3 \\
\hline & 710 & 5.1 & 102 & & 343 & 49.8 & 1.03 & 0.21 & - & 33.9 & 2,1 \\
\hline & 710 & 5.1 & 102 & & 372 & 53.9 & 1.15 & 0.23 & - & 32.3 & 3 \\
\hline \multicolumn{5}{|c|}{$295-K$ average } & 361 & 52.3 & 1.11 & 0.25 & 0.21 & 33.0 & \\
\hline 76 & 710 & 5.1 & 102 & & 538 & 78.0 & 1.50 & 0.43 & - & 37.8 & 2 \\
\hline & 710 & 5.1 & 102 & & 577 & 83.7 & 1.61 & 0.48 & 0.26 & 36.8 & 2,1 \\
\hline & 710 & 5.1 & 102 & & 576 & 83.5 & 1.50 & 0.42 & - & 38.9 & 2,1 \\
\hline \multicolumn{5}{|c|}{$76-K$ average } & 564 & 81.7 & 1.54 & 0.44 & 0.26 & 37.8 & \\
\hline
\end{tabular}

24 - Lay-up: [0/90/06 $\left./ 90 / 0_{6} / 90\right]$; process: pultrusion

\begin{tabular}{|c|c|c|c|c|c|c|c|c|c|c|c|}
\hline \multirow[t]{3}{*}{295} & 1800 & 12.7 & 250 & VE-EL/ & 559 & 81.1 & 1.44 & 0.30 & 0.20 & 38.5 & 1 \\
\hline & 1800 & 12.7 & 250 & MEKP & 552 & 80.1 & 1.45 & 0.34 & 0.22 & 37.6 & 3 \\
\hline & 1800 & 12.7 & 250 & & 536 & 77.8 & 1.40 & 0.31 & 0.19 & 39.4 & 2 \\
\hline \multicolumn{5}{|c|}{$295-K$ average } & 549 & 79.7 & 1.43 & 0.32 & 0.20 & 38.5 & \\
\hline 76 & 1800 & $: 2.7$ & 250 & & 615 & 89.1 & 1.25 & 0.37 & 0.27 & 41.4 & 1,2 \\
\hline & 1800 & 12.7 & 250 & & 570 & 82.7 & 1.36 & 0.38 & 0.30 & 45.1 & 3 \\
\hline & 1800 & 12.7 & 250 & & 690 & 100.1 & 1.26 & 0.44 & 0.29 & 44.3 & 1,2 \\
\hline \multicolumn{5}{|c|}{$76-K$ average } & 625 & 90.7 & 1.29 & 0.40 & 0.29 & 43.6 & \\
\hline
\end{tabular}

25 - Lay-up: $\left[ \pm 82 / \pm 10_{3} / \pm 82 / \pm 10_{3} / \pm 82 / \pm 10_{3} / \pm 82\right]$;

process: filament winding

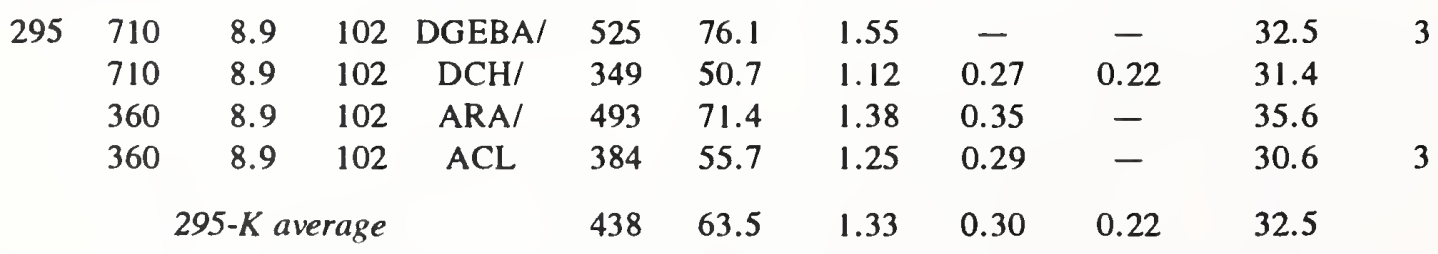


Table 5.1. Results of compression tests (continued).

\begin{tabular}{|c|c|c|c|c|c|c|c|c|c|c|c|c|c|}
\hline \multirow{2}{*}{$\begin{array}{l}\text { Tube } \\
\text { Type }\end{array}$} & \multirow{2}{*}{$\begin{array}{c}\text { Test } \\
\text { Temp. } \\
(\mathrm{K})\end{array}$} & \multicolumn{3}{|c|}{$\begin{array}{l}\text { Tube Dimensions } \\
\qquad(\mathrm{mm})\end{array}$} & \multirow{2}{*}{$\begin{array}{l}\text { Resin } \\
\text { System }\end{array}$} & \multicolumn{2}{|c|}{$\begin{array}{l}\text { Ultimate } \\
\text { Strength }\end{array}$} & \multirow{2}{*}{$\begin{array}{c}\text { Strain to } \\
\text { Failure } \\
(\%)\end{array}$} & \multirow{2}{*}{$\begin{array}{c}\text { Max. } \\
\text { Hoop } \\
\text { Strain } \\
(\%)\end{array}$} & \multirow{2}{*}{$\begin{array}{l}\text { Poisson's } \\
\text { Ratio }\end{array}$} & \multirow{2}{*}{$\begin{array}{c}\text { Axial } \\
\text { Modulus } \\
\text { (GPa) }\end{array}$} & \multirow{2}{*}{$\begin{array}{c}\text { Failure } \\
\text { Loca- } \\
\text { tion" }\end{array}$} & \multirow[t]{2}{*}{ Notes } \\
\hline & & $\ell$ & $t$ & i.d. & & (MPa) & (ksi) & & & & & & \\
\hline & \multirow[t]{4}{*}{76} & 360 & 8.9 & 102 & \multirow{4}{*}{$\begin{array}{c}\text { DGEBA/ } \\
\text { DCH/ } \\
\text { ARA/ } \\
\text { ACL }\end{array}$} & 675 & 97.9 & 1.84 & 0.49 & - & 40.4 & 3 & \\
\hline & & 710 & 8.9 & 102 & & 570 & 82.6 & 1.64 & 0.46 & - & 36.1 & 3 & \\
\hline & & 710 & 8.9 & 102 & & 613 & 88.9 & 1.77 & 0.52 & 0.29 & 36.7 & 3 & \\
\hline & & \multicolumn{3}{|c|}{$76-K$ average } & & 619 & 89.8 & 1.75 & 0.49 & 0.29 & 37.7 & & \\
\hline
\end{tabular}

$26-$ Lay-up: $[ \pm 10]_{n}$;

process: rubber expansion + hand lay-up

$\begin{array}{rlllllllllll}295 & 360 & 5.1 & 102 & \text { CE } & 698 & 101.3 & 1.69 & 0.50 & - & 40.7 & 3 \\ 76 & 360 & 5.1 & 102 & & 976 & 141.5 & 2.45 & 0.76 & - & 43.1 & 3\end{array}$

27 - Lay-up: $\left[90 / \pm 45 / 0_{3} / \pm 45 / 90\right]_{4 S}$;

process: rubber expansion + hand lay-up

\begin{tabular}{|c|c|c|c|c|c|c|c|c|c|c|}
\hline 295360 & 5.1 & 102 & $\mathrm{CE}$ & 221 & 32.1 & 0.81 & 0.13 & - & 26.4 & 1 \\
\hline 76360 & 5.1 & 102 & & 152 & 22.1 & 0.53 & 0.14 & - & 286 & \\
\hline
\end{tabular}

"Failure locations: 1 - end cap, 2 - within 100 mm of end cap, 3 - midsection

Notes: (a) Fracture path ran into tube end (not complete $360^{\circ}$ fracture)

(b) Manufacturing inconsistencies (tube wall delaminated)

(c) Strain gaged and cured following cryogenic test procedures

(d) Cured at $250 \mathrm{~K}\left(77^{\circ} \mathrm{C}\right)$; no gages

(e) No gages; no heat cure

(f) Postcured at $339 \mathrm{~K}\left(66^{\circ} \mathrm{C}\right)$ for $16 \mathrm{~h}$ 


\subsection{Fatigue Tests}

Specimens, loads, and results of the fatigue tests are summarized in Table 5.2. Some tubes were cycled to 10,000 cycles (the number expected during SMES lifetime); others were cycled to 40,000 . Then they were statically loaded to determine residual strength.

All tubes cycled for 10,000 cycles at $60 \%$ ultimate compressive strength (UCS) did not fail and were then loaded in compression to failure. Residual strengths ranging from 93 to $111 \%$ of the average UCS indicate that 10,000 cycles at $60 \%$ had little effect on the strength of the tubes. However, tests conducted for 40,000 cycles at $60 \%$ UCS produced a surprising result: The strongest tube in the program, type 6, failed at 17,601 cycles. The other tube types tested at $60 \%$ UCS survived the full 40,000 cycles and had residual strengths of $115 \%$ and $91 \%$.

Table 5.2. Results of fatigue tests.

\begin{tabular}{|c|c|c|c|c|c|c|c|c|c|c|}
\hline \multirow{2}{*}{$\begin{array}{l}\text { Tube } \\
\text { Type } \\
\text { (Mfg.) } \\
\end{array}$} & \multirow{2}{*}{$\begin{array}{c}\text { Test } \\
\text { Temp. } \\
(\mathrm{K}) \\
\end{array}$} & \multirow{2}{*}{$\begin{array}{l}\text { Cyclic } \\
\text { Stress }\end{array}$} & \multirow{2}{*}{$\begin{array}{c}\% \\
\text { UCS }^{*}\end{array}$} & \multirow{2}{*}{ Cycles } & \multirow{2}{*}{$\begin{array}{l}\text { Strength } \\
(\mathrm{MPa})\end{array}$} & \multirow{2}{*}{$\begin{array}{c}\text { \% Residual } \\
\text { of Avg. } \\
\text { UCS }^{*}\end{array}$} & \multicolumn{2}{|c|}{ Modulus } & \multicolumn{2}{|c|}{ Modulus } \\
\hline & & & & & & & $(\mathrm{GPa})$ & Cycles & $(\mathrm{GPa})$ & Kilocycles \\
\hline \multirow{6}{*}{$\begin{array}{c}2 \\
(\mathrm{~A})\end{array}$} & \multirow[t]{6}{*}{295} & 287 & 60 & $10,000+$ & 469 & 97.9 & 34.5 & 3 & 34.5 & 10 \\
\hline & & 287 & 60 & 40,000 & 549 & 114.6 & 30.3 & 622 & 31.0 & 40 \\
\hline & & 335 & 70 & 19,230 & failed & & 33.8 & 3 & 33.1 & 15 \\
\hline & & 335 & 70 & 14,825 & failed & & 24.1 & 50 & 23.4 & 15 \\
\hline & & 383 & 80 & 488 & failed & & 34.5 & 2 & 33.1 & 0.15 \\
\hline & & 383 & 80 & 8,927 & failed & & 25.5 & 50 & 24.8 & 8.5 \\
\hline \multirow{4}{*}{$\begin{array}{c}4 \\
\text { (A) }\end{array}$} & \multirow[t]{4}{*}{295} & 279 & 60 & $10,000+$ & 516 & 111 & 24.1 & 3 & 24.1 & 10 \\
\hline & & 279 & 60 & $41,135+$ & 424 & 91.2 & 21.4 & 103 & 22.1 & 41 \\
\hline & & 326 & 70 & $40,000+$ & 447 & 96.1 & 23.4 & 3 & 23.4 & 40 \\
\hline & & 372 & 80 & 1,800 & failed & & & & & \\
\hline \multirow{4}{*}{$\begin{array}{c}6 \\
(\mathrm{~A})\end{array}$} & \multirow[t]{4}{*}{295} & 379 & 60 & $10,000+$ & 589 & 93.2 & 38.6 & 3 & 37.9 & 10 \\
\hline & & 379 & 60 & 17,601 & failed & & 31.7 & 250 & 31.7 & 15 \\
\hline & & 442 & 70 & $40,000+$ & 696 & 110.1 & 36.5 & 3 & 35.9 & 40 \\
\hline & & 506 & 80 & 900 & failed & & 34.5 & 186 & 29.6 & 0.85 \\
\hline \multirow{2}{*}{$\begin{array}{c}8 \\
\text { (B) }\end{array}$} & \multirow[t]{2}{*}{295} & 365 & 70 & 2,270 & failed & & 31.0 & 152 & 30.3 & 2.3 \\
\hline & & 417 & 80 & 0 & failed & & & & & \\
\hline \multirow{2}{*}{$\begin{array}{c}10 \\
(\mathrm{~B})\end{array}$} & \multirow[t]{2}{*}{295} & 389 & 70 & 10,057 & failed & & 30.3 & 50 & 29.6 & 7 \\
\hline & & 445 & 80 & 500 & failed & & 30.3 & 1 & 29.6 & 0.45 \\
\hline \multirow{2}{*}{$\begin{array}{c}8 \\
\text { (B) }\end{array}$} & \multirow[t]{2}{*}{76} & 448 & 70 & 14,911 & failed & & 35.9 & 100 & 35.2 & 10.7 \\
\hline & & 384 & 60 & $40,000+$ & 401 & 62.7 & 35.2 & 50 & 35.9 & 40.1 \\
\hline \multirow{2}{*}{$\begin{array}{l}10 \\
\text { (B) }\end{array}$} & \multirow[t]{2}{*}{76} & 499 & 70 & $40,000+$ & 696 & 97.6 & 37.9 & 50 & 38.6 & 40 \\
\hline & & 428 & 60 & $41,000+$ & 630 & 88.4 & 36.5 & 100 & 39.3 & 43.6 \\
\hline \multirow{2}{*}{$\begin{array}{l}18 \\
(\mathrm{~A})\end{array}$} & $295-7$ & 345 & 63 & $40,000+$ & & & 28.3 & 60 & 28.3 & 40 \\
\hline & $\begin{array}{c}6 \\
76\end{array}$ & 345 & 55 & $40,000+$ & & & 35.9 & 1 & 36.5 & 40 \\
\hline
\end{tabular}

- UCS - (average) ultimate compressive strength 
At $70 \%$ UCS, a specimen of tube type 6 survived the 40,000 cycles and had a residual strength of $110 \%$. This result implies that the previous specimen tested at $60 \%$ for 17,601 cycles failed as a result of a manufacturing defect.

Specimens of tube type 2 failed at 1.9 and 1.5 lifetimes at $70 \%$ UCS; specimens of tube type 4 survived the 4 lifetimes and had a residual strength of $96 \%$; both were produced by manufacturer $A$. The two tubes produced by manufacturer $B$ failed much sooner: Tube type 10 lasted for only 1 lifetime, and the tube type 8 lasted for about $1 / 4 \cdot$ lifetime. The high porosity $(\sim 8$ to $9 \%$ ) of these tubes probably shortened their lives.

None of the tubes survived one lifetime at $80 \%$ UCS. Tube type 8 failed on the initial load. Tube type 2 survived 8,927 cycles, longer than any of the others. Again, the tubes from manufacturer $\mathrm{B}$ had the longest fatigue lives.

Tubes manufactured by $B$ were the only small-scale tubes tested at $76 \mathrm{~K}$-at 60 and $70 \%$ UCS. Both tube types tested ( 8 and 10 ) survived 4 lifetimes at $60 \%$ and had residual strengths of 63 and $88 \%$. Tube type 8 failed at 1.5 lifetimes at $70 \%$ UCS, whereas tube type 10 survived 4 lifetimes at $70 \%$ UCS and had a residual strength of $98 \%$. Thus the fatigue resistance of these composites is better at low temperatures.

An initial modulus and a modulus determined in the latter stages of fatigue are shown in Table 5.2. The modulus changed little or not at all during the fatigue, with one exception: The modulus of a specimen of tube type 6 decreased about $5 \mathrm{GPa}$ during its short life (900 cycles).

The two large-scale tubes, which were fatigued simultaneously, survived 40,000 cycles. No serious damage occurred in the 76-K tube. The upper tube (with a thermal gradient along its axis) had areas of hoop fiber breakage and delamination. The hoop fibers broke about 90 $\mathrm{mm}$ above the frost line on the tube, which was adjacent to the top of the Dewar and $37 \mathrm{~cm}$ above the lower end cap. From this area, the delaminations grew upward at $\pm 10^{\circ}$ bends, ranging from $2 \mathrm{~mm}$ to $10 \mathrm{~mm}$ wide. The hoop fibers began breaking at approximately 1,400 cycles. At 7,275 cycles, the delamination had grown to $115 \mathrm{~mm}$ in the direction of the upper end cap, and at 23,000 cycles, the delamination reached the end cap.

Approximately $180^{\circ}$ from the area where the hoop fibers broke, another area at the frost line delaminated, producing strips at $10^{\circ}$ angles from the tube surface. The total length of this delamination was $175 \mathrm{~mm}$. Small, circular delaminations ( $\leq 50 \mathrm{~mm}$ in diameter) occurred in two other areas of the tube, below the frost line; they did not appear to propagate.

The 76-K tube had only two small ( $\leq 50 \mathrm{~mm}$ in diameter) delaminated areas that did not propagate. These delaminations did not affect the modulus change in the 295-to-76-K (upper) tube (see Table 5.2). 


\section{Discussion}

The dominant factors that affected the compression strength of the tubes were manufacturing variations and fiber lay-up. Tube inconsistencies (e.g., resin-rich areas) and defects (e.g., porosity, delamination) due to manufacturing produced the scatter of the measured strengths. In the absence of tube inconsistencies, fiber lay-up was primarily responsible for the strength that was achievable. The data are summarized in Figure 6.1, plots of compressive strengths versus the axial compressive moduli of the tubes at room temperature (a) and at 76 and $4 \mathrm{~K}(\mathrm{~b})$. The axial compressive moduli were obtained by using strain gages oriented at $90^{\circ}$ with respect to each other in the test section of the tube. Thus, these moduli data are not, as a rule, strongly influenced by tube inconsistencies, and accordingly, their data scatter is typically less than $\pm 5 \%$ (see Table 5.1). However, the scatter of compressive-strength data is much larger. In Figure 6.2, the scatter of tube compressive strength data is portrayed in bar-graph format. Scatter is defined as the difference between the maximum and minimum tube strengths divided by the average tube strength. Here, the number of types of tubes (see Table 5.1 and Appendix A) that have data scatter within the specified limitations is summarized. Notice that the distribution of data scatter is, essentially, independent of temperature and that the data scatter for some types of tubes is as high as 20 to $25 \%$. To eliminate data scatter from consideration of the effects of other variables, the compressive-strength data points plotted in Figure 6.1 represent the highest two data points or, in the case of only two data points, the highest data point. The rationale for these procedures is based on the assumption that tube-manufacturing inconsistencies result only in lower compressive strengths.

Of course, there is a clear distinction between the use of the highest tube data to assess the effects of fiber lay-up and other parameters and the use of average or lowest bound data to determine operational stress levels.

A number of composite variables were studied, including tube length-to-diameter and diameter-to-thickness ratios, resin variations, filament winding versus pultrusion, and fiber layup. In addition, the tubes were obtained from five different suppliers. The effects of these variables are discussed below.

\subsection{Fiber Lay-up and Temperature}

Higher compressive moduli were obtained by increasing the number of fibers in $0^{\circ}$ or near $-0^{\circ}$ orientations (parallel to tube axis). Thus, in Figure 6.1, the highest moduli at each temperature were obtained from tubes with most of the fibers in near- $0^{\circ}$ orientations. The tubes that had the highest moduli contained some hoop windings (e.g., tube type 5 in Table 5.1) and had a $d / t$ ratio of 10 . Lower moduli resulted from quasi-isotropic lay-ups, including $\pm 45^{\circ}$ fiber orientations and $80^{\circ}$ to $90^{\circ}$ fiber orientations.

As seen in Figure 6.1, the compressive axial moduli correlate well with the tube compressive strength at both room and low temperatures. In changing from unidirectional to quasiisotropic fiber lay-ups, the compressive axial modulus changed from about 40 to $25 \mathrm{GPa}$ at room temperature and from about 45 to $30 \mathrm{GPa}$ at 76 and $4 \mathrm{~K}$. Compressive-strength changes resulting from differences in the fiber orientation (unidirectional to quasi-isotropic) were about $680 \mathrm{MPa}(100 \mathrm{ksi})$ to $400 \mathrm{MPa}(60 \mathrm{ksi})$ at room temperature and $1000 \mathrm{MPa}$ (145 ksi) to $680 \mathrm{MPa}(100 \mathrm{ksi})$ at low temperatures. 

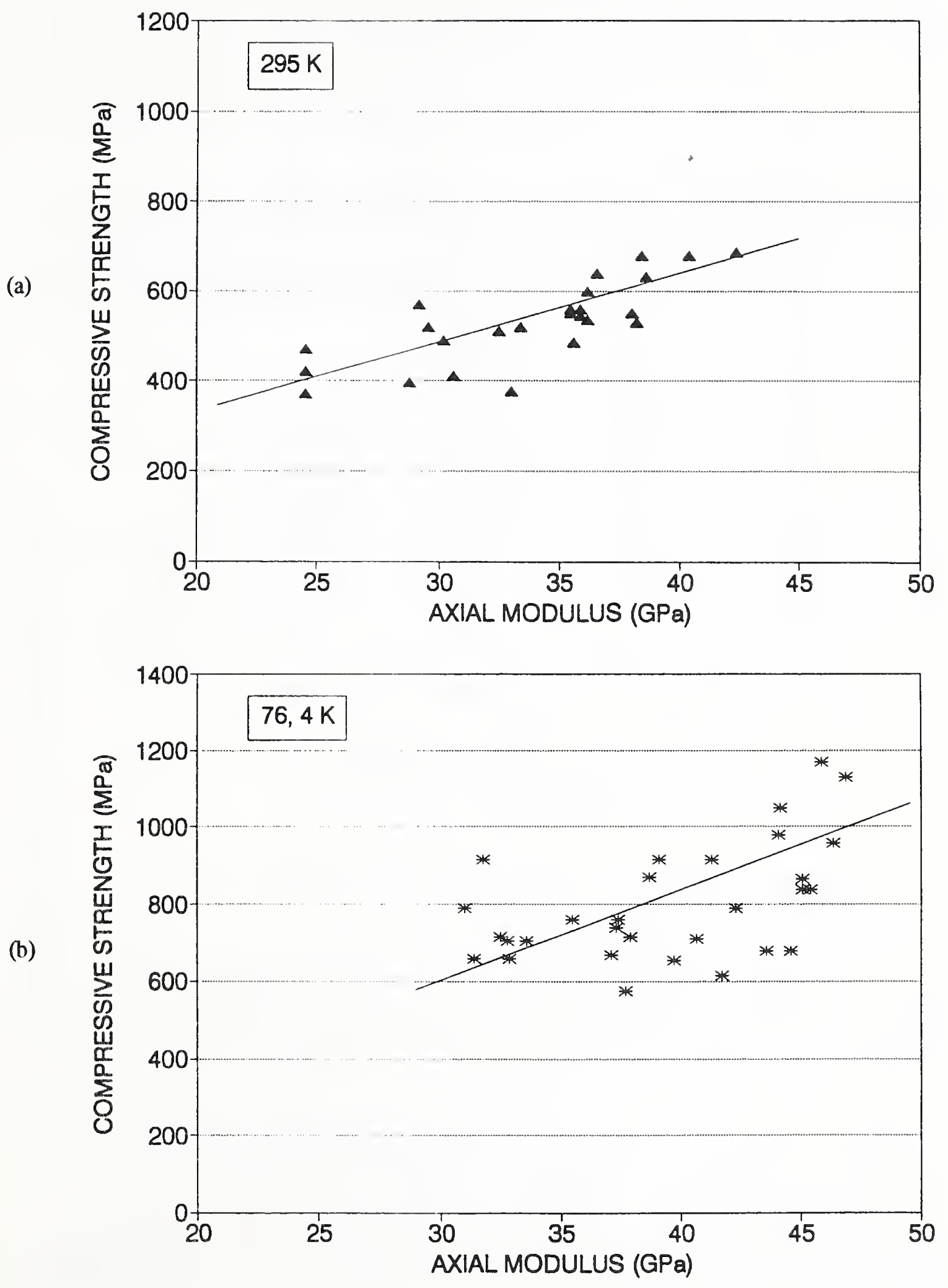

Figure 6.1. Compressive strength versus axial compressive moduli of tubes tested at (a) room temperature and (b) 76 and $4 \mathrm{~K}$. 

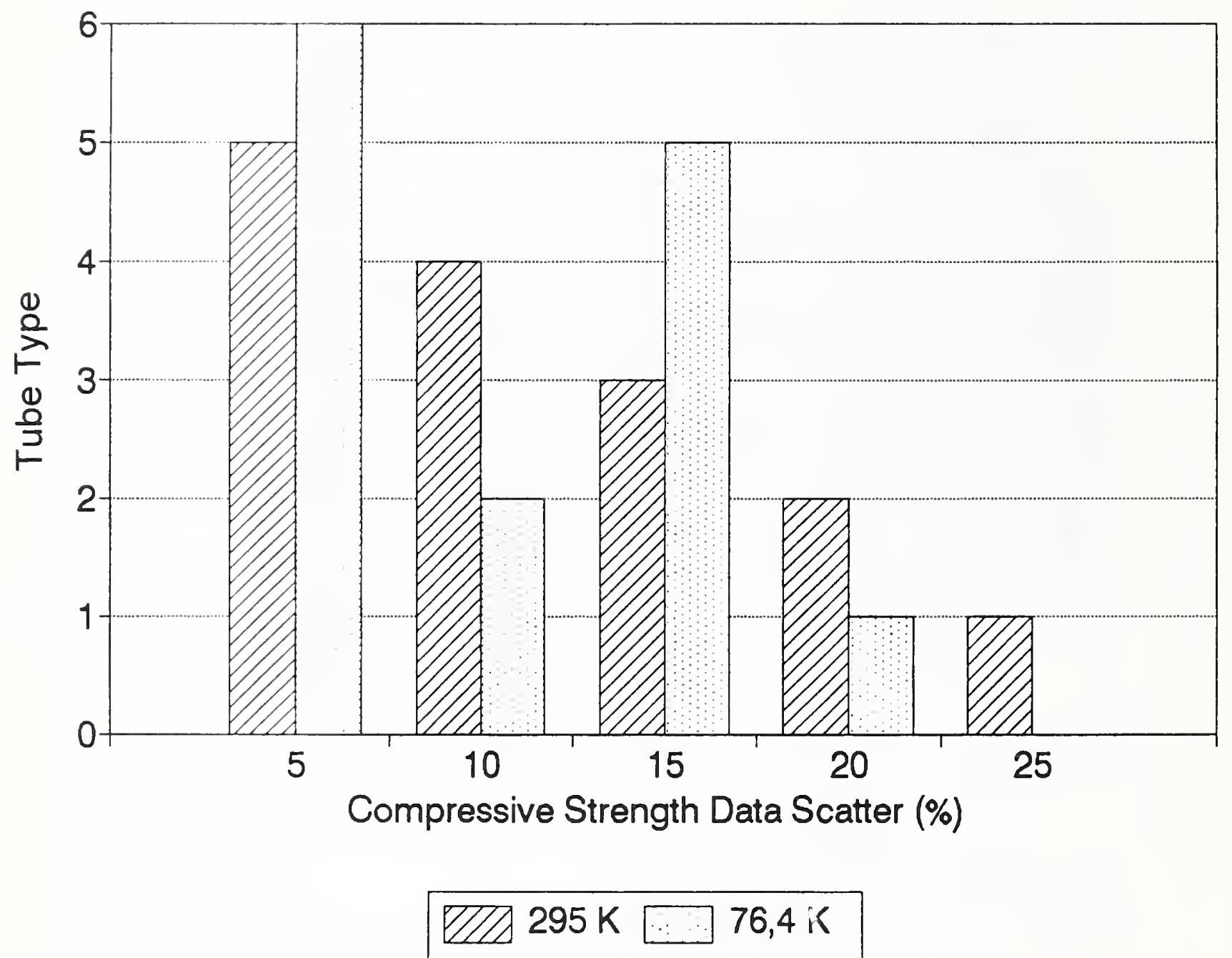

Figure 6.2. Scatter of compressive strengths for various types of tube.

At room temperature, the average value of all compressive-strength data points in Figure 6.1 is $533 \mathrm{MPa}$; at low temperatures, the average value is $806 \mathrm{MPa}$. Thus, at low temperatures $(76,4 \mathrm{~K})$, tube strengths were about $50 \%$ higher than at room temperature. This corresponds very well with the $50^{+} \%$ increase in the tensile strength of E-glass fiber from $295 \mathrm{~K}$ to 76 and $4 \mathrm{~K}$ (see Figure 4.1 in $\S \mathrm{IV}$ ).

The average of the axial compressive moduli at room temperature is $33.9 \mathrm{GPa}$, and at 76 and $4 \mathrm{~K}, 39.1 \mathrm{GPa}$. This represents a $15 \%$ increase on cooling. The compressive modulus of the epoxy-matrix unidirectional composite increased about $10 \%$ from 295 to 76 and $4 \mathrm{~K}$ $(\S \mathrm{V})$, whereas the elastic modulus of typical epoxies increased approximately $100 \%$ ( $\$ I I I)$. Thus, considering that the average moduli data for the tubes includes some quasi-isotropic layups (in which the matrix contributes more to mechanical properties), the $15 \%$ average increase of the tube axial modulus is reasonable. 
At room temperature, the effect on the tube compressive strength of adding $80^{\circ}$ to $90^{\circ}$ windings to a tube with predominantly $0^{\circ}$ to $10^{\circ}$ fiber orientation was difficult to discern from our data set. However, at low temperatures, there was a clear advantage in adding fiber windings in the hoop direction. Since the thermal conductivity should have been lower in those tubes with limited $90^{\circ}$ fiber windings, there appears to be an operational advantage in having a limited number of layers with $80^{\circ}$ to $90^{\circ}$ fiber orientations.

For each tube lay-up, the maximum compressive strengths at room and cryogenic temperatures are shown in Figure 6.3. The tubes with the highest strengths were those with a preponderance of fibers in the $\pm 10^{\circ}$ orientation. At low temperatures, the strongest tubes were those with $\pm 10^{\circ}$ lay-ups supplemented with transverse-wound fibers; the room-temperature data were less distinctive. The compressive strength of a $[ \pm 10]_{n} \mathrm{CE}$ tube was equivalent to that of tubes with transverse fibers.

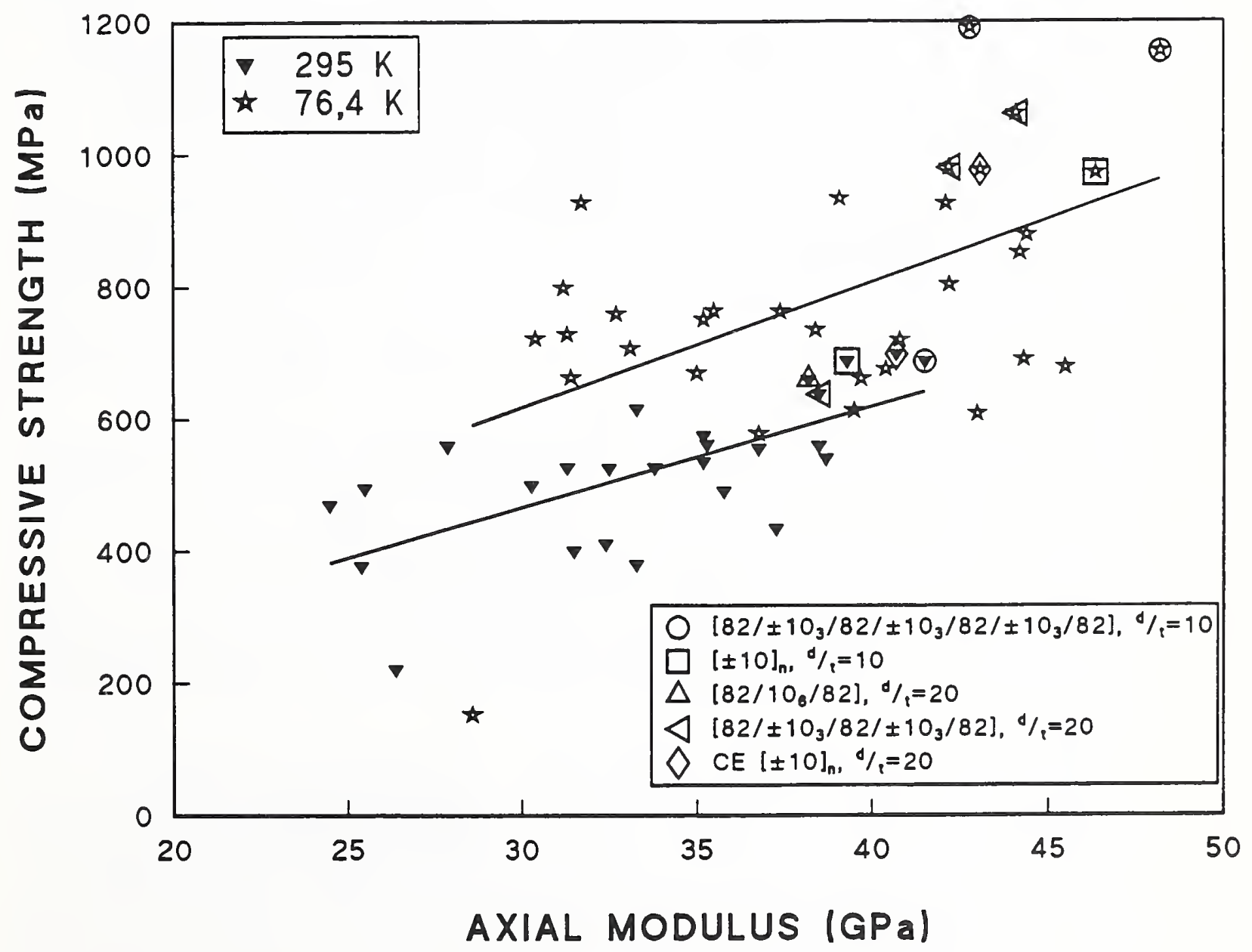

Figure 6.3. Compressive strength versus axial modulus for different lay-ups. 


\subsection{Type of Resin}

Four epoxies, one vinyl ester, and one cyanate ester were used as the matrix materials in the tubes included in this test program. The cyanate-ester data are highlighted in Figure 6.4. The cyanate ester (CE) tubes were manufactured by pressure expansion of an internal rubber tube to reduce porosity and improve fiber straightness. The reader is cautioned that the very limited data (one test per variable) significantly increase the chances for misleading points. Nevertheless, these data raise several issues that deserve to be recognized:

1. At room temperature, the tube made by the rubber-expansion technique from cyanateester resin and near $-0^{\circ}$ fiber orientation had exceptionally good strength. The high strength may reflect the straighter and near $-0^{\circ}$ fiber orientation, yet the compressive strength at $76 \mathrm{~K}$, although higher than that of many other tubes at low temperatures, was not the highest. This high room-temperature compressive strength (698 MPa, $101 \mathrm{ksi}$ ) suggests that additional research be conducted on filament-wound tubes made with a cyanate-ester resin if the cost of the cyanate ester can be reduced to be competitive with the epoxies.

2. At both 295 and $76 \mathrm{~K}$, the compressive strengths of quasi-isotropic-fiber tubes made from the same cyanate ester by the rubber-expansion technique were very low. This is in stark contrast to the results of unidirectional-fiber tubes discussed above. Follow-up macrographs indicated that these tubes had very high porosity and delaminations.

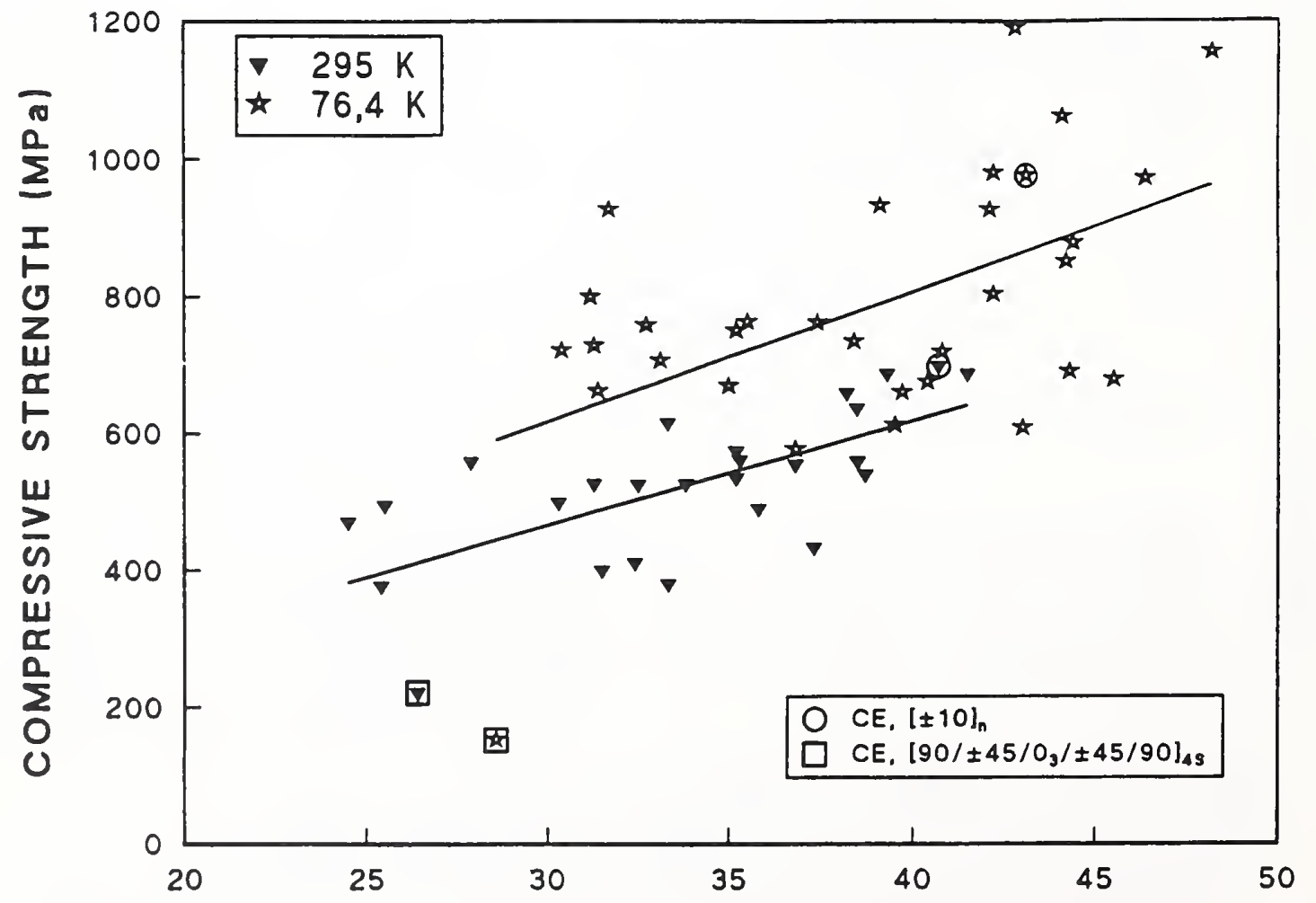

AXIAL MODULUS (GPa)

Figure 6.4. Compressive strength versus axial modulus for cyanate-ester-resin tube, lay-ups $[ \pm 10]_{n}$ and $\left[90 / \pm 45 / 0_{3} / \pm 45 / 90\right]_{4 s}$. 
Compressive data for filament-wound and pultruded tubes made from from vinyl-ester resin are highlighted in Figure 6.5(a) and (b), respectively. Notice that the compressive strengths of the pultruded tubes are lower than those of filament-wound tubes, but those of the epoxy- and vinyl-ester-resin systems are similar. Thus, the vinyl-ester resin system has no strength advantage. However, the supplier of the pultruded tubes indicated that it was significantly easier to manufacture tubes from a vinyl-ester resin system than from an epoxy resin system.

The filament-wound tubes made from vinyl-ester resin with a predominantly unidirectional lay-up $\left(\left[82 / 10_{6} / 82\right]\right)$ produced by one supplier had equivalent strengths but lower axial moduli than those of other unidirectional, epoxy-resin tubes (tube type 21 in Table 5.1).

To summarize, the strengths of tubes constructed from vinyl-ester resin system were lower, or equivalent to, those of comparable epoxy-resin-system tubes. Consideration should be given to the use of vinyl ester only because it may reduce the resin or production costs. The S2-glass tubes had low strengths and low strains to failure. Since their properties are inferior to those of E-glass, there is no advantage to using S2-glass fibers in these tubes.

\subsection{Tube Geometry}

Analyses of the data contained in Table 5.1 for tube types 1,2, 5, and 6 that have predominantly unidirectional fibers suggest that decreasing the tube diameter-to-thickness ratio $(d / t)$ from 20 to 10 results in improved strength and elastic modulus. Specifically, the axial compressive strength increased $16 \%$ and the elastic (axial) modulus increased $8 \%$ when the tube wall thickness increased $5 \mathrm{~mm}(5.1$ to $10.2 \mathrm{~mm})$, independent of test temperature. However, the scatter of data almost doubled for the thicker tubes. Those trends are not seen for the quasiisotropic lay-ups (tube types 3 and 4).

The increase in elastic modulus must be attributed to an increase of fibers with near $-0^{\circ}$ orientations in the thicker tubes. In tubes that contain off-axis fiber windings, the percent fiber in the near $-0^{\circ}$ orientation is greater in tubes with thicker walls.

The increase in strength is partially associated with the increase in elastic modulus. From the slopes of the lines in Figure 6.1, one can see that, approximately, an $8 \%$ increase in elastic modulus produced an $8 \%$ increase in strength at both room and low temperatures. The remaining strength increase must be associated with the increase in structural stability of orthotropic cylinders of larger cross-sectional area.

Limited tests were conducted to assess the effects of tube length, for a given tube diameter, on compressive strength (see Table 5.1). All these tests were conducted at room temperature. Tube lengths varied from $710 \mathrm{~mm}$ (28 in) to $480 \mathrm{~mm}$ (19 in) to $230 \mathrm{~mm}$ (9 in) for 10.2-mm (0.4-in) wall thickness. Three different lay-ups (tube types 1, 3, and 5 in Table 5.1) were included in a total of 16 comparable tests. Average compressive strengths increased from $501 \mathrm{MPa}$ to $518 \mathrm{MPa}$ to $542 \mathrm{MPa}$ for the decreasing tube lengths. Thus, a 3-to-1 reduction of tube length resulted in an approximate $10 \%$ increase in average compressive strength. However, the maximum compressive strengths, such as those used to construct Figures 6.1 through 6.5 , were insensitive to tube length. These results are interpreted to mean that a reduction in tube length serves to reduce the chances of sensing load-limiting defects in the tubes, thus 
(a)

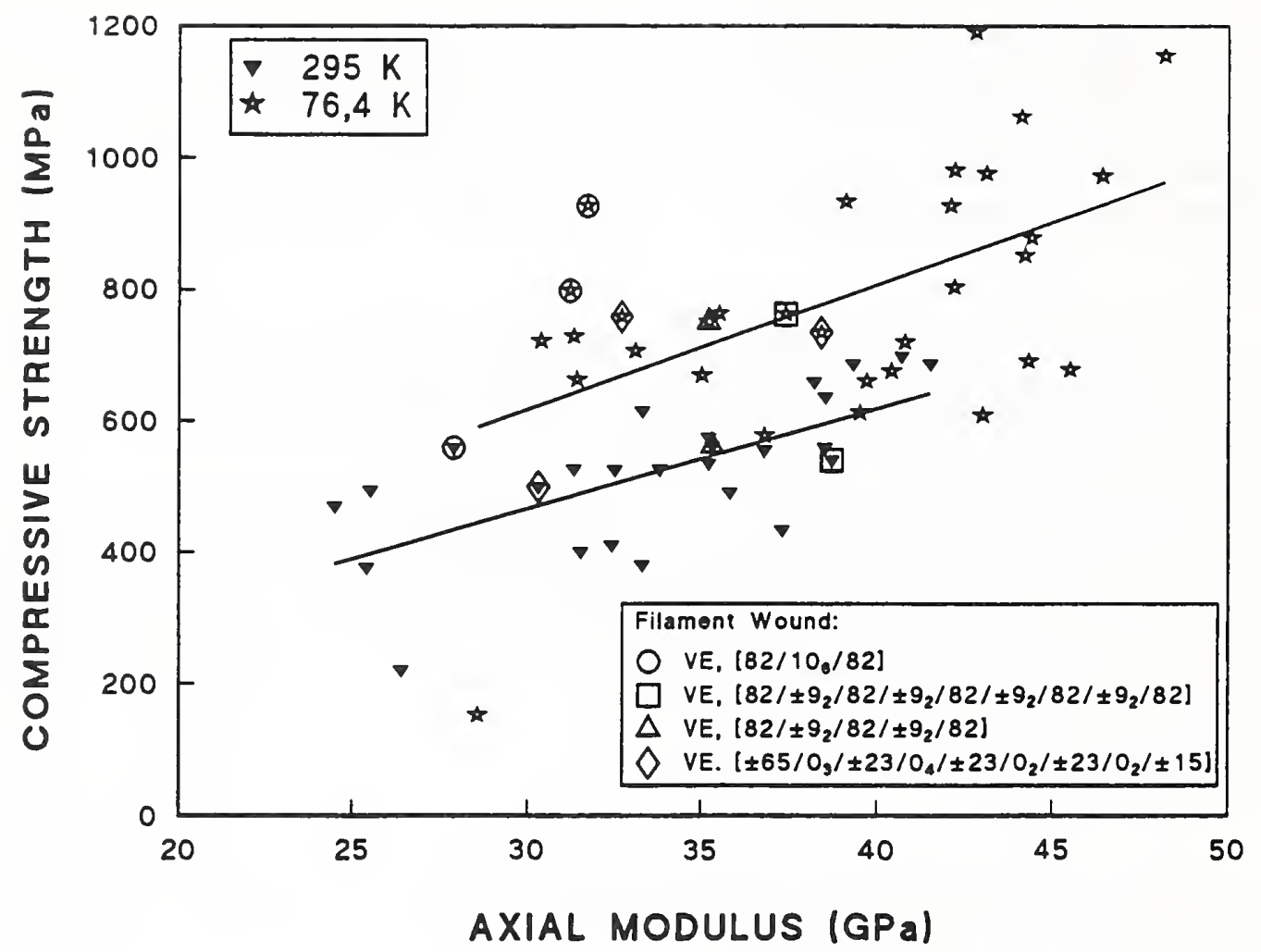

(b)

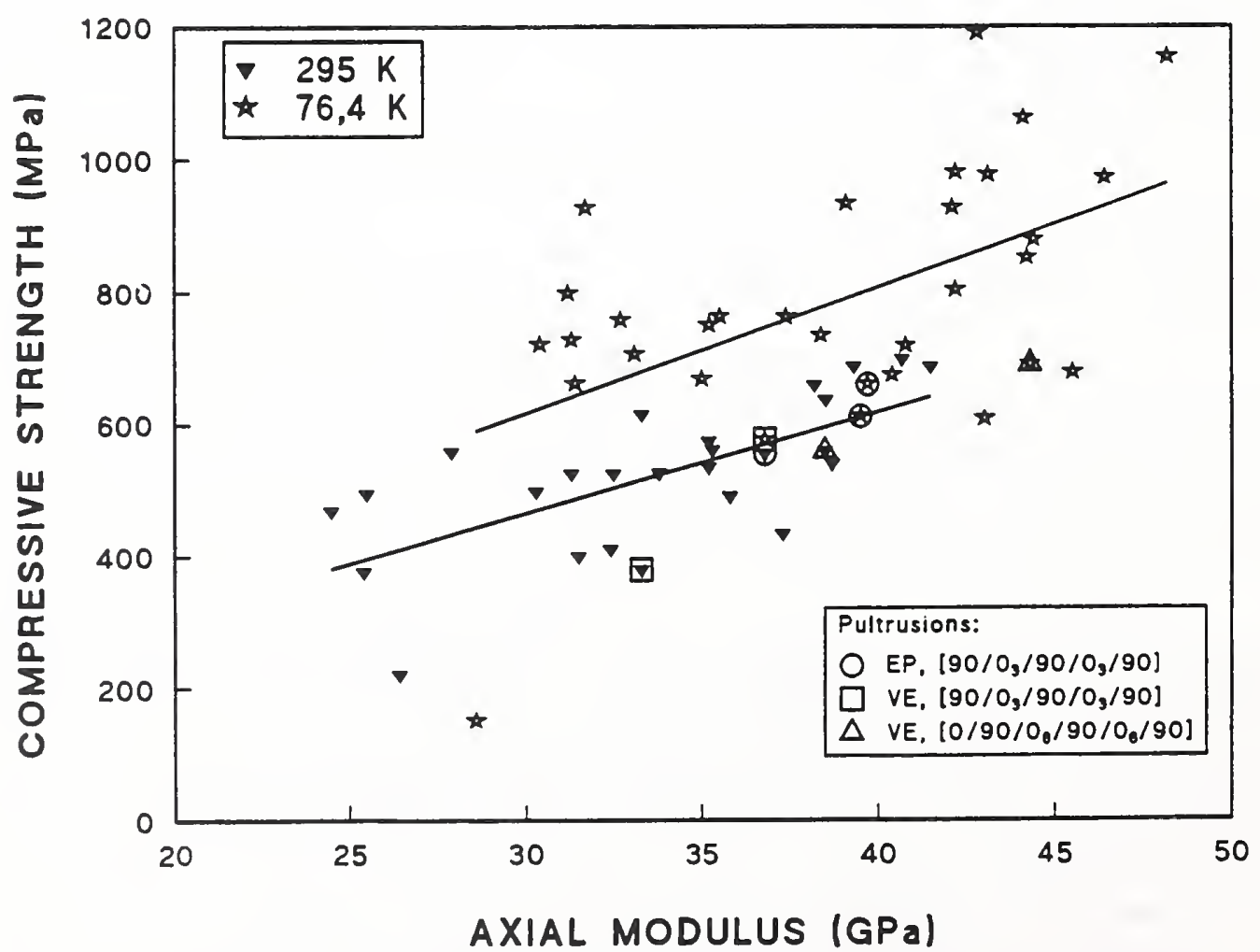

Figure 6.5. Compressive strength versus axial modulus of (a) filament-wound and (b) pultruded tubes. 
increasing the effective average strength. Structurally, the length changes were well within tube buckling limitations, and, thus, no effects on maximum strengths were observed.

In the Phase I work of both SMES teams, the effect of increasing the diameter of the tubes was to lower the compressive strength by 40 to $60 \%$. The large-diameter tube data for this program are highlighted in Figure 6.6. It is apparent that increasing the tube diameter and maintaining constant $d / t$ and $\ell / d$ ratios had no significant influence on compressive strength. Higher, large-scale, filament-wound tube strengths may be achieved if one is able to increase the compressive axial modulus.

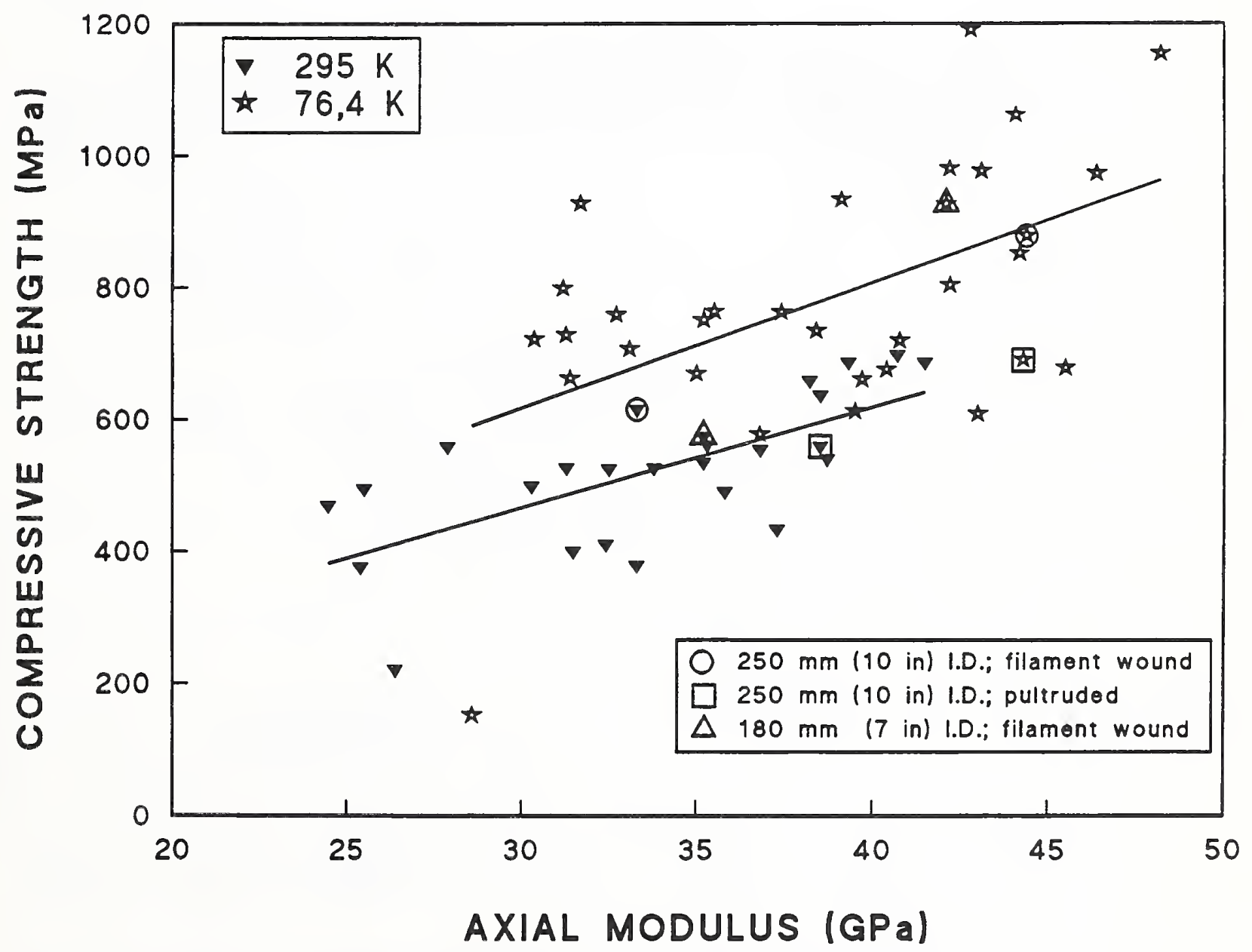

Figure 6.6. Compressive strength versus axial modulus for tubes of different diameters. 


\subsection{Filament Winding versus Pultrusion}

Data from tubes constructed with the pultrusion process are shown in Figure 6.7. In Figure 6.6, the large-scale pultruded tubes are compared with filament-wound tubes. The values all fall short of those for equivalent filament-wound tubes, especially at low temperatures. The reason for the poorer performance of the pultruded tubes is not immediately apparent. The tubes have not been compared for relative differences in porosity (volume percent or size). Fiber alignment should be better in the pultruded tubes. There are distinctions in the hooporiented fibers (see Appendix A). However, as seen from Figures 6.6 and 6.7 at comparable axial modulus levels, the strengths of pultruded tubes are always less than those of filamentwound tubes.

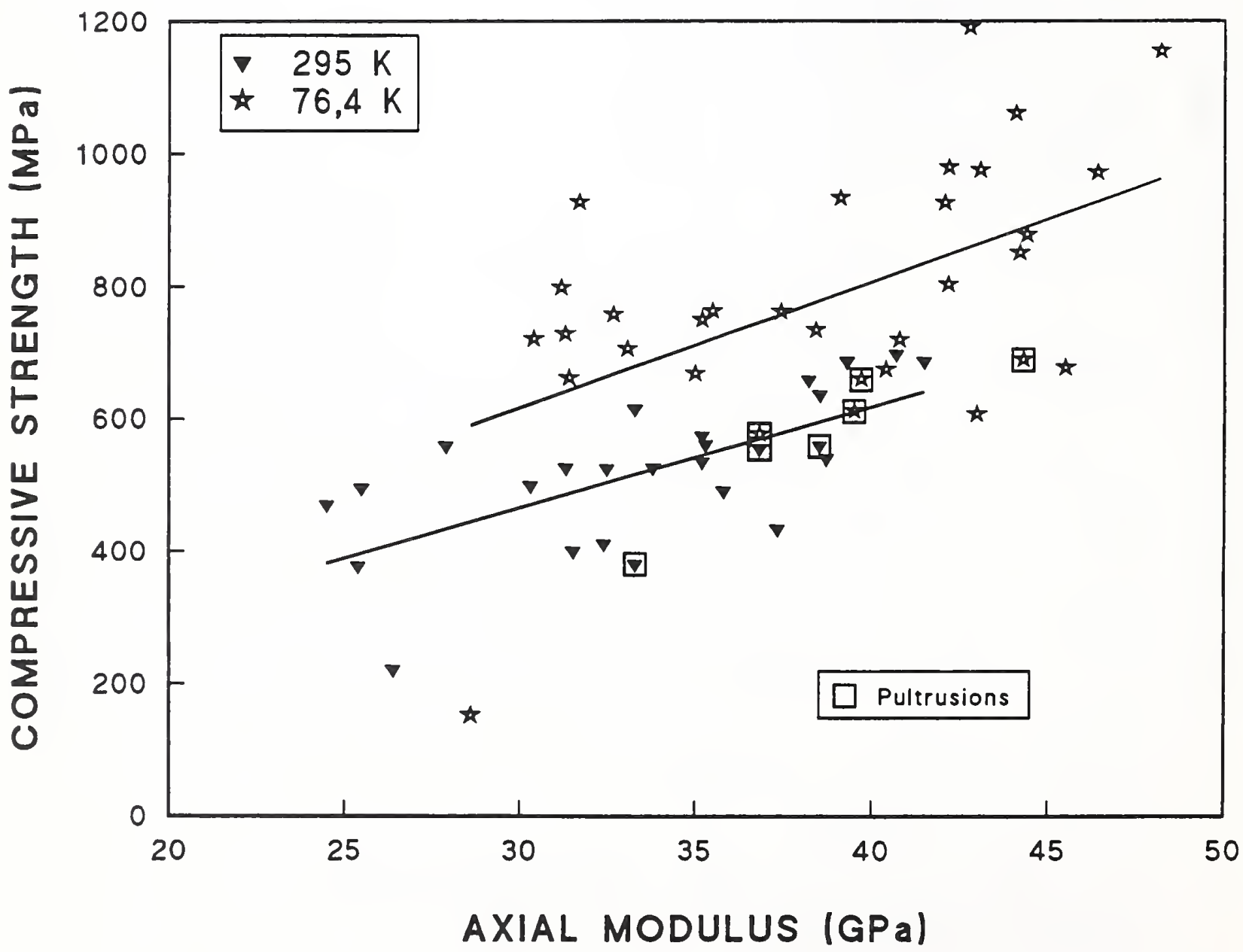

Figure 6.7. Compressive strength versus axial modulus for pultruded tubes. 


\subsection{Strain to Failure}

Most average values of tube strain to failure (summarized in Table 5.1) range from 1.4 to $1.8 \%$ at room temperature for unidirectional (axial) fiber orientations and more than $2.0 \%$ for quasi-isotropic lay-ups. At low temperatures, the strain-to-failure values are higher: the average maximum values are about $30 \%$ higher at $76 \mathrm{~K}$ than at $295 \mathrm{~K}$. In general, for unidirectional-fiber-dominated lay-ups, the correspondence of the strain-to-failure data with composite and manufacturing variables is similar to that of compressive strength, as discussed in $\$ 6.2$ through $\$ 6.4$.

Hoop strain to failure is, very generally, inversely related to the axial modulus. At all temperatures, maximum hoop strain is linearly related to the axial strain to failure, with the exception of room-temperature data from those tubes that do not contain hoop windings (Figure 6.8).

At low temperatures, the lack of hoop windings is not significant, and all data between hoop and axial strains are linearly related. Apparently, at low temperatures, the resin matrix has sufficient elastic stiffness to restrain, circumferentially, the microbuckling tendencies of the axial fibers.

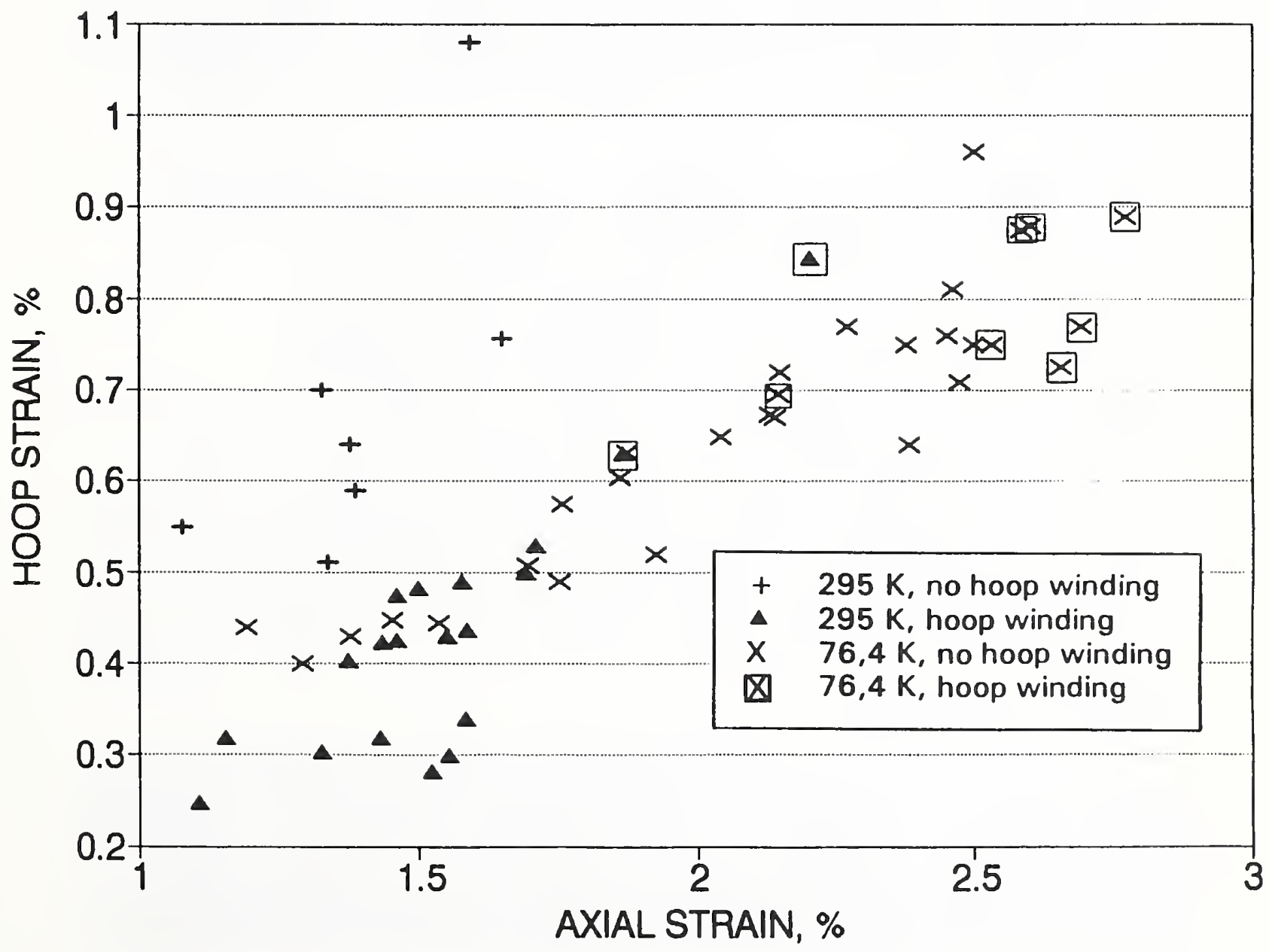

Figure 6.8. Hoop versus axial strain. 


\subsection{Optimization of Strength and Thermal Conductivity}

In $\S \mathrm{V} .3$, we reported thermal conductivity measurements as a function of temperature for $0^{\circ}$ and $90^{\circ}$ fiber orientations. The thermal conductivity of the quasi-isotropic fiber lay-up can be estimated by assuming that the thermal conductivity is half the sum of the $0^{\circ}$ and $90^{\circ}$ values. Then, simple ratios were calculated to compare the strength and thermal conductivity of unidirectional and quasi-isotropic fiber lay-ups; they are presented in Table 6.1. At all temperatures, the thermal conductivities of composites with quasi-isotropic fiber lay-ups were about $20 \%$ less than those of composites with unidirectional fiber lay-ups. Thus, heat leakage was reduced about $20 \%$ in changing to off-axis fiber orientations. However, at room temperature, about $25 \%$ of tube compressive strengths were lost by changing from unidirectional to quasiisotropic lay-ups. At low temperatures, the compressive strength increased about $50 \%$ for unidirectional orientations. Quasi-isotropic lay-ups at low temperatures provided about the same compressive strengths as unidirectional lay-ups at room temperature but about $30 \%$ less strength than unidirectional lay-ups at low temperatures.

To summarize, if one uses a one-tube strut system spanning the temperature range from 4 to $295 \mathrm{~K}$, the weakest portion of the tube is at room temperature, and therefore, one should use a fiber lay-up with predominantly $0^{\circ}$ to $10^{\circ}$ fiber orientations and some at $80^{\circ}$ to $90^{\circ}$. If one uses a two-tube strut system with temperature increments from 4 to $76 \mathrm{~K}$ and 76 to $295 \mathrm{~K}$, both tubes should have predominantly $0^{\circ}$ to $10^{\circ}$ fiber lay-ups if the tube cross-sectional area is reduced in the low-temperature segment. If the tube cross-sectional area is held constant, then a quasi-isotropic lay-up may be used in the low-temperature section to reduce heat leakage.

Table 6.1. Effects of unidirectional and quasi-isotropic fiber lay-ups on ratios of tube strength and thermal conductivity.

\begin{tabular}{lcc}
\hline \multicolumn{1}{c}{ Property } & $\begin{array}{c}\text { Unidirectional } \\
\left(\text { near } 0^{\circ}\right)\end{array}$ & $\begin{array}{c}\text { Qunsi-isotropic } \\
\left(0^{\circ}, 45^{\circ}, 90^{\circ}\right)\end{array}$ \\
\hline $\begin{array}{l}\text { Thermal conductivity } \\
\text { (all temperatures) }\end{array}$ & 1 & 0.8 \\
Compressive strength & & \\
at $295 \mathrm{~K}$ & 1 & 0.75 \\
at $76,4 \mathrm{~K}$ & 1.5 & 1.05 \\
\hline
\end{tabular}

\subsection{Comparison of Strengths}

Tensile and compressive strength data for E-glass and unidirectional plies and tubes are summarized in Table 6.2. From Figure 4.1 of $\S I V$, the tensile strength of E-glass is about 3800 $\mathrm{MPa}(550 \mathrm{ksi})$ at room temperature and $5700 \mathrm{MPa}(825 \mathrm{ksi})$ at $76 \mathrm{~K}$. The tensile strengths of unidirectional plies (adjusted to $100 \%$ fiber volume fraction) are about $65 \%$ less than the fiber strengths (see Tables 1.3 and 1.6 of $\S \mathrm{V}$ ) at both 295 and $76 \mathrm{~K}$. Surprisingly, the compressive strengths of unidirectional plies and tubes with predominately unidirectional fiber orientations 
are nearly identical. Adjusted to $100 \%$ fiber volume percent, the tensile strengths of both equal $1750 \mathrm{MPa}(250 \mathrm{ksi})$ at $76 \mathrm{~K}$, and at room temperature, their strengths are within $7 \%$.

The close similarity of compressive strengths of the unidirectional plies and tubes suggests difficulty in achieving higher strengths by adjusting the fiber lay-up in tubes. However, since the unidirectional ply specimens had rather high porosity, strength improvements may be possible by lowing the porosity content or size in both types of composites.

Table 6.2. Comparison of strengths.

\begin{tabular}{|c|c|c|c|c|c|c|c|c|}
\hline \multirow[t]{2}{*}{ Material } & \multicolumn{2}{|c|}{$\begin{array}{c}\text { Tensile } \\
\text { Strength }\end{array}$} & \multicolumn{2}{|c|}{$\begin{array}{l}\text { Compressive } \\
\text { Strength } \\
(\mathrm{MPa})(\mathrm{ksi})\end{array}$} & \multicolumn{2}{|c|}{$\begin{array}{c}\text { Tensile } \\
\text { Strength } \\
(\mathrm{MPa})(\mathrm{ksi})\end{array}$} & \multicolumn{2}{|c|}{$\begin{array}{l}\text { Compressive } \\
\text { Strength } \\
\text { (MPa) (ksi) }\end{array}$} \\
\hline & \multicolumn{4}{|c|}{ at room temperature } & \multicolumn{4}{|c|}{ at $76 \mathrm{~K}$} \\
\hline E-glass fiber & 3800 & 550 & - & & 5700 & 825 & - & \\
\hline $\begin{array}{l}\text { Unidirectional ply } \\
-75 \text { vol.\% fiber } \\
\text {-extrapolated to } \\
100 \% \text { fiber) }\end{array}$ & $\begin{array}{l}1010 \\
1350\end{array}$ & $\begin{array}{l}146 \\
200\end{array}$ & $\begin{array}{r}800 \\
1075\end{array}$ & $\begin{array}{l}115 \\
155\end{array}$ & $\begin{array}{l}1320 \\
1750\end{array}$ & $\begin{array}{l}190 \\
250\end{array}$ & $\begin{array}{l}1400 \\
1875\end{array}$ & $\begin{array}{l}200 \\
275\end{array}$ \\
\hline $\begin{array}{l}\text { Unidirectional tube } \\
-60 \text { vol.\% fiber } \\
\text {-extrapolated to } \\
100 \text { vol.\% fiber }\end{array}$ & - & & $\begin{array}{r}700 \\
1150\end{array}$ & $\begin{array}{l}100 \\
170\end{array}$ & - & & $\begin{array}{l}1050 \\
1750\end{array}$ & $\begin{array}{l}150 \\
250\end{array}$ \\
\hline
\end{tabular}

\subsection{Factors of Concern for Strut Systems}

Rather large data scatter resulted from compression tests of $10-\mathrm{mm}$ - to $250-\mathrm{mm}$ diameter tubes produced by filament-winding and pultrusion processes. This data scatter has been attributed, primarily, to production-induced irregularities, such as porosity and resin-rich areas. To improve the operating stress levels of struts, better production techniques must be developed and maintained. Some improvement will come naturally from production repetitiveness. Other improvement must be insisted upon by the drafting of tighter procurement specifications.

Qualification (acceptance) criteria for manufactured tubes must also be addressed. Alternate techniques include proof testing, mechanical screening at lower stress levels, or nondestructive evaluation (NDE). In our view, the most practical and least expensive approach is mechanical screening using strain measurements. For example, perhaps guidelines such as those suggested in §IX could be developed. Proof testing requires extensive large-scale testing, and NDE techniques will require significant development. However, once developed, NDE techniques would offer speed and accuracy not available from other methods. 
Other factors that have an impact on these considerations and the establishment of structural safety factors are the degree of structural redundancy (in the strut system) that is factored into the design of SMES plants and the ease of in-service monitoring, repair, and replacement of strut systems.

The thermal intercepts included in the strut systems must also be considered. If the thermal intercept is mechanically attached to each tube, the connection may result in reduced compressive or fatigue strength at the interface. In this case, the entire strut system must be tested to compare system and tube strengths.

Finally, each strut system endures a temperature gradient from 4 to $295 \mathrm{~K}$ with possible other fixed temperatures (from thermal intercepts) along this length. The temperature gradient induces a stress gradient along the tube length. If the thermal gradient is too severe, the resulting stress gradient may be sufficient to focus fatigue strain within this particular tube length. This possibility suggests that strut-system testing should be performed under simulated operating conditions prior to selecting the final design.

\section{References}

[2.1] Davis, J.G., Jr. Fabrication of uniaxial filament-reinforced epoxy tubes for structural applications. Soc. Adv. Mater. Process Eng. Proc. 14: II2A-1; 1968.

[2.2] Swanson, S.R.; Colvin, G.E., Jr.; Haslam, C.L. Measurements of the compressive behavior of AS4/3501-6 and IM7/8551-7 carbon/epoxy, in Proceedings, 33rd international SAMPE symposium. Covina, CA: Society for Advanced Material and Process Engineering; 1988. 1571-1578.

[2.3] Colvin, G.E., Jr.; Swanson, S.R. In-situ compressive strength of carbon/epoxy AS4/ 3501-6 laminates. ASME J. Mater. Technol. 115: 122-128; 1993.

[2.4] Khitrov, V.V.; Kutarzhnov, Y.I. The effect of angle of reinforcement on the carrying capacity of wound tubes in compression. Mech. Compos. Mater. 4: 372-376; 1979.

[2.5] Hahn, H.T.; Williams, J.G. Compression failure mechanisms in unidirectional composites. NASA TM-85834; 1984.

[2.6] Greszczuk, L.B. Microbuckling of laminar reinforced composites, in Composite materials and design. ASTM STP 546. Philadelphia, PA: American Society for Testing and Materials; 1974. 5-29.

[2.7] Sohi, M.M.; Hahn, H.T.; Williams, J.G. The effect of resin toughness and modulus on compressive failure modes of quasi-isotropic graphite/epoxy laminates, in Toughened composites. ASTM STP 937. Philadelphia, PA: American Society for Testing and Materials; 1987. 37-60.

[2.8] Shuart, M.J. Failure of compression-loaded multi-directional composite laminates. AIAA J. 88: 631-641; 1988. 
[2.9] Sinclair, J.H.; Chamis, C.C. Compressive behavior of unidirectional fibrous composites, in Compressing testing of homogenous materials and composites. ASTM STP 808, 155-174. Philadelphia, PA: American Society for Testing and Materials; 1982.

[2.10] Brink, N.O. Mechanical behavior of reinforced plastics at cryogenic temperatures. Soc. Plast. Eng. J. 20: 1123-1131; 1964.

[2.11] Hanson, P.H.; Richards, H.T.; Hickel, R.O. Preliminary investigation of filamentwound glass-reinforced plastics and liners for cryogenic vessels. NASA TN D-2741; 1965.

[4.1] Peters, S.T. Filament winding, in Reference book for composite technology. Lancaster, PA: Technomic Publishing; 1989. 143-178. 


\section{Appendixes}

\section{Appendix A. Tube Construction}

\section{TUBE TYPE 1}

MANUFACTURER:

A

LAY-UP:

$[ \pm 10]_{n}$

WALL THICKNESS:

$10.2 \mathrm{~mm}(0.40 \mathrm{in})$

LENGTH:

$710 \mathrm{~mm}$ (28 in), $480 \mathrm{~mm}$ (19 in), $230 \mathrm{~mm}$ (9 in)

RESIN:

DGEBA/DCH/ARA/ACL

FIBER:

E-glass, $1100 \mathrm{TEX}(450 \mathrm{yd} / \mathrm{lb})$, continuous strand

PROCESS:

filament winding

MEASURED FIBER VOLUME FRACTION: $\quad 0.56$

MEASURED VOID VOLUME FRACTION: $\quad 0.023$

COMMENTS:

- Thickness: 24 plies $\times 0.424 \mathrm{~mm}(0.0167 \mathrm{in}) / \mathrm{ply}=10.2 \mathrm{~mm}(0.401 \mathrm{in})$

- Proprietary resin and cure schedule

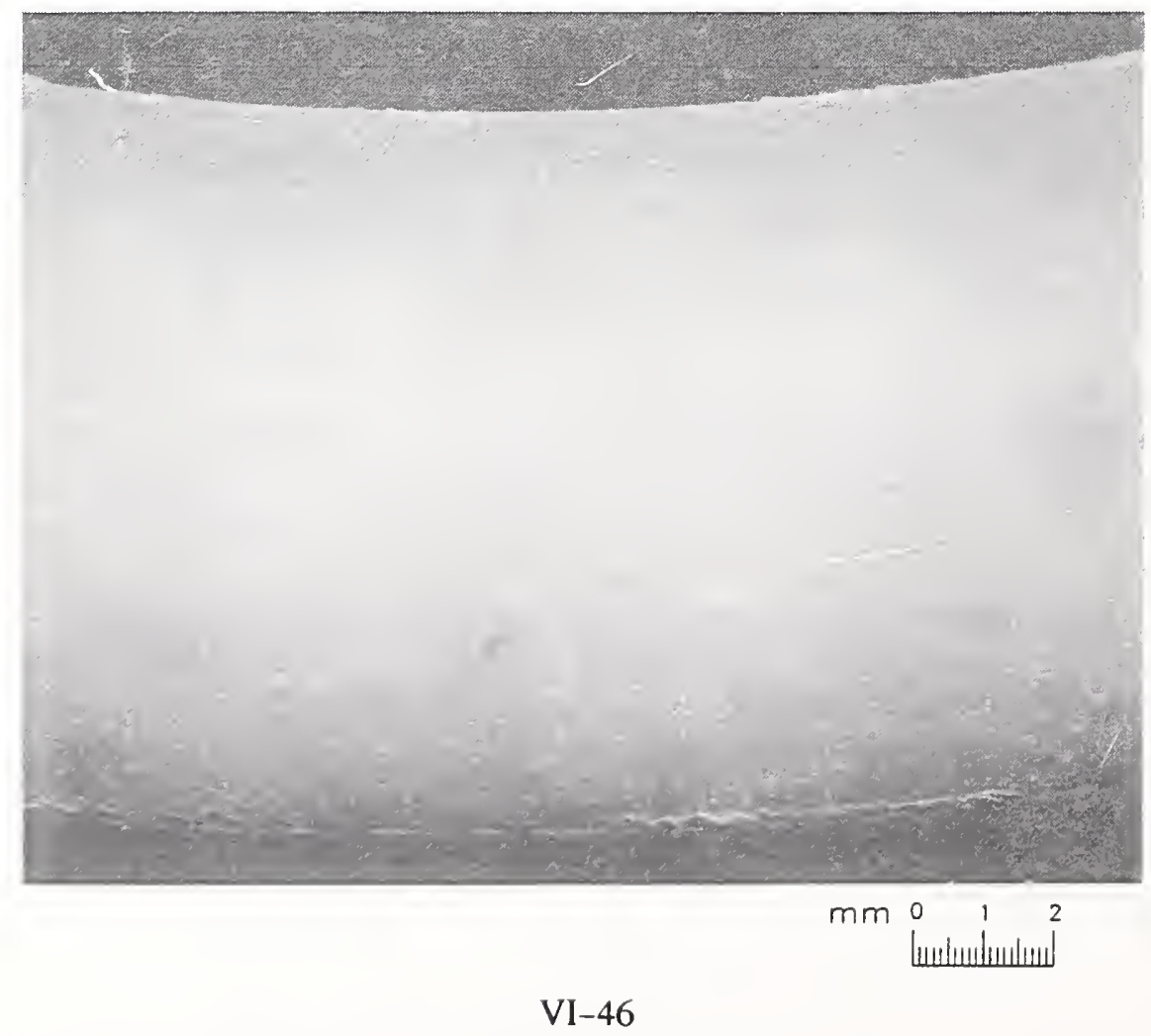




\section{TUBE TYPE 2}

MANUFACTURER:

A

LAY-UP: $\quad[ \pm 10]_{n}$

WALL THICKNESS: $\quad 5.1 \mathrm{~mm}(0.20 \mathrm{in})$

LENGTH: $\quad 710 \mathrm{~mm}(28 \mathrm{in})$

RESIN:

DGEBA/DCH/ARA/ACL

FIBER:

E-glass, 1100 TEX (450 yd/lb), continuous strand

PROCESS: filament winding

MEASURED FIBER VOLUME FRACTION: $\quad 0.53$

MEASURED VOID VOLUME FRACTION: $\quad 0.018$

COMMENTS:

- Thickness: 12 plies $\times 0.424 \mathrm{~mm}(0.0167 \mathrm{in}) / \mathrm{ply}=5.1 \mathrm{~mm}(0.200 \mathrm{in})$

- Proprietary resin and cure schedule

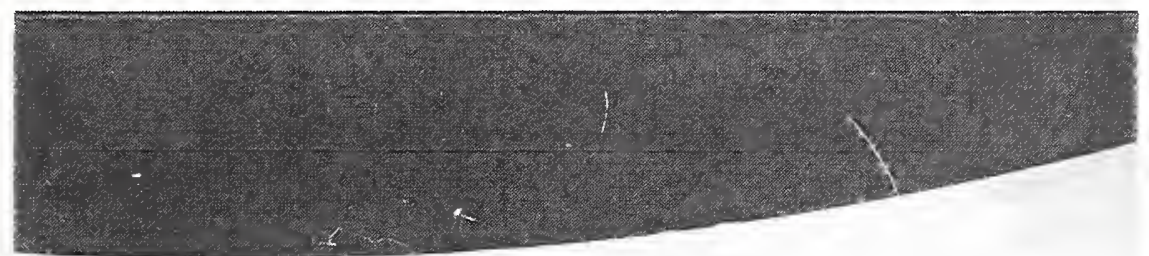




\section{TUBE TYPE 3}

MANUFACTURER:

LAY-UP:

WALL THICKNESS:

LENGTH:

RESIN:

FIBER:

PROCESS:

MEASURED FIBER VOLUME FRACTION:

MEASURED VOID VOLUME FRACTION:

COMMENTS:

- 24 plies;

thickness: $12[ \pm 10]$ plies $\times 0.43 \mathrm{~mm}(0.017 \mathrm{in}) / \mathrm{ply}=5.2 \mathrm{~mm}(0.204 \mathrm{in})-50 \%$ 4 [82] plies $\times 0.43 \mathrm{~mm}(0.017 \mathrm{in}) / \mathrm{ply}=1.7 \mathrm{~mm}(0.068 \mathrm{in})-17 \%$

$8[ \pm 45]$ plies $\times 0.43 \mathrm{~mm}(0.017 \mathrm{in}) / \mathrm{ply}=3.4 \mathrm{~mm}(0.136 \mathrm{in})-33 \%$

- Proprietary resin and cure schedule
A

$\left[82 / \pm 45 / \pm 10_{3} / \pm 45 / 82_{2} / \pm 45 / \pm 10_{3} / \pm 45 / 82\right]$

$10.2 \mathrm{~mm}(0.40 \mathrm{in})$

$710 \mathrm{~mm}$ (28 in), $480 \mathrm{~mm}$ (19 in), $230 \mathrm{~mm}$ (9 in)

DGEBA/DCH/ARA/ACL

E-glass, 1100 TEX (450 yd/lb), continuous strand

filament winding
0.017

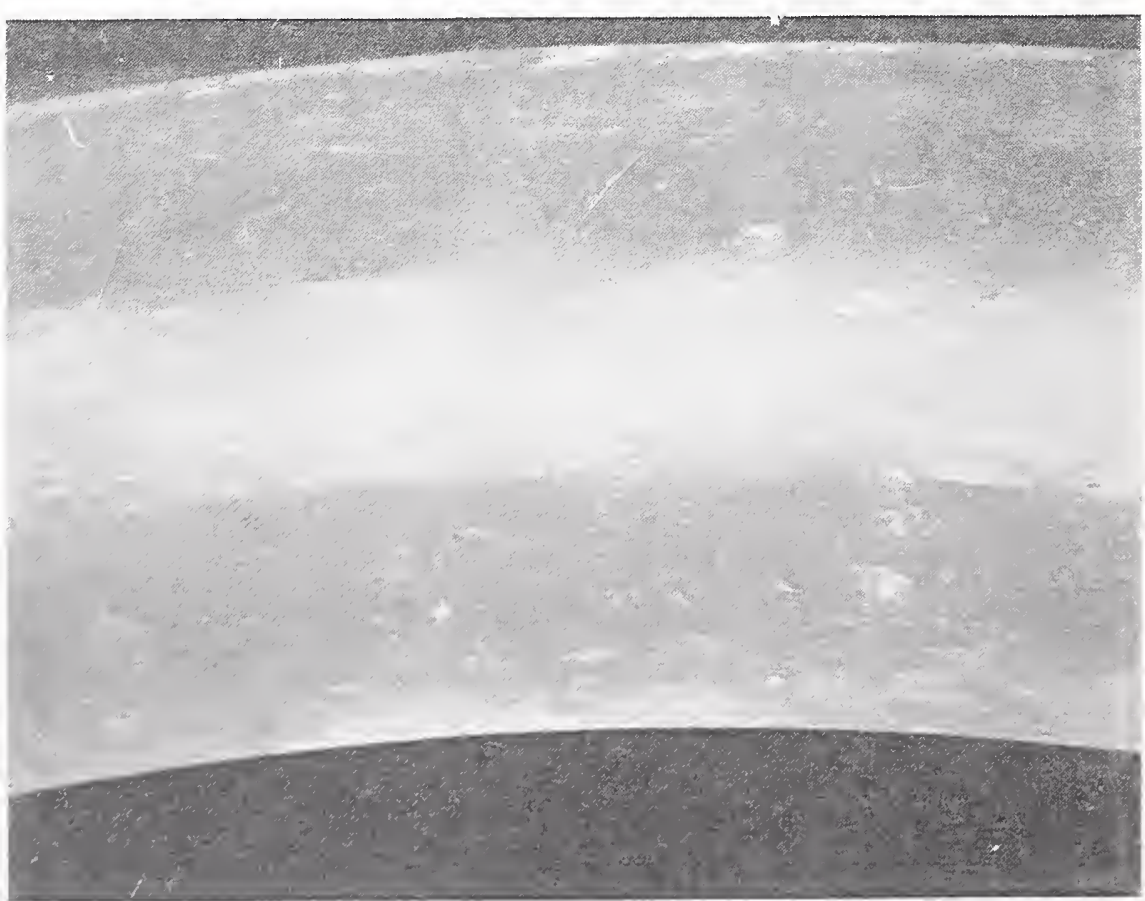

$m m$ 


\section{TUBE TYPE 4}

MANUFACTURER: A

LAY-UP:

$\left[82 / \pm 45 / \pm 10_{3} / \pm 45 / 82\right]$

WALL THICKNESS:

$5.1 \mathrm{~mm}(0.20 \mathrm{in})$

LENGTH:

$710 \mathrm{~mm}$ (28 in)

RESIN:

DGEBA/DCH/ARA/ACL

FIBER:

E-glass, $1100 \mathrm{TEX}$ (450 yd/lb), continuous strand

PROCESS:

filament winding

MEASURED FIBER VOLUME FRACTION: $\quad 0.52$

MEASURED VOID VOLUME FRACTION: $\quad 0.005$

\section{COMMENTS:}

- 12 plies;

thickness: $6[ \pm 10]$ plies $\times 0.43 \mathrm{~mm}(0.017 \mathrm{in}) / \mathrm{ply}=2.6 \mathrm{~mm}(0.102 \mathrm{in})-50 \%$ 2 [82] plies $\times 0.43 \mathrm{~mm}(0.017 \mathrm{in}) / \mathrm{ply}=0.86 \mathrm{~mm}(0.034 \mathrm{in})-17 \%$

$4[ \pm 45]$ plies $\times 0.43 \mathrm{~mm}(0.017 \mathrm{in}) / \mathrm{ply}=1.7 \mathrm{~mm}(0.068 \mathrm{in})-33 \%$

- There are no internal circular wraps [82] on this lay-up

- Proprietary cure and resin schedule

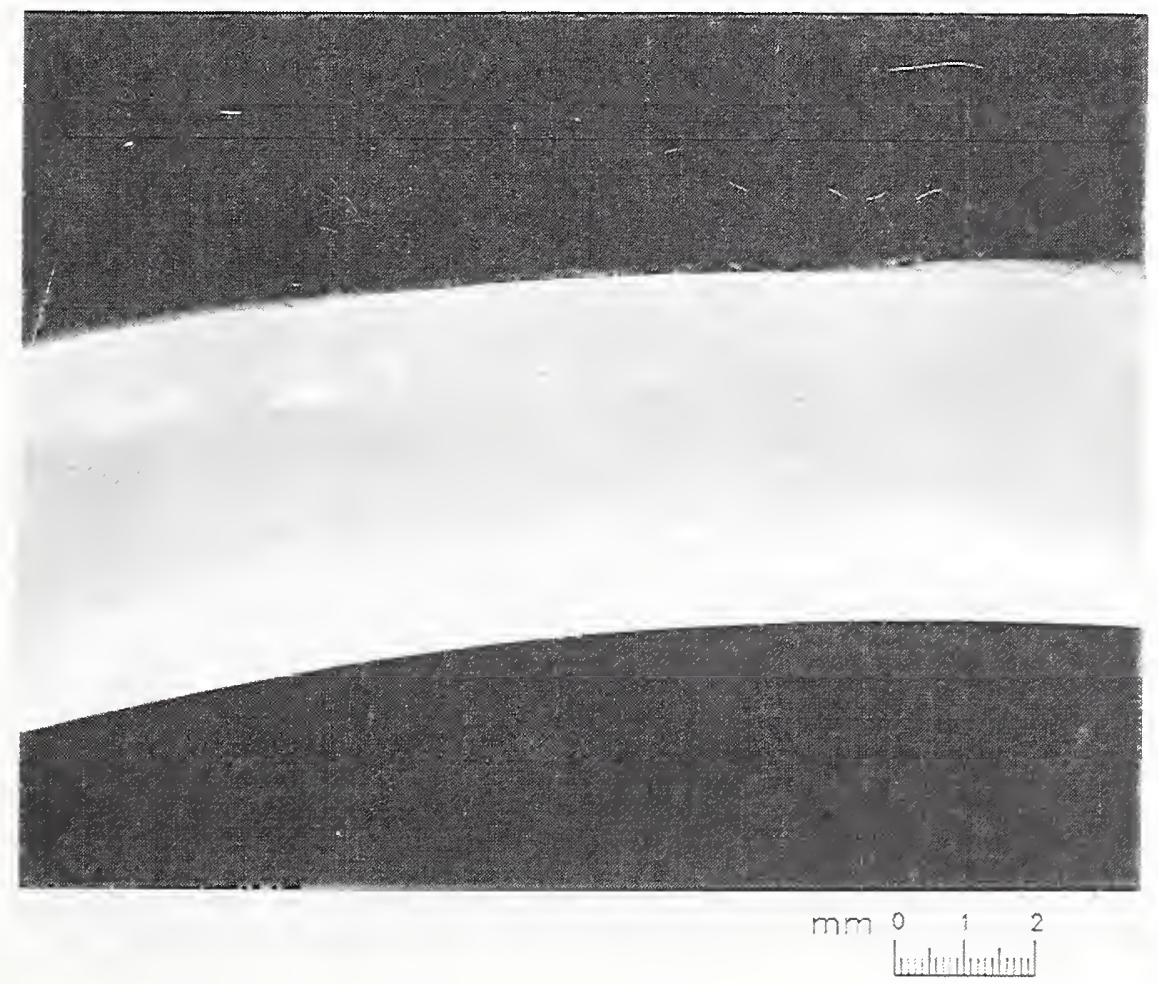




\section{TUBE TYPE 5}

MANUFACTURER: A

LAY-UP:

$\left[82 / \pm 10_{3} / 82 / \pm 10_{3} / 82 / \pm 10_{3} / 82\right]$

WALL THICKNESS:

$10.2 \mathrm{~mm}(0.40 \mathrm{in})$

LENGTH:

$710 \mathrm{~mm}$ (28 in), $480 \mathrm{~mm}$ (19 in), $230 \mathrm{~mm}$ (9 in)

RESIN:

DGEBA/DCH/ARA/ACL

FIBER:

E-glass, 1100 TEX (450 yd/lb), continuous strand

PROCESS:

filament winding

MEASURED FIBER VOLUME FRACTION: $\quad 0.609$

MEASURED VOID VOLUME FRACTION: $\quad<0.005$

\section{COMMENTS:}

- 22 plies;

thickness: $18[ \pm 10]$ plies $\times 0.51 \mathrm{~mm}(0.020 \mathrm{in}) / \mathrm{ply}=9.1 \mathrm{~mm}(0.360 \mathrm{in})-90 \%$ 4 [82] plies $\times 0.025 \mathrm{~mm}(0.010 \mathrm{in}) / \mathrm{ply}=1 \mathrm{~mm}(0.040 \mathrm{in})-10 \%$

- 2 internal hoop winds [82]

- Proprietary resin and cure schedule

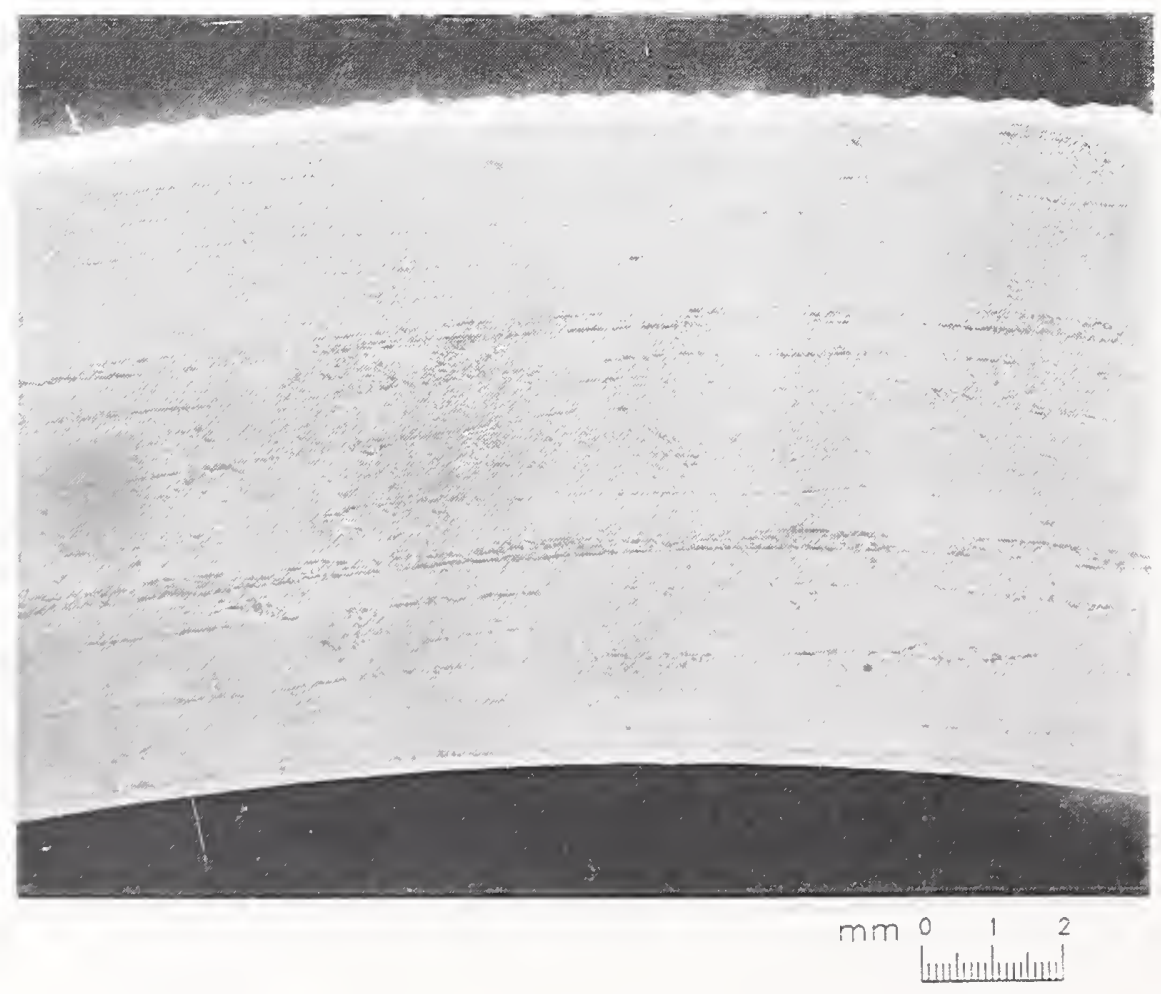




\section{TUBE TYPE 6}

MANUFACTURER:

A

LAY-UP:

$\left[82 / \pm 10_{3} / 82 / \pm 10_{3} / 82\right]$

WALL THICKNESS:

$5.1 \mathrm{~mm}(0.20 \mathrm{in})$

LENGTH:

$710 \mathrm{~mm}(28 \mathrm{in})$

RESIN:

DGEBA/DCH/ARA/ACL

FIBER:

E-glass, 1100 TEX (450 yd/lb), continuous strand

PROCESS:

filament winding

MEASURED FIBER VOLUME FRACTION: $\quad 0.64$

MEASURED VOID VOLUME FRACTION: $\quad<0.005$

\section{COMMENTS:}

- 15 plies:

thickness: $12[ \pm 10]$ plies $\times 0.36 \mathrm{~mm}(0.014 \mathrm{in}) / \mathrm{ply}=4.3 \mathrm{~mm}(0.168 \mathrm{in})-85 \%$

3 [82] plies $\times 0.25 \mathrm{~mm}(0.01 \mathrm{in}) / \mathrm{ply}=0.75 \mathrm{~mm}(0.030 \mathrm{in})-15 \%$

- 1 internal hoop winding [82]

- Proprietary resin and cure schedule
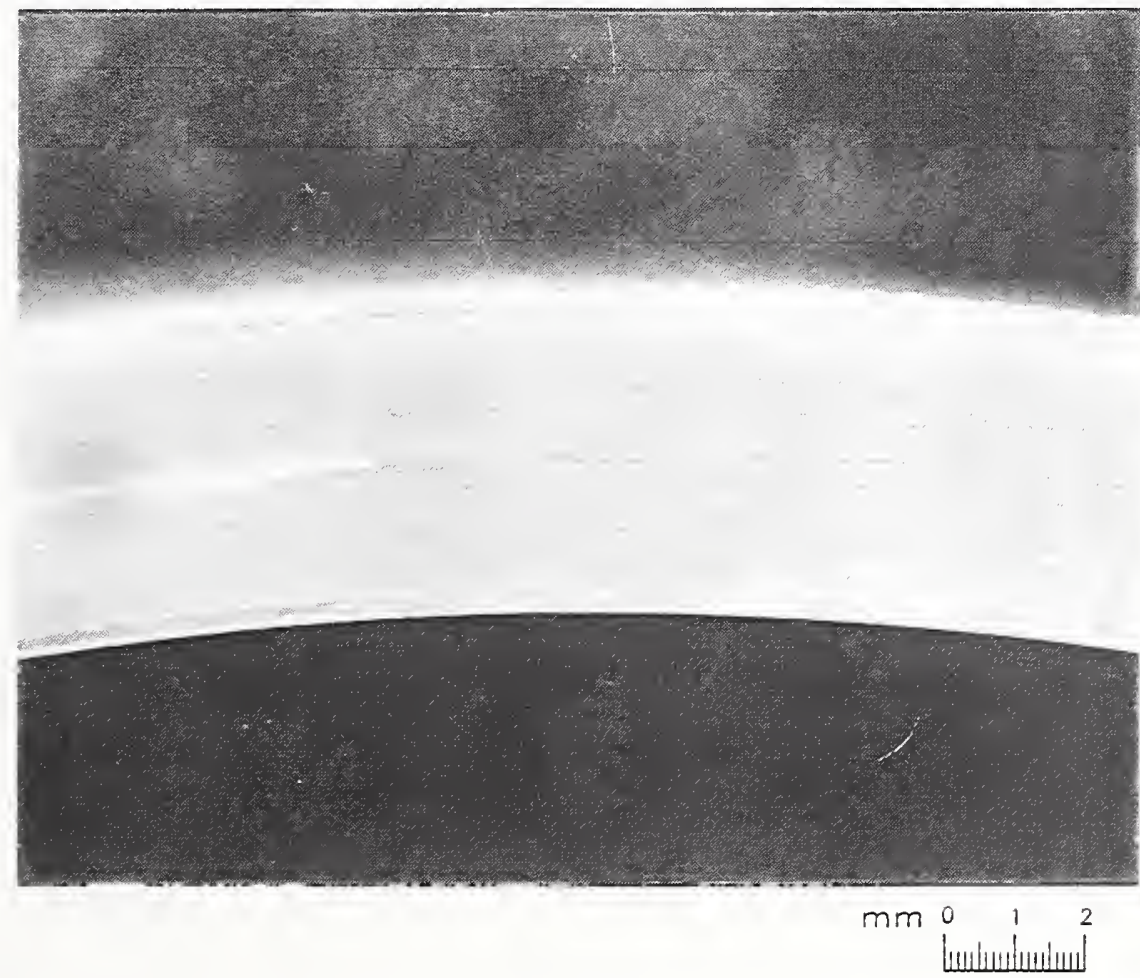


\section{TUBE TYPE 7}

MANUFACTURER: B

LAY-UP:

$\left[82 / \pm 9_{2} / 82 / \pm 9_{2} / 82 / \pm 9_{2} / 82 / \pm 9_{2} / 82\right]$

WALL THICKNESS:

$10.2 \mathrm{~mm}(0.40 \mathrm{in})$

LENGTH:

$710 \mathrm{~mm}$ (28 in)

RESIN:

DGEBA/ARA/DTA

FIBER:

E-glass, $1100 \mathrm{TEX}$ (450 yd/lb), continuous strand

PROCESS:

filament winding

MEASURED FIBER VOLUME FRACTION: $\quad 0.49$

MEASURED VOID VOLUME FRACTION: $\quad 0.071$

COMMENTS:

- Cure: $1 \mathrm{~h}$ at $120^{\circ} \mathrm{C}, 2 \mathrm{~h}$ at $177^{\circ} \mathrm{C}$

- 21 plies; thickness: $\quad 16[ \pm 9]$ plies $\times 0.53 \mathrm{~mm}(0.021 \mathrm{in}) / \mathrm{ply}=8.5 \mathrm{~mm}(0.336 \mathrm{in})-85 \%$ 5 [82] plies $\times 0.30 \mathrm{~mm}(0.012 \mathrm{in}) / \mathrm{ply}=1.5 \mathrm{~mm}(0.060 \mathrm{in})-15 \%$
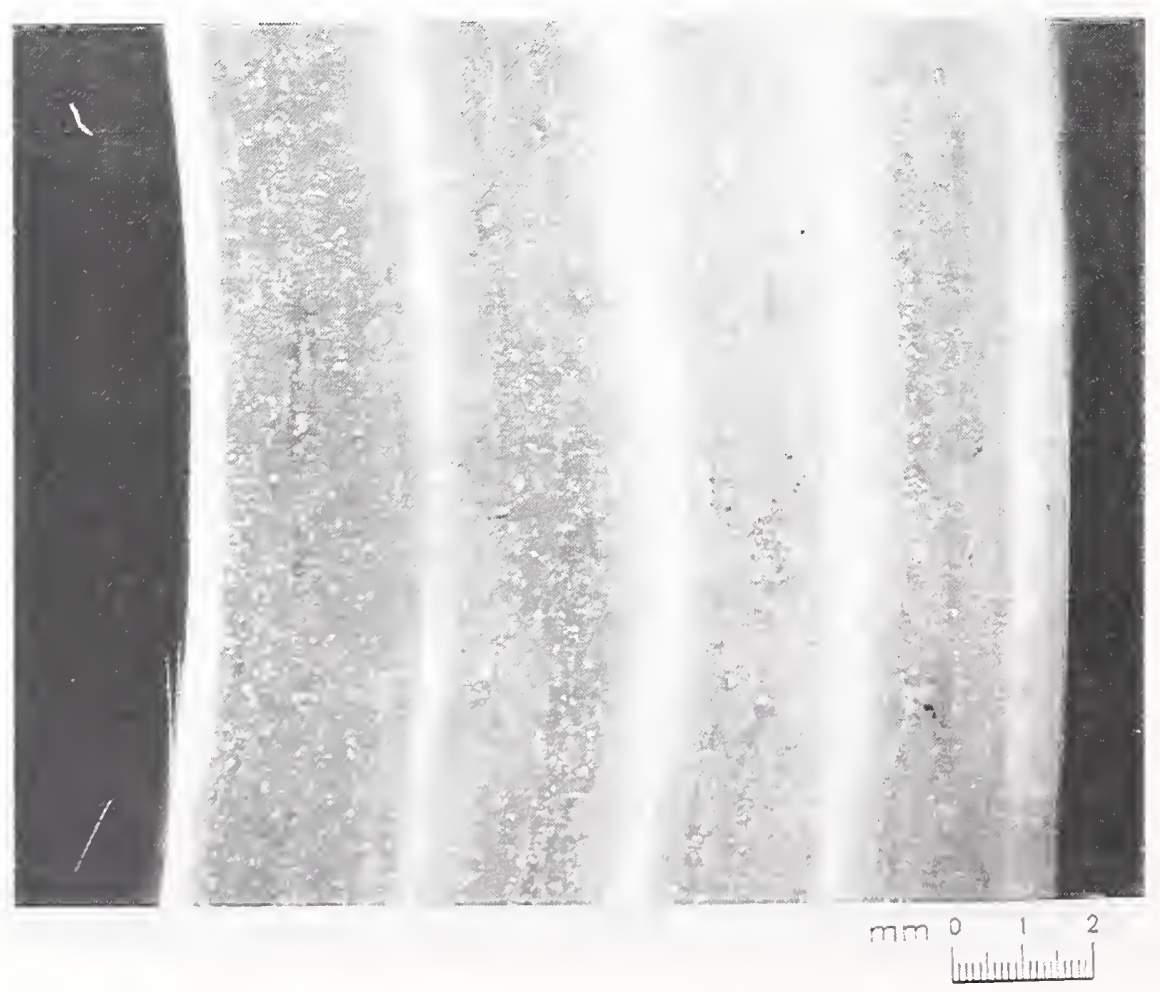


\section{TUBE TYPE 8}

MANUFACTURER: B

LAY-UP: $\quad\left[82 / \pm 9_{2} / 82 / \pm 9_{2} / 82\right]$

WALL THICKNESS: $\quad 5.1 \mathrm{~mm}(0.20 \mathrm{in})$

LENGTH: $\quad 710 \mathrm{~mm}(28 \mathrm{in})$

RESIN: $\quad$ DGEBA/ARA/DTA

FIBER: $\quad$ E-glass, $1100 \mathrm{TEX}$ (450 yd/lb), continuous strand

PROCESS: $\quad$ filament winding

MEASURED FIBER VOLUME FRACTION: $\quad 0.59$

MEASURED VOID VOLUME FRACTION: $\quad 0.052$

COMMENTS:

- Cure: $1 \mathrm{~h}$ at $120^{\circ} \mathrm{C}, 2 \mathrm{~h}$ at $177^{\circ} \mathrm{C}$ )

- 11 plies;

thickness: $8[ \pm 9]$ plies $\times 0.53 \mathrm{~mm}(0.021 \mathrm{in}) / \mathrm{ply}=4.3 \mathrm{~mm}(0.17 \mathrm{in})-84 \%$

3 [82] plies $\times 0.28 \mathrm{~mm}(0.011 \mathrm{in}) / \mathrm{ply}=0.84 \mathrm{~mm}(0.033 \mathrm{in})-16 \%$

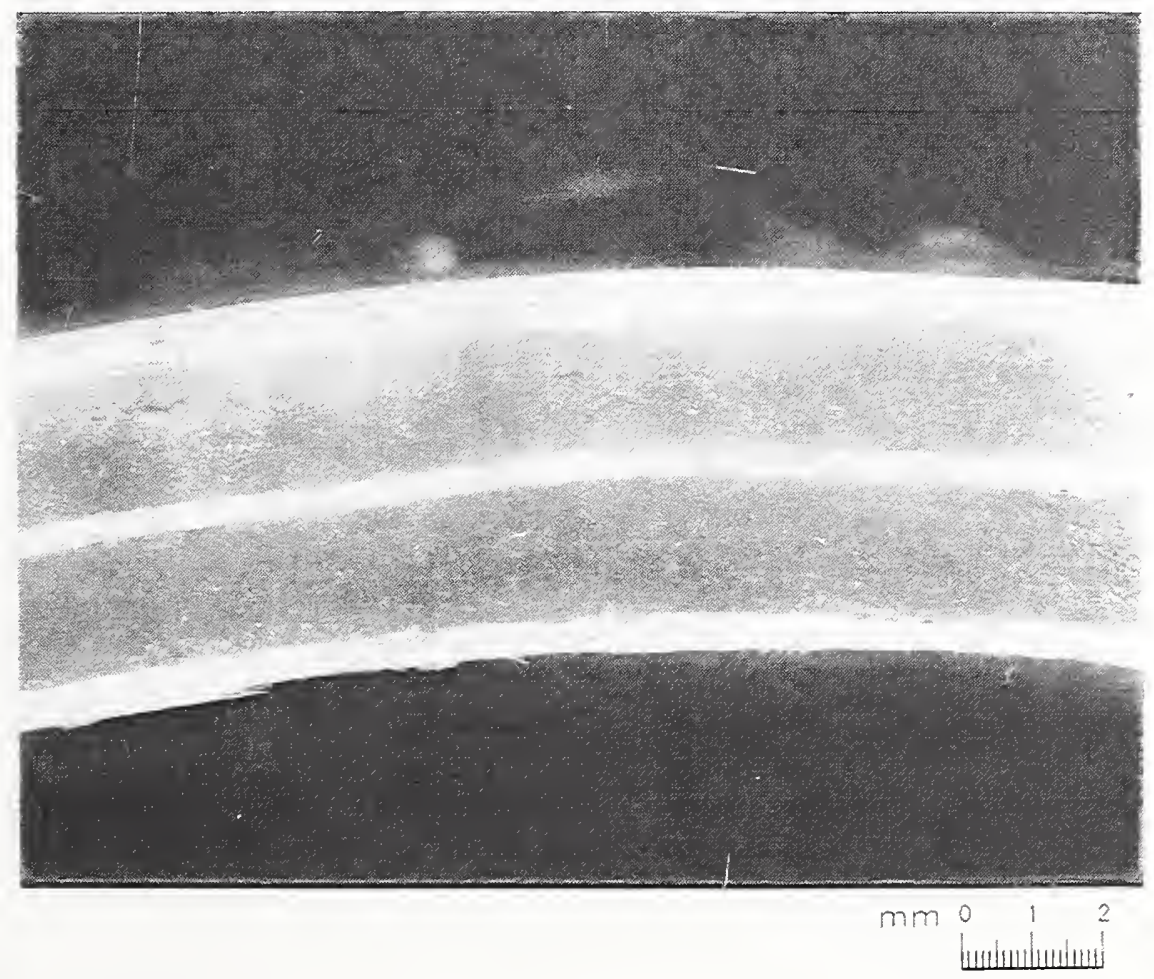




\section{TUBE TYPE 9}

MANUFACTURER: B

LAY-UP:

$\left[82 / \pm 9_{2} / 82 / \pm 9_{2} / 82 / \pm 9_{2} / 82 / \pm 9_{2} / 82\right]$

WALL THICKNESS:

$10.2 \mathrm{~mm}(0.40 \mathrm{in})$

LENGTH:

$710 \mathrm{~mm}(28 \mathrm{in})$

RESIN:

VE-EL/MEKP

FIBER:

E-glass, 1100 TEX (450 yd/lb), continuous strand

PROCESS:

filament winding

MEASURED FIBER VOLUME FRACTION: $\quad 0.55$

MEASURED VOID VOLUME FRACTION: $\quad 0.030$

COMMENTS:

- $24 \mathrm{~h}$ cure at room temperature

- 21 plies;

thickness: $16[ \pm 9]$ plies $\times 0.53 \mathrm{~mm}(0.021 \mathrm{in}) / \mathrm{ply}=8.5 \mathrm{~mm}(0.336 \mathrm{in})-86 \%$ 5 [82] plies $\times 0.28 \mathrm{~mm}(0.011 \mathrm{in}) / \mathrm{ply}=1.4 \mathrm{~mm}(0.055 \mathrm{in})-14 \%$

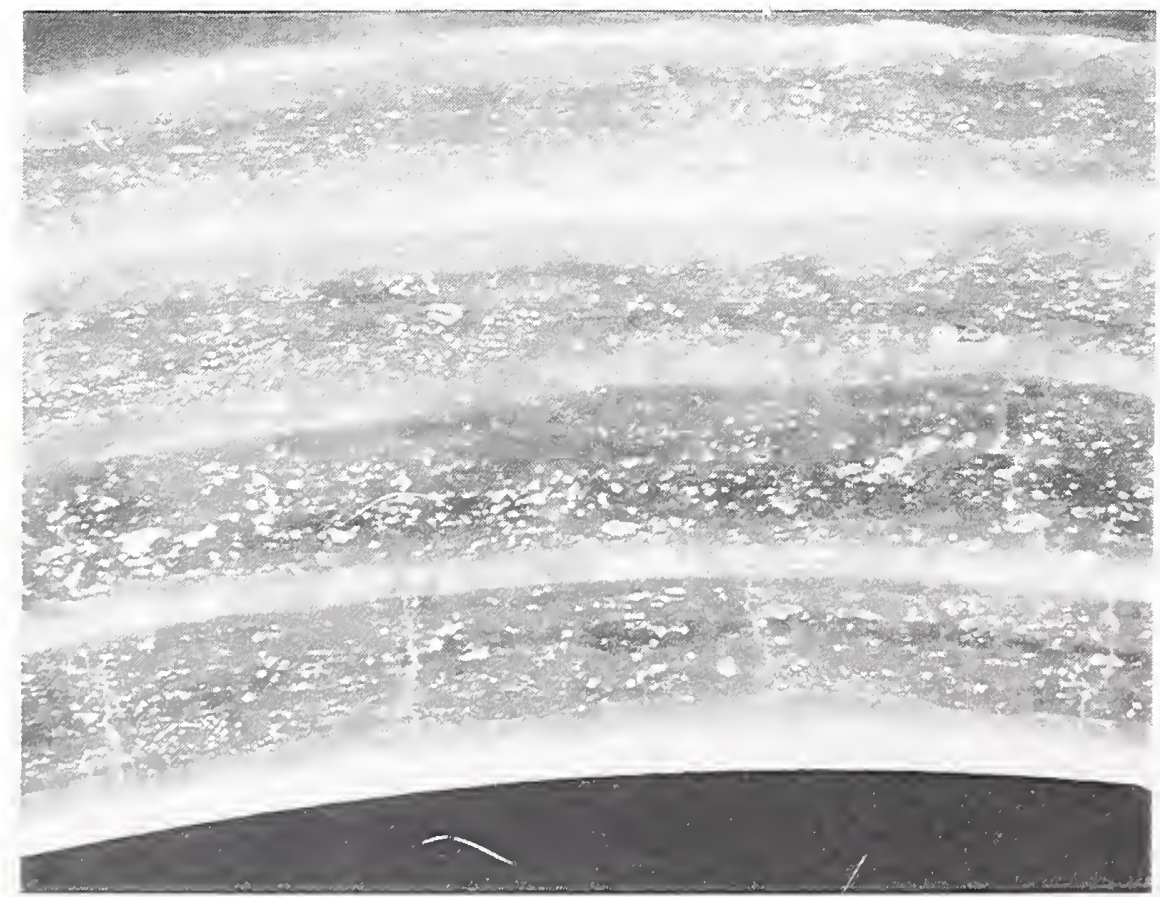




\section{TUBE TYPE 10}

MANUFACTURER: B

LAY-UP:

$\left[82 / \pm 9_{2} / 82 / \pm 9_{2} / 82\right]$

WALL THICKNESS:

$5.1 \mathrm{~mm}(0.20 \mathrm{in})$

LENGTH:

$710 \mathrm{~mm}(28 \mathrm{in})$

RESIN:

VE-EL/MEKP

FIBER:

E-glass, $1100 \mathrm{TEX}(450 \mathrm{yd} / \mathrm{lb})$, continuous strand

PROCESS:

filament winding

MEASURED FIBER VOLUME FRACTION: $\quad 0.63$

MEASURED VOID VOLUME FRACTION: $\quad 0.012$

COMMENTS:

- Cure: $24 \mathrm{~h}$ at room temperature

- 11 plies;

thickness: $8[ \pm 9]$ plies $\times 0.53 \mathrm{~mm}(0.021 \mathrm{in}) / \mathrm{ply}=4.2 \mathrm{~mm}(0.168 \mathrm{in})-83 \%$

3 [82] plies $\times 0.28 \mathrm{~mm}(0.011 \mathrm{in}) / \mathrm{ply}=0.84 \mathrm{~mm}(0.033 \mathrm{in})-17 \%$

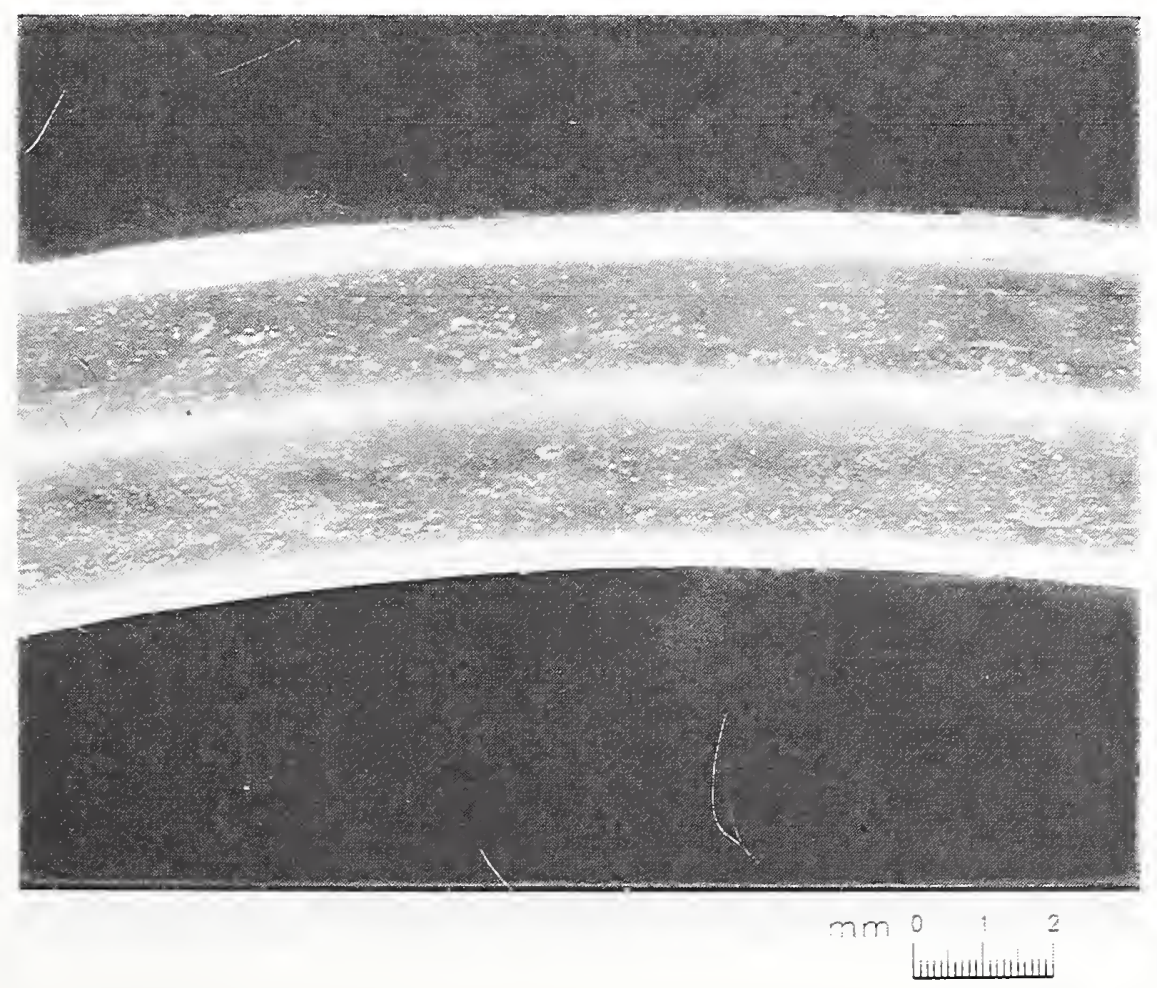




\section{TUBE TYPE 11}

MANUFACTURER:

C

LAY-UP:

$\left[ \pm 65 / 0_{3} / \pm 23 / 0_{4} / \pm 23 / 0_{2} / \pm 23 / 0_{2} / \pm 65\right]$

WALL THICKNESS:

$10.2 \mathrm{~mm}(0.40 \mathrm{in})$

LENGTH:

$710 \mathrm{~mm}(28 \mathrm{in})$

RESIN:

VE-EL/MEKP

FIBER:

E-glass, $1100 \mathrm{TEX}(450 \mathrm{yd} / \mathrm{lb})$, continuous strand

E-glass, $532 \mathrm{~g} / \mathrm{m}^{2}\left(15.7 \mathrm{oz} / \mathrm{yd}^{2}\right)$, unidirectional weft

PROCESS:

filament winding + hand lay-up (unidirectional weft)

MEASURED FIBER VOLUME FRACTION: $\quad 0.52$

MEASURED VOID VOLUME FRACTION: $\quad 0.013$

\section{COMMENTS:}

- 21 layers;

$52 \%$ [0], 19\% [ \pm 65$], 29 \%$ [ \pm 23$]$; thickness not supplied by manufacturer

- This lay-up is similar to the A24 lay-up (50\% [ \pm 10$], 17 \%[90], 33 \%[ \pm 45])$ except that cloth was used to get true zeroes in these tubes

- Proprietary cure schedule

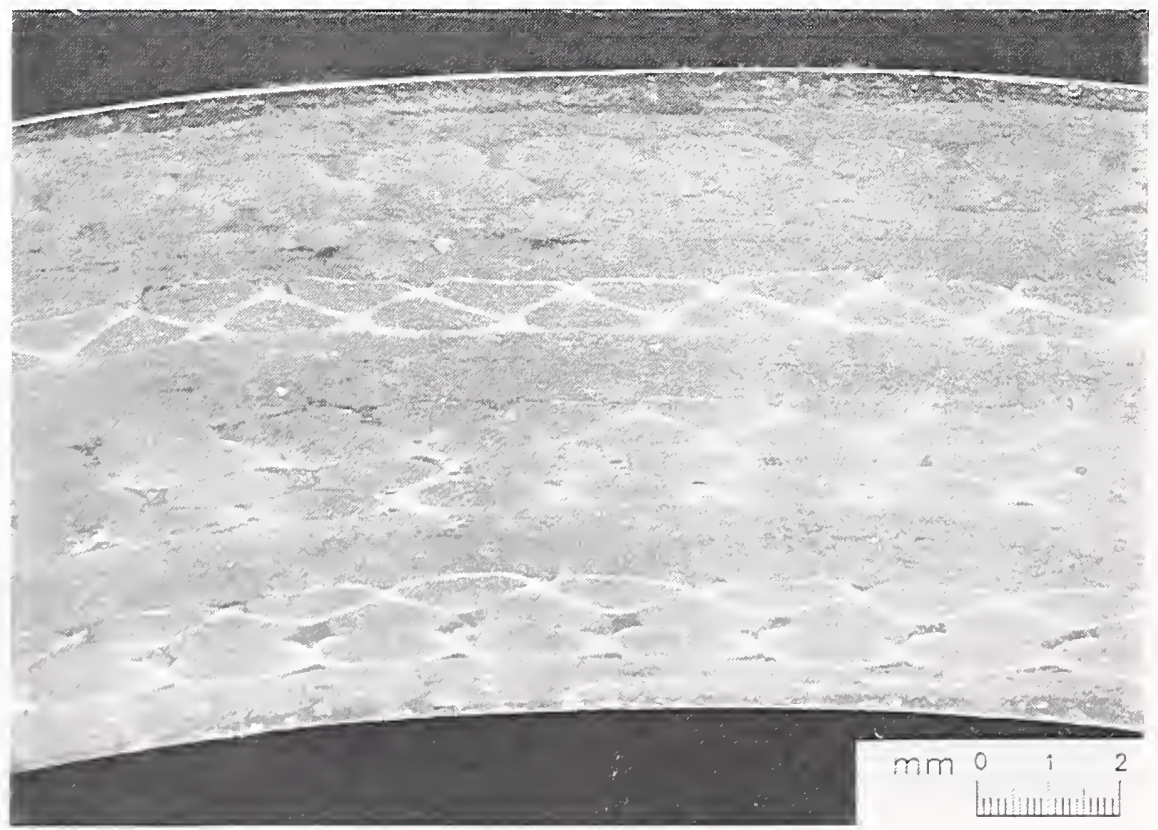




\section{TUBE TYPES 12 and 13}

MANUFACTURER:

LAY-UP:

WALL THICKNESS:

LENGTH:

RESIN:

FIBER:
A

$\left[ \pm 45 / \pm 10_{2} /+45 / \pm 10 /-45 / \pm 45\right]$

$5.1 \mathrm{~mm}(0.20 \mathrm{in})$

$710 \mathrm{~mm}(28 \mathrm{in})$

DGEBA/ARA/DTA

Tube 12: E-glass, $1100 \mathrm{TEX}$ (450 yd/lb), continuous strand Tube 13: same fiber, but under high-tension $(\sim 2-\mathrm{kg})$ fiber

PROCESS: $\quad$ filament winding

MEASURED FIBER VOLUME FRACTION: $\quad 0.59$

MEASURED VOID VOLUME FRACTION: $\quad 0.025$

COMMENTS:

- Except for the fiber tension, tube types 12 and 13 (not shown) are identical.

- 12 plies:

thickness: $6[ \pm 45]$ plies $\times 0.34 \mathrm{~mm}(0.0133 \mathrm{in}) / \mathrm{ply}^{*}=2.0 \mathrm{~mm}(0.080 \mathrm{in})-40 \%$

$6[ \pm 10]$ plies $\times 0.51 \mathrm{~mm}(0.020 \mathrm{in}) / \mathrm{ply}^{*}=3.0 \mathrm{~mm}(0.120 \mathrm{in})-60 \%$

- Proprietary resin and cure schedule

*estimate

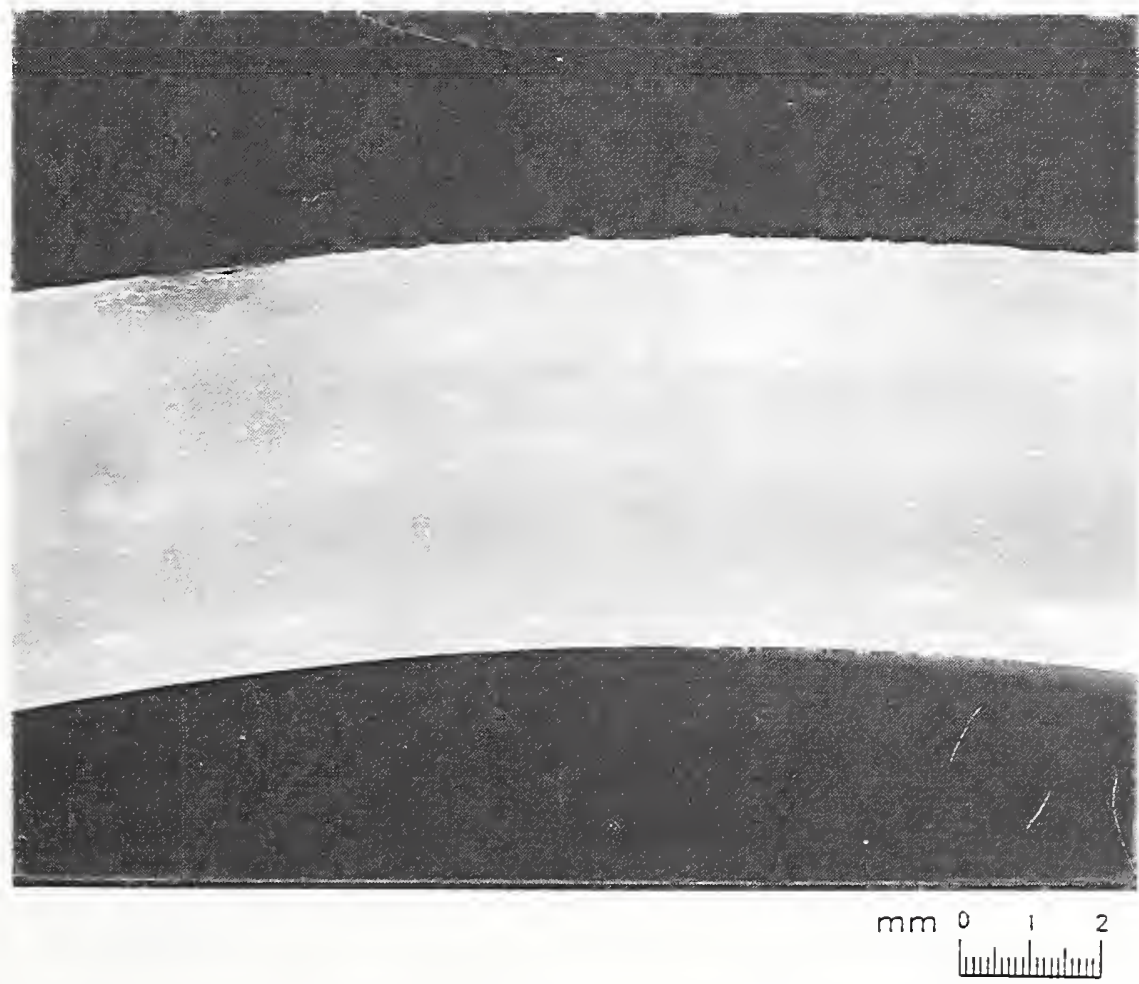




\section{TUBE TYPES 14 and 15}

MANUFACTURER:

LAY-UP:

WALL THICKNESS:

LENGTH:

RESIN:

FIBER:
A

$\left[ \pm 45 / \pm 10_{2} /+45 / \pm 10 /-45 / \pm 45\right]$

$5.1 \mathrm{~mm}(0.20 \mathrm{in})$

$710 \mathrm{~mm}(28 \mathrm{in})$

\section{DGEBF/CTBN/AN}

Tube 14: E-glass, 1100 TEX (450 yd/lb), continuous strand Tube 15: same fiber, but under high-tension $(\sim 2-\mathrm{kg})$ fiber filament winding

PROCESS:

0.58

MEASURED VOID VOLUME FRACTION: $\quad 0.060$

COMMENTS:

- Except for the fiber tension, Tubes 14 and 15 (not shown) are identical.

- 12 plies;

thickness: $6[ \pm 45]$ plies $\times 0.34 \mathrm{~mm}(0.0133 \mathrm{in}) / \mathrm{ply}^{*}=2.0 \mathrm{~mm}(0.080 \mathrm{in})-40 \%$ $6[ \pm 10]$ plies $\times 0.51 \mathrm{~mm}(0.020 \mathrm{in}) / \mathrm{ply}^{*}=3.0 \mathrm{~mm}(0.120 \mathrm{in}-60 \%$ *estimate
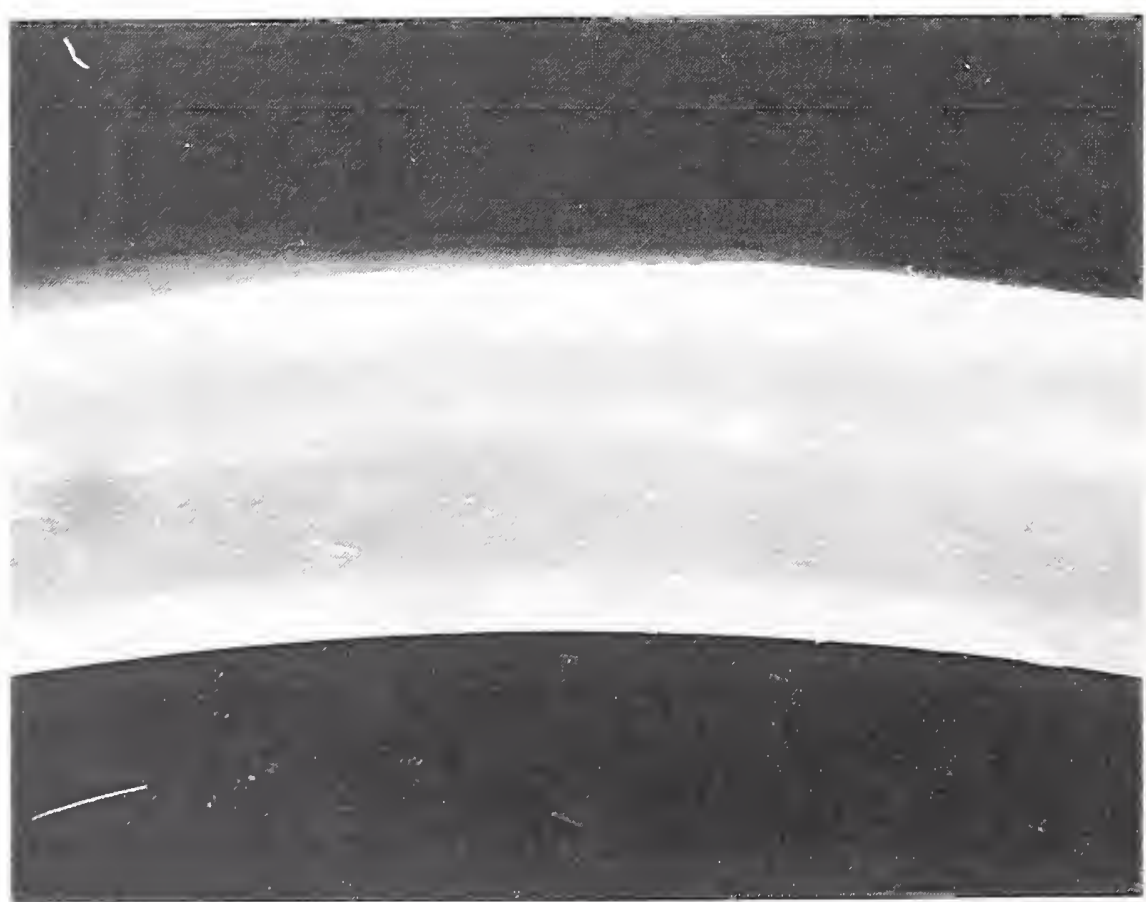

$m \log ^{0}$ 


\section{TUBE TYPE 16}

MANUFACTURER:

A

LAY-UP:

$\left[82 / \pm 10_{6} / 82\right]$

WALL THICKNESS:

$5.1 \mathrm{~mm}(0.20 \mathrm{in})$

LENGTH:

$710 \mathrm{~mm}$ (28 in)

RESIN:

DGEBA/DCH/ARA/ACL

FIBER:

E-glass, $1100 \mathrm{TEX}$ (450 yd/lb), continuous strand

PROCESS:

filament winding

MEASURED FIBER VOLUME FRACTION: $\quad 0.57$

MEASURED VOID VOLUME FRACTION: $\quad 0.074$

\section{COMMENTS:}

- 14 plies;

thickness: $\quad 12[ \pm 10]$ plies $\times 0.38 \mathrm{~mm}(0.015 \mathrm{in}) / \mathrm{ply}=4.6 \mathrm{~mm}(0.180 \mathrm{in})-90 \%$ 2 [82] plies $\times 0.25 \mathrm{~mm}(0.010 \mathrm{in}) / \mathrm{ply}=0.50 \mathrm{~mm}(0.020 \mathrm{in})-10 \%$

- No midwall circular wraps

- Proprietary resin and cure schedule

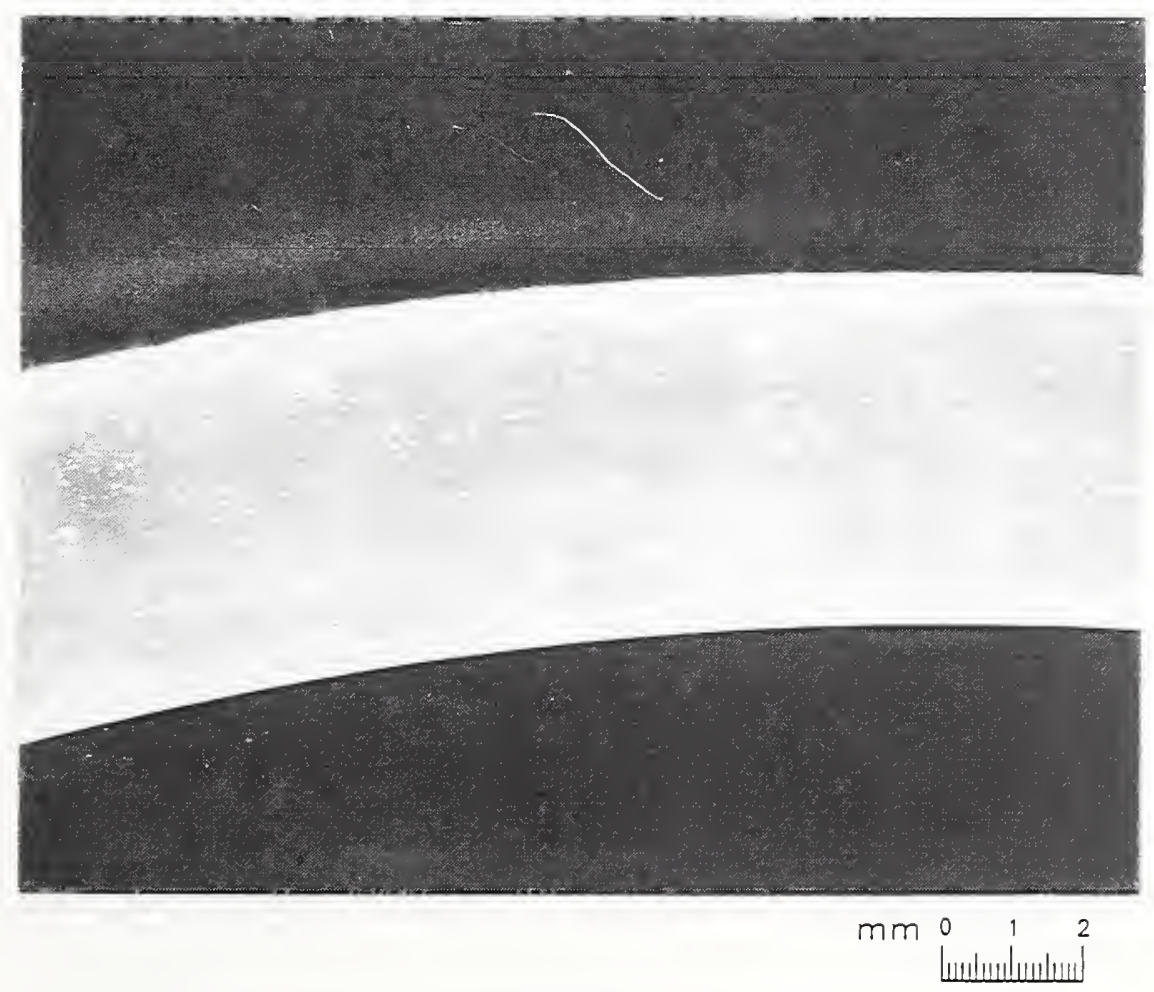

VI-59 


\section{TUBE TYPE 17}

MANUFACTURER:

LAY-UP:

WALL THICKNESS:

LENGTH:

RESIN:

FIBER:

PROCESS:
A

$\left[82 / \pm 10_{6} / 82 / \pm 10_{6} / 82\right]$

$8.9 \mathrm{~mm}(0.35 \mathrm{in})$

$1200 \mathrm{~mm}$ (49 in), 610 (24 in)

DGEBA/DCH/ARA/ACL

E-glass, 1100 TEX (450 yd/lb), continuous strand

filament winding

MEASURED FIBER VOLUME FRACTION: $\quad 0.59$

MEASURED VOID VOLUME FRACTION: $\quad 0.065$

COMMENTS:

- 27 plies;

thickness: $24[ \pm 10] \times 0.34 \mathrm{~mm}(0.0135 \mathrm{in}) / \mathrm{ply}=8.2 \mathrm{~mm}(0.324 \mathrm{in})-92 \%$

3 [82] plies $\times 0.25 \mathrm{~mm}(0.010 \mathrm{in}) / \mathrm{ply}=0.75 \mathrm{~mm}(0.030 \mathrm{in})-8 \%$

- 1 midwall circular ply

- Proprietary resin and cure schedule

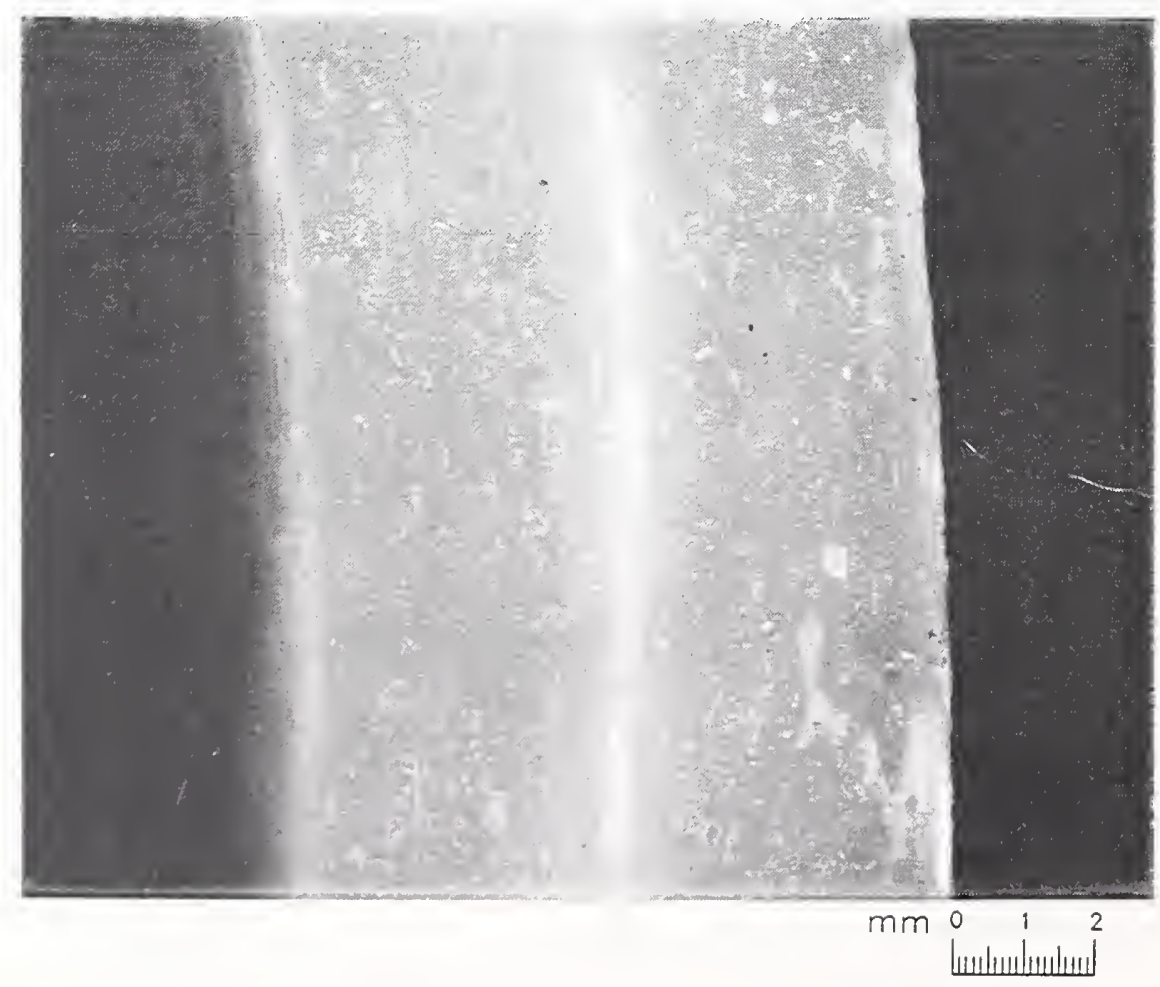

VI-60 


\section{TUBE TYPE 18}

MANUFACTURER:

LAY-UP:

WALL THICKNESS:

LENGTH:

RESIN:

FIBER:

PROCESS:
A

$\left[82 / \pm 10_{6} / 82 / \pm 10_{6} / 82\right]$

$12.7 \mathrm{~mm}(0.500 \mathrm{in})$

$1800 \mathrm{~mm}$ (703 in), $910 \mathrm{~mm}$ (36 in)

DGEBA/DCH/ARA/ACL

E-glass, $1100 \mathrm{TEX}$ (450 yd/lb), continuous strand

filament winding

MEASURED FIBER VOLUME FRACTION: $\quad 0.57$

MEASURED VOID VOLUME FRACTION: $\quad 0.055$

COMMENTS:

- 27 plies;

thickness: $24[ \pm 10]$ plies $\times 0.51 \mathrm{~mm}(0.020 \mathrm{in}) / \mathrm{ply}=12.2 \mathrm{~mm}(0.480 \mathrm{in})-94 \%$

3 [82] plies $\times 0.25 \mathrm{~mm}(0.010 \mathrm{in}) / \mathrm{ply}=0.75 \mathrm{~mm}(0.030 \mathrm{in})-6 \%$

- 1 midwall circular wrap [82]

- Proprietary resin and cure schedule

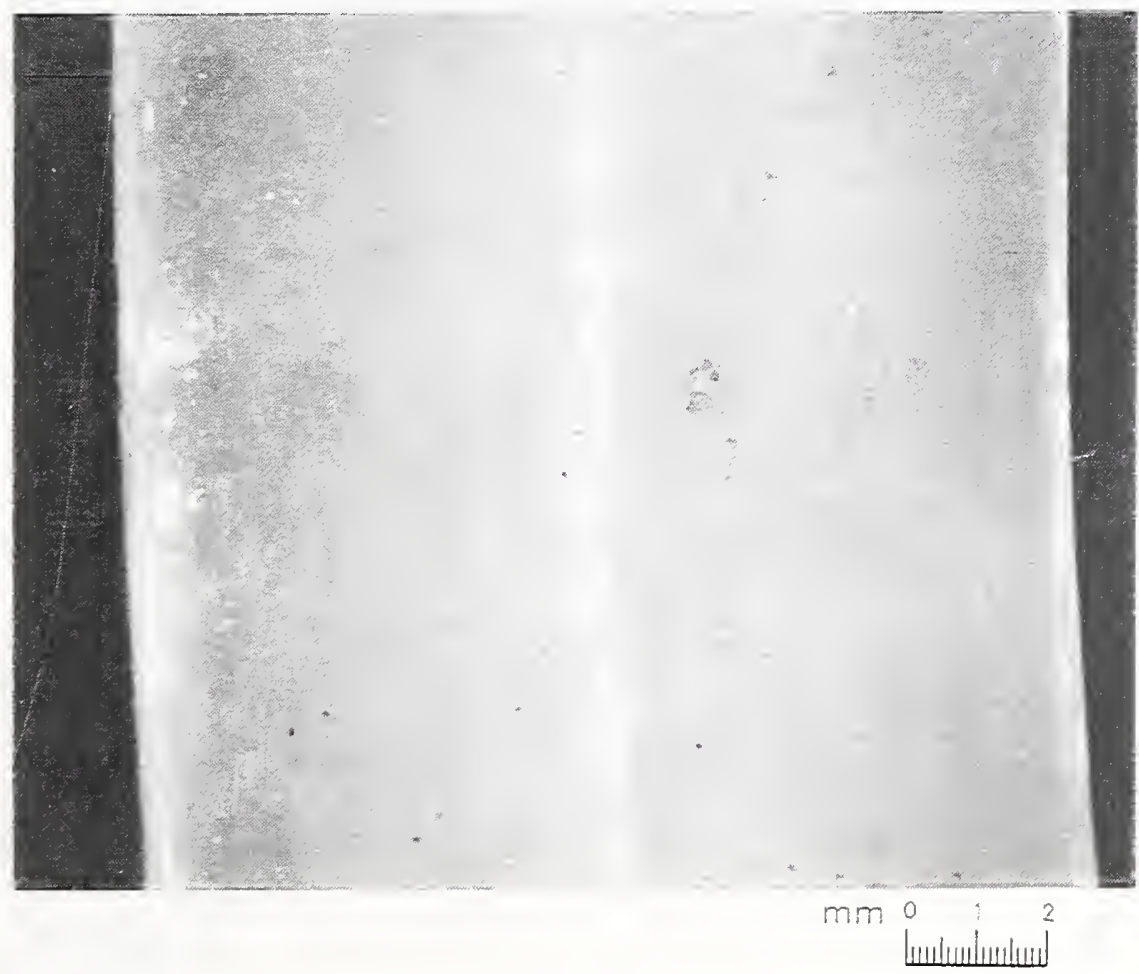




\section{TUBE TYPE 19}

MANUFACTURER:

A

LAY-UP:

$\left[82 / \pm 10_{6} / 82\right]$

WALL THICKNESS:

$5.1 \mathrm{~mm}(0.20 \mathrm{in})$

LENGTH:

$710 \mathrm{~mm}(28 \mathrm{in})$

RESIN:

DGEBF/CTBN/AN

FIBER:

E-glass, 1100 TEX (450 yd/lb), continuous strand

PROCESS:

filament winding

MEASURED FIBER VOLUME FRACTION: $\quad 0.57$

MEASURED VOID VOLUME FRACTION: $\quad 0.054$

COMMENTS:

- Except for fiber type, tubes 19 and 20 are identical.

- 14 plies;

thickness: $12[ \pm 10]$ plies $\times 0.38 \mathrm{~mm}(0.015 \mathrm{in}) / \mathrm{ply}=4.6 \mathrm{~mm}(0.18 \mathrm{in})-90 \%$ 2 [82] plies $\times 0.25 \mathrm{~mm}(0.010 \mathrm{in}) / \mathrm{ply}=0.50 \mathrm{~mm}(0.020 \mathrm{in})-10 \%$

- No midwall circular wraps

- Proprietary resin and cure schedule

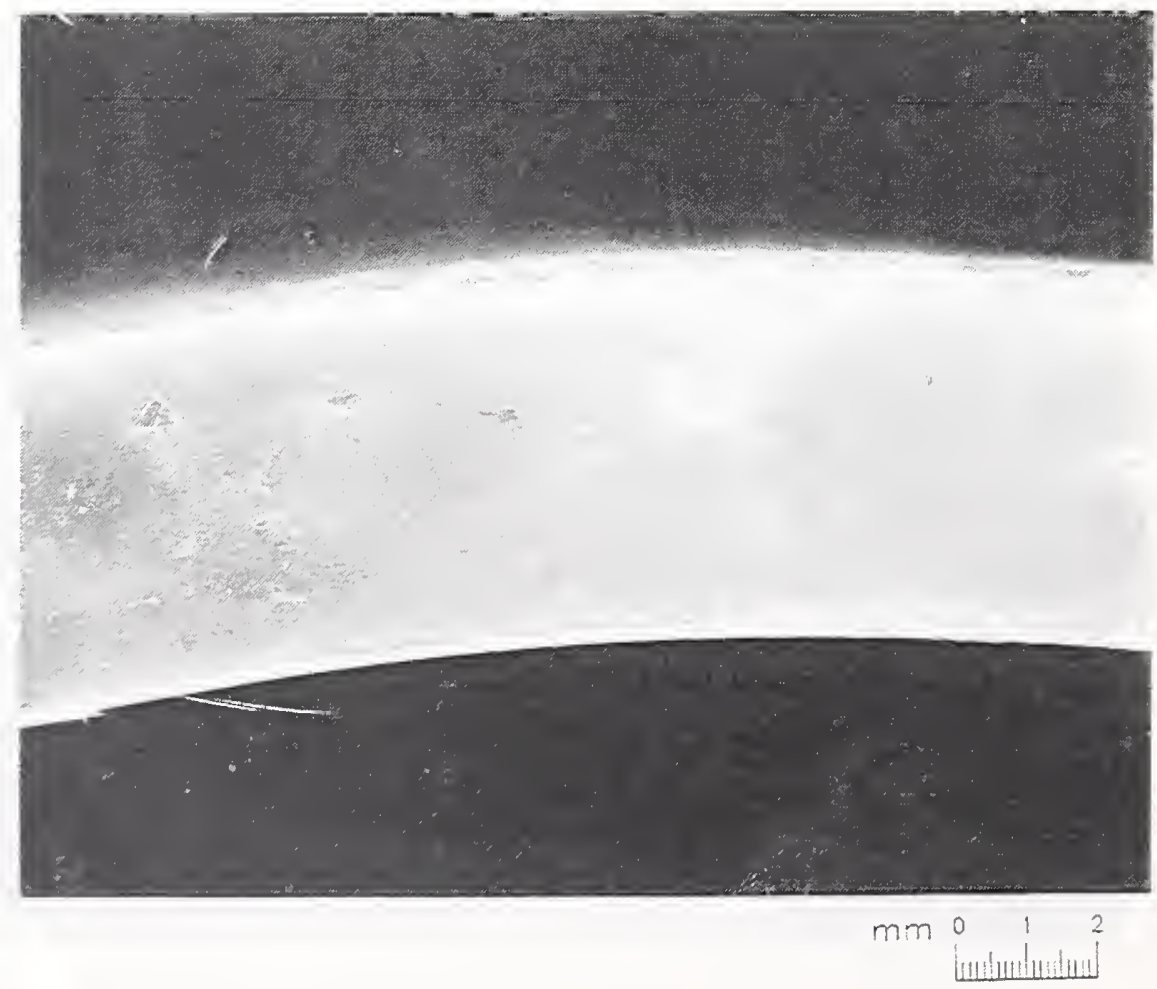




\section{TUBE TYPE 20}

MANUFACTURER:

A

LAY-UP:

$\left[82 / \pm 10_{6} / 82\right]$

WALL THICKNESS:

$5.1 \mathrm{~mm}(0.20 \mathrm{in})$

LENGTH:

$710 \mathrm{~mm}$ (28 in)

RESIN:

DGEBF/CTBN/AN

FIBER:

Tube 20: S2-glass, $1100 \mathrm{TEX}$ (450 yd/lb), continuous strand

PROCESS:

filament winding

MEASURED FIBER VOLUME FRACTION: $\quad 0.54$

MEASURED VOID VOLUME FRACTION: $\quad 0.094$

COMMENTS:

- Except for fiber type, tubes 19 and 20 are identical.

- 14 plies;

thickness: $12[ \pm 10]$ plies $\times 0.38 \mathrm{~mm}(0.015 \mathrm{in}) / \mathrm{ply}=4.6 \mathrm{~mm}(0.18 \mathrm{in})-90 \%$

2 [82] plies $\times 0.25 \mathrm{~mm}(0.010 \mathrm{in}) / \mathrm{ply}=0.50 \mathrm{~mm}(0.020 \mathrm{in})-10 \%$

- No midwall circular wraps

- Proprietary resin and cure schedule

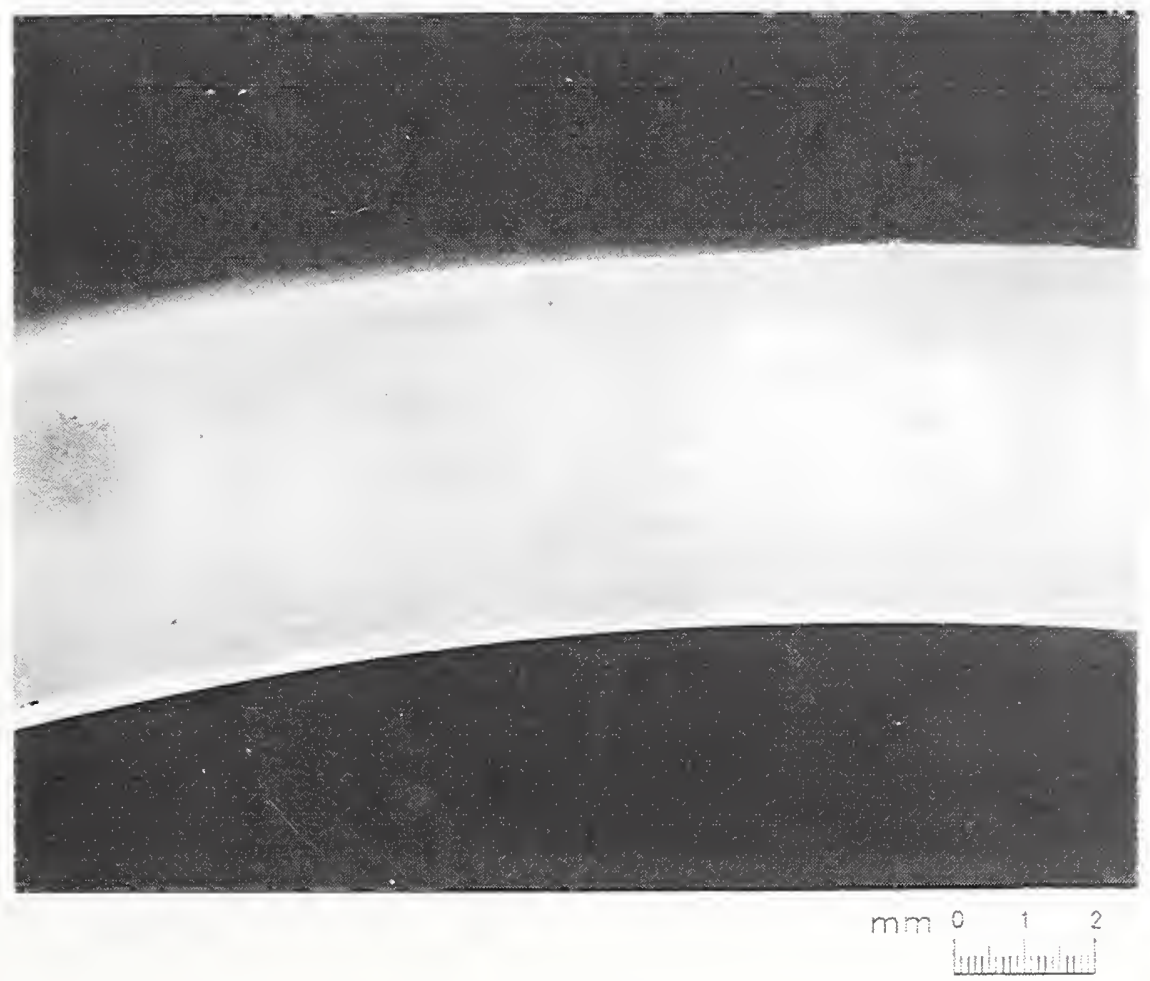




\section{TUBE TYPE 21}

MANUFACTURER:

A

LAY-UP:

$\left[82 / \pm 10_{6} / 82\right]$

WALL THICKNESS:

$5.1 \mathrm{~mm}(0.20 \mathrm{in})$

LENGTH:

$710 \mathrm{~mm}(28 \mathrm{in})$

RESIN:

VE-EL/MEKP

FIBER:

E-glass, 1100 TEX (450 yd/lb), continuous strand

MEASURED FIBER VOLUME FRACTION: $\quad 0.46$

MEASURED VOID VOLUME FRACTION: $\quad<0.005$

COMMENTS:

- 14 plies;

thickness: $12[ \pm 10]$ plies $\times 0.38 \mathrm{~mm}(0.015$ in $) /$ ply $=4.6 \mathrm{~mm}(0.18 \mathrm{in})-90 \%$

2 [82] plies $\times 0.25 \mathrm{~mm}(0.010 \mathrm{in}) / \mathrm{ply}=0.50 \mathrm{~mm}(0.020 \mathrm{in})-10 \%$

- No midwall circular wraps

- Proprietary resin and cure schedule

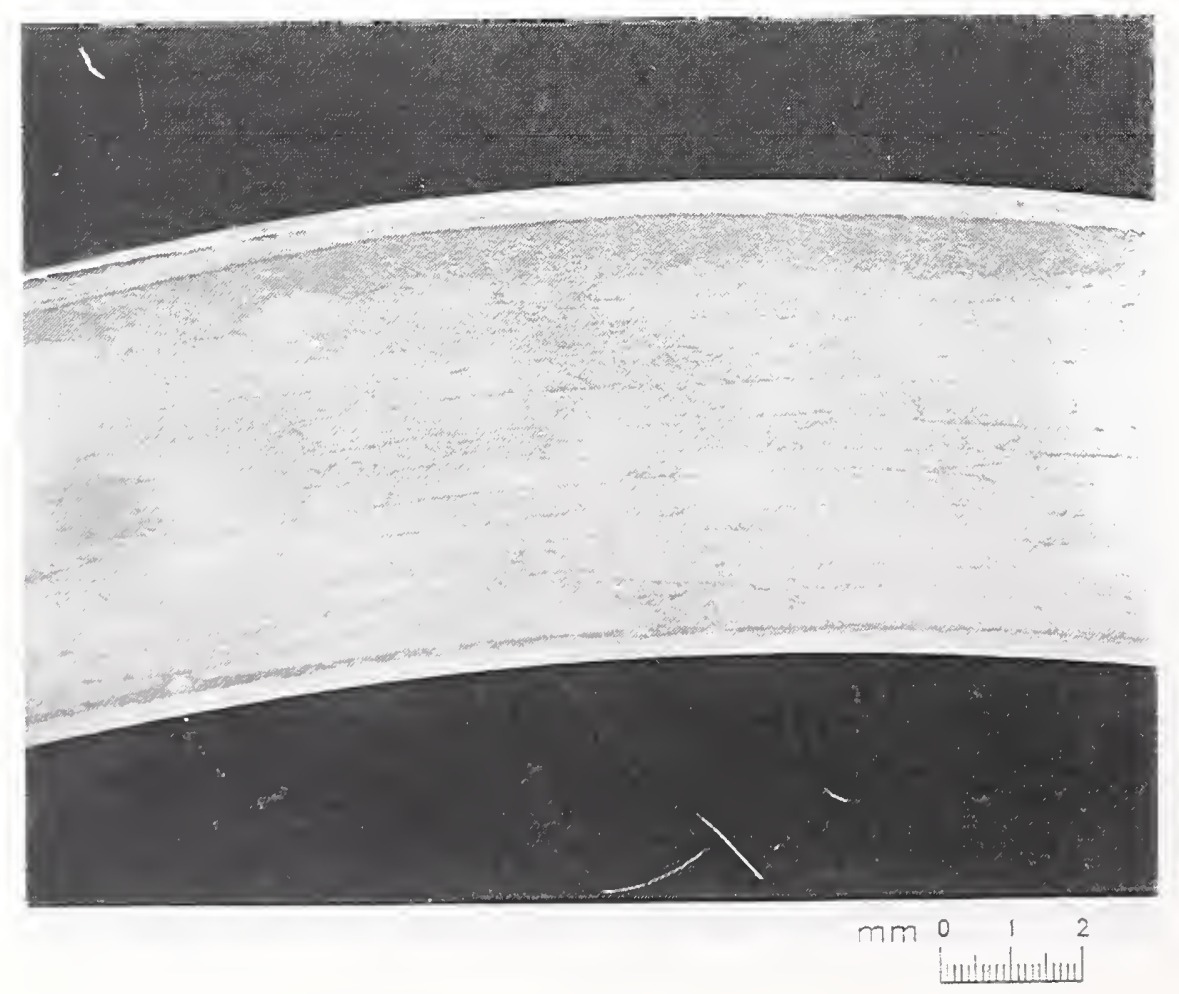




\section{TUBE TYPE 22}

MANUFACTURER: $\quad P$

LAY-UP: $\quad\left[90 / 0_{3} / 90 / 0_{3} / 90\right]$

WALL THICKNESS: $\quad 5.1 \mathrm{~mm}(0.200 \mathrm{in})$

LENGTH: $\quad 710 \mathrm{~mm}$ (28 in)

RESIN: $\quad$ DGEBA-ST/ARA/ACL/F

FIBER: $\quad$ E-glass $(185$ yield $=$ hoop $)(113$ yield $=$ axial $)$

PROCESS: pultrusion

MEASURED FIBER VOLUME FRACTION: $\quad 0.58$

MEASURED VOID VOLUME FRACTION: $\quad 0.015$

COMMENTS:

- 9 plies;

thickness: $6[0]$ plies $\times 0.51 \mathrm{~mm}(0.020 \mathrm{in}) / \mathrm{ply}=3.1 \mathrm{~mm}(0.120 \mathrm{in})-60 \%$

3 [90] plies $\times 0.51 \mathrm{~mm}(0.020 \mathrm{in}) / \mathrm{ply}=1.5 \mathrm{~mm}(0.060 \mathrm{in})-30 \%$

balance $=$ inner/outer mat $283 \mathrm{~g}(10 \mathrm{oz})$ and Nexus $-10 \%$

- 1 midwall circular wrap [90]

- Resin cured in heated mandrel at $82^{\circ} \mathrm{C}$

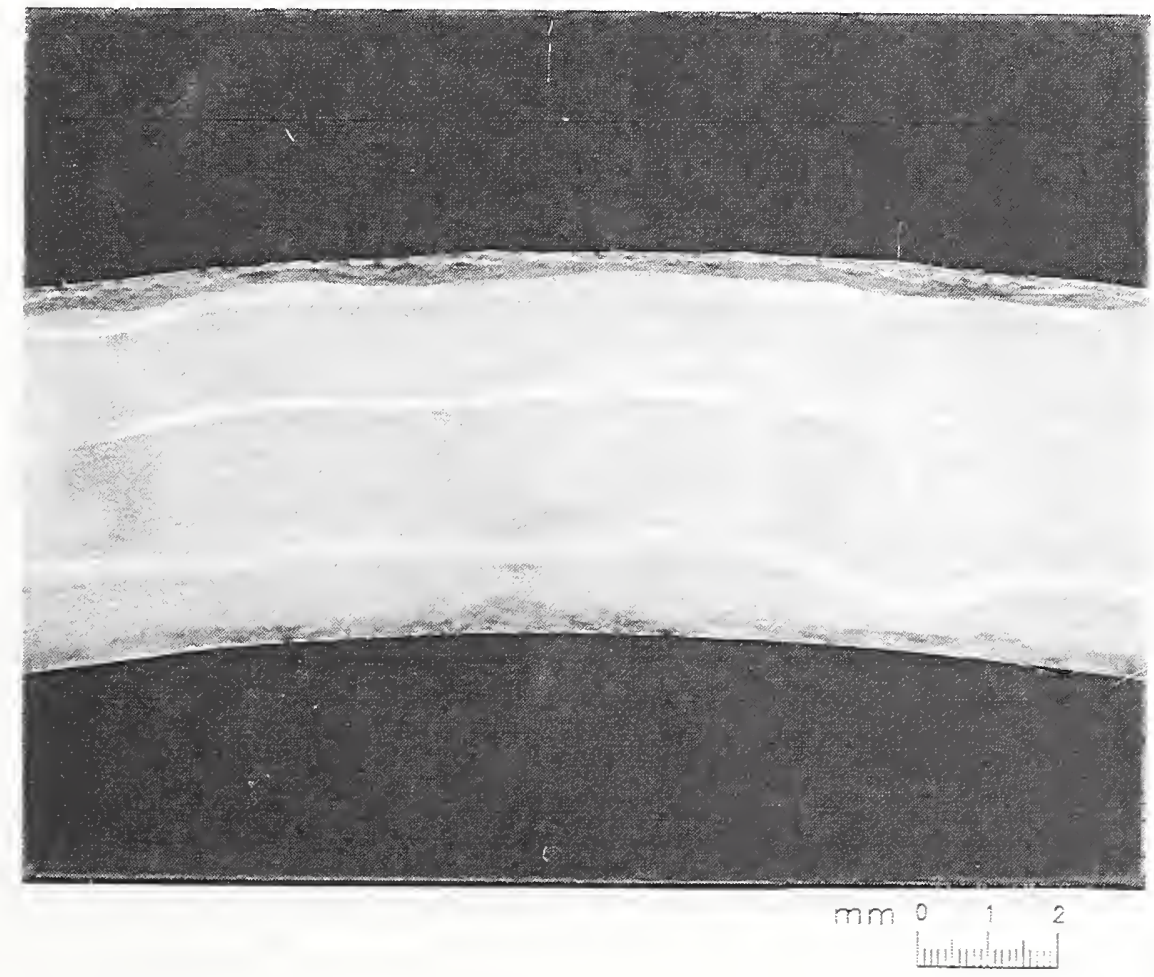




\section{TUBE TYPE 23}

MANUFACTURER:

LAY-UP:

WALL THICKNESS:

LENGTH:

RESIN:

FIBER:

PROCESS:
P

$\left[90 / 0_{3} / 90 / 0_{3} / 90\right]$

$5.1 \mathrm{~mm}(0.20 \mathrm{in})$

$710 \mathrm{~mm}$ (28 in)

VE-EL/MEKP

E-glass $(185$ yield $=$ hoop $)(113$ yield $=$ axial $)$

pultrusion

MEASURED FIBER VOLUME FRACTION: $\quad 0.58$

MEASURED VOID VOLUME FRACTION: $\quad 0.017$

\section{COMMENTS:}

- 9 plies;

thickness: 6 [0] plies $\times 0.51 \mathrm{~mm}(0.020 \mathrm{in}) / \mathrm{ply}=3.1 \mathrm{~mm}(0.120 \mathrm{in})-60 \%$ $3[90]$ plies $\times 0.51 \mathrm{~mm}(0.020 \mathrm{in}) / \mathrm{ply}=1.5 \mathrm{~mm}(0.060 \mathrm{in})-30 \%$ balance $=$ inner/outer mat $283 \mathrm{~g}(10 \mathrm{oz})$ and Nexus $-10 \%$

- 1 midwall circular [90] wrap

- Resin cured in heated mandrel at $82^{\circ} \mathrm{C}$

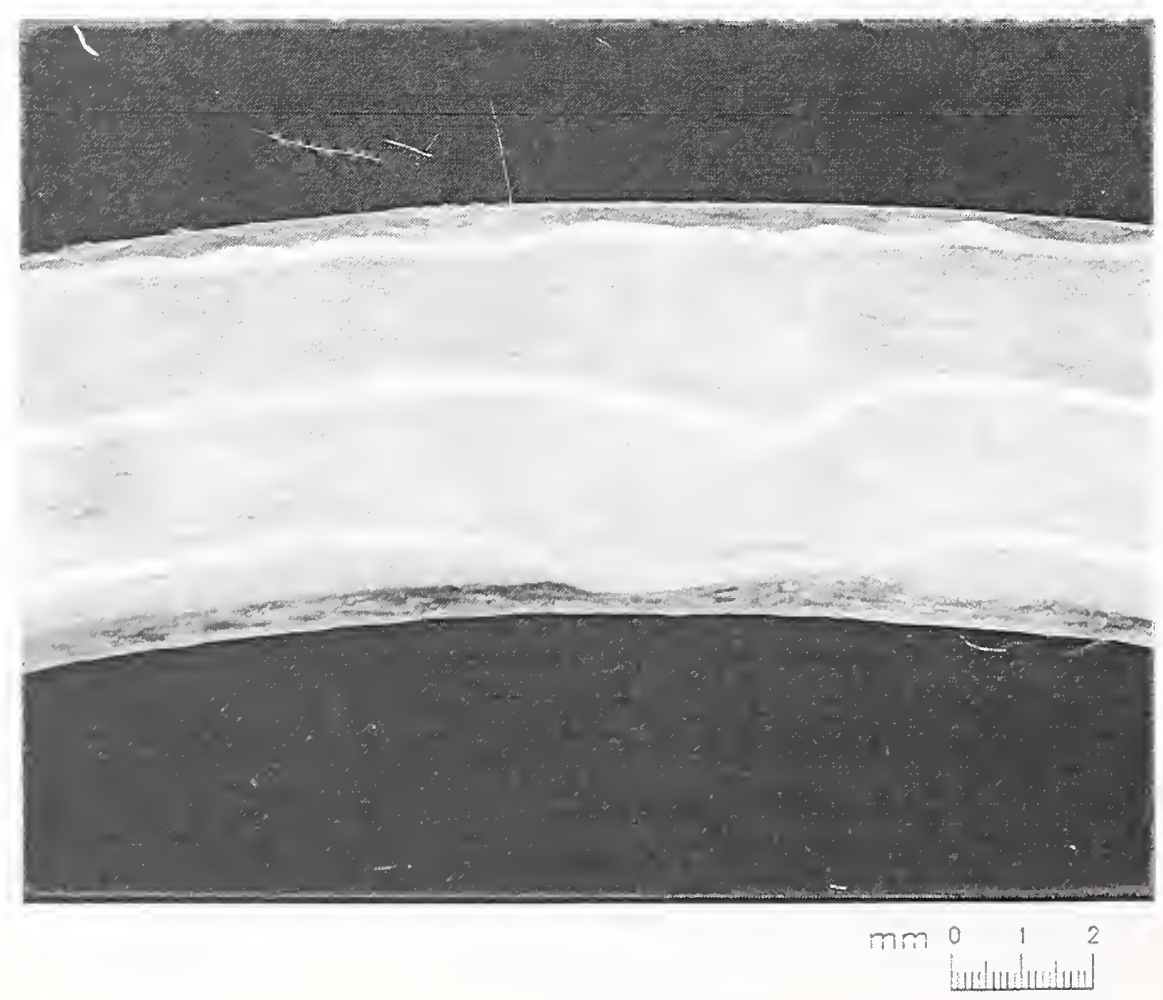




\section{TUBE TYPE 24}

MANUFACTURER: $\quad P$

LAY-UP:

$\left[90 / 0_{6} / 90 / 0_{6} / 90\right]$

WALL THICKNESS:

$12.7 \mathrm{~mm}(0.500 \mathrm{in})$

LENGTH:

$1800 \mathrm{~mm}(70 \mathrm{in})$

RESIN:

VE-EL/MEKP

FIBER:

E-glass $(250$ yield $=$ hoop $)(56$ yield $=$ axial $)$

PROCESS:

pultrusion

MEASURED FIBER VOLUME FRACTION: $\quad 0.60$

MEASURED VOID VOLUME FRACTION: $\quad<0.005$

COMMENTS:

- 15 plies;

thickness: 12 [0] plies $\times 0.86 \mathrm{~mm}(0.034 \mathrm{in}) / \mathrm{ply}=10.3 \mathrm{~mm}(0.408 \mathrm{in})-82 \%$

3 [90] plies $\times 0.51 \mathrm{~mm}(0.020 \mathrm{in}) / \mathrm{ply}=1.5 \mathrm{~mm}(0.060 \mathrm{in})-12 \%$

Nexus and cloth (inside and outside wall) $-6 \%$

- 1 midwall circular wrap [90]

- Resin cured in mandrel at $82^{\circ} \mathrm{C}$

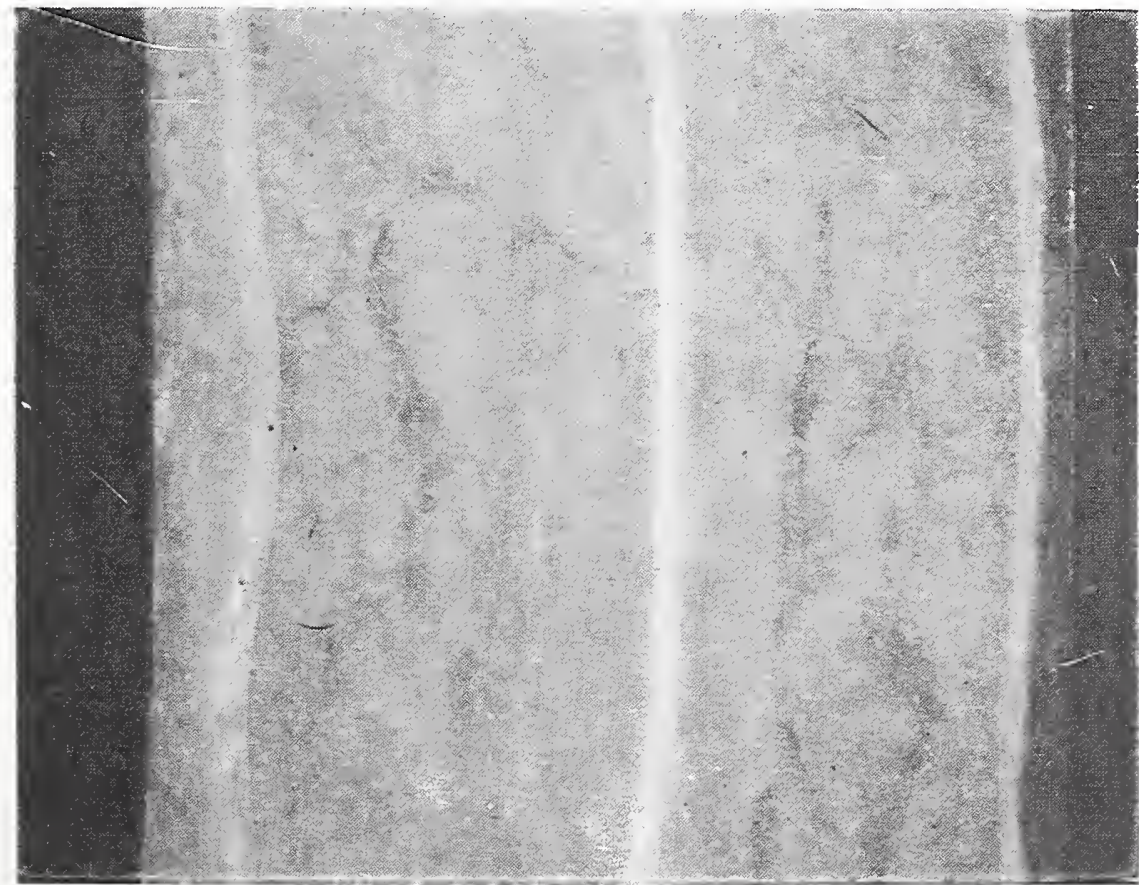

mmo 


\section{TUBE TYPE 25}

MANUFACTURER:

LAY-UP:

WALL THICKNESS:

LENGTH:

RESIN:

FIBER:

PROCESS:
A

$\left[ \pm 82 / \pm 10_{3} / \pm 82 / \pm 10_{3} / \pm 82 / \pm 10_{3} / \pm 82\right]$

$8.9 \mathrm{~mm}(0.35 \mathrm{in})$

$710 \mathrm{~mm}$ (28 in), $360 \mathrm{~mm}(14.0 \mathrm{in})$

DGEBA/DCH/ARA/ACL

$1100 \mathrm{TEX}(450 \mathrm{yd} / \mathrm{lb})$, continuous strand

filament winding

MEASURED FIBER VOLUME FRACTION: $\quad 0.58$

MEASURED VOID VOLUME FRACTION: $\quad 0.054$

COMMENTS:

- 26 plies;

thickness: $18[ \pm 10]$ plies $\times 0.38 \mathrm{~mm}(0.015 \mathrm{in}) / \mathrm{ply}=6.85 \mathrm{~mm}(0.27 \mathrm{in})-77 \%$

$8[ \pm 82]$ plies $\times 0.25 \mathrm{~mm}(0.010 \mathrm{in}) / \mathrm{ply}=2.0 \mathrm{~mm}(0.08 \mathrm{in})-23 \%$

- Proprietary resin and cure schedule

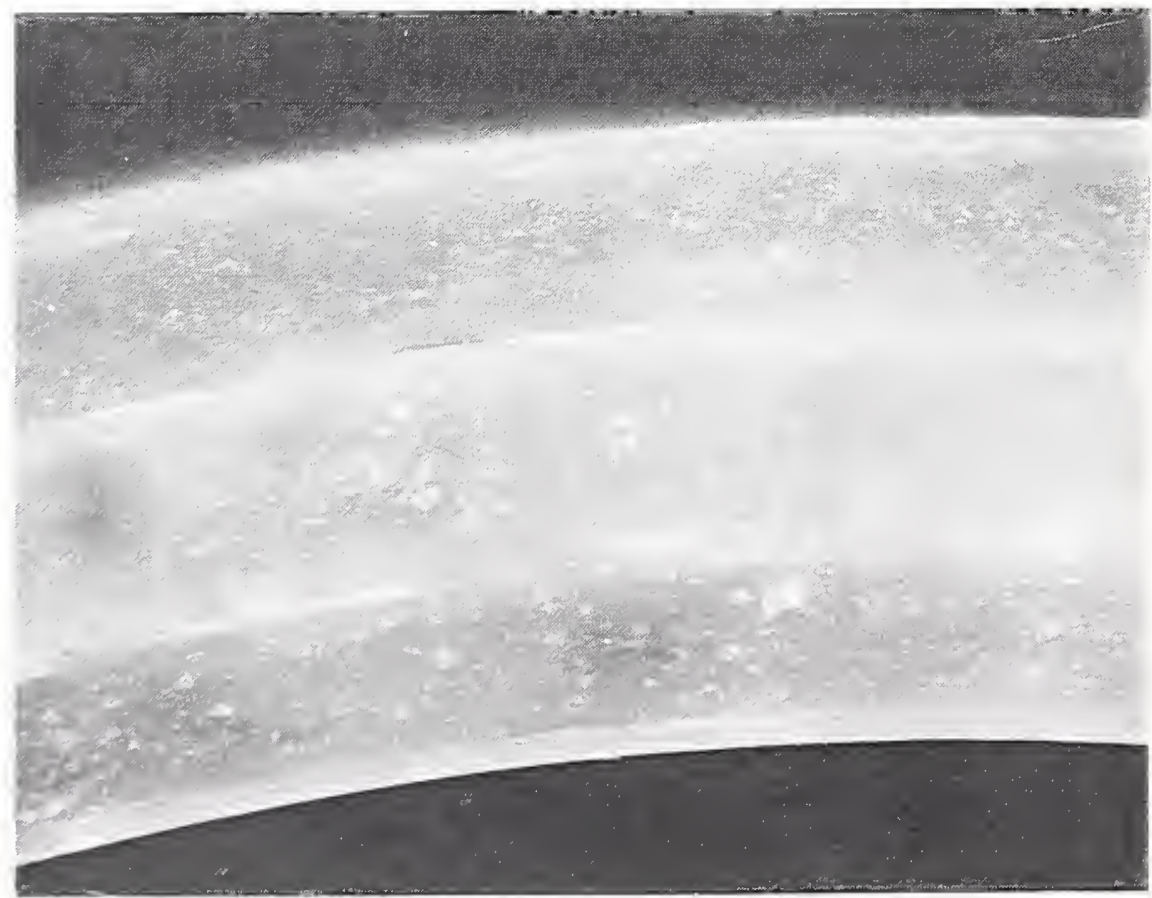

$\min 0$ 


\section{TUBE TYPE 26}

MANUFACTURER: $\quad \mathrm{R}$

LAY-UP: $\quad[ \pm 10]_{n}$

WALL THICKNESS: $\quad 5.1 \mathrm{~mm}(0.20 \mathrm{in})$

LENGTH: $\quad 360 \mathrm{~mm}(14 \mathrm{in})$

RESIN: $\quad$ CE

FIBER: $\quad$ E-glass, $1100 \mathrm{TEX}(450 \mathrm{yd} / \mathrm{lb})$, continuous strand (prepreg)

PROCESS: $\quad$ rubber expansion + hand lay-up

MEASURED FIBER VOLUME FRACTION: $\quad 0.58$

MEASURED VOID VOLUME FRACTION: $\quad 0.080$

COMMENTS:

- 44 plies $[0.12 \mathrm{~mm}(0.005 \mathrm{in}) / \mathrm{ply}]$

- Tube manufactured by rubber-expansion method, cured at $177^{\circ} \mathrm{C}$ for $2 \mathrm{~h}$

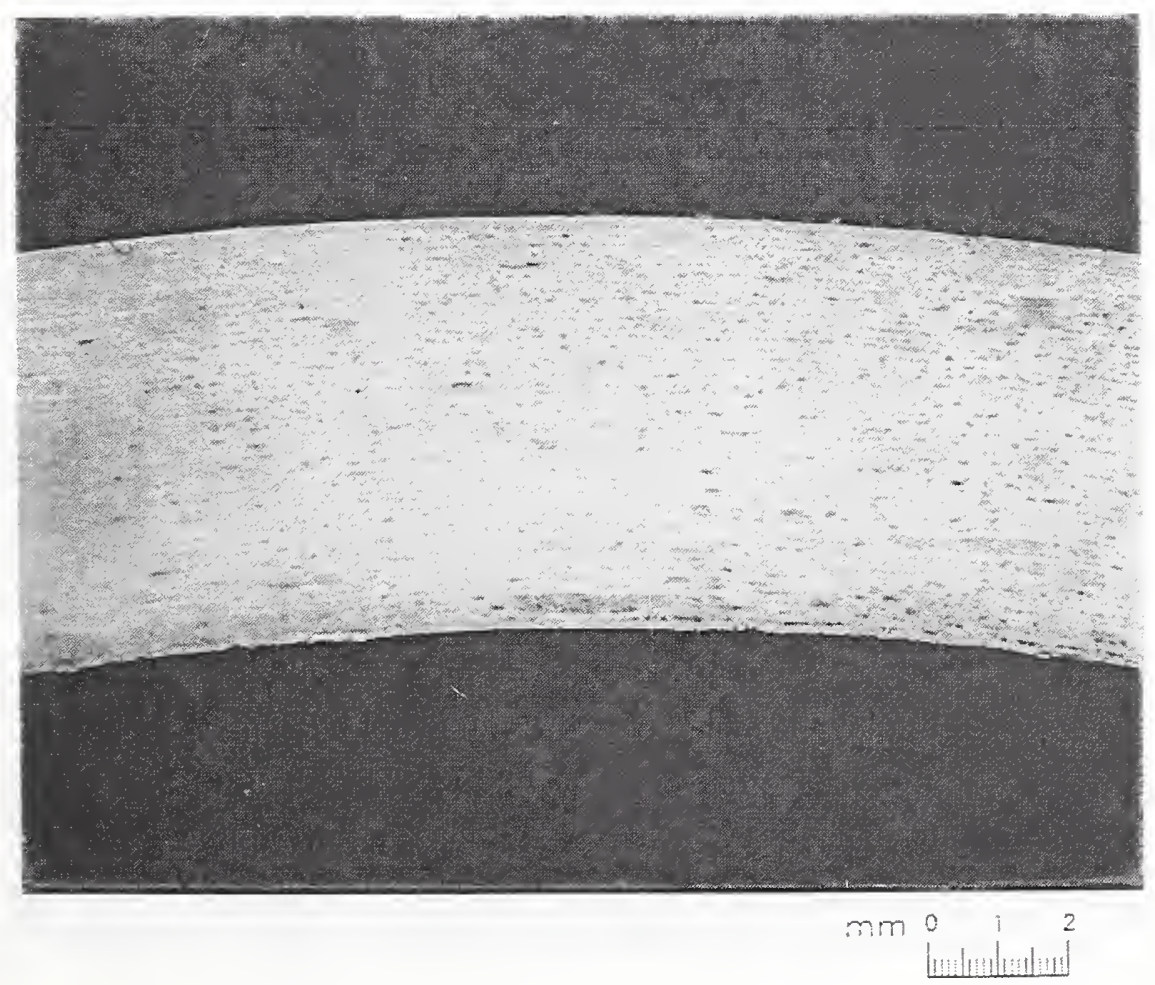




\section{TUBE TYPE 27}

MANUFACTURER: $\quad \mathrm{R}$

LAY-UP:

$\left[90 / \pm 45 / 0_{3} / \pm 45 / 90\right]_{4 S}$

WALL THICKNESS:

$5.1 \mathrm{~mm}(0.20 \mathrm{in})$

LENGTH:

$360 \mathrm{~mm}(14 \mathrm{in})$

RESIN:

CE

FIBER:

E-glass, $1100 \mathrm{TEX}$ (450 yd/lb), continuous strand (prepreg)

PROCESS:

rubber expansion + hand lay-up

MEASURED FIBER VOLUME FRACTION: -

MEASURED VOID VOLUME FRACTION:

COMMENTS:

- 36 plies;

thickness: $8[90]$ plies $\times 0.14 \mathrm{~mm}(0.0056 \mathrm{in}) / \mathrm{ply}=1.12 \mathrm{~mm}(0.044 \mathrm{in})-22 \%$

$16[ \pm 45]$ plies $\times 0.14 \mathrm{~mm}(0.0056 \mathrm{in}) / \mathrm{ply}=2.24 \mathrm{~mm}(0.088 \mathrm{in})-44 \%$

12 [0] plies $\times 0.14 \mathrm{~mm}(0.0056 \mathrm{in}) / \mathrm{ply}=1.68 \mathrm{~mm}(0.066 \mathrm{in})-34 \%$

- Tube manufactured by rubber-expansion method, cured at $177^{\circ} \mathrm{C}$ for $2 \mathrm{~h}$

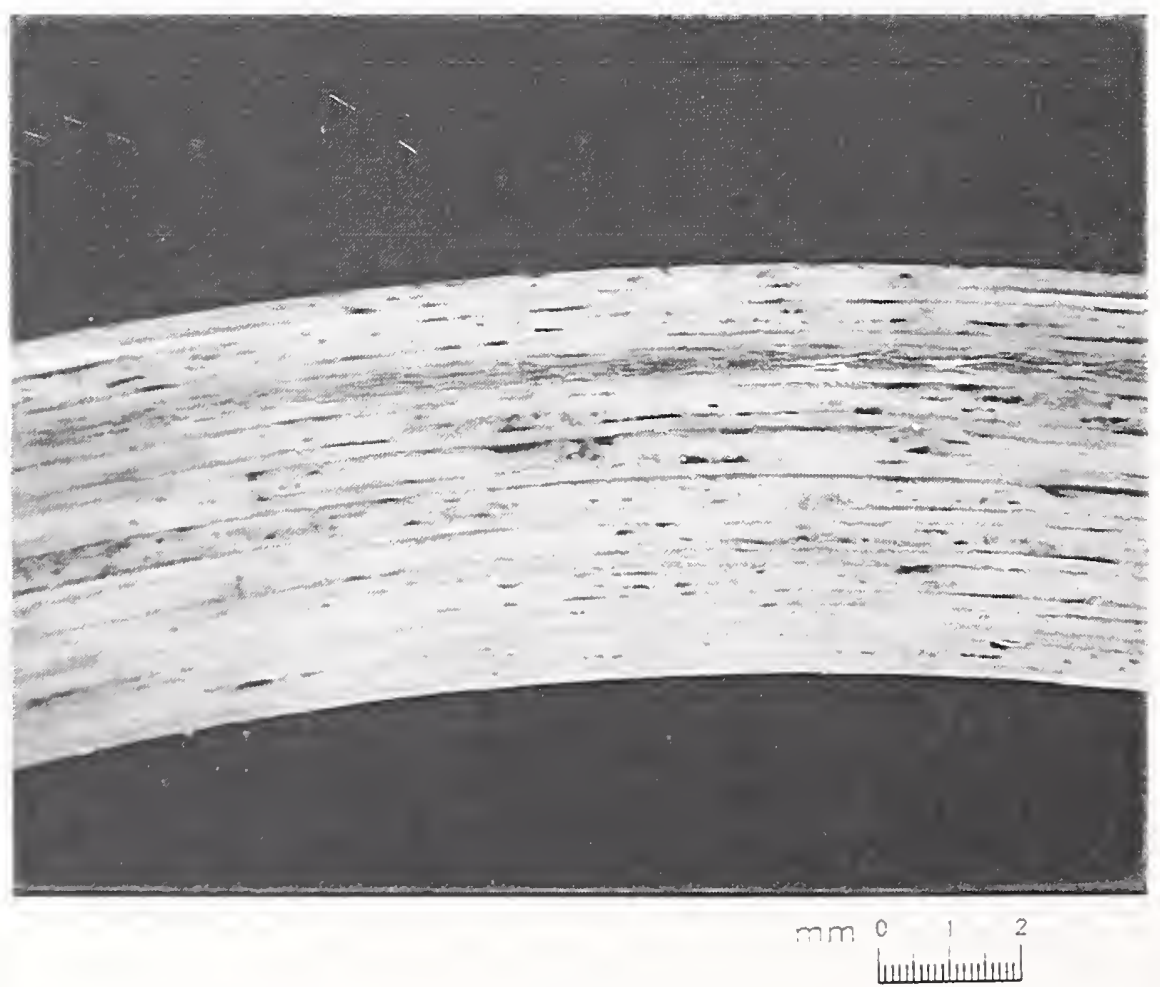

VI-70 


\section{Appendix B. Photographs of Test Equipment and Specimens}

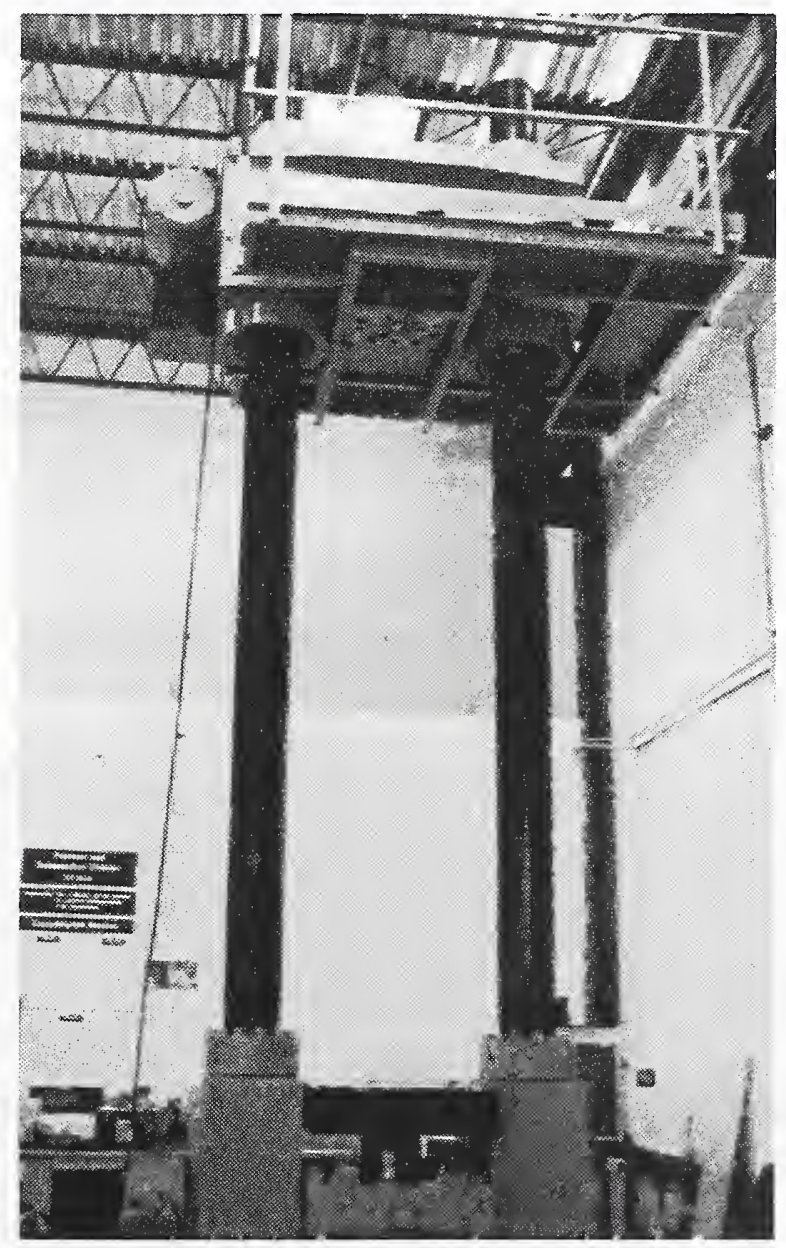

Figure B.1. The 4.5-MN servohydraulic fatigue machine (295 to $4 \mathrm{~K}$ ). 


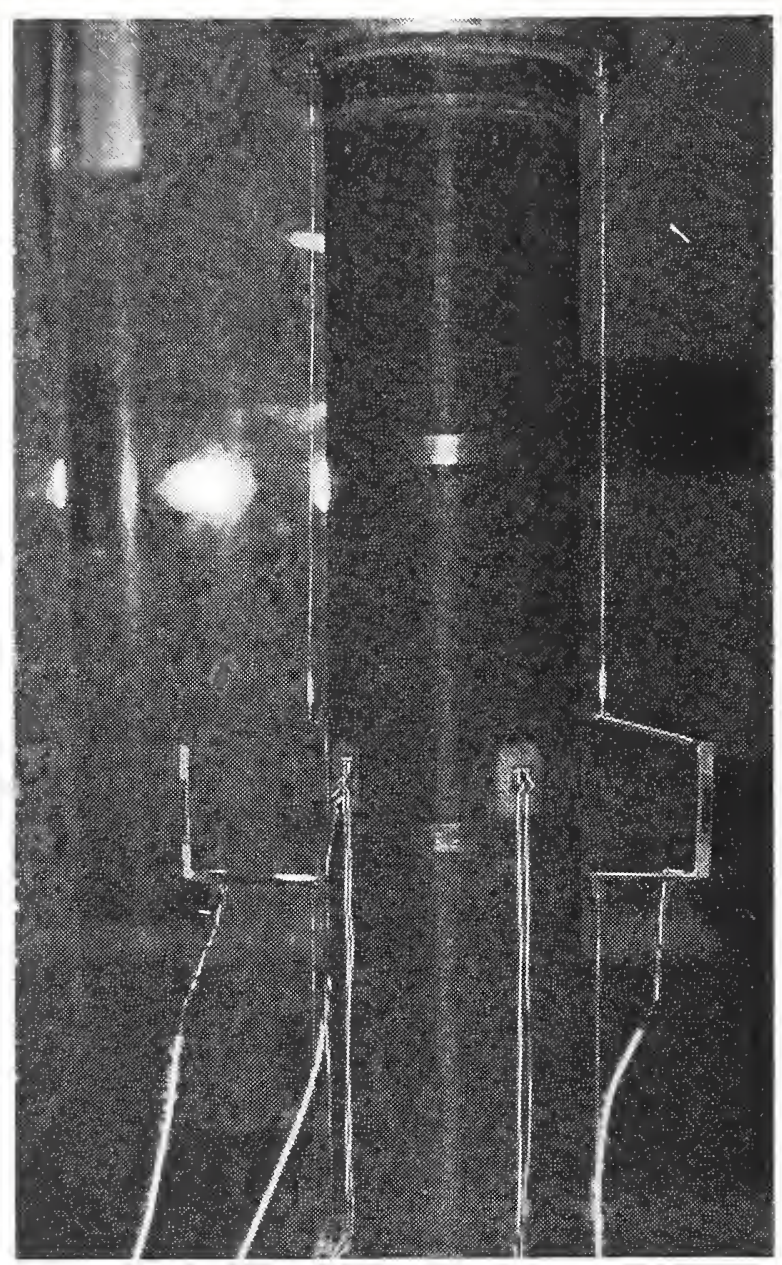

Figure B.2. Specimen ready for $295-\mathrm{K}$ test of ultimate compressive strength.

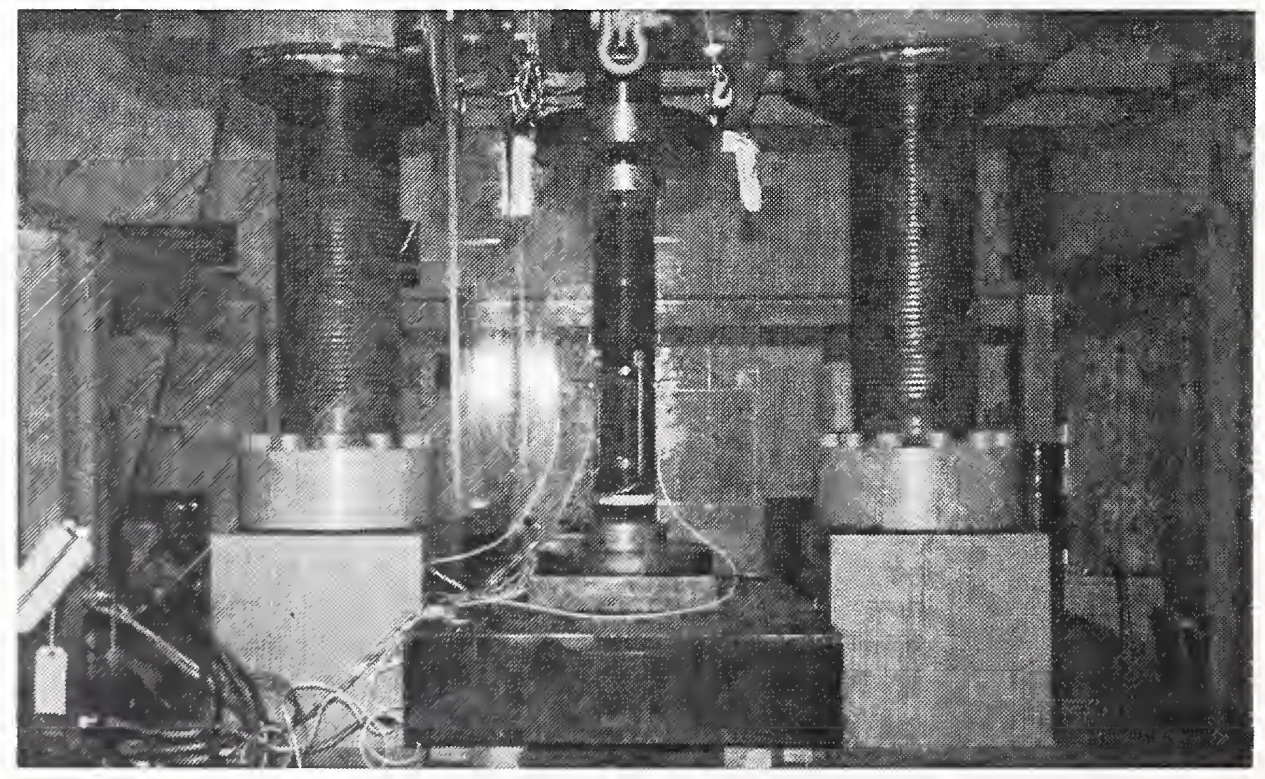

Figure B.3. Specimen in test machine. 

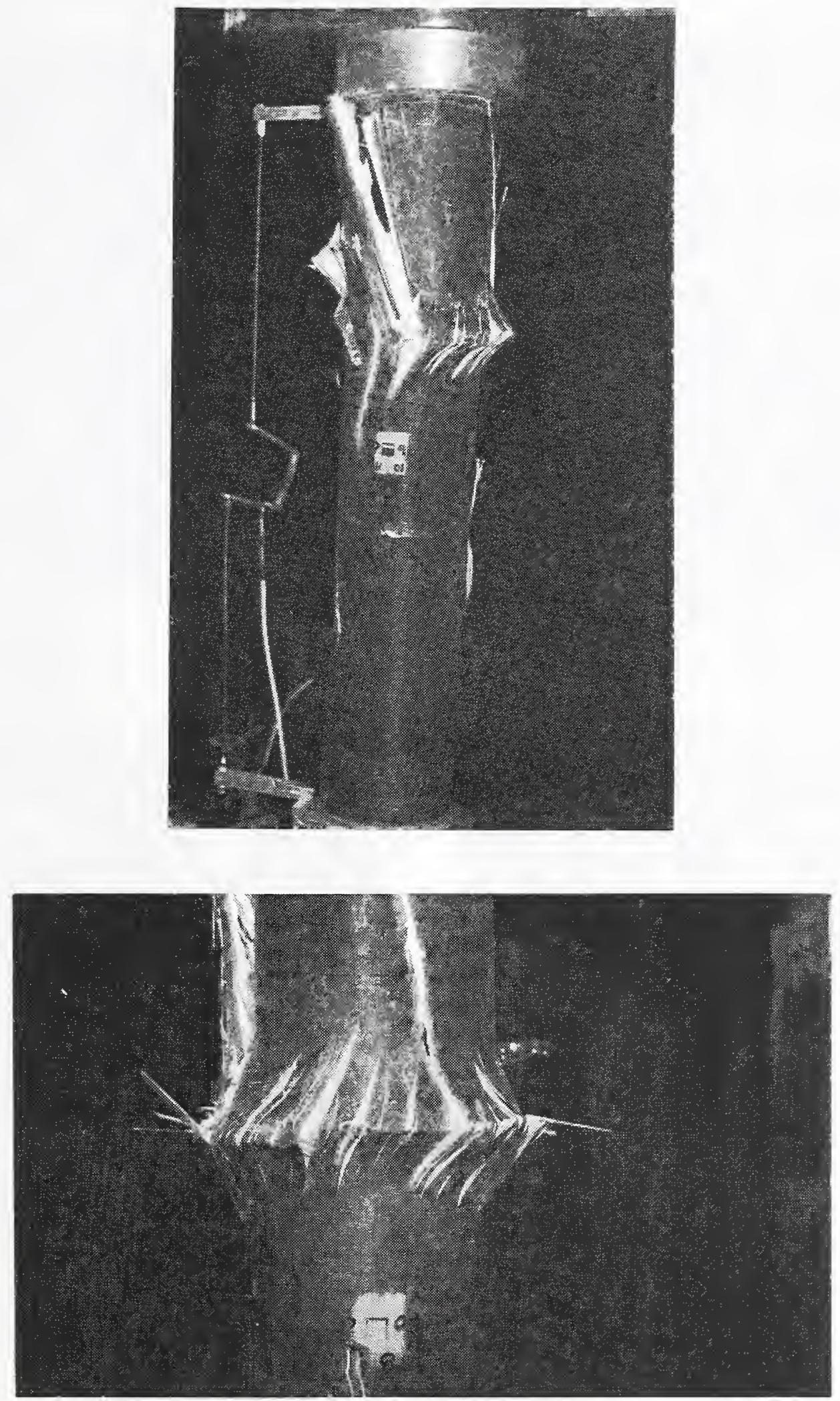

Figure B.4. Typical failure in $[ \pm 10]$ lay-up. 


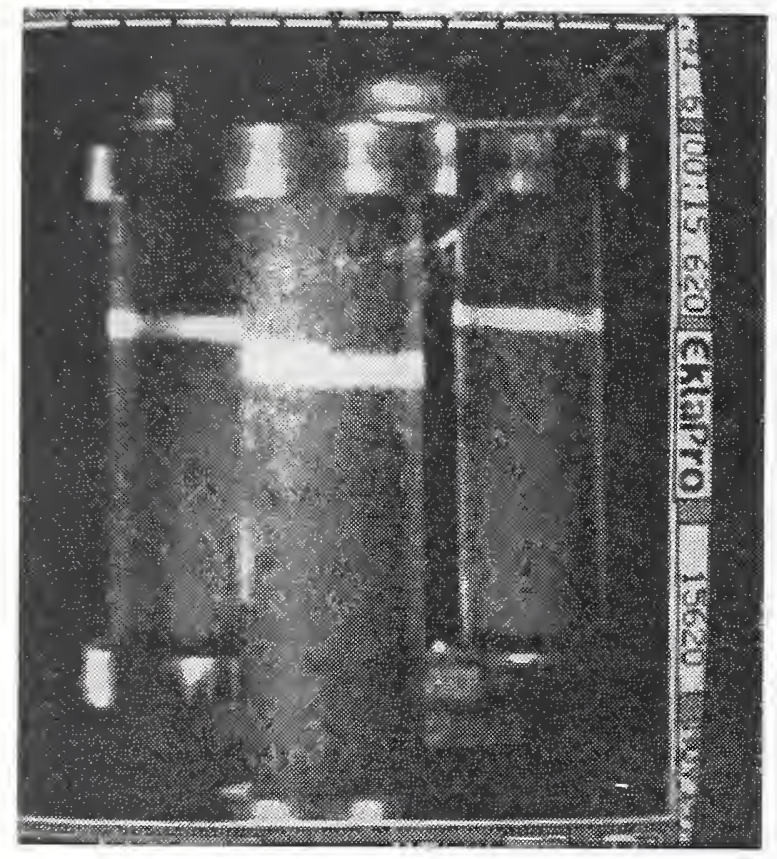

$t=15.620 \mathrm{~s}$

no apparent damage

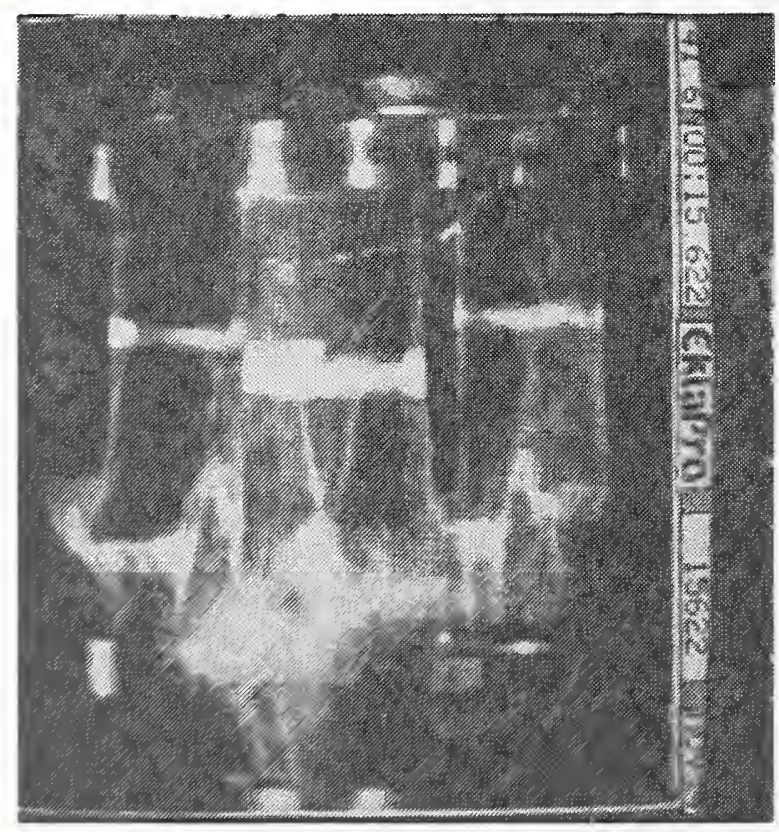

$t=15.622 \mathrm{~s}$

multiple cracks; failure

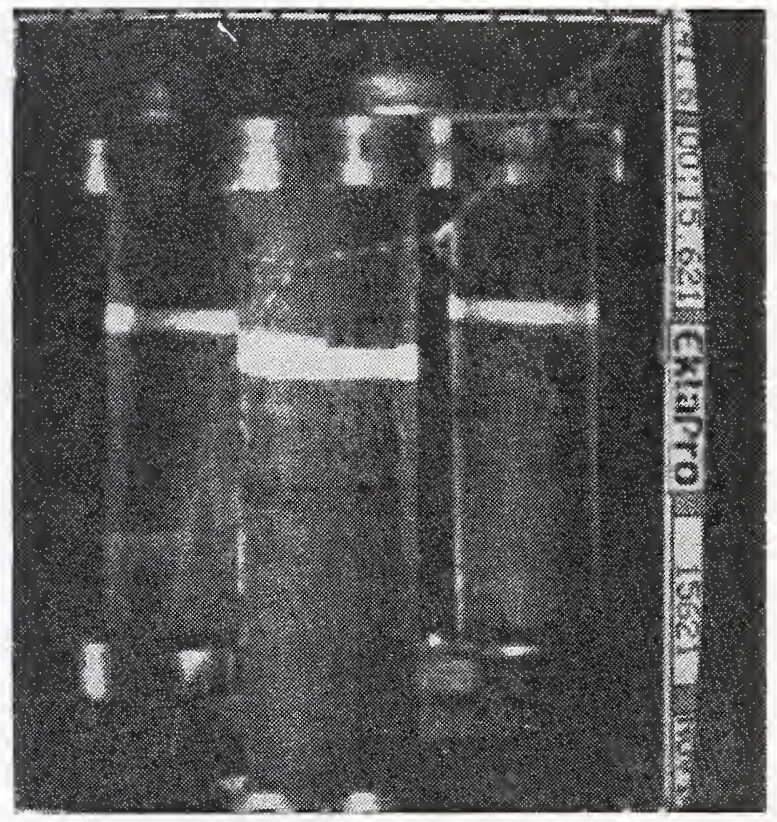

$$
t=15.621 \mathrm{~s}
$$

$\pm 10^{\circ}$ crack and horizontal crack

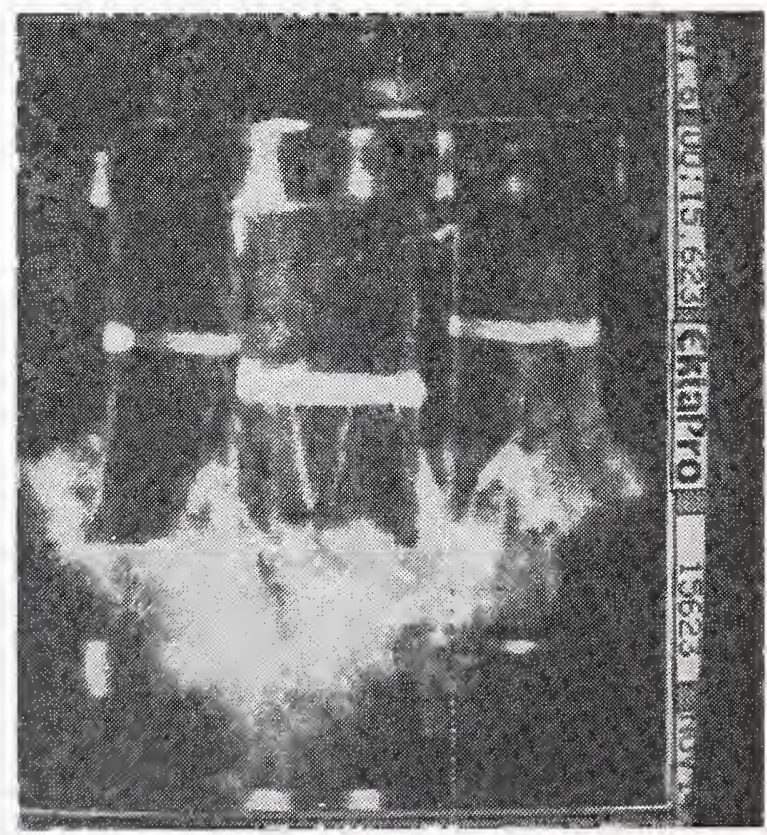

$$
t=15.623 \mathrm{~s}
$$

failure

Figure B.5. Sequence showing instantaneous failure in a $[ \pm 10]$ lay-up. 


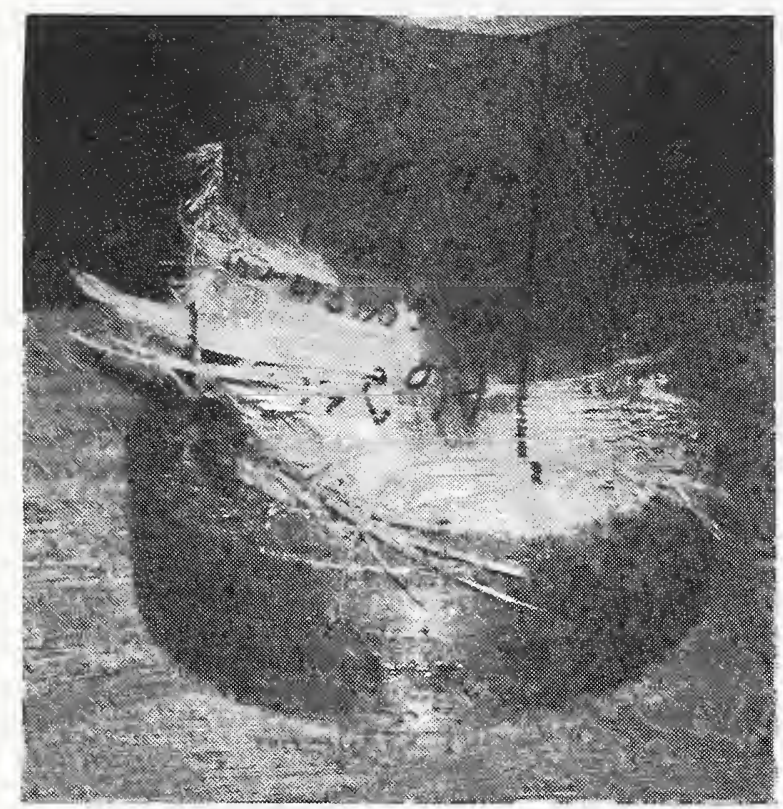

Figure B.6. Typical cap failure of filamentwound tube (type 19).

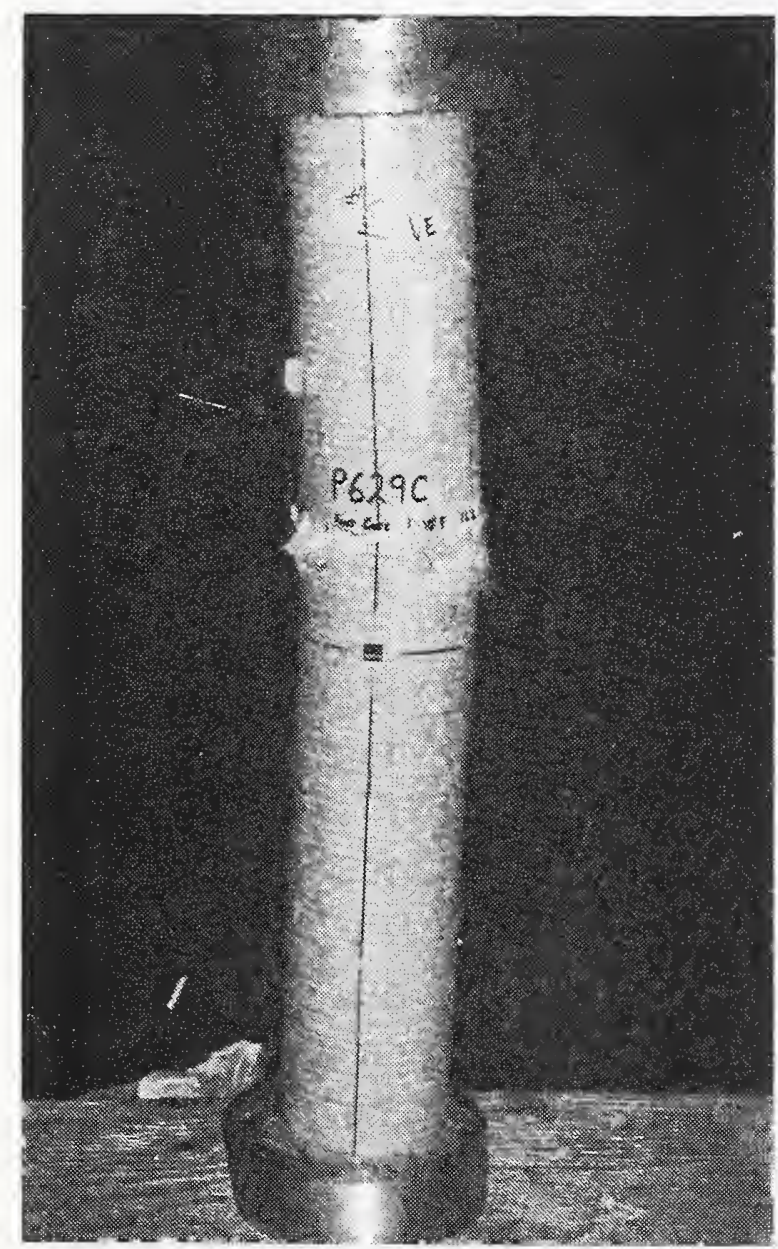

Figure B.8. Test-section failure in pultruded tube (type 23).

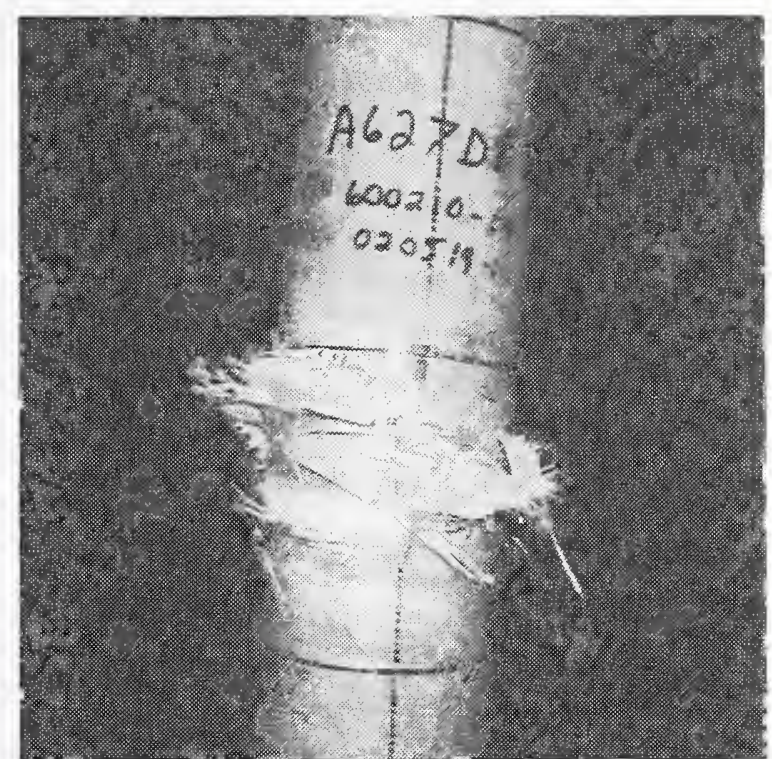

Figure B.7. Typical test-section failure of filament-wound tube (type 19).

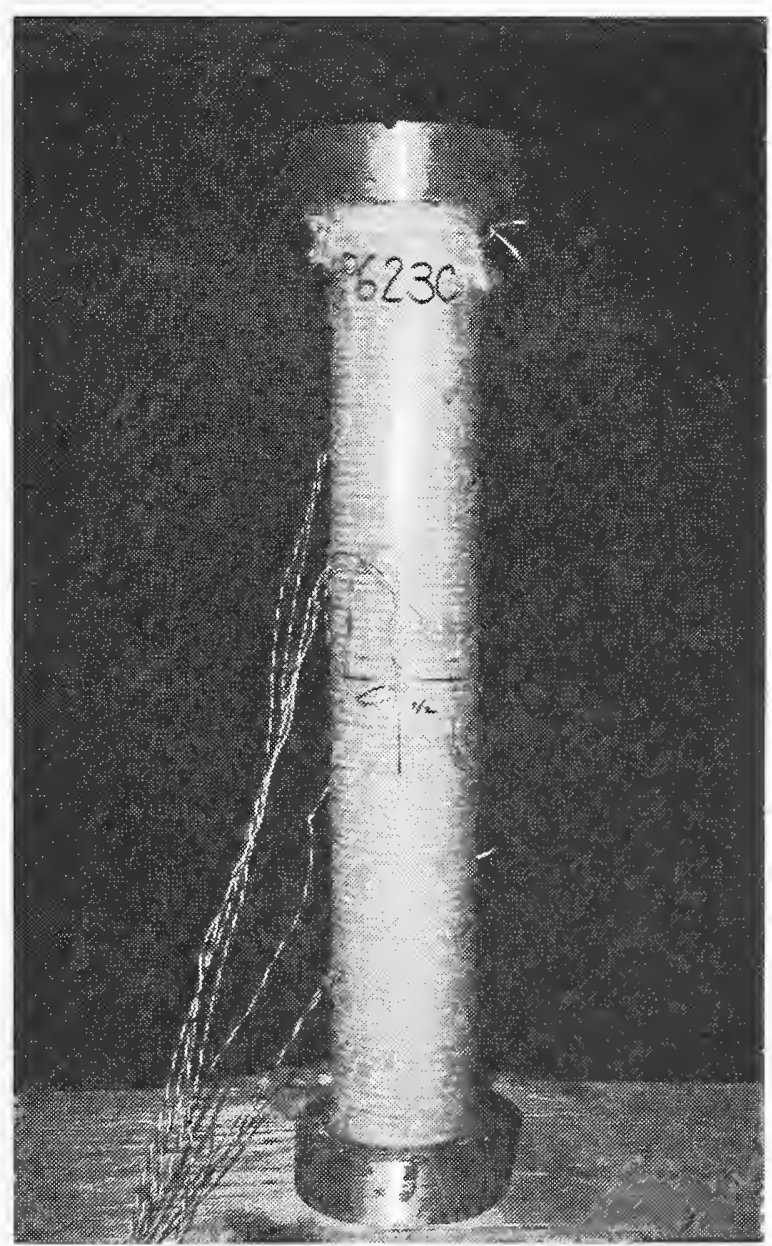

Figure B.9. Test-section failure in pultrudedtube end cap (tube type 23). 


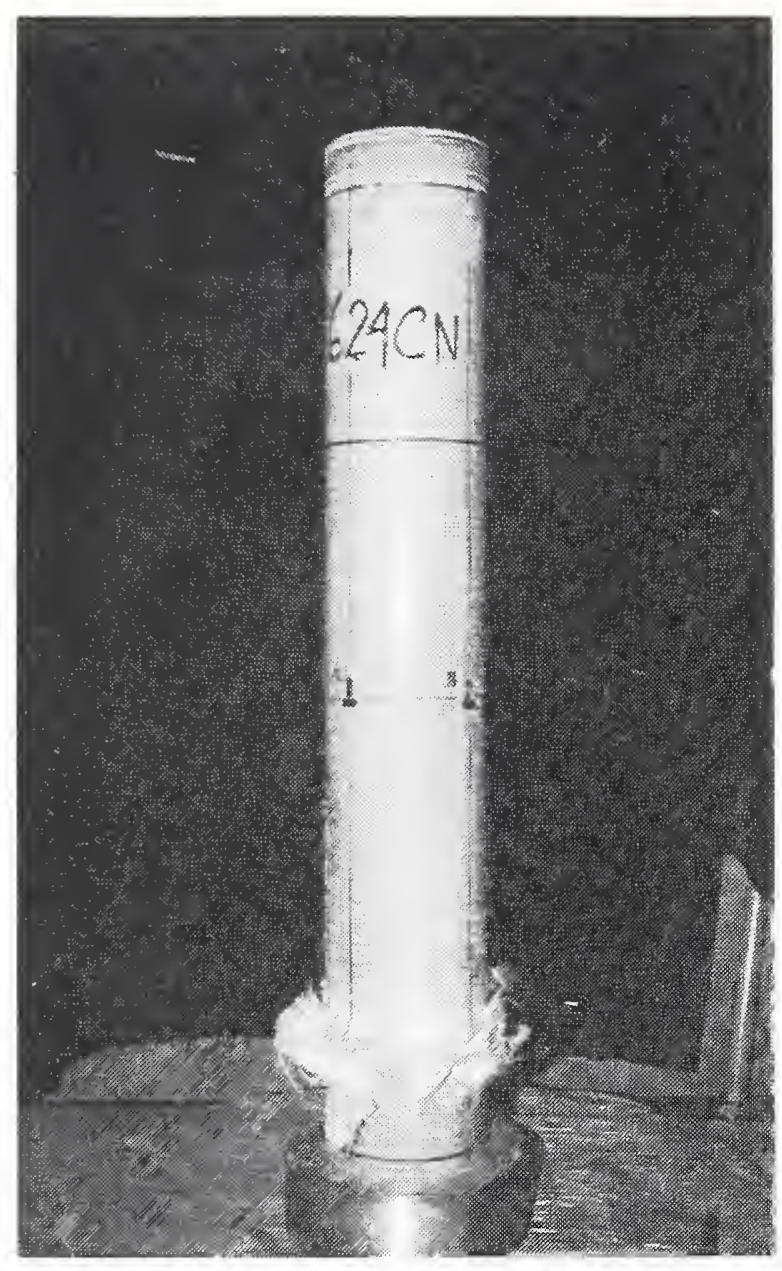

Figure B.10. Pultruded-tube end-cap failure in tube type 23 .

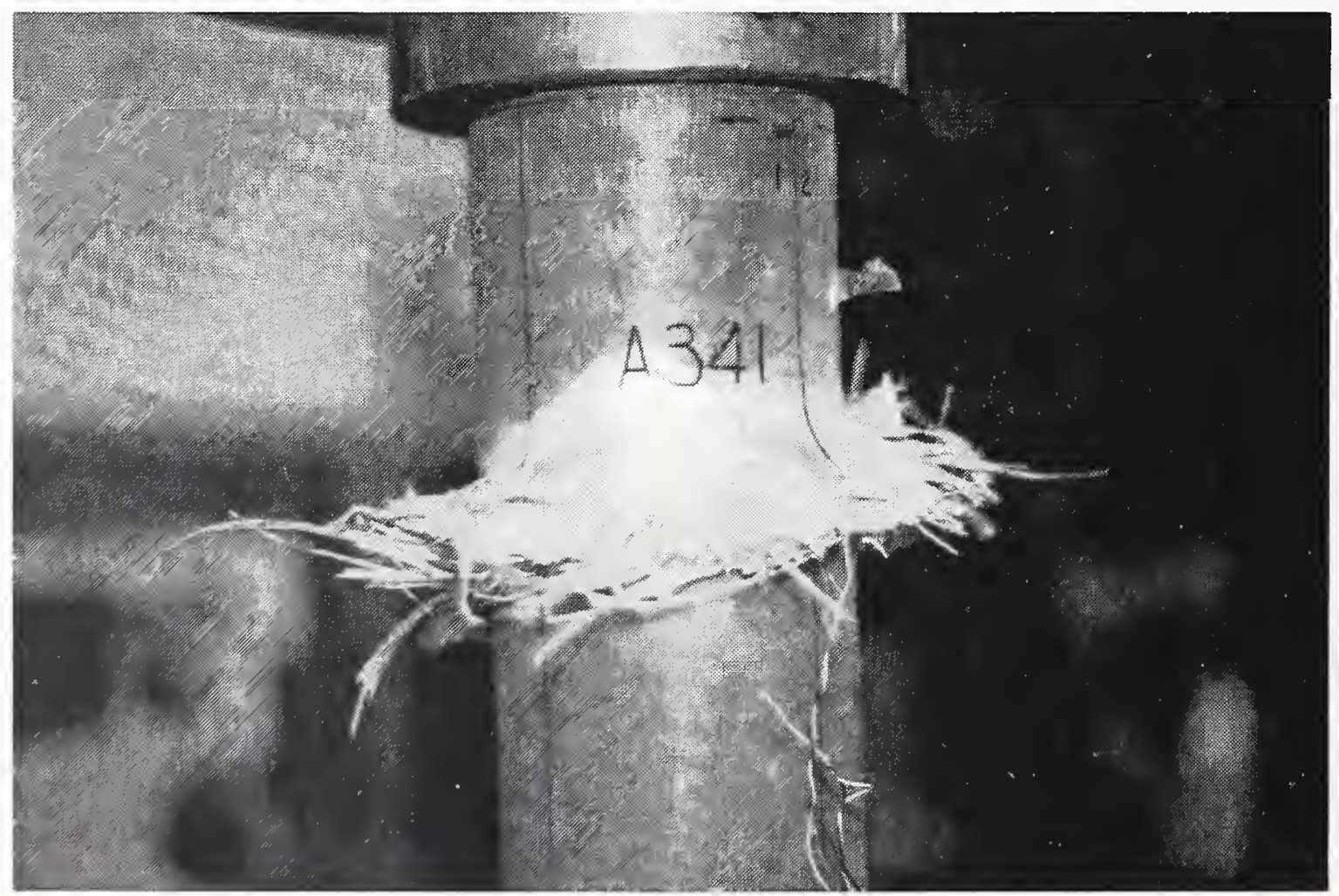

Figure B.11. Pultruded-tube-end failure in tube type 5. 


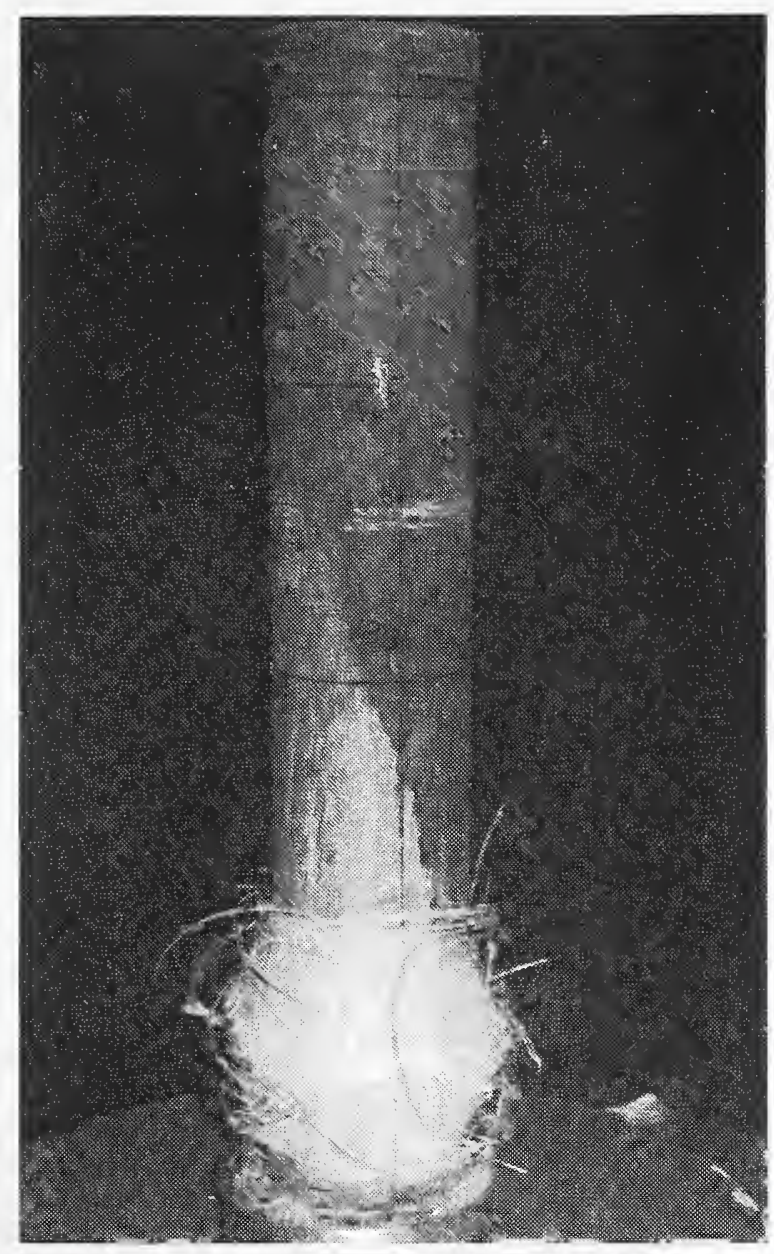

Figure B.12. Failure in tube type 5 at $76 \mathrm{~K}$.

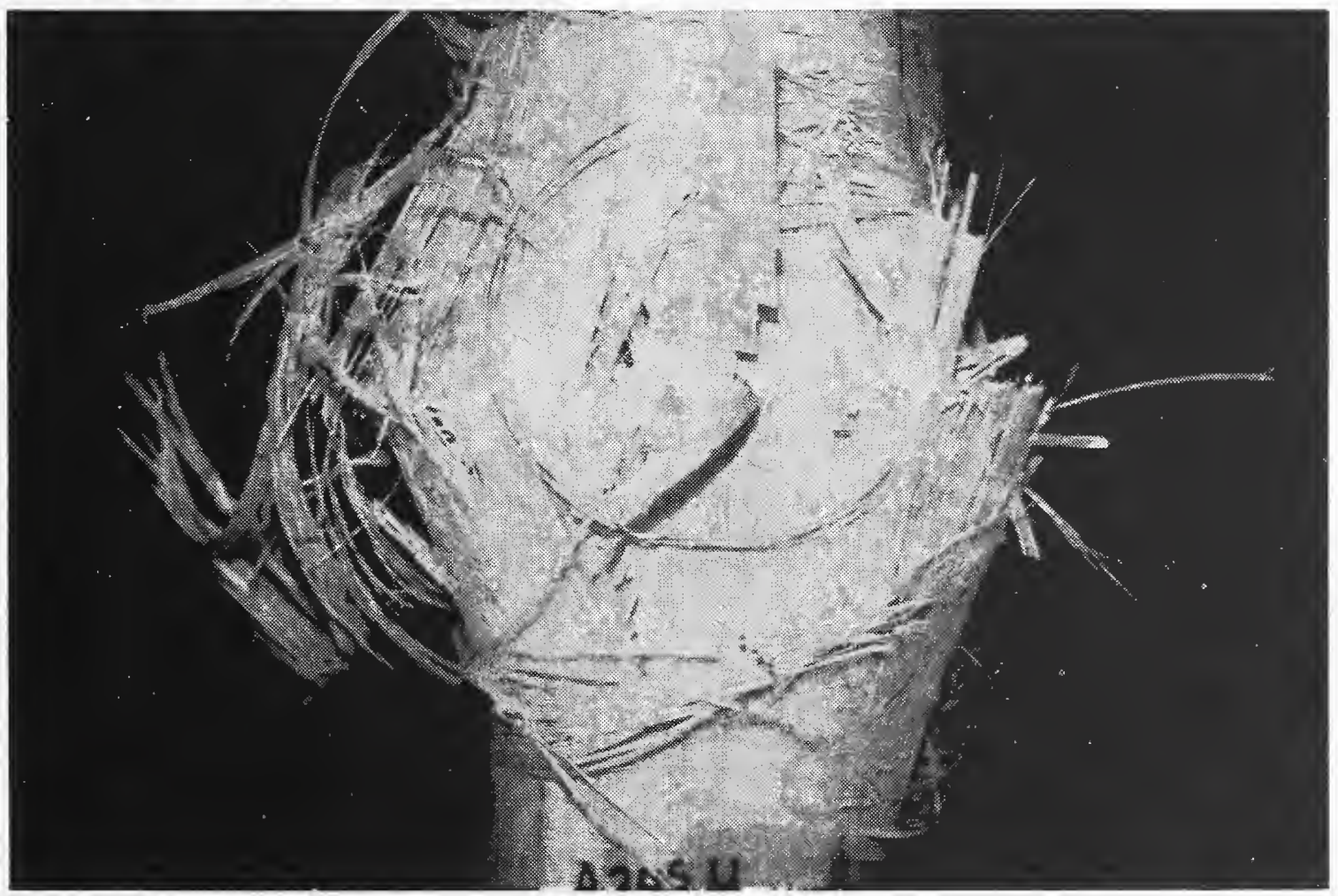

Figure B.13. Failure in tube type 5 at $4 \mathrm{~K}$. 


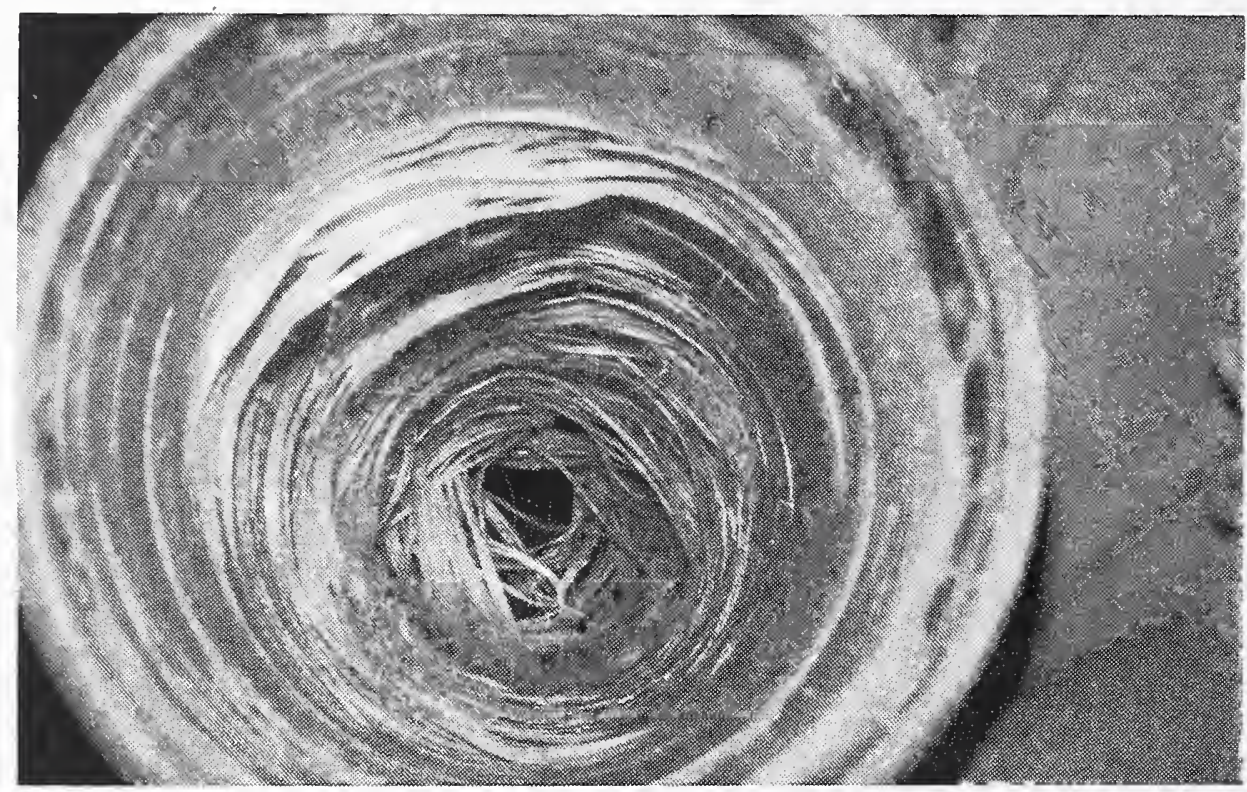

Figure B.14. Internal hoop-ply delamination (tube type 18) at $76 \mathrm{~K}$.
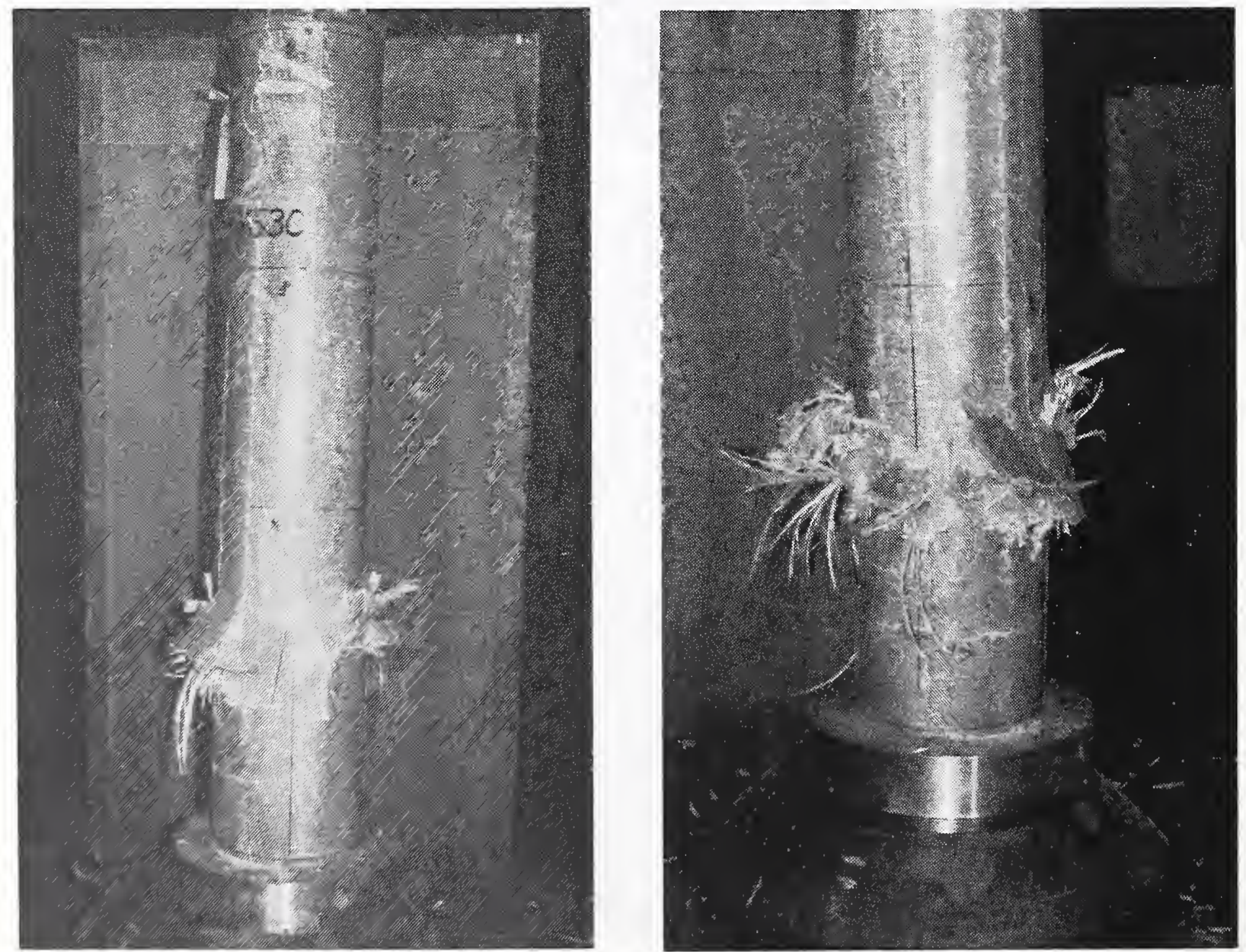

Figure B.15. Test section failure (pultruded tube type 24). 


\section{Analyses of the Response of Fiber-Reinforced Composite Cylinders to Loads}

George S. Springer and László P. Kollár

Stanford University

Stanford, California 


\title{
VII. Analyses of the Response of Fiber-Reinforced Composite Cylinders to Loads
}

\author{
George S. Springer and László P. Kollár \\ Stanford University \\ Stanford, California
}

\begin{abstract}
A method was developed that describes the response of fiber-reinforced composite cylinders to axially varying mechanical and thermal loads. The analysis utilizes a three-dimensional elasticity solution, Fourier's heat conduction law, and the Tsai-Wu failure criterion. The equations governing the problem were derived, and, on the basis of these equations, a computer code was written that provides the stress, strain, and temperature distributions inside the cylinder and the load at which first ply failure occurs. To validate the model and the code, results obtained by the code were compared with existing numerical and experimental results. In every case, good agreement was found between the present and previous results.
\end{abstract}

\section{Introduction}

Numerous analyses have been proposed in the past for calculating the stresses and strains in fiber-reinforced composite cylinders subjected to mechanical and hygrothermal loads. Most of the existing analyses employ shell approximations, as indicated by the recent survey of Noor et al. [1.1].

Analytical solutions taking into account three-dimensional variations in stresses and strains have been developed by Chou and Achenbach, Noor and Rarig, Srinivas, Grigorenko et al., Chandrashekhara and Gopalakrishnan, Hyer et al., Ren, Hyer, Roy and Tsai, Noor and Peters, Spencer et al., Varadan and Bhaskar, and Roy [1.2-1.14]. These investigators analyzed only orthotropic cylinders.

Three-dimensional stress analyses of composite cylinders with arbitrary lay-ups have been presented by Lekhnitskii, Hyer and Rousseau, Hyer et al., Lee and Springer, Spencer et al., Kollár and Springer, and Kollár et al. [1.15-1.21]. Only Kollár et al. considered radially and circumferentially varying stresses $[1.20,1.21]$. All the other investigators took into account stresses that vary only in the radial direction. No analysis seems to be available for generally anisotropic, thick composite cylinders, in which the stresses and strains vary in both the axial and radial directions. Therefore, the objective of this investigation was to develop a stress analysis of composite cylinders in which stresses and strains vary in the axial and radial directions due to imposed hygrothermal and mechanical loads.

An analytical method was employed in this investigation because, for long or for thick composite cylinders, this requires less computational time than finite-element techniques. 


\section{Problem Statement}

We consider a cylinder made of $n$ layers of unidirectional fiber-reinforced composites (Figure 2.1). There are no restrictions on either the number of plies, the orientation (ply angle) of the fibers in each ply, or the material of each ply. The cylinder may be thick, and the lay-up may be unsymmetric. Furthermore, there is no restriction on the length $L$ of the cylinder.

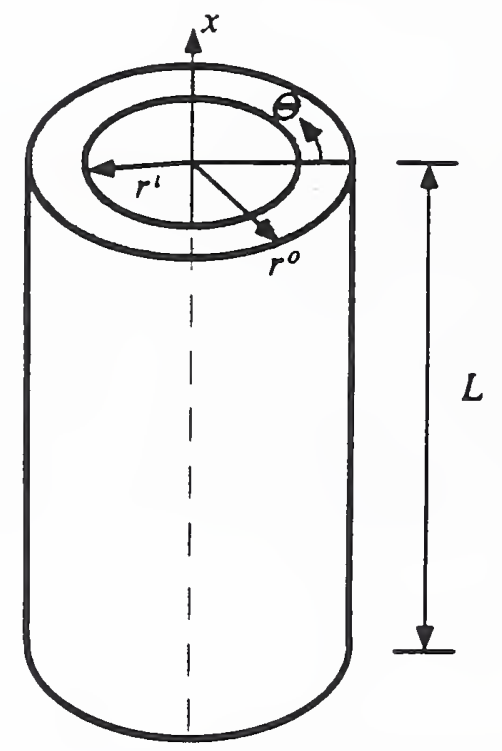

Figure 2.1. Cylinder dimensions.

Each edge may be free, constrained, or built in (Figure 2.2). No loads are applied on a free edge. A constrained edge is in contact with a rigid plate, the surface of the plate being parallel to the plane of the edge. Via this plate, an axial load $N$, a torque $T$, and a shear force $Q$ can be transmitted to the edge. Two cases are of special interest: when the friction between the edge and the plate is negligible and when the edge is fixed ("glued") to the plate (Figure 2.3). In the case of frictionless contact, either the compressive axial load $N$ or the axial displacement $u$ can be prescribed. For a fixed edge, the axial force or displacement $(N$ or $u$ ) and the torque $T$ (or the circumferential displacement $v$ ) can be prescribed.

The edge may also be attached to a structure (built-in edge). This structure may transmit to the cylinder axial forces $N$, radial forces $Q$, a moment $M$, and a torque $T$. The support structure may also cause axial, radial, and circumferential displacements $(u, w, v)$ and a rotation $(\eta)$ of the cylinder's edge (Figure 2.4). In the problem considered here, the applied loads and displacements must be independent of the circumferential direction $\theta$.

To fully describe the problem, at each edge, four of the eight variables (four loads and four displacements) must be given in a consistent manner. Frequently, the applied axial force $N$, moment $M$, shear $Q$, and torque $T$ or the displacements $u, v, w$ and the rotation $\eta$ are readily known. 


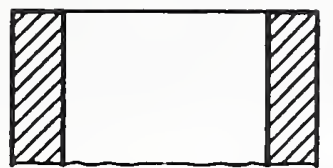

FREE

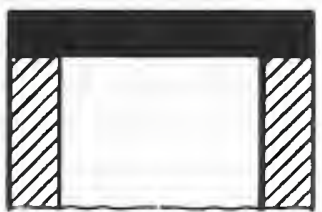

CONSTRAINED

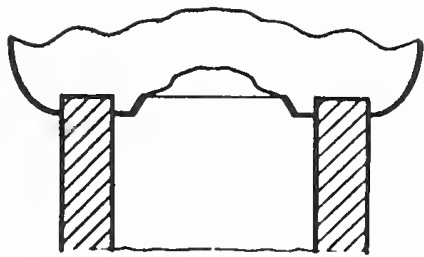

BUILT IN

Figure 2.2. Conditions at the edges of the cylinder.

\section{CONSTRAINED BOUNDARY}

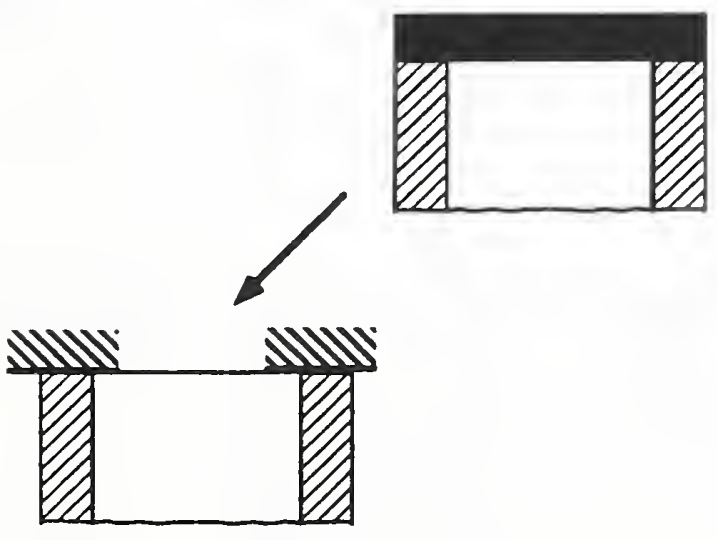

Fixed

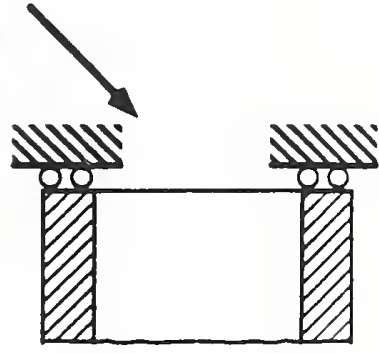

Frictionless

Figure 2.3. Constrained edge of the cylinder.

Surface and hygrothermal loads may also be imposed on the cylinder (Figure 2.5). The forces per unit area on the inner $\left(r=r^{i}\right)$ and outer $\left(r=r^{o}\right)$ surfaces are denoted by $p^{i}$ and $p^{o}$. The temperature $T$ and moisture content $c$ are specified with respect to reference values $T_{r}$ and $c_{r}$,

$$
\Delta T=T-T_{r} \quad \Delta c=c-c_{r},
$$

where $\Delta T$ and $\Delta c$ are the known distribution of temperature and moisture content. In those cases where the temperature or moisture are specified at the two edges of the cylinder $(x=0)$ and $(x=L)$, the axial distribution of temperature and moisture can be calculated as given in Appendix A. Since the temperature and moisture distributions may vary in the axial direction $(x)$, the material properties may also vary along $x$. 
The analysis presented here can be used to calculate the stresses and strains in a composite cylinder subjected to the hygrothermal and mechanical loads described above. The analysis is intended to serve two purposes: (1) to yield results directly when the edge loads (or edge displacements) are known, as is the case, for example, for cylinders with free or fixed edges and (2) to provide the cylinder portion of the analysis needed to describe an entire assembly consisting of a cylinder and its support structure.

\section{BUILT IN BOUNDARY}
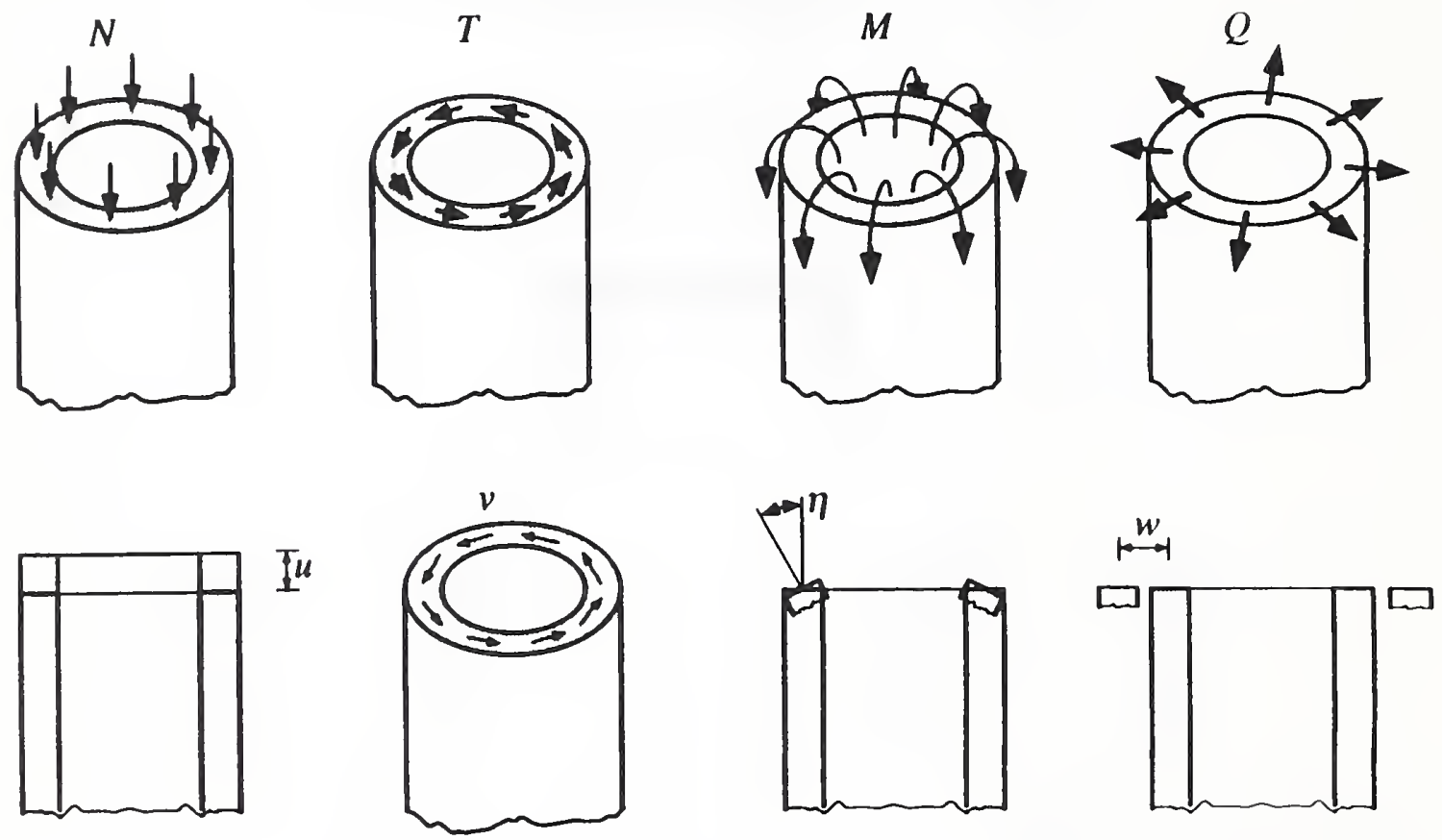

Figure 2.4. Loads and displacements at a built-in edge.
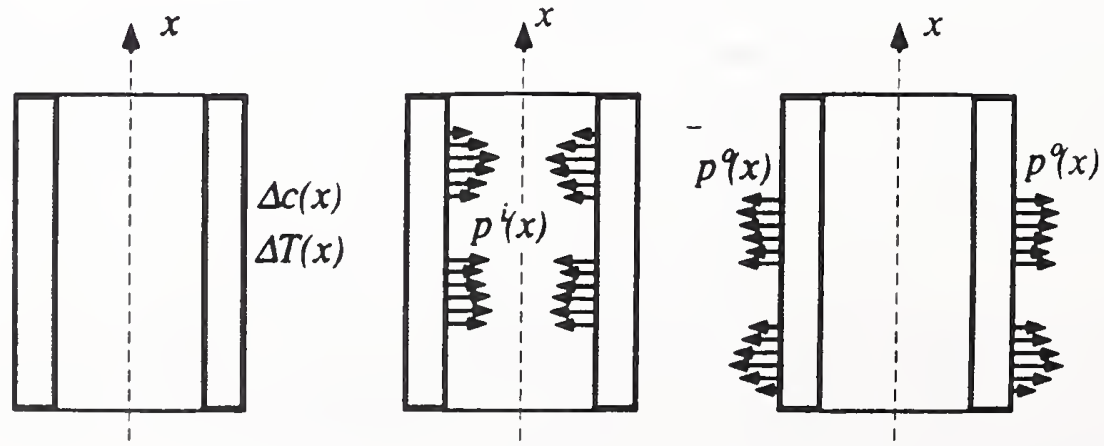

Figure 2.5. Temperature, moisture, and surface loads on a cylinder. 


\section{Governing Equations}

The governing equations are derived by assuming that the material properties are constant. The variations in material properties with position are taken into account in a manner described in $\$ 6$.

In the present analysis, the stresses and strains depend only on the axial and radial coordinates $(x, r)$. Correspondingly, the equations of equilibrium [3.1] are

$$
\begin{gathered}
-\frac{\partial \sigma_{x}}{\partial x}-\frac{\partial \tau_{x \boldsymbol{x}}}{\partial r}-\frac{\tau_{x \boldsymbol{r}}}{r}=0 \\
-\frac{\partial \tau_{\theta r}}{\partial r}-2 \frac{\tau_{\theta r}}{r}-\frac{\partial \tau_{\theta x}}{\partial x}=0 \\
\frac{\sigma_{\theta}}{r}-\frac{\partial \sigma_{r}}{\partial r}-\frac{\sigma_{r}}{r}-\frac{\partial \tau_{r x}}{\partial x}=0,
\end{gathered}
$$

where, as usual, $\sigma$ and $\tau$ represent normal and shear stresses. The strain displacement relationships [3.1] are

$$
\begin{aligned}
\epsilon_{x}=\frac{\partial u}{\partial x} & \gamma_{\theta r}=\frac{\partial v}{\partial r}-\frac{v}{r} \\
\epsilon_{\theta}=\frac{w}{r} & \gamma_{x r}=\frac{\partial u}{\partial r}+\frac{\partial w}{\partial x} \\
\epsilon_{r}=\frac{\partial w}{\partial r} & \gamma_{x \theta}=\frac{\partial v}{\partial x}
\end{aligned}
$$

where $\epsilon$ and $\gamma$ represent the strains, and $u, w, v$ are the displacements in the axial, radial, and circumferential directions $(x, r, \theta)$. Note that although the displacements are independent of $\theta$, the displacement $v$ in the circumferential direction is not zero.

For a linearly elastic material, the stress-strain relationship in the $x, \theta, r$ off-axis coordinate system [3.2] is

$$
\left[\begin{array}{c}
\sigma_{x} \\
\sigma_{\theta} \\
\sigma_{r} \\
\tau_{\theta r} \\
\tau_{x r} \\
\tau_{x \theta}
\end{array}\right]^{\prime}=\left[\begin{array}{cccccc}
C_{11} & C_{12} & C_{13} & 0 & 0 & C_{16} \\
C_{21} & C_{22} & C_{23} & 0 & 0 & C_{26} \\
C_{31} & C_{32} & C_{33} & 0 & 0 & C_{36} \\
0 & 0 & 0 & C_{44} & C_{45} & 0 \\
0 & 0 & 0 & C_{54} & C_{55} & 0 \\
C_{61} & C_{62} & C_{63} & 0 & 0 & C_{66}
\end{array}\right]^{\prime}\left[\begin{array}{c}
\epsilon_{x}-\alpha_{x} \Delta T-\beta_{x} \Delta c \\
\epsilon_{\theta}-\alpha_{\theta} \Delta T-\beta_{\theta} \Delta c \\
\epsilon_{r}-\alpha_{r} \Delta T-\beta_{r} \Delta c \\
\gamma_{\theta r} \\
\gamma_{x r}-\alpha_{x \theta} \Delta T-\beta_{x \theta} \Delta c
\end{array}\right]^{\prime},
$$


where $C_{i j}\left(=C_{j i}, i, j=1, \ldots 6\right)$ are the components of the stiffness matrix, and $\alpha$ and $\beta$ are the thermal and moisture expansion coefficients. The superscript $l$ indicates that Equation 3.3 applies to the $l^{\text {th }}$ layer.

Temperature and moisture affect the strains similarly (see Equation 3.3). Therefore, we include only $\Delta T$ in the subsequent analysis. Changes in the moisture content $\Delta c$ can be treated in the same way as changes in $\Delta T$.

\section{Boundary Conditions}

The temperature, moisture, and surface forces depend only on $x$. Hence, their known distributions can be expressed as

$$
\begin{aligned}
\Delta T & =\Delta T(x) & \Delta c & =\Delta c(x) \\
p^{i} & =p^{i}(x) & p^{o} & =p^{o}(x) .
\end{aligned}
$$

At each edge, the loads $N, T, M$, and $Q$ (see Figure 2.4) are related to the stresses inside the composite at $x=0$ and $x=L$ by the expressions

$$
\begin{array}{ll}
N_{0}=2 \pi \int_{r^{i}}^{r^{\circ}} r \sigma_{x}(0, r) d r & N_{L}=2 \pi \int_{r^{i}}^{r^{\circ}} r \sigma_{x}(L, r) d r \\
T_{0}=2 \pi \int_{r^{i}}^{r^{\circ}} r^{2} \tau_{x r}(0, r) d r & T_{L}=2 \pi \int_{r^{i}}^{r^{\circ}} r^{2} \tau_{x r}(L, r) d r \\
M_{0}=\frac{1}{R} \int_{r^{i}}^{r^{\circ}} r(r-R) \sigma_{x}(0, r) d r & M_{L}=\frac{1}{R} \int_{r^{i}}^{r^{\circ}} r(r-R) \sigma_{x}(L, r) d r \\
Q_{0}=\frac{1}{R} \int_{r^{i}}^{r^{\circ}} r \tau_{x r}(0, r) d r & Q_{L}=\frac{1}{R} \int_{r^{i}}^{r^{\circ}} r \tau_{x r}(L, r) d r
\end{array}
$$

where 0 and $L$ refer to the $x=0$ and $x=L$ edges. The radius of the midsurface is

$$
\mathrm{R}=\frac{1}{2}\left(r^{i}+r^{o}\right)
$$

To satisfy equilibrium, the axial loads on the two edges must be equal, and the torques on the two edges must be equal:

$$
N_{0}=N_{L} \quad T_{0}=T_{L}
$$

The axial $\bar{u}$ and circumferential $\bar{v}$ edge displacements at the midsurface $R$ are the relative displacements between the two edges $(x=0, x=L)$ : 


$$
\begin{aligned}
& \bar{u}=u(L, R)-u(0, R) \\
& \bar{v}=v(L, R)-v(0, R) .
\end{aligned}
$$

The rotations of the cross section $\bar{\eta}$ at the $(x=0)$ and $(x=L)$ edges are defined as

$$
\bar{\eta}_{0}=\frac{u\left(0, r^{\rho}\right)-u\left(0, r^{i}\right)}{r^{0}-r^{i}} \quad \bar{\eta}_{L}=\frac{u\left(L, r^{\rho}\right)-u\left(L, r^{i}\right)}{r^{\rho}-r^{i}} .
$$

The radial edge displacements $\bar{w}$ are equal to the radial displacements of the midsurface:

$$
\bar{w}_{0}=w(0, R) \quad \bar{w}_{L}=w(L, R) .
$$

Here, $\bar{u}, \bar{v}, \bar{\eta}_{0}, \bar{\eta}_{L}, \bar{w}_{0}$, and $\bar{w}_{L}$ are the applied edge displacements, and $u, v$, and $w$ are the axial, circumferential, and radial displacements of the composite.

For convenience, in the subsequent analysis we denote the applied loads by $\Omega$ and the applied displacements by $q$ :

$$
\begin{array}{ll}
\Omega_{1}=M_{0} & q_{1}=\bar{\eta}_{0} \\
\Omega_{2}=Q_{0} & q_{2}=\bar{w}_{0} \\
\Omega_{3}=M_{L} & q_{3}=\bar{\eta}_{L} \\
\Omega_{4}=Q_{L} & q_{4}=\bar{w}_{L} \\
\Omega_{5}=N_{0} & q_{5}=\bar{u} \\
\Omega_{6}=T_{0} & q_{6}=\bar{v} .
\end{array}
$$

We must specify six of the above twelve parameters, such that either a force or a displacement from each row is given. For example, if both edges are free, the six edge forces are zero, and we have

$$
\begin{array}{ll}
\Omega_{1}=M_{0}=0 & \Omega_{2}=Q_{0}=0 \\
\Omega_{3}=M_{L}=0 & \Omega_{4}=Q_{L}=0 \\
\Omega_{5}=N_{0}=0 & \Omega_{6}=T_{0}=0 .
\end{array}
$$

The above conditions imply that the integrals of the appropriate stresses are zero along a free edge.

If both edges are fixed, the following six edge displacements are given:

$$
\begin{array}{ll}
q_{1}=\bar{\eta}_{0}=0 & q_{2}=\bar{w}_{0}=0 \\
q_{3}=\bar{\eta}_{L}=0 & q_{4}=\bar{w}_{L}=0 \\
q_{5}=\bar{u} & q_{6}=\bar{v} .
\end{array}
$$

Instead of $q_{5}$ and $q_{6}$, we may specify $\Omega_{5}\left(=N_{0}\right)$ and $\Omega_{6}\left(=T_{0}\right)$.

If the edge at $x=0$ is fixed, and the edge at $x=L$ is free, we have the following six boundary conditions: 


$$
\begin{array}{ll}
q_{1}=\bar{\eta}_{0}=0 & q_{2}=\bar{w}_{0}=0 \\
\Omega_{3}=M_{L}=0 & \Omega_{4}=Q_{L}=0 \\
\Omega_{5}=N_{0}=0 & \Omega_{6}=T_{0}=0 .
\end{array} .
$$

\section{General Solution of the Governing Equations}

To proceed with the solution, we express the displacements in the form of Fourier series. We will utilize the following two series

1. Unstarred series

$$
\begin{aligned}
u^{l}(x, r) & =\sum_{i=1} u_{i}^{l}(r) \cos i \frac{\pi}{L} x \\
v^{l}(x, r) & =\sum_{i=1} v_{i}^{l}(r) \cos i \frac{\pi}{L} x \\
w^{l}(x, r) & =\sum_{i=1} w_{i}^{l}(r) \sin i \frac{\pi}{L} x
\end{aligned}
$$

2. Starred series

$$
\begin{aligned}
u^{l}(x, r) & =u_{o}^{l *}(r)-\sum_{i=1} u_{i}^{l *}(r) \sin i \frac{\pi}{L} x \\
v^{l}(x, r) & =v_{o}^{l *}(r)-\sum_{i=1} v_{i}^{l *}(r) \sin i \frac{\pi}{L} x \\
w^{l}(x, r) & =w_{o}^{l *}(r)+\sum_{i=1} w_{i}^{l *}(r) \cos i \frac{\pi}{L} x
\end{aligned}
$$

Both of the above series are needed to satisfy all the possible boundary conditions. In the above expressions $u_{i}^{l}(r), v_{i}^{l}(r), w_{i}^{l}(r) ; u_{\mathrm{o}}^{l *}(r), v_{\mathrm{o}}^{l *}(r), w_{\mathrm{o}}^{l *}(r)$ and $u_{\mathrm{o}}^{l *}(r), v_{\mathrm{o}}^{l *}(r), w_{\mathrm{o}}^{l_{*}}(r)$ are, as yet, unknown functions.

Since the solution is sought in terms of these types of series, it is required that the applied temperature and applied surface forces (pressure) also be expressed in similar forms. The appropriate expressions for temperature and pressure are given in Appendix B. By combining the expressions for displacements (Equations 5.1 and 5.2) and temperature (Equations B.1 and B.3) with the definition of strain (Equation 3.2) and the constitutive relationship (Equation 3.3), we obtain the stresses as functions of the displacements. The stress components corresponding to the different terms in the displacement series are listed in Table 5.1.

\subsection{Displacements, $0^{\text {th }}$ Terms}

The $0^{\text {th }}$ terms in the displacements result in stresses that depend on the radial coordinate $r$ only. Appropriate expressions for the $0^{\text {th }}$ terms were given by Kollár and Springer [5.1]. For 
Table 5.1. The displacement, temperature, and stress terms.

The displacements

\begin{tabular}{l|l|l|l}
$u^{l}(x, r)$ & $u_{0}^{l *}(r)$ & $u_{i}^{l}(r) \cos i \frac{\pi}{L} x$ & $-u_{i}^{l *}(r) \sin i \frac{\pi}{L} x$ \\
$v^{l}(x, r)$ & $v_{0}^{l *}(r)$ & $v_{i}^{l}(r) \cos i \frac{\pi}{L} x$ & $-v_{i}^{l *}(r) \sin i \frac{\pi}{L} x$ \\
$w^{l}(x, r)$ & $w_{0}^{l *}(r)$ & $w_{i}^{l}(r) \sin i \frac{\pi}{L} x$ & $w_{i}^{l *}(r) \cos i \frac{\pi}{L} x$
\end{tabular}

The corresponding temperature terms

\begin{tabular}{|l|c|c|c}
$\Delta T(x)$ & $\Delta T_{0}$ & $\Delta T_{i} \sin i \frac{\pi}{L} x$ & $\Delta T_{i}^{*} \cos i \frac{\pi}{L} x$
\end{tabular}

The corresponding stress terms

\begin{tabular}{|c|c|c|c|}
\hline$\sigma_{x}^{l}(x, r)$ & $\sigma_{x 0}^{l e}(r)$ & $\sigma_{x i}^{l}(r) \sin i \frac{\pi}{L} x$ & $\sigma_{x i}^{l i}(r) \cos i \frac{\pi}{L} x$ \\
\hline$\sigma_{\theta}^{l}(x, r)$ & $\sigma_{\theta_{0}}^{l *}(r)$ & $\sigma_{\theta_{i}}^{l}(r) \sin i \frac{\pi}{L} x$ & $\sigma_{\theta i}^{l \theta}(r) \cos i \frac{\pi}{L} I$ \\
\hline$\sigma_{r}^{l}(x, r)$ & $\sigma_{r o}^{l *}(r)$ & $\sigma_{r i}^{l}(r) \sin i \frac{\pi}{L} x$ & $\sigma_{r i}^{l *}(r) \cos i \frac{\pi}{L} x$ \\
\hline$\tau_{\theta_{r}}^{l}(x, r)$ & $\tau_{\theta \text { ro }}^{l}=0$ & $\tau_{d_{r i}}^{l}(r) \cos i \frac{\pi}{L} x$ & $\tau_{\theta r i}^{l *}(r) \sin i \frac{\pi}{L} x$ \\
\hline$\tau_{x \boldsymbol{r}}^{l}(x, r)$ & $\tau_{x \mathrm{ro}}^{l *}=0$ & $\tau_{\theta+i}^{l}(r) \cos i \frac{\pi}{L} x$ & $\tau_{x r i}^{l *}(r) \sin i \frac{\pi}{L} x$ \\
\hline$\tau_{x \theta}^{l}(x, r)$ & $T_{x \theta_{0}}^{l \theta}(r)$ & $\tau_{x \theta i}^{l}(r) \sin i \frac{\pi}{L} x$ & $\tau_{x \theta i}^{l \cdot}(r) \cos i \frac{\pi}{L} x$ \\
\hline
\end{tabular}


the present problem of a closed cylinder (in which the temperature is independent of $r$ and onwhich there are no applied surface shear forces), Kollár and Springer's expressions reduce to

$$
\begin{aligned}
u_{o}^{l *}= & u_{a}^{l} x \\
v_{o}^{l *}= & v_{a}^{l} x r \\
w_{o}^{l *}= & A_{1}^{l}\left(\frac{r}{R}\right)^{\lambda}+A_{2}^{l}\left(\frac{r}{R}\right)^{-\lambda}+\delta_{2} R f_{o}(r) \\
& +\delta_{3} R^{2} f_{1}(r)+\Delta R f_{o}(r)
\end{aligned}
$$

The parameters $\delta_{2}, \delta_{3}, \Delta \lambda$, and $f_{i}(r)(i=0,1)$ are defined in Table 5.2. The unknown constants are $u_{a}^{l}, v_{a}^{l}, A_{1}^{l}$, and $A_{2}^{l}$. Thus, for an $n$-ply laminate, there are $4 \times n$ constants. The number of these unknown constants can be reduced by the use of the continuity conditions at the interfaces. At each interface, the displacements must be equal and the radial normal stresses must be equal. These conditions are expressed as

$$
\begin{aligned}
u_{a}^{l} & =u_{a}^{l+1} \\
v_{a}^{l} r^{l+1} & =v_{a}^{l+1} r^{l+1} \\
w_{o}^{l *}\left(r^{l+1}\right) & =w_{o}^{l+1 *}\left(r^{l+1}\right) \quad l=1,2, \ldots, n-1 \\
\sigma_{r o}^{l *} & =\sigma_{r o}^{l+1 *}
\end{aligned}
$$

where $r^{l+1}$ denotes the inner radius of the $(l+1)^{t h}$ layer. The shear stresses $l_{\theta \text { ro }}^{l_{*}}$ and $\tau_{x r o}$ are zero, and they need not be considered. By applying the continuity conditions at every interface (total number of interfaces $=n-1$ ), all but four of the unknowns can be determined.

Table 5.2. Definition of the parameters in Equation 5.3.

$$
\begin{aligned}
& \delta_{2}=u_{a}\left(C_{21}-C_{31}\right) \\
& \delta_{3}=v_{a}\left(C_{26}-2 C_{36}\right) \\
& \Delta=\Delta T_{0}\left[\alpha_{x}\left(C_{21}-C_{31}\right)+\alpha_{\theta}\left(C_{22}-C_{32}\right)+\alpha_{r}\left(C_{23}-C_{33}\right)+\alpha_{r \theta}\left(C_{26}-C_{36}\right)\right] \\
& \lambda=\sqrt{\frac{C_{22}}{C_{33}}} \\
& f_{i}(r)=\frac{1}{(3+1)^{2} C_{33}-C_{22}}\left(\frac{r}{R}\right)^{i+1} \text { if }(i+1)^{2} C_{33}-C_{22} \neq 0 \\
& f_{i}(r)=\frac{\ln \frac{r}{R}}{2(i+1) C_{33}}\left(\frac{r}{R}\right)^{i+1} \text { if }(i+1)^{2} C_{33}-C_{22}=0
\end{aligned}
$$




\subsection{Displacements, $i^{\text {th }}$ Term of the Unstarred Series}

We consider the following unstarred terms of the displacements (Equation 5.1) and the temperature (Equation B.1),

$$
\begin{array}{ll}
u_{i}^{l}(r) \cos i \frac{\pi}{L} x & \\
v_{i}^{l}(r) \cos i \frac{\pi}{L} x & \Delta T_{i} \sin i \frac{\pi}{L} x \\
w_{i}^{l}(r) \sin i \frac{\pi}{L} x &
\end{array}
$$

By substituting these expressions and the expressions for the strains and stresses (Equations 3.2 and 3.4) into Equations 3.1, we obtain the following sixth-order ordinary differential equation system:

$$
\left[\begin{array}{lll}
\Theta_{11} & \Theta_{12} & \Theta_{13} \\
\Theta_{21} & \Theta_{22} & \Theta_{23} \\
\Theta_{31} & \Theta_{32} & \Theta_{33}
\end{array}\right]\left[\begin{array}{c}
u_{i}^{l}(r) \\
v_{i}^{l}(r) \\
w_{i}^{l}(r)
\end{array}\right]=\Delta T_{i}\left[\begin{array}{l}
B_{1} \\
B_{2} \\
B_{3}
\end{array}\right]
$$

where $\theta_{i j}, B_{1}, B_{2}$, and $B_{3}$ are given in Table 5.3. The solution of this system of equations is presented in Appendix $C$.

The final solution for the displacements is

$$
\left[\begin{array}{l}
u_{i}^{l}(r) \\
v_{i}^{l}(r) \\
w_{i}^{l}(r)
\end{array}\right]=\sum_{j=1}^{6} C_{j}^{l}\left[\begin{array}{c}
u_{i j}^{l, h o m}(r) \\
v_{i j}^{l, h o m}(r) \\
w_{i j}^{l, h o m}(r)
\end{array}\right]+\left[\begin{array}{c}
u_{i}^{l, i n h}(r) \\
v_{i}^{l, i n h}(r) \\
w_{i}^{l, i n h}(r)
\end{array}\right]
$$

the homogeneous (superscript hom) and inhomogeneous (superscript inh) displacements are given by Equations C.8 and C.9. The terms $C_{1}^{l}$ through $C_{6}^{l}$ are six unknown constants. Thus, for an $n$-ply laminate, there are $6 \times n$ unknowns. The number of these unknowns can be reduced by applying the following displacement and stress continuity conditions at each interface:

$$
\begin{aligned}
& u_{i}^{l}\left(r^{l+1}\right)=u_{i}^{l+1}\left(r^{l+1}\right) \\
& v_{i}^{l}\left(r^{l+1}\right)=v_{i}^{l+1}\left(r^{l+1}\right) \quad l=1,2, \ldots, n-1 \\
& w_{i}^{l}\left(r^{l+1}\right)=w_{i}^{l+1}\left(r^{l+1}\right) \\
& \sigma_{r i}^{l}\left(r^{l+1}\right)=\sigma_{r i}^{l+1}\left(r^{l+1}\right) \\
& \tau_{\theta r i}^{l}\left(r^{l+1}\right)=\tau_{\theta r i}^{l+1}\left(r^{l+1}\right) \\
& l=1,2, \ldots, n-1 \\
& \tau_{x r i}^{l}\left(r^{l+1}\right)=\tau_{x r i}^{l+1}\left(r^{l+1}\right)
\end{aligned}
$$


Table 5.3. Definition of the symbols in Equation 5.6.

$$
\begin{aligned}
& \Theta_{11}=\frac{i^{2} \pi^{2}}{L^{2}} C_{11}-C_{55} \frac{1}{r} \frac{\partial}{\partial r}-C_{55} \frac{\partial^{2}}{\partial r^{2}} \\
& \Theta_{12}=\frac{i^{2} \pi^{2}}{L^{2}} C_{16}-C_{45} \frac{\partial^{2}}{\partial r^{2}} \\
& \Theta_{13}=\frac{1 \pi}{L} \frac{1}{r}\left(-C_{12}-C_{55}\right)-\frac{i \pi}{L}\left(C_{13}+C_{55}\right) \frac{\partial}{\partial r} \\
& \Theta_{21}=\frac{i^{2} \pi^{2}}{L^{2}} C_{16}-2 C_{45} \frac{1}{r} \frac{\partial}{\partial r}-C_{45} \frac{\partial^{2}}{\partial r^{2}} \\
& \Theta_{22}=\frac{i^{2} \pi^{2}}{L^{2}} C_{66}+C_{44} \frac{1}{r^{2}}-C_{44} \frac{1}{r} \frac{\partial}{\partial r}-C_{44} \frac{\partial^{2}}{\partial r^{2}} \\
& \Theta_{23}=-\frac{i \pi}{L}\left(C_{26}+2 C_{45}\right) \frac{1}{r}-\frac{i \pi}{L}\left(C_{36}+C_{45}\right) \frac{\partial}{\partial r} \\
& \Theta_{31}=\frac{1 \pi}{L}\left(-C_{12}+C_{13}\right) \frac{1}{r}+\frac{1 \pi}{L}\left(C_{13}+C_{55}\right) \frac{\partial}{\partial r} \\
& \Theta_{32}=\frac{i \pi}{L}\left(-C_{26}+C_{36}-C_{45}\right) \frac{1}{r}+\frac{i \pi}{L}\left(C_{36}+C_{45}\right) \frac{\partial}{\partial r} \\
& \Theta_{33}=\frac{i^{2} \pi^{2}}{L^{2}} C_{55}+C_{22} \frac{1}{r^{2}}-C_{33} \frac{1}{r} \frac{\partial}{\partial r}-C_{33} \frac{\partial^{2}}{\partial r^{2}}
\end{aligned}
$$

$$
\begin{aligned}
& B_{1}=-\frac{i \pi}{L} q_{1} \\
& B_{2}=-\frac{i \pi}{L} q_{2} \\
& B_{3}=-\frac{1}{r} q_{3}
\end{aligned}
$$

$$
\begin{aligned}
& q_{1}=C_{11} \alpha_{x}+C_{12} \alpha_{\theta}+C_{13} \alpha_{r}+C_{16} \alpha_{x \theta} \\
& q_{2}=C_{16} \alpha_{x}+C_{26} \alpha_{\theta}+C_{36} \alpha_{r}+C_{66} \alpha_{x \theta} \\
& q_{3}=\left(C_{13}-C_{12}\right) \alpha_{x}+\left(C_{23}-C_{22}\right) \alpha_{\theta}+\left(C_{33}-C_{23}\right) \alpha_{r}+\left(C_{36}-C_{26}\right) \alpha_{x \theta}
\end{aligned}
$$

VII-12 
At the inner and outer surfaces, the shear stresses $\tau_{\theta r}$ and $\tau_{x r}$ are zero, yielding four more conditions:

$$
\begin{array}{ll}
\tau_{\theta r}^{1}\left(r^{i}\right)=0 & \tau_{\theta r}^{n}\left(r^{0}\right)=0 \\
\tau_{x r}^{1}\left(r^{i}\right)=0 & \tau_{x r}^{n}\left(r^{o}\right)=0 .
\end{array}
$$

By applying Equations 5.8 at every interface and the above four shear stress conditions, all but two of the $6 \times n$ constants can be evaluated.

\subsection{Displacements, $i^{\text {th }}$ Term of the Starred Series}

Here we consider the following starred terms of the displacements (Equation 5.2) and temperature (Equation B.3):

$$
\begin{array}{ll}
-u_{i}^{l *}(r) \sin i \frac{\pi}{L} x & \\
-v_{i}^{l *}(r) \sin i \frac{\pi}{L} x & \Delta T_{i}^{*} \cos i \frac{\pi}{L} x \\
w_{i}^{l *}(r) \cos i \frac{\pi}{L} x
\end{array}
$$

We now make use of the following identities:

$$
\begin{aligned}
& \cos \left(i \frac{\pi}{L} x+\frac{\pi}{2}\right)=-\sin i \frac{\pi}{L} x \\
& \sin \left(i \frac{\pi}{L} x+\frac{\pi}{2}\right)=\cos i \frac{\pi}{L} x
\end{aligned}
$$

If we replace $i(\pi / L) x$ with $i(\pi / L) x+\pi / 2$ in the unstarred expression (Equation 5.5), the unstarred and starred series become identical. Thus, the solutions obtained for the unstarred series can readily be applied to the solution of the starred series by the following simple substitution:

$$
\underbrace{i \frac{\pi}{L} x}_{\text {unstarred" }} \longrightarrow \underbrace{i \frac{\pi}{l} x+\frac{\pi}{2}}_{\text {"starred" }}
$$

Equations 5.1 and 5.2 together with Equations 5.3 and 5.7 provide the displacement field inside the cylinder. The unknown constants that appear in these equations are determined with the aid of the boundary conditions, as described in the next section. 


\section{Solutions for the Displacements, Stresses, and Strains}

To obtain solutions for the displacements, stresses, and strains that arise under general loading conditions, we first consider seven special cases. The solution for the general problem is then obtained by combining the solutions of these seven special cases.

\subsection{Case 0}

Consider a cylinder on which are applied known surface loads $p^{i}, p^{o}$ and a known temperature distribution $\Delta T$. The two edges of the cylinder are assumed to be hinged (see Table 6.1). Hinged support requires that the following conditions be satisfied:

$$
\begin{array}{ll}
q_{2}=\bar{w}_{0}=0 & q_{4}=\bar{w}_{L}=0 \\
\Omega_{1}=M_{0}=0 & \Omega_{3}=M_{L}=0 \\
\Omega_{5}=N_{0}=0 & \Omega_{6}=T_{0}=0 .
\end{array}
$$

The conditions given by Equation 6.1 are met if the displacements, temperature distribution, and surface loads specified in Table 6.1 are used. In the expressions for the displacements, there are two as-yet undetermined constants. These constants are determined from the relationships given in Table 6.1.

\subsection{Cases 1 and 2}

Consider a cylinder on which there is no temperature distribution $(\Delta T=0)$, and on which there are no surface loads $\left(p^{i}=p^{o}=0\right)$. In Case 1 , a unit axial load is applied $\left(N_{0}=1\right)$ and the applied torque is zero $\left(T_{0}=0\right)$. In Case 2 , a unit torque is applied $\left(T_{0}=1\right)$ and the axial load is zero $\left(N_{0}=0\right)$. The displacements that satisfy these conditions are given in Table 6.2. These displacements contain four unknown constants, which can be determined with the conditions given in Table 6.2.

\subsection{Cases 3 through 6}

Consider a cylinder on which the applied temperature distribution is zero $(\Delta T=0)$. We place different prescribed surface loads on the cylinder. In Case $3 \mathrm{a}$, a uniform radial tensile load is applied on the outer surface of the cylinder (Table 6.3). In Case 3b, a uniform radial compressive load is applied on the outer surface of the cylinder. In Cases $4 a, b$ through $6 a, b$, tensile and compressive radial forces are applied on the outer surface of the cylinder in the manner illustrated in Tables 6.4 through 6.6. In each case (Cases 3 through 6), the sum (Case "a" plus Case "b") of the surface forces is zero. The displacements corresponding to each of these cases are given in Tables 6.3 through 6.6. The conditions required to determine the unknowns in the displacements are also given in these tables.

For each case (Cases 3 through 6), $\Delta T=0, p^{i}=p^{o}=0$. However, the solutions obtained for each of these cases are different because the edge loads are different. 
Table 6.1. Loads and displacements in the $0^{\text {th }}$ Case.

\begin{tabular}{|c|c|c|}
\hline \multicolumn{3}{|l|}{ Case 0} \\
\hline \multicolumn{2}{|l|}{ temperature } & $\Delta T=\sum_{i=1} \Delta T_{i} \sin i \frac{\pi}{L} x$ \\
\hline \multicolumn{2}{|l|}{ surface load } & $p^{i}=\sum_{i=1} p_{i}^{i} \sin i \frac{\pi}{I} x$ \\
\hline \multicolumn{2}{|c|}{ prescribed edge loads } & $p^{0}=\sum_{i=1} p_{i}^{0} \sin i \frac{\pi}{L} x$ \\
\hline \multirow[t]{3}{*}{ displacements } & $u$ & $U_{0}^{l}=\sum_{i=1} u_{i}^{l}(r) \cos i \frac{\pi}{I} I$ \\
\hline & $v$ & $V_{0}^{l}=\sum_{i=1} v_{i}^{l}(r) \cos i \frac{\pi}{I} x$ \\
\hline & $w$ & $W_{0}^{l}=\sum_{i=1} w_{i}^{l}(r) \sin i \frac{\pi}{I} I$ \\
\hline \multicolumn{2}{|c|}{ number of unknowns } & 2 in each term \\
\hline \multicolumn{2}{|c|}{ conditions to determine } & $\sigma_{r i}^{l}\left(r^{i}\right)=p_{i}^{i}$ \\
\hline \multicolumn{2}{|l|}{ the unknowns } & $\sigma_{r i}^{n}\left(r^{0}\right)=p_{i}^{0}$ \\
\hline
\end{tabular}


Table 6.2. Loads and displacements in the First and Second Cases.

\begin{tabular}{|c|c|}
\hline Case 1 and 2 & \\
\hline temperature & $\Delta T=0$ \\
\hline $\begin{array}{l}\text { surface load } \\
\text { prescribed edge loads }\end{array}$ & $\begin{array}{l}\begin{array}{l}p^{\prime}=0 \\
p^{\circ}=0\end{array} \\
\left.\left.\begin{array}{l}N_{0}=1 \\
T_{0}=0\end{array}\right\} \text { case } 1 \quad \begin{array}{l}N_{0}=0 \\
T_{0}=1\end{array}\right\} \text { case } 2\end{array}$ \\
\hline $\begin{array}{c}u \\
v \\
\\
w\end{array}$ & $\begin{array}{l}k=1\} \text { case } 1 \\
k=2\} \text { case } 2\end{array}$ \\
\hline number of unknowns in the displacement & 4 \\
\hline $\begin{array}{l}\text { conditions to } \\
\text { determine the constants }\end{array}$ & $\begin{array}{l}\sigma_{r_{0}}^{1 *}\left(r^{i}\right)=0 \\
\begin{array}{l}\sigma_{r o}^{n *}\left(r^{0}\right)=0 \\
\left.\left.\begin{array}{l}N_{0}=1 \\
T_{0}=0\end{array}\right\} \text { case } 1 \quad \begin{array}{l}N_{0}=0 \\
T_{0}=1\end{array}\right\} \text { case } 2\end{array}\end{array}$ \\
\hline
\end{tabular}


Table 6.3. Loads and displacements in the Third Case.

\begin{tabular}{|c|c|c|c|}
\hline Case 3 & & a & \\
\hline & Case 3 & Case $3 a$ & Case $3 b$ \\
\hline temperature & $\Delta T=0$ & $\Delta T=0$ & $\Delta T=0$ \\
\hline $\begin{array}{l}\text { surface load } \\
\text { prescribed edge loads }\end{array}$ & $\begin{array}{l}p^{i}=0 \\
p^{0}=0\end{array}$ & $\begin{array}{l}p^{\prime}=0 \\
p^{0}=1=\sum_{i=1} p_{j}^{0} \sin i \frac{\pi}{L} x\end{array}$ & $\begin{array}{l}p^{1}=0 \\
p^{0}=-1 \\
N_{0}=0 \\
T_{0}=0\end{array}$ \\
\hline $\begin{array}{r}\text { displacements } \\
\\
v \\
w\end{array}$ & $\begin{array}{l}U_{3}^{l} \\
V_{3}^{l} \\
W_{3}^{l}\end{array}$ & $\begin{array}{l}\sum_{i=1} u_{i}^{l}(r) \cos i \frac{\pi}{2} x \\
\sum_{i=1} v_{i}^{l}(r) \cos i \frac{\pi}{2} x \\
\sum_{i=1} w_{i}^{l}(r) \sin i \frac{\pi}{2} x\end{array}$ & $\begin{array}{l}u_{0}^{l *} \\
v_{0}^{l *} \\
w_{0}^{l *}\end{array}$ \\
\hline number of unknowns & & 2 for each Fourier term & 4 \\
\hline $\begin{array}{l}\text { conditions to } \\
\text { determine the } \\
\text { unknowns }\end{array}$ & & $\begin{array}{l}\sigma_{r i}^{1}\left(r^{i}\right)=0 \\
\sigma_{r i}^{n}\left(r^{0}\right)=p_{i}^{0}\end{array}$ & $\begin{array}{l}\sigma_{r 0}^{1 *}\left(r^{i}\right)=0 \\
\sigma_{r 0}^{n *}\left(r^{0}\right)=-1 \\
N_{0}=0 \\
T_{0}=0\end{array}$ \\
\hline
\end{tabular}

Remark

$p_{i}^{0}= \begin{cases}\frac{4}{i \pi} & \text { if } i=1,3,5 \\ 0 & \text { if } i=2,4,6\end{cases}$ 
Table 6.4. Loads and displacements in the Fourth Case.

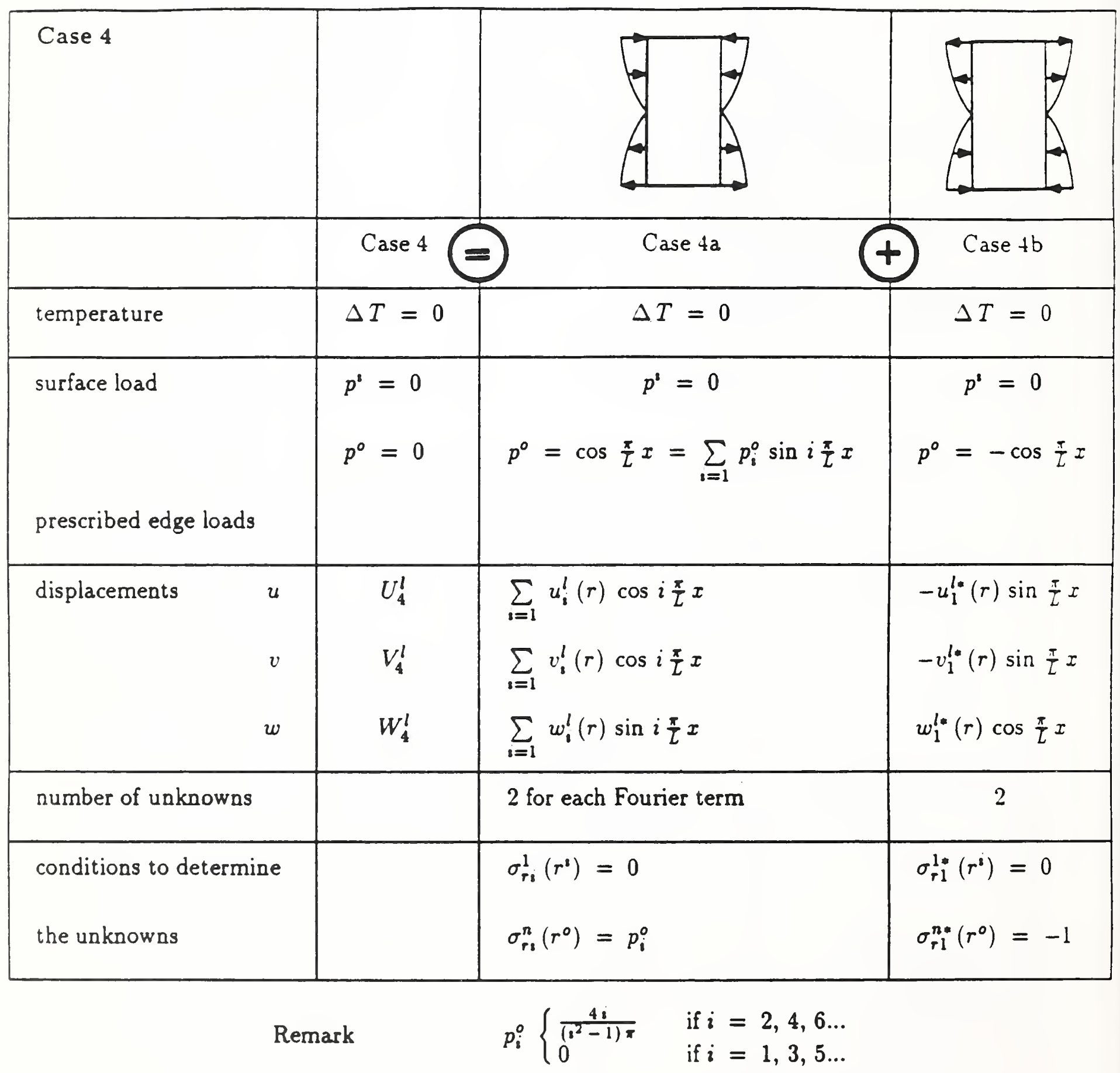


Table 6.5. Loads and displacements in the Fifth Case.

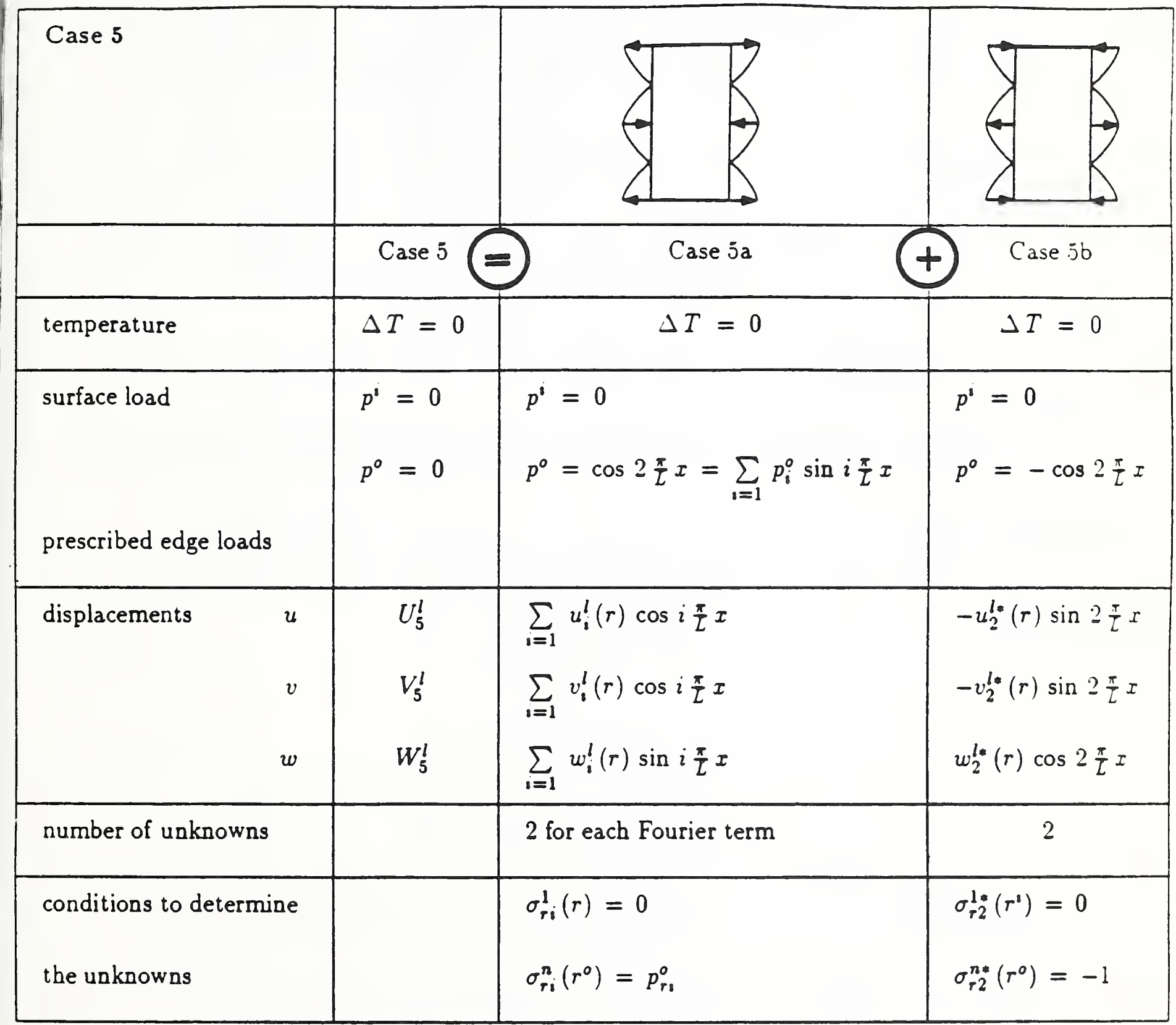

Remark

$p_{i}^{o}= \begin{cases}\frac{4 i}{\left(3^{2}-4\right) \pi} & \text { if } 1,3,5, \ldots \\ 0 & \text { if } 2,4,6, \ldots\end{cases}$ 
Table 6.6. Loads and displacements in the Sixth Case.

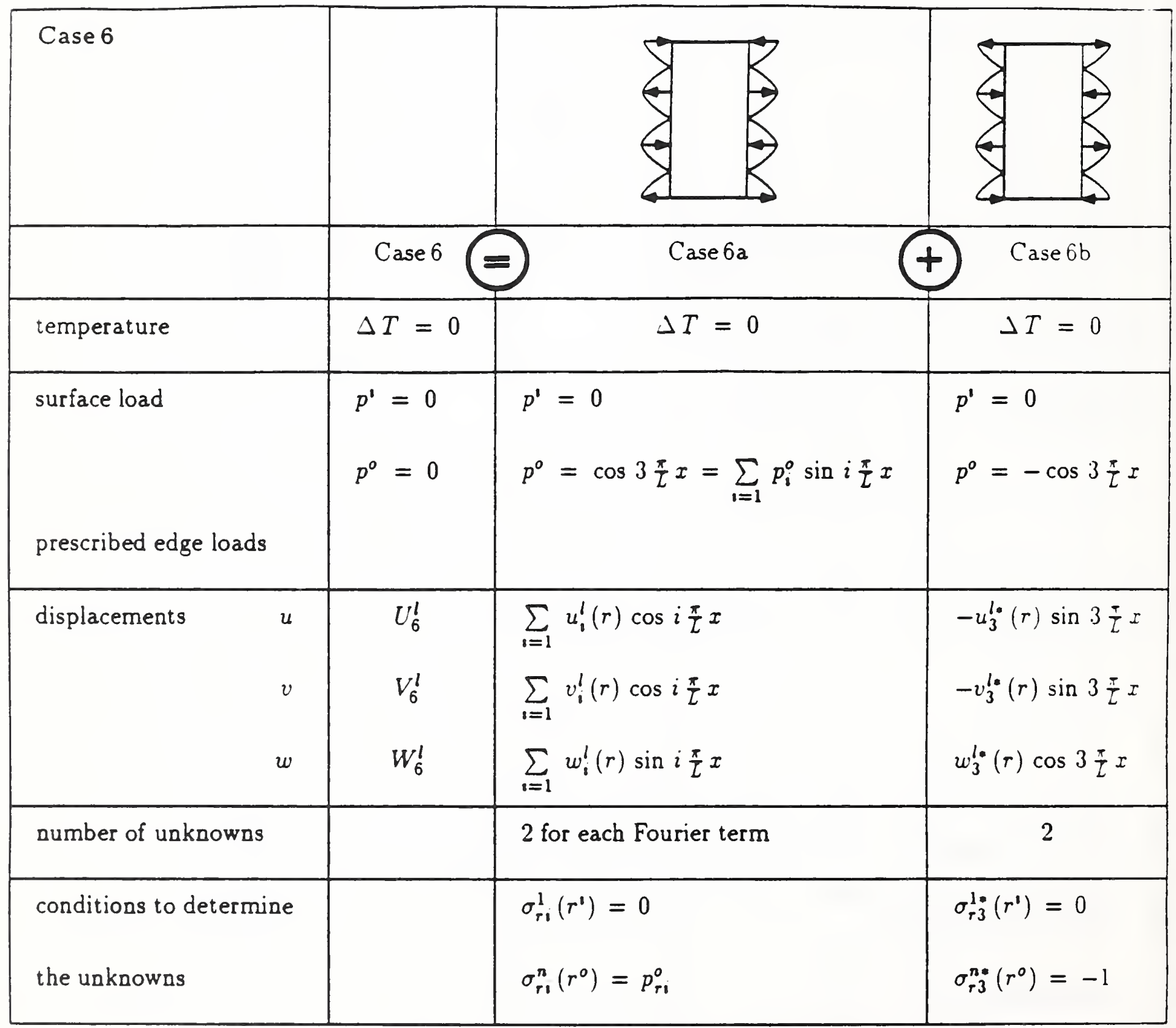

Remark

$$
p_{i}^{o}=\left\{\begin{array}{cl}
\frac{4 i}{\left(r^{2}-9\right) \pi} & \text { if } i=2,4,6 \ldots \\
0 & \text { if } i=1,3,5 \ldots
\end{array}\right.
$$




\subsection{General Solutions}

By using the seven special cases (Cases 0 through 6) above, the solution for the general loading condition is established as follows: The six edge displacements and the six edge forces have been denoted previously by $q_{1}$ through $q_{6}$ and by $\Omega_{1}$ through $\Omega_{6}$ (see Equation 4.10 ). The edge displacements resulting from Cases 0 through 6 are denoted by $q_{1 j}, q_{2 j}, \ldots q_{6 j}$, and the edge forces by $\Omega_{1 j}, \Omega_{2 j}, \ldots \Omega_{6 j}$, where $j$ takes on the values from 0 through 6 . By using the displacements summarized in Tables 6.1 through $6.6, q_{1 j}-q_{6 j}$ and $\Omega_{1 j}-\Omega_{6 j}$ can be determined by using Equations 4.3 through 4.10.

Expressions for the "general" edge displacements and "general" edge forces are formed in the following manner:

$$
\begin{aligned}
q_{i} & =q_{i o}+\sum_{j=1}^{6} q_{i j} X_{j} \\
\Omega_{i} & =\Omega_{i o}+\sum_{j=1}^{6} \Omega_{i j} X_{j}
\end{aligned}
$$

The terms $X_{j}(j=1,2, \ldots 6)$ are six unknown constant multipliers that are determined by substituting the above expressions for $q_{i}$ and $\Omega_{i}$ into the appropriate boundary conditions given by Equations 4.11, 4.12, or 4.13.

The "general" displacements are then obtained by summing the displacements for Case 0 through Case 6, with each term being multiplied by the appropriate multiplier:

$$
\begin{aligned}
& u^{l}=U_{o}^{l}+\sum_{j=1}^{6} U_{j}^{l} X_{j} \\
& v^{l}=V_{o}^{l}+\sum_{j=1}^{6} V_{j}^{l} X_{j} \\
& w^{l}=W_{o}^{l}+\sum_{j=1}^{6} W_{j}^{l} X_{j}
\end{aligned}
$$

where $U_{0}^{l}, V_{0}^{l}, W_{0}^{l}$ through $U_{6}^{l}, V_{6}^{l}, W_{6}^{l}$ are the displacements corresponding to the seven cases (Cases 0 through 6) given in Tables 6.1 through 6.6.

From the known values of the displacements, the stresses and strains can be calculated from Equations 3.2 and 3.3.

\subsection{Method of Solution}

The equations presented in this paper form a complete set for calculating the displacements, stresses, and strains under specified hygrothermal and mechanical loading conditions. Solutions of these equations require extensive analytical and numerical calculations. 
The analytical calculations were performed with a symbolic manipulator "Mathematica" [6.1]. These calculations resulted in a set of algebraic equations that are used to determine the unknown constants in the displacements. A computer code, using MATLAB [6.2], was then written for obtaining solutions of these equations and for determining the constants. The code is designated CYLINDER 2. The input parameters required and the output parameters provided by the code are given in Tables 6.7 and 6.8 .

Table 6.7. Input parameters required for the CYLINDER 2 computer code.

Geometry

- inner radius, $r^{i}$

- thickness of one ply, $h_{0}$

- number of ply groups, $n$

-number of plies in each ply group, $m_{i}$

-direction (angle) of fibers in each ply group, $\phi_{i}$

Material (On-Axis) Properties

Stiffness parameters

- longitudinal Ycung's modulus, $E_{x}$

- transverse in-plane Young's modulus, $E_{y}$

- transverse out-of-plane Young's modulus, $E_{z}$

- shear moduli, $G_{y z}, G_{x z}, G_{x y}$

- Poisson's ratios, $\nu_{y z}, \nu_{x z}, \nu_{x y}$

Thermal and moisture expansion coefficients

- longitudinal expansion coefficients, $\alpha_{x}, \beta_{x}$

- transverse in-plane expansion coefficients, $\alpha_{y}, \beta_{y}$

- transverse out-of-plane expansion coeffcients, $\alpha_{z}, \beta_{z}$

Strength parameters

- longitudinal tensile strength, $X$

- longitudinal compressive strength, $X^{\prime}$

- transverse tensile strength, $Y$

- transverse compressive strength, $Y^{\prime}$

- longitudinal shear strength, $S$

Surface Load

- radial load on the inner surface, $p^{i}$

- radial load on the outer surface, $p^{\rho}$

Temperature and Moisture Load

$\Delta T, \Delta c$

Prescribed Edge Loads or Edge Displacements

- moment or rotation at the lower edge, $M_{0}$ or $\bar{\eta}_{0}$

- shear force or radial displacement at the lower edge, $Q_{0}$ or $\bar{w}_{0}$

- moment or rotation at upper edge, $M_{L}$, or $\bar{\eta}_{L}$

- shear force or radial displacement at the upper edge, $Q_{L}$ or $\bar{w}_{L}$

- normal force or relative axial displacement of the edges, $N_{0}$ or $\bar{u}$

- torque or relative circumferential displacements of the edges, $T_{0}$ or $\bar{v}$ 
Table 6.8. Output parameters required for the CYLINDER 2 computer code.

- displacements of the midsurface

- stresses in each ply, $\sigma_{x}, \sigma_{\theta}, \sigma_{r}, \tau_{\theta r}, \tau_{x r}, \tau_{x \theta}$

- strains in each ply, $\epsilon_{x}, \epsilon_{\theta}, \epsilon_{r}, \gamma_{\theta r}, \gamma_{x r}, \gamma_{x \theta}$

- strengths ratio in each ply, $R$

- edge displacements and forces

- temperature and moisture distribution

As was noted previously, the equations were derived for constant material properties. Solutions for problems in which the material properties vary in the axial direction were incorporated into the code by dividing the cylinder into sections along its length. In each section, the displacements and the forces were "matched" at the interfaces of the adjacent sections.

In principle, the calculation procedure outlined in this paper is simple. In practice, there are two problems:

1. For long cylinders, an excessive number of Fourier terms may be required to achieve the required accuracy.

2. In some loading cases, there is an "overshoot" in the Fourier series near the edges of the cylinders. (This is the so-called Gibbs effect.)

To reduce the need for a large number of Fourier terms, the following computational procedure was built into the algorithm: If the cylinder is long, it is analyzed in three separate parts (Figure 6.1). First, the displacements in the entire cylinder are calculated, those resulting from the surface loads, the temperature load, and the edge loads $N_{0}$ and $T_{0}$. Then the displacements near the edges are calculated, those due to imposed loads $\Omega_{3}$ and $\Omega_{4}$ on the upper edge and $\Omega_{1}$ and $\Omega_{2}$ on the lower edge. The displacements obtained for these three parts are then added together to give the final displacement of the cylinder.

The Gibbs effect was minimized by extending the Fourier series over a length $L^{\prime}$, which is longer than the length $L$ of the actual cylinder (Figure 6.2). The actual edge loads are applied at $x=0$ and $x=L$, and the solutions for $\mathrm{x}<0$ and $x>L$ are ignored.

Note that both of the above numerical steps are built into the code and are "opaque" to the user. 


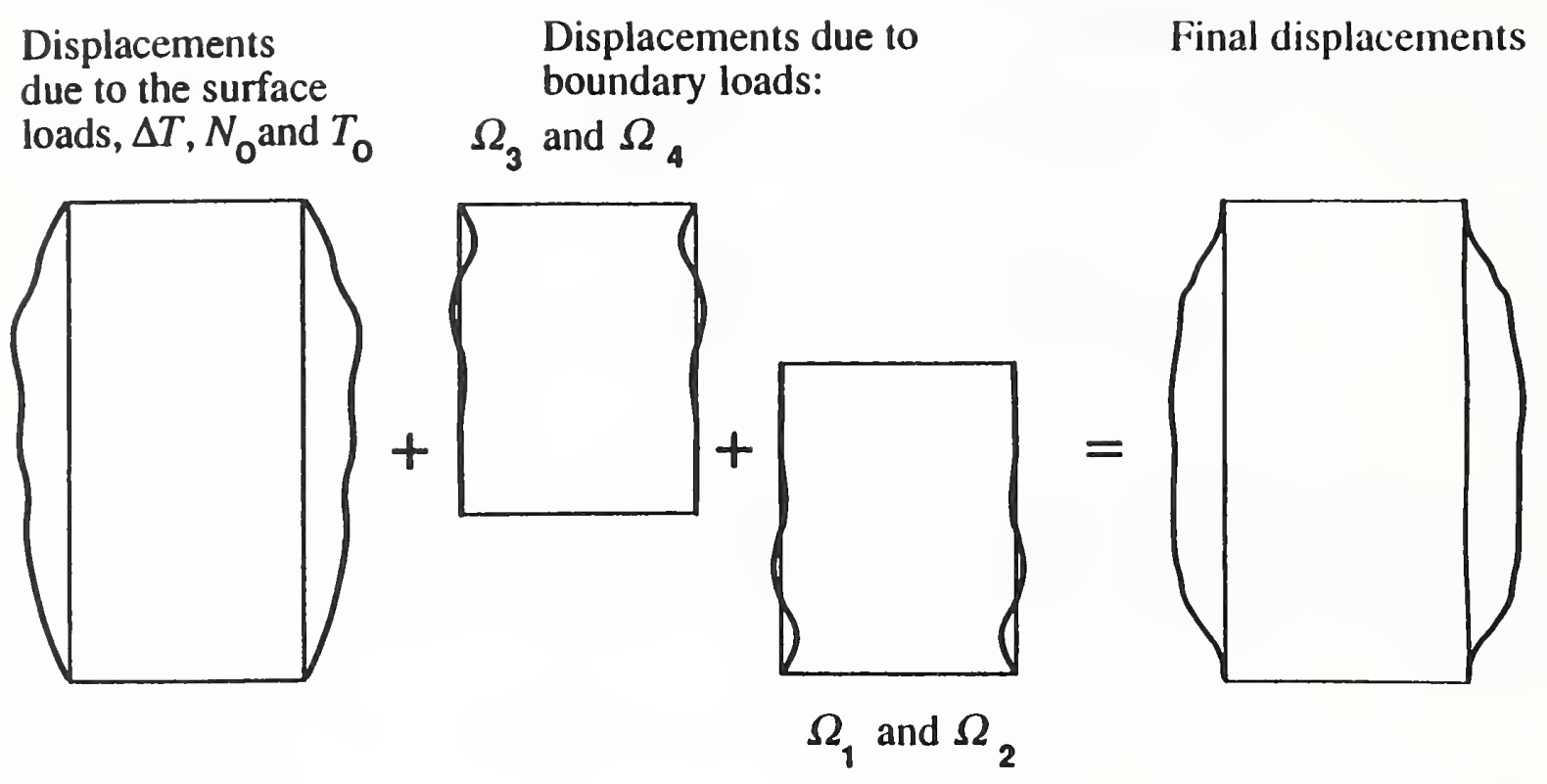

Figure 6.1. Analysis of long cylinders.

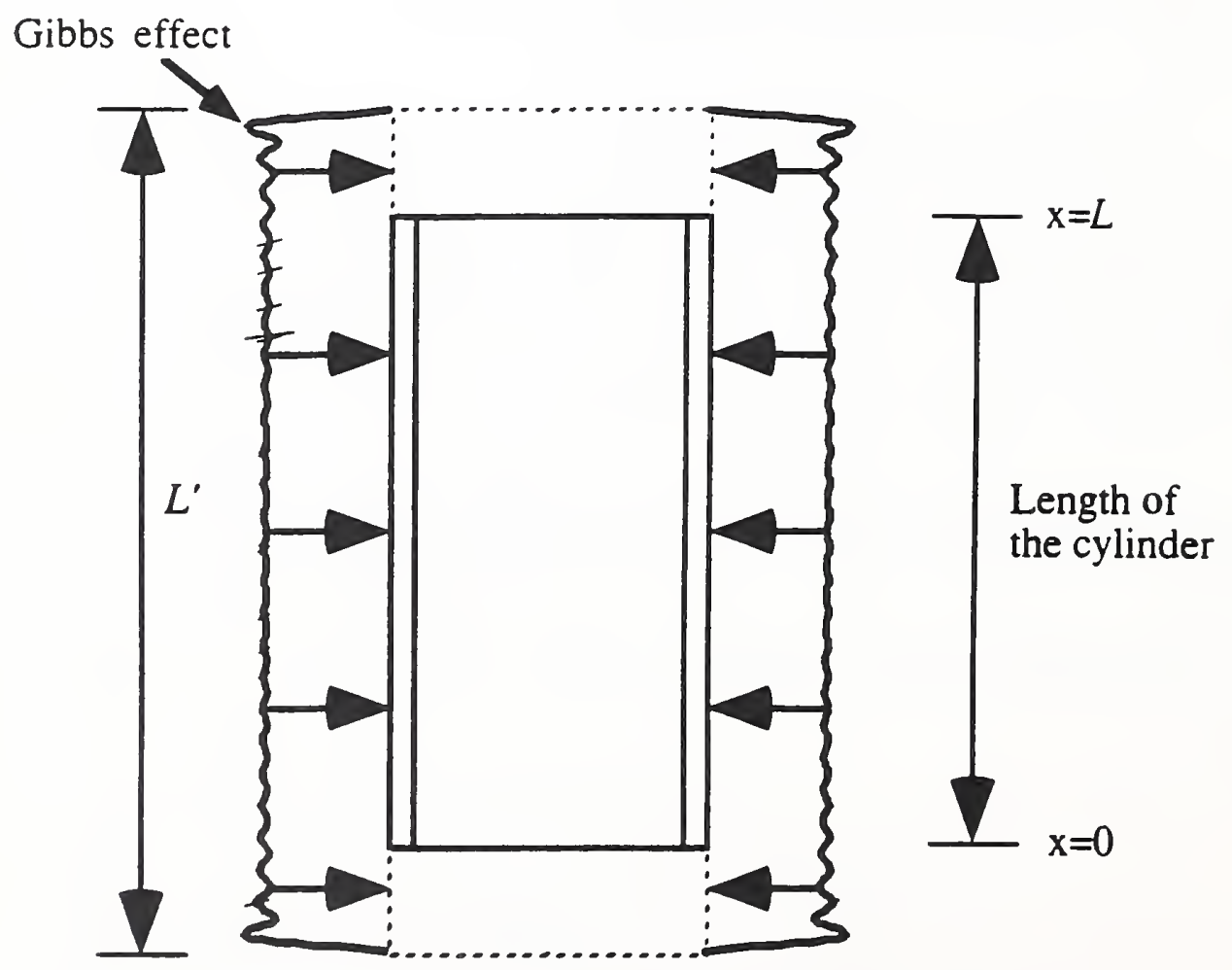

Figure 6.2. Illustration of the minimization of the Gibbs effect. 


\section{Sample Problems}

Solutions to sample problems were obtained, first to assess the accuracy of the CYLINDER 2 code and second to illustrate the type of information that can be generated by this code. Two types of problems were investigated: (1) a composite cylinder subjected to displacement and (2) a composite cylinder subjected to temperature load.

A composite cylinder (inner radius $r^{i}=5.1 \mathrm{~mm}$, length $L=230 \mathrm{~mm}$, and wall thickness $h=5.1 \mathrm{~mm}$ ) is fixed between two rigid surfaces (Figure 7.1). The laminate lay-up is $\left[80_{20} / 45_{20}\right]$. The cylinder is made of an E-glass-epoxy composite. The material properties utilized in this study, from Tsai [7.1], are summarized in Table 7.1.

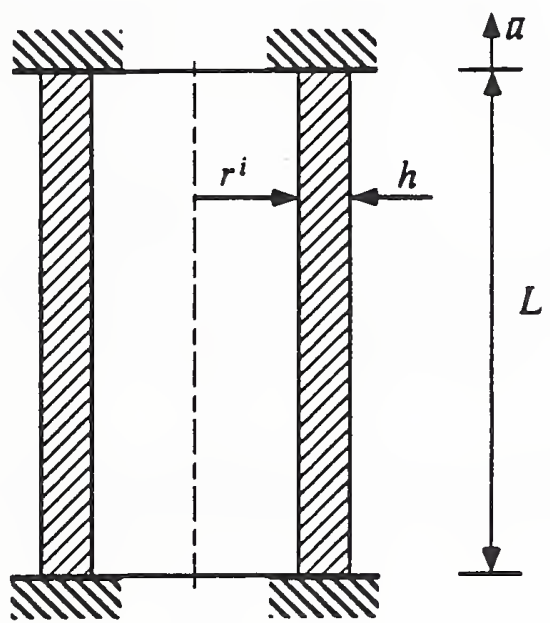

Figure 7.1. Description of the composite cylinder used in the sample problem.

Table 7.1. Material properties of E-glass-epoxy composite.

Stiffness Parameters

- longitudinal Young's modulus

$E_{x}=39 \mathrm{GPa}$

- transverse in-plane Young's modulus

$E_{y}=8.3 \mathrm{GPa}$

- transverse out-of-plane Young's modulus

$E_{z}=8.3 \mathrm{GPa}$

- shear moduli

- Poisson's ratios

$G_{y z}=G_{x z}=G_{z y}=4 \mathrm{GPa}$

$\nu_{y z}=0.4$

$\nu_{x z}=\nu_{x y}=0.26$

Thermal Expansion Coefficients

- longitudinal

$\alpha_{x}=8.6 \times 10^{-6} /{ }^{\circ} \mathrm{C}$

- transverse

$\alpha_{y}=\alpha_{z}=22.1 \times 10^{-6} /{ }^{\circ} \mathrm{C}$ 


\subsection{Composite Cylinder Subjected to Axial Displacement}

A compressive displacement, $\bar{u}=-0.64 \mu \mathrm{m}$ was applied on the top surface. The stresses and displacements were calculated by the CYLINDER 2 code. The calculated stress components through the thickness at $x=25 \mathrm{~mm}$ are plotted in Figure 7.2. The stresses were also calculated by the finiteelement method (and the corresponding AXIMPACT code) of Karaoglan and Springer [7.1]. These stresses are also included in Figure 7.2. Two points are noted: First, there is good agreement between the results of the AXIMPACT and the CYLINDER 2 codes. Second, there are $\sigma_{r}, \tau_{0 r}$, and $\tau_{x r}$ stresses present in the cylinder. To calculate these stresses, a three-dimensional analysis is needed. Two-dimensional analyses would not provide these stresses.

The displacements in the midsurface were also calculated by the AXIMPACT and by the CYLINDER 2 codes. Again, the results are in good agreement, as illustrated in Figure 7.3.

\subsection{Composite Cylinder Subjected to Temperature Load}

We consider the cylinder prescribed in the previous section. The two edges are fixed, and the displacements at the edges are zero. The cylinder is subjected to a temperature load that varies linearly along the axis from 310 to $366 \mathrm{~K}$ (Figure 7.4).

The stresses and displacements were calculated by the CYLINDER 2 CODE. The calculated stresses through the thickness at $x=25 \mathrm{~mm}$ are plotted in Figure 7.5; the displacements at the midsurface are given in Figure 7.6. 


$$
\begin{aligned}
& \text { E-glass-Epoxy } \\
& {\left[80_{20} / 45_{20}\right]} \\
& r^{i}=50 \mathrm{~mm} \\
& L=230 \mathrm{~mm} \\
& h=5.1 \mathrm{~mm}
\end{aligned}
$$
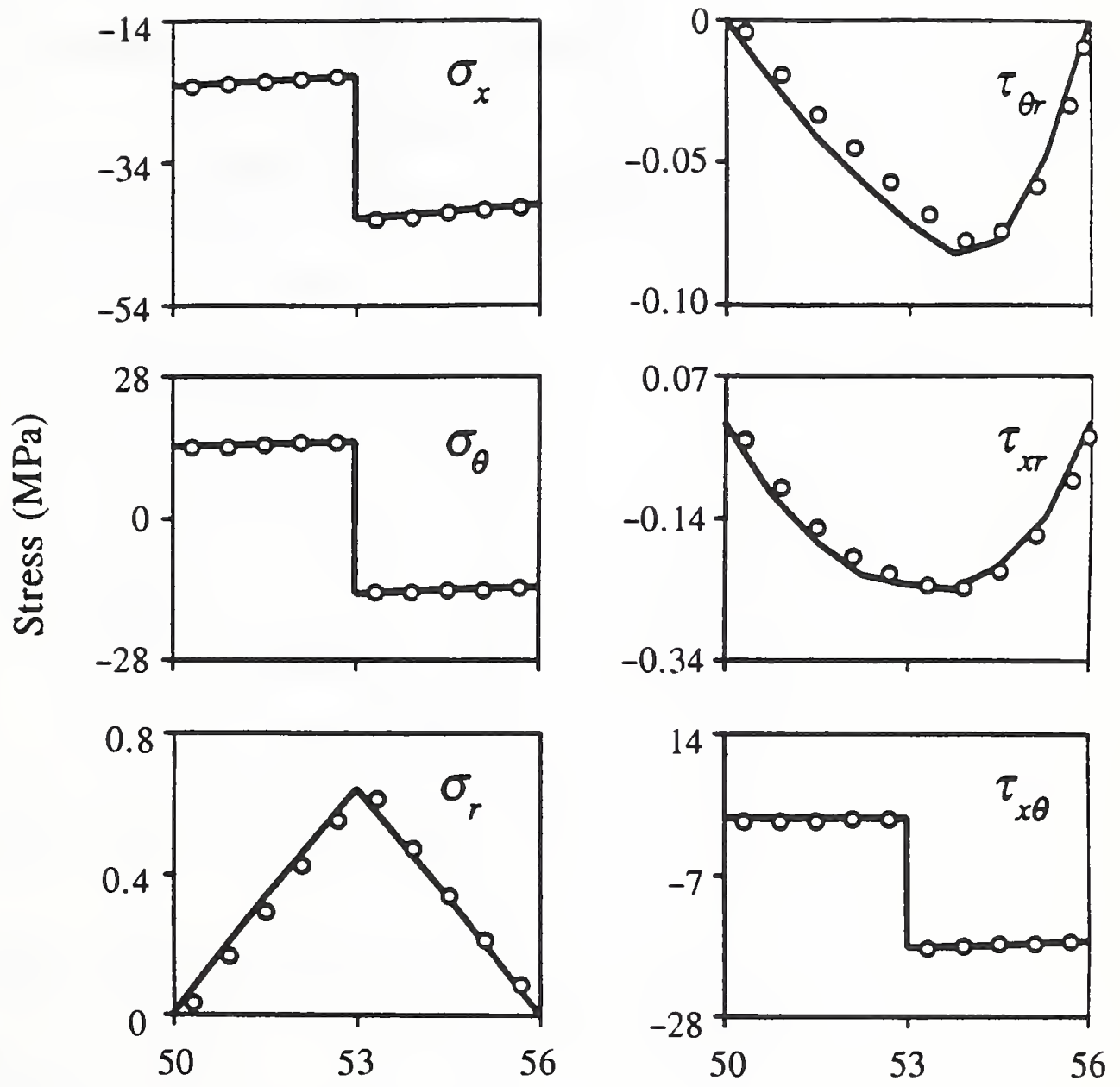

Radial Position, $r(\mathrm{~mm})$

Figure 7.2. Stress distributions through the thickness at $x=25 \mathrm{~mm}$ for the cylinder shown in Figure 7.1. Edge displacement $\bar{u}=-0.64 \mu \mathrm{m}$. Results computed by the CYLINDER 2 (solid lines) and AXIMPACT (circles) codes. 
E-glass-Epoxy

$\left[80_{20} / 45_{20}\right]$

$r^{i}=50 \mathrm{~mm}$

$L=230 \mathrm{~mm}$

$h=5.1 \mathrm{~mm}$
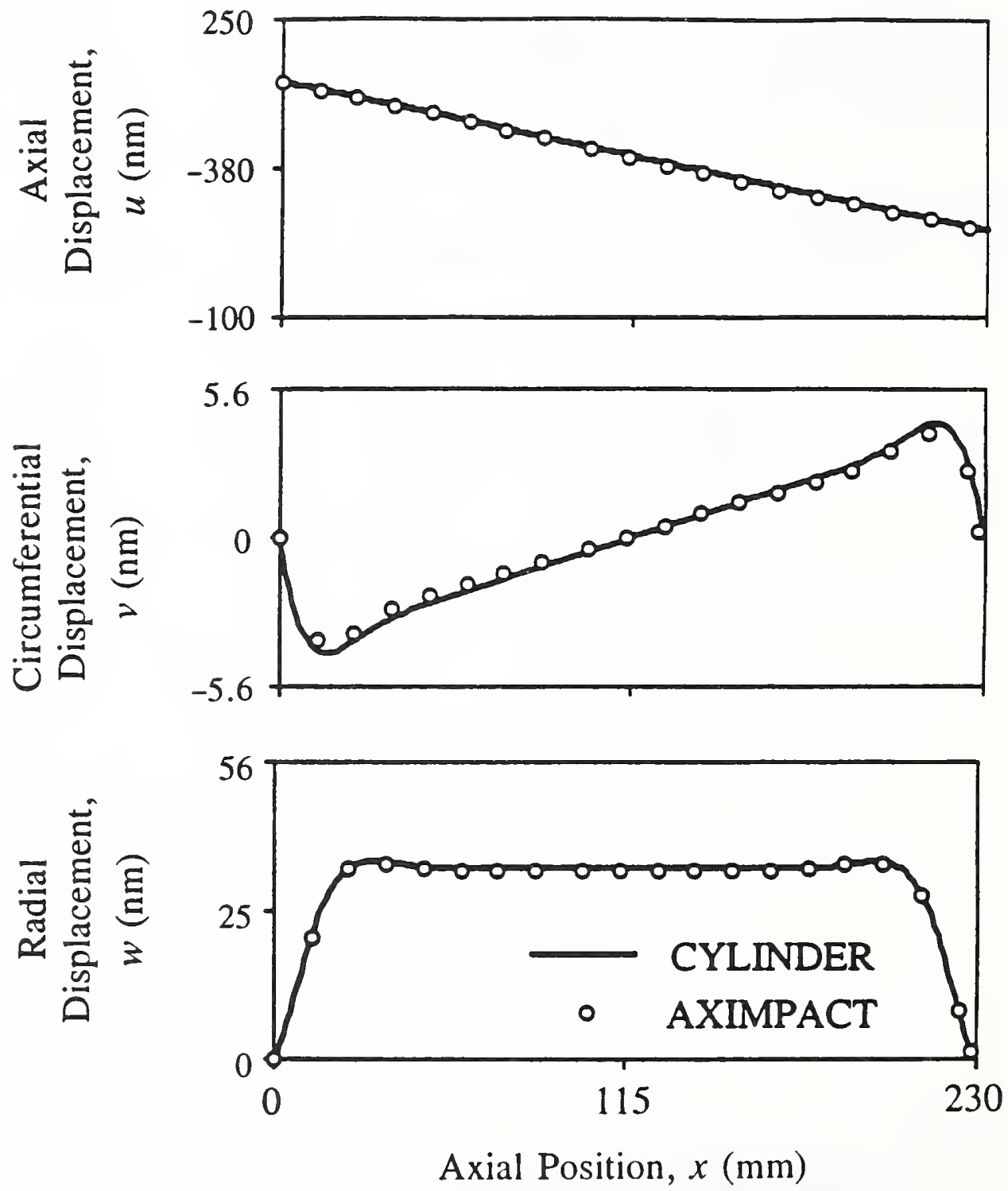

Figure 7.3. Axial, radial, and circumferencial displacements at the midsurface for the cylinder shown in Figure 7.1. Edge displacement $\bar{u}=-0.64 \mu \mathrm{m}$. Results computed by the CYLINDER 2 (solid lines) and AXIMPACT (circles) codes. 

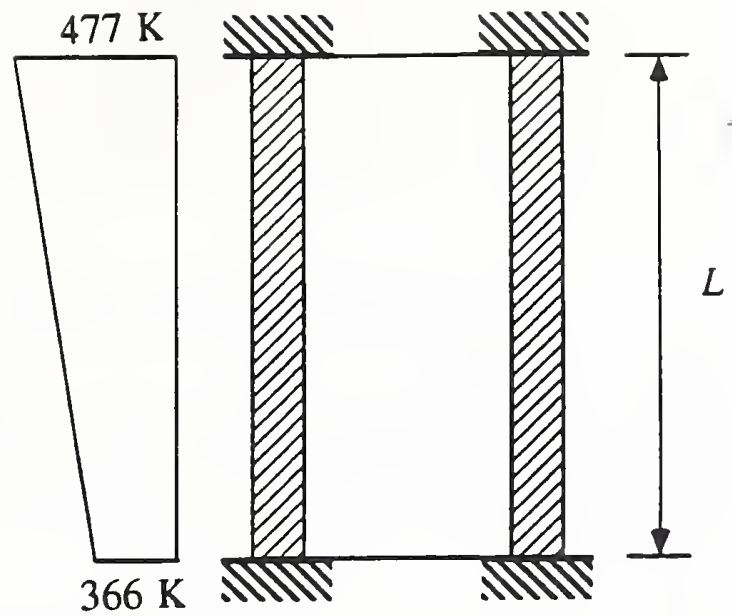

Figure 7.4. Temperature distribution along the cylinder in the sample problem.

E-glass-Epoxy
$\left[80_{20} / 45_{20}\right]$
$r^{i}=50 \mathrm{~mm}$
$L=230 \mathrm{~mm}$
$h=5.1 \mathrm{~mm}$
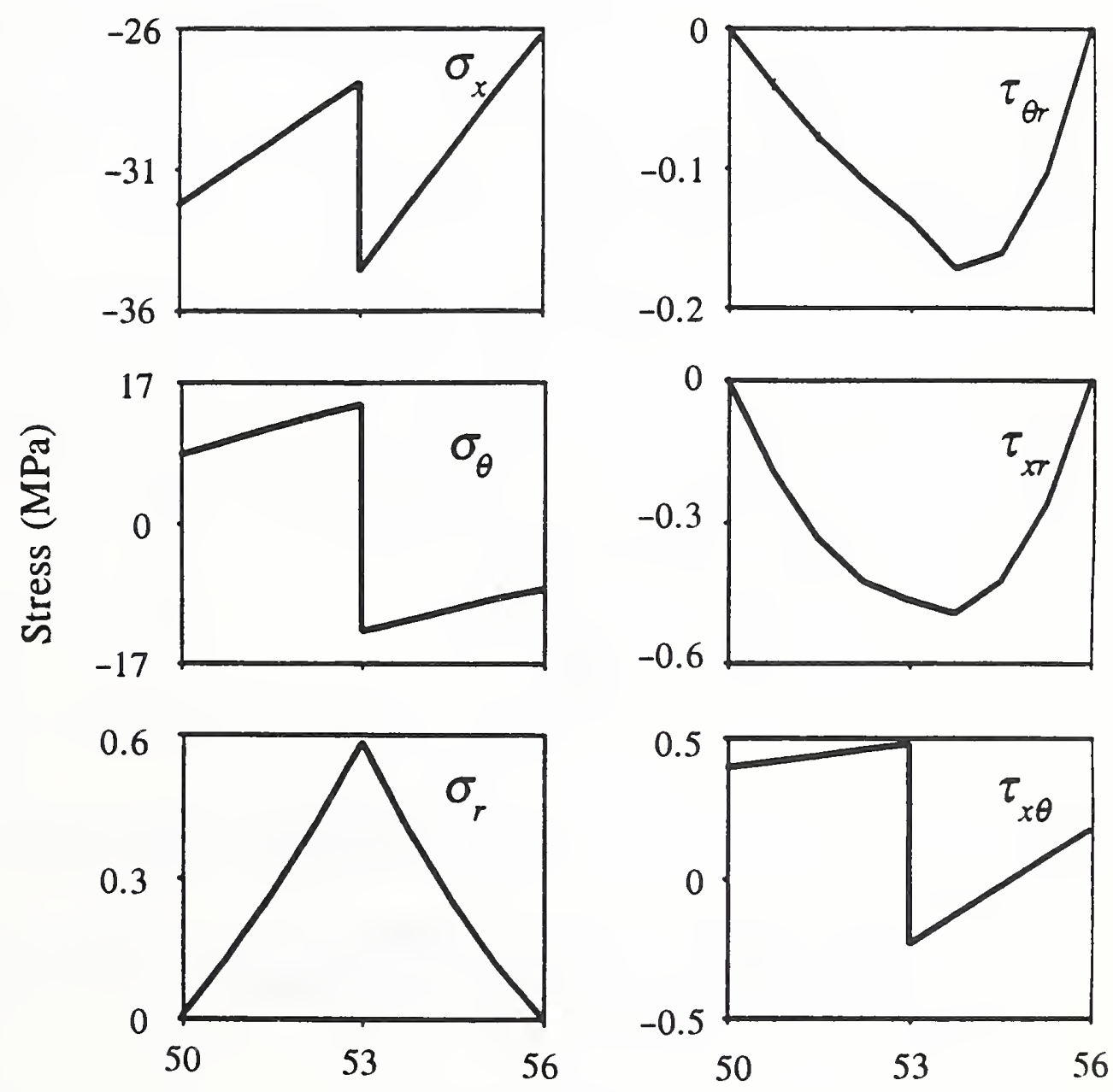

Radial Position, $r(\mathrm{~mm})$

Figure 7.5. Stress distributions through the thickness at $x=25 \mathrm{~mm}$ for the cylinder subjected to the temperature load shown in Figure 7.4. 
E-glass-Epoxy $\left[80_{20} / 45_{20}\right]$

$r^{i}=50 \mathrm{~mm}$

$L=230 \mathrm{~mm}$

$h=5.1 \mathrm{~mm}$
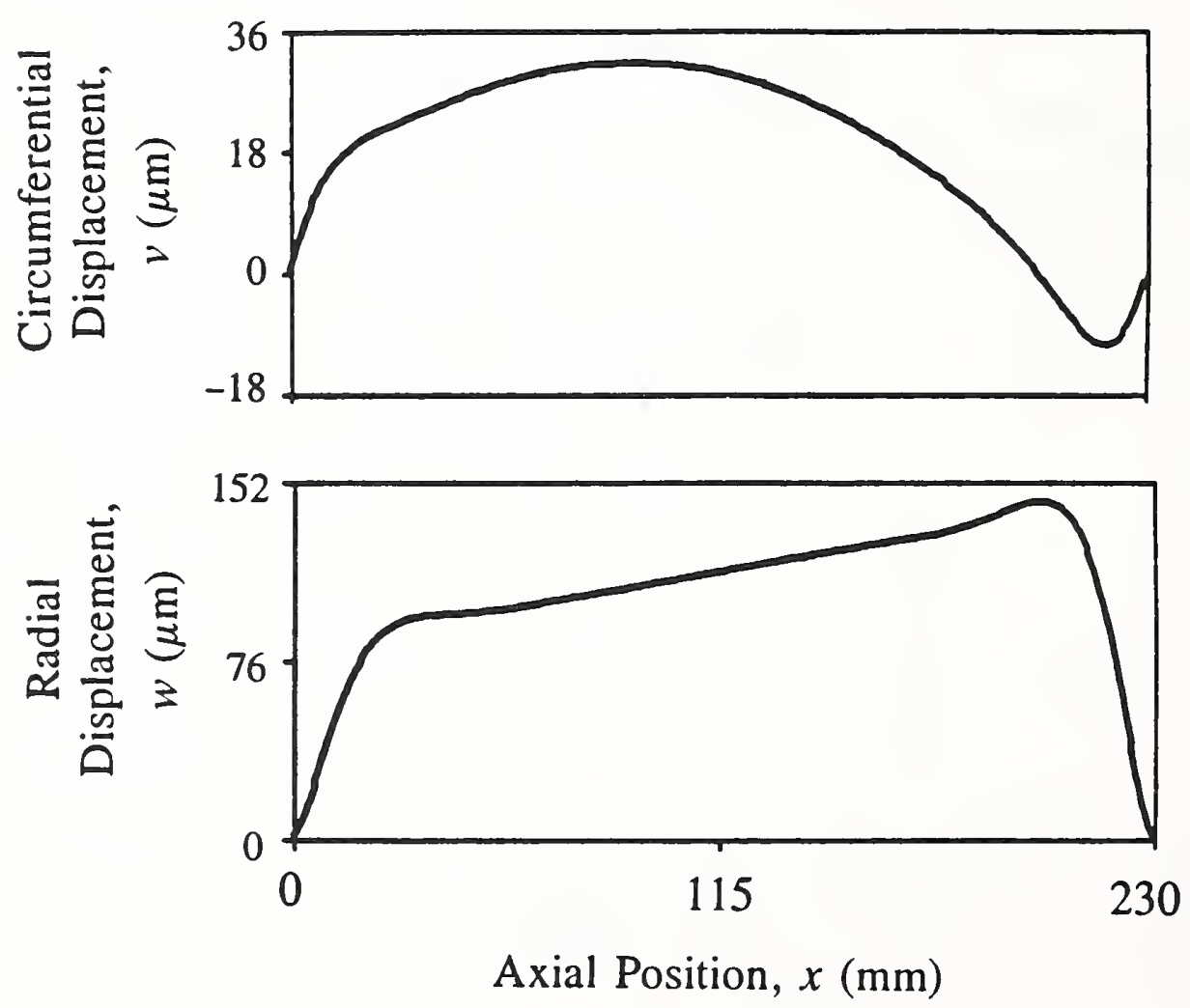

Figure 7.6. Axial, radial, and circumferential displacements at the midsurface for the cylinder subjected to the temperature load shown in Figure 7.4. 


\section{Failure of Axially Loaded Composite Cylinders}

The model described previously was evaluated by generating results and comparing these with data obtained at NIST.

The cylinder specifications are given in Table 8.1 .

The stresses and strains in each cylinder were calculated by the model and the failure (first ply failure) by the three-dimensional quadratic Tsai-Wu failure criterion (Table 8.2). Both the stress analysis and the failure criterion are incorporated in the CYLINDER 2 code, and this code was used to generate numerical results. The material properties used in the calculations are listed in Table 8.3.

Table 8.1. Test cylinders.

\begin{tabular}{|c|c|c|c|c|c|}
\hline $\begin{array}{l}\text { Cyl. } \\
\text { No. }\end{array}$ & $\begin{array}{c}\text { Inner } \\
\text { Diameter } \\
(\mathbf{m m})\end{array}$ & $\begin{array}{c}\text { Thickness, } \\
h \\
(\mathrm{~mm})\end{array}$ & $\begin{array}{c}\text { Length, } \\
L \\
(\mathrm{~mm})\end{array}$ & Lay-up & $\begin{array}{l}\text { E-Glass-Reinforced } \\
\text { Resin }\end{array}$ \\
\hline 1 & 100 & 10.2 & 230 & {$\left[90 / \pm 10_{6} / 90 / \pm 10_{6} / 90 / \pm 10_{6} / 90\right]$} & DGEBA/DCH/ARA/ACL \\
\hline 2 & 100 & 10.2 & 480 & {$\left[90 / \pm 10_{6} / 90 / \pm 10_{6} / 90 / \pm 10_{6} / 90\right]$} & DGEBA/DCH/ARA/ACL \\
\hline 3 & 100 & 10.2 & 710 & {$\left[90 / \pm 10_{6} / 90 / \pm 10_{6} / 90 / \pm 10_{6} / 90\right]$} & DGEBA/DCH/ARA/ACL \\
\hline 4 & 100 & 5.1 & 710 & {$\left[90_{2} / \pm 10_{9} / 90_{2} / \pm 10_{9} / 90_{2}\right]$} & DGEBA/DCH/ARA/ACL \\
\hline 5 & 100 & 5.1 & 710 & {$\left[90_{2} / \pm 10_{18} / 90_{2}\right]$} & DGEBA/DCH/ARA/ACL \\
\hline 6 & 180 & 8.9 & 610 & {$\left[90_{2} / \pm 10_{17} / 90_{2} / \pm 10_{17} / 90_{2}\right]$} & DGEBA/DCH/ARA/ACL \\
\hline 7 & 180 & 8.9 & 1200 & {$\left[90_{2} / \pm 10_{17} / 90_{2} / \pm 10_{17} / 90_{2}\right]$} & DGEBA/DCH/ARA/ACL \\
\hline 8 & 250 & 13 & 910 & {$\left[90_{2} / \pm 10_{24} / 90_{2} / \pm 10_{24} / 90_{2}\right]$} & DGEBA/DCH/ARA/ACL \\
\hline 9 & 100 & 10.2 & 230 & {$[ \pm 10]_{12}$} & DGEBA/DCH/ARA/ACL \\
\hline 10 & 100 & 10.2 & 480 & {$[ \pm 10]_{12}$} & DGEBA/DCH/ARA/ACL \\
\hline 11 & 100 & 10.2 & 710 & {$[ \pm 10]_{12}$} & DGEBA/DCH/ARA/ACL \\
\hline 12 & 100 & 5.1 & 710 & {$[ \pm 10]_{6}$} & DGEBA/DCH/ARA/ACL \\
\hline 13 & 100 & 10.2 & 230 & {$\left[90 / \pm 45 / \pm 10_{3} / \pm 45 / 90_{2} / \pm 45 / 10_{3} / \pm 45 / 90\right]$} & DGEBA/DCH/ARA/ACL \\
\hline 14 & 100 & 10.2 & 480 & {$\left[90 / \pm 45 / \pm 10_{3} / \pm 45 / 90_{2} / \pm 45 / 10_{3} / \pm 45 / 90\right]$} & DGEBA/DCH/ARA/ACL \\
\hline 15 & 100 & 10.2 & 710 & {$\left[90 / \pm 45 / \pm 10_{3} / \pm 45 / 90_{2} / \pm 45 / 10_{3} / \pm 45 / 90\right]$} & DGEBA/DCH/ARA/ACL \\
\hline 16 & 100 & 5.1 & 710 & {$\left[90 / \pm 45 / \pm 10_{3} / \pm 45 / 90\right]$} & DGEBA/DCH/ARA/ACL \\
\hline 17 & 100 & 10.2 & 710 & {$\left[90_{12} / \pm 9_{46} / 90_{12} / \pm 9_{45} / 90_{11} / \pm 9_{42} 90_{11} / \pm 9_{41} / 90_{11}\right]$} & VE-EL/MEKP \\
\hline 18 & 100 & 5.1 & 710 & {$\left[90_{11} / \pm 9_{42} / 90_{11} / \pm 9_{42} / 90_{11}\right]$} & VE-EL/MEKP \\
\hline 19 & 100 & 10.2 & 710 & {$\left[ \pm 65_{13} / 0_{75} / \pm 23_{13} / 0_{100} / \pm 23_{13} / 0_{50} / \pm 65_{13}\right]$} & VE-EL/MEKP \\
\hline 20 & 100 & 10.2 & 710 & {$\left[90_{12} / \pm 9_{46} / 90_{12} / \pm 9_{45} / 99_{11} / \pm 9_{42} / 90_{11} / \pm 9_{41} / 90_{11}\right]$} & DGEBF/CTBN/AN \\
\hline 21 & 100 & 5.1 & 710 & {$\left[90_{11} / \pm 9_{42} / 90_{11} / \pm 9_{42} / 90_{11}\right]$} & DGEBF/CTBN/AN \\
\hline
\end{tabular}


Table 8.2. Three-dimensional Tsai-Wu quadratic failure criterion.

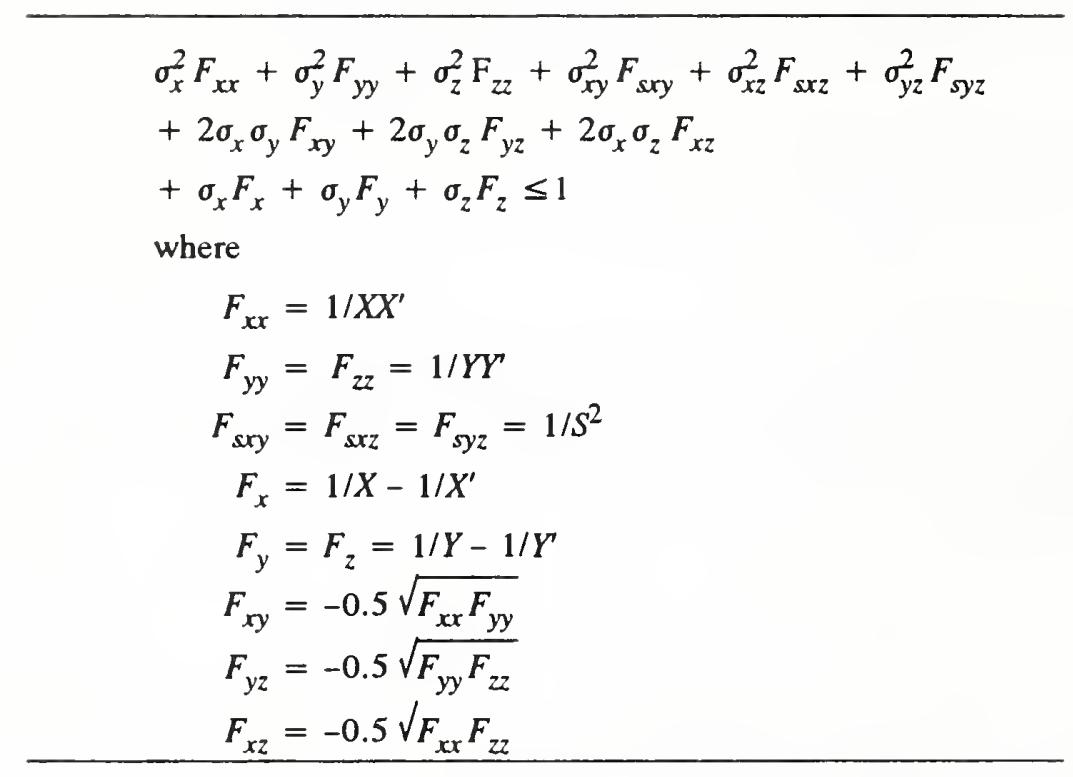

Note: $X, X^{\prime}, Y, Y^{\prime}$, and $S$ are given in Table 8.3.

Table 8.3 Properties of E-glass-reinforced resins used in the calculations.

\begin{tabular}{|c|c|c|c|}
\hline Property & $\begin{array}{c}\text { DGEBA/DCH } \\
\text { ARA/ACL }\end{array}$ & $\begin{array}{l}\text { VE-El/ } \\
\text { MEKP }\end{array}$ & $\begin{array}{c}\text { DGEBF/ } \\
\text { CTBN/AN }\end{array}$ \\
\hline \multicolumn{4}{|l|}{ Stiffness Parameters } \\
\hline - longitudinal Young's modulus, $E_{x}(\mathrm{GPa})$ & 38.6 & 46 & 46 \\
\hline - transverse in-plane Young's modulus, $E_{y}(\mathrm{GPa})$ & 8.3 & 6.9 & 6.9 \\
\hline - transverse out-of-plane Young's modulus, $E_{z}(\mathrm{GPa})$ & 8.3 & 6.9 & 6.9 \\
\hline $\begin{array}{l}\text { - shear moduli, } G_{y z}(\mathrm{GPa}) \\
\qquad G_{x z}=G_{x y}(\mathrm{GPa})\end{array}$ & $\begin{array}{l}3 \\
4\end{array}$ & $\begin{array}{l}2.5 \\
2.5\end{array}$ & $\begin{array}{l}2.5 \\
2.5\end{array}$ \\
\hline - Poisson's ratios, $\begin{aligned} & \nu_{y z} \\
\nu_{z x} & =\nu_{y x}\end{aligned}$ & $\begin{array}{l}0.4 \\
0.3\end{array}$ & $\begin{array}{l}0.4 \\
0.3\end{array}$ & $\begin{array}{l}0.4 \\
0.3\end{array}$ \\
\hline \multicolumn{4}{|l|}{ Thermal Expansion Coefficients $\left(10^{-6} / \mathrm{K}\right)$} \\
\hline - longitudinal coefficient, $\alpha_{x}$ & 8.61 & 8.61 & 8.61 \\
\hline - transverse coefficients, $\alpha_{y}=\alpha_{z}$ & 22.1 & 22.1 & 22.1 \\
\hline \multicolumn{4}{|l|}{ Strength Parameters (MPa) } \\
\hline - longitudinal tensile strength, $X$ & 1060 & 1160 & 1160 \\
\hline - longitudinal compressive strength, $X^{\prime}$ & 883 & 670 & 660 \\
\hline - transverse tensile strength, $Y$ & 17 & 83 & 76 \\
\hline - transverse compressive strength, $Y^{\prime}$ & 190 & 140 & 130 \\
\hline - longitudinal shear strength, $S$ & 76 & 83 & 76 \\
\hline
\end{tabular}




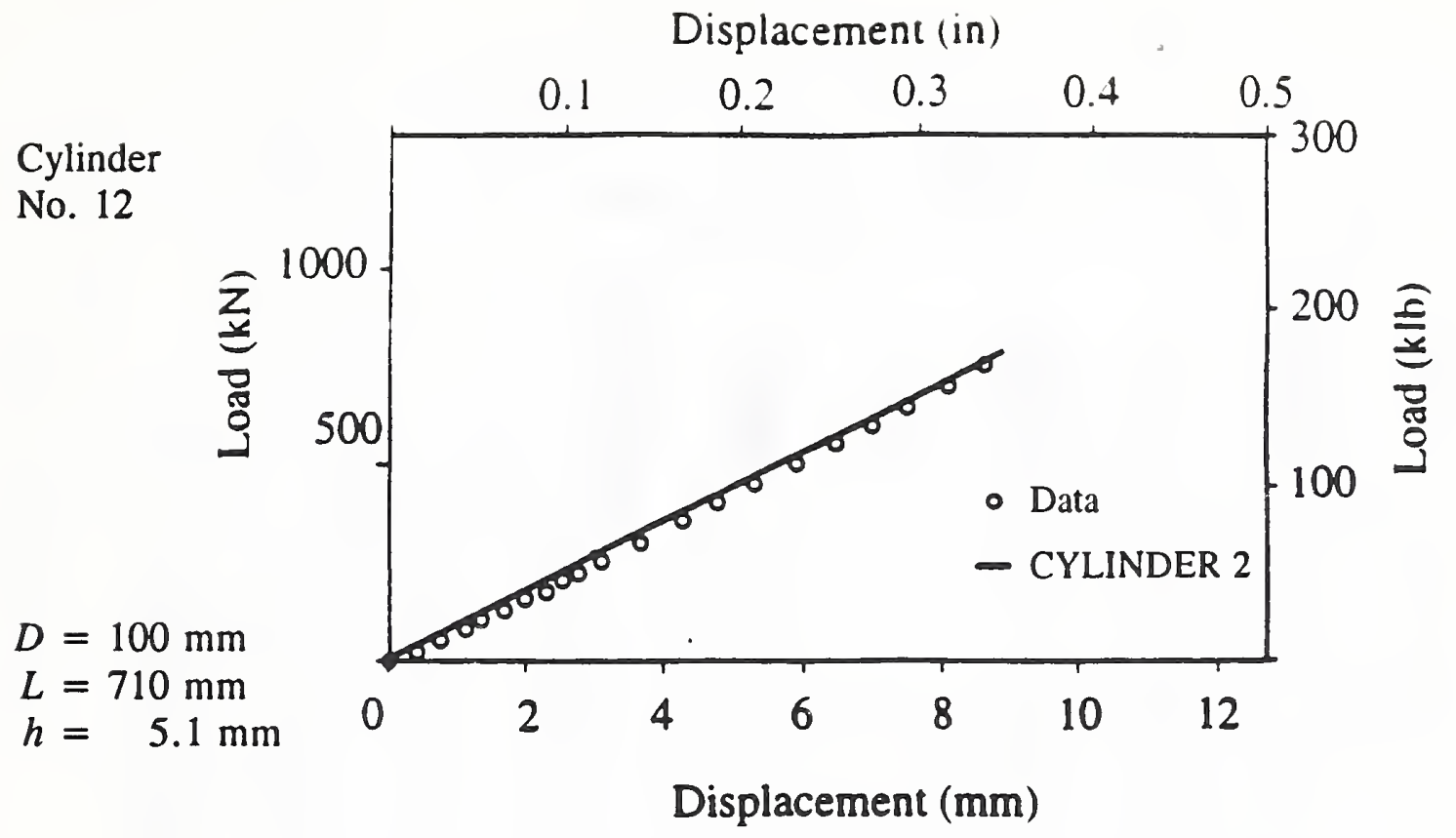

Figure 8.1. The load versus displacement for an E-glass epoxy cylinder (see Table 8.1).

The load versus displacement was measured for every cylinder. Here, we include one typical result (Figure 8.1). In this figure, both the data and the values calculated by the CYLINDER 2 code are shown. There is excellent agreement between the calculated and measured displacements.

The failure strengths of the cylinders are shown in Figures 8.2 and 8.3. The failure strength is defined as

$$
\sigma_{f}=F / A,
$$

where $F$ is the failure load and $A$ is the load-bearing area of the cylinder. In these figures, the spread in the data is also indicated.

Some of the cylinders were identical in all respect except their length (cylinders 1-3, $6-7,9-11,13-15)$. Both the data and the analytical results show that, for the lengths investigated, the length did not significantly affect the failure load.

The first ply failure loads calculated by the CYLINDER 2 code are shown in Figures 8.2 and 8.3. The calculation was performed for two situations: First, it was assumed in the calculations that there were no manufacturing-induced thermal stresses in the cylinder, stresses caused by temperature changes during curing $\left(\Delta T_{c}=0\right)$. Second, the calculations were performed by assuming that thermal stresses were present in the cylinder, introduced by a $100 \mathrm{~K}$ temperature change during curing $\left(\Delta T_{c}=-100 \mathrm{~K}\right)$. The first set of results $\left(\Delta T_{c}=0\right)$ is presented at the top of Figures 8.2 and 8.3 ; the second set $\left(\Delta T_{c}=-100 \mathrm{~K}\right)$, at the bottom of Figures 8.2 and 8.3. The agreements between the measured and calculated failure loads are quite good, especially in view of the wide spread in the data and the uncertainties in the material properties needed to perform the calculations. 

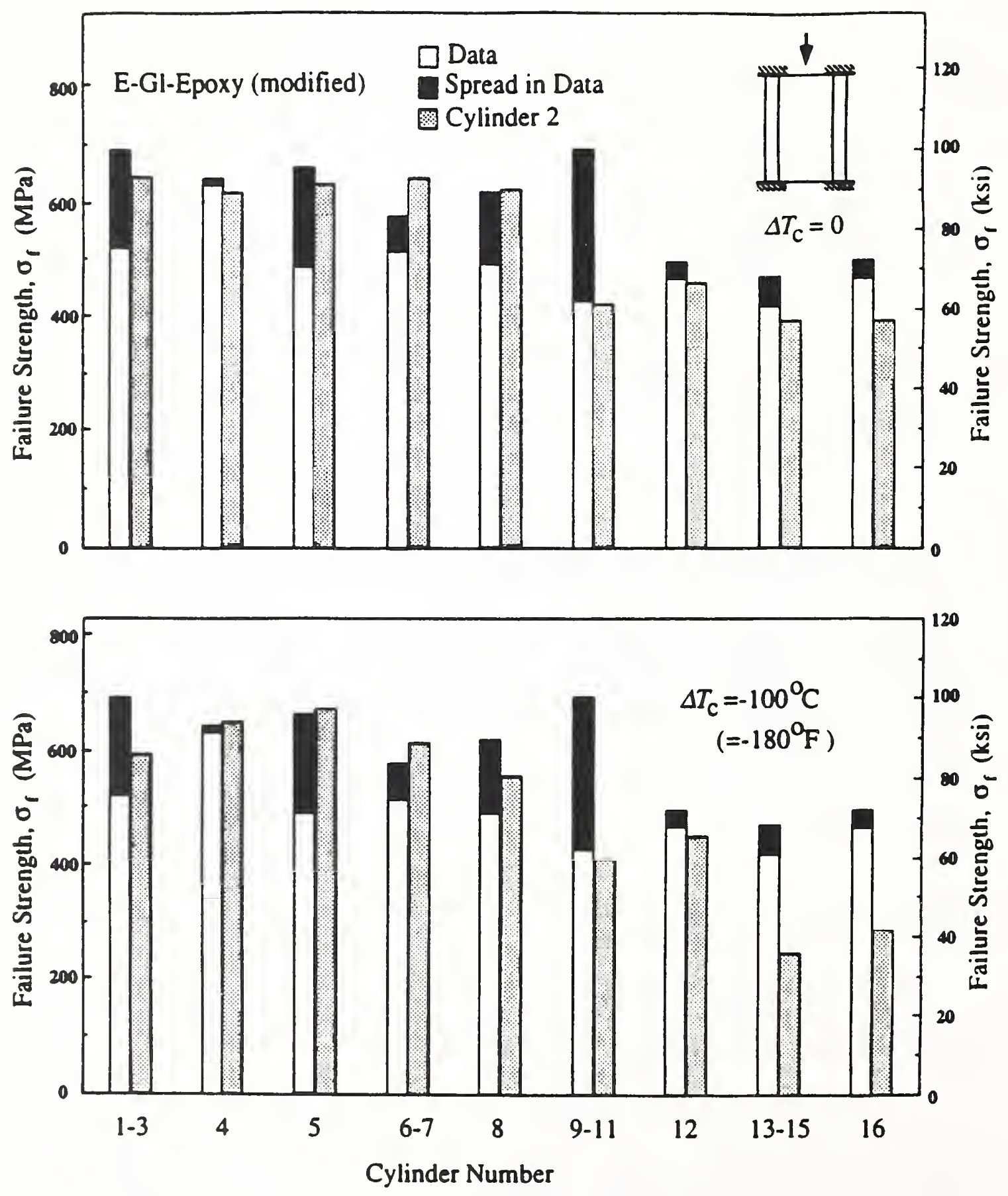

Figure 8.2. Failure strengths of E-glass-reinforced DGEBA/DCH/ARA/ACL cylinders (see Table 8.1). Open columns: data; shaded columns: results calculated by the CYLINDER 2 code for $\Delta T_{c}=0$ (top) and for $\Delta T_{c}=-100 \mathrm{~K}$ (bottom). 

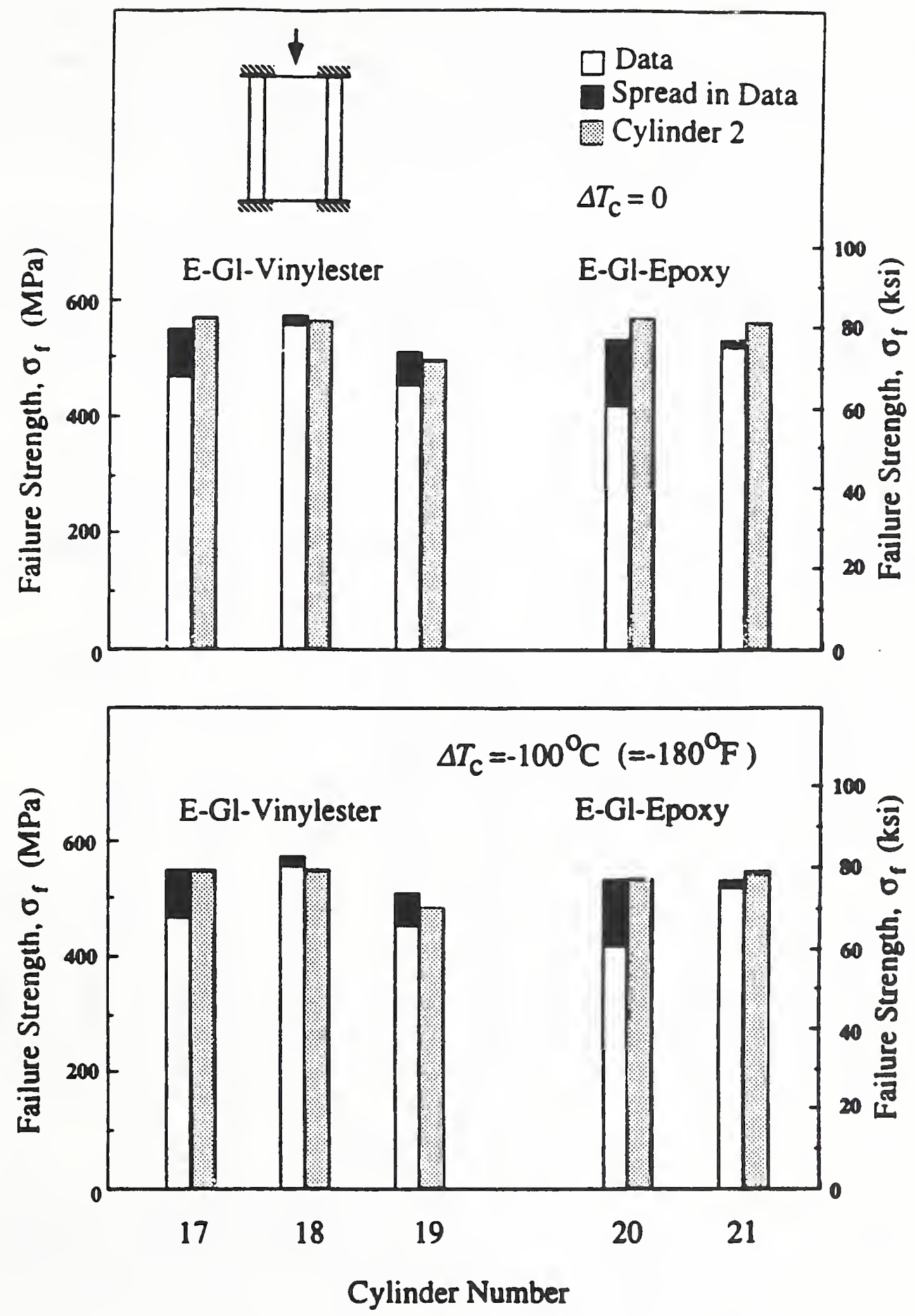

Figure 8.3. Failure strengths of E-glass-reinforced VE-EL/MEKP and DGEBF/CTBN/AN cylinders (see Table 8.1). Open columns: data; shaded columns: results calculated by the CYLINDER 2 code for $\Delta T_{c}=0$ (top) and for $\Delta T_{c}=-100 \mathrm{~K}$ (bottom). 
Two observations can be made regarding Figures 8.2 and 8.3: First, it is seen that the calculated first ply failure load approximates reasonably well the ultimate failure load. This suggests that, in the cylinders included in this study, ultimate failure occurred shortly after first ply failure took place. Second, manufacture-induced thermal stresses may change the failure load significantly. This is further illustrated by Figures 8.4 and 8.5 , where the first ply loads calculated by the CYLINDER 2 code are compared for $\Delta T_{c}=0$ and $\Delta T_{c}=100 \mathrm{~K}$. The results in Figures 8.4 and 8.5 show tht the effect of the thermal stress depends upon the material, the cylinder geometry, and the lay-up.

The aforementioned results suggest that the model and the CYLINDER 2 computer code can be used to describe the behavior of composite cylinders subjected to axial loading, and they can provide estimates of the deflection of a cylinder and the load at which failure occurs. Thus, the code should serve as a useful tool in the design of composite cylinders.

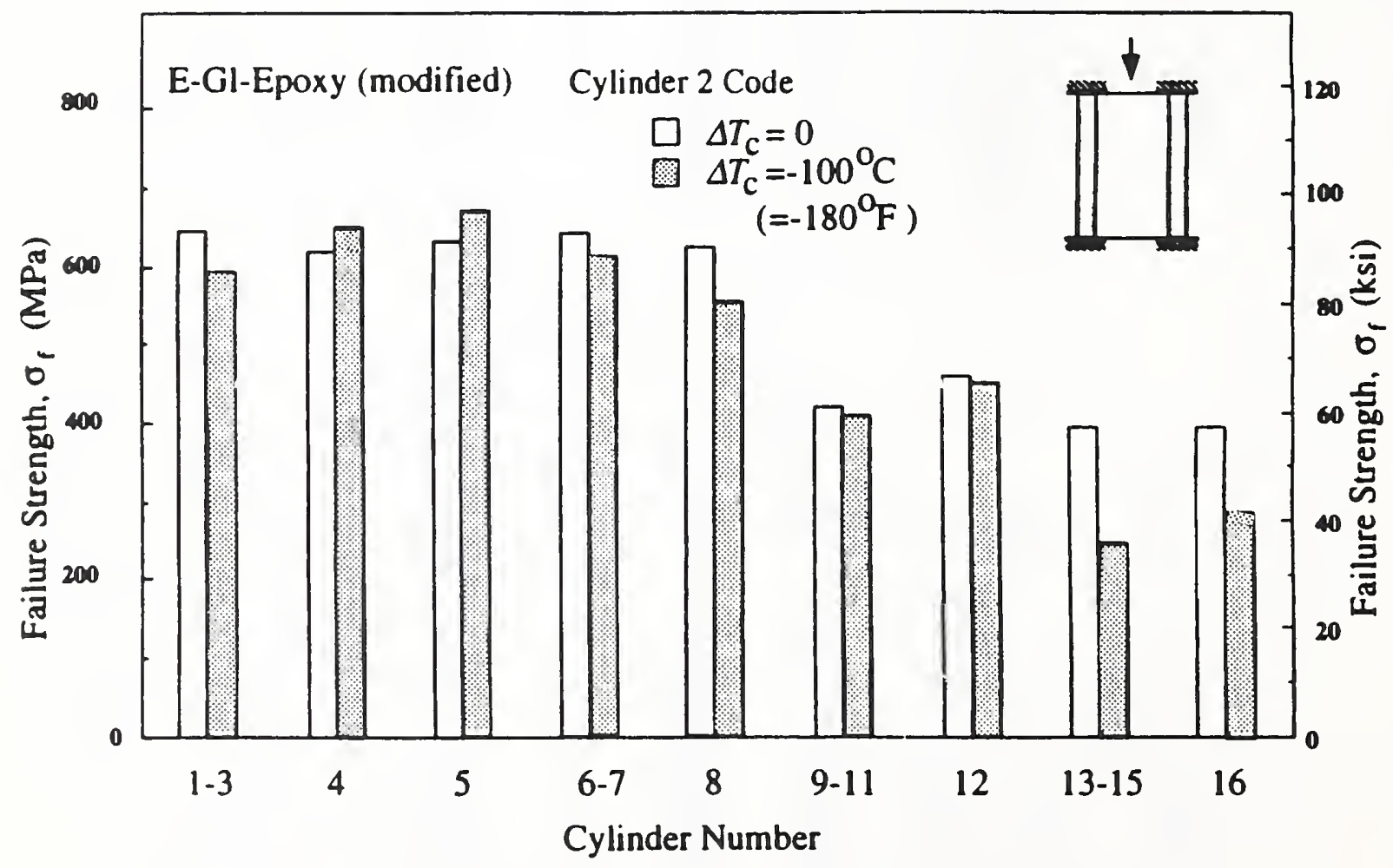

Figure 8.4. Failure strengths of E-glass-reinforced DGEBA/DCH/ARA/ACL cylinders (see Table 8.1) calculated by the CYLINDER 2 code for no thermal stresses $\left(\Delta T_{c}=0\right)$ and for thermal stresses induced by a $100 \mathrm{~K}$ temperature difference $\left(\Delta T_{c}=-100 \mathrm{~K}\right)$. 


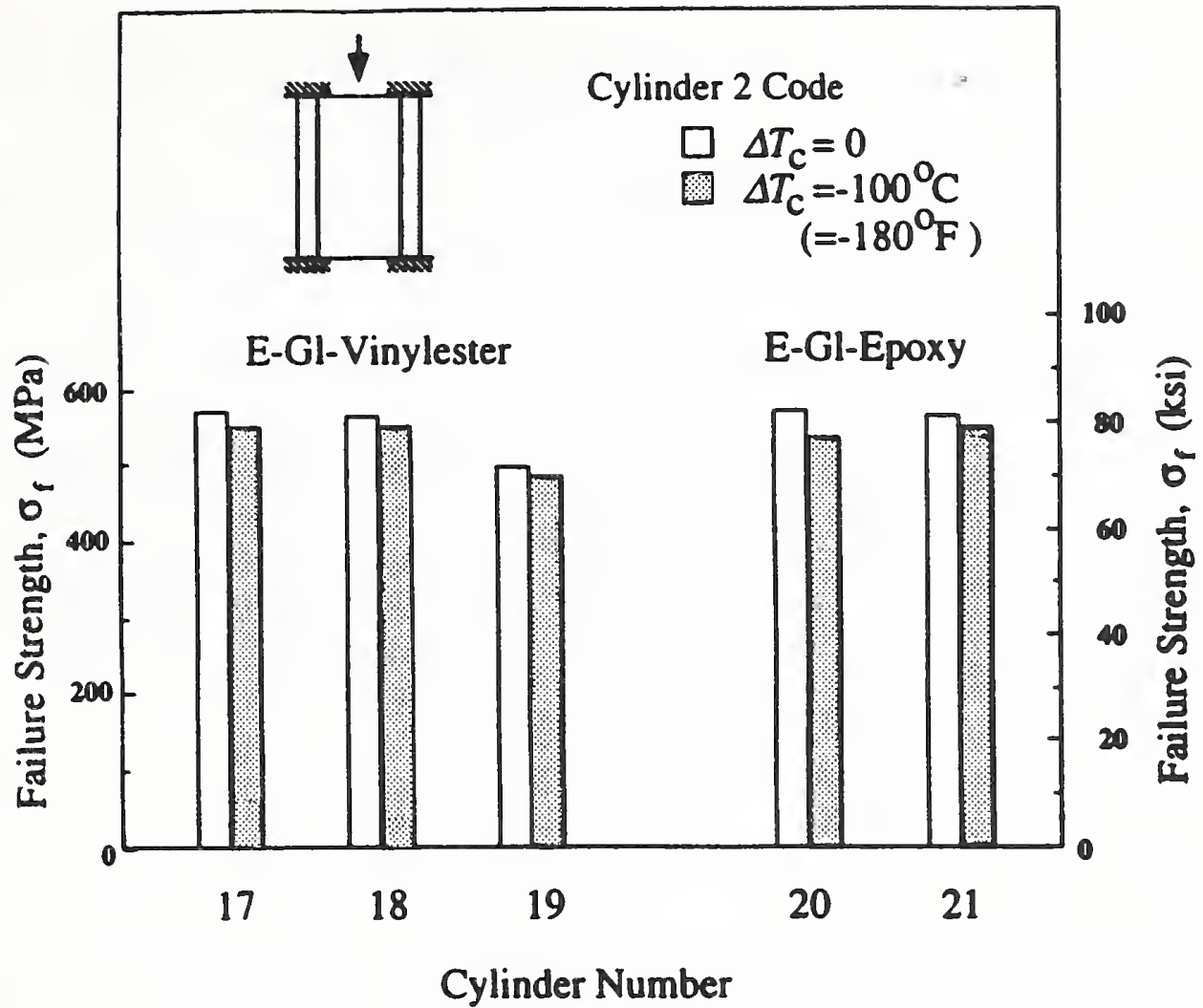

Figure 8.5. Failure strengths of E-glass-reinforced VE-EL/MEKP and DGEBA/CTBN/AN cylinders (see Table 8.1) calculated by the CYLINDER 2 code for no thermal stresses $\left(\Delta T_{c}=0\right)$ and for thermal stresses induced by a $100 \mathrm{~K}$ temperature difference $\left(\Delta T_{c}=-100 \mathrm{~K}\right)$.

\section{References}

[1.1] Noor, A.K; Burton, W.S.; Peters, J.M. Assessment of computational models for multilayered composite cylinders. Int. J. Solids Struct. 27: 1269-1286; 1991.

[1.2] Chou, F.H.; Achenbach, J.D. Three dimensional vibration of orthotropic cylinders. J. Eng. Mech. ASCE 98(EM4): 813-822; 1972.

[1.3] Noor, A.K.; Rarig, P.L. Three dimensional solutions of laminated cylinders. Comput. Methods Appl. Mech. Eng. 3: 319-334; 1974.

[1.4] Srinivas, S. Analysis of laminated, composite, circular, cylindrical shells with general boundary conditions. NASA T-R-412; 1974.

[1.5] Grigorenko, Ya.; Vasilenko, A.T.; Pankratova, N.D. Computation of the stressed state of thick-walled inhomogeneous anisotropic shells. Prikladanaya Mekhanika 10: 86-93 (in Russian); Sov. Appl. Mech. 10: 523-528 (English translation); 1974.

[1.6] Chandrashekhara, K.; Gopalakrishnan, P. Elasticity solution for a multilayered transversely isotropic circular cylindrical shell. J. Appl. Mech. 49: 108-114; 1982. 
[1.7] Hyer, M.W.; Cooper, D.E.; Cohen, D. Stresses and deformations in cross-ply composite tubes subjected to uniform temperature change. J. Therm. Stresses 9: 97-117; 1986.

[1.8] Ren, J.G. Exact solutions for laminated cylindrical shells in cylindrical bending. Comput. Sci. Techn. 29: 169-187; 1987.

[1.9] Hyer, M.W. Hydrosttic response of thick laminated composite cylinders. J. Reinf. Plast. Compos. 7: 321-340; 1988.

[1.10] Roy, A.K.; Tsai, S.W. Design of thick composite cylinders. J. Pressure Vessel Tech. ASME 110: 255-262; 1988.

[1.11] Noor, A.K.; Peters, J.M. Stress, vibrations and buckling of multilayered cylinders. J. Struct. Eng. 115: 69-88; 1989.

[1.12] Spencer, A.J.M.; Watson, P.; Rogers, T.G. Stress analysis of anisotropic laminated circular cylindrical shells. ASME, AMD-113: 57-60; 1990.

[1.13] Varadan, T.K.; Bhaskar, K. Bending of laminated orthotropic cylindrical shells An elastic approach. Compos. Struct. 17: 141-156; 1991.

[1.14] Roy, A.K. Response of thick laminated composite rings to thermal stresses. Compos. Struct. 18: 125-138; 1991.

[1.15] Lekhnitskii, S.G. Theory of elasticity of an anisotropic body. Moscow, Russia: Mir; 1981 .

[1.16] Hyer, M.W.; Rosseau, C.Q. Thermally induced stresses and deformations in angle-ply composite tubes. J. Compos. Mater. 21: 454-480; 1987.

[1.17] Hyer, M.W.; Rosseau, C.Q.; Tompkins, S.S. Thermally induced twist in graphite-epoxy tubes. J. Eng. Mater. Technol., ASME 110: 83-88; 1988.

[1.18] Lee, S.Y.; Springer, G.S. Filament winding cylinders: I. Process model. J. Compos. Mater. 24: 1270-1298; 1990.

[1.19] Spencer, A.J.M.; Watson, P.; Rogers, T.G. Mathematical analysis of the springback effect in laminted thermoplastic channel sections. Compos. Mfg. 2: 253-258; 1991.

[1.20] Kollár, L.P.; Springer, G.S. Stress analysis of anisotropic laminated cylinders and cylindrical segments. Int. J. Solids Struct. 29: 1499-1517; 1992.

[1.21] Kollár, L.P.; Patterson, J.M.; Springer, G.S. Composite cylinders subjected to hygrothermal and mechanical loads. Int. J. Solids Struct. 29: 1519-1534; 1992.

[3.1] Love, A.E.H. A treatise on the mathematical theory of elasticity, 4th Ed. New York, NY: Dover; 1944.

[3.2] Tsai, S.W. Composite design, 4th Ed. Dayton, OH: Think composites; 1988.

[5.1] Kollár, L.P.; Springer, G.S. Stress analysis of anisotropic laminated cylinders and cylindrical segments. Int. J. Solids Struct. 29: 1499-1517; 1992.

[6.1] Wolfram, S. Mathematica. A system for doing mathematics by computer. Redwood City, CA: Addison-Wesley; 1991.

[6.2] MATLAB, user's guide. Natick, MA: The Math Works, Inc.; 1991.

[7.1] Karaoglan, L.; Springer, G.S. Axial impact of composites. Comput. Struct. 49(5): 807-824; 1993. 


\section{Appendixes}

\section{Appendix A. Axial Temperature Distribution in the Cylinder}

For simplicity, we include only the temperature in the analysis with the understanding that the moisture content can be included in an identical manner as the temperature.

We consider a cylinder of length $L$, where the temperatures specified at the two edges $(x=0$ and $x=L)$ are denoted at $T_{0}$ and $T_{L}$. Heat transfer is only by conduction along the length of the cylinder. The objective is to find the function of the steady-state temperature distribution $T(x)$ along the axis of the cylinder.

The equation of heat conduction in this case $[\mathrm{A} .1]$ is

$$
\frac{\partial}{\partial x}\left(K \frac{\partial T}{\partial x}\right)=0
$$

where $K$ is the thermal conductivity, which is a function of the temperature.

Equation A.1 can be reduced to a simpler form by introducing a new variable:

$$
\zeta=\int_{T_{0}}^{T} K d T
$$

The first derivative of $\zeta$ with respect to $x$ is

$$
\frac{\partial \zeta}{\partial x}=K \frac{\partial T}{\partial x}
$$

Introducing Equation A.3 into A.1, the equation of heat conduction becomes

$$
\frac{\partial^{2} \zeta}{\partial x^{2}}=0
$$

Equation A.4 is a linear, second-order differential equation, the general solution of which is

$$
\zeta=d_{1} x+d_{o} .
$$

The unknown constants, $d_{1}$ and $d_{0}$, can be determined from the following boundary conditions: 


$$
\begin{aligned}
& \text { at } x=0 \quad \zeta=\int_{T_{0}}^{T_{0}} K d T=0 \\
& \text { at } x=L \quad \zeta=\int_{T_{0}}^{T_{L}} K d T,
\end{aligned}
$$

where the second integral is evaluated numerically.

The relationship between the temperature and the axial position is (see Equations A.5, A.6, and A.2)

$$
x=L \frac{\int_{T_{0}}^{T} K d T}{\int_{T_{0}}^{T_{L}} K d T}
$$

The thermal conductivity of a unidirectional ply on the basis of the thermal conductivities of the matrix $k_{m}$ and the fiber $k_{f}$ are given by Springer and Tsai [A.2],

$$
\begin{gathered}
k_{11}^{l}=k_{m}^{l}\left[v_{f}^{l} \frac{k_{f}^{l}}{k_{m}^{l}}+\left(1-v_{f}\right)\right] \\
k_{22}^{l}=k_{m}^{l}\left\{\left(1-2 \sqrt{v_{f}^{l} / \pi}\right)+\frac{1}{B}\left[\pi-\frac{4}{\sqrt{1-\left(B^{2} v_{f}^{l} / \pi\right)}} \tan ^{-1} \frac{\sqrt{1-\left(B^{2} v_{f}^{l} / \pi\right)}}{1+\sqrt{B^{2} v_{f}^{l} / \pi}}\right]\right\} \\
B=2\left(\frac{k_{m}^{l}}{k_{f}^{l}}-1\right)
\end{gathered}
$$

where $k_{11}^{l}$ and $k_{12}^{l}$ are the thermal conductivities of the ply in the fiber direction and perpendicular to the fiber; $k_{\mathrm{f}}^{l}$ and $k_{\mathrm{m}}^{l}$ are the thermal conductivities of the fiber and matrix, respectively; $v_{\mathrm{f}}^{l}$ is the fiber volume fraction; and $l$ refers to the $l^{\text {th }}$ ply in the cylinder. Thermal conductivity of an $n$-ply cylinder in the axial direction is 


$$
K=\sum_{l=1}^{n} A_{0}^{l}\left(k_{11}^{l} \cos ^{2} \varphi^{l}+k_{22}^{l} \sin ^{2} \varphi^{l}\right)
$$

where $A_{0}^{l}$ is the cross-sectional area of the $l^{\text {th }}$ layer and $\varphi^{l}$ is the angle between the fibers of the $l^{\text {th }}$ ply and the axis of the cylinder.

References

[A.1] Carslaw, H.S.; Jaeger, J.C. Conduction of heat in solids. Oxford, United Kingdom: Clarendon Press; 1959.

[A.2] Springer, G.S.; Tsai, S.W. Thermal conductivities of unidirectional materials, J. Compos. Mater. 1: 166-173; 1967. 


\section{Appendix B. The Series of the Temperature and Surface Loads} series: ${ }^{*}$

The given temperature $\Delta T(x)$ can be expressed in the form of the following two

1. Unstarred (sine) series

$$
\Delta T=\sum_{i=1}\left[\Delta T_{i} \sin i \frac{\pi}{L} x\right],
$$

where the coefficients of the Fourier series are

$$
\Delta T_{i}=\frac{2}{L} \int_{0}^{L} \Delta T \sin i \frac{\pi}{L} x d x
$$

2. Starred (cosine) series

$$
\Delta T=\sum_{i=0}\left[\Delta T_{i}^{*} \cos i \frac{\pi}{L} x\right],
$$

where

$$
\Delta T_{i}^{*}= \begin{cases}\frac{1}{L} \int_{0}^{L} \Delta T d x & \text { if } i=0 \\ \frac{2}{L} \int_{0}^{L} \Delta T \cos i \frac{\pi}{L} x d x & \text { if } i=1,2, \ldots\end{cases}
$$

The surface loads on the inner $\left[p^{i}(x)\right]$ and outer $\left[p^{o}(x)\right]$ surfaces can also be represented by either a sine or a cosine series, ${ }^{*}$ as follows:

1. Unstarred (sine) series

$$
\begin{gathered}
p^{i, o}=\sum_{i=1} p_{i}^{i, o} \sin i \frac{\pi}{L} x, \\
\text { where } p_{i}^{i, o}=\frac{2}{L} \int_{o}^{L} p^{i, o} \sin i \frac{\pi}{L} x d x .
\end{gathered}
$$

${ }^{*}$ All equations are from Korn and Korn [B.1]. 
2. Starred (cosine) series

$$
p^{i, o}=\sum_{i=0} p_{i}^{i, o *} \cos i \frac{\pi}{l} x,
$$

where

$$
p_{i}^{i, o *}= \begin{cases}\frac{1}{L} \int_{o}^{L} p^{i, o} d x & \text { if } i=0 \\ \frac{2}{L} \int_{o}^{L} p^{i, o} \cos i \frac{\pi}{L} x d x & \text { if } i=1,2 \ldots,\end{cases}
$$

These equations (B.5-B.8) apply at both the inner (superscript $i$ ) and outer (superscript $o$ ) surfaces.

\section{Reference}

[B.1] Korn, G.A.; Korn, T.M. Mathematical handbook for scientists and engineers, 2nd Ed. New York, NY: McGraw-Hill; 1968. 


\section{Appendix C. Solution of the Differential Equation $\mathbf{5 . 6}$}

In this appendix we describe the solution of Equation 5.6. First we rearrange Equation 5.6 into the following form:

$$
\begin{aligned}
& {\left[\left(\frac{i \pi}{L}\right)^{2} \mathbf{0}_{A}+\frac{i \pi}{L}\left(\frac{1}{r} \mathbf{0}_{B}+\frac{\partial}{\partial r} \mathbf{0}_{D}\right)+\frac{1}{r^{2}} \mathbf{0}_{C}+\frac{1}{r} \frac{\partial}{\partial r} \mathbf{0}_{E}+\frac{\partial^{2}}{\partial r^{2}} \mathbf{0}_{F}\right] \mathbf{a}(r)} \\
& =\Delta T_{i}\left[\frac{i \pi}{L} \mathrm{~b}_{A}+\frac{1}{r} \mathrm{~b}_{B},\right.
\end{aligned}
$$

where $\mathbf{0}_{A}$ through $\mathbf{0}_{F}$ are matrices and $\mathbf{b}_{A}$ and $\mathbf{b}_{B}$ are vectors given in Table $C .1$, and $\mathbf{a}(r)$ is the vector of the unknown functions,

$$
\mathbf{a}(r)=\left[\begin{array}{l}
u_{i}^{l}(r) \\
v_{i}^{l}(r) \\
w_{i}^{l}(r)
\end{array}\right] .
$$

Equation C.1 is a sixth-order differential equation system having a singularity at $r=0$.

We have obtained solutions to the above set of differential equations by three different methods:

1. by the Frobenius method, which is applicable when $\mathrm{ri} \pi / L$ is small;

2 . by an asymptotic solution method, which is applicable when $r i \pi / L$ is large;

3. by an approximate method, which is applicable for all values of $r i \pi / L$.

In addition to being applicable to a wide range of $r i \pi / L$ values, the last method also proved to require less computer time than the other two. Therefore, this method was built into the CYLINDER 2 code. The basic steps of this method are as follows: We replace the functions $1 / r$ and $1 / r^{2}$ in Equation C. 1 by $1 / r_{k}$ and $1 / r_{k}^{2}$, where $r_{k}$ is the radius of the midsurface of the $l^{\text {th }}$ ply:

$$
r_{k}=\left(r^{l}+r^{l+1}\right) / 2
$$

We obtain from Equation C.1

$$
\begin{aligned}
& {\left[\left(\left(\frac{i \pi}{L}\right)^{2} \mathbf{0}_{A}+\frac{i \pi}{L r_{k}} \mathbf{0}_{B}+\frac{1}{r_{k}^{2}} \mathbf{0}_{C}\right)+\frac{\partial}{\partial r}\left(\frac{i \pi}{L} \mathbf{0}_{D}+\frac{1}{r_{k}} \mathbf{0}_{E}\right)+\frac{\partial^{2}}{\partial r^{2}} \mathbf{0}_{F}\right] \mathbf{a}(r)} \\
& =\Delta T_{i}\left[\frac{i \pi}{L} \mathrm{~b}_{A}+\frac{1}{r_{k}} \mathrm{~b}_{B}\right] .
\end{aligned}
$$


Table C.1. Definitions of symbols in Equation C.1.

$$
\begin{gathered}
0_{A}=\left[\begin{array}{ccc}
C_{11} & C_{16} & 0 \\
C_{16} & C_{66} & 0 \\
0 & 0 & C_{55}
\end{array}\right] \quad 0_{B}=\left[\begin{array}{ccc}
0 & 0 & -\left(C_{12}+C_{55}\right) \\
0 & 0 & -\left(C_{26}+2 C_{45}\right) \\
-C_{12}+C_{13} & -C_{26}+C_{36}-C_{45} & 0
\end{array}\right] \\
0_{C}=\left[\begin{array}{ccc}
0 & 0 & 0 \\
0 & C_{44} & 0 \\
0 & 0 & C_{22}
\end{array}\right] \quad 0_{D}=\left[\begin{array}{ccc}
0 & 0 & -\left(C_{13}+C_{55}\right) \\
C_{13}+C_{55} & C_{36}+C_{45} & -\left(C_{36}+C_{45}\right) \\
0
\end{array}\right] \\
0_{E}=\left[\begin{array}{ccc}
-C_{55} & 0 & 0 \\
-2 C_{45} & -C_{44} & 0 \\
0 & 0 & -C_{33}
\end{array}\right] \quad 0_{F}=\left[\begin{array}{ccc}
-C_{55} & -C_{45} & 0 \\
-C_{45} & -C_{44} & 0 \\
0 & 0 & -C_{33}
\end{array}\right] \\
\mathrm{b}_{A}=\left[\begin{array}{c}
-q_{1} \\
-q_{2} \\
0
\end{array}\right] \quad \mathrm{b}_{B}=\left[\begin{array}{c}
0 \\
0 \\
-q_{3}
\end{array}\right]
\end{gathered}
$$

Note: $q_{1}, q_{2}$, and $q_{3}$ are given in Table 5.3 .

\section{General Solution of the Homogeneous Equation}

When the temperature difference is zero $\left(\Delta T_{i}=0\right)$, Equation C.4 reduces to a homogeneous equation. For this homogeneous equation we seek a solution in the form

$$
\mathrm{a}(r)=e^{\lambda\left(\frac{r}{r_{k}}-1\right)} \mathrm{d},
$$

where $\lambda$ and the three elements of vector $\mathbf{d}$ are, as yet, unknown constants. By substituting Equation $C .5$ and $\Delta T_{i}=0$ into Equation C.4, after algebraic manipulation, we obtain

$$
\left[\mathbf{H}_{1}+\lambda \mathbf{H}_{2}+\lambda^{2} \mathbf{H}_{3}\right] \mathbf{d}=\mathbf{0} \text {, }
$$


where

$$
\begin{aligned}
& \mathrm{H}_{1}=\left(r_{k} i \frac{\pi}{L}\right)^{2} 0_{A}+\left(r_{k} i \frac{\pi}{L}\right) 0_{B}+0_{C} \\
& \mathrm{H}_{2}=r_{k} i \frac{\pi}{L} 0_{D}+0_{E} \\
& \mathrm{H}_{3}=0_{F} .
\end{aligned}
$$

Equation C.6 is a generalized eigenvalue problem that can be reduced to a linear eigenvalue problem (see ref. C.1) and can be solved by any standard method (e.g., by MATLAB). The eigenvalue problem (Equation C.6) has six eigenvalues, denoted by $\lambda_{1}$ through $\lambda_{6}$. The corresponding eigenvectors are denoted by $d_{1}$ through $d_{6}$. The six solutions of the homogeneous equations are

$$
\mathbf{a}_{j}^{\text {hom }}(r)=\left[\begin{array}{c}
u_{i j}^{\text {l, hom }}(r) \\
v_{i j}^{l, h o m}(r) \\
w_{i j}^{l, h o m}(r)
\end{array}\right]=e^{\lambda j\left(\frac{r}{r_{k}}-1\right)} \mathrm{d}_{j} \quad j=1,2, \ldots, 6
$$

Note that if $C_{22}=C_{33}$, then two of the $\lambda-s$ (and the corresponding $\mathbf{d}-s$ ) are identical, and two of the above solutions are not independent.

The missing independent solution can be found hy the method given by Korn and Korn [C.2]. Here we chose a different method; namely, we changed slightly the value of the stiffness $C_{22}$. This procedure does not significantly affect the results, hut it increases the computational efficiency.

\section{A Particular Solution of the Inhomogeneous Equation}

A particular solution of the inhomogeneous equation (Equation C.4) is

$$
\mathbf{a}^{i n h}=\left[\begin{array}{c}
u_{i}^{l, i n h} \\
v_{i}^{l, i n h} \\
w_{i}^{l, i n h}
\end{array}\right]=\Delta T_{i} r_{k} \mathbf{H}_{1}^{-1}\left[\frac{r_{k} i \pi}{L} \mathbf{b}_{A}+\mathbf{b}_{B}\right] \text {, }
$$

where $\mathbf{H}_{\mathbf{1}}$ is defined by Equation C.7. 


\section{General Solution of the Inhomogeneous Equation}

We obtain the general solution of the inhomogeneous equation as the sum of the linear combination of the six homogeneous solutions and the inhomogeneous solution:

$$
\sum_{j=1}^{6} C_{j}^{l}\left[\begin{array}{l}
u_{i j}^{l, h o m} \\
v_{i j}^{l, h o m} \\
w_{i j}^{l, h o m}
\end{array}\right]+\left[\begin{array}{l}
u_{i}^{l, i n h} \\
v_{i}^{l, i n h} \\
w_{i}^{l, i n h}
\end{array}\right]
$$

where $C_{j}^{l}(j=1,2, \ldots 6)$ are six arbitrary constants.

To obtain the particular solution of a given problem, we have to determine the six constants $\left(C_{1}^{l}, C_{2}^{l}, \ldots C_{6}^{l}\right)$ from the continuity and boundary conditions.

\section{References}

[C.1] Wilkinson, J.H. The algebraic eigenvalue problem. Oxford, United Kingdom: Clarendon Press; 1965.

[C.2] Korn, G.A.; Korn, T.M. Mathematical handbook for scientists and engineers, 2nd Ed. New York, NY: McGraw-Hill; 1968. 


\section{Appendix D. Sample Problems for the CYLINDER 2 Code}

In the next three sections we will consider sample problems to show the running capabilities of the CYLINDER 2 computer code. The three problems considered were

1. Cylinder subjected to mechanical and temperature loads prescribed along the axis. The material properties do not vary with $x$.

2. Cylinder subjected to temperature load prescribed at the edges. The material properties do not vary with $x$.

3. Cylinder subjected to an axial end load. The material properties may vary with $x$.

Cylinder Subjected to Mechanical and Temperature Loads Prescribed along the Axis. The Material Properties Do Not Vary with $x$.

We consider a cylinder whose material properties and geometrical data used in the calculation are given in Table 7.1. We determine the displacements and stresses for three different loading conditions as follows:

1. specified end shortening, $\bar{u}=0.64 \mu \mathrm{m}$;

2. linearly distributed surface pressure on the inner surface; pressure varies from -100 to $-280 \mathrm{kPa}$;

3. temperature load varying linearly with $x$ between 366 and $477 \mathrm{~K}$.

Before the running, the code and the geometrical and material properties must be specified in the file "xdata," and the boundary and loading conditions due to the end shortening in the file "xdataloads." These files are presented in Tables D.1 and D.2.

To run the code, type

$\gg$ cyli Return

Then the following "inputlist" appears on the screen:

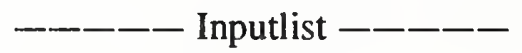

1) New running

2) New load for an old case

0) Quit

Select an input number: 
Table D.1 File "xdata" for a cylinder specified in section 7 .

8 xdata

\begin{tabular}{|c|c|}
\hline 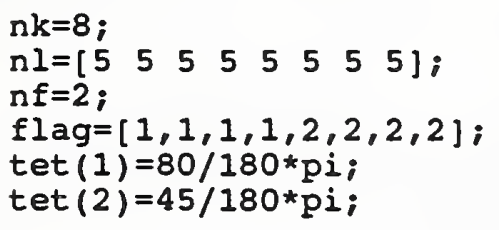 & $\begin{array}{l}\text { qnumber of groups in one lamina } \\
\text { qnumber of plies in groups } \\
\text { qnumber of ply orientations } \\
\text { qwhich plyorientation in a group? } \\
\text { \&lst plyorientation } \\
\text { \&2nd plyorientation }\end{array}$ \\
\hline $\begin{array}{l}\text { thick } \\
\mathrm{Rm}=2 ;\end{array}$ & $\begin{array}{c}\text { 8thickness of one ply } \\
\text { oradius of the mold } \\
\text { \&length of the }\end{array}$ \\
\hline
\end{tabular}

\& Elastic properties of a ply

$E_{x}=.56 e 7 ; E_{Y}=.12 e 7 ; E_{z}=.12 e 7 ;$

Gxy $=.6 e 6 ;$ Gxz $=.6 e 6 ;$ Gyz $=.6 e 6$;

nux $=.26 ;$ nuxz $=.26 ;$ nuyz $=.4 ;$

\& Coefficients of thermal expansion

alf $x=4.78 e-6 ;$ alf $y=12.28 e-6 ;$ alf $z=12.28 e-6$;

o Strength properties

$x x=154 \mathrm{e} ; \quad x x c=88.45 e 3 ; y y=4.495 e 3 ; y y c=17.11 \mathrm{e} ; ; s s=10.44 \mathrm{e} 3 ;$

o Multipliers in Tsai-Wu

$F x x=0 ; F Y y=0 ; F z z=0 ; F x=0 ; F y=0 ; F z=0 ; F s s=0 ; F s=0 ; F x y=0 ; F x z=0 ; F y z=0$;

$F \times x=1 / x \times / x x c$;

FYY $=1 /$ YY $/$ YYC;

FZZ=FYY;

\&six six

8siy siy

osiz siz

$F x=1 / x x-1 / x x c ;$

$F Y=1 / Y Y-1 / Y_{Y C} ;$

$8 s i x$

\&siy

$\mathrm{FZ}=\mathrm{FY}$;

\&siz

Fss $=1 / s s / s s ;$

$\mathbf{F} \boldsymbol{g}=0$;

Fxy $=.5 * \operatorname{sqrt}\left(\right.$ Fxx ${ }^{*}$ Fyy $)$;

$8 F Y z=0$;

FXz $=$ FXY;

8sixy

ssixy sixy and sixz sixz

82 six siy

82 six siz

82 siy siz 
Table D.2. File "xdataload" for a cylinder fixed at both edges with specified end shortening $\overline{\mathrm{u}}=-0.64 \mu \mathrm{m}$

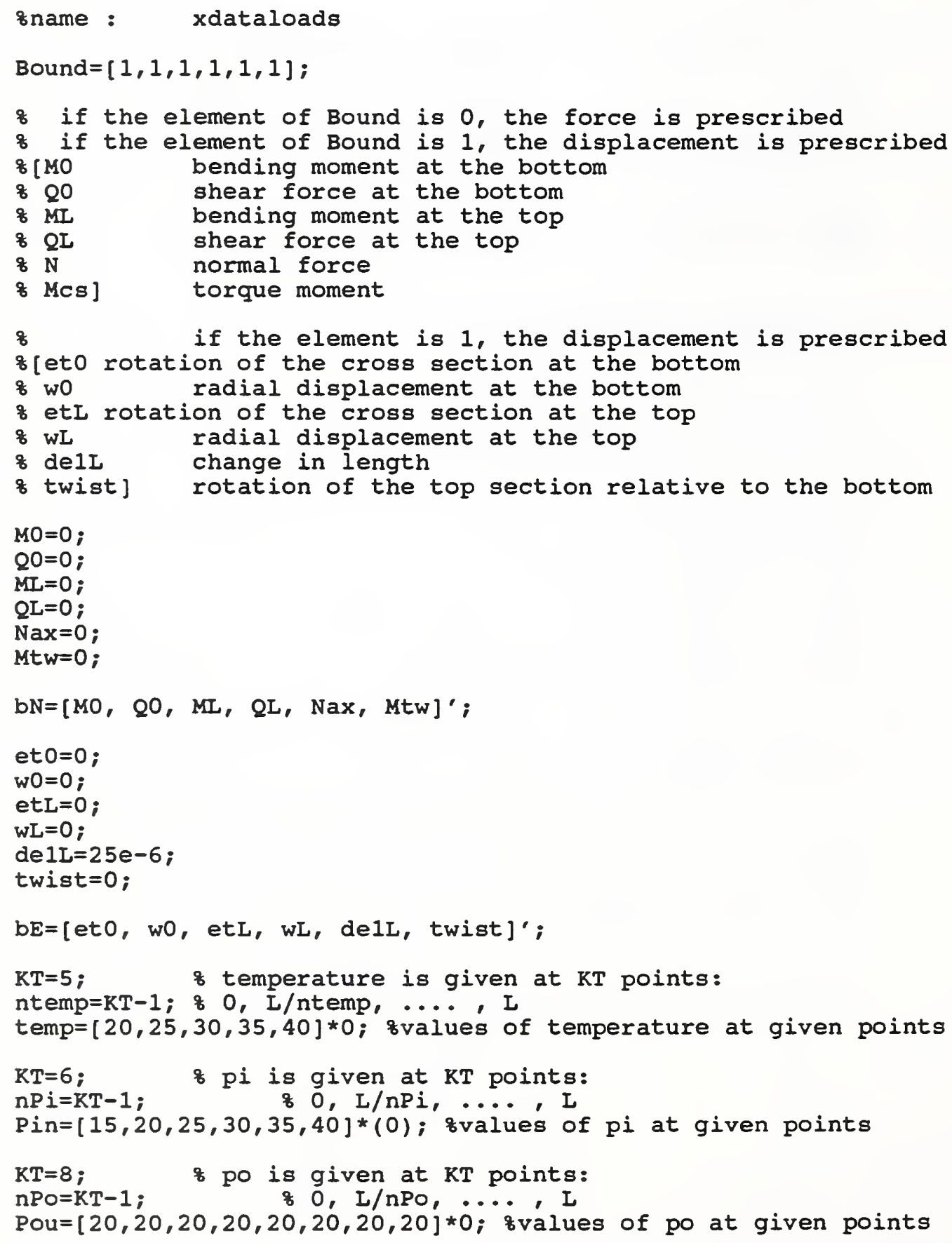


Type

$$
\gg 1 \text { Return }
$$

and

Title of the work: appears.

Type

$$
\gg \text { Laszlo-1 Return }
$$

If the computation is over, the following "outputlist" appears on the screen:

$$
\text { - - - - Outputlist }
$$

1) Data.

2) Displacements and global internal forces.

3) Stresses at a cross section.

4) Load factor, critical cross section.

5) New loads/boundary conditions.

6) Calculation of temperature distribution.

0) Quit

Select an output number:

By entering $1,2, \ldots 6$ or 0 , the item specified in the outputlist appears on the screen.

Type

$$
\gg 1 \text { Return }
$$

and the results given in Figure D.1 appear on the screen.

Type

$$
\gg \text { Return }
$$

and we get Figure D.2. 
Lay-up: $\left[80_{5} / 80_{5} / 80_{5} / 80_{5} / 45_{5} / 45_{5} / 45_{5} / 45_{5}\right]$

inner radius: $50 \mathrm{~mm}$

length: $230 \mathrm{~mm}$

total thickness: $5 \mathrm{~mm}$

thickness of one ply: $130 \mu \mathrm{m}$

\section{Elastic Constants}

$E_{x}=39 \mathrm{GPa}$

$$
\begin{aligned}
G_{x y} & =4.1 \mathrm{GPa} \\
G_{x z} & =4.1 \mathrm{GPa} \\
G_{y z} & =4.1 \mathrm{GPa}
\end{aligned}
$$$$
\nu_{x y}=0.26
$$$$
\nu_{x z}=0.26
$$$$
E_{z}=8.3 \mathrm{GPa}
$$$$
\nu_{y z}=0.4
$$

\section{Strength Constants}
$X=1060 \mathrm{MPa}$
$Y=31 \mathrm{MPa}$
$S=72 \mathrm{MPa}$
$X^{\prime}=610 \mathrm{MPa}$
$Y^{\prime}=118 \mathrm{MPa}$
$F_{x y}{ }^{*}=-1 / 2$

\section{Coefficients of Thermal Expansion}

$\alpha_{x}=8.6 \times 10^{-6} /{ }^{\circ} \mathrm{C}$ (in plane, fiber direction)

$\alpha_{x}=22.1 \times 10^{-6} /{ }^{\circ} \mathrm{C}$ (in plane, perpendicular to the fiber)

$\alpha_{x}=22.1 \times 10^{-6} /{ }^{\circ} \mathrm{C}$ (out of plane)

\section{Boundary Displacements and Forces}

at the lower ( 0$)$ and upper $(L)$ edges

radial displacement: $w_{0}=3.17 \times 10^{-24} \mathrm{~m} ; w_{L}=-9.14 \times 10^{-24} \mathrm{~m}$

slope of the meridian: $\eta_{0}=-5.58 \times 10^{-22} ; \eta_{L}=-4.83 \times 10^{-22}$

relative axial displacement of the edges: $d_{0 L}=6.4 \times 10^{-7} \mathrm{~m}$

relative twist of the edges: $\mathrm{f}_{O L}=3.78 \times 10^{-22}$

shear force: $Q_{r 0}=12.6 \mathrm{~N} / \mathrm{m} ; Q_{r L}=12.6 \mathrm{~N} / \mathrm{m}$

bending moment: $M_{0}=0.137 \mathrm{~N} \cdot \mathrm{m} / \mathrm{m} ; M_{L}=0.137 \mathrm{~N} \cdot \mathrm{m} / \mathrm{m}$

normal force: $N_{a x}=-5.63 \mathrm{~kg}$

torque moment: $M_{\text {tor }}=-0.757 \mathrm{~N} \cdot \mathrm{m}$

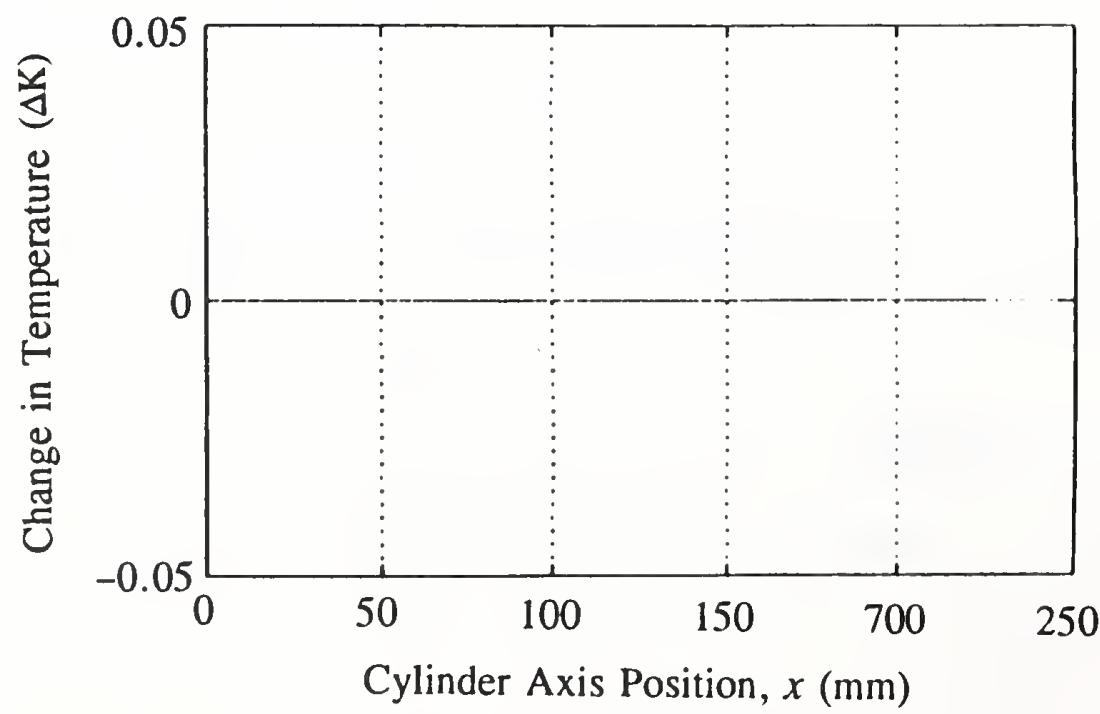

Figure D.1. Input data, edge loads, and edge displacements for a cylinder with specified end shortening. 


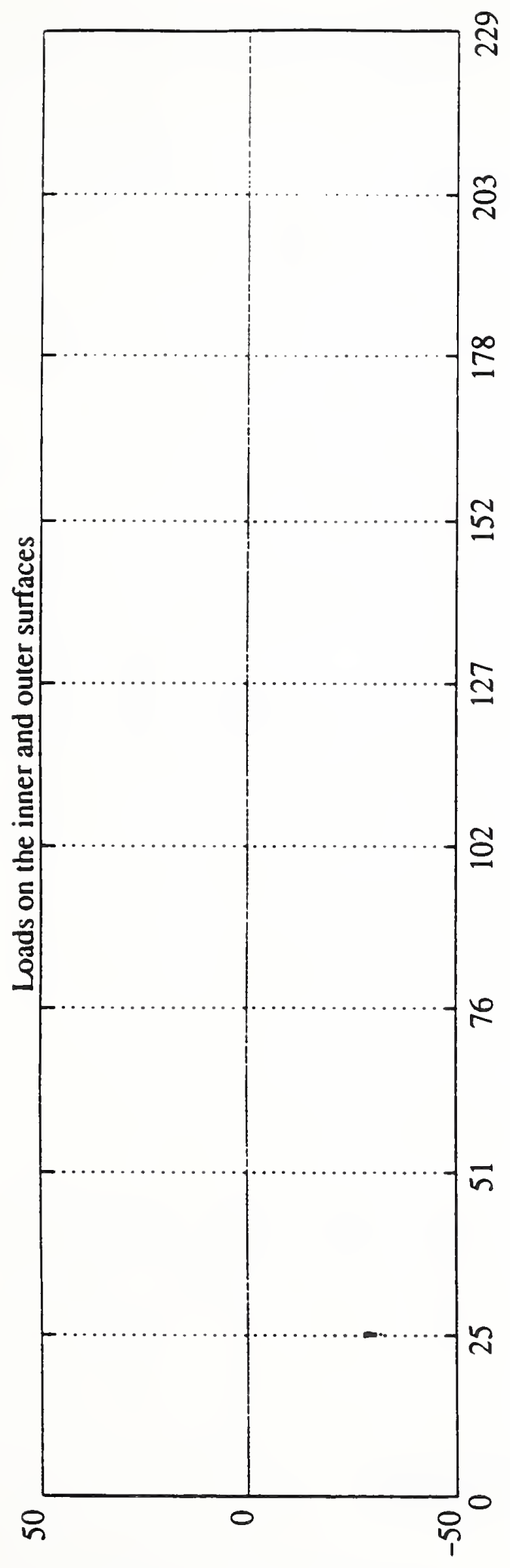

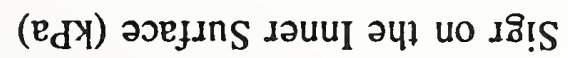

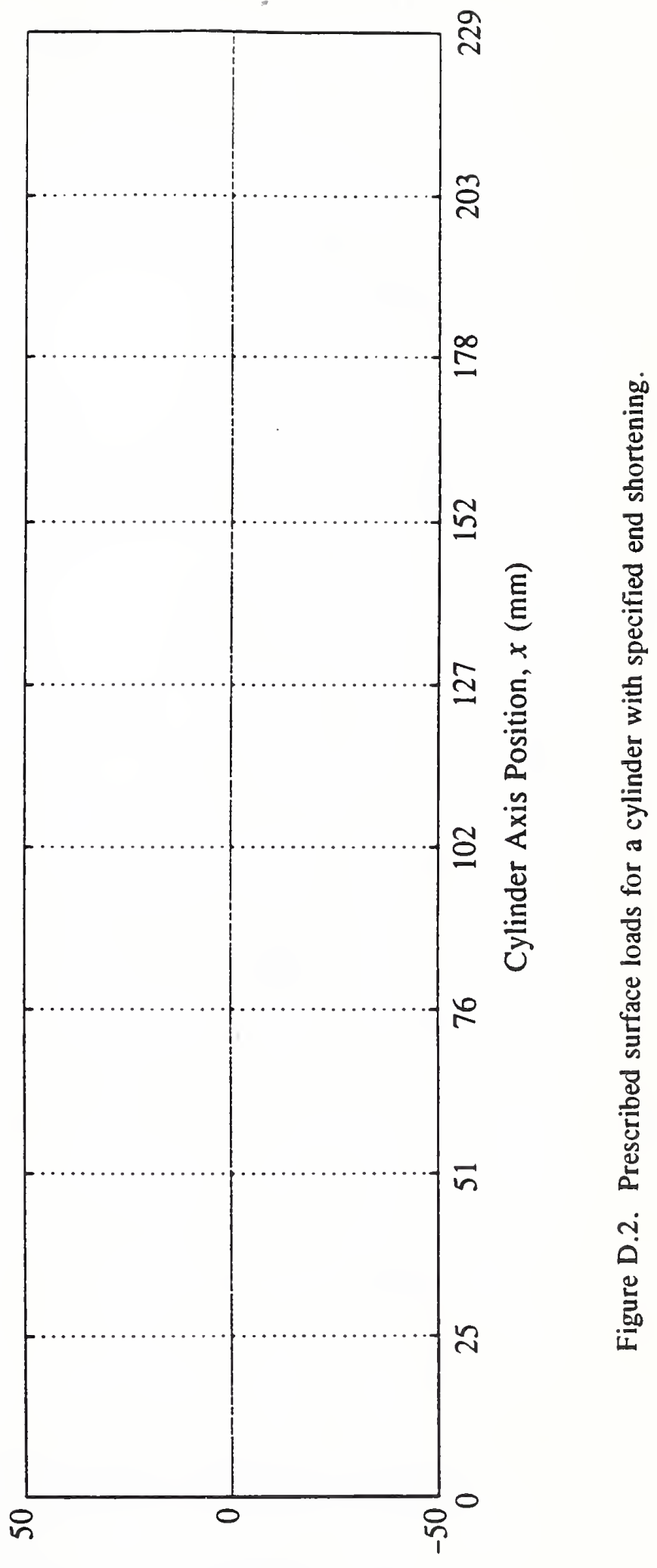

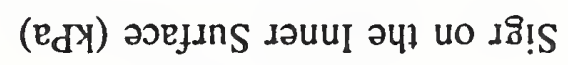


Type

$$
\gg 2 \text { Return }
$$

and we get Figure D.3.

Type

$$
\gg \text { Return }
$$

and we get Figure D.4.

Type

$$
\gg 3 \text { Return }
$$

and

$$
x \text { coordinate }=\text { appears. }
$$

Type

$$
\gg 1 \text { Return }
$$

and we get Figure D.5.

Type

$$
\gg \text { Return }
$$

and we get Figure D.6.

Type

$$
\gg 4 \text { Return }
$$

and we get Figure D.7.

Type

$$
\gg \text { Return }
$$

and we get Figure D.8.

To get results for the second loading case, we have to modify and store the file "xdataloads." The new file is given in Table D.3.

Type

$$
\gg 5 \text { Return }
$$

and Did you change the "xdataloads" file? $(\mathrm{y} / \mathrm{n})$ appears.

Type

$$
\gg \text { y Return }
$$




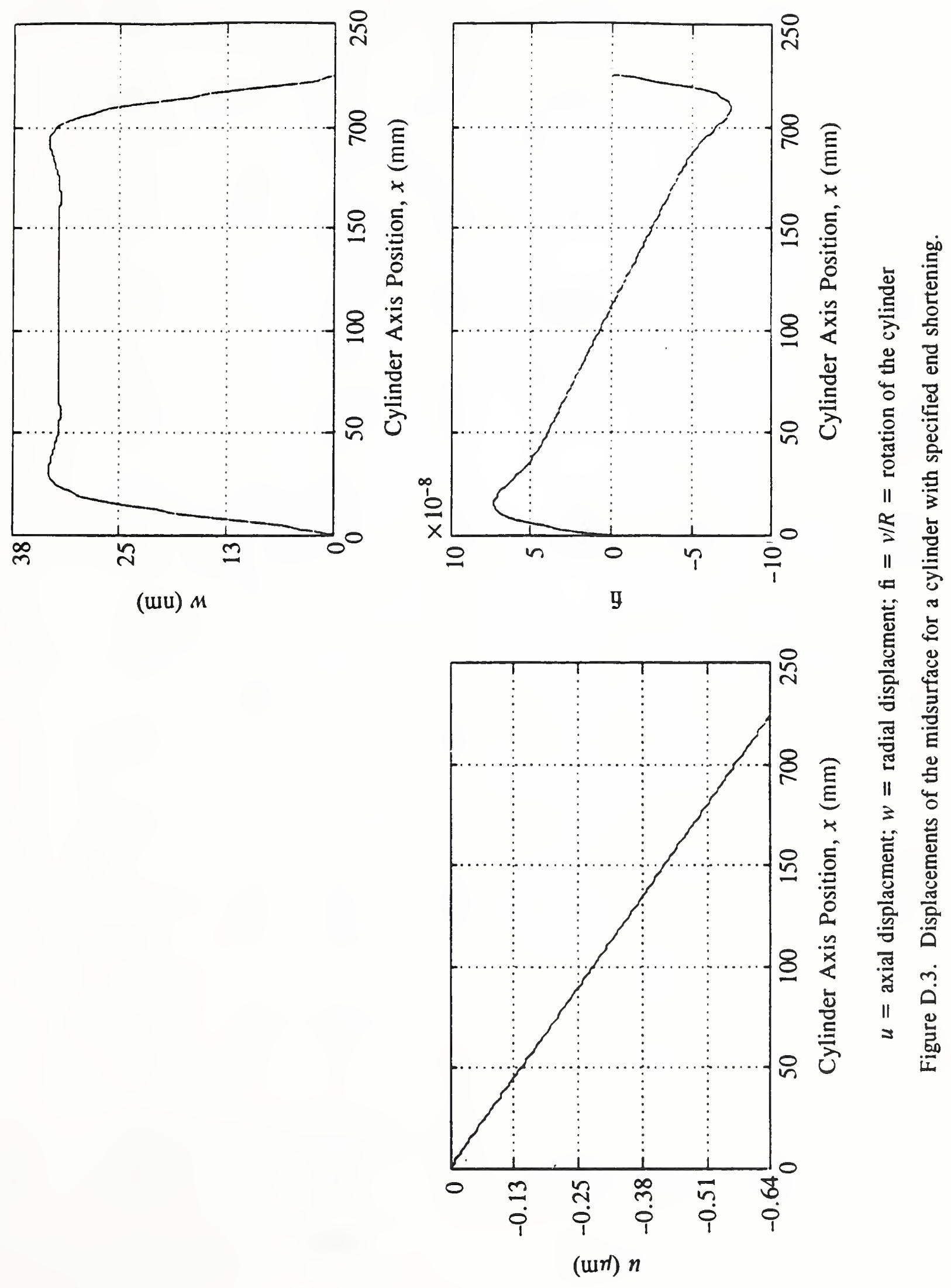



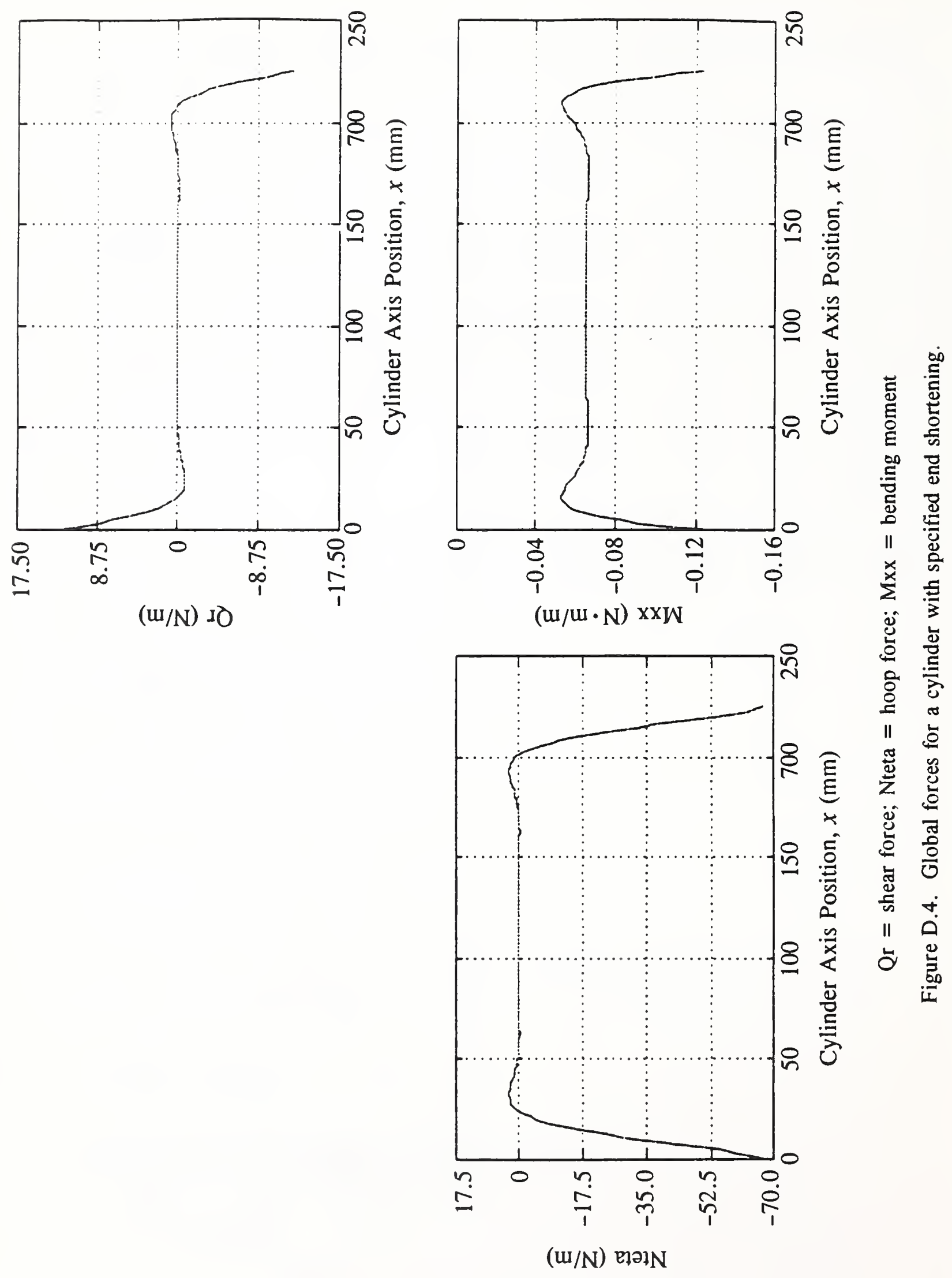


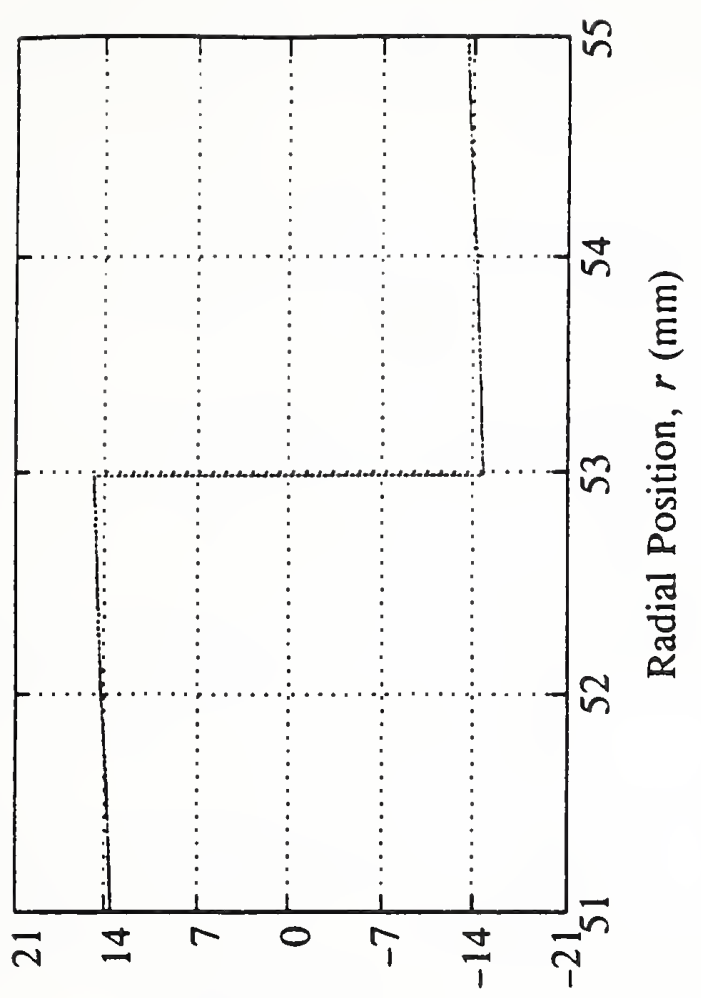

(ЕdY) ㄲาวชి!S

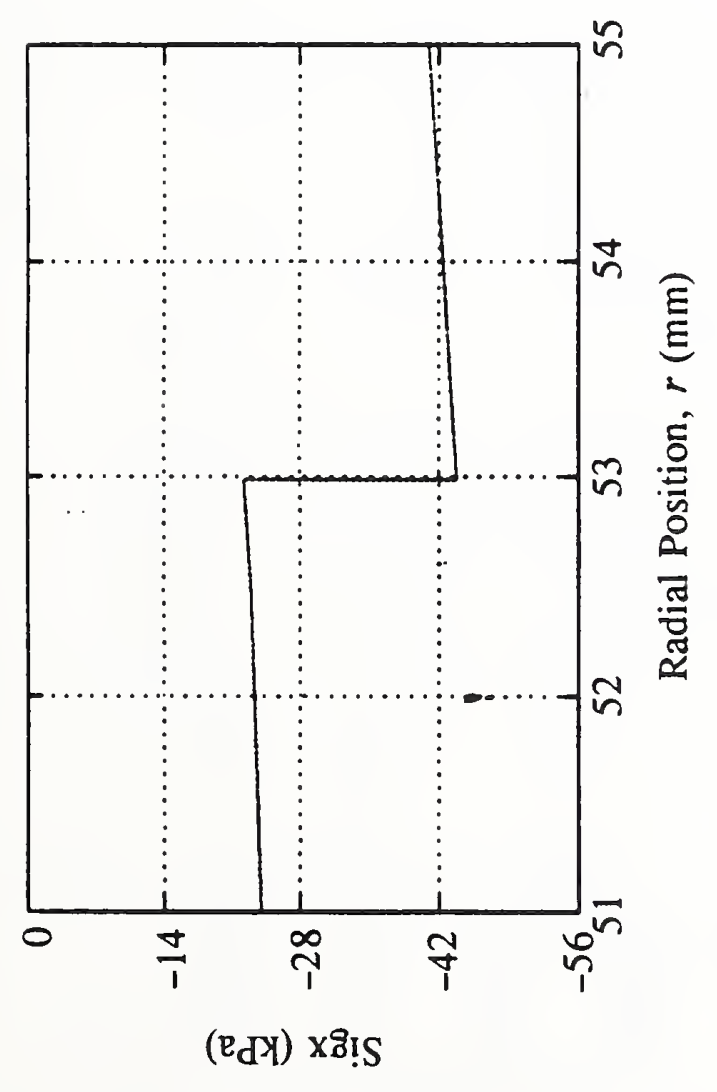

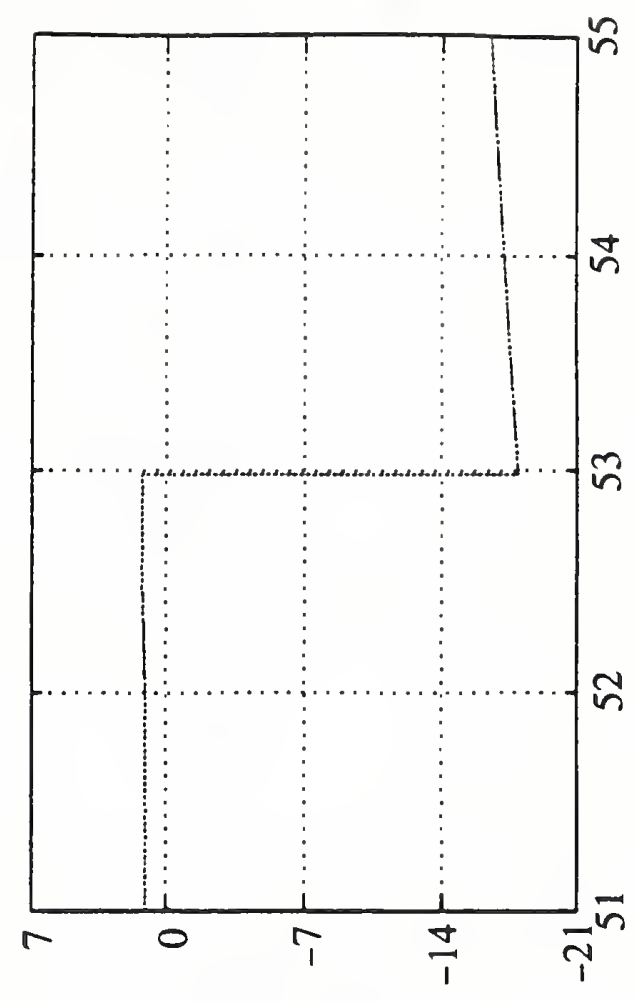

(ह

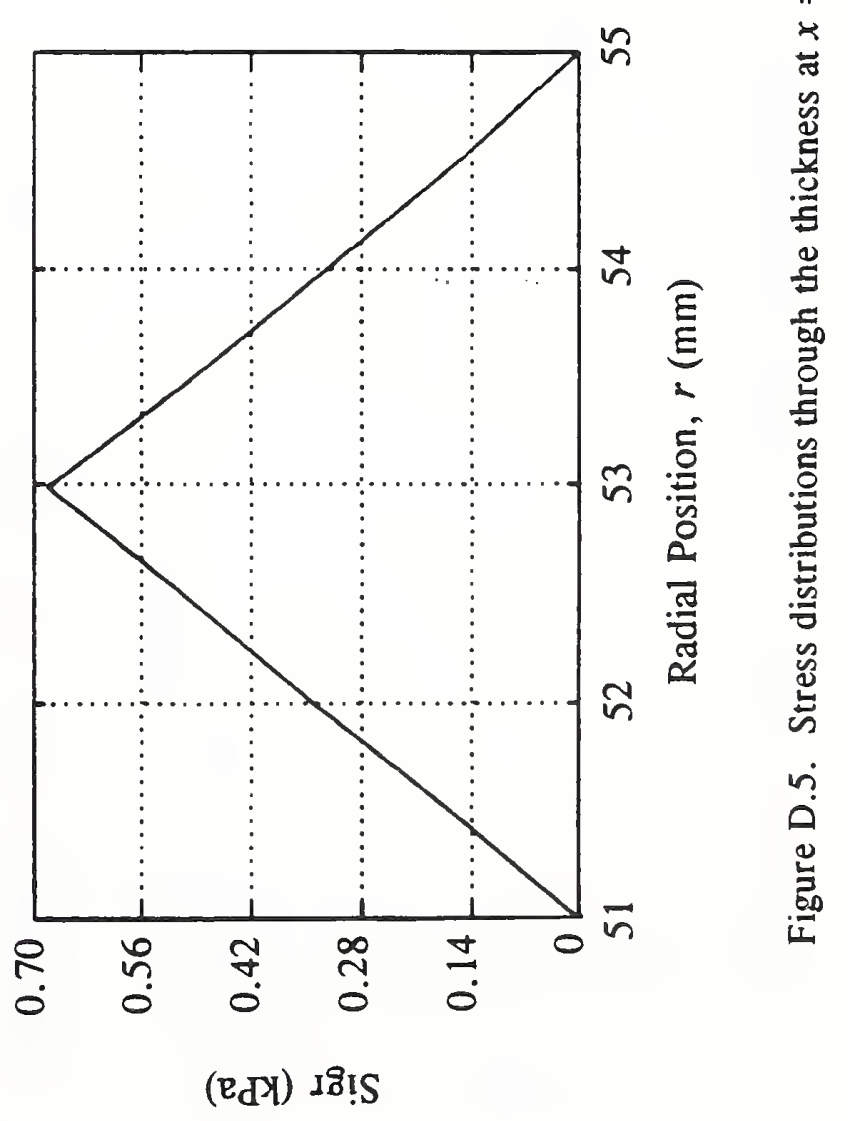



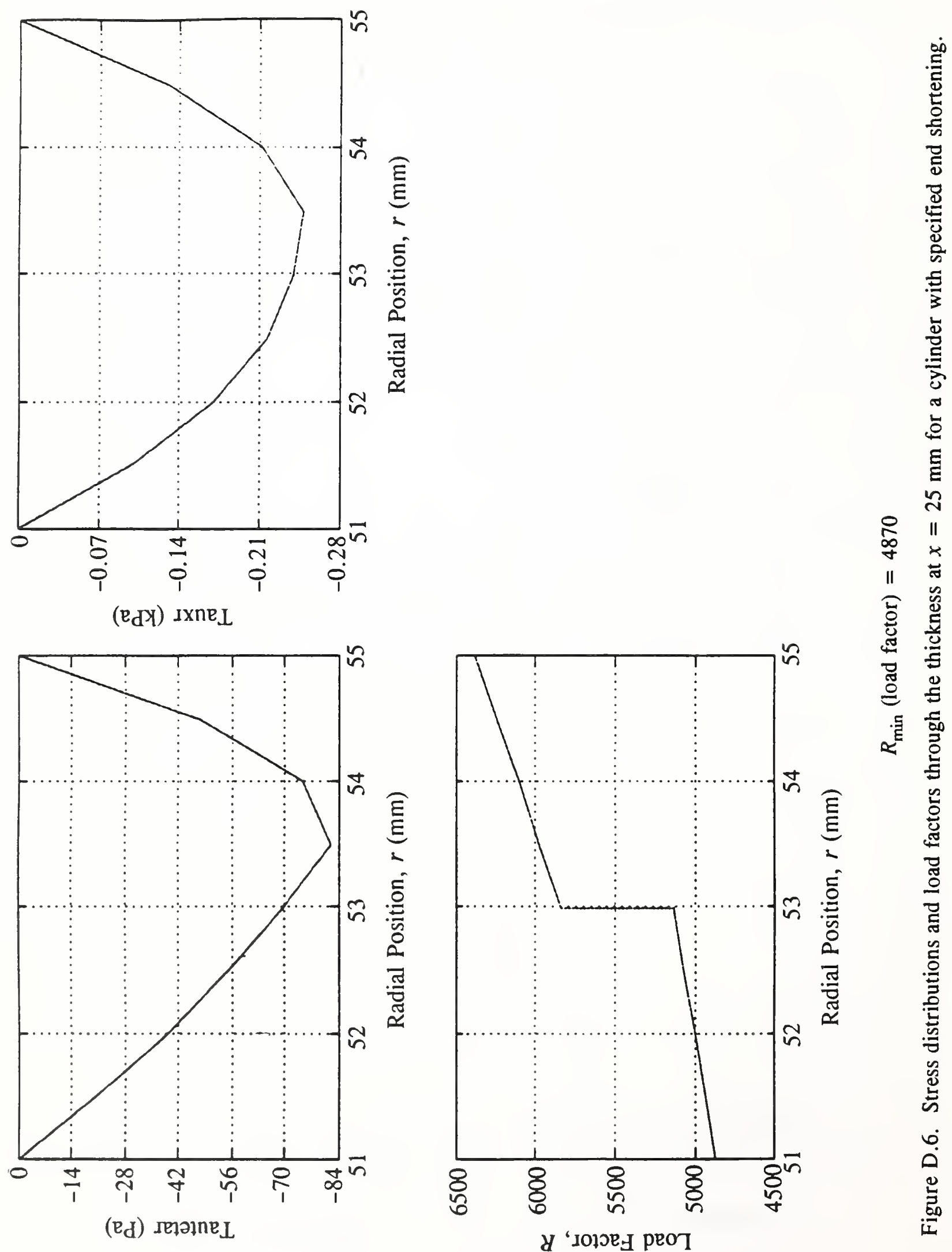


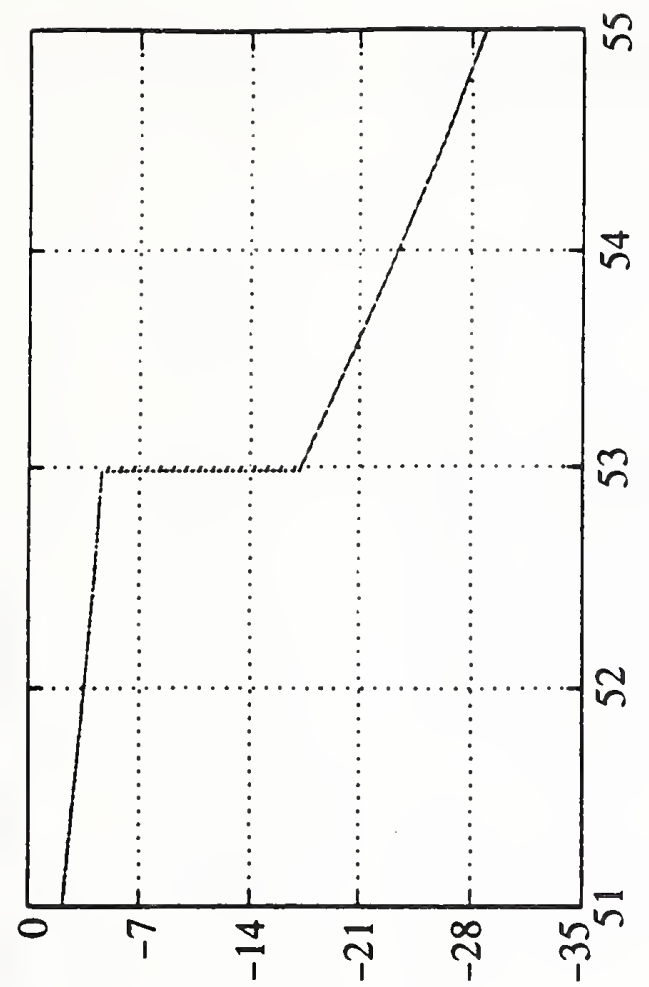

(다) ำวภิ!S

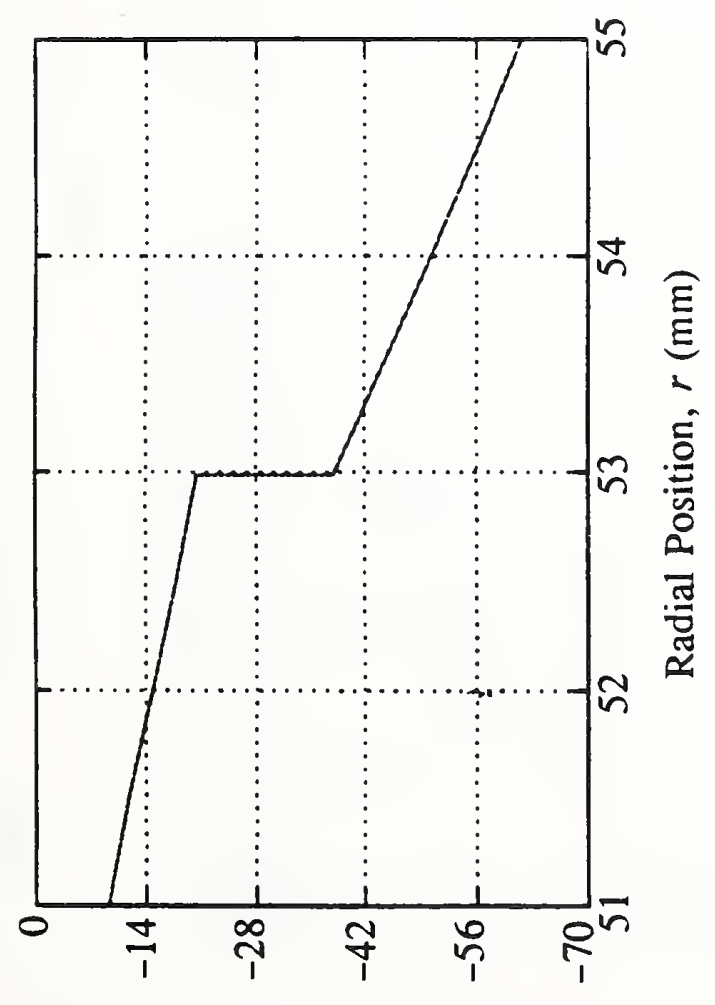

(घ

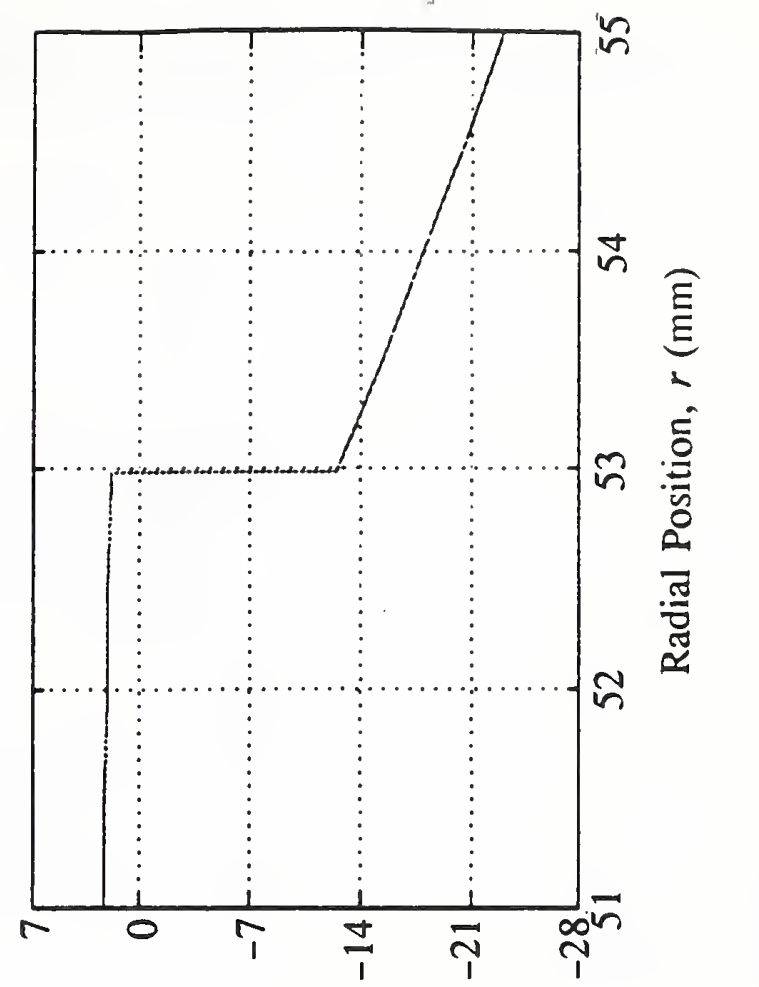

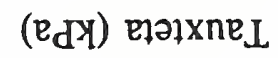

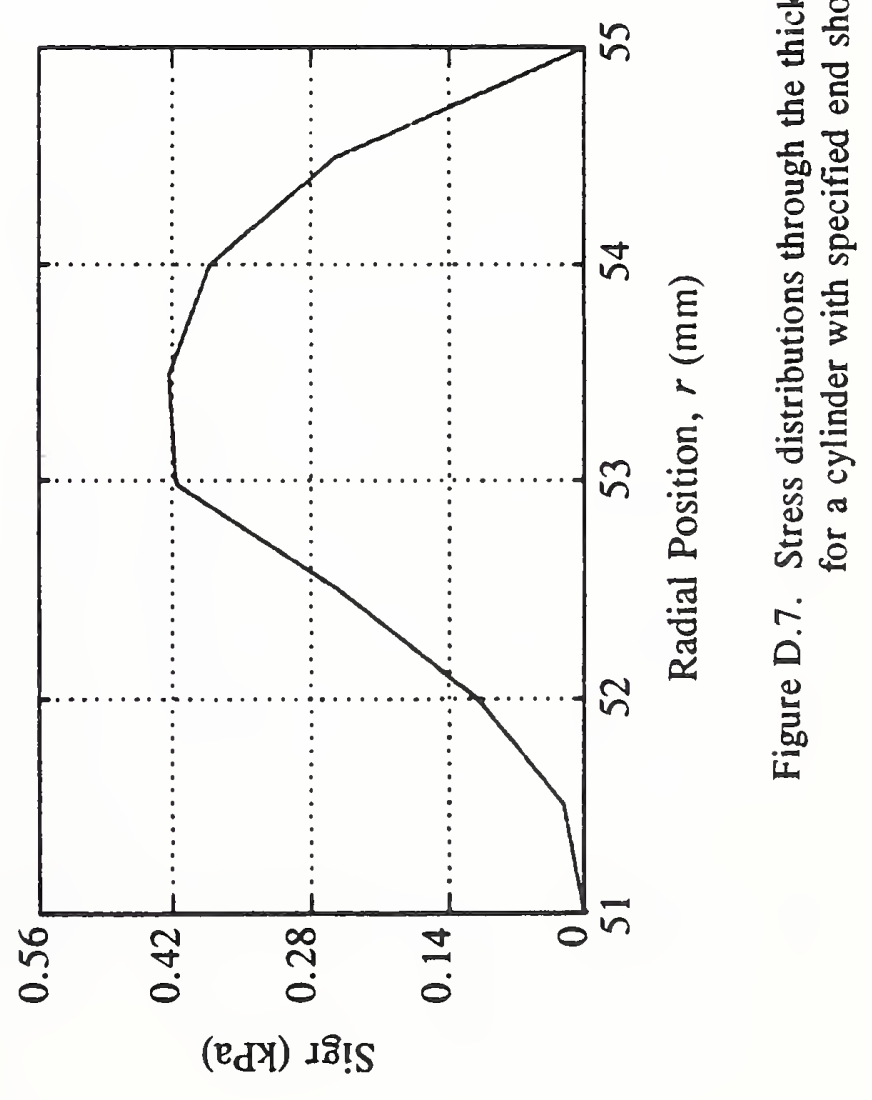



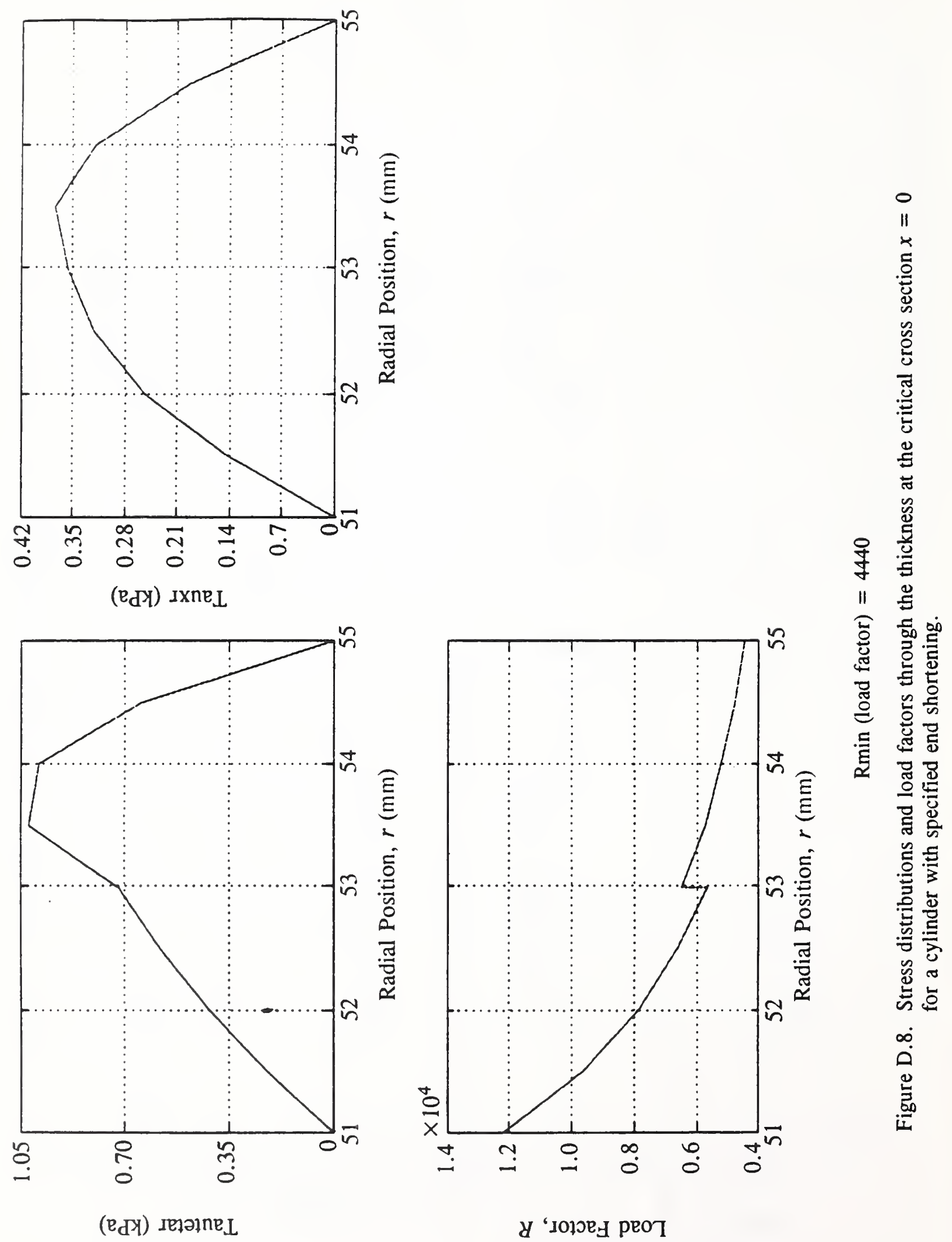
Table D.3. File "xdataload" for a cylinder fixed at both edge日 subjected to linearly varying surface load on the inner surface.

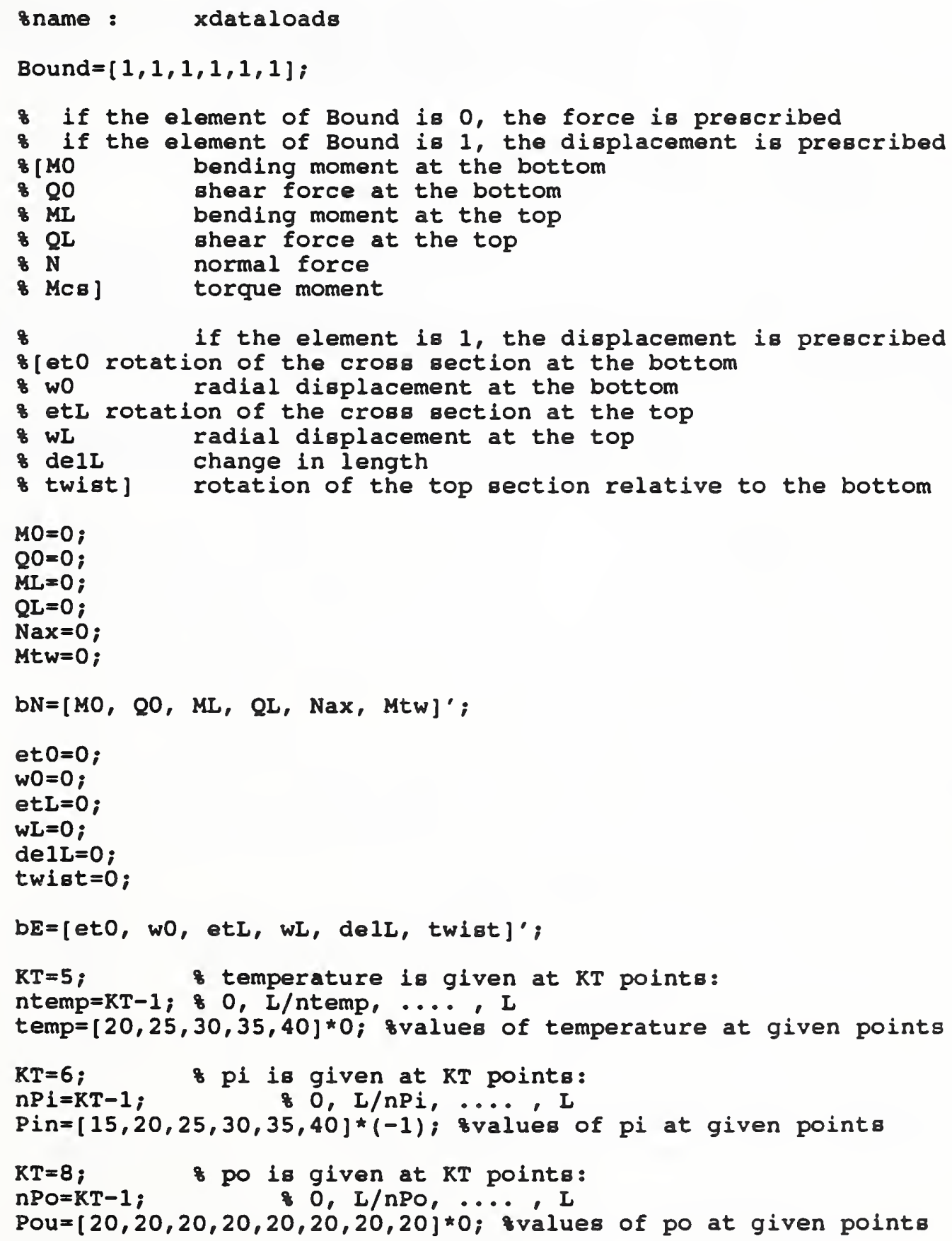


After several seconds we get back the "output list," and the results can be obtained in a similar way as for the first loading case. The results are presented in Figures D. 8 through D.14.

We have to modify and store the file "xdataloads" to get results for the third loading case. The new file is given in Table D.4; the results, obtained similarly to the results of the second loading case, are given in Figures D.15 through D.19.

\section{Cylinder Subjected to Temperature Load Prescribed at the Edges.}

The Material Properties Do Not Vary with $x$.

We consider the cylinder described in the previous section, the temperature of which at the bottom is $252 \mathrm{~K}$ and at the top, $433 \mathrm{~K}$. Before running the code, the thermal conductivity data of the matrix and those of the fiber must be prescribed at equidistant temperatures in the file "xdatconductivity." This file, which is given in Table D.5, also contains the fiber volume fraction and the temperature of the lower and upper ends of the cylinder.

To calculate the temperature distribution, we have to use option 6 from the "outputlist."

Type

$$
\gg 6 \text { Return }
$$

We obtain Figure D.20 on the screen.

Then type

$$
\gg \text { Return }
$$

to get the temperature distribution along the axis of the cylinder, as can be seen in Figure D.21.

To get the results due to this temperature load, we used options 1 through 3 of the "outputlist." The results are presented in Figures

Before quitting, we change the file "xdataloads" back to that given in Table D.2.

Type

$$
\gg 5 \text { Return }
$$

and

$$
\text { Did you change the "xdataloads" file? }(\mathrm{y} / \mathrm{n}) \text { appears. }
$$

Type

$$
\gg y \quad \text { Return }
$$

Type

$$
\gg 0 \text { Return }
$$


Lay-up: $\left[80_{5} / 80_{5} / 80_{5} / 80_{5} / 45_{5} / 45_{5} / 45_{5} / 45_{5}\right]$

inner radius: $50 \mathrm{~mm}$

total thickness: $5 \mathrm{~mm}$

length: $230 \mathrm{~mm}$

thickness of one ply: $130 \mu \mathrm{m}$

\section{Elastic Constants}

$E_{x}=39 \mathrm{GPa}$
$E_{y}=8.3 \mathrm{GPa}$
$E_{z}=8.3 \mathrm{GPa}$

$$
\begin{aligned}
G_{x y} & =4.1 \mathrm{GPa} \\
G_{x z} & =4.1 \mathrm{GPa} \\
G_{y z} & =4.1 \mathrm{GPa}
\end{aligned}
$$$$
\nu_{x y}=0.26
$$$$
\nu_{x z}=0.26
$$$$
\nu_{y z}=0.4
$$

\section{Strength Constants}
$X=1060 \mathrm{MPa}$
$Y=31 \mathrm{MPa}$
$S=72 \mathrm{MPa}$
$X^{\prime}=610 \mathrm{MPa}$
$Y^{\prime}=118 \mathrm{MPa}$
$F_{x y}{ }^{*}=-1 / 2$

\section{Coefficients of Thermal Expansion}

$\alpha_{x}=8.6 \times 10^{-6} /{ }^{\circ} \mathrm{C}$ (in plane, fiber direction)

$\alpha_{x}=22.1 \times 10^{-6} /{ }^{\circ} \mathrm{C}$ (in plane, perpendicular to the fiber)

$\alpha_{x}=22.1 \times 10^{-6} /{ }^{\circ} \mathrm{C}$ (out of plane)

\section{Boundary Displacements and Forces}

at the lower ( 0$)$ and upper $(L)$ edges

radial displacement: $w_{0}=0 ; w_{L}=0$

slope of the meridian: $\eta_{0}=-1.69 \times 10^{-20} ; \eta_{L}=4.74 \times 10^{-20}$

relative axial displacement of the edges: $d_{0 L}=6.86 \times 10^{-22} \mathrm{~m}$

relative twist of the edges: $\mathrm{fi}_{0 L}=-5.42 \times 10^{-20}$

shear force: $Q_{r 0}=684 \mathrm{~N} / \mathrm{m} ; Q_{r L}=-2.24 \mathrm{kN} / \mathrm{m}$

bending moment: $M_{0}=-2.28 \mathrm{~N} \cdot \mathrm{m} / \mathrm{m} ; M_{L}=-10.0 \mathrm{~N} \cdot \mathrm{m} / \mathrm{m}$

normal force: $N_{a x}=60.5 \mathrm{~kg}$

torque moment: $M_{\text {tor }}=36.7 \mathrm{~N} \cdot \mathrm{m}$

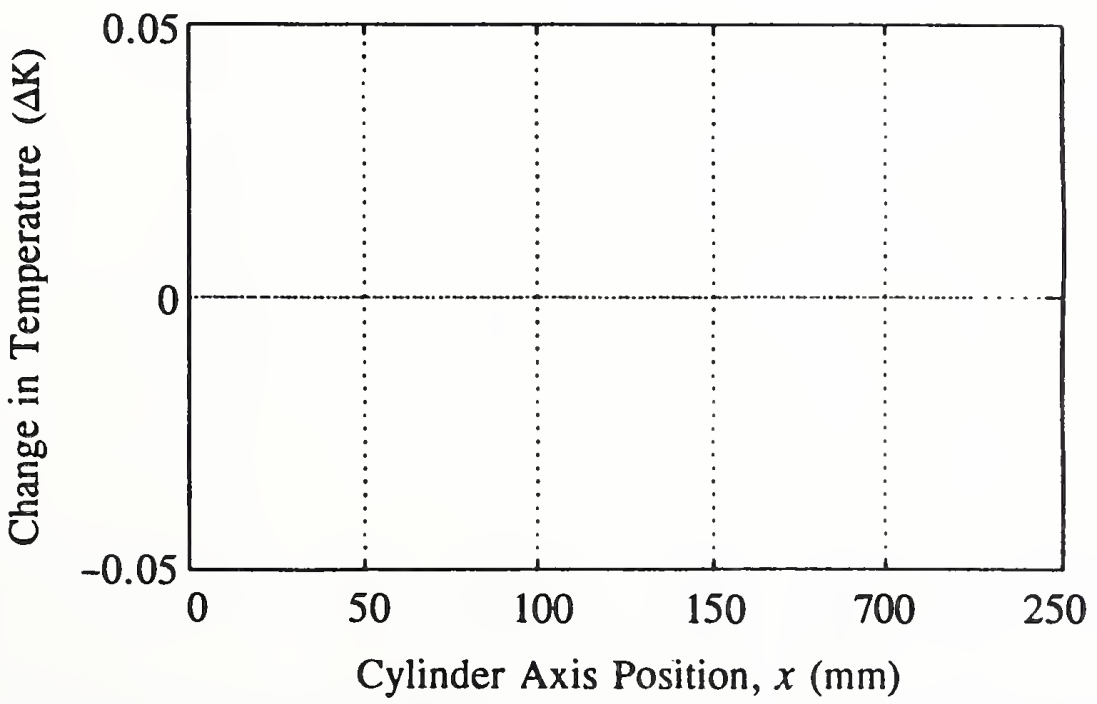

Figure D.9. Input data, edge loads, and edge displacements for a cylinder subjected to surface load. 

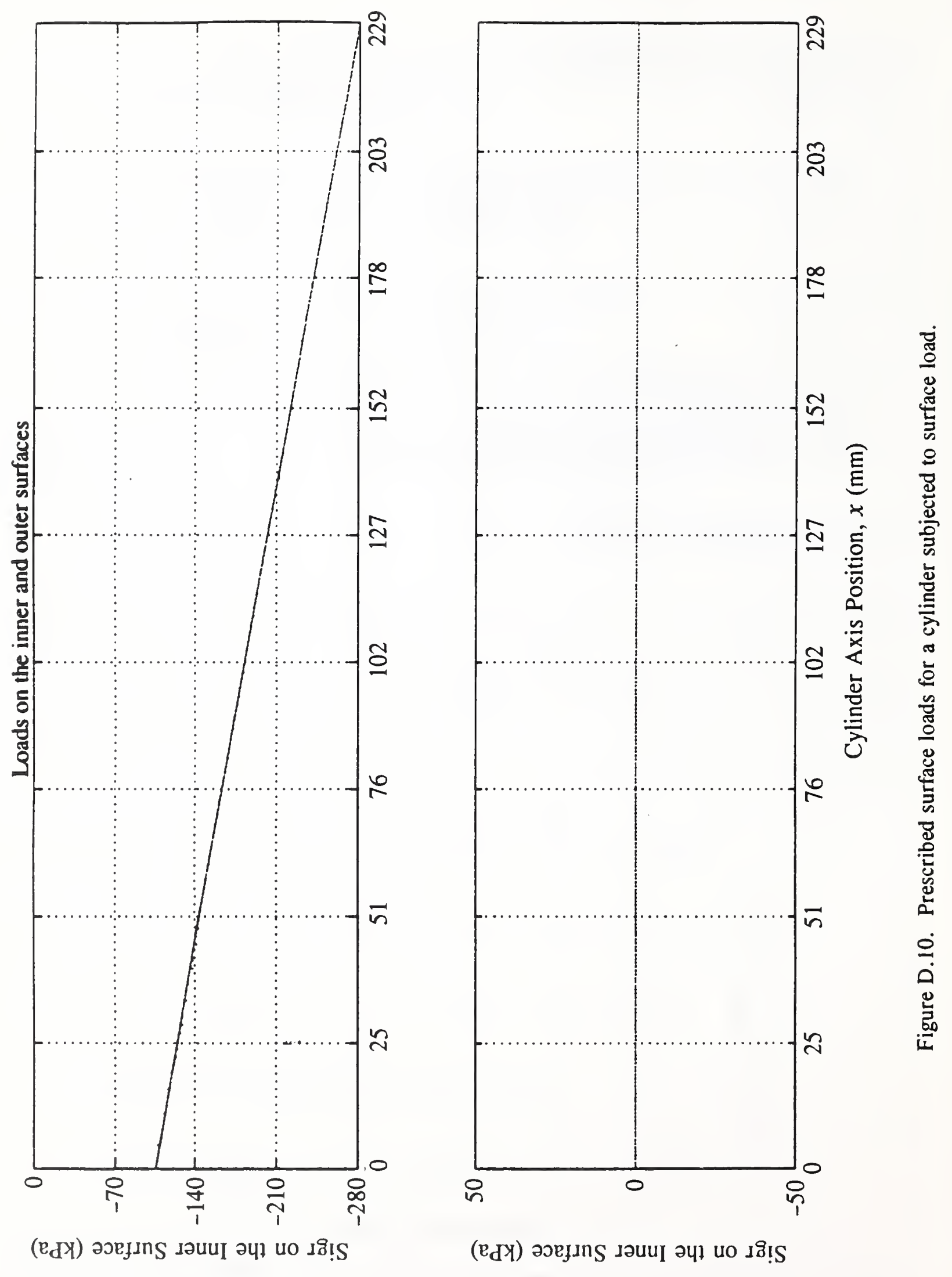


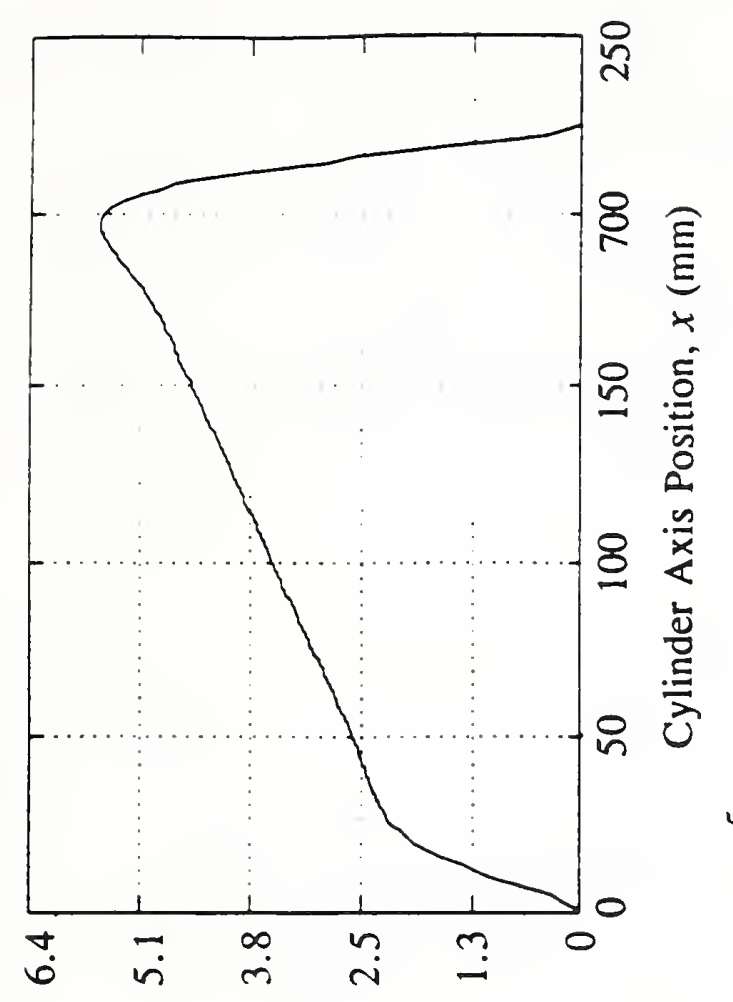

(un) $M$

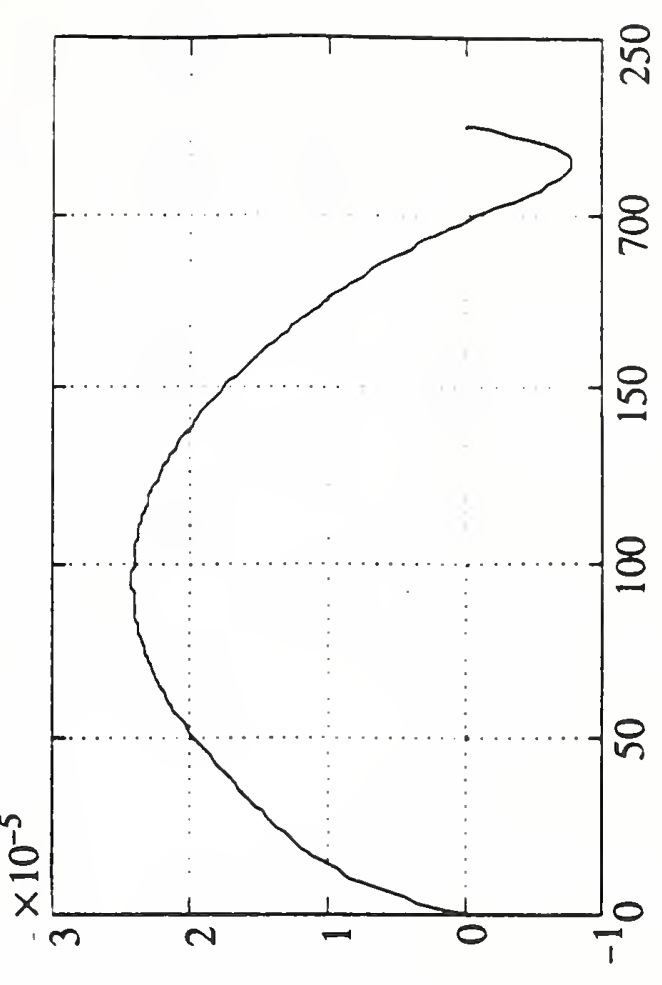

y

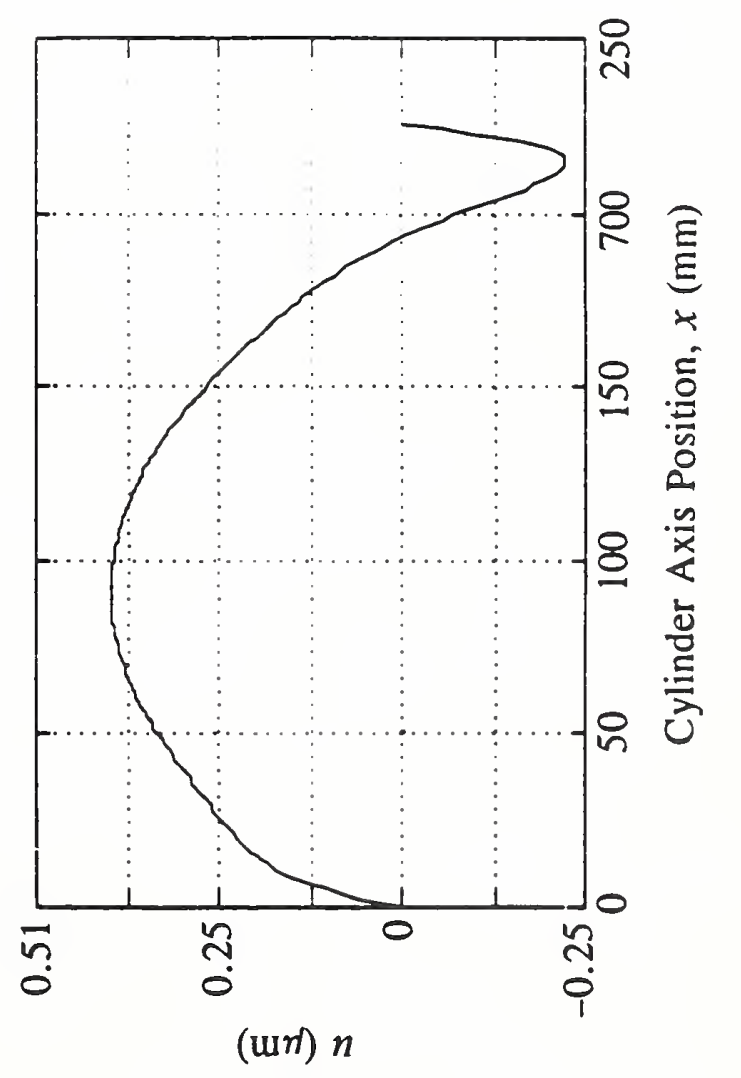

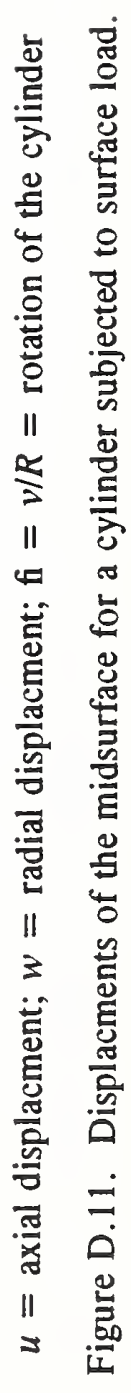



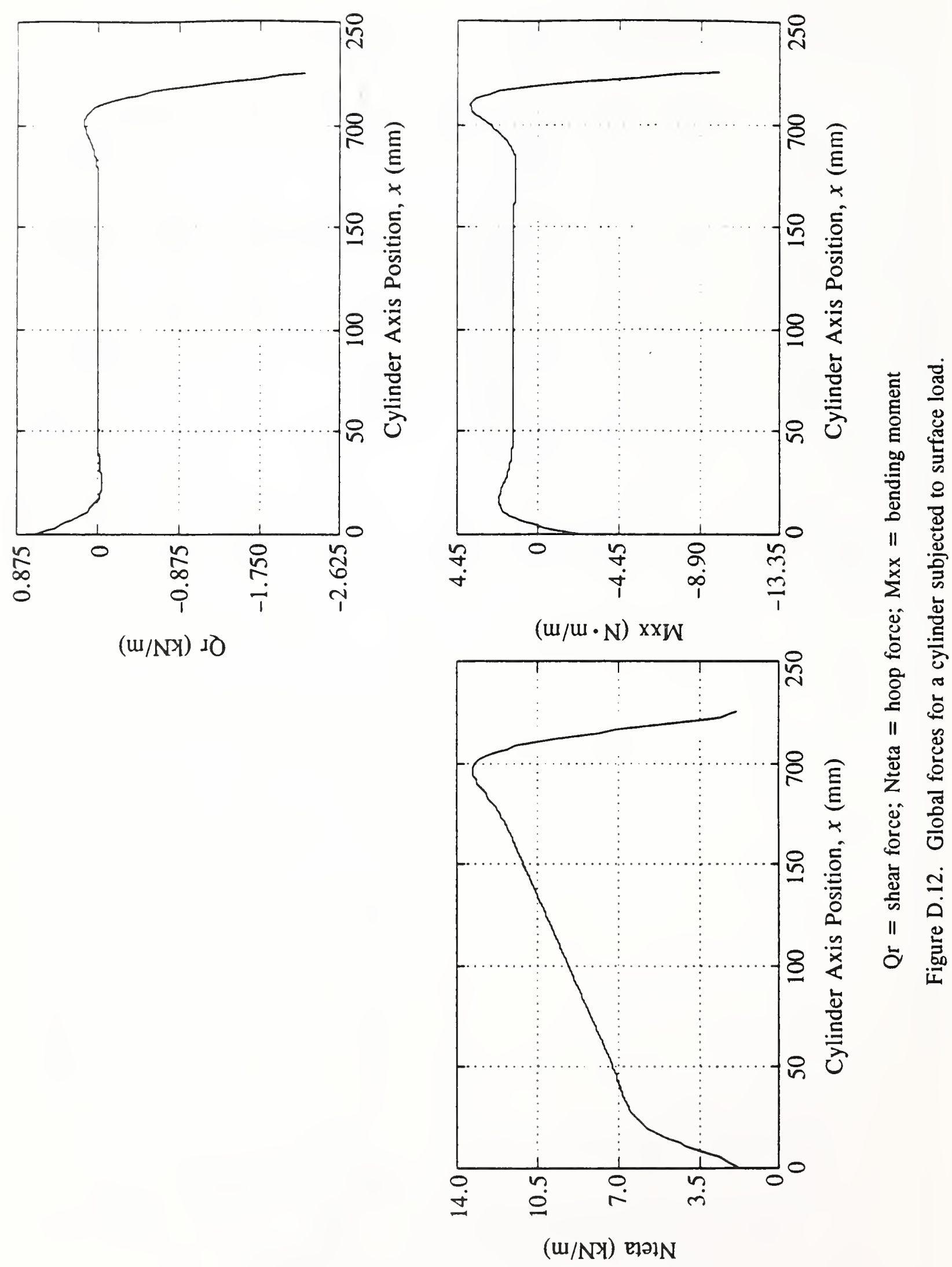

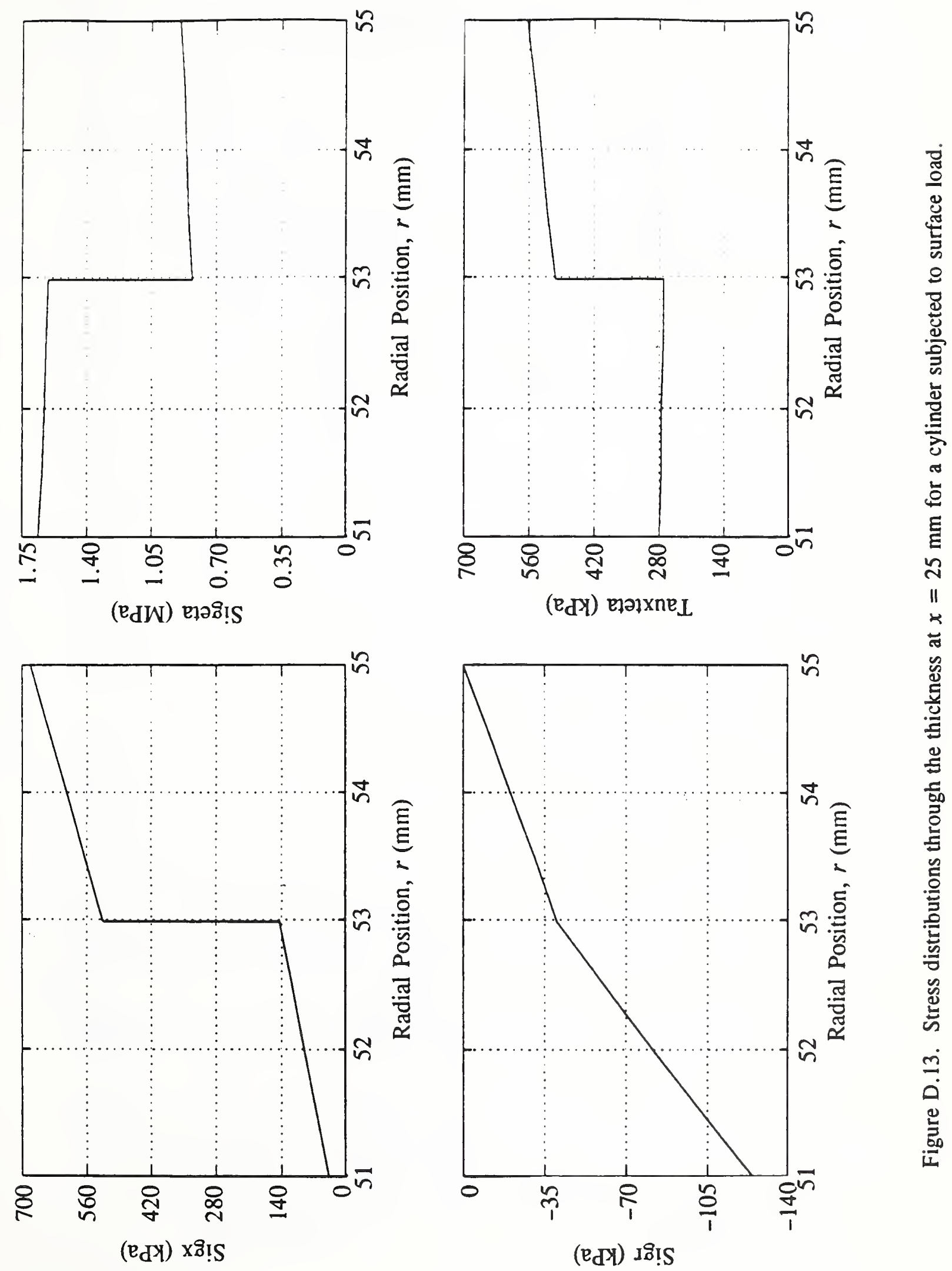

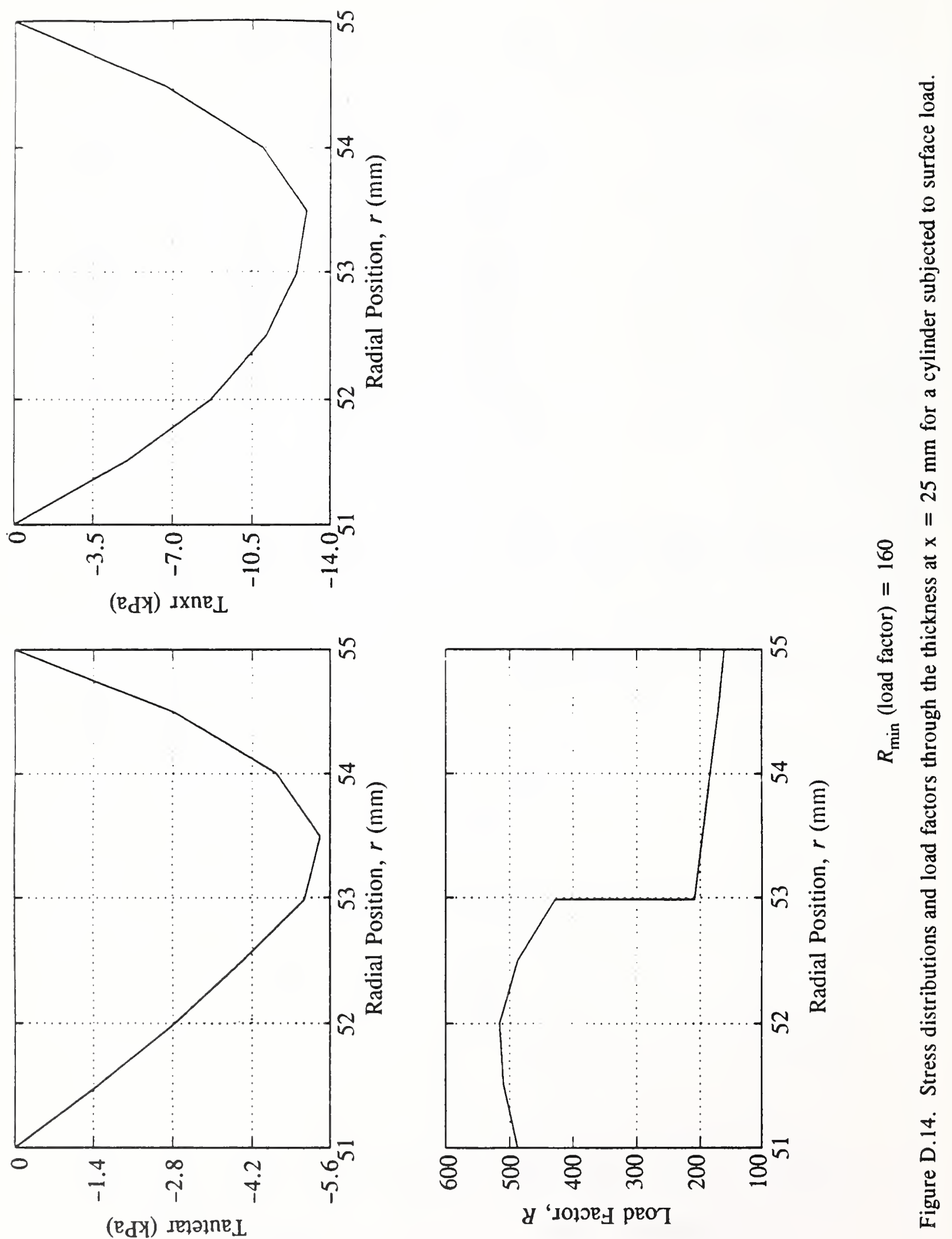
Table D.4. File "xdataload" for a cylinder fixed at both edges

subjected to linearly varying temperature distribution

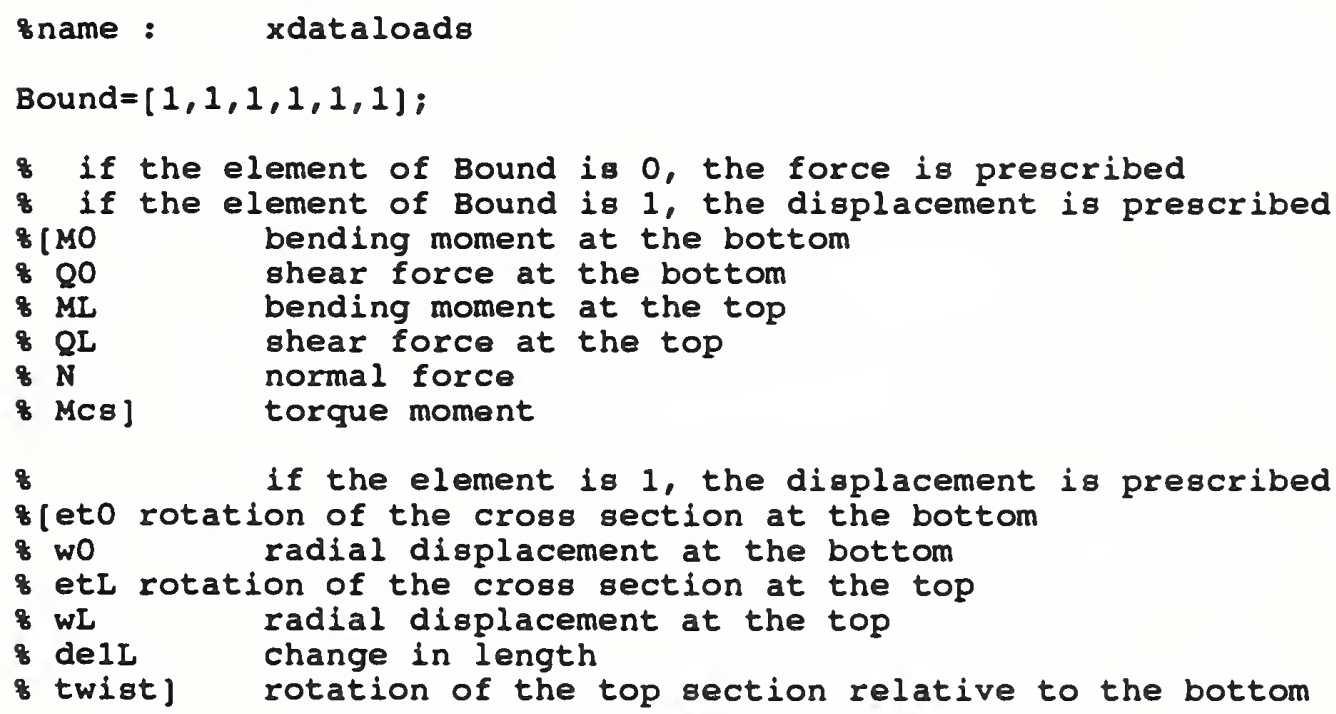


Lay-up: $\left[80_{5} / 80_{5} / 80_{5} / 80_{5} / 45_{5} / 45_{5} / 45_{5} / 45_{5}\right]$

inner radius: $50 \mathrm{~mm}$

length: $230 \mathrm{~mm}$

\section{Elastic Constants}

$E_{x}=39 \mathrm{GPa}$

$E_{y}=8.3 \mathrm{GPa}$

$E_{z}=8.3 \mathrm{GPa}$ total thickness: $5 \mathrm{~mm}$ thickness of one ply: $130 \mu \mathrm{m}$

\section{Strength Constants}
$X=1060 \mathrm{MPa}$
$Y=31 \mathrm{MPa}$
$S \quad=72 \mathrm{MPa}$
$X^{\prime}=610 \mathrm{MPa}$
$Y^{\prime}=118 \mathrm{MPa}$

$$
\begin{aligned}
& \nu_{x y}=0.26 \\
& \nu_{x z}=0.26 \\
& \nu_{y z}=0.4
\end{aligned}
$$

\section{Coefficients of Thermal Expansion}

$\alpha_{x}=8.6 \times 10^{-6} /{ }^{\circ} \mathrm{C}$ (in plane, fiber direction)

$\alpha_{x}=22.1 \times 10^{-6} /{ }^{\circ} \mathrm{C}$ (in plane, perpendicular to the fiber)

$\alpha_{x}=22.1 \times 10^{-6} /{ }^{\circ} \mathrm{C}$ (out of plane)

\section{Boundary Displacements and Forces}

at the lower $(0)$ and upper $(L)$ edges

radial displacement: $w_{0}=-1.10 \times 10^{-20} \mathrm{~m} ; w_{L}=-2.20 \times 10^{-20} \mathrm{~m}$

slope of the meridian: $\eta_{0}=7.86 \times 10^{-19} ; \eta_{L}=5.69 \times 10^{-19}$

relative axial displacement of the edges: $d_{0 L}=1.76 \times 10^{-19} \mathrm{~m}$

relative twist of the edges: $\mathrm{fi}_{O L}=0$

shear force: $Q_{r 0}=31.7 \mathrm{kN} / \mathrm{m} ; Q_{r L}=-55.8 \mathrm{kN} / \mathrm{m}$

bending moment: $M_{0}=188 \mathrm{~N} \cdot \mathrm{m} / \mathrm{m} ; M_{L}=284 \mathrm{~N} \cdot \mathrm{m} / \mathrm{m}$

normal force: $N_{a x}=-5.27 \mathrm{Mg}$

torque moment: $M_{\text {tor }}=179 \mathrm{~N} \cdot \mathrm{m}$

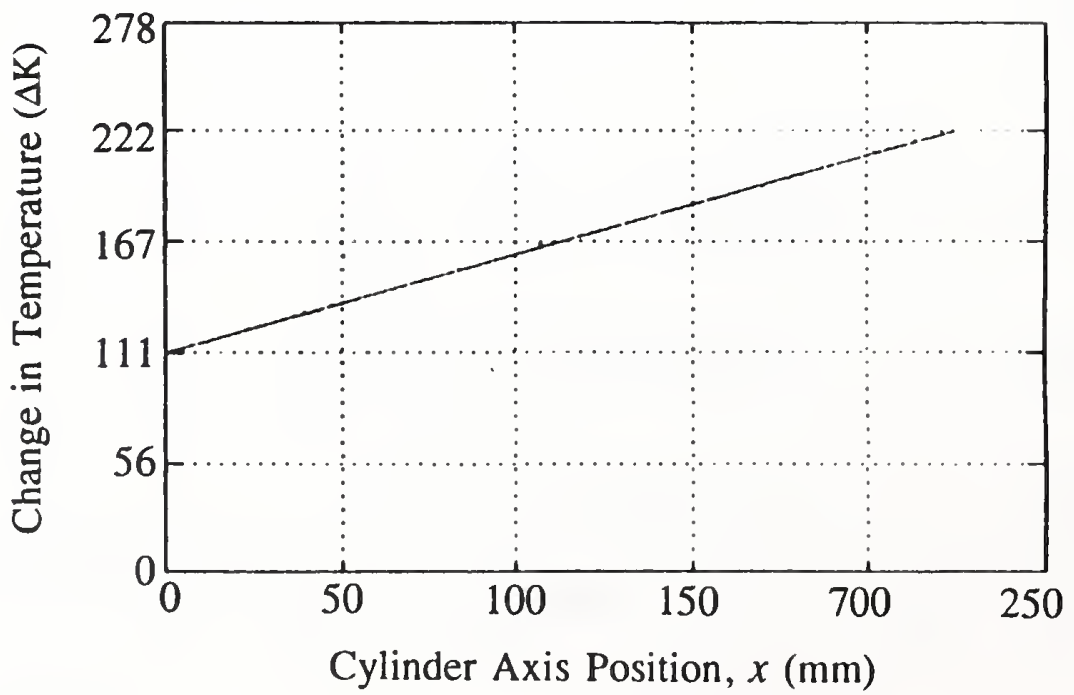

Figure D.15. Input data, edge loads, and edge displacments subjected to temperature load. 

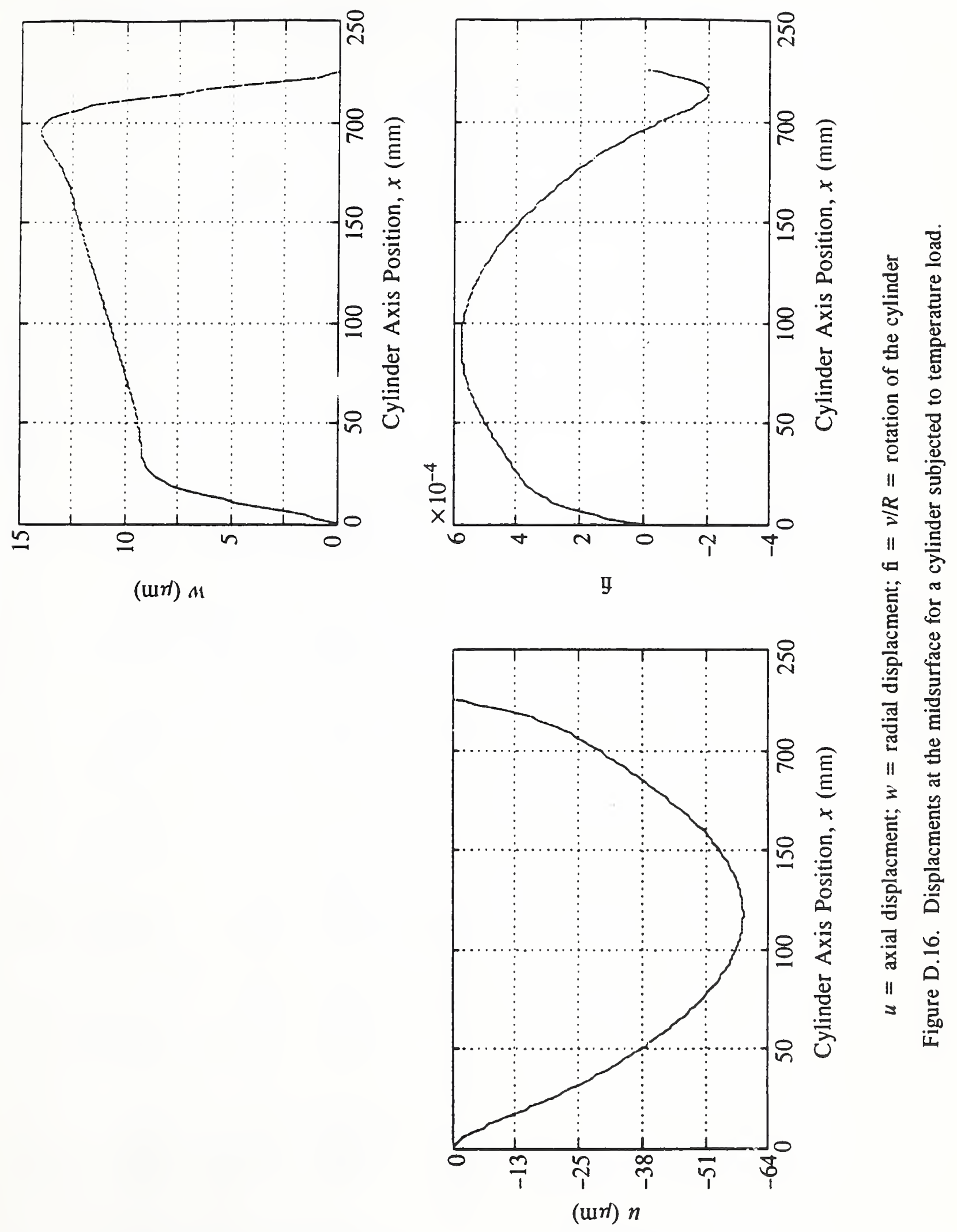
(U/NY) IO
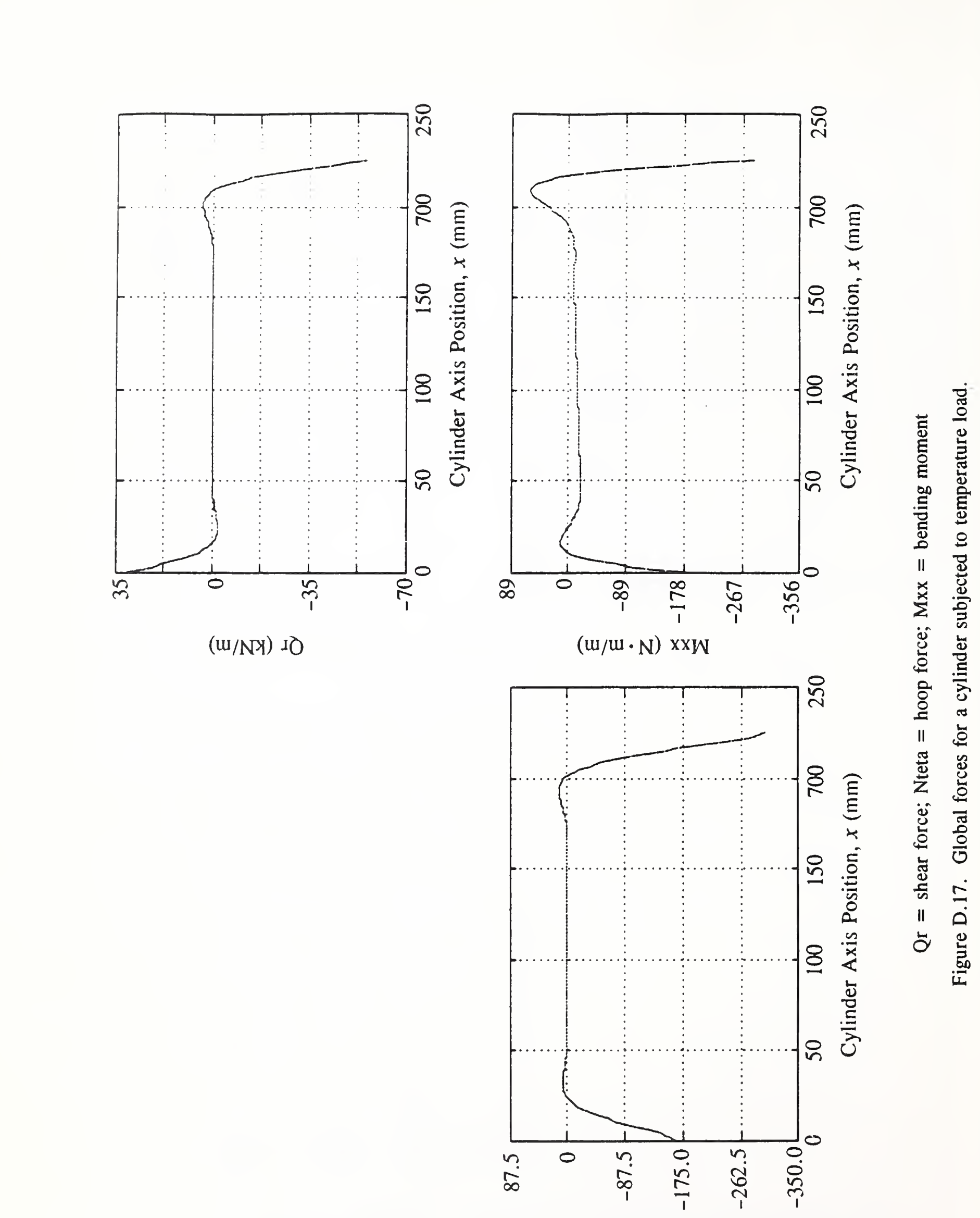

(W/NY) ๒IวIN 


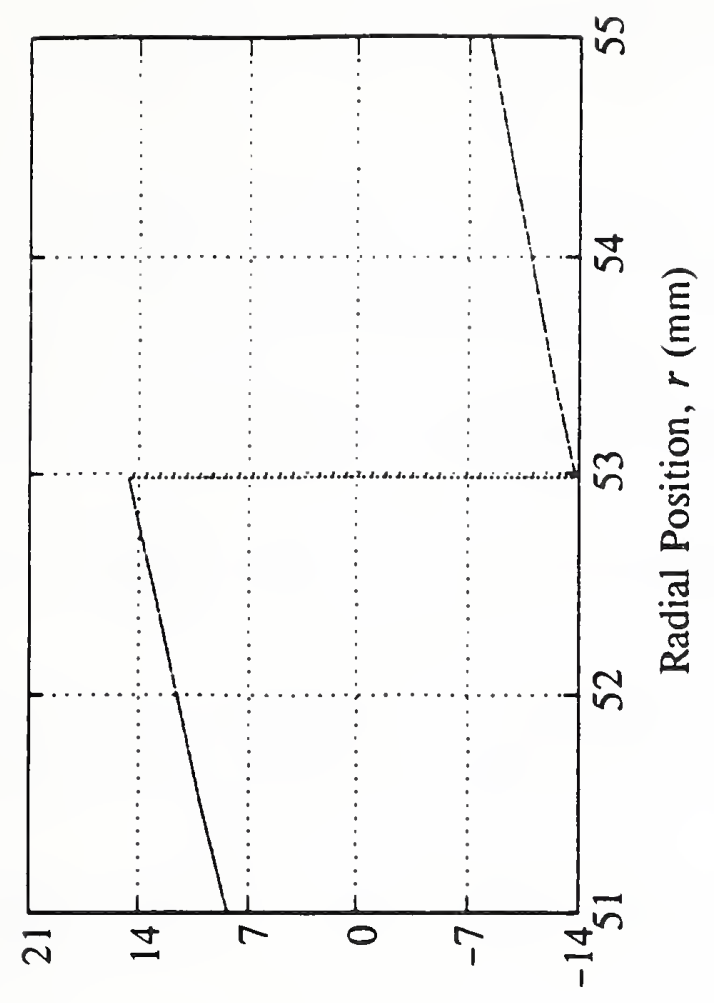

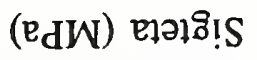

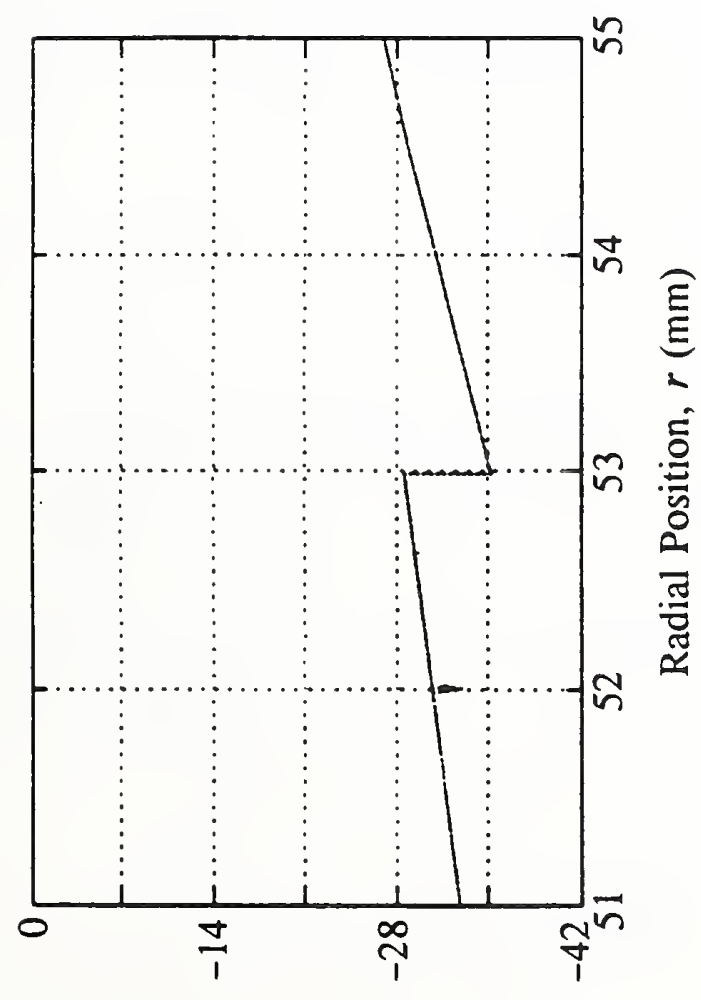

(घ)WW) xô!

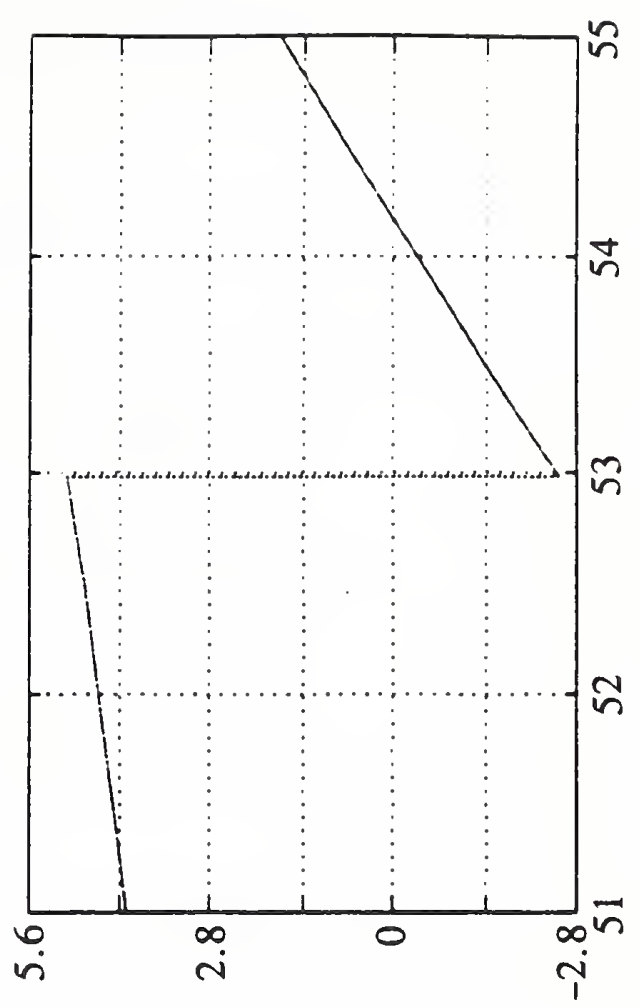

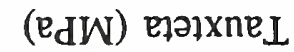

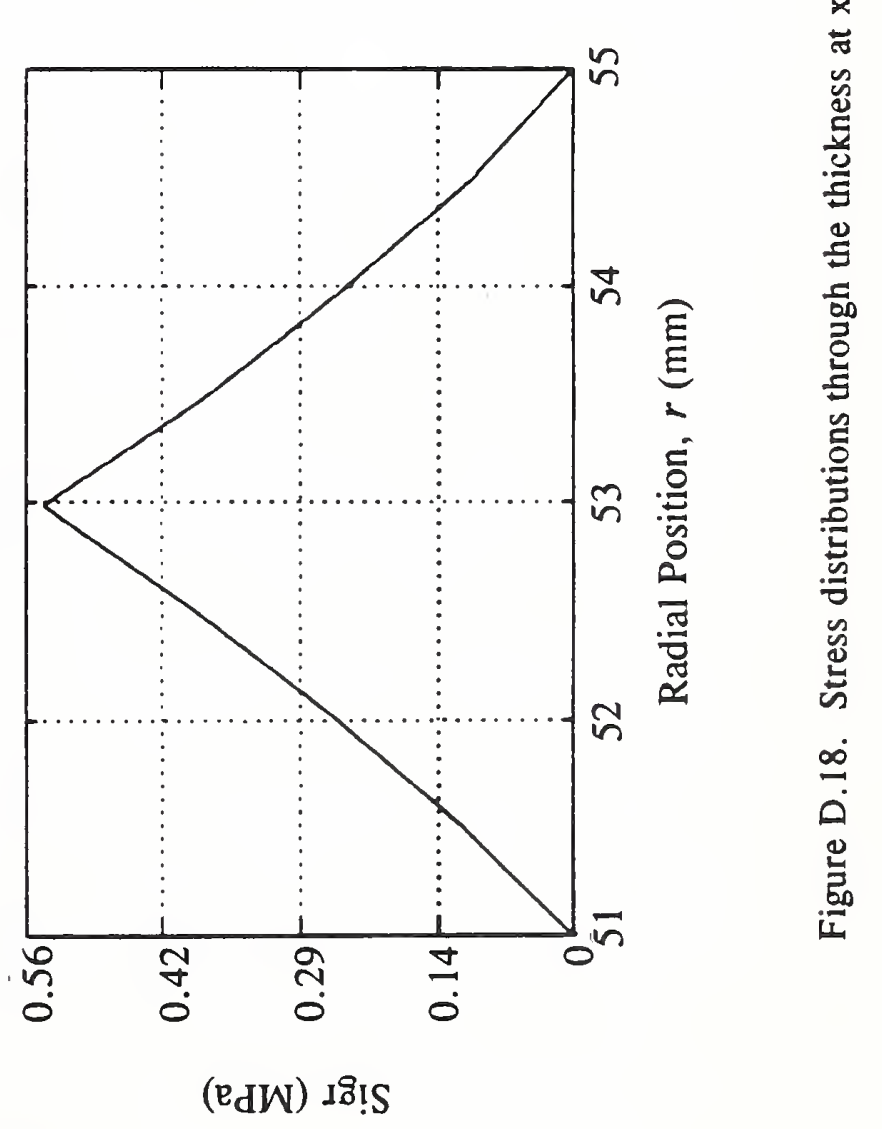




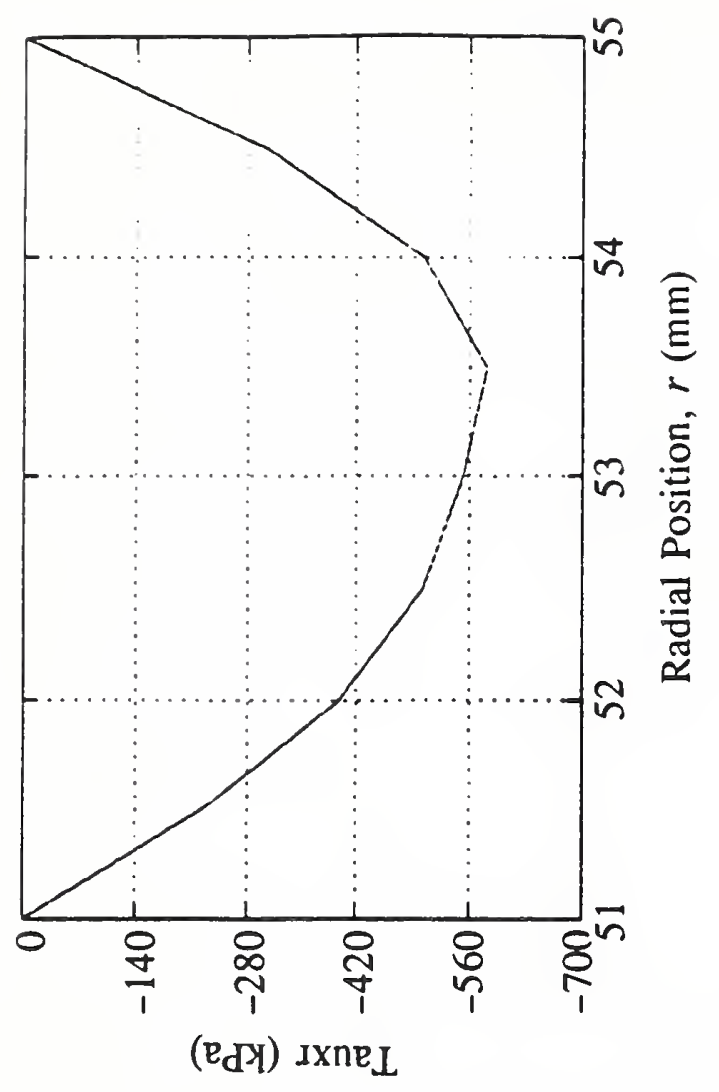

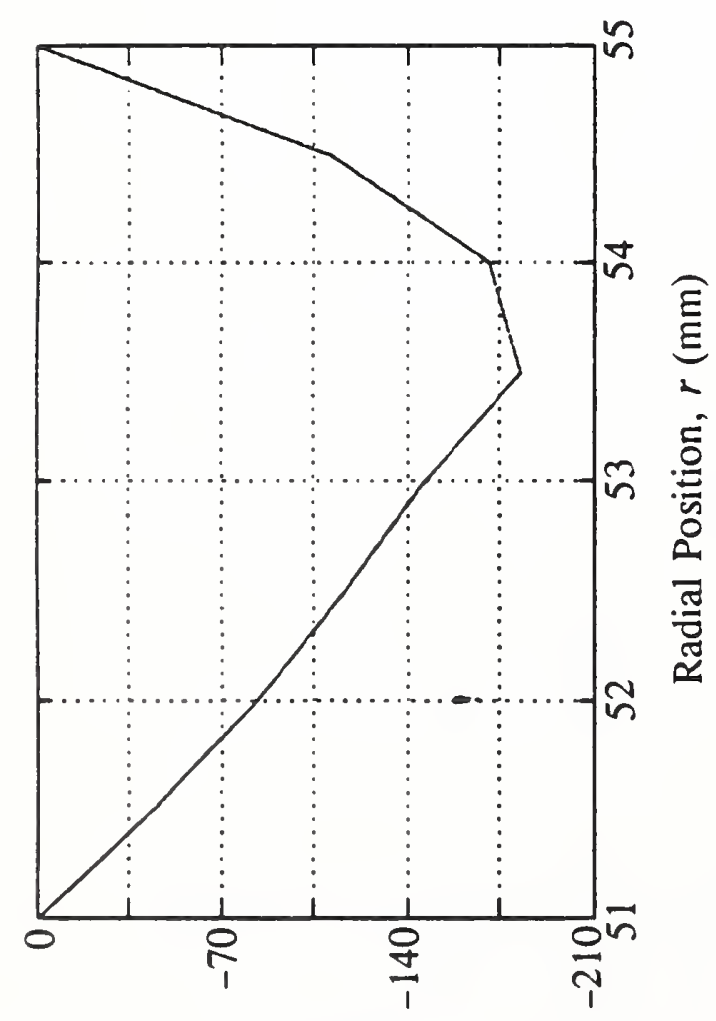

(edX) IEฺฺกए

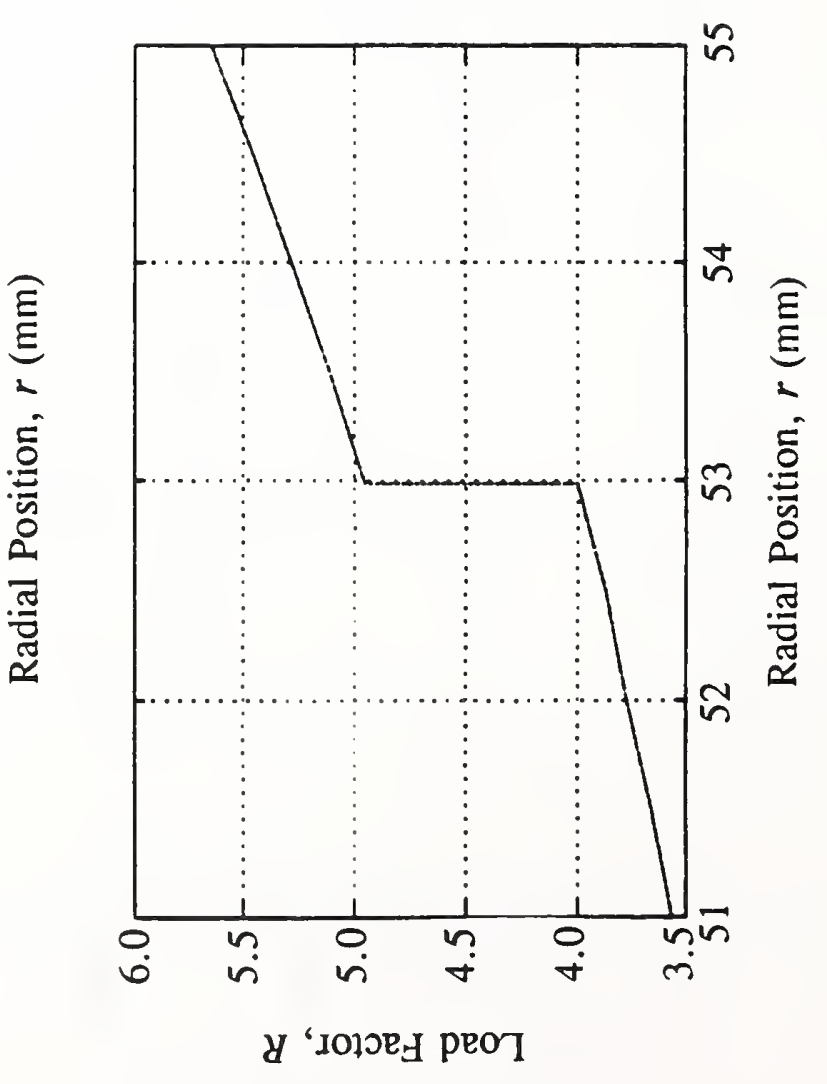

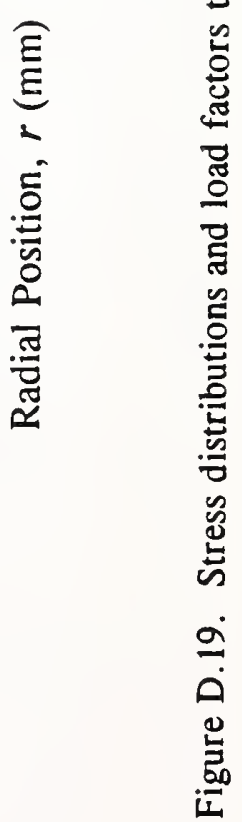


Table D.5. File "xdataconductivity" contains the thermal conductivity data for the matrix and the fiber at equidistant temperatures.

8 xdataconductivity

\& The thermal conductivity is given at $\mathrm{Kn}$ different temperatures.

o the lowest temperature is $\mathrm{Ta}$, the upper is $\mathrm{Tb}$. There are

\& $\mathrm{Kn}-2$ equid. points in between.

\& Vector hamkm contains the thermal conductivity

of the matrix

\& Vectors hamkfpa, and hamkfpe contain the thermal conductivities

of the fiber parallel and perpendicular to the fiber axis,

\& for $\mathrm{Kn}$ different temperatures

\% The program calculate the temperature at KT points.

8 The temperature is given at the lower and upper edges (T1 and Tu).

\& $[\mathrm{T} a<=\mathrm{T} 1<\mathrm{Tu}<=\mathrm{Tb}]$

$\mathrm{Kn}=23$;

hamKm= [ ] i

\% number of points (thermal conductivity)

hamKfpa=[]; \& thermal conductivity of fibers parallel to fib. direction

ramkfpe=l]; o thermal conductivity of fibers perpendicular to fib. direction

\begin{tabular}{|c|c|c|c|c|c|c|c|c|c|c|c|c|}
\hline $\begin{array}{l}\text { h: } \\
\text { hamKfpa= } \\
\text { hamRfpe }=[.032 \\
\text { hamKm }=[.008 \\
0.1794] ;\end{array}$ & $\begin{array}{r}5 \\
.103 \\
.067 \\
.063\end{array}$ & $\begin{array}{r}2 \\
.17 \\
.12 \\
.08\end{array}$ & & $\begin{array}{r}35 \\
.228 \\
.168 \\
.101\end{array}$ & $\begin{array}{r}50 \\
.277 \\
.208 \\
.12\end{array}$ & $\begin{array}{r}65 \\
.332 \\
.248 \\
.138\end{array}$ & $\begin{array}{r}80 \\
.368 \\
.270 \\
.150\end{array}$ & & $\begin{array}{r}95 \\
424 \\
309 \\
162\end{array}$ & $\begin{array}{l}110 \\
.463 \\
.332 \\
.1687\end{array}$ & $\begin{array}{r}125 \\
.497 \\
.353 \\
0.17\end{array}$ & $\begin{array}{r}140 \\
.528] \\
.371] \\
745^{3}\end{array}$ \\
\hline $\begin{array}{l}\text { 8 } \mathrm{T}: \\
\text { hamRfpa= [ hamRfpa } \\
\text { hamRfpe= [ hamRfpe } \\
\text { hamRm }=\text { [ hamRm } 0.18 \\
0.1862 \quad 0.183\end{array}$ & $\begin{array}{l}155 \\
.555 \\
.387 \\
834 \\
30\end{array}$ & $\begin{array}{r}170 \\
.579 \\
.402 \\
0.186 \\
0.1\end{array}$ & $\begin{array}{r}185 \\
.602 \\
.416 \\
5 \\
.789\end{array}$ & $\begin{array}{rr}5 & 200 \\
2 & .623 \\
6 & .428 \\
0.1887\end{array}$ & $\begin{array}{r}215 \\
.629 \\
.428 \\
7.1735 \\
0.173\end{array}$ & $\begin{aligned} & 230 \\
. & 636 \\
. & 429 \\
0 . & 1900 \\
9 & \end{aligned}$ & $\begin{array}{r}245 \\
.643 \\
.429 \\
0.16\end{array}$ & $\begin{array}{l}260 \\
.65 \\
.429 \\
0.190 \\
6801\end{array}$ & $\begin{array}{l}275 \\
.657 \\
9.429 \\
04\end{array}$ & $\begin{array}{l}290 \\
.663 \\
.43 \\
0.1899\end{array}$ & $\begin{array}{l}305 \\
.67 \\
.43 \\
.\end{array}$ & $\begin{array}{l}320 \\
.675] i \\
.43] i \\
.1885\end{array}$ \\
\hline
\end{tabular}

$v f=.63 ;$

$\mathrm{Ta}=10 ; \mathrm{Tb}=320$;

\& fiber volume frac.

$\mathrm{KT}=40$;

$\mathrm{T} 1=5 ; \mathrm{Tu}=320$;

\& temperature at $\mathrm{KT}$ points

matrix $=$ 'Derakane $8084^{\prime}$

$a=.02992 ; b=-.33242 ; c=-.00592 ; d=.03143 ; e=0.00087 ;$

$\mathrm{T}=5: 15: 320$;

$\operatorname{hamRm}=\left(a+c * \log (T)+e^{*}(\log (T)) \cdot-2\right) \cdot /(1+b * \log (T)+d *(\log (T)) \cdot-2)$;

subplot (111):

$\mathrm{T}=\left[\begin{array}{ll}-10 \mathrm{~T} & \mathrm{~T}\end{array}\right.$

hamKm=[(2*hamkm(1)-hamkm(2)) hamKm ];

plot ( $T$, hamKm) ; grid;

title (matrix) 


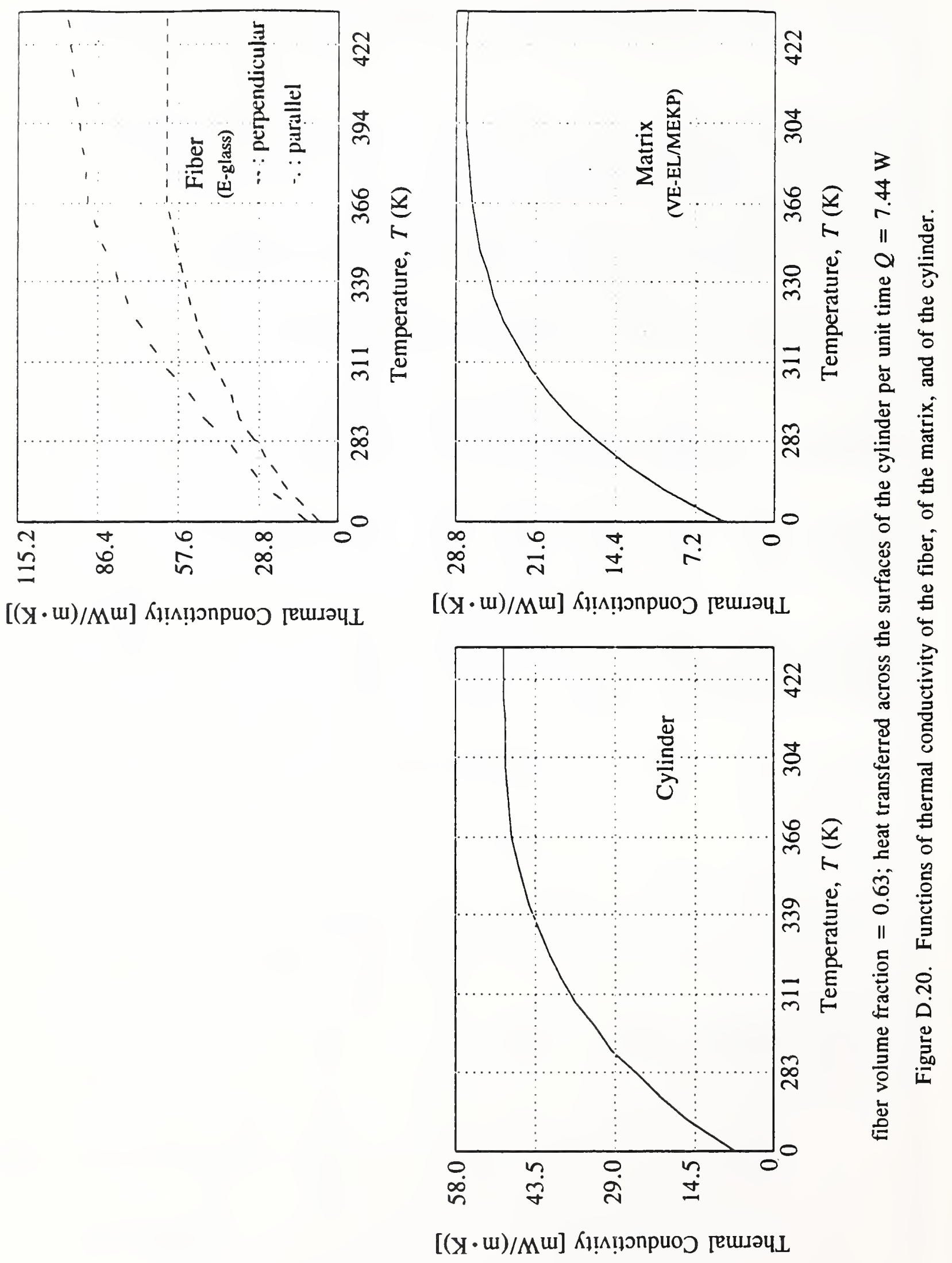




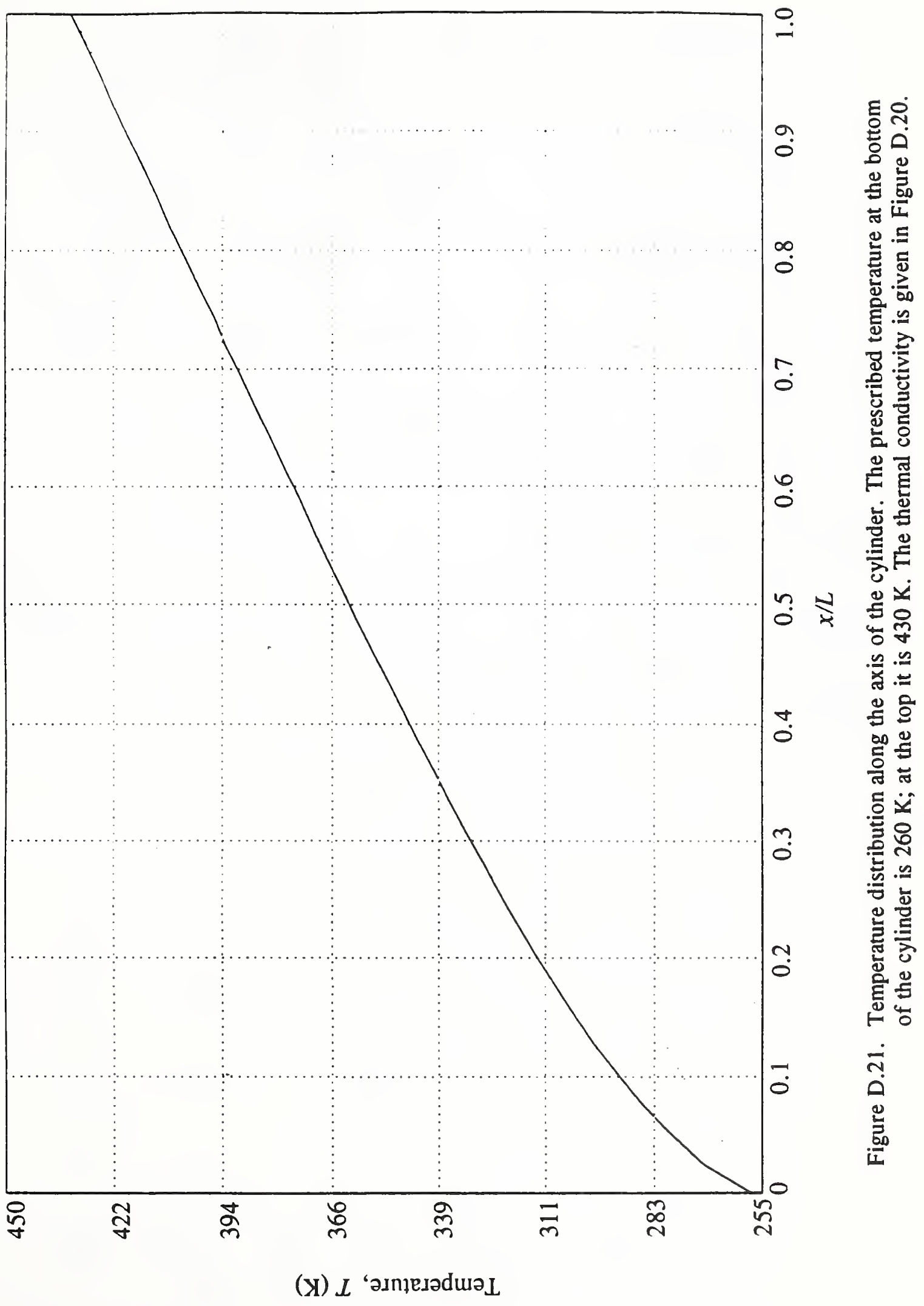


Lay-up: $\left[80_{5} / 80_{5} / 80_{5} / 80_{5} / 45_{5} / 45_{5} / 45_{5} / 45_{5}\right]$

inner radius: $50 \mathrm{~mm}$

length: $230 \mathrm{~mm}$

total thickness: $5 \mathrm{~mm}$

thickness of one ply: $130 \mu \mathrm{m}$

Elastic Constants

$E_{x}=39 \mathrm{GPa}$
$E_{y}=8.3 \mathrm{GPa}$
$E_{z}=8.3 \mathrm{GPa}$

$G_{x y}=4.1 \mathrm{GPa}$
$G_{x z}=4.1 \mathrm{GPa}$
$G_{y z}=4.1 \mathrm{GPa}$

$$
\begin{aligned}
& \nu_{x y}=0.26 \\
& \nu_{x z}=0.26 \\
& \nu_{y z}=0.4
\end{aligned}
$$

Strength Constants
$X=1060 \mathrm{MPa}$
$Y=31 \mathrm{MPa}$
$X^{\prime}=610 \mathrm{MPa}$
$Y^{\prime}=118 \mathrm{MPa}$
$S=72 \mathrm{MPa}$
$F_{x y}{ }^{*}=-1 / 2$

\section{Coefficients of Thermal Expansion}

$\alpha_{x}=8.6 \times 10^{-6} /{ }^{\circ} \mathrm{C}$ (in plane, fiber direction)

$\alpha_{x}=22.1 \times 10^{-6} /{ }^{\circ} \mathrm{C}$ (in plane, perpendicular to the fiber)

$\alpha_{x}=22.1 \times 10^{-6} /{ }^{\circ} \mathrm{C}$ (out of plane)

\section{Boundary Displacements and Forces}

at the lower $(0)$ and upper $(L)$ edges

radial displacement: $w_{0}=1.03 \times 10^{-21} \mathrm{~m} ; w_{L}=0$

slope of the meridian: $\eta_{0}=-3.25 \times 10^{-19} ; \eta_{L}=2.30 \times 10^{-18}$

relative axial displacement of the edges: $d_{0 L}=-8.81 \times 10^{-20} \mathrm{~m}$

relative twist of the edges: $\mathrm{fi}_{O L}=0$

shear force: $Q_{r 0}=6.21 \mathrm{kN} / \mathrm{m} ; Q_{r L}=-43.3 \mathrm{kN} / \mathrm{m}$

bending moment: $M_{0}=-66.4 \mathrm{~N} \cdot \mathrm{m} / \mathrm{m} ; M_{L}=-210 \mathrm{~N} \cdot \mathrm{m} / \mathrm{m}$

normal force: $N_{a x}=-3.24 \mathrm{Mg}$

torque moment: $M_{\text {tor }}=114 \mathrm{~N} \cdot \mathrm{m}$

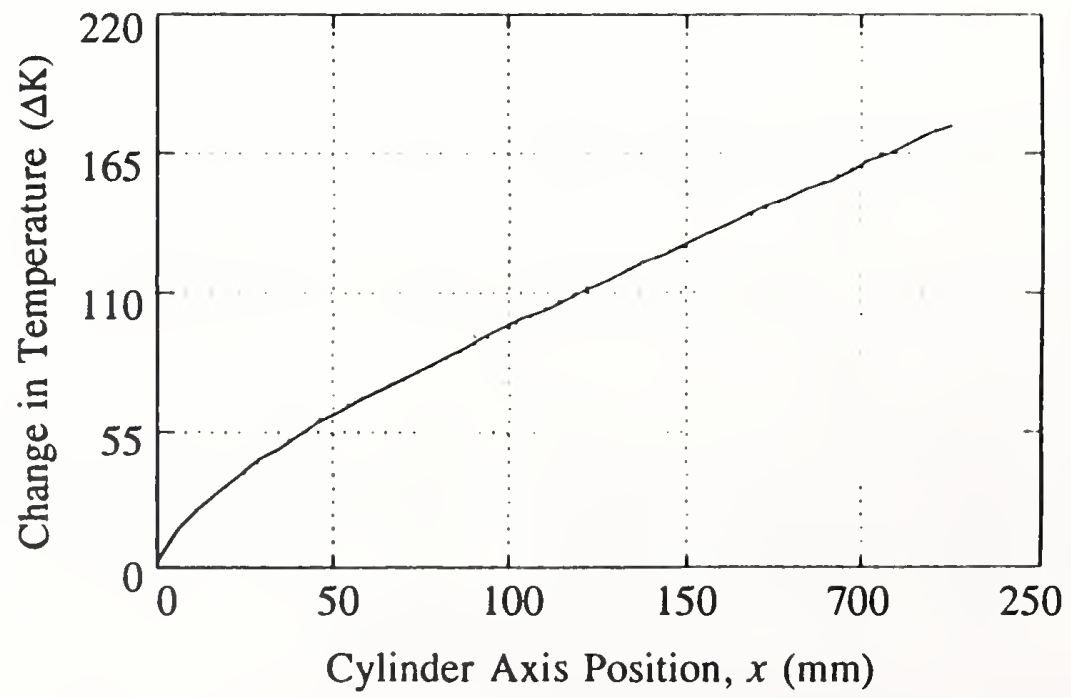

Figure D.22. Input data, edge loads, and edge displacements for a cylinder with prescribed temperatures at the edges. 

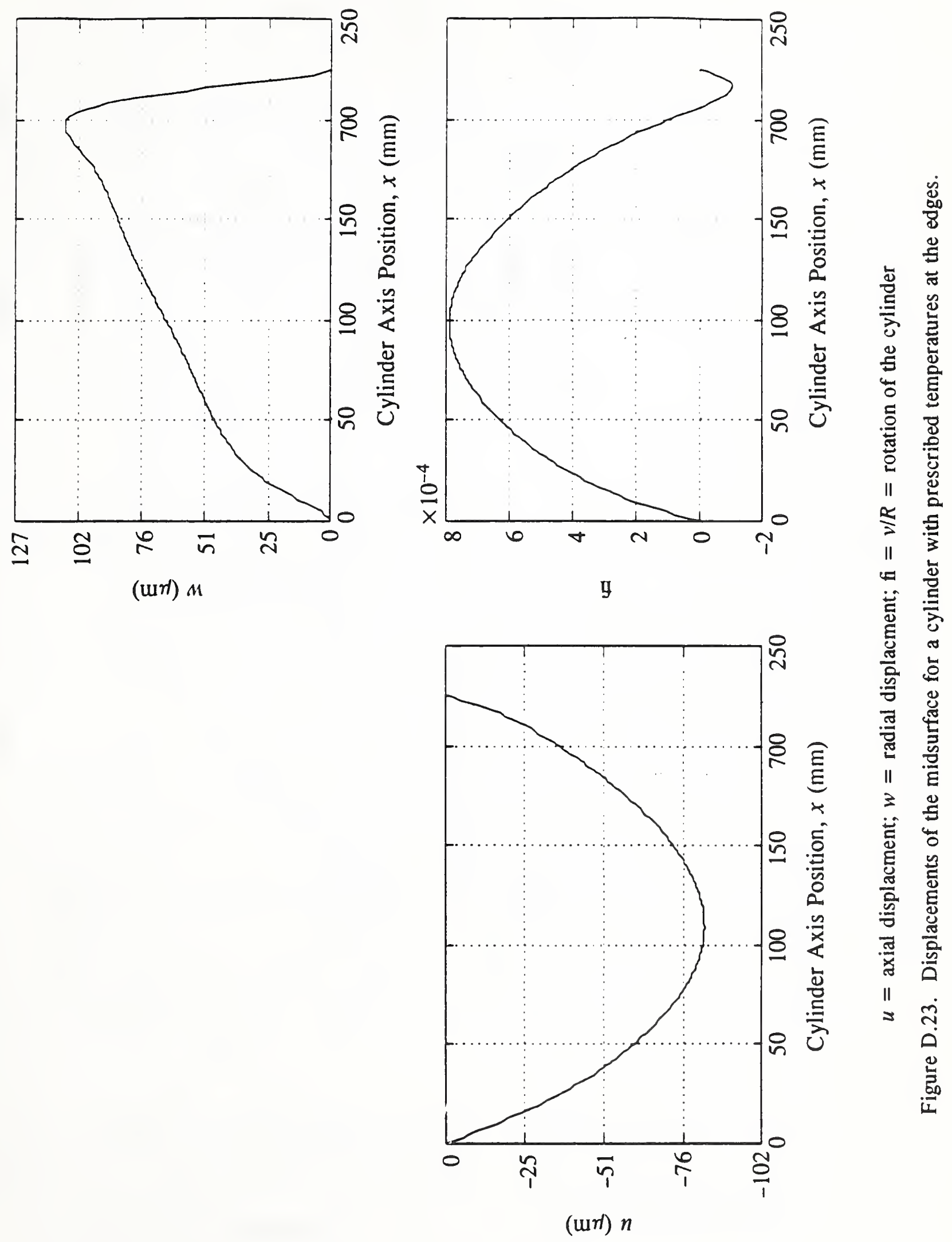


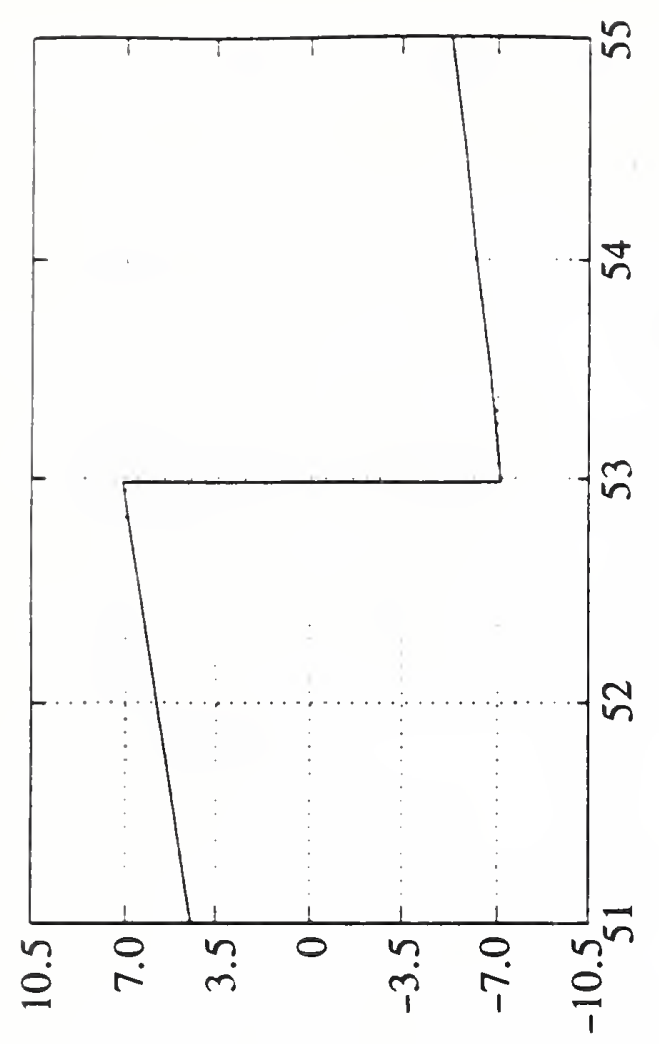

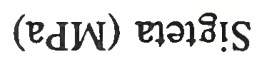

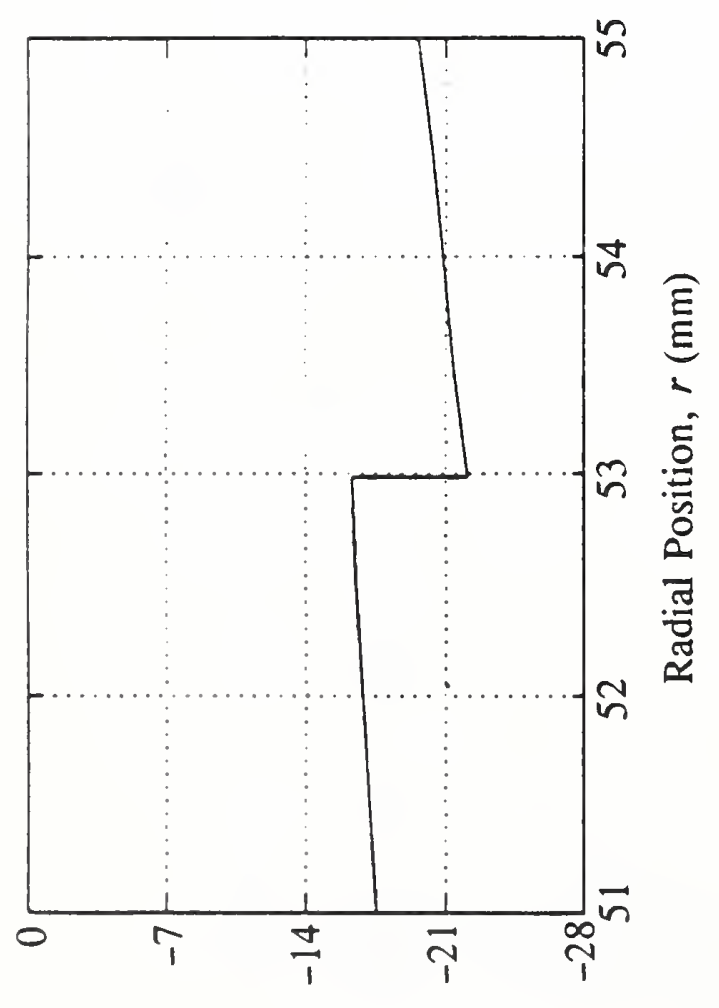

(อ $\mathrm{dW}) \times 8 \% ! 5$
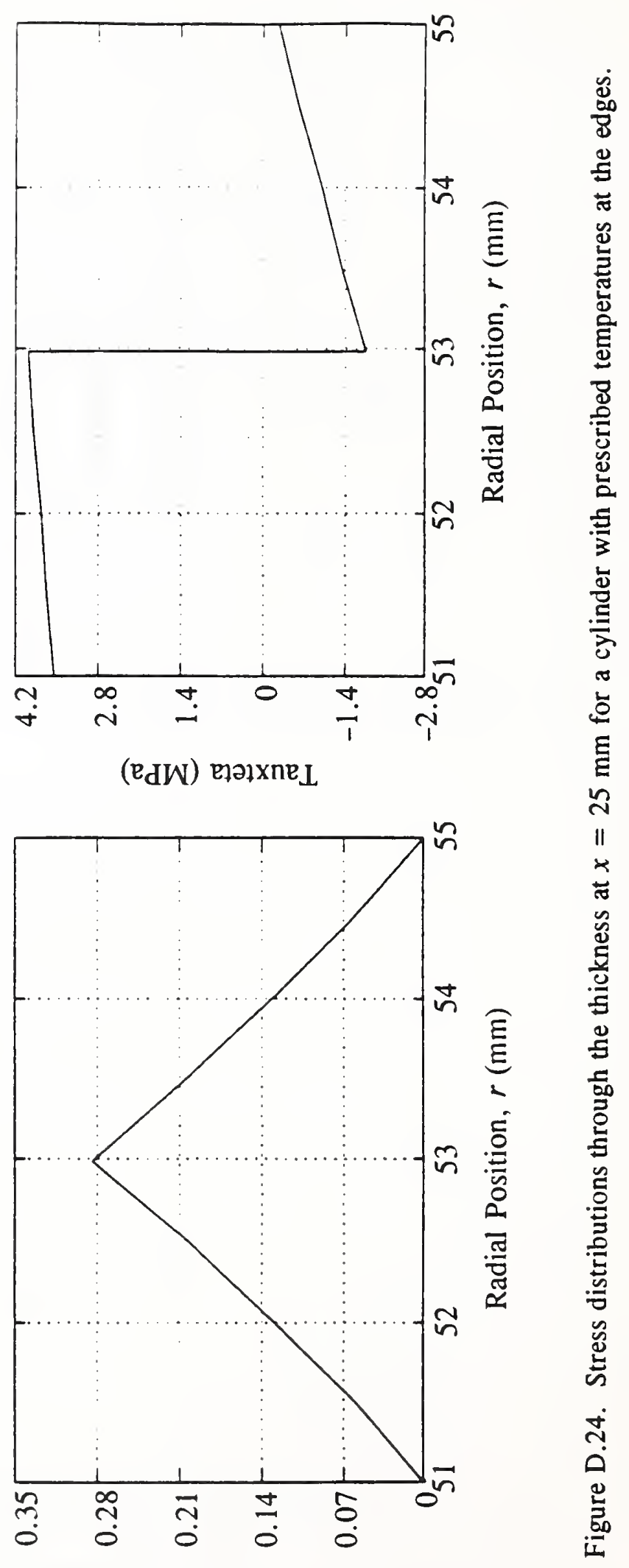

(dW) $18 ึ ! S$ 

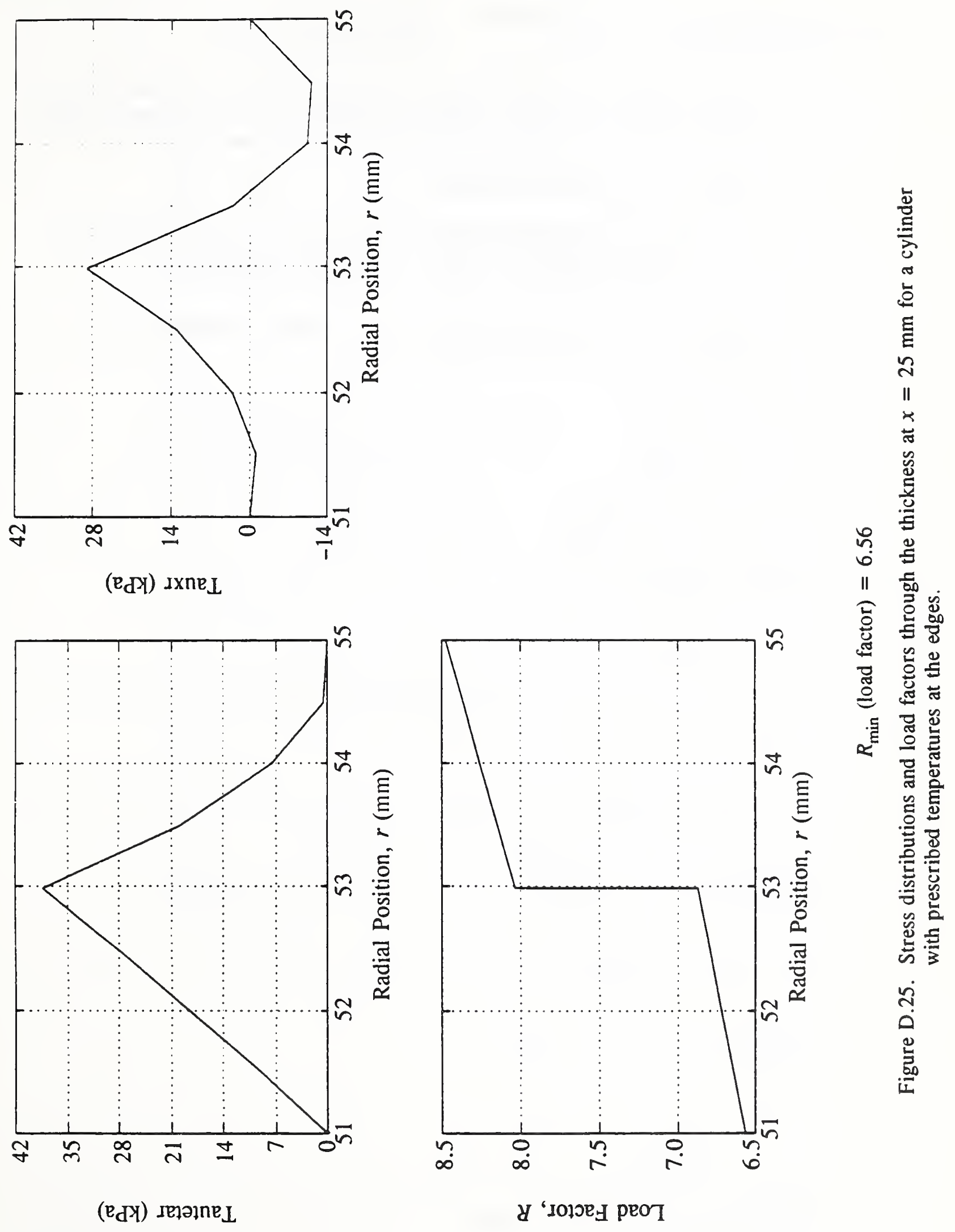


\section{Cylinder Subjected to an Axial End Load.}

\section{The Material Properties Vary with $x$.}

We consider a cylinder with a total length of $480 \mathrm{~mm}$. The lay-up is the same as in the previous cases, and the material properties on the bottom $240 \mathrm{~mm}$ are also the same, whereas the elastic constants on the top $240 \mathrm{~mm}$ are half those of the bottom part.

The cylinder is subjected to a unit load $N_{0}=1$.

To analyze the whole 480 -mm-long cylinder, first we have to solve the two sections with constant material properties separately. The bottom section, designated "Laslo-1," was treated in the previous section.

To run the top section, we have to change the elastic properties in the file "xdata" (Table D.6). Then type

$$
\begin{aligned}
& \gg \text { cyli Return } \\
& \gg 1 \text { Return } \\
& \gg \text { Laszlo-2 Return }
\end{aligned}
$$

After getting the "outputlist," type

$$
\gg 0 \quad \text { Return }
$$

To calculate the whole cylinder, we have to store file "moredataload" (Table D.7).

To run the whole cylinder, type

$$
\gg \text { more Return }
$$

and Title of the work: appears.

Type

$$
\gg \text { DUGO Return }
$$

and

$$
\text { Number of cylinders appears. }
$$

Type

$$
\gg 2 \text { Return }
$$

and
Name of the 1th cylinder:
appears. 
Table D.6. File "xdata" for the "top" part of the cylinder with varying material propertieg.

8 xdata
$n k=8$;
$\mathrm{nl}=\left[\begin{array}{llllllll}5 & 5 & 5 & 5 & 5 & 5 & 5 & 5\end{array}\right]$;
znumber of groups in one lamina
$\mathrm{nf}=2$;
inumber of plies in one group
$f \mathrm{ag}=[1,1,1,1,2,2,2,2]$; $\operatorname{tet}(1)=80 / 180 * \mathrm{pi}$;
tet $(2)=45 / 180 * \mathrm{pi}$;
inumber of ply orientations
thickne日s $=.005$;
$\mathrm{Rm}=2$;
\$which plyorientation in a group?
slst plyorientation
₹2nd plyorientation
$\mathrm{L}=9$;
thickness of one ply
iradius of the mold
ilength of the cylinder

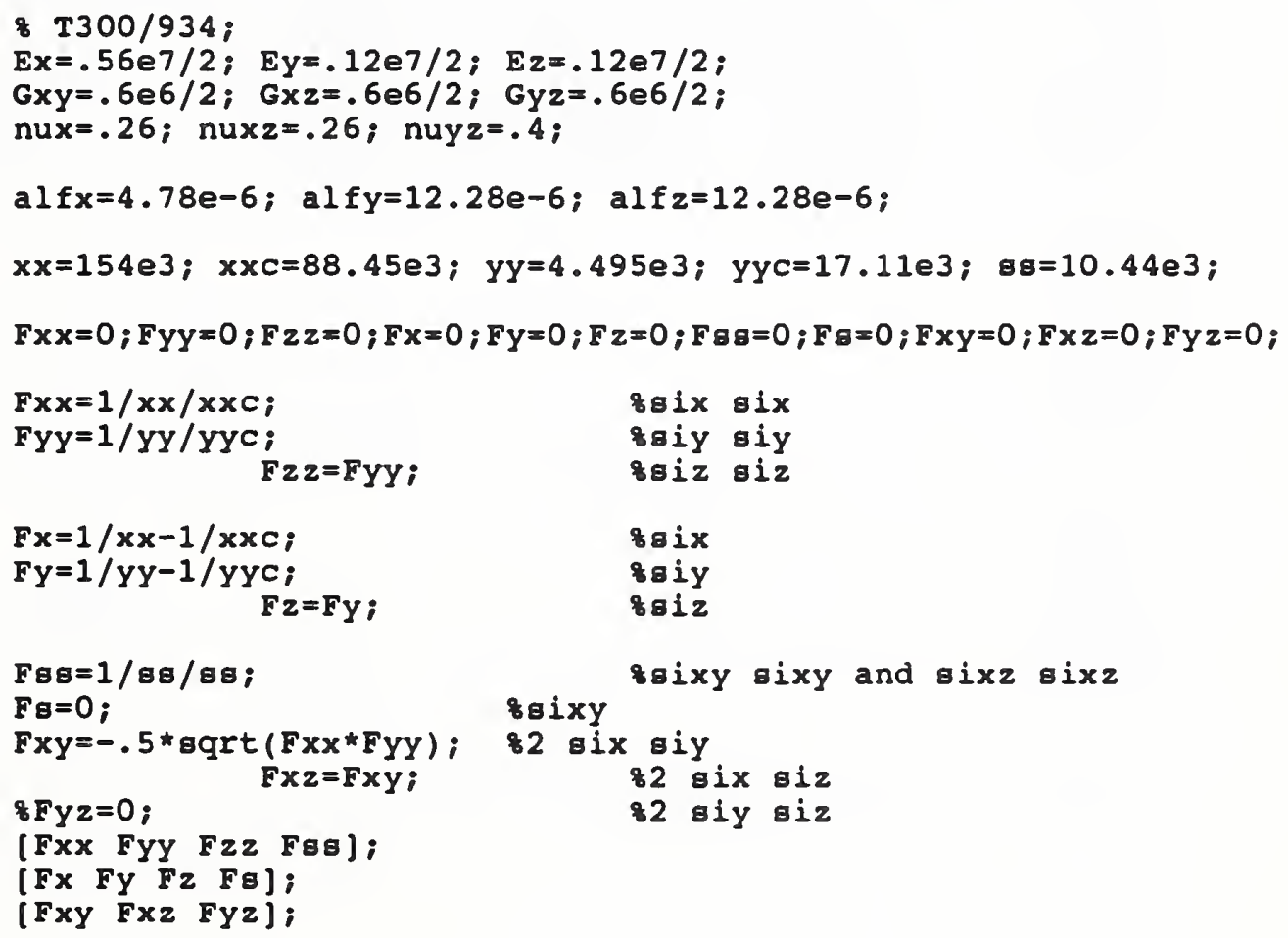


Table D.7. File "moredataload" for a cylinder fixed at both edges and subjected to a tensile force $\eta_{0}=1$

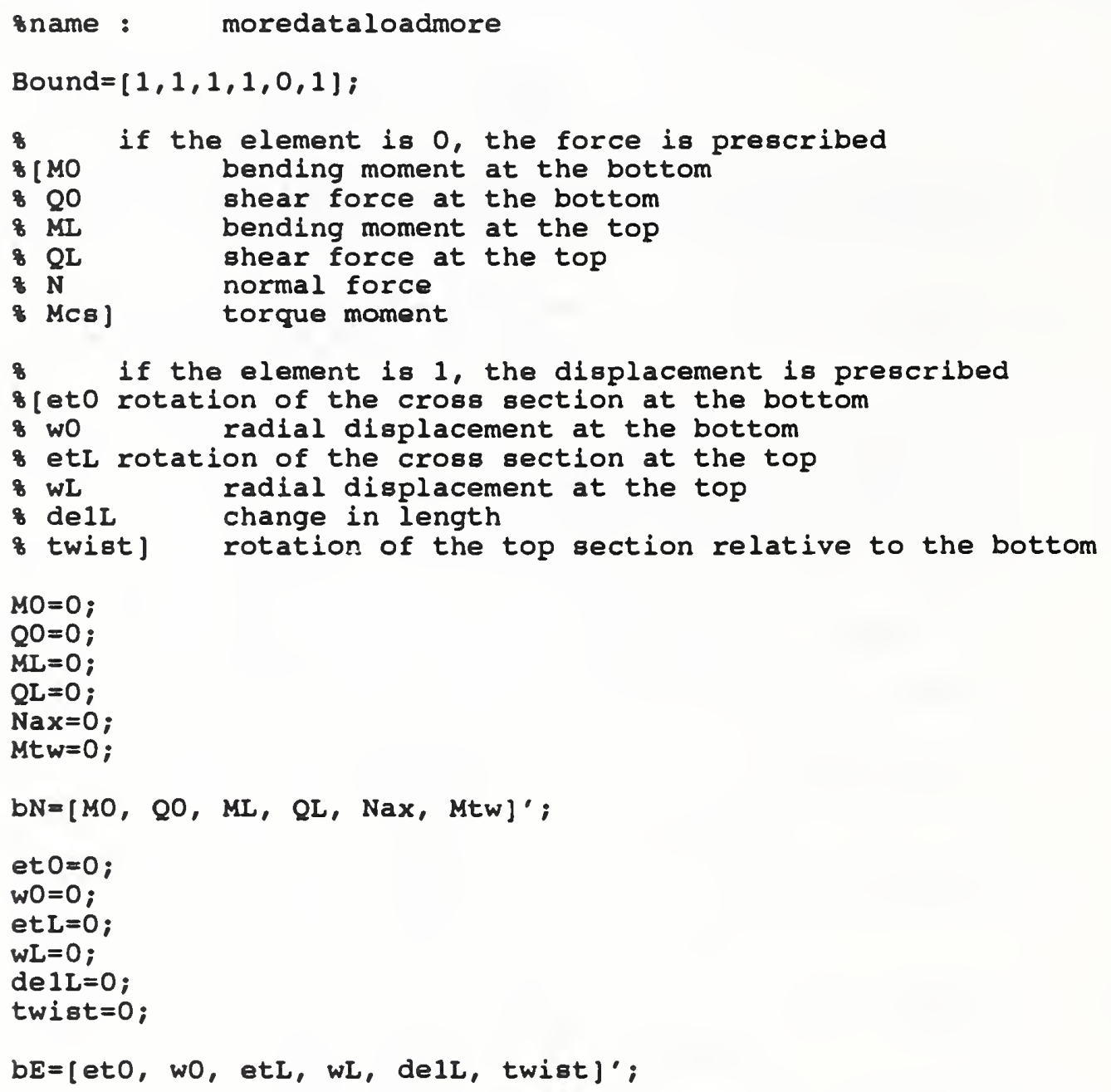


Type

$$
\gg \text { Laszlo-1 Return }
$$

and Name of the 2th cylinder: appears.

Type

$$
\gg \text { Laszlo-2 Return }
$$

and

$$
\text { -THE 1th CYLINDER - DUGO appears. }
$$

Type

$$
\gg \text { Return }
$$

Then the "outputlist" for the bottom section appears. (Do no use options 5 and 6!) We printed the results as before; they are in Figures D.26 and D.27.

Type

$$
\gg \quad 0 \quad \text { Return }
$$

and

$$
\text { -THE 2th CYLINDER - DUGO }
$$

appears.

Type

$$
\gg \text { Return }
$$

Then the "outputlist" for the top section appears. The results are printed in Figures D.28 and D.29. 
Lay-up: $\left[80_{5} / 80_{5} / 80_{5} / 80_{5} / 45_{5} / 45_{5} / 45_{5} / 45_{5}\right]$

inner radius: $50 \mathrm{~mm}$

length: $230 \mathrm{~mm}$ total thickness: $5 \mathrm{~mm}$

thickness of one ply: $130 \mu \mathrm{m}$

\section{Elastic Constants}

$$
\begin{aligned}
& E_{x}=39 \mathrm{GPa} \\
& E_{y}=8.3 \mathrm{GPa} \\
& E_{z}=8.3 \mathrm{GPa}
\end{aligned}
$$$$
\begin{aligned}
G_{x y} & =4.1 \mathrm{GPa} \\
G_{x z} & =4.1 \mathrm{GPa} \\
G_{y z} & =4.1 \mathrm{GPa}
\end{aligned}
$$

$$
\begin{aligned}
& \nu_{x y}=0.26 \\
& \nu_{x z}=0.26 \\
& \nu_{y z}=0.4
\end{aligned}
$$

\section{Strength Constants}

$$
X=1060 \mathrm{MPa}
$$$$
X^{\prime}=610 \mathrm{MPa}
$$

$$
\begin{aligned}
& S=72 \mathrm{MPa} \\
& F_{x y}{ }^{*}=-1 / 2
\end{aligned}
$$

\section{Coefficients of Thermal Expansion}

$\alpha_{x}=8.6 \times 10^{-6} /{ }^{\circ} \mathrm{C}$ (in plane, fiber direction)

$\alpha_{x}=22.1 \times 10^{-6} /{ }^{\circ} \mathrm{C}$ (in plane, perpendicular to the fiber)

$\alpha_{x}=22.1 \times 10^{-6} /{ }^{\circ} \mathrm{C}$ (out of plane)

\section{Boundary Displacements and Forces}

at the lower ( 0$)$ and upper $(L)$ edges

radial displacement: $w_{0}=3.87 \times 10^{-25} \mathrm{~m} ; w_{L}=-3.66 \times 10^{-9} \mathrm{~m}$

slope of the meridian: $\eta_{0}=-1.32 \times 10^{-23} ; \eta_{L}=-1.13 \times 10^{-7}$

relative axial displacement of the edges: $d_{0 L}=5.13 \times 10^{-8} \mathrm{~m}$

relative twist of the edges: $\mathrm{f}_{O L}=4.54 \times 10^{-10}$

shear force: $Q_{r 0}=-1.01 \mathrm{~N} / \mathrm{m} ; Q_{r L}=-0.189 \mathrm{~N} / \mathrm{m}$

bending moment: $M_{0}=11.0 \mathrm{mN} \cdot \mathrm{m} / \mathrm{m} ; M_{L}=6.16 \mathrm{mN} \cdot \mathrm{m} / \mathrm{m}$

normal force: $N_{a x}=0.45 \mathrm{~kg}$

torque moment: $M_{\text {tor }}=0.06 \mathrm{~N} \cdot \mathrm{m}$

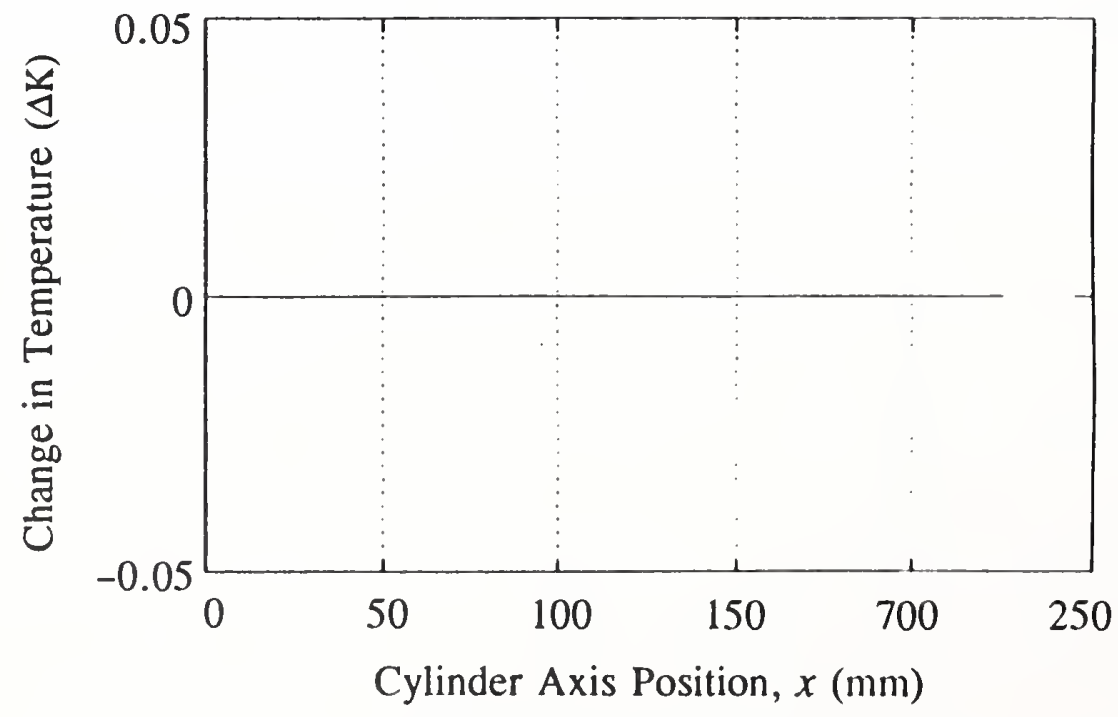

Figure D.26. Input data, edge loads, and edge displacements for the bottom section of a cylinder with varying material properties. 

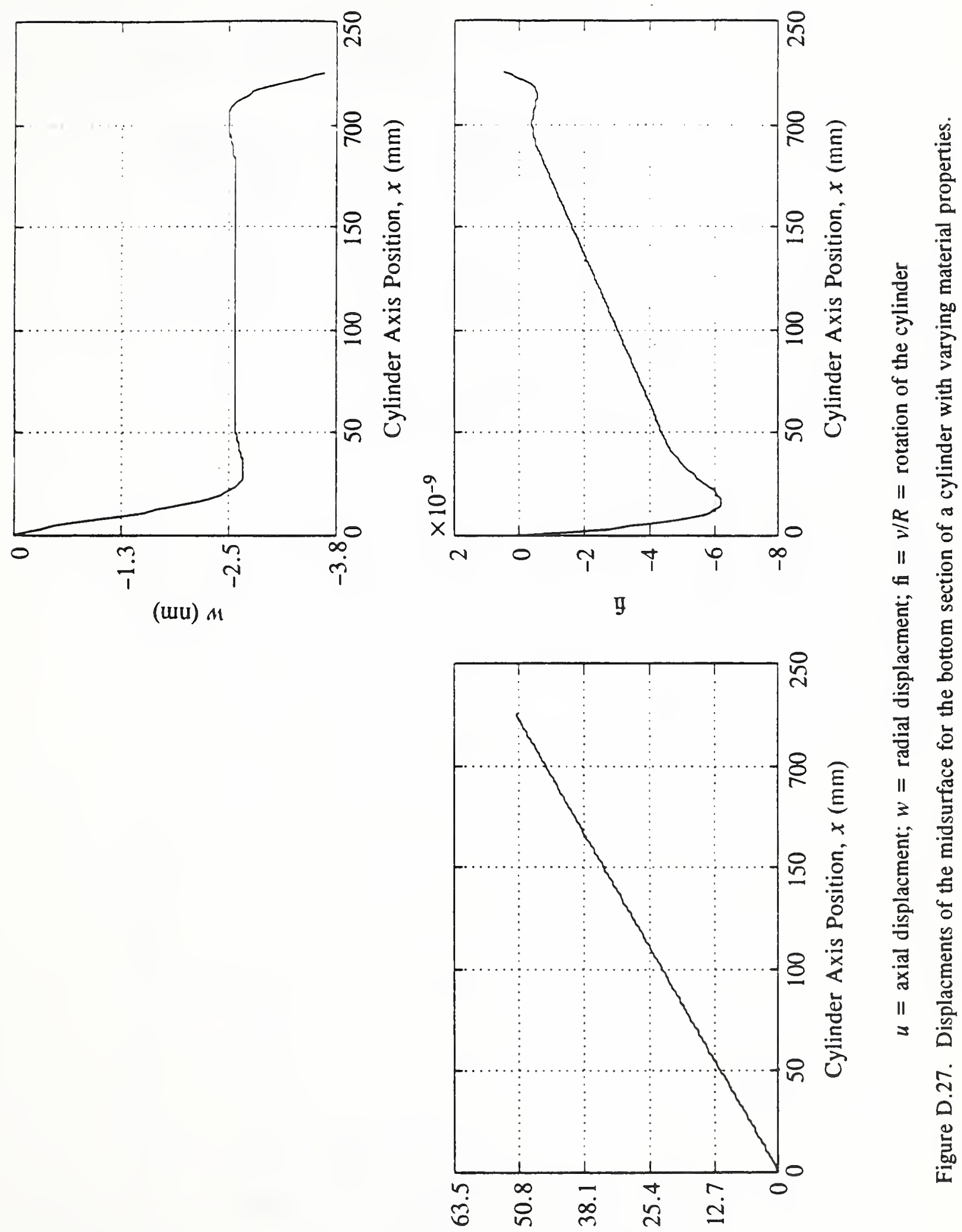

(uu) $n$ 
Lay-up: $\left[80_{5} / 80_{5} / 80_{5} / 80_{5} / 45_{5} / 45_{5} / 45_{5} / 45_{5}\right]$

inner radius: $50 \mathrm{~mm}$

length: $230 \mathrm{~mm}$ total thickness: $5 \mathrm{~mm}$

thickness of one ply: $130 \mu \mathrm{m}$

\section{Elastic Constants}

$E_{x}=19 \mathrm{GPa}$

$$
\begin{aligned}
& G_{x y}=2.1 \mathrm{GPa} \\
& G_{x z}=2.1 \mathrm{GPa} \\
& G_{y z}=2.1 \mathrm{GPa}
\end{aligned}
$$$$
E_{y}=4.1 \mathrm{GPa}
$$

$$
\begin{aligned}
& \nu_{x y}=0.26 \\
& \nu_{x z}=0.26 \\
& \nu_{y z}=0.4
\end{aligned}
$$

\section{Strength Constants}
$X=1060 \mathrm{MPa}$
$Y=31 \mathrm{MPa}$
$S=72 \mathrm{MPa}$
$X^{\prime}=610 \mathrm{MPa}$
$Y^{\prime}=118 \mathrm{MPa}$
$F_{x y}{ }^{*}=-1 / 2$

\section{Coefficients of Thermal Expansion}

$\alpha_{x}=8.6 \times 10^{-6} /{ }^{\circ} \mathrm{C}$ (in plane, fiber direction)

$\alpha_{x}=22.1 \times 10^{-6} /{ }^{\circ} \mathrm{C}$ (in plane, perpendicular to the fiber)

$\alpha_{x}=22.1 \times 10^{-6} /{ }^{\circ} \mathrm{C}$ (out of plane)

\section{Boundary Displacements and Forces}

at the lower $(0)$ and upper $(L)$ edges

radial displacement: $w_{0}=-3.66 \times 10^{-9} \mathrm{~m} ; w_{L}=1.37 \times 10^{-24} \mathrm{~m}$

slope of the meridian: $\eta_{0}=-1.13 \times 10^{-7} ; \eta_{L}=1.51 \times 10^{-22}$

relative axial displacement of the edges: $d_{0 L}=1.03 \times 10^{-7} \mathrm{~m}$

relative twist of the edges: $\mathrm{f}_{O L}=-4.54-10^{-10}$

shear force: $Q_{r 0}=-0.189 \mathrm{~N} / \mathrm{m} ; Q_{r L}=1.01 \mathrm{~N} / \mathrm{m}$

bending moment: $M_{0}=6.16 \mathrm{mN} \cdot \mathrm{m} / \mathrm{m} ; M_{L}=0.432 \mathrm{~N} \cdot \mathrm{m} / \mathrm{m}$

normal force: $N_{a x}=0.45 \mathrm{~kg}$

torque moment: $M_{\text {tor }}=0.06 \mathrm{~N} \cdot \mathrm{m}$

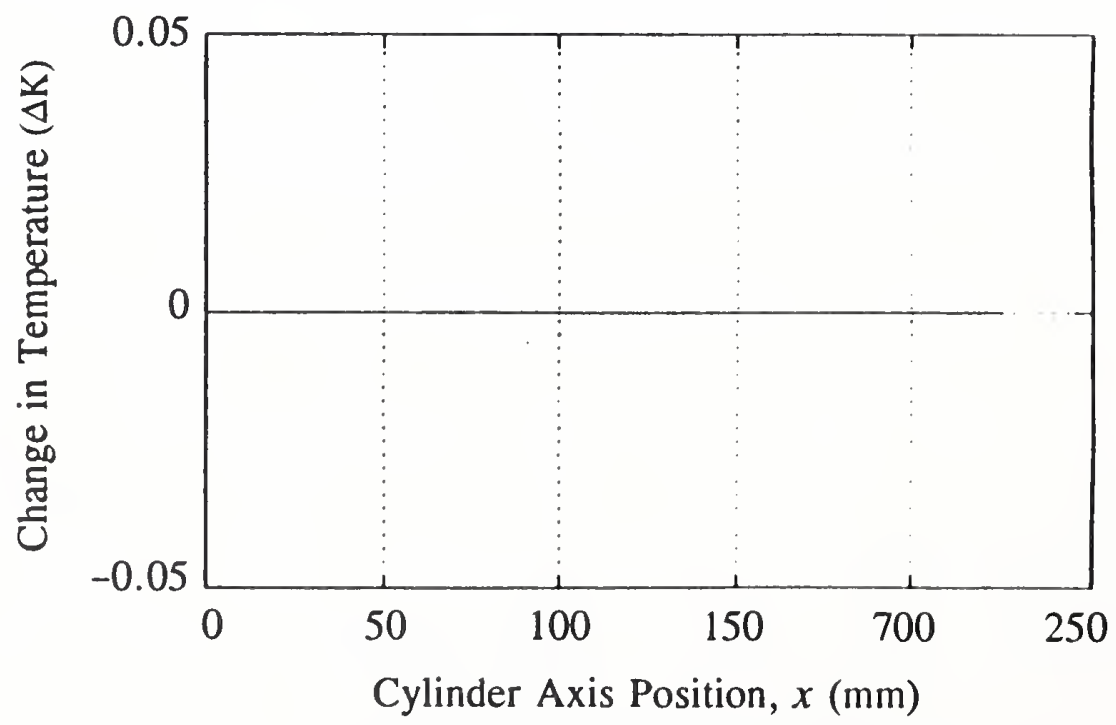

Figure D.28. Input data, edge loads, and edge displacements for the top section of a cylinder with varying material properties. 

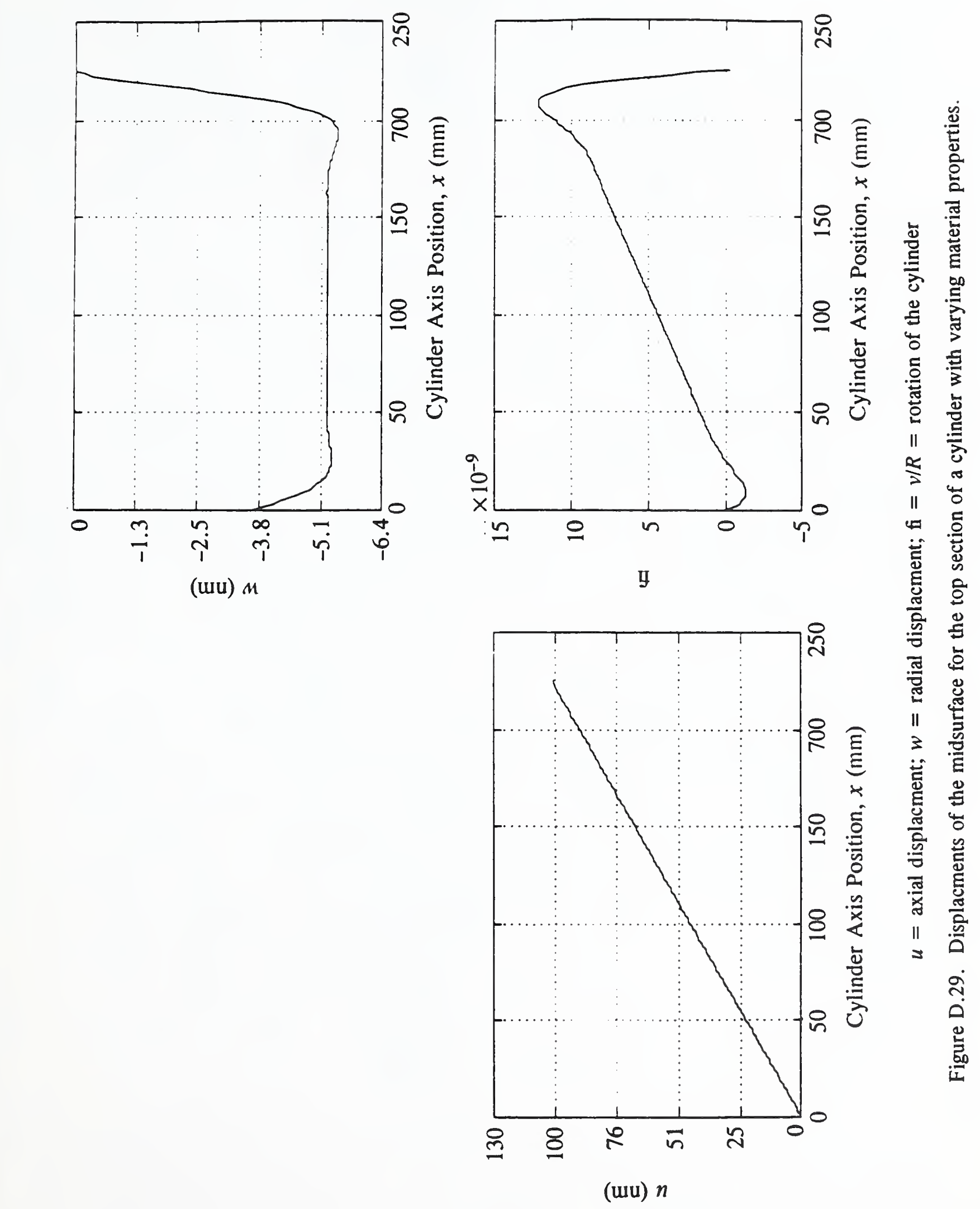



\section{Analysis of End-Fitting-Induced Strains in Axially Loaded Glass-Epoxy Cylinders}

M. W. Hyer and T. W. Knott

Virginia Polytechnic Institute and State University

Blacksburg, Virginia 
I

I

I

I

I

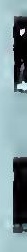

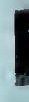

,

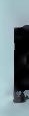

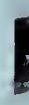

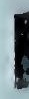

1 


\title{
VIII. Analysis of End-Fitting-Induced Strains in Axially Loaded Glass-Epoxy Cylinders
}

\author{
M. W. Hyer and T. W. Knott \\ Virginia Polytechnic Institute and State University \\ Blacksburg, Virginia
}

\begin{abstract}
This section discusses the strain response of axially loaded, thick, glass-epoxy composite cylinders. Two stacking sequences are considered: $[ \pm 10]_{6 n}$ and $\left[81 / \pm 45 / \pm 10_{3} / \pm 45 / 82\right]_{n}(n=1,2)$. The cylinders are representative of the type intended for the support structure of the proposed superconducting magnetic energy storage system (SMES). As such, the strain response at room temperature and at $4 \mathrm{~K}$ were computed. The strain levels at room temperature were $25 \%$ higher than at $4 \mathrm{~K}$. Finite-element analyses were used throughout. The influence of two end-fitting designs and two wall thickness, $n=1$ and $n=2$, were investigated. Design had little effect on strain response and magnitude. Both thermally induced and load-induced intra- and interlaminar strains are considered.
\end{abstract}

\section{Introduction}

The loading of a cylinder or tube in axial compression, while at first glance is quite straightforward, requires attention to the details of load introduction at the ends of the cylinder. Preparation of the cylinder and the design of the additional fittings and fixturing that transfer the load from the surrounding environment, be it a loading frame or another structural component, to the ends of the cylinder are the primary points that must be addressed. For a cylinder in simple axial compression, the end fitting will most likely control failure. Even with the utmost care in design, the end fitting will introduce stress or strain concentrations into the cylinder that, under normal circumstances, will exceed the strains due to any other effect. Whether the end fitting makes use of bolts, pins, or other mechanical fasteners to introduce the load into the cylinder wall via holes in the wall, or whether it is of the more conventional design which transmits the load by bearing on the end of the cylinder, there will be stress concentration effects. This is assuming that the ends of the cylinder have been prepared properly. Proper preparation involves something as simple as assuring the ends of the cylinder have been machined flat and parallel, to something as complicated as designing additional thickness into the cylinder wall and perhaps tapering the thickness as a function of axial distance away from the ends. If the ends of the cylinder have not been given a certain level of attention, stress concentration effects can be more pronounced. 


\section{Procedures}

Here the issue of the end fitting for the glass-epoxy SMES support cylinders will be addressed in the following context: A number of cylinders with an inner radius of $50.0 \mathrm{~mm}$ ( $2.00 \mathrm{in})$ have been tested to failure using a simple aluminum bearing-type end fitting. The failure loads and failure modes for these cylinders have been recorded. The approach here will be to conduct an analysis of the end region of these cylinders by considering the details of the end fitting used in the testing. The strain levels introduced into the cylinders as a result of this design and representative load and temperature levels will be determined. This end fitting will be referred to as the baseline design. Two cylinder wall constructions that were tested will be considered, namely lay-up no. 1 and lay-up no. 2 . Lay-up no. 1 has stacking sequence $[ \pm 10]_{6 n}, n=1,2$, and lay-up no. 2 has the stacking sequence $\left[81 / \pm 45 / \pm 10_{3} / \pm 45 / 82\right]_{n}$, $n=1,2$. Lay-up no. 1 is quite stiff in the axial direction and soft in the circumferential direction. Lay-up no. 2 is stiffer in the circumferential direction than the first construction, with some accompanying sacrifice of axial stiffness. Two wall thickness, $n=1$ and $n=2$, of these constructions are considered: $5.08 \mathrm{~mm}$ and $10.2 \mathrm{~mm}(0.2$ and $0.4 \mathrm{in})$. The thicker wall probably is more representative of actual cylinder designs. With these cylinder dimensions, the mean ratios of cylinder radius to wall thickness are 10.5 for the thinner cylinder and 5.5 for the thicker cylinder, the latter most certainly being classified as a thick-walled cylinder and the former one being in the thick-walled category by most standards. Note that the cylinder wall lay-up no. 1 was intended to be all unidirectional, whereas lay-up no. 2 was intended to consist of $0^{\circ}, 90^{\circ},+45^{\circ}$, and $-45^{\circ}$ plies. Due to the process by which the cylinders were made, $0^{\circ}$ and $90^{\circ}$ plies were not possible.

With the strains in the walls of cylinders with these two lay-ups and two thicknesses computed, attention will then focus on an alternative end-fitting design. These same two cylinder wall constructions and the same representative loading conditions will be studied to determine the effect of the alternative end-fitting design on wall strain levels. The alternative design discussed is intended only to show a possible trend in the effect of end-fitting design on wall strain levels. The alternative design has not been constructed, nor is it necessarily being proposed. It should be noted that strains. not stresses, are being reported because strains can be measured directly in an experiment, while experimental stress measurements require knowing the constitutive properties of the material being tested. This requires further testing which may lead to additional fixturing.

An important issue in the end-fitting analysis is the material properties of the glassepoxy. This is particularly the case at temperatures other than room temperature, where material-property data are, in general, not readily available for fiber-reinforced materials. Also, the different manufacturing techniques commonly used to fabricate tubes result in different material properties, even though the specifications for the material properties may be the same for each manufacturing technique. Lack of the ability to control resin deposition exactly, to control tension in the tows in a filament-winding process, to minimize void formation, to closely monitor consolidation, to control the environment, and a host of other issues result in anomalies that lead to deviations from the specified material properties. Thus, experimentally determined material-property measurements of a particular sample of material may not result in representative data. The approach herein will be to compute the material properties from micromechanical models that utilize constituent properties, that is, fiber properties and matrix properties, that have been measured experimentally. It will be assumed that the fiber volume fraction of the 
glass fibers is $60 \%$ and there are no voids. Ply properties will be determined from these micromechanical models, and then macromechanical models will be used to smear ply, or layer, properties to determine properties for the cylinder wall that will, at least for the purposes of computing the overall strain response of the cylinder, be assumed to be constant throughout the wall thickness. Appendix A discusses the micromechanical modeling, and Appendix B discusses the macromechanical modeling.

Figure 2.1 is a schematic diagram of one end of the cylinder with the end fitting attached. Figure 2.1(a) illustrates the baseline end-fitting design; Figure 2.1(b) illustrates the alternative design. With the baseline design, which is rather simple, the cylinder end fits into a groove that is slightly wider than the thickness of the cylinder wall. The cylinder is then potted in place with an aluminum-filled epoxy potting compound. Since the axial compressive loading forces the cylinder into the groove, the potting serves only to keep the end fitting attached the cylinder for purposes of handling. Because during testing of the cylinders the end fittings bear on some other surface, a steel baseplate is included in the analysis to represent the effect of the surrounding load-bearing environment on the cylinders. The steel baseplate is assumed to have an axial dimension of $76 \mathrm{~mm}$ (3.00 in).

With the baseline end fitting, the region where the cylinder exits the groove is expected to be the location of the highest strains. In simplest terms, the strains at the end-fitting exit will be a combination of axial compressive strains that result from the overall compressive load, and bending strains that result from the deformation of the cylinder wall as it exits the end fitting. The bending effects can be explained as follows: Away from the end fitting, as a result of Poisson effects, the axial compressive force causes the cylinder to become larger in diameter. The end fitting, being quite stiff in the radial direction, prevents the portion of the cylinder in the end fitting from becoming larger in diameter. In fact, the diameter within the end fitting is basically the unloaded diameter of the cylinder. Thus, as the cylinder exits the end fitting, its diameter goes from the unloaded diameter to the larger, loaded diameter. It is this sudden change in diameter, or rather, the freedom to have this sudden change, that leads to high strain concentrations as the cylinder exits the end fitting. Likewise, if the cylinder and end fitting are subjected to a temperature change, the end fitting will expand or contract radially in a different manner than the cylinder itself. Away from the end fitting, the cylinder can deform freely. Where the cylinder exits the end fitting, the transition from one deformed state to the other results in bending strains in the cylinder wall.

Typically, the region away from the end of a cylinder is called the membrane region; it is characterized by the fact that no cylinder wall bending is taking place in that region and the strains, and hence stresses, are independent of the radial position within the wall. Furthermore, in the membrane region, the strains are also usually independent of the axial position. A simple force-divided-by-area analysis will provide an accurate estimate of the response of the cylinder in the membrane region. In contrast, the region near the end of a cylinder is usually characterized by the rapid change in strain levels with both the radial and the axial coordinate. This region is referred to as a boundary-layer region. The axial distance covered by the boundarylayer region is generally a function of material properties and cylinder radius. For geometrically nonlinear problems, the boundary-layer length also depends on the load level. For the thick cylinders considered here, geometrically nonlinear effects will not be important. Thus load-level effects on the boundary-layer length will not be an issue. Generally, but not always, the shorter the boundary layer, the more severe the strains are within it. As can be surmised, in this study the boundary layer is the region where the cylinder exits the end fitting and the alternative 


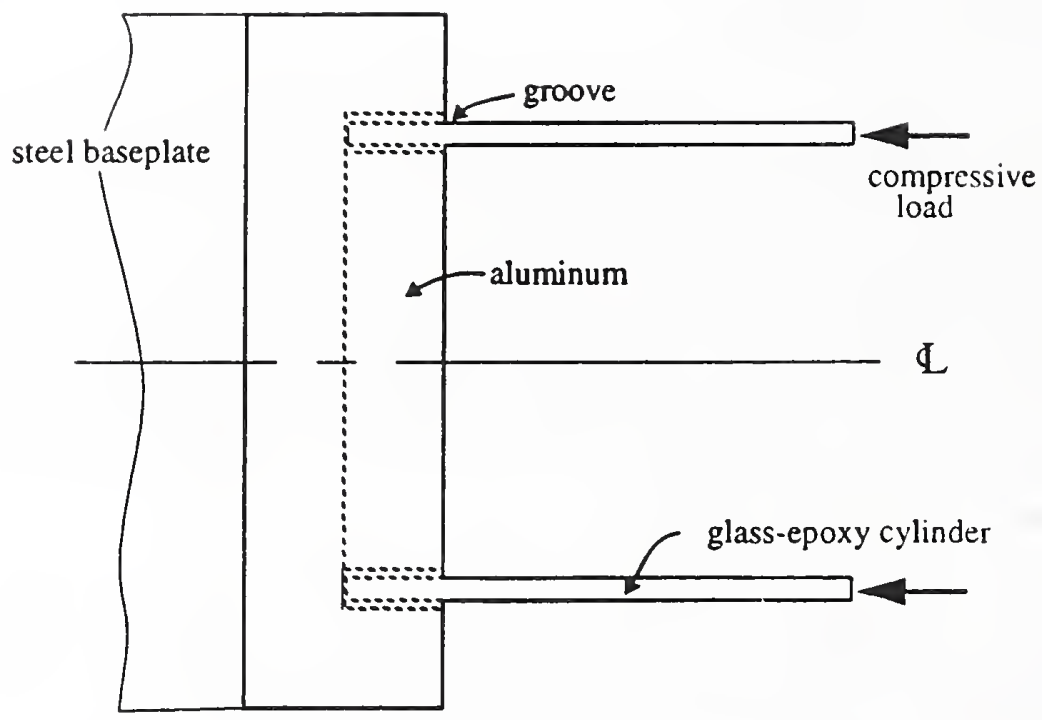

(a) Baseline design

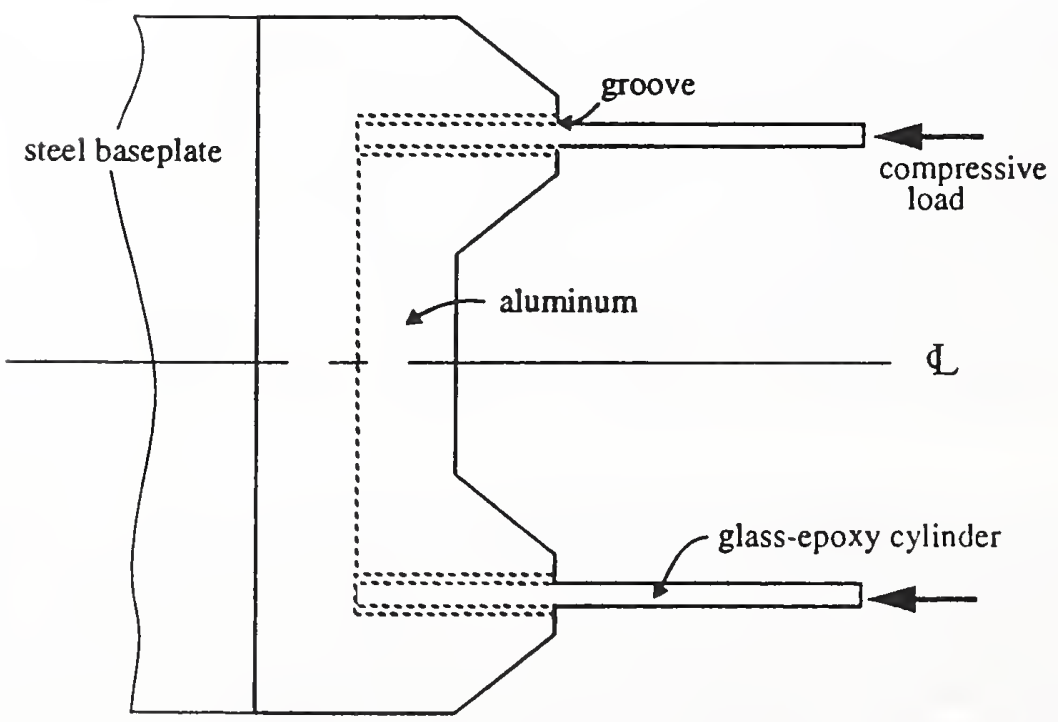

(b) Alternative design

Figure 2.1. Two end-fitting designs. 


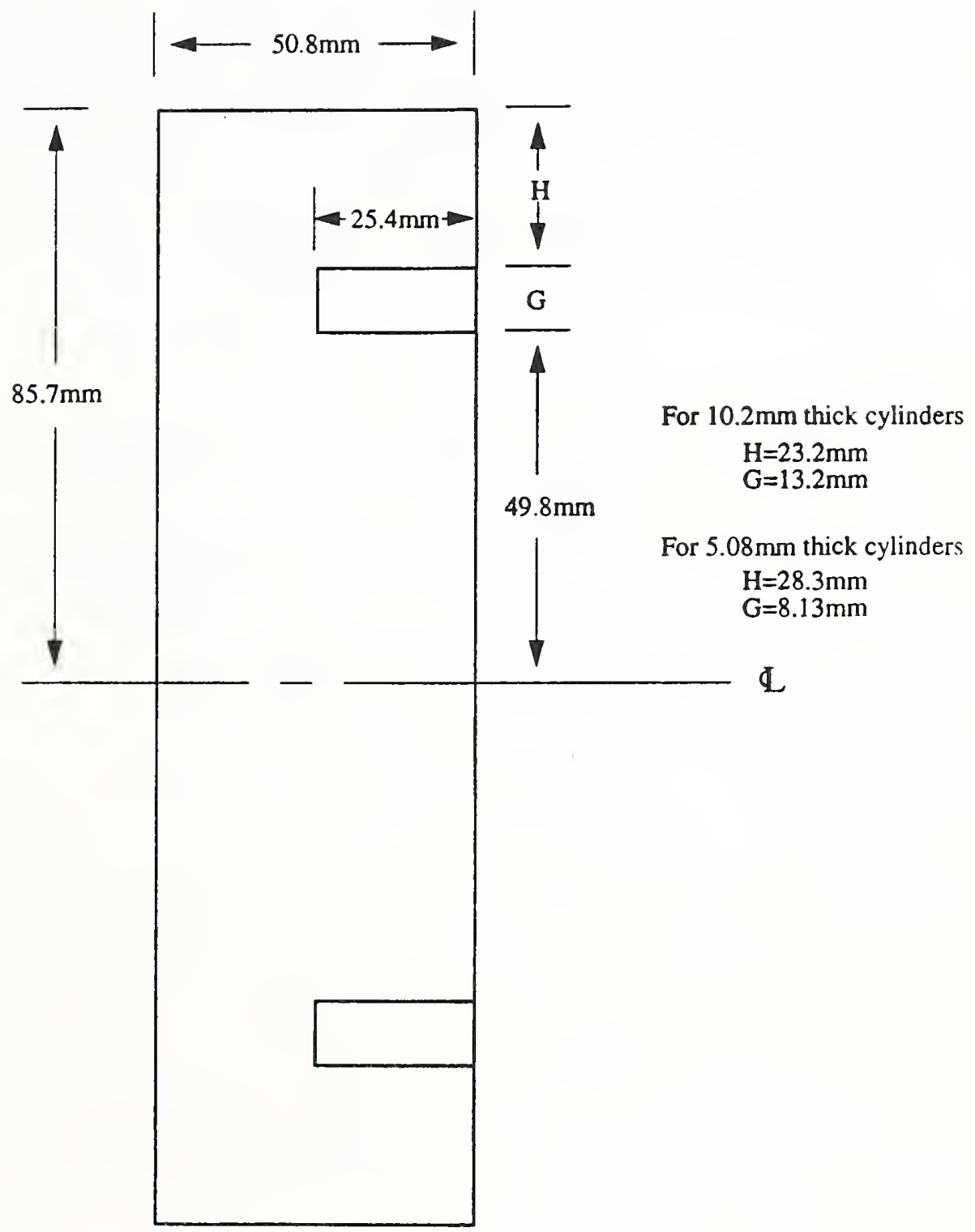

Figure 2.2. Baseline end-fitting design (section). 


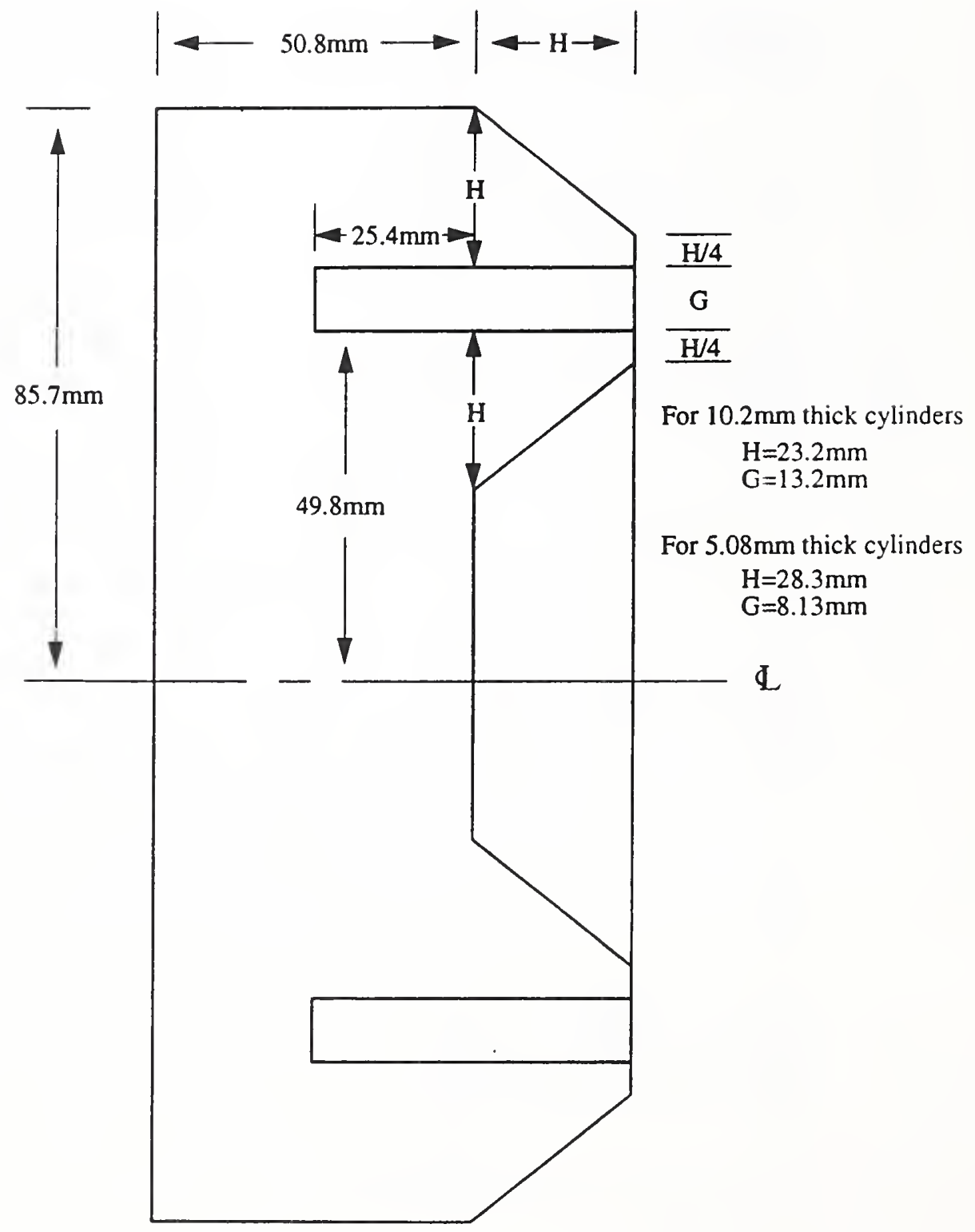

Figure 2.3. Details of alternative end-fitting design (section). 
design is focusing on changing the boundary-layer region of the cylinder. The alternative design attempts to change the length of the boundary layer, and thus perhaps the severity of the strains within it.

Figure 2.2 provides details of the baseline end fittings as they were configured for testing cylinders with an inner radius of $50.8 \mathrm{~mm}(2.00 \mathrm{in})$. Since the two cylinder wall thicknesses considered had the same inner radii, the two cylinder thickness were accommodated by simply adjusting the width of the groove in the end fitting. As mentioned earlier, in the testing that has taken place, the end-fitting material was aluminum. The alternative design is being kept simple by using the taper dimensions given in Figure 2.3. Because the length of the tapered section and the width of the narrow end of the tapered section could both be varied, the number of alternative end-cap designs is innumerable. Hence the scheme of Figure 2.3 serves only to limit the study and provide insight as to whether the geometry of the taper shown in that figure has any effect whatsoever.

In the actual application of the cylinders, one end will be immersed in liquid helium, at a temperature of $4 \mathrm{~K}\left(-452^{\circ} \mathrm{F}\right)$, while the other end will be at ambient temperature, here taken to be room temperature, $297 \mathrm{~K}\left(77^{\circ} \mathrm{F}\right)$. As a result, there is a temperature gradient along the length of the cylinder. Here the temperature of the cylinder and end fitting will be assumed to be spatially uniform, either at room temperature or at $4 \mathrm{~K}$. This assumption, which makes the analysis considerably simpler, is not felt to compromise the results. To determine the influence on the strains in the cylinders due to the extreme environment of $4 \mathrm{~K}$, the strains for the two laminates, the two wall thicknesses, and the baseline and alternative end fittings will be computed for the case of cooling the cylinder from room temperature to $4 \mathrm{~K}$ but with no axial load applied $\left(\Delta T=-293 \mathrm{~K},-529^{\circ} \mathrm{F}\right)$. For this case, all strains, and hence all stresses, are assumed to be zero at room temperature. After the discussion of temperature effects, the strains for the case of applying a load that results in $689-\mathrm{MPa}(100-\mathrm{ksi})$ mean axial compressive stress will be presented. These strains will be presented for the case of using material properties evaluated at $4 \mathrm{~K}$. The sum of the thermally induced strains at $4 \mathrm{~K}$ and the load-induced strains at $4 \mathrm{~K}$ will thus represent the situation at the cold end of a loaded cylinder. To represent the effects at the warm end of the inner loaded cylinder, the strains resulting from the 689-MPa mean axial compressive stress and room-temperature properties are presented.

Note that the residual curing strains are not included. As the epoxy tends to become stiffer at lower temperatures, a worst-case estimate of the residual strains due to cooling from cure rather than cooling from room temperature can be obtained by using the thermally induced strains at $4 \mathrm{~K}$ and ratioing them using the difference between cure temperature and room temperature. 


\section{Results}

Results of the finite element analysis of the various cases discussed above follow:

The commercially available, general-purpose code ABAQUS [3.1] was used to obtain all results. The pre- and postprocessing software PATRAN [3.2] was used for model development and exhibiting undeformed and deformed geometries. Representative undeformed and deformed geometries, the finite element discretization for the baseline and alternative end-fitting designs, are shown in Figures 3.1(a) and 3.1(b), respectively. The deformed and undeformed geometries provide insight into the aforementioned length of the boundary layer and the bending effects within this region. Following the deformations, the strains in the cylinder are shown in graphical form. The graphical results were prepared with the data analysis package DAN [3.3]. The strains are shown on a ply-byply basis. Within the plarie of each ply the principal material system intralaminar strains are illustrated, with $\epsilon_{1}$ being the strain in the fiber direction, and $\epsilon_{2}$ being the strain perpendicular to the fiber direction (referred to here as transverse strain), and $\gamma_{12}$ being the shear strain. Since they represent worst-case strains, only the strains in the extreme inner and outer plies at each fiber orientation are considered. These strains are shown as a function of axial length along the cylinder.

(a)

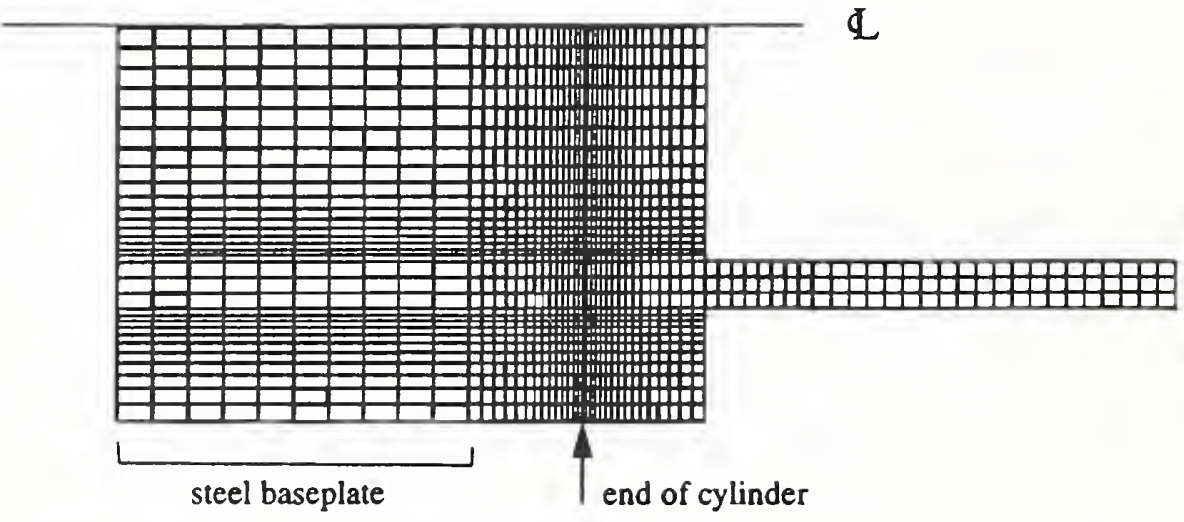

(b)

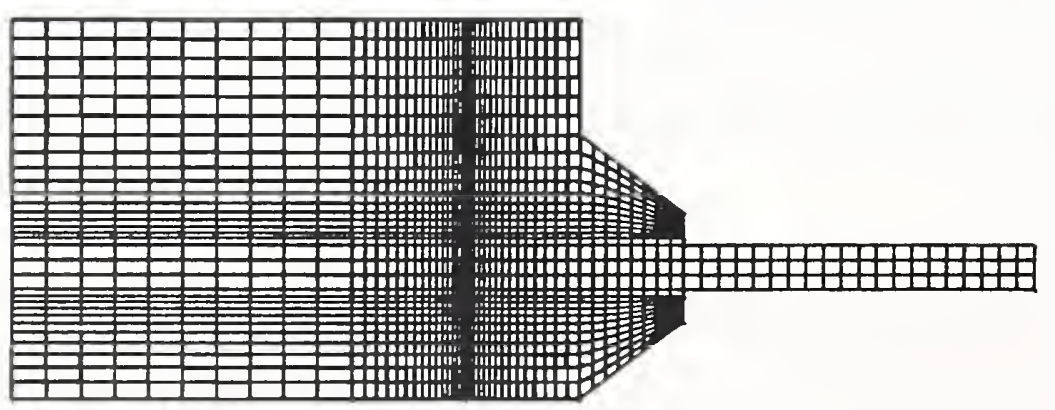

Figure 3.1. Finite element meshes: (a) baseline design, (b) alternative design. 
The coordinate system used to describe the cylinder is shown in Figure 3.2. The radial direction $(r)$ is as would be expected in a cylindrical coordinate system, and the circumferential coordinate $(\theta)$ is not shown. Note that the location $x=0$ is at the end of the cylinder within the end fitting. For the baseline end fitting, the cylinder exits the end fitting at $x=25.4 \mathrm{~mm}$ ( $1 \mathrm{in})$; with the alternative design, the cylinder exits the end fitting at a larger value of $x$. Two through-the-thickness, or interlaminar, strains are reported. One strain is the interlaminar normal strain in the radial direction $\left(\epsilon_{r}\right)$, which represents the tendency of the plies to move apart or together, depending on the sign of the strain. The other interlaminar strain is the shear strain $\left(\gamma_{r x}\right)$. This strain represents the tendency of the plies to slide by each other in the axial direction. Neither of these strains is resisted by fiberreinforcing effects; rather, it is strictly the strength and stiffness of the epoxy that prevent these strains from becoming excessive. In addition to considering the inner and outer plies, the interlaminar strains at the cylinder midply location are also shown. The midply location is added because the interlaminar strains can be maximum at this radial location. For all the strains, the character just into the membrane region is depicted. As will be seen, and as mentioned above, the strains do not change with axial or radial coordinate in this region.
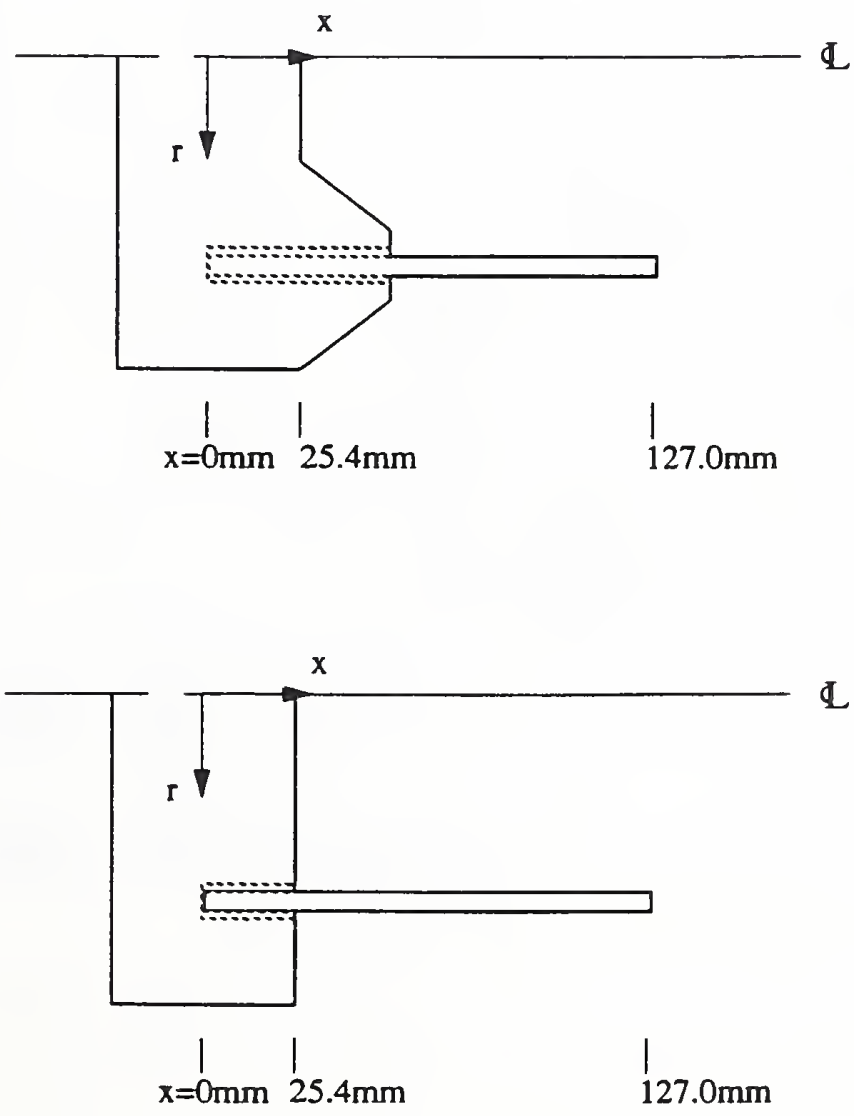

Figure 3.2. Coordinate system used in the analysis. 
The smeared material properties used in the analyses for the glass-epoxy are given in Tables 3.1 and 3.2, while Table 3.3 lists the material properties used for the aluminum. The material properties of the filled-epoxy compound to bond the cylinder into the groove in the aluminum end fitting are shown in Table 3.4. The results for the thick cylinders are discussed in some detail. Since there is considerable similarity between the responses of the thin and thick cylinders, the thin cylinders are not discussed. The results for the thin cylinders are included in Appendix C. Herein, for ease of notation, lay-up no. 1 , which has stacking sequence $[ \pm 10]_{6 n}$ will be denoted by [10], and lay-up no. 2 , which has stacking sequence $\left[82 / \pm 45 / \pm 10_{3} / \pm 45 / \pm 82\right]_{n}$ will be denoted by $[82 / 45 / 10]$.

Table 3.1. Smeared properties for [10] laminate.

\begin{tabular}{|c|c|c|c|c|}
\hline \multirow{2}{*}{ Property } & \multicolumn{4}{|c|}{ Temperature } \\
\hline & $4 \mathrm{~K}$ & $\left(-452^{\circ} \mathrm{F}\right)$ & $297 \mathrm{~K}$ & $\left(77^{\circ} \mathrm{F}\right)$ \\
\hline$E_{x}, \mathrm{GPa}(\mathrm{Msi})$ & 48.8 & $(7.08)$ & 42.2 & $(6.12)$ \\
\hline$E_{r}, \mathrm{GPa}(\mathrm{Msi})$ & 31.9 & $(4.62)$ & 15.6 & $(2.26)$ \\
\hline$E_{\theta}, \mathrm{GPa}(\mathrm{Msi})$ & 31.1 & $(4.51)$ & 15.1 & $(2.20)$ \\
\hline$\nu_{x r}, \mathrm{GPa}(\mathrm{Msi})$ & 0.261 & & 0.236 & \\
\hline$\nu_{x \theta}, \mathrm{GPa}(\mathrm{Msi})$ & 0.306 & & 0.332 & \\
\hline$\nu_{r \theta}, \mathrm{GPa}(\mathrm{Msi})$ & 0.340 & & 0.302 & \\
\hline$G_{x r}, \mathrm{GPa}(\mathrm{Msi})$ & 8.71 & $(1.26)$ & 4.05 & $(0.587)$ \\
\hline$G_{x \theta}, \mathrm{GPa}(\mathrm{Msi})$ & 9.80 & $(1.42)$ & 5.19 & $(0.752)$ \\
\hline$G_{r \theta}, \mathrm{GPa}(\mathrm{Msi})$ & 6.21 & $(0.901)$ & 2.86 & $(0.415)$ \\
\hline$\epsilon_{x}^{T}$ & -15 & $\times 10^{-6}$ & & \\
\hline$\epsilon_{r}^{T}$ & -530 & $\times 10^{-6}$ & & \\
\hline$\epsilon_{\theta}^{T}$ & -52 & $\times 10^{-6}$ & & \\
\hline$\alpha_{x}, \mathrm{~K}^{-1}\left({ }^{\circ} \mathrm{F}^{-1}\right)$ & & & $6.30 \times 10^{-6}$ & $\left(3.50 \times 10^{-6}\right)$ \\
\hline$\alpha_{r}, \mathrm{~K}^{-1}\left({ }^{\circ} \mathrm{F}^{-1}\right)$ & & & $26.1 \times 10^{-6}$ & $\left(14.5 \times 10^{-6}\right)$ \\
\hline$\alpha_{\theta}, \mathrm{K}^{-1}\left({ }^{\circ} \mathrm{F}^{-1}\right)$ & & & $25.7 \times 10^{-6}$ & $\left(14.3 \times 10^{-6}\right)$ \\
\hline
\end{tabular}


Table 3.2. Smeared properties for [82/45/10] laminate.

\begin{tabular}{l|cc|cc}
\hline \hline \multirow{2}{*}{ Property } & \multicolumn{4}{|c}{ Temperature } \\
\cline { 2 - 5 } & $4 \mathrm{~K}$ & $\left(-452^{\circ} \mathrm{F}\right)$ & $297 \mathrm{~K}$ & $\left(77^{\circ} \mathrm{F}\right)$ \\
\hline \hline$E_{x}, \mathrm{GPa}(\mathrm{Msi})$ & 39.2 & $(5.68)$ & 29.4 & $(4.27)$ \\
$E_{r}, \mathrm{GPa}(\mathrm{Msi})$ & 32.3 & $(4.68)$ & 16.0 & $(2.32)$ \\
$E_{\theta}, \mathrm{GPa}(\mathrm{Msi})$ & 32.8 & $(4.75)$ & 19.8 & $(2.87)$ \\
$\nu_{x r}, \mathrm{GPa}(\mathrm{Msi})$ & 0.253 & & 0.218 & \\
$\nu_{x \theta}, \mathrm{GPa}(\mathrm{Msi})$ & 0.346 & & 0.368 & \\
$\nu_{r \theta}, \mathrm{GPa}(\mathrm{Msi})$ & 0.282 & & 0.205 & \\
$G_{x r}, \mathrm{GPa}(\mathrm{Msi})$ & 7.74 & $(1.12)$ & 3.58 & $(0.520)$ \\
$G_{x \theta}, \mathrm{GPa}(\mathrm{Msi})$ & 12.3 & $(1.78)$ & 7.97 & $(1.16)$ \\
$G_{r \theta}, \mathrm{GPa}(\mathrm{Msi})$ & 6.82 & $(0.990)$ & 3.15 & $(0.457)$ \\
$\epsilon_{x}^{T}$ & $-232 \times 10^{-6}$ & & \\
$\epsilon_{r}^{T}$ & $-5550 \times 10^{-6}$ & & \\
$\epsilon_{\theta}^{T}$ & $-3900 \times 10^{-6}$ & & \\
$\alpha_{x}, \mathrm{~K}^{-1}\left({ }^{\circ} \mathrm{F}^{-1}\right)$ & & & $8.89 \times 10^{-6}$ & $\left(4.94 \times 10^{-6}\right)$ \\
$\alpha_{r}, \mathrm{~K}^{-1}\left({ }^{\circ} \mathrm{F}^{-1}\right)$ & & & $28.3 \times 10^{-6}$ & $\left(15.7 \times 10^{-6}\right)$ \\
$\alpha_{\theta}, \mathrm{K}^{-1}\left({ }^{\circ} \mathrm{F}^{-1}\right)$ & & & $16.5 \times 10^{-6}$ & $\left(9.19 \times 10^{-6}\right)$ \\
\hline \hline
\end{tabular}

Table 3.3. Properties of aluminum.

\begin{tabular}{l|cc|cc}
\hline \multirow{2}{*}{ Property } & \multicolumn{4}{|c}{ Temperature } \\
\cline { 2 - 5 } & $4 \mathrm{~K}$ & $\left(-452^{\circ} \mathrm{F}\right)$ & $297 \mathrm{~K}$ & $\left(77^{\circ} \mathrm{F}\right)$ \\
\hline \hline$E, \mathrm{GPa}(\mathrm{Msi})$ & 77.2 & $(11.2)$ & 68.9 & $(10.0)$ \\
$\nu$ & 0.343 & & 0.300 & \\
$G, \mathrm{GPa}(\mathrm{Msi})$ & 28.7 & $(4.21)$ & 26.5 & $(3.85)$ \\
$\epsilon^{T}$ & $-4200 \times 10^{-6}$ & & \\
$\alpha, \mathrm{K}^{-1}\left({ }^{\circ} \mathrm{F}^{-1}\right)$ & & & $23.4 \times 10^{-6}$ & $\left(13.0 \times 10^{-6}\right)$ \\
\hline \hline
\end{tabular}


Table 3.4. Properties of filled epoxy.

\begin{tabular}{l|cc|cc}
\hline \hline \multirow{2}{*}{ Property } & \multicolumn{4}{|c}{ Temperature } \\
\cline { 2 - 5 } & $4 \mathrm{~K}$ & $\left(-452^{\circ} \mathrm{F}\right)$ & $297 \mathrm{~K}$ & $\left(77^{\circ} \mathrm{F}\right)$ \\
\hline \hline$E, \mathrm{GPa}(\mathrm{Msi})$ & 6.89 & $(1.00)$ & 3.45 & $(0.50)$ \\
$\nu$ & 0.400 & & 0.400 & \\
$G, \mathrm{GPa}(\mathrm{Msi})$ & 2.46 & $(0.36)$ & 1.23 & $(0.18)$ \\
$\epsilon^{T}$ & $-800 \times 10^{-6}$ & & \\
$\alpha, \mathrm{K}^{-1}\left({ }^{\circ} \mathrm{F}^{-1}\right)$ & & & $39.6 \times 10^{-6}$ & $\left(22.0 \times 10^{-6}\right)$ \\
\hline \hline
\end{tabular}

\section{1 [10] Cylinders}

First discussed is the response of the thick [10] cylinder to a temperature change from room temperature to $4 \mathrm{~K}$. Material properties evaluated at $4 \mathrm{~K}$ are used. Then the response of the thick [10] cylinder to an applied axial load is discussed, the material properties at $4 \mathrm{~K}$ being used. Finally the response of the thick [10] cylinder to an applied axial load using room-temperature properties is discussed. Comparison of the last two cases enables evaluation of the influence of temperature-dependent material properties on cylinder response. The responses of the thick [82/45/10] cylinders to these same three conditions are then considered. Comparison of the three conditions for the two lay-ups enables evaluation of lamination effects on cylinder response.

\subsubsection{Thermally Induced Strain - Baseline-Design End Fitting}

The deformations in the cylinder with the baseline-design end fitting that result from lowering the temperature from room temperature to $4 \mathrm{~K}$ can be assessed by comparing the outline of the undeformed mesh in Figure 3.1(a) with the deformed mesh in Figure 3.3. As can be seen, lowering the temperature causes the portion of the cylinder away from the end fitting to decrease in radius. The end fitting contracts radially, but not much differently than the cylinder, thus leading to just a slight bending of the cylinder wall as it exits the end fitting. The thermal dilatation properties of the cylinder wall (Table 3.1) relative to the dilatational properties of the aluminum end fitting (Table 3.3) are responsible for this slight inward bending of the cylinder wall.

The strains due to lowering the temperature from room level to $4 \mathrm{~K}$ for the thick [10] cylinder with a baseline end fitting are illustrated in Figures 3.4 and 3.5. Throughout, the strains shown are the total strains minus thermal dilatation effects. It is this difference that is used directly in Hooke's Law to compute the stresses. The principal material intralaminar strains at a representative radial position within the $+10^{\circ}$ ply at the outer radial location and at a representative radial position within the $-10^{\circ}$ ply at the inner radial location are shown in Figure 3.4, the fiber-direction and transverse strains being at the top and center, respectively, 


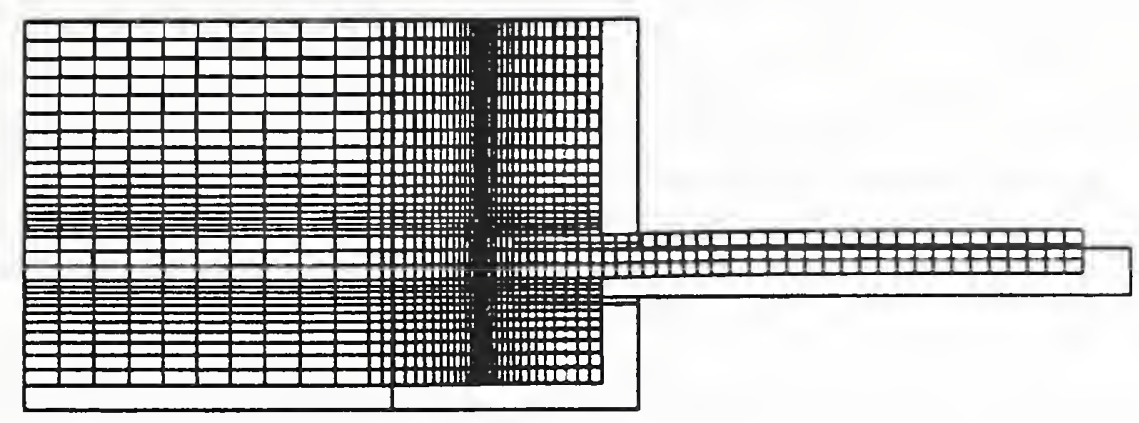

Figure 3.3. Deformations due to temperature change of thick [10] cylinder with baseline-design end fitting $(\Delta T=-294 \mathrm{~K})$.

of the figure, and the shear strains being at the bottom. (The term 'representative radial position' within the outer ply is used to mean that the strains at the Gauss point closest to the outer radius are what are reported. A similar interpretation in terms of Gauss-point location is associated with the inner-ply location.) The strains in each ply are due to two effects: (1) the differences in thermal-expansion properties from one ply to the next within the cylinder wall and (2) the aforementioned difference in thermal-expansion properties between the aluminum end fitting and the cylinder wall.

As can be seen in Figure 3.4, the most severe tensile strain in the fiber direction $\left(\epsilon_{1}\right)$ occurs at the outer-ply location just as the cylinder wall exits the end fitting. The strain level is 1000 microstrain, and from the deformed mesh it is clear why the most severe tensile strain occurs here. At this same axial location, but on the outer-ply location of the cylinder, the strain is practically zero. Higher fiber-direction compressive strains occur within the end fitting, particularly near the end of the cylinder. These are due to the thermally induced contraction of the aluminum in the axial direction being greater than the thermally induced contraction of the cylinder in the axial direction. As can be seen in the membrane region, the strains at the innerand outer-ply locations do not vary with axial location.

The intralaminar transverse strains $\left(\epsilon_{2}\right)$ are low but are most severe within the end fitting, the strains being tensile and of similar magnitude at the inner- and outer-ply locations. Since the 2 direction within a ply is approximately circumferential, the small tensile strain reflects the fact that upon cooling to $4 \mathrm{~K}$ the cylinder tends to shrink only slightly more in the circumferential direction than does the end fitting. An examination in Tables 3.1 and 3.3 of the circumferential dilatational strain at $4 \mathrm{~K}$ for the [10] lay-up and the dilatational strain for the aluminum shows this to be the case.

The principal material system intralaminar shear strains $\left(\gamma_{12}\right)$ in the inner and outer plies are shown in the lower portion of Figure 3.4. With shear strains, the magnitude, not the sign, is important. As can be seen, the largest shear strain occurs in the outer ply just as the cylinder exits the end fitting. However, strains of similar magnitude occur throughout the membrane region, away from the boundary layer. This indicates that the influence on residual shear strain of cooling from room temperature to $4 \mathrm{~K}$ within the cylinder material is about as severe as the conditions encountered where the cylinder exits the end fitting. Note that the levels of 
fiber direction and transverse strain away from the end fitting are close to zero, indicating negligible residual thermal effects on these strain components within the material upon cooling from room temperature to $4 \mathrm{~K}$.

The interlaminar strains due to cooling from room temperature to $4 \mathrm{~K}$ are shown in Figure 3.5. The interlaminar strains are shown at three radial locations, the inner-ply location, the midply location, and the outer-ply location. As mentioned, the midply location is added to the discussion of these strains because the interlaminar strains, particularly the interlaminar
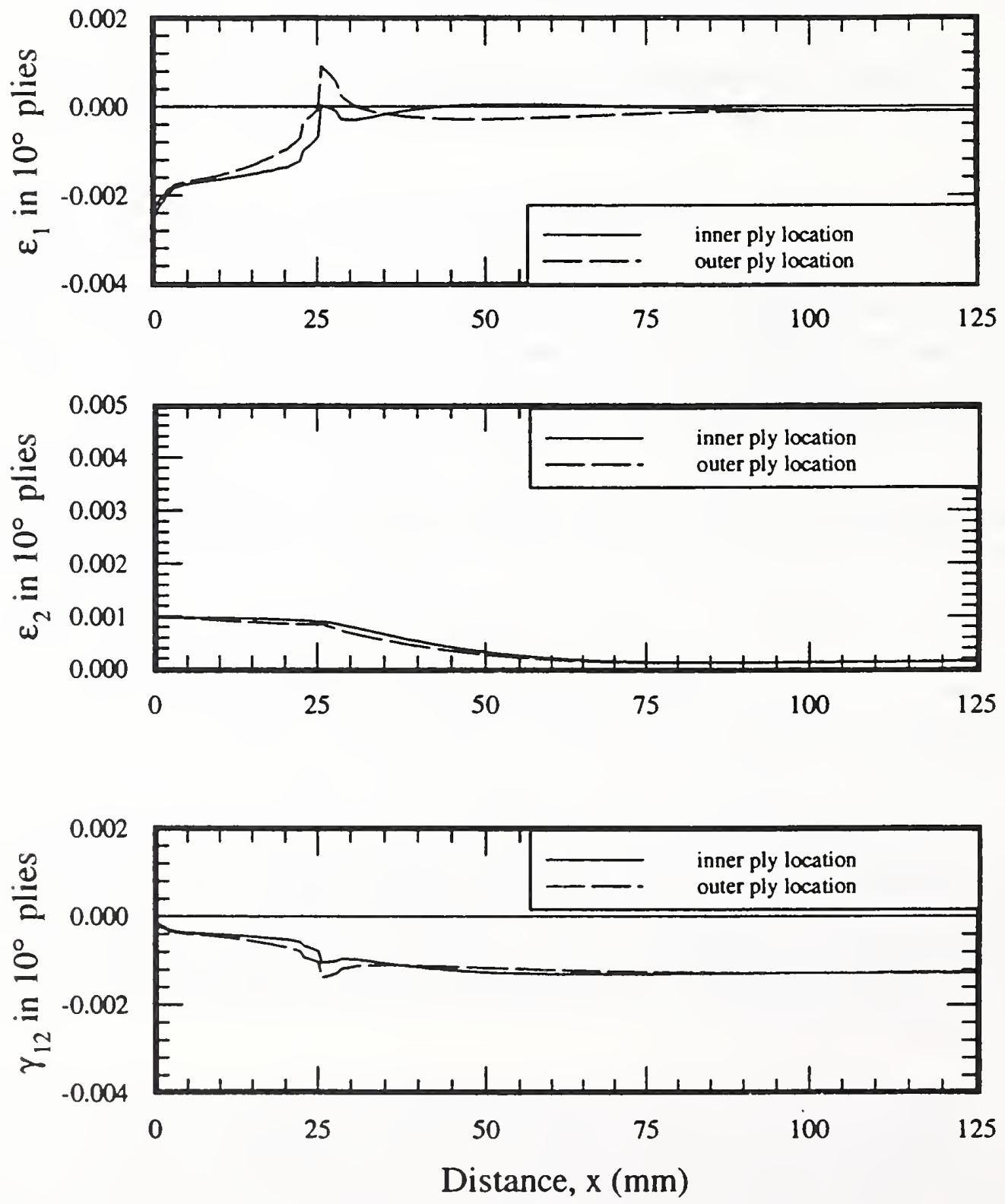

Figure 3.4. Intralaminar strains due to a temperature change of $-294 \mathrm{~K}$. in thick [10] cylinder with baseline-design end fitting. 
shear strain, can be maximum in the center of the cylinder wall. Here the interlaminar radial strain is both tensile and compressive, the tensile strain being of more importance since it implies the plies have the tendency to move apart in the thickness direction, leading to delamination. The magnitude of the tensile radial strain is about 1600 microstrain, the peak values occurring near the end of the cylinder, within the end fitting. The interlaminar shear strain is high at the end of the cylinder, within the end fitting. The interlaminar shear strain has a local peak as it exits the end fitting. Care must be taken when considering the peak values of strain, both here with the interlaminar shear strains, and in general, with all the strains. The finite element model considers no filets or radiusing effects with geometry nor yielding with material behavior. All corners, both interior and exterior, are perfect $90^{\circ}$ angles. In theory, right angles lead to infinite stress concentrations. However, in reality, the corners are not perfect right angles, and even for sharp corners nonlinear material behavior such as yielding or crazing occurs, which redistributes the load and reduces the actual stress concentration. Here perfect right angles are assumed to occur at the end of the cylinder, that is, at the base of the groove and in the potting material as the cylinder exits the end fitting. With the potting material and the cylinder wall forming a right-angle fillet just as the cylinder exits the end fitting, high strain concentrations will be computed.
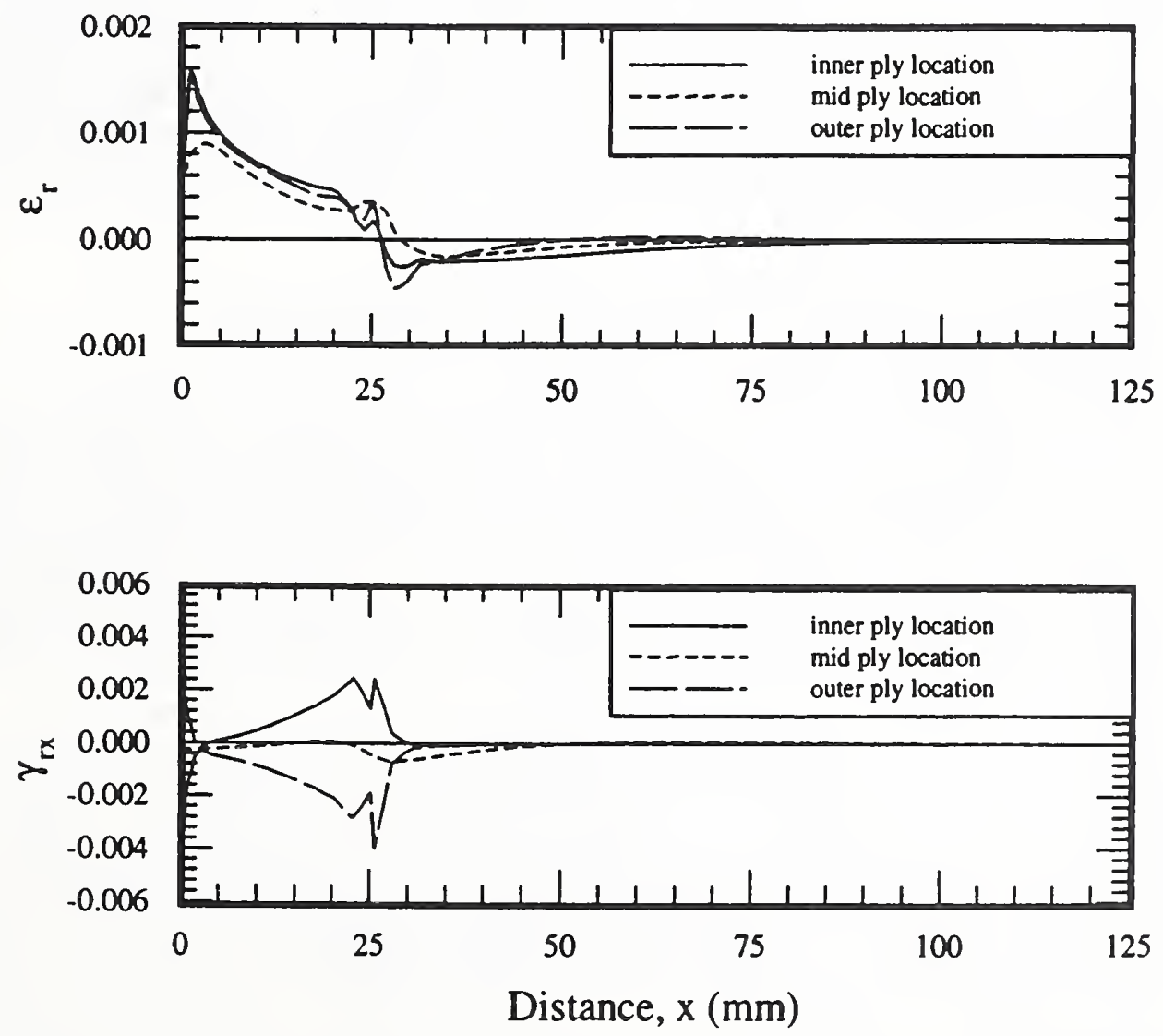

Figure 3.5. Interlaminar strains due to a temperature change of $-294 \mathrm{~K}$ in thick [10] cylinder with baseline-design end fitting. 


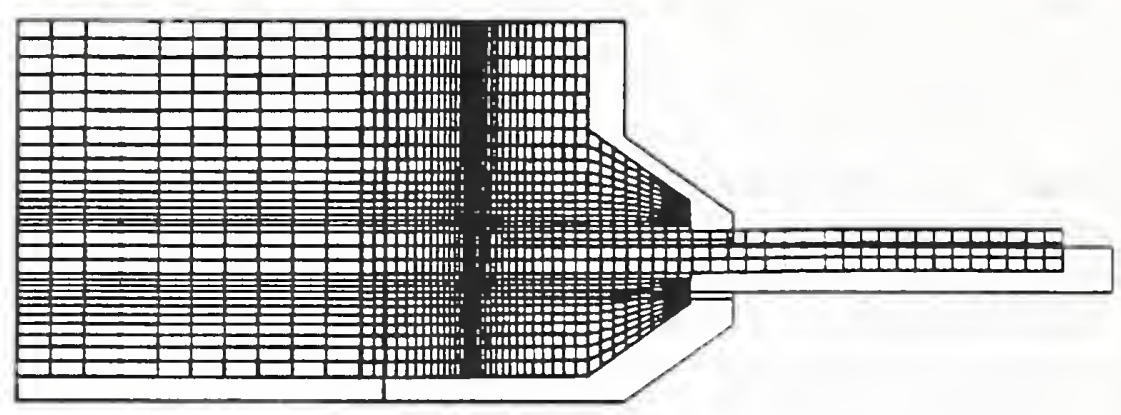

Figure 3.6. Deformation due to temperature change of thick [10] cylinder with alternative-design end fitting $(\Delta T=-294 \mathrm{~K})$.

\subsubsection{Thermally Induced Strain: Alternative-Design End Fitting}

The thermally induced deformations in the cylinder with the alternative end fitting are illustrated in Figure 3.6. The influence on the thermally induced strains of introducing the alternative end fitting is illustrated in Figures 3.7 and 3.8. These figures should be compared with Figures 3.4 and 3.5. Note: similar scales are used for ease of comparison. As can be observed, the primary effect of the alternative end fitting is to shift, in the axial direction, the location of the peak strains. In fact, the peak strains are shifted by $23.2 \mathrm{~mm}(0.915 \mathrm{in})$, the length of the tapered extension to the end fitting. Any reduction in strain levels is difficult to detect. If thermally induced strains were the only strains to contend with, and knowing that the alternative design most likely costs more to manufacture, it would not be worth the additional expense of the alternative end fitting.

\subsubsection{Strains due to Load and Low-Temperature Material Properties - Baseline-Design End Fitting}

The application of an axial load results in strains that depend on the axial and radial coordinates differently than the strains resulting from thermally induced effects. The deformed geometry due to the applied load for the baseline end-fitting design (using the material properties of glass-epoxy evaluated at $4 \mathrm{~K}$ ) is shown in Figure 3.9. The applied load results in an average axial stress level of $689 \mathrm{MPa}$. With an applied load, as opposed to a temperature decrease, away from the end fitting, the cylinder expands. The constraining effect of the endfitting results in the cylinder wall bending outward.

The fiber-direction compressive strain, the upper part of Figure 3.10, is maximum just as the cylinder wall exits the end cap. The sharp peak in the fiber-direction strain response shows the magnitude of the maximum compressive strain to be about 17,500 microstrain. This is a large value, and it occurs at the outer ply of the wall. An examination of the deformed geometry in Figure 3.9 reveals why the maximum compressive strain is located at the outer radial location. Away from the end fitting, in the membrane region, the fiber-direction compressive strain is about 13,500 microstrain. This represents a strain concentration effect due to the end fitting of about $1.3(17,500 / 13,500)$. The inner-ply location within the wall does not experience as large a fiber-direction compressive strain. Within the end fitting, the fiberdirection compressive strains are not large. Note that compared to the thermally induced fiberdirection compressive strain shown in Figure 3.4, the load-induced fiber-direction strain is considerably larger throughout the cylinder. 

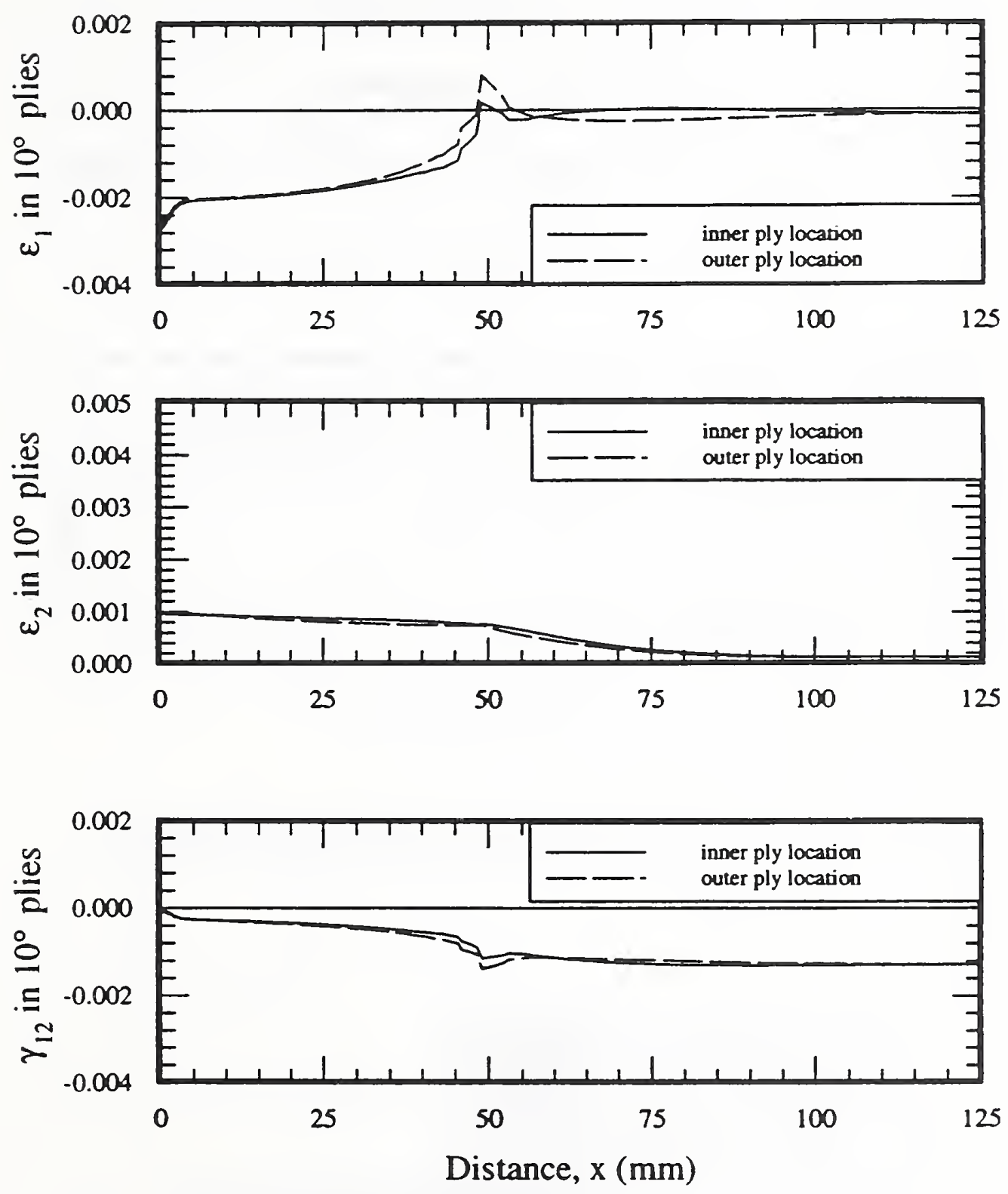

Figure 3.7. Intralaminar strains due to a temperature change of $-294 \mathrm{~K}$ in thick [10] cylinder with alternative-design end fitting. 

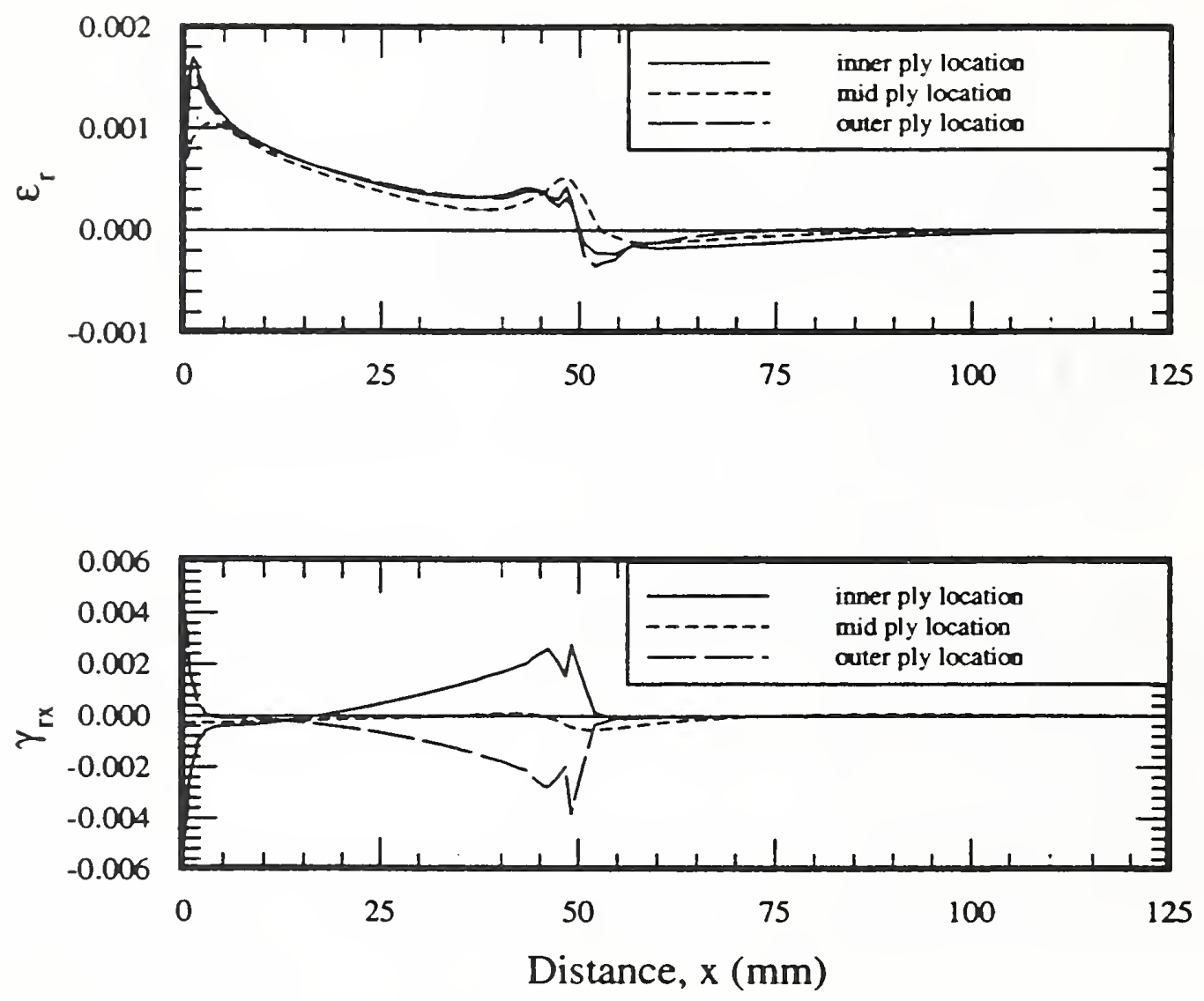

Figure 3.8. Interlaminar strains due to a temperature change of $-294 \mathrm{~K}$ in thick [10] cylinder with alternative-design end fitting.

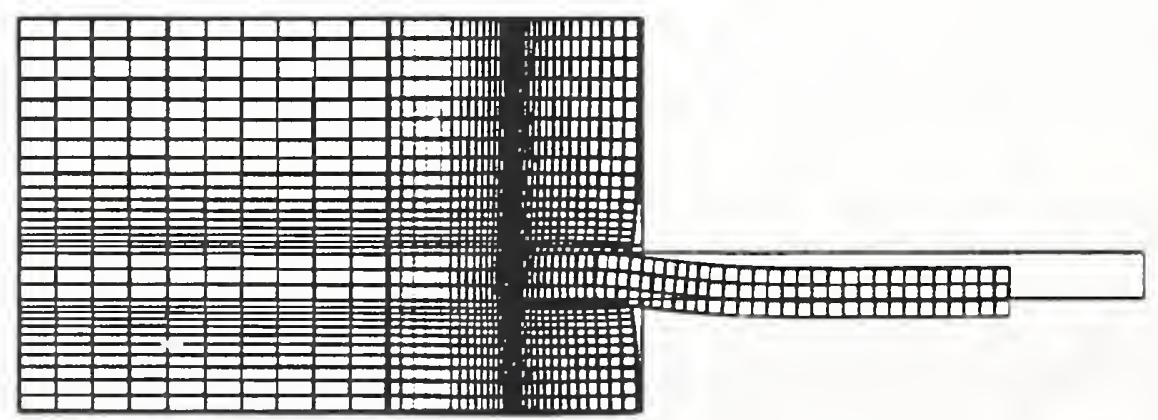

Figure 3.9. Deformation due to applied load of thick [10] cylinder with baseline-design end fitting, low-temperature properties. 

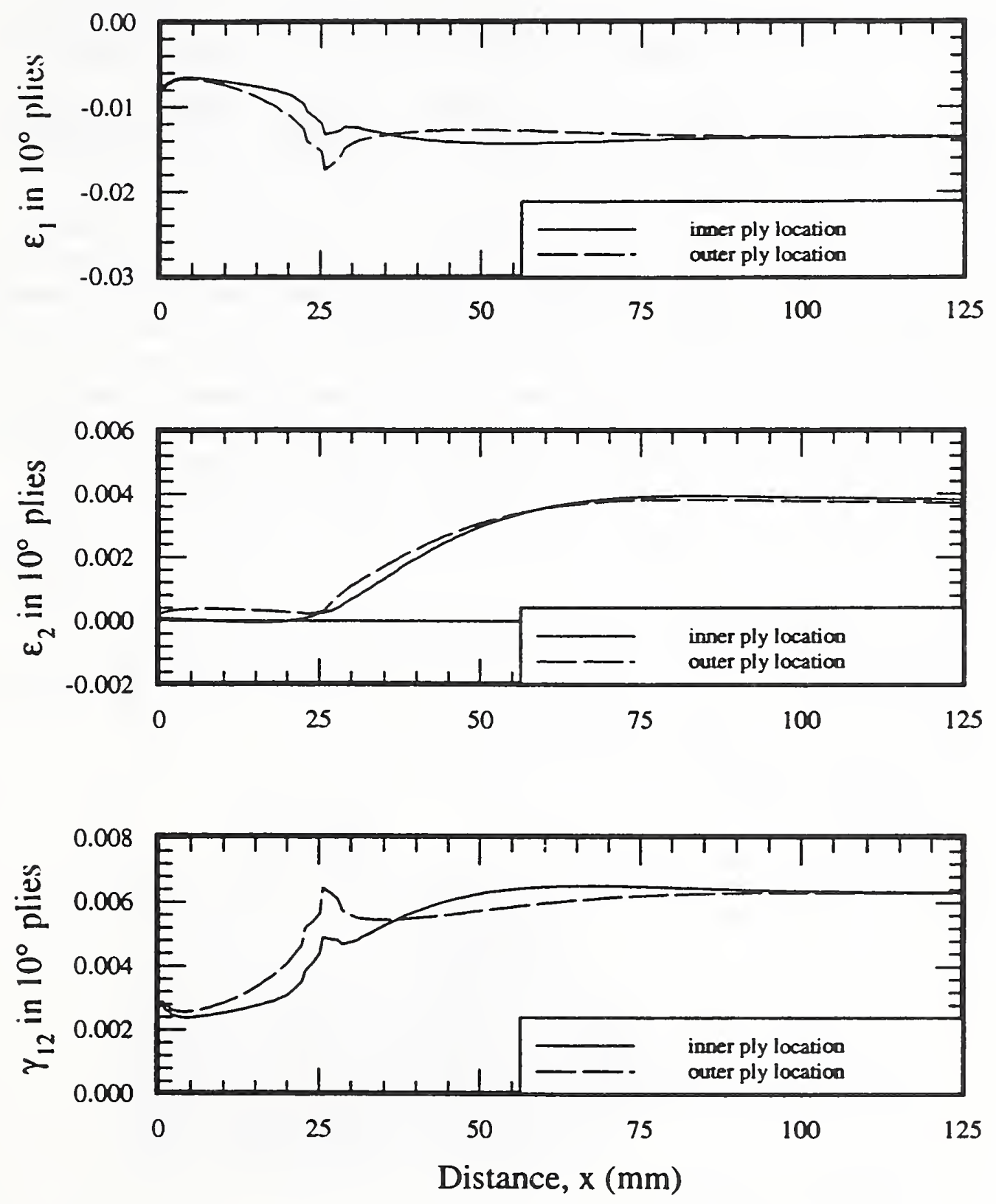

Figure 3.10. Intralaminar strains due to applied axial stress of $-689 \mathrm{MPa}$ in thick [10] cylinder with baseline-design end fitting, 4-K properties. 
The transverse strain, the center portion of Figure 3.10, is tensile and has a maximum value well away from the end fitting, within the membrane region of the cylinder. The maximum level of this tensile strain is just under 4000 microstrain. The low level of transverse strain within the end fitting, the transverse strain again being practically circumferential, is indicative of the effect of the end fitting becoming only slightly larger in circumference owing to the applied load and thus restraining the cylinder within it from circumferential expansion.

The intralaminar shear strain $\gamma_{12}$, shown in the lower portion of Figure 3.10 , exhibits a peak just as the cylinder exits the end fitting. However, this peak is not too different than the level of strain experienced in the membrane region, a level of about 6300 microstrain.

The load-induced interlaminar strains, $\epsilon_{r}$ and $\gamma_{r x}$, due to the axial load and material properties evaluated at $4 \mathrm{~K}$, are illustrated in Figure 3.11. As the cylinder exits the end fitting, the radial strain experiences a large tensile value, the peak value being about 5800 microstrain. Interestingly enough, the peak value is outside the end fitting by $2.5 \mathrm{~mm}(0.1 \mathrm{in})$. In the membrane region, the radial strain is similar at all three radial locations and has a magnitude of about 3800 microstrain. The interlaminar shear strains show an extreme peak at the exit to the end fitting, the actual spike being due to the aforementioned idealized fillet geometry. Without the spike, the maximum value of interlaminar shear strain occurs about $5 \mathrm{~mm}(0.2 \mathrm{in})$ inside the end fitting. This peak value of the shear strain is about 10,000 microstrain.
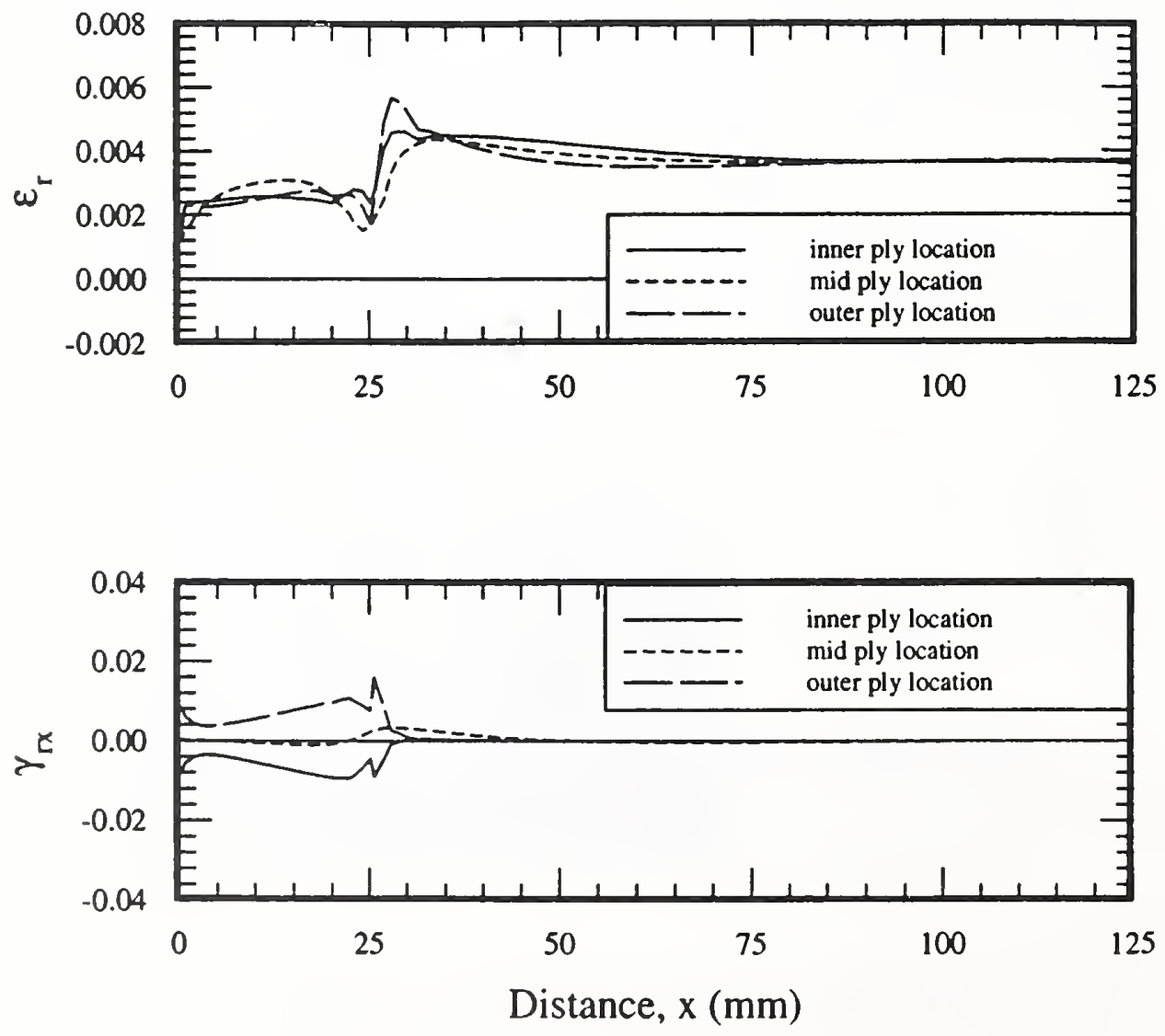

Figure 3.11. Interlaminar strains due to applied axial stress of $-689 \mathrm{MPa}$ in thick [10] cylinder with baseline-design end fitting, 4-K properties. 


\subsubsection{Strains due to Load and Low-Temperature Material Properties - Alternative-Design End Fitting}

The deformations that result from applying the load to a cylinder with the alternative end fitting and material properties evaluated at $4 \mathrm{~K}$ are illustrated in Figure 3.12. The deformations where the cylinder exits the taper are similar to the deformations associated with the baseline end fitting (Fig. 3.9). The strains that result from the application of the axial load with the alternative end fitting and the material properties evaluated at $4 \mathrm{~K}$ are shown in Figures 3.13 and 3.14. To assess the effect of the end fitting, these figures should be compared to Figures 3.10 and 3.11. Comparing the fiberdirection compressive strains for the two end fittings (Figs. 3.10 and 3.13), again it is seen that the primary effect of the alternative end fitting is to shift the location of the peak fiber-direction strain axially an amount equal to the length of the tapered addition. It is difficult to detect any reduction in the magnitude of the peak strain. The magnitude of the transverse strain is also virtually unaffected by the alternative end fitting design. The shear strain is influenced somewhat, but because the fiberdirection compressive strain and the transverse strain are not influenced, the apparent influence of the tapered end fitting on the shear strain is considered of secondary value.

The influence of the alternative end-cap design on the interlaminar strains (Fig. 3.14), when compared with the interlaminar strains due to the baseline design (Fig. 3.11), is negligible. The peak value of the interlaminar radial strain is reduced slightly, but the interlaminar shear strain, not counting the spike, actually increases.

In summary, it appears that the particular alternative end-cap design studied is not effective in reducing the strain-concentration effects in the $[10]$ cylinder.

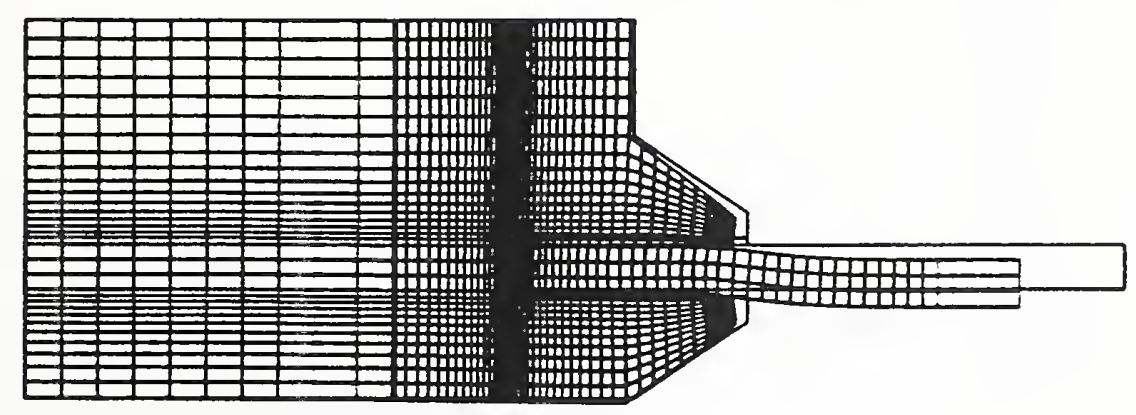

Figure 3.12. Deformation due to applied load of thick [10] cylinder with alternative-design end fitting, low-temperature properties. 

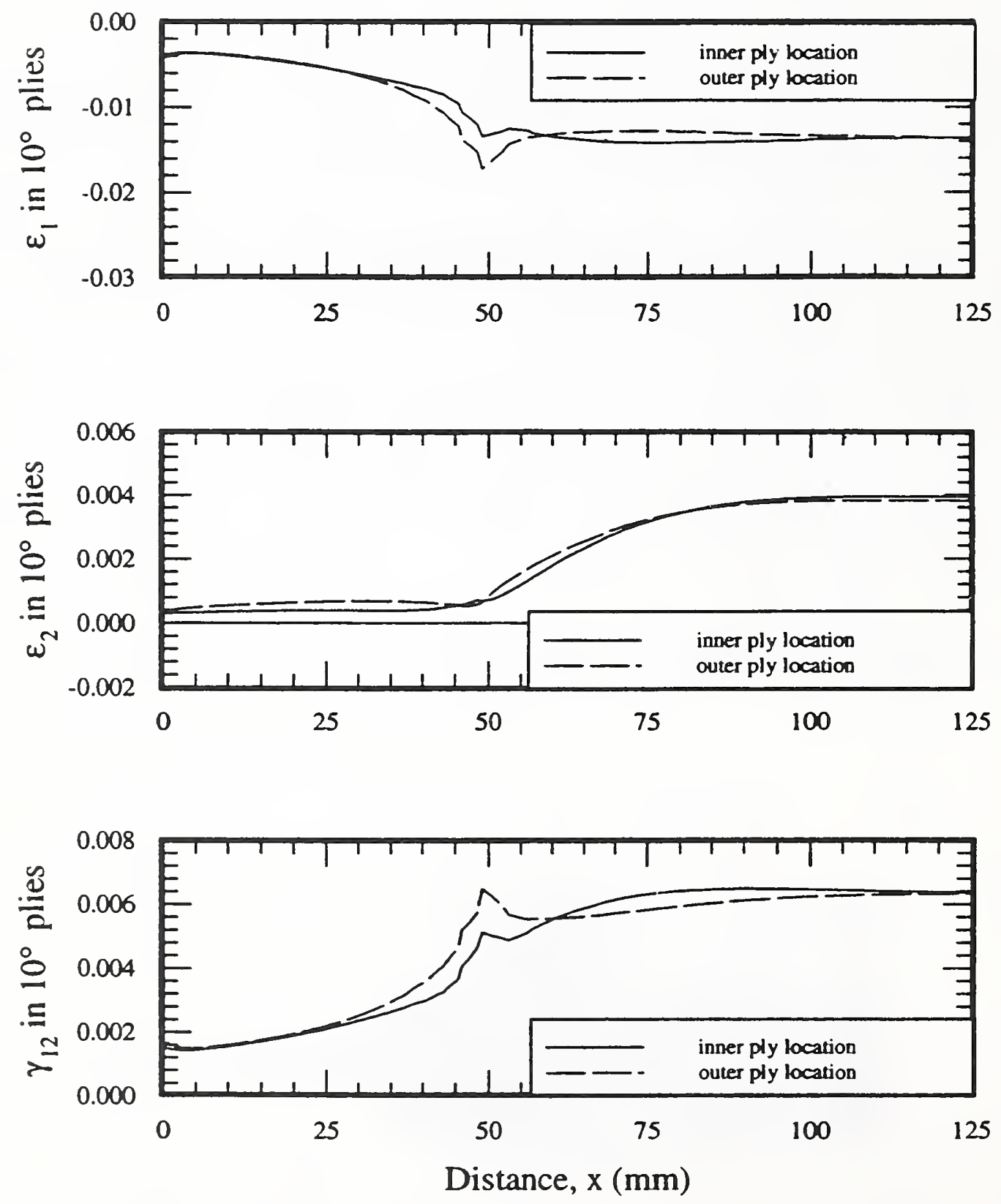

Figure 3.13. Intralaminar strains due to applied axial stress of $-689 \mathrm{MPa}$ in thick [10] cylinder with alternative-design end fitting, 4-K properties. 

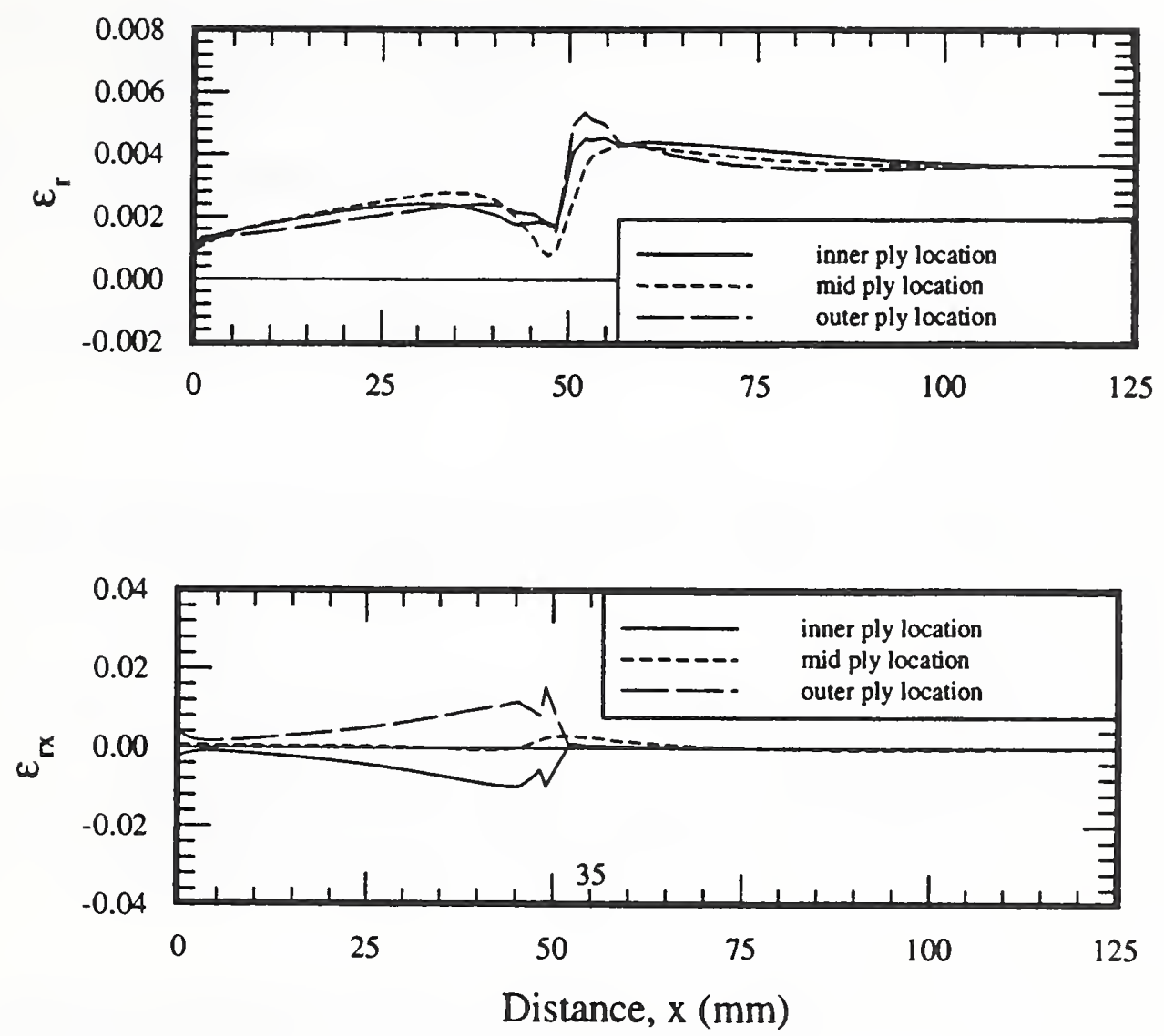

Figure 3.14. Interlaminar strains due to applied axial stress of $-689 \mathrm{MPa}$ in thick [10] cylinder with alternative-design end fitting, 4-K properties.

\subsubsection{Strains due to Load and Room-Temperature Material Properties - Baseline-Design End Fitting}

Since the one end of the SMES support cylinder is operating at noncryogenic temperatures, it is of interest to determine whether the difference in material properties between $4 \mathrm{~K}$ and room temperature has any effect on the load-induced strains. As can be seen from the material properties given in Tables 3.1 and 3.2, glass-epoxy is softer at room temperature than at $4 \mathrm{~K}$, owing to the stiffening of the epoxy matrix at lower temperatures. Within the scale of the figures, the deformed geometry for the case of room-temperature properties cannot be distinguished from the deformed geometry for the case properties at $4 \mathrm{~K}$. Thus, for the remainder of the discussion, deformed geometries will not be shown.

The load-induced strains due to the baseline end-fitting design and room-temperature properties are illustrated in Figures 3.15 and 3.16. These figures should be compared, respectively, to the previously discussed Figures 3.10 and 3.11, illustrating the strains for the 4-K temperature conditions. 

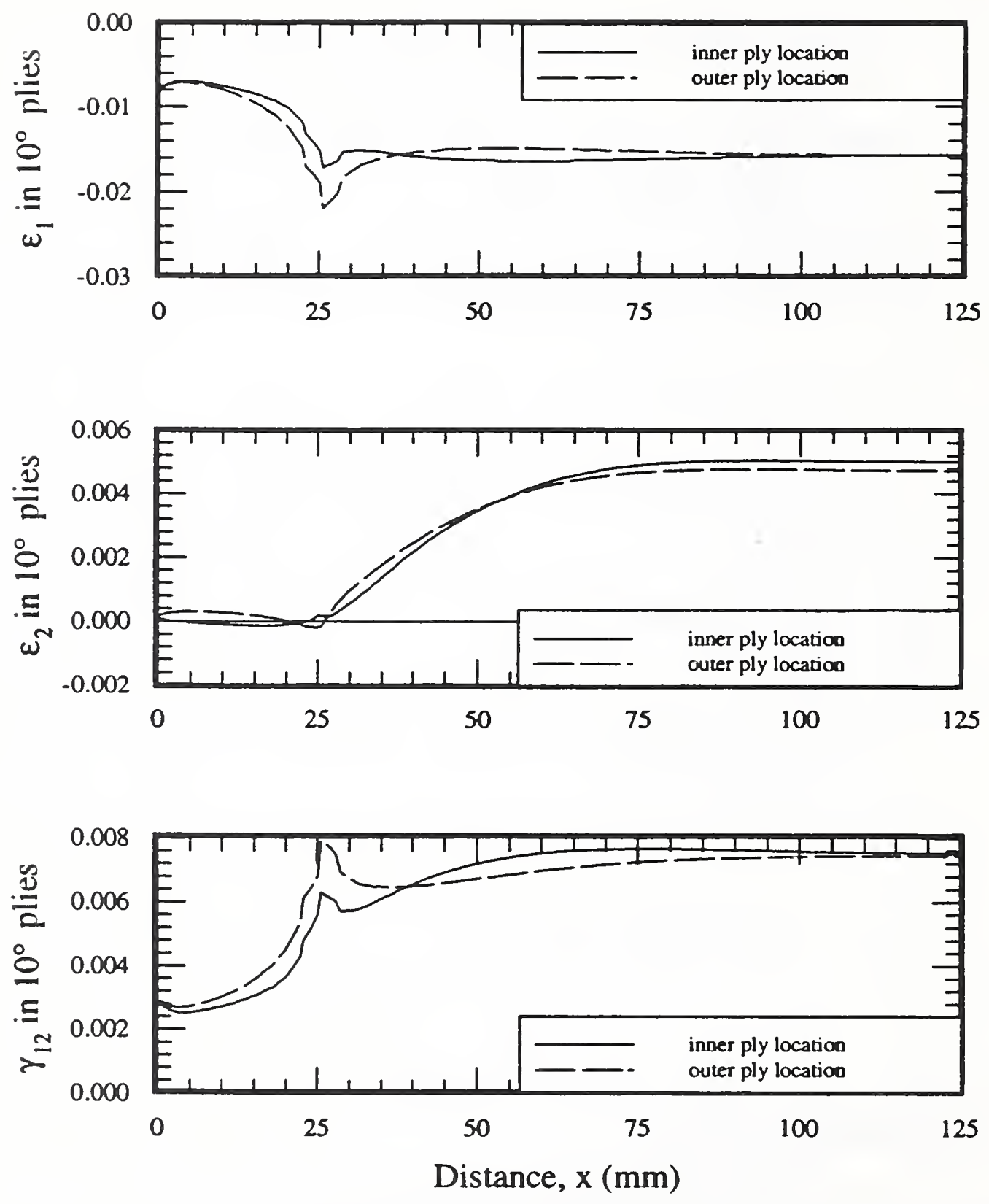

Figure 3.15. Intralaminar strains due to applied axial stress of $-689 \mathrm{MPa}$ in thick [10] cylinder with alternative-design end fitting, 297-K properties.

A close comparison of Figures 3.10 and 3.15 indicates that fiber-direction strains are greater when the cylinder is loaded at room temperature. However, the general character of the strains at the inner- and outer-ply locations does not depend on the temperature. For both temperatures, the most severe compressive strain occurs in the outer plies as the cylinder exits the end fitting. Further examination of these two figures shows that the transverse strains in the membrane region are $25 \%$ larger at room temperature than at $4 \mathrm{~K}$. The strain concentration factor for the fiber-direction compressive strain in the $10^{\circ}$ ply is about 1.4 , slightly higher than for the lower temperature case. The shearing strains are also about $25 \%$ larger at room temperature than at $4 \mathrm{~K}$. 

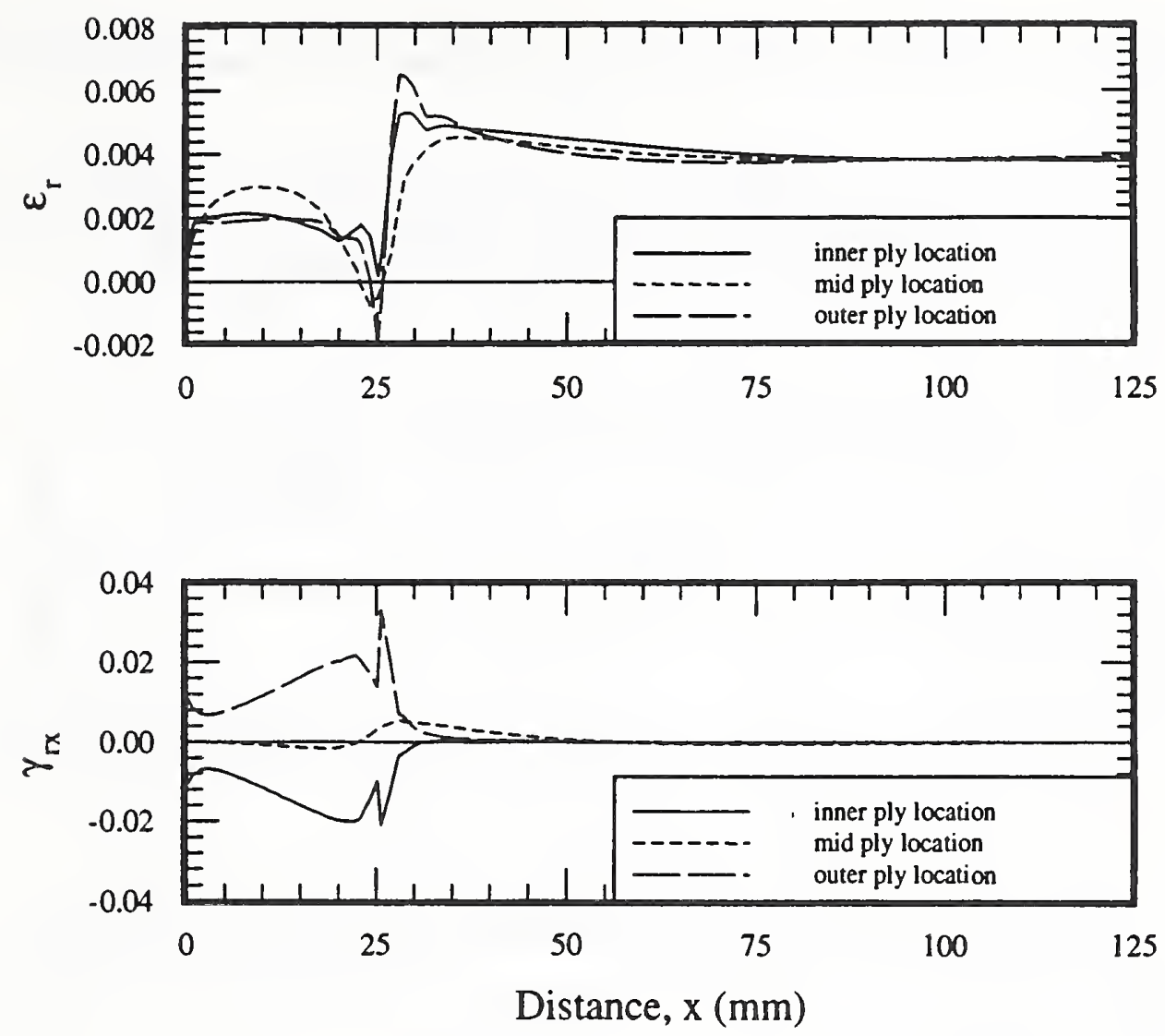

Figure 3.16. Interlaminar strains due to applied axial stress of $-689 \mathrm{MPa}$ in thick [10] cylinder with alternative-design end fitting, 297-K properties.

Examination of the interlaminar shear strains using the room temperature properties (Fig. 3.16) shows that the tensile radial strain at room temperature has increased relative to the strains at $4 \mathrm{~K}$ (Fig. 3.11). The interlaminar shear strain, not counting the spikes, at room temperature has also increased relative to the shear strain at $4 \mathrm{~K}$. This can be easily seen by comparing lower portions of Figures 3.11 and 3.16. As mentioned earlier, the interlaminar strains depend solely on epoxy properties, and with these being softer at room temperature, more strain should be expected.

\subsubsection{Strains due to Load and Room-Temperature Material Properties - Alternative-Design End Fitting}

As with the properties at $4 \mathrm{~K}$, using room-temperature material properties with the alternative end fitting does little more than shift, relative to the baseline case, the characteristics of the strainversus-axial-position relation toward the membrane region by a distance equal to the length of the tapered addition. This can be seen by comparing Figures 3.17 and 3.18 with Figures 3.15 and 3.16. Comparison of Figure 3.17 with Figure 3.13 shows that room-temperature conditions with the alternative end cap result in greater fiber-direction compressive strains, greater transverse strains, and 
greater shear strains. In addition, and as might be expected, the interlaminar strains at room temperature with the alternative end cap (Fig. 3.18) are larger than the interlaminar strains at $4 \mathrm{~K}$ (Fig. 3.14).

It would appear that the differences between material properties at $4 \mathrm{~K}$ and those at room temperature manifest themselves in the strain response of the cylinder, both with the baseline end cap and with the alternative design, being about $25 \%$ larger at room temperature.
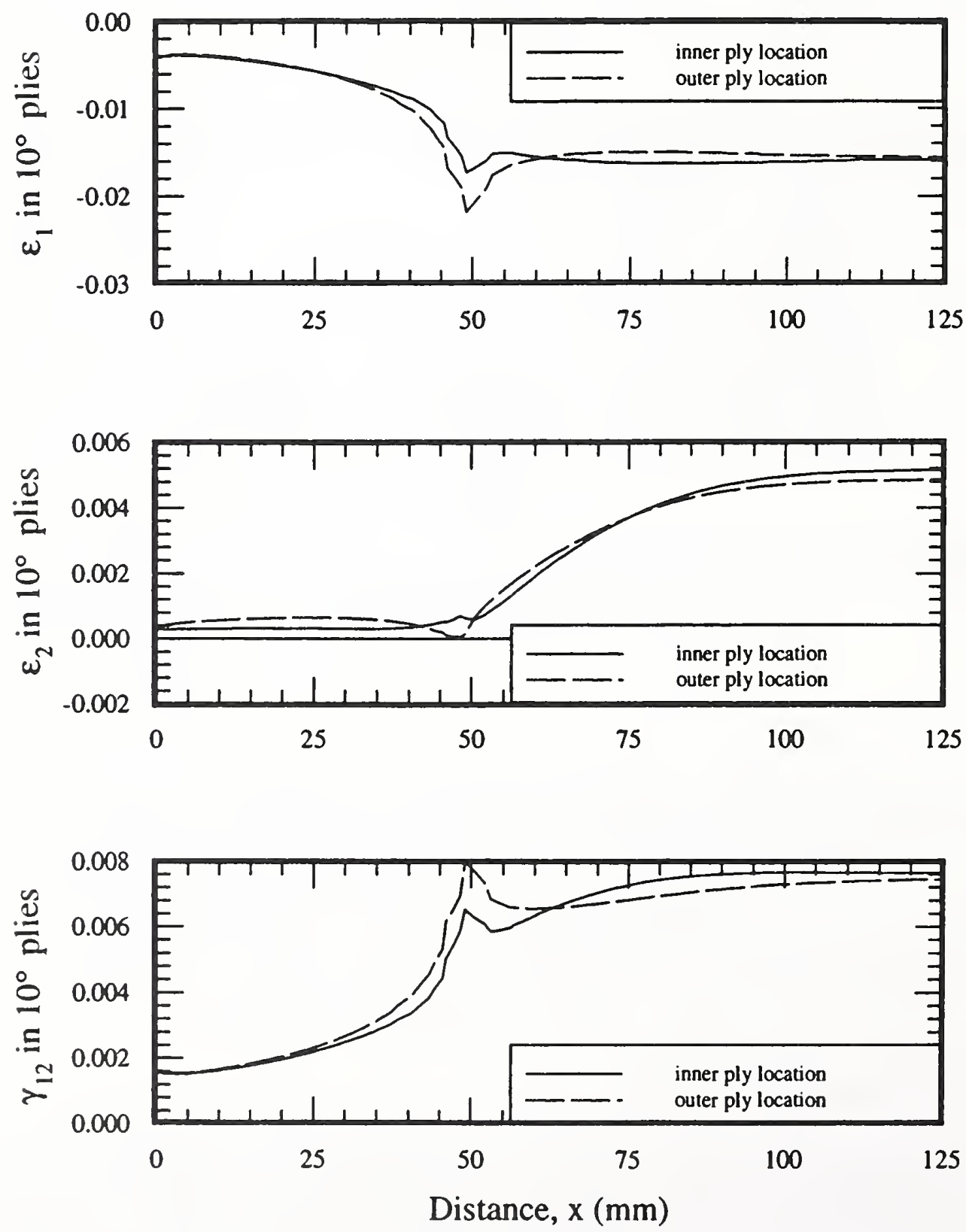

Figure 3.17. Intralaminar strains due to applied axial stress of $-689 \mathrm{MPa}$ in thick [10] cylinder with alternative-design end fitting, 297-K properties. 

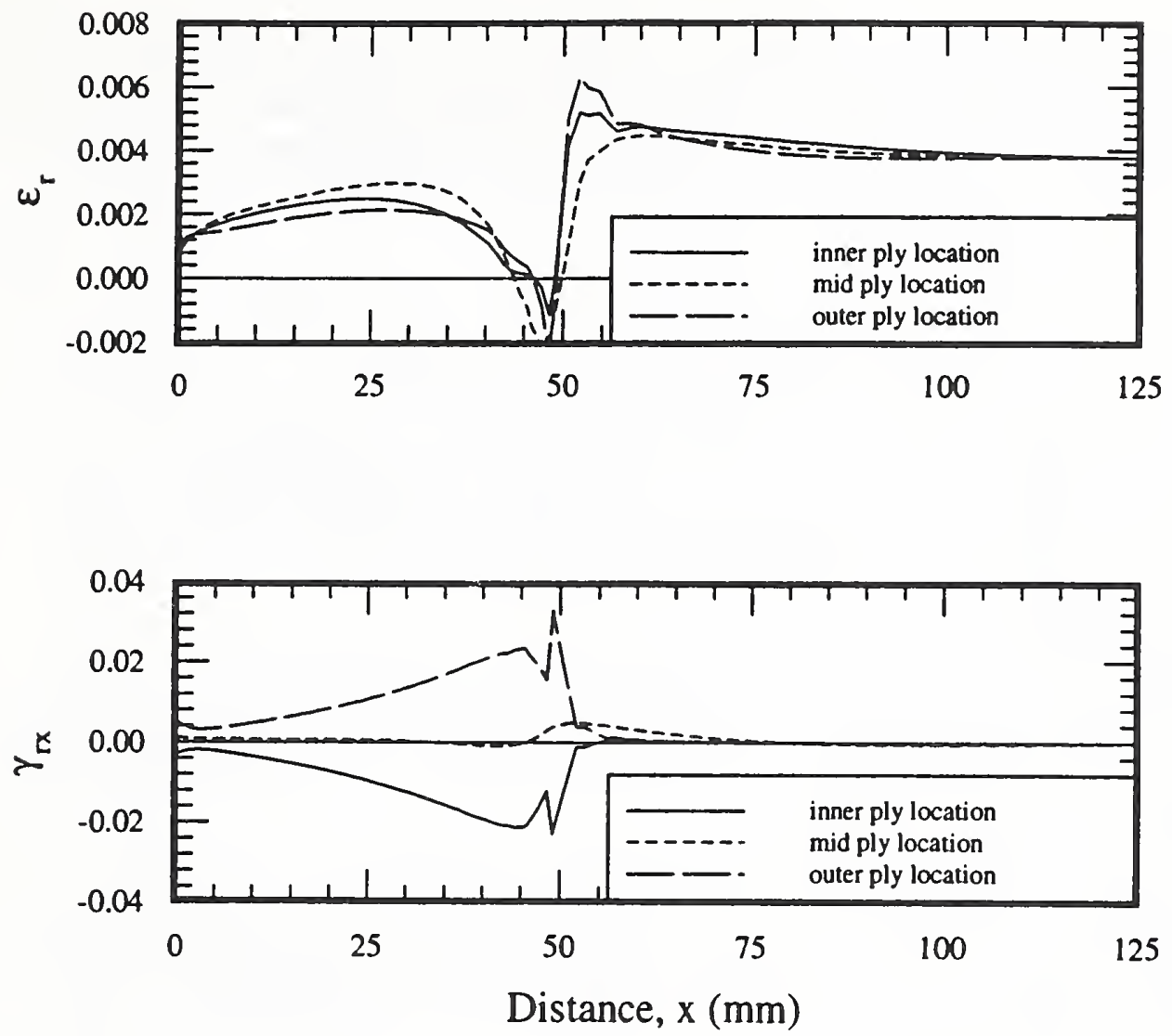

Figure 3.18. Interlaminar strains due to applied axial stress of $-689 \mathrm{MPa}$ in thick [10] cylinder with alternative-design end fitting, 297-K properties.

\section{$3.2[82 / 45 / 10]$ Cylinders}

\subsubsection{Thermally Induced Strains - Baseline-Design End Fitting}

As in the case of the [10] laminate (Fig. 3.4), the thermally induced fiber-direction strain $\left(\epsilon_{1}\right)$ at $4 \mathrm{~K}$ in the $10^{\circ}$ ply for the [82/45/10] laminate is maximum within the end fitting (Fig. 3.19). In fact, the strain in this ply looks much like the strain in the $10^{\circ}$ ply of the [10] laminate as it is cooled to $4 \mathrm{~K}$ from room temperature. The magnitude and character of the strains, including the transverse strain $\left(\epsilon_{2}\right)$ are not similar; transverse strain is maximum (about 1500 microstrain) in the membrane region for the $[82 / 45 / 10]$ laminate. The shear strain $\left(\gamma_{12}\right)$ for the $10^{\circ}$ ply for the two laminates looks similar, but the magnitudes are different, the strain being smaller in the [82/45/10] laminate.

In the $45^{\circ}$ ply in the $[82 / 45 / 10]$ laminate (Fig. 3.20), the fiber-direction strain is about the same magnitude as it is in the $10^{\circ} \mathrm{ply}$, although the strain is higher in the membrane region for the $[82 / 45 / 10]$ laminate than it is for the [10] laminate. This higher membrane-region strain reflects a higher residual thermal strain at $4 \mathrm{~K}$ in the [82/45/10] laminate than in the [10] laminate. The same can be said of the transverse strain, residual effects at $4 \mathrm{~K}$ being higher in the [82/45/10] laminate than in the [10] laminate (Fig. 3.21). Note the similarity in the character with $x$ of the fiber direction 
and transverse strains. This is characteristic of the $45^{\circ}$ angle. The shear strain $\left(\gamma_{12}\right)$ in the $45^{\circ}$ ply is much larger than the shear strain in the $10^{\circ}$ ply. Increased shear strains in off-axis plies are common owing to the shear-extension coupling peculiar to fiber-reinforced materials. As the cylinder exits the end fitting, the shear strain in the $45^{\circ}$ ply peaks at about 3200 microstrain.
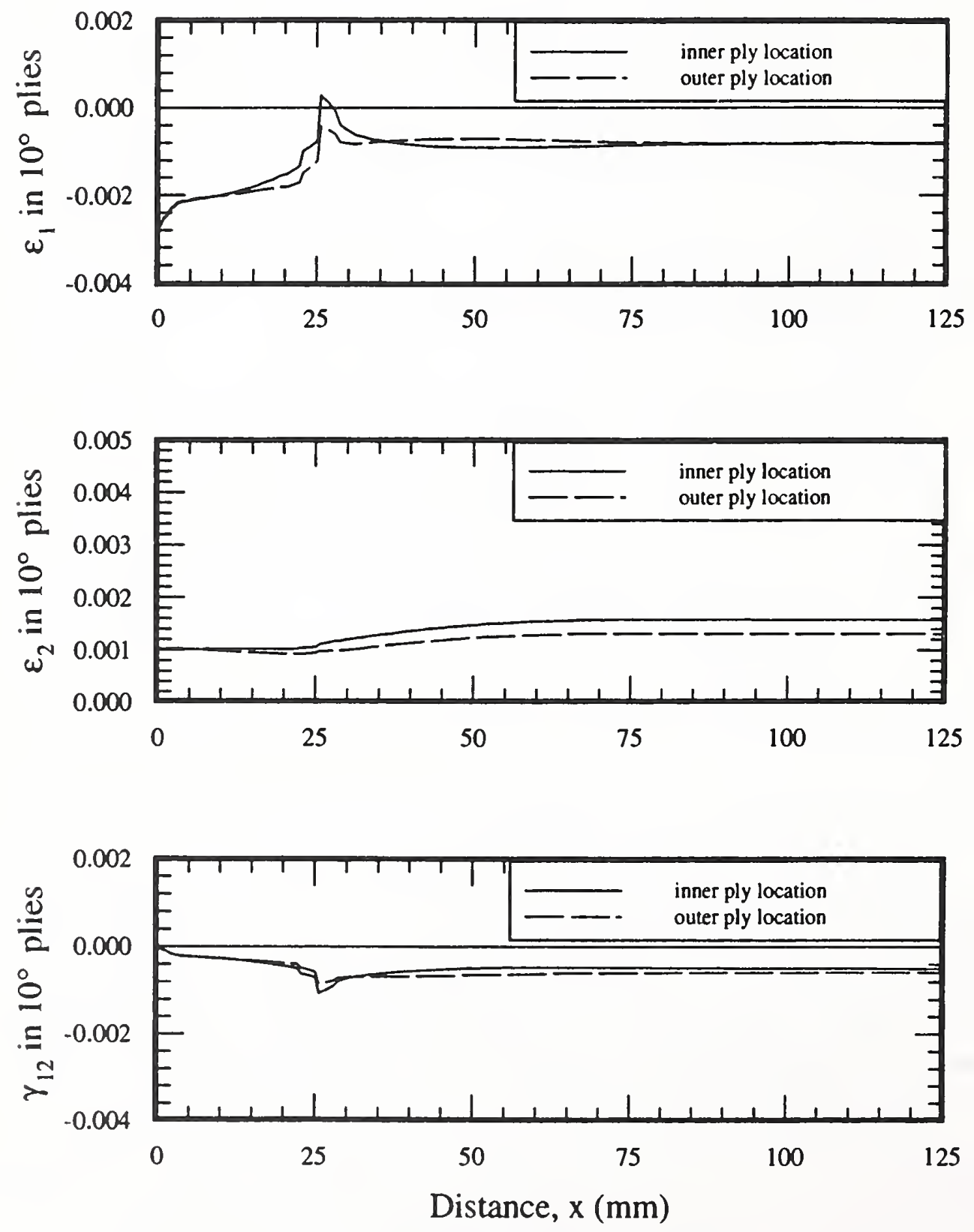

Figure 3.19. Intralaminar strains due to applied axial stress of $-294 \mathrm{MPa}$ in $\pm 10^{\circ} \mathrm{plies}$ of thick [82/45/10] cylinder with baseline-design end fitting. 

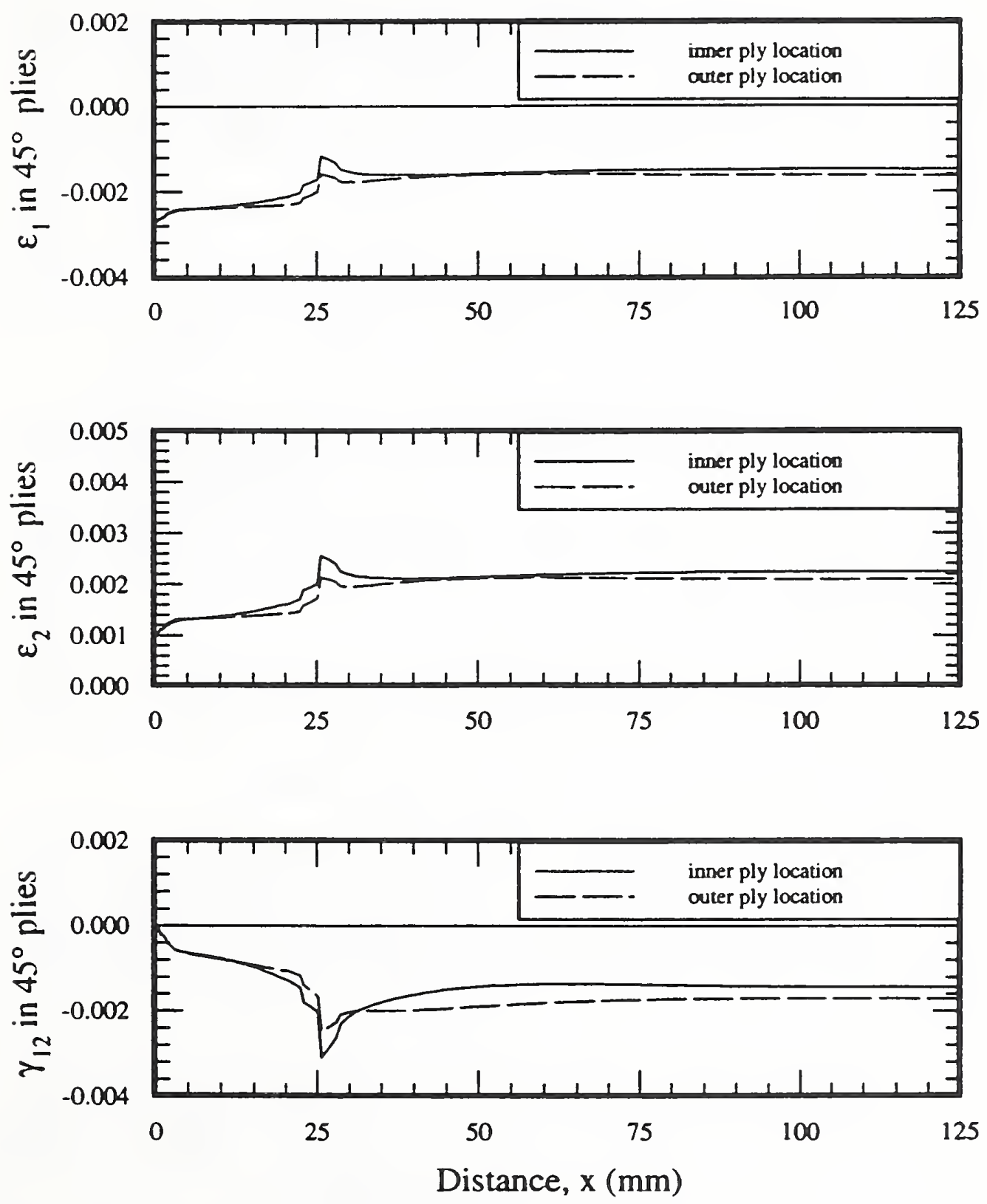

Figure 3.20. Intralaminar strains due to a temperature change of $-294 \mathrm{~K}$ in $\pm 45^{\circ}$ plies of thick [82/45/10] cylinder with baseline-design end fitting. 

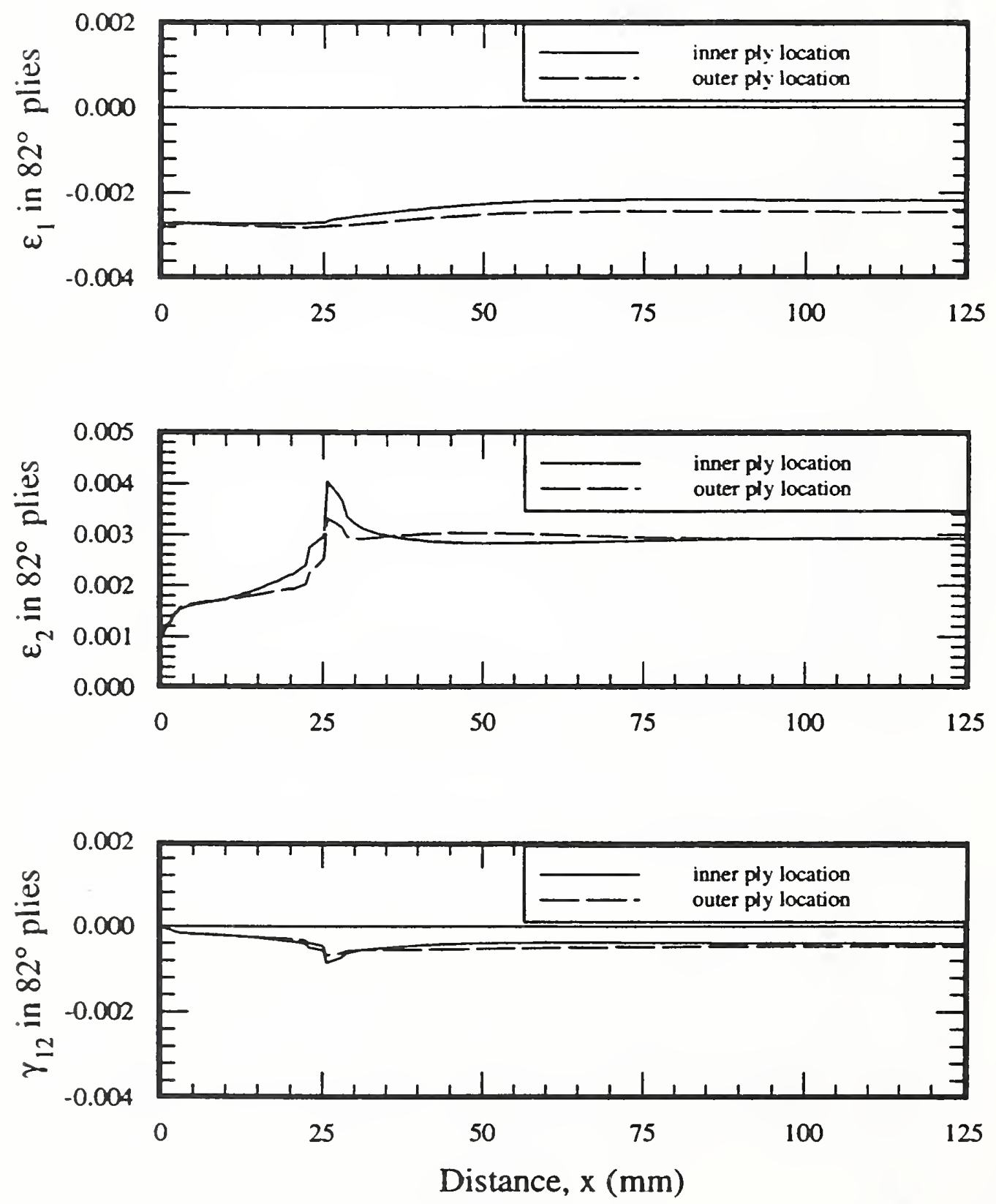

Figure 3.21. Intralaminar strains due to a temperature change of $-294 \mathrm{~K}$ in $\pm 82^{\circ}$ plies of thick [82/45/10] cylinder with baseline-design end fitting. 

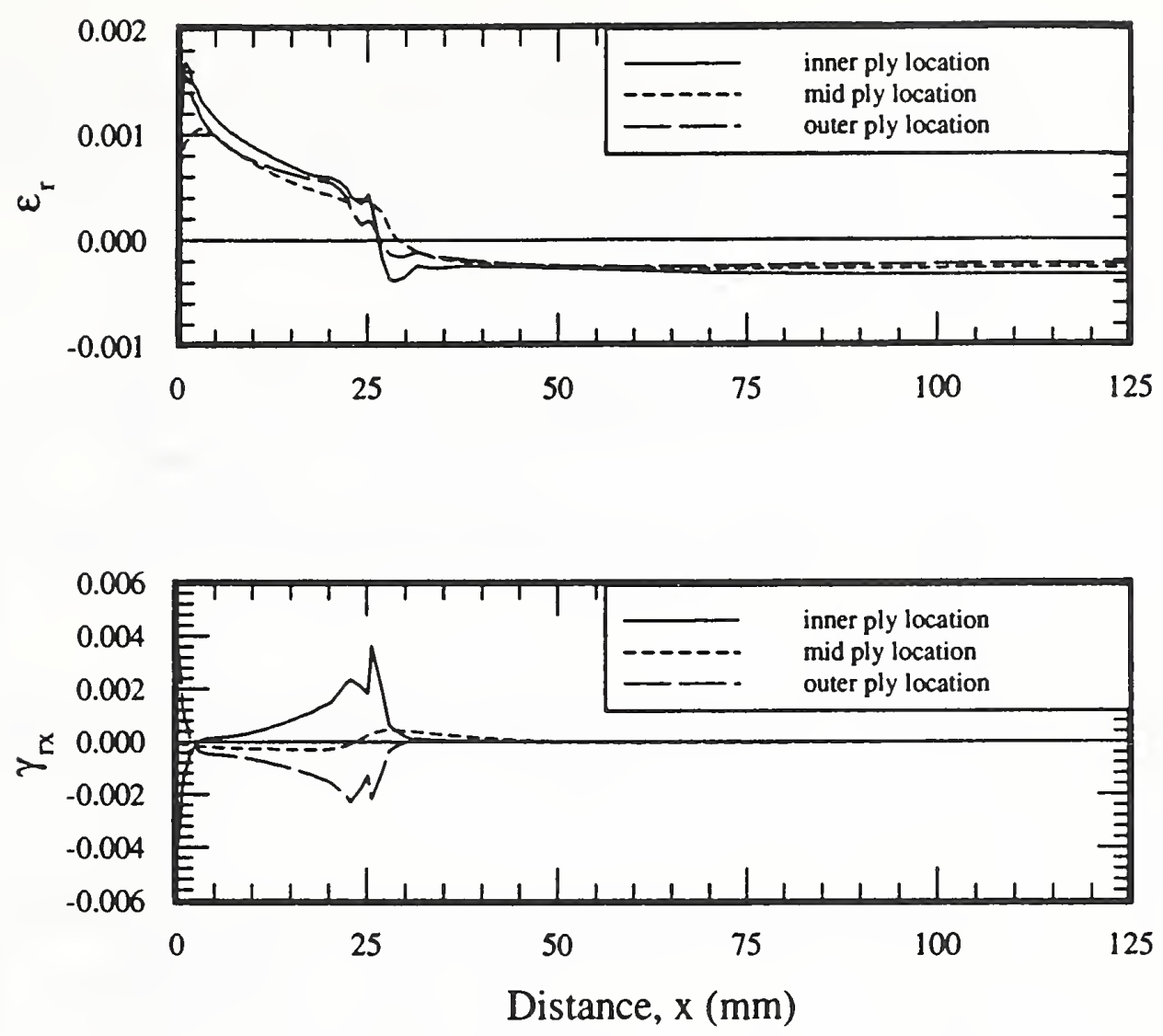

Figure 3.22. Interlaminar strains due to a temperature change of $-294 \mathrm{~K}$ in thick [82/45/10] cylinder with baseline-design end fitting.

In the $[82 / 45 / 10]$ laminate, the character of the fiber-direction and transverse strains in the $82^{\circ}$ ply (Fig. 3.22) is the same as the character of the transverse and fiber-direction strains, respectively, in the $10^{\circ} \mathrm{ply}$, although the magnitudes are not similar. This reversal of trends for these two plies will be seen throughout the discussion. The shear strains in the two plies, however, have similar character and magnitude. In the $82^{\circ} \mathrm{ply}$, the transverse strain reaches a value of 4000 microstrain at the inner-ply location just as the cylinder exits the end fitting.

Interestingly enough, the interlaminar radial strains in the [82/45/10] laminate (Fig. 3.22) are practically identical to the interlaminar radial strains in the [10] laminate (Fig. 3.5). The interlaminar shear strains are similar but not to the degree the radial strains are similar. Despite the differences in intralaminar properties of the two laminates, apparently the baseline end fitting generates approximately the same interlaminar strain response in the two laminates.

\subsubsection{Thermally Induced Strain - Alternative-Design End Fitting}

Figures 3.23 through 3.26 document the strains resulting from the cool-down to $4 \mathrm{~K}$ and the alternative end fittings. Comparison of the strains resulting from cool-down and the baseline end fitting in Figures 3.19 through 3.22 indicates that the alternative end fitting does very little to alter the 
thermally induced strains other than to shift the characteristics in the axial direction a distance equal to the length of the added taper of the design. It appears that the taper of the design is not enough to alter the strain distribution, although more tapering could result in stresses in the end fitting that may exceed the yield stress of the aluminum. This issue was not pursued here.
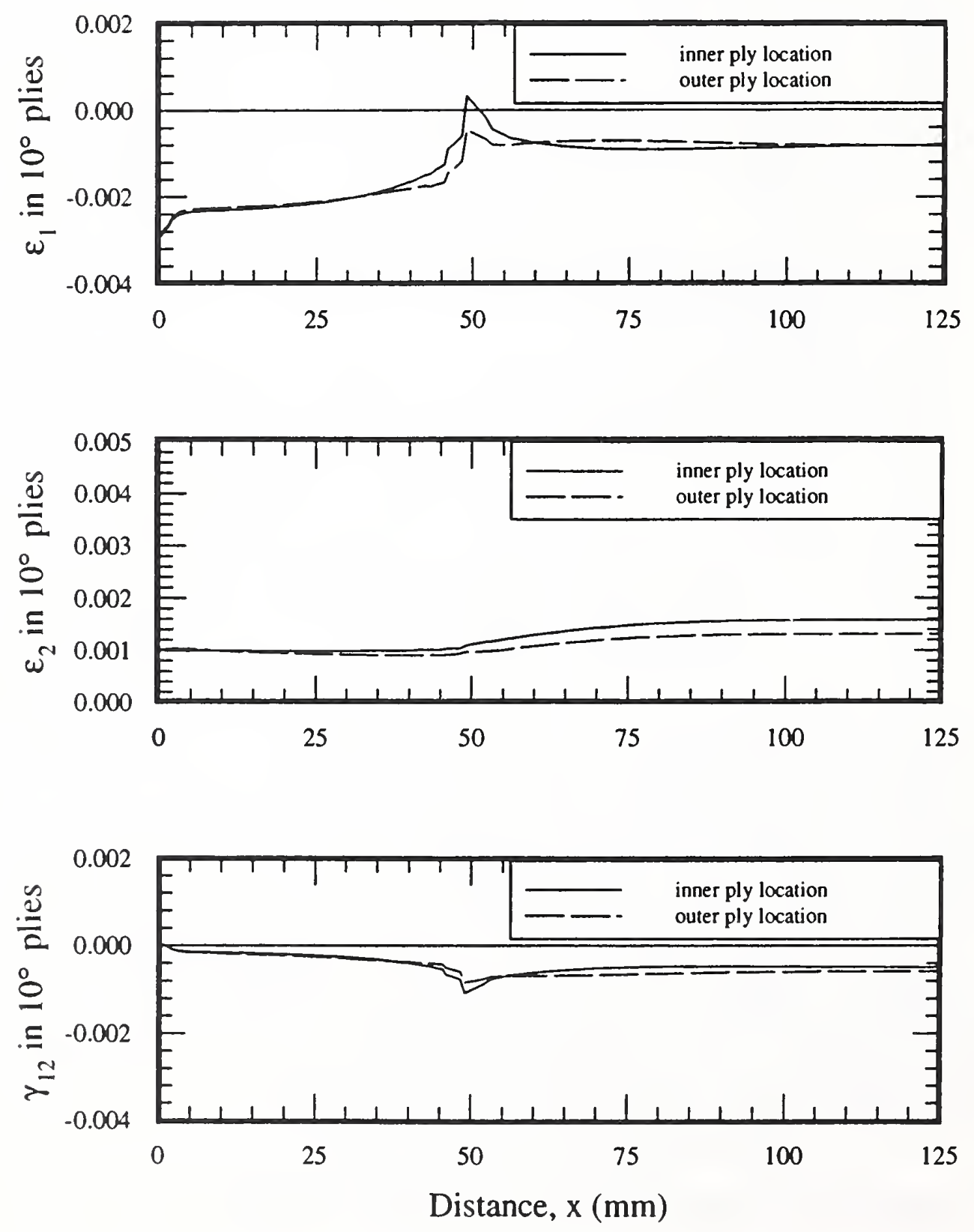

Figure 3.23. Intralaminar strains due to a temperature change of $-294 \mathrm{~K}$ in $\pm 10^{\circ}$ plies of thick [82/45/10] cylinder with alternative-design end fitting. 

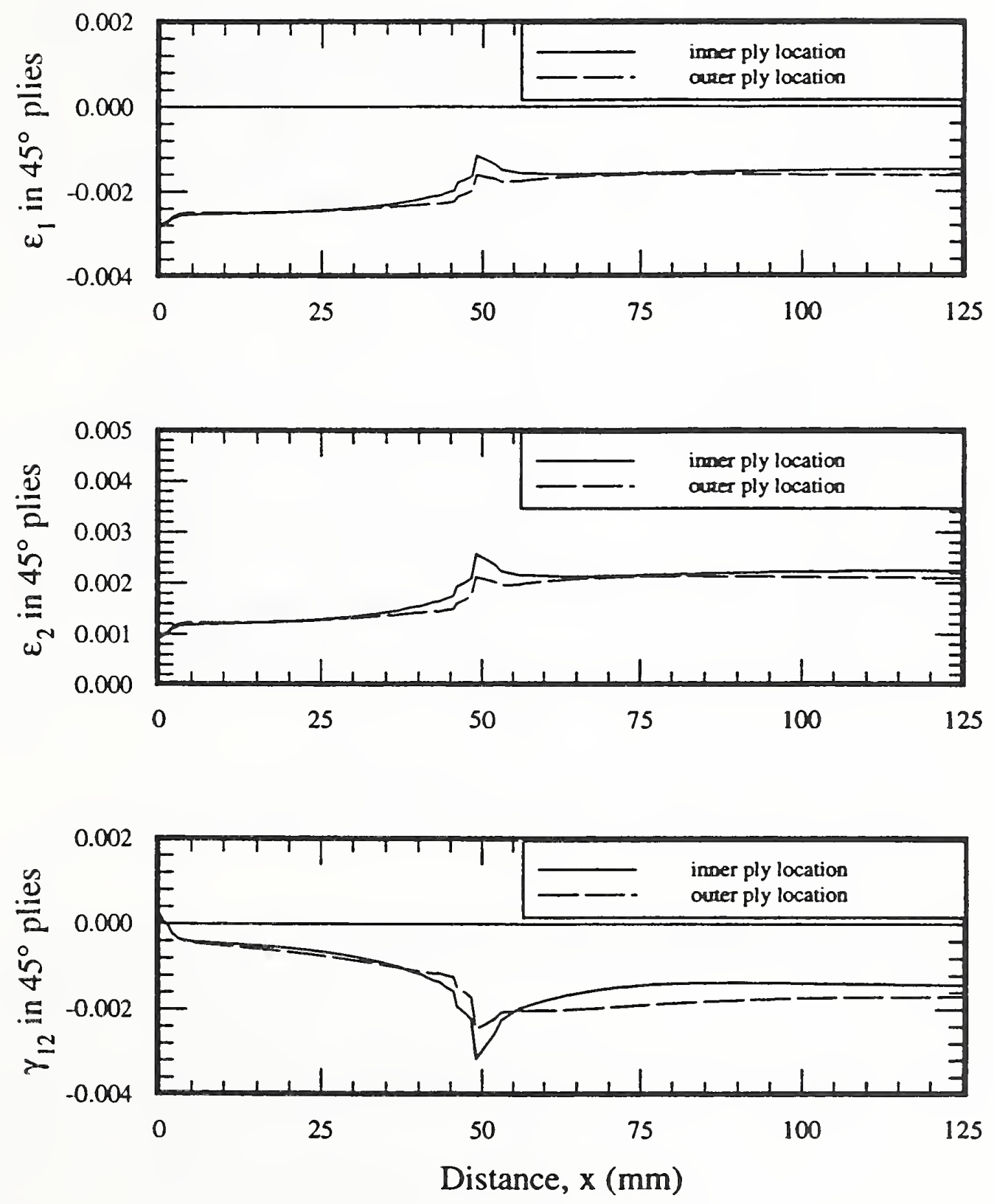

Figure 3.24. Intralaminar strains due to a temperature change of $-294 \mathrm{~K}$ in $\pm 45^{\circ}$ plies of thick [82/45/10] cylinder with alternative-design end fitting. 

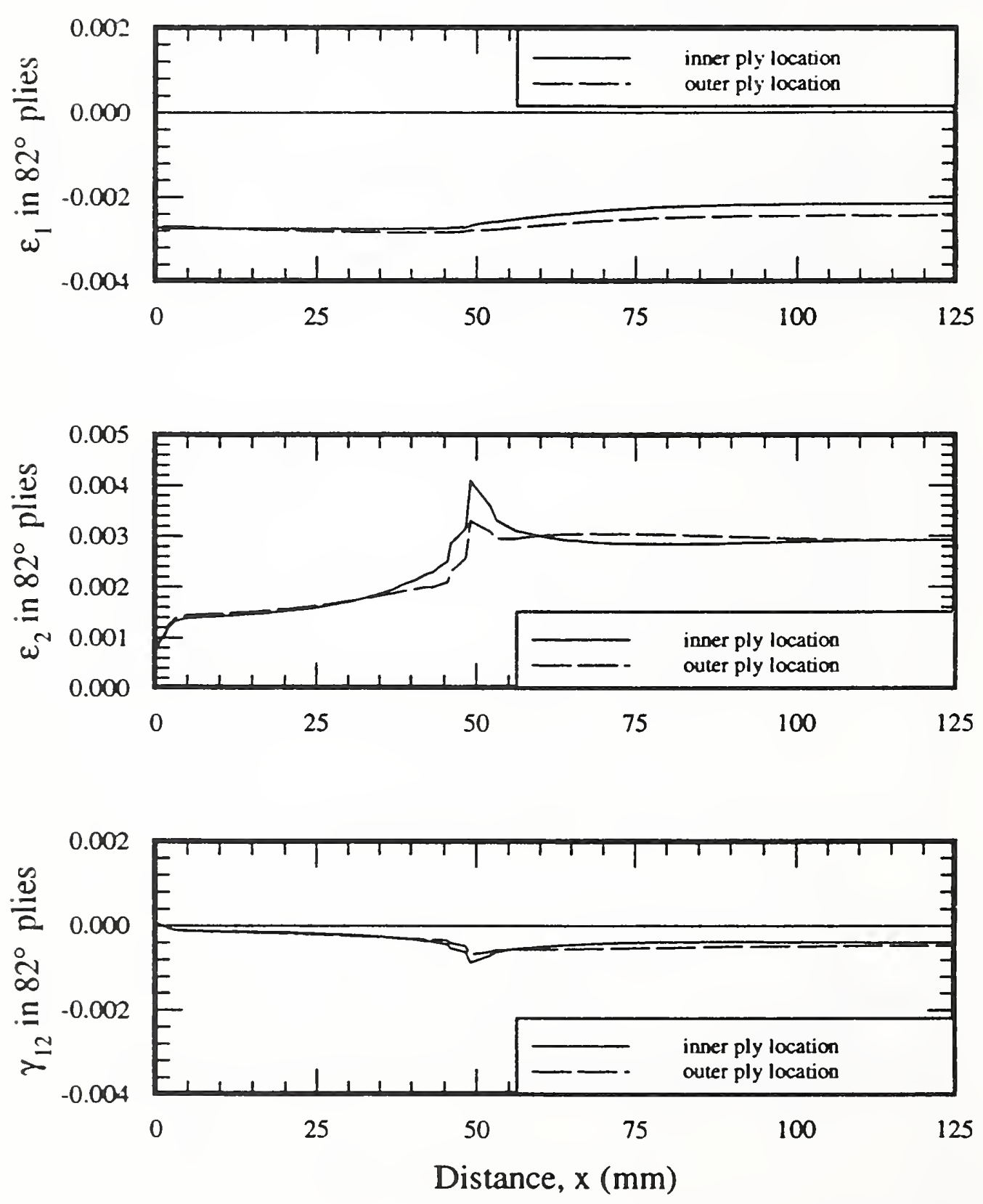

Figure 3.25. Intralaminar strains due to a temperature change of $-294 \mathrm{~K}$ in $\pm 82^{\circ}$ plies of thick $[82 / 45 / 10]$ cylinder with alternative-design end fitting. 

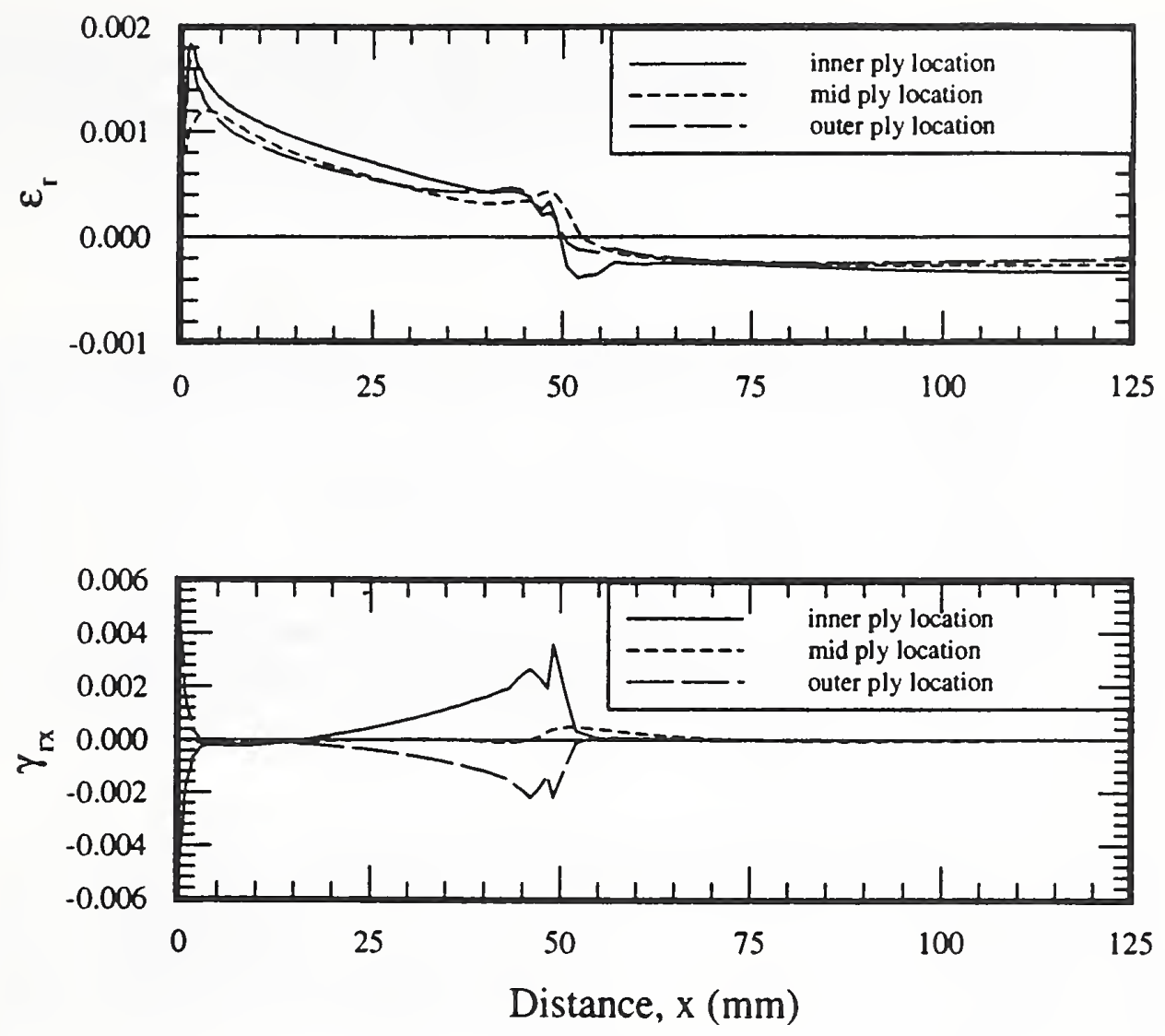

Figure 3.26. Interlaminar strains due to a temperature change of $-294 \mathrm{~K}$ in thick [82/45/10] cylinder with alternative-design end fitting.

\subsubsection{Strains due to Load and Low-Temperature Material Properties - Baseline-Design End Fitting}

The effects on the strains of loading the thick [82/45/10] cylinder, using material properties evaluated at $4 \mathrm{~K}$, are illustrated in Figures 3.27 through 3.30; Figure 3.27 illustrates the strains in the $10^{\circ}$ plies. As with the [10] laminate (Figs. 3.10 and 3.11), the average applied axial stress is $689 \mathrm{MPa}$. It is seen that the magnitude of the fiber-direction compressive axial strain in the $10^{\circ} \mathrm{ply}$ at the outer radius is somewhat over 20,000 microstrain, a rather high number. This high strain occurs just as the cylinder exits the end fitting, and this level exceeds the strain level in the [10] laminate. The concentration factor for these strains is about 1.3 . With the $[82 / 45 / 10]$ laminate, the $82^{\circ}$ plies and the $+45^{\circ}$ and $-45^{\circ}$ plies do not contribute much to the axial stiffness; thus, a softer laminate and greater strains result for the same applied load level. The transverse strains (the center portion of Fig. 3.27) are larger than they are for the [10] laminate. Interestingly enough, even though the second laminate has considerable circumferential stiffness compared to the first laminate, the circumferential strains, as represented by the transverse strains in the $10^{\circ} \mathrm{ply}$, are still quite large, 5600 microstrain tensile to be specific, in the membrane portion of the cylinder. For this second laminate, as with the 
first laminate, the aluminum end cap restrains the portion of the cylinder within from expanding circumferentially, as evidenced by the low transverse strains in the cylinder within the end cap. The shear strains in the $10^{\circ}$ ply in the [82/45/10] laminate (the lower portion of Fig. 3.27) are somewhat greater than in the [10] laminate.
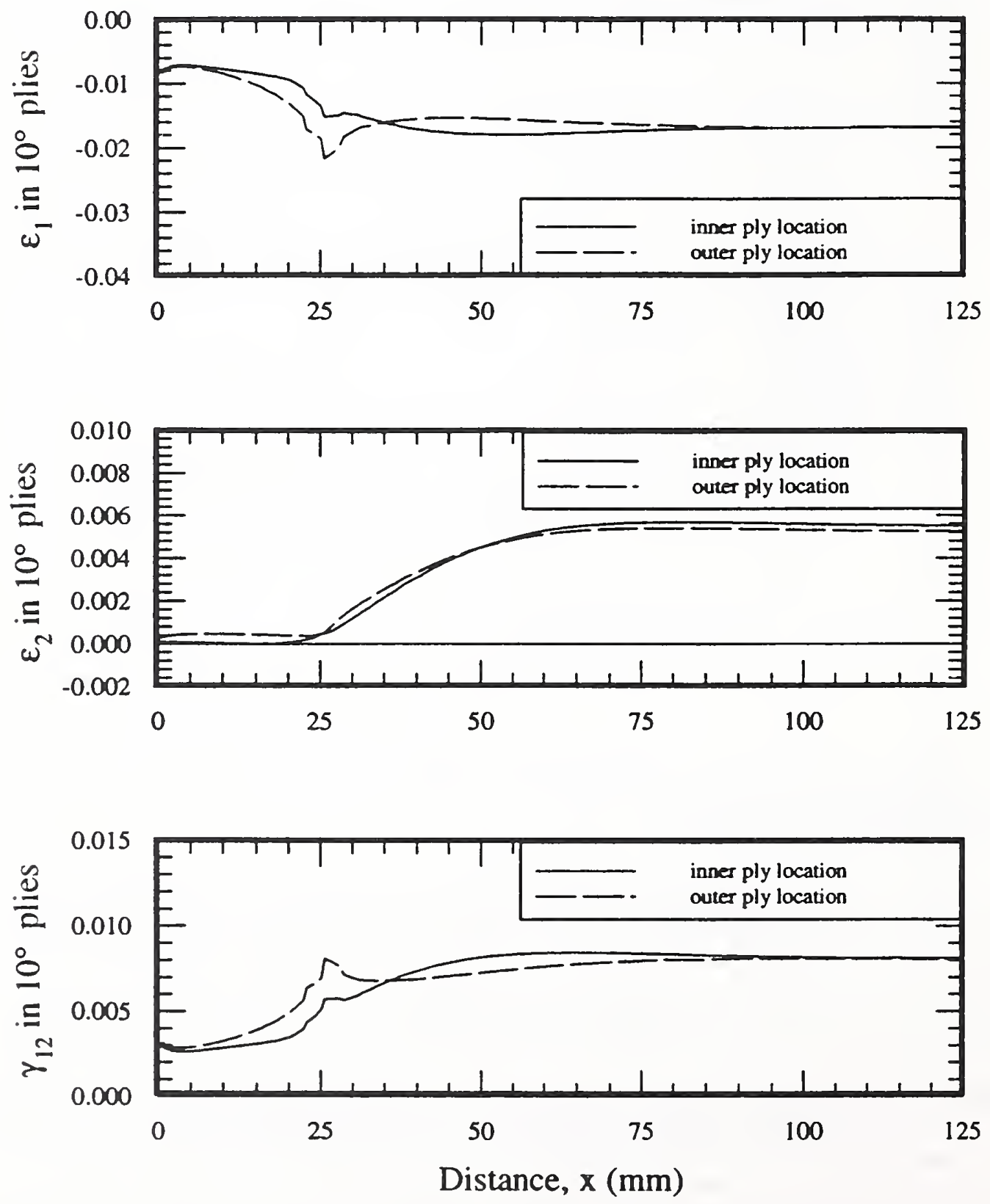

Figure 3.27. Intralaminar strains due to an applied axial stress of $-689 \mathrm{MPa}$ in $\pm 10^{\circ}$ plies of thick [82/45/10] cylinder with baseline-design end fitting, 4-K properties. 
In the $45^{\circ}$ plies (Fig. 3.28), the shear strain dominates the response, peaking to a level of about 25,000 microstrain as the cylinder exits the end cap. This strain component also exists at a significant level in the membrane region, again reflecting the high shear-extension coupling present in off-axis plies of fiber-reinforced material. The transverse strain level $(10,000$ microstrain) is also somewhat large, except it is compressive in nature.
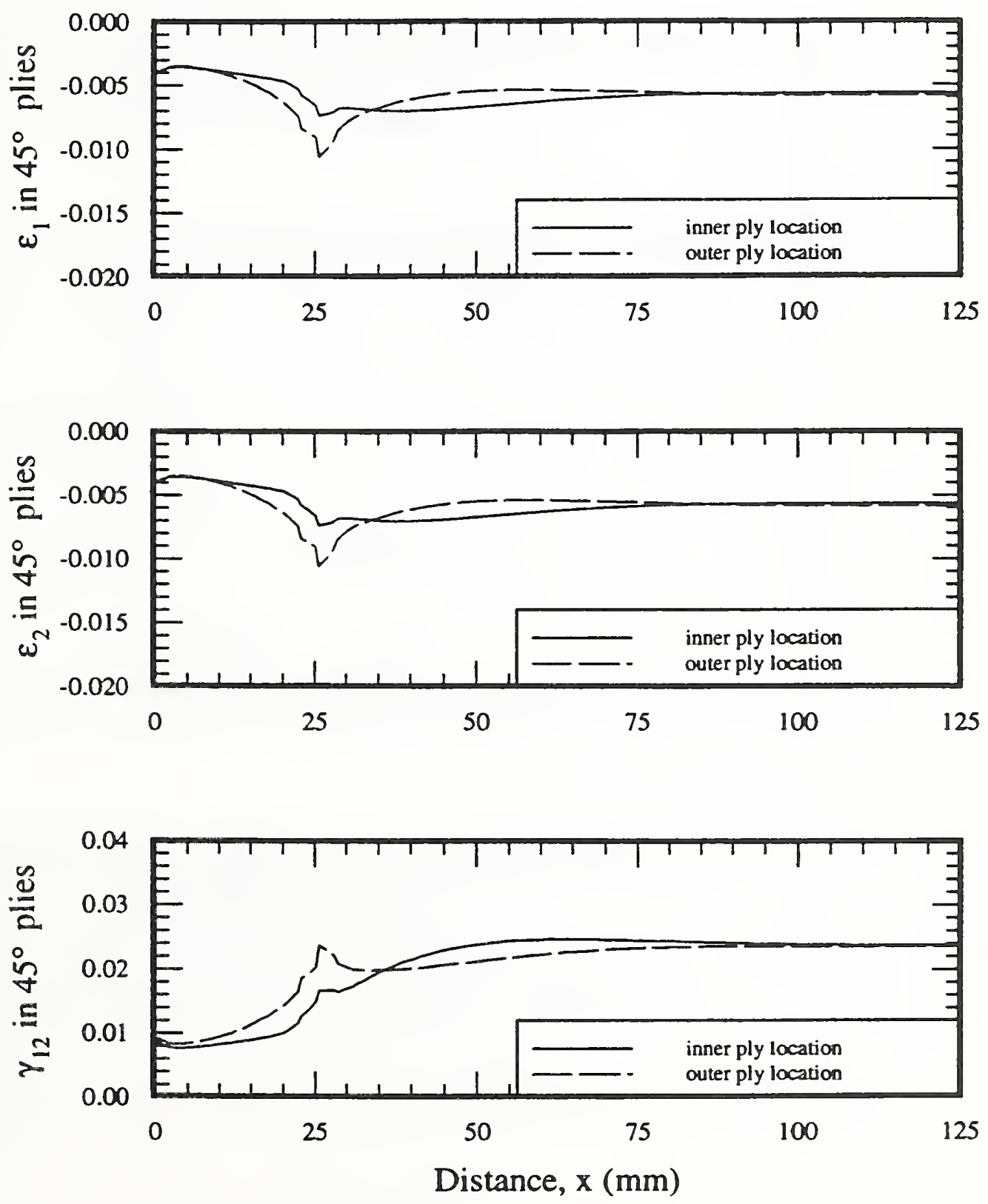

Figure 3.28. Intralaminar strains due to an applied axial stress of $-689 \mathrm{MPa}$ in $\pm 45^{\circ}$ plies of thick [82/45/10] cylinder with baseline-design end fitting, 4-K properties. 
The fiber-direction strain in the $82^{\circ}$ ply (Fig. 3.29) is very similar to the transverse strain in the $10^{\circ}$ ply (Fig. 3.27 ), whereas the transverse strain in the $82^{\circ}$ ply is similar to the fiber-direction strain in the $10^{\circ} \mathrm{ply}$, an interchange of characteristics seen previously with this laminate.
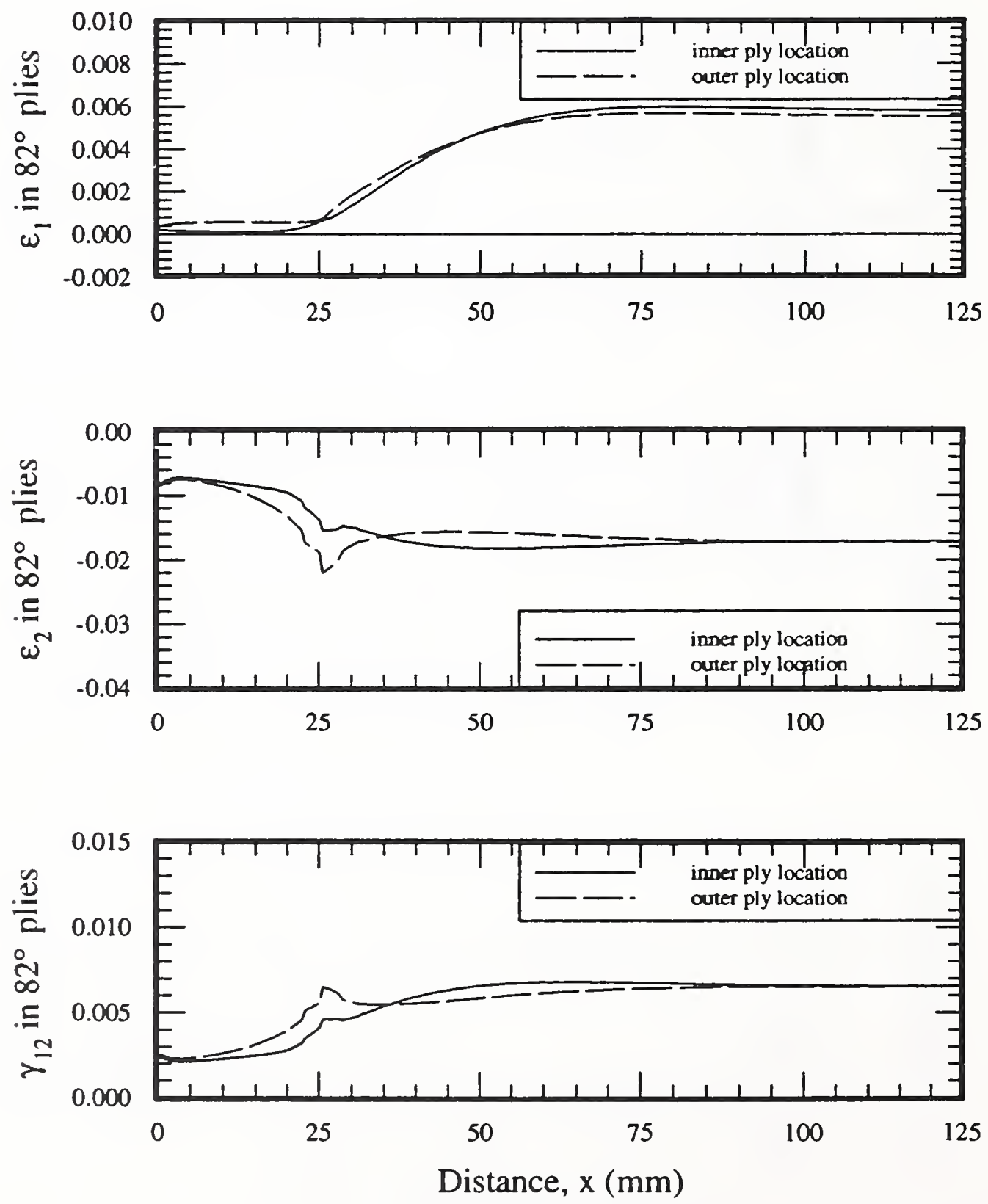

Figure 3.29. Intralaminar strains due to an applied axial stress of $-689 \mathrm{MPa}$ in $\pm 82^{\circ}$ plies of thick [82/45/10] cylinder with baseline-design end fitting, 4-K properties. 

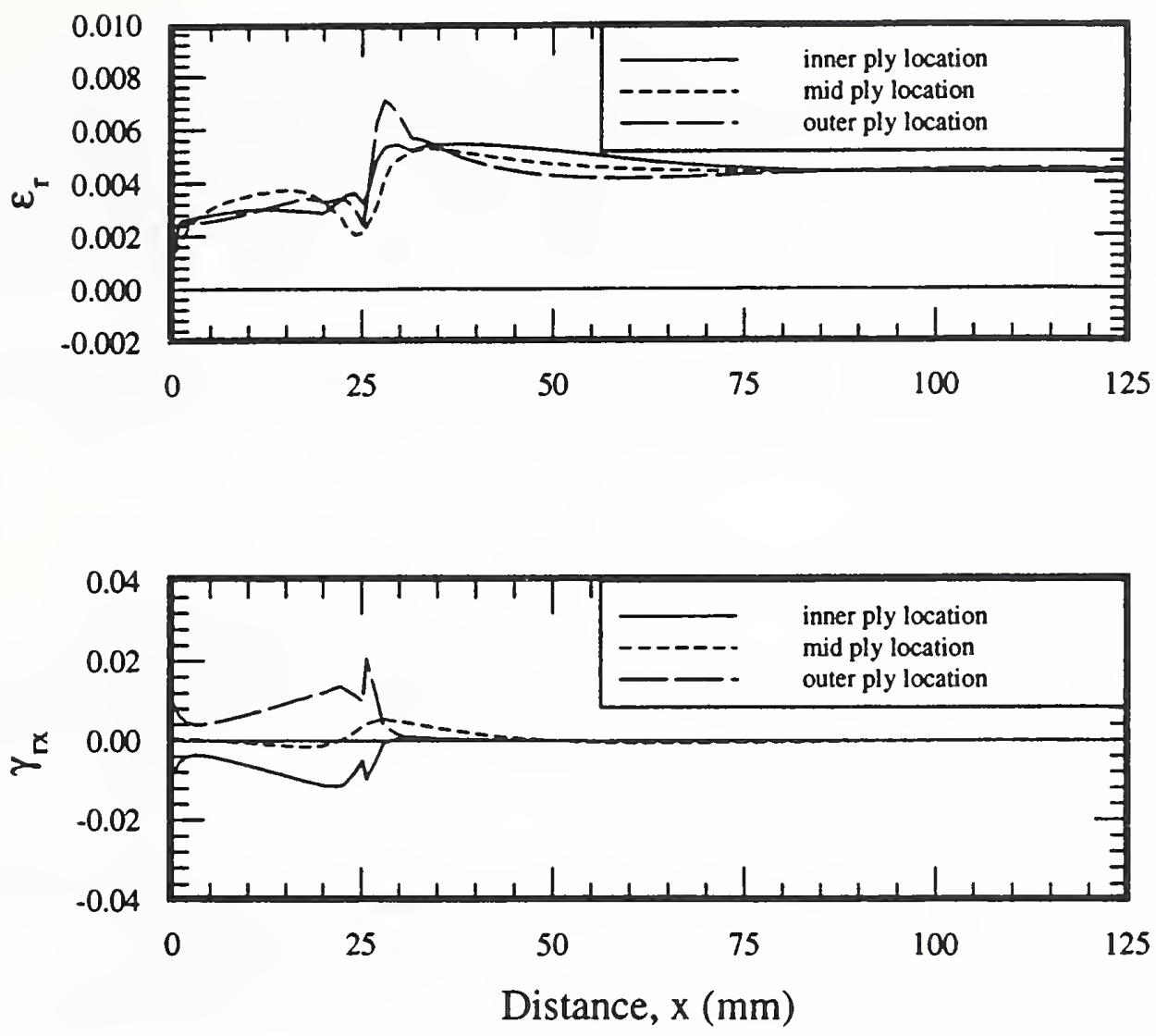

Figure 3.30. Interlaminar strains due to an applied axial stress of $-689 \mathrm{MPa}$ in thick [82/45/10] cylinder with baseline-design end fitting, 4-K properties.

The interlaminar strains in the $[82 / 45 / 10]$ laminate (Fig. 3.30), although very similar in character to the interlaminar strains in the [10] laminate (Fig. 3.11) show increased levels relative to that laminate. The interlaminar radial strain (the upper portion of Fig. 3.30) has a larger tensile value for this second laminate than for the first laminate under identical loading. This high level, when combined with the high in-plane strain components near the exit from the end fitting, could result in a serious condition. In the [82/45/10] laminate, the interlaminar shear strain is about 15,000 microstrain, not counting the spike, just inside of the end fitting. 


\subsubsection{Strains due to Load and Low-Temperature Material Properties - Alternative-Design End Fitting}

As before, an examination of the alternative end cap with the [82/45/10] laminate, with material properties evaluated at $4 \mathrm{~K}$ (Figs. 3.31 through 3.34), shows little difference in the cylinder response compared with that of the baseline end fitting (Figs. 3.27 through 3.30). Alterations in the length of the boundary layer just do not exist, although there is a slight reduction in the peak interlaminar radial strain just at the exit to the end fitting, for example, when comparing the top portions of Figures 3.30 and 3.34.
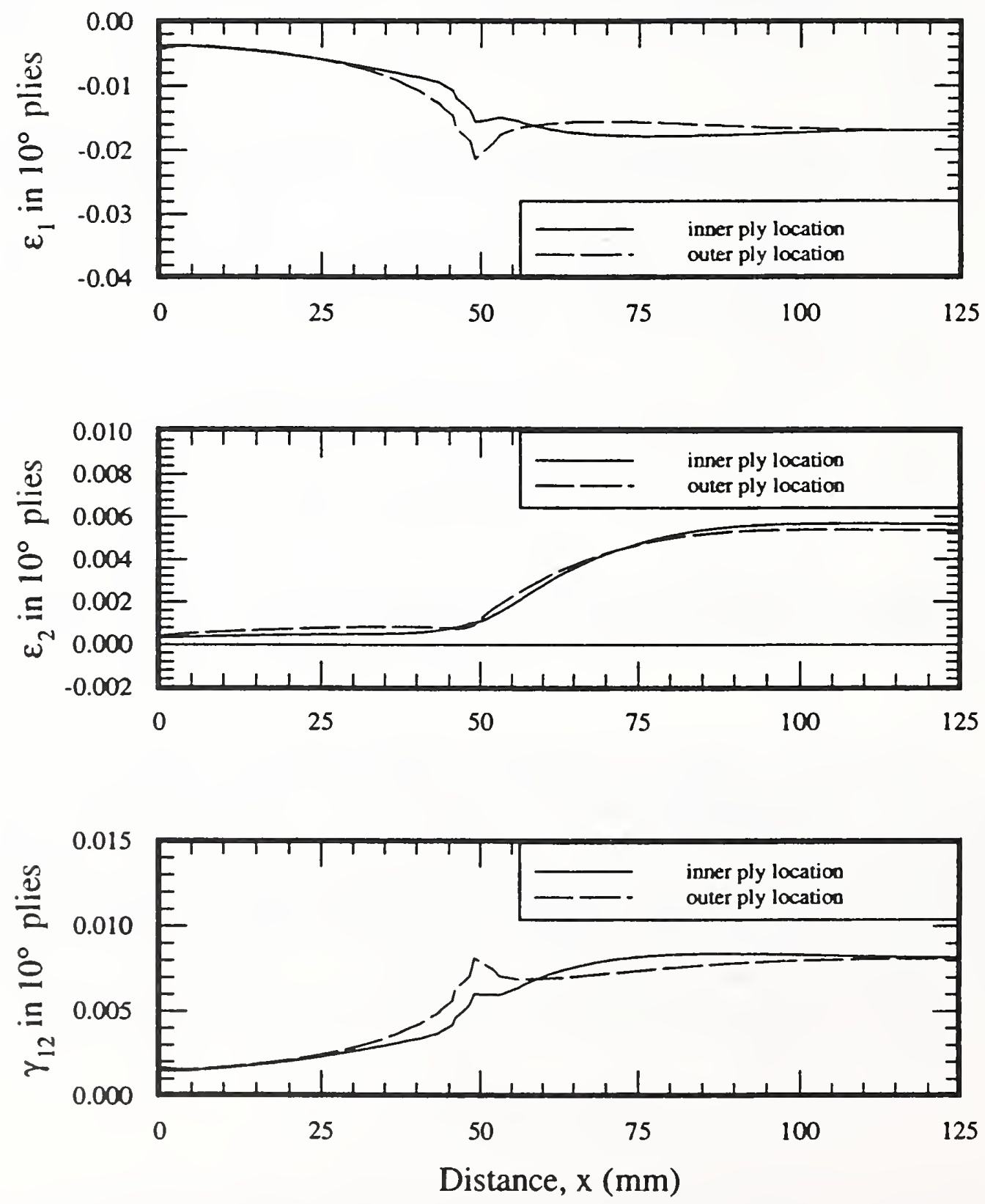

Figure 3.31. Intralaminar strains due to an applied axial stress of $-689 \mathrm{MPa}$ in $\pm 10^{\circ} \mathrm{plies}$ of thick [82/45/10] cylinder with alternative-design end fitting, 4-K properties. 

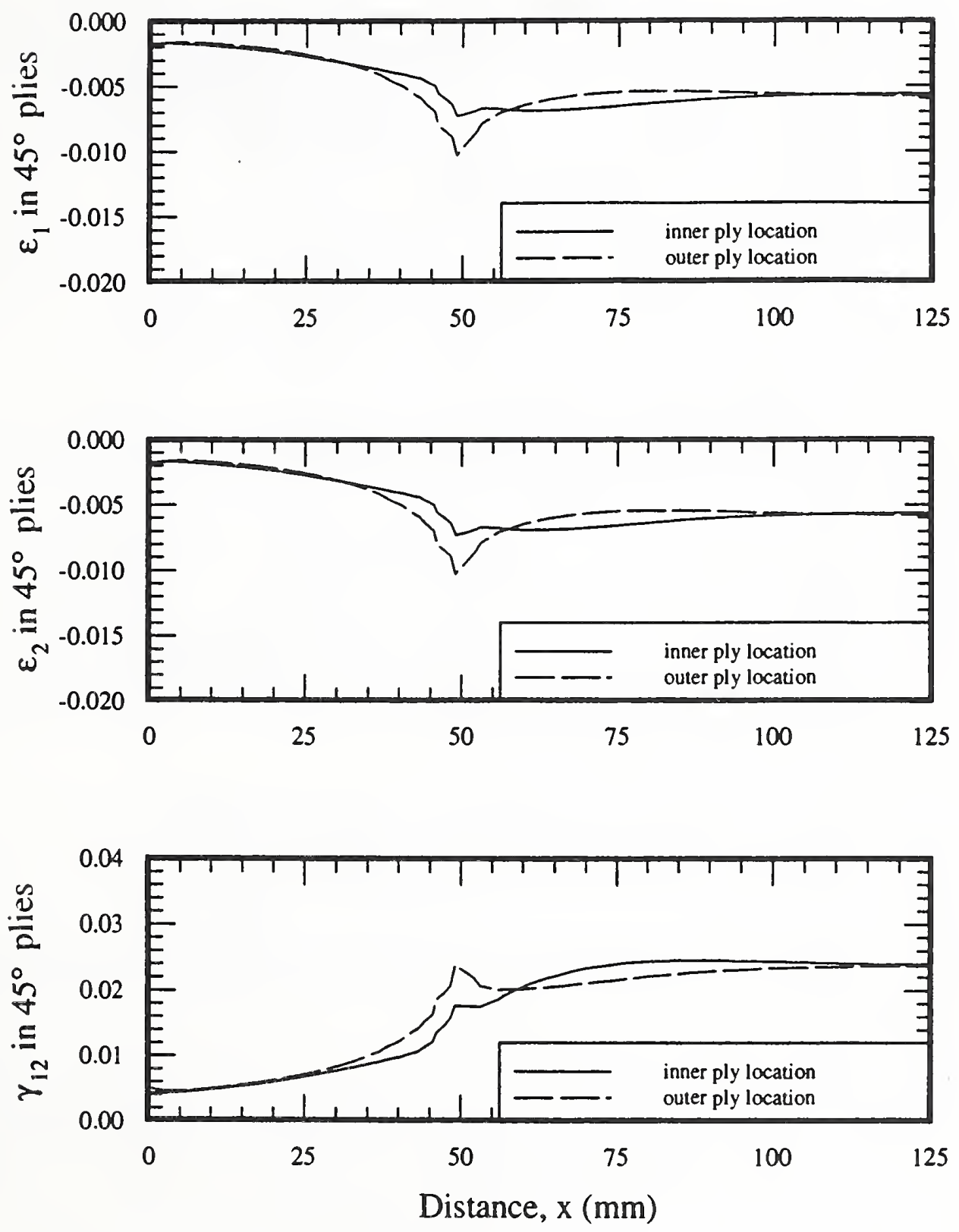

Figure 3.32. Intralaminar strains due to an applied axial stress of $-689 \mathrm{MPa}$ in $\pm 45^{\circ}$ plies of thick [82/45/10] cylinder with alternative-design end fitting, 4-K properties. 

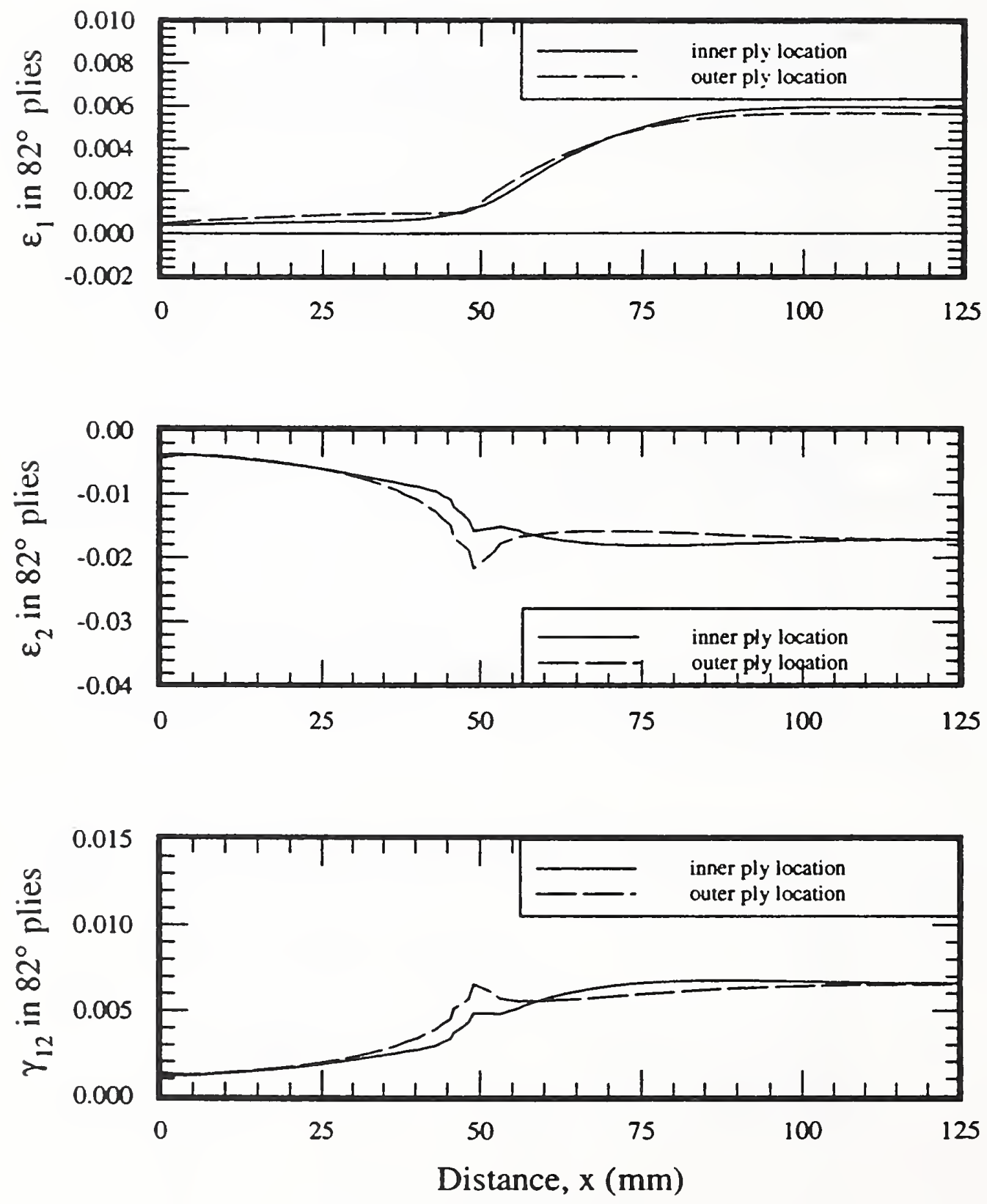

Figure 3.33. Intralaminar strains due to an applied axial stress of $-689 \mathrm{MPa}$ in $\pm 82^{\circ}$ plies of thick [82/45/10] cylinder with alternative-design end fitting, 4-K properties. 

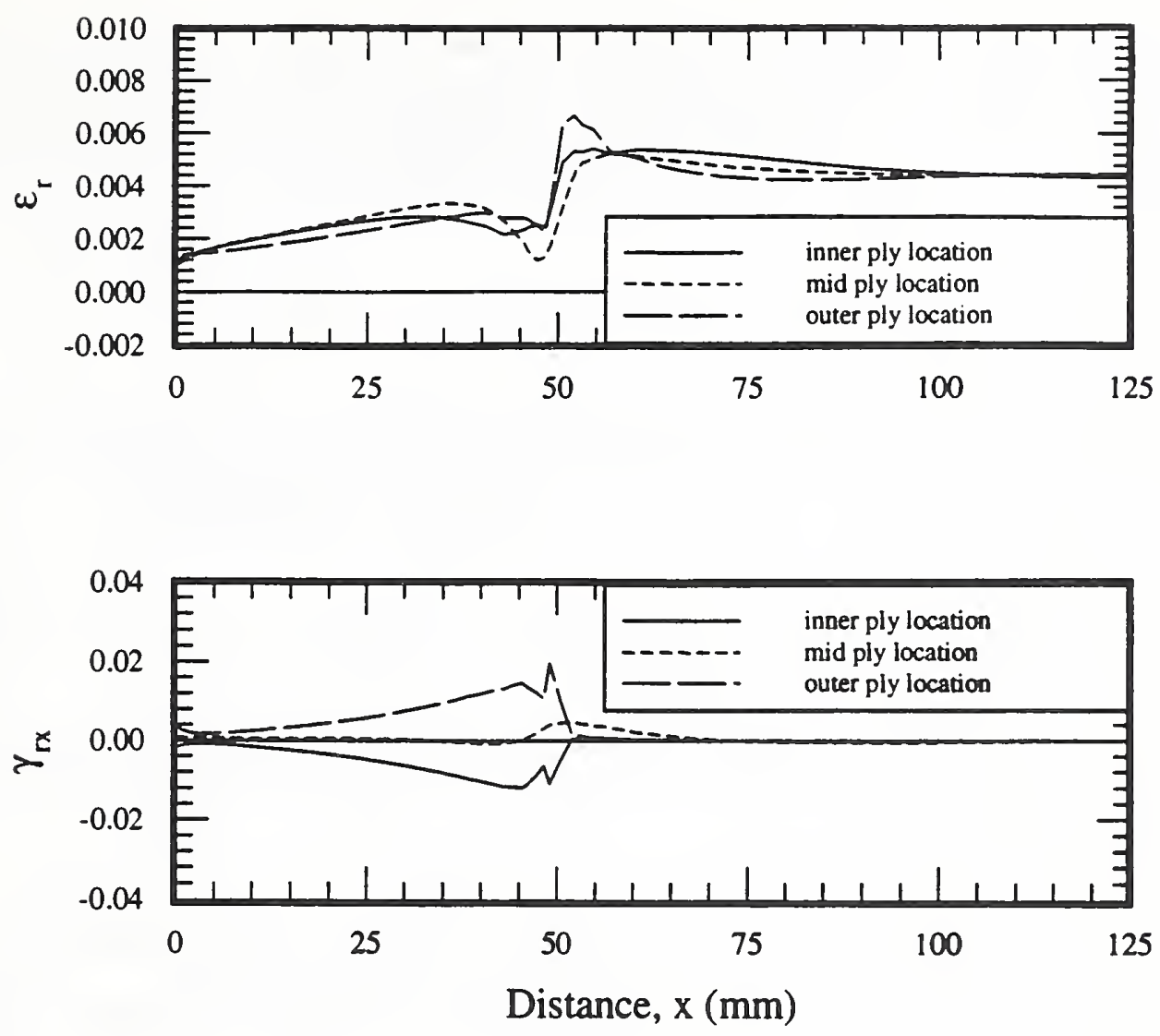

Figure 3.34. Interlaminar strains due to an applied axial stress of $-689 \mathrm{MPa}$ in thick [82/45/10] cylinder with alternative-design end fitting, 4-K properties.

\subsubsection{Strains due to Load and Room-Temperature Material Properties - Baseline-Design End Fitting}

Using room-temperature material properties in the analysis of the load-induced strains in the $[82 / 45 / 10]$ laminate due to the baseline end fitting results in the response shown in Figures 3.35 through 3.38. These results can be compared with those of the baseline end fitting for the same laminate using properties at $4 \mathrm{~K}$ (Figs. 3.27 through 3.30) or with those of the [10] laminate at room temperature (Figs. 3.15 and 3.16). Compared to the same laminate but using properties at $4 \mathrm{~K}$ (Fig. 3.27), the fiber-direction compressive strain in the $10^{\circ}$ ply (Fig. 3.35) is greater by about a factor of 1.5. The concentration factor for these strains is about 1.4. This effect of less stiff material properties at room temperature, as compared to $4 \mathrm{~K}$, was seen with the [10] laminate, although the factor was not as high as 1.5. This larger factor for the [82/45/10] laminate is felt to be due to its overall softer axial properties. This is also reflected in Figures 3.27 and 3.35 in the transverse and shear strains. This factor of 1.5 is quite evident in the strains in the $45^{\circ} \mathrm{ply}$. By comparing any one of the three strain components in Figure 3.36 with the corresponding strain component in Figure 3.28, the factor of 1.5 becomes evident. What is important is the large value ( $>30,000$ microstrain) of shear strain in $45^{\circ} \mathrm{ply}$ in the membrane region (the lower part of Fig. 3.36). Clearly, this can lead to 
microcracking in the plane of the ply, a condition that can lead to the loss of stability of the compressively loaded fiber. Fortunately, the transverse strain is a large compressive value, indicating that perhaps the fiber is stabilized by compressive stresses perpendicular to the fiber direction. Figure 3.37 shows the reverse role of the fiber direction and transverse strains in the $90^{\circ}$ ply as compared to the $10^{\circ}$ ply (Fig. 3.35).
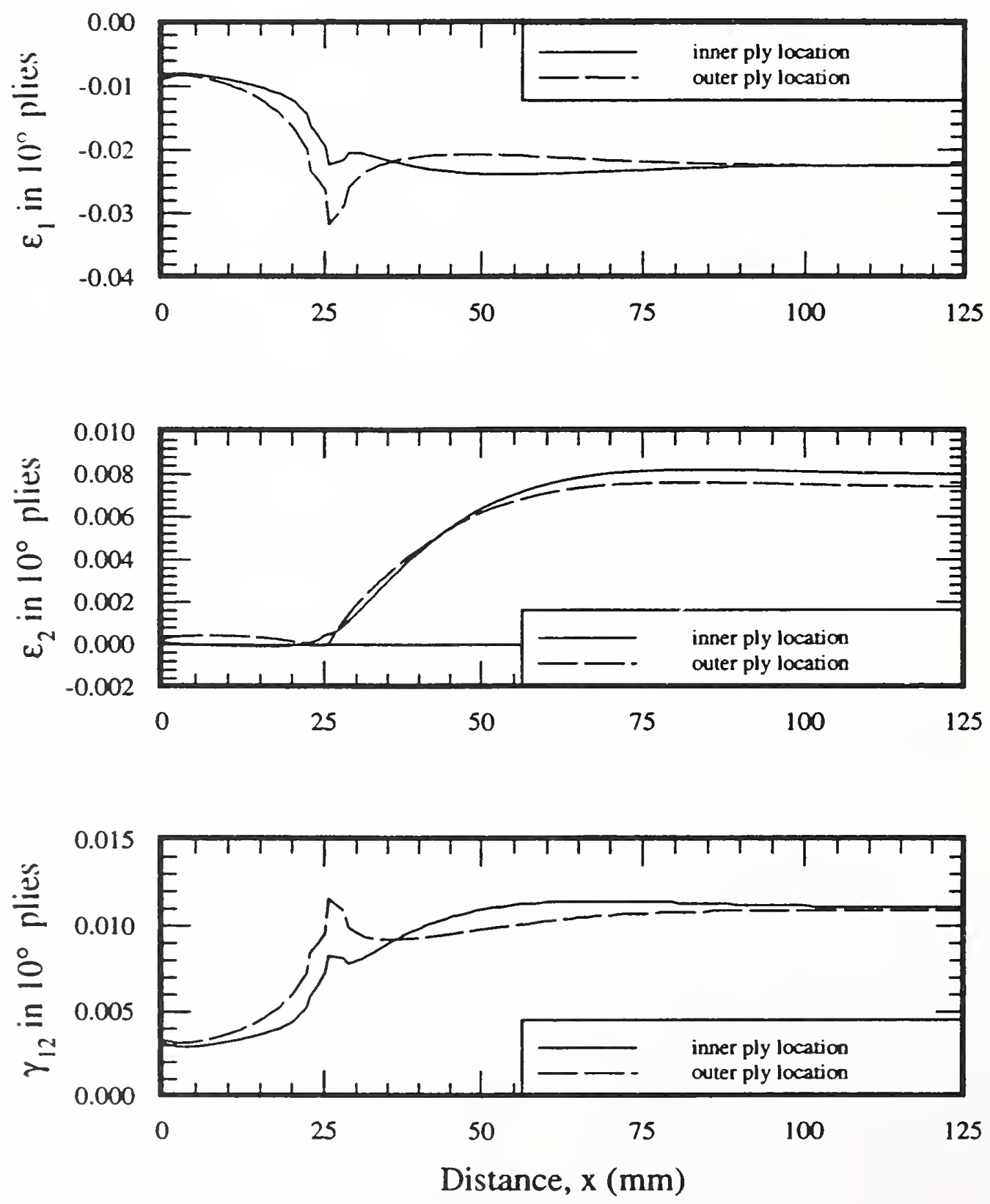

Figure 3.35. Intralaminar strains due to an applied axial stress of $-689 \mathrm{MPa}$ in $\pm 10^{\circ}$ plies of thick [82/45/10] cylinder with baseline-design end fitting, 297-K properties. 

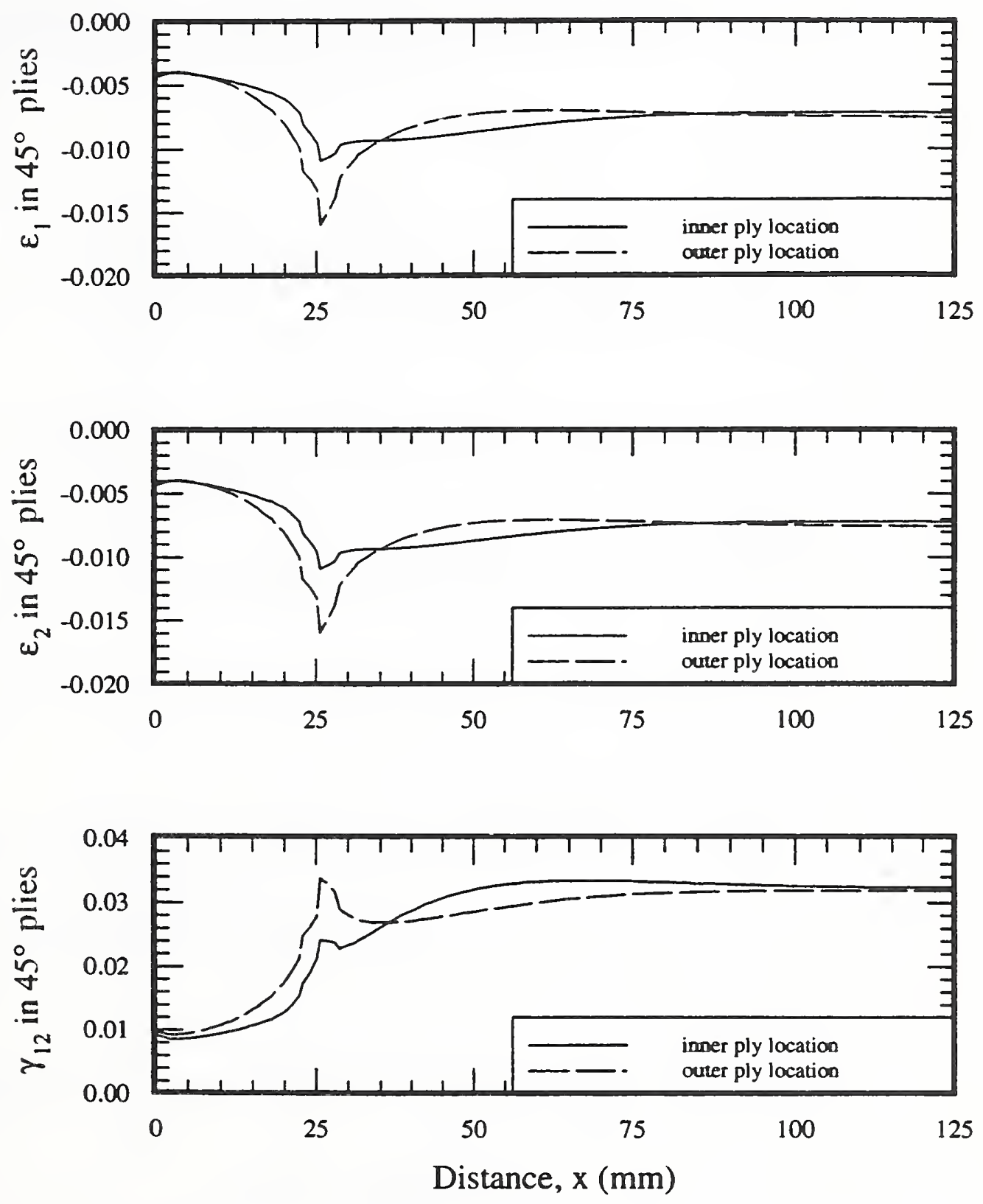

Figure 3.36. Intralaminar strains due to an applied axial stress of $-689 \mathrm{MPa}$ in $\pm 45^{\circ}$ plies of thick [82/45/10] cylinder with baseline-design end fitting, 297-K properties. 

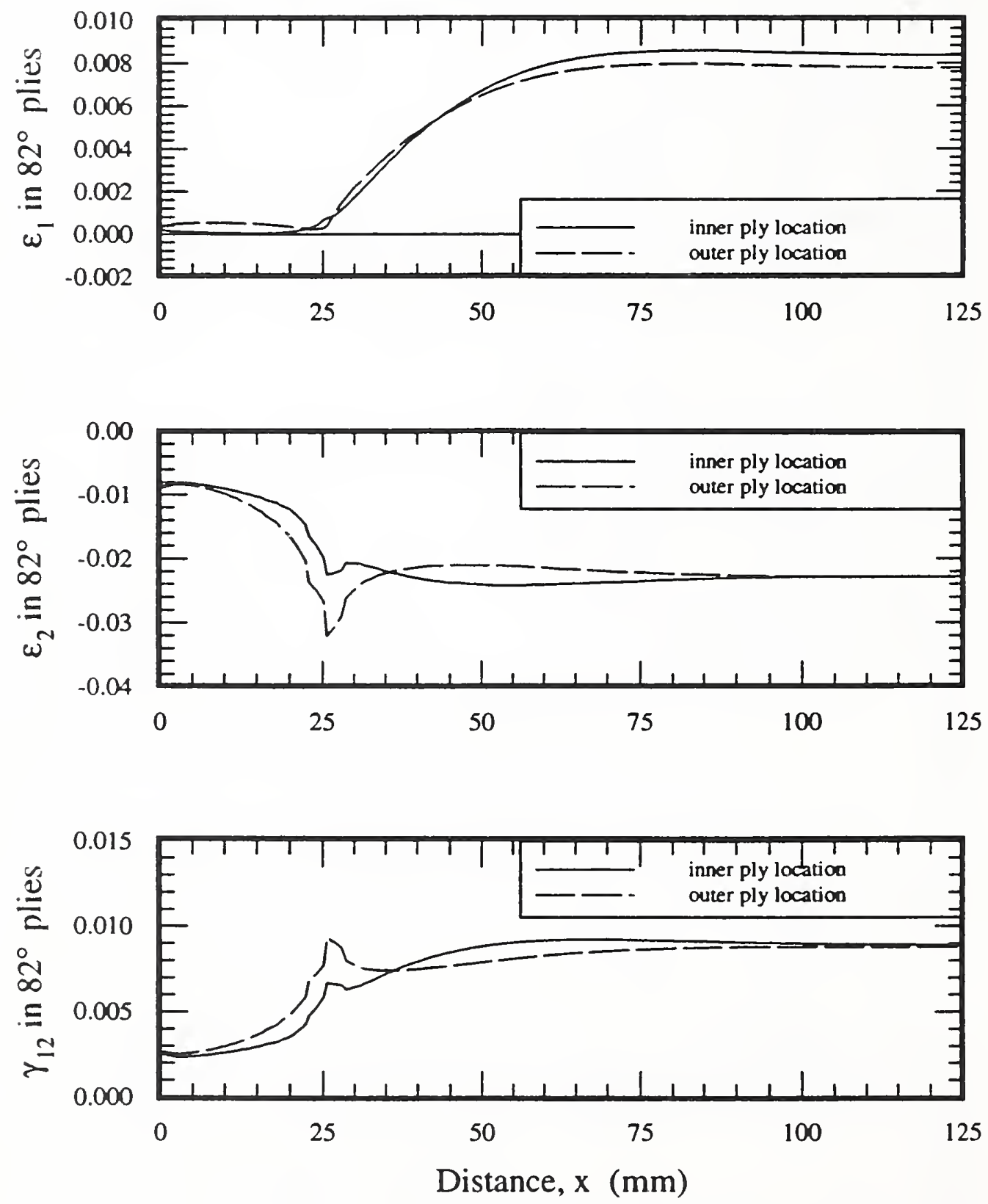

Figure 3.37. Intralaminar strains due to an applied axial stress of $-689 \mathrm{MPa}$ in $\pm 82^{\circ}$ plies of thick [82/45/10] cylinder with baseline-design end fitting, 297-K properties. 
Finally, as might be expected, the interlaminar strains for the [82/45/10] laminate with roomtemperature properties (Fig. 3.38) are considerably greater than the case of properties evaluated at $4 \mathrm{~K}$ (Fig. 3.30) or the [10] cylinder with properties evaluated at $297 \mathrm{~K}$. Discounting the spike, the interlaminar shear strain at the outer radius is about 30,000 microstrain, a large number. Such a large strain could lead to failure of the cylinder just as it enters the end cap.
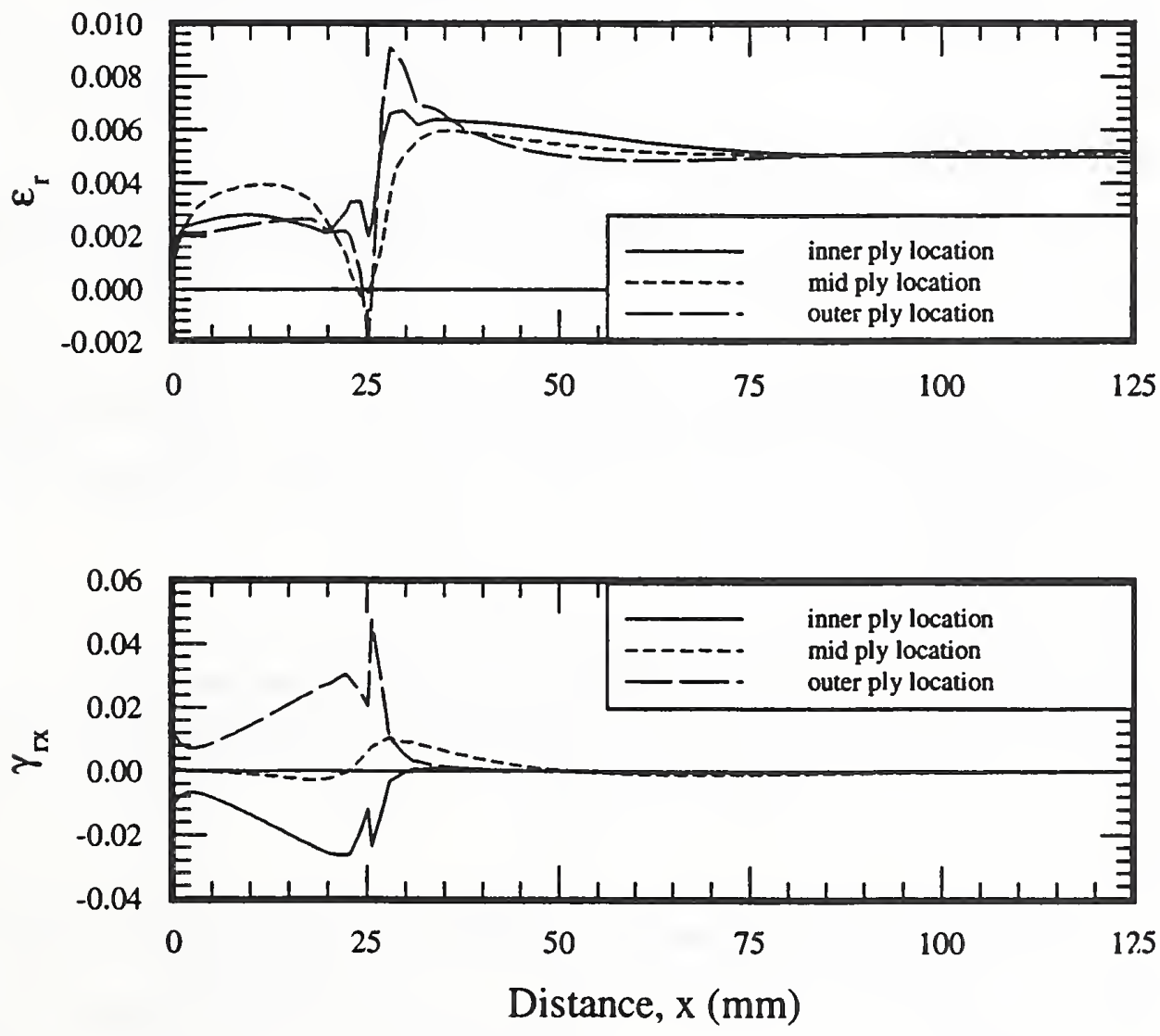

Figure 3.38. Interlaminar strains due to an applied axial stress of $-689 \mathrm{MPa}$ in thick [82/45/10] cylinder with baseline-design end fitting, 297-K properties. 


\subsubsection{Strains due to Load and Room-Temperature Material Properties - Alternative-Design End Fitting}

The effect of the alternative end fitting on the [82/45/10] cylinder under axial load and with room-temperature material properties, as it has been throughout the discussion (Figs. 3.39 through 3.42 ) is to simply shift the strain characteristics in the axial direction an amount equal to the length of the tapered section. In light of this, it can be said that for these thick cylinders, the taper as it is designed has no noticeable effect on the distribution or magnitude of the strains.
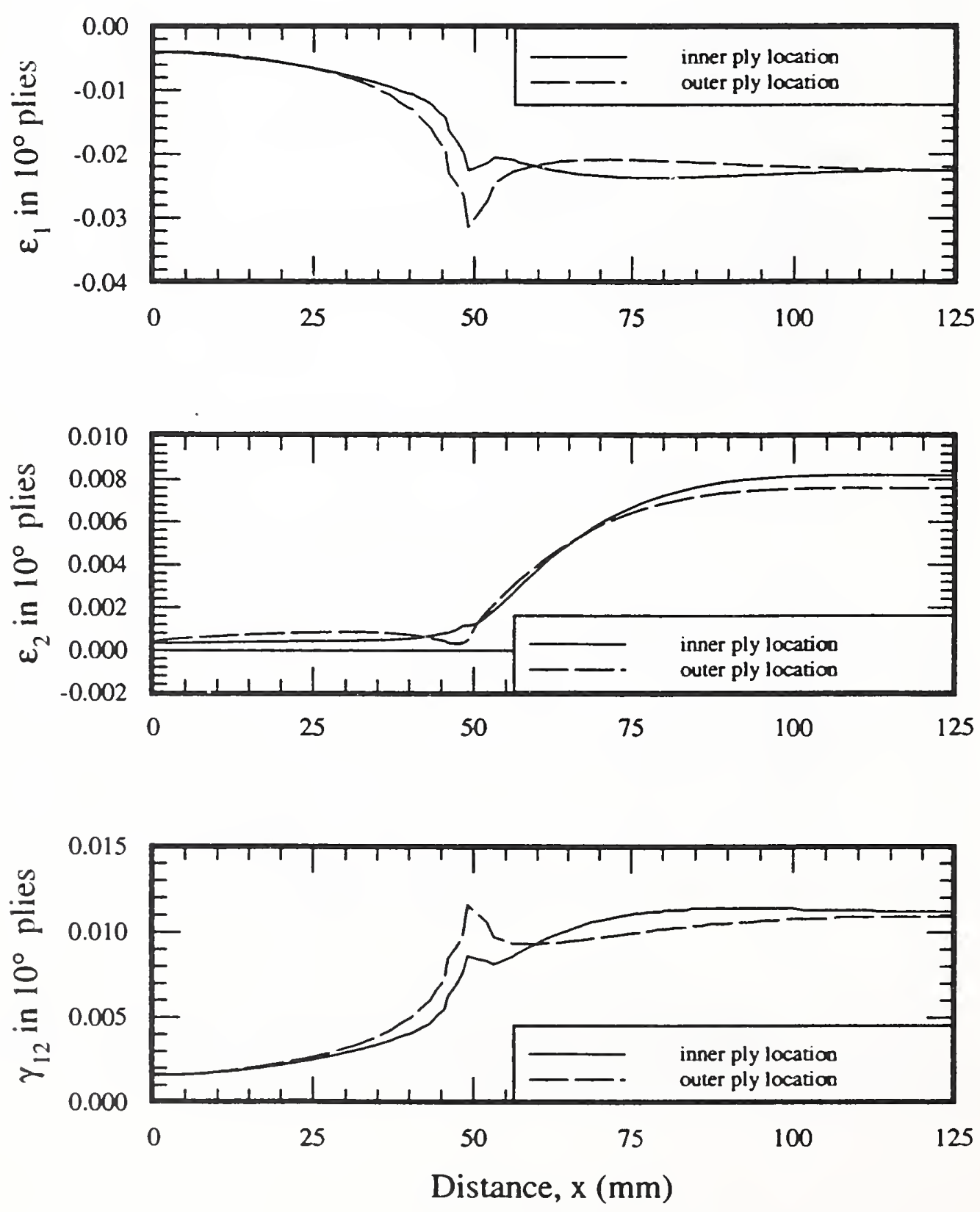

Figure 3.39. Intralaminar strains due to an applied axial stress of $-689 \mathrm{MPa}$ in $\pm 10^{\circ}$ plies of thick [82/45/10] cylinder with alternative-design end fitting, 297-K properties. 

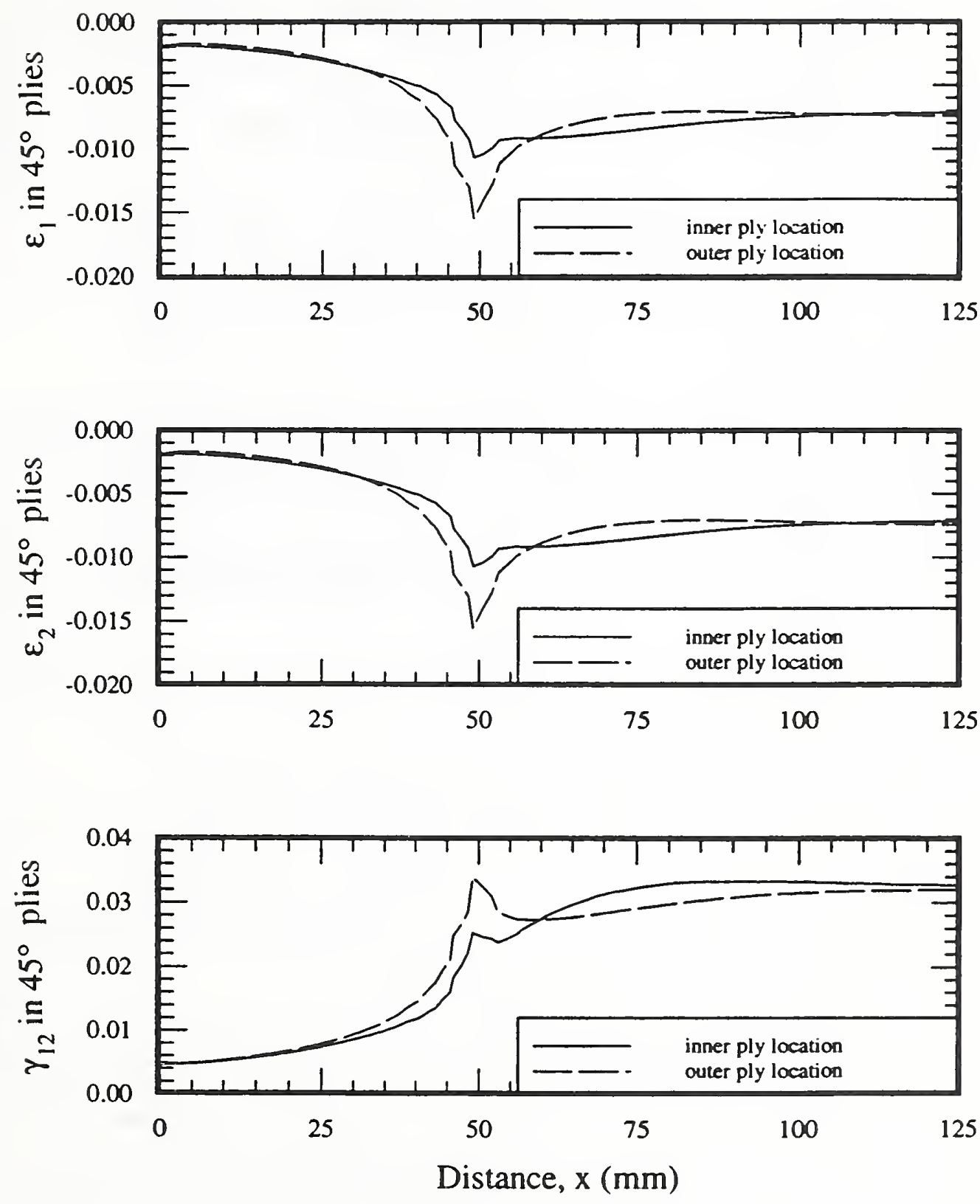

Figure 3.40. Intralaminar strains due to an applied axial stress of $-689 \mathrm{MPa}$ in $\pm 45^{\circ}$ plies of thick [82/45/10] cylinder with alternative-design end fitting, 297-K properties. 

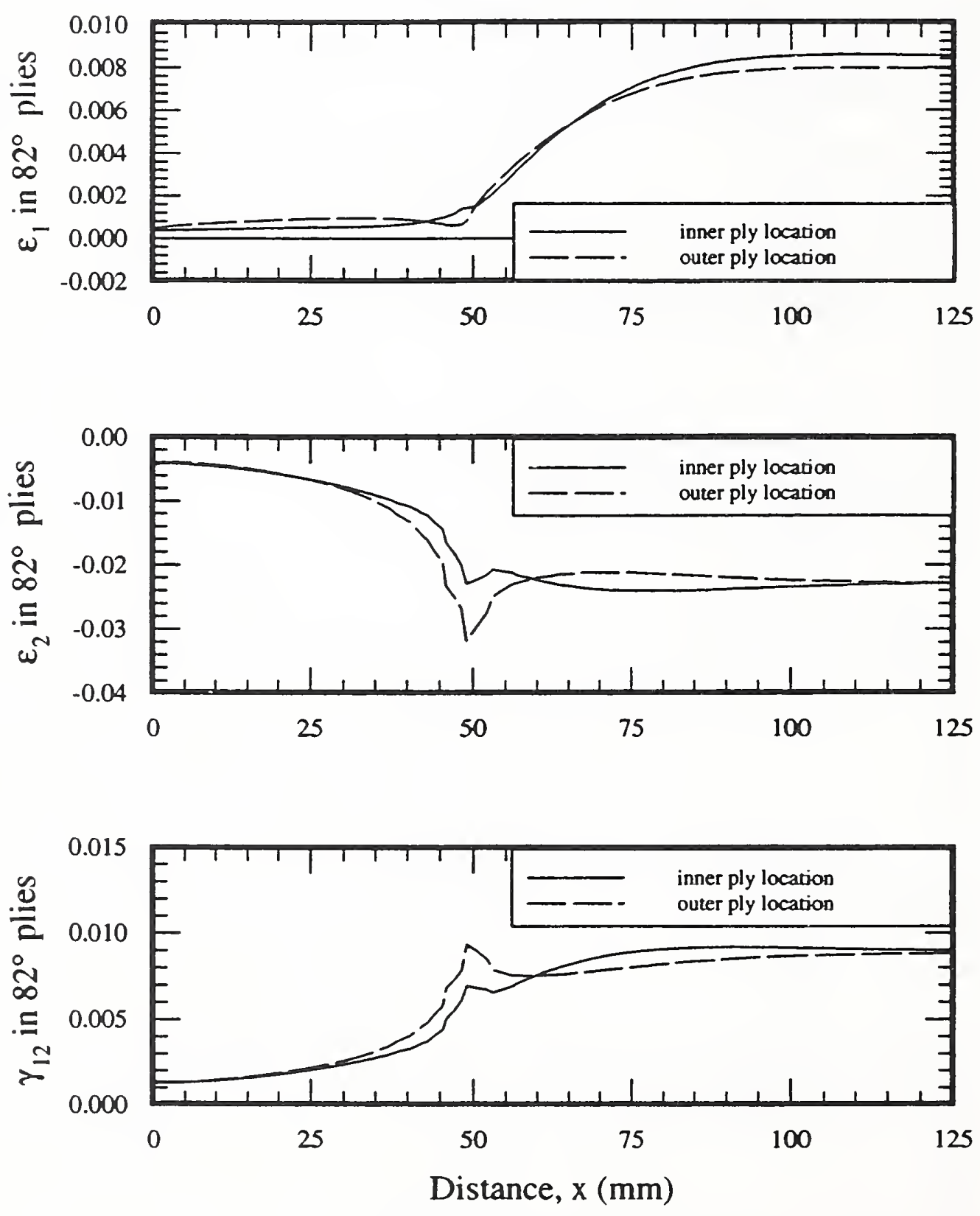

Figure 3.41. Intralaminar strains due to an applied axial stress of $-689 \mathrm{MPa}$ in $\pm 82^{\circ}$ plies of thick [82/45/10] cylinder with alternative-design end fitting, $297-\mathrm{K}$ properties. 

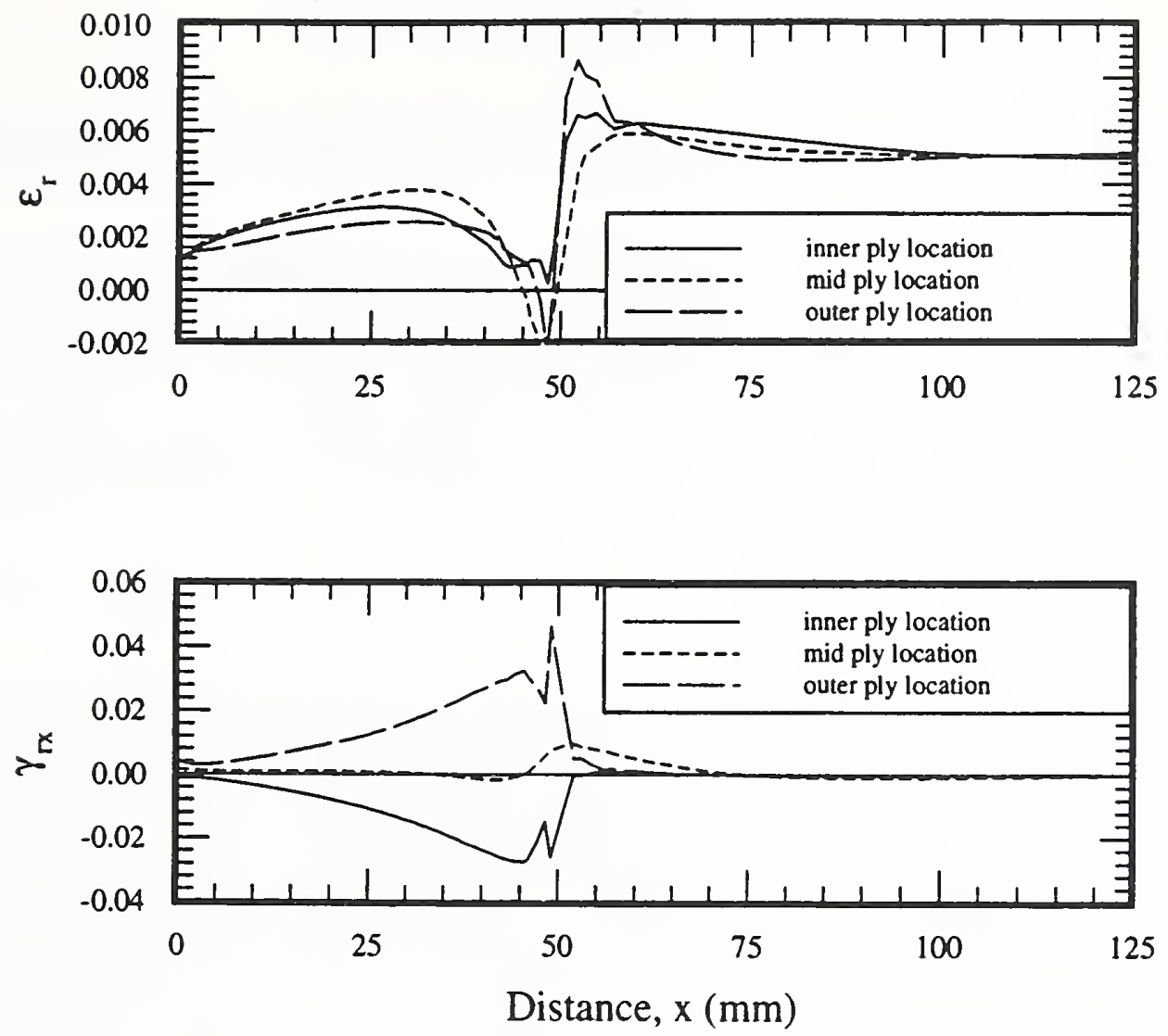

Figure 3.42. Intralaminar strains due to an applied axial stress of $-689 \mathrm{MPa}$ in thick [82/45/10] cylinder with alternative-design end fitting, $297-\mathrm{K}$ properties.

\subsection{Thinner Cylinders}

The strains in the thinner [10] and [82/45/10] cylinders are practically identical to the strains in the thicker cylinders for the same temperature and loading conditions and the same end-fitting design. As a result, there is no need to specifically discuss those results; they are presented in Appendix C. For convenience and comparison with the thicker cylinders, although it leads to unorthodox figure numbering, in Appendix $\mathrm{C}$ the figure numbers for the various strain responses for the thinner cylinder are identical to the figure numbers for the thicker cylinder in the main text, except the figures numbers are preceded by a 'C.' For example, in Appendix C Figures C. 4 and C.5 illustrate the strain response due to a temperature change in the thinner [10] cylinder with the baseline-design end fitting. Figures 3.4 and 3.5 have shown the strain response due to a temperature change in the thicker [10] cylinder with the baseline-design end fitting. 


\section{Conclusions}

The results presented in this report have illustrated several important features of the strain response of axially loaded glass-epoxy cylinders:

1. The strain levels at room temperature $(297 \mathrm{~K})$ are $25 \%$ higher than the strain response at $4 \mathrm{~K}$.

2. The alternative-design end fitting discussed had little, if any, effect on the strain, both in terms of the character of the response and the magnitude of the strain levels.

3. The strain-concentration factors for the fiber-direction compressive strains in the outer $10^{\circ}$ plies ranged from 1.3 to 1.4 .

\section{Possible Future Activities}

The last three months of last year's program saw a considerable generation of data from the testing of a number of cylinders. Because these tests occurred in the latter part of the program, there was not time to correlate measured strains with numerical predictions. It would be quite useful to continue to study the data and correlate it with numerical predictions representing these latter tests (e.g., on the pultruded cylinders), particularly the predictions of strains near the end fitting and particularly tests of the latter lay-ups. In the earlier tests, strains from the end-fitting location were not recorded and thus were not available for comparison with the numerical predictions. This end-fitting region is a region of high strain; the strain concentration factor for several of the earlier lay-ups was predicted to be between 1.3 and 1.5. It would be useful to focus on this specific area of all the cylinders that have been tested and to study the data carefully.

\section{References}

[3.1] ABAQUS users manual. Providence, RI: Hibbitt, Karsen and Sorensen; 1989.

[3.2] PATRAN. Costa Mesa, CA: PATRAN Division of PDA Engineering, California; 1989.

[3.3] DAN. Los Alamos, NM: Triakis, Inc.; 1991. 


\section{Appendixes}

\section{Appendix A. Determination of Ply Properties from Micromechanics}

When the material properties of the constituent fiber and matrix materials are known, there are a number of approaches to determining the material properties of a ply of composite. These approaches can be divided into roughly three categories: closed-form elasticity, strength-of-materials models, and numerical models based on a detailed representation of the geometry of the microstructure. Although the first two approaches are more attractive because of their simplicity, the third approach generally gives better results. In fact, for the so-called transverse properties, numerical modeling of the microstructure is preferred because oversimplifications often characterize the closedform-elasticity or strength-of-materials models. This oversimplification often leads to poor correlation between the predicted and measured properties.

Here, the material properties of a ply of glass-epoxy at room temperature and at $4 \mathrm{~K}$ are computed with a combination of approaches. Finite-element representations of the microstructure will be used to estimate all but one material property. A closed-form elasticity solution will be used to estimate that one property. Figure A.1 illustrates a ply and the principal material coordinate system generally associated with a ply. The 1 direction is the fiber direction, 2 is transverse to the fiber direction in the plane of the ply, and 3 is perpendicular to the ply. It will be assumed that the ply is transversely isotropic in the 2-3 plane. With this a number of material properties are equal, namely, $E_{3}=E_{2}, \nu_{13}=\nu_{12}$, and $\alpha_{3}=\alpha_{2}$, the latter being the coefficients of thermal expansion. If thermal dilatation, $\epsilon^{T}$, is used, as it is here, then $\epsilon_{2}^{T}=\epsilon_{3}^{T}$.

A study of the cross section of a ply reveals that in a well-made composite there is a regularity to the spacing of the fibers. One idealization to the spacing is the square-packed array shown in Figure A.2(a). Another idealization to the spacing is the hexagonal-packed array, as shown in Figure A.2(b). The nomenclature derives from the fact that if the centers of adjacent fibers are joined with lines, squares and hexagons result. It is assumed that the square or hexagonal arrays encompass large areas and one need only consider a representative region, or unit cell, to be able to represent the response of the entire cross section. The shaded region in Figure A.3(a) illustrates a unit cell that can be associated with the square-packing arrangement, and the shaded regions of Figure A.3(b) illustrate unit cells associated with the hexagonal array. Other unit cell arrangements can be associated

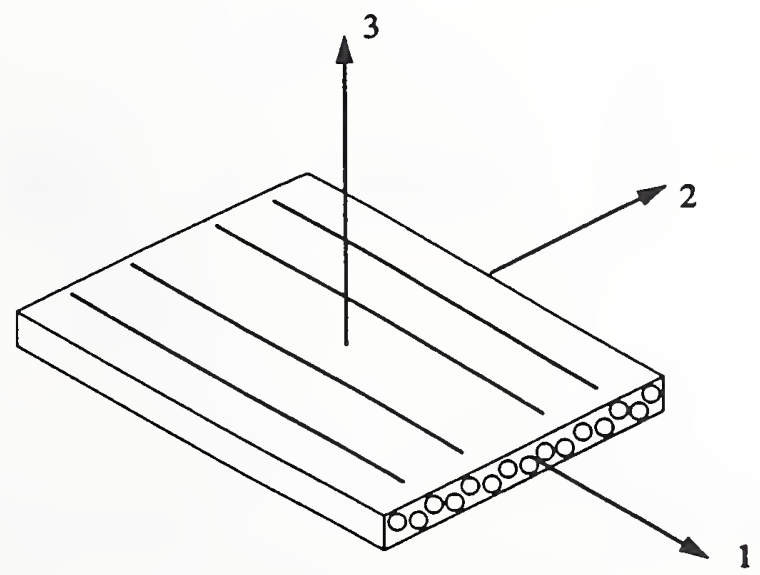

Figure A.1. A ply and the principal material coordinate system. 
with either packing array. The reduced quarter-cell rectangular subregion within the square array, as opposed to the entire unit cell, can often be used for many micromechanical studies. The similar reduced region in the hexagonal array can also often be used instead of the entire unit cell. An important question is: Which packing array should be used? Here for some properties, both will be considered, and the results will be compared. Strictly speaking, the hexagonal array tends to be more representative of the packing observed within a cross section. The modeling is somewhat more complicated, so it will be interesting to compare the results of the hexagonal array with results from the simpler square array, although much of the comparison will be left to the reader. The unit cell approach will be used to estimate $E_{1}, \nu_{12}\left(=\nu_{13}\right), E_{2}\left(=E_{3}\right), \nu_{23}, G_{23} \alpha_{1}, \alpha_{2}\left(=\alpha_{3}\right)$, or alternatively, $\epsilon_{2}^{T}\left(=\epsilon_{3}^{T}\right.$ ). The modulus $G_{12}$ will be determined by an elasticity approach that does not use either the square-packed or hexagonally packed arrays. For determining the ply properties, the glassfiber and epoxy-matrix properties at room temperature and at $4 \mathrm{~K}$ are given in Table A.1. As can be seen from the table, it is assumed that both the fiber and the matrix are isotropic.

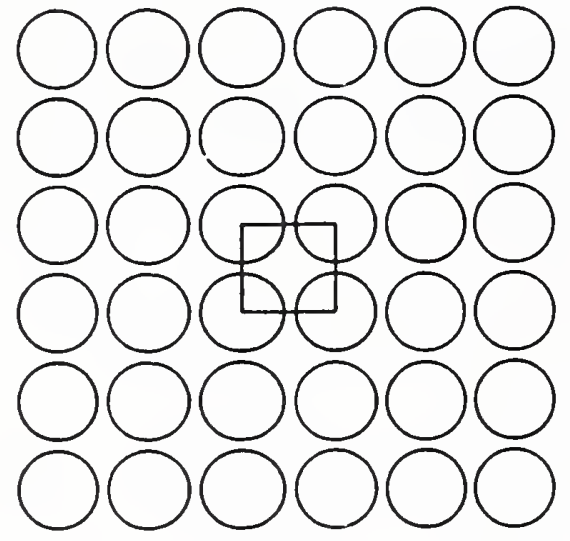

(a) Square-packed array.

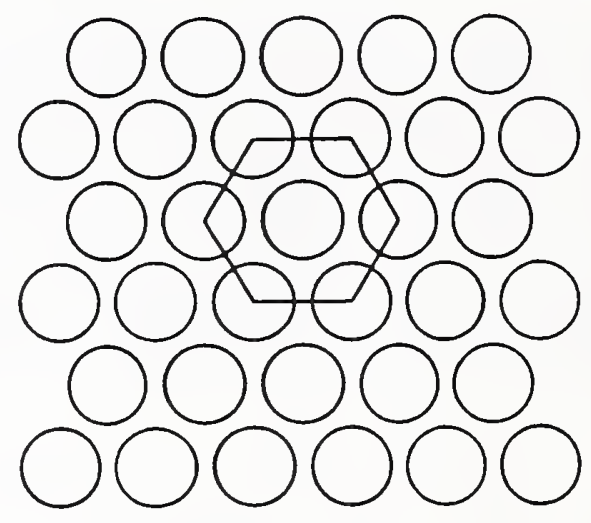

(b) Hexagonally packed array.

Figure A.2 Cross-section idealization.

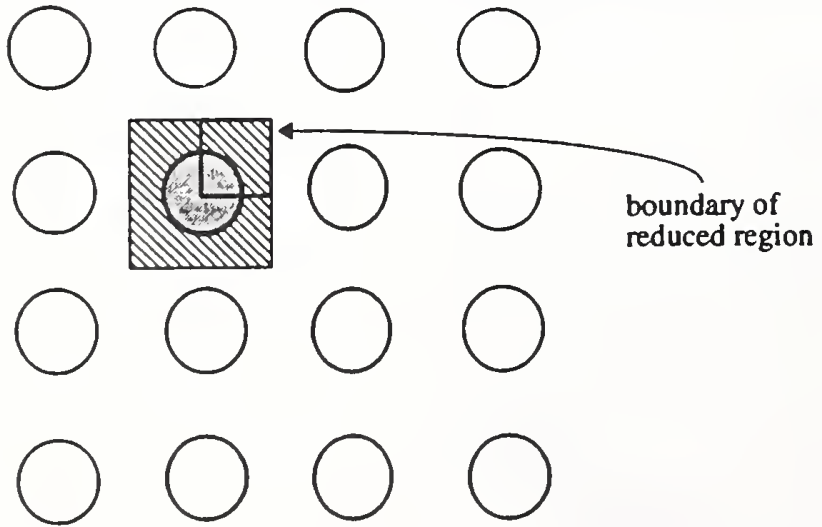

(a) Square-packed array.

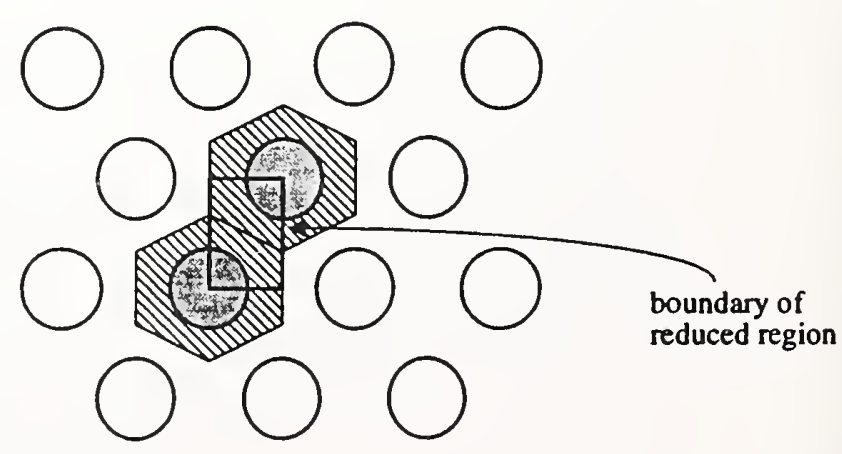

(b) Hexagonally packed array.

Figure A.3. Unit cells. 
Table A.1. Constituent properties.

\begin{tabular}{cl}
\hline & Room Temperature $(297 \mathrm{~K})$ \\
\hline glass fiber: & $\begin{array}{l}E=72.4 \mathrm{GPa}(10.5 \mathrm{Msi}) ; \nu=0.2 \\
\alpha=5.00 \times 10^{-6} / \mathrm{K} \quad\left(2.78 \times 10^{-6} /{ }^{\circ} \mathrm{F}\right)\end{array}$ \\
epoxy matrix: $\quad \begin{array}{l}3.23 \mathrm{GPa}(0.468 \mathrm{Msi}) ; \nu=0.38 \\
\alpha=50.0 \times 10^{-6} / \mathrm{K} \quad\left(27.8 \times 10^{-6} /{ }^{\circ} \mathrm{F}\right)\end{array}$ \\
\hline
\end{tabular}

To obtain numerical results from the unit-cell models, a finite-element discretization is used. The quarter model discretization for the square-packed array is shown in Figure A.4; the corresponding model for the hexagonally packed array is given in Figure A.5. The finite-element model uses a generalized plane deformation analysis, an analysis which assumes that the strains, and through Hooke's Law, the stresses, do not vary with the 1 direction. Hence the unit-cell cross section is sufficient for studying the response since it represents the situation anywhere along the 1 axis. Actually, the analysis considers the unit cell to be bounded in the 1 direction by two parallel planes, each perpendicular to the 1 axis, the two planes being a unit distance apart. The fiber volume fraction $V^{F}$ ) of the unit cell is controlled by what elements represent fiber and what elements represent matrix. With the finite-element meshes of Figures A.4 and A.5, changing fiber volume fraction is quite simple and the meshes were made to study $0.20,0.40$, or 0.60 fiber volume fraction.

To determine $E_{1}$ and $\nu_{12}$, a force is applied in the axial (1) direction to move the two bounding planes apart. The force to move the planes apart and the ensuing deformations with in the unit cell are used to determine these two material properties. As other boundary conditions on the unit cell when this force is applied (referring to Figure A.6), lines OA and OC are considered lines of symmetry. Line $\mathrm{AB}$ can displace but must remain straight and vertical, while line $\mathrm{BC}$ can displace but must remain straight and horizontal. The displacement in the 2 direction of line $\mathrm{AB}$ relative to the known movement in the 1 direction of the two boundary planes defines Poisson's ratio $\nu_{12}$. The known movement apart of the two bounding planes, the force to move them, and the cross-sectional area $\mathrm{OABC}$ defines $E_{1}$. With a known force applied in the 1 direction, the square-array unit-cell cross section deforms as shown in Figure A.7(a). Similar deformations occur with the hexagonal unit cell.

To determine transverse modulus $E_{2}$, with lines $\mathrm{OB}$ and $\mathrm{OC}$ being lines of symmetry, line $\mathrm{AB}$ is displaced a known amount in the 2 direction, while line $\mathrm{BC}$ is allowed to move but must remain straight and horizontal. The displacement of line $B C$ relative to the known displacement of line $\mathrm{AB}$ defines Poisson's ratio $\nu_{23}$. The force to move line $\mathrm{AB}$ the known amount, along with the known displacement and the dimensions of the unit cell, provide information to determine $E_{2}$. As stated above, for a transversely isotropic ply, $E_{3}$ equals $E_{2}$. Figure A.7(b) illustrates the transverse deformation of the square-array unit cell. 


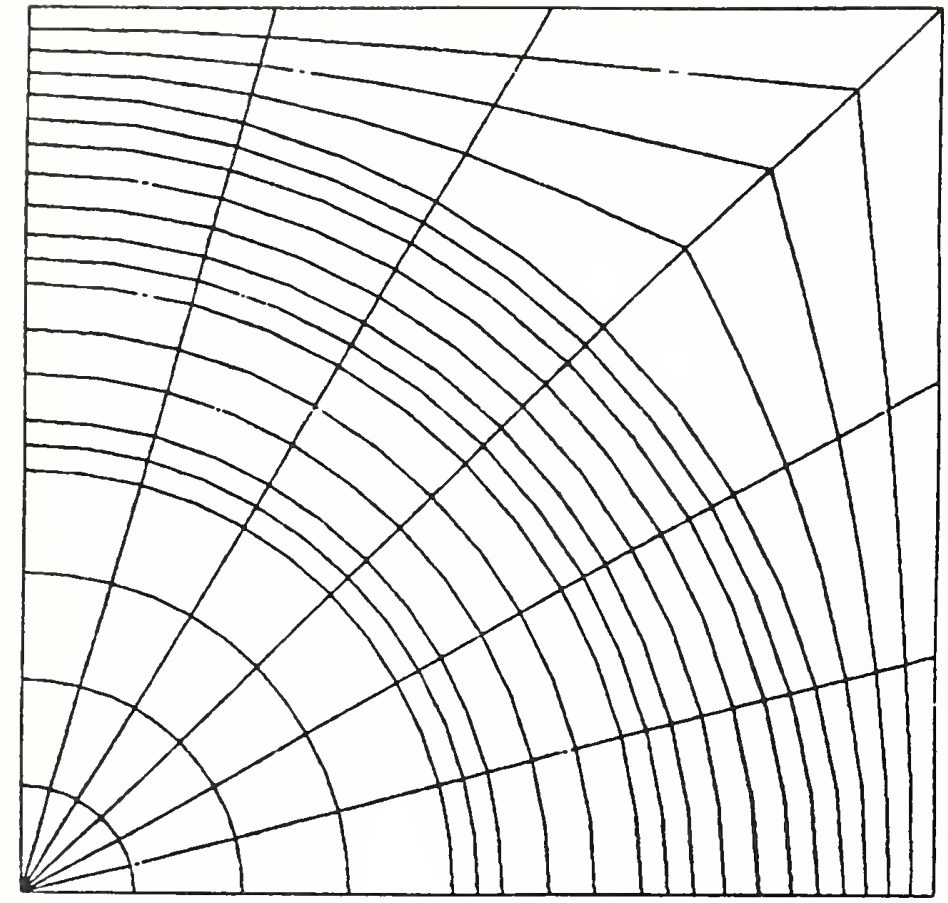

Figure A.4. Finite-element representation of the square-packed array (1/4 model).
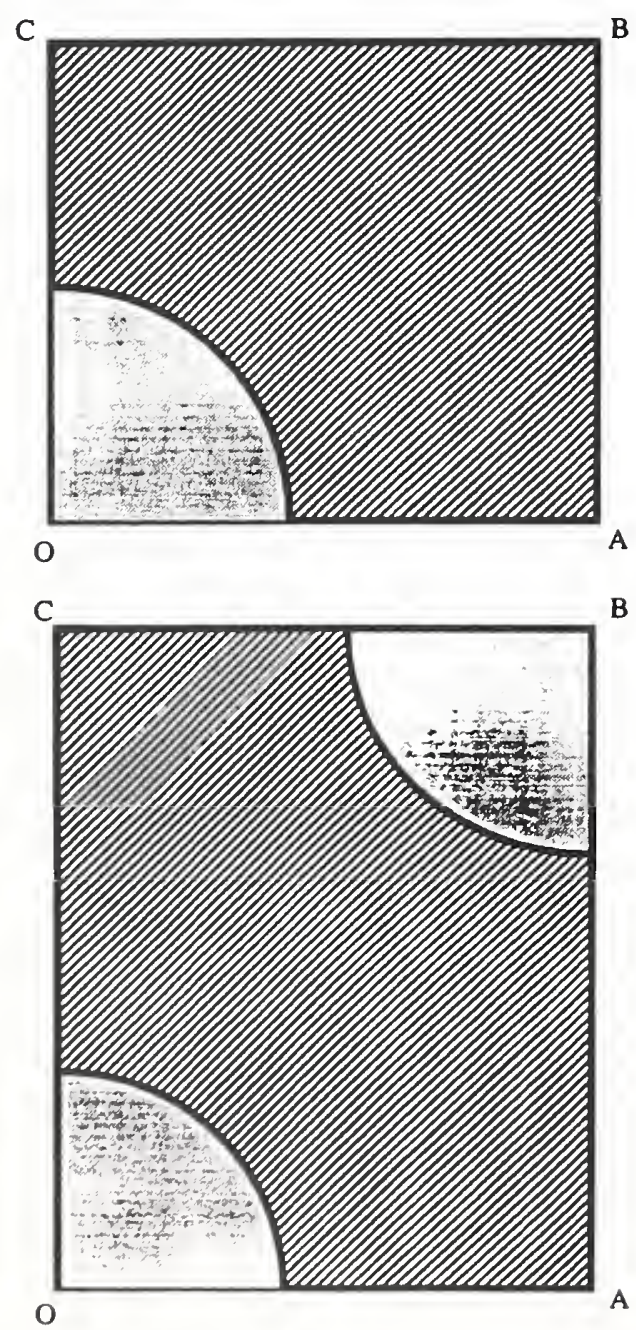

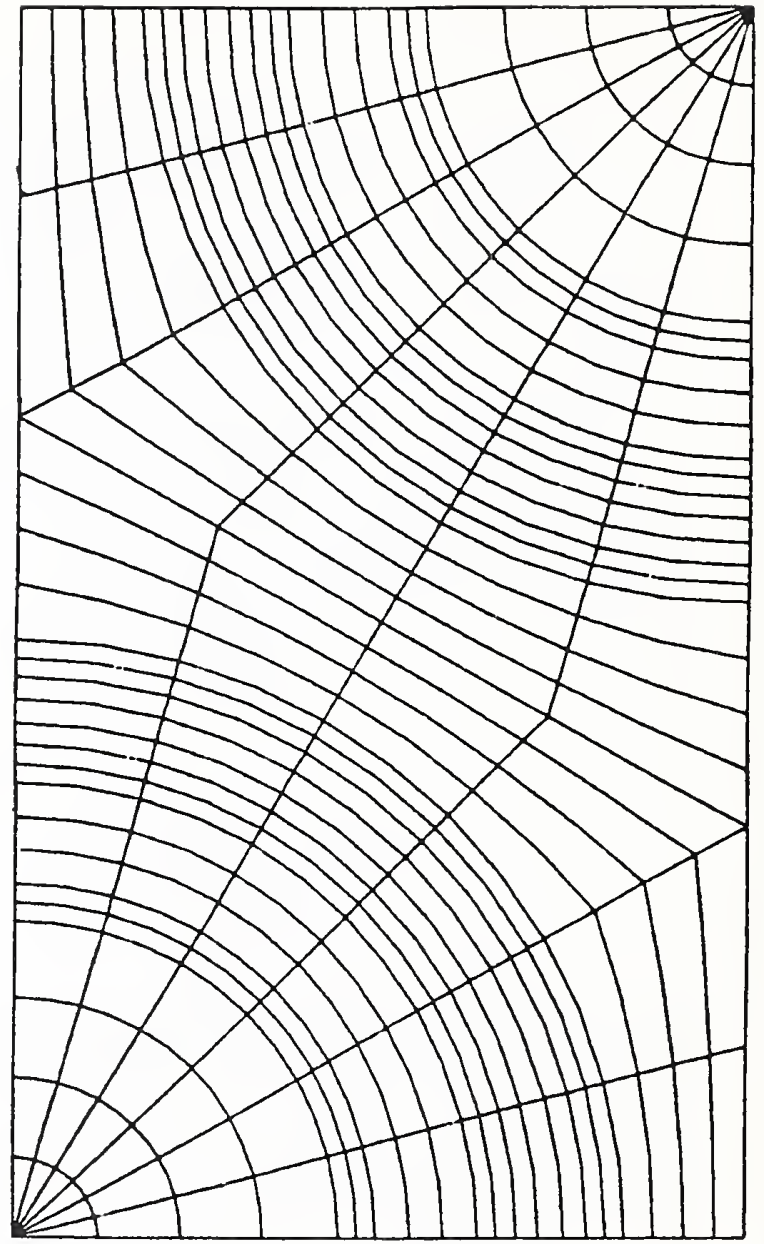

Figure A.5. Finite-element representation of the hexagonally packed array (partial model).

Figure A.6. Boundaries of unit-cell models. 


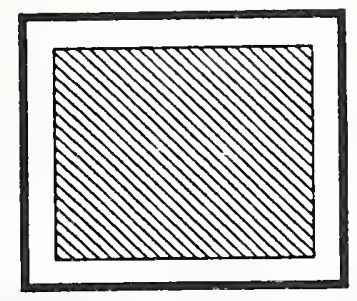

(a) Known axial force.

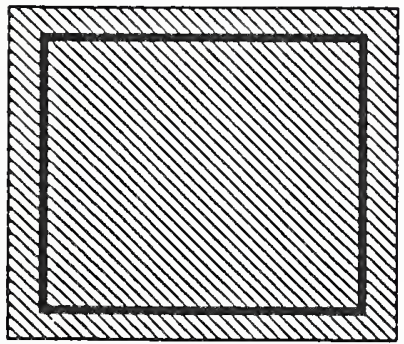

(c) Known temperature change.

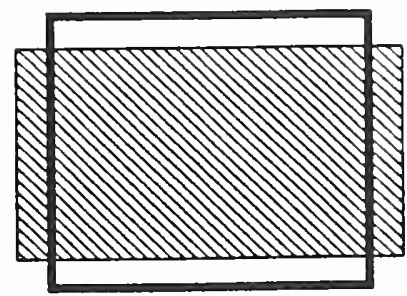

(b) Known transverse deformation.

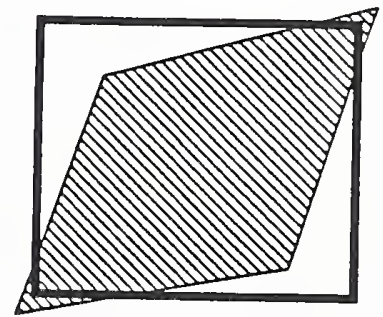

(d) Known shear deformation.

Figure A.7. Cross-sectional deformations of unit cell resulting from known conditions.

The thermal dilatations $\epsilon_{1}^{T}$ and $\epsilon_{2}^{T}\left(=\epsilon_{3}^{T}\right)$ are determined as follows: With lines OA and OC being lines of symmetry, as each constituent is given the thermal dilatation corresponding to a particular temperature change, lines $\mathrm{AB}$ and $\mathrm{BC}$ are required to remain straight and parallel to their original positions (see Figure A.6). The bounding planes perpendicular to the 1 direction are free to move but must remain perpendicular to the 1 direction. Thus, the distance the two bounding planes move and the distance line $\mathrm{AB}$ moves can be used to determine $\epsilon_{1}^{T}$ and $\epsilon_{2}^{T}$, respectively, for the ply.

To determine the ply shear transverse modulus, as $G_{23}$ is often referred to, the quartersymmetry model of the past figures cannot be used. Another quarter model can be used, but it is a model of antisymmetry. Alternatively, the entire unit-cell model can be used, and it can be given a known shear deformation, as shown in Figure A.6(d). The bounding planes, of course, are free to move but must remain planar. The forces required to produce the shear deformations, along with model geometry, can be used to determine the transverse shear modulus.

Finally, the axial shear modulus ( $G_{12}$, as it is sometimes referred to) can be computed using a technique known as the composite cylinder assemblage (CCA). By using CCA, the effective moduli of a composite material can be determined through the requirement that a composite cylinder, consisting of a single fiber of radius $b$ in an annulus of matrix of outer radius $c$ within a composite and a similar cylinder of an equivalent homogeneous medium must have the same average stress and strain for a given loading. For an applied shear strain $\left(\gamma_{12}\right)$ the boundary conditions on a composite cylinder are 


$$
\begin{aligned}
& u_{1}(S)=\frac{\gamma_{12}}{2} x_{2} \\
& u_{2}(S)=\frac{\gamma_{12}}{2} x_{1} \\
& u_{3}(S)=0
\end{aligned}
$$

The elasticity solution is solved using a semi-inverse method in which the displacements in both the fiber and the matrix of the composite cylinder are assumed to have the form

$$
\begin{aligned}
& u_{1}=\phi\left(x_{2}, x_{3}\right)-\frac{\gamma_{12}}{2} x_{2}-\frac{\gamma_{12}}{2} x_{3} \\
& u_{2}=\frac{\gamma_{12}}{2} x_{1} \\
& u_{3}=0
\end{aligned}
$$

The fact that the assumed form of the displacements does not satisfy the boundary conditions on the end of the composite cylinder produces insignificant end effects in a long cylinder. Using the strain-displacement relations, the stress-strain relations, and the equilibrium equations leads to the governing equation for $\phi$, which for isotropic or transversely isotropic constituents is the LaPlace equation:

$$
\nabla^{2} \phi=0
$$

Application of the boundary conditions is facilitated by writing the governing equation in the cylindrical coordinate system. This leads to the solution

$$
\begin{aligned}
& \phi^{m}(r, \theta)=\left(\mathrm{A}^{m} r+\frac{\mathrm{B}^{m}}{r}\right) \cos \theta \\
& \phi^{f}(r, \theta)=\left(\mathrm{A}^{f} r+\frac{\mathrm{B}^{f}}{r}\right) \cos \theta .
\end{aligned}
$$

in the matrix and fiber respectively. The constants $\mathrm{A}^{m}, \mathrm{~B}^{m}, \mathrm{~A}^{f}$, and $\mathrm{B}^{f}$ are then determined by considering

- finite displacement $u_{1}$ at the center of the fiber, $r=0$

- continuity of $u_{1}$ at the fiber matrix interface, $r=b$

- continuity of traction $T_{1}$ at the fiber matrix interface, $r=b$

- the applied displacement on the boundary of the composite cylinder, $r=c$

Once the constants are determined, the function $\phi$ is completely defined. Then examining the tractions at $r=c$, the only nonzero traction is given by

$$
T_{1}(c)=G_{m} \frac{\partial \phi^{m}}{\partial r}
$$


For the equivalent homogeneous material, the traction at $r=c$ is

$$
T_{1}(c)=G_{12} \gamma_{12} \cos \theta
$$

Thus, since the two tractions must be the same, the effective axial shear modulus of the composite cylinder consisting of transversely isotropic or isotropic phases is given by

$$
G_{12}=G_{m}\left\{\frac{\left(G_{m}+G_{f}\right)+V^{F}\left(G_{f}-G_{m}\right)}{\left(G_{m}+G_{f}\right)+V^{F}\left(G_{m}-G_{f}\right)}\right\},
$$

where $V^{F}$ is the fiber volume fraction.

Table A.2 lists the room-temperature properties of a ply of glass-epoxy at three volume fractions $\left(V^{F}\right)$ as computed with the above-described procedures. The coefficients of thermal expansion are given. Table A.3 lists the properties of a ply of glass-epoxy at three volume fractions for a temperature of $4 \mathrm{~K}$. Here, the thermal dilatations that result relative to room temperature, rather than the coefficients of thermal expansion, are listed. 
Table A.2. Material properties of glass-epoxy at room temperature (297 K).

\begin{tabular}{|c|c|c|c|c|c|c|}
\hline Property & \multicolumn{2}{|c|}{$V^{F}=0.20$} & \multicolumn{2}{|c|}{$V^{F}=0.40$} & \multicolumn{2}{|c|}{$V^{F}=0.60$} \\
\hline \multicolumn{7}{|l|}{ Square Array } \\
\hline$E_{1}, \mathrm{GPa}(\mathrm{Msi})$ & 17.0 & $(2.47)$ & 31.0 & $(4.49)$ & 44.8 & $(6.49)$ \\
\hline$E_{2}, \mathrm{GPa}(\mathrm{Msi})$ & 5.20 & $(0.754)$ & 8.51 & $(1.23)$ & 15.6 & $(2.56)$ \\
\hline$G_{23}, \mathrm{GPa}(\mathrm{Msi})$ & 1.26 & $(0.183)$ & 2.59 & $(0.244)$ & 2.83 & $(0.411)$ \\
\hline$\nu_{12}$ & 0.338 & & 0.300 & & 0.262 & \\
\hline$\nu_{23}$ & 0.508 & & 0.431 & & 0.303 & \\
\hline$\alpha_{1}, \mathrm{ppm} / \mathrm{K}\left(\mathrm{ppm} /{ }^{\circ} \mathrm{F}\right)$ & 12.3 & $(6.83)$ & 8.19 & $(4.55)$ & 6.61 & $(3.67)$ \\
\hline$\alpha_{2}, \mathrm{ppm} / \mathrm{K}\left(\mathrm{ppm} /{ }^{\circ} \mathrm{F}\right)$ & 50.3 & $(28.0)$ & 38.3 & $(21.3)$ & 25.9 & $(14.4)$ \\
\hline \multicolumn{7}{|l|}{ Hexagonal Array } \\
\hline$E_{1}, \mathrm{GPa}(\mathrm{Msi})$ & 17.1 & $(2.48)$ & 30.9 & $(4.48)$ & 44.8 & $(6.50)$ \\
\hline$E_{2}, \mathrm{GPa}(\mathrm{Msi})$ & 4.97 & $(0.721)$ & 7.27 & $(1.06)$ & 12.0 & $(1.74)$ \\
\hline$G_{23}, \mathrm{GPa}(\mathrm{Msi})$ & \multicolumn{2}{|c|}{ did not compute } & \multicolumn{2}{|c|}{ did not compute } & \multicolumn{2}{|c|}{ did not compute } \\
\hline$\nu_{12}$ & \multicolumn{2}{|l|}{0.338} & \multicolumn{2}{|l|}{0.300} & \multicolumn{2}{|l|}{0.264} \\
\hline$\nu_{23}$ & \multicolumn{2}{|l|}{0.531} & \multicolumn{2}{|l|}{0.513} & \multicolumn{2}{|l|}{0.450} \\
\hline$\alpha_{1}, \mathrm{ppm} / \mathrm{K}\left(\mathrm{ppm} /{ }^{\circ} \mathrm{F}\right)$ & 12.3 & $(6.81)$ & 8.19 & $(4.55)$ & 6.54 & $(3.64)$ \\
\hline$\alpha_{2}, \mathrm{ppm} / \mathrm{K}\left(\mathrm{ppm} /{ }^{\circ} \mathrm{F}\right)$ & 50.2 & $(27.9)$ & 38.4 & $(21.4)$ & 26.6 & $(14.8)$ \\
\hline \multicolumn{7}{|l|}{ Theory of Elasticity } \\
\hline$G_{12}, \mathrm{GPa}$ (Msi) & 1.70 & $(0.247)$ & 2.56 & $(0.369)$ & 4.10 & $(0.595)$ \\
\hline
\end{tabular}


Table A.3. Material properties of glass-epoxy at $4 \mathrm{~K}$.

\begin{tabular}{|c|c|c|c|c|c|}
\hline Property & $V^{F}=0.20$ & $V^{F}=$ & $=0.40$ & $V^{F}=$ & $=0.60$ \\
\hline \multicolumn{6}{|l|}{ Square Array } \\
\hline$E_{1}, \mathrm{GPa}(\mathrm{Msi})$ & $(3.26)$ & 36.8 & $(5.34)$ & 51.0 & $(7.40)$ \\
\hline$E_{2}, \mathrm{GPa}(\mathrm{Msi})$ & $(1.84)$ & 19.8 & $(2.86)$ & 31.8 & $(4.62)$ \\
\hline$G_{23}, \mathrm{GPa}(\mathrm{Msi})$ & $(0.48)$ & 3.94 & $(0.57)$ & 6.16 & $(0.89)$ \\
\hline$\nu_{12}$ & 0.358 & 0.315 & & 0.274 & \\
\hline$\nu_{23}$ & 0.504 & 0.446 & & 0.341 & \\
\hline$\epsilon_{2}^{T^{*}}, \mathrm{ppm} / \mathrm{K}\left(\mathrm{ppm} /{ }^{\circ} \mathrm{F}\right)$ & $-3710 \times 10^{-6}$ & -2220 & $\times 10^{-6}$ & -1550 & $\times 10^{-6}$ \\
\hline$\epsilon_{1}^{T^{*}}, \mathrm{ppm} / \mathrm{K}\left(\mathrm{ppm} /{ }^{\circ} \mathrm{F}\right)$ & $-9820 \times 10^{-6}$ & -7690 & $\times 10^{-6}$ & -5280 & $\times 10^{-6}$ \\
\hline \multicolumn{6}{|l|}{ Hexagonal Array } \\
\hline$E_{1}, \mathrm{GPa}(\mathrm{Msi})$ & $(3.27)$ & 36.8 & $(5.33)$ & 51.0 & $(7.40)$ \\
\hline$E_{2}, \mathrm{GPa}(\mathrm{Msi})$ & $(1.77)$ & 17.3 & $(2.50)$ & 26.3 & $(3.82)$ \\
\hline$G_{23}, \mathrm{GPa}(\mathrm{Msi})$ & did not compute & did not $\mathrm{c}$ & ompute & did not & ompute \\
\hline$\nu_{12}$ & 0.358 & 0.316 & & 0.275 & \\
\hline$\nu_{23}$ & 0.524 & 0.515 & & 0.451 & \\
\hline$\epsilon_{1}^{T^{*}}, \mathrm{ppm} / \mathrm{K}\left(\mathrm{ppm} /{ }^{\circ} \mathrm{F}\right)$ & $-3700 \times 10^{-6}$ & -2220 & $\times 10^{-6}$ & -1520 & $\times 10^{-6}$ \\
\hline$\epsilon_{2}^{T^{*}}, \mathrm{ppm} / \mathrm{K}\left(\mathrm{ppm} /{ }^{\circ} \mathrm{F}\right)$ & $-9820 \times 10^{-6}$ & -7700 & $\times 10^{-6}$ & -5360 & $\times 10^{-6}$ \\
\hline \multicolumn{6}{|l|}{ Theory of Elasticity } \\
\hline$G_{12}, \mathrm{GPa}(\mathrm{Msi})$ & $(0.598)$ & 5.91 & $(0.857)$ & 8.83 & $(1.28)$ \\
\hline
\end{tabular}

*Relative to room temperature (297 K) 


\section{Appendix B. Equivalent Laminate Properties}

Although it is theoretically possible to model an unlimited number of layers, say 1000 layers, in a laminate, it may not always be within the computational capacity that is available. Lumping or smearing the layers into a single, equivalent, thicker layer may be required if numerical results are to be obtained. Thus, a smearing scheme must be developed. There are a number of approaches for determining smeared properties. This appendix focuses on one approach.

The goal of smearing the elastic properties is to determine the effective moduli and Poisson's ratios that can be used to analyze a thick, multilayer cylinder as a thick, single layer. Specifically, Young's moduli in the axial, circumferential, radial directions, $E_{x}, E_{\theta}$, and $E_{r}$, and Poisson's ratios $\nu_{x \theta}, \nu_{x r}$, and $\nu_{\theta r}$ are needed. As a check, $\nu_{\theta x}, \nu_{r x}$, and $\nu_{r \theta}$ can be computed and compared with the values obtained using the well-known relation between moduli and Poisson's ratios:

$$
\frac{\nu_{i j}}{E_{i}}=\frac{\nu_{j i}}{E_{j}} \quad i, j=x, \theta, r
$$

In addition, the three shear moduli $G_{x \theta}, G_{r \theta}$, and $G_{x r}$ are needed. For problems involving thermal effects, the coefficients of thermal expansion in the $x, \theta$, and $r$ directions, $\alpha_{x}, \alpha_{\theta}$, and $\alpha_{r}$ are needed.

When constructing models for determining equivalent properties, it is most straightforward to keep in mind the definitions of the individual properties. For example, the definitions of $E_{x}, \nu_{x \theta}$, and $\nu_{x r}$ require that the laminate be subjected to a simple tensile stress in the axial direction and the extension in the axial direction, and contractions in the circumferential and radial directions be determined. To determine $E_{x}, \nu_{r x}$, and $\nu_{r \theta}$, a laminate is subjected to simple tension in the radial direction and that the extension in that direction and the contractions in the axial and circumferential directions must be determined. For modeling purposes, these simple loads are applied within the context of assumptions regarding laminate-deformation response. In this work, the primary kinematic assumption is that straight lines normal to the layers before deformation remain straight and normal after deformation. Although similar to the Kirchhoff hypothesis, it will be assumed that the length of these normals can change. In what follows, use will be made of an element, or cube, of thickness $t$ cut from a thick, multilayer cylinder. Such a cube is shown in Figure B.1. Despite the fact a cylinder is described by polar coordinates, for the sake of simplicity the undeformed element is shown as a cube instead of the usual cylindrical volume element. To keep in mind that it is actually a cylindrical element, the $y$ axis is denoted as $\theta$. The use of a cube is of no consequence in the results to follow. 


\section{$\uparrow$}

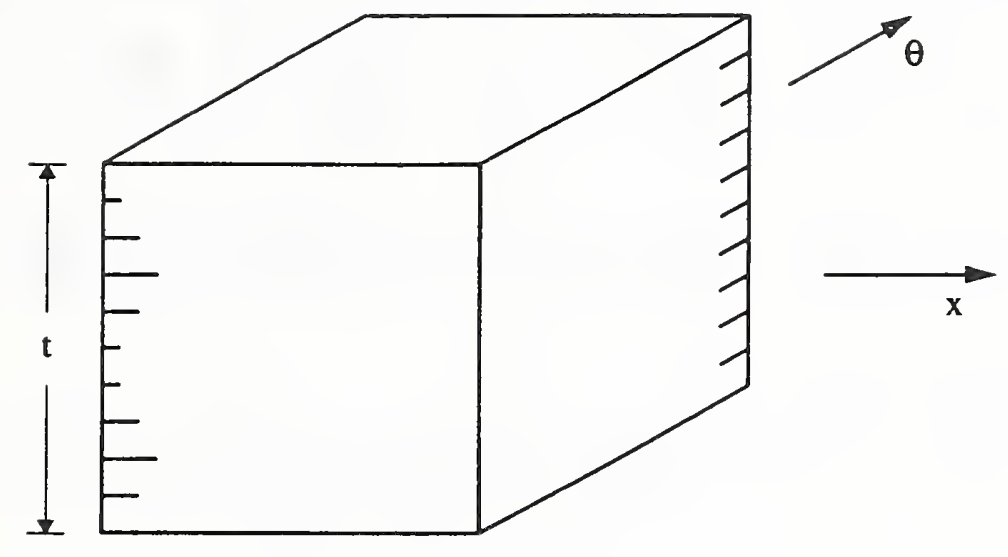

Figure B.1. Cubic element cut from thick laminate.

\section{Determination of Equivalent of $E_{r}, \nu_{r x}$, and $\nu_{r \theta}$}

To compute the equivalent properties $E_{r}, \nu_{r x}$, and $\nu_{r \theta}$, the so-called out-of-plane properties, assume an element of the laminate is subjected to a known stress $\sigma_{r}$. This is shown in Figure B.2.

Due to the assumption of the normals remaining straight, the corners of the cube remain orthogonal when a $\sigma_{r}$ is applied. The cube elongates in the $r$ direction and contracts in the $x$ and $\theta$ directions. Because the normals are assumed to remain straight, the contraction strains in the $x$ and $\theta$ directions are independent of thickness. Call these strains $\epsilon_{x}^{0}$ and $\epsilon_{\theta}^{0}$. If several layers in the cube are oriented at an angle other than $0^{\circ}$ or $90^{\circ}$, then there might be an over change in the orthogonal corners in the $x-\theta$ plane. Call this angle change $\gamma_{x \theta}^{0}$. Here we shall assume the laminate is balanced so there is no change in the right angles in the $x-\theta$ plane, that is, $\gamma_{x \theta}^{0}=0$. With these considerations, the stress-strain relation for a single layer is

$$
\begin{aligned}
& \sigma_{x}=C_{11} \epsilon_{x}^{0}+C_{12} \epsilon_{\theta}^{0}+C_{13} \epsilon_{r}+C_{16} \gamma_{x \theta}^{0} \\
& \sigma_{\theta}=C_{12} \epsilon_{x}^{0}+C_{22} \epsilon_{\theta}^{0}+C_{23} \epsilon_{r}+C_{26} \gamma_{x \theta}^{0} \\
& \sigma_{r}=C_{13} \epsilon_{x}^{0}+C_{23} \epsilon_{\theta}^{0}+C_{33} \epsilon_{r}+C_{36} \gamma_{x \theta}^{0}
\end{aligned},
$$

where, despite $\gamma_{x \theta}^{0}$ being zero, it is included in the appropriate way as a reminder of the assumption that it is assumed to be zero. In the above, the $C_{i j}$ are the transformed stiffnesses of the layer in the $x-\theta-r$ coordinate system. Owing to the thinness of a layer, it is assumed that within each layer the radial strain $\epsilon_{r}$ is independent of $z$. However, $\epsilon_{r}$ can vary from layer to layer. Solving the third of Equation B.2 for $\epsilon_{r}$ leads to 


$$
\epsilon_{r}=\left(\frac{\sigma_{r}}{C_{33}}\right)-\left(\frac{C_{13}}{C_{33}}\right) \epsilon_{x}^{0}-\left(\frac{C_{23}}{C_{33}}\right) \epsilon_{\theta}^{0}-\left(\frac{C_{36}}{C_{33}}\right) \gamma_{x \theta}^{0}
$$

Substituting this into the other two of Equation B.2, and now setting $\gamma_{x \theta}^{0}$ to zero, yields

$$
\begin{aligned}
& \sigma_{x}=\left(C_{11}-\frac{C_{13}^{2}}{C_{33}}\right] \epsilon_{x}^{0}+\left(C_{12}-\frac{C_{13} C_{23}}{C_{33}}\right) \epsilon_{\theta}^{0}+\left(\frac{C_{13}}{C_{33}}\right) \sigma_{r} \\
& \sigma_{\theta}=\left(C_{12}-\frac{C_{13} C_{23}}{C_{33}}\right) \epsilon_{x}^{0}+\left[C_{22}-\frac{C_{23}^{2}}{C_{33}}\right) \epsilon_{\theta}^{0}+\left(\frac{C_{23}}{C_{33}}\right) \sigma_{r} .
\end{aligned}
$$

Redefining the stiffness terms results in

$$
\begin{aligned}
& \sigma_{x}=\bar{Q}_{11} \epsilon_{x}^{0}+\bar{Q}_{12} \epsilon_{\theta}^{0}+\bar{Q}_{17} \sigma_{r} \\
& \sigma_{\theta}=\bar{Q}_{12} \epsilon_{x}^{0}+\bar{Q}_{22} \epsilon_{\theta}^{0}+\bar{Q}_{27} \sigma_{r} .
\end{aligned}
$$

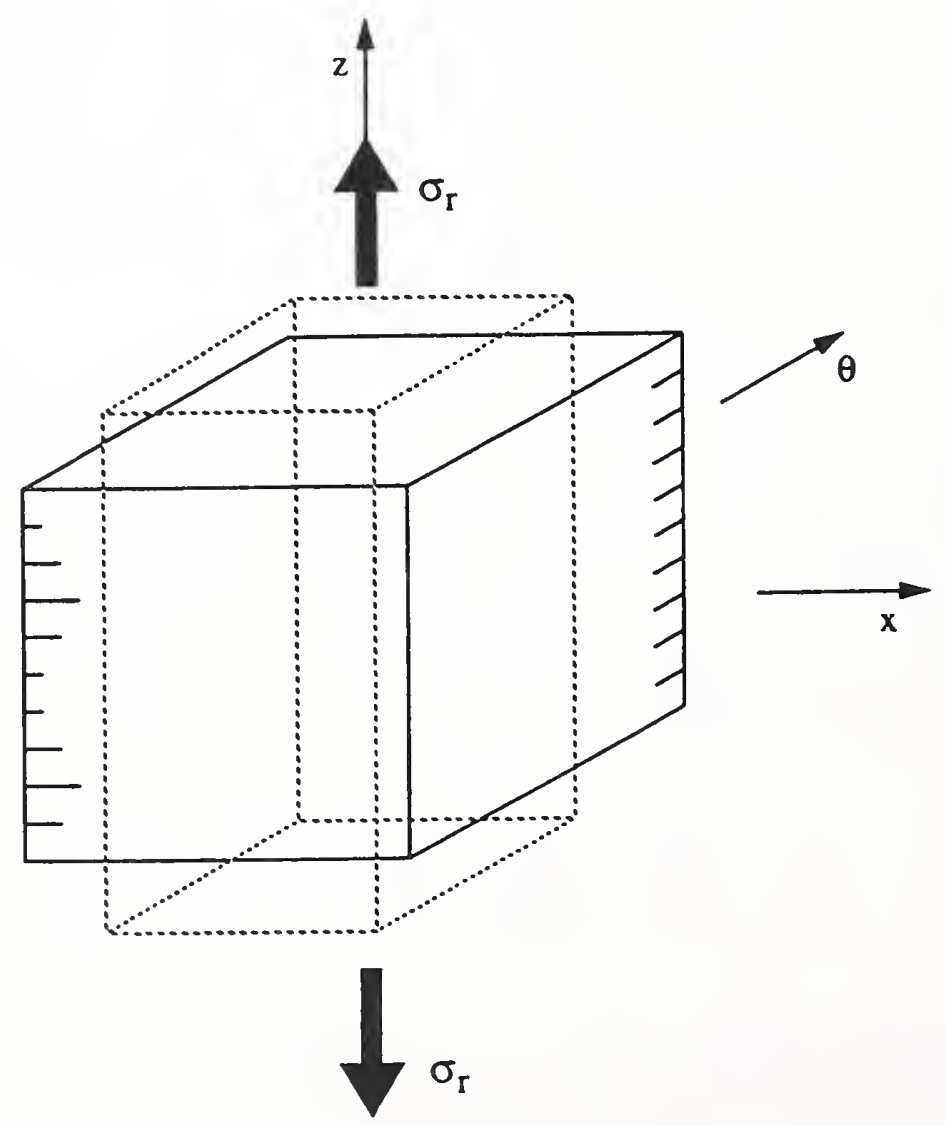

Figure B.2. Element subjected to stress $\sigma_{r}$. 
Recall that the element considered $r e$ is being subjected to a known value of $\sigma_{r}$. Owing to the requirement of continuity of this component of stress at each layer interface, and to the fact that $\epsilon_{r}$ is assumed to be independent of $z$ within each layer, $\sigma_{r}$ is independent of $z$ and is the same in each layer. Furthermore, there is no net stress resultant in either the $x$ or $\theta$ directions. This latter fact is useful if the above equations are integrated through the thickness of the element to yield

$$
\begin{aligned}
& N_{x}=\int_{-\frac{t}{2}}^{\frac{t}{2}} \sigma_{x} d z=\left[\int_{-\frac{t}{2}}^{\frac{t}{2}} \bar{Q}_{11} d z\right) \epsilon_{x}^{0}+\left[\int_{-\frac{t}{2}}^{\frac{t}{2}} \bar{Q}_{12} d z\right) \epsilon_{\theta}^{0}+\left[\int_{-\frac{t}{2}}^{\frac{t}{2}} \bar{Q}_{17} d z\right) \sigma_{r} \\
& N_{\theta}=\int_{-\frac{t}{2}}^{\frac{t}{2}} \sigma_{\theta} d z=\left[\int_{-\frac{1}{2}}^{\frac{t}{2}} \bar{Q}_{12} d z\right) \epsilon_{x}^{0}+\left(\int_{-\frac{t}{2}}^{\frac{t}{2}} \bar{Q}_{22} d z\right) \epsilon_{\theta}^{0}+\left(\int_{-\frac{t}{2}}^{\frac{t}{2}} \bar{Q}_{27} d z\right) \sigma_{r},
\end{aligned}
$$

$t$, again, being the thickness of the element. The above integrations are possible because $\epsilon_{x}^{0}, \epsilon_{\theta}^{0}$, and $\sigma_{r}$ are independent of $z$. Setting $N_{x}$ and $N_{\theta}$ to zero yields a relation for $\epsilon_{x}^{0}$ and $\epsilon_{\theta}^{0}$, namely

$$
\begin{aligned}
& \epsilon_{x}^{0}=\left(\frac{A_{12} A_{27}-A_{22} A_{17}}{A_{11} A_{22}-A_{12}^{2}}\right) \sigma_{r}=A_{x r} \sigma_{r} \\
& \epsilon_{\theta}^{0}=\left(\frac{A_{12} A_{17}-A_{11} A_{27}}{A_{11} A_{22}-A_{12}^{2}}\right) \sigma_{r}=A_{\theta r} \sigma_{r}
\end{aligned}
$$

In the above

$$
A_{i j}=\int_{-\frac{t}{2}}^{\frac{1}{2}} \bar{Q}_{i j} d z=\sum_{k=1}^{N} \bar{Q}_{i j}^{(k)} t_{k}
$$

$t_{k}$ being the thickness of the $k^{\text {th }}$ layer, there being $N$ layers in the element of Figure B.2.

With $\sigma_{r}, \epsilon_{x}^{0}$, and $\epsilon_{\theta}^{0}$ known, the radial strain $\epsilon_{r}^{(k)}$ for the $k^{\text {th }}$ layer can be determined from Equation B.3:

$$
\epsilon_{r}^{(k)}=\left(\frac{\sigma_{r}}{C_{33}^{(k)}}\right)-\left(\frac{C_{13}^{(k)}}{C_{33}^{(k)}}\right) \epsilon_{x}^{0}-\left(\frac{C_{23}^{(k)}}{C_{33}^{(k)}}\right) \epsilon_{\theta}^{0}
$$

The thickness change of the $k^{\text {th }}$ layer is

$$
\Delta t_{k}=\epsilon_{r}^{(k)} t_{k}
$$

and the total thickness change of the element is 


$$
\Delta t=\sum_{k=1}^{N} \Delta t_{k}
$$

The average radial strain, $\epsilon_{r}^{0}$, is given by

$$
\epsilon_{r}^{0}=\frac{\Delta t}{t}=\frac{\sum_{k=1}^{N} \Delta t_{k}}{t}=\frac{\sum_{k=1}^{N} \epsilon_{r}^{(k)} t_{k}}{t} .
$$

With this loading, the definition of Young's modulus in the radial direction follows directly, namely,

$$
E_{r}=\frac{\sigma_{r}}{\epsilon_{r}^{0}}=\frac{t}{\sum_{k=1}^{N}\left[\frac{1}{C_{33}^{(k)}}-\left(\frac{C_{13}^{(k)}}{C_{33}^{(k)}}\right) A_{x r}-\left(\frac{C_{23}^{(k)}}{C_{33}^{(k)}}\right) A_{\theta r}\right) t_{k}}
$$

Equations B.7 through B.12 were used to arrive at Equation B.13. Also, by definition, the two Poisson's ratios become

$$
\begin{aligned}
& \nu_{r x}=-\frac{\epsilon_{x}^{0}}{\epsilon_{r}^{0}}=-\frac{A_{x r} t}{\sum_{k=1}^{N}\left[\frac{1}{C_{33}^{(k)}}-\left(\frac{C_{13}^{(k)}}{C_{33}^{(k)}}\right) A_{x r}-\left(\frac{C_{23}^{(k)}}{C_{33}^{(k)}}\right) A_{\theta r}\right) t_{k}} \\
& \nu_{r \theta}=-\frac{\epsilon_{\theta}^{0}}{\epsilon_{r}^{0}}=-\frac{A_{\theta r} t}{\sum_{k=1}^{N}\left(\frac{1}{C_{33}^{(k)}}-\left(\frac{C_{13}^{(k)}}{C_{33}^{(k)}}\right) A_{x r}-\left(\frac{C_{23}^{(k)}}{C_{33}^{(k)}}\right) A_{\theta r}\right) t_{k}} .
\end{aligned}
$$

\section{Determination Equivalent of $E_{x,} E_{\theta}, \nu_{x \theta}, \nu_{r x}$, and $\nu_{\theta r}$}

If $N_{x}$ and $N_{\theta}$ are reinterpreted in terms of an average stress $\sigma_{x}^{0}$ and $\sigma_{\theta}^{0}$ and only stresses in the $x$ or $\theta$ directions are applied, that is, $\sigma_{r}=0$, then Equation B.6 takes the form

$$
\begin{aligned}
& N_{x}=\sigma_{x}^{0} t=A_{11} \epsilon_{x}^{0}+A_{12} \epsilon_{\theta}^{0} \\
& N_{\theta}=\sigma_{\theta}^{0} t=A_{12} \epsilon_{x}^{0}+A_{22} \epsilon_{\theta}^{0} .
\end{aligned}
$$


With $\sigma_{x}^{0} \neq 0$ and $\sigma_{\theta}^{0}=0$, these expressions lead directly to the definition of Young's modulus in the $x$ direction and a Poisson's ratio. These definitions are

$$
\begin{gathered}
E_{x}=\frac{\sigma_{x}^{0}}{\epsilon_{x}^{0}}=\frac{A_{11} A_{22}-A_{12}^{2}}{A_{22} t} \\
\nu_{x \theta}=-\frac{\epsilon_{\theta}^{0}}{\epsilon_{x}^{0}}=\frac{A_{12}}{A_{22}} .
\end{gathered}
$$

Conversely, with $\sigma_{x}^{0}=0$ and $\sigma_{\theta}^{0} \neq 0$,

$$
\begin{gathered}
E_{\theta}=\frac{\sigma_{y}^{0}}{\epsilon_{\theta}^{0}}=\frac{A_{11} A_{22}-A_{12}^{2}}{A_{11} t} \\
\nu_{\theta x}=-\frac{\epsilon_{x}^{0}}{\epsilon_{\theta}^{0}}=\frac{A_{12}}{A_{11}} .
\end{gathered}
$$

For the case of $\sigma_{x}^{0} \neq 0$ and $\sigma_{\theta}^{0}=0$, the radial strain in the $k^{\text {th }}$ layer is given by Equation B.3 as

$$
\epsilon_{r}^{(k)}=\left[\frac{C_{23}^{(k)}}{C_{33}^{(k)}} \nu_{x \theta}-\frac{C_{13}^{(k)}}{C_{33}^{(k)}}\right] \epsilon_{x}^{0} .
$$

The total thickness change of the element, due to $\sigma_{x}^{0}$, is

$$
\Delta t=\sum_{k=1}^{N} \epsilon_{r}^{(k)} t_{k} .
$$

The average radial strain for this situation, $\epsilon_{r}^{0}$, is

$$
\epsilon_{r}^{0}=\frac{\Delta t}{t}=\frac{\sum_{k=1}^{N} \epsilon_{r}^{(k)} t^{k}}{t} .
$$

Then by definition

$$
\nu_{x r}=-\frac{\epsilon_{r}^{0}}{\epsilon_{x}^{0}}=-\frac{1}{t} \sum_{k=1}^{N}\left[\frac{C_{23}^{(k)}}{C_{33}^{(k)}} \nu_{x \theta}-\frac{C_{13}^{(k)}}{C_{33}^{(k)}}\right] t_{k} .
$$


For the case of $\sigma_{x}^{0}=0$ and $\sigma_{\theta}^{0} \neq 0, \nu_{\theta r}$ can be determined and is given by

$$
\nu_{\theta r}=-\frac{\epsilon_{r}^{0}}{\epsilon_{\theta}^{0}}=-\frac{1}{t} \sum_{k=1}^{N}\left(\frac{C_{13}^{(k)}}{C_{33}^{(k)}} \nu_{\theta x}-\frac{C_{23}^{(k)}}{C_{33}^{(k)}}\right) t_{k} .
$$

\section{Determination of Equivalent $G_{r x}, G_{r \theta}, G_{x \theta}$}

To compute the equivalent shear modulus $G_{x r}$, consider the cube in Figure B. 1 with shear stresses applied. This is shown in Figure B.3, the shear stresses $\tau_{\theta z}$ and $\tau_{x z}$ being applied at the outermost layers and on the faces through the thickness of the element. Because each layer is in equilibrium, the shear stresses in the $k^{\text {th }}$ layer are

$$
\begin{aligned}
& \tau_{\theta z}^{(k)}=\tau_{\theta z} \\
& \tau_{x z}^{(k)}=\tau_{x z} .
\end{aligned}
$$
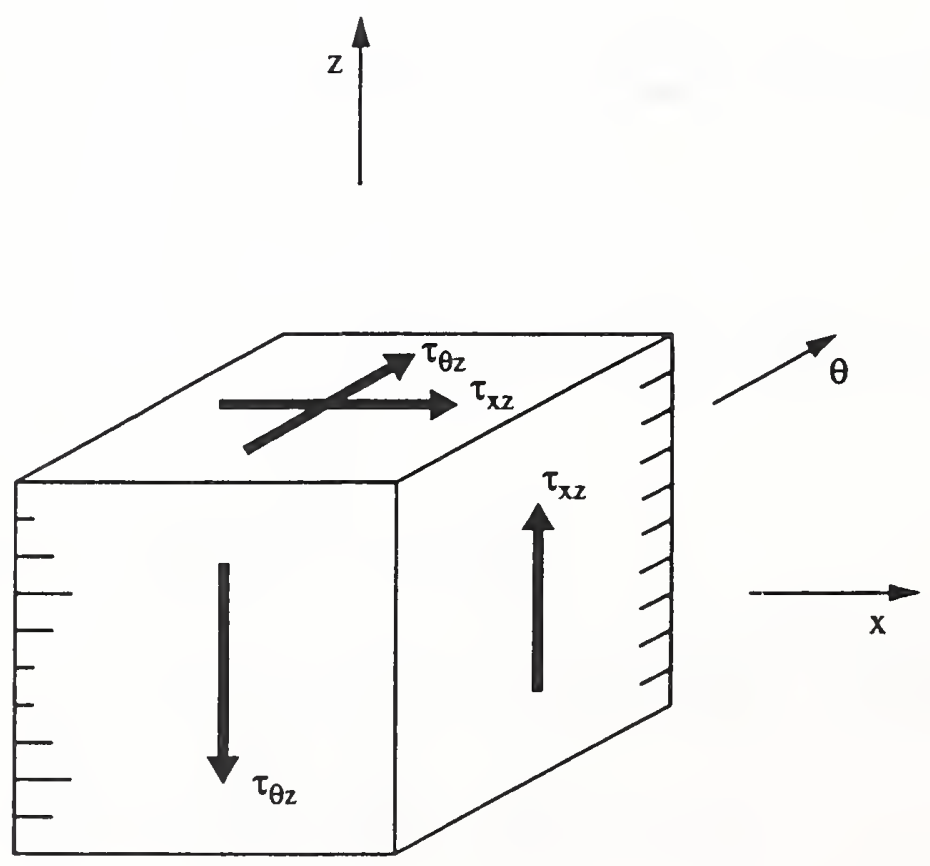

Figure B.3. Element subject to shear stresses. 
The strain-stress relation for the $k^{\text {th }}$ layer is

$$
\begin{aligned}
& \gamma_{\theta z}^{(k)}=S_{44}^{(k)} \tau_{\theta z}^{(k)}+S_{45}^{(k)} \tau_{x z}^{(k)} \\
& \gamma_{x z}^{(k)}=S_{45}^{(k)} \tau_{\theta z}^{(k)}+S_{55}^{(k)} \tau_{x z}^{(k)},
\end{aligned}
$$

where $S_{44}^{(k)}, S_{45}^{(k)}$, and $S_{55}^{(k)}$ are the transformed compliances for the $k^{\text {th }}$ layer and $\gamma_{\theta z}^{(k)}$ and $\gamma_{k z}^{(k)}$ are the shear strains in the $k^{\text {th }}$ layer. Examining the shear deformations of the $k^{\text {th }}$ layer, from geometry

$$
\Delta_{x}^{(k)}=\gamma_{x z}^{(k)} t_{k}
$$

where, as observed in exaggerated form in Figure B.4, $\Delta_{x}^{(k)}$ is the displacement in the $x$ direction of the upper surface of the $k^{\text {th }}$ layer relative to the bottom surface. With a similar definition in the $y$ direction,

$$
\Delta_{\theta}^{(k)}=\gamma_{\theta z}^{(k)} t_{k}
$$

With several layers stacked upon one another, as in Figure B.5, the displacement in the $x$ direction of the top surface of the cubic element in Figure B. 3 relative to the bottom surface is

$$
\Delta_{x}=\sum_{k=1}^{N} \Delta_{x}^{(k)}=\sum_{k=1}^{N} \gamma_{x z}^{(k)} t_{k} .
$$

Likewise,

$$
\Delta_{\theta}=\sum_{k=1}^{N} \Delta_{\theta}^{(k)}=\sum_{k=1}^{N} \gamma_{\theta z}^{(k)} t_{k}
$$

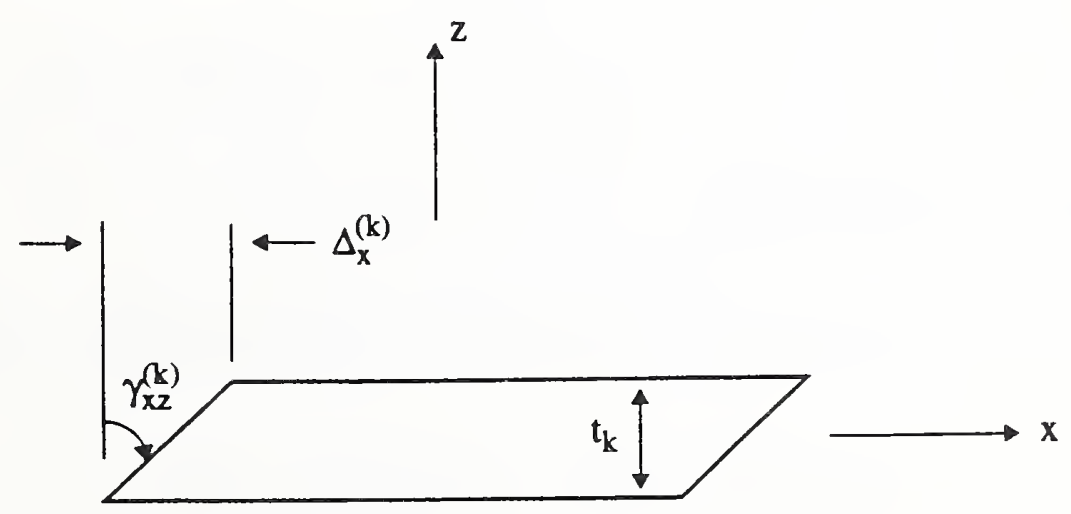

Figure B.4. Deformation of $k^{\text {th }}$ layer due to $\tau_{x z}$. 


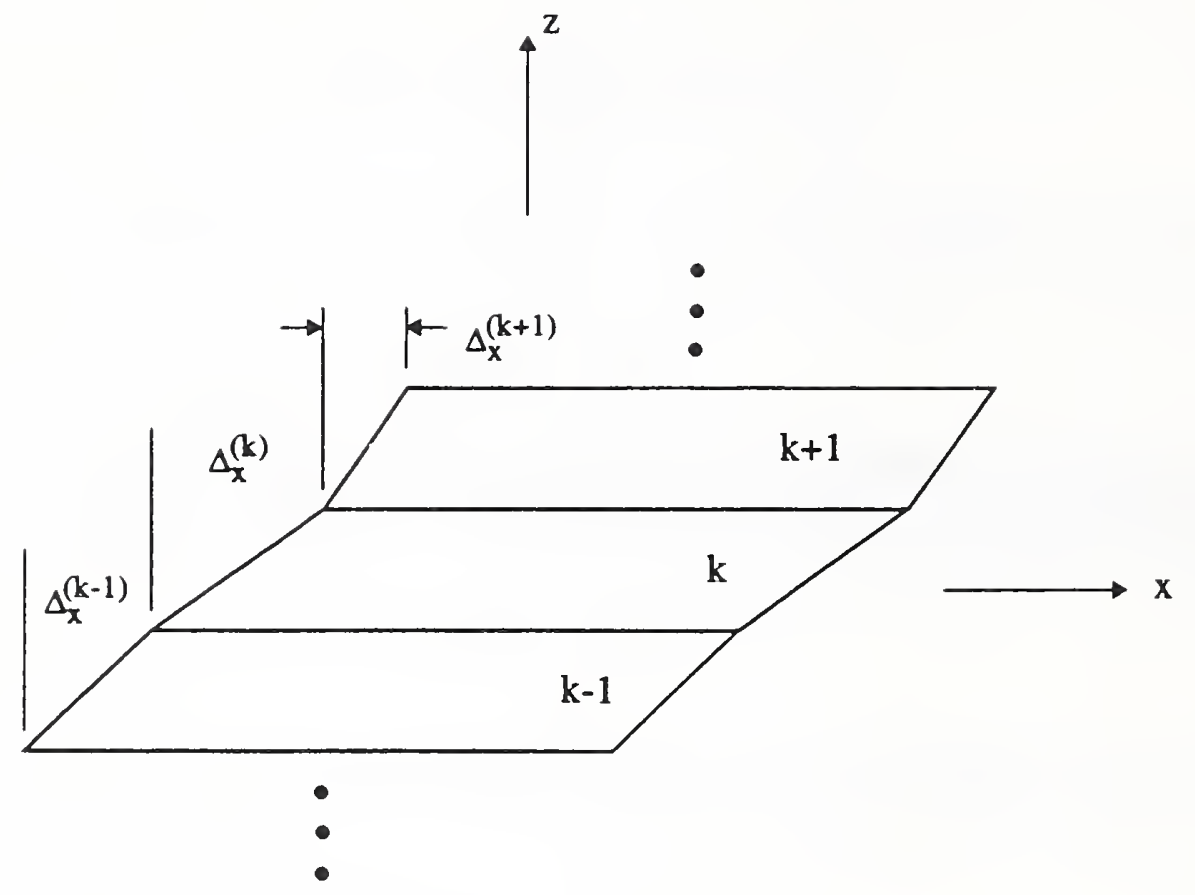

Figure B.5. Deformations of several layers due to $\tau_{x z}$.

The overall shear strains in the cube are defined to be

$$
\gamma_{x z}^{0}=\frac{\Delta_{x}}{t} \quad \text { and } \quad \gamma_{\theta z}^{0}=\frac{\Delta_{\theta}}{t}
$$

or

$$
\begin{aligned}
& \gamma_{\theta z}^{0}=\frac{1}{t} \sum_{k=1}^{N} \gamma_{\theta z}^{(k)} t_{k} \\
& \gamma_{x z}^{0}=\frac{1}{t} \sum_{k=1}^{N} \gamma_{x z}^{(k)} t_{k} .
\end{aligned}
$$

Using Equation B.24 in Equation B.30,

$$
\begin{aligned}
& \gamma_{\theta z}^{0}=\frac{1}{t}\left[\sum_{k=1}^{N} S_{44}^{(k)} t_{k} \tau_{\theta z}^{(k)}+\sum_{k=1}^{N} S_{45}^{(k)} t_{k} \tau_{x z}^{(k)}\right) \\
& \gamma_{x z}^{0}=\frac{1}{t}\left(\sum_{k=1}^{N} S_{45}^{(k)} t_{k} \tau_{\theta z}^{(k)}+\sum_{k=1}^{N} S_{55}^{(k)} t_{k} \tau_{x z}^{(k)}\right) .
\end{aligned}
$$


By virtue of Equation B.23

$$
\begin{aligned}
& \gamma_{\theta z}^{0}=\left(\frac{1}{t} \sum_{k=1}^{N} S_{44}^{(k)} t_{k}\right) \tau_{\theta z}+\left(\frac{1}{t} \sum_{k=1}^{N} S_{45}^{(k)} t_{k}\right) \tau_{x z} \\
& \gamma_{x z}^{0}=\left(\frac{1}{t} \sum_{k=1}^{N} S_{45}^{(k)} t_{k}\right) \tau_{\theta z}+\left(\frac{1}{t} \sum_{k=1}^{N} S_{55}^{(k)} t_{k}\right) \tau_{x z} .
\end{aligned}
$$

But for a balanced laminate,

$$
\sum_{k=1}^{N} S_{45}^{(k)} t_{k}=0
$$

so

$$
\gamma_{\theta z}^{0}=\bar{S}_{44} \tau_{\theta z} \quad \text { and } \quad \gamma_{x z}^{0}=\bar{S}_{55} \tau_{x z}
$$

with the smeared compliances $\bar{S}_{44}$ and $\bar{S}_{55}$ defined as

$$
\bar{S}_{44}=\frac{1}{t} \sum_{k=1}^{N} S_{44}^{(k)} t_{k} \quad \text { and } \quad \bar{S}_{55}=\frac{1}{t} \sum_{k=1}^{N} S_{55}^{(k)} t_{k} .
$$

Inverting these relations, the smeared shear moduli are

$$
G_{z \theta}=\frac{1}{S_{44}} \text { and } G_{z x}=\frac{1}{S_{55}} .
$$

\section{Determination of Equivalent $\boldsymbol{G}_{\boldsymbol{x} \theta}$}

For a layer subjected to just a shear stress $\tau_{x \theta}$, the stress-strain relation is

$$
\tau_{x \theta}=C_{16} \epsilon_{x}+C_{26} \epsilon_{\theta}+C_{36} \epsilon_{r}+C_{66} \gamma_{x \theta} .
$$

When the layer is part of the cubic element, the sides of the element remaining straight, the stress-strain relation for a layer becomes

$$
\tau_{x \theta}=C_{16} \epsilon_{x}^{0}+C_{26} \epsilon_{\theta}^{0}+C_{36} \epsilon_{r}+C_{66} \gamma_{x \theta}^{0}
$$


For an in-plane shear loading of the cube of Figure B.1, it is safe to say as a good approximation

$$
\sigma_{r}=0 \text {. }
$$

Using this in the $3^{\text {rd }}$ of Equation B.2, solving for $\epsilon_{r}$, and substituting $\epsilon_{r}$ into Equation B.38 results in

$$
\begin{aligned}
\tau_{x \theta}= & {\left[C_{16}-\frac{C_{13} C_{36}}{C_{33}}\right] \epsilon_{x}^{0}+\left(C_{26}-\frac{C_{23} C_{36}}{C_{33}}\right) \epsilon_{\theta}^{0} } \\
& +\left(C_{66}-\frac{C_{36} C_{36}}{C_{33}}\right) \gamma_{x \theta}^{0} .
\end{aligned}
$$

Rewriting

$$
\tau_{x \theta}=\bar{Q}_{16} \epsilon_{x}^{o}+\bar{Q}_{26} \epsilon_{\theta}^{o}+\bar{Q}_{66} \gamma_{x \theta}^{o}
$$

The $\bar{Q}_{\mathrm{S}}$ being the redefined, namely reduced, stiffnesses. Integrating through the thickness, the average shear stress is given by

$$
\tau_{x \theta}^{0}=\frac{1}{t} \int_{-\frac{t}{2}}^{\frac{1}{2}} \tau_{x \theta} d z
$$

From Equation B.41

$$
\tau_{x \theta}^{0}=\frac{1}{t} \int_{-\frac{t}{2}}^{\frac{1}{2}}\left(\bar{Q}_{16} \epsilon_{x}^{0}+\bar{Q}_{26} \epsilon_{\theta}^{0}+\bar{Q}_{66} \gamma_{x \theta}^{0}\right) d z
$$

or

$$
\begin{aligned}
\tau_{x \theta}^{o}= & \left(\frac{1}{t} \int_{-\frac{t}{2}}^{\frac{t}{2}} \bar{Q}_{16} d z\right) \epsilon_{x}^{o}+\left(\frac{1}{t} \int_{-\frac{t}{2}}^{\frac{t}{2}} \bar{Q}_{26} d z\right) \epsilon_{\theta}^{o} \\
& +\left(\frac{1}{t} \int_{-\frac{1}{2}}^{\frac{t}{2}} \bar{Q}_{66} d z\right) \gamma_{x y}^{o}
\end{aligned}
$$

For balanced laminate, the first two integrals are zero, resulting in

$$
\tau_{x \theta}^{0}=\left\{\frac{1}{t} \int_{-\frac{t}{2}}^{\frac{t}{2}} \bar{Q}_{66} d z\right\} \gamma_{x \theta}^{0} .
$$




$$
\tau_{x \theta}^{0}=\left\{\frac{1}{t} \int_{-\frac{t}{2}}^{\frac{t}{2}} \bar{Q}_{66} d z\right\} \gamma_{x \theta}^{0}
$$

Thus, the equivalent in-plane shear modulus is

$$
G_{x \theta}=\frac{A_{66}}{t},
$$

where

$$
A_{66}=\int_{-\frac{t}{2}}^{\frac{t}{2}} \bar{Q}_{66} d z
$$

\section{Determination of Equivalent Coefficients of Thermal Expansion $\alpha_{x}^{0}$ and $\alpha_{\theta}^{0}$}

For a layer that is part of the cube of Figure B.1, if thermal expansion effects are important, the stress-strain relation for a layer, for example, B.2, becomes

$$
\begin{aligned}
\sigma_{x}= & C_{11}\left(\epsilon_{x}^{0}-\alpha_{x} \Delta T\right)+C_{12}\left(\epsilon_{\theta}^{0}-\alpha_{\theta} \Delta T\right) \\
& +C_{13}\left(\epsilon_{r}-\alpha_{r} \Delta T\right)+C_{16}\left(\gamma_{x \theta}^{0}-\alpha_{x \theta} \Delta T\right) \\
\sigma_{\theta}= & C_{12}\left(\epsilon_{x}^{0}-\alpha_{x} \Delta T\right)+C_{22}\left(\epsilon_{\theta}-\alpha_{\theta} \Delta T\right) \\
& +C_{23}\left(\epsilon_{r}-\alpha_{r} \Delta T\right)+C_{26}\left(\gamma_{x \theta}^{0}-\alpha_{x \theta} \Delta T\right) \\
\sigma_{r}= & C_{13}\left(\epsilon_{x}^{0}-\alpha_{x} \Delta T\right)+C_{23}\left(\epsilon_{\theta}^{0}-\alpha_{\theta} \Delta T\right) \\
& +C_{33}\left(\epsilon_{r}-\alpha_{r} \Delta T\right)+C_{36}\left(\gamma_{x \theta}^{0}-\alpha_{x \theta} \Delta T\right),
\end{aligned}
$$

where the quantities $\alpha_{x}, \alpha_{\theta}, \alpha_{r}$, and $\alpha_{x \theta}$ are the transformed coefficients of thermal expansion for the layer. (Alternatively, the $\alpha \Delta T$ s can be interpreted as dilatation due to thermal effects.) For a temperature change within the cube, though $\sigma_{x}$ and $\sigma_{\theta}$ will not be zero, $\sigma_{r}$ can be approximated as zero. Thus, using the third of Equation B.48 in the first two and using the definitions of the $Q_{\mathrm{s}}$ previously introduced, Equation B. 48 becomes

$$
\begin{aligned}
& \sigma_{x}=\bar{Q}_{11}\left(\epsilon_{x}^{0}-\alpha_{x} \Delta T\right)+\bar{Q}_{12}\left(\epsilon_{\theta}^{0}-\alpha_{\theta} \Delta T\right)+\bar{Q}_{16}\left(\gamma_{x \theta}^{0}-\alpha_{x \theta} \Delta T\right) \\
& \sigma_{\theta}=\bar{Q}_{12}\left(\epsilon_{x}^{0}-\alpha_{x} \Delta T\right)+\bar{Q}_{22}\left(\epsilon_{\theta}^{0}-\alpha_{\theta} \Delta T\right)+\bar{Q}_{26}\left(\gamma_{x \theta}^{0}-\alpha_{x \theta} \Delta T\right) .
\end{aligned}
$$


As in Equation B.6, integrating through the thickness and making use of the fact that the laminate is balanced

$$
\begin{aligned}
& N_{x}=A_{11} \epsilon_{x}^{0}+A_{12} \epsilon_{\theta}^{0}-N_{x}^{T} \Delta T \\
& N_{\theta}=A_{12} \epsilon_{x}^{0}+A_{22} \epsilon_{\theta}^{0}-N_{\theta}^{T} \Delta T
\end{aligned}
$$

where

$$
\begin{aligned}
& N_{x}^{T}=\int_{-\frac{t}{2}}^{\frac{t}{2}}\left(\bar{Q}_{11} \alpha_{x}+\bar{Q}_{12} \alpha_{\theta}+\bar{Q}_{16} \alpha_{x \theta}\right) d z \\
& N_{\theta}^{T}=\int_{-\frac{t}{2}}^{\frac{t}{2}}\left(\bar{Q}_{12} \alpha_{x}+\bar{Q}_{22} \alpha_{\theta}+\bar{Q}_{26} \alpha_{x \theta}\right) d z .
\end{aligned}
$$

These quantities are strictly material properties but are defined as equivalent unit thermal stress resultants. For a particular temperature change and no applied loads $\left(N_{x}=N_{\theta}=0\right)$, the laminate coefficients of thermal expansion in the $x$ and $\theta$ directions, $\alpha_{x}^{0}$ and $\alpha_{y}^{0}$, are defined as

$$
\begin{aligned}
& \alpha_{x}^{0}=\epsilon_{x}^{0} / \Delta T \\
& \alpha_{\theta}^{0}=\epsilon_{\theta}^{0} / \Delta T,
\end{aligned}
$$

or

$$
\begin{aligned}
& \alpha_{x}^{0}=\left(A_{22} N_{x}^{T}-A_{12} N_{\theta}^{T}\right) /\left(A_{11} A_{22}-A_{12}^{2}\right) \\
& \alpha_{\theta}^{0}=\left(A_{11} N_{\theta}^{T}-A_{12} N_{x}^{T}\right) /\left(A_{11} A_{22}-A_{12}^{2}\right) .
\end{aligned}
$$

\section{Determination of Equivalent Through-the-thickness Coefficient of Thermal Expansion $\alpha_{z}^{0}$}

To determine the equivalent through-the-thickness thermal-expansion coefficient, consider again the cube of Figure B.1 subjected to a temperature change. Using the fact that only balanced laminates are being considered, that for a temperature change $\sigma_{r}$ can be approximated as zero, and Equation B.52, the third of Equation B.48 yields, for a layer,

$$
\epsilon_{r}=\left[\alpha_{r}+\frac{C_{36}}{C_{33}} \alpha_{x \theta}-\frac{C_{13}}{C_{33}}\left(\alpha_{x}^{0}-\alpha_{x}\right)-\frac{C_{23}}{C_{33}}\left(\alpha_{\theta}^{0}-\alpha_{\theta}\right)\right] \Delta T .
$$

Assuming $\epsilon_{r}$ is constant within a given layer, the change in thickness of the $k^{\text {th }}$ layer, due to temperature change $\Delta T$, is 


$$
\Delta t^{(k)}=\epsilon_{r}^{(k)} t^{(k)}
$$

where it is clear the thickness strain is a function of the particular layer, that is,

$$
\begin{aligned}
\epsilon_{r}^{(k)}= & {\left[\alpha_{r}^{(k)}+\frac{C_{36}^{(k)}}{C_{33}^{(k)}} \alpha_{x \theta}^{(k)}-\frac{C_{13}^{(k)}}{C_{33}(k)}\left(\alpha_{x}^{0}-\alpha_{x}^{(k)}\right)\right.} \\
& \left.-\frac{C_{23}^{(k)}}{C_{33}^{(k)}}\left(\alpha_{\theta}^{0}-\alpha_{\theta}^{(k)}\right)\right] \Delta T .
\end{aligned}
$$

The total change in thickness of all layers is

$$
\Delta t=\sum_{k=1}^{N} \Delta t^{(k)}=\sum_{k=1}^{N} \epsilon_{r}^{(k)} t^{(k)} .
$$

Defining the through the thickness coefficient of thermal expansion $\alpha_{z}^{0}$ to be

$$
\alpha_{z}^{0}=\frac{\Delta t}{t} / \Delta T,
$$

then

$$
\begin{aligned}
\alpha_{z}^{0}= & \frac{1}{t} \sum_{k=1}^{N}\left\{\alpha_{r}^{(k)}+\frac{C_{36}^{(k)}}{C_{33}^{(k)}} \alpha_{x \theta}^{(k)}-\frac{C_{13}^{(k)}}{C_{33}^{(k)}}\left(\alpha_{x}^{0}-\alpha_{x}^{(k)}\right)\right. \\
& \left.-\frac{C_{23}^{(k)}}{C_{33}^{(k)}}\left(\alpha_{\theta}^{0}-\alpha_{\theta}^{(k)}\right)\right\} t^{(k)}
\end{aligned}
$$

With the material properties of each layer defined in the $x-\theta-r$ coordinate system, and knowing the thickness of each layer, the equivalent single thick-layer properties can be computed using the above approach. 


\section{Appendix C. Strain Response for Thinner Laminates}

This appendix contains the strains as a function of axial distance from the end of the cylinder for the thinner laminate. The figure numbers for the particular temperature, load condition, and laminate coincide with the corresponding-figure numbers in the main text, except for being preceded by a 'C.'
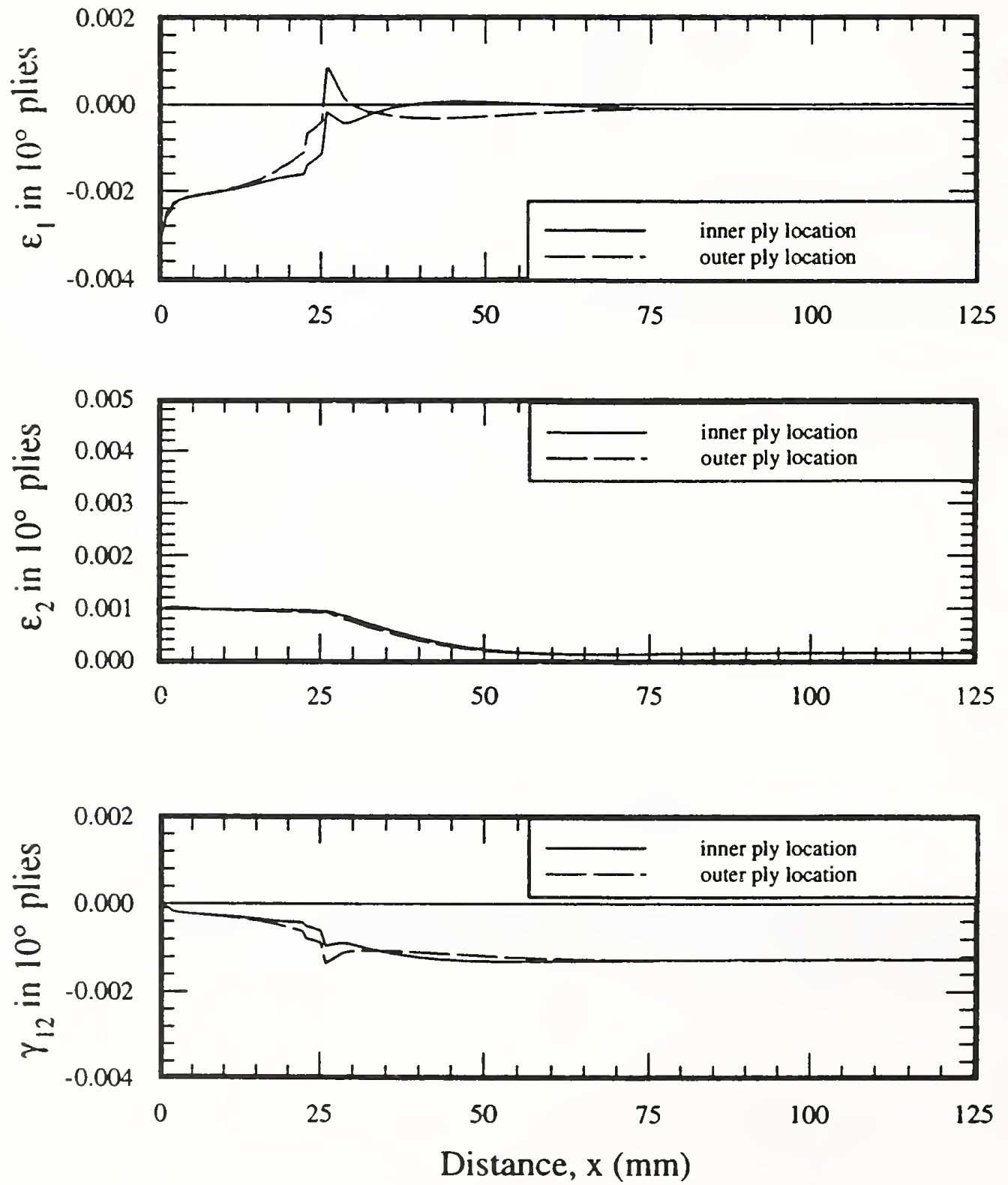

Figure C.4. Intralaminar strains due to a temperature change of $-294 \mathrm{~K}$ in thin [10] cylinder with a baseline-design end fitting. 

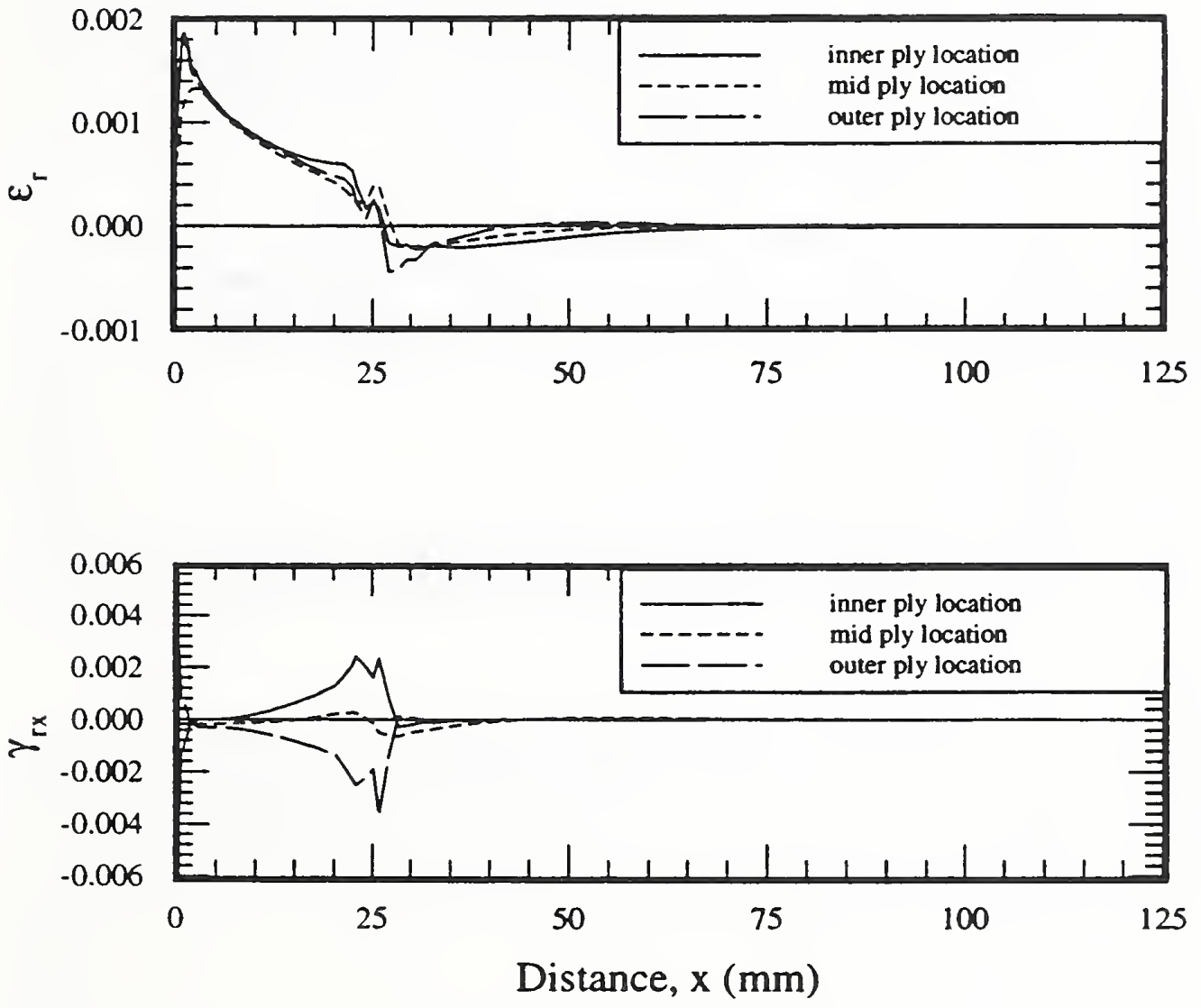

Figure C.5. Interlaminar strains due to a temperature change of $-294 \mathrm{~K}$ in thin [10] cylinder with baseline-design end fitting. 

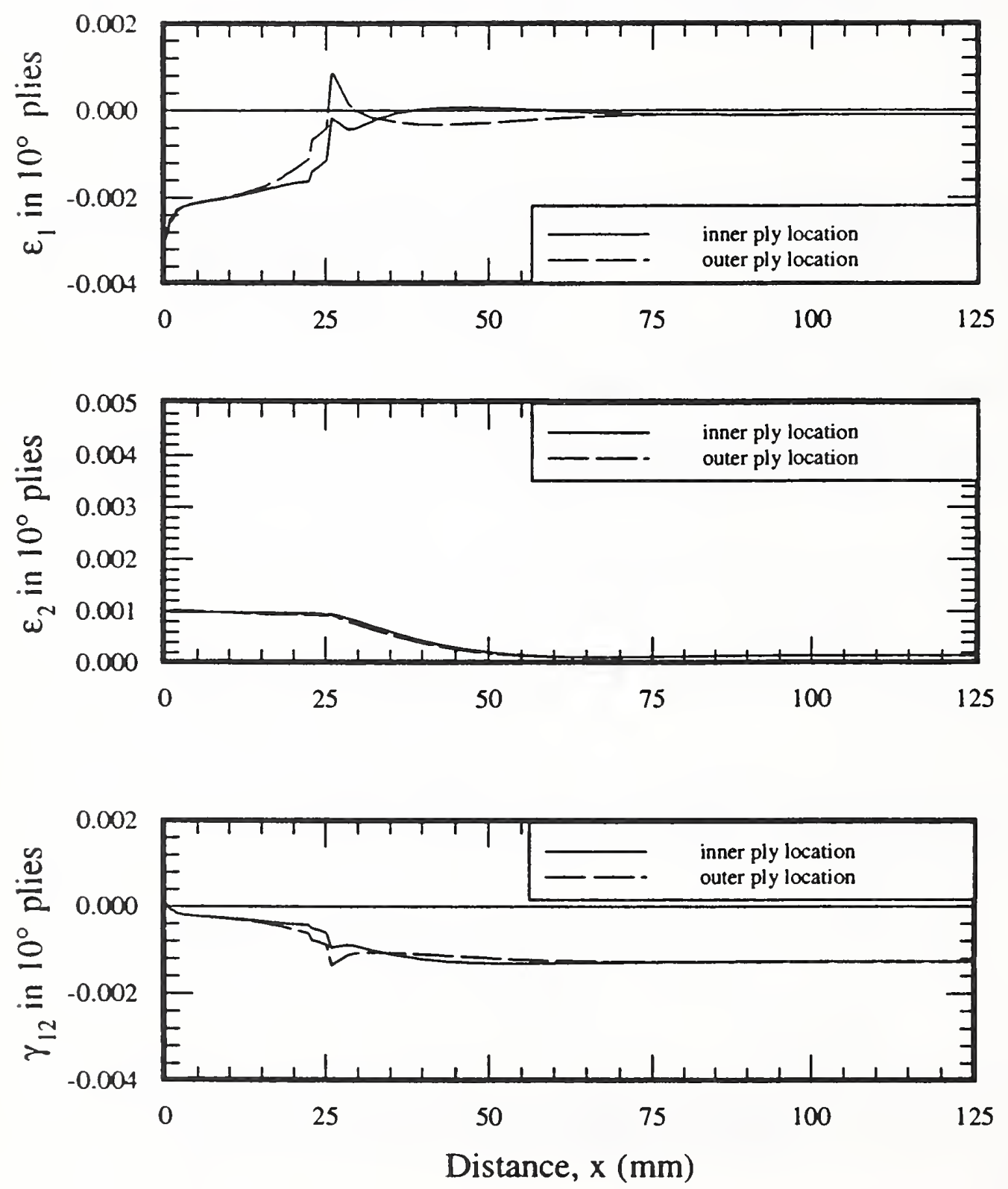

Figure C.7. Intralaminar strains due to a temperature change of $-294 \mathrm{~K}$ in thin [10] cylinder with alternative-design end fitting. 

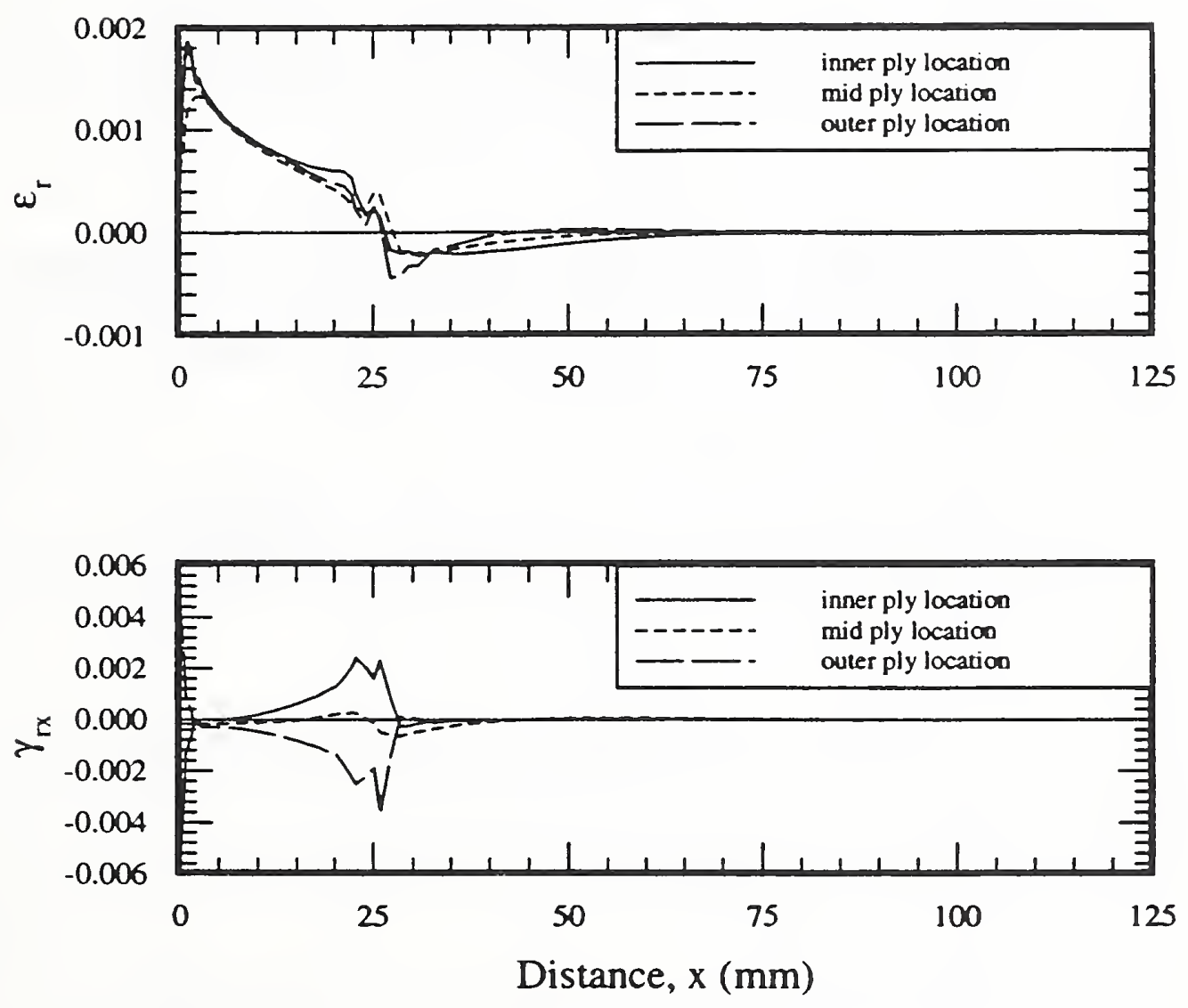

Figure C.8. Interlaminar strains due to a temperature change of $-294 \mathrm{~K}$ in thin [10] cylinder with alternative-design end fitting. 

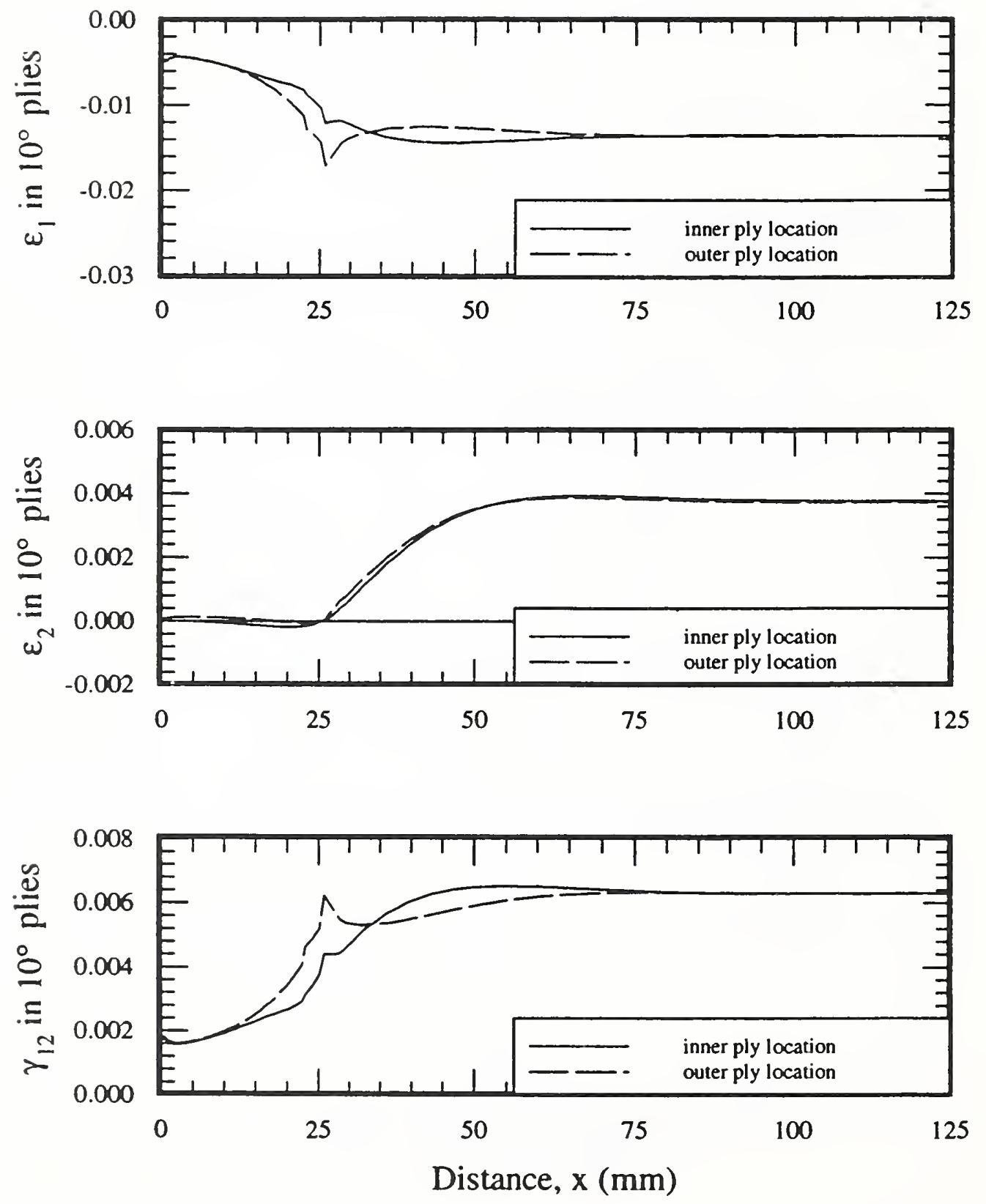

Figure C.10. Intralaminar strains due to applied axial stress of $-689 \mathrm{MPa}$ in thin [10] cylinder with baseline-design end fitting, 4-K properties. 

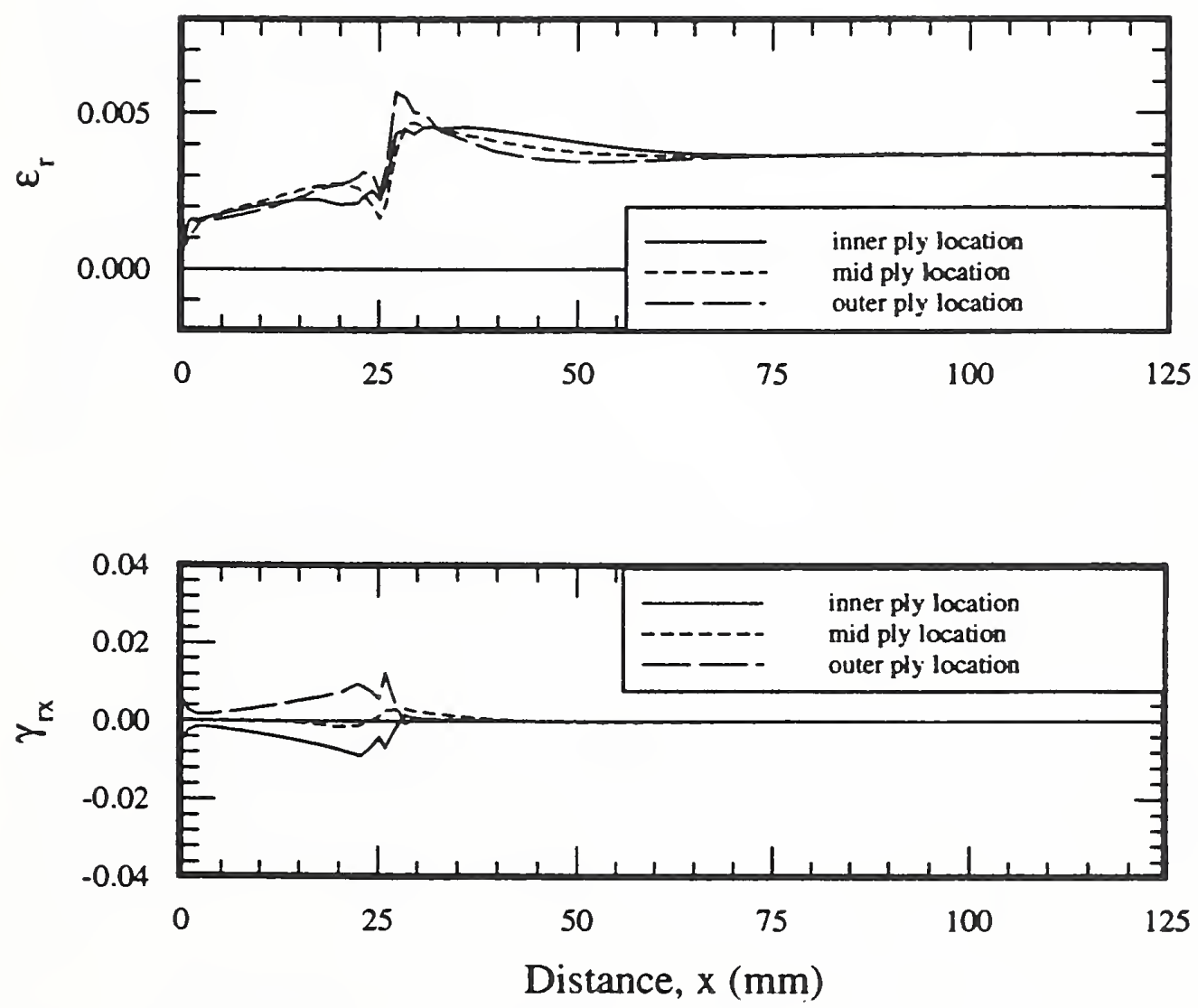

Figure C.11. Interlaminar strains due to applied axial stress of $-689 \mathrm{MPa}$ in thin [10] cylinder with baseline-design end fitting, 4-K properties. 

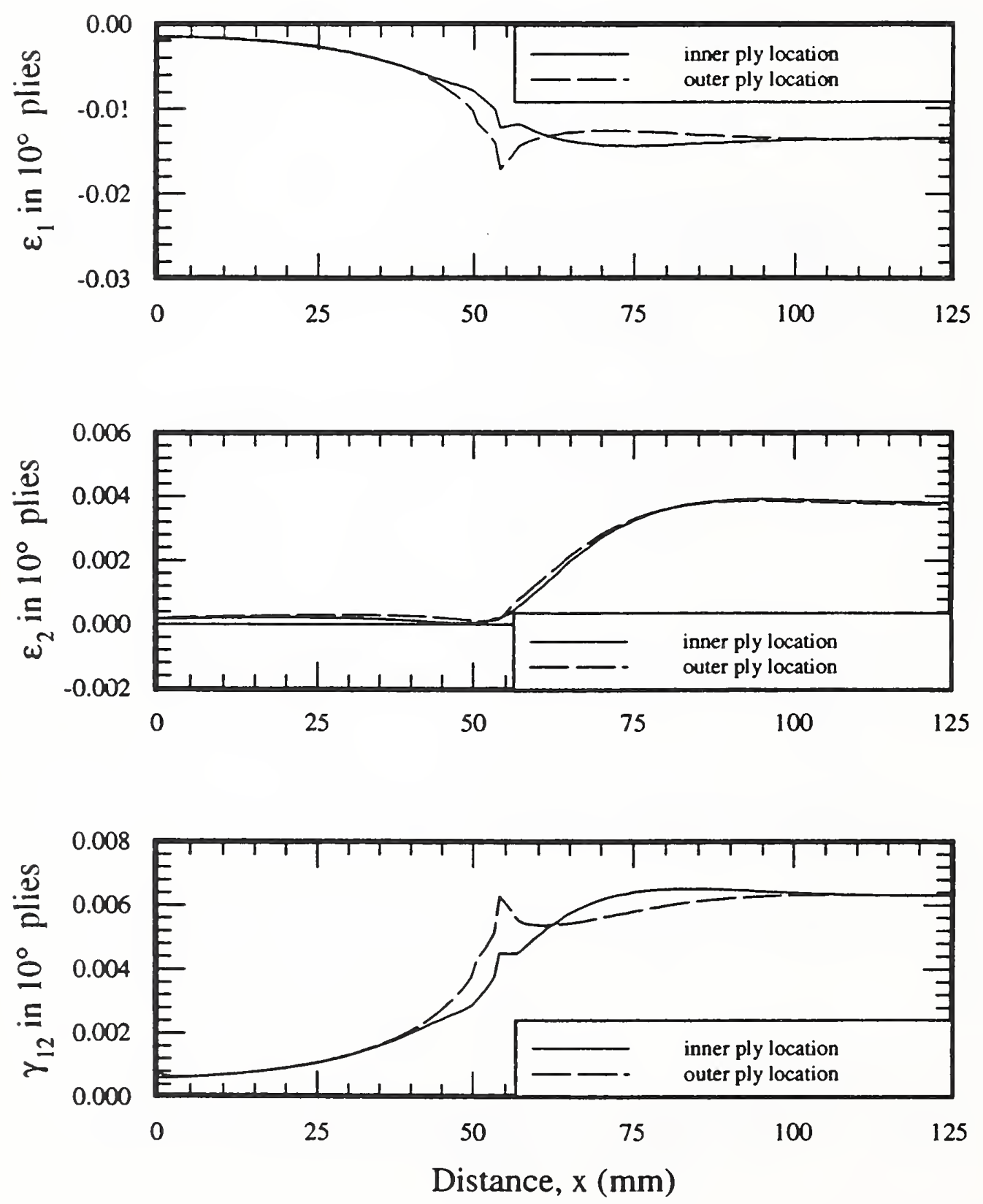

Figure C.13. Intralaminar strains due to applied axial stress of $-689 \mathrm{MPa}$ in thin [10] cylinder with alternative-design end fitting, 4-K properties. 

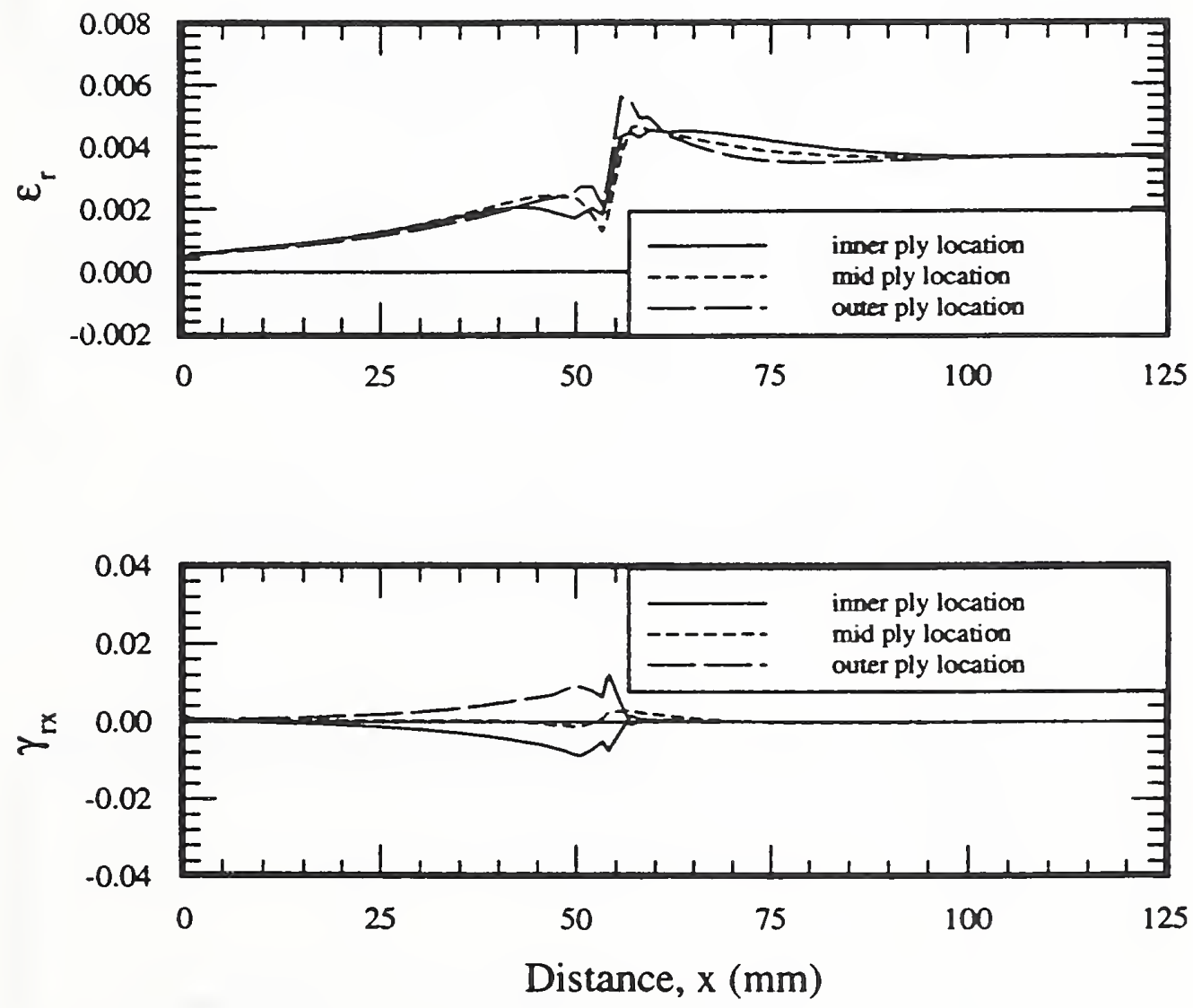

Figure C.14. Interlaminar strains due to applied axial stress of $-689 \mathrm{MPa}$ in thin [10] cylinder with alternative-design end fitting, 4-K properties. 

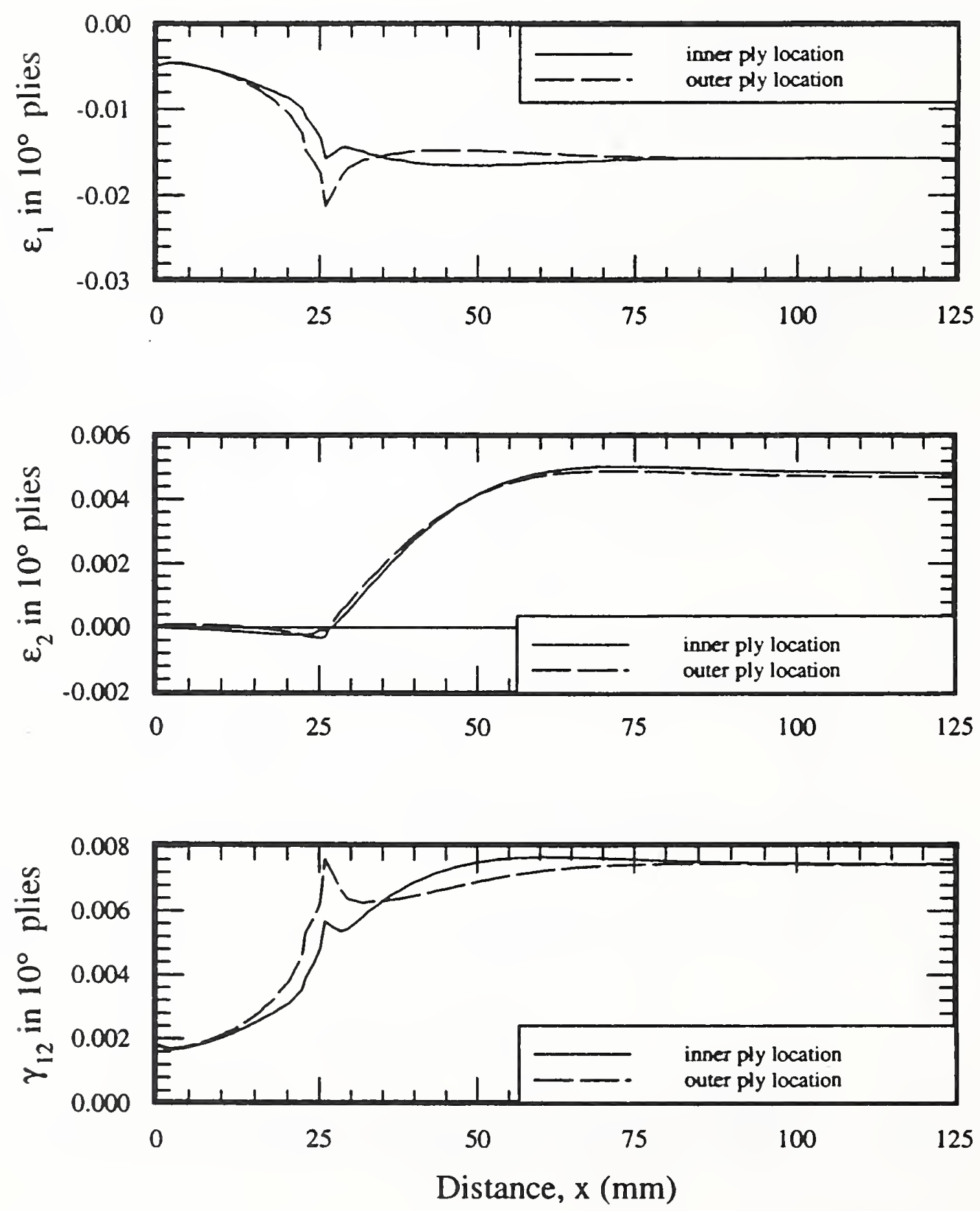

Figure C.15. Intralaminar strains due to applied axial stress of $-689 \mathrm{MPa}$ in thin [10] cylinder with baseline-design end fitting, 297-K properties. 

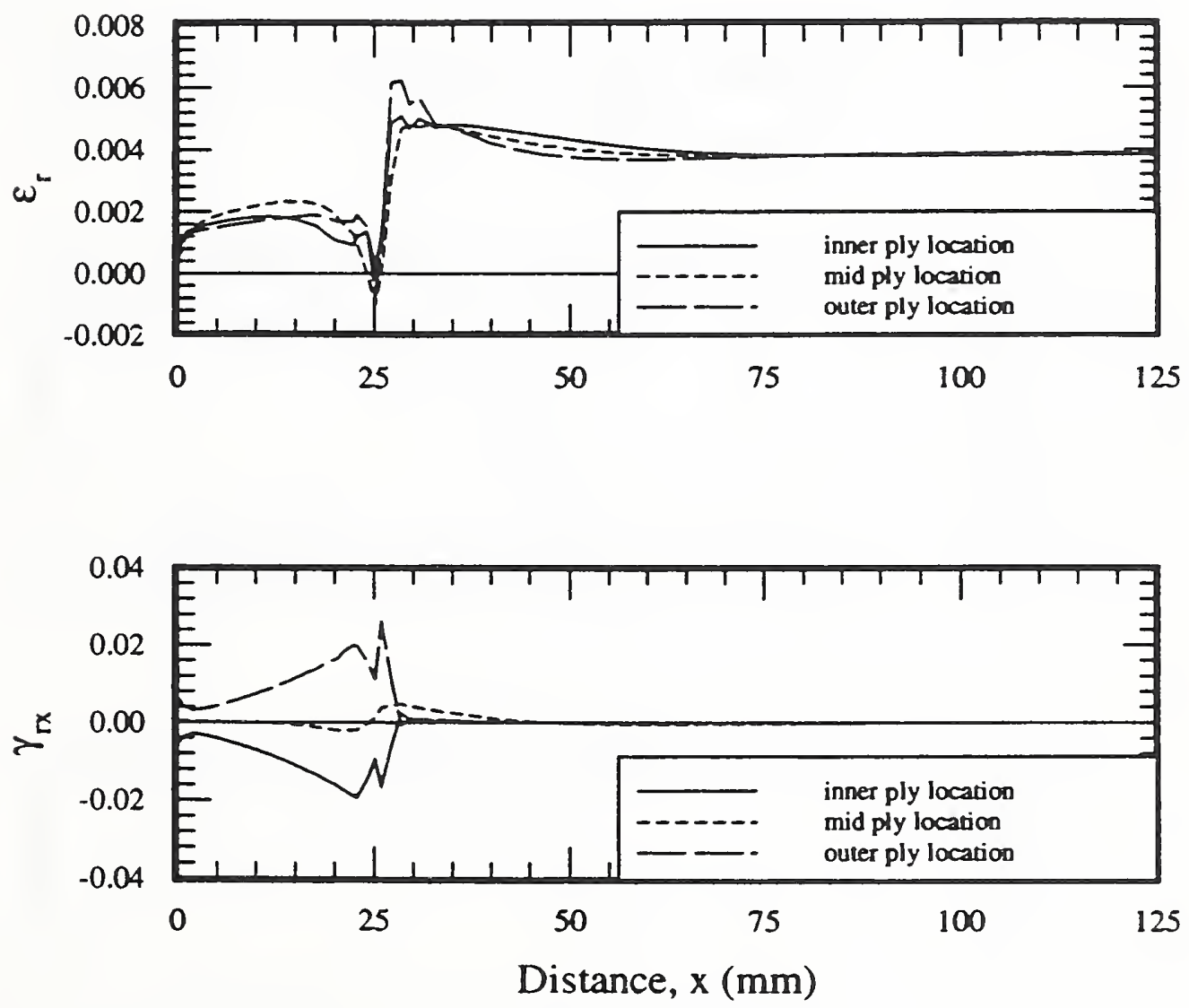

Figure C.16. Interlaminar strains due to applied axial stress of $-689 \mathrm{MPa}$ in thin [10] cylinder with baseline-design end fitting, 297-K properties. 

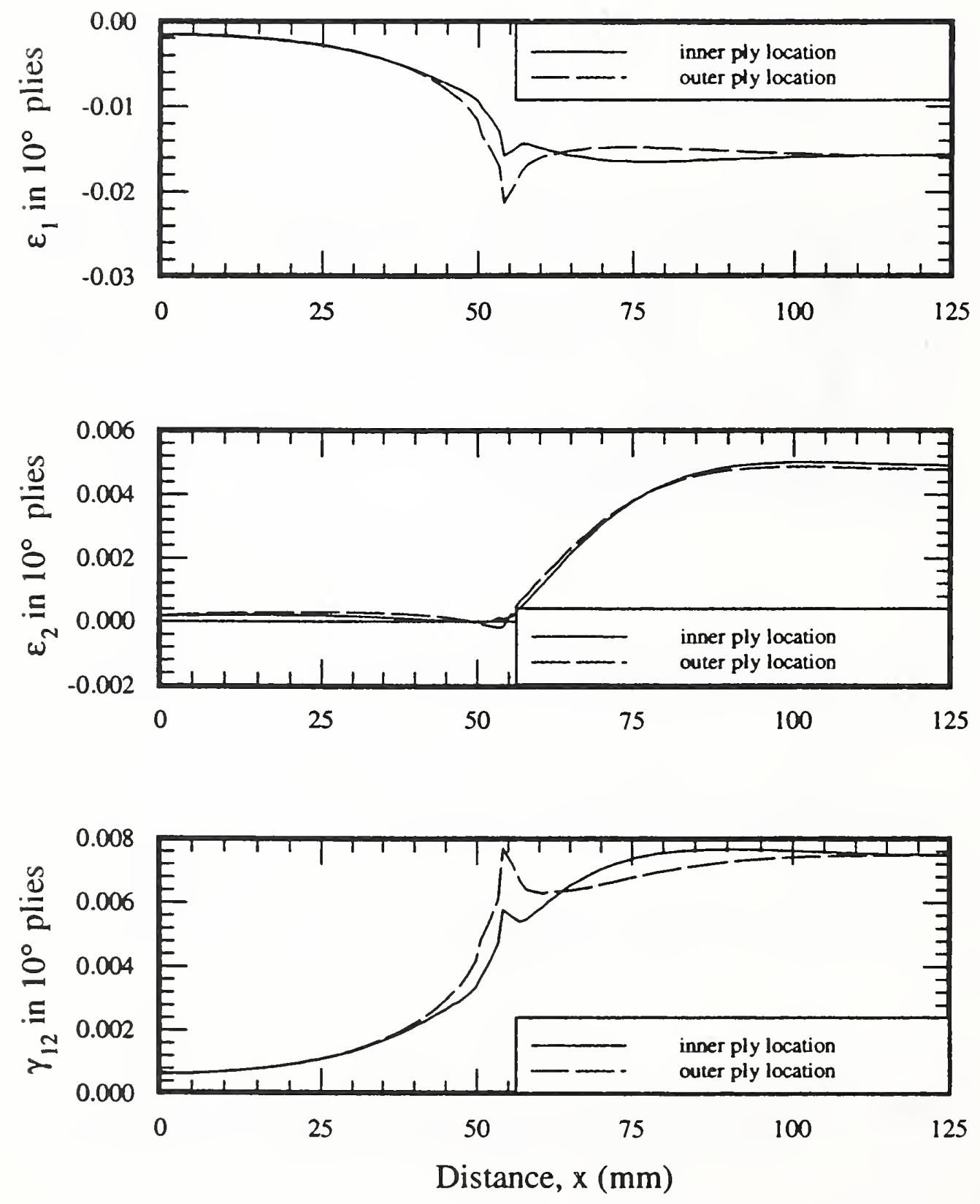

Figure C.17. Intralaminar strains due to applied axial stress of $-689 \mathrm{MPa}$ in thin [10] cylinder with alternative-design end fitting, 297-K properties. 

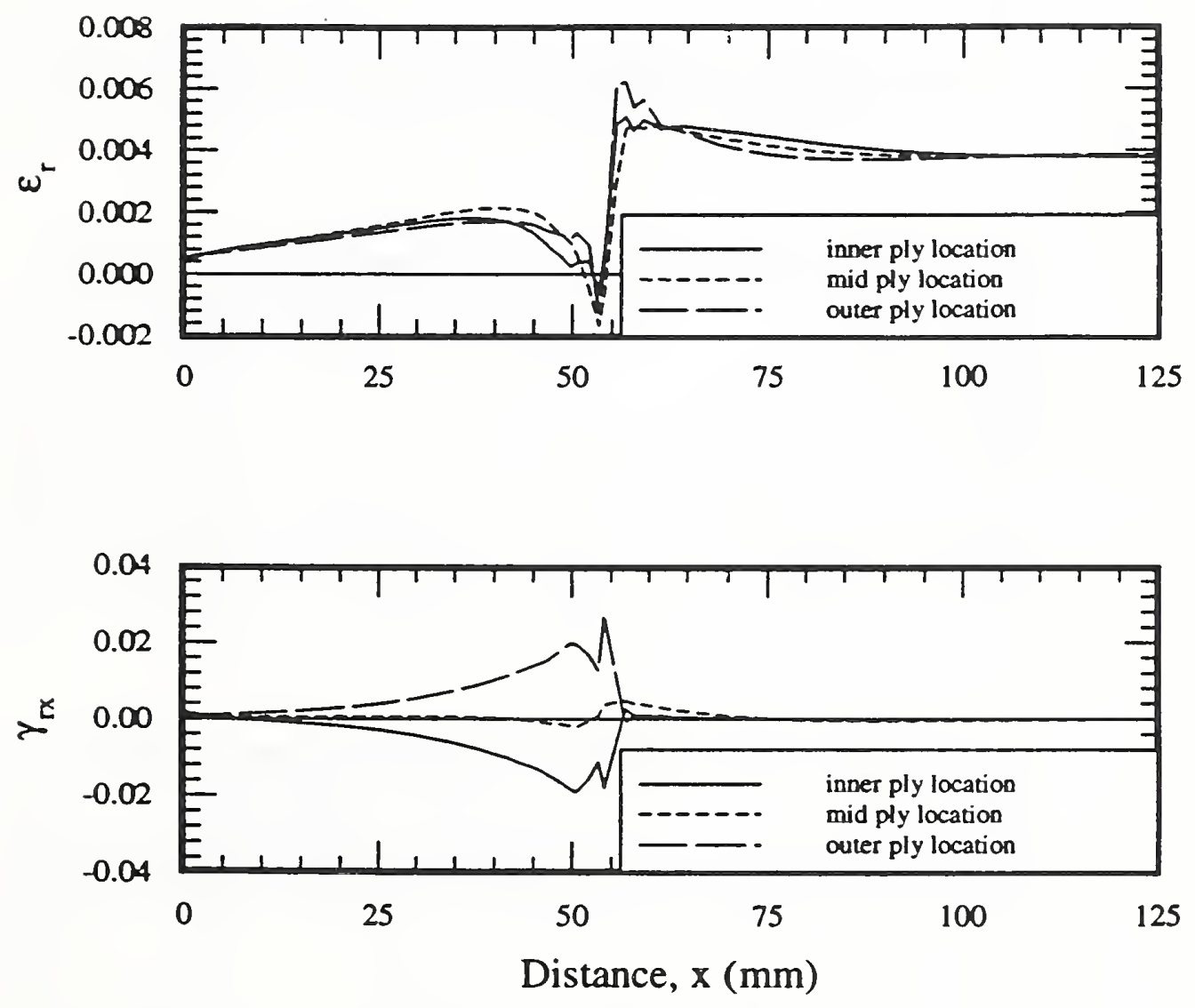

Figure C.18. Interlaminar strains due to applied axial stress of $-689 \mathrm{MPa}$ in thin [10] cylinder with alternative-design end fitting, 297-K properties. 

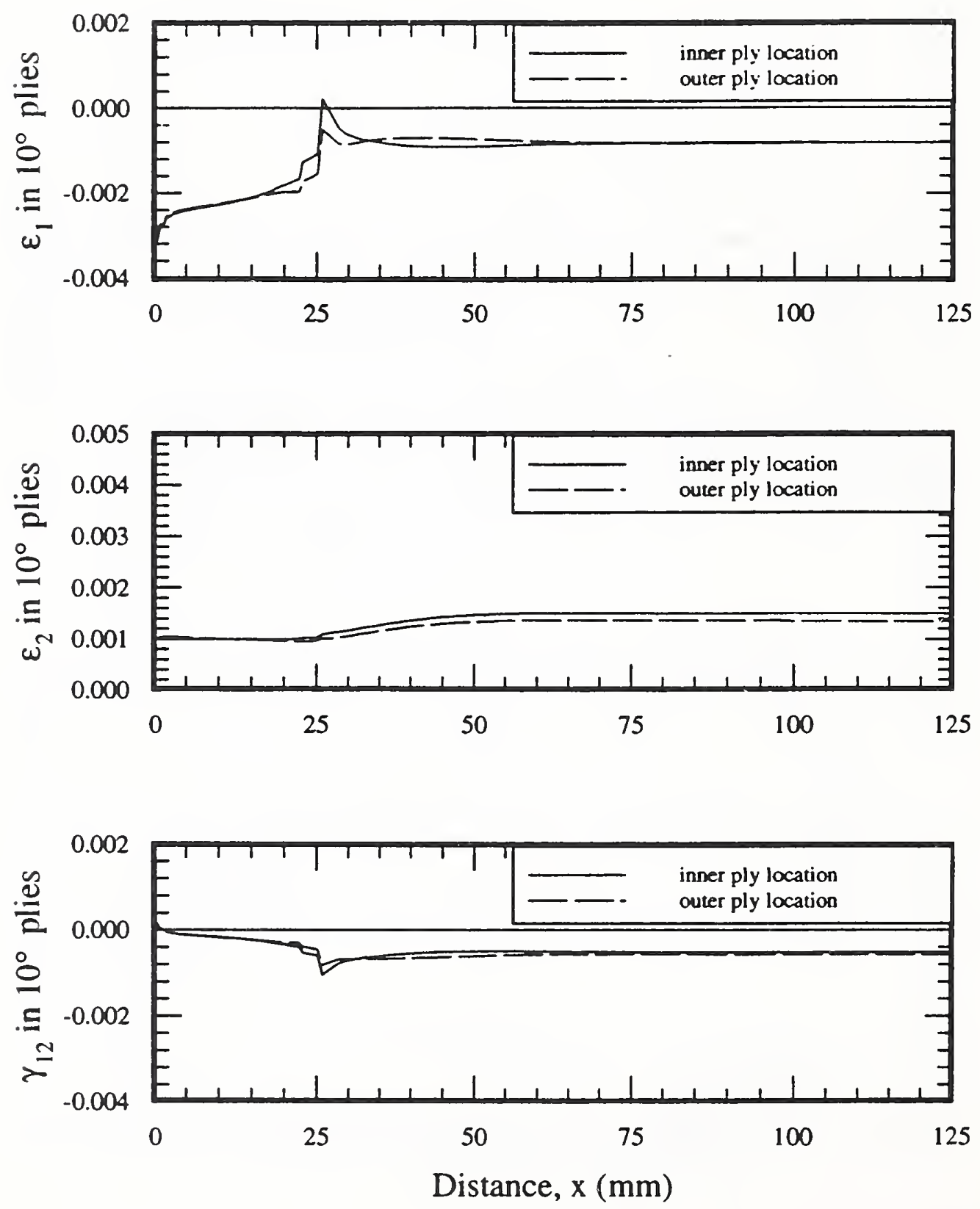

Figure C.19. Intralaminar strains due to a temperature change of $-294 \mathrm{~K}$ in $\pm 10^{\circ}$ plies of thin $[82 / 45 / 10]$ cylinder with baseline-design end fitting. 

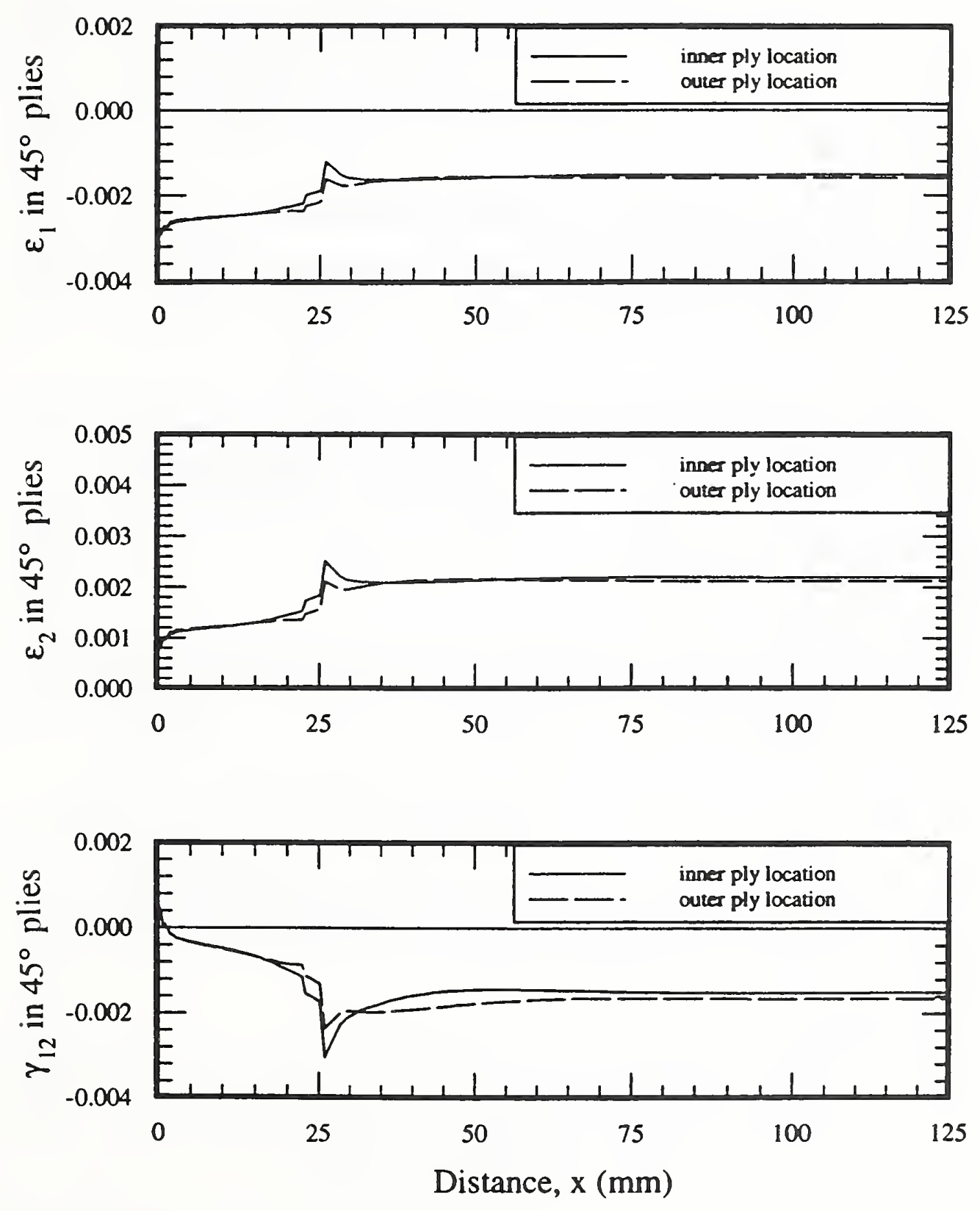

Figure C.20. Intralaminar strains due to a temperature change of $-294 \mathrm{~K}$ in $\pm 45^{\circ}$ plies of thin $[82 / 45 / 10]$ cylinder with baseline-design end fitting. 

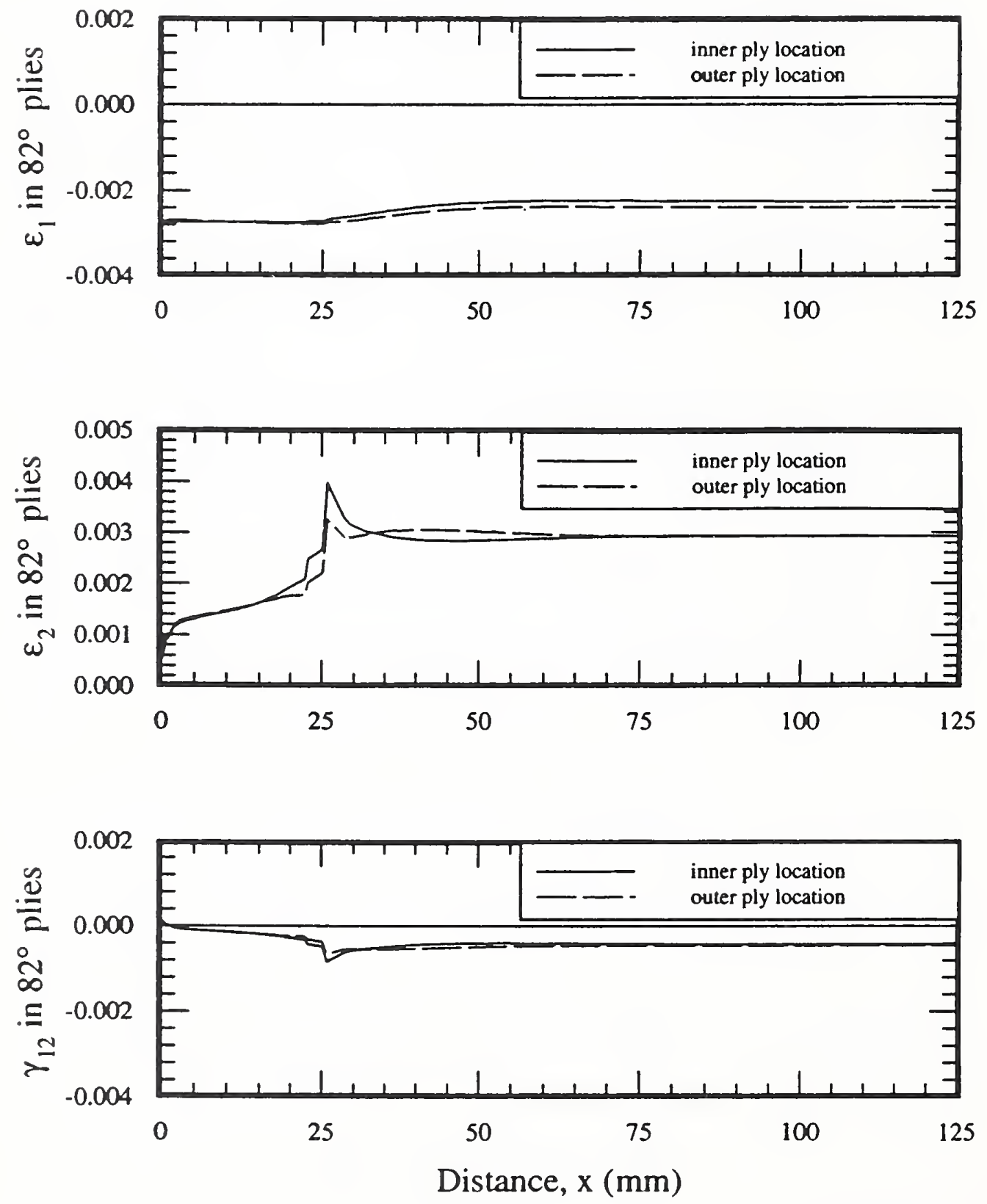

Figure C.21. Intralaminar strains due to a temperature change of $-294 \mathrm{~K}$ in $\pm 82^{\circ}$ plies of thin [82/45/10] cylinder with baseline-design end fitting. 

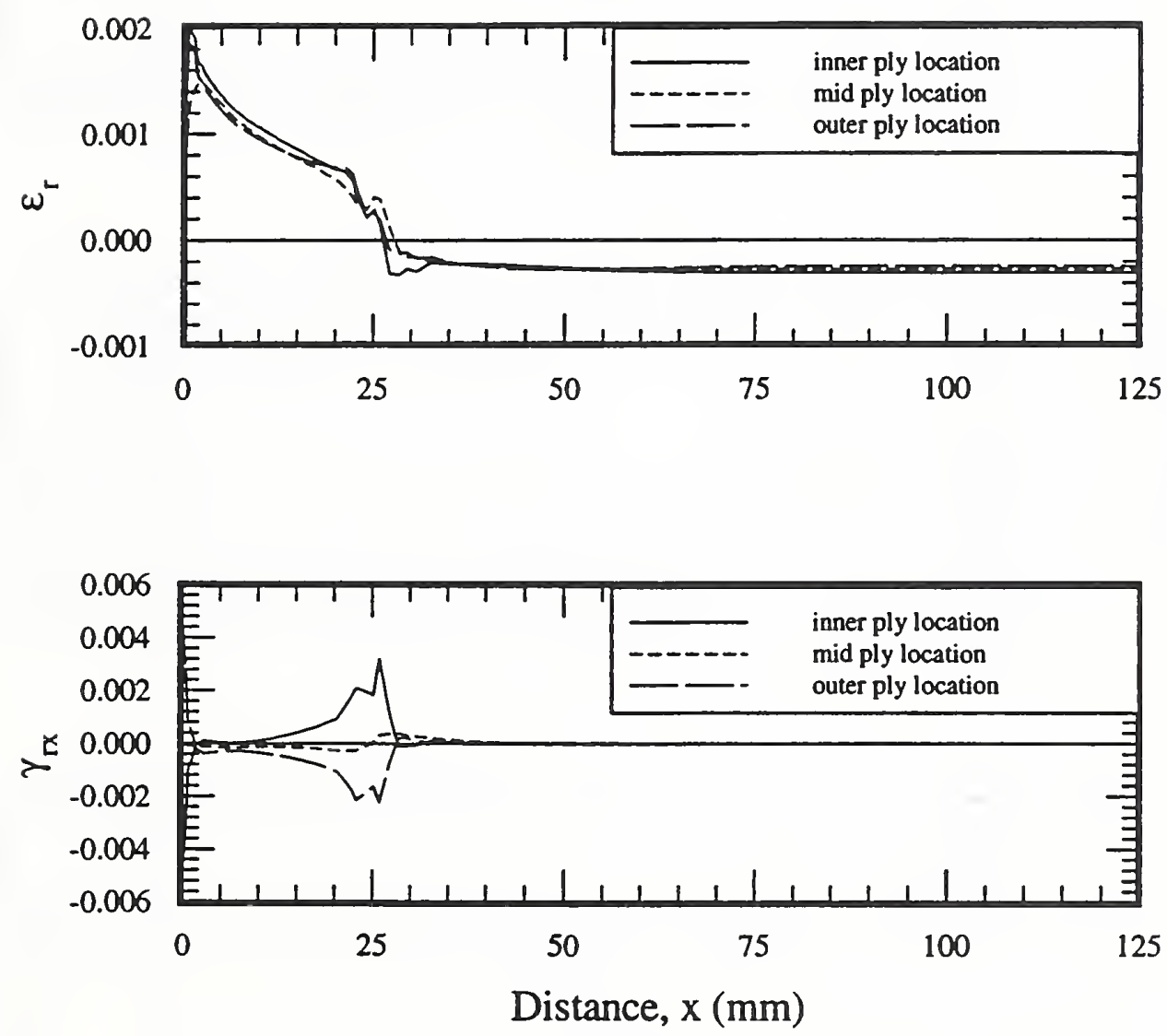

Figure C.22. Interlaminar strains due to a temperature change of $-294 \mathrm{~K}$ in thin [82/45/10] cylinder with baseline-design end fitting. 

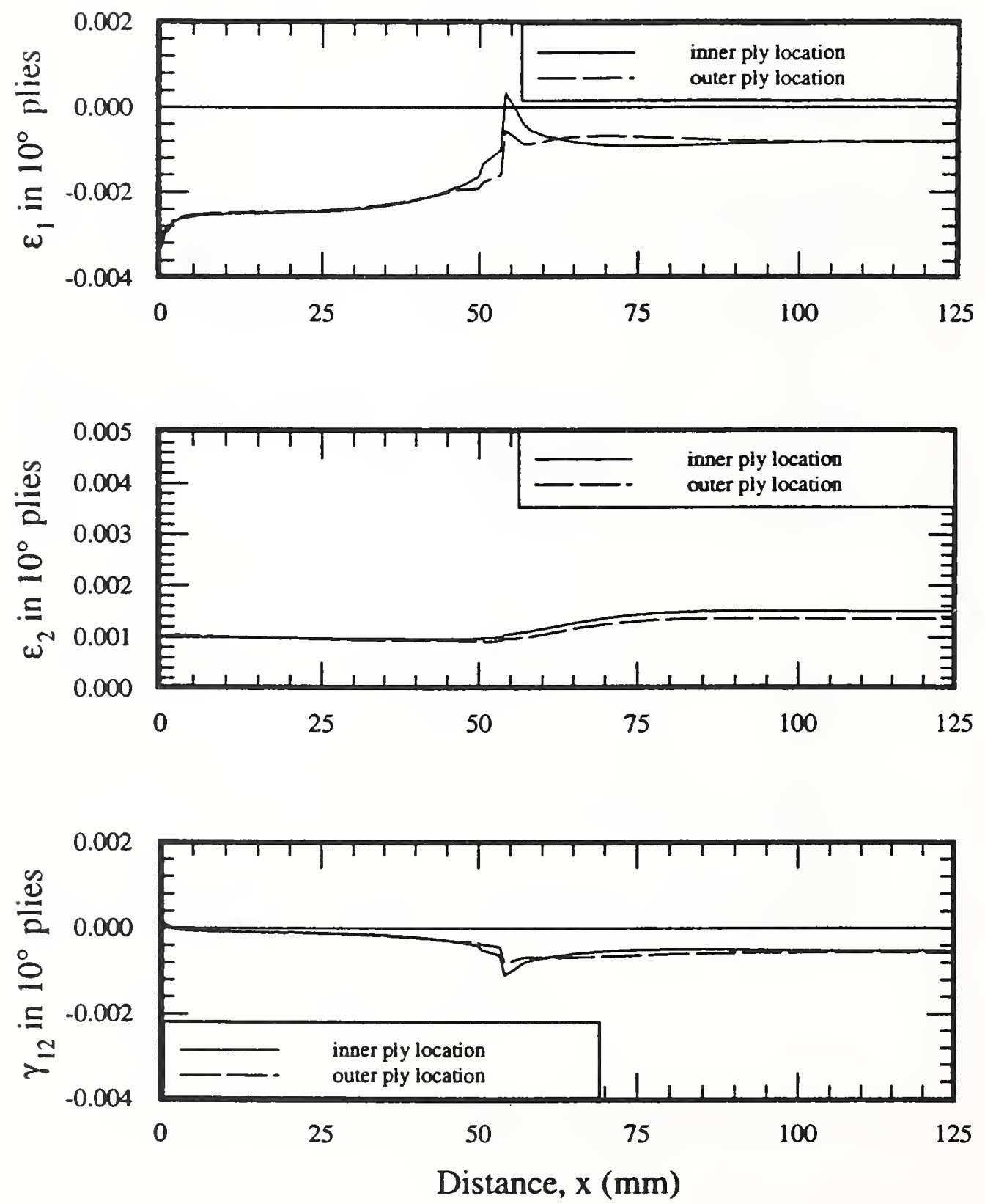

Figure C.23. Intralaminar strains due to a temperature change of $-294 \mathrm{~K}$ in $\pm 10^{\circ}$ plies of thin $[82 / 45 / 10]$ cylinder with alternative-design end fitting. 

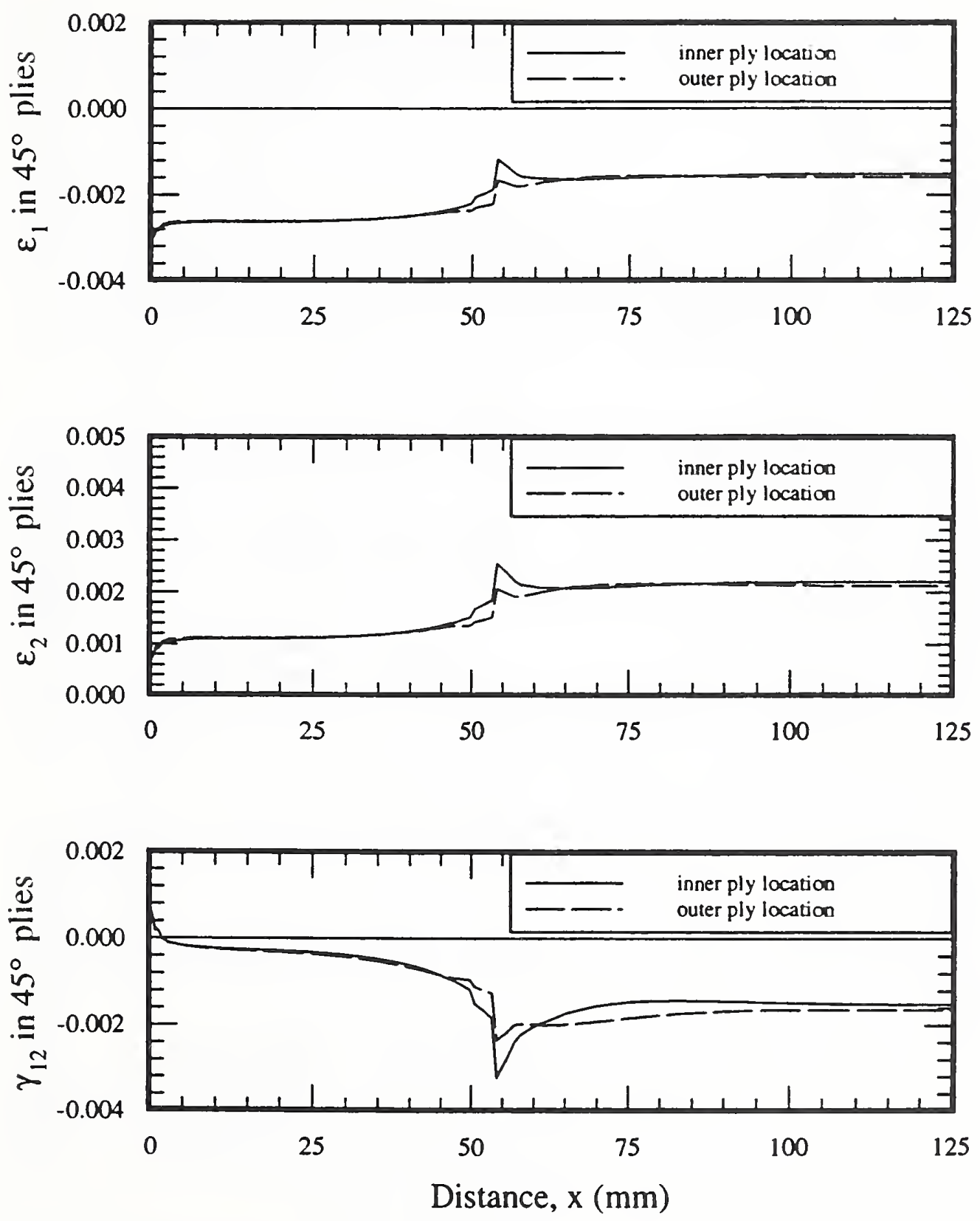

Figure C.24. Intralaminar strains due to a temperature change of $-294 \mathrm{~K}$ in $\pm 45^{\circ}$ plies of thin [82/45/20] cylinder with alternative-design end fitting. 

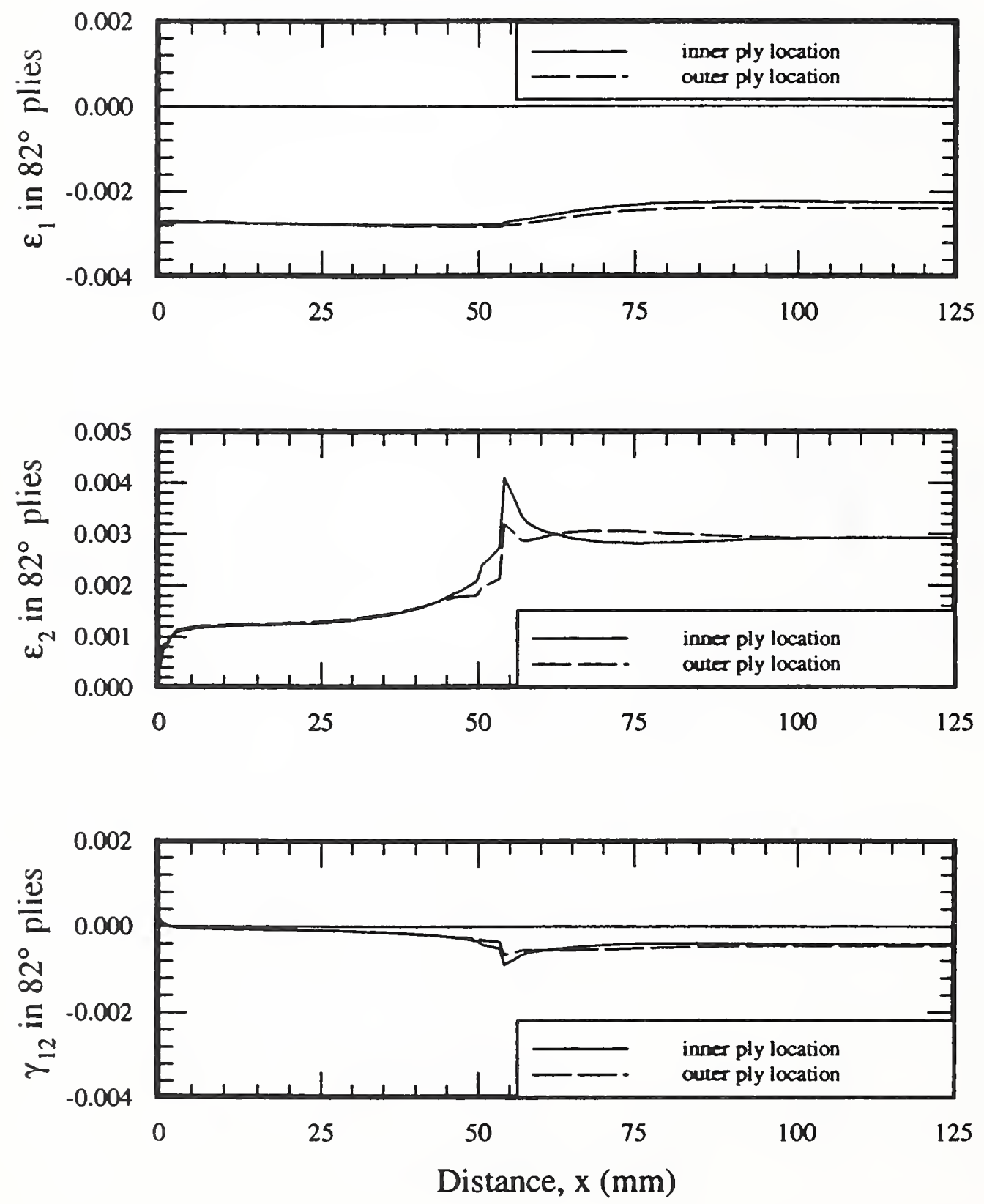

Figure C.25. Intralaminar strains due to a temperature change of $-294 \mathrm{~K}$ in $\pm 82^{\circ}$ plies of thin $[82 / 45 / 20]$ cylinder with alternative-design end fitting. 

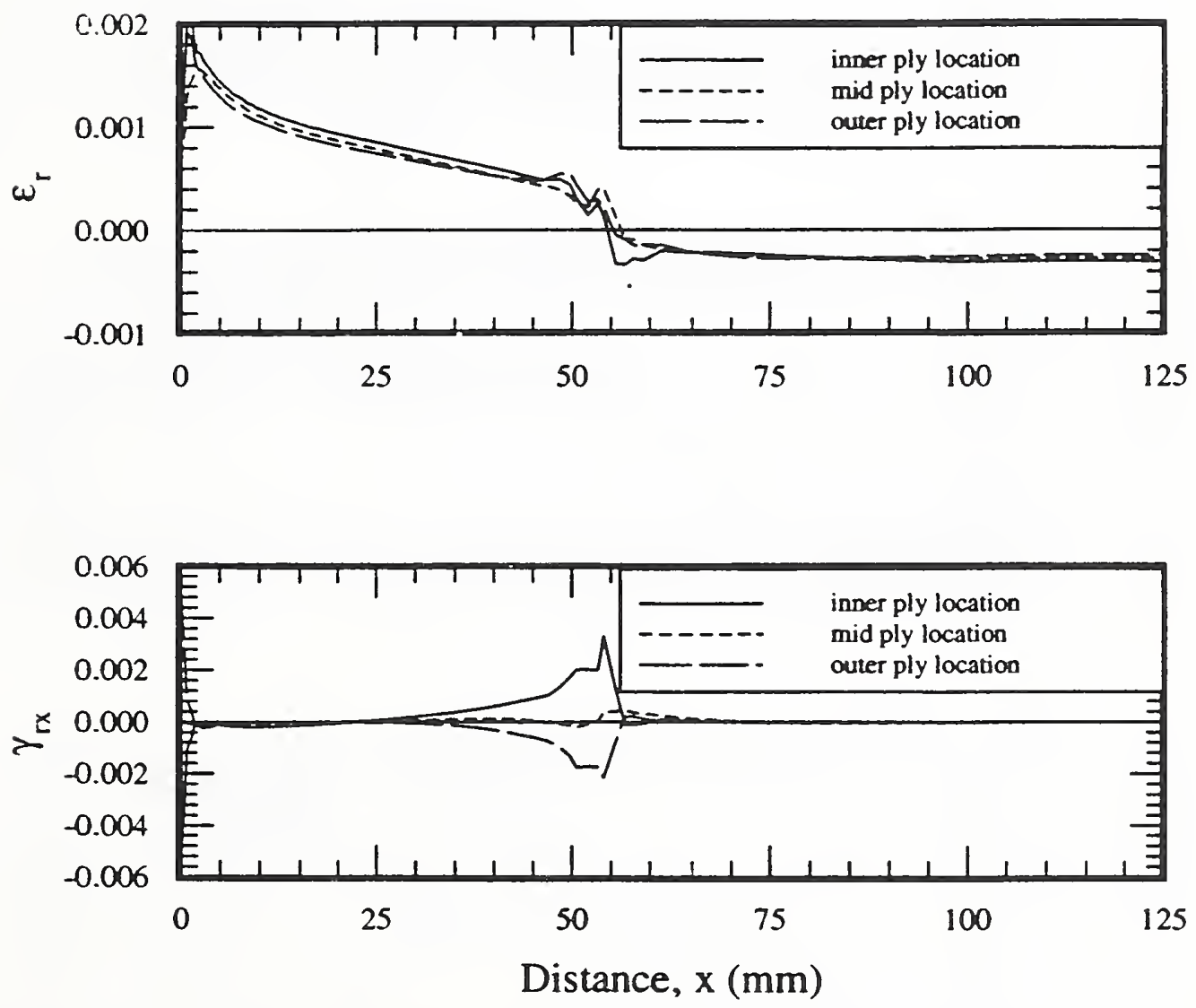

Figure C.26. Interlaminar strains due to a temperature change of $-294 \mathrm{~K}$ in thin $[82 / 45 / 10]$ cylinder with alternative-design end fitting. 

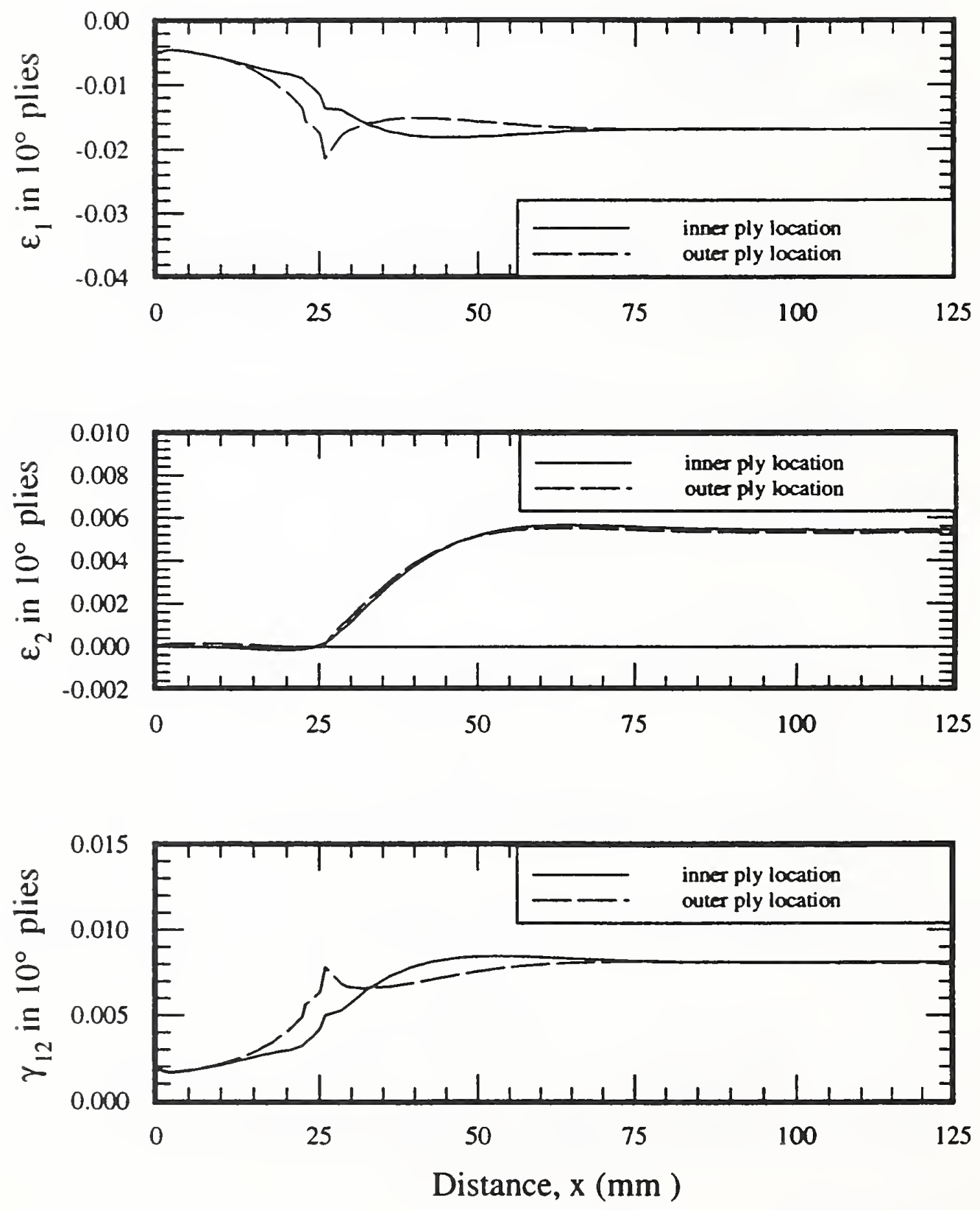

Figure C.27. Intralaminar strains due to an applied axial stress of $-689 \mathrm{MPa}$ in $\pm 10^{\circ}$ plies of thin $[82 / 45 / 10]$ cylinder with baseline-design end fitting, 4-K properties. 

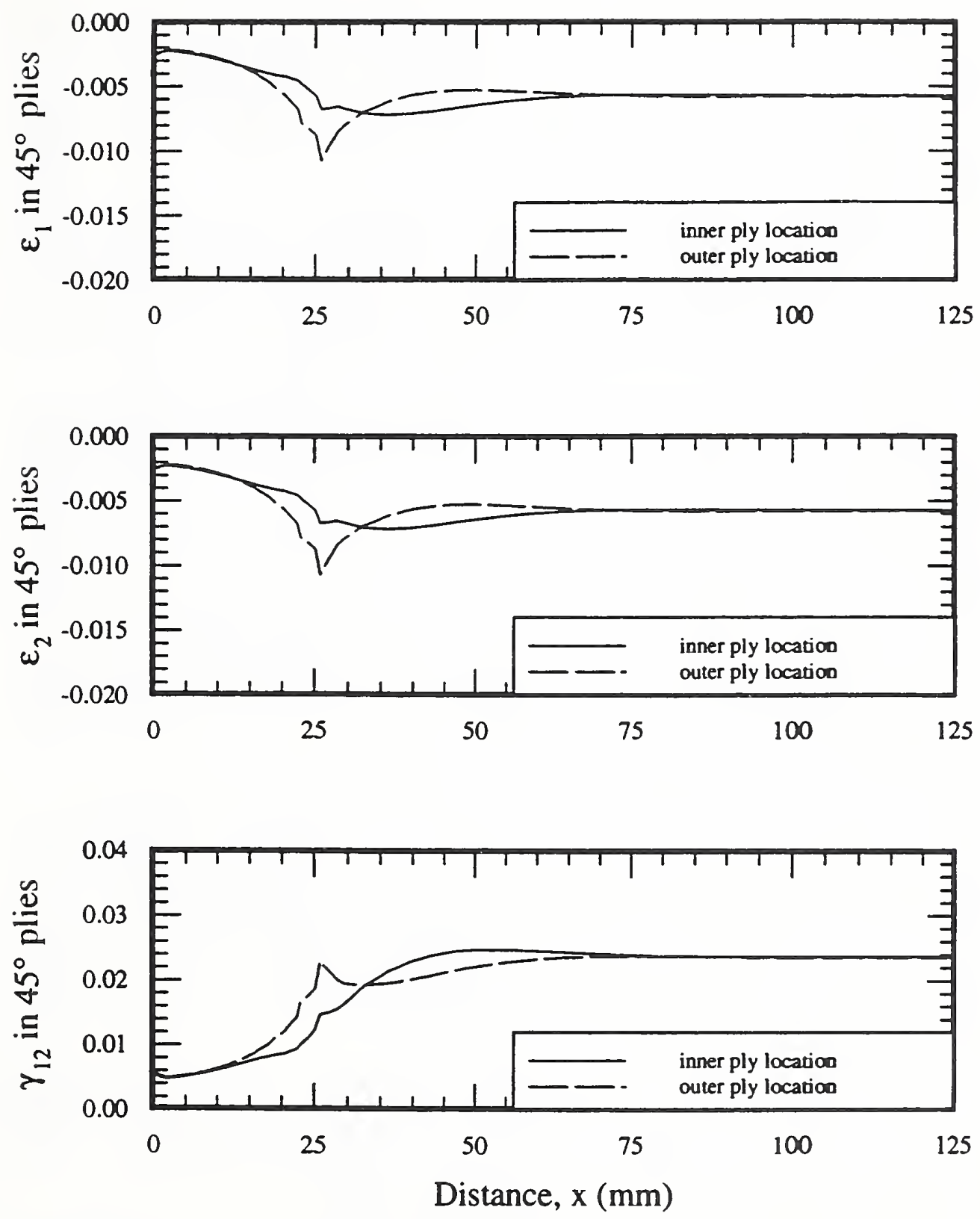

Figure C.28. Intralaminar strains due to an applied axial stress of $-689 \mathrm{MPa}$ in $\pm 45^{\circ}$ plies of thin $[82 / 45 / 10]$ cylinder with baseline-design end fitting, $4-\mathrm{K}$ properties. 

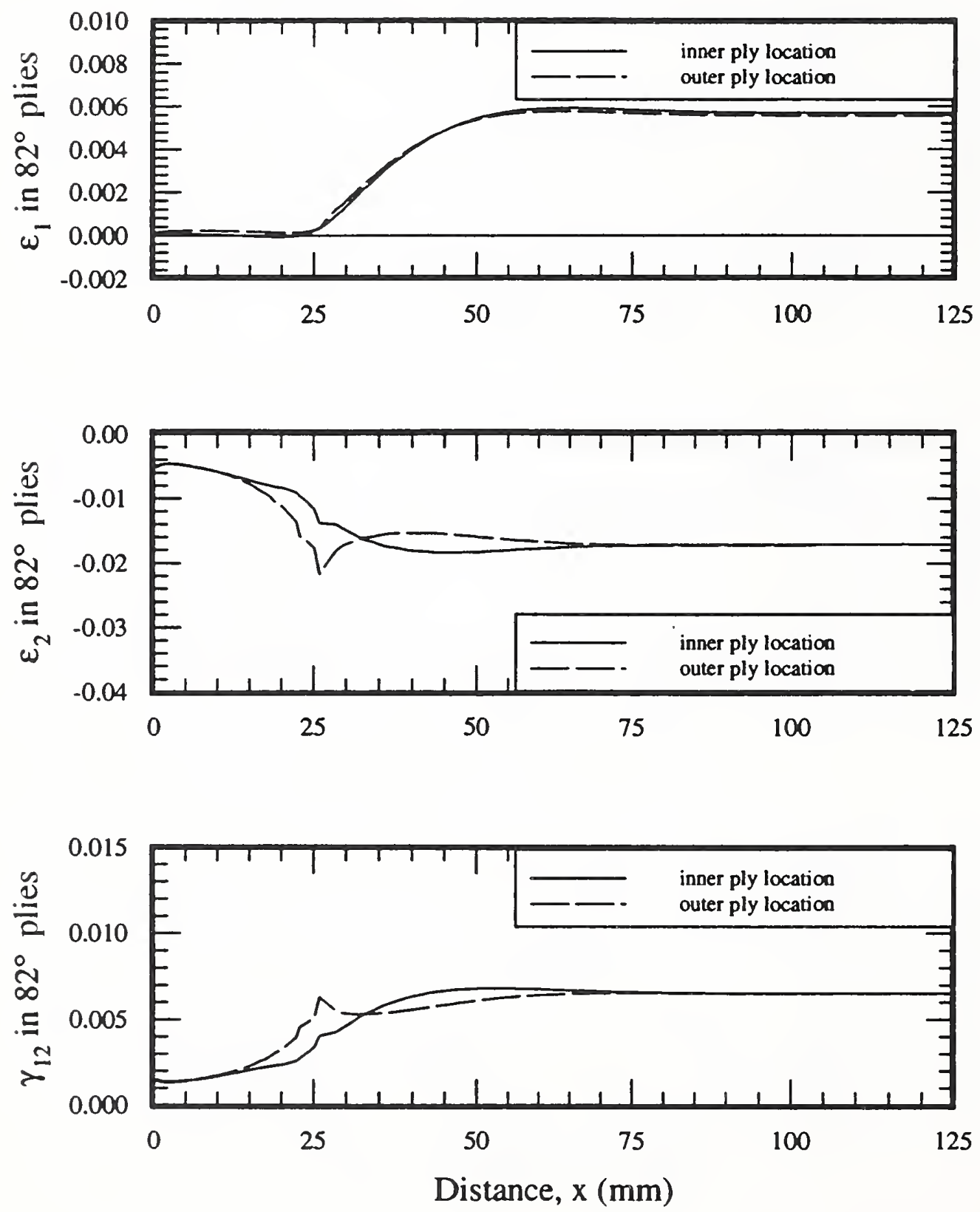

Figure C.29. Intralaminar strains due to an applied axial stress of $-689 \mathrm{MPa}$ in $\pm 82^{\circ}$ plies of thin [82/45/10] cylinder with baseline-design end fitting, 4-K properties. 

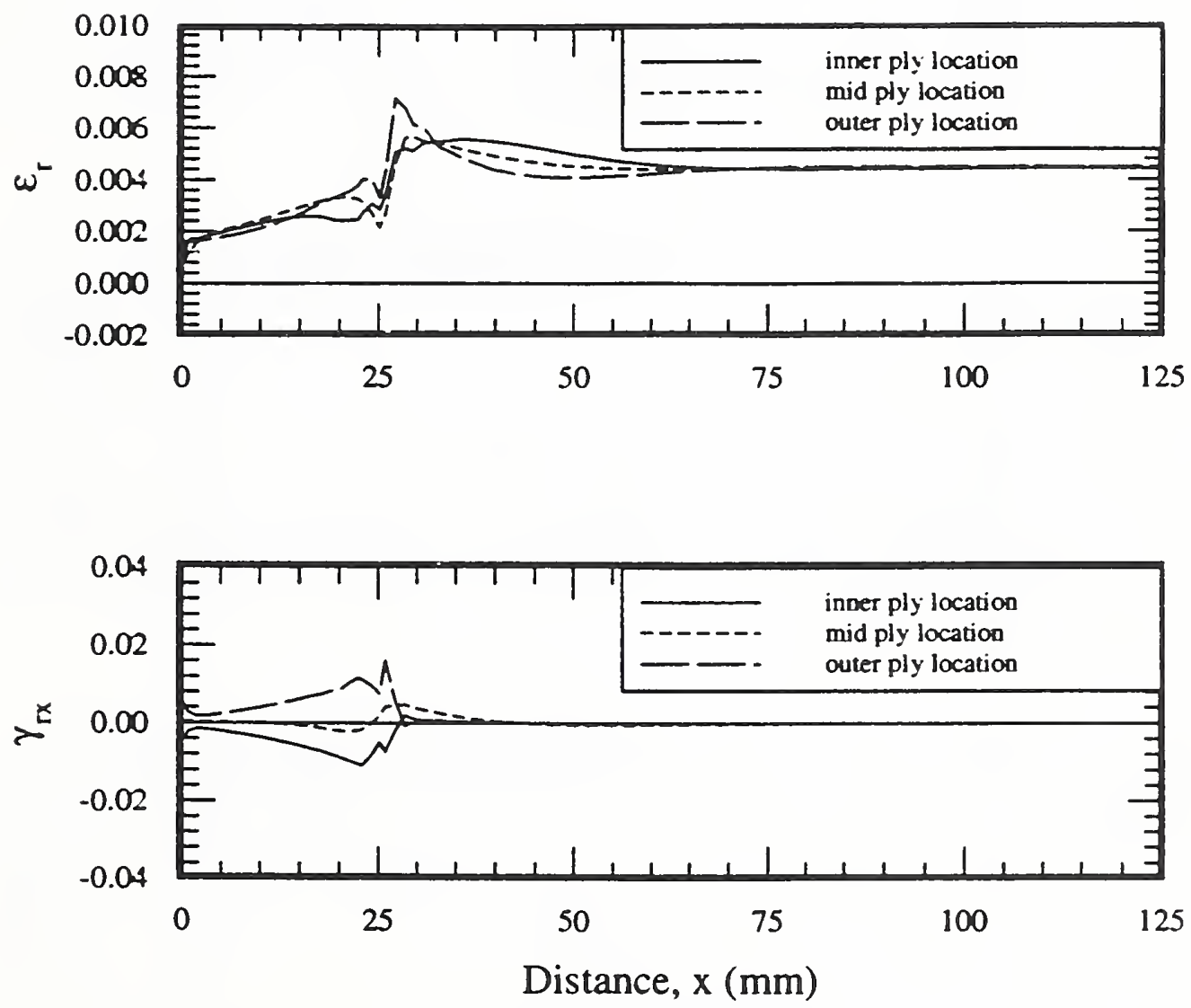

Figure C.30. Interlaminar strains due to an applied axial stress of $-689 \mathrm{MPa}$ in thin $[82 / 45 / 10]$ cylinder with baseline-design end fitting, $4-\mathrm{K}$ properties. 

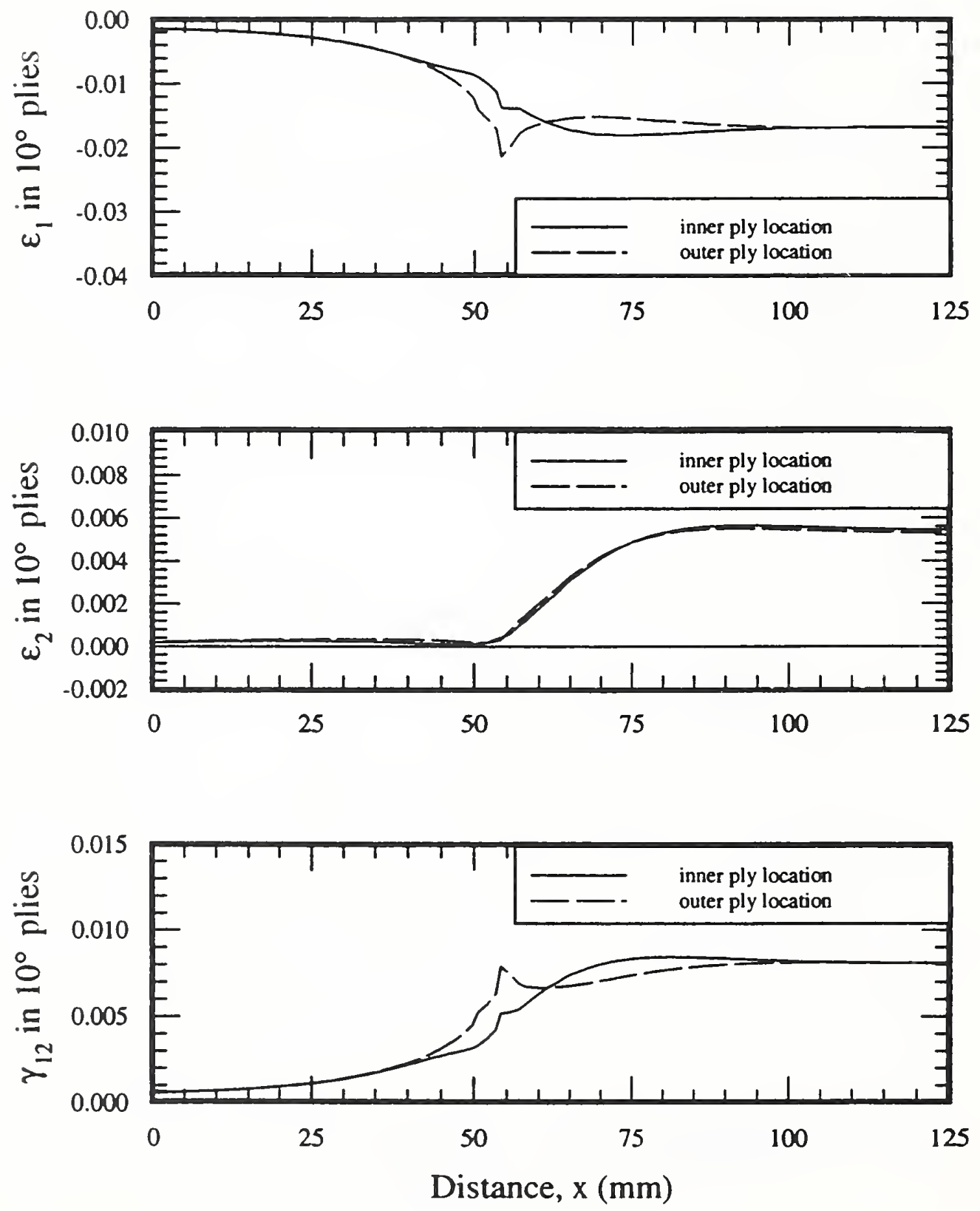

Figure C.31. Intralaminar strains due to an applied axial stress of $-689 \mathrm{MPa}$ in $\pm 10^{\circ}$ plies of thin [82/45/10] cylinder with alternative-design end fitting, 4-K properties. 

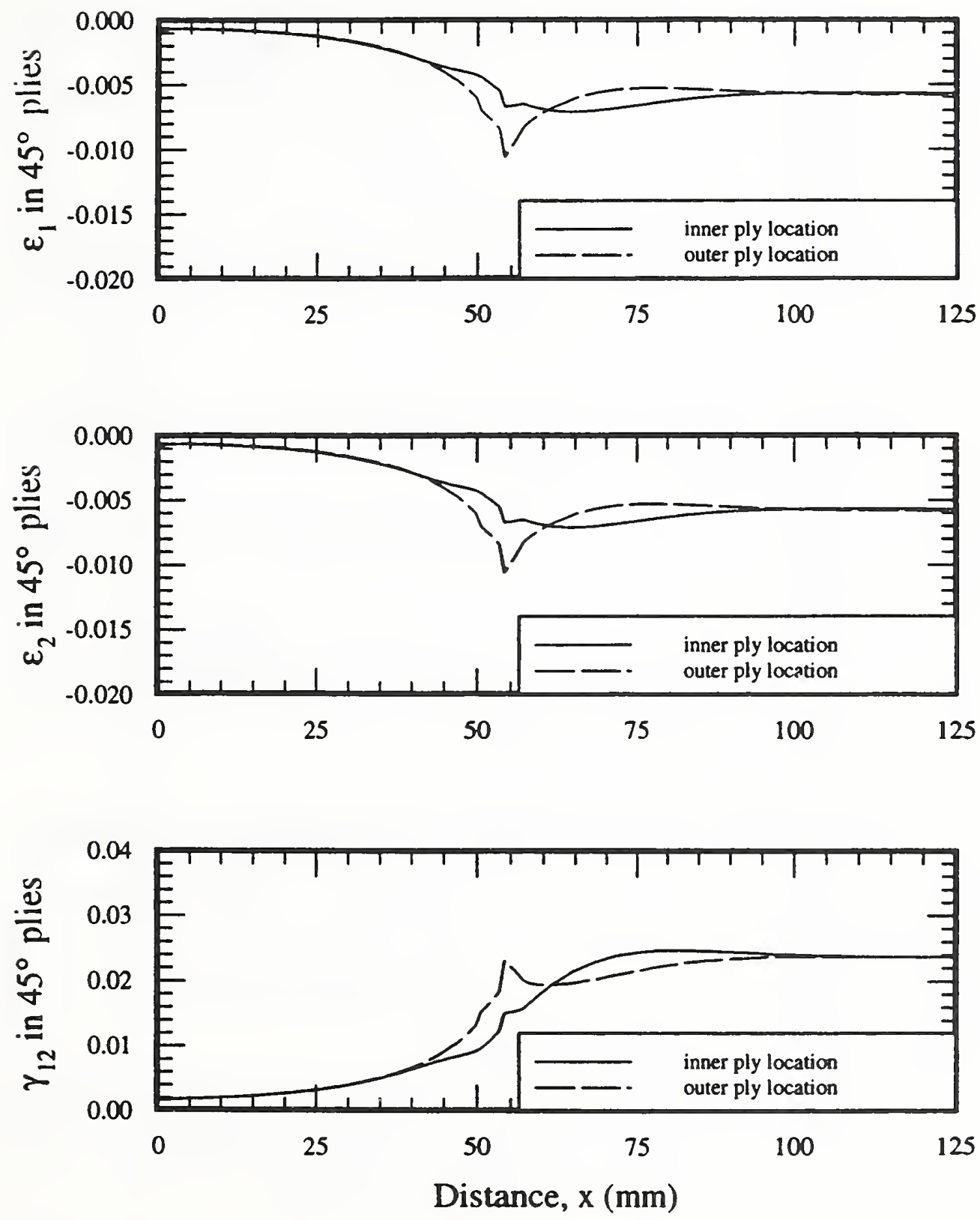

Figure C.32. Intralaminar strains due to an applied axial stress of $-689 \mathrm{MPa}$ in $\pm 45^{\circ}$ plies of thin $[82 / 45 / 10]$ cylinder with alternative-design end fitting, 4-K properties. 

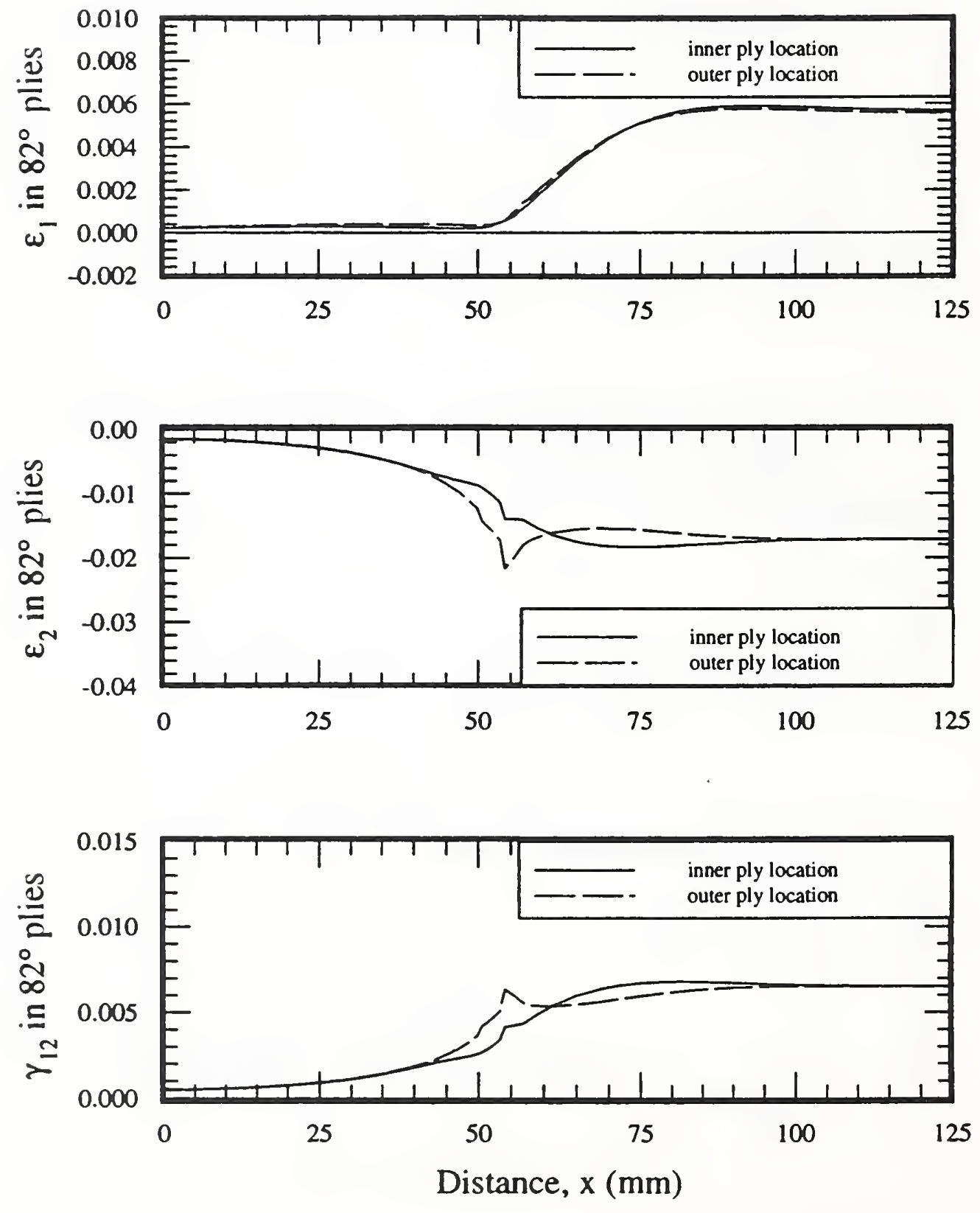

Figure C.33. Intralaminar strains due to an applied axial stress of $-689 \mathrm{MPa}$ in $\pm 82^{\circ}$ plies of thin $[82 / 45 / 10]$ cylinder with alternative-design end fitting, $4-\mathrm{K}$ properties. 

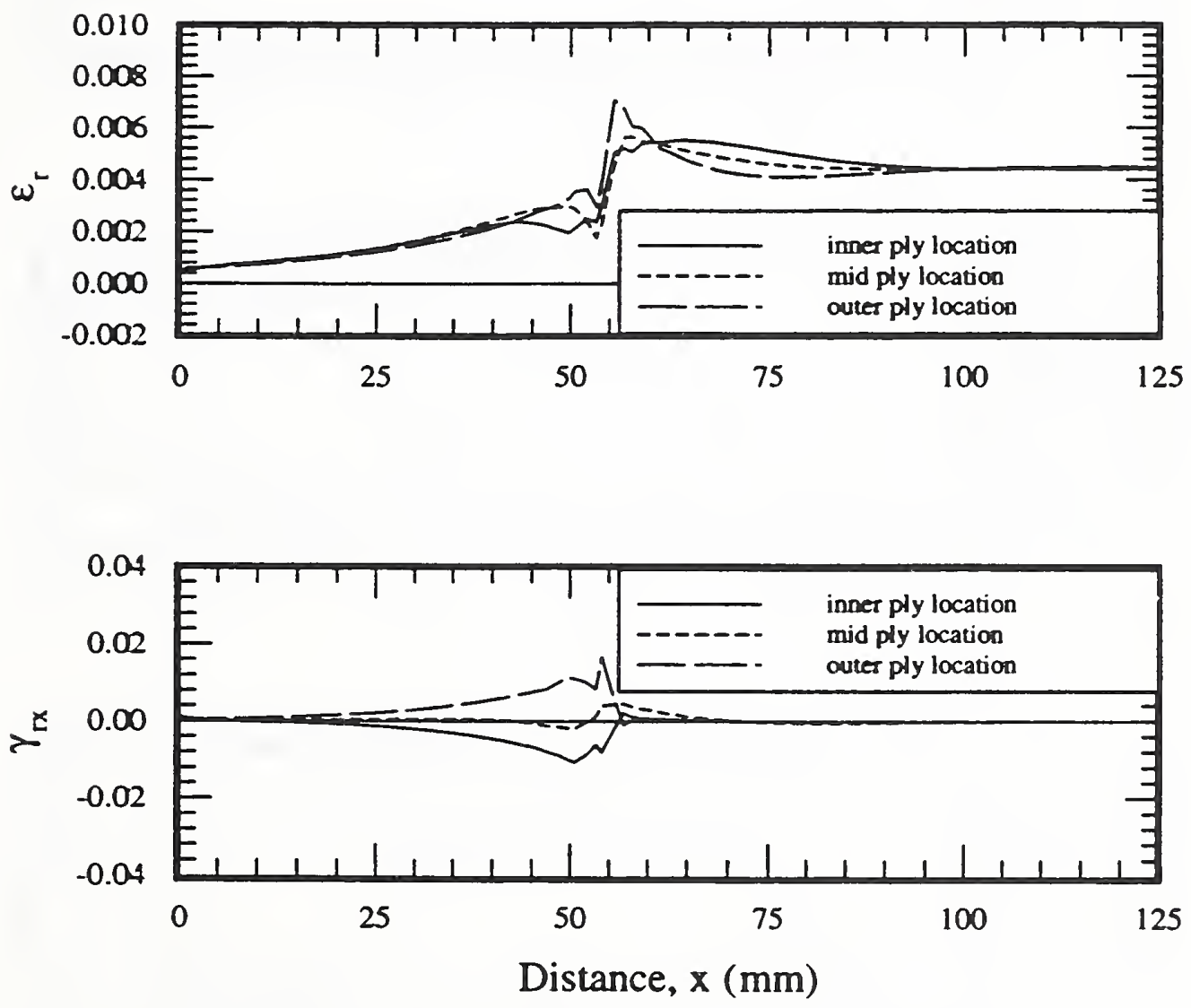

Figure C.34. Interlaminar strains due to an applied axial stress of $-689 \mathrm{MPa}$ in thin [82/45/10] cylinder with alternative-design end fitting, 4-K properties. 

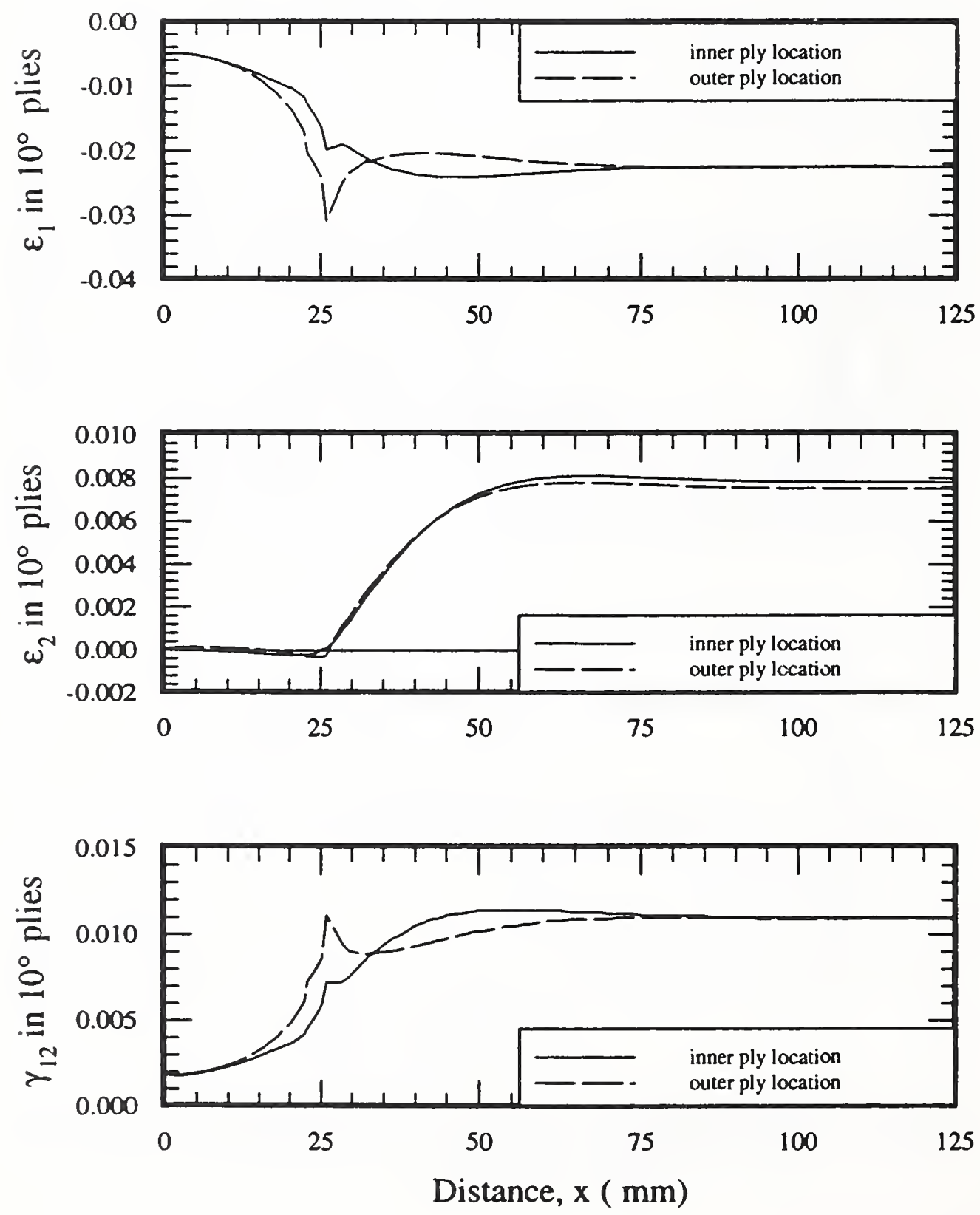

Figure C.35. Intralaminar strains due to an applied axial stress of $-689 \mathrm{MPa}$ in $\pm 10^{\circ}$ plies of thin [82/45/10] cylinder with baseline-design end fitting, 297-K properties. 

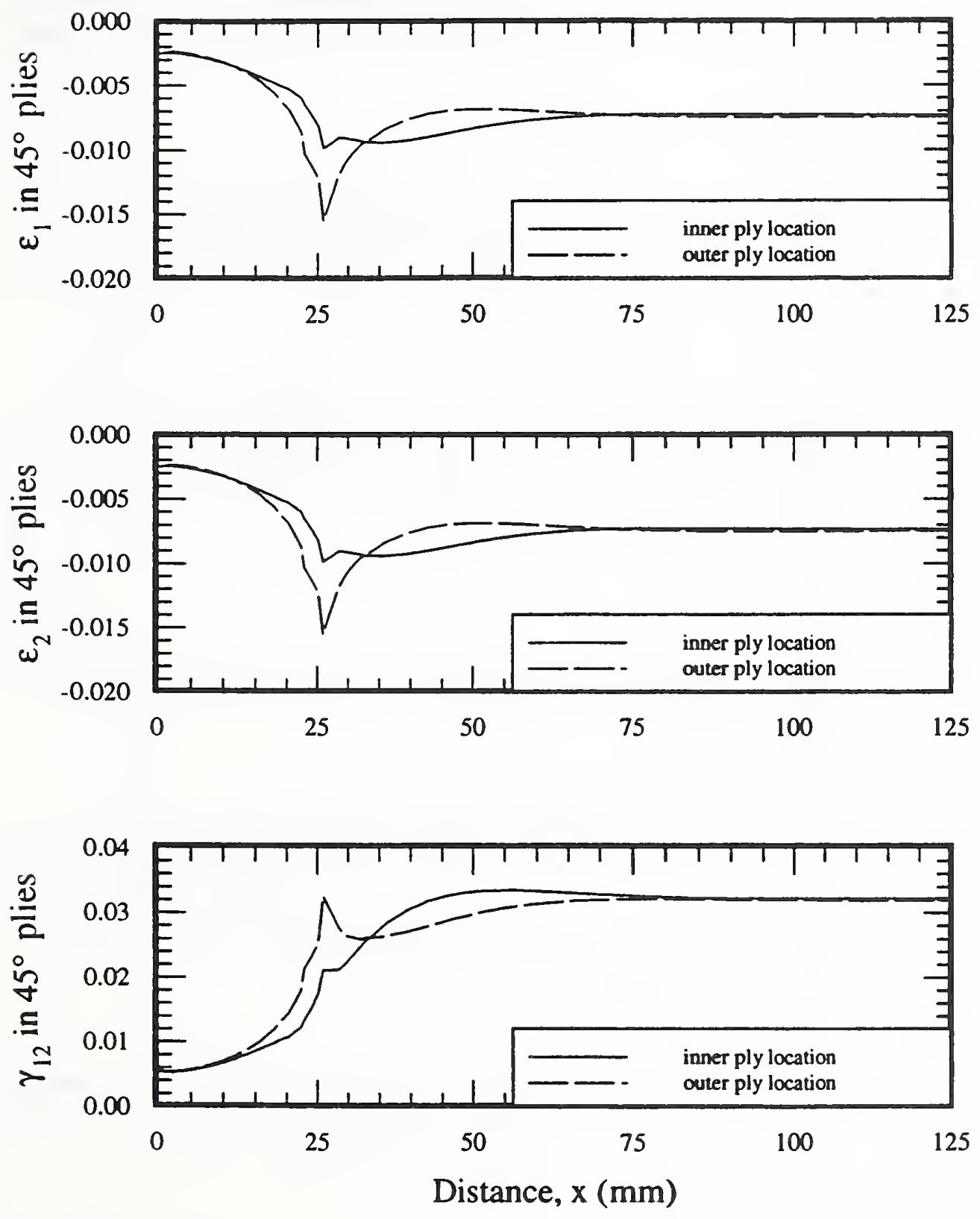

Figure C.36. Intralaminar strains due to an applied axial stress of $-689 \mathrm{MPa}$ in $\pm 45^{\circ}$ plies of thin [82/45/10] cylinder with baseline-design end fitting, 297-K properties. 

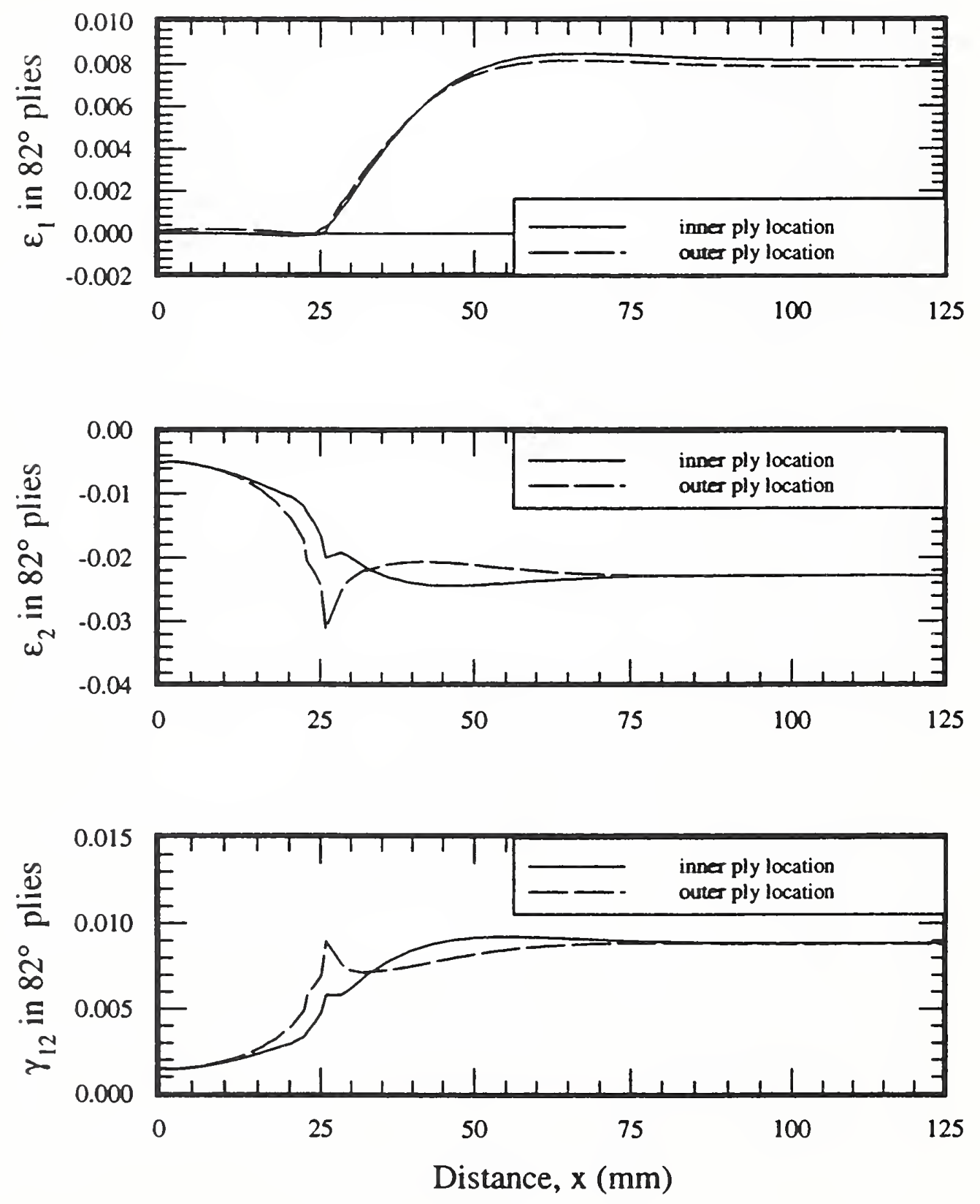

Figure C.37. Intralaminar strains due to an applied axial stress of $-689 \mathrm{MPa}$ in $\pm 82^{\circ}$ plies of thin [82/45/10] cylinder with baseline-design end fitting, 297-K properties. 

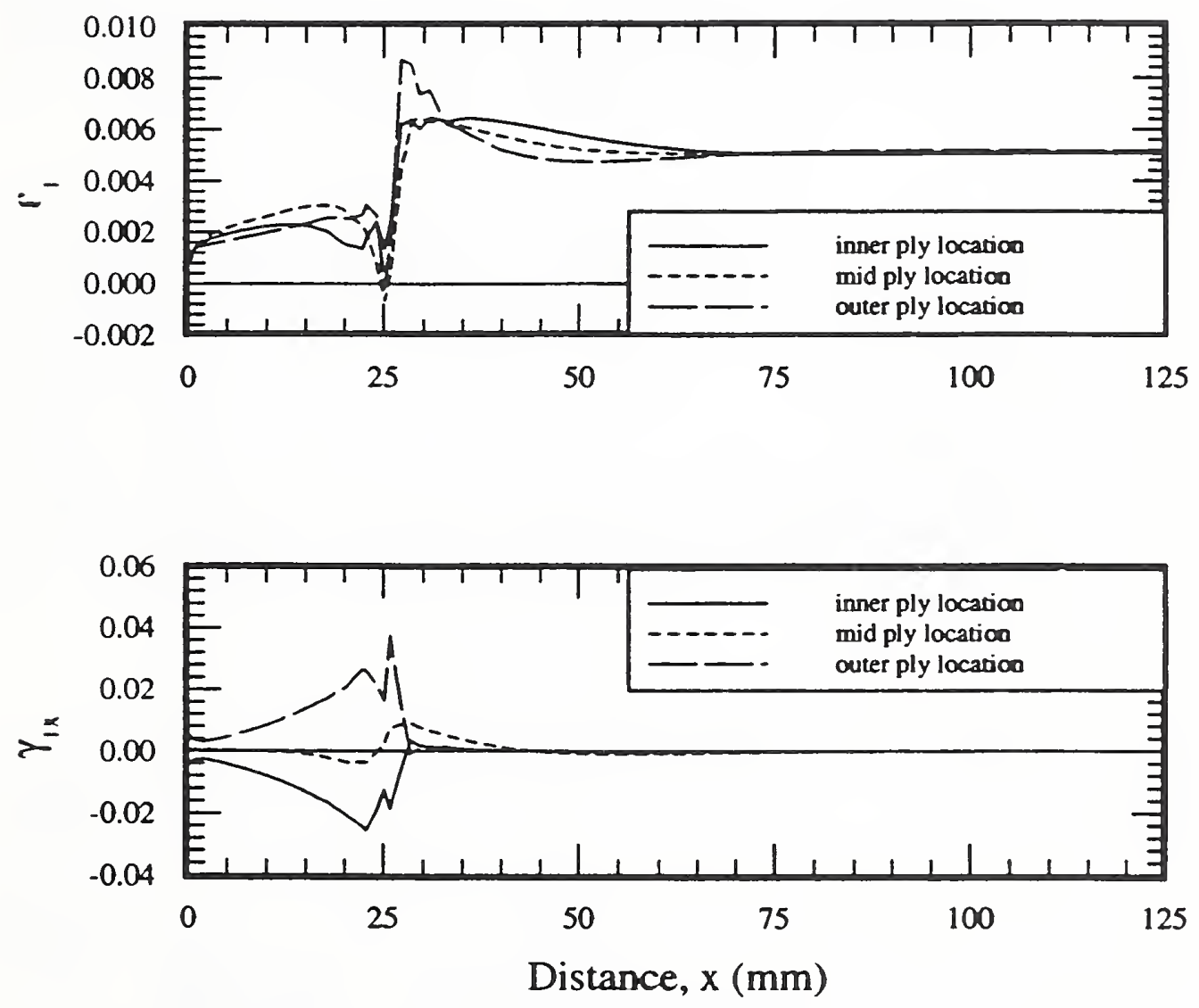

Figure C.38. Interlaminar strains due to an applied axial stress of $-689 \mathrm{MPa}$ in thin [82/45/10] cylinder with baseline-design end fitting, 297-K properties. 

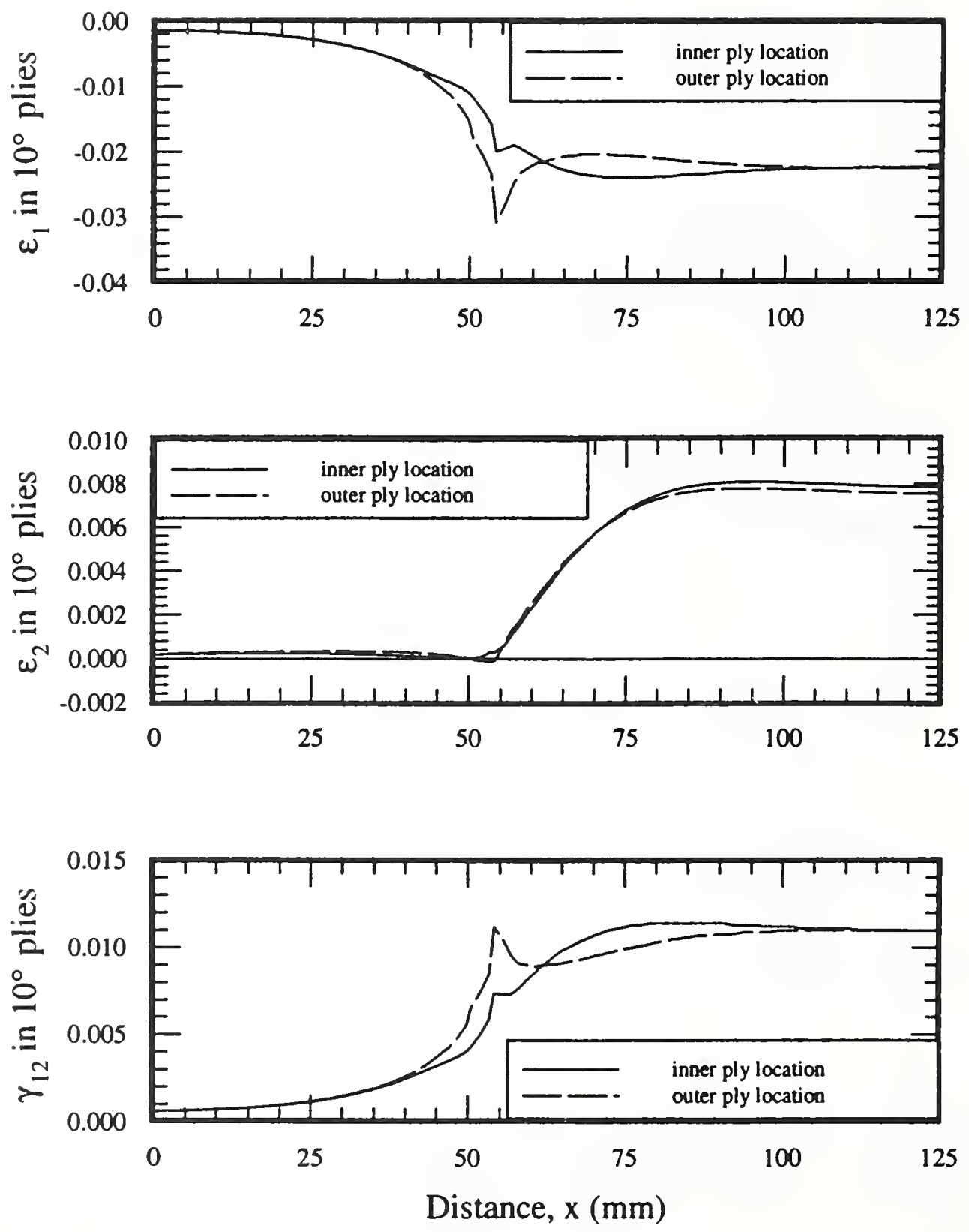

Figure C.39. Intralaminar strains due to an applied axial stress of $-689 \mathrm{MPa}$ in $\pm 10^{\circ}$ plies of thin $[82 / 45 / 10]$ cylinder with alternative-design end fitting, 297-K properties. 

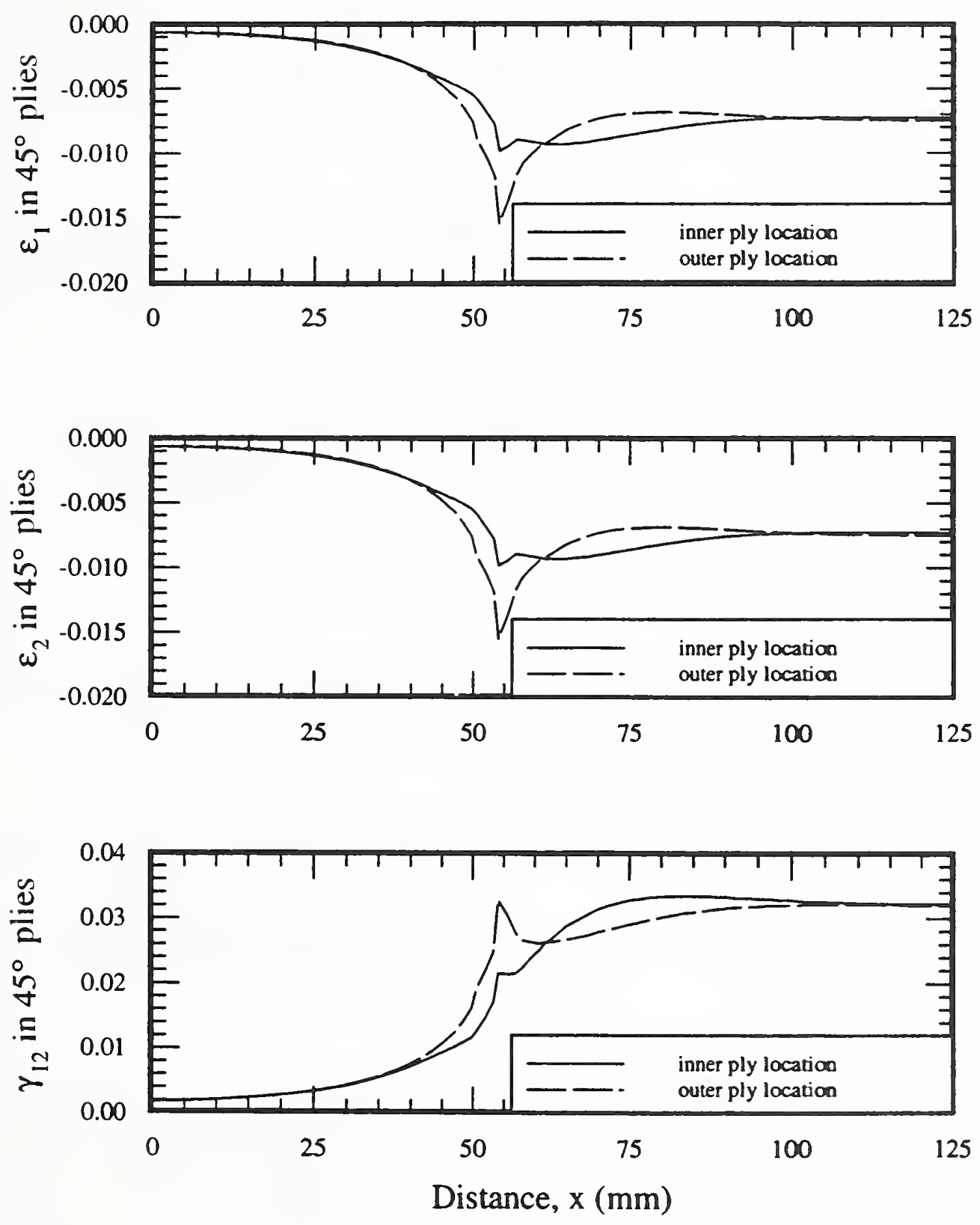

Figure C.40. Intralaminar strains due to an applied axial stress of $-689 \mathrm{MPa}$ in $\pm 45^{\circ}$ plies of thin $[82 / 45 / 10]$ cylinder with alternative-design end fitting, 297-K properties. 

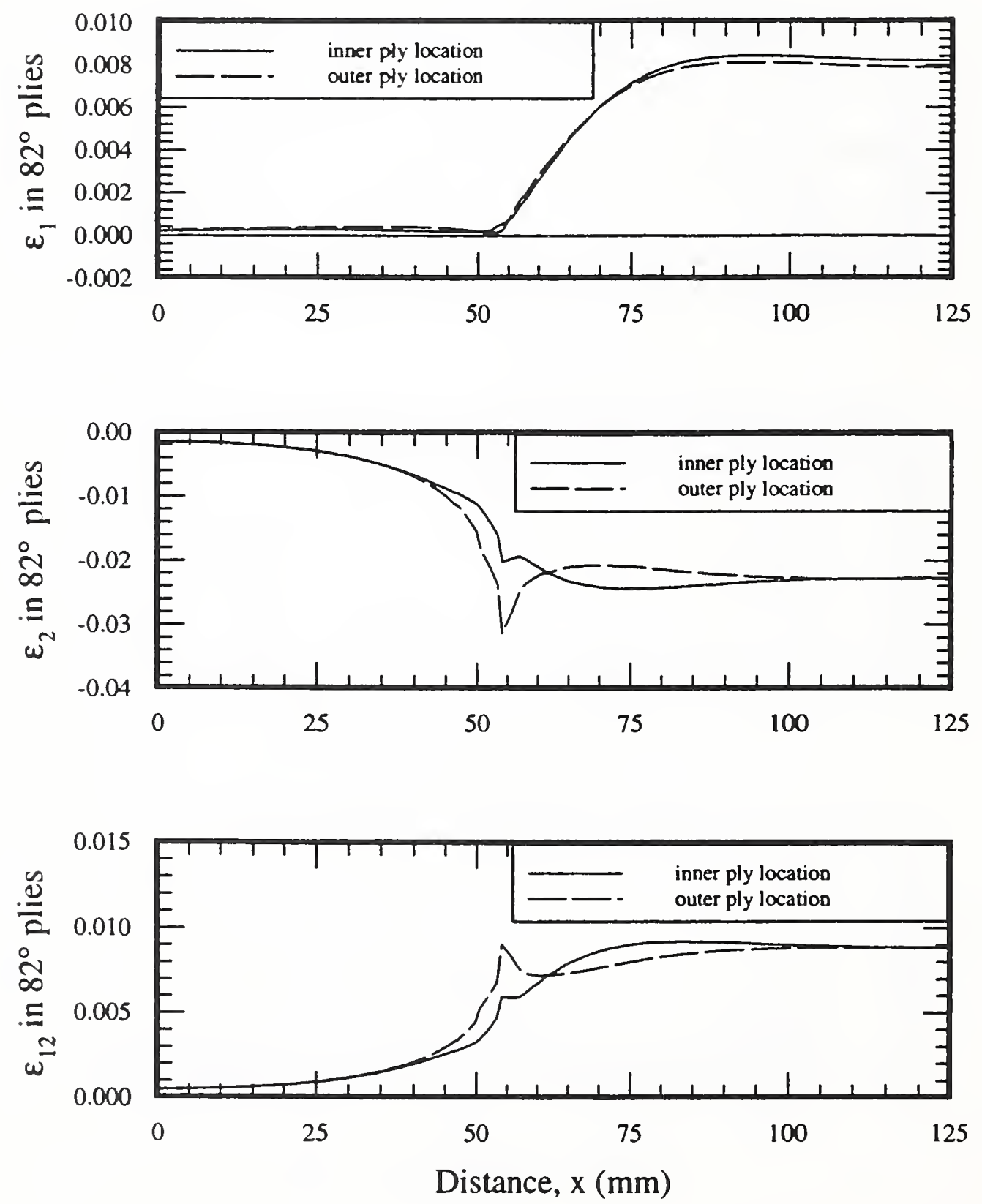

Figure C.41. Intralaminar strains due to an applied axial stress of $-689 \mathrm{MPa}$ in $\pm 82^{\circ}$ plies of thin [82/45/10] cylinder with alternative-design end fitting, 297-K properties. 

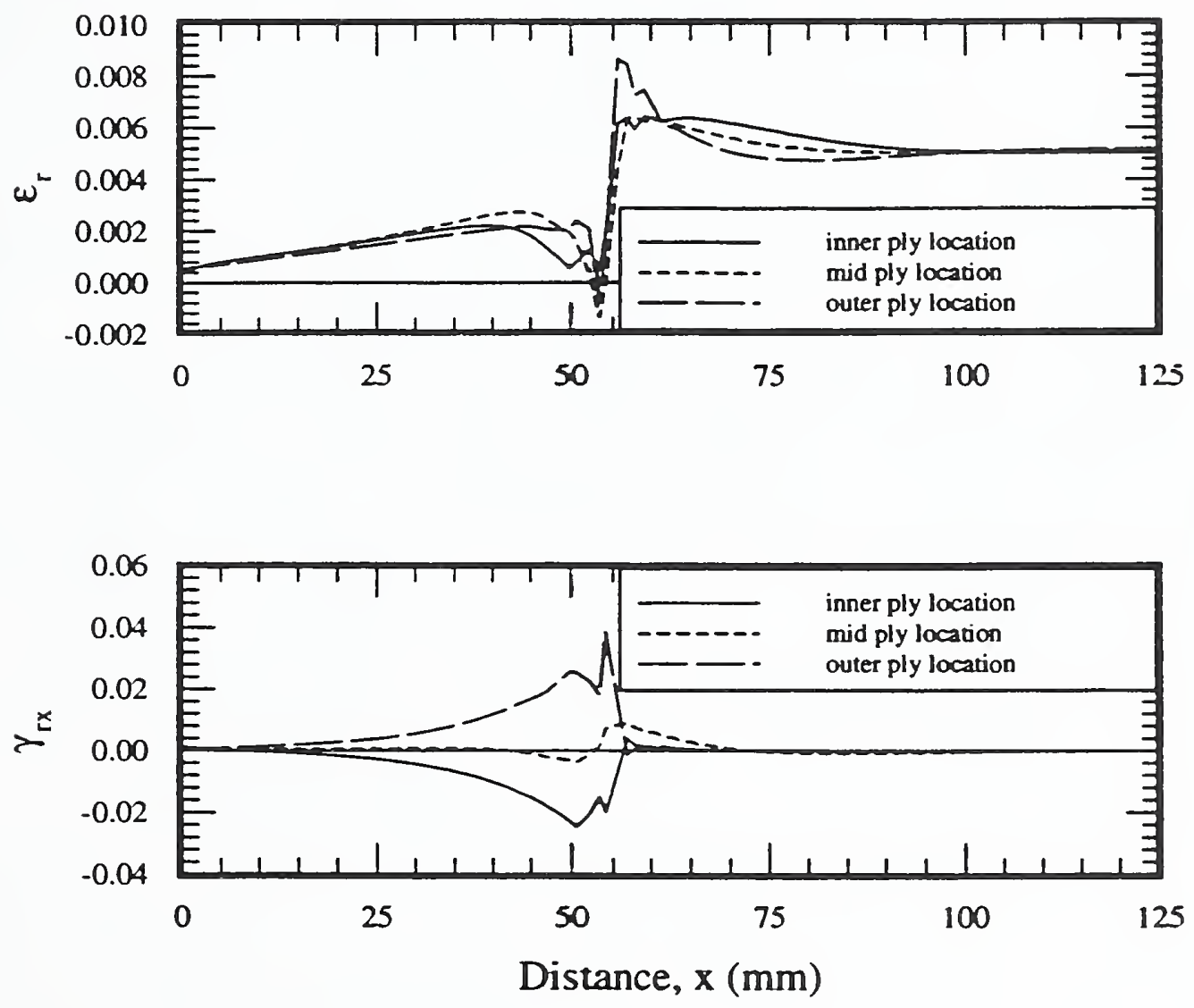

Figure C.42. Interlaminar strains due to an applied axial stress of $-689 \mathrm{MPa}$ in thin [82/45/10] cylinder with alternative-design end fitting, 297-K properties. 


\title{
IX. Failure and Design for SMES Fiberglass Tubes
}

\author{
Marc Regelbrugge \\ Lockheed Palo Alto Laboratories \\ Palo Alto, California
}





\title{
IX. Failure and Design Analyses for SMES Fiberglass Tubes
}

\author{
Marc Regelbrugge \\ Lockheed Palo Alto Laboratories \\ Palo Alto, California
}

\section{Introduction}

Subscale fiberglass tubes were studied to gain insight into their modes of failure under compression and their compressive strength and to develop an engineering basis for design of full-scale SMES support tubes. The database gathered through subscale tube tests at the National Institute of Standards and Technology (NIST) was used as the source of data describing both failure modes and engineering properties of the candidate fiberglass materials. All the tubes in this study were produced by filament winding and $1100 \mathrm{TEX}(450 \mathrm{yd} / \mathrm{lb})$, continuous-strand E-glass. The tube types referred to in this section are distinguished in Table 1.1. Additional information regarding construction and micrographs of the tube cross sections are given in $\$$ VI.8, pages VI-46 through VI-54.

Table 1.1. Description of tube types.

\begin{tabular}{|c|c|c|c|c|c|c|}
\hline $\begin{array}{l}\text { Tube } \\
\text { Type }\end{array}$ & $\begin{array}{l}\text { Manu- } \\
\text { facturer }\end{array}$ & Lay-up & Resin & $\begin{array}{c}\text { Wall } \\
\text { Thickness } \\
(\mathrm{mm})\end{array}$ & $\begin{array}{c}\text { Fiber } \\
\text { Volume } \\
\text { Fraction }\end{array}$ & $\begin{array}{c}\text { Void } \\
\text { Volume } \\
\text { Fraction }\end{array}$ \\
\hline 1 & A & {$[ \pm 10]_{n}$} & DGEBA/DCH/ARA/ACL & 10.2 & 0.56 & 0.023 \\
\hline 2 & A & {$[ \pm 10]_{n}$} & DGEBA/DCH/ARA/ACL & 5.1 & 0.53 & 0.018 \\
\hline 3 & A & $\begin{array}{l}{\left[82 / \pm 45 / \pm 10_{3} / \pm 45 / 82_{2} /\right.} \\
\left. \pm 45 / \pm 10_{3} / \pm 45 / 82\right]\end{array}$ & DGEBA/DCH/ARA/ACL & 10.2 & 0.54 & 0.017 \\
\hline 4 & A & {$\left[82 / \pm 45 / \pm 10_{3} / \pm 45 / 82\right]$} & DGEBA/DCH/ARA/ACL & 5.1 & 0.52 & 0.005 \\
\hline 5 & A & $\begin{array}{l}{\left[82 / \pm 10_{3} / 82 / \pm 10_{3} / 82 /\right.} \\
\left. \pm 10_{3} / 82\right]\end{array}$ & DGEBA/DCH/ARA/ACL & 10.2 & 0.609 & $<0.005$ \\
\hline 6 & A & {$\left[82 / \pm 10_{3} / 82 / \pm 10_{3} / 82\right]$} & DGEBA/DCH/ARA/ACL & 5.1 & 0.64 & $<0.005$ \\
\hline 7 & B & $\begin{array}{l}{\left[82 / \pm 9_{2} / 82 / \pm 9_{2} / 82 / \pm 9_{2} /\right.} \\
\left.82 / \pm 9_{2} / 82\right]\end{array}$ & DGEBA/ARA/DTA & 10.2 & 0.49 & 0.071 \\
\hline 9 & B & $\begin{array}{l}{\left[82 / \pm 9_{2} / 82 / \pm 9_{2} / 82 / \pm 9_{2} /\right.} \\
\left.82 / \pm 9_{2} / 82\right]\end{array}$ & VE-EL/MEKP & 10.2 & 0.55 & 0.030 \\
\hline
\end{tabular}

The failure-mode study consisted of qualitative photomicrographic examinations of failed tube specimens and a quantitative assessment of tube failure-strain characteristics. Methods were developed and applied to extract material-level elastic properties and strain-to-failure limits from NIST data taken from subscale tube compression tests. These properties and strain limits were used in design studies to indicate the best tube winding parameters for maximizing strength with minimal crosssectional area. 
The results of these studies are presented in the following sections, along with some discussion of key design aspects for development of full-scale SMES fiberglass support tubes. The photomicrographic study of failure modes is presented first, followed by the extraction of material properties and failure strains from tube test data. Design studies based on the extracted material properties are then described. Key results from these studies include:

1. Significant nonuniformities and irregularities in layer thickness and distribution are evident in the construction of the subscale, filament-wound compression tubes. These nonuniformities most likely contribute, in large measure, to the observed scatter in failure stress and strain data. SMES tubes will have to be designed by considering the deleterious and nondeterministic effects of nonuniform construction.

2. Transverse and shearing strain limits at the material-layer level can be identified from tube test data. These limits provide lower-bound strength estimates useful for appropriately conservative design of full-scale tubes. However, given the construction irregularities noted above, some screening procedure will probably be required to ensure adequate performance of each tube in service.

3. A best-fit procedure for estimating transversely isotropic layer elastic properties from test data was developed and applied with some success to subscale tube test data. This methodology may prove useful in screening full-scale tube specimens for certain kinds of irregularities (e.g., missing layers).

4. Both fiber- and transverse-direction strains (shear and tension) contribute to failure of subscale tubes. Fiber dominance can be enhanced by the addition of a relatively small number of hoopwound layers. Use of intermediate-angle (e.g., 30-60 off-axis) layers provides some benefit only over a small range of failure-strain envelopes, when transverse and shear-strain capabilities are assumed to be high.

Although the results and projections of these studies provide some engineering basis for design of full-scale SMES tubes, the practical issues associated with irregularities in the mass production of such tubes will tend to dominate both their design and performance. As such, the discussions that follow should be reviewed primarily for the qualitative assessment of failure mechanisms and their effect on tube design, rather than as a quantitative derivation of design parameters for future SMES tubes.

\section{Photomicrographic Failure Analysis}

Scanning-electron and optical (metallographic) micrographs were taken from sections of three failed tube specimens (types 2, 3, and 5). The examinations were intended to identify key features of the failure modes of these tubes, to assess the quality of matrix-fiber interface bonding, and to examine internal crack-propagation paths.

To provide a coherent photographic record, the actual micrographs are presented in Appendix A (§IX.8) with observations and commentary for each photograph on the same page as the photo. Review of this material will assist in developing a comprehensive view of the character of the micromechanical fracture behavior of these tubes. The observations presented below summarize the results of the micrographic examinations: 
1. All specimens show considerable variation (in excess of $100 \%$ ) in layer thicknesses, with some layers dropping off altogether in certain regions.

2. Considerable variation in fiber volume fraction is also evident among different regions of the same specimen. This variation is particularly pronounced in the angled layers $\left( \pm 45^{\circ}\right.$ and $\left.82^{\circ}\right)$, where matrix-rich regions abound.

3. Electron micrographs show considerable variation in failure-surface morphology and in the degree of fiber-matrix adhesion depending on local matrix volume fraction. Matrix disbonds from fibers usually occur over small fractions (less than 25\%) of the fiber's lateral surface. This is typical of matrix failure due to transverse tension or shear.

4. All specimens exhibit transverse cracks (i.e., cracks normal to the fiber direction) in the $\pm 10^{\circ}$ layers. These transverse cracks run primarily in a radial direction when viewed in micrographs taken of the tube cross sections, and they propagate between fiber edges through regions with the highest fiber volume fractions (matrix stress concentrations caused by fiber-matrix load transfer are most severe in regions with relatively high fiber content).

5. Transverse cracks usually run through the entire thickness of contiguous $10^{\circ}$ layers. Their behavior at interfaces with layers oriented at other angles (notably $\pm 45^{\circ}$ and $82^{\circ}$ ) varies among specimen types, although the cracks generally do not propagate readily through the hoop-wound $\left(82^{\circ}\right)$ layers.

6. Specimens of tube type 3 (lay-up $\left[82 / \pm 45 / \pm 10_{3}\right]_{n}$ ) show considerable delamination near the failure region. The delamination occurs primarily at interfaces between $45^{\circ}$ and $10^{\circ}$ layers, although some delaminations between $10^{\circ}$ layers and between $45^{\circ}$ and $82^{\circ}$ layers are evident as well. The delamination growth is associated with transverse cracking in the $45^{\circ}$ layers.

7. Specimens of tube types 2 and 3 show very high void content. Voids are distributed fairly evenly throughout the specimens examined, and they seem to be associated with propagation, initiation, or termination of some transverse cracks. However, transverse cracks propagate more often than not through void-free regions, because these regions are typically associated with the lowest matrix volume fraction and highest matrix transverse-stress concentrations.

From these observations, several characteristic aspects of the failure of the fiberglass compression tubes can be inferred: Most notably, the general failure of the $\pm 10^{\circ}$ dominated tubes appears to follow cracks forming transverse to the fibers throughout the tube, not just in the final failure region. These cracks may be caused by transverse tension, shear stresses, or both in the $10^{\circ}$ layers. Also, regularly spaced transverse cracks were observed in the outer $82^{\circ}$ layers of the coldtested $\left[ \pm 10_{3} / 82\right]$ tube. These cracks are visible even in unmagnified examinations of the tube's outer surface. Their regular spacing hints that they are caused by differential thermal contractions between the surface $82^{\circ}$ layer and underlying $10^{\circ}$ layers.

\section{Analysis of Failure Strain Data}

Midsection strain-gage readings at tube failure were collected from Reference 3.1 for all specimens listed in Table 3.1. Strain data collected from gages at the ends of the tubes were not used because the circumferential restraint of the end caps caused considerably lower hoop strains to be measured there than in the tube test sections. Table 3.2 lists the maximum, minimum, and average values for the axial and hoop strains measured for each tube tested and reported in Reference 3.1. The coefficient of variation (standard deviation normalized by the mean) is also listed as a measure of 
the scatter found in the test data. The smallest coefficient of variation is less than $1 \%$ for axial-strain measurements from specimen no. 1 of tube type 2, and the largest is more than $21 \%$ for hoop-strain measurements taken from specimen no. 3 of tube type 1. In general, the scatter in a specimen's hoopstrain data is greater than that in its axial-strain data, but this is not true of all specimens.

Table 3.1 Strain-at-failure data for subscale tubes tested at NIST [3.1].

\begin{tabular}{|c|c|c|c|c|c|}
\hline \multirow{2}{*}{$\begin{array}{l}\text { Tube } \\
\text { Type }\end{array}$} & \multirow{2}{*}{$\begin{array}{c}\text { Tube } \\
\text { Length } \\
\text { (mm) }\end{array}$} & \multirow{2}{*}{$\begin{array}{c}\text { Test } \\
\text { Temp. } \\
\text { (K) }\end{array}$} & \multirow{2}{*}{$\begin{array}{l}\text { Specimen } \\
\text { Number }\end{array}$} & \multicolumn{2}{|c|}{ Failure Strain } \\
\hline & & & & axial & hoop \\
\hline \multirow[t]{12}{*}{1} & 710 & 295 & 1 & -14088 & 5743 \\
\hline & & & 1 & -14388 & 5479 \\
\hline & & & 1 & -14438 & 5210 \\
\hline & & & 1 & -14934 & 5782 \\
\hline & & & 1 & -15375 & 5728 \\
\hline & & & 1 & -14978 & 4226 \\
\hline & & & 2 & -11786 & 5989 \\
\hline & & & 2 & -11582 & 4727 \\
\hline & & & 2 & -11443 & 5009 \\
\hline & & & 2 & -11350 & 5769 \\
\hline & & & 3 & -13358 & 4810 \\
\hline & & & 3 & -13799 & 6505 \\
\hline \multirow[t]{9}{*}{1} & 230 & 295 & 1 & -13376 & 6020 \\
\hline & & & 1 & -12144 & 6410 \\
\hline & & & 1 & -12115 & 5462 \\
\hline & & & 1 & -11599 & 6482 \\
\hline & & & 2 & -16485 & 7198 \\
\hline & & & 2 & -17787 & 8186 \\
\hline & & & 2 & -17640 & 7558 \\
\hline & & & 2 & -17530 & 8040 \\
\hline & & & 2 & -18377 & 7897 \\
\hline \multirow[t]{4}{*}{1} & 480 & 295 & 1 & -13585 & \\
\hline & & & 1 & -13613 & \\
\hline & & & 1 & -12824 & \\
\hline & & & 1 & -12799 & \\
\hline \multirow[t]{5}{*}{2} & 710 & 295 & 1 & -12462 & 5232 \\
\hline & & & 1 & -12519 & 6138 \\
\hline & & & 2 & -14081 & 6210 \\
\hline & & & 2 & -12499 & 6432 \\
\hline & & & 2 & -12226 & 5582 \\
\hline
\end{tabular}


Table 3.1 Strain-at-failure data for subscale tubes tested at NIST [3.1] (continued).

\begin{tabular}{|c|c|c|c|c|c|}
\hline \multirow{2}{*}{$\begin{array}{l}\text { Tube } \\
\text { Type }\end{array}$} & \multirow{2}{*}{$\begin{array}{l}\text { Tube } \\
\text { Length } \\
(\mathrm{mm})\end{array}$} & \multirow{2}{*}{$\begin{array}{c}\text { Test } \\
\text { Temp. } \\
(\mathrm{K})\end{array}$} & \multirow{2}{*}{$\begin{array}{c}\text { Specimen } \\
\text { Number }\end{array}$} & \multicolumn{2}{|c|}{ Failure Strain } \\
\hline & & & & axial & hoop \\
\hline \multirow[t]{6}{*}{2} & 710 & 76 & 3 & -19275 & 6115 \\
\hline & & & 3 & -19470 & 5871 \\
\hline & & & 3 & -17896 & 6152 \\
\hline & & & 3 & -19031 & 5737 \\
\hline & & & 3 & -19702 & 6726 \\
\hline & & & 3 & -18640 & 6042 \\
\hline \multirow[t]{11}{*}{2} & 710 & 4 & 4 & -21118 & 5884 \\
\hline & & & 4 & -20361 & 7227 \\
\hline & & & 4 & -19507 & 5385 \\
\hline & & & 4 & -20679 & 7007 \\
\hline & & & 4 & -21509 & 6665 \\
\hline & & & 4 & -19995 & 5908 \\
\hline & & & 5 & -14600 & 4907 \\
\hline & & & 5 & -14331 & 4712 \\
\hline & & & 5 & -14697 & 5444 \\
\hline & & & 5 & -14209 & 4810 \\
\hline & & & 5 & -14258 & 4467 \\
\hline \multirow[t]{16}{*}{3} & 710 & 295 & 1 & -12580 & 3458 \\
\hline & & & 1 & -11805 & 4154 \\
\hline & & & 1 & -10400 & 4041 \\
\hline & & & 1 & -11504 & 4401 \\
\hline & & & 2 & -18287 & 8280 \\
\hline & & & 2 & -17468 & 7316 \\
\hline & & & 2 & -19889 & 7448 \\
\hline & & & 2 & -20221 & 6090 \\
\hline & & & 2 & -18993 & 6255 \\
\hline & & & 2 & -16665 & 8647 \\
\hline & & & 2 & -18915 & 6987 \\
\hline & & & 3 & -13240 & 5619 \\
\hline & & & 3 & -15451 & 5145 \\
\hline & & & 3 & -16129 & 5042 \\
\hline & & & 3 & -13469 & 5137 \\
\hline & & & 3 & -16477 & 5491 \\
\hline \multirow[t]{3}{*}{3} & 710 & 76 & 4 & -26147 & 9448 \\
\hline & & & 4 & -25415 & 8692 \\
\hline & & & 4 & -30518 & 9741 \\
\hline \multirow[t]{4}{*}{3} & 480 & 295 & 1 & -20155 & \\
\hline & & & 1 & -20067 & \\
\hline & & & 1 & -20601 & \\
\hline & & & 1 & -21208 & \\
\hline
\end{tabular}


Table 3.1 Strain-at-failure data for subscale tubes tested at NIST [3.1] (continued).

\begin{tabular}{|c|c|c|c|c|c|}
\hline \multirow{2}{*}{$\begin{array}{l}\text { Tube } \\
\text { Type }\end{array}$} & \multirow{2}{*}{$\begin{array}{l}\text { Tube } \\
\text { Length } \\
(\mathrm{mm})\end{array}$} & \multirow{2}{*}{$\begin{array}{c}\text { Test } \\
\text { Temp. } \\
(\mathrm{K}) \\
\end{array}$} & \multirow{2}{*}{$\begin{array}{c}\text { Specimen } \\
\text { Number }\end{array}$} & \multicolumn{2}{|c|}{ Failure Strain } \\
\hline & & & & axial & hoop \\
\hline \multirow[t]{3}{*}{3} & 230 & 295 & 2 & -19325 & 8272 \\
\hline & & & 2 & -21279 & 8148 \\
\hline & & & 2 & -20180 & 8564 \\
\hline \multirow[t]{10}{*}{4} & 710 & 295 & 1 & -17280 & 7539 \\
\hline & & & 1 & -19770 & 6802 \\
\hline & & & 1 & -18712 & 7152 \\
\hline & & & 1 & -17251 & 8276 \\
\hline & & & 2 & -21116 & 8090 \\
\hline & & & 2 & -14748 & 8506 \\
\hline & & & 2 & -16580 & 8310 \\
\hline & & & 2 & -13692 & 9617 \\
\hline & & & 2 & -12808 & 8553 \\
\hline & & & 2 & -25008 & 7992 \\
\hline \multirow[t]{5}{*}{4} & 710 & 76 & 3 & -25000 & 8411 \\
\hline & & & 3 & -23999 & 8521 \\
\hline & & & 3 & -25000 & 8179 \\
\hline & & & 3 & -20996 & 8887 \\
\hline & & & 3 & -25000 & 9094 \\
\hline \multirow[t]{3}{*}{4} & 710 & 4 & 4 & -22803 & 7813 \\
\hline & & & 4 & -22729 & 7740 \\
\hline & & & 4 & -21387 & 6616 \\
\hline \multirow[t]{9}{*}{5} & 710 & 295 & 1 & -14871 & 5584 \\
\hline & & & 1 & -16949 & 5047 \\
\hline & & & 1 & -17693 & 5124 \\
\hline & & & 2 & -15686 & 4480 \\
\hline & & & 2 & -13733 & 4382 \\
\hline & & & 2 & -13879 & 4053 \\
\hline & & & 2 & -13293 & 5078 \\
\hline & & & 2 & -15442 & 3674 \\
\hline & & & 2 & -15845 & 4626 \\
\hline \multirow[t]{6}{*}{5} & 710 & 76 & 3 & -21839 & 5615 \\
\hline & & & 3 & -21949 & 6445 \\
\hline & & & 3 & -18554 & 6738 \\
\hline & & & 3 & -20154 & 6702 \\
\hline & & & 3 & -22327 & 5554 \\
\hline & & & 3 & -21423 & 5517 \\
\hline \multirow[t]{4}{*}{5} & 230 & 295 & 4 & -16959 & \\
\hline & & & 4 & -14917 & \\
\hline & & & 4 & -16345 & \\
\hline & & & 4 & -18223 & \\
\hline
\end{tabular}


Table 3.1 Strain-at-failure data for subscale tubes tested at NIST [3.1] (continued).

\begin{tabular}{|c|c|c|c|c|c|}
\hline \multirow{2}{*}{$\begin{array}{l}\text { Tube } \\
\text { Type }\end{array}$} & \multirow{2}{*}{$\begin{array}{l}\text { Tube } \\
\text { Length } \\
(\mathrm{mm})\end{array}$} & \multirow{2}{*}{$\begin{array}{c}\text { Test } \\
\text { Temp. } \\
\text { (K) }\end{array}$} & \multirow{2}{*}{$\begin{array}{c}\text { Specimen } \\
\text { Number }\end{array}$} & \multicolumn{2}{|c|}{ Failure Strain } \\
\hline & & & & axial & hoop \\
\hline \multirow[t]{4}{*}{5} & 480 & 295 & 2 & -11010 & \\
\hline & & & 2 & -11275 & \\
\hline & & & 2 & -12421 & \\
\hline & & & 2 & -12583 & \\
\hline \multirow[t]{12}{*}{6} & 710 & 295 & 1 & -16870 & 5286 \\
\hline & & & 1 & -16577 & 4443 \\
\hline & & & 1 & -18311 & 5933 \\
\hline & & & 1 & -15637 & 5310 \\
\hline & & & 1 & -14893 & 5945 \\
\hline & & & 1 & -18896 & 6238 \\
\hline & & & 2 & -14893 & 2710 \\
\hline & & & 2 & -16418 & 3271 \\
\hline & & & 2 & -13208 & 4492 \\
\hline & & & 2 & -14697 & 4834 \\
\hline & & & 2 & -17285 & 4333 \\
\hline & & & 2 & -14001 & 3735 \\
\hline \multirow[t]{8}{*}{6} & 710 & 76 & 3 & -30835 & 6738 \\
\hline & & & 3 & -27661 & 6934 \\
\hline & & & 3 & -30078 & 5029 \\
\hline & & & 3 & -23462 & 8691 \\
\hline & & & 3 & -22485 & 6738 \\
\hline & & & 4 & -24683 & 8838 \\
\hline & & & 4 & -25269 & 8642 \\
\hline & & & 4 & -24097 & 7813 \\
\hline
\end{tabular}


Table 3.2. Strain-at-failure statistics for subscale tubes tested at NIST [3.1].

\begin{tabular}{|c|c|c|c|c|c|c|c|c|}
\hline $\begin{array}{l}\text { Tube } \\
\text { Type }\end{array}$ & $\begin{array}{l}\text { Specimen } \\
\text { Number }\end{array}$ & $\begin{array}{l}\text { Tube } \\
\text { Length } \\
\text { (mm) }\end{array}$ & $\begin{array}{c}\text { Test } \\
\text { Temp. } \\
\text { (K) }\end{array}$ & $\begin{array}{l}\text { Direc- } \\
\text { tion }\end{array}$ & Maximum & Minimum & Average & C.V. \\
\hline 1 & 1 & 710 & 295 & $\begin{array}{l}\text { axial } \\
\text { hoop }\end{array}$ & $\begin{array}{r}-0.015375 \\
0.005782\end{array}$ & $\begin{array}{r}-0.014088 \\
0.004226\end{array}$ & $\begin{array}{r}-0.014700 \\
0.005361\end{array}$ & $\begin{array}{l}0.0323 \\
0.1114\end{array}$ \\
\hline 1 & 2 & 710 & 295 & $\begin{array}{l}\text { axial } \\
\text { hoop }\end{array}$ & $\begin{array}{r}-0.011786 \\
0.005989\end{array}$ & $\begin{array}{r}-0.011350 \\
0.004727\end{array}$ & $\begin{array}{r}-0.011540 \\
0.005373\end{array}$ & $\begin{array}{l}0.0164 \\
0.1120\end{array}$ \\
\hline 1 & 3 & 710 & 295 & $\begin{array}{l}\text { axial } \\
\text { hoop }\end{array}$ & $\begin{array}{r}-0.013799 \\
0.006505\end{array}$ & $\begin{array}{r}-0.013358 \\
0.004810\end{array}$ & $\begin{array}{r}-0.013579 \\
0.005658\end{array}$ & $\begin{array}{l}0.0230 \\
0.2119\end{array}$ \\
\hline 1 & 1 & 230 & 295 & $\begin{array}{l}\text { axial } \\
\text { hoop }\end{array}$ & $\begin{array}{r}-0.013376 \\
0.006482\end{array}$ & $\begin{array}{r}-0.011599 \\
0.005462\end{array}$ & $\begin{array}{r}-0.012308 \\
0.006093\end{array}$ & $\begin{array}{l}0.0613 \\
0.0767\end{array}$ \\
\hline 1 & 2 & 230 & 295 & $\begin{array}{l}\text { axial } \\
\text { hoop }\end{array}$ & $\begin{array}{r}-0.018377 \\
0.008186\end{array}$ & $\begin{array}{r}-0.016485 \\
0.007198\end{array}$ & $\begin{array}{r}-0.017564 \\
0.007776\end{array}$ & $\begin{array}{l}0.0391 \\
0.0512\end{array}$ \\
\hline 1 & 1 & 480 & 295 & $\begin{array}{l}\text { axial } \\
\text { hoop }\end{array}$ & $\begin{array}{r}-0.013613 \\
0.000000\end{array}$ & $\begin{array}{r}-0.012799 \\
0.000000\end{array}$ & $\begin{array}{r}-0.013205 \\
0.000000\end{array}$ & $\begin{array}{l}0.0345 \\
0.0000\end{array}$ \\
\hline 2 & 1 & 710 & 295 & $\begin{array}{l}\text { axial } \\
\text { hoop }\end{array}$ & $\begin{array}{r}-0.012519 \\
0.006138\end{array}$ & $\begin{array}{r}-0.012462 \\
0.005232\end{array}$ & $\begin{array}{r}-0.012491 \\
0.005685\end{array}$ & $\begin{array}{l}0.0032 \\
0.1127\end{array}$ \\
\hline 2 & 2 & 710 & 295 & $\begin{array}{l}\text { axial } \\
\text { hoop }\end{array}$ & $\begin{array}{r}-0.014081 \\
0.006432\end{array}$ & $\begin{array}{r}-0.012226 \\
0.005582\end{array}$ & $\begin{array}{r}-0.012935 \\
0.006075\end{array}$ & $\begin{array}{l}0.0774 \\
0.0726\end{array}$ \\
\hline 2 & 3 & 710 & 76 & $\begin{array}{l}\text { axial } \\
\text { hoop }\end{array}$ & $\begin{array}{r}-0.019702 \\
0.006726\end{array}$ & $\begin{array}{r}-0.017896 \\
0.005737\end{array}$ & $\begin{array}{r}-0.019002 \\
0.006107\end{array}$ & $\begin{array}{l}0.0344 \\
0.0559\end{array}$ \\
\hline 2 & 4 & 710 & 4 & $\begin{array}{l}\text { axial } \\
\text { hoop }\end{array}$ & $\begin{array}{r}-0.021509 \\
0.007227\end{array}$ & $\begin{array}{r}-0.019507 \\
0.005835\end{array}$ & $\begin{array}{r}-0.020528 \\
0.006421\end{array}$ & $\begin{array}{l}0.0357 \\
0.0972\end{array}$ \\
\hline 2 & 5 & 710 & 4 & $\begin{array}{l}\text { axial } \\
\text { hoop }\end{array}$ & $\begin{array}{r}-0.014697 \\
0.005444\end{array}$ & $\begin{array}{r}-0.014209 \\
0.004467\end{array}$ & $\begin{array}{r}-0.014419 \\
0.004868\end{array}$ & $\begin{array}{l}0.0150 \\
0.0742\end{array}$ \\
\hline 3 & 1 & 710 & 295 & $\begin{array}{l}\text { axial } \\
\text { hoop }\end{array}$ & $\begin{array}{r}-0.012580 \\
0.004401\end{array}$ & $\begin{array}{r}-0.010400 \\
0.003458\end{array}$ & $\begin{array}{r}-0.011572 \\
0.004013\end{array}$ & $\begin{array}{l}0.0781 \\
0.0996\end{array}$ \\
\hline 3 & 2 & 710 & 295 & $\begin{array}{l}\text { axial } \\
\text { hoop }\end{array}$ & $\begin{array}{r}-0.020221 \\
0.008647\end{array}$ & $\begin{array}{r}-0.016665 \\
0.006090\end{array}$ & $\begin{array}{r}-0.018634 \\
0.007289\end{array}$ & $\begin{array}{l}0.0681 \\
0.1308\end{array}$ \\
\hline 3 & 3 & 710 & 295 & $\begin{array}{l}\text { axial } \\
\text { hoop }\end{array}$ & $\begin{array}{r}-0.016477 \\
0.005619\end{array}$ & $\begin{array}{r}-0.013240 \\
0.005042\end{array}$ & $\begin{array}{r}-0.014953 \\
0.005287\end{array}$ & $\begin{array}{l}0.1008 \\
0.0477\end{array}$ \\
\hline 3 & 4 & 710 & 76 & $\begin{array}{l}\text { axial } \\
\text { hoop }\end{array}$ & $\begin{array}{r}-0.030518 \\
0.009741\end{array}$ & $\begin{array}{r}-0.025415 \\
0.008692\end{array}$ & $\begin{array}{r}-0.027360 \\
0.009294\end{array}$ & $\begin{array}{l}0.1009 \\
0.0582\end{array}$ \\
\hline 3 & 1 & 480 & 295 & $\begin{array}{l}\text { axial } \\
\text { hoop }\end{array}$ & $\begin{array}{r}-0.021208 \\
0.000000\end{array}$ & $\begin{array}{r}-0.020067 \\
0.000000\end{array}$ & $\begin{array}{r}-0.020508 \\
0.000000\end{array}$ & $\begin{array}{l}0.0255 \\
0.0000\end{array}$ \\
\hline 3 & 2 & 230 & 295 & $\begin{array}{l}\text { axial } \\
\text { hoop }\end{array}$ & $\begin{array}{r}-0.021279 \\
0.008564\end{array}$ & $\begin{array}{r}-0.019325 \\
0.008148\end{array}$ & $\begin{array}{r}-0.020261 \\
0.008328\end{array}$ & $\begin{array}{l}0.0483 \\
0.0256\end{array}$ \\
\hline
\end{tabular}


Table 3.2. Strain-at-failure statistics for subscale tubes tested at NIST [3.1] (continued).

\begin{tabular}{|c|c|c|c|c|c|c|c|c|}
\hline $\begin{array}{l}\text { Tube } \\
\text { Type }\end{array}$ & $\begin{array}{l}\text { Specimen } \\
\text { Number }\end{array}$ & $\begin{array}{c}\text { Tube } \\
\text { Length } \\
(\mathrm{mm})\end{array}$ & $\begin{array}{c}\text { Test } \\
\text { Temp. } \\
\text { (K) }\end{array}$ & $\begin{array}{c}\text { Direc- } \\
\text { tion }\end{array}$ & Maximum & Minimum & Average & C.V. \\
\hline 4 & 1 & 710 & 295 & $\begin{array}{l}\text { axial } \\
\text { hoop }\end{array}$ & $\begin{array}{r}-0.019770 \\
0.008276\end{array}$ & $\begin{array}{r}-0.017251 \\
0.006802\end{array}$ & $\begin{array}{r}-0.018253 \\
0.007442\end{array}$ & $\begin{array}{l}0.0668 \\
0.0849\end{array}$ \\
\hline 4 & 2 & 710 & 295 & $\begin{array}{l}\text { axial } \\
\text { hoop }\end{array}$ & $\begin{array}{r}-0.025008 \\
0.009617\end{array}$ & $\begin{array}{r}-0.012808 \\
0.007992\end{array}$ & $\begin{array}{r}-0.017325 \\
0.008511\end{array}$ & $\begin{array}{l}0.2758 \\
0.0688\end{array}$ \\
\hline 4 & 3 & 710 & 76 & $\begin{array}{l}\text { axial } \\
\text { hoop }\end{array}$ & $\begin{array}{r}-0.025000 \\
0.009094\end{array}$ & $\begin{array}{r}-0.020996 \\
0.008179\end{array}$ & $\begin{array}{r}-0.023999 \\
0.008618\end{array}$ & $\begin{array}{l}0.0722 \\
0.0428\end{array}$ \\
\hline 4 & 4 & 710 & 4 & $\begin{array}{l}\text { axial } \\
\text { hoop }\end{array}$ & $\begin{array}{r}-0.022803 \\
0.007813\end{array}$ & $\begin{array}{r}-0.021387 \\
0.006616\end{array}$ & $\begin{array}{r}-0.022306 \\
0.007390\end{array}$ & $\begin{array}{l}0.0357 \\
0.0908\end{array}$ \\
\hline 5 & 1 & 710 & 295 & $\begin{array}{l}\text { axial } \\
\text { hoop }\end{array}$ & $\begin{array}{r}-0.017693 \\
0.005584\end{array}$ & $\begin{array}{r}-0.014871 \\
0.005047\end{array}$ & $\begin{array}{r}-0.016504 \\
0.005252\end{array}$ & $\begin{array}{l}0.0886 \\
0.0553\end{array}$ \\
\hline 5 & 2 & 710 & 295 & $\begin{array}{l}\text { axial } \\
\text { hoop }\end{array}$ & $\begin{array}{r}-0.015845 \\
0.005078\end{array}$ & $\begin{array}{r}-0.013293 \\
0.003674\end{array}$ & $\begin{array}{r}-0.014646 \\
0.004382\end{array}$ & $\begin{array}{l}0.0773 \\
0.1101\end{array}$ \\
\hline 5 & 3 & 710 & 76 & $\begin{array}{l}\text { axial } \\
\text { hoop }\end{array}$ & $\begin{array}{r}-0.022327 \\
0.006738\end{array}$ & $\begin{array}{r}-0.018554 \\
0.005517\end{array}$ & $\begin{array}{r}-0.021041 \\
0.006095\end{array}$ & $\begin{array}{l}0.0680 \\
0.0974\end{array}$ \\
\hline 5 & 4 & 230 & 295 & $\begin{array}{l}\text { axial } \\
\text { hoop }\end{array}$ & $\begin{array}{r}-0.018223 \\
0.000000\end{array}$ & $\begin{array}{r}-0.014917 \\
0.000000\end{array}$ & $\begin{array}{r}-0.016611 \\
0.000000\end{array}$ & $\begin{array}{l}0.0827 \\
0.0000\end{array}$ \\
\hline 5 & 2 & 480 & 295 & $\begin{array}{l}\text { axial } \\
\text { hoop }\end{array}$ & $\begin{array}{r}-0.012583 \\
0.000000\end{array}$ & $\begin{array}{r}-0.011010 \\
0.000000\end{array}$ & $\begin{array}{r}-0.011822 \\
0.000000\end{array}$ & $\begin{array}{l}0.0673 \\
0.0000\end{array}$ \\
\hline 6 & 1 & 710 & 295 & $\begin{array}{l}\text { axial } \\
\text { hoop }\end{array}$ & $\begin{array}{r}-0.018896 \\
0.006238\end{array}$ & $\begin{array}{r}-0.014893 \\
0.004443\end{array}$ & $\begin{array}{r}-0.016864 \\
0.005526\end{array}$ & $\begin{array}{l}0.0907 \\
0.1180\end{array}$ \\
\hline 6 & 2 & 710 & 295 & $\begin{array}{l}\text { axial } \\
\text { hoop }\end{array}$ & $\begin{array}{r}-0.017285 \\
0.004834\end{array}$ & $\begin{array}{r}-0.013208 \\
0.002710\end{array}$ & $\begin{array}{r}-0.015084 \\
0.003896\end{array}$ & $\begin{array}{l}0.1006 \\
0.2069\end{array}$ \\
\hline 6 & 3 & 710 & 76 & $\begin{array}{l}\text { axial } \\
\text { hoop }\end{array}$ & $\begin{array}{r}-0.030835 \\
0.008691\end{array}$ & $\begin{array}{r}-0.022485 \\
0.005029\end{array}$ & $\begin{array}{r}-0.026904 \\
0.006826\end{array}$ & $\begin{array}{l}0.1409 \\
0.1901\end{array}$ \\
\hline 6 & 4 & 710 & 4 & $\begin{array}{l}\text { axial } \\
\text { hoop }\end{array}$ & $\begin{array}{r}-0.025269 \\
0.008838\end{array}$ & $\begin{array}{r}-0.024097 \\
0.007813\end{array}$ & $\begin{array}{r}-0.024683 \\
0.008431\end{array}$ & $\begin{array}{l}0.0237 \\
0.0645\end{array}$ \\
\hline
\end{tabular}


The strain data were transformed into axes aligned with each of the tube layer orientations according to the well-known transformation relation for planar engineering strains:

$\begin{array}{ccccc}\epsilon_{1} & \cos ^{2} \theta & \sin ^{2} \theta & \sin \theta \cos \theta & \epsilon_{\mathrm{x}} \\ \epsilon_{2}=\sin ^{2} \theta & \cos ^{2} \theta & -\sin \theta \cos \theta & \epsilon_{\mathrm{y}} \\ \gamma_{12} & -2 \sin \theta \cos \theta & 2 \sin \theta \cos \theta & \cos ^{2}-\sin ^{2} \theta & \gamma_{\mathrm{x} \theta}\end{array}$.

Here, $\epsilon_{x}, \epsilon_{y}$, and $\gamma_{x \theta}$ are the axial, hoop, and (engineering) twisting strains in the tube's axis system, respectively, and $\epsilon_{1}, \epsilon_{2}$, and $\gamma_{12}$ are the fiber-direction, transverse, and (engineering) shear strains in a layer oriented at an angle $\theta$ with respect to the tube's axis.

A key assumption enabling the use of the above transformation relation is that strains measured at a tube's surface are constant through the thickness of the tube. This assumption is not strictly true for thick tubes (such as the subscale test specimens), but the variation of strain through the thickness of a typical tube is much smaller than the observed variability of strain measurements, so through-thickness variations of strain were neglected in favor of the above, simplified analysis.

Alternative, stress-based measures of failure are more difficult to apply than the strain measures described above. Layer elastic properties are needed to convert strain data into stress predictions, and since these properties are somewhat uncertain (see §IX.4), additional uncertainty and scatter would arise in the study of failure stresses at the layer level. Instead, the present study focused on establishment of strain-to-failure limits as a possible criterion for further design studies and verification of tube capability.

Table 3.3 presents the results of the data analysis. The data presented correspond to averages taken across several specimens. Average, worst-case maximum, and worst-case minimum failure strains are listed along with the coefficients of variation for the samplings indicated.

For strength design of SMES support tubes, it is prudent to focus on the failure of the axially aligned layers as they carry the operative compressive loads. Off-axis layers are useful to reduce transverse strains and to stabilize the axial layers, but these effects will contribute primarily to increasing the apparent strength of the axial layers. Thus, if failure predictors can be developed based on the stress or strain state of the axially aligned layers (in the present case these are layers oriented at $\pm 10^{\circ}$ to the tube axis), then resulting designs will address the load-carrying capability of the tubes directly. 
Table 3.3(a). Strain at failure in $10^{\circ}$ layers from subscale tubes tested at NIST [3.1].

Tube types 1 and 2 (room temperature) - 26 specimens

$\begin{array}{llll}\text { Strains: } & \text { Fiber } & \text { Transverse } & \text { Shear } \\ \text { Max.: } & -0.010834 & 0.007403 & 0.008986 \\ \text { Min.: } & -0.017585 & 0.003647 & 0.005578 \\ \text { Ave.: } & -0.013334 & 0.005467 & 0.006842 \\ \text { C.V. (\%): } & 15.598 & 17.513 & 14.734\end{array}$

Tube types 1 and $2(76 \mathrm{~K})-6$ specimens

\begin{tabular}{llll} 
Strains: & Fiber & Transverse & \multicolumn{1}{c}{ Shear } \\
Max.: & -0.017171 & 0.005929 & 0.009038 \\
Min.: & -0.018905 & 0.004990 & 0.008224 \\
Ave.: & -0.018245 & 0.005350 & 0.008588 \\
C.V. (\%): & 3.462 & 6.100 & 3.234
\end{tabular}

Tube types 1 and $2(4 \mathrm{~K})-11$ specimens

$\begin{array}{llll}\text { Strains: } & \text { Fiber } & \text { Transverse } & \text { Shear } \\ \text { Max.: } & -0.013636 & 0.006395 & 0.009636 \\ \text { Min.: } & -0.020659 & 0.003902 & 0.006404 \\ \text { Ave.: } & -0.017044 & 0.005007 & 0.008026 \\ \text { C.V. (\%): } & 18.262 & 16.747 & 17.439\end{array}$

Tube types 3 and 4 (room temperature) - 29 specimens

$\begin{array}{llll}\text { Strains: } & \text { Fiber } & \text { Transverse } & \text { Shear } \\ \text { Max.: } & -0.009965 & 0.008914 & 0.011286 \\ \text { Min.: } & -0.024013 & 0.002974 & 0.004940 \\ \text { Ave.: } & -0.016154 & 0.006158 & 0.008120 \\ \text { C.V. (\%): } & 20.684 & 25.684 & 19.296\end{array}$

Tube types 3 and $4(76 \mathrm{~K})-8$ specimens

\begin{tabular}{llll} 
Strains: & Fiber & Transverse & \multicolumn{1}{c}{ Shear } \\
Max.: & -0.020095 & 0.008527 & 0.013770 \\
Min.: & -0.029304 & 0.007179 & 0.010220 \\
Ave.: & -0.024230 & 0.007842 & 0.011674 \\
C.V. (\%): & 10.490 & 6.056 & 8.703
\end{tabular}

Tube types 3 and $4(4 \mathrm{~K})-3$ specimens

\begin{tabular}{llcl} 
Strains: & Fiber & Transverse & \multicolumn{1}{c}{ Shear } \\
Max.: & -0.020543 & 0.006890 & 0.010472 \\
Min.: & -0.021880 & 0.005772 & 0.009578 \\
Ave.: & -0.021411 & 0.006494 & 0.010156 \\
C.V. (\%): & 3.516 & 9.651 & 4.944 \\
\hline
\end{tabular}


Table 3.3(a). Strain at failure in $10^{\circ}$ layers from subscale tubes tested at NIST [3.1] (continued).

Tube types 5 and 6 (room temperature) - 21 specimens

$\begin{array}{llcc}\text { Strains: } & \text { Fiber } & \text { Transverse } & \text { Shear } \\ \text { Max.: } & -0.012674 & 0.005480 & 0.008596 \\ \text { Min.: } & -0.018138 & 0.002179 & 0.006020 \\ \text { Ave.: } & -0.015056 & 0.004080 & 0.006966 \\ \text { C.V. (\%): } & 10.405 & 20.958 & 10.509\end{array}$

Tube types 5 and $6(76 \mathrm{~K})$ - 11 specimens

$\begin{array}{llll}\text { Strains: } & \text { Fiber } & \text { Transverse } & \text { Shear } \\ \text { Max.: } & -0.017791 & 0.007721 & 0.012850 \\ \text { Min.: } & -0.029702 & 0.003970 & 0.008650 \\ \text { Ave.: } & -0.022797 & 0.005519 & 0.010306 \\ \text { C.V. (\%): } & 17.106 & 17.855 & 13.462\end{array}$

Tube types 5 and $6(4 \mathrm{~K})-3$ specimens

\begin{tabular}{llll} 
Strains: & Fiber & Transverse & \multicolumn{1}{c}{ Shear } \\
Max.: & -0.023135 & 0.007827 & 0.011598 \\
Min.: & -0.024246 & 0.006851 & 0.010914 \\
Ave.: & -0.023684 & 0.007432 & 0.011326 \\
C.V. (\%): & 2.347 & 6.920 & 3.203
\end{tabular}

All Tubes (room temperature) - 76 specimens

$\begin{array}{llcl}\text { Strains: } & \text { Fiber } & \text { Transverse } & \text { Shear } \\ \text { Max.: } & -0.009965 & 0.008914 & 0.011286 \\ \text { Min.: } & -0.024013 & 0.002179 & 0.004940 \\ \text { Ave.: } & -0.014886 & 0.005347 & 0.007364 \\ \text { C.V. (\%): } & 18.678 & 27.375 & 18.008\end{array}$

All Tubes $(76 \mathrm{~K})$ - 25 specimens

$\begin{array}{llcc}\text { Strains: } & \text { Fiber } & \text { Transverse } & \text { Shear } \\ \text { Max.: } & -0.017171 & 0.008527 & 0.013770 \\ \text { Min.: } & -0.029702 & 0.003970 & 0.008224 \\ \text { Ave.: } & -0.022163 & 0.006222 & 0.010332 \\ \text { C.V. (\%): } & 16.731 & 21.471 & 15.242\end{array}$

All Tubes (4 K) - 17 specimens

\begin{tabular}{llcl} 
Strains: & Fiber & Transverse & \multicolumn{1}{c}{ Shear } \\
Max.: & -0.013636 & 0.007827 & 0.011598 \\
Min.: & -0.024246 & 0.003902 & 0.006404 \\
Ave.: & -0.018986 & 0.005698 & 0.008984 \\
C.V. (\%): & 19.707 & 21.726 & 19.878 \\
\hline
\end{tabular}


Table 3.3(a). Strain at failure in $10^{\circ}$ layers from subscale tubes tested at NIST [3.1] (continued).

All Tubes $(76 \mathrm{~K}, 4 \mathrm{~K})-42$ specimens

$\begin{array}{llcc}\text { Strains: } & \text { Fiber } & \text { Transverse } & \text { Shear } \\ \text { Max.: } & -0.013636 & 0.008527 & 0.013770 \\ \text { Min.: } & -0.029702 & 0.003902 & 0.006404 \\ \text { Ave.: } & -0.020877 & 0.006010 & 0.009786 \\ \text { C.V. (\%): } & 19.162 & 21.762 & 18.119\end{array}$

Tube types $1,2,5$, and 6 (room temperature) - 47 specimens

$\begin{array}{llcc}\text { Strains: } & \text { Fiber } & \text { Transverse } & \text { Shear } \\ \text { Max.: } & -0.010834 & 0.007403 & 0.008986 \\ \text { Min.: } & -0.018138 & 0.002179 & 0.005578 \\ \text { Ave.: } & -0.014103 & 0.004847 & 0.006898 \\ \text { C.V. (\%): } & 14.475 & 23.538 & 12.880\end{array}$

Tube types $1,2,5$, and $6(76 \mathrm{~K}, 4 \mathrm{~K})-31$ specimens

$\begin{array}{llll}\text { Strains: } & \text { Fiber } & \text { Transverse } & \text { Shear } \\ \text { Max.: } & -0.013636 & 0.007827 & 0.012850 \\ \text { Min.: } & -0.029702 & 0.003902 & 0.006404 \\ \text { Ave.: } & -0.019961 & 0.005490 & 0.009264 \\ \text { C.V. (\%): } & 20.308 & 18.757 & 18.042\end{array}$


Table 3.3(b). Strain at failure in $45^{\circ}$ layers from subscale tubes tested at NIST [3.1].

\begin{tabular}{lccc}
\hline \hline & \multicolumn{4}{l}{ Tube types 3 and 4 (room temperature) -29 specimens } \\
Strains: & Fiber & Transverse & Shear \\
Max.: & -0.002038 & -0.002037 & 0.033000 \\
Min.: & -0.008508 & -0.008508 & 0.014440 \\
Ave.: & -0.004998 & -0.004998 & 0.023744 \\
C.V. $(\%):$ & 29.668 & 29.668 & 19.296
\end{tabular}

Tube types 3 and $4(76 \mathrm{~K})-8$ specimens

\begin{tabular}{llll} 
Strains: & \multicolumn{1}{c}{ Fiber } & Transverse & \multicolumn{1}{c}{ Shear } \\
Max.: & -0.006055 & -0.006054 & 0.040260 \\
Min.: & -0.010389 & -0.010388 & 0.029882 \\
Ave.: & -0.008194 & -0.008194 & 0.034132 \\
C.V. (\%): & 14.416 & 14.416 & 8.703
\end{tabular}

Tube types 3 and $4(4 \mathrm{~K})-3$ specimens

\begin{tabular}{llll} 
Strains: & Fiber & Transverse & \multicolumn{1}{c}{ Shear } \\
Max.: & -0.007386 & -0.007385 & 0.030616 \\
Min.: & -0.007495 & -0.007495 & 0.028004 \\
Ave.: & -0.007458 & -0.007458 & 0.029696 \\
C.V. (\%): & 0.846 & 0.846 & 4.944 \\
\hline
\end{tabular}


Table 3.3(c). Strain at failure in $82^{\circ}$ layers from subscale tubes tested at NIST [3.1].

\begin{tabular}{|c|c|c|c|}
\hline \multicolumn{4}{|c|}{ All tubes (room temperature) - 50 specimens } \\
\hline Strains: & Fiber & Transverse & Shear \\
\hline Max.: & 0.009047 & -0.010047 & 0.01019 \\
\hline Min.: & 0.002279 & -0.024200 & 0.004462 \\
\hline ve.: & 0.005412 & -0.015820 & 0.006898 \\
\hline V. $(\%)$ & 31.252 & 17.655 & 18.307 \\
\hline
\end{tabular}

All tubes $(76 \mathrm{~K})-19$ specimens

$\begin{array}{llll}\text { Strains: } & \text { Fiber } & \text { Transverse } & \text { Shear } \\ \text { Max.: } & 0.008756 & -0.017935 & 0.012440 \\ \text { Min.: } & 0.004170 & -0.029916 & 0.007816 \\ \text { Ave.: } & 0.006678 & -0.023581 & 0.009832 \\ \text { C.V. (\%): } & 21.435 & 14.466 & 12.838\end{array}$

All tubes $(4 \mathrm{~K})-6$ specimens

\begin{tabular}{llll} 
Strains: & Fiber & Transverse & \multicolumn{1}{c}{ Shear } \\
Max.: & 0.008018 & -0.020702 & 0.010480 \\
Min.: & 0.005931 & -0.024439 & 0.008654 \\
Ave.: & 0.007142 & -0.022726 & 0.009704 \\
C.V. (\%): & 10.333 & 6.121 & 6.989
\end{tabular}

Tube types 3 and 4 (room temperature) - 29 specimens

$\begin{array}{llll}\text { Strains: } & \text { Fiber } & \text { Transverse } & \text { Shear } \\ \text { Max.: } & 0.009047 & -0.010047 & 0.010198 \\ \text { Min.: } & 0.003066 & -0.024200 & 0.004462 \\ \text { Ave.: } & 0.006292 & -0.016289 & 0.007338 \\ \text { C.V. (\%): } & 25.435 & 20.664 & 19.296\end{array}$

Tube types 3 and $4(76 K)-8$ specimens

\begin{tabular}{llll} 
Strains: & \multicolumn{1}{c}{ Fiber } & Transverse & \multicolumn{1}{c}{ Shear } \\
Max.: & 0.008756 & -0.020265 & 0.012440 \\
Min.: & 0.007367 & -0.029533 & 0.009234 \\
Ave.: & 0.008036 & -0.024424 & 0.010548 \\
C.V. (\%): & 6.036 & 10.475 & 8.703
\end{tabular}

Tube types 3 and $4(4 K)-3$ specimens

\begin{tabular}{llll} 
Strains: & Fiber & Transverse & \multicolumn{1}{c}{ Shear } \\
Max.: & 0.007064 & -0.020702 & 0.009460 \\
Min.: & 0.005931 & -0.022054 & 0.008654 \\
Ave.: & 0.006663 & -0.021580 & 0.009176 \\
C.V. (\%): & 9.532 & 3.527 & 4.944 \\
\hline
\end{tabular}


Table 3.3(c). Strain at failure in $82^{\circ}$ layers from subscale tubes tested at NIST [3.1] (continued).

Tube types 5 and 6 (room temperature) - 21 specimens

$\begin{array}{llll}\text { Strains: } & \text { Fiber } & \text { Transverse } & \text { Shear } \\ \text { Max.: } & 0.005623 & -0.012775 & 0.007766 \\ \text { Min.: } & 0.002279 & -0.018281 & 0.005440 \\ \text { Ave.: } & 0.004196 & -0.015172 & 0.006292 \\ \text { C.V. (\%): } & 20.576 & 10.399 & 10.509\end{array}$

Tube types 5 and $6(76 \mathrm{~K})-11$ specimens

$\begin{array}{llll}\text { Strains: } & \text { Fiber } & \text { Transverse } & \text { Shear } \\ \text { Max.: } & 0.007904 & -0.017935 & 0.011610 \\ \text { Min.: } & 0.004170 & -0.029916 & 0.007816 \\ \text { Ave.: } & 0.005690 & -0.022969 & 0.009312 \\ \text { C.V. (\%): } & 17.338 & 17.076 & 13.462\end{array}$

Tube types 5 and $6(4 \mathrm{~K})-3$ specimens

\begin{tabular}{llll} 
Strains: & Fiber & Transverse & \multicolumn{1}{c}{ Shear } \\
Max.: & 0.008018 & -0.023316 & 0.010480 \\
Min.: & 0.007032 & -0.024439 & 0.009860 \\
Ave.: & 0.007621 & -0.023873 & 0.010232 \\
C.V. (\%): & 6.823 & 2.352 & 3.203
\end{tabular}

Tube types 5 and $6(76 \mathrm{~K}, 4 \mathrm{~K})-14$ specimens

\begin{tabular}{llll} 
Strains: & \multicolumn{1}{c}{ Fiber } & Transverse & Shear \\
Max.: & 0.008018 & -0.017935 & 0.011610 \\
Min.: & 0.004170 & -0.029916 & 0.007816 \\
Ave.: & 0.006104 & -0.023162 & 0.009510 \\
C.V. (\%): & 19.838 & 14.974 & 12.350
\end{tabular}


A side benefit of using strain-based failure criteria is that they provide a means by which to interrogate a tube's construction nondestructively. By using the strain transformation in Equation 3.1, ratios of fiber- and shear-direction strains to transverse layer strains can be predicted for a layer wound at an angle $\varphi$ to the tube axis by using only the measured tube Poisson's ratio $\left(\nu_{\mathrm{x} \theta}\right)$ as a parameter:

$$
\begin{aligned}
-\frac{\epsilon_{L}}{\epsilon_{T}} & =\frac{\nu_{x \theta} \sin ^{2} \varphi-\cos ^{2} \varphi}{\sin ^{2} \varphi-\nu_{x \theta} \cos ^{2} \varphi} \\
\frac{\gamma_{L T}}{\epsilon_{T}} & =\frac{2\left(1+\nu_{x \theta}\right) \sin \varphi \cos \varphi}{\nu_{x \theta} \cos ^{2} \varphi-\sin ^{2} \varphi}
\end{aligned}
$$

Thus, as limiting strain values are established, the allowable strength of any tube can be predicted from a low-load, elastic test in which tube axial and hoop strains are measured versus applied load. The measured Poisson's ratio can then be used to predict which strain component will exceed its allowable value first in the as-constructed tube. This strain component can be calibrated with respect to the applied load to arrive at the prediction of allowable strength. For such a scheme to work in practice, sufficiently conservative allowable strain limits must be established a priori.

For the specimens tested at room temperature, the averaged ultimate compressive fiber-direction strain in $10^{\circ}$ layers varied from $1.33 \%$ to $1.61 \%$. The averaged ultimate transverse strain in the $10^{\circ}$ layers was between $0.41 \%$ and $0.61 \%$. The averaged (engineering) shear strain in the $10^{\circ}$ layers varied from $0.68 \%$ to $0.81 \%$. Much greater variations were noted in the maximum and minimum failure-strain values. The experimental variation is illustrated graphically in Figures 3.1 and 3.2, which show fiber and shear strains at failure in $10^{\circ}$ layers plotted against transverse layer strains. These figures show fiber strains at failure ranging from $1 \%$ to nearly $2.5 \%$, transverse strains at failure between $0.2 \%$ and $0.9 \%$, and shear strains at failure from $0.5 \%$ to $1.1 \%$. Data from cryogenic tube tests show generally increased strain levels at failure, although with the large scatter and small specimen size, meaningful statistics are difficult to extract.

Review of the failure strain data indicates that possible mechanisms of failure in the tested tubes are related to some limiting value of fiber-direction strain and/or some combination of transverse tension and shear strains. The lower-bound $1 \%$ fiber strain appears at first look to be a useful criterion for design, although the $\left[ \pm 10_{3} / 82\right]$ lay-up tubes offer an empirically higher allowable of roughly $1.25 \%$. Thus, a $1 \%$ fiber strain allowable may be overly conservative by up to $25 \%$. Limiting layer shear strain to values below $0.5 \%$ also appears to provide a conservative lower bound for design. As operating temperature decreases, both fiber and matrix strain allowables appear to increase to roughly $1.3 \%$ and $0.7 \%$ strain, respectively.

An interesting aspect of the failure strain data is that matrix transverse tension and shear strains tend to be coupled, in that transverse compression could support much higher shear strains at failure than transverse tension. Therefore, a combined-loading allowable formulation (e.g., Tsai-Wu tensor theory) could prove more determinative in the establishment of strain allowables than the maximum-strain limitations studied here. This should be explored through more extensive analysis of the failure strain data, although a major difficulty in using Tsai-Wu theory is that it is stress-tensor based, and the layer stresses can be evaluated from strain data only by using postulated values of layer-material elastic properties. 


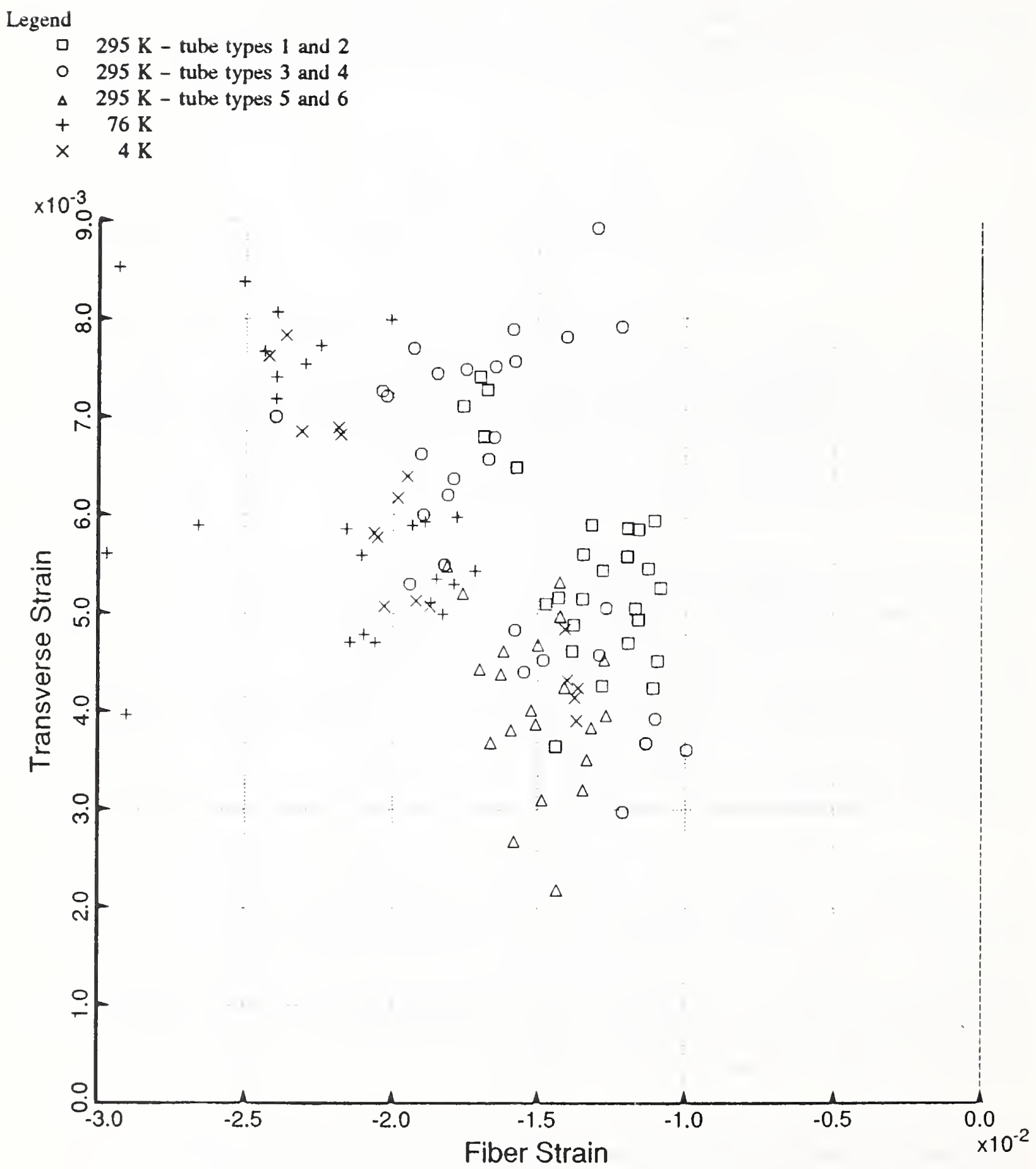

Figure 3.1. Fiber failure strains in $10^{\circ}$ layers from subscale SMES tube tests. 
Legend

D $295 \mathrm{~K}$ - tube types 1 and 2

- $295 \mathrm{~K}$ - tube types 3 and 4

$\triangle 295 \mathrm{~K}$ - tube types 5 and 6

$+76 \mathrm{~K}$

$\times \quad 4 \mathrm{~K}$

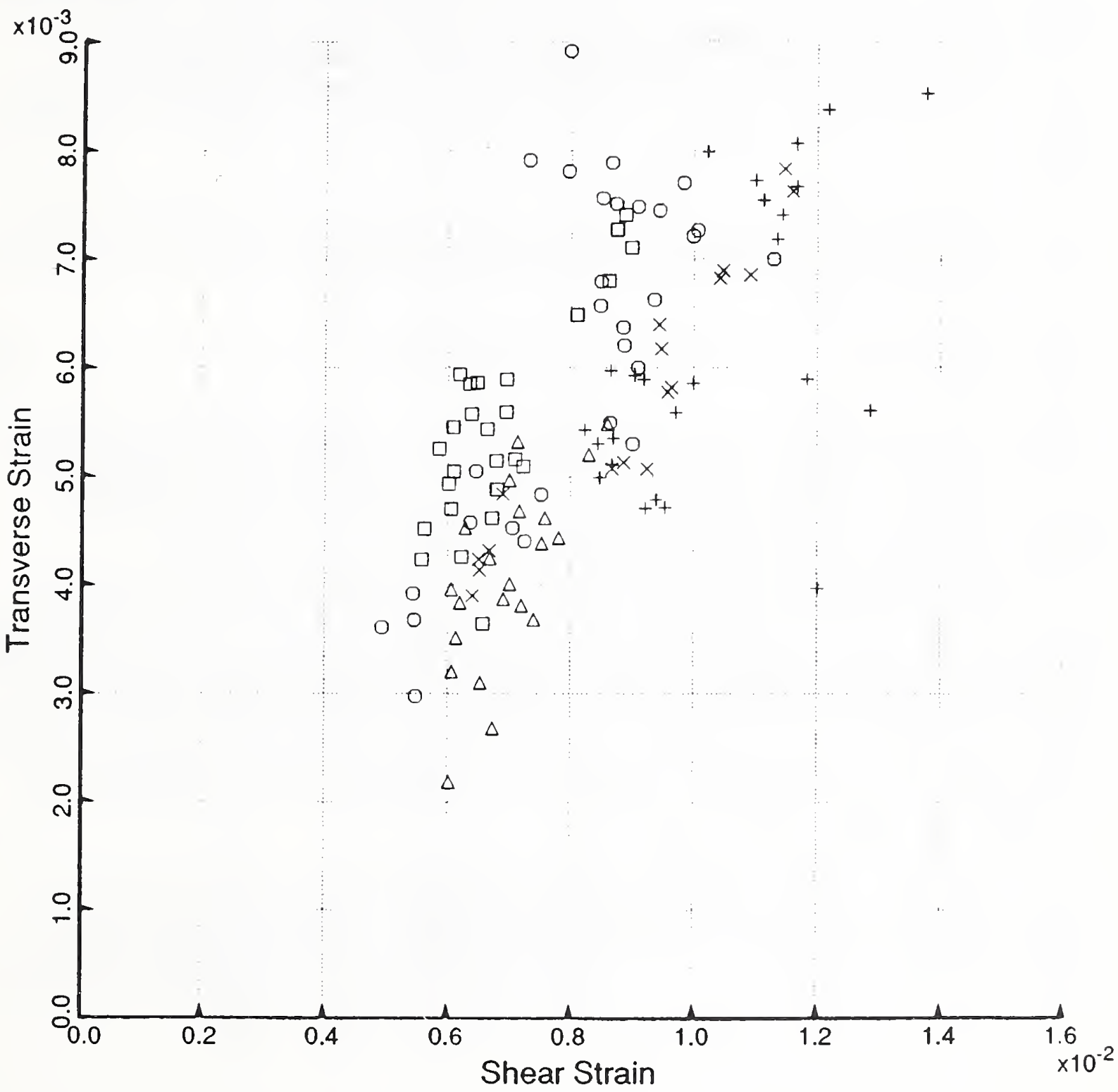

Figure 3.2. Shear failure strains in $10^{\circ}$ layers from subscale SMES tube tests. 


\section{Material Elastic Properties Estimation}

Design of SMES support tubes requires some knowledge of material elastic and strength properties. Although the strength properties can be assessed directly from failure strain data (in the context of a maximum-strain failure criterion), material elastic properties can be inferred only from tests of tube constructions. This is because one must characterize a minimum of four elastic constants in order to predict the elastic response of the orthotropic fiberglass tube materials. Estimation of the four elastic constants-fiber-direction modulus $\left(E_{L}\right)$, transverse modulus $\left(E_{T}\right)$, shear modulus $\left(G_{L T}\right)$ and Poisson's ratio $\left(\nu_{L T}\right)$-enables a transversely isotropic theory of orthotropic materials to be invoked in design of tube constructions. Unfortunately, measurement of these quantities is nearly impossible through tube testing because the layer properties are obscured in the measured composite properties.

To address this problem, a best-fit procedure was developed to estimate layer elastic properties for tested tubes of known construction. This procedure varies postulated layer elastic properties according to a gradient-descent algorithm derived to minimize $J$ :

$$
J=\left(\frac{E_{x}}{E_{0}}-1\right)^{2}+\left[\frac{\nu_{x \theta}}{\nu_{0}}-1\right)^{2},
$$

where $E_{x}$ and $\nu_{x \theta}$ are computed from postulated layer properties according to laminated plate theory, and $E_{0}, \nu_{0}$ are test data. Thus, the properties $\left(E_{L}, E_{T}, G_{L T}\right.$, and $\left.\nu_{L T}\right)$ predicted by the method are those which most closely predict the observed behavior of the tube under test. The FORTRAN program that implements this method for computation is listed in Appendix B (§IX.8).

Because the properties are underdetermined, some uncertainty is present in all predictions. Indeed, the procedure is not even guaranteed to converge to globally minimum errors, but will instead minimize the error between predicted and measured values in a neighborhood around the initial guess. However, experience with the method has shown surprisingly good performance in predicting fiberdirection modulus $\left(E_{L}\right)$ and major Poisson's ratio $\left(\nu_{L T}\right)$, and somewhat larger uncertainty in predicting transverse $\left(E_{T}\right)$ and shear $\left(G_{L T}\right)$ moduli. This result is not surprising since the measured quantities depend most directly on fiber-direction modulus and major Poisson's ratio in the axially dominant tube constructions tested to date.

Material elastic properties extracted using the method are listed in Table 4.1. Measured moduli and Poisson's ratios are averages taken from Reference 3.1. The moduli predicted for tube types $5,6,7$, and 9 were based on the assumption that $92.9 \%$ of the tube was wound at $\pm 10^{\circ}$, rather than the amount predicted by the lay-up designation, which would have been $85.7 \%$ at $\pm 10^{\circ}$. This assumption is borne out by the micrographic examinations described in §IX.2. 
Table 4.1. Measured and predicted material elastic properties for subscale tubes tested at NIST.

\begin{tabular}{|c|c|c|c|c|c|c|c|c|c|c|}
\hline \multirow{2}{*}{$\begin{array}{l}\text { Tube } \\
\text { Type }\end{array}$} & \multirow{2}{*}{$\begin{array}{l}\text { Specimen } \\
\text { Number }\end{array}$} & \multirow{2}{*}{$\begin{array}{c}\text { Tube } \\
\text { Length } \\
(\mathrm{mm}) \\
\end{array}$} & \multirow{2}{*}{$\begin{array}{c}\text { Test } \\
\text { Temp. } \\
(\mathrm{K}) \\
\end{array}$} & \multicolumn{3}{|c|}{ Measured [3.1] } & \multicolumn{4}{|c|}{ Predicted Values } \\
\hline & & & & $E_{0}^{*}$ & $\nu_{0}$ & $E_{L}{ }^{*}$ & $E_{T}^{*}$ & $G_{L T}^{*}$ & $\nu_{L T}$ & $J$ \\
\hline 1 & 1 & 230 & 295 & 38.621 & 0.470 & 40.441 & 5.451 & 1.259 & 0.261 & $9.980 \mathrm{E}-09$ \\
\hline 1 & 2 & 230 & 295 & 39.310 & 0.430 & 41.041 & 6.014 & 2.043 & 0.256 & 8.668E-09 \\
\hline 2 & 1 & 710 & 295 & 36.552 & 0.360 & 37.903 & 6.847 & 3.405 & 0.247 & $9.510 \mathrm{E}-09$ \\
\hline 2 & 2 & 710 & 295 & 35.862 & 0.380 & 37.276 & 6.448 & 2.740 & 0.249 & $9.908 \mathrm{E}-09$ \\
\hline 2 & 3 & 710 & 76 & 40.000 & 0.350 & 41.414 & 7.372 & 4.273 & 0.248 & $9.934 \mathrm{E}-09$ \\
\hline 2 & 4 & 710 & 4 & 42.069 & 0.350 & 43.552 & 7.579 & 4.603 & 0.248 & $8.691 \mathrm{E}-09$ \\
\hline 2 & 5 & 710 & 4 & 42.069 & 0.360 & 43.607 & 7.379 & 4.257 & 0.249 & 8.592E-09 \\
\hline 1 & 1 & 710 & 295 & 40.000 & 0.360 & 41.462 & 7.179 & 3.939 & 0.249 & $9.028 \mathrm{E}-09$ \\
\hline 1 & 2 & 710 & 295 & 37.241 & 0.430 & 38.890 & 5.838 & 1.801 & 0.255 & $8.448 \mathrm{E}-09$ \\
\hline 1 & 3 & 710 & 295 & 35.862 & 0.380 & 37.276 & 6.448 & 2.740 & 0.249 & $9.908 \mathrm{E}-09$ \\
\hline 3 & 2 & 230 & 295 & 22.069 & 0.400 & 36.841 & 4.221 & 0.720 & 0.242 & $9.974 \mathrm{E}-09$ \\
\hline 4 & 1 & 710 & 295 & 22.759 & 0.340 & 37.172 & 4.139 & 2.023 & 0.243 & $9.278 \mathrm{E}-09$ \\
\hline 4 & 2 & 710 & 295 & 25.517 & 0.390 & 40.924 & 6.910 & 1.368 & 0.253 & $9.667 \mathrm{E}-09$ \\
\hline 4 & 3 & 710 & 76 & 31.034 & 0.370 & 47.090 & 11.152 & 2.832 & 0.265 & $9.103 \mathrm{E}-09$ \\
\hline 4 & 4 & 710 & 4 & 33.103 & 0.360 & 49.297 & 12.703 & 3.560 & 0.267 & 9.804E-09 \\
\hline 3 & 1 & 710 & 295 & 26.207 & 0.330 & 41.097 & 6.777 & 2.961 & 0.250 & 9.939E-09 \\
\hline 3 & 2 & 710 & 295 & 24.138 & 0.360 & 38.986 & 5.468 & 1.846 & 0.247 & 9.738E-09 \\
\hline 3 & 3 & 710 & 295 & 24.828 & 0.340 & 39.600 & 5.793 & 2.429 & 0.248 & $9.178 \mathrm{E}-09$ \\
\hline 5 & 3 & 230 & 295 & 40.690 & 0.320 & 45.152 & 7.566 & 1.425 & 0.258 & 8.907E-09 \\
\hline 6 & 1 & 710 & 295 & 37.931 & 0.280 & 41.848 & 6.972 & 3.506 & 0.250 & 8.485E-09 \\
\hline 6 & 2 & 710 & 295 & 38.621 & 0.270 & 42.545 & 7.021 & 4.167 & 0.249 & $8.635 \mathrm{E}-09$ \\
\hline 6 & 3 & 710 & 76 & 41.379 & 0.280 & 45.641 & 7.600 & 3.872 & 0.251 & 9.303E-09 \\
\hline 6 & 4 & 710 & 4 & 41.379 & 0.300 & 45.772 & 7.697 & 2.637 & 0.254 & $9.849 \mathrm{E}-09$ \\
\hline 5 & 1 & 710 & 295 & 42.759 & 0.320 & 47.441 & 7.938 & 1.519 & 0.258 & $9.315 \mathrm{E}-09$ \\
\hline 5 & 2 & 710 & 295 & 42.069 & 0.300 & 46.538 & 7.821 & 2.689 & 0.254 & $9.449 \mathrm{E}-09$ \\
\hline 5 & 3 & 710 & 76 & 47.586 & 0.330 & 52.876 & 8.752 & 1.084 & 0.261 & 8.822E-09 \\
\hline 7 & 1 & 710 & 295 & 29.655 & 0.290 & 34.731 & 7.731 & 0.000 & 0.276 & $1.231 \mathrm{E}-06$ \\
\hline 9 & 1 & 710 & 295 & 37.241 & 0.280 & 43.738 & 9.372 & 0.006 & 0.262 & $8.816 \mathrm{E}-09$ \\
\hline 7 & 2 & 710 & 295 & 31.724 & 0.280 & 37.352 & 7.690 & 0.000 & 0.264 & $2.709 \mathrm{E}-07$ \\
\hline 9 & 2 & 710 & 295 & 35.172 & 0.290 & 41.062 & 9.545 & 0.000 & 0.274 & $1.572 \mathrm{E}-08$ \\
\hline 7 & 3 & 710 & 295 & 28.966 & 0.240 & 34.297 & 5.548 & 1.633 & 0.251 & 9.864E-09 \\
\hline 9 & 3 & 710 & 295 & 33.103 & 0.300 & 38.428 & 9.510 & 0.000 & 0.285 & 9.685E-09 \\
\hline
\end{tabular}

* Modulus values listed are in GPa. 
Examination of Table 4.1 shows an average predicted fiber-direction modulus for roomtemperature tests of $39.86 \mathrm{GPa}$ with an $8 \%$ coefficient of variation. The other predictions are somewhat less uniform, although the predicted Poisson's ratio is very close to 0.25 . Higher moduli are predicted for cryogenic tests, and a Poisson's ratio near 0.27 is predicted for tubes types 7 and 9 . Anomalous values appear for specimens 1,2, and 3 of tube type 5, which, owing to their very high measured moduli, predict much higher material moduli than the average (two standard deviations above the mean).

On the basis of the predictions listed in Table 4.1, the values in Table 4.2 were used for material elastic properties in design and modeling studies:

Table 4.2. Predicted layer elastic properties.

\begin{tabular}{cc}
\hline Property & \multicolumn{1}{c}{ Value } \\
\hline$E_{L}$ & $39.1 \mathrm{GPa}(5.68 \mathrm{Msi})$ \\
$E_{T}$ & $5.96 \mathrm{GPa}(865 \mathrm{ksi})$ \\
$G_{L T}$ & $2.25 \mathrm{GPa}(327 \mathrm{ksi})$ \\
$\nu_{L T}$ & 0.250 \\
\hline
\end{tabular}

\section{Tube Design Studies}

By using the maximum strain failure criterion and strain allowable limits spanning the range of those observed in tube tests at NIST, tube designs minimizing cross-sectional area were derived using the PANDA optimization program [5.1]. Optimal, 10-cm-diameter tube lay-ups were derived for an applied axial compression of $22.27 \mathrm{MN}(500,000 \mathrm{lbf})$ by using the elastic properties described in §IX.4 and varying fiber-direction strain allowables from $1.3 \%$ to $1.7 \%$ and transverse-direction strain allowables from $0.4 \%$ to $0.6 \%$. Tube-winding angles were constrained to lie between $10^{\circ}$ and $82^{\circ}$, and a discrete layer thickness of $0.40 \mathrm{~mm}(0.016 \mathrm{in})$ was enforced.

The PANDA program optimizes designs based on a minimum-weight objective (in this case, minimum cross-sectional area) with allowable strain values input as design constraints. Layer thicknesses and winding angles are varied according to a constrained, gradient-based optimization method known as the Method of Feasible Directions. PANDA uses the CONMIN optimization program developed by G. N. Vanderplaats [5.2]. CONMIN is used widely throughout the aerospace industry for design optimization. A further refinement was introduced into the PANDA program to produce designs that obeyed the $0.40-\mathrm{mm}$ single-layer thickness constraint. Hence, the designs provided by the PANDA studies should be able to be built with current methods.

Note that optimization for minimum cross-sectional area implies minimization of thermal conductivity for designs that are dominated by axial loadings, as the SMES tubes are. However, material characteristics may indicate slightly greater winding angles if conductivity is used as the design objective. Since increasing the winding angles places a premium on off-axis material strength, and since the variability of material strength is so great as seen from the subscale tube tests, no explicit optimization for minimal thermal conductivity was done. 
Figures 5.1 through 5.3 show optimum tube wall thicknesses for $\left[ \pm 10^{\circ}{ }_{n} / \pm \theta_{m}\right]$ tube lay-ups given fiber and transverse failure strain constraints. The fiber strain allowable values are listed in the legends of these figures by the " $\mathrm{L}=\mathrm{x} . \mathrm{xx} \%$ " values, and the transverse strain allowables are listed in the legends as the "T=x.xx\%" values. The notable aspect of these results is that minimum-thickness tubes result from combinations of $10^{\circ}$ and $82^{\circ}$ layers until strain allowables get very high. For allowable transverse strains of $0.6 \%$ and allowable fiber strains of $1.7 \%$, the optimal design comprises only $10^{\circ}$ layers.

Figures 5.4 through 5.6 show the ratio of $10^{\circ}$ ("axial") layers to off-axis angle-wound layers for different off-axis winding angles and failure strain allowables. This is the ratio $\mathrm{n} / \mathrm{m}$ in the laminate designation $\left[ \pm 10_{n} / \pm \theta_{m}\right]$. Some variation of this ratio appears for winding angles between $20^{\circ}$ and $35^{\circ}$. This is due to the sensitivity of designs in this regime to local minima and the interactions between the postulated transverse and shear strain allowables. Nonetheless, these figures clearly show that wall thickness is reduced as fiber strain allowables are increased.

Figures 5.7 through 5.9 indicate the critical constraint that drives the design optimum. Values of +2 in these figures indicate that the transverse tensile strain allowable is the critical limit, whereas values of -1 indicate that fiber compressive strain allowables are critical. Generally, designs with off-axis winding angles in excess of $60^{\circ}$ show the fiber strain limit to be the controlling factor, and designs using off-axis angles in the $20^{\circ}$ to $50^{\circ}$ range are controlled by transverse strain limits. As transverse tensile strain allowables become very high, it becomes possible to develop fiber-dominated designs in the range of off-axis angles from $20^{\circ}$ to $50^{\circ}$.

Figure 5.10 summarizes the results of the design study by showing that minimum wall thicknesses are generally lower when high fiber strain allowables are employed to provide fiber-dominated designs. Off-axis wound layers are needed to achieve these large fiber strains without violating transverse tensile strain limits. Thus, the best designs appear to be axially dominated with few hoop-wound layers to reduce the transverse tension fields. This indicates that the designs currently being pursued by NIST $\left(\left[ \pm 10_{n} / \pm 82\right]\right)$ and pultruded designs in which the angle of the axial layers can be reduced even further offer the best prospects for maximum strength and minimum conductivity.

The design study described above did not address directly the question raised regarding the interaction of layer transverse tensile and shear strain allowables. This interaction should be pursued further, as it may indicate that slightly lower off-axis angles are more beneficial due to their effect on transverse shearing strains. Indeed, this effect may be observed in the generally higher shear strains at failure in the $10^{\circ}$ layers of tubes with $45^{\circ}$ off-axis layers. In any event, the PANDA optimization procedure may be applied equally well to a more extensive study in which shear-strain allowable values serve as parameters for derivation of candidate designs. 


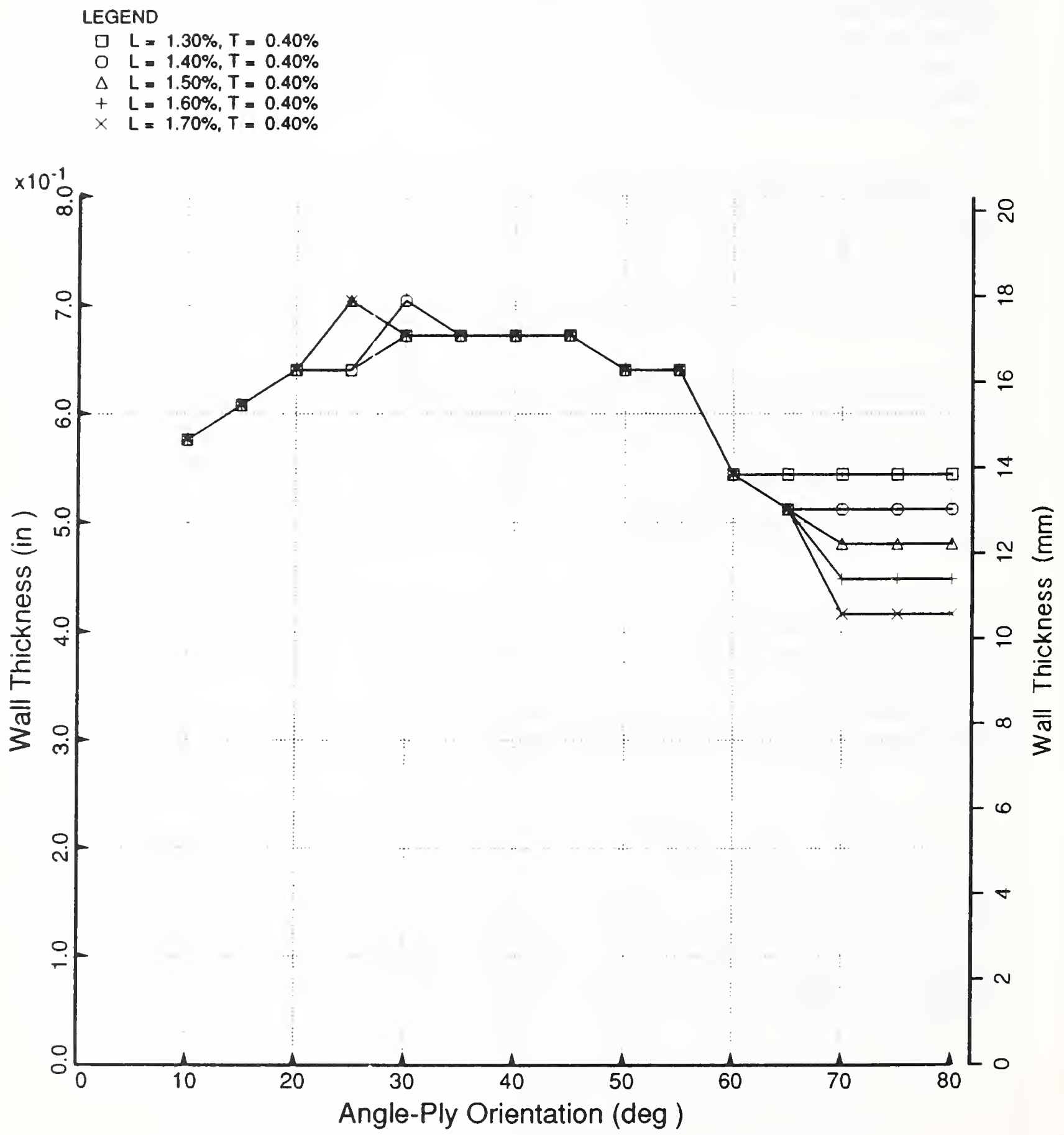

Figure 5.1. Optimum tube wall thicknesses for $\left[ \pm 10^{\circ}(n) / \pm \theta(m)\right]$ tube lay-ups with a transverse $(T)$ failure strain constraint of $0.40 \%$ and different fiber failure strain constraints $(L)$. 

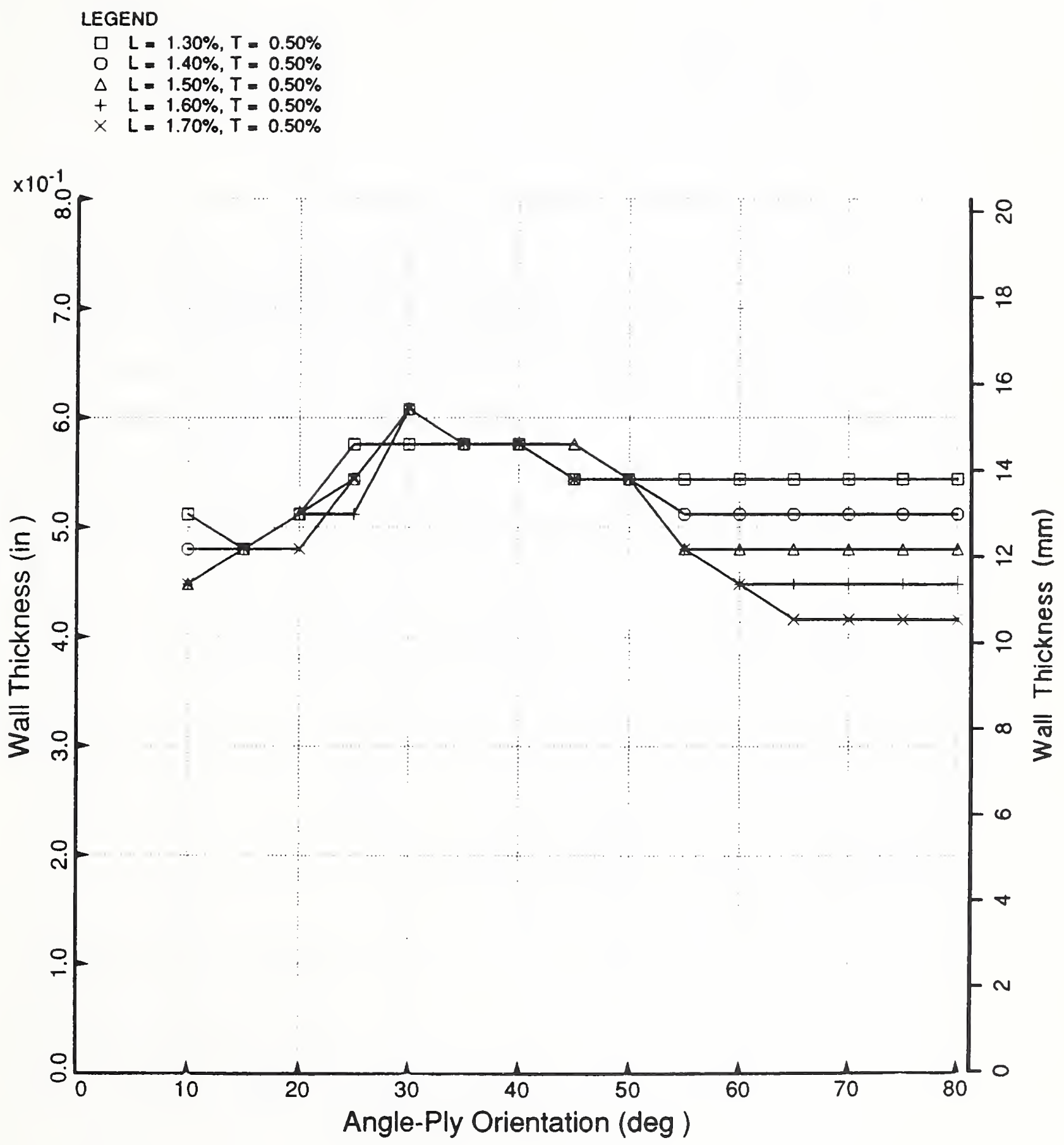

Figure 5.2. Optimum tube wall thicknesses for $\left[ \pm 10^{\circ}(n) / \pm \theta(m)\right]$ tube lay-ups with a transverse $(T)$ failure strain constraint of $0.50 \%$ and different fiber failure strain constraints $(L)$. 

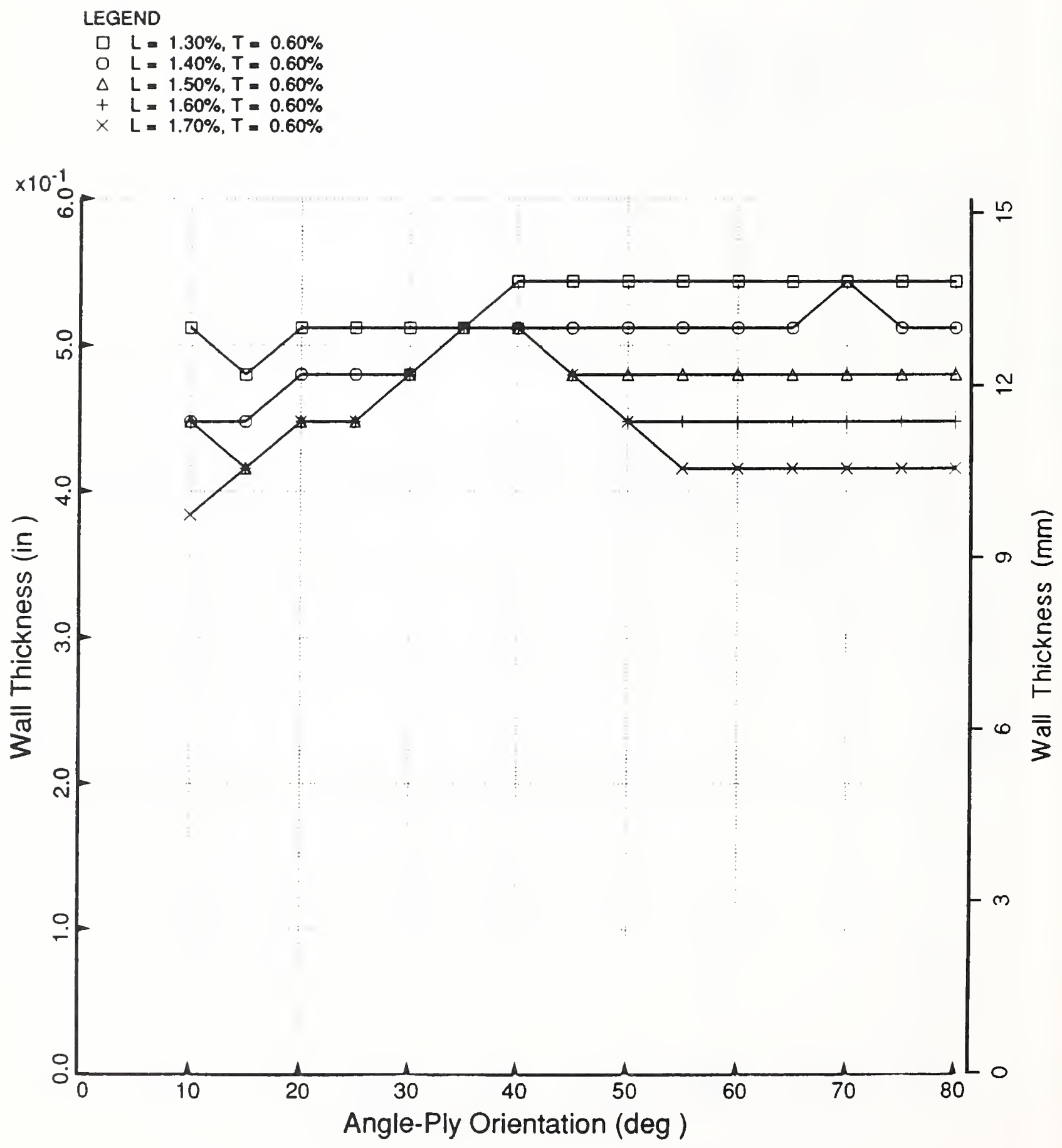

Figure 5.3. Optimum tube wall thicknesses for $\left[ \pm 10^{\circ}(n) / \pm \theta(m)\right]$ tube lay-ups with a transverse $(T)$ failure strain constraint of $0.60 \%$ and different fiber failure strain constraints $(L)$. 


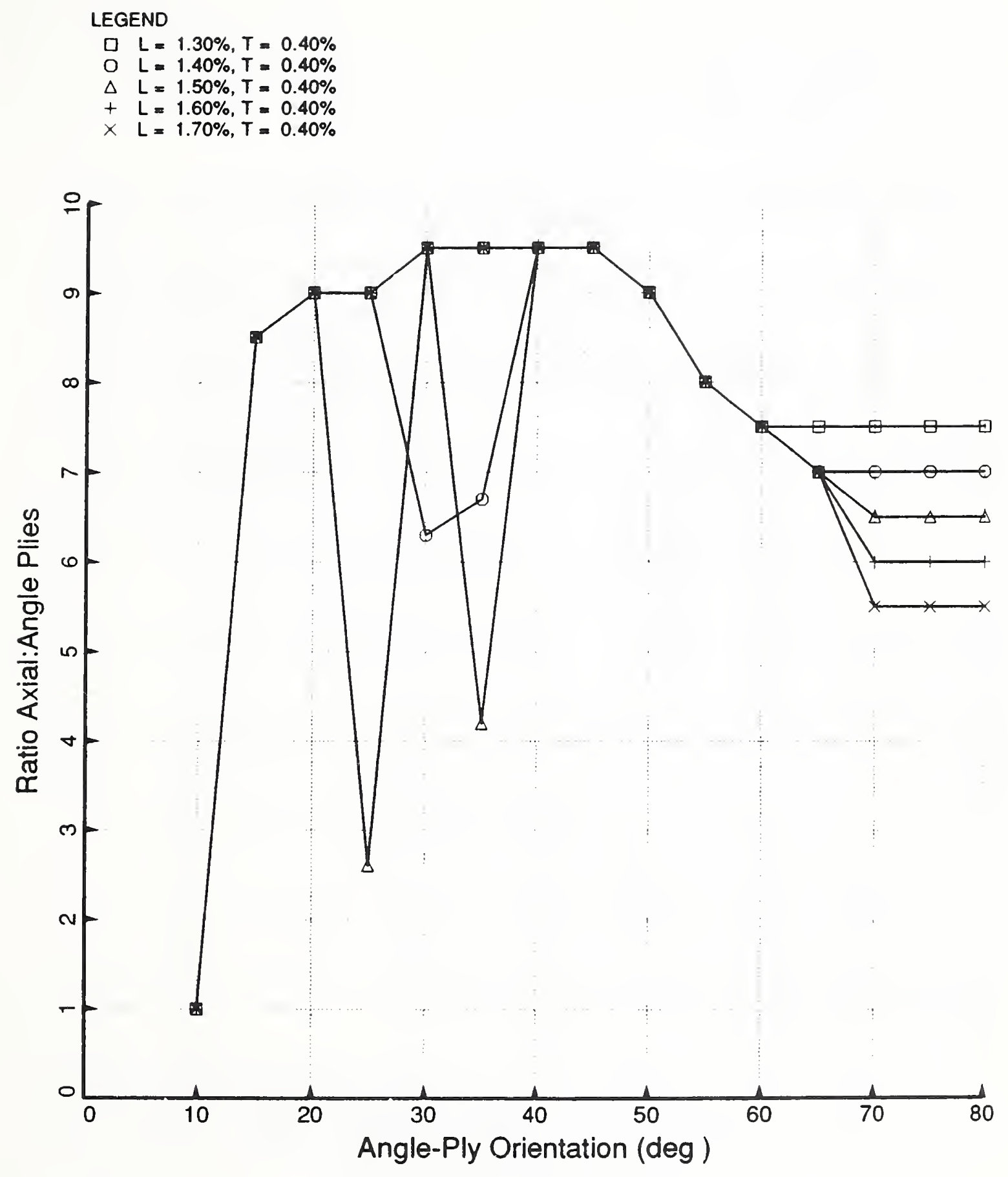

Figure 5.4. The ratio of $10^{\circ}$ layers to off-axis angle-wound layers for different off-axis winding angles and fiber strain allowables $(T=0.40 \%)$. 


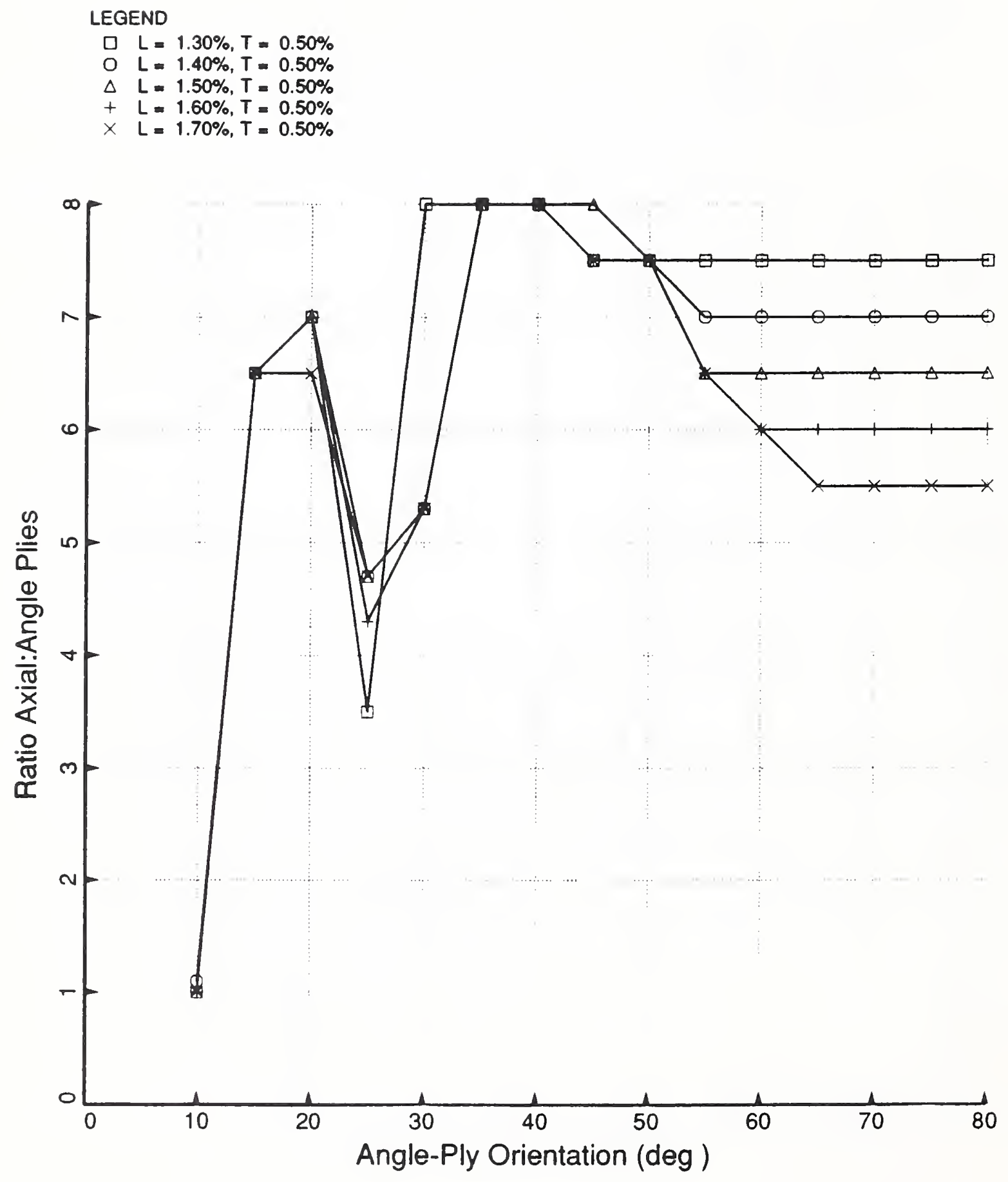

Figure 5.5. The ratio of $10^{\circ}$ layers to off-axis angle-wound layers for different off-axis winding angles and failure strain allowables $(T=0.50 \%)$. 


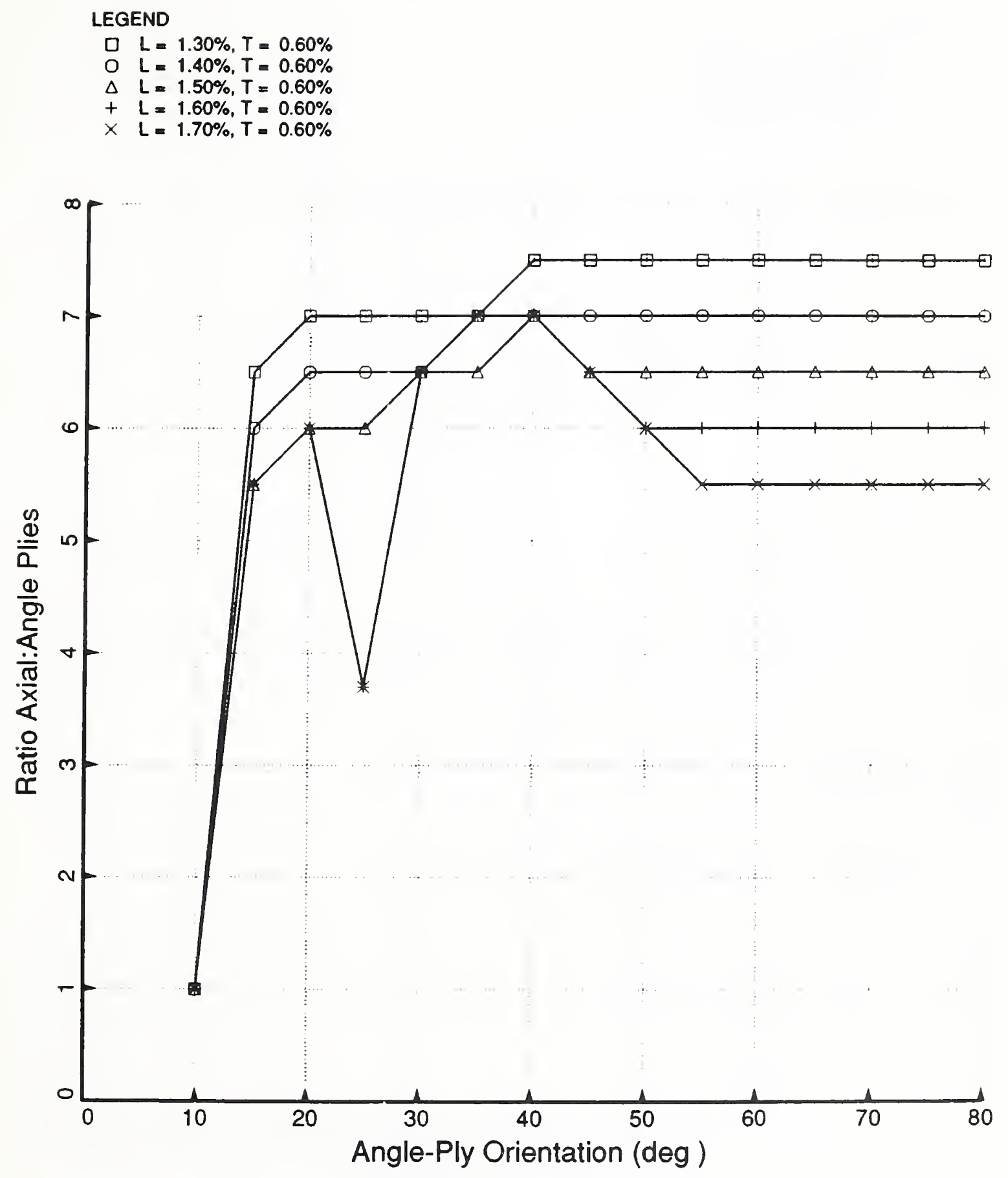

Figure 5.6. The ratio of $10^{\circ}$ layers to off-axis angle-wound layers for different off-axis winding angles and failure strain allowables $(T=0.60 \%)$. 

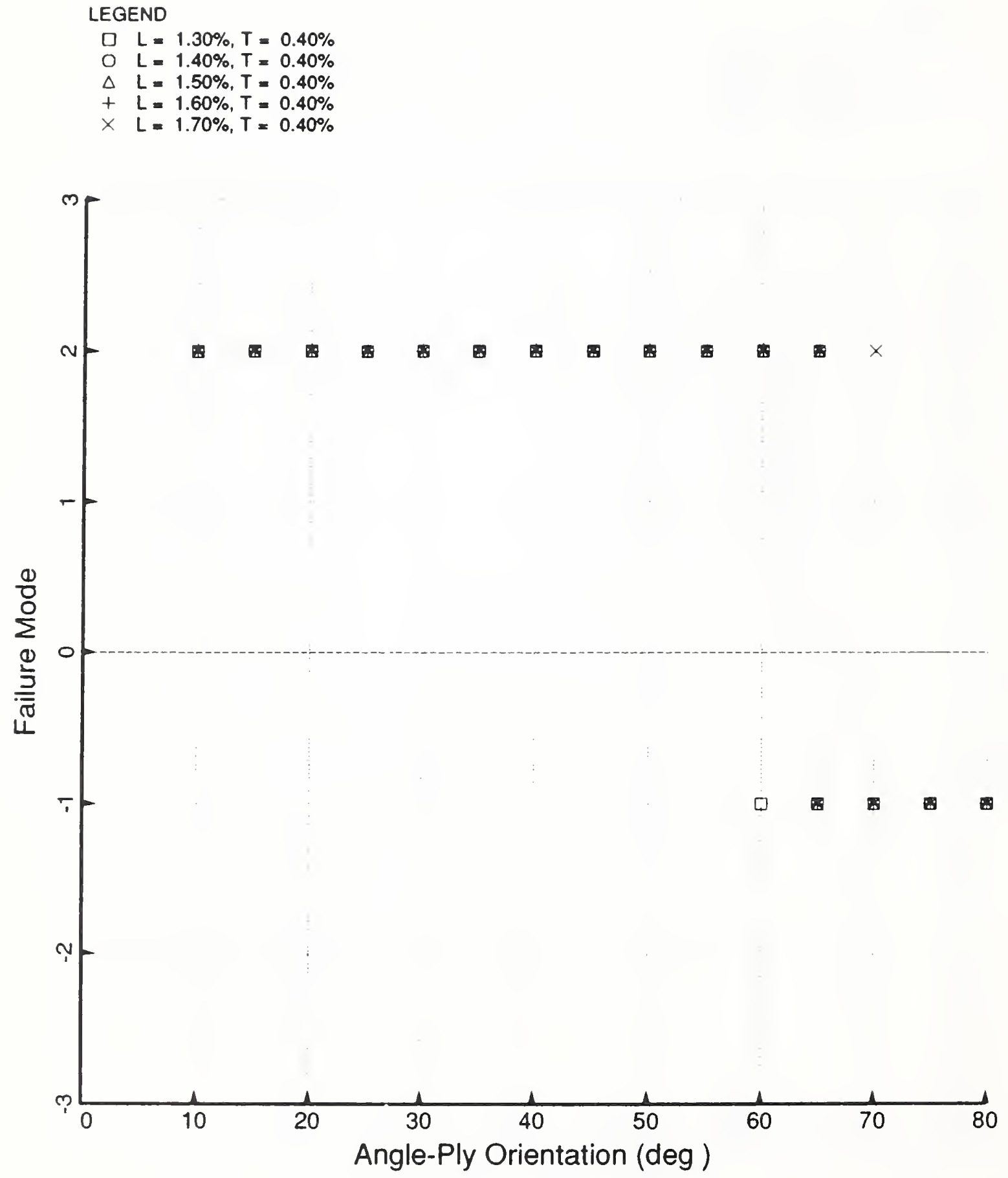

Figure 5.7. The critical constraint that drives the design optimum (at $T=0.40 \%$ ). Values of +2 : the transverse tensile strain allowable is the critical limit. Values of -1: fiber compressive strain allowables are critical. 


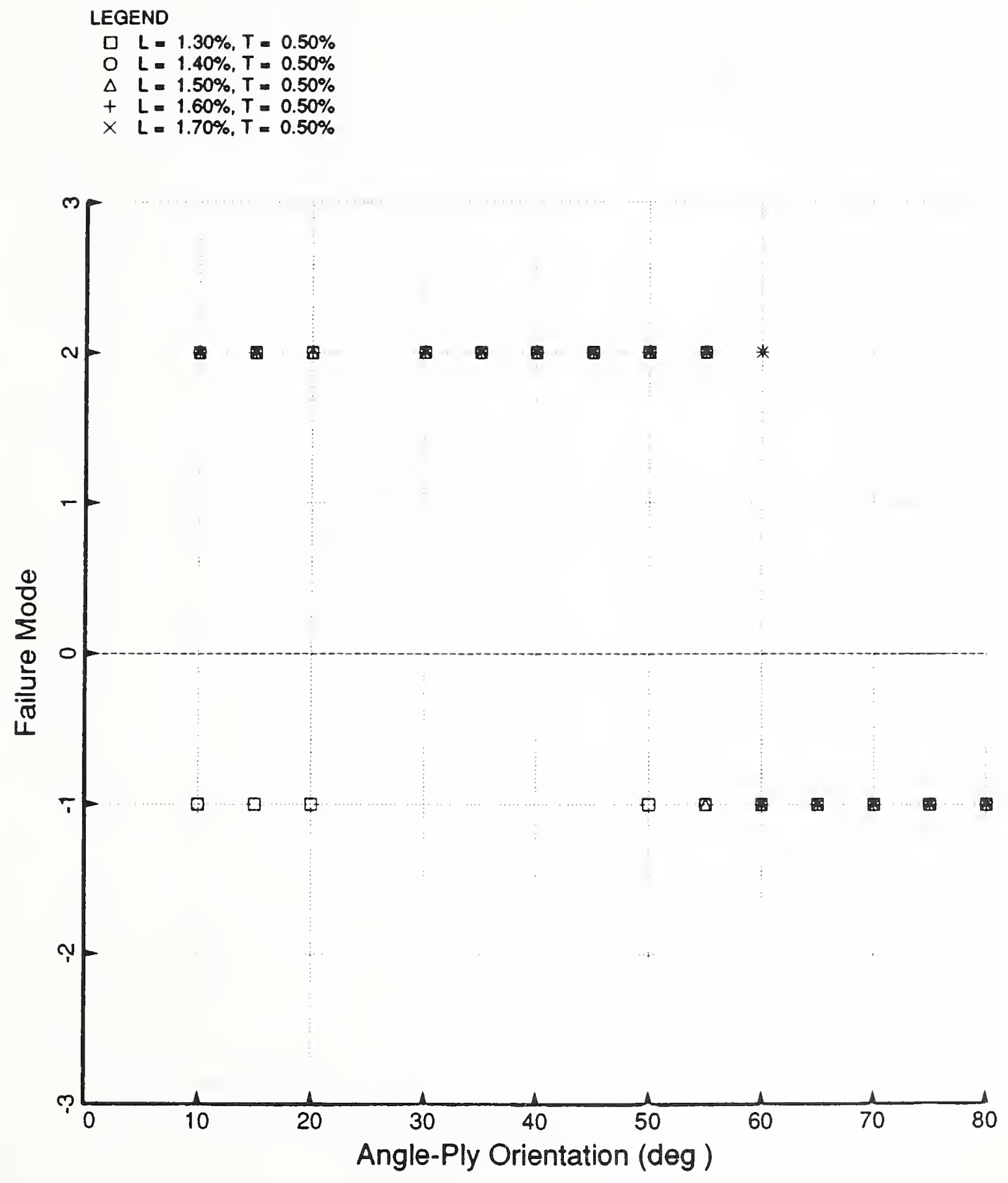

Figure 5.8. The critical constraint that drives the design optimum (at $T=0.50 \%$ ). Values of +2 : the transverse tensile strain allowable is the critical limit. Values of -1 : fiber compressive strain allowables are critical. 


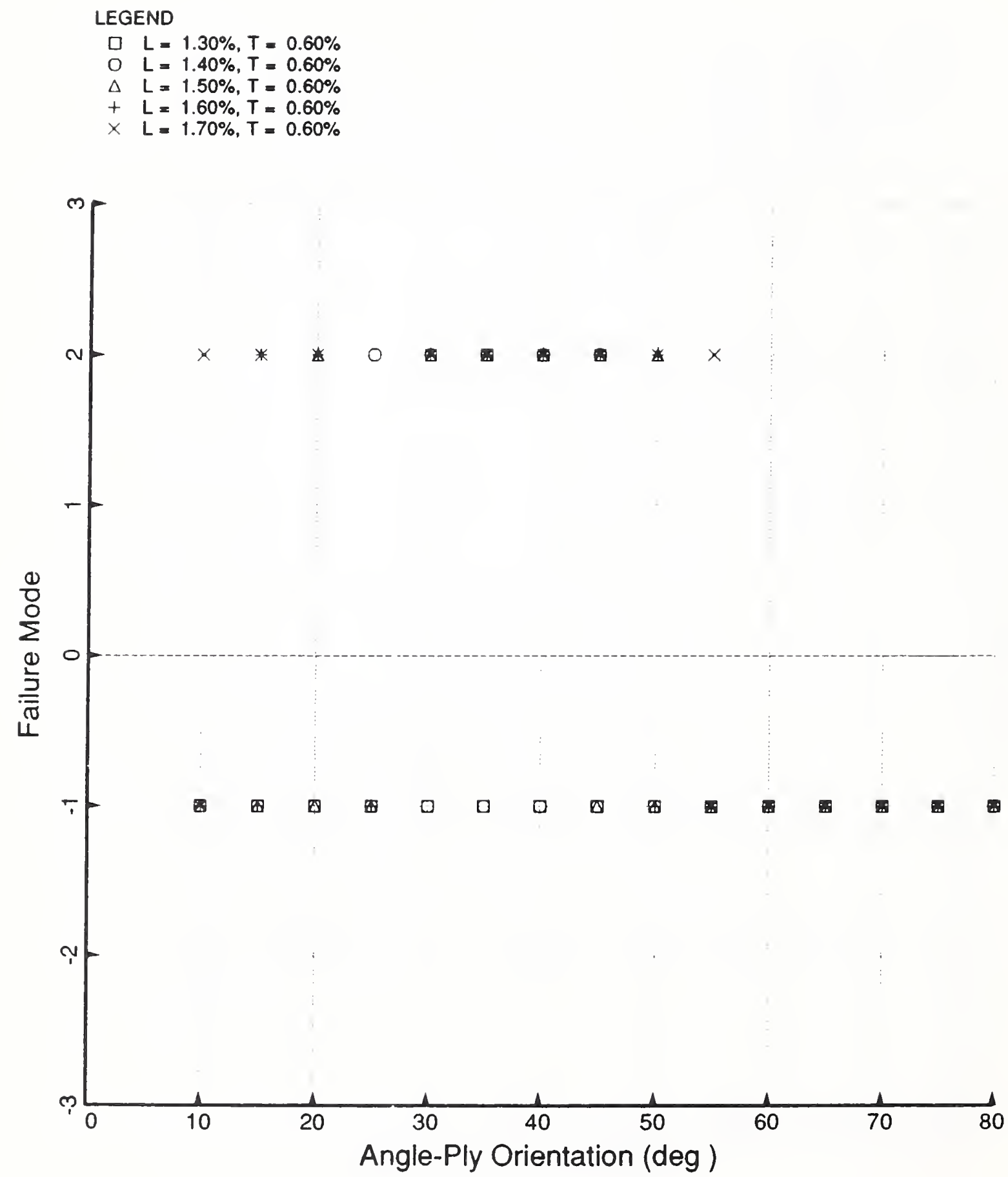

Figure 5.9. The critical constraint that drives the design optimum (at $T=0.60 \%$ ). Values of +2 : the transverse tensile strain allowable is the critical limit. Values of -1 : fiber compressive strain allowables are critical. 

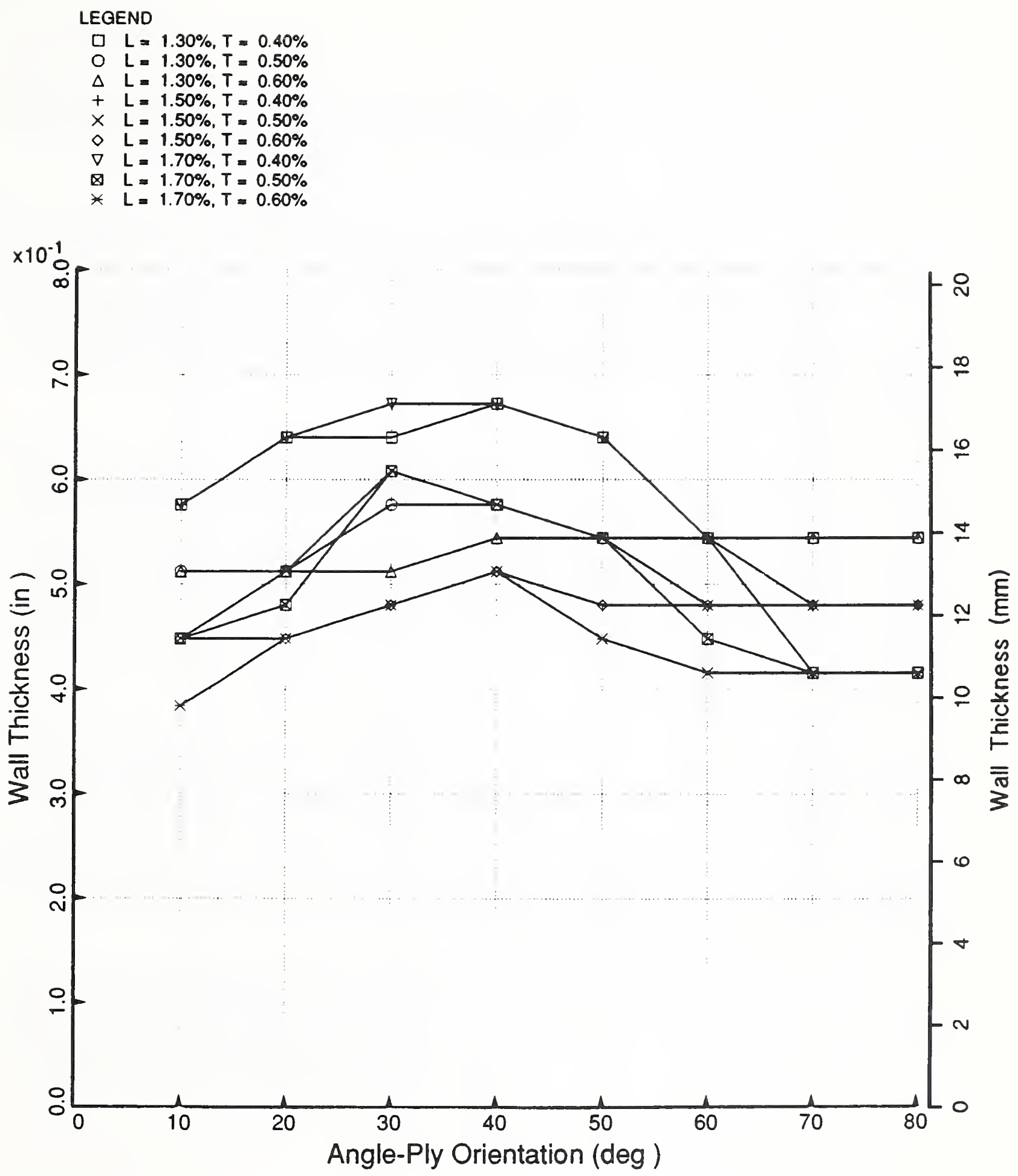

Figure 5.10. Summary of the design study. 


\section{Conclusions}

The mechanical performance of SMES support tubes will be limited by two major factors:

(1) the ability to mass-produce tubes of high quality, low void content and uniform construction and

(2) the room-temperature strength of matrix materials used in the tube construction.

As seen from the tube test data, tube failures occur over a wide range of load and strain states. Micrographic examinations of failed tube specimens also show considerable nonuniformity in tube construction. A reasonable conjecture to be considered (and one typical of composite constructions) is that variability of quality and homogeneity of construction are responsible for a substantial portion of the scatter evident in the experimental results. This means that the higher the tube quality, the better it will be expected to perform in service. In any event, tube quality will determine the margin above the allowables, and consistently high quality may even enable higher allowables to be defined.

Improving matrix strengths at room temperature will improve tube capability by reducing the occurrence of transverse cracks in the axially wound layers. Improvement at cryogenic temperatures will also be beneficial, although of secondary importance, because the tube is loaded uniaxially, and unless tapered tubes are to be considered, the warm end of the tube will see the same stresses as the cold end. It is highly likely that improving matrix strengths by 25 to $30 \%$ will yield a one-for-one increase in tube axial strength. This is predicted by the fact that the mean fiber-direction strain at failure is 25 to $30 \%$ higher than the minimum observed strain. By removing damage nucleation sites through matrix strengthening (or better construction, for that matter), high achievable fiber strains will result in higher tube capability.

More work needs to be done in the analysis of test data for material properties and in the use of these properties for design of full-scale SMES support tubes. Currently, tube design is dominated by the fact that minimum winding angles of $10^{\circ}$ are available from filament-winding procedures. Reducing the axial misalignment of "axial" fibers will result in much lower transverse and shear strains in the axial layers which will, in turn, increase tube strength. Since it is beyond the scope of this study to design the full-scale SMES support tubes, it should suffice to say that the methodologies developed under this study and presented here provide some practical means by which to design and validate SMES tube constructions.

Finally, the development of screening methods for assessing the capability of as-built SMES tubes should be pursued. Even in the best of circumstances, lot-to-lot variability will be present in tubes built for the SMES application, and the costs of construction will prohibit both extensive proof testing and frequent replacement of tubes failed in service. Therefore, prudent conservatism and judicious review will be needed not only in the design of SMES tubes, but also in evaluating their suitability for service. 


\section{References}

[3.1] McColskey, J.D. SMES tubes compression data (preliminary); private communication. Materials Reliability Division, National Institute of Standards and Technology, Boulder, CO; 1992 April.

[5.1] Bushnell, D. PANDA-Interactive program for minimum weight design of stiffened cylindrical panels and shells. J. Comput. Struct. 16:167-185; 1983.

[5.2] Vanderplaats, G.N. CONMIN-A FORTRAN program for constained function minimization. NASA TM X 62-282; updated 1975 March. 


\section{Appendixes}

\section{Appendix A. Micrographic Examination of Failed SMES Tube Samples}

Photomicrographs of sections of three failed SMES tubes are presented along with the tube type, specimen I.D., test temperature, photograph identification number, magnification level, and a short description of the features of each micrograph. Tube types 2 and 5 were $710 \mathrm{~mm}$ long; tube type 3 was $480 \mathrm{~mm}$ long. The major points of description are summarized in $\S \mathrm{IX} .2$

Photo I.D. Nos. Tube Type Specimen Test Temp. Magnification
$92.1616,92.1617$
2
$5-\mathrm{A}$
$4 \mathrm{~K}$
N/A

These photographs show the tube's failed section. The failure is characterized by substantial transverse splitting and fiber-layer brooming delaminations.

The central aspect of interest in this sample is to determine the order of the failure process. In particular, the inner-surface photograph clearly shows transverse cracking in the $\left[ \pm 10^{\circ}\right]$ layers extending well beyond the delaminated region. As such, it seems unlikely that the transverse cracking is caused by the failure; rather, it should precede the overall failure. This hypothesis is investigated further by examination of specimen $B$, which exhibits transverse cracks in a region well removed from the major delaminations.

Photo I.D. Nos. Tube Type Specimen Test Temp. Magnification
$92.1618,92.1619$
2
5-B
$4 \mathrm{~K}$
N/A

These photos show a region of the tube away from the major failure (very near the other end, in fact). The interesting aspect of this area is the large transverse crack extending along the length of the specimen. This crack branches at several points between the $+10^{\circ}$ and $-10^{\circ}$ directions; it is associated with some small delaminations.

The regions marked "MET" in the photograph were the sites sectioned for metallographic mounts 92.0367 and 92.0368 . The major interests in these sites were to determine the extent of the crack through the sample thickness and the coupling of crack paths between the $+10^{\circ}$ and $-10^{\circ}$ directions. Also of interest was the relationship between delamination and transverse cracking in an area removed from the major failure. 


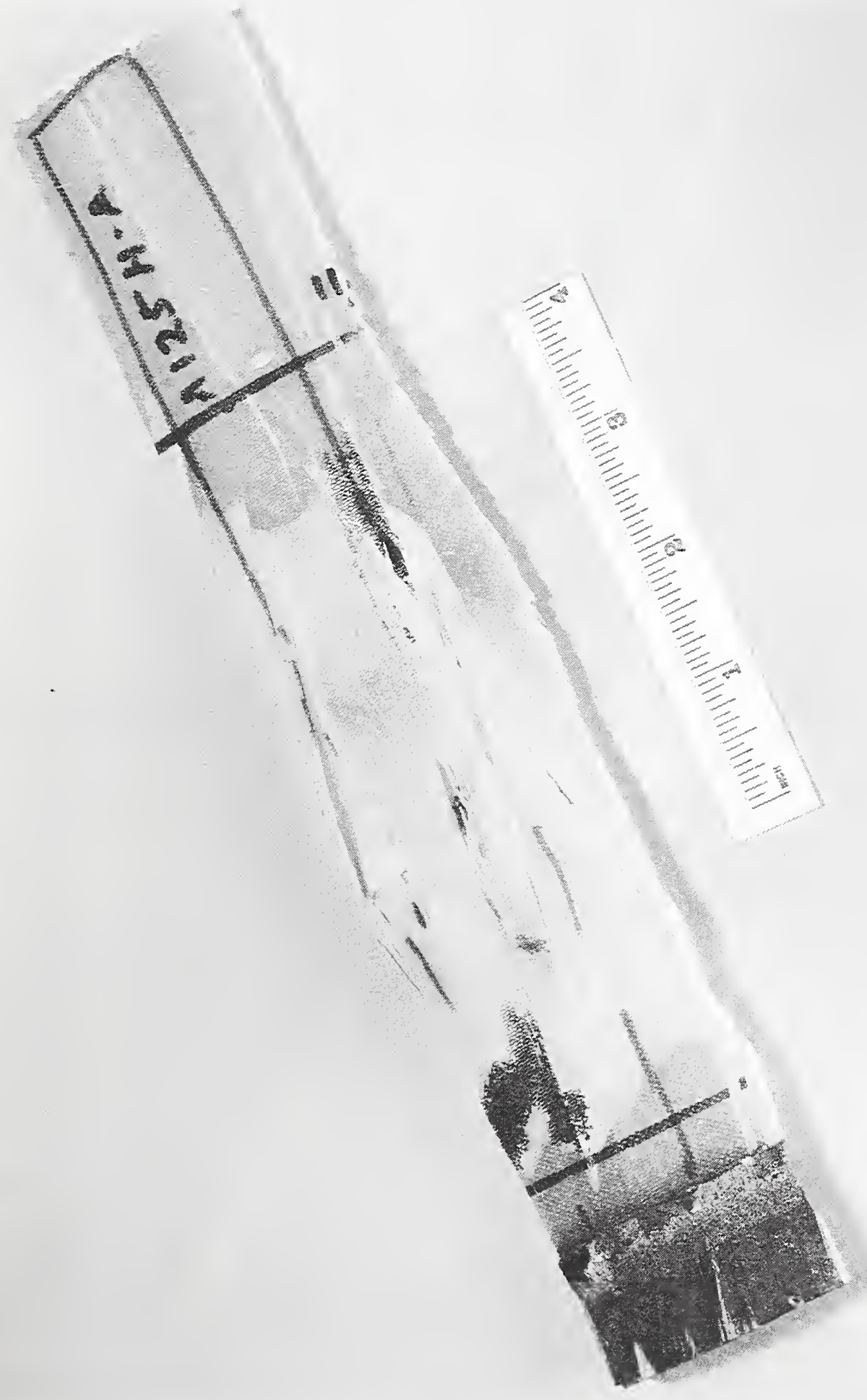

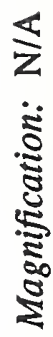

$\checkmark$

ลั่

氙

เ

ڤัँّ

है

$\frac{0}{0}$
$\frac{\sigma}{\sigma}$
$\ddot{2}$
$\vdots$
$\vdots$
$\vdots$
$\frac{0}{2}$

IX-37 


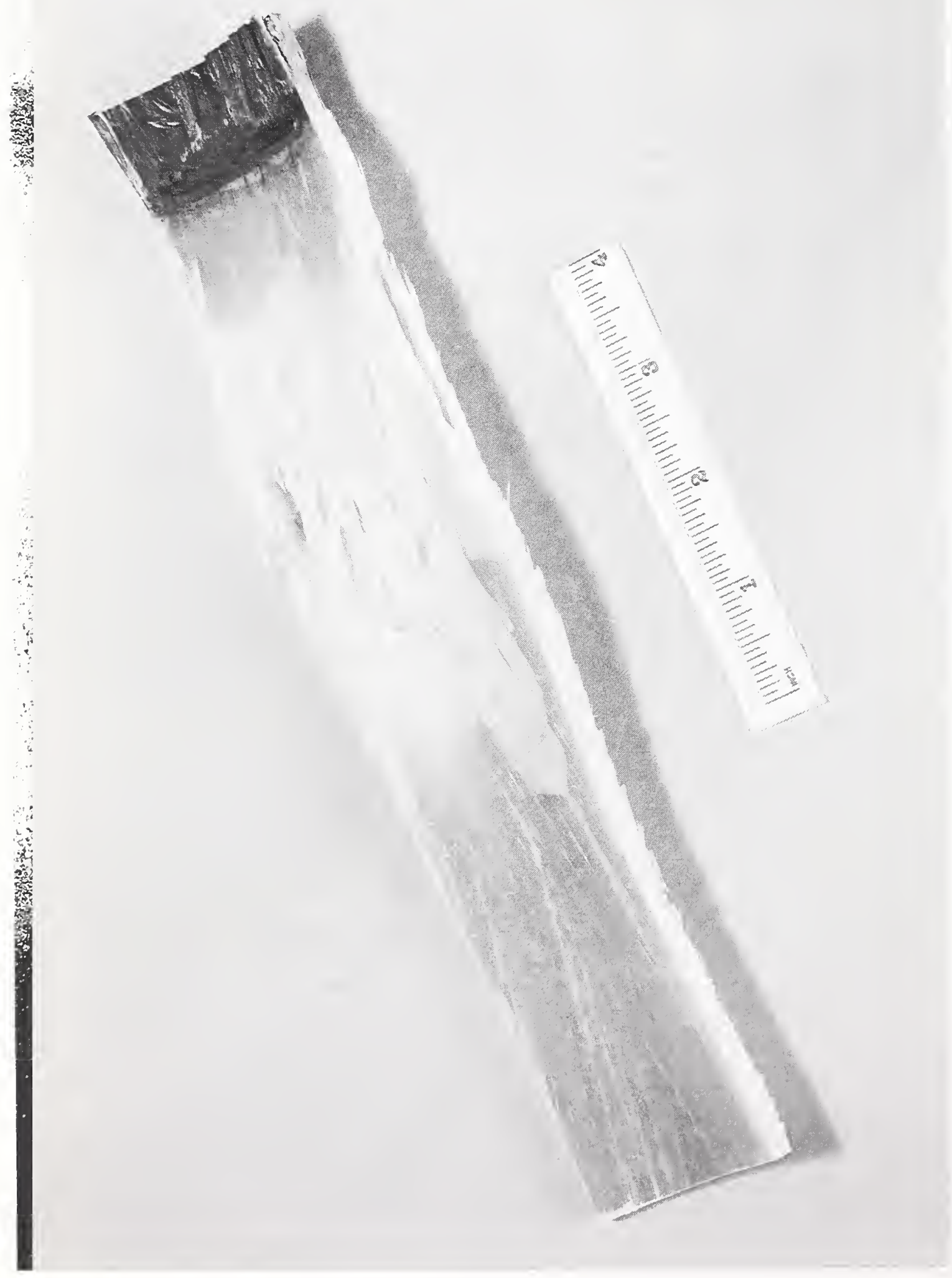

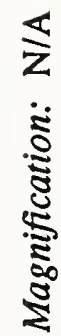

$\frac{1}{\sigma}$

今ั

氙

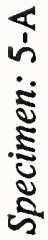

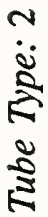

$\frac{7}{2}$

¿̊

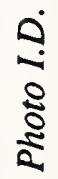

IX-38 


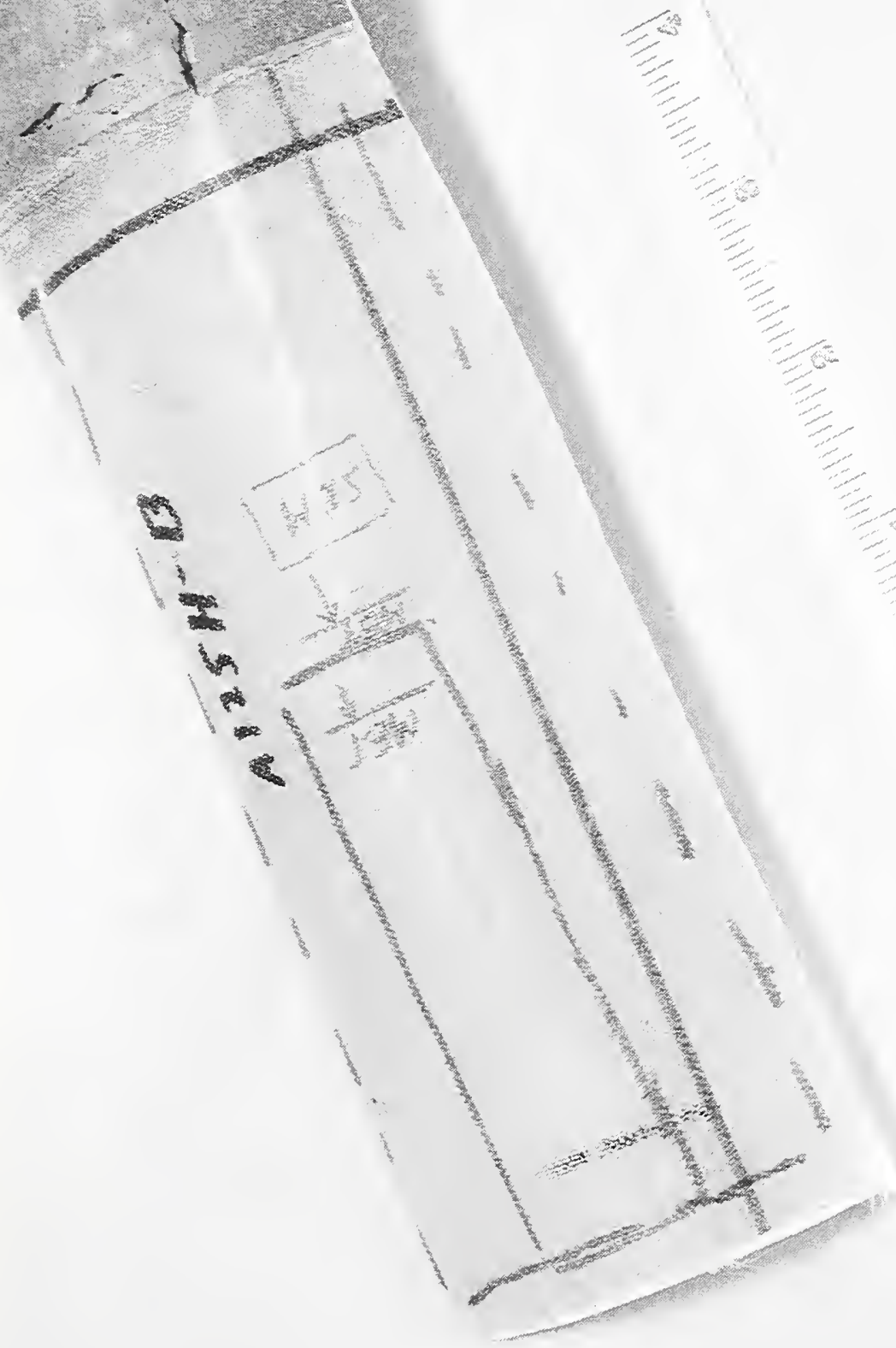




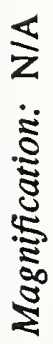

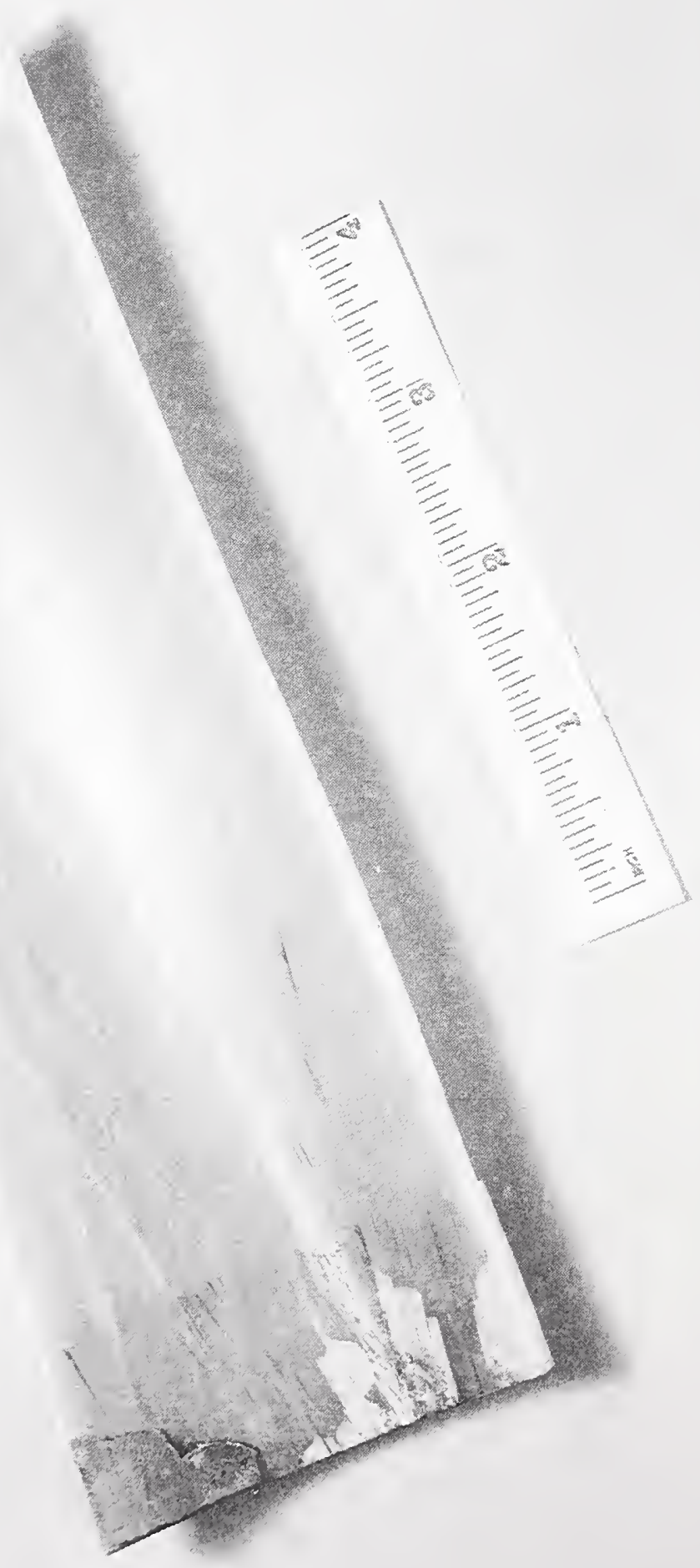

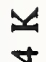

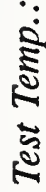

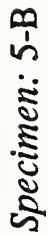

N

हิ

ङ

$\frac{\frac{a}{6}}{2}$

$\ddot{z}$

$\frac{0}{2}$ 
Photo I.D. Nos. Tube Type Specimen Test Temp. Magnification $92.1622,92.1623 \quad 3 \quad 1-B \quad 295 \mathrm{~K} \quad$ N/A

These photos show the failed region of the sample exhibiting significant delamination. The extent of the failure region in the type $3\left(\left[ \pm 82 / \pm 45 / \pm 10_{3}\right]_{n}\right)$ specimens was considerably more local than that in the type $1\left([ \pm 10]_{n}\right)$ specimens.

The interest in this region was to assess the extent of delamination along the length of the specimen away from the fractured end. The inner-diameter photo shows the surfaces selected for metallographic examination (labeled "MET").

The scanning electron micrographs (I.D. No.s 2CXQ through 2CXA+) were taken of the outer diameter of this specimen from the edge of the fractured region into its interior. The SEM specimen was taken from subsurface layers of this region in the location illustrated in the copy on page IX -45 .

$\begin{array}{ccccc}\text { Photo I.D. Nos. } & \text { Tube Type } & \text { Specimen } & \text { Test Temp. } & \text { Magnification } \\ 92.1620,92.1621 & 5 & 3-\mathrm{A} & 76 \mathrm{~K} & \text { N/A }\end{array}$

These photos show a region away from the failed region of this tube. The failure mode in this region is transverse cracking, much like that in the type 1 tube specimens. This effect is highly visible in both outer- and inner-surface photos.

Metallographic specimens were made to examine the sections labelled "MET" in the outer-diameter photograph. One metallographic specimen (mount \# 92.0369) was taken from the region bordering the visible delamination in the upper region of the tube segment, the other (mount \# 92.0370) was taken from a the region exhibiting transverse cracking, but no visible delamination, and located closer to the potted end of the tube specimen. 


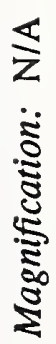

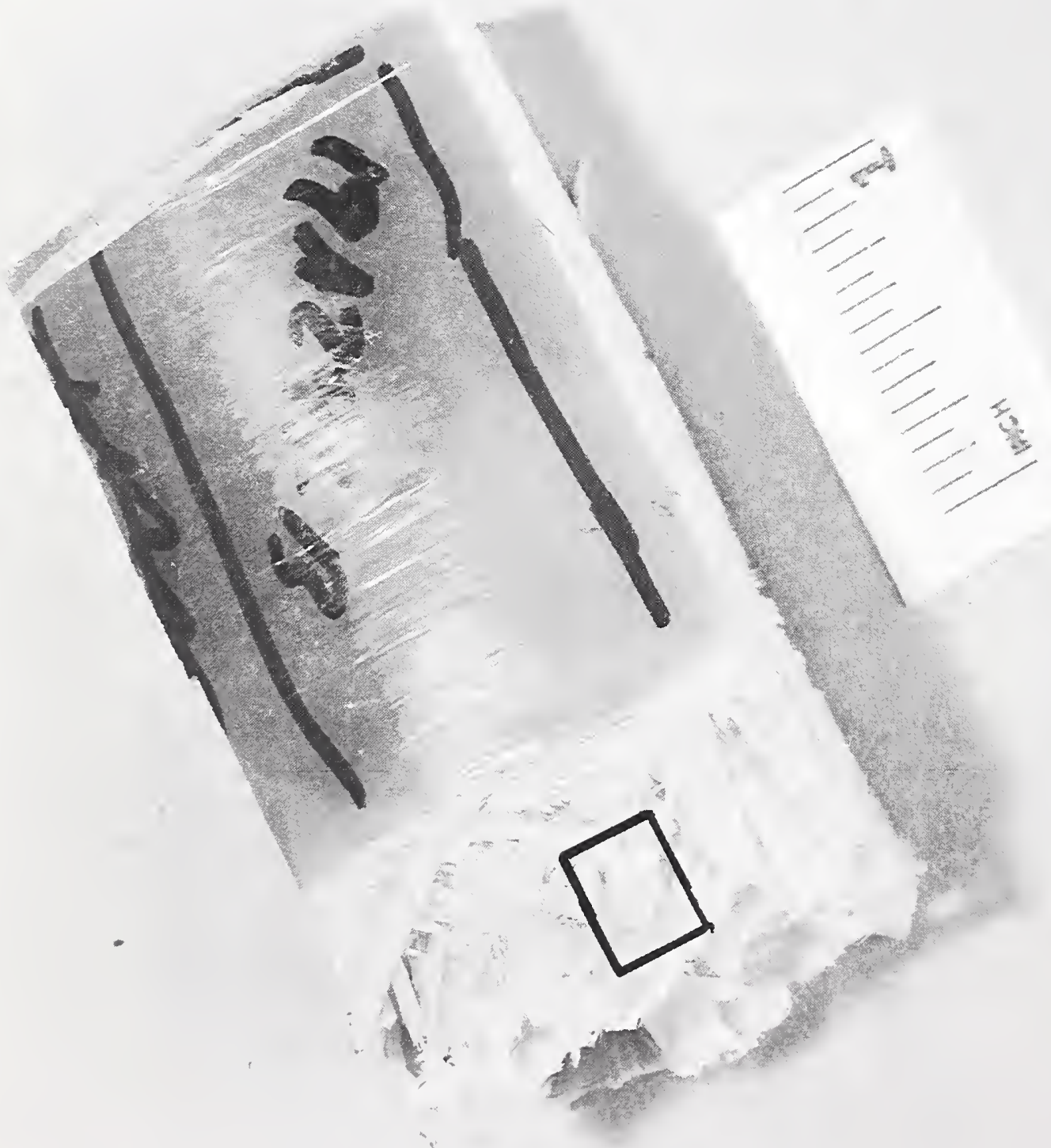

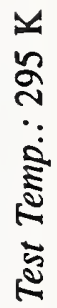

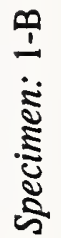

है

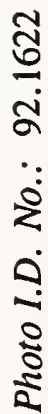




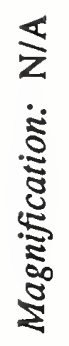

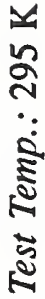

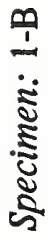

हैं
है
है

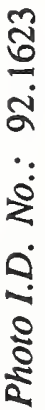




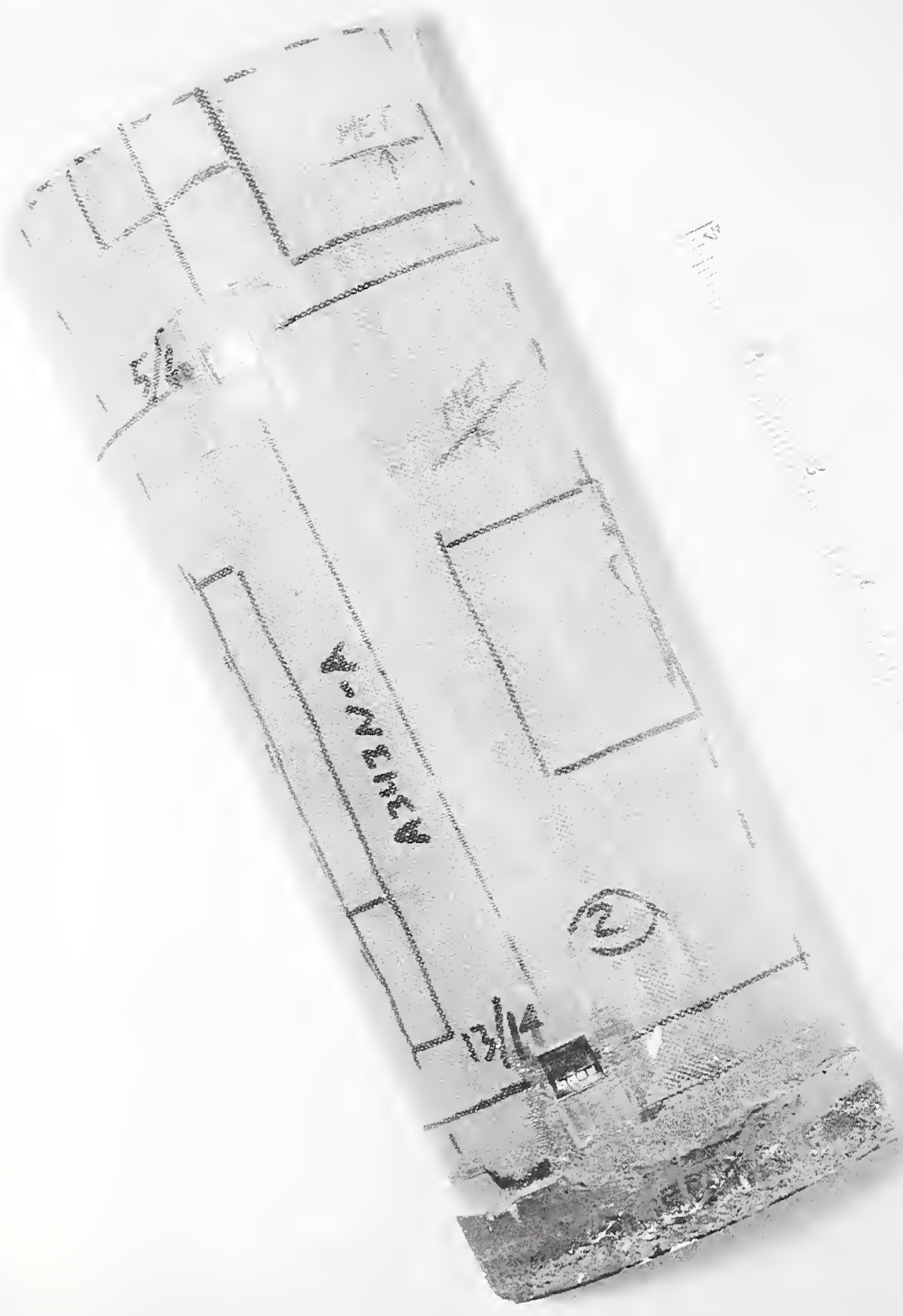

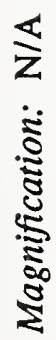

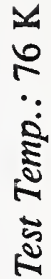

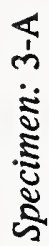

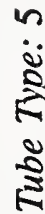

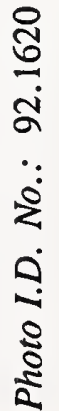




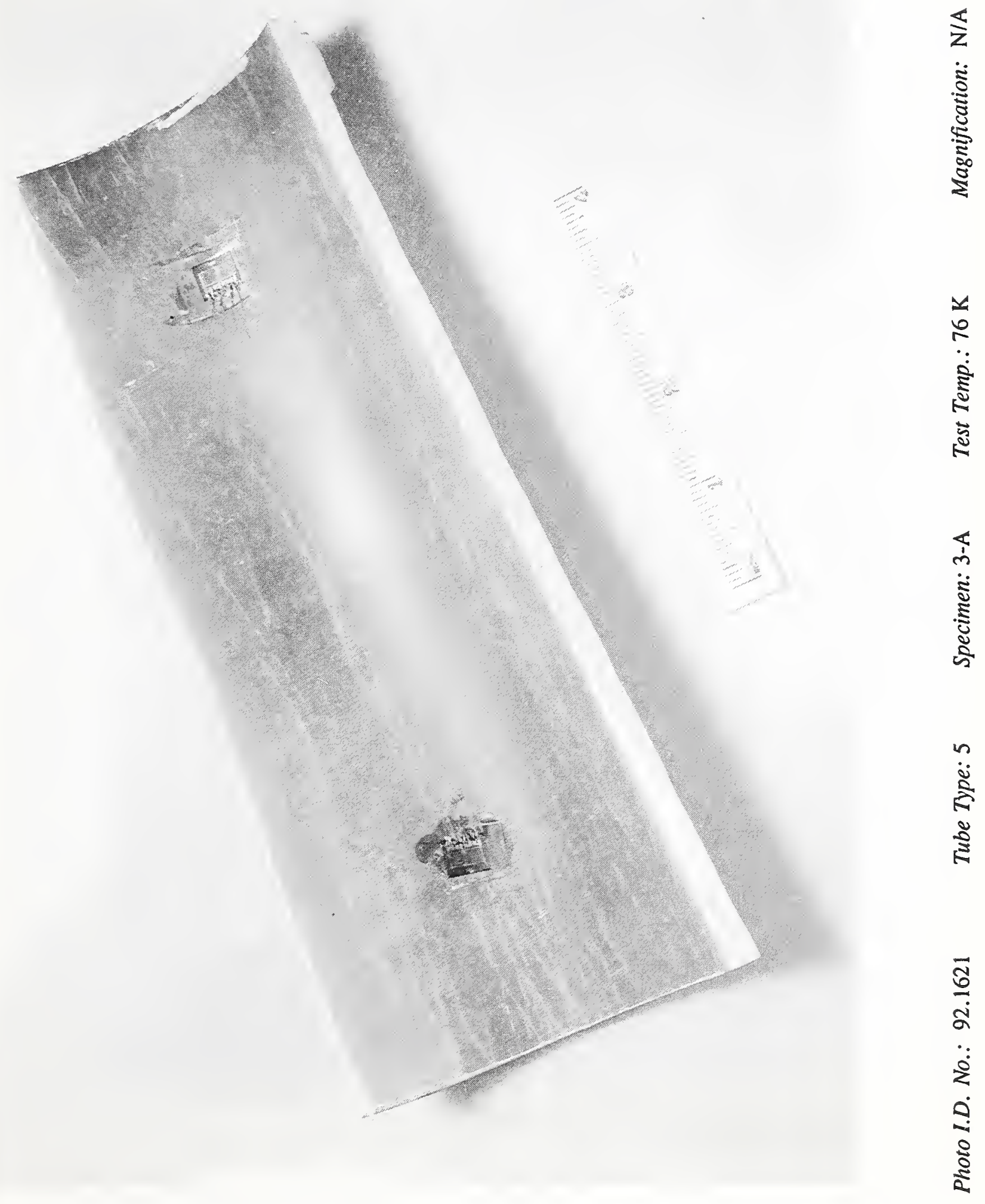

IX -45 


$\begin{array}{ccccc}\text { Photo I.D. No. } & \text { Tube Type } & \text { Specimen } & \text { Test Temp. } & \text { Magnification } \\ \text { SEM 2CXQ } & 3 & 1-\mathrm{B} & 295 \mathrm{~K} & 20 \times\end{array}$

This photo shows the central region of the SEM specimen from which all higher magnification SEM micrographs were taken. This region was chosen because it is a delamination surface that also shows fractures in both the $45^{\circ}$ and $10^{\circ}$ layers.

The specimen surface lies across the $\left[ \pm 45^{\circ}\right]$ layers just outside the tube midsurface. It lies below the exposed cross-plied region in photograph 92.1618 and is not visible in that photo. The top half of the photograph shows a delaminated surface at $+45^{\circ}$, whereas the lower third of the photo shows a similar delaminated surface, but in the $-45^{\circ}$ layer. In between the two surface regions lies a region containing a crack along the $-45^{\circ}$ direction, extending through the thickness of the specimen into the visible upper surface of $\mathrm{a}+10^{\circ}$ layer. The crack is also associated with visible fiber breakages in the $-45^{\circ}$ layer.

The copy of the SEM photograph below indicates the regions in which the higher magnification SEM micrographs were taken. This should be used as a guide when considering the features visible in the more detailed photographs. 


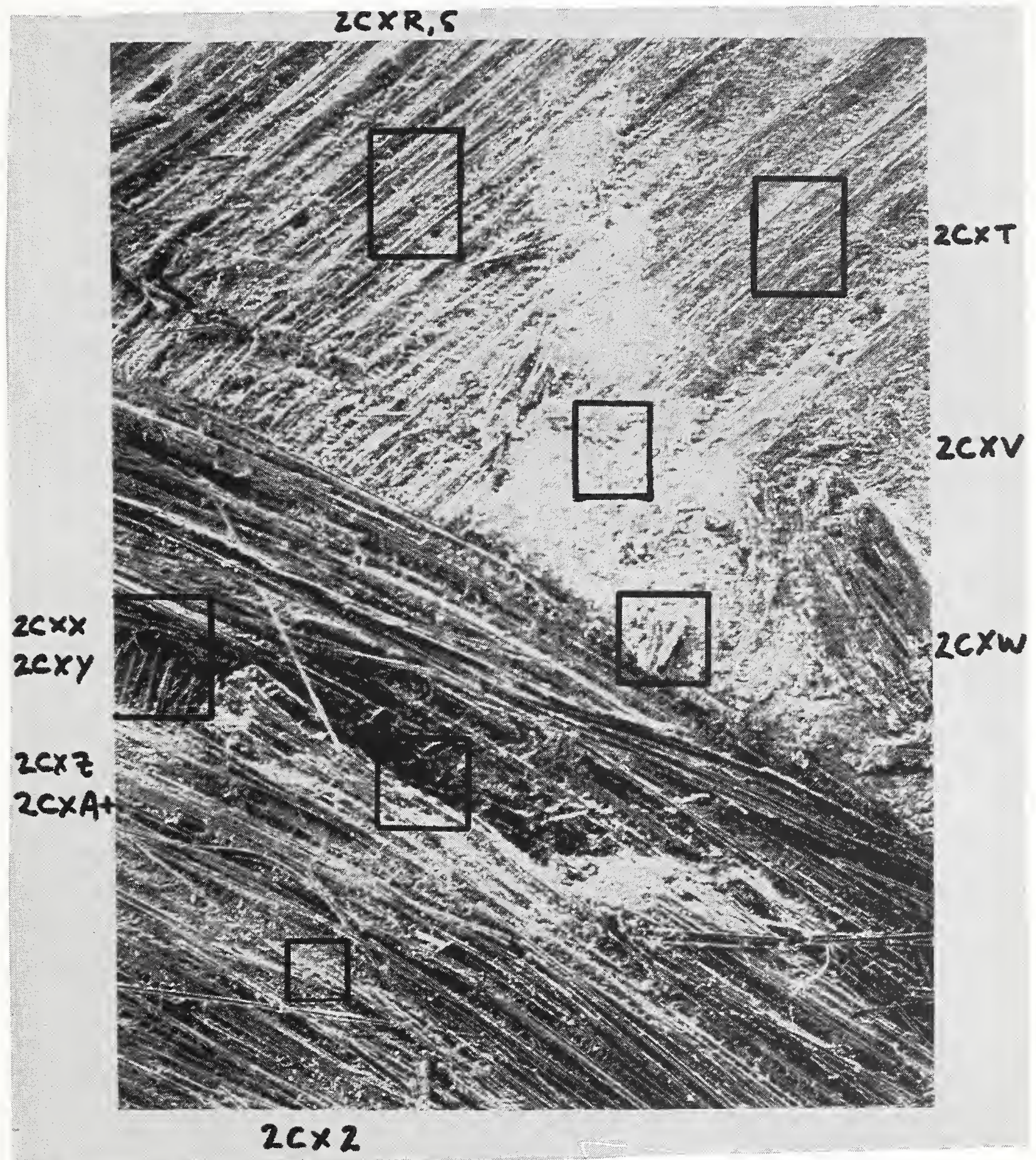

Photo I.D. No.: 2CXQ Tube Type: 3 Specimen: 1-B Test Temp.: $295 \mathrm{~K}$ Magnification: 20X 
Photo I.D. Nos. Tube Type Specimen SEM 2CXR, 2CXS

3

$1-B$

Test Temp. Magnification

$295 \mathrm{~K} \quad 200 \times, 500 \times$

These micrographs show a delaminated region within the $+45^{\circ}$ layer occurring approximately in the center of the layer's thickness. The fracture surface is characterized by visible fibers, some portions of which appear well-covered by matrix material. There is also considerable evidence of matrix-matrix fracture away from fibers. This is best examined in the $500 \times$ micrograph $(2 \mathrm{CXS})$.

It is clear from these photographs that delaminations involve matrix failures both at the fiber-matrix interface and within matrix-rich regions between fibers. The degree of fiber-matrix adhesion is not well-characterized by examination of these regions. Some fibers appear to be well-bonded and embedded into the matrix, whereas at least one fiber has been pulled out of the matrix along its length, as is visible in the higher magnification micrograph. 
Photo I.D. No.: 2CXR

Tube Type: 3

Specimen: 1-B

Test Temp.: $295 \mathrm{~K}$

Magnification: $200 \times$

Photo I.D. No.: 2CXS

Tube Type: 3

Specimen: 1-B

Test Temp.: $295 \mathrm{~K}$

Magnification: $500 \times$
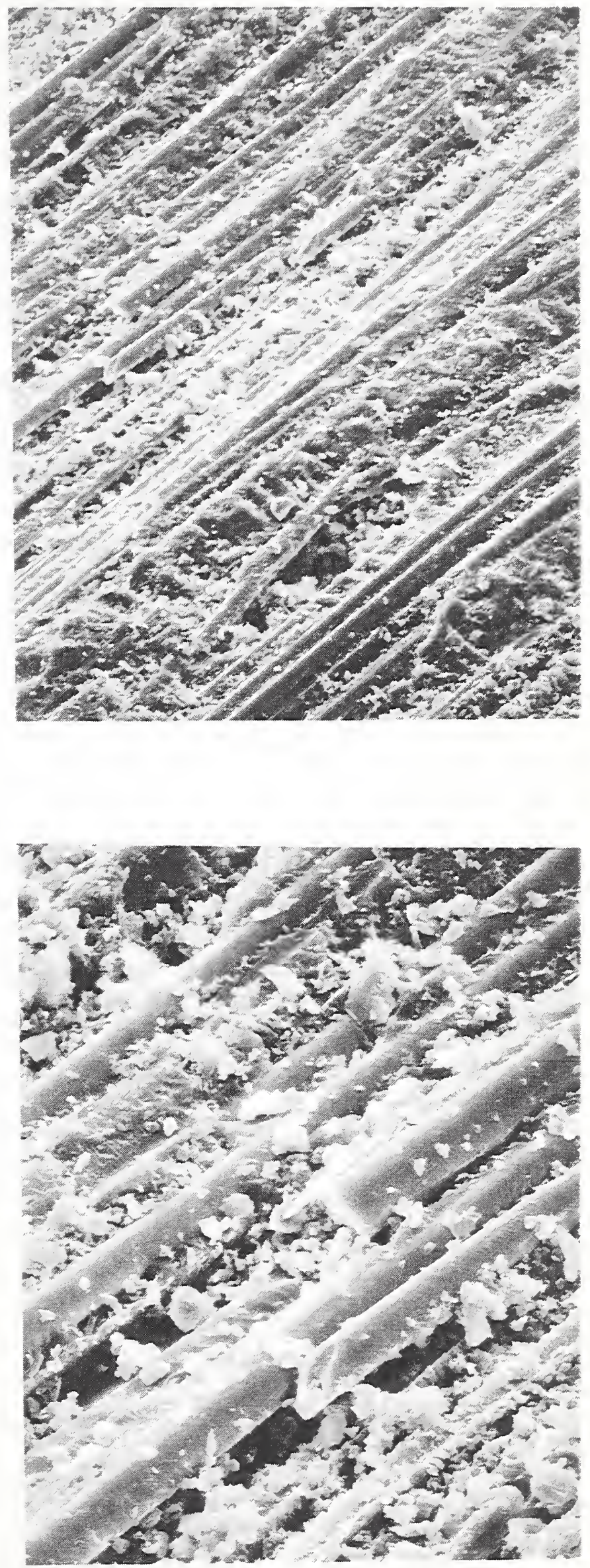
Photo I.D. Nos. Tube Type Specimen Test Temp. Magnification

SEM 2CXT, 2CXU $3 \quad$ 1-B $295 \mathrm{~K} \quad 200 \times, 500 \times$

These micrographs show a delaminated region within the $+45^{\circ}$ layer characterized primarily by failure in a matrix-rich region. In this region, fibers are generally not exposed by the fracture as much as in the region of micrographs $2 \mathrm{CXR}$ and $2 \mathrm{CXS}$. This is most probably due to the presence of a matrix-rich region here, where a fiber-rich region appears in the previous micrographs.

The high-magnification micrograph shows considerable matrix damage due to the fracture. One fiber, or its matrix sheath, is seen to have buckled or have been pulled out of the delamination surface by the fracture itself. The fibers visible in this photo appear very well-immersed in matrix material, indicating fairly good fibermatrix adhesion. 
Photo I.D. No.: 2CXT

Tube Type: 3

Specimen: 1-B

Test Temp.: $295 \mathrm{~K}$

Magnification: $200 \times$

Photo I.D. No.: 2CXU

Tube Type: 3

Specimen: 1-B

Test Temp.: $295 \mathrm{~K}$

Magnification: $500 \times$
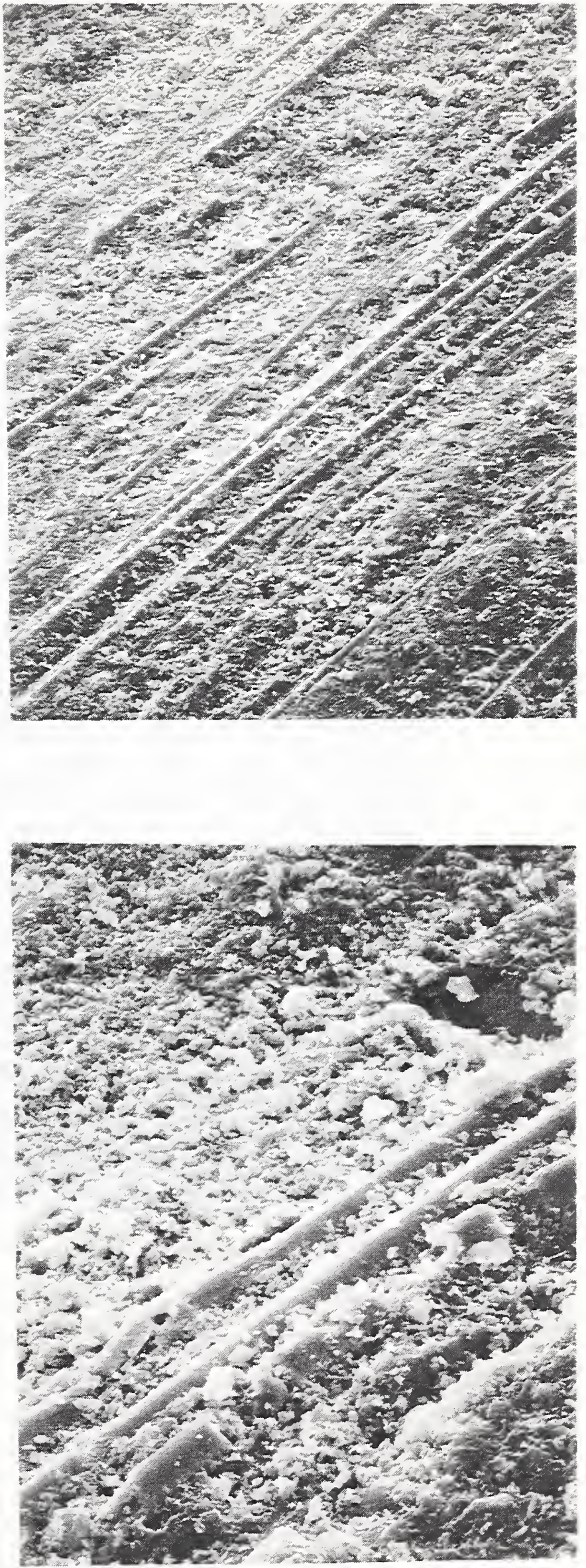


\section{Photo I.D. No. Tube Type Specimen Test Temp. Magnification $\begin{array}{lllll}\text { SEM 2CXW } & 3 & 1-\mathrm{B} & 295 \mathrm{~K} & 200 \times\end{array}$}

This micrograph shows a bundle of fibers pulled transversely from the $+45^{\circ}$ layer. Within the bundle, poor matrix-fiber adhesion is observed in some regions and good adhesion is evident in others. The bundle appears to have separated cleanly along the underside at the layer boundary between the $+45^{\circ}$ and $-45^{\circ}$ layers. A matrix-rich failure region can be seen to the right of the fiber bundle.

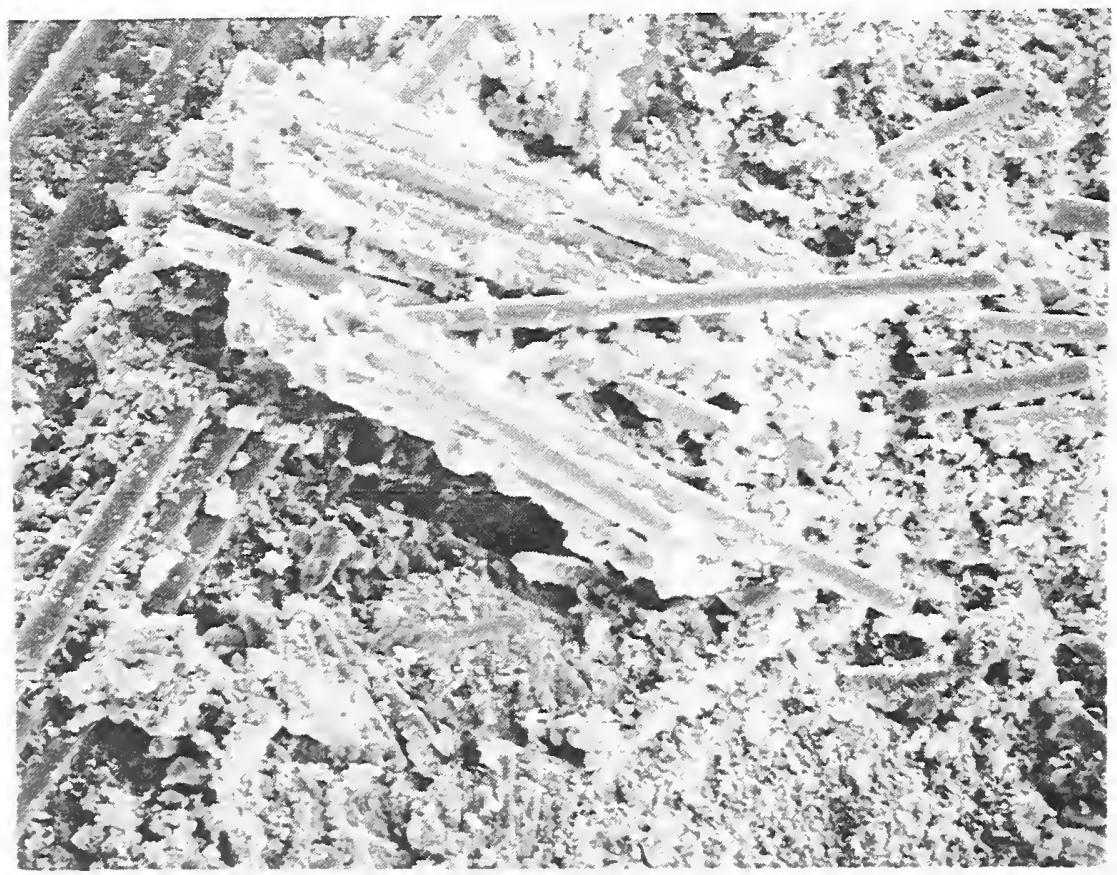


Photo I.D. Nos. Tube Type Specimen Test Temp. Magnification $\begin{array}{lllll}\text { SEM 2CXX, 2CXY } 3 & 1-\mathrm{B} & 295 \mathrm{~K} & 200 \times, 500 \times\end{array}$

These micrographs show the area of fracture in the $-45^{\circ}$ layer opening up to a $+10^{\circ}$ layer below. The fibers visible in the upper portion of the lower magnification photograph lie in the $-45^{\circ}$ layer.

Close examination of the $10^{\circ}$ fibers in the high-magnification photo reveals very good matrix-fiber adhesion, with the exposed surface showing considerable shear failure in the matrix material. This is not surprising, given the magnitude of the interfacial shearing present at the $10^{\circ} / 45^{\circ}$ boundary.

The large, partially detached, matrix-rich region just below the $-45^{\circ}$ layer shows surfaces characteristic of shear and tensile fractures, underscoring the coupled nature of the stress fields acting on the matrix. That such large, isolated regions of pure matrix material are present in the composite is noteworthy in itself, since the character of local failures may be influenced greatly by the load redistribution to fibers around the edges of such regions. 
Photo I.D. No.: 2CXX

Tube Type: 3

Specimen: 1-B

Test Temp.: $295 \mathrm{~K}$

Magnification: $200 \times$

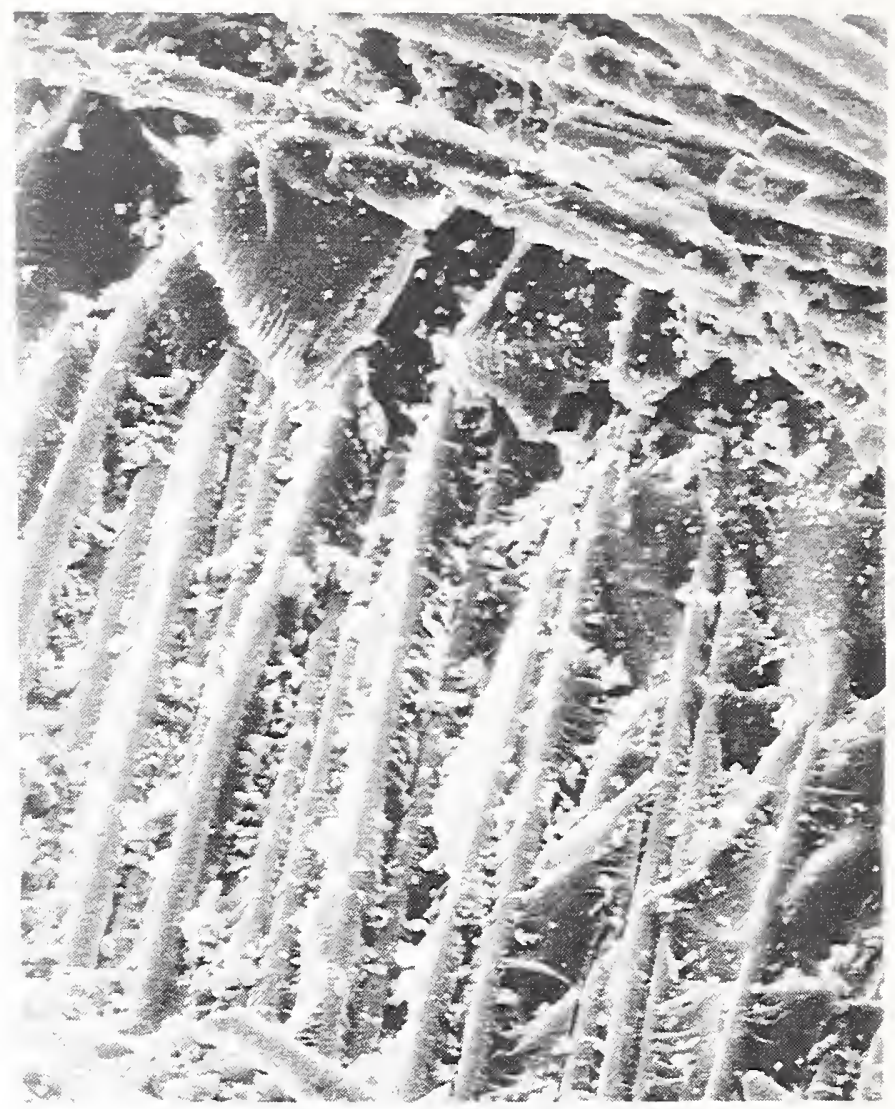

Photo I.D. No.: 2CXY

Tube Type: 3

Specimen: 1-B

Test Temp.: $295 \mathrm{~K}$

Magnification: $500 \times$

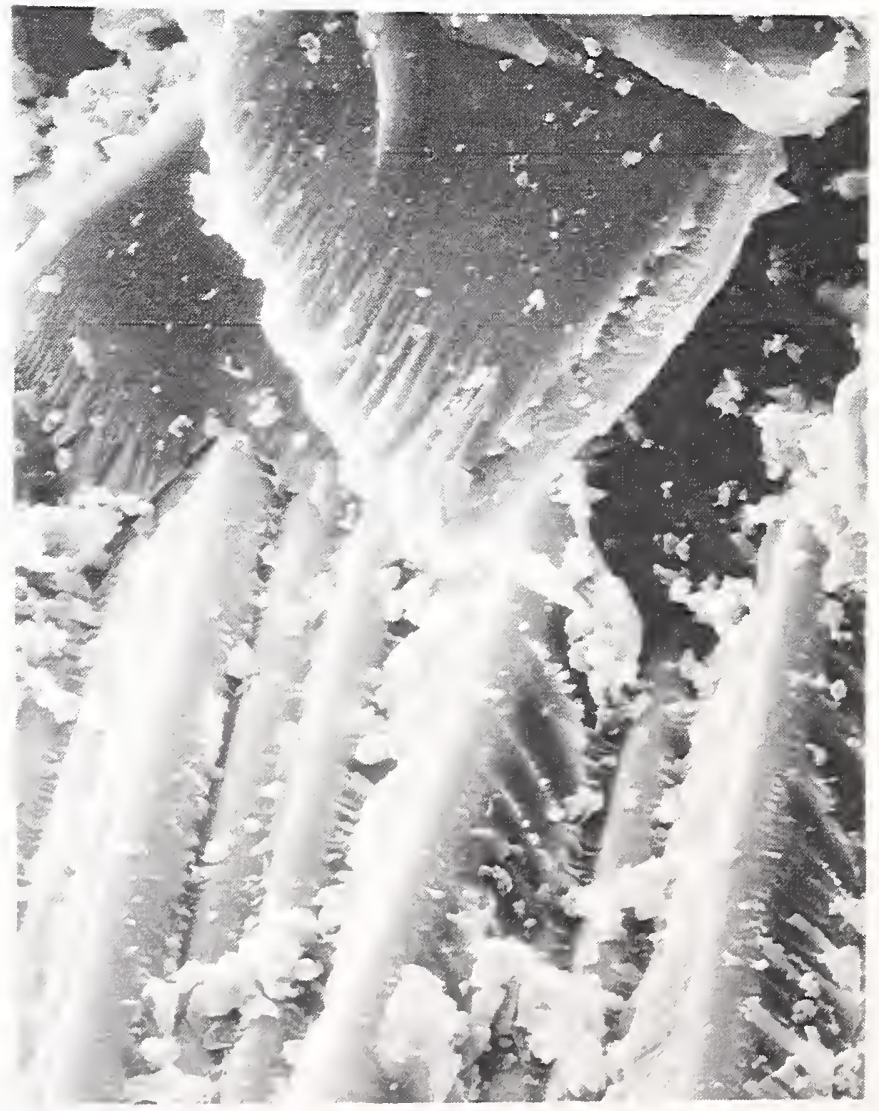


Photo I.D. Nos. Tube Type Specimen Test Temp. Magnification SEM 2CXZ, 2CXA+ $3 \quad 1-\mathrm{B} \quad 295 \mathrm{~K} \quad 200 \times, 500 \times$

These micrographs show the fracture in the $-45^{\circ}$ layer leading to a delaminated region running up and to the right under the layer. The delamination propagates visibly through a matrix-rich region, and the delaminated surface is characterized by the expected tensile matrix failure.

The degree of embedment of the visible fibers in the matrix on the surface of the $-45^{\circ}$ layer indicates good fiber-matrix adhesion in this region. Some clumps of matrix material from the overlying $+45^{\circ}$ layer are also visibly attached to the underlying $-45^{\circ}$ fibers in the lower magnification photo. These clumps are composed exclusively of matrix material, indicating that they also formed matrix-rich regions in the specimen. That they have remained attached to the $-45^{\circ}$ layer is also indicative of good adhesion between the fiber and matrix. 
Photo I.D. No.: $2 \mathrm{CXZ}$

Tube Type: 3

Specimen: 1-B

Test Temp.: $295 \mathrm{~K}$

Magnification: $200 \times$

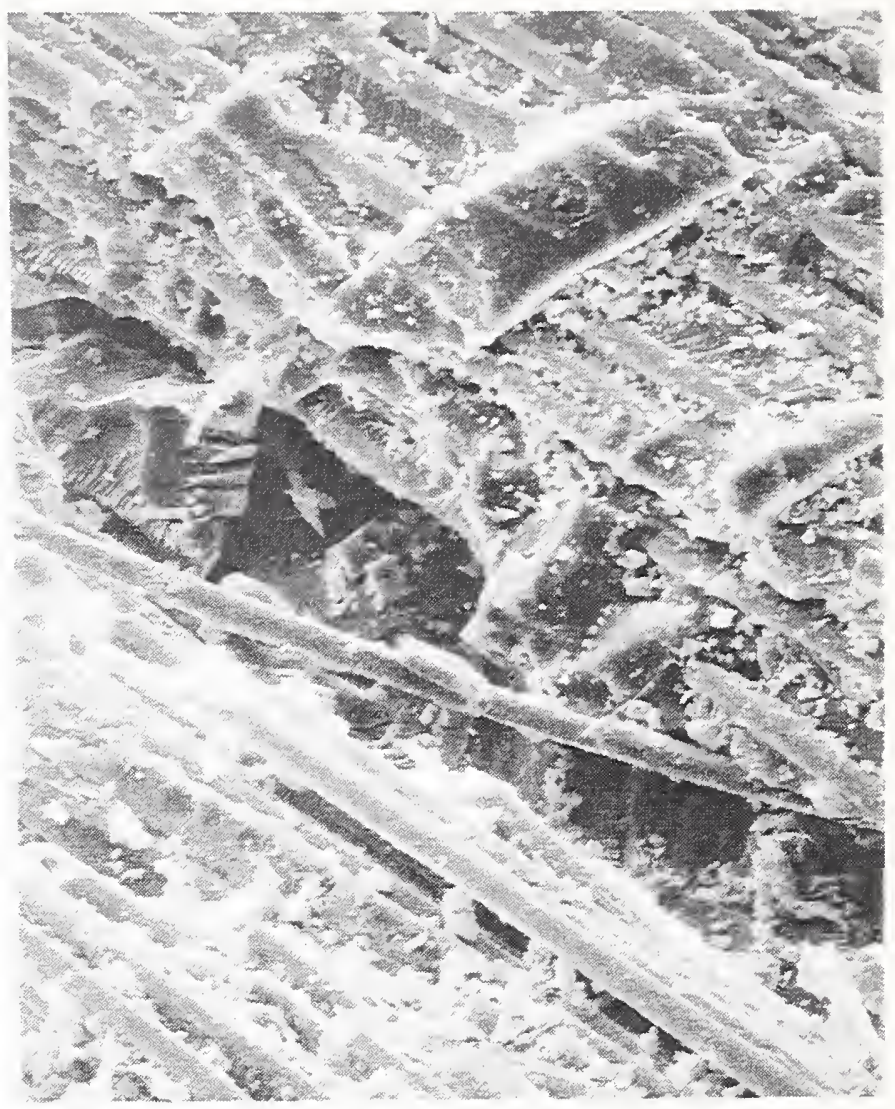

Photo I.D. No.: 2CXA+

Tube Type: 3

Specimen: 1-B

Test Temp.: $295 \mathrm{~K}$

Magnification: $500 \times$

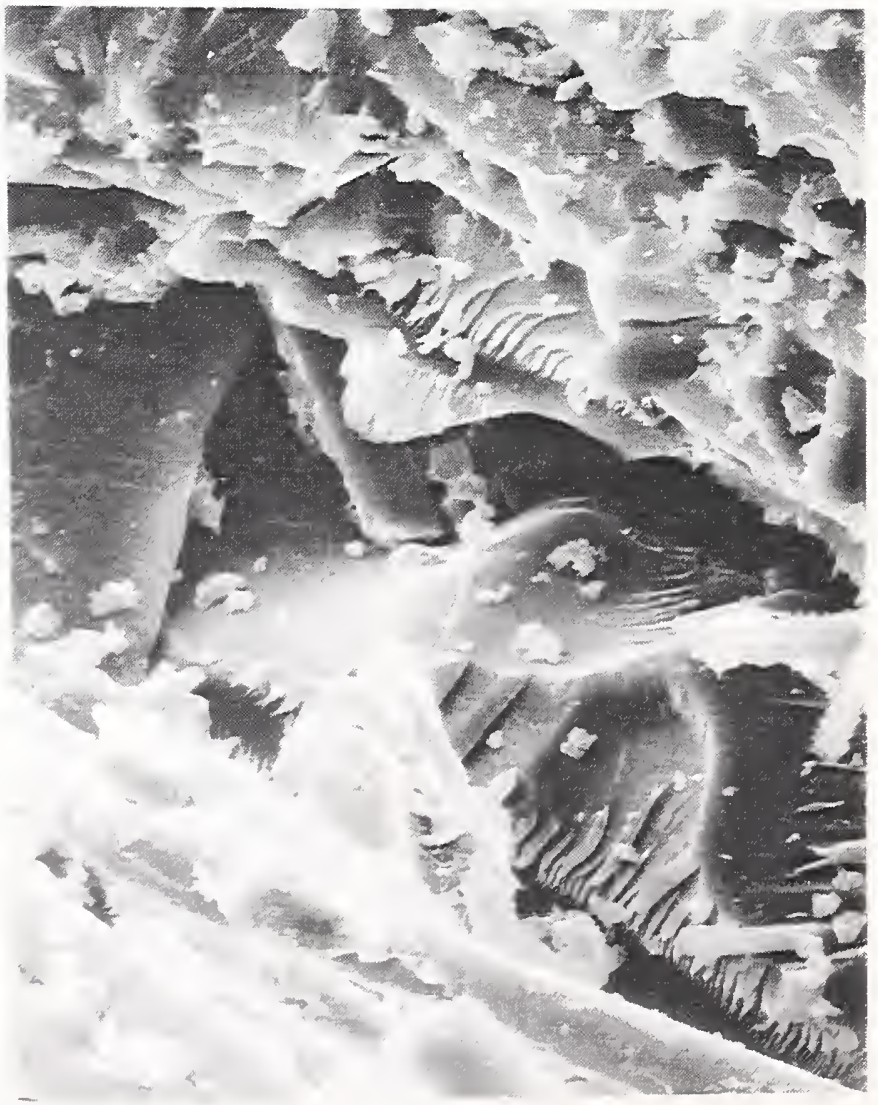


Photo I.D. No. Tube Type Specimen Test Temp. Magnification

$\begin{array}{lllll}\text { SEM } 2 C X 2 & 3 & 1-B & 295 \mathrm{~K} & 200 \times\end{array}$

This micrograph shows fibers on the surface of the $-45^{\circ}$ layer, some of which have been pulled out of the layer by the delamination which exposed the layer. Some of the fibers shown in this photo have considerable amounts of remaining matrix material attached along their length, indicating fairly good fiber-matrix adhesion. Some delamination is also visible below the well-bonded group of fibers running through the center of the region.

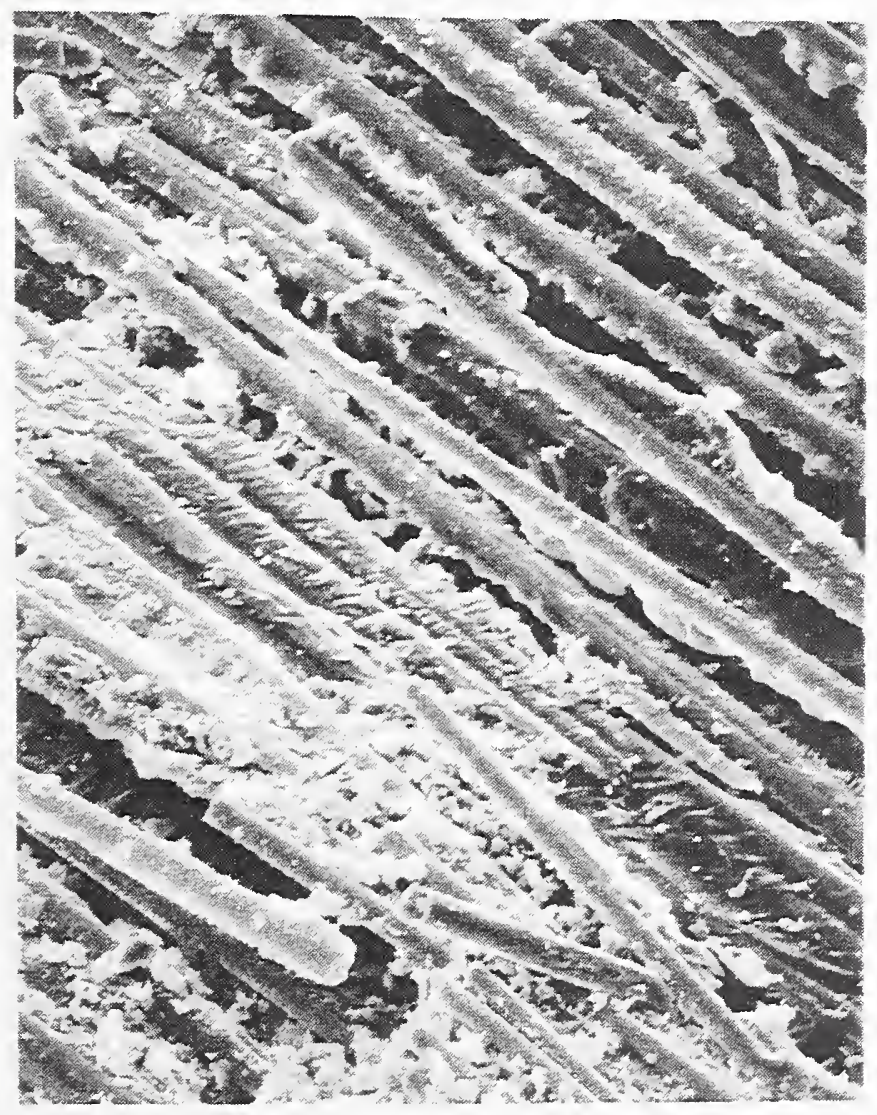




\section{Photo I.D. No. Tube Type Specimen Test Temp. Magnification \\ $\begin{array}{lllll}92.2237 & 2 & 5-\mathrm{B} & 4 \mathrm{~K} & 15 \times\end{array}$}

This optical micrograph shows the cross section of the specimen in the region of a visible transverse crack.

The fiber volume fraction in the bulk specimen is seen to be high, but a very large void content is also evident. Four very large voids are visible in the specimen, along with hundreds of smaller voids ranging in size from roughly $30 \mu \mathrm{m}$ to $250 \mu \mathrm{m}$. The smaller voids are located throughout the cross section, without noticeable aggregation either at layer boundaries or within layer interiors. The larger voids are always present at interlayer boundaries.

The fiber layers are seen to vary considerably in thickness along the circumference of the tube. Visual examination indicates that the thinner layer regions have higher fiber volume fractions than the thicker layer regions. Rough measurements indicate up to $100 \%$ variation in the layer thickness, from about $330 \mu \mathrm{m}(0.013 \mathrm{in})$ to about $660 \mu \mathrm{m}(0.026 \mathrm{in})$. These variations occur over circumferential distances of the order of $0.5 \mathrm{~cm}(0.20 \mathrm{in})$ and are seen to be associated with the crack path in only one case.

The central transverse fracture propagates entirely through the section with much branching and many associated parallel cracks. The fracture runs through the largest void visible in the region, although it is noteworthy that the void visible to the right of the fracture line in the center of the section thickness completely breaks the transverse continuity of a single layer without any evidence of cracks propagating through or around it. This indicates that the voids may not necessarily serve as initiation sites for the transverse fractures-an observation supported by the path of the major fracture through void-free regions of several layers.

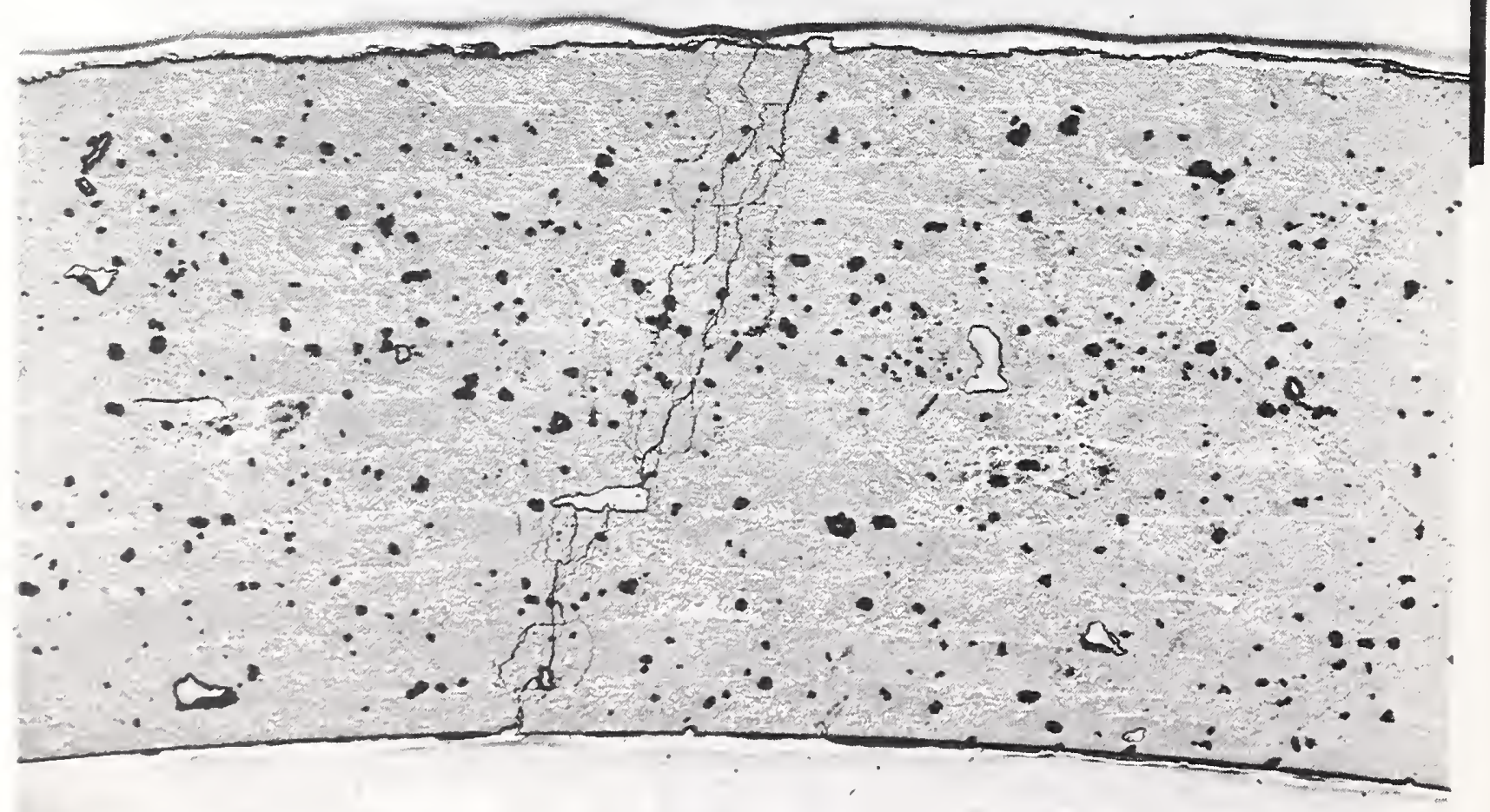




$\begin{array}{ccccc}\text { Photo I.D. No. } & \text { Tube Type } & \text { Specimen } & \text { Test Temp. } & \text { Magnification } \\ 92.2238 & 2 & 5-\mathrm{B} & 4 \mathrm{~K} & 100 \times\end{array}$

This micrograph shows the path of the transverse fracture near the outersurface region of the specimen. Two complete layers, one at $+10^{\circ}$ and one at $-10^{\circ}$, are shown through their thicknesses. The thickness of each layer is approximately $400 \mu \mathrm{m}(0.016 \mathrm{in})$.

The character of the transverse fracture is well-established in this micrograph. The crack propagates between fibers everywhere, and it seems to prefer a path through regions of relatively densely packed fibers. Since the fibers are sites of large transverse-stress concentrations in the matrix material, the observed fracture path seems indicative of matrix tensile failure occurring between closely spaced fibers.

The micrograph also shows branching of the fracture into small, interlaminar delamination cracks at layer boundaries. This behavior is somewhat evident through the entire thickness of the specimen, but the delaminations seem not to propagate very far beyond the immediate neighborhood of the through-thickness crack path.

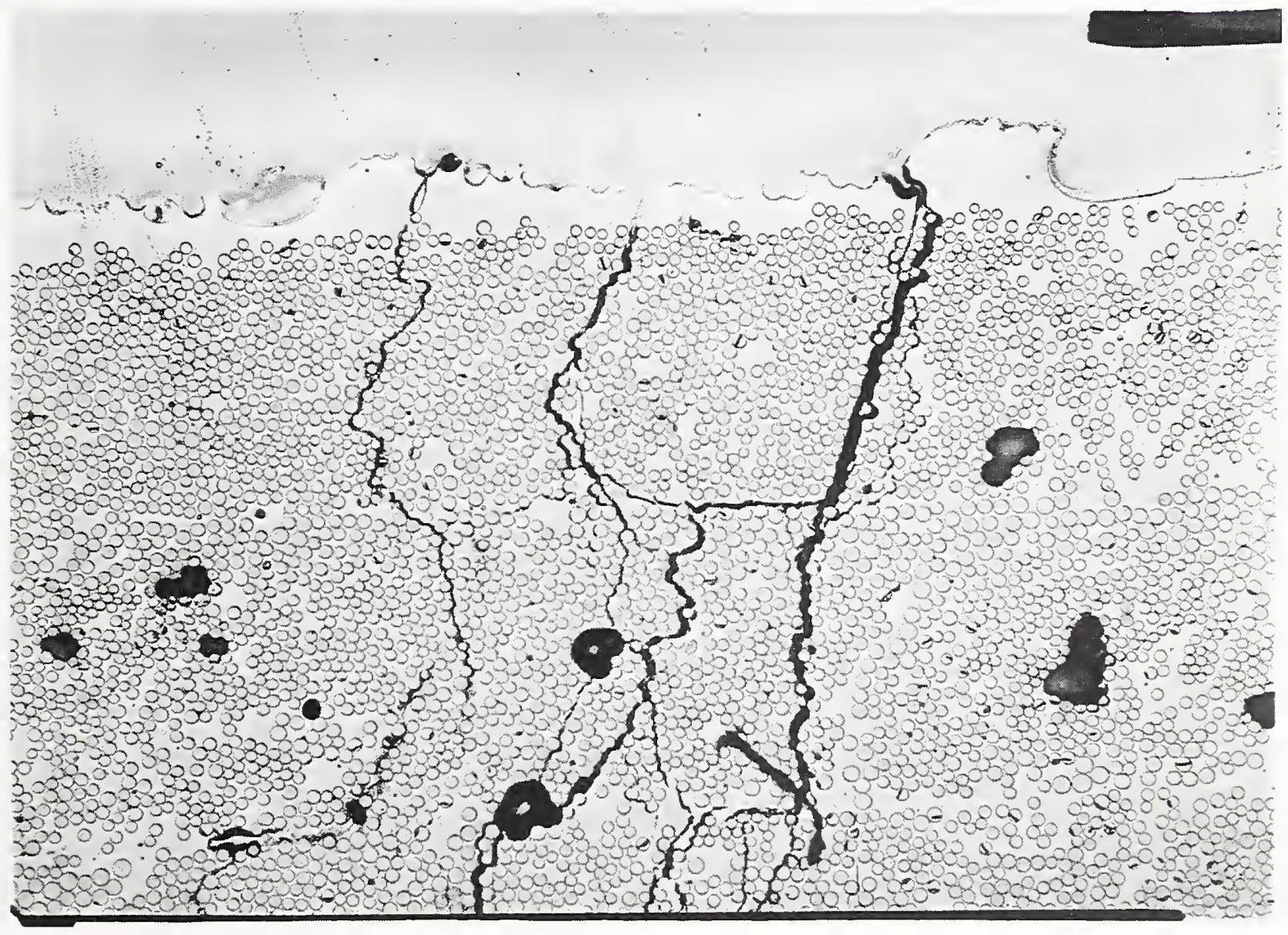




$\begin{array}{ccccc}\text { Photo I.D. No. } & \text { Tube Type } & \text { Specimen } & \text { Test Temp. } & \text { Magnification } \\ 92.2239 & 2 & 5-\mathrm{B} & 4 \mathrm{~K} & 100 \times\end{array}$

This photo shows the path of the transverse fracture into a thick-layer region from a relatively thin layer above. Crack propagation through the matrix-rich region slightly left of the center of the photo appears indicative of a shear failure of the matrix in this region, as evidenced by the occurrence of many parallel branches of the crack.

Again, the crack is observed to propagate through regions with relatively densely packed fibers. This micrograph also shows two parallel crack paths running through voids roughly $85 \mu \mathrm{m}(0.0033 \mathrm{in})$ in diameter. Also, branching along the interlayer boundaries is evident, although the delaminations do not tend to grow beyond the region bounded by the through-thickness, transverse cracks.

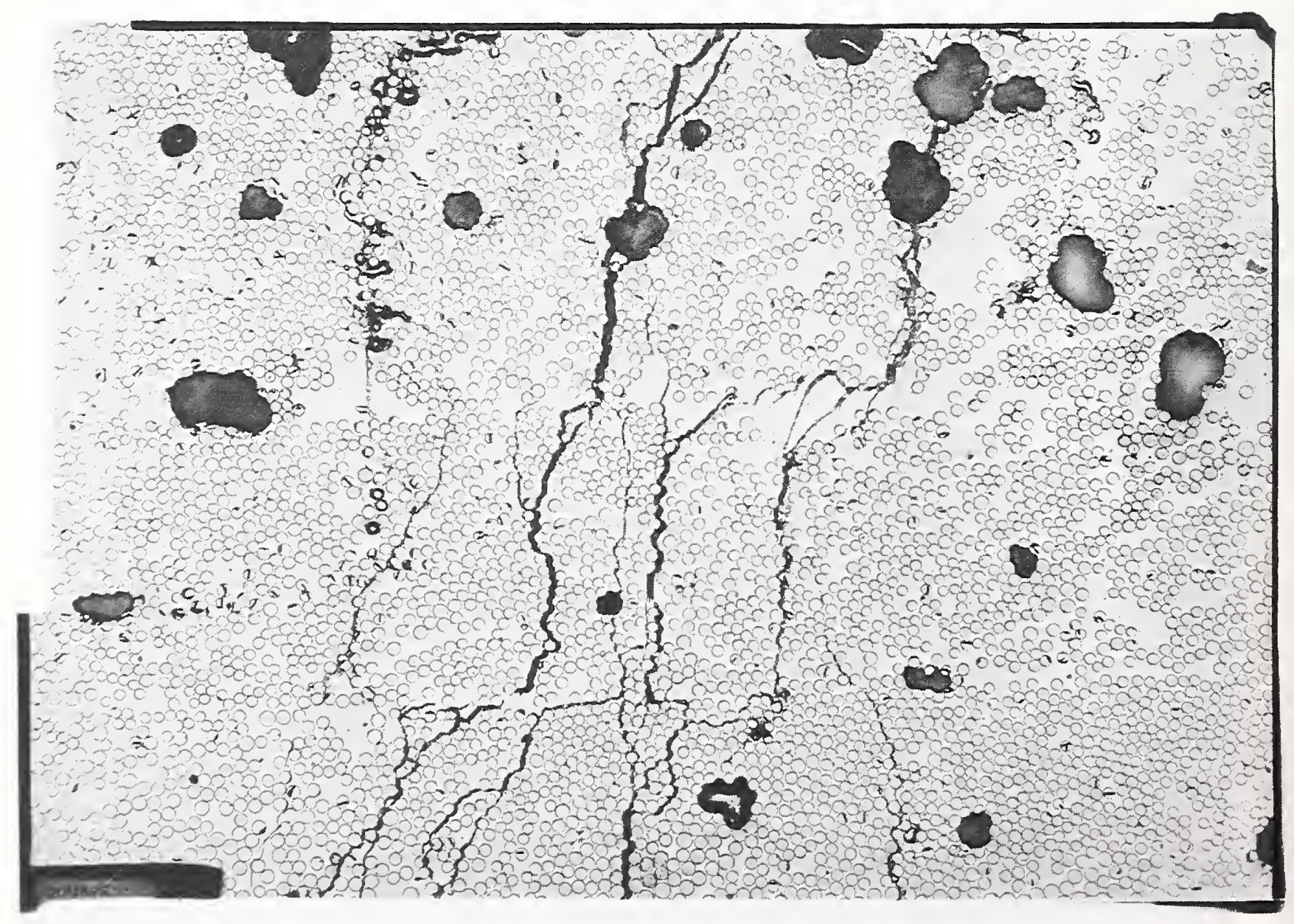




\section{Photo I.D. No. Tube Type Specimen Test Temp. Magnification \\ $\begin{array}{lllll}92.2230 & 3 & 1-\mathrm{B} & 295 \mathrm{~K} & 10 \times\end{array}$}

This photo shows nearly the entire thickness of the specimen. The failure is characterized by widespread delamination occurring mainly on boundaries between the $10^{\circ}$ and $45^{\circ}$ layers, although some delaminations are evident between the $+10^{\circ}$ and $-10^{\circ}$ layers as well. The $10^{\circ}$ layers also show considerable transverse cracking through their thicknesses.

As in the previous specimen, considerable layer thickness variability is evident along with regions of drastically reduced fiber volume fraction, especially in the $82^{\circ}$ layers. Void content is also high, especially in the $10^{\circ}$ layers.

Of particular interest is the drop-off at a $10^{\circ}$ layer near the top center area of the micrograph. The drop-off region is associated with a large void and considerable transverse cracking in the $45^{\circ}$ layer immediately above.

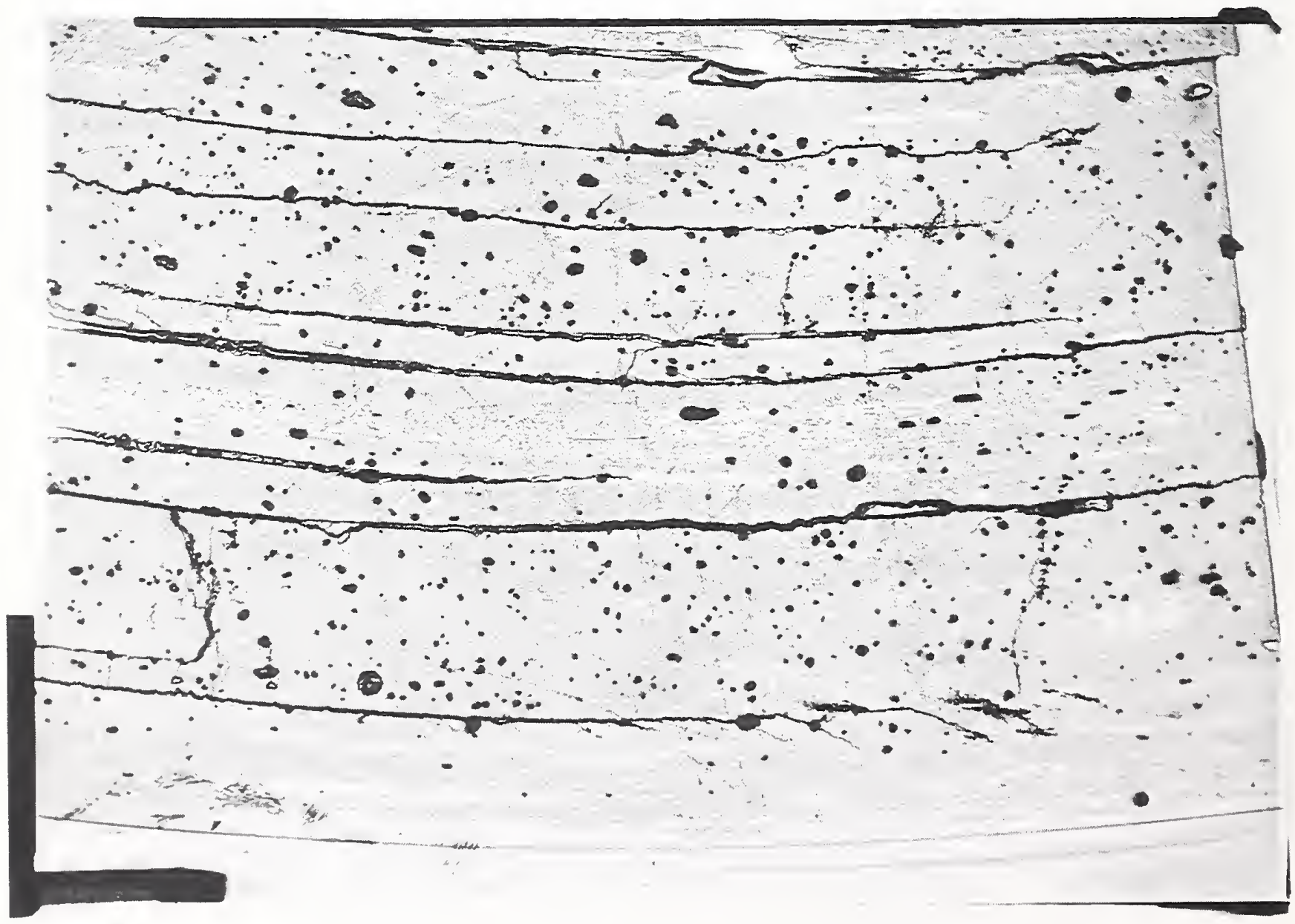




\section{Photo I.D. No. Tube Type Specimen Test Temp. Magnification \\ $\begin{array}{lllll}92.2231 & 3 & 1-\mathrm{B} & 295 \mathrm{~K} & 31.5 \times\end{array}$}

This micrograph shows the region of the specimen around the $10^{\circ}$ layer dropoff. Large voids, as long as $1 \mathrm{~mm}$, and considerable transverse cracking are visible in the $45^{\circ}$ layer immediately above the dropped layer. The transverse cracks coalesce into a delamination running to the left at the interface between the $45^{\circ}$ and $10^{\circ}$ layers. Cracks in the $45^{\circ}$ layer are visible for a large distance along the delamination.

The layer drop-off region is characterized by low fiber volume fraction, but it does not appear to have a significantly higher void content than other regions of the specimen, with the exception of the two large voids through which the delamination runs at the layer interface.

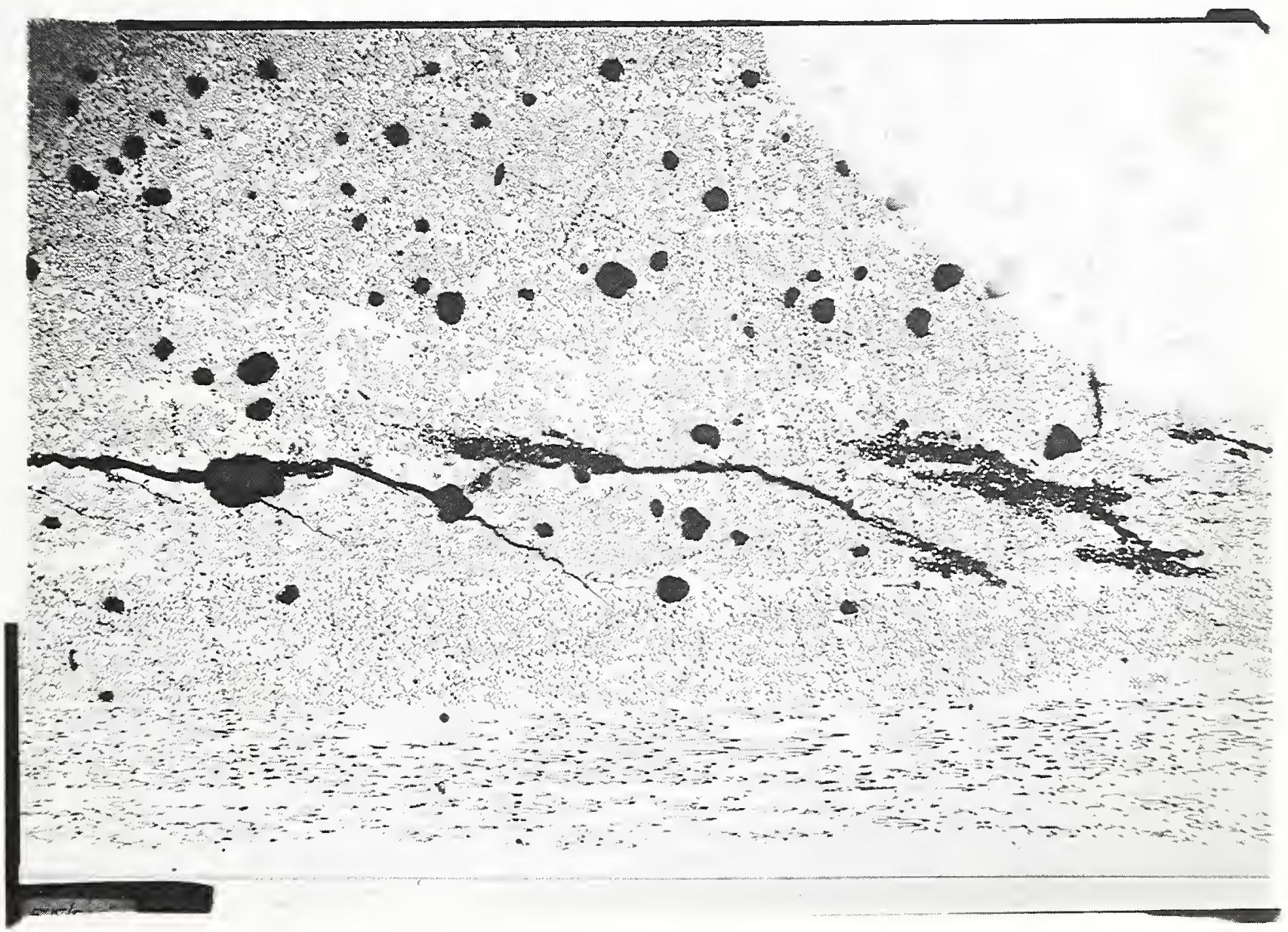




\section{Photo I.D. No. Tube Type Specimen Test Temp. Magnification \\ $92.2232 \quad 3 \quad 1-\mathrm{B} \quad 295 \mathrm{~K} \quad 63 \times$}

This micrograph shows detail of the delaminated region between the outermost $10^{\circ}$ layer and its adjacent $45^{\circ}$ layer. The branching transverse cracks extending upward into the $45^{\circ}$ layer are most probably the result of the delamination because the $10^{\circ}$ layer is displaced downward and to the left after delamination, indicating tension normal to the branching cracks during failure. Also, the branching cracks would tend to stop delamination, were they to have preceded it, because they would tend to relieve the tensile stress across the layer interface. The next page contains a micrograph of the same area at higher magnification.

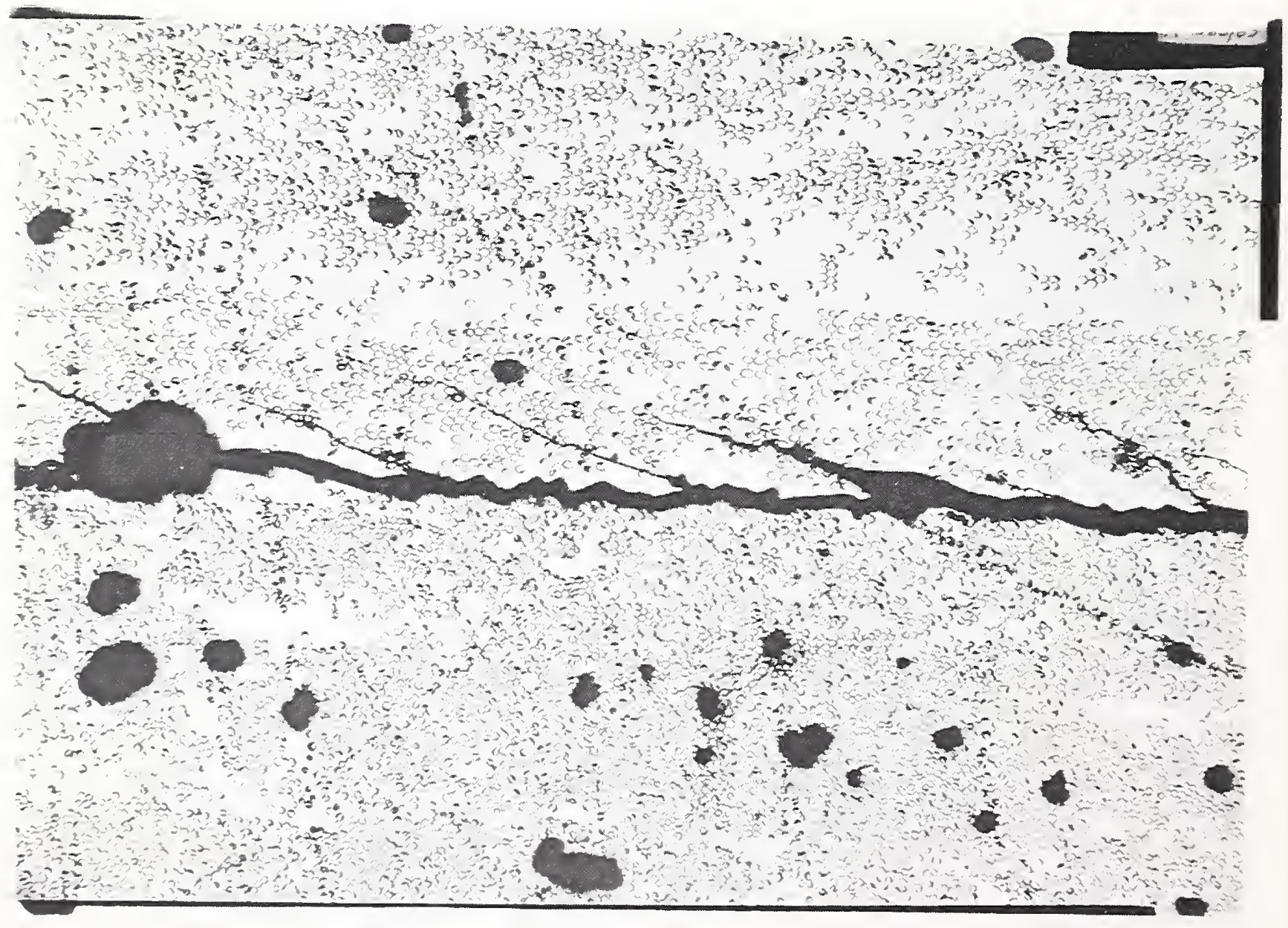




\section{Photo I.D. No. Tube Type Specimen Test Temp. Magnification \\ $92.2233 \quad 3 \quad 1-B \quad 295 \mathrm{~K} \quad 200 \times$}

This high-magnification photo of the area shown on the previous page shows the branching cracks to run primarily through matrix-rich regions, in contrast to the transverse cracking observed in the $[ \pm 10]_{\mathrm{n}}$ specimen which ran through regions dense in fibers. This further supports the hypothesis that such cracking results from the catastrophic event of the delamination.

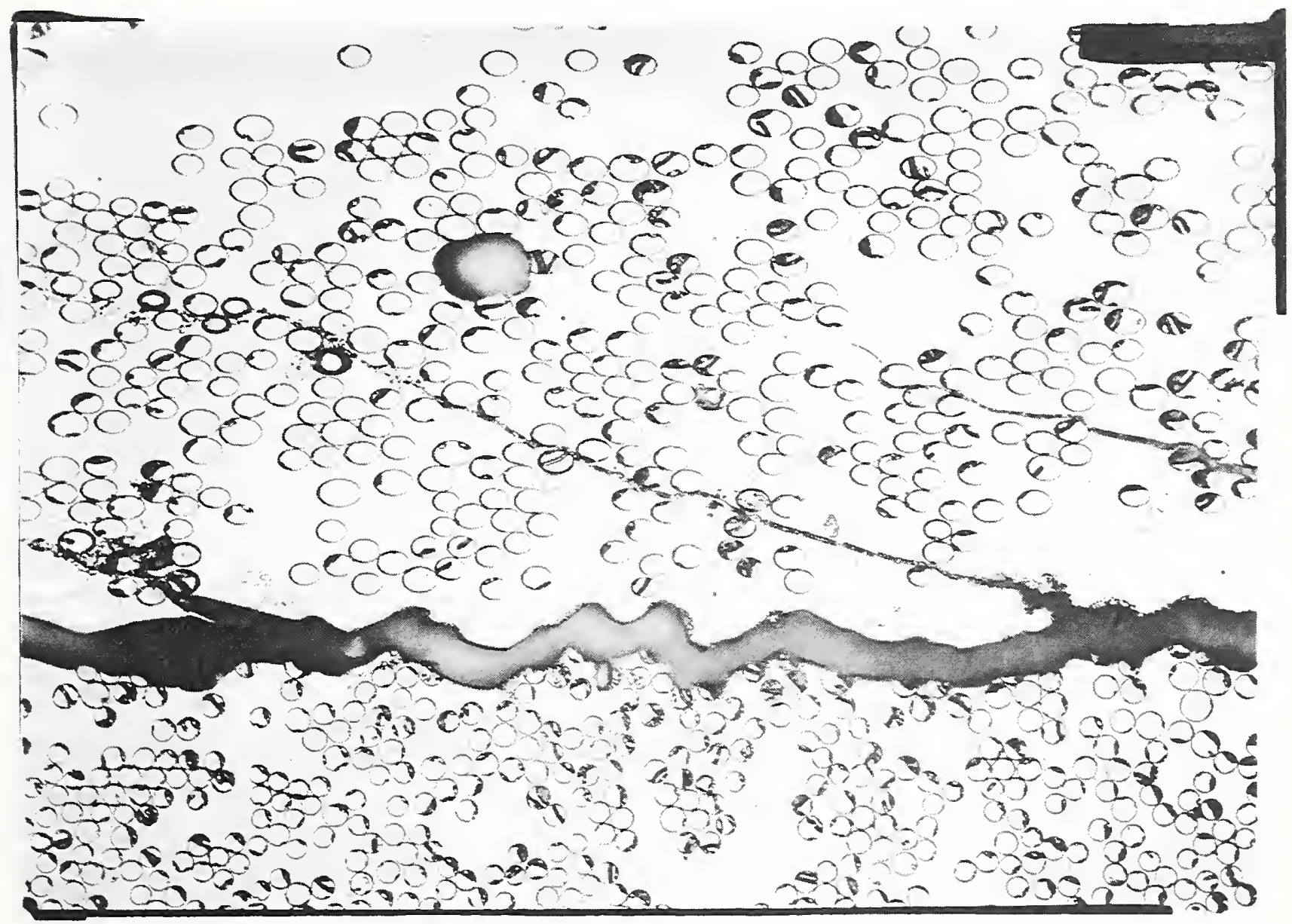


$\begin{array}{ccccc}\text { Photo I.D. No. } & \text { Tube Type } & \text { Specimen } & \text { Test Temp. } & \text { Magnification } \\ 92.3334 & 3 & 1-\mathrm{B} & 295 \mathrm{~K} & 50 \times\end{array}$

This micrograph shows detail of a region near the inner surface of the tube specimen where substantial delamination between the $45^{\circ}$ and $81^{\circ}$ layers has taken place, and where a large transverse crack in the $45^{\circ}$ layer turns into a short delamination between adjacent $\pm 45^{\circ}$ layers. A micrograph at higher magnification appears on the next page.

The transverse crack running through the thickness of the $45^{\circ}$ layer appears to be characterized by matrix shear and tension, with failures propagating between fiber edges. This is substantiated by the primarily leftward displacement of the region below the delamination-a direction consistent with such forces.

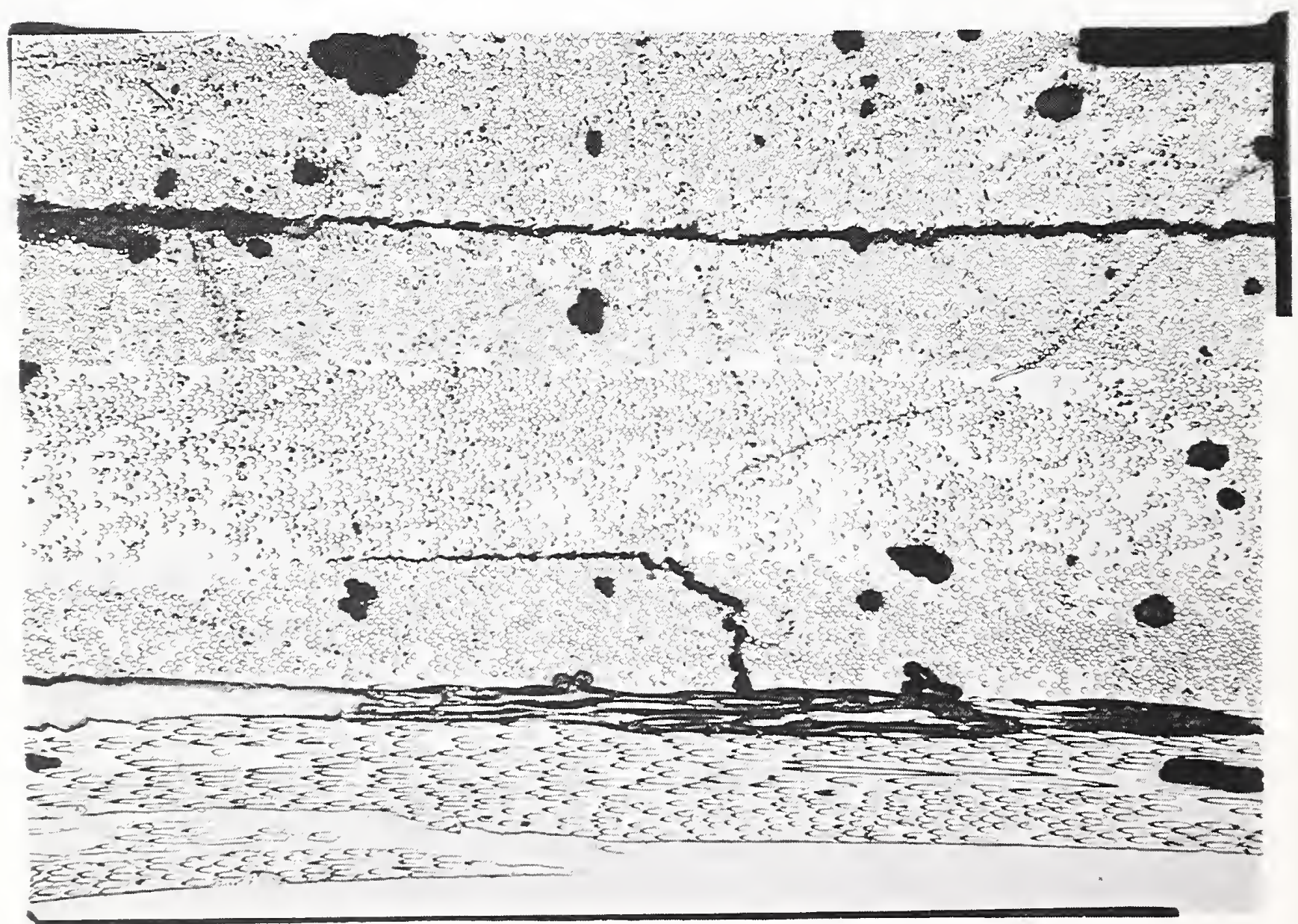




\section{Photo I.D. No. Tube Type Specimen Test Temp. Magnification $92.3335 \quad 3 \quad 1-B \quad 295 \mathrm{~K} \quad 160 \times$}

This micrograph at higher magnification of the specimen area shown on the previous page suggests that the delamination occurred before the through-thickness crack. It very clearly shows deformed regions of matrix material in the layer above (outside) the delamination which indicate that the lower $45^{\circ}$ layer moved initially to the right of the upper layer, pulling matrix material to the right and downward as the delamination progressed. At some point, perhaps at final failure, this shearing produced sufficient force to fracture the lower layer out to the delaminated surface layer. The likely presence of voids in the path of the transverse crack near the surface layer interface may have precipitated the fracture at this location.

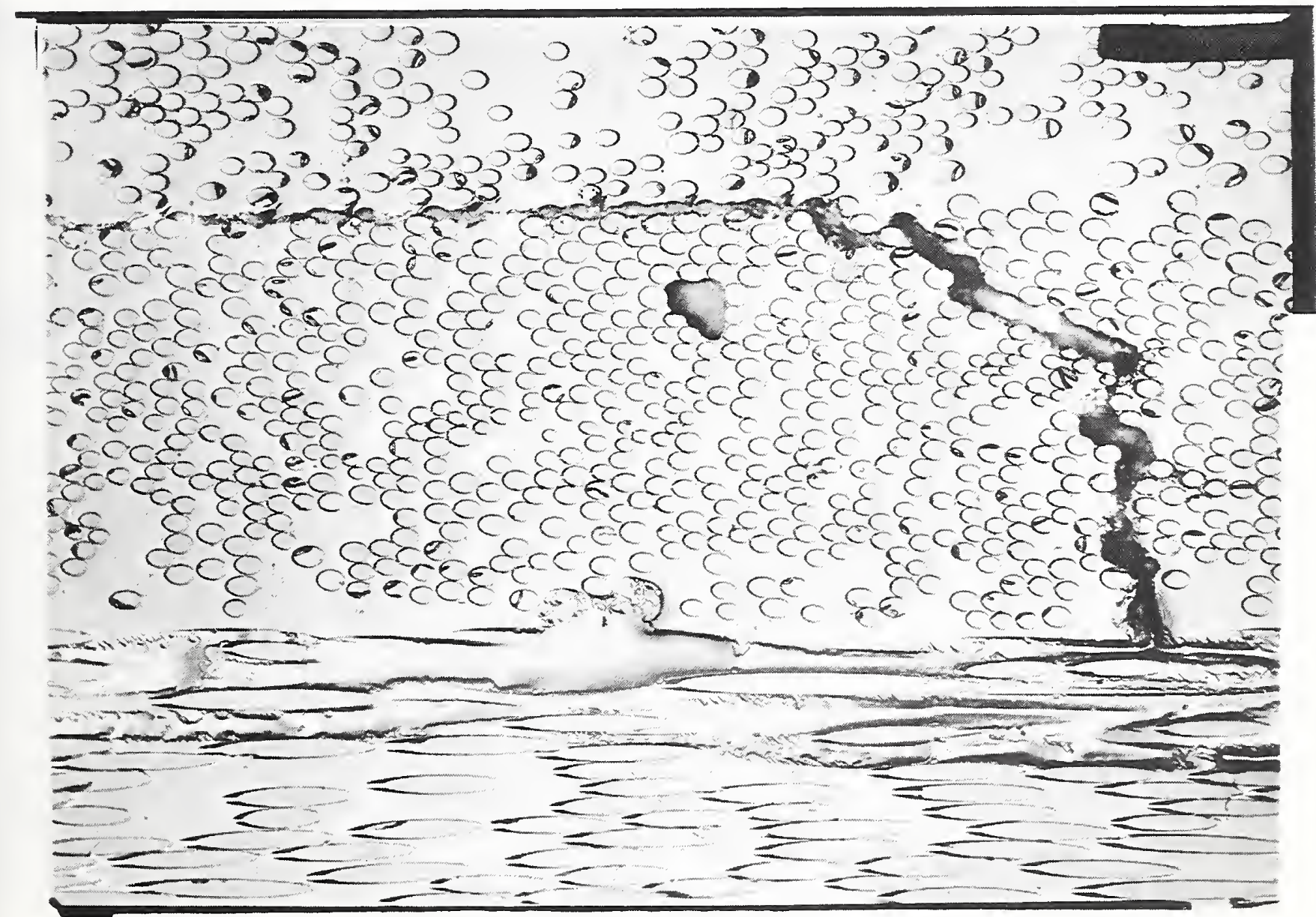




$\begin{array}{ccccc}\text { Photo I.D. No. } & \text { Tube Type } & \text { Specimen } & \text { Test Temp. } & \text { Magnification } \\ 92.2236 & 3 & 1-\mathrm{B} & 295 \mathrm{~K} & 160 \times\end{array}$

This micrograph shows a delamination between adjacent $\pm 10^{\circ}$ layers. The delamination runs mostly through the matrix-rich region between layers. Deformation of the matrix region at the top of the delamination indicates a shearing failure in which the lower $10^{\circ}$ layer was pulled down and to the left from the upper layer. Whether this delamination preceded or followed the major failure of the tube cannot be determined.

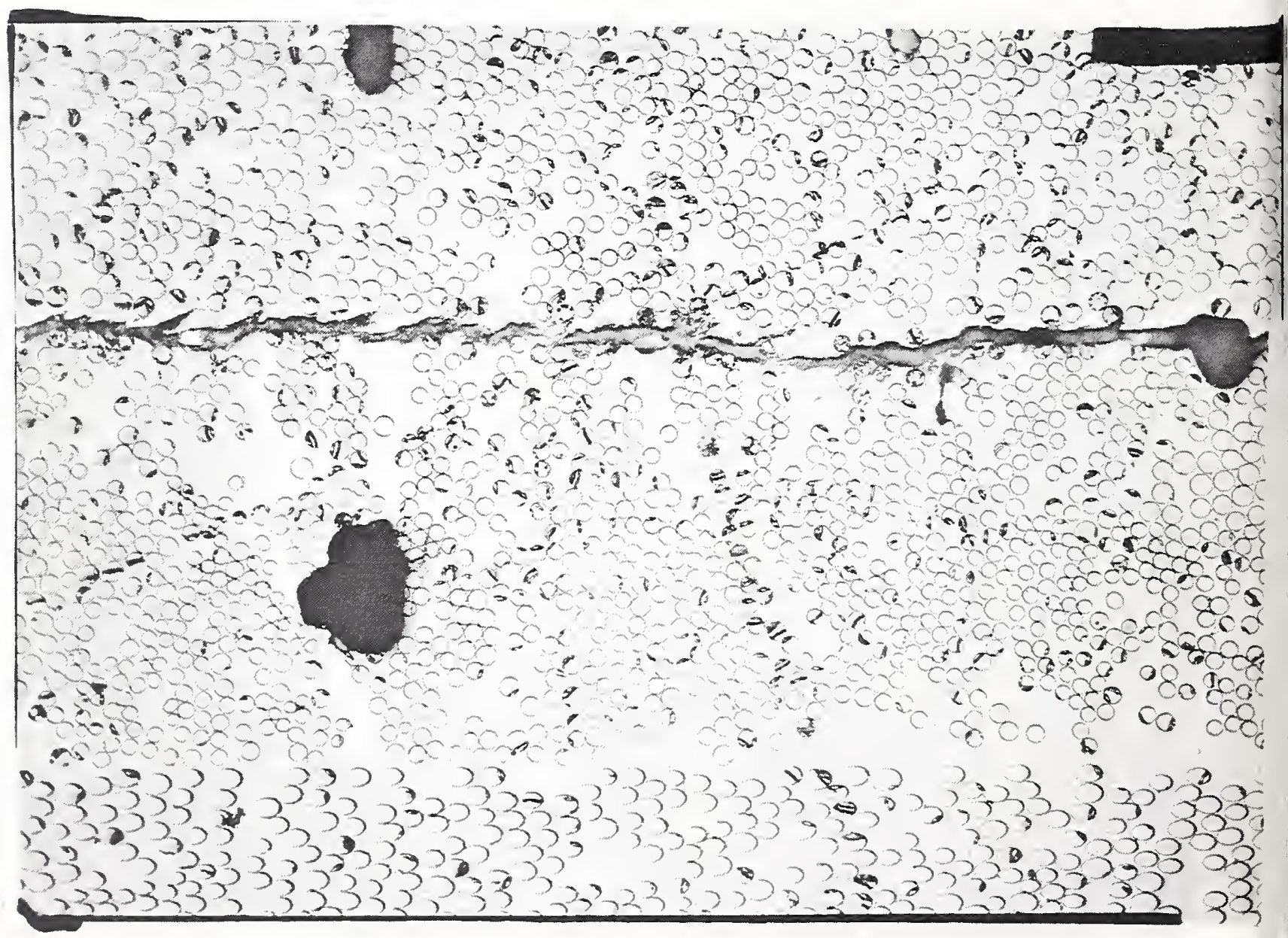


$\begin{array}{ccccc}\text { Photo I.D. No. } & \text { Tube Type } & \text { Specimen } & \text { Test Temp. } & \text { Magnification } \\ 92.2241 & 5 & 3-\mathrm{A} & 76 \mathrm{~K} & 12 \times\end{array}$

This micrograph shows the section of the specimen taken from the lower edge of the tube's visibly delaminated region. Remarkably lower void content is evident in this specimen, unlike the other types. Layer thickness is seen to be significantly variable (up to $\sim 100 \%$ ), as in other specimens.

The aspect of primary interest in this specimen is the transverse cracking, evident throughout the thickness of the specimen. An interesting aspect of this cracking is the observation that most cracks do not propagate directly through the thickness of the hoop-wound $\left(82^{\circ}\right)$ layers. Instead, the cracks seem either to propagate diagonally across the $82^{\circ}$ layers or to be arrested entirely by the $82^{\circ}$ layer. This phenomenon is examined further in the following micrographs.

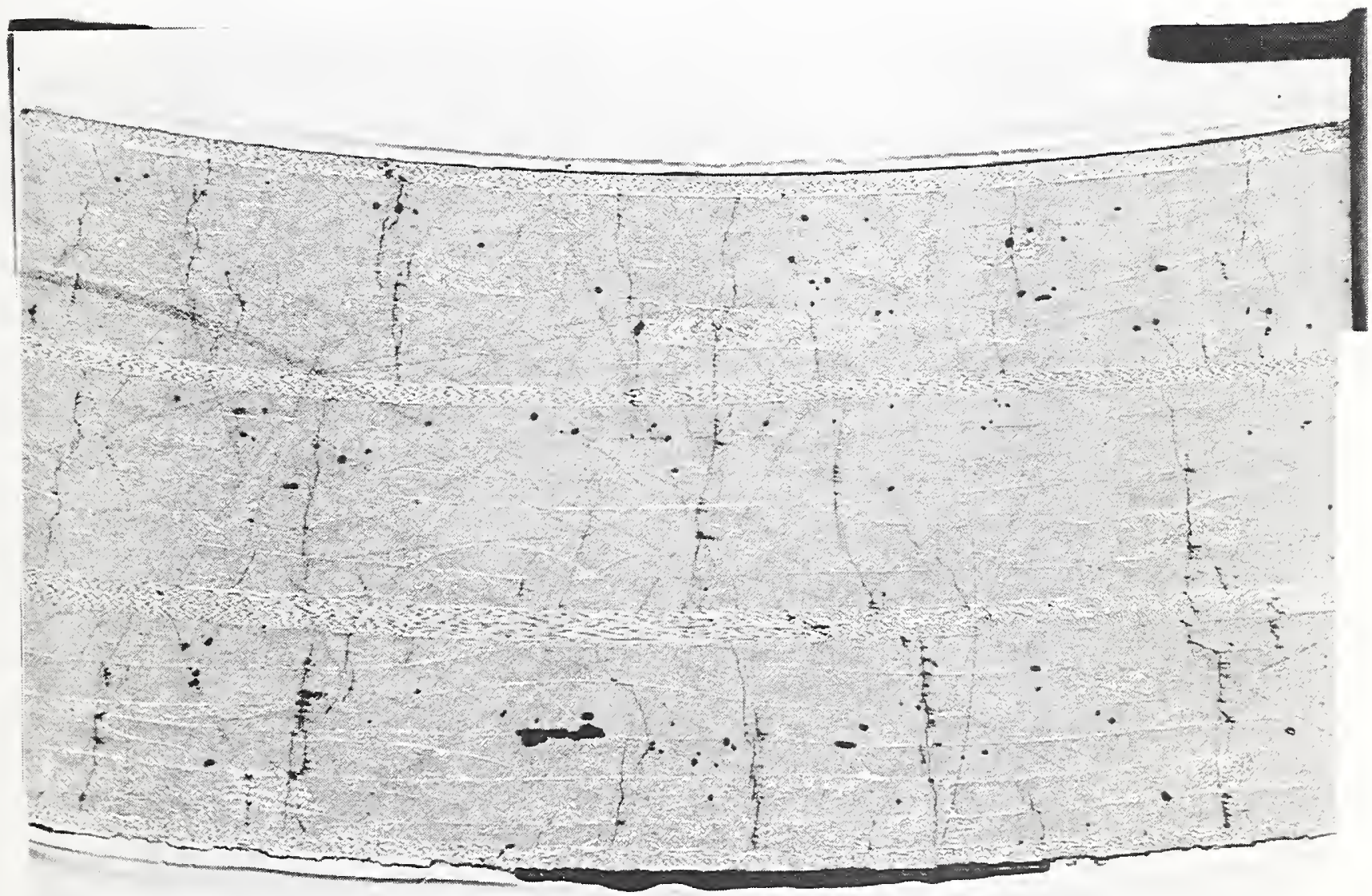


$\begin{array}{ccccc}\text { Photo 1.D. No. } & \text { Tube Type } & \text { Specimen } & \text { Test Temp. } & \text { Magnification } \\ 92.2242 & 5 & 3-\mathrm{A} & 76 \mathrm{~K} & 40 \times\end{array}$

This micrograph shows a region between the two outermost $82^{\circ}$ layers of the specimen. A transverse crack runs vertically through six $[ \pm 10]$ layers from the outermost $82^{\circ}$ layer to the next. The character of the transverse crack is very similar to that observed in the $[ \pm 10]$ (type 2 ) specimens, in that the crack tends to propagate through regions densely packed with fibers.

The crack branches at several locations, the most notable is at the outer surface of the sixth $10^{\circ}$ layer from the specimen surface, where the crack branches into a delamination running to the left through a fiber-rich interlayer interface for a distance of about $625 \mu \mathrm{m}(0.025 \mathrm{in})$. The crack path does not appear to be continuous through the embedded $82^{\circ}$ layer, but seems to be associated with a diagonal crack through this layer that connects with another transverse through crack in the next-inner $10^{\circ}$ layer.

This micrograph also illustrates the variability of fiber volume fraction within both $10^{\circ}$ and $82^{\circ}$ layers, but especially within the $82^{\circ}$ layers. 


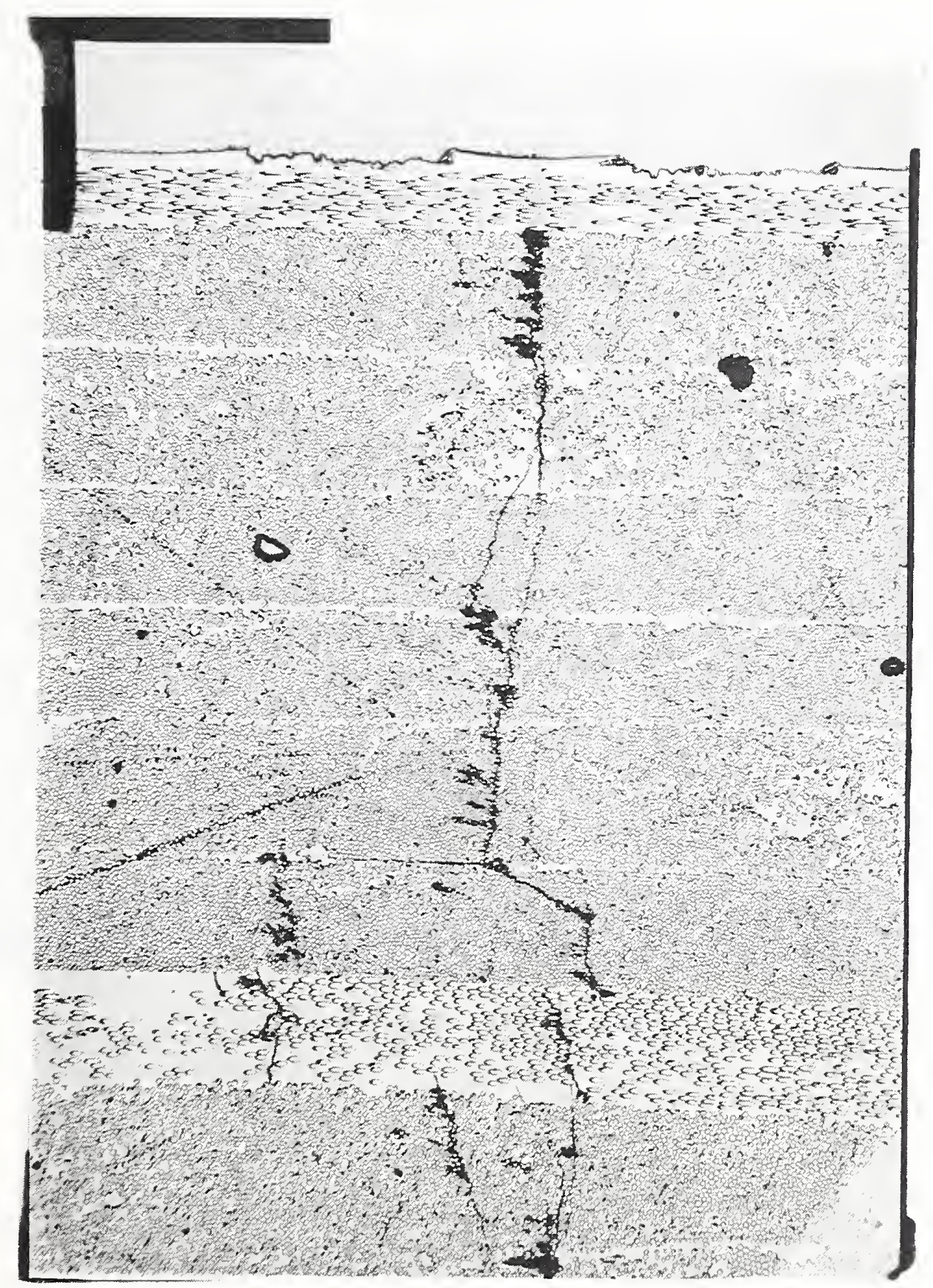

Photo I.D. No.: 99.2242 Tube Type: 5 Specimen: 3-A Test Temp. $76 \mathrm{~K}$ Magnification: $40 \times$ 
$\begin{array}{ccccc}\text { Photo I.D. No. } & \text { Tube Type } & \text { Specimen } & \text { Test Temp. } & \text { Magnification } \\ 92.2243 & 5 & 3-\mathrm{A} & 76 \mathrm{~K} & 80 \times\end{array}$

This micrograph shows further detail of the branching crack, its associated delamination, and nearby cracks through the adjacent $82^{\circ}$ layer. The crack above the $82^{\circ}$ layer is evidently terminated in a void at the $10^{\circ} / 82^{\circ}$ layer interface. This void is small by the standards of the other specimen types: it is roughly $50 \mu \mathrm{m}(0.002 \mathrm{in})$ long.

The crack through the $82^{\circ}$ layer appears connected with the crack in the inner $10^{\circ}$ layer, the top of which is just visible at the bottom of the photograph. There appears to be no connection at this section between the crack propagating upward through the $[ \pm 10]$ layers and the crack through the $82^{\circ}$ layer.

The crack above the $82^{\circ}$ layer branches at several locations into a network of smaller transverse cracks. The delamination running along the $10^{\circ}$ interlayer boundary follows the inner surface of the outer visible $10^{\circ}$ layer very closely, even into the matrix-rich region well to the left of the main crack path. This observation would support the hypothesis that transverse (or delaminating) tensile cracks form in the matrix material at fiber boundaries, either due to the degree of the transverse stress concentration caused by the fiber or due to a weakened interface caused by low fiber-matrix adhesion strengths. 


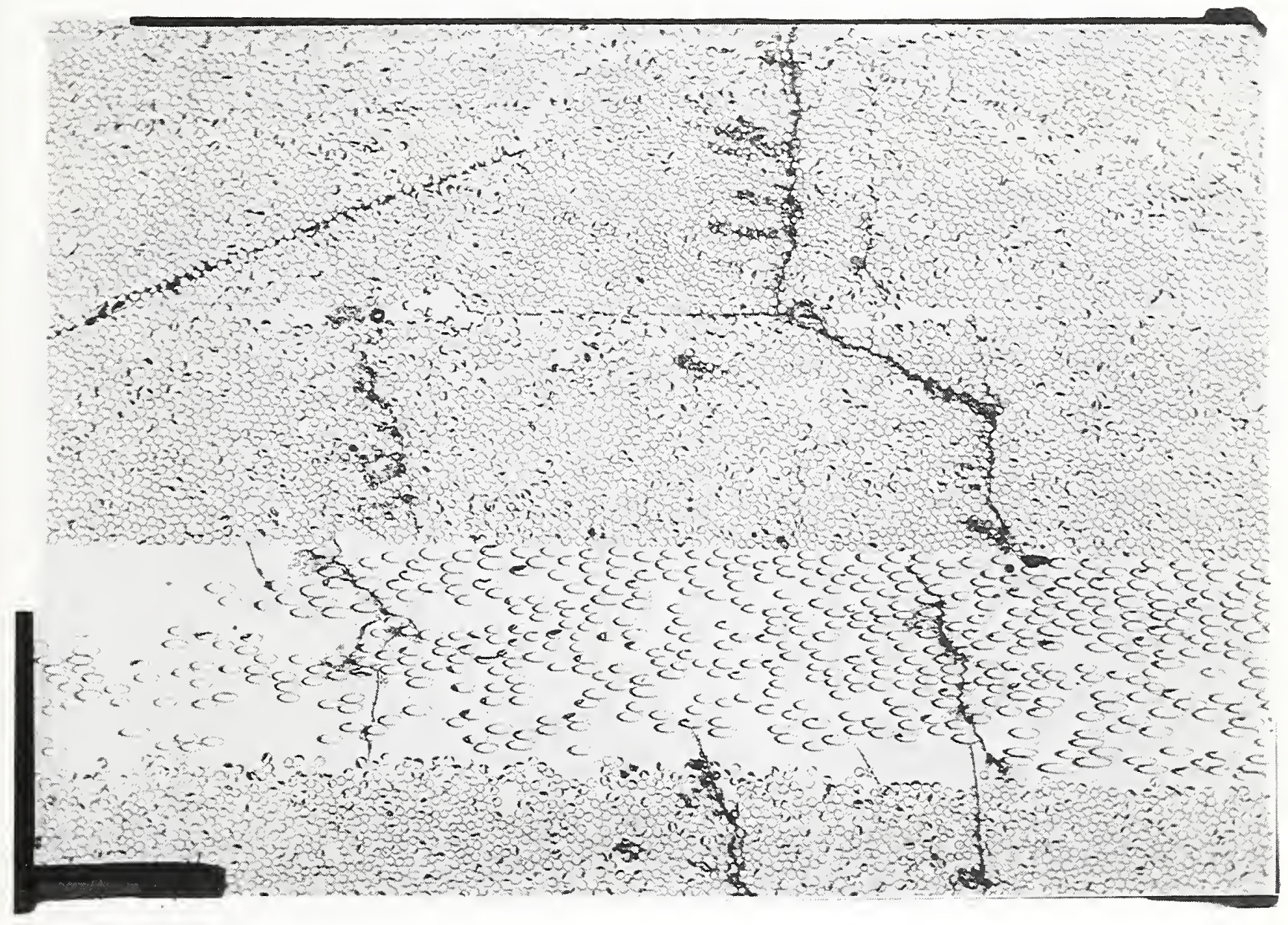

Photo I.D. No.: 99.2243 Tube Type: 5 Specimen: $3-\mathrm{A}$ Test Temp.: $76 \mathrm{~K}$ Magnification: $80 \times$ 
Photo I.D. No. Tube Type Specimen Test Temp. Magnification

$\begin{array}{lllll}92.2245 & 5 & 3-\mathrm{A} & 76 \mathrm{~K} & 12 \times\end{array}$

This micrograph, and the one following, were taken from a region of the specimen away from the final failure and delaminated regions, but exhibiting visible transverse cracking in the $[ \pm 10]$ layers. This cracking is evident through the thickness of the specimen, and it appears with some fairly regularly spaced cracking observed on the surface of the $82^{\circ}$ layer. As typical of specimens from this tube, void content is relatively low, although considerable variations can be seen in layer thickness and local fiber content.

Transverse cracking in the $10^{\circ}$ layers appears, as it does in the other specimens, with crack paths progressing directly through the entire thicknesses of the $10^{\circ}$ layers and with some branching. The cracks are seen not to propagate directly through the $82^{\circ}$ layers. Considerably fewer transverse cracks are noticed in this region of the tube than in that corresponding to micrograph 92.2241 , and none of the cracks in the present specimen are visibly associated with a delamination.

The notable aspect of this micrograph is the fairly regularly spaced occurrence of transverse cracks in the outer surface $82^{\circ}$ layer. These cracks occur roughly every $3 \mathrm{~mm}(0.13 \mathrm{in})$, and they may be associated with the visibly whitened striations visible on the surface of the specimen in photograph 92.1620. (Note that the metallographic specimen's surface is oriented at an angle to the tube axis.) That these cracks are so regularly spaced may indicate their cause to be related to thermal stresses. 


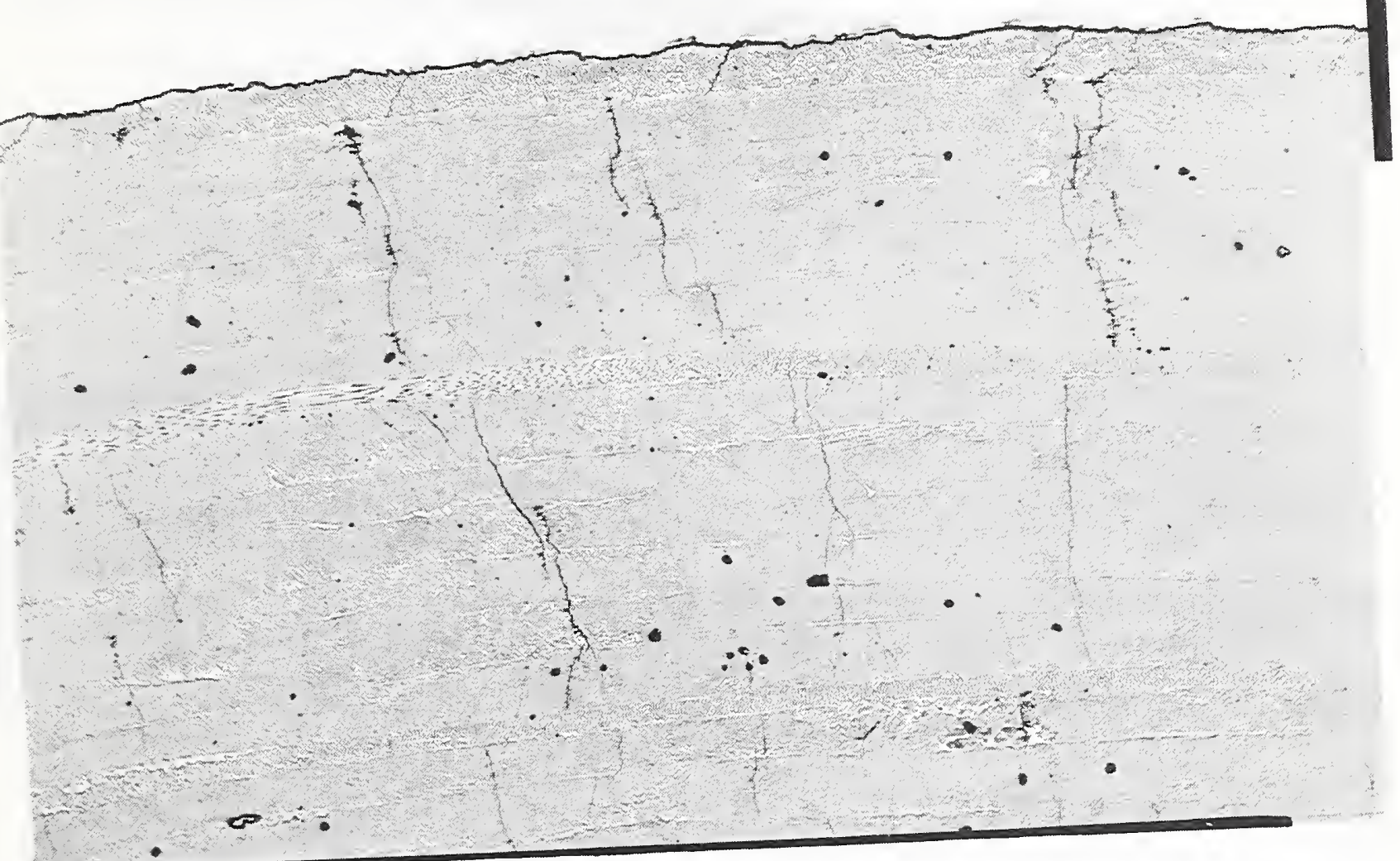

Photo I.D. No.: 99.2245 Tube Type: 5 Specimen: $3-\mathrm{A} \quad$ Test Temp.: $76 \mathrm{~K}$ Magnification: $12 \times$ 


\section{Photo I.D. No. Tube Type Specimen Test Temp. Magnification $\begin{array}{lllll}92.2246 & 5 & 3-\mathrm{A} & 76 \mathrm{~K} & 62.5 \times\end{array}$}

This micrograph shows detail of the central area of photo no. 92.2245 at the surface.

The transverse crack running down through the $10^{\circ}$ layers shows a morphology similar to those documented previously, with the transverse failure appearing to propagate through regions of dense fiber concentration. Of interest in this crack is the occurrence of two voids; one at the interface between the surface $81^{\circ}$ layer and the subsurface $10^{\circ}$ layer, and one adjacent to a matrix-rich line just below the interface. The crack initiates and terminates at the first void, and it propagates directly through the second.

The transverse crack in the surface $81^{\circ}$ layer propagates through the entire layer thickness and then along the topmost fibers of the subsurface $10^{\circ}$ layer for a distance of roughly $120 \mu \mathrm{m}$ (0.005 in). Generally, little coupling is evident between the surface-layer transverse cracks and those running through the $10^{\circ}$ layers.

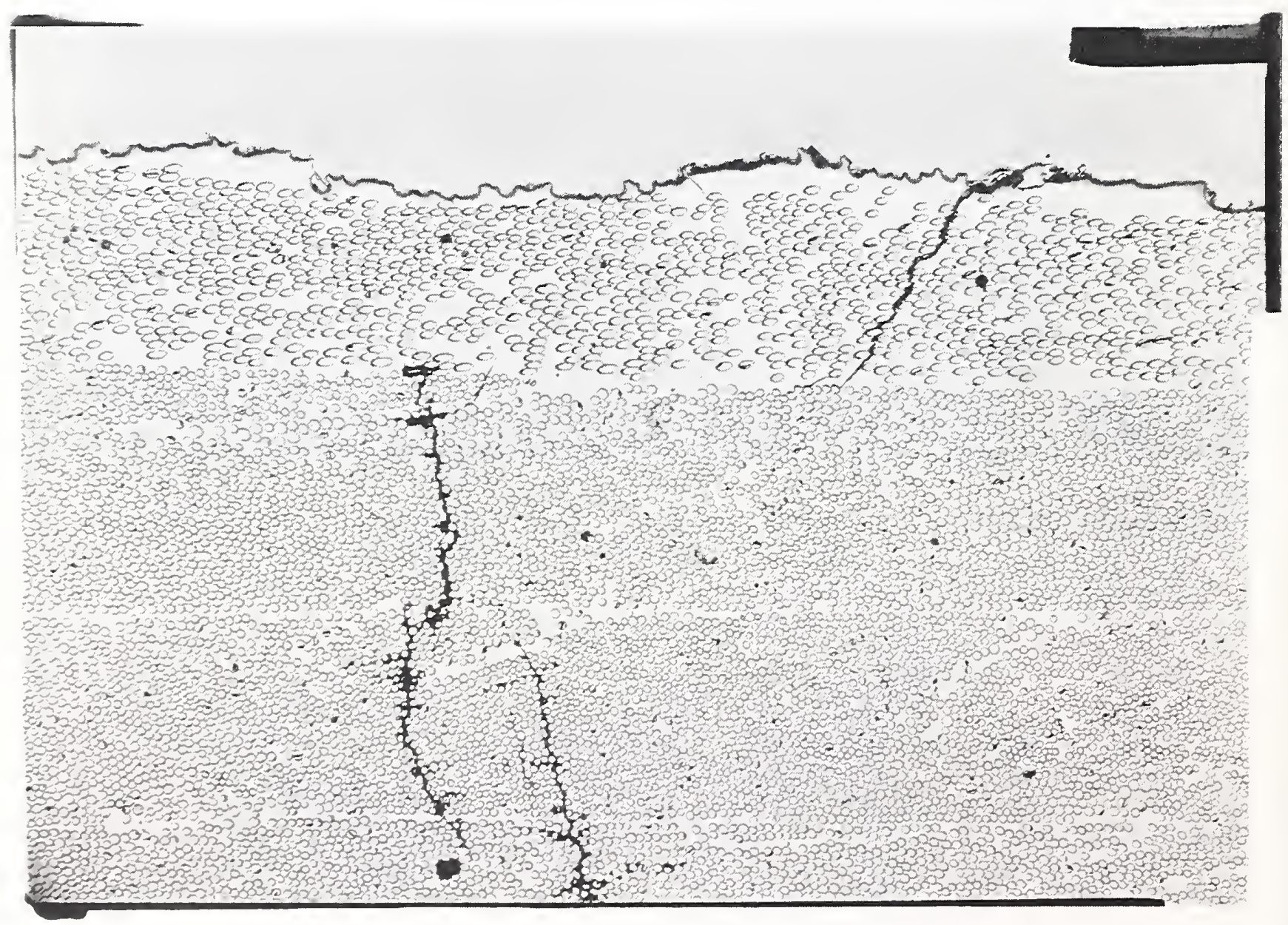




\section{Appendix B. FORTRAN Program for Estimating Layer Elastic Properties of Fiberglass Tubes}

The FORTRAN program and subroutines for computing best-fit 2-D laminated-plate material properties from NIST test data:

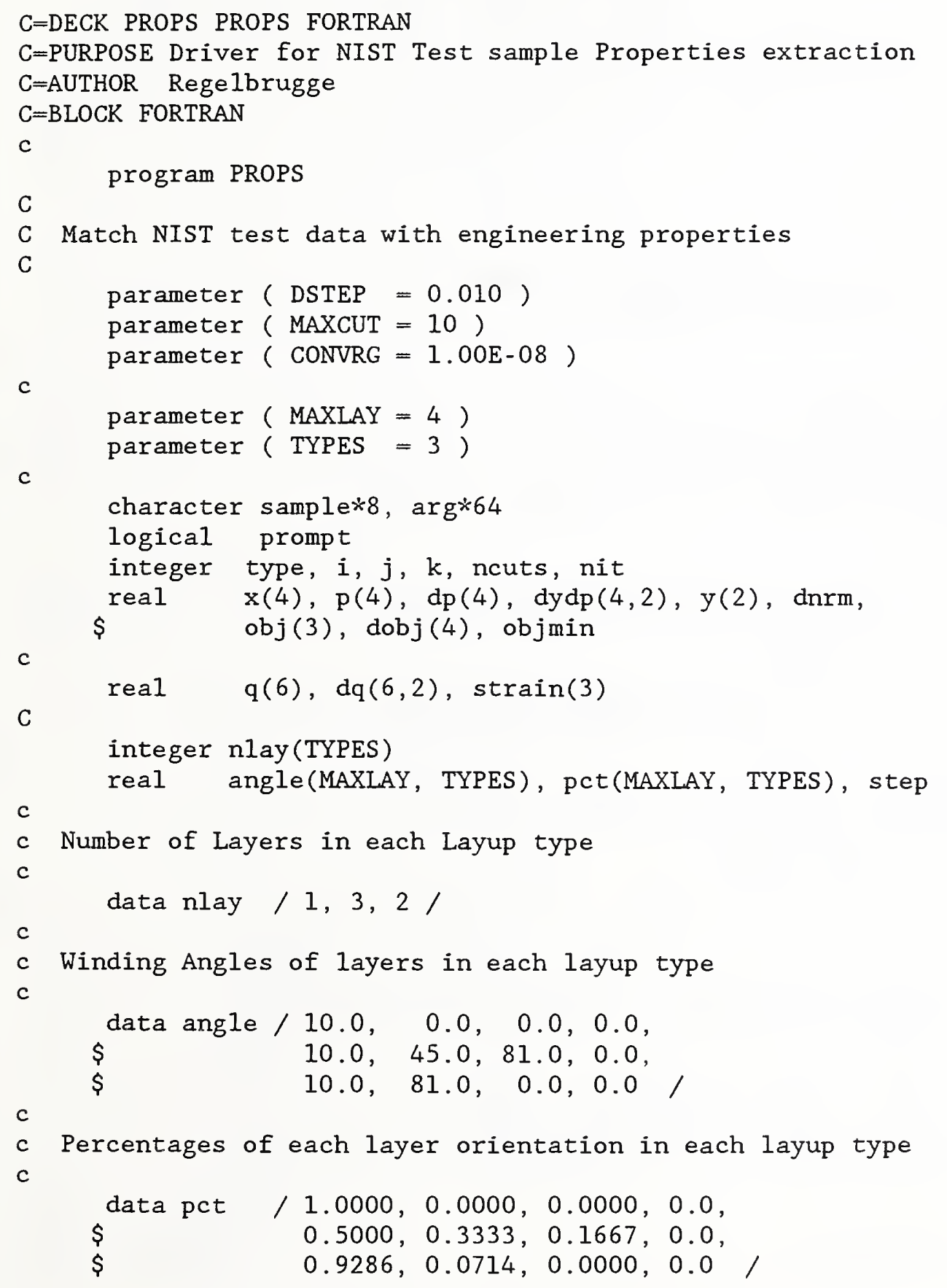




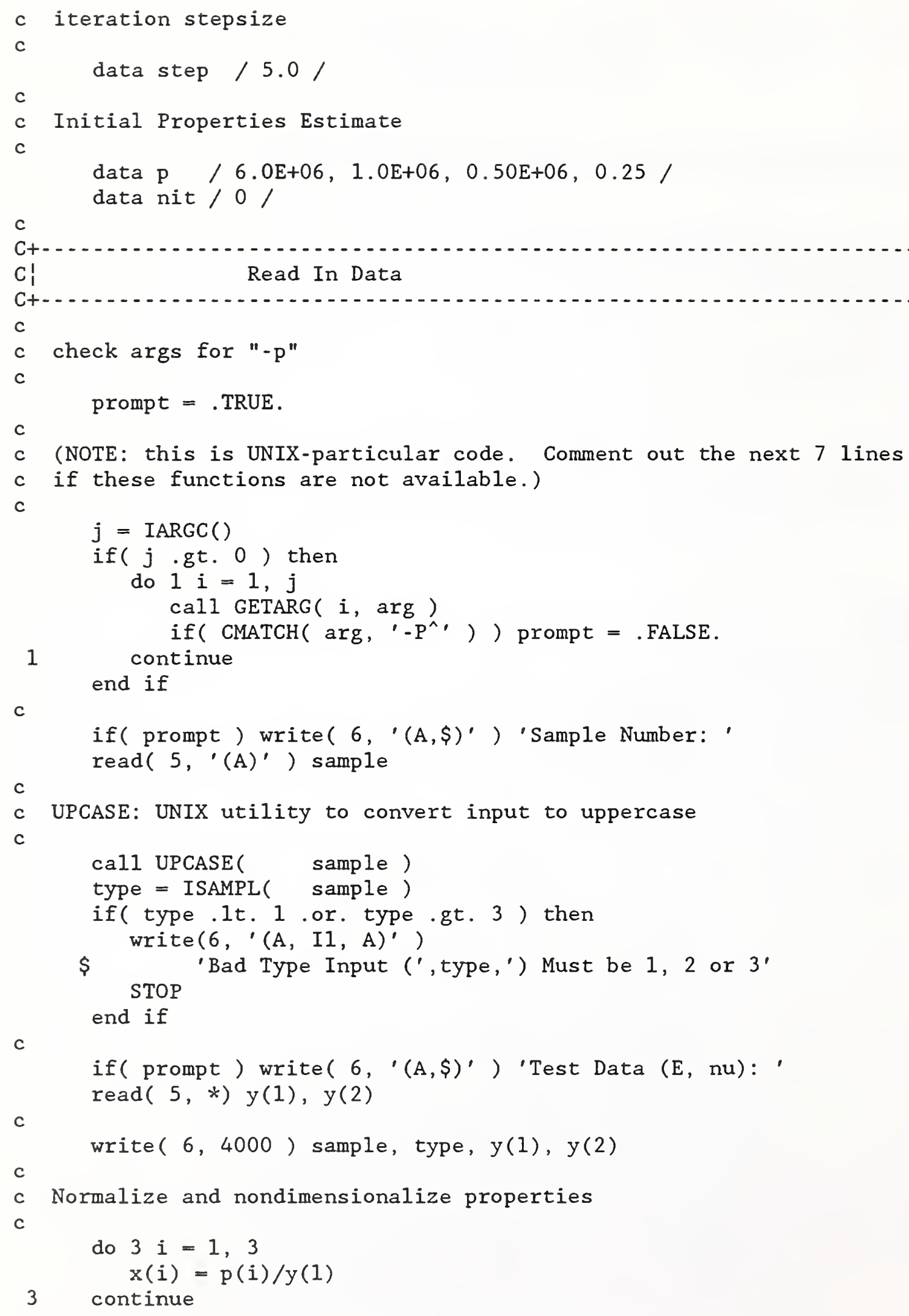




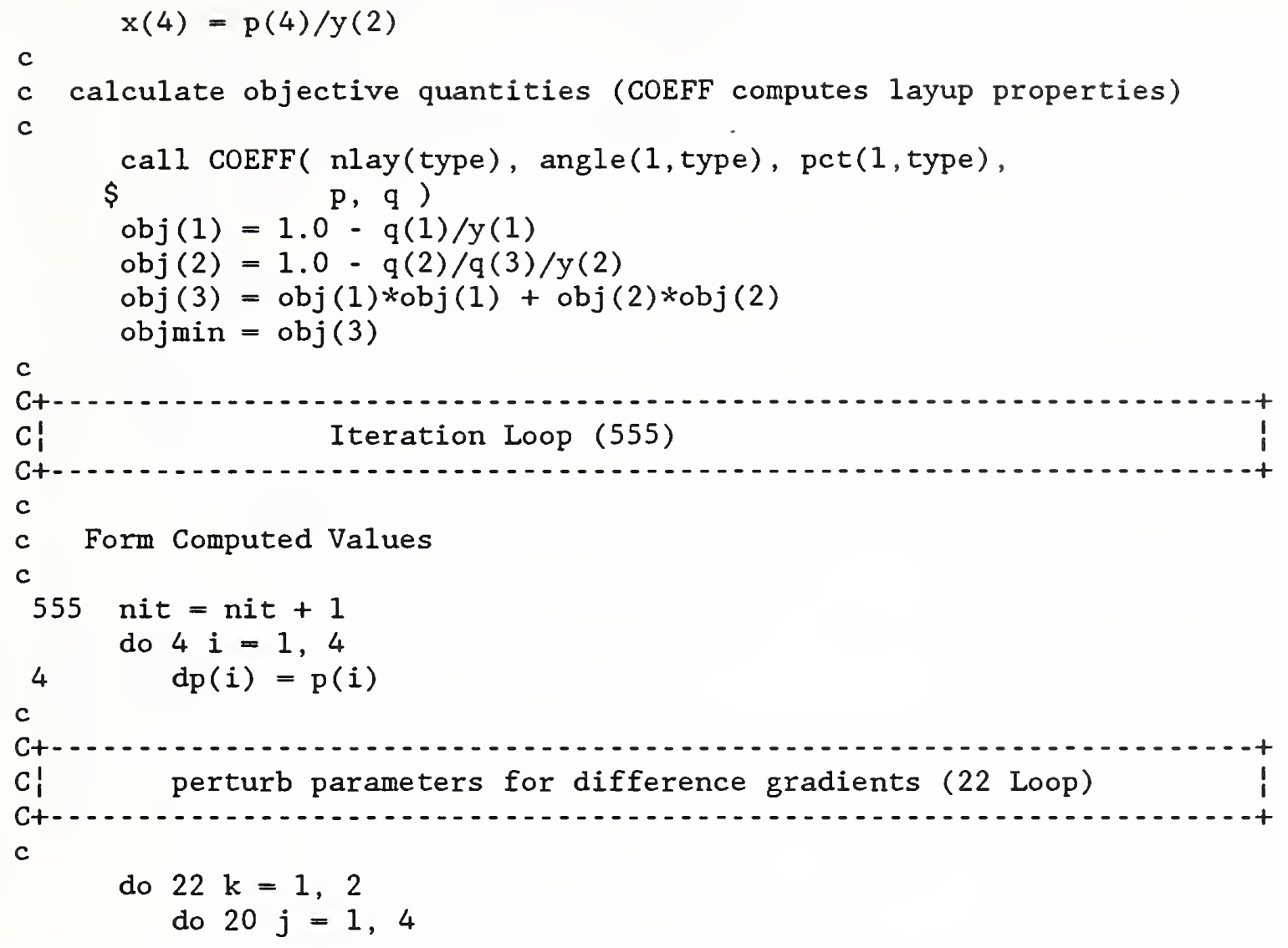

4

Iteration Loop (555)

c

c a) perturb parameters to compute gradient of objective

c

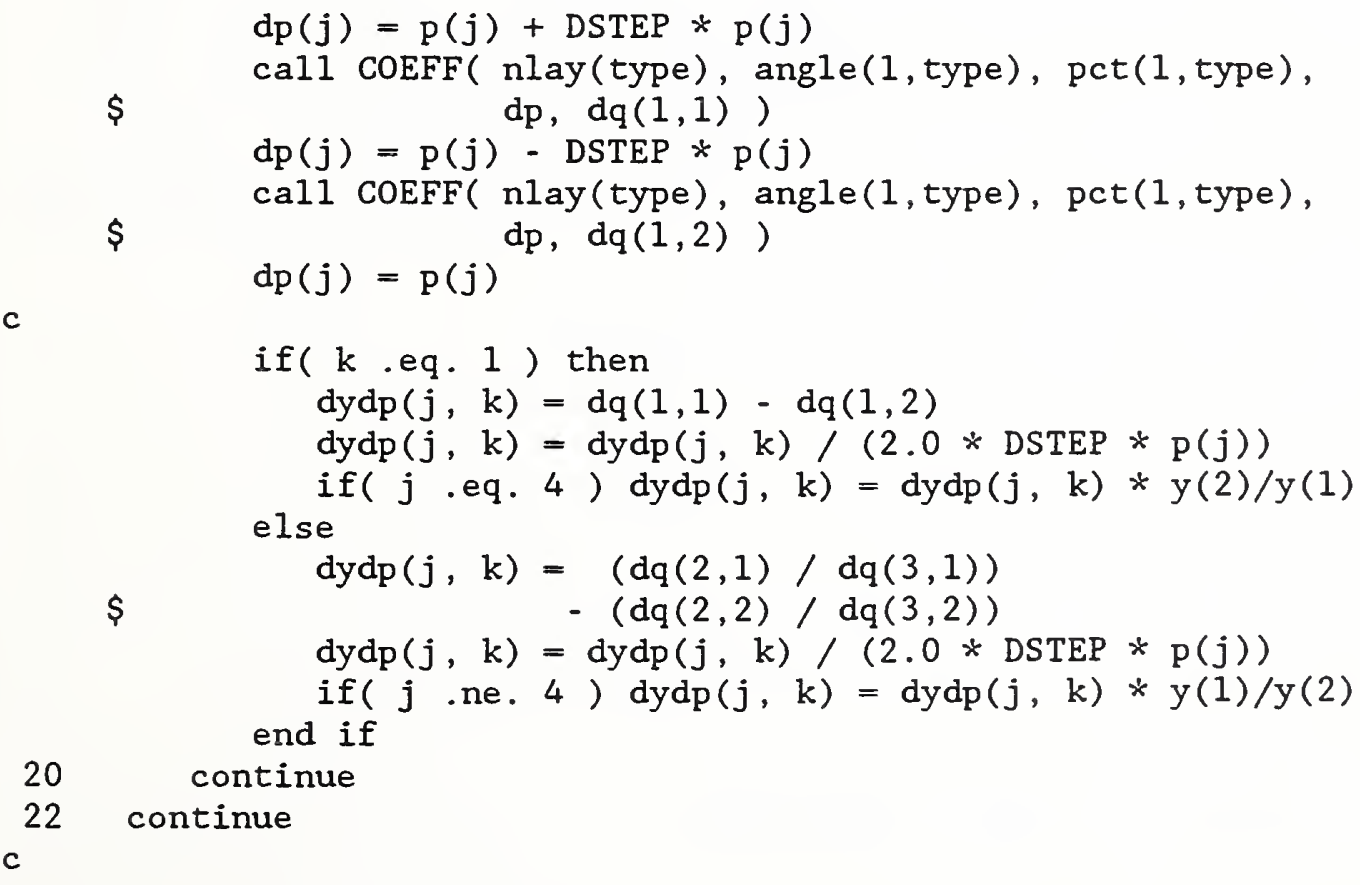


c

c

27 dnrm

dnrm $=0.0$

do $27 \mathrm{j}=1,4$

$\operatorname{dobj}(j)=2.0 *(\operatorname{obj}(1) * \operatorname{dydp}(j, 1)+\operatorname{obj}(2) * \operatorname{dydp}(j, 2))$

27 continue

c

C) update parameters (111 Loop)

C

$$
\text { ncuts }=0
$$

111 do $135 j=1,4$

if $(j$.eq. 4$) d p(j)=p(j)+y(2) * \operatorname{dob} j(j) *($ step*DSTEP $)$

if $(j$.ne. 4$) \operatorname{dp}(j)=p(j)+y(1) * \operatorname{dob} j(j) *($ step*DSTEP $)$

135 continue

c

c update property estimates

c

call COEFF( nlay(type), angle(1,type), pct(1, type), dp, q )

c

c calculate new objective quantities

c

$\operatorname{obj}(1)=1.0-q(1) / y(1)$

$\operatorname{obj}(2)=1.0-q(2) / q(3) / y(2)$

$\operatorname{obj}(3)=\operatorname{obj}(1) * o b j(1)+o b j(2) * o b j(2)$

c

C| Iteration Criteria: cut step, continue or exit

c

c Adjust step if objective increases or exit if MAXCUT exceeded

c

if (obj(3) .ge. objmin) then

step $=0.5 *$ step

ncuts $=$ ncuts +1

if ( ncuts .1t. MAXCUT) GO TO 111

143 do $143 j=1,4$

Go TO 777

c

c continue or exit

c

else

objmin $=$ obj (3)

$p(j)=\operatorname{dp}(j)$

if (objmin .1t. CONVRG) GO TO 777

c 
write $(4,3999)$ nit, $\operatorname{obj}(1), \operatorname{obj}(2),(p(i), i=1,4)$

c

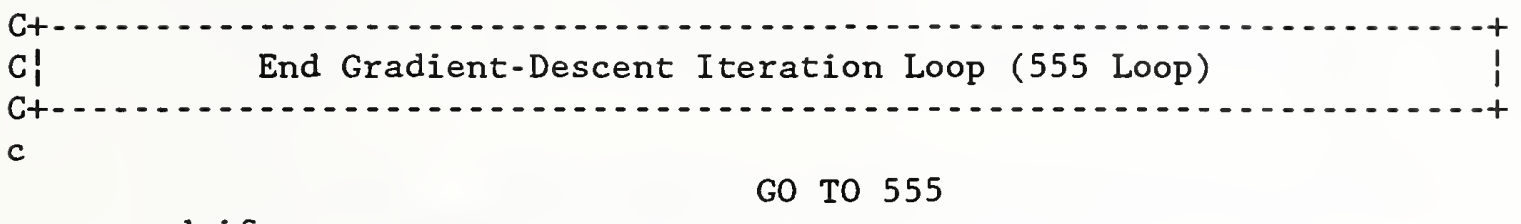

end if

c Exit, but first compute final properties

c

777 call COEFF( nlay(type), angle(1, type), pct $(1$, type), p, q ) write $(6,4001)(\mathrm{p}(\mathrm{i}), \mathrm{i}=1,4)$

write $(6,4002)(\operatorname{obj}(i), i=1,3)$

c

STOP

c

c Formats

c

3999 format (' ', I4, 6(1X,1PE11.4))

4000 format(' Sample: ',A,' (Type ', I1,'):'

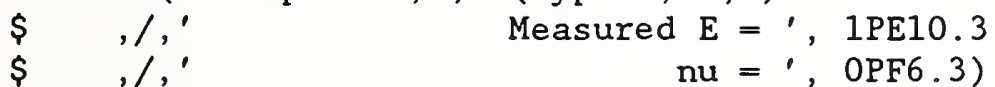

4001 format( ' Best-Fit Properties:'

$\$ \quad, /,^{\prime} \quad \mathrm{EL}=1,1 \mathrm{PE} 10.3$

$\$ \quad, /, '$ ET $=', 1$ PE10.3

$\$ \quad, /,^{\prime}$ GLT $=,, 1$ PE10.3

$\$ \quad, /, ' \quad$ nuLT $=$ ', OPF6.3)

4002 format ('Error-Metric: ', 1PE10.3, ' + ',

$\$$ 1PE10.3, ${ }^{\prime}={ }^{\prime}$,

$\$ \quad 1 P E 10.3$

c

$\left.\$ \quad, /,^{\prime} \quad(\mathrm{E})+(\mathrm{nu})=(\text { total })^{\prime}\right)$

C=END FORTRAN 


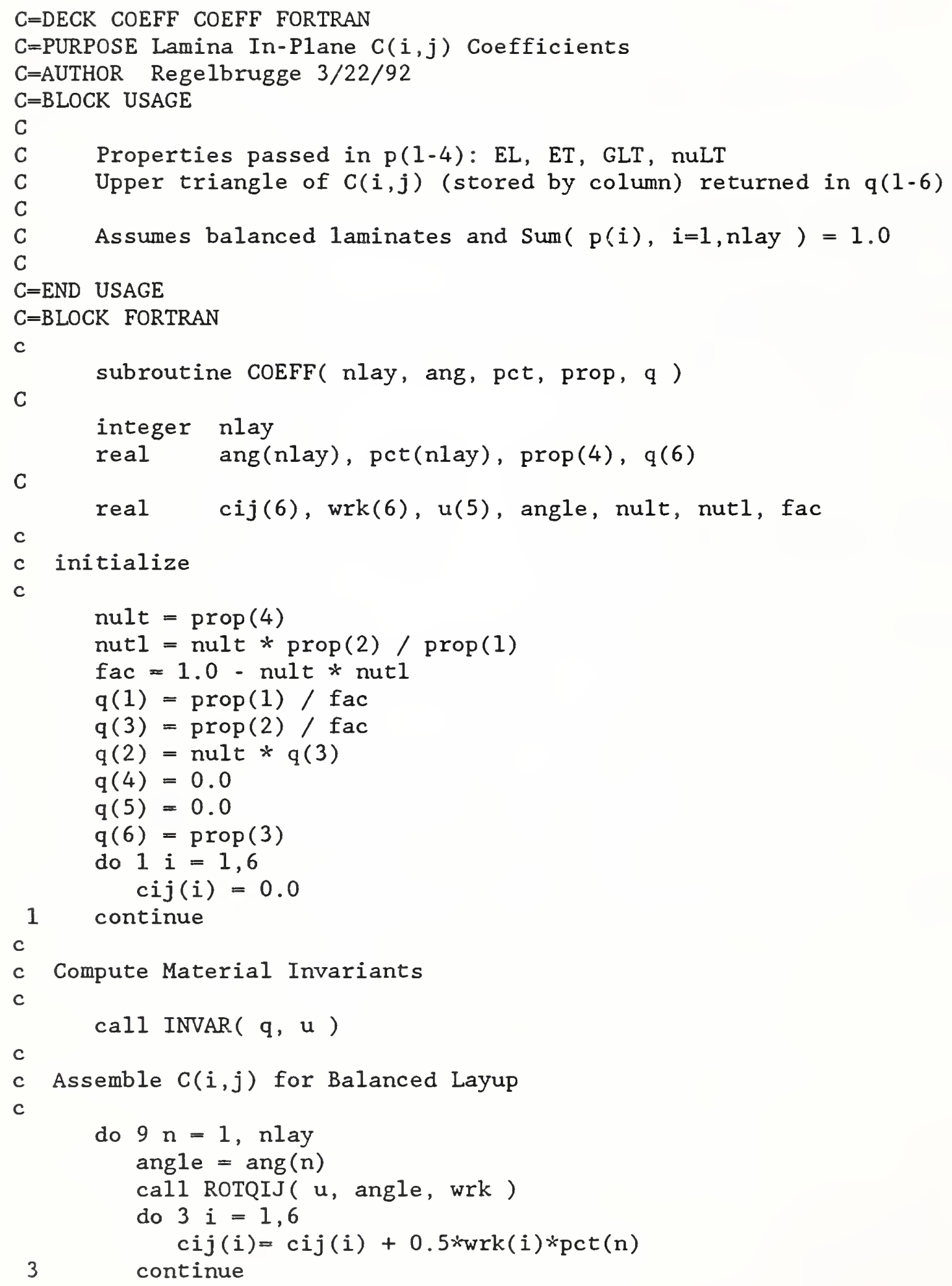


call ROTQIJ ( u, -angle, wrk )

do $4 i=1,6$

$$
\operatorname{cij}(i)=\operatorname{cij}(i)+0.5 * \operatorname{wrk}(i) * \operatorname{pct}(n)
$$

4 continue

9 continue

c

do $10 i=1,6$

$q(i)=\operatorname{cij}(i)$

10 continue

c

return

end

$C=$ END FORTRAN 


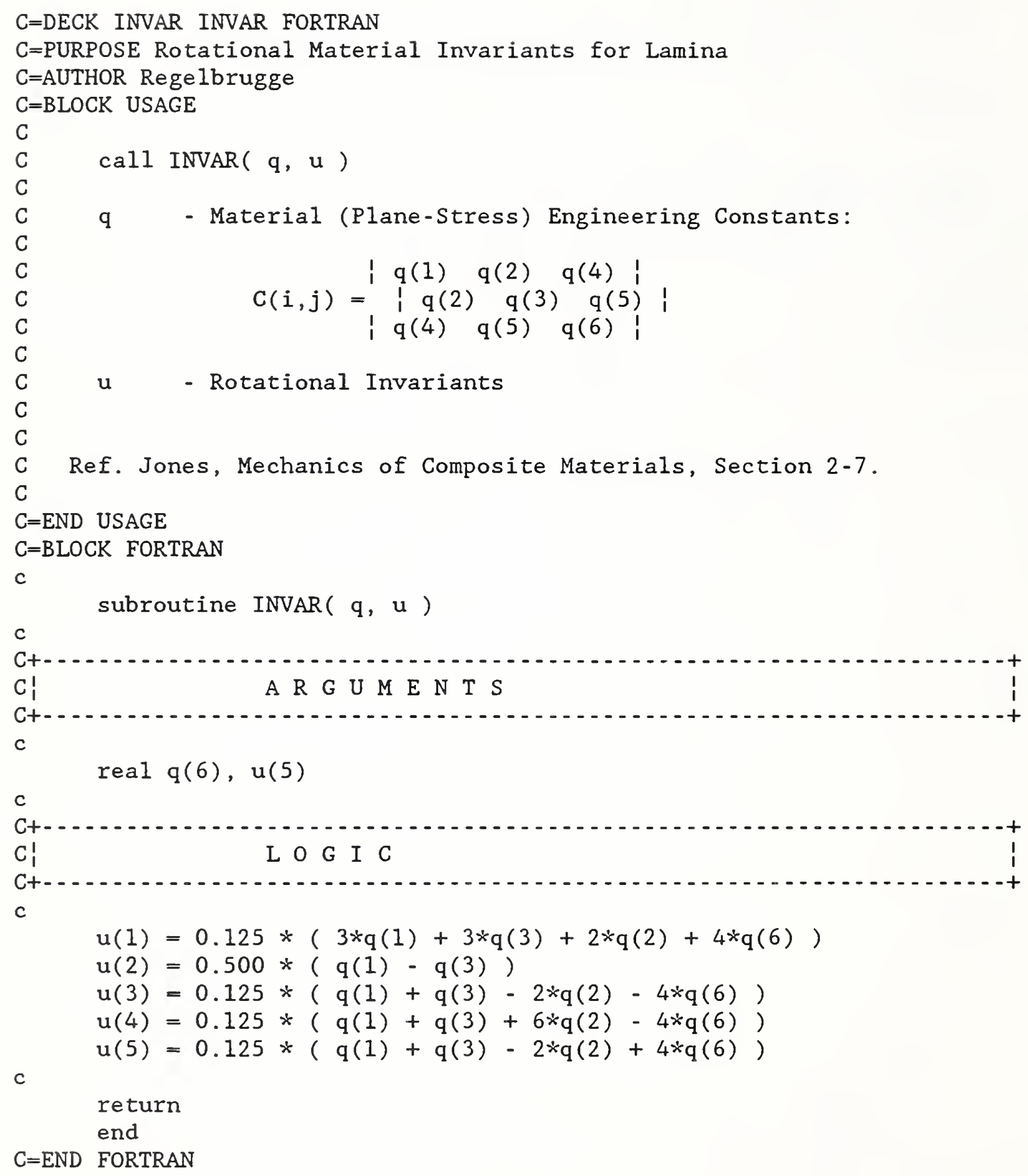




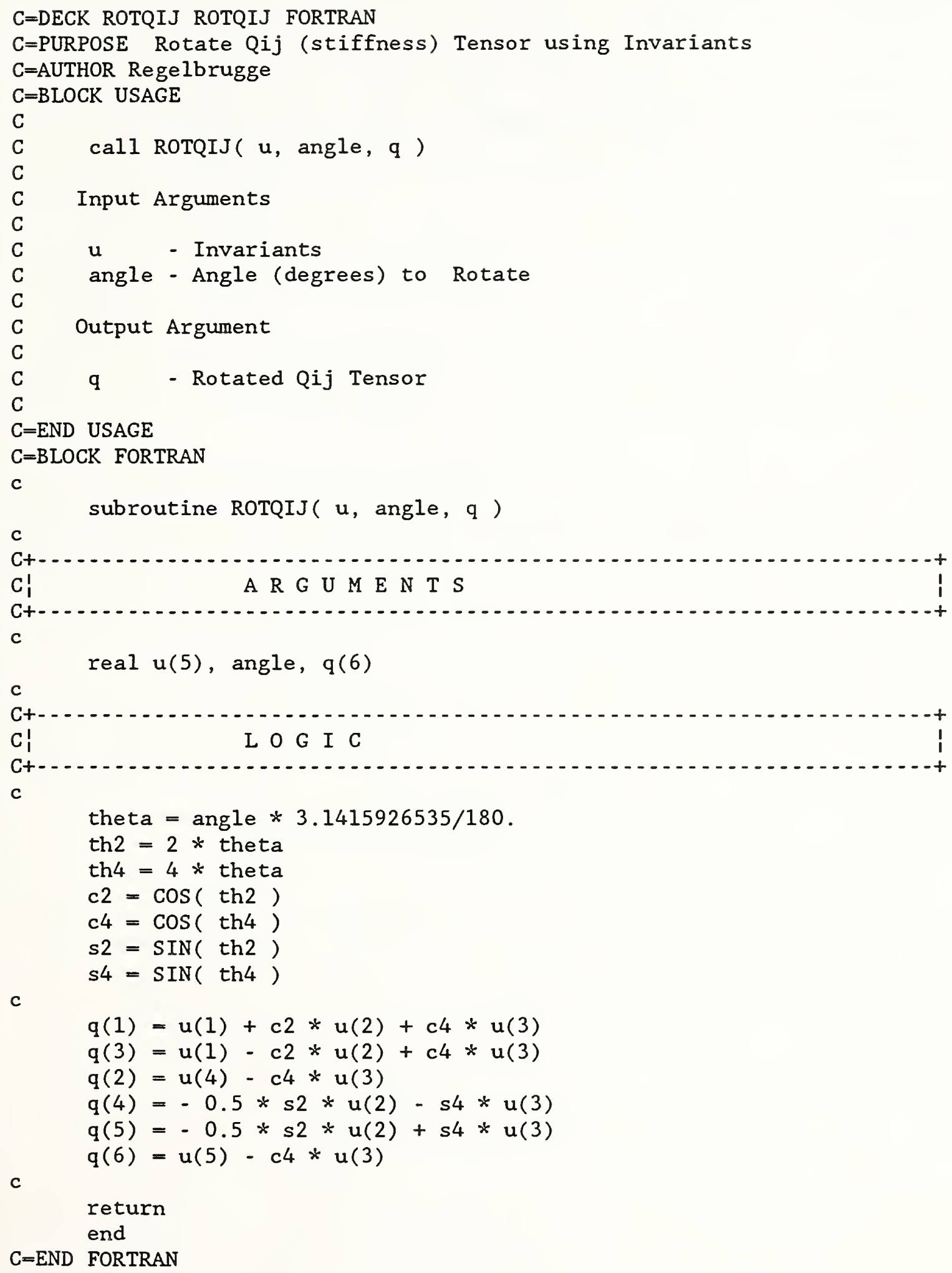

c

$\mathrm{q}(1)=\mathrm{u}(1)+\mathrm{c} 2 * \mathrm{u}(2)+\mathrm{c} 4 * \mathrm{u}(3)$

$\mathrm{q}(3)=u(1)-c 2 * u(2)+c 4 * u(3)$

$\mathrm{q}(2)=\mathrm{u}(4)-\mathrm{c} 4 * \mathrm{u}(3)$

$\mathrm{q}(4)=-0.5 * \mathrm{~s} 2 * \mathrm{u}(2)-s 4 * \mathrm{u}(3)$

$\mathrm{q}(5)=-0.5 * \mathrm{~s} 2 * \mathrm{u}(2)+\mathrm{s} 4 * \mathrm{u}(3)$

$q(6)=u(5)-c 4 * u(3)$

c

return

end

C=END FORTRAN 


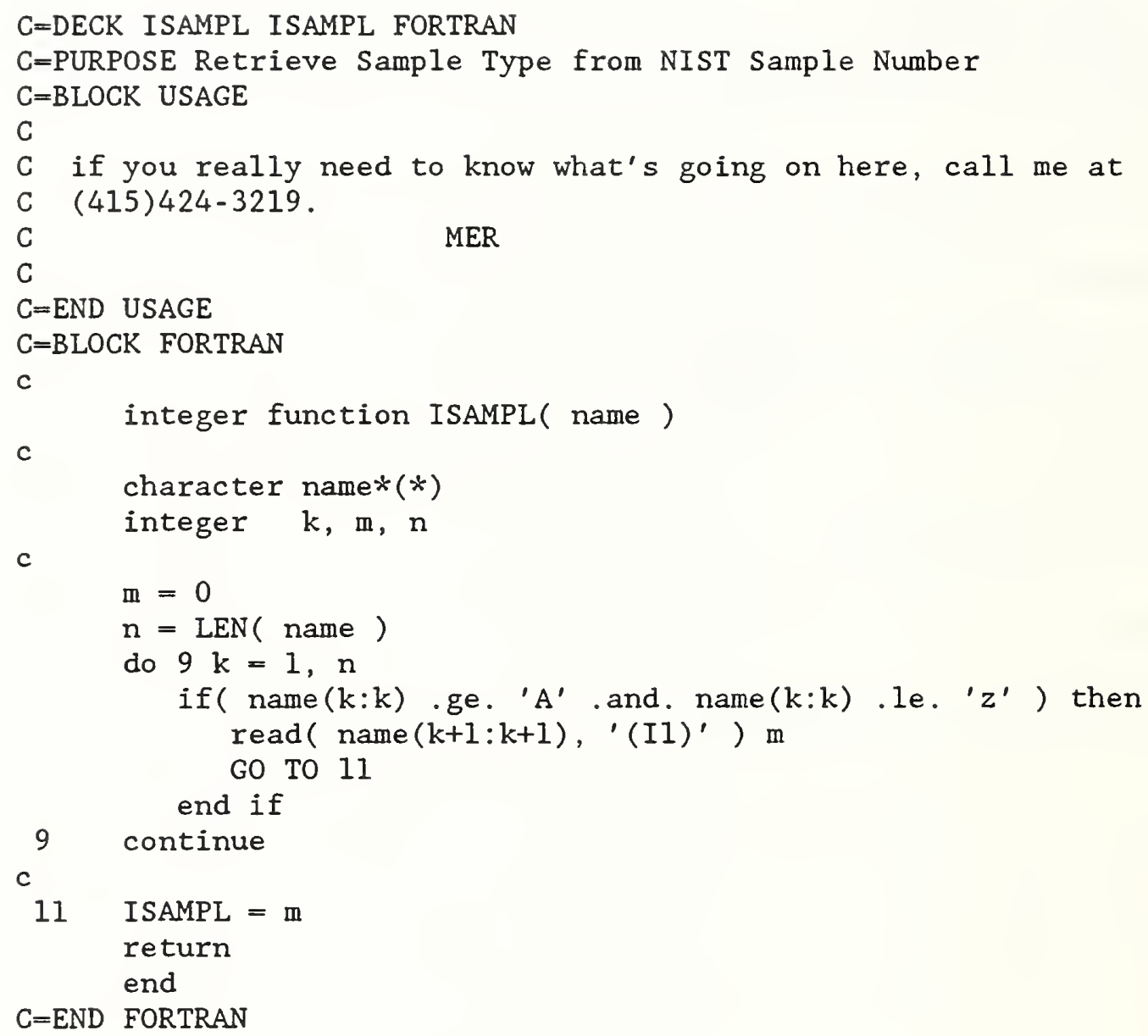


